\title{
Research in the
}

\section{Archiva

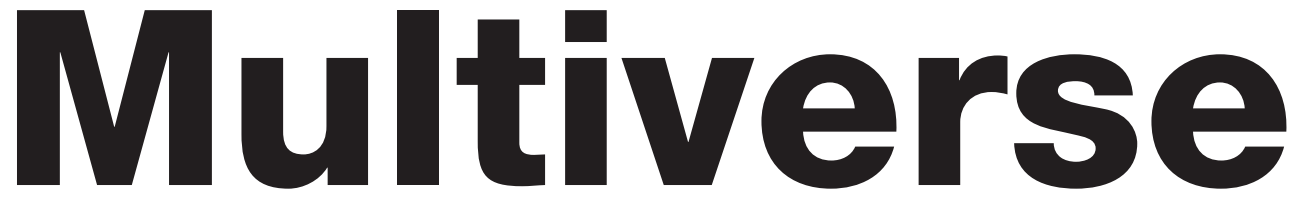

Edited by Anne J Gilliland, Sue McKemmish and Andrew J Lau

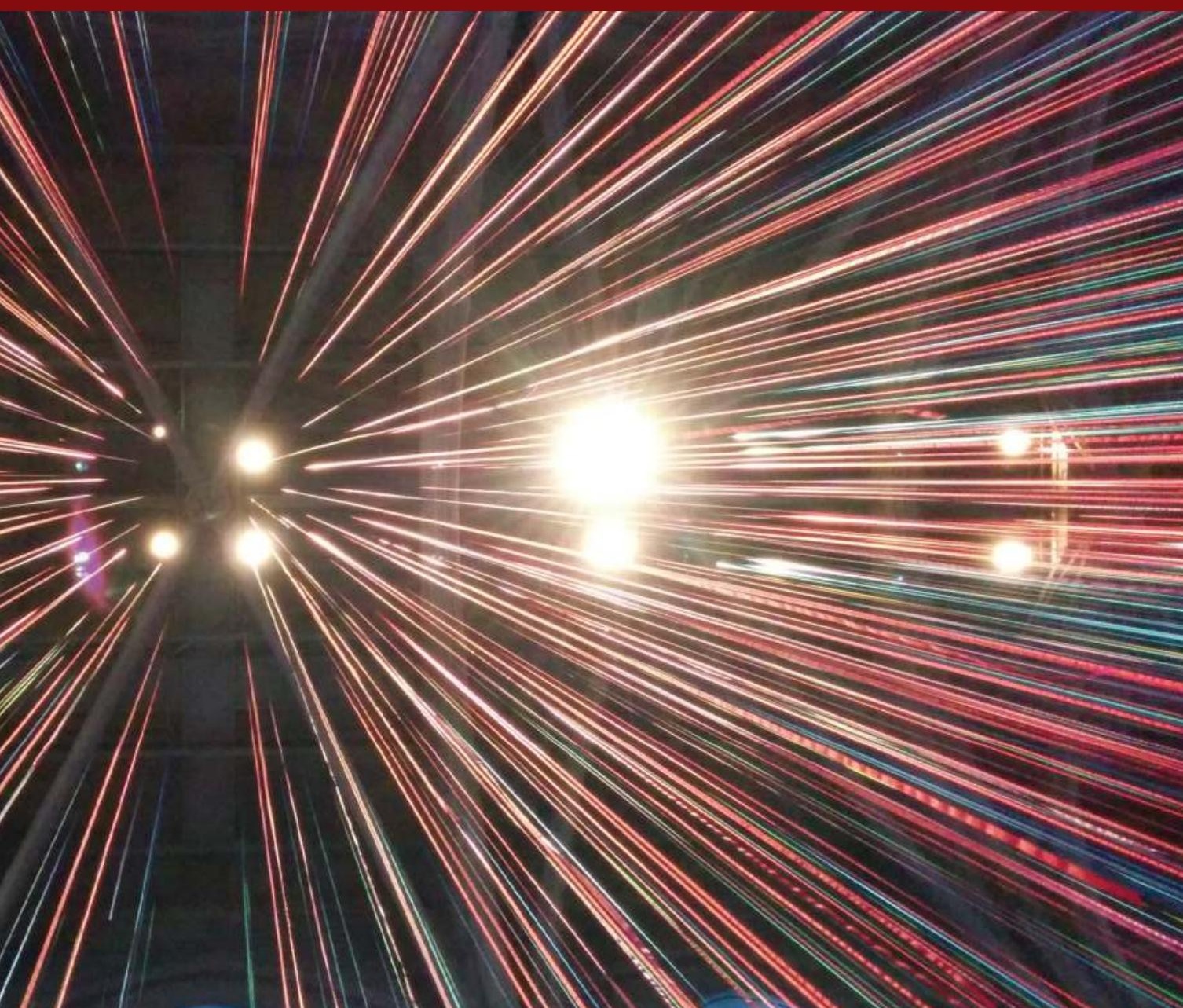




\section{Research in the Archival Multiverse}





\section{Research in the}

\section{Archival Multiverse}

Edited by

Anne J Gilliland, Sue McKemmish and Andrew J Lau

- MONASHUniversity

Publishing 


\section{Research in the Archival Multiverse}

(C) Copyright 2017

(C) Copyright of this collection in its entirety is held by the editors, Anne J. Gilliland, Sue McKemmish and Andrew J Lau.

(C) Copyright of the individual chapters is held by the respective authors.

All rights reserved. Apart from any uses permitted by Australia's Copyright Act 1968, no part of this book may be reproduced by any process without prior written permission from the copyright owners. Inquiries should be directed to the publisher.

Monash University Publishing

Matheson Library and Information Services Building

40 Exhibition Walk

Monash University

Clayton, Victoria 3800, Australia

www.publishing.monash.edu

Monash University Publishing brings to the world publications which advance the best traditions of humane and enlightened thought.

Monash University Publishing titles pass through a rigorous process of independent peer review.

www.publishing.monash.edu/books/ram-9781876924676.html

Series: Social Informatics

Series Editor-in-Chief: Sue McKemmish

Design: Les Thomas

Cover image: (C) Andrew J Lau

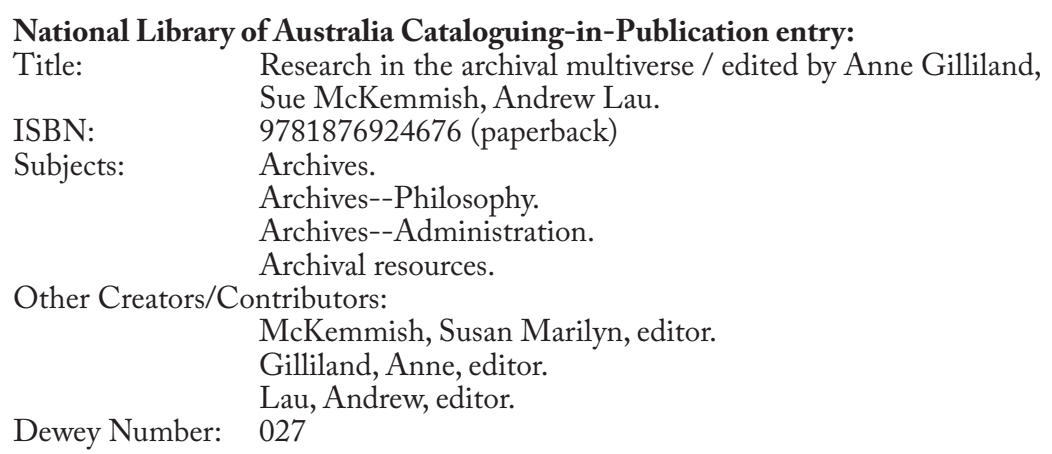




\section{CONTENTS}

Part I: Introduction $\ldots \ldots \ldots \ldots \ldots \ldots \ldots \ldots \ldots \ldots$

Dedication: Archives and Indigenous Communities .......... 2

Shannon Faulkhead and Kirsten Thorpe

Preface...................................... 16

Anne J. Gilliland, Sue McKemmish and Andrew J Lau

Acknowledgements....................... 30

Chapter 1: Archival and Recordkeeping Traditions in the

Multiverse and Their Importance for Researching

Situations and Situating Research ........... 31

Anne J. Gilliland

Part II: Archival Intellectual Context and Theoretical

Frameworks .......................... 74

Chapter 2: The Archival Method................ 75

Luciana Duranti and Giovanni Michetti

Chapter 3: A History of Archival Ideas and Practice in China ... 96 Zhiying Lian

Chapter 4: Recordkeeping in the Continuum ........... 122 Sue McKemmish

Chapter 5: Deciphering and Interpreting an Archival Fonds and Its Parts . . . . . . . . . . . . . 161 Heather MacNeil

Chapter 6: The Archival Multiverse and Eddies in the Spacetime Continuum . . . . . . . . . . . . . . . . . . . . 198 Frank Upward 
Chapter 7: Archival Turns and Returns ............ 228 Eric Ketelaar

Chapter 8: Memory Research / Archival Research . . . . . . . . 269 Jeannette A. Bastian

Chapter 9: When is a Record? . . . . . . . . . . . . . 288 Amelia Acker

Chapter 10: Beyond Pillars of Evidence . . . . . . . . . . . 324 Jamie A. Lee

Chapter 11: Race and Culture . . . . . . . . . . . 352 Kelvin L. White

Chapter 12: Introducing Critical Race Theory to Archival Discourse ....................... 382 Anthony W. Dunbar

Chapter 13: Without a Trace ....................411 Anjali Arondekar

\section{Part III: Overviews of the Application of Research} Methods in Archival Studies . . . . . . . . . . . . . 432

Chapter 14: Historical Case Studies of Pre-Modern European Archives .......................... 433 Randolph C. Head

Chapter 15: Respect My Authority . . . . . . . . . . . 456 Wendy Duff and Kate Cumming

Chapter 16: Negotiated Methodologies . . . . . . . . . . . . . . 479 Shannon Faulkhead 
Chapter 17: Grounded Theory .................. 516 Jenny Bunn

Chapter 18: Looking at Archival Concepts and Practice in the Light of Speech Act Theory........... 537 Pekka Henttonen

Chapter 19: Archival Mediation. ................. 558 Anneli Sundqvist

Chapter 20: Archival IR...................... 581 Jonathan Furner and Anne J. Gilliland

Chapter 21: The Use of Models and Modelling in Recordkeeping Research and Development. . . . . . . . . . . 632 Hans Hofman

Chapter 22: Capacities and Complexities.............. 659 Joanne Evans

Chapter 23: Designing Expert Systems for Archival Evaluation and Processing of Computer-Mediated Communications... 686 Anne J. Gilliland

Part IV: Research Case Studies $\ldots \ldots \ldots \ldots \ldots \ldots \ldots 723$

Chapter 24: Contemporary Archival Diplomatics as a Method of Inquiry .................... 724 Heather MacNeil

Chapter 25: Exploring Social Complexity ............ 762 Leisa Gibbons 
Chapter 26: Investigating Socio-cultural Aspects of the

Implementation of an International Archival

Descriptive Standard in Korea ................ 789

Eunha (Anna) Youn

Chapter 27: Bibliometric Analysis as a Tool in Understanding the Development of Archival Thought . . . . . . . . . 811 Kimberly Anderson

Chapter 28: Public Digital Records Management in the Ugandan

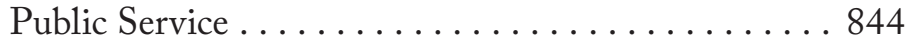

David Luyombya

Chapter 29: Documenting Communities of Practice......... 868 Karen F. Gracy

Chapter 30: Aboriginal Community Archives............. 900 Kirsten Thorpe

Chapter 31: Examinations of Injustice . . . . . . . . . . 935 Lorraine Dong, Joel A. Blanco-Rivera, Michelle Caswell and Joanna Steele

Chapter 32: Examining Activism in Practice............ 968 Joy R. Novak

Chapter 33: Using Grounded Theory to Understand the Archival Needs of Geologists ..................... 998 Sarah Ramdeen and Alex H. Poole

Chapter 34: Applying QMethod to Archival Research. . . . . . . 1028 Amber L. Cushing

Part V: Biographical Notes on Contributors. . . . . . . . 1053 


\section{Part I}

\section{Introduction}




\section{DEDICATION: \\ ARCHIVES AND INDIGENOUS \\ COMMUNITIES}

\section{Our Knowing Allison Boucher Krebs \\ (September 8, 1951 - January 26, 2013)}

Shannon Faulkhead and Kirsten Thorpe

Allison B. Krebs (1956-2013) was a member of the Sault Ste. Marie Tribe of Chipperva Indians, a Ph.D. student at the Information School at the University of Washington, and member of the Indigenous Information Research Group at the University of Washington, Chair of the Native American Archives Roundtable of the Society of American Archivists, and served on the Steering and Strategic Planning Committees of the Association of Tribal Archives, Libraries and Museums (ATALM). Allison was a visiting scholar at Monash University in Australia, a Documenting Endangered Languages Fellow of the National Science Foundation, and earned an MLS as a Knowledge River Scholar at the University of Arizona. She also graduated in the first class of women from Yale College. Her research centred on Indigenous knowledge ecology. ${ }^{1}$

Every time an old man dies in Africa, it is as if a library has burnt down (En Afrique, quand un vieillard meurt, c'est une bibliothèque qui brûle). ${ }^{2}$

A library was burnt down. On the 26 January 2013 we lost our dear friend, colleague and sister Ally.

The incongruity of Ally leaving this world on Australia Day is not lost to us. This is a day of controversy and contradictions within Australia. On

1 This biography is how Ally described herself. Ally Krebs, "Native America's Twentyfirst-century Right to Know," Archival Science, 12 no.2 (June 2012): 186.

2 A. Hampâté Bâ, "Amadou Hampâté Bâ," African Studies Centre, http://www.ascleiden. $\mathrm{nl} /$ ?q=content/webdossiers/amadou-hamp\%C3\%A2t\%C3\%A9-b\%C3\%A2] 
26 January 1788 the first fleet from Great Britain arriving on the shores of the Eora people at Sydney Cove heralded Australia as a colony of Great Britain. This beginning of Australia was also the beginning of the dislocation, massacres, policing, and decimation of the Indigenous peoples of Australia. The 1888 centenary Australia Day celebrations saw Indigenous leaders protest by boycotting the celebrations, the 1938 Australia Day was declared a Day of Mourning, and by the 1988 bicentenary, Australia Day was widely seen as Invasion Day with protests across Australia. Then 1992 saw Survival Day concerts become a part of Australia Day celebrations to celebrate our Ancestors and peoples' achievements and survival. For many therefore, 26 January is a day of mixed emotions - sadness and pride, pain and joy, confusion and clarity, mourning and celebration.

The news of Ally's passing became tangled in with the emotions already being felt by us as Indigenous Australians. Whilst much of Australia did not know Ally, we did and the sadness and pain we felt can never be expressed. We had been in the process of collaborating on a chapter for this book titled 'Archival Research and Indigenous Communities: Searching for the Root of Indigenous Research Frameworks'. The abstract of that unfinished article framed its contents:

Indigenous peoples' views, definitions and understandings of 'archive' are often different and more diverse than those emanating from traditionally based Western archival science. This difference has shown to provide significant contributions to the field's literature and research; often these contributions are in response to improving Indigenous peoples' interactions with archives. Despite these contributions, there is a dismissiveness of Indigenous research and engagement due to it being 'difficult', 'problematic' and 'complex'. This chapter will endeavour to challenge this assumption by providing: examples of Indigenous archival research that is collaborative, reflective and outcome based; new insights into how Indigenous research ethics can benefit archival science; and through the discussion we will introduce key concepts and terms that have guided positive and transformative research in archives and recordkeeping with Indigenous people, such as consent, relationships, trust, participation, co-creator, co-ownership, and reciprocity.

An outsider looking at the abstract might think this was going to be a theoretically dense chapter, but to be truthful we chose this topic - about which we are all passionate - to have some fun. Too often in our writings we 
spend our time - and much of our word limit - explaining and redefining common concepts from our worldview. We saw this as an opportunity to write for fun, and fun we had. Writing, but not as much as we should have. With Ally in the USA 'somewhere' (that woman was always on the go), Kirsten in Sydney, and Shannon in Melbourne, our Skype calls were often loud and joyous. Kirsten's mother Robin always knew the days when we would have the Skype calls with Ally, as the house filled with engaged conversations and lots of laughter. Rarely did the Skype conversations progress the chapter, although there were many vague promises to do something before our next meeting. What we did achieve was so much more. We provided advice to each other. We shared news. We solved problems personal and professional. We shared the silly and the offensive. We lived each other's hurts and joys. We cried. But we laughed so much more. Ally's laugh ... how could you not miss that laugh? Ally's passion outweighed her laugh though.

Whilst each of us met Ally separately, we established a quick and strong bond as we shared the same passion about Indigenous engagement with archives and Indigenous knowledge held in archival repositories. We had a deep sense of respect and trust and mutual understanding. This passion was not just about practicalities either. Many of the conversations we had with Ally concerned the spiritual and emotional connections between Indigenous communities and records - these connections underpinned everything and had to be at the forefront of everything we did. Ally's other archival passion was for the importance of archival collections being created and managed with respect to Indigenous protocols. Ally was part of the First Archivist Circle that, in 2006, issued the groundbreaking Protocols for Native American Archival Materials. ${ }^{3}$ The Protocols articulate guidelines for the culturally responsive care of American Indian archival holdings in nontribal repositories. A major aim of the Protocols is to stimulate a discussion amongst archivists, librarians and Native Americans on professional policy and practice that respect and acknowledge Indigenous rights and beliefs regarding those holdings. They help archivists to understand the living connection between people and records, and the obligations that need to be met to manage collections in ways that respect connection to place and community. When discussing the concept of "yarning in the archive" (discussed further below), Ally suggested: 
Perhaps this is our title as well as the concept of an Indigenous archive, a sense of how an archive needs to be - that an archive needs to be a yarning, a conversation, with all the tacit protocols involved in a conversation between people, the respect in engagement that allows a conversation to continue over time, to be returned to, to grow and deepen, within a shared creative space. Yarning implicitly acknowledges the various contributors, embraces their contributions. It is by nature co-creative.

Ally would speak of Indigenous engagement with archives with such vibrancy, discussing the need for archives to be illuminated and animated, whilst keeping a deep focus on the spiritual connections that need to be respected. She was also generous and respectful in her belief that many of the past injustices, and current inconsiderate practices within archives, with respect to Indigenous peoples can be reconciled with better acceptance of cultural understandings different to our own - always mixed in with a good dose of humour. An example of this humour was evidenced at the beginning of our unfinished chapter:

'We suspect that it will be a much larger step than you believe.'

Although this quote from Langford is referring to the next step in knowledge sharing and respect in archaeology, as Indigenous peoples we often feel that when non-Indigenous people are attempting to understand us, our ways, and how we operate, they move into that space with detached trepidation and (at times) resentment that their worldview is about to be turned on its head. That is not our intention with this chapter, but just in case we feel a disclaimer is necessary:

Warning: Reading this chapter may be dangerous for your archival practitioner worldview.

Ally was forever citing The United Nations Declaration on the Rights of Indigenous Peoples 2007 and its associated mandates as providing important mechanisms for Indigenous peoples to assert and explore rights to the management of their heritage. It was rare that she did not 'mention' in conversation - if not pull out and hand around - the Australian Human Rights Commission's Community Guide to the UN Declaration on the Rights of Indigenous Peoples as something that we as archivists should be using as

$4 \quad$ R. Langford, “Our Heritage - Your Playground,” Australian Archaeology, 16 (1983): 6. 
a guide to our practice. Best practice guidelines for ethical research with Indigenous communities also provide frameworks for engagement with Indigenous peoples and communities and assist Indigenous people in becoming active agents in matters relating to the management of their history and heritage. These mandates are important both in relation to the management of existing collections and of any archives created in the future. Indigenous people and communities are seeking greater input into research that is informed by them, and as a consequence, are actively involved in decisions about the management of records and data created during the research process. Within these contexts, Ally was a passionate advocate of the potential for archives to be created and managed with Indigenous people as key players in the archival process, not merely as subjects or 'captives of the archives. ${ }^{5}$

In a special issue of the journal Archival Science, "Keeping Cultures Alive: Archives and Indigenous Human Rights", Ally explored recent initiatives in the archives, library and museum fields. In her paper, "Native America's $21^{\text {st }}$ century right to know", she reflected on the three decades that have passed since Native American studies and rights activist Vine Deloria Jr. first argued that Native Americans had "The Right to Know" about their past(s) and relevant materials held in libraries, archives and museums. Asserting that these rights were enshrined within the United States Federal Government's treaty responsibilities, Deloria called for the establishment and funding of tribal libraries and archival services. Ally extensively researched two initiatives, funded - at least partially - by the US Federal Government. The first was the Institute of Museum and Library Service (IMLS) grants to Indian tribes scheme, and the second, the Fourth Museum, the off-site outreach component of the National Museum of the American Indian in Washington, D.C. In line with her vision for the future, Ally placed these initiatives within a broader Indigenous knowledge ecology. She wrote passionately of the rights of Indigenous people to access and re-engage with historical materials held in archives. She also wrote poetically about the importance of these reconnections for communities and for reviving Indigenous knowledge systems that had been subjugated through cultural genocide:

In fact, though, these Indigenous knowledge systems are infinitely patient, assuming a posture of dormancy, waiting for the proper

5 Henrietta Fourmile, "Who Owns the Past?: Aborigines as Captives of the Archives," Aboriginal History 13 (1989): 1-8. 


\section{Dedication}

combination and events and agents to align to catalyse a dramatic and seemingly inexplicable change. They are ticking away within a half-life beyond the perception of those who deny their very existence. Their proximity infects those living around them as they restlessly animate their destiny with unperceived dimensions. ${ }^{6}$

There is a deep sense of obligation that Indigenous people feel towards managing archives in appropriate ways that encompasses spiritual, emotional and physical connections to records. There is a network of rights and responsibilities that exist within communities that are usually articulated in oral form:

A responsibility and belief that seems to be shared by all Indigenous nations of Australia is the connection between the land and the people. This is both a physical and an emotional connection that relates to specific areas of land with stories and oral knowledge dating back thousands of years telling us of the responsibility of belonging to the land. These stories explain land boundaries and methods of caring for people and land, for flora and fauna, and for places of significance, such as cemeteries. The responsibility also includes caring for the oral stories necessary for land care and ownership. ${ }^{7}$

These obligations and responsibilities to the care of records extend beyond the physical manifestation of the archive. They include obligations to respect and honour ancestors.

The topic of spiritual care of records is one that we discussed frequently. It was akin to debriefing about the messages that we receive, especially when something is not quite right about a collection or record, or reminders when we are on track about what our work is really about (being caretakers). Some of these messages and reminders teach us that ancestors are still connected even though they are not physically here with us. During these discussions, we also discussed the metaphysical presence of multiple dimensions of time and how on earth this could be explained on paper. We did not solve this in the time we had together.

Indigenous peoples have both rights to as well as responsibilities for Indigenous knowledge in all its manifestations. We have a seven

6 Krebs, "Native America's Twenty-first-century Right to Know," 186

7 Shannon Faulkhead and Jim Berg. Power and the Passion: Our Ancestors Return Hom, Melbourne, Victoria: Koorie Heritage Trust Inc., 2010, p. xvi. 
generational perspective. We have seemingly infinite patience and perseverance that trusts in the animation of our knowledge systems for the sustenance of our peoples. We think strategically, taking seriously the responsibility for connecting the hearts and the minds of all our relations. We perceive the fragmented captives contained in collections, in archives, in recordings, and we will their return, countering willful cultural genocide with willful cultural sovereignty. We recognize that we each have gifts to engage. And we trust that collectively we can catalyze changes of form that others cannot even imagine, let alone will into being, for the well-being of all. ${ }^{8}$

Ally looked to a future in which community driven, controlled and supported libraries, archives and museums will be reciprocally altered and reformed in meaningful and profound ways, eloquently arguing that it is for these Indigenous museums, libraries and archives to set the agenda:

It is these Indigenous institutions, their founders, administrators, staff and communities that will be writing the next chapters of policies and procedures sourced from deep within the multi-verse of Indian Country. It is these institutions and their founding communities that live their protocols. It is these institutions that will struggle with integrity to align their policies and procedures with their ways of knowing. It is here, at the grassroots of Indian Country that the face of the information fields of libraries, archives and museums will be altered, shape shifting in meaningful ways in service of peoplehood, in service of a relational accountability built from ground up in a new sprouting of trust. ${ }^{9}$

Ally concluded her paper in the special issue of Archival Science on Archives and Indigenous Human Rights with a series of challenging questions:

The question then, for those of us who care, is: how do we support the work that needs to be done? How do we create space for conversations to take place, for dialogue to develop, for trust to grow? How do we learn to listen respectfully and reflect? How do we manage to keep from feeling threatened when some of the basic values that we hold self-evident are fundamentally challenged? 


\section{Dedication}

What will it take to transform the information practices, policies and procedures around Indigenous information and knowledge held outside Indigenous communities? ${ }^{10}$

In proposing a "short answer" to these questions, Ally wrote that it would "take time, respect, and patience from both sides of the divide", and "open hearts and minds ready to search for win/win solutions located potentially outside the comfort zones of existing practice", as well as "conversation, lots of it". 11

Another question that we often engaged with was one that was posed in the Archival Education and Research Initiative (AERI)'s Pluralizing the Archival Curriculum Group's influential article, "Educating for the Archival Multiverse" (of which Ally and Shannon were among the co-authors):

How do we move from an archival universe dominated by one cultural paradigm to an archival multiverse; from a world constructed in terms of "the one" and "the other" to a world of multiple ways of knowing and practicing, of multiple narratives co-existing in one space? An important related question is How do we accept that there may be incommensurable ontologies and epistemologies between communities that surface in differing cultural expressions and notions of cultural property and find ways to accept and work within that reality? ${ }^{12}$

Being from different countries we often found similarities and differences between our homes. One of the similarities that we found was the worldview that everything is interconnected. While many peoples have the same or similar view, many of the systems adopted by Western knowledge systems are aimed at keeping everything neat and tidy. There is a failure to recognise that it is often in the messiness that amazing things are to be found - a bit like finding 'just the right thing' in an opportunity shop way down the bottom of a throw-out pile:

We do not deny that community research partnerships are messy, complex and time consuming, but this experience can also make the research fun and beneficial on so many levels. One of the reasons for the perception of messiness is because there is not and cannot be a step-

10 Krebs, "Native America's Twenty-first-century Right to Know," 189.

11 Krebs, "Native America's Twenty-first-century Right to Know," 189.

12 The Archival Education and Research Institute (AERI) Pluralizing the Archival Curriculum Group (PACG), "Educating for the Archival Multiverse," The American Archivist 74 (Spring/Summer 2011): 73. 
by-step guidebook to community partnerships. Each one is different because each partnership is different. For research and community partnerships to succeed, confusion needs to be reflected on, and worked through, and the messiness understood. ${ }^{13}$

Earlier we mentioned 'yarning':

Academic conversation has barely begun to discuss formally the utilization of yarning as a feasible method of conducting research among Aboriginal Peoples across Australia. However, Indigenous communities have utilised yarning as a workable method to share, explore and learn for many previous generations and will continue to do so. ${ }^{14}$

So often this term is viewed derogatorily as in 'telling a yarn' or fib. Yet for the many cultures, communities and families who engage in orality as their primary source of knowledge sharing, it is a vital way of life. Conversation, yarning and reflection about our work and our research has protected our sanity and provided an outlet for thoughts and ideas that were yet unformed or that we believe to be madness.

Come into my kitchen

And rest your feet and weary mind

You can settle and I will listen

You feel the pain and all the suffering

You carry the load, for what? For nothing

Get out of your head and into this world

You can't solve the problems, no, no

At least not on your own

Not on your own ${ }^{15}$

For us it became an ongoing discussion, reflecting on our engagement with the archival profession and archival discourse. Connecting with other Indigenous people working in the field of archives and libraries is often a source of nourishment for us. Being able to share experiences and perspectives,

13 Karen Adams and Shannon Faulkhead, "This is Not a Guide to Indigenous Research Partnerships: But it Could Help," Information, Communication E Society 15 no.7 (2012): 1017.

14 C. Dean, "A Yarning Place in Narrative Histories," History of Education Review, 39 no.2, (2010): 6.

15 Tiddas, "Inside My Kitchen," Inside My Kitchen - EP, Universal Music Australia Pty Ltd., 1992. 


\section{Dedication}

lessons learnt about projects and ways that we can build sustainable research and programs.

Yarning is a recognised narrative research method that involves selfreflection and deep discussion about a particular issue. For this paper we specifically utilised collaborative yarning. Collaborative yarning in research involves exploring similar or different ideas in explaining concepts and this can lead to new information and understandings. ${ }^{16}$

In a research setting, yarning is a way of making deep connections, establishing relationships about 'who' you are and where you are from, and sharing ideas about the future in a culturally safe way:

Yarning is conducive to an Indigenous way of doing things; its strength is in the cultural security that it creates for Indigenous people participating in research. Yarning is a process that cuts across the formality of identity as a researcher and demands the human to human interaction where both are knowers and learners in the process. ${ }^{17}$

As a research method, yarning provides a space for knowledge sharing and a relaxed forum for discussing deep issues of common interest to a group.

The adoption and engagement of the "archival multiverse" as a concept is one that the three of us saw as a positive step forward. It felt as if for the first time the onus was removed from us to explain our worldview, and that it gave permission to everyone to explore his or her own. They were freed to explore the paradigms that have directed and/or restricted their archival perceptions. The archival multiverse not only enlarges the archival perception, it also allows multiple views of purpose, code and existence. Thus it allows for multiple views of archiving and is inclusive of various minority or underrepresented groups. There is another perspective of it that benefits our communities, however. It supports individual groups in achieving their particular needs - needs with which other groups may not be concerned, but which, in this inclusive world, are supported and recognised by all. At the same time, however, archival protocols created through consultative processes among groups or communities and that are culturally safe and respectful must also be practical and implementable. So we need to identify how best to achieve this.

16 Adams and Faulkhead, "This is not a Guide," 1019.

17 D. Bessarab and B. Ng'andu, "Yarning as a Legitimate Method in Indigenous Research," International Journal of Critical Indigenous Studies, 3 no.1 (2010): 47. 
We aspire to an archival profession that addresses Indigenous archival concerns as a fundamental right and as more than "special needs" - respecting them as asserting a valid set of rights and approaches for the management of material in culturally-sensitive ways. We acknowledge that Indigenous archival needs do not fit into the box of the dominant worldview of the archival field. We know that communities do things differently, and expect that things should be done differently. Often the concerns of Indigenous and other marginalised communities do not fit with how things are taught in the professional curricula. They are also very rarely showcased in research that comes out of the archival world, something that this book has sought to address.

In this multiverse, it is important that we, as Indigenous people, connect too. That we create opportunities to share and exchange information and build trusted relationships. We need to do this in ways that suit our communication styles - through conversation and discussion, agreement and disagreement, listening and understanding. Our work is often not centred in the academic value of a conference presentation or publication - considered to be a one-way production of 'knowledge'- but in the heartfelt value of our work to community, to the continuing thriving of community. In the US, Vizenor calls this "survivance," while Holm calls it peoplehood. ${ }^{18}$ Ally called it resilience and persistence. And even when we are engaged in those formats of communication or performance, our real conversations are taking place in private moments on the periphery of the conference spaces or through implications that can be read by the attuned between the lines of the journal article (this chapter being one of the exceptions.) Recognising these forms of literary warrant is critical, because in the western world, when something is not written, if it is only discussed in the hallways or exists as scribbled as notes in the margin, then it is treated as invisible by academia, and silent by those staunchly traditional archivists who need to hear it the most. But how can they be helped to hear it?

We aspire to create a safe space for our collective knowledge generation. As Ally observed when corresponding with us:

There is something here about tacit knowledge of what is safe and unsafe, tacit understanding of the deeper operation of protocols. This was something I was deeply aware of coming to visit in your part of

18 T. Holm, J.D. Pearson and B. Chavis, "Peoplehood: A Model for the Extension of Sovereignty in American Indian Studies," Wicazo Sa Review, 18 no.1 (June 6, 2003): $7-24$. 


\section{Dedication}

the world. Although I was invited as an Indigenous person, even more frightening as a sort of Indigenous spokesperson, I was also painfully aware of how little I knew of the protocols appropriate to your part of the world. It was like operating within invisible force fields, a little like walking on egg shells, always afraid to step too hard unintentionally. The best way I can describe this is a tacit knowledge ... that I did not have the appropriate tacit knowledge, that I could easily turn into a bull in a china shop unintentionally shattering all the unspoken agreements that I hold most important.

Perhaps this is why many of our deepest conversations take place on the margins, on the periphery, of all the other bulls thrashing around knocking things over right and left and then stomping on them as they race off in some other direction, not even aware of the damage they have done. ${ }^{19}$

We will miss her continuing engagement in that conversation, but Ally's words will continue to inspire Indigenous communities, archival institutions, the archival profession, and archival scholarship to act, and to be responsive to Indigenous requests for the appropriate management of their history and heritage. It was Ally who first brought the concept of the multiverse and its possibilities for archival thought, practice and education to the others working with her in the Pluralizing the Archival Curriculum Group. She shared her deep understanding of the emotional and spiritual dimensions of archiving. Modern Western archival theory and practice has privileged and celebrated only the physical and intellectual dimensions of recordkeeping. Ally inspires archivists and archival researchers to explore all four dimensions present in all forms of recordkeeping and archiving in the archival multiverse, and to contribute to an enriched and more inclusive recordkeeping and archival theory and practice.

We knew the woman, but we never heard her whole story. Ally's passing caused the most devastating emotion. We knew her. We knew who she was. Where she came from. Where she hoped to go. So while our time together was always full, it was not long enough.

19 Personal correspondence from Ally Krebs. 


\section{Introducing Ourselves}

With Ally we were three Indigenous women. Two from Australia, and one from the United States of America, all archivists and all passionate about improving archival science so that our communities no longer face the dilemmas and heartache of the past and present. For the past four to six years our paths have crisscrossed to the point where we could no longer ignore one another (even if we wanted to) and developed a friendship, a familyship, and decided that we needed to work together to share and educate.

\section{Shannon Faulkhead}

I am Shannon Faulkhead. I am a Koorie ${ }^{20}$ woman from Mildura in the north-west corner of the state of Victoria in Australia. According to my family and community I am of Indigenous Australian, English, German, Spanish, and African descent. I am proud of where I come from - however as I was born and continue to live on the continent to which the Indigenous Australians belong, I have chosen to identify with my Indigenous Australian ancestry and be an active member of the Koorie community. Much of my working life has involved working with and within the Victorian Koorie community. Through this and being a part of the Victorian Koorie community I observed three understandings at work. The first is the destructive power and continuing negative impact that colonial-invasion narratives about Indigenous Australians have had upon the Indigenous and Victorian communities. The second is the positive power that narratives have in combating negative stereotypes and in creating community pride. And thirdly there are certain narratives that require a shared space that acknowledge both Indigenous and non-Indigenous knowledge to enable them to present a comprehensive and holistic cultural heritage in perpetuity. Many of these understandings have stemmed from or resulted in archives, and how archives should be defined. This has meant that much of academic existence has centred in and around Indigenous peoples and archives.

20 Koorie is a term of self-identification used by some Indigenous Australian people from Victoria and southern parts of New South Wales, meaning 'our people', 'man' or 'person'. Although using this term myself, I recognise and respect that this is not a blanket term adopted by all Indigenous Australian peoples from this region. Many prefer to use their own clan, nation, or state title, or the generic terms 'Indigenous Australian' or 'Australian Aboriginal and Torres Strait Islander'. 


\section{Kirsten Thorpe}

I am Kirsten Thorpe. I am a descendant, on my mother's side, of the Worimi people of Port Stephens, New South Wales. I came to the field of archives through serendipity after my mother Robin was reading the Aboriginal newspaper The Koori Mail and noticed an advertisement for a Cadet Aboriginal Archivist. In 1999 I undertook a year of training at State Records NSW, and completed Post Graduate Studies in Archives and Records. My interest in archives was in some ways to do with a sense of frustration - knowing that an important collection existed behind the walls of the NSW State Archives - but that Aboriginal people and communities did not know that the records existed. Throughout my formal archival studies, I always found myself questioning archival studies literature and its reliance on Western concepts of records and archives. I was challenged about the questions of ownership of the records, notions of "truth" embedded in material recorded in official records, and the positionality of people documented in archival collections.

One of my passions in working with Aboriginal communities and archives has been around the concept of "Taking Knowledge Back Home." I have been driven by an urge to connect archives with communities so that records can be revitalised and re-contextualised. The concept of breathing life back into records resonates deeply with me. However, traditional archival practice falls short in being able to meet the needs of communities who are wishing to have deep engagement with archival records. I firmly believe the profession needs to engage in meaningful and collaborative research to change practices so that they can be reflective of a diversity of needs. 


\title{
PREFACE
}

\author{
Anne J. Gilliland, Sue McKemmish and Andrew J Lau
}

\section{Genesis and Scope of this Book}

When Anne Gilliland and Sue McKemmish edited the special 2004 double issue of Archival Science on archival research methods, it was clear that the articles included in the issue only provided the first tantalising glimpse of the complex and wide-ranging work that had begun to emerge in archival and recordkeeping scholarship since the early 1990s. In their own article in that issue, Gilliland and McKemmish outlined several factors that had contributed to this expansion in scholarship. These included the growth in doctoral education, forums for presenting and publishing research, the numbers and size of graduate archival education programs, availability of diverse funding for research, transdisciplinary and international research collaborations, and application of innovative research methods and tools appropriate for investigating increasingly complex and wide-ranging research questions. The double issue rapidly became a touchstone for research design in archival and recordkeeping programs. However, the editors were aware from the start that something much more comprehensive was needed, hence the idea for this book. Gilliland and McKemmish, together with co-editor Andrew Lau, sought to put together a volume that would be authored not only by some of the most influential scholars of our time, but also by some of the best and brightest of new scholars emerging in the field. The Archival Education and Research Institute (AERI) ${ }^{1}$ provided rich grounds for

1 The Archival Education and Research Institute (AERI) is one component of a broader ongoing collaborative initiative, Building the Future of Archival Education and Research. Phases I and II of AERI were funded through grants from the US Institute for Museum and Library Services (IMLS). The initiative seeks to stimulate the growth of archival studies as a field within the United States and worldwide by nurturing and promoting state-of-the-art scholarship, as well as encouraging curricular and pedagogical innovation. Centred at UCLA, it is led by an international consortium of academic institutions that offer or plan to develop a doctoral specialisation in archival studies. Founding members were UCLA; University of Michigan; University of Pittsburgh; University of Maryland; University of Texas at Austin; University of North 
identifying such individuals, as did the recommendations of our colleagues in universities around the world, based upon their experiences with research collaborations and with their own students.

The book lays out questions and methods that are exemplary of the current state of archival and recordkeeping research in the archival multiverse, encompassing the pluralism of evidentiary texts, ${ }^{2}$ memory-keeping practices and institutions, bureaucratic and personal motivations, community perspectives and needs, and cultural and legal constructs. ${ }^{3}$ It is relevant also to many other fields, including those that have engaged with the Archive in its broadest sense, history, memory studies, ethnic studies, gender studies, anthropology, sociology, business administration, digital humanities, systems analysis and design, and information seeking, retrieval and use. We have not attempted to replicate recent in-depth work that has addressed the evolving scope, frameworks, and infrastructure of archival and recordkeeping research or indeed practice. Instead we would refer readers to that work as complementary to this volume. ${ }^{4}$ The book vividly illustrates how much the field has developed in recent years, even though there is considerably more research than it has been possible for us to capture within a single volume. Indeed,

Carolina, Chapel Hill; University of Wisconsin, Madison; and Simmons College.

AERI has also received significant in-kind support from universities elsewhere in North America, Europe, Asia and Australia, especially Monash University in Melbourne. See https://aeri.website.

2 We have used the term 'evidentiary texts' here to be inclusive of records as they exist in multiple cultural contexts (i.e., the societal record), because the term 'records' could be read as pertaining only to institutional/bureaucratic forms of recordkeeping.

3 AERI Pluralizing the Archival Curriculum Group (PACG). "Educating for the Archival Multiverse," American Archivist 74 no.1 (Spring/Summer 2011): 69-102, quote p. 73.

4 See Anne J. Gilliland and Sue McKemmish, "Introduction" and "Building an Infrastructure for Archival Research," Archival Science 3-4 (2004): 143-147; 149-199 [republished in Spanish: Anne, Gilliland y Sue McKemmish. "Introducción” y "Construir una Infrastructura para la investigación archivística," Tendencias 5 (2006); 9-62. Republished in Lezen! Teksten over het archief, Y. Bos-Rops et al. eds. (Amsterdam: Stichting Archiefpublicaties, 2009), pp.101-150; Sue McKemmish and Anne J. Gilliland, "Archival and Recordkeeping Research: Past, Present and Future," in Research Methods: Information Management, Systems, and Contexts, Kirsty Williamson and Graeme Johanson, eds. (Prahran, Vic: Tilde University Press, 2012), pp.80-112; and Julie McLeod, Sue Childs and Rachel Hardiman, Transforming Information and Records Management through Research \& Development? Proceedings of the 3rd Northumbria International Witness Seminar Conference. School of Computing, Engineering, and Information Sciences, Northumbria University, 2010, http://www.academia.edu/918450/ Transforming_Information_and_Records_Management_through_Research_and_ Development; Rachel Hardiman, "Under the Influence: The Impact of Philosophy on Archives and Records Management," chapter 6 in Caroline Brown, ed. Archives and Recordkeeping: Theory into Practice (London: Facet Publishing, 2014), pp.171-225. 
as broad as it is, the book cannot hope to reflect all of the dynamism in the research currently being undertaken within the academy, in professional practice, and in individual sectors and communities, especially in response to accelerating technological implementations, political upheavals around the world, the pursuit of human rights and social justice, and a flood of revelations about government and corporate recordkeeping, accountability measures, and intelligence gathering.

We had several goals for this book beyond simply providing a compendium of examples of research design and methods employed in archival and recordkeeping studies: to review the conceptual lineage of the field, as seen through different epistemological lenses and archival traditions; to underscore that theoretical framing and conceptual clarity are important in both theoretical and applied research; to provide literary warrant for a range of methods that have been adopted or adapted in archival and recordkeeping research and to provide rigorous examples of how they have been applied; to demonstrate the diversity of settings in which the research is, or might be, undertaken; and to draw attention to the kinds of challenges and dilemmas that emerge when working within a pluralised research paradigm. Some of the authors have chosen to address the notion of the archival multiverse directly in their chapters, while the specific foci of others demonstrate the plurality of perspectives and approaches that can be simultaneously understood as valid and valuable in contemporary archival and recordkeeping research.

As this book demonstrates, archives and recordkeeping have continuously and symbiotically intertwined with evolving information and communications technologies, and this intertwining has spawned fertile areas of systems, media, and policy-oriented research and development, particularly following the inception of electronic recordkeeping in the second half of the twentieth century and later of the Web, social media, mobile computing, and personal digital archives (see, for example, chapters by Acker, Evans, Furner \& Gilliland, Gilliland, Luyombya, and MacNeil). ${ }^{5}$ At the same time, research in archival studies has also been particularly concerned with issues relating to memory, identity, accountability, rights, culture and heritage, grand and counter-narratives, and inter-community power dynamics and equity concerns (see, for example, chapters by Arondekar, Bastian, Dunbar, Gibbons, Ketelaar, Lee, Upward, White, Faulkhead, Thorpe, and Dong, Blanco-Rivera, Caswell \& Steele). Not surprisingly, many different

5 For more details on these developments, see Anne J. Gilliland, Conceptualizing Twentyfirst-century Archives (Chicago, IL: Society of American Archivists, 2014). 
methodological approaches have accompanied the different epistemological framings of archival and recordkeeping studies. ${ }^{6}$ This book takes a serious look at several of them, acknowledging the distinctive value of each for studying particular phenomena in the field, but with the understanding that more will inevitably emerge in time and also that frameworks and models will shift or be supplanted over time. While a given framework or model might advance a contemporary state of awareness, it subsequently might also provide a piton or a rebuttal point for those who are reformulating old concepts, or discovering new forms of knowledge. Moreover, frameworks and models themselves should be subject to evaluation and their variability and confounding factors examined in order to challenge claims that might be made about their universality.

It should be emphasised that research modes customarily associated with the humanities, arts, social sciences, and engineering and technological fields, at the very least, should all be considered to be equally legitimate when applied appropriately and rigorously. This means that not all research in archival and recordkeeping studies will necessarily exhibit characteristics associated with the kinds of empirical or historical research that is most common in the social sciences, for example, a research hypothesis, data-gathering, or experimental design. Some research may rather employ theory-building, analytical reasoning, or even the creative arts (e.g., storytelling or film-making).

Although the book demonstrates the breadth of archival and recordkeeping studies, it can only suggest the differing degrees of sustained trajectories of research or replication of studies within individual areas of the field. Some of the review chapters (for example, Hofman on modelling and Sundqvist on user studies) speak to the few areas where an increasingly substantial and cumulative body of work is being developed by multiple researchers. Many others address nascent areas where little research has been undertaken as yet but where there is strong potential (for example, Youn's research looking at the socio-cultural implications of implementing international standards in particular national or cultural contexts).

Similarly, the book references methods or techniques that have been commonly used in archival and recordkeeping studies (for example, historiography, surveys, interviews and focus groups). It also discusses some methods that have taken on their distinctive formulation specifically through their application in the field (for example, Duranti \& Michetti and MacNeil on

6 See McKemmish and Gilliland. "Archival and Recordkeeping Research: Past, Present and Future," for a more complete exposition of the range of methods currently being used in archival studies research. 
contemporary archival diplomatics, Duff \& Cumming on literary warrant analysis, and Gracy on the ethnography of the archive). Several chapters treat or mention different implementations of one particular method (for example, Anderson and Gilliland on bibliometrics, and Bunn and Ramdeen \& Poole on grounded theory development). Finally, some chapters are doubly hermeneutic in the sense that the expository process of delineating how a method drawn from another field might be applied in archival studies itself has the potential to critique and augment the conceptualisation of that method within its parent field (for example, Furner and Gilliland's discussion of archival information retrieval [IR]). We have also chosen to include reprints of four articles that we consider to be of particular importance. MacNeil's critique of contemporary archival diplomatics, Gracy's exposition of archival ethnography, Arondekar's discussion of the application of frameworks drawn from sexuality studies as well as postcoloniality, and Dunbar's introduction of Critical Race Theory (CRT). Each has been seminal either as the first such treatment in the field, and/or as a piece that has become a touchstone within the field.

None of this is to suggest that all methods discussed here are equally applicable in every situation, however, or that equal amounts of research have been conducted or should be conducted in every one of the areas covered in the book. And although research may be driven by methods, intellectual stances, or societal problems, it is important to remember that archival and recordkeeping studies, with its roots in archival science, has classically been driven by professional problems or concerns. In problem-centric research, a method is a means to an end and not an end in itself. It is also important that the voice of the researcher comes through and is not eclipsed by, or hidden behind, the method employed. That said, the growth in research that is explicitly driven by an epistemological or ideological stance is welcomed and we can only imagine the new research vistas that it will continue to open up.

\section{About this Book}

When we commenced work on the ambitious project of compiling a monograph that would highlight the diversity of research that was occurring in the field, as well as of epistemological approaches and methods being applied, it seemed to be a natural illustration of the archival multiverse concept. It was not until the book was actually well underway, however, that we realised that the original articulation of the archival multiverse (to which all of the editors had been party) had overlooked making an overt assertion about the plural and pluralising nature of research in that multiverse. We 
also became aware that new facets of the multiverse and perspectives about its nature were emerging simply from the unprecedented juxtaposition of diverse and sometimes diverging scholarship and conceptualisations being discussed by the authors of different chapters. This book both illustrates and operationalises the archival multiverse in action in research, thereby addressing this critical omission from the original definition. At the risk of sounding pedantic, therefore, we would offer the following friendly restatement of the original definition:

the pluralism of evidentiary texts, memory-keeping practices and institutions, bureaucratic and personal motivations, community perspectives and needs, and cultural and legal constructs with which archival professionals and academics must be prepared, through graduate education and through research and development, to engage.

Such a restatement underscores three central characteristics that we hope this book illustrates. The first of these is the pluralism that is evident in research today, including pluralism in terms of the subjects (as illustrated in Table 1), modes, and sites of engagement; methods and frameworks employed; reflexivity; and ethical considerations). The second is the picture of a simultaneously cumulative and evolving disciplinary pluralism that emerges when research trends are viewed over time. This pluralism points to the vibrancy and relevance of archival and recordkeeping studies as a research field (see, for example, chapters by Gilliland on the multiverse of archival and recordkeeping traditions; Lian and McKemmish delineating the lineage and contingencies of the archival and recordkeeping traditions in China and Australia respectively; Duranti and Michetti on the history of the nineteenth century European development of the 'archival method' out of the philological and historical disciplines; Head on the plurality of descriptive approaches in pre-modern European archives; MacNeil on the conceptual and methodological alliances between the Anglo-American tradition of textual criticism and the European theory of archival arrangement; and Upward and Gibbons on the application of philosophies of emergence to evolving records continuum theory). ${ }^{7}$ We would add an important caveat to these two characteristics, however, which is that although they evidence a previously under-demonstrated heterogeneity in our field, that heterogeneity remains bounded in problematic ways by its strongly Western and academic orientation. Chapters by Arondekar,

7 For further discussion of this diversity, see McKemmish and Gilliland. "Archival and Recordkeeping Research: Past, Present and Future." 
Faulkhead and Thorpe all discuss the epistemological, methodological and socio-political limitations of this orientation for the archival multiverse.

This latter observation leads us to a third characteristic - the multiple kinds of actual or potential relationships between research and praxis in the archival multiverse. The professional domain of archival science is an integral applied component of archival and recordkeeping research, addressing through practice, policy and research "the characteristics of records in their social and cultural contexts and how they are created, used, selected and transferred through time," and focusing on the concerns, principles, techniques, technologies and ethics of archival and recordkeeping practice. Examples of research areas with applied applications in this book include systems design and development outlined in chapters by Evans and Gilliland; potential information retrieval implementations discussed in the chapter by Furner \& Gilliland; ethnographically-derived understandings of archival practices and priorities as illustrated by Gracy; modelling techniques delineated by Hofman; practitioner understandings of and attitudes toward archival activism by Novak; user studies reviewed and analysed by Sundqvist; innovation and ethics in practice described by Thorpe; and adoption and adaptation of metadata standards investigated by Youn. As archival and recordkeeping studies as a field has become more robust within the academy over the past two decades, however, an unfortunate by-product has been the widening of the gap between the academy and professional practice. ${ }^{8}$ The unprecedented growth and growing prominence of academically based scholarship has begun to overshadow important theoretical and empirical contributions emanating out of the archival profession. Such contributions by 'scholar-practitioners' drove conceptual and technological development throughout much of the twentieth century and remain essential to furthering sound archival practice. ${ }^{9}$ While the growth of research in the academy has been well chronicled and is amply represented in this book, growth of research in practice has been slower and under-acknowledged. We particularly welcome, therefore, the contributions to the book of several exceptional new scholar-practitioners and also note that an increasing body of research referenced by chapters in the book involves academic-community partnerships and academic-professional institution collaborations. ${ }^{10}$

8 We should note that this has also been an unanticipated effect of developing an academic-centric research forum such as AERI.

9 See, for example, the work of David Bearman, Brien Brothman, Terry Cook, Adrian Cunningham, Verne Harris, Chris Hurley, Hilary Jenkinson, Michael Piggott, Barbara Reed, Theodore Schellenberg and Hugh Taylor.

For example, Kimberly Anderson, Kate Cumming, Joy Novak and Kirsten Thorpe. 
Table 1. Characterising the Archival and Recordkeeping Research Landscape ${ }^{11}$

\begin{tabular}{|c|c|c|}
\hline $\begin{array}{l}\text { Couture \& Ducharme } \\
\qquad(1998)^{*}\end{array}$ & Gilliland \& McKemmish (2004) & $\begin{array}{l}\text { AERI research presentations } \\
(2009-2012)\end{array}$ \\
\hline $\begin{array}{l}\text { Archives and archival } \\
\text { science - the nature of the } \\
\text { Archive, archival goals and } \\
\text { the usefulness of archives }\end{array}$ & $\begin{array}{l}\text { Building; evaluating, reflecting } \\
\text { on: }\end{array}$ & $\begin{array}{l}\text { Anthropological data collection } \\
\text { and repatriation }\end{array}$ \\
\hline $\begin{array}{l}\text { Archives and society - role } \\
\text { and place of archives, archival } \\
\text { science and the profession }\end{array}$ & Archival education & Archaeological recordkeeping \\
\hline $\begin{array}{l}\text { Archival issues - ethics, } \\
\text { access, privacy }\end{array}$ & Archival history & $\begin{array}{l}\text { Archival description and } \\
\text { recordkeeping metadata }\end{array}$ \\
\hline Archival functions & Archival media & $\begin{array}{l}\text { Archival education, training and } \\
\text { pedagogy }\end{array}$ \\
\hline $\begin{array}{l}\text { History of archives and } \\
\text { archival science }\end{array}$ & Archival practice & $\begin{array}{l}\text { Archival implications of social } \\
\text { media }\end{array}$ \\
\hline $\begin{array}{l}\text { Management of archival } \\
\text { programs and services }\end{array}$ & $\begin{array}{l}\text { Archival research methods and } \\
\text { techniques }\end{array}$ & Archives and human rights \\
\hline Technologies & Archival systems & Archives and postcoloniality \\
\hline $\begin{array}{l}\text { Types of media and archives; } \\
\text { electronic records }\end{array}$ & $\begin{array}{l}\text { Archival theory, ideas and } \\
\text { concepts }\end{array}$ & Arts and performing arts archives \\
\hline \multirow[t]{11}{*}{ Types of archival institutions } & Archival tools and technology & Business records and recordkeeping \\
\hline & $\begin{array}{l}\text { Archival use and usability (by } \\
\text { specific user groups) }\end{array}$ & $\begin{array}{l}\text { Community recordkeeping } \\
\text { practices }\end{array}$ \\
\hline & $\begin{array}{l}\text { Archives and recordkeeping } \\
\text { metadata }\end{array}$ & $\begin{array}{l}\text { Community-based archives and } \\
\text { community-centric archival policy }\end{array}$ \\
\hline & $\begin{array}{l}\text { Archives and recordkeeping } \\
\text { policy }\end{array}$ & $\begin{array}{l}\text { Criminal justice, counter-terrorism } \\
\text { and recordkeeping }\end{array}$ \\
\hline & $\begin{array}{l}\text { Development of descriptive } \\
\text { models and schemas }\end{array}$ & Decolonisation of the Archive \\
\hline & Electronic recordkeeping & $\begin{array}{l}\text { Diasporic and expatriate records } \\
\text { and identity concerns }\end{array}$ \\
\hline & $\begin{array}{l}\text { Ethnography of archival } \\
\text { collaboration }\end{array}$ & Digital curation \\
\hline & Ethnography of archival practice & Digital forensics \\
\hline & Ethnography of the archive & Digital heritage convergences \\
\hline & $\begin{array}{l}\text { Impact on the record of organisa- } \\
\text { tional and technological change } \\
\text { and vice versa }\end{array}$ & Digital humanities convergences \\
\hline & $\begin{array}{l}\text { Psychology and ethnology of } \\
\text { recordkeeping and use, including } \\
\text { socialisation into document } \\
\text { creation and use }\end{array}$ & $\begin{array}{l}\text { Digitisation and associated policy } \\
\text { concerns, e.g., copyright }\end{array}$ \\
\hline
\end{tabular}

11 McKemmish and Gilliland, "Archival and Recordkeeping Research," 82. 


\begin{tabular}{|c|c|c|}
\hline & $\begin{array}{l}\text { Sociology and politics of the } \\
\text { record and recordkeeping }\end{array}$ & $\begin{array}{l}\text { Electronic recordkeeping systems } \\
\text { and approaches }\end{array}$ \\
\hline & $\begin{array}{l}\text { Emergent areas of research } \\
\text { related to archival globalisation }\end{array}$ & Evidence studies \\
\hline & $\begin{array}{l}\text { Exploration of ways to diversify } \\
\text { the archival paradigm and } \\
\text { understand associated power and } \\
\text { empowerment issues }\end{array}$ & $\begin{array}{l}\text { Globalisation and other global } \\
\text { concerns }\end{array}$ \\
\hline & $\begin{array}{l}\text { Assessment of the impact of } \\
\text { global research and international } \\
\text { standards emanating from } \\
\text { research upon local archival } \\
\text { traditions and theory, as well as } \\
\text { marginalised communities }\end{array}$ & Health records and recordkeeping \\
\hline & $\begin{array}{l}\text { Post-colonial issues: "The West } \\
\text { vs. the Rest" }\end{array}$ & $\begin{array}{l}\text { History of archives and archival } \\
\text { practices }\end{array}$ \\
\hline & $\begin{array}{l}\text { Evaluation, comparison and } \\
\text { potential reconciliation of } \\
\text { conflicting conceptual models and } \\
\text { descriptive schema }\end{array}$ & $\begin{array}{l}\text { Indigenous knowledge, culture and } \\
\text { the Archive }\end{array}$ \\
\hline & $\begin{array}{l}\text { Records law and policy, including } \\
\text { reconciliation of different } \\
\text { traditions }\end{array}$ & Legislative analysis \\
\hline & $\begin{array}{l}\text { Ontological, semantic, and ethno- } \\
\text { methodological issues relating } \\
\text { to developing understanding of } \\
\text { emergent media forms }\end{array}$ & Memory and identity studies \\
\hline & $\begin{array}{l}\text { Addressing terminological differ- } \\
\text { ence within the archival field and } \\
\text { between it and other fields inter- } \\
\text { ested in some of the same issues. }\end{array}$ & Metadata modelling \\
\hline & & $\begin{array}{l}\text { Moving image archives (analogue } \\
\text { and digital) }\end{array}$ \\
\hline & & Museum archives \\
\hline & & $\begin{array}{l}\text { Personal recordkeeping and digital } \\
\text { archives }\end{array}$ \\
\hline & & $\begin{array}{l}\text { Scientific recordkeeping and data } \\
\text { archives }\end{array}$ \\
\hline & & $\begin{array}{l}\text { Social justice, human rights, truth } \\
\text { and reconciliation commissions }\end{array}$ \\
\hline & & $\begin{array}{l}\text { The social life of records and } \\
\text { documents }\end{array}$ \\
\hline & & $\begin{array}{l}\text { Transformative research by } \\
\text { and with Indigenous and other } \\
\text { communities partners }\end{array}$ \\
\hline & & Trusted digital repositories \\
\hline
\end{tabular}

* See Couture \& Ducharme, 2005. The article was first published in French as Couture, Carole, and Ducharme, Daniel. "La recherche en archivistique: un état de la question" in the journal Archives 30 nos.3-4 (1998): 11-38. 


\section{How to Approach the Book}

The book is divided into three sections. The first addresses the intellectual lineage, contemporary conceptual landscape, and possible future directions of archival and recordkeeping studies. Authors present these aspects by drawing upon a range of different national archival traditions, and cultural and critical frameworks. While each chapter in this section stands on its own, when read together, the chapters allow the reader to discern critical shifts and differences over time and across geographies and epistemological approaches. The second section discusses the nature, strengths and weaknesses, and potential areas of application of particular methodological approaches. In so doing, it treats an unprecedented range of methods but deliberately avoids situating them under familiar rubrics such as quantitative and qualitative, in order to encourage contemplation of less traditional ways in which they might be applied individually or in a multi-method research design. The final section comprises case studies of actual implementations of particular methods. In these case studies, the authors describe their own research experiences and reflect upon their choice of methods and research design. We asked them to make their meta-assumptions about their research explicit rather than tacit and to frame their case studies within the broader discourse, philosophy and values in which they were situated. We also asked authors in this section to discuss explicitly their stance at the outset and how that might have evolved in the process of the research. We hope that such an approach will prove useful to researchers who are interested in undertaking similar research, or in using a similar research design or the same methods. It is also in concert with the overarching philosophy of the archival multiverse - where all actors should be aware of their own motivations, preconceptions, perspectives and cultural context, how these come into play in their work, and how these shift during processes of engagement in research and with others.

Readers will become aware that we have made no attempt to control the terminology or definitions of terminology that are provided by the book's various authors, nor have we included a glossary. Since this book is representative of the archival multiverse and we believe that it is very important to demonstrate how different archival traditions are at work, we have instead directed our authors to state specifically how they are understanding the terms of art that they are using. In some cases this has also involved co-authors who come from different archival and recordkeeping traditions negotiating terminology and conceptual definitions as a part of 
the authorial process. As a result, one way in which this book can be read is to contemplate whether and when different authors are arriving at similar or different conceptualisations, why and by what means. For example, their definitions reflect at times the archival or recordkeeping tradition within which they are working, and at times they reflect the construction they come to put upon them in the course of the research - an option always available to the researcher that may not necessarily be available to the practitioner or even the standards developer. This definitional license exposes nuances and diversity, as well as divergences in conceptualisation that hopefully not only speak to the multiverse, but also demonstrate places where there is an absence of consensus or of clarity (for example, areas that have been historically governed by assumptions, assertions or fuzziness) that might be ripe for exploration through research.

Every chapter also includes a list of references. Although we resisted the temptation to do our own bibliometric analysis on the scatter of the literature cited, it is illuminating to examine both the range and the overlap of scholarship upon which authors are drawing. Certainly a wide-ranging selection is being brought in from other domains. In terms of literature from our own field, there are clearly some canonical texts and indeed quotations that are repeatedly cited. However, these are not always understood in the same ways by all authors, perhaps because the authors come from different archival traditions but also likely reflecting the authors' own critical questioning of and quest to clarify canonical understandings. Thus this book taken in its entirety, while not making claims for comprehensiveness, nevertheless provides an important meta-review of many relevant literatures.

We would be remiss if we did not also point out some of the areas that this book does not cover or cover adequately for a variety of reasons from the unavailability of potential authors to the underdeveloped state of the area. These include policy aspects, community archives, digital humanities, the role of spirituality in recordkeeping and records use, emergent technological developments such as machine translation of primary sources, storytelling, and, ironically perhaps, given the lineage of the field, historical approaches. Talking about the epistemological stance and the research design and methods and theoretical frameworks that one has employed comes more naturally to some areas than others, and it proved particularly difficult (with the notable exception of Head's chapter) when putting this book together to find scholars employing historical methods who were prepared to discuss their intellectual process in this way. 


\section{Where to from Here? Enduring Challenges of a Multiverse Approach to Research}

We wish to conclude with a few points that struck us as this book came together. Firstly, one can discern interesting generational shifts in research focus, methodological choices, and professional engagement within the field. These shifts illustrate how far the field has come over the past several decades but also perhaps reflect a transition that has taken place in the backgrounds of academics. While many senior academics had prior or even simultaneous careers as archival practitioners and also academic training in fields such as history, literature or religious studies, increasing numbers of new scholars have no professional background but have instead been trained as career academics within archival and recordkeeping programs. At the same time, this volume includes chapters by several individuals who, having recently completed doctoral programs, have chosen to return to the field as practitioners who will also actively pursue research in practice. Both of these developments herald, we hope, an even more rigorous and diversified research future for archival studies but at the same time raise important questions about the nature of future education and research relationships between the academy and the professional field.

Secondly, writing about our research in ways that both problematise the context within which we are working and explicate it so that it is understandable and compelling to those in other domains can add considerable overhead in terms of effort, time and definitional work. This overhead needs to be explicitly acknowledged and anticipated in the authorial process and in the space and stylistic conventions to which authors must conform when writing for publications in other domains.

Thirdly, while there has been an enormous increase in the amount of research being undertaken all around the world, it is clear that at least two important barriers to its dissemination are limiting how much researchers are aware of developments elsewhere within the field. One of these barriers is language of publication. The most prominent research venues are predominantly English language journals (and we are very conscious that English was chosen as the language for this book even though it is designed to address the archival multiverse). While English is becoming increasingly dominant as the lingua franca of academia in Northern and Central Europe and parts of Asia, this largely submerges research published in much of the rest of the non-English-speaking world. The historically influential IberoHispanic and French archival traditions, the wealth of current research 
being published by Chinese scholars in Chinese journals, not to mention the literatures of the Arab world, Africa, other parts of Asia and many other regions, in the absence of knowledgeable translation programs, are effectively invisible to the English-speaking community, as well as vice versa. The pressure for academics to publish in English and in international journals can also result in ruptures of what we maintain should be an organic relationship between the academic field and professional practice, where the professional field increasingly does not read the same literature, attend the same conferences and institutes, or even work or publish in the same languages. The dominance of one language or one culture's editorial practices can also shape published research into the epistemological structures and semantics that tend to accompany that language. The impact of this process (often unseen or unacknowledged by those who bring these to bear) on the expression and understandings of major concepts can be particularly insidious. As the range of definitions for the same terms or concepts as expressed in English and used by the authors of this book demonstrate, the cultural nuances and resonances of the terms and concepts can confuse authors who are writing outside their own language. It can also result in mistranslation of key terms and semantics used in the literature and in standards from English into other languages.

Another barrier is created by limitations in the dissemination of scholarly knowledge within the academy, within the professional field, and to communities outside both. The most obvious factors are the small numbers of major peer-reviewed venues for presenting research. Very few of the major professional archival conferences include a peer-reviewed track or publish peerreviewed proceedings. This limits the incentives for academics to participate. Despite increased recognition of research in archival and recordkeeping studies within iSchools, it has proven difficult to get that research accepted by the major information journals and conferences - largely because peer reviewers are unfamiliar with the archival domain and the extent and relevance of existing research within that domain. Complicating this scenario are the parameters imposed by their institutions and even states about where to publish and limitations of online access to certain journals, especially in less-developed nations where only one journal subscription service may be supported (thus limiting the numbers of titles available and sometimes also resulting in the universities insisting that their faculty publish in those titles). Many archival institutions, especially in the community sector, are not resourced to subscribe to the journal databases of the large commercial publishers who increasingly dominate publication in the field, especially of highly ranked or cited journals, 
and the fees for downloading single articles are a disincentive for individuals outside the academy to access them. An up-to-date and widely accepted ranking is not available across the field and many key archival journals have not been indexed by scholarly services. For these reasons, and also to attain greater international prominence, as already mentioned, universities may require their archival faculty to publish in English-language or international journals only, in journals that are central to a broader field such as information science or history but are not widely read within archival and recordkeeping studies, or only in A or $\mathrm{A}^{*}$ journals or journals indexed in a service such as Elsevier's Scopus or Thompson-Reuter's Web of Science. These are particularly vexing problems for a field that is still trying to establish its credibility and intellectual centredness within the academy.

Finally, putting this book together has made us, as editors, more conscious of our differing epistemological outlooks, and how our own research has been approached from a range of perspectives, personal research inclinations, and methods. We encouraged authors to include critical selfreflection as part of their methodological descriptions, and that to consider and analyse from a situated position the experience and process of designing, conducting, and evaluating research. We needed to do the same. As editors, we made a conscious effort not to assume a transcendent view of the field - a comprehensive "view from nowhere" - but to recognise the conceptual and practical limits of this project from the outset. This stance requires that we acknowledge our embeddedness throughout the process of compiling this volume, including (but certainly not limited to) its framing in terms of reflexive research practices, the numerous editorial conversations with authors in shaping their contributions, and our own reliance as editors on the vibrant network of archival researchers - whether academic or practitioner or some combination thereof - and the research that is produced.

More than a collation of research methods for handy reference, therefore, this volume advocates for reflexive research practice as a means by which to lay bare the fuzziness and messiness of research. Whereas research in the form of published research papers and juried conference presentations provide a view of archival and recordkeeping studies framed in terms of research questions and findings, reflexive research practice reveals the context of a study and chains of situations, choices, and decisions that influence the trajectories of the studies themselves. Such elucidations from the position of the researcher are instructive for others, who may be inspired to apply or adapt the methodologies, design approaches and individual methods explored here for their own research. 


\section{ACKNOWLEDGEMENTS}

We would like to thank all the authors of the papers in this book for their willingness to take on writing about aspects of research and theory that are too infrequently covered in archival studies but that are much needed. We would also like to thank Monash University Publishing and especially Tom Denison and the Editorial Board for the Social Informatics monograph series, and the Centre for Organisational and Social Informatics in the Faculty of Information Technology at Monash for all their support of this endeavour.

We would like to acknowledge the Archival Education and Research Initiative (AERI) community, out of which the idea for this book originated, and which provided such a fertile source of expertise, authors, and advisors from around the globe on various aspects of the book's development; and also the United States Institute for Museum and Library Services (IMLS), which has graciously funded AERI for the past six years.

Finally, we would like to pay tribute to and remember Allison Krebs, who, as a key member of AERI's Pluralizing the Archival Curriculum Group, first proposed applying and adapting the concept of the Multiverse within the archival context.

Anne J. Gilliland

Sue McKemmish

Andrew J Lau

Los Angeles, Melbourne and Oakland 


\title{
ARCHIVAL AND RECORDKEEPING TRADITIONS IN THE MULTIVERSE AND THEIR IMPORTANCE FOR RESEARCHING SITUATIONS AND SITUATING RESEARCH
}

\author{
Anne J. Gilliland
}

\begin{abstract}
This chapter provides a brief introduction to the history of archival practices and ideas and how these came together over the past three centuries to form an internationally recognised body of theoretical principles, definitions and best practices that is central to the professional field of archival science around the globe. It places archival science in relation to the broader conceptualisations of recordkeeping and archival studies. It then introduces the concept of the archival multiverse and discusses the ways in which considerable plurality in ideas and practices has continued not only to co-exist but also to emerge, both inside and outside the profession and to challenge and expand its core notions. It argues that this plurality is one of the hallmarks of the archival multiverse and is, therefore, a critical variable that should be accounted for in conducting and presenting situated research. With reference to research being conducted as part of the Archival Education and Research Initiative (AERI), the chapter concludes with suggestions as to how such plurality of archival and recordkeeping traditions, ideas, practices and histories could be better explicated and accounted for, particularly in theoretical and applied research around the globe. It also proposes several fertile areas for research, including research supporting grand challenges identified by different institutions, nations and international bodies, as well as grand challenges facing the archival and recordkeeping field itself.
\end{abstract}




\section{Introduction}

In the past two decades, archival and recordkeeping ${ }^{1}$ research has gained prominence as a rapidly growing and generative research presence within the academy. Such research encompasses a diversity of disciplinary, transdisciplinary, professional and technological studies of topics relevant to notions of the Archive as well as of archives as professionally construed. It has addressed philosophical, cultural and media aspects of the Archive and its societal functions in a "multicultural, pluralistic, and increasingly interconnected and globalised world," ${ }^{2}$ as well as of archives and recordkeeping in and over time and space and across communities. In many respects, the state of the field today is the natural outcome of the interactions of long and varied epistemological and practical trajectories addressing things archival (in this expansive sense) within different disciplinary, professional and community spaces. This chapter first provides an introduction to the history of archival practices and ideas and how these came together over the past three centuries to form what has been referred to as "the archival paradigm" - "a set of assumptions, principles and practices that are common to the archival community [that] are a model for its activities and outlooks". ${ }^{4}$ It defines archival science and then places it in relation to broader conceptualisations of recordkeeping and archival studies. It introduces the concept of the archival multiverse and discusses some of the ways in which considerable plurality in ideas and practices have continued not only to co-exist but also to emerge and cross-fertilise, both inside and outside the professional field, and to challenge and expand its core notions. It argues that this plurality is one of the hallmarks of the archival multiverse and is,

$1 \quad$ Although the term "recordkeeping" as used in the records continuum sense encompasses archival aspects of the management of records, it is not always understood as such outside continuum contexts. Hence this chapter uses the construction (really a misconstruction) "archival and recordkeeping" to underscore that it is referring to a spectrum of conceptualisations of the keeping of records and archives.

2 Sue McKemmish and Anne J. Gilliland. "Archival and Recordkeeping Research: Past, Present and Future," in Research Methods: Information Management, Systems, and Contexts, Kirsty Williamson and Graeme Johanson, eds. (Prahran, Vic: Tilde University Press, 2012), pp.80-112.

3 Anne J. Gilliland, "Enduring Paradigm, New Opportunities: The Value of the Archival Perspective in the Digital Environment," in Michèle V. Cloonan, Preserving Our Heritage: Perspectives from Antiquity to the Digital Age (Neals-Schuman, ALA Editions, 2014), pp.150-161 [excerpted and updated from Gilliland-Swetland, Anne J. Enduring Paradigm, New Opportunities: The Value of the Archival Perspective in the Digital Environment (Washington, D.C.: Council on Library and Information Resources, 2000)]. Gilliland, "Enduring Paradigm." 
therefore, a critical variable that should be accounted for in conducting and presenting situated research in archival and recordkeeping studies. With reference to research being conducted as part of the Archival Education and Research Initiative (AERI), the chapter concludes with suggestions as to how such plurality of archival and recordkeeping traditions, ideas, practices and histories could be better explicated and accounted for, particularly in theoretical and applied research across the globe, and proposes several fertile areas for research including research supporting grand challenges identified by different institutions, nations and international bodies, as well as grand challenges facing the archival and recordkeeping field itself.

It should be noted that one of the difficulties in writing such an overview is that it must inevitably be written from the perspective and with the assumptions of the tradition(s) in which an author is most deeply versed, which in this case is the U.S. archival tradition (to this end, a note on the history and influence of U.S. approaches to records and archives has been included at the end of this chapter). Moreover it cannot possibly address in detail the situations in any or all specific contexts - there is much research that needs to be done to begin to address archival and recordkeeping selfknowledge in this respect. While every chapter in this book also reflects something of the tradition within which it is written, readers are particularly encouraged to read this chapter together with chapters 2, 3 and 4 in order to get a better sense of the diversity and dynamics of some of the traditions that are exerting important influences on archival theory and practice today. Chapter 2 discusses how the archival body of knowledge in Europe originated in the legal disciplines and developed over the centuries into a humanistic science through integration with philological and historical disciplines. Chapter 3 traces the lineage of Chinese archival practice and ideas and the social, political, economic and cultural contexts that have influenced and shaped them in different periods of Chinese history. Chapter 4 explores an Australian recordkeeping tradition that has evolved out of British colonial archival practices. It reflects on the evolution of records continuum thinking and practice with reference to their formative historical, societal, juridical and geo-political influences, and the philosophies and theories that frame them.

\section{The Emergence of 'the Archival Paradigm' and Recent Critiques}

First defined by American physicist and philosopher of science Thomas Kuhn, a paradigm is a formal model or pattern of beliefs, outlooks, assertions, 
values, and practices regarding a particular activity or phenomenon. The archival paradigm (or paradigms, if one views the different frameworks and traditions within which archivists have operated as distinct paradigms rather than as different manifestations and phases of a unifying paradigm) first took shape in a series of regulations and manuals published in France, Prussia, the Netherlands, Spain, Italy, and elsewhere in Western Europe in the eighteenth and nineteenth centuries. Emanating chiefly out of the experiences of those working in archives holding state and municipal records, it supports an evidence-based approach to the management of those records. It is fundamentally concerned with the organisational and individual functions, processes, and contexts through which records and knowledge are created and preserved as well as the ways in which records individually and collectively reflect those functions, processes, and contexts in and over time. The paradigm operates on multiple conceptual, functional, and professional levels as a framework for archival theorising as well as for archival practice. In this framework theory develops out of practice (inductive) and practice develops out of theory (deductive). It represents both a particular perspective on information and knowledge management and a distinctive professional ethos. $^{5}$

In some of the world's earliest societies as well as in contemporary societies that have maintained their oral traditions, many of which are not located in the Global North, the recording of important knowledge, events, judgments, agreements and transactions has often occurred in non-tangible forms. These include ritual, recitation, song and dance. In terms of tangible forms, petroglyphs that are thought to represent some kind of image or symbol have been discovered in Africa and can be dated to the Middle Paleolithic era (70,000 BCE). Some of the earliest evidentiary and memory-making texts were created up to 40,000 years ago in Aboriginal Australia in the form of pictograms on rocks and in caves. In societies with oral traditions, intangible forms were/are also complemented by or interact with material objects such as the quipus used by early Andean societies, Central African power figures, ${ }^{6}$

5 Anne J. Gilliland, Conceptualizing Twenty-first-century Arcbives (Chicago, IL: Society of American Archivists, 2014) See also Theo Thomassen, "The Development of Archival Science and its European Dimension," in The Archivist and the Archival Science. Seminar for Anna Christina Ulfsparre... (Lund: Landsarkivet, 1999), pp.67-74, http://daz.hr/zad/ arhol/the-development-of-archival-science-and-its-european-dimension/

6 See, for example, Metropolitan Museum, Power Figure (Nkisi N'Kondi: Mangaaka), http://www.metmuseum.org/collections/search-the-collections/320053. It is, unfortunately, not unusual to see such records collected and described as art objects or other forms of historical or anthropological artefacts or simply as curiosities 
the strings of wampum and winter counts created by Native American Eastern Woodlands and Plains tribes respectively, or talking sticks used by several Indigenous cultures but in particular by Native American tribes in the Northwest coast of North America. Designated individuals, often in hereditary positions or positions confined to members of an endogenous caste, such as quipucamayocs, ${ }^{7}$ winter count keepers, and West African griots or jalis served and in living traditions continue to serve as custodians of Traditional Knowledge, community historians, storytellers, and recordkeepers, even as embodied 'living archives.'

Archaeological finds from the Mesopotamian region are among the most widely cited indicators that tangible recordkeeping has been an integral component of human bureaucratic processes and business transactions in that part of the world since at least $3200 \mathrm{BCE} .^{8}$ Ancient records, historical compilations, and other forms of documentation recorded variously using bone, shell, stone, clay and cast metal tablets, silk, and papyrus and palm leaves have also been found in Mesopotamia and east across Asia as well as around the Mediterranean. These reveal ancient royal, mercantile and agricultural recordkeeping activity, including systematised and sophisticated methods of creating, organising, retrieving and referring to records. Family and personal recordkeeping, albeit almost always created by and documenting élites,

within museums, especially those of countries or cultures other than the creators, decontextualised from and frequently ignoring their original and continuing status as records. At the same time, this is an example of the many different ways that records have been and come to be regarded and appropriated over time.

7 Paul Beynon-Davies, "Informatics and the Inca," International Journal of Information Management 27 (207): 306-318; Gary Urton, "Tying the Truth in Knots:

Trustworthiness and Accountability in the Inka Khipu" in Brooke Harrington, ed. Deception: Methods, Motives, Contexts and Consequences, (Palo Alto, CA: Stanford University Press, 2009), pp.154-182, and "Tying the Archive in Knots, or: Dying to Get into the Archive in Ancient Peru," Journal of the Society of Archivists 32 no.1 (2011): 5-20.

8 Ernst Posner, Archives in the Ancient World (Cambridge, MA: Harvard University Press, 1972); Giovanni Pettinato, The Archives of Ebla: An Empire Inscribed in Clay, (New York, NY: Doubleday, 1981); James Gregory Bradsher, "Ebla’s Royal Archives," Information Development 1 no.4 (1985): 238-243; Alfonso Archi, "Archival Record-keeping at Ebla, 2400-2350 B.C." in Ancient Archives and Archival Traditions: Concepts of RecordKeeping in the Ancient World, Maria Brosius, ed. (Oxford: Oxford University Press, 2003), pp.17-36; Maria Brosius, "Ancient Archives and Concepts of Record-Keeping: An Introduction," in Ancient Archives and Archival Traditions, 1-16; Anne J. Gilliland, "Reflections on the Value of Metadata Archaeology for Recordkeeping in a Global, Digital World," Journal of the Society of Archivists 32 no.1 (April 2011): 97-112; Geoffrey Yeo, "Posner's Archives in the Ancient World Revisited: A New Look at Some Old Records," plenary paper presented at the Seventh International Conference on the History of Records and Archives (ICHORA 7), Amsterdam, August 2015, http:// ichora.org/?page_id=315. 
included genealogies, estate records, mercantile enterprises, personal and family accounts, diaries, correspondence, and portraits. Literate individuals such as scribes, priests, court historians and imperial historiographers, as well as the oft-referenced Greek archons had official recordkeeping and history-writing roles. In recent years, as a result of new archaeological evidence and the analysis of writings and other depictions contemporary with these civilisations, we have learned more about those who performed these roles but there remains considerable debate about the extent to which their status, roles and practices might approximate those we associate with modern-day professional archivists and recordkeepers.

It was out of Europe, and the legacy of the Roman Empire's legal and recordkeeping systems, that many of the world's Civil Law juridical frameworks and centralised registrarial approaches that came to dominate their governance and recordkeeping structures emanated. They were further adopted and spread with the founding and expansion of the early Christian churches (both Catholic and Orthodox). Trade and colonial empires as well as Christian evangelisation deployed these recordkeeping structures even further around the globe. Civil registration was used to great effect in nineteenth and twentieth century developments of health, welfare and education services as well as in government in many European countries. This is not to say that the specific implementations of these structures were always the same. Bureaucratic, business, spiritual and liturgical, and scholarly interpretive traditions were historically exercised in various locally-specific ways ${ }^{9}$ as were bureaucratic linguistic practices (e.g., use of ecclesiastical, vernacular, dialectical, or 'office' language, or some hybrid of some or all ${ }^{10}$ ). This local specificity continues today to shape archival ideas and practices in different European countries and regions.

The modern concept of archival work as a science (i.e., the notion that a designated, trained archivist employs a professionally sanctioned body of archival theory, practice and methodology to work with organisational recordkeeping and documentation activities) did not really begin to emerge until the multi-ethnic empires in Western Europe began to break apart into nation states based around political, geographic or cultural identity (a

9 An excellent exposition of diverse pre- and early Modern practices can be found in Interdisciplinary Essays on European Knowledge Culture, 1400-1900, special issue of Archival Science, Randolph Head, ed. 10 no.3 (September 2010).

10 See, for example, Tatiana Nikolaeva Nikolova-Houston, Margins and Marginality: Marginalia and Colophons in South Slavic Manuscripts During the Ottoman Period, 1393 1878, doctoral dissertation (Austin, TX: University of Texas, 2008). 
process that continued well into the twentieth century). By the seventeenth century, these states and often their colonies had formed stable centres of government, and the records of rulers and nobility, military leaders and traders, that had often been carried from place to place in archives chests or deposited in a temple, monastery, mosque, castle, military fortress or barracks, trading house or other sacred or secure structures for safekeeping, began to be consolidated into centralised repositories. At the same time, independent archives were maintained by many religious traditions and religious records continue today to offer parallel, or at least supplementary documentation regarding individuals and social life and cultures within a state. ${ }^{11}$ These religious records have subsequently proven to be particularly important in the recovery of evidence and information contained in official records lost or destroyed during conflicts or eliminated by dictatorial or sectarian regimes. Aguirre and Villa-Flores, for example, note that in post-independence Latin American states: "The reconstruction of religious practices but also of social relations, family life, distribution of wealth and property, mentalities and many other such topics have been greatly advanced by the abundance and accessibility of religious archives." ${ }^{12}$ In the wars that erupted with the collapse of the former Yugoslavia, archivists risked their lives to microfilm or to rescue records from churches and mosques in conflict zones, knowing that they contained information that often paralleled that in destroyed or lost state records and that might prove vital to establishing the identity, citizenship and marital status, inheritance and even existence of individuals from that region.

The Age of Enlightenment or Age of Reason that began in Europe in the late seventeenth century and continued through the eighteenth century was characterised by an emphasis on rationality, empiricism, scientific rigour, and the promotion of democracy, freedom, reason and religious tolerance. ${ }^{13}$ It was also characterised by the colonial ambitions and trading empires of western European nations. Vast bureaucracies were developed to administer

11 See, for example, Arndt Brendecke, “'Arca, Archivillo, Archivo': The Keeping, Use and Status of Historical Documents about the Spanish Conquista," Archival Science 10 no.3 (2010): 267-283; Bernard Lewis, From Babel to Dragomans: Interpreting the Middle East: Interpreting the Middle East (Oxford: Oxford University Press, 2004), p.415; and Carlos Aguirre and Javier Villa-Flores, "Introduction," in From the Ashes of History: Loss and Recovery of Archives and Libraries in Modern Latin America (Raleigh, NC: Editorial A Contra corriente, 2015), pp.11-38.

12 Aguirre and Villa-Flores, "Introduction," p.16.

13 Of course these did not often extend in the same ways to the peoples who were colonised by the empires established by many of these nations. 
colonies; manage the flow of information within colonial empires; support trade, slaving, and the extraction of other material assets; and enumerate, subjugate, 'civilise,' convert, enslave, transport, and even eradicate those who were colonised. Recordkeeping infrastructures were integral to many aspects of colonial administration, including communication and information management, financial accounting, and documenting and monitoring the colonised and their activities.

From the late eighteenth century and particularly in the nineteenth century in Western Europe, key archival principles began to be codified through a series of laws and regulations and practices systematised accordingly. In 1790, Carlos IV of Spain introduced Ordinances governing the handling of the recently created Archivo General de Indias (General Archive of the Indies) in Seville and proposing that its contents be maintained according to original order. In France in 1794, the Messidor Decree asserted the principle of accessibility of archives to the public and the value of records as documents of historical value. Most prominent were the French and Prussian articulations of Respect des Fonds, the Sanctity of Original Order, and the Principle of Provenance. Collectively, such principles reflected the hierarchies that generated the records as well as the registry systems that supported the work- and information flow in European government bureaucracies. They also formalised existing archival ideas about the cumulative nature of archives and their organic and ongoing relationship to the authority and activities by which they were created. The principles enshrined the primacy of provenance (construed as the creator or collector responsible for the generation or aggregation of the archives) in the collective arrangement, description and access as well as management of records in fonds or groups. Records within fonds were to be kept in the original order in which they were created or received, and fonds of different provenances were not to be intermixed. ${ }^{14}$ This codification and systematisation was taken a step further in 1910 at the International Congress of Librarians and Archivists in Brussels when the above-mentioned principles for archival arrangement and description, enshrined by the Dutch archivists, Muller, Feith and Fruin, in their so-called Dutch Manual, ${ }^{15}$ were ratified as the guiding principles for archival practice not only in Europe, but internationally (meaning, in

14 For a detailed explanation of each, see Gilliland, "Enduring Paradigm."

15 Samuel Muller, Johan A. Feith and Robert Fruin. Manual for the Arrangement and Description of Archives, Eric Ketelaar, Theo Thomassen and Peter Horsman, trans. of second edition (Chicago: Society of American Archivists Classic Series, 2003). 
effect, in those countries with representatives attending the Congress). ${ }^{16}$ However, Ketelaar is quick to remind us that this did not mean that there was uniformity in European archival practices, either then or today. Quoting a Dutch colleague remarking that, "Europe is united by its differences," he asserts that:

That makes it difficult and dangerous to refer to the European concept or theory. Any archivist from Europe feels flattered and exasperated when asked by a non-European audience to present the European position. Roman law, the Church, Napoleonic occupation, the Habsburg Empire - they provided European nations and states with a supranational framework having a great impact on national norms and systems, but they did not replace national, regional, or local archival cultures. ${ }^{17}$

Although one can also identify significant differences between archival practice within a life cycle-based organisational records managementarchival tradition, a records continuum-based recordkeeping tradition, and a "special collections" collecting tradition that has close historical associations with librarianship, these principles continue today to overarch and guide the archival and recordkeeping field today. Together they lie at the heart of the archival paradigm. They are embedded within many professional standards (especially descriptive standards), including national and sector standards and those promulgated worldwide by the International Council on Archives. Training in or implementation of these standards in turn are frequently required when accrediting archival and recordkeeping education and training programs, hiring professional staff, applying for government grants, or seeking repository certification.

At the same time as they promote consistency as well as interaction and exchange between institutions, sectors and nations, however, the principles promote a hegemony that is highly problematic when viewed from more plural perspectives than those out of which they historically emerged. While Yeo recently provided an articulate critique of the limitations of these principles and the need for their reconceptualisation to support what he

16 The Manual has already been published in German, Italian and French. This decision, however, prompted translation and publication of the Dutch Manual in Bulgarian, English (American edition), Chinese and Brazilian in succeeding decades, thus also spreading its influence to countries that had no delegates at the 1910 Congress. See Peter Horsmann, Eric Ketelaar and Theo Thomassen, "New Respect for the Old Order: The Context of the Dutch Manual," American Archivist 66 (2003): 265-267.

17 Eric Ketelaar, "The Difference Best Postponed? Cultures and Comparative Archival Science," Archivaria 44 (1997): 146. 
termed archival "third order" openness and flexibility in relation to digital affordances,$^{18}$ he did not address their power dimensions. Fundamental to the principles is an unchallenged construct of singular agency in records that has far-reaching implications for modern archival and recordkeeping practices. Recognising only one creator as the provenance of records denies agency to other parties, even the general citizenry, who are involved in the creation of other events and transactions documented in the records. It thereby substantially limits what rights those parties should or might have in decisions relating to all aspects of recordkeeping. ${ }^{19}$ Similarly embedded in Respect des Fonds and the Principle of Original Order is the notion of a singular fixed original order which fails to take into account both the dynamic nature of recordkeeping, and the existence of the multiple contexts of those documented in the records. Again they privilege the perspective of the singular creator in archival descriptive systems. Another consideration is embedded in the practice of collective description. Although responding to the dual concerns of retaining and explaining records in context and processing massive volumes of records (that can also be quite similar in structure and content), collective description fails to bring to light the traces of and about individuals that often exist only at the item, or within-item levels and that can be so important in reconstructing the lives and activities of non-élites.

In the twentieth century, archival science played an increasingly important applied role in managing the burgeoning records created by evolving government and organisational bureaucracies and recordkeeping technologies. Archival science in Europe, former European colonies and the United States was typically framed within a life cycle view which holds that records move through predictable stages in their lives (creation and capture within an organisational recordkeeping system; storage and maintenance semi-active, inactive; disposition-transfer to an archives or discarding and destruction), with each stage associated with particular activities, agents (records creators, records managers, archivists) and levels and types of use

18 Geoffrey Yeo, "Bringing Things Together: Aggregate Records in a Digital Age," Archivaria 74 (Fall 2012): 43-91.

19 The subjugating effects of this construct has been called out by Henrietta Fourmile, an Indigenous Australian writer who refers to the presence of Indigenous people in the archive as literally "captives of the archives." Fourmile, "Who Owns the Past? Aborigines as Captives of the Archives," Aboriginal History 13 (1989): 1-2. See also Anne J. Gilliland and Sue McKemmish, "The Role of Participatory Archives in Furthering Human Rights, Reconciliation and Recovery," Atlanti: Review for Modern Archival Theory and Practice 24 (2014): 79-88. 
(initially high, then progressively lower as records become inactive, until they are either disposed of or are preserved by an archives where they are subject to use by secondary users). The life cycle model is another component that was viewed until the end of the 1980s as fundamental to archival ideas, legislation, and programs in many parts of the world. The need to work with records being produced in digital form began to highlight key conceptual deficiencies in as well as the paper-orientation of this model. Archivists and other recordkeepers increasingly realised that they needed to be actively engaged in the design of recordkeeping systems as well as all other aspects of the life of a born-digital record rather than being passive recipients of whatever survived the vicissitudes of the increasing digital workplace if they were going to have any hope of identifying, capturing, preserving and making a trustworthy and complete record accessible in the long- and even the near-term. Networking of digital records and their collaborative creation and storage across juridical, institutional and public-private boundaries (e.g., in the Cloud) further challenged both the life cycle model and core archival and recordkeeping principles. The development and implementation of the records continuum model in Australia in the 1990s, as delineated by McKemmish in Chapter 4, has influenced conceptualisations of the life of records and dynamics of recordkeeping in several other countries also, notably in Scandinavia. It has also influenced corporate records management, nationally and internationally (in part because of the incorporation of continuum thinking into international records management standards).

Other new worldviews, concepts and archival methods have emerged since the 1990s as a result of applied and theoretical research addressing the nature and management of records created in digital and non-textual media, and the influence of postmodern, postcolonial, gender and sexuality studies, and other critical, historical, political and cultural theoretical movements, as well as Indigenous ways of knowing. These include Indigenous frameworks for contemporary recordkeeping, Queered methodologies for archival practice, and methods such as contemporary archival diplomatics, business process analysis and literary warrant analysis. ${ }^{20}$ The role of archives, whether viewed as enabling, collusive or surveilling, became a central focus of postmodern and postcolonial theorists, community memory initiatives, and human rights and social justice activists. And by the twenty-first century a similar

20 However it should be noted that despite the increase in research rooted in Indigenous epistemologies and post-colonial approaches, much critical and cultural theory continues to reflect a strongly Western orientation. 
focus was clearly evident within the archival field itself. ${ }^{21}$ A growing body of research has been examining the integral role played by archivists and other recordkeepers in colonial, military and other oppressive administrative bureaucracies historically and still today, and not only by western powers. For example, new Japanese business practices, spread outside Japan during the Meiji period and through Japanese expansionism, eradicated much of the traditional and Chinese-influenced practices of Korea. These in turn were overlaid with American recordkeeping practices introduced during U.S. military and political engagement in the Korean peninsula. ${ }^{22}$ Recordkeeping and archives have also been implicated around the globe in political repression in Cold War 'hotspots' and post-colonial dictatorships such as those in Latin America. ${ }^{23}$ It is certainly the case that the latter twentieth century, with the rise of civil rights movements, the Cold War and the regimes and conflicts it nurtured, and the bloody conflicts that erupted in many former Communist European countries with the end of the Cold War, contributed substantially to a new archival focus on social and political documentation. Another developing area of research pursues the pervasive ways in which recordkeeping principles and practices historically privileged official recordkeepers and perpetuated oppressive practices towards women. Government administrators and authority figures (in effect the creators of records that would be recognised in recording their provenance) were historically almost always male in most parts of the world. The records and recordkeeping infrastructures they administered also supported male-dominated systems (government, military, church, courts, property, etc.) that tended to (and in some jurisdictions still) ignore or submerge the presence of women, whether free or slaves, or grant them few rights.

\section{Situating Archival and Recordkeeping Studies}

It is necessary at this point to step back and address the variation in nomenclature that is employed to describe and, by implication, to delineate the field itself. The most commonly used term worldwide, "archival science"

21 For more discussion on this subject, see the articles contained in the 2014 special issue of Archival Science edited by Michelle Caswell on human rights archives.

22 Eunha (Anna) Youn, "Archival Traditions in Korean History: From Medieval Practice to the Contemporary Public Records Management Act." Archival Science 13 (2013): 2344; and "Investigating Socio-cultural Aspects of the Implementation of an International Archival Descriptive Standard in Korea," chapter 26 in this volume.

23 Carlos Aguirre and Javier Villa-Flores, From the Ashes of History: Loss and Recovery of Archives and Libraries in Modern Latin America (Raleigh, NC: Editorial A Contra corriente, 2015). 
[author's emphasis], directly reflects the European Enlightenment thinking that dominated the modern formation of the field. Duranti and MacNeil define archival science as "a body of concepts and methods directed toward the study of records in terms of their documentary and functional relationships and the ways in which they are controlled and communicated,"24 and it is within this conceptualisation that Duranti and Giovanni Michetti write about "the archival method" in this book. ${ }^{25}$ Historically this conceptualisation of the field has been directed to the principles, processes and methods associated with the domain of archival practice. ${ }^{26}$

The establishment in 1777 of an archival education program at the University of Naples marked the beginnings of professional education in archival science in Europe. Western Europe was to be the cradle for such education, which was centred around the ideas and practices native to the archives of state and church in countries such as Prussia, France, Austria and Spain, as well as a growing body of scholarly and legal techniques of document analysis and authentication. The formation of the École des Chartes and subsequently other national archival education programs further formalised the body of knowledge that had come to be known as archival science.

During the nineteenth century, archival science and the archival method gained scholarly traction in the Western academy as a subfield of modern scientific history pioneered by Leopold von Ranke at the University of Berlin, while still maintaining important links to philology and law. As Ketelaar has noted, the term "archivistics," often used interchangeably with archival science, especially in Europe, avoids any confusion with the natural sciences (and by implication, sheds some of the positivistic resonance of "archival science"). ${ }^{27}$ This history notwithstanding, in 2001, Terry Cook, arguing that archival science is neither universal nor immutable, wrote that:

24 Luciana Duranti and Heather MacNeil, "The Protection of the Integrity of Electronic Records: An Overview of the UBC-MAS Research Project," Archivaria 42 (Fall 1996): 46-67.

25 See Luciana Duranti and Giovanni Michetti, “The Archival Method," chapter 2 in this volume.

26 "n. A systematic body of theory that supports the practice of appraising, acquiring, authenticating, preserving, and providing access to recorded materials," Richard PearceMoses, comp. Glossary of Archival and Records Terminology, Richard Pearce-Moses, ed. (Chicago: Society of American Archivists, 2005).

27 "Archival science is a science in the German sense of Wissenschaft, but to avoid confusion with the natural sciences in the Anglo-Saxon meaning, I personally use the term "archivistics," being the equivalent to the Dutch archivistiek, the German Archivistik, the French archivistique, and the Italian and Spanish archivistica. Archivistics consists of theory, practice, and methodology." Eric Ketelaar, "Archivistics 
To North American and Australian archivists, the term "archival science" is so foreign that it finds no place in their extensive published glossaries, and, until very recently and under the impact of imported European ideas, rarely has been mentioned in their professional discourse. Conversely, for many European archivists, "archival science" is deeply ingrained as part of their professional mindset. For example, three leading archivists from three European countries, who have used "archival science" in the title of recent articles designed to explore aspects of the meaning of archival science, do not really define the term, or even explain it, but simply assume that their readers will know what they mean. The term sometimes seem to encompass in such writing, to this untrained North American eye, all the professional knowledge that forms the intellectual discipline of archives, including archival theory, archival history, archival strategy, archival methodology, even diplomatics or aspects of records management. But archival science seems most often equated by these writers with what North Americans think of as "archival theory," and, more specifically, with concepts concerning the arrangement and description of archives in order to protect their provenance or contextual integrity. ${ }^{28}$

The central and distinctive preoccupations of the field in any definition are the record and the notion of evidence, something that is strongly emphasised today in the Australian conceptualisation of government recordkeeping, which derives from a records continuum, rather than a life cycle approach:

The making and maintaining of complete, accurate and reliable evidence of business transactions in the form of recorded information. Recordkeeping includes the creation of records in the course of business activity, the means to ensure the creation of adequate records, the design, establishment and operation of recordkeeping systems and the management of records used in business (traditionally regarded as the domain of records management) and as archives (traditionally regarded as the domain of archives administration). ${ }^{29}$

Research Saving the Profession”, in: American Archivist 63 (2000): 324. See also Ketelaar, Eric. “Archivistics: Science or Art?” In Jennie Hill (ed.), The Future of Archives and Recordkeeping. A Reader (London: Facet, 2011), p. 93.

28 Terry Cook, "Archival Science and Postmodernism," Archival Science 1 (2001): 3-24, http://www.mybestdocs.com/cook-t-postmod-p1-00.htm.

29 National Archives of Australia. Glossary. Term adapted from Standards Australia, AS 4390, Part 1, Clause 4.19; and Part 3, Foreword. http://www.naa.gov.au/recordsmanagement/publications/glossary.aspx\#r 
Or the broader concept of recordkeeping in the continuum defined as:

... a range of intertwined recordkeeping and archiving processes and activities carried out by records managers and archivists for current, regulatory and historical recordkeeping purposes. These purposes include the roles that recordkeeping plays in and through space and time in governance and accountability, remembering and forgetting, shaping identity and providing value-added sources of information. In classificatory terms "recordkeeping" in this usage subsumes records management and archival administration. It also encompasses the personal and corporate recordkeeping activities undertaken by individuals in their everyday lives, in families, work or community groups, and in organisations of all kinds. ${ }^{30}$

"Archival studies" (or more broadly "archival and recordkeeping studies" as used throughout this chapter) is an emerging term in North America that refers to the growing multidisciplinary research and conceptual domain that:

addresses, on one or more levels (societal, organisational, community, group, individual):

texts (regardless of their media and format) that serve to record, document, control and narrate;

axiomatic (cross-cutting) constructs such as memory, culture, identity, accountability, authenticity, enterprise, narrative, and power relations; and

processes such as recordkeeping, selecting/appraising, preserving, remembering, forgetting, (re)presenting, interpreting, and storytelling. ${ }^{31}$

In the second half of the twentieth century, education and training programs began to be established in countries around the world. Sometimes these were as a result of the influence of colonial or former colonial or political

30 Sue McKemmish, Franklyn Herbert Upward and Barbara Reed, "The Records Continuum Model," in Encyclopedia of Library and Information Sciences, Third Edition, Marcia J. Bates and Mary Niles Maack, eds. (Taylor and Francis: New York, 2009).

31 Kelvin White and Anne J. Gilliland. "Promoting Reflexivity and Inclusivity in Archival Education, Research and Practice," Library Quarterly 80 no.3 (July 2010): 231-248; Anne J. Gilliland and Kelvin White, "Perpetuating and Extending the Archival Paradigm: The Historical and Contemporary Roles of Professional Education and Pedagogy," InterActions: UCLA Journal of Education and Information Studies Vol.5, Issue 1 (2009). http://interactions.gseis.ucla.edu/ 
relationships; sometimes they were nurtured by UNESCO initiatives or by distance education programs offered by European, North American and Australian universities; sometimes they developed completely independently in response to local needs. With an increasing focus on archival science as a distinct profession and pressure on the profession to address technology concerns, there was a concomitant shift in the latter half of the twentieth century as the field's academic base in North America, Australia, and some parts of Europe and Asia away from history programs. Instead they aligned themselves with professional information programs such as those in library and information science and information and knowledge management. ${ }^{32}$

From the mid-1990s, those professional programs began diversifying in scope while converging with computer science, management, communications, business, and digital humanities programs, among others, around information phenomena, technologies and contexts. The 1990s also gave rise to the emergent concept of the $i \mathrm{School}$ (i.e., information School), which would make rapid gains in the early years of the new millennium. ${ }^{33} 34$ The iSchools' blending of professional, disciplinary and technological areas led to a heightened emphasis on scholarship and a notable trend toward methodological pluralism. Not only were the methods traditionally associated with each of these fields brought into proximity with one another, but there was also a growing awareness that the complexity of information phenomena under investigation demanded multi-method approaches as well as methodological adaptation and innovation. This expansion in scope and approach contributed considerably to a fertile and more receptive climate for scholarship in archival and recordkeeping studies than previously. ${ }^{35}$

32 That said, in some regions, for example in central and southeastern Europe, archivists are still drawn from history or sometimes, theological, philological or linguistic studies, and their scholarship often has a strong historical orientation.

33 Many former library and information science programs have transformed themselves since the 1990s into information studies or information sciences programs, whether or not they are formally members of the $i$ Schools Caucus.

34 iSchools "take it as given that expertise in all forms of information is required for progress in science, business, education, and culture. This expertise must include understanding of the uses and users of information, the nature of information itself, as well as information technologies and their applications," http://ischools.org/about/ charter/the-purpose-of-the-ischools/.

35 See White and Gilliland, "Promoting Reflexivity and Inclusivity"; and Anne Gilliland and Kelvin White, "Perpetuating and Extending the Archival Paradigm: The Historical and Contemporary Roles of Professional Education and Pedagogy," InterActions: UCLA Journal of Education and Information Studies 5 no.1 (2009), http:// interactions.gseis.ucla.edu/. 
As discussed by Ketelaar in this volume, also occurring over the past several decades has been the so-called "archival turn" in the humanities, arts, and social sciences that has had at its centre postmodern and postcolonial epistemological, political and cultural constructions of the Archive. However, such scholarship has often been critical of or simply uninformed about the evolving conceptual bases and practices of archival science, as also has the developing fields of digital humanities and data archiving. The expansion of archival and recordkeeping studies (in particular, the challenging and redefinition of fundamental concepts within archival science such as "the record" and the "the archive," as well as of assertions about evidence, authority, and historical truth) in part constitutes an academic response to, and to some extent, incorporation of, the archival turn. In so doing, scholars in archival and recordkeeping studies have increasingly, and sometimes controversially, incorporated epistemological frameworks and textual and anthropological methods being applied by postmodern, postcolonial, and race, ethnicity and gender studies scholars, among others, that eschew the positivist claims of validity that underpin methodological approaches employed in scientific history, diplomatics or legal theorising about evidence. ${ }^{36}$

While archival and recordkeeping studies programs and departments within the academy still largely focus on preparing professionals for archival and recordkeeping careers, and increasingly also on doctoral education which will prepare future academics and researchers-in-practice, archival and recordkeeping studies as a rubric additionally serves as a potential bridge with scholars engaged in the "archival turn." Similarly, there is also a growing number of important areas of intersection with other professional

36 It is worth noting that while today many of these epistemological approaches are still criticised as lacking in rigour and validity within the academy, modern scientific history also went through its own tribulations in terms of achieving epistemological recognition. Political theorist and historian, and early proponent of objective value pluralism Isaiah Berlin noted that by the early seventeenth century, French philosopher and mathematician René Descartes:

had already denied to history any claim to be a serious study. Those who accepted the validity of the Cartesian criterion of what constitutes rational method could (and did) ask how they could find the clear and simple elements of which historical judgments were composed, and into which they could be analysed; where were the definitions, the logical transformation rules, the rules of inference, the rigorously defined conclusions? While this confused amalgam of memories and travelers' tales, fables and chroniclers' stories, moral reflections and gossip, might be a harmless pastime, it was beneath the dignity of serious men seeking what alone is worth seeking - the discovery of truth in accordance with the principles and rules which alone guarantee scientific validity.

Isaiah Berlin, "History and Theory: The Concept of Scientific History," History and Theory 1 no.1 (1960): 1-31. 
fields and communities that are influencing the scope and conceptualisation of archival studies. Figure 1.1 provides one of many possible representations of how the global situation of contemporary archival studies and intersecting fields and communities might be viewed at this moment. This particular representation places archival studies at the centre of the domain. ${ }^{37}$ If recordor indeed memorykeeping were to be placed in the centre, or if the same figure were to be drawn during previous periods or within a single country or region, the other fields and communities might be differently placed, or others that are entirely different might be represented.

Figure 1.1: One Representation of Contemporary Archival Studies and Intersecting Fields and Communities

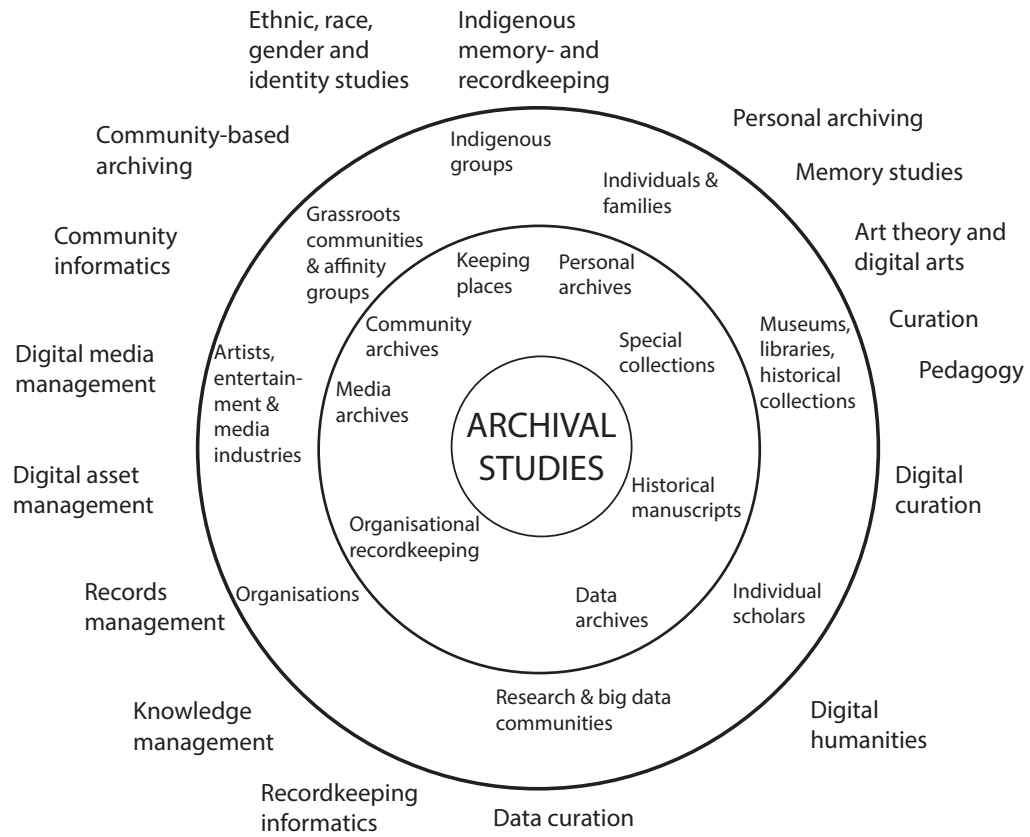

\section{The Archival Multiverse}

As already discussed, professional archival and recordkeeping standards and practices reference a paradigm comprising a core set of principles, concepts and also ethics, largely articulated in Western Europe and the

37 Taken from Anne J. Gilliland, "An Archival Paradigm for the Twenty-first Century," invited paper, Desafios arquivisticos contemporâneos: seminário em torno dos 40 anos de CPDOC, Rio de Janeiro, Brazil, August 2013. 
Northern hemisphere over the past three centuries, although drawing more recently upon Australian records continuum thinking and practices, especially as a result of Australian influence on the ISO Records Management standards. This paradigm provides a basis for communication and standardisation across the archival and recordkeeping communities worldwide and serves as its epistemological representation when interacting with other fields that share common interests or goals. ${ }^{38}$ The benefit of having such a paradigm is that it allows a field to state what it is and what it is not, as well as what its primary objects of concern are and are not in order to demarcate professional domain and expertise. Expanding its scope, or making its boundaries more liminal, however, runs the risk of stretching the theoretical and applied space occupied by a paradigm to the point where it becomes difficult to see any core or to discern its limits. Nevertheless, as already illustrated, professional, technological, intellectual or ideological developments and cultural differences and ethical exigencies, as well as the resilience and resistance of many marginalised archival and recordkeeping practices will always push against an overly constraining paradigm. So too does the framework of the archival multiverse since it indicates ways in which this core perpetuates privileging the mainstream archival and recordkeeping practices of governments, the corporate sector, religious and cultural institutions, and contributes to the continued marginalisation of local, community and Indigenous practices. ${ }^{39}$

In July 2009, the inaugural Archival Education and Research Institute (AERI) was held at the University of California, Los Angeles (UCLA). ${ }^{40}$ This landmark event was the first of what went on to become annual institutes that have brought together faculty and doctoral students from

\section{Gilliland, "Enduring Paradigm."}

39 Gilliland, "Enduring Paradigm."

40 AERI is one component of a broader ongoing collaborative initiative, Building the Future of Archival Education and Research. Phases I and II of AERI were funded through grants from the US Institute for Library and Museum Services (IMLS). The initiative seeks to stimulate the growth of archival studies as a field within the United States and worldwide by nurturing and promoting state-ofthe-art scholarship, as well as encouraging curricular and pedagogical innovation. Centred at UCLA, it is led by an international consortium of academic institutions that offer or plan to develop a doctoral specialisation in archival studies. Founding members were UCLA; University of Michigan; University of Pittsburgh; University of Maryland; University of Texas at Austin; University of North Carolina, Chapel Hill; University of Wisconsin, Madison; and Simmons College. AERI has also received significant in-kind support from universities elsewhere in North America, Europe, Asia and Australia, especially Monash University in Melbourne. See aeri.website. 
around the globe whose scholarship is focused in archival and recordkeeping studies, broadly conceived. AERI, expanded in recent years into the Archival Education and Research Initiative has evolved into a forum and international community where those engaged in archival research and education can present and critique research, network with their peers. ${ }^{41}$ They also work together both inside and outside the annual institutes to develop the infrastructure necessary to make archival and recordkeeping studies a robust presence within the academy and a rigorous source of theoretical and empirical support for the archival profession. It was through this infrastructure-building work that an AERI working group formulated the concept of the archival multiverse as central to an overarching pluralising framework within which to situate professional and research education and scholarship in archival and recordkeeping studies. ${ }^{42}$ That framework was codified in an influential 2011 article co-authored by twenty-six faculty members and doctoral students from academic institutions around the world and published in The American Archivist. The article defined the archival multiverse as encompassing:

The pluralism of evidentiary texts, memory-keeping practices and institutions, bureaucratic and personal motivations, community perspectives and needs, and cultural and legal constructs with which archival professionals and academics must be prepared, through graduate education, to engage. ${ }^{43}$

One of the least explored aspects of the archival multiverse ${ }^{44}$ is the plurality of archival traditions with distinct epistemological, ontological, ideological, practical, even linguistic aspects at work within the contemporary professional archival and recordkeeping landscapes as well as within different communities of records that carry out record- and

41 Indeed, several of the multi-authored papers in this book are the result of collaborations that were formed as a result of AERI attendance.

42 Please see the dedication to this book, and its discussion of the role played by the late Allison Krebs in introducing the utility of the concept of the multiverse in the archival context.

43 Archival Education and Research Institute (AERI) Pluralizing the Archival Curriculum Group (PACG), "Educating for the Archival Multiverse," American Archivist (Spring/Summer 2011): 73.

44 Defined here in the same way as in the Preface of this book as the pluralism of evidentiary texts, memory-keeping practices and institutions, bureaucratic and personal motivations, community perspectives and needs, and cultural and legal constructs with which archival professionals and academics must be prepared, through graduate education and through research and development, to engage. 
memory-keeping functions outside professional archival purview. ${ }^{45}{ }^{46}$ This often under-recognised plurality has important implications both for research in comparative archivistics that seeks to identify and understand differences and similarities across traditions and settings, and for appropriately framing, designing and interpreting research situated within one or more traditions or settings, whether those be of the past, the present or the future. ${ }^{47}$ As the introduction to this chapter as well as the wide range of diverging definitions and arguments provided by individual chapters in this volume suggest, the conceptualisations and manifestations of records and archives, as well as of actions and transactions, practices, concepts and assumptions associated with archives and recordkeeping, can vary significantly - and for significant reasons. Professional archival and recordkeeping traditions in different countries and sectors have converged around certain aspects of theory

45 Bastian coined the term "community of memory" during her own research into the loss of access of the people of the Virgin Islands to their archives and the effect that had upon their ability "to write their own history and construct their collective memory." Such an experience, she argues "also concentrates our attention on the general relationships between records and the communities that create them, between records and memory and between memory and access ... It suggests that, in order to use records as reliable indicators of an entire society, both the subjects as well as the creators of the records must be seen as active participators in a process in which record creating is defined as much by place, people, and community as it is by the act of creation itself." Jeannette A. Bastian, Owning Memory: How a Caribbean Community Lost Its Archives and Found Its History (Contributions in Librarianship and Information Science), Libraries Unlimited, 2003, pp.1-2. In other words, archives are but one very small component of a complex of interacting and interdependent ways in which society remembers and retains and maintains memory-Harris' "archival sliver." Verne Harris, "The Archival Sliver: A Perspective on the Construction of Social Memory in Archives and Their Transition from Apartheid to Democracy," in Carolyn Hamilton et al., eds. Refiguring the Archive, Dordrecht: Kluwer, 2002, pp.135-151. See also Sue McKemmish, Anne GillilandSwetland, and Eric Ketelaar, "Communities of Memory': Pluralising Archival Research and Education Agendas," Archives and Manuscripts 33 (2005): 146-175 and Jeannette A. Bastian and Ben Alexander (eds.), Community Archives. The Shaping of Memory (London, Facet 2009).

46 Published work in comparative archivistics has primarily been authored by those who have been deeply engaged in national archives and government recordkeeping and national and international juridical frameworks such as Frank Burke and Eric Ketelaar.

47 Eric Ketelaar writes that, "Archiving and archivalization are influenced by social, religious, cultural, political and economic contexts. These may vary in any given time and in any given place. That challenges archivistics to be a comparative science. Comparative archivistics is more than treating and teaching a subject from an international and multicultural perspective, since it asks for ethnography followed by ethnology, for 'what' followed by 'why'. Comparative research should be carried out in the present, cross-cultural and cross-societal, but also in the past." Ketelaar, "What is Archivistics or Archival Science?" http://cf.hum.uva.nl/bai/home/eketelaar/ information.html. Ketelaar further expands on this in "The Difference Best Postponed? Cultures and Comparative Archival Science," Archivaria 44 (1997): 142-148. 
and practice as a result of influential figures and publications, the need to address technological developments, and the design and implementation of professional standards and best practices. In other respects, though, they have retained elements distinctive to their own contexts or have even diverged. They may reference the same paradigm, but they may approach it with different semantics, through different linguistic expressions, and from different ontological, epistemological and political perspectives. Moreover, they may function within entirely different epistemologies. For example, in certain societies and institutions, the traditions must take into account spiritual, metaphysical or emotional dimensions which are often entirely absent from internationally recognised best practices and standards. ${ }^{48}$

An uncritical and unanalysed acceptance of the notion of an inclusive and universally and similarly understood paradigm is an inaccurate and misleading basis for comparative archivistics, for trans-tradition theorising, for applied developments such as standards design, and indeed for archival and recordkeeping education and nurturing ethical understandings. Professional best practices and international standards and glossaries notwithstanding, each archival and recordkeeping tradition, as both Ketelaar and Eastwood have noted, ${ }^{49}$ whether regional, national, or within a particular community

48 For example, Confucian and Taoist ideas about propriety, humaneness, rite, loyalty and filial piety; Hindu ethical and metaphysical ideas about social and cosmic purpose; and the medicine wheel used in certain Native American spiritual traditions to represent harmony and connections between people and ways. See Meung Hoan Noh, "Confucian Community Construction and Records/Archives Management of Joseon Dynasty - focused on the Meaning of the Uigwe Production, Preservation, and Use," paper presented at the Archival Education and Research Institute, University of Michigan, Ann Arbor, July 2012, https://aeri2012.wordpress.com/conference-schedule/ paper-presentations/community-archiving/; Eunha Youn, Standardization of Archival Description in Korea: Examining the Understanding, Adoption, and Implementation of ISAD (G), Ph.D. dissertation (Los Angeles: University of California Los Angeles, 2011); Marcia H. Chappell, "The Place of Reference Service in Ranganathan's Theory of Librarianship," Library Quarterly 46 no.4 (1976): 378-96; Marisa Elena Duarte and Miranda Belarde-Lewis, "Imagining: Creating Spaces for Indigenous Ontologies," Cataloging \& Classification Quarterly special issue on Indigenous Knowledge Organization, 53 nos.5-6 (2015): 677-702; Scott Cline, “'To the Limits of Our Integrity': Reflections on Archival Being," American Archivist 72 no.2 (2009): 331-43. See also a critique of the records continuum in Anne J. Gilliland, Conceptualizing Twentyfirst-century Archives (Chicago, IL: Society of American Archivists, 2014) and Anne J. Gilliland and Marika Cifor, eds. special issue of Archival Studies on Affect and the Archive (forthcoming, 2016).

49 See Ketelaar, "What is Archivistics or Archival Science?" and "The Difference Best Postponed?"; and Terry Eastwood, "Reflections on the Development of Archives in Canada and Australia," in Sue McKemmish and Frank Upward, eds. Archival Documents Providing Accountability Through Recordkeeping (Melbourne: Ancora Press, 1993), pp.27-39. 'Archival tradition' is defined here as the manifestations, behaviours and understandings resulting from the historical, cultural and 
or institutional setting, retains a high degree of contingency on a distinctive fusion of legal, cultural, social, historical, political and economic factors. ${ }^{50}$ Professional and conceptual archival discourse and the legal frameworks in which that discourse is situated often draw definitional parameters around what is "a record" and what is "an archive" that exclude certain practices and settings in which record- and memory-keeping take place. To take the case of the definition of a record - the International Council on Archives, drawing directly from the 2001 International Organization for Standardization (ISO) standard, Information and Documentation: Records Management. 15489-1. Part 1 defines it as:

Information created, received, and maintained as evidence and information by an organization or person, in pursuance of legal obligations or in the transaction of business and inclusive of records of continuing value (archives). ${ }^{51}$

Successive InterPARES Projects investigating the creation and preservation of electronic records over the past two decades have cumulatively defined a record as:

n., A document made or received in the course of a practical activity as an instrument or a by-product of such activity, and set aside for action or reference. (See also: Ontology A) [and] n., A document made or received and set aside in the course of a practical activity. [InterPARES 1 Glossary]. ${ }^{52}$

These particular examples, because they all developed through formalised international consensus-building processes, are arguably among the closest articulations of the ideas that are fundamental to the elusive archival paradigm and the primary business of archives and recordkeeping. It could be questioned, however, the extent to which even these are based upon common understandings, since subtle semantics of terminology often get lost in translations between different languages or are even removed by over-zealous publication editors in the name of editorial consistency. For

socio-political lineage and influences, accumulated and evolving ideas, recordand memory-keeping practices, relevant juridical framings and requirements and experiences particular to a specific local, national or regional formalised professional archival environment.

50 See, for example, Youn's chapter in this volume regarding the Korean archival tradition.

51 International Council on Archives, Multilingual Archival Terminology Database, http:// www.ciscra.org/mat/termdb/term/60.

52 InterPARES3 Project, Terminology Database, http://www.interpares.org/ip3/ip3_ terminology_db.cfm. 
example, as already discussed, the Australian term "recordkeeping" and the term "record-keeping" used in the United States and in many other locations have very different meanings. Many other languages do not even have separate words for archives and records, or archiving and record-keeping, never mind the ability to distinguish between recordkeeping and record-keeping. This is a particularly important example because of the prominence of English (in different national forms) in the articulation of archival and recordkeeping concepts. There are also additional or alternate definitions being applied at different national, institutional and community levels. Yeo challenges many of the attempts to define records in terms of evidence or information and argues that they fail to encompass the relationship of records also to memory and cultural identity. He offers instead a definition of records as "persistent representations of activities, created by participants or observers or their authorized proxies" that is multidisciplinary and embraces "a wide spectrum of understanding." ${ }^{33}$ Many records are routinely understood, collected and exhibited as artefacts and thus find their way into museums, galleries and private collections. They might be early manifestations of what would in time become a more formalised written recordkeeping tradition (as is the case with early Chinese records inscribed on bone and shell), but they could equally exist in the present, especially in community and counter-archival settings that ascribe a particular value to objects qua records. An example would be the use of cloth arpilleras made by Chilean women to document their own experiences under the repressive Pinochet regime when opposition voices were violently silenced ${ }^{54}$ and the proliferation of this practice by women in other post-dictatorial and post-conflict contexts as diverse as Spain, Burma, South Africa and Northern Ireland. ${ }^{55}$

Even with such variation and attempts at broadening, fixity, persistency (in the sense of "enduring") and textuality (narrowly defined) nevertheless remain hallmarks of many professional definitions. ${ }^{56}$ Such definitions exist

53 Geoffrey Yeo, "Concepts of Record (1): Evidence, Information, and Persistent Representations," American Archivist 70 (2007): 315-343; quotes pp.342 and 343.

54 Kristen Walker "Chilean Women's Resistance in the Arpillera Movement", CETRI Le Sud en Mouvement (30 October, 2008), http://www.cetri.be/Chilean-Women-sResistance-in-the?lang=fr. See also Cachando Chile: Reflections on Chilean Culture, "Chilean Arpilleras: A Chapter of History Written on Cloth," (September 11, 2010), https://cachandochile.wordpress.com/2010/09/11/chilean-arpilleras-a-chapter-ofhistory-written-on-cloth/.

55 Roberta Bačić, "Arpilleras that Cry Out, Sing and Challenge," Magazine of the United Nations Development Program (n.d.), pp.22-22, http://escolapau.uab.cat/img/programas/ musica/chilean_tapestries.pdf.

56 In February 2015, the United States Supreme Court struck down a lower court 
in large part to address as unambiguously as possible, practical, legal and technological requirements. But we should not lose sight of the fact that at the same time they effectively create problematic bifurcations. Institutionalised and professionalised record- and memory-keeping practices are depicted as paradigmatically evolving in relation to tangible forms and formats of the bureaucratic record. The practices of oral societies and other communities that do not predominantly use textual forms of record- and memorykeeping are portrayed as centred exclusively around intangible or embodied record- and memory-keeping structures (for example, those employing storytelling, ritual, song or dance). ${ }^{57}$ Such bifurcations fail to acknowledge how the function of record- and memory-keeping may be carried out through intangible, non-fixed forms that do not necessarily conform to western notions about time or permanence. These kinds of bifurcations also, as Wareham has demonstrated, over-simplify the complex realities of societal recordkeeping and remembering. They underplay the prevalence of so-called tangible forms in societies in which oral traditions are more dominant; and of intangible forms in many other societies (characterised as literate) throughout time. They also submerge situations where oral and artefactual traditions have been maintained or have emerged specifically to ensure the presence and preservation of voice, as an expression of identity, or as a form of resistance (for example, among African slaves and their descendant communities ${ }^{58}$ or in certain communities of women). Different

ruling based upon the Sarbanes-Oxley Act that makes it a crime to destroy, alter or cover up "any record, document, or tangible object with the intent to ... impede or obstruct" the investigation of "any matter within federal jurisdiction." The National Oceanic and Atmospheric Administration had accused a fisherman of catching under-sized fish and agued that he destroyed evidence when he disposed of two of the fish in question. The court ruled that the "government overreached by deploying the financial fraud law against a commercial fisherman. The majority said tangible objects should be read to mean documents or computer hard drives not undersized fish." Justice Ruth Bader Ginsburg remarked in a summary of her opinion from the bench, "Fish one may fry, but may one falsify, or make a false entry in the sea-dwelling creatures?" Krishnadev Calamur, "Supreme Court Sides With Fisherman In Case Of The Missing Fish,” National Public Radio broadcast (February 25, 2015), http://www.npr.org/sections/thetwoway/2015/02/25/389005176/supreme-court-sides-with-fisherman-in-case-of-themissing-fish. The Society of American Archivists' Glossary clearly asserts that, "a record has fixed content, structure and context." Richard Pearce-Moses, comp., "Records," Glossary (Chicago: Society of American Archivists, 2005). http:// www2.archivists.org/glossary/terms/r/record.

57 These are discussed in several other chapters of this book, including those by Faulkhead, Thorpe and White.

58 See for example, Kelvin L. White, The Dynamics of Race and Remembering in a 'Colorblind' Society: A Case Study of Racial Paradigms and Archival Education in Mexico, 
record- and memory-keeping epistemologies and practices have inevitably interacted and been cross-influenced throughout human history. As already discussed, the amount of real dynamism that exists in the content and context as well as the fixity of records as they move through time and space is often over-simplified or under-conceptualised. Indeed it has taken the transition into pervasive digital and networked recordkeeping to force more complexification and nuance into professional conceptualisations of fixity, temporality and materiality as these pertain to the definition of a record. ${ }^{59}$ One would assume, by extension, that this should lead the professional field to reconsider its historical rejection of "intangible" practices such as those of predominantly oral communities as well as within "literate" societies as recordkeeping practices.

There are other examples of hybrid of layered traditions resulting, for example, from a desire to adopt "modern" or enterprising practices, or through the implementation of new technologies and international standards. Such impositions rarely completely eradicate all traces of prior practices, although they can severely damage, disempower or disable them, especially if a prior system of recordkeeping goes unrecognised or is disrespected by those imposing the new one. ${ }^{60}$ This is not the only dubious or at least permeable binary that has been set up through such definitions. McKemmish and Piggott discuss what they call the "binary opposition" between personal and organisational archives. ${ }^{61}$ Others include the binaries between organisational and collecting archives, and between professional and community archiving practices. Community archives, which have become an increasingly present element in the archival and recordkeeping landscapes in many parts of the world, exemplify a range of less conventional and hybrid models of recordand memory-keeping, especially for communities who have traditionally been submerged, ignored or silenced through official archives and their practices. $^{62}$

doctoral dissertation (Los Angeles: University of California Los Angeles, 2008).

59 Amelia Acker's chapter, "When is a Record? A Research Framework for Locating Electronic Records in Infrastructure," in this volume provides an excellent exemplar of this complexification.

60 Evelyn Wareham, "From Explorers to Evangelists: Archivists, Recordkeeping, and Remembering in the Pacific Islands," Archival Science 2 (2002): 187-207.

61 Sue McKemmish and Michael Piggott, "Toward the Archival Multiverse: Challenging the Binary Opposition of the Personal and Corporate Archive in Modern Archival Theory and Practice", Archivaria 76 (2013): 111-144.

62 See, for example, Andrew Flinn, "The Impact of Independent and Community Archives on Professional Archival Thinking and Practice." In Hill, J., ed. The Future of Archives and Recordkeeping (London: Facet Publishing, 2011); Anne 
The position that this chapter advances, therefore, of there being multiple traditions and possibly multiple paradigms, and of the need for a tolerance of multiplicity, is certainly a multiverse position. Extracting from the previous discussion, this chapter argues that for several reasons, the field needs to be aware of different conceptualisations and nuances:

- There must be concern about cultural resilience. Both recordkeeping and information technology can be strongly hegemonic forces. There is considerable incentive for all to resolve to a single set of practices in order to facilitate exchange of data, records and knowledge. Employing the same practices also makes adoption of pre-packaged solutions simpler (of course, this is never truly the case, since local variations and workarounds intervene in all sorts of unofficial or unacknowledged ways). Education can also be streamlined to prepare professionals in the same practices and modes of thinking. All of this, however, intentionally submerges and ultimately kills off local ways of thinking and doing. Local ecologies remain important.

- When we are all doing things in the same way, or are approaching our work from the same perspective (e.g., based on our class, gender identity or sexual orientation) we all tend to err with the same biases, and therefore systematically disempower the same communities and experiences everywhere. A certain amount of heterogeneity in the backgrounds of archivists and archival researchers perhaps diminishes the likelihood of this effect.

- As experiences with community-based archiving have demonstrated, not everyone needs archives or recordkeeping to behave in the same way or to achieve the same objectives.

- As the various Indigenous protocols for archival materials and practices remind us, there is a need to promote mutual understanding and respect between communities that have very different epistemologies, ontologies, experiences, needs and well-being and to acknowledge incommensurabilities.

J. Gilliland and Andrew Flinn. "The Wonderful and Frightening World of Community Archives: What Are We Really Talking About?” Keynote address, Nexus, Confluence, and Difference: Community Archives meets Community Informatics: Prato CIRN Conference Oct 28-30 2013, Larry Stillman, Amalia Sabiescu, Nemanja Memarovic, eds, Centre for Community Networking Research, Centre for Social Informatics, Monash University. http://www.ccnr.infotech.monash.edu.au/ assets/docs/prato2013_papers/gilliland_flinn_keynote.pdf; Rebecka Sheffield, The Emergence, Development and Survival of Four Lesbian and Gay Archives, Ph.D. dissertation (Toronto: University of Toronto, 2015). 
- Widely different legal systems (e.g., civil law, common law, Islamic law [sharia], tribal law, canon law, positive vs. natural law), constructed through different and often conflicting worldviews, belief systems and power structures exist around the world and in different communities. Archives and recordkeeping juridical notions and instituional stances and practices are closely associated with these frameworks and also how those frameworks view or treat alternate or different frameworks.

- The professional archival paradigm and even more inclusive models such as the records continuum omit spiritual elements that are integral in many other cultures, and fail to acknowledge that not only Indigenous but also Judaeo-Christian, Confucian and other religious assumptions and spiritual beliefs permeate relevant behaviour, social relations and business practices. They affect, for example, what trust means and how it is established in any kind of relationship or action, or even who can communicate with who and how; or notions of originality or authenticity.

- The affects of records and recordkeeping practices for different individuals and communities in and over time are underrecognised and thus under-addressed.

- As archival and recordkeeping education has become increasingly focused on the implementation and effects of technology and standards in an increasingly global archival and recordkeeping ecosystem, not enough emphasis is being placed on understanding the history and reasons for different archival traditions.

\section{AERI and Societal Grand Challenges}

Understandings and awareness of the multiple traditions and plurality of the archival multiverse need to be supported by the implementation of pluralised research and education agendas that address, among other things, the situated contexts of archival and recordkeeping thinking and practices. The AERI community has argued that concerted transformative research and development relating to archival and recordkeeping imperatives, frameworks, processes, technologies and standards can contribute in significant ways to addressing many of society's most pressing grand challenges. ${ }^{63}$ It can also

63 Anne J. Gilliland and Sue McKemmish. "Recordkeeping Metadata, the Archival Multiverse, and Societal Grand Challenges," Proceedings of the International Conference 
raise and address grand challenges specific to recordkeeping. Recordkeeping is a fundamental infrastructural component of administrative, economic, scientific, technical, clinical, developmental, educational, governance, social, community and personal systems and evidentiary functions. As a growing body of research in recent decades has amply demonstrated, recordkeeping is also integral to key societal processes such as cultural and community sustainability, identity formation, reconciliation and recovery, and remembering and forgetting. Discourse about grand challenge research has argued that research that is most likely to be effective will be "authoritative," "collaborative" and "competitive," 64 and that what it will take is "awareness, acceptance and adaptation" ${ }^{\prime 5}$ - important precepts for the archival and recordkeeping research field to bear in mind. AERI's ongoing Research Grand Challenges initiative, begun in 2011, has four goals:

1. To identify ways in which recordkeeping is an integral consideration in societal grand challenges, ${ }^{66}$ citizen-centred grand challenges ${ }^{67}$

on Dublin Core and Metadata Applications 2012, Kuching, Malaysia, September 2012, 106-115, http://dcpapers.dublincore.org/pubs/article/view/3661.

64 N.C. Roberts, "Wicked Problems and Network Approaches to Resolution," International Public Management Review, 1 no.1 (2000).

65 Wei-Ning Xiang, "Editorial: Working with Wicked Problems in Socio-ecological Systems: Awareness, Acceptance and Adaptation," Landscape and Urban Planning 110 (2013): 1-4, http://www.spatialcomplexity.info/files/2013/01/Working-with-wickedproblems-2013.pdf.

66 "Societal grand challenges and what are sometimes referred to as 'wicked problems' are complex, multifaceted and widely recognized fundamental problems with broad applicability that require extraordinary breakthroughs and the engagement of multiple areas of expertise to address." U.S. National Science Foundation Advisory Committee for Cyberinfrastructure Taskforce on Grand Challenges, 2011, www.nsf. gove/cise/aci/taskforces/TaskForceReport_GrandChallenges.pdf. "Grand Challenges Can: Help create the industries and jobs of the future; Expand the frontiers of human knowledge about ourselves and the world around us; Help tackle important problems related to energy, health, education, the environment, national security, and global development; and Serve as a "North Star" for collaboration between the public and private sectors." 21st Century Grand Challenges, U.S. White House Office of Science and Technology Policy, https://www.whitehouse.gov/administration/eop/ostp/grandchallenges.

67 PublicSphere.org has been promoting a civil society platform to "help build a more effective platform for these efforts, to help address humankind's shared problems such as environmental degradation, human rights abuses, economic injustice and war that other sectors, notably government and business are seemingly powerless to stem," PublicSphere.org. 
wicked problems, ${ }^{68}$ super-wicked problems,${ }^{69}$ and messes and social messes $^{70}$ that have been identified by other communities, as well as by different nations and internationally;

2. To suggest some specific aspects of grand challenges that might be addressed through research in archival and recordkeeping studies;

3. To draw attention to complex, interdependent and persistent problems that might be characterised as grand challenges facing the field of archival and recordkeeping studies itself; and

4. To identify infrastructure that needs to be developed within the field to support such research.

In work that has been conducted so far in AERI sub-groups addressing several selective areas (Corporate Governance and Social Responsibility, Climate Change, Global Health, Human Rights and Social Justice, the Information Society, and Peace and Security), it has become apparent that similar concerns often surface across multiple areas, even if they are positioned

68 Wicked problems have been characterised as follows: The solution depends on how the problem is framed and vice versa (i.e., the problem definition depends on the solution). Stakeholders have radically different world views and different frames for understanding the problem. The constraints that the problem is subject to and the resources needed to solve it change over time. The problem is never solved definitively. Solutions require widescale changes in mindsets and behaviours. Many wicked problems can be found in economic, environment, political and health arenas and include climate change, public health crises, international drug trafficking, social injustice, and nuclear weapons. See Horst W. J. Rittel and Melvin M. Webber, "Dilemmas in a General Theory of Planning," Policy Sciences 4 (1973): 155-169 and Xiang, "Editorial: Working with Wicked Problems".

69 In an article on climate change, Levin, Cashore, Bernstein and Auld defined super wicked problems as having the following characteristics: Time is running out; no central authority; those seeking to solve the problem are also causing it; and policies discount the future irrationally. Kelly Levin, Benjamin Cashore, Steven Bernstein and Graeme Auld, "Overcoming the Tragedy of Super Wicked Problems: Constraining our Future Selves to Ameliorate Global Climate Change," Policy Sciences 45 no.2 (2012): 123-152.

70 Horn (2007) explains that, "A Social Mess is a set of interrelated problems and other messes. Complexity - systems of systems - is among the factors that makes Social Messes so resistant to analysis and, more importantly, to resolution.” He identifies several characteristics:

no unique "correct" view of the problem; different views of the problem and contradictory solutions; most problems are connected to other problems; data are often uncertain or missing; multiple value conflicts; ideological and cultural constraints; political constraints; economic constraints; often a-logical or illogical or multi-valued thinking; numerous possible intervention points; consequences difficult to imagine; considerable uncertainty, ambiguity; great resistance to change; and, problem solver(s) are out of contact with the problems and potential solutions.

Horn, "Mess Map Table of Contents," http://www.stanford.edu/ rhorn/a/kmap/mess/ tocMessMaps.html 
differently depending upon the interests and perspectives of the framers. By implication, therefore, any given concern might call for very different kinds of research in terms of the nature and scope of the questions being investigated, the backgrounds and expertise of the researchers, the research designs being applied, and whether or not the research has a finite goal or is ongoing. This finding also indicates that there is a core set of recordkeeping/ archival concerns that surface repeatedly in connection with multiple grand challenges, thus suggesting some particularly fertile areas where a research concentration might prove to be transformative. These include cultural and community considerations relating to recordkeeping, archives and memory (e.g., those concerned with cultural sustainability); the role and use of records in supporting accountability, sustainability, decision-making and program assessment; education and capacity building in archival and recordkeeping skills; best practices and standards development; compliance management; scalable systems and services infrastructure development; metadata implementations and implications; promoting open access to archives while addressing privacy and security concerns/vulnerabilities; and, perhaps most prominently, global integration of archival systems and holdings.

\section{Conclusion}

Notwithstanding this discussion of a grand challenge research agenda, the history and nature of the diversity in archival and recordkeeping traditions is a rich subject for research in its own right. It is also an important aspect to acknowledge and account for when undertaking research within or across traditions or settings. The increasingly postcolonial, and in some cases neocolonial and re-colonised and globalised world that we inhabit today is a very different place from Western Europe a century and more ago from whence many archival ideas and practices emerged, or even from the 1990s when the first international standards for archival description and code of ethics were developed. There is a need for critical examination of the tensions not just between global interactivity and exchangeability and local practices and individuality, but also of the power dimensions at work in these developments and the extent to which pluralism and liberation can be built into them. As archival and recordkeeping concerns and technologies inevitably become more globally engaged and oriented, it is going to be essential to understand the dimensions and impacts of encounters between different traditions and on the communities of affected stakeholders. It also necessitates that we prioritise looking at old principles in new ways, and at how then to implement 
the conclusions that we reach when practises and systems have become so embedded and have such an installed technical base that they are going to be very difficult to change or even to throw out and start anew. For example, as we come to understand more about the operations and interaction effects of parallel and hybrid recordkeeping systems and also seek to address the rights of co-creators of and other stakeholders in the records thereby created, whether we are interested in recordkeeping informatics, Indigenous memorykeeping, or postcolonial archival studies, we are immediately challenged to address dominant notions of single provenance, agency and authority.

There are various reasons why archival researchers have not been more engaged with this plurality, among the more obvious being difficulties in finding out about and accessing research and professional literatures across language and dissemination venues (as discussed in the Preface); the historically small number of archival and recordkeeping scholars within many countries and communities; and the paucity of forums where archival scholars might come together to present their work and to have it critiqued by an international audience. There is another problem, already alluded to in this chapter - that of conceptual and definitional drift and an associated paucity of critical reflexivity and semantic precision. Over time archival and recordkeeping practices can shift in sometimes obvious, sometimes subtle, but often in unrecognised or unacknowledged ways. ${ }^{71}$ Sometimes it is because of a translation problem (much of the research and standards literature is published in English or other European languages and finely nuanced meaning can get lost in translation. It might also be the case that no conceptual or semantic but instead a misleading linguistic equivalent exists in the other culture). Sometimes it is because archivists have grasped too quickly at phenomena that appear at first glance to be familiar, but turn out to have quite different aspects within their own setting. And sometimes it results from a lack of knowledge of the field's own history, cultural, political and institutional biases, and professional thinking. ${ }^{72}$ International standards

71 Ketelaar, for example, speaks of "the risks of archivists using a metaphor or borrowing a term from another domain and giving it a specific archival meaning, a meaning which, moreover, can change over time." Ketelaar, “The Difference Best Postponed?", 142-143.

72 Ketelaar gives the example of the International Council on Archives' (ICA) General International Standard Archival Description, which "uses terms and definitions formulated specifically for the purposes of ISAD $(G)$, leaving it to the users to find equivalents in their own institutional or national idiom. $\operatorname{ISAD}(\mathrm{G})$ defines "series" and "fonds" without suggesting that these are equivalent to the French série, the German Serie, the Russian $\Omega \mathrm{OH} \Delta$, the Dutch fonds, or the French fonds. There are many more terms in the professional archival terminology which are only understandable in another language when one knows and fully understands the professional, cultural, 
creation and translation have also contributed to this effect because standards development tends to be dominated by representatives from Western and English-speaking countries who themselves may not be completely familiar with the different traditions and semantics at work around the world.

Things are changing, however. Beyond what they are learning from archaeological evidence, anthropological and sociological research of prior and existing recordkeeping systems, archival and recordkeeping professionals, scholars and educators, who traditionally were closely tied to their own institutions and nations, are now interacting through a growing number of international conferences, standards setting and systems design endeavours, international scholarly and educational exchanges and collaborations, and consulting and activist careers. In so doing, they are realising that there are distinctive differences, some subtle, some very apparent, between how the field is understood and practised in different regions, nations and communities. Because of this increased interaction and scholarly communication, it has also become easier to discern influences and influencers across different traditions. However we do not as yet understand what is likely to happen to local traditions with globalisation. Will they persist, in part or in whole? Will they be eclipsed as globalisation diminishes or even makes extinct the concept of the nation state and technology imposes more procedural and linguistic uniformity? Might local traditions even be liberated or invigorated by the erosion of the nation state or the accessibility of easy to use and access technology and networked media? Will we learn more from each other through these interactions and thereby augment our current armament of ideas and practices?

There are many other fertile areas for research beyond those alluded to in the preceding discussion. For example, what are the implications for archives and recordkeeping concerns such as access, privacy, security, intelligence and ownership of the United States controlling so much of the world's information infrastructure, both technically and legally? How does the concept of agency manifest itself in different traditions? Who has it? Who doesn't? How does this play out in theories and practices? Is it possible to determine that what appear to be almost parallel intellectual or theoretical

\footnotetext{
legal, historical, and sometimes political background of the term. "Evidential value," "inventory," "estray" - these are terms for which a simple translation in a dictionary is not enough. They can only be understood when their conceptual framework is explained - in an encyclopaedia rather than in a dictionary, as Jenkinson assumed - and comprehended." Ketelaar, “The Difference Best Postponed?”, 143. See also Jean Dryden, "A Tower of Babel: Standardizing Archival Terminology," Archival science 5 no.1 (2005): 1-16 and Bogdan-Florin Popovici, "Archival Science: a South-East European Approach," Atlanti 18 (Trieste 2008), pp. 379-388.
} 
developments in quite distinct traditions are in fact the result of the influence of one or two tradition-crossing events or individuals, e.g., the publication and translation of the Dutch Manual in different countries, the work of influential archival and recordkeeping consultants such as David Bearman, Eric Ketelaar or Trudy Peterson, or the in-person visits to other countries of prominent theorists/theorists-practitioners such as Theodore Schellenberg or Terry Cook. Or instead are such developments more inevitabilities or collective realisations when circumstances in recordkeeping and the field get to a certain point, e.g., when addressing the implications of electronic recordkeeping and now ubiquitous digital networking; the sweeping social changes of the second half of the twentieth century; or the social justice and reconciliation movements that began at the end of the twentieth century as it moved away from World War II and the Cold War and responded to massive more localised inter-ethnic conflicts?

Such research in archival and recordkeeping studies is important in part precisely because it unsettles some of our own complacency and confidence in our professional knowledge base and its associated assumptions. This is exactly as it should be. As historian Will Durant famously noted, "Inquiry is fatal to certainty." Sometimes research will tell us things that the field does not want to hear because of this destabilising of certainty, or because of the changes in institutional infrastructure, standards, funding, education and training, and ethics that it might take to respond to findings. However, there needs to be a recognition that surfacing unpalatable truths and building bases of data that can support, debunk or simply complicate long-held assumptions and assertions are a part of our role as archival scholars. Sometimes it helps us to arrive at a common understanding of needs and priorities. Sometimes it simply helps us to understand ourselves and our stances better. In any case, as Ketelaar has stated, "we should not postpone the differences, but instead bring them to light, describe, investigate, test them. Suggesting uniformity, where there are differences, would be counterproductive."73

\section{A Brief Note on the U.S. Archival Tradition}

The development of the U.S. archival tradition and contemporary attitudes and practices of American archivists merits some specific explication because of its narrative of American exceptionalism and the early rejection of centralised control over records that shaped archival practice in many other countries. It has been observed that, "From the start, a tension has been built

73 Ketelaar, “The Difference Best Postponed?”, 146. 
into America's relations with the outside world - a tension between idealism and practicality." ${ }^{144}$ Often regarded as a country without any rigorous sense of archival theory, this statement perhaps characterises the American archival field's unique blend of democratic ideals, individualism, entrepreneurialism, and pragmatism - not pragmatism in the sense of Peirce and James, but in the sense of practical orientation. Resistance to centralised recordkeeping and archival systems as being overly controlling and limiting on states' and individual rights and on enterprise and innovation (in this respect quite similar to prevalent contemporary attitudes on the imposition of certain standards and regulatory frameworks), became a distinctive hallmark of the United States as it evolved after its origins in the American colonies. ${ }^{75}$

England's colonies in America gained independence from the English Crown in 1776 following the Revolutionary War. Similarly to other countries such as Korea there were concerns about the risks to unique records posed by fire, natural disaster, war or rebellion. As a young and expanding nation, there was also a need to disseminate materials across long distances sometimes in the absence of reliable or safe transportation and roads. The early practice, therefore, was to make print versions of important documents and distribute them in multiple copies. This practice also addressed the issue of public distrust in central government as the only historical authority since individual citizens could have their own copies of records that pertained to them. ${ }^{76}$

The first archives in the United States developed almost incidentally. They were by-products of efforts by private citizens - generally wealthy men who were avocational historians - to gather, publish and disseminate documents important to colonial history, as well as the papers of prominent early American figures and families. The originals of the published documents ended up being retained in private historical societies, particularly in the former colonial states of Massachusetts and New York, precursors of the collecting and philanthropic tradition that was to become so characteristic of the American archival field. By the mid-nineteenth century, in the Midwest region of the country, state government records were increasingly housed in state historical societies or state libraries that might be privately funded, or receive some modicum of state

74 James Fallows, “Idealism \& Practicality," The Atlantic Ideas Tour, http://www.theatlantic. com/ideastour/idealism/.

75 Anne J. Gilliland, "Professional, Institutional and National Identities in Dialog: The Development of Descriptive Practices in the First Decade of the U.S. National Archives," Festschrift for David Gracy, special issue of Information E Culture 49 no.1 (2014): 54-73.

76 It should be noted that this practice was directly counter to ideas about establishing the authoritativeness and authenticity of original records through techniques such as diplomatics that developed in Europe. 
funding for managing state records. The first publicly-funded state archives were established in the southern states in the wake of the U.S. Civil War (the first being Alabama in 1901), and were closely tied in with the desire to preserve the legacy of the White, pre-Emancipation South.

When the National Archives was established in 1934, therefore, it was confronted with a massive backlog of idiosyncratically created and haphazardly stored Federal Government records that had never been subject to systematic or standardised workflow systems, and challenged by a burgeoning collecting culture influenced by the bibliographically-oriented practices of the Library of Congress. The National Archives needed to define a body of practices that would help them make decisions about how to appraise, accession, arrange and describe records. ${ }^{77}$ Theodore Schellenberg's appraisal framework, first published as an internal circular within the National Archives, was designed specifically to address the nature and needs of U.S. Federal Government records. However, as one of the first articulations of archival theory within the U.S. context, it also exerted an important influence on other American institutional as well as collecting archives, many of whom were experiencing similar issues regarding which materials to accession or select. ${ }^{78}$ Under the leadership of Deputy Archivist (later National Archivist) Solon Buck, the Archives also carefully studied and then selectively adopted or adapted archival practices from different countries in Europe such as England, France, Germany, Italy, Russia and Poland, depending on what they felt might work in the context of the current state of U.S. Federal records and records-creating agencies. Inevitably these practices were somewhat decontextualised from how they had originated and were instantiated in their original European contexts. The end result was the National Archives' concept of the record group and Oliver Wendell Holmes "levels of archival arrangement."

It is important to bear in mind that the mandate of the U.S. National Archives has always been to work with records generated by the Executive branch of the Federal Government. The National Archives has no authority over government archives at the levels of individual states or municipalities, which function independently and not as part of any national system. It is also distinct from the other major archival sectors, by far the largest of which are

77 Gilliland, "Professional, Institutional and National Identities in Dialog," 57-58.

78 Theodore R. Schellenberg, The Appraisal of Modern Public Records: National Archives Bulletin 8 (Washington, D.C.: National Archives and Records Service, 1956). See also Philip Brooks, "The Selection of Records for Preservation," American Archivist 3, no. 4 (October 1940): 221-34 and G. Philip Bauer, The Appraisal of Current and Recent Records, Staff Information Paper 13 (Washington, D.C.: National Archives and Records Service, 1946). 
college and university archives that tend to function within the collecting tradition and often as part of academic or research library special collections. Other sectors include corporate archives and records management, private historical societies, a large diversity of religious archives, museum archives, film and other forms of media archives, and also a rapidly growing number of grassroots, community-based archives. ${ }^{79}$ These non-governmental archives, historical repositories, memory institutions and community-initiated projects have played a central role in articulating and implementing documentary approaches to diversify and systematise archival collection development. Perhaps the best-known approach was the documentation strategy, enunciated in the 1980s and influenced by the work of West German archivist Hans Booms ${ }^{80}$ as well as by social historians and the Civil Rights movement. Also responding to the possibilities and challenges of emerging technologies, documentation strategists advocated developing trans-institutional, transdisciplinary plans to collect and also create materials that would provide well-rounded documentation of places, racial and ethnic communities, political movements, and social and scientific phenomena. ${ }^{81}$ Another area of more recent engagement has been in archival activism and social justice. Community archives in particular have focused on surfacing and advocating for the presence and experiences of populations that have been historically under-represented or submerged in official records, including those of women, ethnic and racial, immigrant and LGBTQ communities.

79 Archives and collections established by African Americans were probably the first form of community archives in the United States after the U.S. Civil War. The American Jewish Historical Society founded in 1892 claims to be "the oldest ethnic cultural archive in the United States," http://www.ajhs.org/about.

80 Hans Booms, "Society and the Formation of a Documentary Heritage: Issues in the Appraisal of Archival Sources," trans. Hermina Joldersma and Richard Klumpenhouwer, Archivaria 24 (Summer 1987): 69-107 [first presented by Booms in his opening address to the German Archives Conference and then published in German as "Gesellschaftsordnung und Überlieferungsbildung: Zur Problematik archivarischer Quellenbewertung," Archivalische Zeitschrift 68 (1972): 3-40].

81 See, for example, F. Gerald. Ham, "Archival Strategies for the Post-custodial Era," American Archivist 44 no. 3 (Summer 1981): 207-216; Larry J. Hackman and Joan Warnow-Blewett. "The Documentation Strategy Process: A Model and a Case Study," American Archivist 50 (Winter 1987): 12-29; Helen Samuels, Varsity Letters: Documenting Modern Colleges and Universities (Metuchen: Scarecrow Press, 1992); Richard J. Cox, "The Documentation Strategy and Archival Appraisal Principles: A Different Perspective," Archivaria 38 (Fall 1994): 11-36; Timothy L. Ericson, “'To Approximate June Pasture', The Documentation Strategy in the Real World," Archival Issues 22 no.1 (1997): 5-20; and Dominique Daniel, "Documenting the Immigrant and Ethnic Experience in American Archives," American Archivist 73 (2010): 82-104. 
Not deploying formal registry or pré-archivage systems in government or business administration has set the American archival tradition not only outside, but also largely unaware and unappreciative of the kinds of recordkeeping workflows and archival expectations that are so embedded in many other archival traditions as to be practically assumed to be universal. At the same time, however, the National Archives as well as many U.S. archival consultants have played influential roles in the development of archival and records management systems and practices, as well as of records and information management and access legislation, in countries around the world. And U.S. archival and historical manuscript professionals, as well as the Society of American Archivists and the Library of Congress have been instrumental in the development, maintenance and exporting of archival descriptive standards (both $\mathrm{MaRC}$ and $\mathrm{EAD}$-based) and the articulation of International Council on Archives (ICA) descriptive standards. The Society of American Archivists' Code of Ethics also considerably influenced the first code of ethics to be adopted by ICA. One could reasonably ask what the effects have been on the recordkeeping and archival traditions of other countries of these kinds of interactions and influences. Perhaps giving even more pause for thought, what has been the impact of recent U.S. dominance in finance, trade and information technology development and implementation, of its far-reaching legal prosecutions and intelligence activities, and of its military presence in many countries, as these relate to understandings about and safeguards ensuring accountability, authority, compliance, security and individual rights? Much of the world's information infrastructure today, including software used to create and manage digital records, is designed and managed by United States' companies. What are the implications of this given the limited mutual understandings of the different archival and recordkeeping practices at work in the U.S. and elsewhere?

To conclude on perhaps a more productive note, given that today's recordkeeping in many cases is no longer as integral as previously to business and workflow control, and that the creation of records has consequently become an added activity that is often overlooked, is there something other countries can learn from American experiences about the nature and necessity of the record? Similarly, does such a culture largely constructed of autonomous archives have the effect of including more voices and perspectives in the national historical narrative, or are professional, intellectual, "white" and heteronormative biases so deeply engrained that they tend to be present in these archives even in the absence of a national system, centralised control or wholesale adoption of standards? 


\section{References}

Acker, Amelia. "When is a Record? A Research Framework for Locating Electronic Records in Infrastructure," in Anne J. Gilliland, Sue McKemmish and Andrew J. Lau, eds. Research in the Archival Multiverse (Melbourne: Monash University Press, 2015).

Aguirre, Carlos and Javier Villa-Flores, eds. From the Ashes of History: Loss and Recovery of Archives and Libraries in Modern Latin America (Raleigh, NC: Editorial A Contra corriente, 2015).

Archi, Alfonso. "Archival Record-keeping at Ebla, 2400-2350 B.C." in Ancient Archives and Archival Traditions: Concepts of Record-Keeping in the Ancient World, Maria Brosius, ed. (Oxford: Oxford University Press, 2003): 17-36.

Archival Education and Research Institute (AERI) Pluralizing the Archival Curriculum Group (PACG). "Educating for the Archival Multiverse," American Archivist 74 no.1 (Spring/Summer 2011): 69-102.

Bačić, Roberta. "Arpilleras that Cry Out, Sing and Challenge," Magazine of the United Nations Development Program (n.d.), pp.22-22, http://escolapau.uab.cat/img/ programas/musica/chilean_tapestries.pdf.

Bastian, Jeannette A. Owning Memory: How a Caribbean Community Lost Its Archives and Found Its History (Contributions in Librarianship and Information Science, Libraries Unlimited, 2003).

Bastian, Jeannette A. and Ben Alexander (eds.), Community Archives. The Shaping of Memory (London, Facet 2009).

Bauer, G. Philip. The Appraisal of Current and Recent Records, Staff Information Paper 13 (Washington, D.C.: National Archives and Records Service, 1946).

Berlin, Isaiah. "History and Theory: The Concept of Scientific History," History and Theory 1, no. 1 (1960): 1-31.

Beynon-Davies, Paul. "Informatics and the Inca," International Journal of Information Management 27 (207): 306-318.

Booms, Hans. "Society and the Formation of a Documentary Heritage: Issues in the Appraisal of Archival Sources," trans. Hermina Joldersma and Richard Klumpenhouwer, Archivaria 24 (Summer 1987): 69-107.

Bradsher, James Gregory. “Ebla’s Royal Archives,” Information Development 1 no.4 (1985): 238-243.

Brendecke, Arndt. “'Arca, Archivillo, Archivo': The Keeping, Use and Status of Historical Documents about the Spanish Conquista," Archival Science 10 no.3 (2010): 267-283.

Brooks, Philip. "The Selection of Records for Preservation," American Archivist 3 no. 4 (October 1940): 221-34.

Brosius, Maria. "Ancient Archives and Concepts of Record-Keeping: An Introduction," in Ancient Archives and Archival Traditions: Concepts of Record-Keeping in the Ancient World, M. Brosius, ed. (Oxford: Oxford University Press, 2003): 1-16.

Cachando Chile: Reflections on Chilean Culture, "Chilean Arpilleras: A Chapter of History Written on Cloth," (September 11, 2010), https://cachandochile.wordpress. com/2010/09/11/chilean-arpilleras-a-chapter-of-history-written-on-cloth/.

Calamur, Krishnadev "Supreme Court Sides With Fisherman In Case Of The Missing Fish,” National Public Radio broadcast (February 25, 2015), http://www.npr. org/sections/thetwo-way/2015/02/25/389005176/supreme-court-sides-withfisherman-in-case-of-the-missing-fish.

Chappell, Marcia H. "The Place of Reference Service in Ranganathan's Theory of Librarianship," Library Quarterly 46 no.4 (1976): 378-96. 
Cline, Scott. "To the Limits of Our Integrity': Reflections on Archival Being," American Archivist 72 no.2 (2009): 331-43.

Cook, Terry. "Archival Science and Postmodernism," Archival Science 1 (2001): 3-24, http:// www.mybestdocs.com/cook-t-postmod-p1-00.htm.

Cox, Richard J. "The Documentation Strategy and Archival Appraisal Principles: A Different Perspective," Archivaria 38 (Fall 1994): 11-36.

Daniel, Dominique. "Documenting the Immigrant and Ethnic Experience in American Archives," American Archivist 73 (2010): 82-104.

Dryden, Jean. "A Tower of Babel: Standardizing Archival Terminology," Archival science 5 no.1 (2005): 1-16.

Duarte, Marisa Elena and Miranda Belarde-Lewis, "Imagining: Creating Spaces for Indigenous Ontologies," Cataloging E Classification Quarterly special issue on Indigenous Knowledge Organization, 53 nos.5-6 (2015): 677-702.

Duranti, Luciana and Heather MacNeil. "The Protection of the Integrity of Electronic Records: An Overview of the UBC-MAS Research Project," Archivaria 42 (Fall 1996): 46-67.

Duranti, Luciana and Giovanni Michetti. "The Archival Method," in Research and the Archival Multiverse (Melbourne: Monash University Press, 2015).

Ericson, Timothy L. “'To Approximate June Pasture', The Documentation Strategy in the Real World," Archival Issues 22 no.1 (1997): 5-20.

Fallows, James. "Idealism \& Practicality," The Atlantic Ideas Tour, http://www.theatlantic. com/ideastour/idealism/.

Faulkhead, Shannon. "Negotiated Methodologies: Designing Research Respectful of Academic and Indigenous Traditions," in Research in the Archival Multiverse, Anne J. Gilliland, Sue McKemmish and Andrew J Lau, eds. (Melbourne: Monash University Press, 2016).

Faulkhead, Shannon. Narratives of Koorie Victoria, Ph.D. Thesis, (Faculty of Arts, Monash University, 2008).

Flinn, Andrew. "The Impact of Independent and Community Archives on Professional Archival Thinking and Practice." In Hill, J., ed. The Future of Archives and Recordkeeping (London: Facet Publishing, 2011).

Fourmile, Henrietta. "Who Owns the Past? Aborigines as Captives of the Archives," Aboriginal History 13 (1989): 1-2.

Eastwood, Terry. "Reflections on the Development of Archives in Canada and Australia," in Sue McKemmish and Frank Upward, eds. Archival Documents: Providing Accountability Through Recordkeeping (Melbourne: Ancora Press, 1993): 27-39.

Gilliland, Anne J. "An Archival Paradigm for the Twenty-first Century," invited paper, Desafios arquivisticos contemporâneos: seminário em torno dos 40 anos de CPDOC, Rio de Janeiro, Brazil, August 2013.

Gilliland, Anne J. Conceptualizing Twenty-first-century Archives (Chicago, IL: Society of American Archivists, 2014).

Gilliland, Anne J. "Enduring Paradigm, New Opportunities: The Value of the Archival Perspective in the Digital Environment," in Michèle V. Cloonan, Preserving Our Heritage: Perspectives from Antiquity to the Digital Age (Neals-Schuman, ALA Editions, 2014), pp.150-161.

Gilliland, Anne J. "Professional, Institutional and National Identities in Dialog: The Development of Descriptive Practices in the First Decade of the U.S. National Archives," Festschrift for David Gracy, special issue of Information E Culture 49 no.1 (2014): 54-73.

Gilliland, Anne J. "Reflections on the Value of Metadata Archaeology for Recordkeeping in a Global, Digital World," Journal of the Society of Archivists 32 


\section{Chapter I}

no.1 (April 2011): 97-112.

Gilliland, Anne J. and Marika Cifor, eds. Archival Studies special issue on Affect and the Archive (forthcoming, 2016).

Gilliland, Anne J. and Andrew Flinn. "The Wonderful and Frightening World of Community Archives: What Are We Really Talking About?” Keynote address, Nexus, Confluence, and Difference: Community Archives meets Community Informatics: Prato CIRN Conference Oct 28-30 2013, Larry Stillman, Amalia Sabiescu, Nemanja Memarovic, eds, Centre for Community Networking Research, Centre for Social Informatics, Monash University. http://www.ccnr.infotech.monash.edu.au/assets/ docs/prato2013_papers/gilliland_flinn_keynote.pdf.

Gilliland, Anne J. and Sue McKemmish. "Recordkeeping Metadata, the Archival Multiverse, and Societal Grand Challenges," Proceedings of the International Conference on Dublin Core and Metadata Applications 2012, Kuching, Malaysia, September 2012, 106-115, http://dcpapers.dublincore.org/pubs/article/view/3661.

Gilliland and Sue McKemmish, "The Role of Participatory Archives in Furthering Human Rights, Reconciliation and Recovery," Atlanti: Review for Modern Archival Theory and Practice 24 (2014): 79-88.

Gilliland, Anne J. and Kelvin White. "Perpetuating and Extending the Archival Paradigm: The Historical and Contemporary Roles of Professional Education and Pedagogy," InterActions: UCLA Journal of Education and Information Studies 5, no. 1 (2009), http:// interactions.gseis.ucla.edu/.

Hackman, Larry J. and Joan Warnow-Blewett. “The Documentation Strategy Process: A Model and a Case Study," American Archivist 50 (Winter 1987): 12-29.

Ham, F. Gerald. "Archival Strategies for the Post-custodial Era," American Archivist 44 no. 3 (Summer 1981): 207-216.

Harris, Verne. "The Archival Sliver: A Perspective on the Construction of Social Memory in Archives and Their Transition from Apartheid to Democracy," in Carolyn Hamilton et al., eds. Refiguring the Archive, Dordrecht: Kluwer, 2002, pp.135-151.

Head, Randolph. Interdisciplinary Essays on European Knowledge Culture, 1400-1900, special issue of Archival Science, 10 no.3 (September 2010).

Horn, Robert E. "Mess Map Table of Contents," http://www.stanford.edu/ rhorn/a/ $\mathrm{kmap} / \mathrm{mess} /$ tocMessMaps.html

Horsmann, Peter, Eric Ketelaar and Theo Thomassen, "New Respect for the Old Order: The Context of the Dutch Manual," American Archivist 66 (2003): 265-267.

International Council on Archives, Multilingual Archival Terminology Database, http:// www.ciscra.org/mat/termdb/term/60.

International Project on Permanent Records in Electronic Systems (InterPARES) 3 Project, Terminology Database, http://www.interpares.org/ip3/ip3_terminology_ db.cfm.

Ketelaar, Eric. “Access: The Democratic Imperative," Archives and Manuscripts 34 no.2 (2006): 62-81.

Ketelaar, Eric. "Archivalisation and Archiving”, Archives and Manuscripts 27 no.1 (1999): 54-61

Ketelaar, Eric. "Archivistics Research Saving the Profession,'”'American Archivist 63 (2000): 324.

Ketelaar, Eric. "Archivistics: Science or Art?" In Jennie Hill (ed.), The Future of Archives and Recordkeeping. A Reader (London: Facet, 2011), pp. 89-100.

Ketelaar, Eric. "The Difference Best Postponed? Cultures and Comparative Archival Science," Archivaria 44 (1997): 142-148.

Ketelaar, Eric. "Sharing: Collected Memories in Communities of Records," Archives and Manuscripts 33 no.1 (2005): 44-61. 
Ketelaar, Eric. "What is Archivistics or Archival Science?" http://fketelaa.home.xs4all.nl/ information.html.

Levin, Kelly, Benjamin Cashore, Steven Bernstein and Graeme Auld, "Overcoming the Tragedy of Super Wicked Problems: Constraining our Future Selves to Ameliorate Global Climate Change," Policy Sciences 45 no.2 (2012): 123-152.

Lewis, Bernard. From Babel to Dragomans: Interpreting the Middle East: Interpreting the Middle East (Oxford: Oxford University Press, 2004),

McKemmish, Sue and Anne J. Gilliland. "Archival and Recordkeeping Research: Past, Present and Future," in Research Methods: Information Management, Systems, and Contexts, Kirsty Williamson and Graeme Johanson, eds. (Prahran, Vic: Tilde University Press, 2012), pp.80-112.

McKemmish, Sue, Anne Gilliland-Swetland, and Eric Ketelaar, "Communities of Memory': Pluralising Archival Research and Education Agendas," Archives and Manuscripts 33 (2005): 146-175.

McKemmish, Sue and Michael Piggott. "Toward the Archival Multiverse: Challenging the Binary Opposition of the Personal and Corporate Archive in Modern Archival Theory and Practice", Archivaria 76 (2013): 111-144.

Muller, Samuel, Johan A. Feith and Robert Fruin. Manual for the Arrangement and Description of Archives, Eric Ketelaar, Theo Thomassen and Peter Horsman, trans. of second edition (Chicago: Society of American Archivists Classic Series, 2003).

National Archives of Australia. Glossary. http://www.naa.gov.au/records-management/ publications/glossary.aspx\#r.

Nikolaeva Nikolova-Houston, Tatiana. Margins and Marginality: Marginalia and Colophons in South Slavic Manuscripts During the Ottoman Period, 1393-1878, doctoral dissertation (Austin, TX: University of Texas, 2008).

Noh, Meung-Hoan. "Confucian Community Construction and Records/Archives Management of Joseon Dynasty - focused on the meaning of the Uigwe Production, Preservation, and Use," paper presented at AERI 2012, University of California Los Angeles, July 2012, https://aeri2012.wordpress.com/conference-schedule/paperpresentations/community-archiving/.

Pearce-Moses, Richard, comp. Glossary of Archival and Records Terminology, Richard PearceMoses, ed. (Chicago: Society of American Archivists, 2005).

Pettinato, Giovanni. The Archives of Ebla: An Empire Inscribed in Clay, (New York, NY: Doubleday, 1981).

Popovici, Bogdan-Florin. “Archival Science: a South-East European Approach," Atlanti 18 (Trieste 2008), pp. 379-388.

Posner, Ernst. Archives in the Ancient World (Cambridge, MA: Harvard University Press, 1972).

Rittel, Horst W. J. and Melvin M. Webber, "Dilemmas in a General Theory of Planning," Policy Sciences 4 (1973): 155-169.

Roberts, N.C. "Wicked Problems and Network Approaches to Resolution," International Public Management Review, 1 no.1 (2000).

Samuels, Helen. Varsity Letters: Documenting Modern Colleges and Universities (Metuchen, NJ: Scarecrow Press, 1992).

Schellenberg, Theodore R. The Appraisal of Modern Public Records: National Archives Bulletin 8 (Washington, D.C.: National Archives and Records Service, 1956).

Sheffield, Rebecka. The Emergence, Development and Survival of Four Lesbian and Gay Archives, Ph.D. dissertation (Toronto: University of Toronto, 2015).

Thomassen, Theo. "The Development of Archival Science and its European Dimension," in The Archivist and the Archival Science. Seminar for Anna Christina Ulfsparre ... (Lund: 


\section{Chapter I}

Landsarkivet, 1999), 67-74, http://daz.hr/zad/arhol/the-development-of-archivalscience-and-its-european-dimension/

Thorpe, Kirsten. "Aboriginal Community Archives: A Case Study in Ethical Community Research," in Research in the Archival Multiverse, Anne J. Gilliland, Sue McKemmish and Andrew J Lau, eds. (Melbourne: Monash University Press, 2015).

United States National Science Foundation Advisory Committee for Cyberinfrastructure Taskforce on Grand Challenges, 2011, www.nsf.gove/cise/aci/taskforces/

TaskForceReport_GrandChallenges.pdf.

United States White House Office of Science and Technology Policy. 21st Century Grand Challenges, U.S. https://www.whitehouse.gov/administration/eop/ostp/grandchallenges.

Urton, Gary. "Tying the Archive in Knots, or: Dying to Get into the Archive in Ancient Peru," Journal of the Society of Archivists 32 no.1 (2011): 5-20.

Urton, Gary. "Tying the Truth in Knots: Trustworthiness and Accountability in the Inka Khipu" in Brooke Harrington, ed. Deception: Methods, Motives, Contexts and Consequences, (Palo Alto, CA: Stanford University Press, 2009): 154-182.

Walker, Kristen. "Chilean Women's Resistance in the Arpillera Movement" CETRI Le Sud en Mouvement (30 October, 2008), http://www.cetri.be/Chilean-Women-s-Resistancein-the?lang-fr.

Wareham, Evelyn. "From Explorers to Evangelists: Archivists, Recordkeeping, and Remembering in the Pacific Islands," Archival Science 2 (2002): 187-207.

White, Kelvin L. The Dynamics of Race and Remembering in a 'Colorblind' Society: A Case Study of Racial Paradigms and Archival Education in Mexico, Ph.D. dissertation (Los Angeles: University of California Los Angeles, 2008).

White, Kelvin. "Ethnic Studies as a Theoretical Framework for Archival and Recordkeeping Research," in Research in the Archival Multiverse, Anne J. Gilliland, Sue McKemmish and Andrew J Lau, eds. (Melbourne: Monash University Press, 2016).

White, Kelvin and Anne J. Gilliland. "Promoting Reflexivity and Inclusivity in Archival Education, Research and Practice," Library Quarterly 80 no.3 (July 2010): 231-248.

Xiang, Wei-Ning. "Editorial: Working with Wicked Problems in Socio-ecological Systems: Awareness, Acceptance and Adaptation," Landscape and Urban Planning 110 (2013): 1-4, http://www.spatialcomplexity.info/files/2013/01/Working-with-wickedproblems-2013.pdf.

Yeo, Geoffrey. "Bringing Things Together: Aggregate Records in a Digital Age," Archivaria 74 (Fall 2012): 43-91.

Yeo, Geoffrey. "Concepts of Record (1): Evidence, Information, and Persistent Representations," American Archivist 70 (2007): 315-343.

Yeo, Geoffrey. "Posner's Archives in the Ancient World Revisited: A New Look at Some Old Records," plenary paper presented at the Seventh International Conference on the History of Records and Archives (ICHORA 7), Amsterdam, August 2015, http:// ichora.org/?page_id=315.

Youn, Eunha (Anna). "Archival Traditions in Korean History: From Medieval Practice to the Contemporary Public Records Management Act.” Archival Science 13 (2013): 23-44.

Youn, Eunha. "Investigating Socio-cultural Aspects of the Implementation of an International Archival Descriptive Standard in Korea," in Research in the Archival Multiverse, Anne J. Gilliland, Sue McKemmish and Andrew J Lau, eds. (Melbourne:

Monash University Press, 2016)

Youn, Eunha. Standardization of Archival Description in Korea: Examining the Understanding, Adoption, and Implementation of ISAD (G), Ph.D. dissertation (Los Angeles: University of California Los Angeles, 2011). 


\section{Part II}

Archival Intellectual Context and Theoretical Frameworks 
Chapter 2

\title{
THE ARCHIVAL METHOD
}

\section{Luciana Duranti and Giovanni Michetti}

\begin{abstract}
The archival body of knowledge had its origin in the legal disciplines and developed over the centuries through integration with philological and historical disciplines. In other words, archival science grew out of humanistic knowledge and reached its maturity as a humanistic science, which addressed its own research questions using a method derived from its body of interdisciplinary theory, the "archival method," as it was called when developed in the $19^{\text {th }}$ century. In the past half century, archival science has slowly but consistently moved towards the social sciences, primarily because of the AngloSaxon tendency to include archival programs of education in schools of library and information studies. The movement to the social sciences is less common in other parts of the world, but it has had a strong impact on the nature of the discipline and on the character of the research conducted by its specialists. Increasingly, social sciences methods are used to solve archival problems and to address archival research questions. Although these methods are valid for certain types of issues, primarily of a practical nature, they have shown significant weaknesses in addressing issues that affect the core of archival theory and which, at this time of fast and seemingly uncontrollable use of new and emerging technologies, loom larger and larger. The purpose of this chapter is to revisit the archival method, examine its origin, development and characteristics, look at the theoretical ideas upon which it is based and at its constituent analytical processes, and identify its place in research in the Archival Multiverse.
\end{abstract}




\section{Introduction}

"It is probably as much a mistake to ask a working historian to discuss [what History is] as to ask a painter to give his views on aesthetics." (Norman Stone) ${ }^{1}$

This statement can also be used to refer to archivists: asking them about the archival method is like asking painters about aesthetics. Paraphrasing Michael Duchein, they may answer that it is easier to put the archival method into practice than to define it. ${ }^{2}$ The following discussion of the archival method is rooted in the belief that - having accepted the truth of the above statement - it is nevertheless fundamental to the development of archival scholarship to engage actively and continuously in a discourse around this topic. Without ever claiming to have reached a definitive answer, such discourse should aim to progressively refine this area of inquiry and begin clearing the vagueness surrounding it. This chapter will examine its origin, development and characteristics, look at the theoretical ideas upon which it is based and at its constituent analytical processes, and identify its place in research in the Archival Multiverse.

The requirement to articulate the essence of the archival method comes from outside the archival domain: as suggested, archivists probably do not feel the need to define, describe, and demonstrate the method of inquiry they use. Even if they might not go so far as to say that the archival method is the archival method is the archival method, they may still think that their method is what it is, and that is all. ${ }^{3}$ But archivists do not operate in isolation and are often confronted with the work, assumptions and worldviews of different professionals in the vast domain of Information Science, where environments, purposes, functions, even terminology, may be shared. Such

1 The original citation is: "It is probably as much a mistake to ask a working historian to discuss this theme as to ask a painter to give his views on aesthetics." Norman Stone, "Grim Eminence," London Review of Books, January 20, 1983, 982, as cited in Richard J. Evans, In Defence of History (London: Granta Books, 2000), 10.

2 French archivist and historian Michel Duchein, writing about respect des fonds, remarked in his seminal essay: "Like many principles, however, it is easier to state than to define and easier to define than to put into practice." Michel Duchein, "Theoretical Principles and Practical Problems of Respect des fonds in Archival Science," Archivaria 16 (Summer 1983): 64.

3 Gertrude Stein's “a rose is a rose is a rose" referred to the archetypical elements of objects and to evoking imagery and emotions by their name. She used this expression in her Geography and Plays (1922). Since then, it has permeated popular culture and has been used as an authoritative image for the law of identity. 
proximity thereby creates a need to (re)define the cornerstones of the archival discipline in order to position it correctly in the broad information field. This chapter intends to offer a contribution to the identification of such a position.

A discipline - Trevor Livelton writes - "denotes a form of study with a distinct methodology used to gain knowledge. A discipline encompasses both a way of gaining knowledge - rules of procedure that discipline the scholar's search - and the resulting knowledge itself." ${ }^{\prime 4}$ Differently stated, a discipline is "a systematic and ordered study based upon clearly defined models and rules of procedure," or - more generally - "a body of knowledge that is often expressed in practice and continually changed and expanded through research."

Archival scholarly literature comprises a wealth of writings on archival functions: authors may disagree on the scope of some "minor" functions but they all identify the same core archival functions, which they regard as the ground upon which archivists build their scientific, professional and educational profiles. These functions are Appraisal and Acquisition, Arrangement and Description, Retention and Preservation, Management and Administration, and Reference and Access. Authors may also argue as to whether each of these pairs should be regarded as a single unitary function or as two distinct functions. They may debate whether there is something missing in the list - for example, depending on the conceptual framework developed in their country, some may add Records Classification and Scheduling, or Outreach and Advocacy, while others may simply label the entities above in a different way, such as Records Management or Governance and Public Service or Relations. They may even discuss which, among archival activities, belong to each function (i.e., all of the activities aimed to accomplish one purpose ${ }^{7}$ ) or what activities (i.e., a series of acts or actions aimed to one purpose ${ }^{8}$ ) are archival in nature, but they all substantially agree on what are the sets of

4 Trevor Livelton, Archival Theory, Records, and the Public (Lanham, MD, \& London: The Society of American Archivists and The Scarecrow Press, 1996), 44.

5 Anthony Snodgrass, An Archaeology of Greece: The Present State and Future Scope of a Discipline (Berkeley, CA: University of California Press, 1987).

6 Margaret O. Doheny, Christina Cook, and Mary Stopper, The Discipline of Nursing: An Introduction, $2^{\text {nd }}$ edition (Norwalk, CT: Appleton \& Lange, 1987).

7 The InterPARES Project, Terminology Database, http://www.interpares.org/ip3/ip3_ terminology_db.cfm?letter=f\&term=28. See also Luciana Duranti, Diplomatics: Nerw Uses for an Old Science (Lanham, MD: Society of American Archivists and Association of Canadian Archivists in association with Scarecrow Press, 1998), 90, note 10.

8 The InterPARES Project, http://www.interpares.org/ip3/ip3_terminology_ db.cfm?letter $=$ a\&term $=967$. 
activities by which archivists fulfil their mission. ${ }^{9}$ They all seem to miss a fundamental item though, the "stone guest"10 - the Research function, the systematic investigation of physical and/or abstract entities. What is its place in the list of archival endeavours? Is it a meta-function or a distinct function operating at the same level as the others? Or is it rather a sub-function of a specific function? Is Research a separated endeavour that can drive archival functions, or is it rather an integral part of them. These authors believe the latter to be true: we regard Research as the foundation of each and every archival activity within each and every function, even routine ones, as it permeates and guides them all. Be it theoretical or empirical, Research is an integral component of archival work. This has been recognised by archival writers since the $16^{\text {th }}$ century, from Charles du Molin to Bonifacio Baldassarre to Alberto Barisone and Michele Battagia ${ }^{11}$ and by all archival

9 According to the Society of American Archivists "[ $t$ ]he identity of a profession is founded on an exclusive body of knowledge". Archival core knowledge encompasses all archival functions, that is, "Appraisal and Acquisition, Arrangement and Description, Preservation, Reference and Access, Outreach and Advocacy, Management and Administration, Records and Information Management, and Digital Records and Access Systems." Society of American Archivists, Curriculum, http://www2.archivists. org/gpas/curriculum.

10 This is a translation of the Italian expression convitato di pietra, referring to a "looming but invisible presence, silent and therefore disturbing and unpredictable, of which everyone is aware but which no one mentions" (translated from Treccani.it. L'enciclopedia italiana, http://www.treccani.it/vocabolario/convitato/).

11 Carolus Molinaeus (= Charles Du Molin), In regulas Cancellariae Romanae hactenus in Regno Franciae usus receptus commentarius analiticus. Ludguni, 1552 [this work was later incorporated in his Opera quae extant omnia, Lutetiae Parisiorum, sumptibus N. Buon, 1612]; Jacob von Rammingen, Von der Registratur und jbren Gebäwen und Regimenten dessgleichen von jhren Bawmeistern und Verwaltern und jrer qualificationen und habitibus..., Heidelberg, durch Johannem Maior, MDLXXI; Annales ecclesiastici, auctore Caesare Baronio..., tomus decimus, Lucae, Typis leonardi Venturini, MDCCXLI [Baronio's work was compiled in 1578 and first published between 1588 and 1607]; Ahasver Fritsch, De Jure Archivi et cancellariae, Jena, 1664 [this work is included in his Opuscula varia Norimbergae, sumptibus Joannis Stein, 1731]; Disputatio solennis juridica de Archivis... subjicit Franciscus Michael Neveu de Windtschlee ad diem 9-19 Nov. A MDCLVIII, Argentorati, literis Johannis wilhelmi Tidemann, 1668; Nicolaus Glussianus, Methodus Archiviorum, seu modus eadem texendi ac disponendi, Mediolani, ex Typographia Francisci Vigoni, in foro piscario veteri, 1684; Dissertatio de iure archivorum...submitted in juleo majori die 16 juniii anno MDCLXXXVIII Friedrich Ernst Rinckhamer, hal. Sax., autor. Helmstadii, typis Georg-wolfgangi Hammii acad.typogr.; and Jus ecclesiasticum universum, brevi methodo ad discentium utilitatem explicatum, seu lucubrationes canonicae in quinque libros Decretalium Gregorii IX Pontificis Maximi... authore R.B. Francisco Schmalzgrueber, Neapoli, 1738, prostant Venetiis apud Josephum Bortoli; Lester K. Born, "The 'De Archivis commentarius' of Alberto Barisoni, 1587-1667," Archivalische Zeitschrift," 50-51 (1955): 12-22; Lester K. Born, "Baldassarre Bonifacio and His Essay 'De Archivis', The American Archivist 4 (1941): 221-237 [Bonifacio's work was published in 1631]; J.G. de Chevrières, Le nouvel archiviste, contenant une nouvelle méthode de 
schools and programs, worldwide, where research is embedded in programs of education. ${ }^{12}$

However, one cannot help wondering whether the integration and close identification of archival research with archival work, a phenomenon that began in the $16^{\text {th }}$ century and continued well into the second half of the $20^{\text {th }}$ century, was a primary reason why archival scholarship did not thrive as it should have. Archival theorist Giorgio Cencetti once defined the archivist as "the technician of historical research." ${ }^{13}$ His argument was that archivists regard research as a professional function if not the core of all functions. Thus, the subject of their research is not determined by personal interest, but by the nature of the material for which they are responsible, the archival institution's description priorities, the requests of the users, and the needs of the many services in which they are expert. This definition of archival research as an integral component of archival work, characterised by its obligation to present problems neither ideologically nor theoretically, but rather driven by the very nature of the material, created a dichotomy not only between archival research and historical research but also between "archival scholarship" and "scholarship in the archival discipline." This dichotomy has endured to our days and will be discussed later in the chapter.

\section{Archival Research}

Today, when we talk about research, we usually refer to scholarly research, i.e., research based on a clearly identified sound methodology following explicitly stated rules. This type of research is usually carried out either by

ranger un chartier dont l'ordre chronologique est la base..., Paris, l'Autheur, 1775; Michele Battagia, Discorso sull'antichita' ed utilita' degli archive, nonche' sulla dignita' degli archivisti. Tipografia di Alvisopoli: Venezia, 1817, 30.

12 See for example: Giovanni Vittani, "La formazione dell'archivista," in Annuario del R. Archivio di Stato di Milano 1917, reprinted in Giovanni Vittani, Scritti di diplomatica e archivistica (Milano: Cisalpino Goliardica, 1974), 154; Eugenio Casanova, Archivistica (Siena: Lazzeri, 1928), 468; Sir Hilary Jenkinson, "Roots," in Selected Writings of Sir Hilary Jenkinson, eds. Roger Ellis and Peter Walne (Gloucester: Alan Sutton, 1980), 372; Aurelio Tanodi, Manual de archivologia hispanoamericana (Cordoba, Argentina: Universidad National de Cordoba, 1961); Leopoldo Sandri, "L'archivistica," in Atti, Congresso ANAI, Este, Ottobre 1966, XXVII (1967), 424; Giorgio Cencetti, Scritti archivistici (Roma: Il Centro di ricerca editore, 1970); Bruno Delmas, "Bilancio e prospettive dell'archivistica francese alle soglie del terzo millennio," in L'Archivistica alle soglie del 2000, ed. Oddo Bucci (Macerata: Pubblicazioni dell'Università di Macerata, 1992), 85; Elio Lodolini, Archivistica. Principi e Problemi, 8th edition (Milano: Franco Angeli, 1998), 243-251.

13 Cencetti, Scritti archivistici, 17. 
academics and students in the context of dedicated educational programs or by teams led either by academics or professionals in the context of research projects. Archival research is the term commonly used to refer to research aiming to explore archival matters. Despite the easy and generic wording, this definition is unclear: what are the boundaries of archival matters, of archival objects, activities, or issues? This is a fundamental question the answer to which is at the core of archival identity. This question will be explored here, not in general, but specifically focusing on the methodology that archivists use to better understand objects, activities and issues pertaining to their domain: the Archival Method.

In the archivists' traditional sphere of competence, some research endeavours are mainly directed towards examining physical and/or abstract entities they easily identify as archival (e.g., records, fonds) ${ }^{14}$ and rely on well-recognised methods aimed at achieving their specific purposes (e.g., describing the archival materials or preserving them). Other research endeavours deal with entities that are the object of investigation of other disciplines (e.g., costs, human behaviour, technology) and borrow methods developed by them to create new knowledge useful to the archivists' endeavours that then become part of their intellectual armament. In other words, some research focuses on objects, methods and activities that are already recognised as part of the archival field, while other research aims at increasing archival knowledge through the investigation of different domains and/or the use of different methodologies. Examples of the former type of research are studies that investigate either an integral part of archival practice itself, like those supporting the appraisal or arrangement of a given body of records, or that aim to develop archival theory and methods in light of changes related to records and their context of creation, use and preservation such as those seeking to support continuing digital authenticity. Examples of the latter type of research are studies on users, on organisational culture, or on policies and legislation, cost-benefit analyses of specific processes and, in general, investigations of phenomena surrounding archival practice.

Both types of research qualify as archival because, if they are beneficial to the archival endeavour and augment archival knowledge in terms of

14 For the purposes of this chapter, the definitions of record and fonds are the following:

"Record: A document made or received in the course of a practical activity as an instrument or a by-product of such activity, and set aside for action or reference;" "Fonds: The whole of the records created (meaning made or received and set aside for action or reference) by a physical or juridical person in the course of carrying out its activities." The InterPARES2 Project, The InterPARES 2 Project Glossary, http://www.interpares.org/ip2/ display_file.cfm?doc=ip2_glossary.pdf\&CFID $=3306160 \&$ CFTOKEN=9372284. 
theory, methodology and practice, they belong to the archival domain. From this point of view, we may paraphrase Terentius' motto by saying "We are archivists; nothing archival is foreign to us, ${ }^{15}$ although this still leaves us with the problem of figuring out what "archival" means with regard to the object and method of research. Going back to the above examples, a research study on organisational culture is not archival per se, even if it might produce outcomes indirectly related to archival objects; however, a research study on organisational culture that has the aim of understanding how such culture affects documentary processes is without doubt archival research in terms of its object, if not necessarily its method. The same holds true with regard to knowledge areas and methods which encroach or overlap with our own. For example, while there is no doubt that records classification theory and method are a well-established area of knowledge and investigation in the archival realm, what about business process analysis? Concepts and methods from this area are designed to serve general purposes, i.e., they might be applied to identify business needs and determine solutions to business problems in any sector, including archives; however, most archivists wouldn't mention this kind of knowledge as a component of the archival intellectual armament. What really makes the difference is the goal of the research: if the theories and methods we decide to use are directed towards the understanding and management of archival entities, the research using them will have an archival imprint. In other words, 'external' knowledge borrowed to work on or to analyse 'internal' entities produces 'internal' knowledge. This holds true not only for the outcomes of the research but also for the theories and methods used to reach them, which are changed by the use that we make of them and by the purpose of such use. This is a fundamental point because this is the way a science keeps growing and renewing itself.

The "autonomous" internal core of archival knowledge coming to us from ancient times is very small. The accruals to it were not so frequent in the following few centuries, but they usually occurred at times of significant change in the conception of the state. ${ }^{16}$ It was towards the end of the $19^{\text {th }}$

15 Gaius Terentius Varro, Heautontimorumenos, line 77: "Homo sum, humani a me nihil alienum puto" (Engl. "I am a human being, nothing human is foreign to me").

16 The purpose of archives has changed over time, and the body of knowledge needed to control archival material has changed accordingly. Since ancient times, the characterising feature of archives was their ability to ensure the certainty of the law and provide evidence of facts and acts. This remained the main function of archives through the Middle Ages. Archives were the place in which public records were kept in order to make full faith and credit. In the 1500s, the birth of modern States strengthened the political function of archives, and they became the foundation of power and authority. 
century that the archival discipline began flourishing and has continued since. It did so by borrowing from different fields: the debate between historians and archivists led to the affirmation of the historical method of analysis; the strong relationship with library science affected retrieval practices and theories; the first attempts to introduce mechanisation in public administration changed documentation processes; information science changed the relationship of archives with technology; social sciences questioned archival behaviour, processes, and policies; and post-modernism spurred a debate about archival identity and purposes. However, the core of archival knowledge dating back to Sumerian times has not changed, and neither has the body of concepts and principles that accrued around that core between the $16^{\text {th }}$ and the $19^{\text {th }}$ centuries, because the core has controlled the use of knowledge taken from other disciplines and shaped the outcome of interactions with them. For example, using a modelling technique to represent procedures for protecting electronic evidence ${ }^{17}$ as part of an archival research project is not simply borrowing from another field: if the project is successful and demonstrates the usefulness of the modelling technique, the resulting models will become part of the knowledge that future archivists will acquire. Similarly, social sciences techniques used to investigate users' behaviour become archival tools when they help archivists to understand how to develop better finding aids, to re-interpret their role in the digital age, or to improve their communication skills.

Therefore, the access to them became more and more limited and there was no growth of archival knowledge, as security was all that was needed. It was between the end of the $18^{\text {th }}$ and the beginning of $19^{\text {th }}$ century that archival knowledge began acquiring the characteristics of a discipline because archives started to be used by other than their creators as historical resources. The French Revolution established the principle that archives belonged to the people and the state had the responsibility of keeping them on its behalf and for its benefit. In order to fulfil such responsibility the state had to ensure that the people could have access to archives, and this required that they be properly arranged. This is why it was the state that first articulated the principles of respect des fonds and respect for original order, thereby producing the most significant growth in the archival body of knowledge in centuries.

17 The project carried out by the United States Department of Defense in conjunction with the University of British Columbia between 1995 and 1997 was aimed at investigating methods capable of guaranteeing the reliability and authenticity of electronic records by using principles of diplomatics and archival science. This investigation led to a formal modelling of archival functions and objects using IDEF (Integrated Definition) modelling language: the nature, properties and boundaries of functions and objects were rigorously defined according to formal graphic diagrams which constituted the first serious attempt to apply modelling techniques to the archival domain. Luciana Duranti, Terry Eastwood and Heather MacNeil, Preservation of the Integrity of Electronic Records (Dordrecht: Kluwer Academic Publishers Group, 2002), http://www.interpares. org/UBCProject/index.htm. 
Archival knowledge grows from the use of the instruments of different disciplines in a process that continuously broadens and refines its core without altering it. In other words, there is an evolving and deepening but established body of knowledge commonly recognised as archival; and in addition there is a complex system of theories, methods and practices surrounding it. The latter propel the discipline and may be later assimilated into it as part of its core. This means that archivists must study concepts, laws and models from various fields to foster useful transfers to their own field, to encourage the development of archival theory in emerging areas of endeavour and investigation, to eliminate the duplication of theoretical efforts in different fields, and to promote consistency of scientific knowledge. However, in order to develop the body of knowledge of archival science, when archivists bring those extraneous concepts, laws and models into the archival system, they have to make them consistent with all of its parts (i.e., confront them with archival theory, methods, practice and scholarship), subject them to a feedback process, and insert them into the fundamental structure of the system. Only in this way will they be able to maintain the integrity and continuity of their discipline while at the same time fostering its enrichment and growth, an integrity and a continuity that are vital to their ability to preserve all records, regardless of medium.

In other words, a discipline may indeed be a melting pot of principles and methods taken from other disciplines. What is essential to maintaining its integrity is that those entities coming from outside are gathered into a cohesive system. A discipline must have its own methodology - even if resulting from a variety of processes taken and adapted from other disciplines (often called reference disciplines) - which constitutes, and is assessed and recognised as, an integral part of its body of knowledge. ${ }^{18}$ A methodology may be regarded as a coherent system of concepts, rules, processes and procedures adopted by a

18 "Reference disciplines theories and methods are assessed, not merely adopted." Peter G. W. Keen, "MIS Research: Reference Disciplines and Cumulative Tradition", in Proceedings of the First International Conference on Information Systems, ed. Ephraim R. McLean (Philadelphia, PA: Society for Management Information Systems, 1980) 9-18. There is a vast literature on the concept of discipline that proposes very different definitions and interpretations. Liles et al. build upon the analysis of the existing definitions and suggest that a discipline must have six basic characteristics: (1) a focus of study, (2) a world view or paradigm, (3) a set of reference disciplines used to establish the discipline, (4) principles and practices associated with the discipline, (5) an active research or theory development agenda, and (6) the deployment of education and promotion of professionalism. Apart from highlighting the presence of the last two characteristics - most authors don't mention them explicitly, while they are indeed interesting criteria to take into account - this sort of checklist may be compared with the archival body of knowledge, and it may be easily recognised that such body 
discipline or - more generally - used in the context of an area of knowledge for solving a problem.

\section{The Archival Method}

We wish to dwell on archival methodology because, as Bloomfield writes, an investigator must understand the methodology s/he uses: "He must see the reasons for its existence, be aware of its limitations, and be able to follow it, through all difficulties and seemingly endless amassments of material, consistently to a conclusion, good or bad." ${ }^{19}$

Francesco Bonaini (1806-1874), who was a professor at the University of Florence and the first director and superintendent of the Tuscan Archives, writing about the research that archivists undertake to discover the original order of the records in a fonds, stated that:

The evidence of facts, the sequence of events is recorded into documents. These, more or less, had an arrangement and a title. Therefore, first rule: respect the fact; second: re-establish it, if it were altered. ${ }^{20}$

Bonaini called this method "historical" (metodo storico) not because it served history, but because its roots were in history. ${ }^{21}$ That method may be considered as the Italian contribution to a new approach to archives which spread, more or less simultaneously, throughout Europe, albeit taking different names: in France respect des fonds, in Germany Provenienz Prinzip, in Italy metodo

constitutes a discipline. See Donald H. Liles, Mary E. Johnson, Laura M. Meade, Ryan Underdown, "Enterprise Engineering: A Discipline?" (paper presented at the Conference of the Society for Enterprise Engineering, Orlando, June 1995).

19 Note that Bloomfield refers to the method of science here but the statement may actually be applied to any methodology, assuming that the scientific method is indeed a methodology. And he continues: "In all sciences there are many who can do this; it requires, at this day, no gift of genius. In the sciences that deal with man, however, there is a second demand, much harder to fulfil, to wit, that the scholar divest himself (for the time being, at least) of all the prejudices and preconceptions of his person, of his social group, or even of all mankind. So rare is this ability that it has grown commonplace to say that our social sciences are merely systematized expositions of tribal belief." Leonard Bloomfield, review of Language, Its Nature, Development, and Origin by Otto Jespersen, The American Journal of Philology 43, no. 4 (1922): 370.

20 "La testimonianza dei fatti, la successione delle vicende rimane nei documenti; i quali, più o meno bene, ebbero un ordine, una denominazione. Prima regola dunque: rispettare il fatto; seconda: ristabilirlo, ove si trovasse alterato." Francesco Bonaini, Relazione al Ministero dell'Istruzione pubblica, 1867, as cited in Elio Lodolini, Storia dell'archivistica italiana. ${ }^{\text {rd }}$ edition (Milano: Franco Angeli, 2004), 174. 
storico. While these are not identical concepts, they all belong to the same cultural environment that was seeking to affirm a new understanding of documentary phenomena. ${ }^{22}$

A century later, another Italian scholar, Giorgio Cencetti, stated that what Bonaini called the historical method, although born as a method of arrangement, "should rather be considered something more and allencompassing, a fundamental principle of archival doctrine, and the necessary condition for using an archives." ${ }^{23}$ Indeed, the historical method was born as a procedure, a technique. As Horsman states, "pragmatism gave birth to the notion - not pure theory. [ ... ] The method was spontaneously born as a practical solution for problems in archival arrangement." ${ }^{24}$ Cencetti then made the crucial step forward: moving from practice to theory, he recognised how the historical method could serve as a fundamental instrument for developing new theory and methods, thus making of it the pillar on which all archival knowledge rests. He elevated the historical method from a method supporting "archival scholarship" to a method supporting "scholarship in the archival discipline." Cencetti decided to call the historical method "the archival method" because it emanates from the very nature of the archival material that is the object of its study. In the process, Cencetti not only changed a name, he changed the entire archival worldview.

What is then the archival method? Archivists, in fulfilling their professional functions, cast their research questions in a juridical as well as an historical framework. There are of course other dimensions to those questions - for example, there is a more general societal environment that determines the role and the nature of both archives and archivists; there is a technical context that raises relevant issues and affects research agendas; there is an economic profile that usually underlies archival actions. However, these additional dimensions contribute to define the juridical as well as the historical framework.

Records are the primary objects of archival investigation: in order to understand their nature and meaning, and to determine how to control

22 "Respect des fonds is not a French invention, despite Fenyo blaming them for it. Many European countries had anticipated its practical application in the early nineteenth century and before". Peter Horsman, "The Last Dance of the Phoenix, or the Rediscovery of the Archival Fonds," Archivaria 54 (Fall 2002): 6.

23 "Sorto come sistema di ordinamento, il metodo storico, a ben considerarlo, si rivela come qualcosa di più ampio, cioè come principio basilare di tutta la dottrina archivistica [...] e condizione necessaria per l'utilizzazione dell'archivio." Giorgio Cencetti, "Inventario bibliografico e inventario archivistico," in Scritti Archivistici, 63.

24 Peter Horsman, "The Last Dance," 6-7. 
them, archivists who conduct archival research focus on the records creator as the main source of information about the records. More precisely, archival researchers are interested in the documentary manifestation of records creators: the life and history of creators, be they persons or organisations, interest them only inasmuch as these help in defining the context of the records and gaining knowledge about each creator's interaction with its fonds. Stella writes that archival research is stimulated by the nature and characteristics of the material it studies. ${ }^{25}$ Cencetti refines this idea by saying that the archival questions are indeed always the same, as they aim "to determine the records creator's functions, to study how they were fulfilled, and to ascertain the way they were documented."26 This, however, does not mean that the archival researcher ignores the historical dimension of archival research. The central distinction between historical and archival inquiry does not reside in the historical sensitivity or capacity of the scholar, but in the approach, as mentioned above.

In 1905, Benedetto Croce wrote:

Intuition and conception, poetry and philosophy, imagination and reasoning, are the presuppositions of the historical function; there is not one moment, in its process, in which the two elements, document and interpretation, fact and idea, intuition and conception, are separate; the distinction of the three phases - the collection of historical material (heuristics), the analysis of it (criticism), and its interpretation (understanding) - has only an empirical value. The three moments are not three but one: at its first move history finds, critiques, and interprets; and it finds because it critiques and interprets. ${ }^{27}$

25 Vittorio Stella, "La storiografia, l'archivistica, il lavoro d'archivio e l'archivista," in Antologia di scritti archivistici, ed. Romualdo Giuffrida (Roma: Ministero per i beni culturali e ambientali, 1985), 34-5. See also Leopoldo Cassese, "Del metodo storico in archivistica," Società XI (1955): 885. It should be noted that Stella makes a generalisation while some historians think that their work is driven by the material itself: "The historian's questions should be formulated not by some present theory but from the historical sources themselves." Geoffrey R. Elton, Return to Essentials: Some Reflections on the Present State of Historical Study (Cambridge, UK: Cambridge University Press, 1991), 68. Richard J. Evans adds, "these views are echoed by the senior American historian Oscar Handlin, who argued for the 'rejection of theory as a guide' and saw its influence as deeply damaging to standards of historical scholarship." Evans, In Defense of History, 232, note 2 .

26 Giorgio Cencetti, "Inventario bibliografico e inventario archivistico," in Scritti Archivistici, 65.

27 Benedetto Croce, Logica, teoria del concetto puro (Bari: Laterza, 1905), 61. 
Croce was an idealist philosopher, so his statement must be put in context, as it embodies his fundamental intellectual stance, the "philosophy of spirit," which should by no means be regarded as the way in which historians approach history today. ${ }^{28}$ Regardless, it is useful to reflect on Croce's words in order to define archival research per negativum: the archival approach is different from the historical approach because its theory and methodology are not an indivisible unit, or an inextricable tangle of threads. Rather, they constitute a coherent system in which the former, theory, guides the latter, methodology.

Theoretical ideas about the nature of a fonds, for example - generally or in the abstract - dictate the archival methodology by which a particular fonds is examined by the archivist, which in turn determines the resulting scholarly product. ${ }^{29}$

This means that archival research conducted according to the archival method, rooted in archival theory, is non-evaluative in nature, but not because it is not touched by problems related to truth, ethics, and value: "indeed, ... archivists themselves, like historians and other scholars, are constantly involved in processes that shape the "stuff" from which history is made." 30 Archival research is non-evaluative in the sense that it finds objectivity not by virtue of some external criterion but by adopting established (albeit historically set) principles and methods belonging to the archival doctrine itself.

This is the reason why the outcomes of archival research - for example, the acquisition of a specific body of records, the arrangement given to the records in a fonds, the attribution of previously unidentified documents, the selection conducted on a fonds, the determination of what social media products have a record nature, the development of a preservation policy - can provide support to any kind of research, historical or otherwise,

28 Rather, there is an intense debate among historians, with many voices and many worldviews. Echoes of Croce's philosophy may still be found but one can hardly consider them as a major trend. From the same idealistic milieu comes the pivotal principle established by Giorgio Cencetti, upon which Italian archival theory is built: "everything is archives," which means that there is no distinction between current records and historical records, there is a seamless continuity from active to semi-active and inactive records, and records belong to archives since they were born, due to their original bond with all the records of the creator. Giorgio Cencetti, "Il fondamento teorico della dottrina archivistica," in Scritti archivistici, 38-46.

29 Livelton, Archival Theory, 45.

30 Francis X. Blouin Jr. and William G Rosenberg, Processing the Past (Oxford, UK: Oxford University Press, 2011) 142. 
subsequently carried out by the users of the archives, who can trust the outcomes of research undertaken by archivists in the course of their work. ${ }^{31}$ In this regard, Antonio Cassese, a legal historian, wrote about the autonomy of archival research from history and historiography even as it was at the same time a part of the same cultural system. Adolf Brenneke, in his Archivkunde, also emphasised the difference between the reality and history of an archival fonds (revealed through the application of the archival method) and that of the body that produced it (revealed through the application of the historical method), but then he proposed to solve the discrepancy by assigning a creative function to the archivist that allows the reconciliation of the two, by reorganising the archival fonds in a way that clearly reflects the activities of the creator. Angelika Menne-Haritz strongly objected to such a method of arrangement on the grounds that it would destroy evidence and emphasised the difference between the purposes of archival research and that of historical research. ${ }^{32}$ This fundamental distinction between historical and archival method applies to both archival scholarship and scholarship in the archival discipline. In fact, even when its purpose is to further develop archival theory and methods in light of technological innovation or changes in legal, administrative, ethical or other frameworks that might result in new types of records, new uses of records, or new issues related to records creation, record-keeping and records preservation, archival research finds its conceptual and methodological autonomy in the object of its study - the phenomena and structures of records and record aggregations, which are not examined for their content, but for the meaning of their characteristics, form, organisation, and administrative, functional, procedural and documentary contexts, as archival theory dictates.

Over the centuries, the relationship between archival and historical scholarship has been very close. For example, in the case of European archival education, the École des Chartes in France was founded in 1821 to teach research methods based on history and the auxiliary sciences of history (i.e., the philological sciences) to archivists. The school supported the idea that archival scholarship focuses its questions, as well its method, on the records nature, structure and formative processes. It acknowledged that the archival method of research had consisted since its origin in the archivist

31 Leopoldo Cassese, "Del metodo storico in archivistica," 878-85.

32 Adolf Brenneke, Archivkunde (Leipzig: Koeheler \& Amelang, 1953); Angelika Menne-Haritz, "Appraisal or Documentation: Can We Appraise Archives by Selecting Content?” The American Archivist 57 (1994): 532. 
making himself/herself "contemporary to the records", ${ }^{33}$ and understanding the administrative competences, procedures and routines resulting from the records, as well as the relationship between records and actions. Between evaluating its object of study and understanding it, the archival method definitely chooses the latter and does so based on its necessary interpretive framework, archival theory, according to which all records that enter an archival fonds are equally important because they are equally necessary to the existence of the whole, and all archival fonds are equally important because they are equally necessary to the formation of the global documentary heritage.

\section{Evidence and Reality}

Many contemporary archival writers are impatient with this nonevaluative model, and even more with the frequent analogy between the archival scholar and the legal scholar that derives from it. In 1991, historian Carlo Ginzburg stated: "In the last twenty-five years words like proof, or even truth ... have acquired in the social sciences an unfashionable ring, evoking positivist implications. ${ }^{34}$ But Ginzburg believes that this hostility is due to a confusion, which he attempts to clarify. "There is an element in positivism ${ }^{35}$ that must be unequivocally rejected: the tendency to simplify the relationship between evidence and reality," he writes. This happens when researchers examine a record and assess its trustworthiness in terms of authenticity and reliability. If they find that the record is not authentic (i.e., it is not what it purports to be) or is unreliable (i.e., authentic but inaccurate or incomplete) they do not trust its content. If they find that the record is either authentic or reliable (even if not authentic), they take it as trustworthy evidence of what it states, that is, of something other than the record. Now, this is wrong, because the record in itself is not regarded as evidence per se, but rather as "a transparent medium - as an open window that gives us direct access to reality." ${ }^{36}$ Indeed, archival researchers following the archival method do not take for granted the relationship between a record and what it talks about (i.e., its content), and also do not take a direct approach to reality.

33 Arnaldo D’Addario, Lezioni di Archivistica (Bari: Adriatica Editrice, 1972), 53.

34 Carlo Ginzburg, "Checking the Evidence: The Judge and the Historian," Critical Inquiry 18 (1991): 83.

35 Simply stated, positivism is a philosophy according to which the social world, just like the physical world, operates according to absolute laws. Its founder is considered to be Auguste Comte (1798-1857). See John J. Macionis, Sociology. 14th Edition (Boston: Pearson, 2012), 11.

36 Ginzburg, "Checking the Evidence," 82-3. 
Research guided by the archival method is inferential in nature, because it occurs within the interpretive framework of archival theory, according to which proof, truth, and evidence are extra-textual. Menne-Haritz writes that:

Evidence means patterns of processes, aims and mandates, procedures and results, as they can be examined. It consists of signs, of signals, not primarily of words. It might be in symbols, like crosses or lines showing that a person who, according to a job description, has to assume responsibility for a certain task has actually seen the document, has read it, and is aware of the decisions behind it. It might be the location of a certain piece of text in the upper left corner of a record, giving it the function of an address. It might even be the following up of records in a file, indicating a sort of working order. Or it might be a certain cover of a folder or some specific handwriting telling where the folder originated. All those are nonverbal signs that must be interpreted in context to disclose their meaning. To one who understands them, they will tell how processes worked and who was responsible for which decision. ${ }^{37}$

Evidence as described by Menne-Haritz is what archival scholars look for when applying the archival method to analyse archival material, seeking to reconstruct relationships among internal and external elements of the records, their structure and their context. To do so they employ tools drawn from the philological disciplines which, since the beginning of archival research, have been auxiliary instruments of archival scholars. In fact, these disciplines help to distinguish evidence from the criteria for assessing that evidence. This is an essential scholarly activity when the aim of the archival researcher is not only to understand but, on the basis of this understanding, to make a decision, either as part of an archival function (e.g., arrangement or selection), or in order to produce a conceptual, methodological or policy finding (e.g., a new definition of record, a new metadata application profile for authenticity, or a policy for the use of social media). These philological disciplines include philology of any language, palaeography, chronology, sigillography, heraldry, codicology, toponymy, epigraphy and textual criticism, among others, but the most important among them for the application of the archival method is diplomatics.

French historian Marc Bloch wrote that: 
1681, the year of publication of the de Re Diplomatica, was truly a great one in the history of the human mind, for the criticism of the documents of archives was definitely established. Moreover, it was in every respect the decisive moment in the history of the critical method. ${ }^{38}$

Diplomatics as a body of concepts and methods for the analysis of records and the assessment of their trustworthiness has been amply discussed in the archival literature, ${ }^{39}$ and its usefulness for the understanding of digital records is the subject of chapters of this book (see McNeil, Chapter 24) thus we will limit our comments to the way in which the use of diplomatics in archival scholarship supports that claim of objectivity that the archival scholar makes when using the archival method of inquiry. In fact, when diplomatics guides the conduct of an archival function, it has no other concern than to discern records elements, attributes and relationships, whatever they may be, so that they can be recorded as identifying factors for whatever archival purpose will follow. Moreover, when diplomatics guides the development of a new concept or method, it has no other concern than observing, recognising and explaining so that such understanding can provide the foundation for the addition of new knowledge to the archival system of knowledge. Thus, archival scholars, when adopting the archival method of inquiry, employ the interpretive framework of both archival theory (what archivists work with) and methodology (how they work) in conjunction with the tools of philological disciplines, because their purpose is to implement and further develop the science of the record, archival science.

In fact, a science, as Bloch pointed out when speaking of history, is not defined entirely in terms of its object - "the what." Its confines can also be defined by the nature of its method - "the how". ${ }^{40}$ While historians are not capable of observing the facts that they study (historical knowledge is always indirect), archivists are, because their facts are "the what" (i.e., the records and their aggregations) and "the how" (i.e., activities and functions) of archives. Therefore, the critical method of history, which relies on the same philological disciplines that the archival method uses as its primary tools,

38 Marc Bloch, The Historian's Craft (New York: Vintage Books, 1953), 81.

39 Duranti, Diplomatics: New Uses for an Old Science; Heather MacNeil, Trusting Records: Legal, Historical, and Diplomatic Perspectives (Dordrecht, Netherlands: Kluwer Academic Publishing Group, 2000); Luciana Duranti, “Diplomatics," in Encyclopedia of Library and Information Science, ed. Marcia Bates, Mary Niles Maack and Miriam Drake (New York, Basel, Hong Kong: Marcel Dekker, 2009); Janet Turner, "Experimenting with New Tools: Special Diplomatics and the Study of Authority in the United Church of Canada," Archivaria 30 (Summer 1990): 91-103.

Bloch, The Historian's Craft, 47. 
must move beyond the analysis towards its ultimate justification, which is interpretation. ${ }^{41}$ The archival method, on the other hand, can integrate the results of the analysis with its archival conceptual and methodological understanding to reach rigorous conclusions, thereby getting very close to the process of understanding used by a judge in a legal proceeding. ${ }^{42}$

\section{Conclusion}

Is the archival method sufficient for archival research to meet all the challenges presented by the contemporary and future archival landscape in terms of building a scholarship that addresses them thoroughly, rigorously, and creatively? Probably not by itself, but it is the pillar that will support the whole effort and the necessary validation of any scholarship resulting from the use of other methods.

The future of archival scholarship and of scholarship in the archival discipline is based on research that is international in scope, multidisciplinary in approach, interdisciplinary in content, and interactive in process. As we undertake our investigations in a world where societies are engaged in an active dialogue between their need to understand and appreciate one another and their desire to maintain distinctive social and cultural identities, we need comparative studies of the documentary cultures of past and present societies that are able to identify both common and distinctive issues and their interdependence, and to find solutions to their problems.

Technological change has created great uncertainty, widened the gulf between the rich and the poor, and given rise to a mistrust of public institutions and private organisations, with the consequent requirement to increase accountability and transparency. In searching for solutions, we need research that brings together the scientific community, social science scholars and humanists to study ways of addressing these new social issues and their manifestations, so that all relevant factors can be taken into account.

As we try to build a scholarly world that celebrates both individual and collaborative efforts aimed at the creative interaction of distinct disciplines for the development of new interdisciplinary knowledge, we need research that analyses the challenges posed to the control of and access to records by juridical and social diversity, literacy problems, internationalism, globalisation and the delegation of state powers to supra and sub-state entities.

41 Bloch, The Historian's Craft, 155.

42 Bloch, The Historian's Craft, 139. 
As we carry out archival work in a world that values contributions to the solution of local problems, to the enrichment of community culture and to the enhancement of life by pushing forwards the frontiers of knowledge by means of risk-taking initiatives, we need research that seeks cooperation with local and regional community groups to meet their intellectual, social, cultural and economic needs in terms of records programs, strategies, policies and initiatives. This research will have to facilitate the transfer of knowledge from academia to the community, encourage scholars' openness and connectivity, and ensure their accountability, while at the same time keeping scholarly endeavours linked to pressing concrete issues of a specific nature and a manageable dimension that must be addressed consistently with the more general, global ones.

The large spectrum of research outlined above, to be successful, must simultaneously nurture disciplinary archival scholarship and foster its diversity by keeping at its core the archival method of investigation, maintaining its traditional linkages with the methods of history and jurisprudence, and venturing onto the shores of both allied and distant disciplines to borrow from them what is needed to complement and integrate the archival body of knowledge.

\section{References}

D'Addario, Arnaldo. Lezioni di Archivistica. Bari: Adriatica Editrice, 1972.

Annales ecclesiastici, auctore Caesare Baronio ... , tomus decimus. Lucae: typis Leonardi Venturini, 1741.

Battagia, Michele. Discorso sull'antichità ed utilità degli archivj, non che sulla dignità degli archivisti. Venezia: Tipografia di Alvisopoli, 1817.

Bloch, Marc. The Historian's Craft. New York: Vintage Books, 1953.

Bloomfield, Leonard. Review of Language, Its Nature, Development, and Origin by Otto Jespersen, The American Journal of Philology 43, no. 4 (1922): 370-3.

Blouin, Francis X. Jr. and William G Rosenberg. Processing the Past. Oxford, UK: Oxford University Press, 2011.

Born, Lester K. “The 'De Archivis commentarius' of Alberto Barisoni, 1587-1667.” Archivalische Zeitschrift 50-51 (1955): 12-22.

Born, Lester K. "Baldassarre Bonifacio and His Essay 'De Archivis'." The American Archivist 4 (1941): 221-37.

Brenneke, Adolf. Archivkunde: Ein Beitrag zur Theorie und Geschichte des Europaischen Archivwesens. Leipzig: Koehler \& Amelang, 1953.

Casanova, Eugenio. Archivistica. Siena: Lazzeri, 1928.

Cassese, Leopoldo. “Del metodo storico in archivistica.” Società XI (1955): 878-85.

Cencetti, Giorgio. Scritti archivistici. Roma: Il Centro di ricerca editore, 1970.

Chevrières, J.G. de. Le nouvel archiviste, contenant une nouvelle méthode de ranger un chartier dont l'ordre chronologique est la base. Paris: l'auteur, Cailleau, Lacombe, veuve Duchesne, 1775. 
Croce, Benedetto. Logica, teoria del concetto puro. Bari: Laterza, 1905.

D’Addario, Arnaldo. Lezioni di Archivistica. Bari: Adriatica Editrice, 1972.

Delmas, Bruno. "Bilancio e prospettive dell'archivistica francese alle soglie del terzo millennio.” In L'Archivistica alle soglie del 2000, edited by Oddo Bucci. Macerata: Pubblicazioni dell'Università di Macerata, 1992.

Doheny, Margaret O., Christina Cook, and Mary Stopper. The Discipline of Nursing: An Introduction, 2nd edition. Norwalk, CT: Appleton \& Lange, 1987.

Duchein, Michel. "Theoretical Principles and Practical Problems of Respect des Fonds in Archival Science.” Archivaria 16 (Summer 1983): 64-82.

Duranti, Luciana. Diplomatics: New Uses for an Old Science. Lanham, MD, \& London: Society of American Archivists and Association of Canadian Archivists in association with Scarecrow Press, 1998.

Duranti, Luciana. "Diplomatics." In Encyclopedia of Library and Information Science, edited by Marcia Bates, Mary Niles Maack and Miriam Drake. New York, Basel, Hong Kong: Marcel Dekker, 2009.

Duranti, Luciana, Terry Eastwood and Heather MacNeil. Preservation of the Integrity of Electronic Records. Dordrecht, Netherlands: Kluwer Academic Publishers Group, 2002.

Elton, Geoffrey R. Return to Essentials: Some Reflections on the Present State of Historical Study. Cambridge, UK: Cambridge University Press, 1991.

Evans, Richard J. In Defence of History. London: Granta Books, 2000.

Fritsch, Ahasver. De Jure Archivi et cancellariae. Jena, 1664.

Ginzburg, Carlo. "Checking the Evidence: The Judge and the Historian." Critical Inquiry 18 (1991): 79-92.

Glussianus, Nicolaus. Methodus Archiviorum, seu modus eadem texendi ac disponendi. Mediolani: ex Typographia Francisci Vigoni, in foro Piscario veteri, 1684.

Horsman, Peter. "The Last Dance of the Phoenix, or the Re-discovery of the Archival Fonds." Archivaria 54 (Fall 2002): 1-23.

InterPARES Project, www.interpares.org/ip3/ip3_terminology_db.cfm.

Jenkinson, Hilary. “Roots.” In Selected Writings of Sir Hilary Jenkinson, edited by Roger Ellis and Peter Walne, Gloucester: Alan Sutton, 1980.

Keen, Peter G. W. "MIS Research: Reference Disciplines and Cumulative Tradition." In Proceedings of the First International Conference on Information Systems, edited by Ephraim R. McLean. Philadelphia, PA: Society for Management Information Systems, 1980, 9-18.

Liles, Donald H., Mary E. Johnson, Laura M. Meade, and Ryan Underdown. "Enterprise Engineering: A Discipline?" Paper presented at the Conference of the Society for Enterprise Engineering, Orlando, June 1995.

Livelton, Trevor. Archival Theory, Records, and the Public. Lanham, MD \& London: The Society of American Archivists and The Scarecrow Press, 1996.

Lodolini, Elio. Archivistica. Principi e Problemi. $8^{\text {th }}$ edition. Milano: Franco Angeli, 1998.

Lodolini, Elio. Storia dell'archivistica italiana. $3^{\text {rd }}$ edition. Milano: Franco Angeli, 2004.

Macionis, John J. Sociology. 14 ${ }^{\text {th }}$ Edition. Pearson: Boston, 2012.

MacNeil, Heather. Trusting Records: Legal, Historical, and Diplomatic Perspectives.

Dordrecht, Netherlands: Kluwer Academic Publishers Group, 2000.

Menne-Haritz, Angelika. "Appraisal or Documentation: Can We Appraise Archives by Selecting Content." The American Archivist 57 (1994): 528-42.

Molinaeus, Carolus [Du Molin, Charles]. In regulas Cancellariae Romanae hactenus in Regno Franciae usus receptus commentarius analiticus. Ludguni, 1552. 


\section{Chapter 2}

Neveu, Michael. Disputatio solennis juridica de archivis ... subjicit Franciscus Michael Neveu de Windtschlee Ad diem 9./19. Nov. A[nno] MDCLXVIII. Strasbourg: Argentorati, literis Johannis Wilhelmi Tidemann, 1668.

Rammingen, Jacob von. Von der Registratur und jhren Gebäwen und Regimenten dessgleichen von jhren Bawmeistern und Verwaltern und jrer qualificationen und habitibus ..., Heidelberg; durch Johannem Maior, 1571.

Rinckhamer, Friderich Ernst. Dissertatio de iure archivorum ... submittet in juleo majori die 16. Junii Anno MDCLXXXVIII Friderich Ernst Rinckhamer, Hal. Sax. Autor. Helmstadi: typis Georg-Woflgangi Hammii Acad.Typogr., 1688.

Sandri, Leopoldo. "L'archivistica," in Atti, Congresso ANAI, Este, Ottobre 1966, XXVII (1967).

Schmalzgrueber, R.B. Francisco. Jus ecclesiasticum universum, brevi methodo ad discentium utilitatem explicatum, seu lucubrationes canonicae in quinque libros Decretalium Gregorii IX Pontificis Maximi, Neapoli; prostant Venetiis apud Josephum Bortoli, 1738.

Society of American Archivists. Curriculum, http://www2.archivists.org/gpas/curriculum.

Snodgrass, Anthony. An Archaeology of Greece: The Present State and Future Scope of a Discipline. Berkeley, CA: University of California Press, 1987.

Stella, Vittorio. "La storiografia, l'archivistica, il lavoro d'archivio e l'archivista." In Antologia di scritti archivistici, edited by Romualdo Giuffrida. Roma: Ministero per i beni culturali e ambientali, 1985, 34-5.

Tanodi, Aurelio. Manual de archivologia hispanoamericana. Cordoba, Argentina: Universidad National de Cordoba, 1961.

Turner, Janet. "Experimenting with New Tools: Special Diplomatics and the Study of Authority in the United Church of Canada." Archivaria 30 (Summer 1990): 91-103.

Vittani, Giovanni. Scritti di diplomatica e archivistica. Milano: Cisalpino-Goliardica, 1974. 
Chapter 3

\title{
A HISTORY OF ARCHIVAL IDEAS AND PRACTICE IN CHINA
}

\author{
Zhiying Lian
}

\begin{abstract}
This chapter analyses the lineage of Chinese archival practice and ideas and the social, political, economic and cultural contexts that have influenced and shaped them during the three phases of Chinese history (ancient China, modern China and contemporary China). It also summarises changes during this time in the role of archivists, in how the value of records and archives are regarded, and the shifting focus of the archival community and framing of the archival paradigm. The chapter concludes that today's China is undergoing major digital as well as social transition. As has been the trend in other countries, today's Chinese archival community needs to exercise its agency in developing Chinese archival ideas and practices and also adapting them to address these transitions. It needs to rethink current definitions of archives, records and archives management, as well as the roles of archivists, archival education and research within the new societal ethos. It also needs to study such aspects as archives and memory, community archives, and issues of rights, power and accountability that have not been paid much attention in the official paradigm. A careful balancing of the Chinese archival tradition and traditional archival ideas with a receptivity to ideas and practices developed by archival communities outside China will be necessary in order to enhance mutual understanding and learning in the archival multiverse.
\end{abstract}

\section{Introduction}

The history of archival ideas and practices in China can be traced back to the Shang Dynasty $\left(17^{\text {th }}-11^{\text {th }}\right.$ century $\left.\mathrm{BCE}\right)$, many centuries before the beginnings of the Roman Empire. Archaeological research shows that the archives inscribed on bones or tortoise shells excavated in the ruins of Yin, the capital of late Shang Dynasty, were arranged separately according to 
medium and to time period. Considering the age of the Chinese archival tradition, little literature addresses its extensive history except for the textbook Zhongguodanganshiyeshi (The History of Chinese Archives Work) edited by Xueheng Zhou, which mainly introduces archives and archival practice from the Shang Dynasty to the 1990s. ${ }^{1}$ Current research usually divides this history into three phases according to the general Chinese historical periodisation: ancient (before 1840), modern (1840-1949) and contemporary (1949-). As for what archivists today know about ancient Chinese archival ideas and practices, they are mainly introduced to particular archival ideas and practices within specific dynasties. For example, Aiguo Zhao wrote of the archives compilations in Ming and Qing dynasties, ${ }^{2}$ and Lan Wang analysed the ideas on archival classification and appraisal of the Qin and Han Dynasties. ${ }^{3}$ But such research is scanty. Jihong Liang argued that ancient Chinese archival ideas were scattered across the rules and regulations of different dynasties and the work of some historians, and that the Chinese archival community, influenced by western academic practices and professionalisation of the field, ignores the research on the ancient Chinese archival tradition. ${ }^{4}$ With regard to the modern Chinese archival tradition, the arrangement of the Ming-Qing archives at that time, reform regarding the records and archives of the National Government, and the archival ideas arising from both drew some researchers' attention. For example, Huichao Zhang teased out the history of the arrangement of the Ming-Qing archives in the Republic of China, and summarised some historians' ideas about the value of archives, archival arrangement and the compilation of archives. ${ }^{5}$ Rongxiao Fu analysed the background and process of the reform of records and archives management by the National Government and its effect on the birth of modern Chinese archival science in the 1930s. ${ }^{6}$ With regard

1 Xueheng Zhou, Zhongguo Danganshiyeshi [History of the Development of Chinese Archives] (Beijing: Renmin University Press, 1994).

2 Aiguo Zhao, "MingQingdanganwenxianbianzuanpingshu [Review of the Archives Compilation of Ming and Qing Dynasties]," Archives Bulletin 1 (1994): 52-54, 67.

3 Lan Wang, "Cong'Ceshu',"Bixiao"kanxianqinlianghandedanganxuesixiang [Analysis on the Archival Ideas of Qin and Han Dynasties from "Ceshu" and "Bixiao"]," Archival Study 1 (2000): 9-15.

4 Jihong Liang, Zhongguogudaidanganxuedexuanshuchuangtongyujiazhi [Academic Tradition and Values of Ancient Chinese Archival Science], http://theory.people.com. cn/GB/17698974.html.

5 Huichao Zhang, Mingguoshiqi Mingqingdanganzhengliyanjiu [Research on the Arrangement of the Ming-Qing Dynastic Archives during the National Government] (Shanghai: World Publication Corporation, 2011)

6 Rongxiao Fu, "Lunsanshiniandainanjingguominzhengfudewenshudangangaige [Research on the Reform of Records and Archives Management of National 
to contemporary Chinese archival ideas and practice, some literature has analysed the achievements that contemporary archival science has made and related reasons or experiences, ${ }^{7}$ but there remains a lack of critical examination and reflection on the development of contemporary archival ideas and practices.

It is, therefore, difficult to describe and analyse in any detail Chinese archival ideas and practices in different periods within the space of a single paper. This chapter analyses the lineage of Chinese archival ideas and practice from the perspective of the social structure of China. It examines the three major phases in the history of Chinese archival ideas and practice, and addresses, by means of analysis and induction, several questions in order to provide a lens through which the wider archival community can know more about the Chinese archival tradition and thus enhance its understanding, as well as to promote self-reflection on the part of the Chinese archival community about Chinese archival history: 1) Within what kind of social structures have Chinese archival ideas and practices formed during different phases of Chinese history? Conversely, how does social structure, including the political, economic and cultural contexts during different phases, influence Chinese archival ideas and practices? and, 2) What characteristics do Chinese archival ideas and practices during different phases exhibit? What changes are there in the role of archivists, the value of records and archives, the focus of the archival community and the overall framing of the archival paradigm during the long history of Chinese archival traditions? This chapter concludes that the current Chinese archival community should exercise more agency in developing archival ideas and practice to adapt to digital and social transitions in China today.

\section{Tracing Terminology}

The terms 'records' and 'archives' that are core to this chapter are defined differently in different countries, so it is necessary at the outset to provide definitions of how they are employed in China.

The term 'archives' in the Chinese archival tradition appeared in the early Qing Dynasty (1644-1911), while the term 'records' appeared in late

Government in 1930s]," Archives Bulletin 1 (2005): 87-90.

7 See Huiling Feng, "Zhouxianghuihuang(zhishi)-Danganxuelilundefazhangyufanrong [To Glory (No.10)-the Development and Boom of Archival Theory]," China Archives 10 (1999): 5-7.; Caifu Li, "Fazhangshiqidezhongguodanganxue:Deshi, Tedianjiqichengyin[ Developing Chinese Archival Science: Achievements, Limitations, Characteristics and the Reasons]," Zhejiang Archives 12 (2001): 12-13. 
Qing Dynasty and was popularly used in administrations of the National Government in China. ${ }^{8}$ Before that, the terms 'records' and 'archives' were not differentiated and the same word was used to refer to both 'records' and 'archives' without semantic distinction. That word differed, however, in different dynasties. For example, the Shang Dynasty used the term Diance, while the Tang and Song Dynasties used Andu or Wendu. Until the 1980s, the term 'records' mainly referred to written materials created by governments. After that time, however, more kinds of records were being created in a range of kinds of organisations as a result of the opening of China to the world and new economic policies. In 1987, Zhaowu Chen argued that 'records' refers to the concept of 'big record' and not just to written government records. Instead, 'records' should refer to any recorded materials created in the course of organisational or personal social interactions and communications that document different phenomena, business, the expression of ideas, and socialising. He also asserted that archives are a type of records. ${ }^{9}$ In 1991, he refined this definition and argued that records are materials recording information and are created by organisations or persons in the course of doing business. ${ }^{10}$ This evolution of the definition of records also reflects the shift in the Chinese archival paradigm from 'official' to 'societal,' almost at the same time as West German archivist Hans Booms' influential discussion of the societal approach was first published in English in $1987 .{ }^{11}$ This definition has been widely accepted, ${ }^{12}$ suggesting that the essence of records lies

8 The Second History Archives of China, Mingguoshiqiwenjianyudanganguanlicailiao [Materials on the Records and Archives Management of the National Go the idea of theds is a continuuhen dress to the agechival ideas and traditionses. Sometimes it simply helps us to understand Government] (Beijing: Archives Press, 1987).

9 Zhaowu Chen, "Zailundangandedingyi - Jianlunwenjiandedingyiheyingdongzhouqiwenti [Re-visiting the Definition of Archives - and Discussion of the Definition of Records and the Movement Cycle of Records]," Archives Bulletin 2 (1987): 21-25.

10 Zhaowu Chen, "Wenjian [Records]," Archives Bulletin 4 (1991): 72-73.

11 Hans Booms, "Society and the Formation of a Documentary Heritage: Issues in the Appraisal of Archival Sources," Archivaria 24 (Summer 1987): 69-107 [first presented by Booms in his opening address to the German Archives Conference and then published in German as "Gesellschaftsordnung und Überlieferungsbildung: Zue Problematik archivarischer Qvellenbewertung,' Archivalische Zeitschrift 68 (1972): 3-40].

12 For example, The Encyclopedia of Chinese Library, Information and Archival Science, published in 1993, defines records as recorded materials in all kinds of media that are used by administrations, organisations, enterprises and persons to deal with business and to communicate information in any form in social activities. The Dictionary of Archival Science, published in 1994, defines records as all the materials recording information created by organisations or persons during the process of their dealing with business. Cibai, a Chinese comprehensive dictionary published in 1999 defines records as all materials recording information created by organisations or persons to fulfil legal responsibilities or to deal with business. General Terminology for Archives Work (DA/ 
in the act of recording, whether the records are in oral or written form, or whether their media be bones, tortoise shell, stone, metal, bamboo, paper or electronic.

With the introduction of the idea of the life cycle of records in the 1980s, the term 'big records' came into currency and was further developed. Big records include current records, archives kept in Danganshi (archives rooms) and archives kept in archives (i.e., the institutions that collectively keep and manage the archives within a specified scope ${ }^{13}$ ), while 'current records' on their own are sometimes referred to as 'small records'. ${ }^{14}$ Danganshi is an internal department of a government agency (e.g., the Shanghai Municipal Development and Reform Commission) or an organisation. It keeps the archival records of the government agency or the organisation and only serves that agency or organisation. According to the Archives Law of China, those archives having permanent or long-term value that have been kept in Danganshi for 10 or 20 years should be selected and transferred to the national archives (also known as 'comprehensive archives') which are established by the State according to administrative divisions or historical periods and which manage many kinds of archives within their specified scope. ${ }^{15}$ There are three conditions for current records to become archives: 1) The records must have been processed; 2) The records must have value for future social activities; and 3) The records must have been filed and kept according to certain rules. ${ }^{16}$ The third condition is mainly applicable to the records created by organisations or administrations. Similar to the definition of records, the term 'archives' is broadly framed. According to the Archives Law of China:

T1-2000), the professional standard published in 2000, defines records as all kinds of recorded information created by administrations, organisations or persons during the process of their fulfilling legal responsibilities or dealing with businesses.

13 See DA/T1 - 2000, "General Terminology for Archives Work," http://www.nmgepb. gov.cn/tslm/dagl/hb/201009/t20100930_66211.html

14 Some examples of Chinese literature discussing "big records" and "small records" includes Xia Yang, "Lunwenjiandegainian [Discussion of the Definition of Records]," Archives Bulletin 1 (2000): 24-26; Pingcai Wu, "Dawenjianguan: Dandaizhongguodanganxuedebirangxuanzhe [The Perspective of Big Records: The Inevitable Choice of Contemporary Chinese Archival Science]," Archives Management 5 (2008): 34-37; and Liangen Pan and Dongbing Liu, "Guanyudawenjiangainiandebanxi [Analysing the Concept of Big Records]," Archives Management 5 (2008): 4-10.

15 See DA/T1 - 2000, "General Terminology."

16 Zhaowu Chen and Baorong He, Danganguanli [Archives Management] (Beijing: Renmin University, 1996), 12-14. 
'archives' means historical records in various forms, including writings in different languages, pictures, diagrams, audio-visual, etc., whose preservation is of value to the State and society and which have been or are being directly formed by State organs, public organisations and individuals in their political, military, economic, scientific, technological, cultural, religious and other activities. ${ }^{17}$

\section{Chinese Archival Tradition under Imperial Rule and Confucianism}

Up until the National Government period (1925-1948 CE), when professional education for archivists was established, records and archives management was mainly based on experience and handed-down knowledge, as well as on rules and regulations stipulated by different dynasties. Nevertheless, a formal recordkeeping and archives function can be identified.

During the Shang Dynasty a designated official, the Shiguan, was in charge of the augury, and was also responsible for recording the king's words and activities and keeping the records (at the time engraved on bones or tortoise shells). ${ }^{18}$ The Shiguan is, therefore, regarded as the earliest form of archivist in China. He usually had wide knowledge about history, astronomy, medicine and literature and was of high social status. From the Han Dynasty (202-220 BCE) until the Qing Dynasty (1636-1911 CE), responsibilities were divided between the Shiguan, who was primarily charged with recording the emperor's words and activities, and sometimes with making archives compilations and writing history, and the $L i$ (a government clerk put in charge of the management and keeping of records and archives). A Shiguan during this period should be well-educated, good at writing, and have extensive knowledge about history, literature and science. From the Tang Dynasty (618-907 CE) onwards, Shiguan also needed to pass $K e j u$ (an imperial examination). The qualifications to be a $L i$ were less demanding. $L i$ had low social status and were usually appointed through a hereditary system. ${ }^{19}$ Their experience and knowledge of records and archives management was passed from generation to generation within a family, and

17 Archives Law of the People's Republic of China, available at http://www.saac.gov.cn/ xxgk/2010-02/05/content_1560.htm.

18 Xueheng Zhou, Zhongguo Danganshiyeshi [History of the Development of Chinese Archives] (Beijing: Renmin University Press, 1994), 18.

19 Zhou, ZhongguoDanganshiyeshi, 114. 
constituted a specialised skill that provided a living for them. Others knew little about their knowledge, and scant written documentation of it or their experience was created and kept.

From around $500 \mathrm{BCE}$ until the early twentieth century, all aspects of records and archives management in China were deeply influenced by Confucianism. Confucianism began during the Eastern Zhou Dynasty and over time its cultural influence extended also into Japan, Korea and Vietnam. ${ }^{20}$ In the Han Dynasty (202-220 BCE), when Confucianism became the dominant political ideology, it provided justification for the dynasty's highly centralised authority. From then until the end of the Qing Dynasty (1636-1912), the purpose of records and archives management was to maintain imperial power. ${ }^{21}$ Confucian intellectuals dominated the bureaucracy, and Confucian ideals, including centralisation, rigid social levels, and the values of Ren (Humaneness), Yi (Justice), Li (Etiquette), Zhi (Knowledge) and Xin (Integrity) were promoted. Regulations were stipulated by the dynastic rulers about the kinds and formats of records and the colour of paper to be used, ${ }^{22}$ and a stringent taboo system ${ }^{23}$ controlling how records were to be written was implemented. Selected records created by the emperor, the government and the ruling class were to be kept collectively and permanently in a safe place

20 See, for example, Meung-Hoan Noh, "Confucian Community Construction and Records/Archives Management of Joseon Dynasty - focused on the meaning of the Uigwe Production, Preservation, and Use," paper presented at AERI 2012, University of California Los Angeles, July 2012, http://aeri2012.wordpress.com/conferenceschedule/paper-presentations/community-archiving/.

21 For example, Confucianism proposed the divine right of Huangdi (emperors) which claimed Tian (Heaven) was the master of everything in the world and the emperor was this son who could communicate with Heaven and was Heaven's representative in the world. Everyone, therefore, should be obedient and loyal to the emperor. See Hongbo Meng, "Tangchaonujiasixiangdechanshengtantao [A Discussion about the Creation of Confucianism in the Han Dynasty]," Tangdu Journal 6 (2008): 39-43.

22 There were many kinds of records, some kinds of which could only be used by the emperor, and some which could only be used by the prince. Different kinds of records had different formats. For example, if an official needed to submit some records to the emperor, then he should express his respect, loyalty and subservience to the emperor. The records, therefore, usually began with sentence such as: "your servant submits the record at the risk of death" and added some words further expressing his subservience before his name. The colour of paper used also reflected the Confucian hierarchy, since government officials at different levels should use paper of different colours for different uses. See Zhou, Zhongguo Danganshiyeshi, 120.

23 That is, the names of the emperor, the emperor's family members, ancestors, and the saints, or the names of the writer's own parents, ancestors and the leaders could not appear in the records. See Xin Wang, "ZhongguoGuodaibihuizhiduyanjiu [Research on the Taboo System of Ancient Chinese Records]," Yunnan Archives 5 (2011): 23-24. 
and only the emperor and authorised officers could access them. These rules and regulations reinforced control over records and archives and reflected the main ideas driving early Chinese archival practises. In the Tang (618$907 \mathrm{CE}$ ) and Song (960-1279 CE) Dynasties, the rules and regulations were relatively sophisticated. For example, in the Tang Dynasty, strict rules and regulations governed the creation, transmission and filing of records, and the appraisal and destruction of archives. It also established a rigorous supervision and inspection system of records and archives management. ${ }^{24}$ A number of archives were created during these dynasties and were stored collectively in locations such as Lantai and Dongguan in the Han Dynasty, Jiageku in the Song, Ming and Qing Dynasties, and Huangshicheng in the Ming and Qing Dynasties.

To maintain imperial power, the emperors of every dynasty attached importance to the writing of history that could provide justification for their rule, ${ }^{25}$ so the most important task of archives management in imperial China was to support this activity. Archives compilations ${ }^{26}$ were usually created and were so valued by the emperors that they themselves sometimes supervised the activity. ${ }^{27}$ Official archives compilations were guided by Confucianism: virtues in accordance with Confucian culture were praised, and behaviours contrary to Confucian culture were criticised as vices. The compilations, therefore, helped to spread Confucian culture and provide behavioural norms for the people, which in turn strengthened dynastic rule. Besides these official archives compilations and histories, some officials who were permitted to access the archives also created archives compilations ${ }^{28}$ and

24 Zhou, Zhongguo Danganshiyeshi, pp.172-176.

25 Ancient official archives compilations mainly include Shengxun (compilations of the emperors' edicts and words admonishing their officials), Shilu (compilations of the important official activities of the emperors), Qijuzhu (compilations of the words and activities of emperors), and the compilations of codes and criminal laws.

26 Creating an archives compilation involves selecting, conducting textual research on, compiling and reviewing archives regarding a particular topic. See Xichen Cao, Danganwenxianbianzhuangxue [Archives Compilations] (Beijing: Renmin University Press, 1990), 44.

27 For example, in 1729 Yongzheng, one of the emperors of the Qing Dynasty, supervised the compilation Dayijuemilu of the archives about the trial of an anti-Qing activity. Yuanzhang Zhu, the first emperor of Ming Dynasty, supervised the compilation Mingdahao of criminal cases. See Cao, Danganwenxianbianzhuangxue, 67, 73.

28 For example, Confucius compiled Shangshu (the Book of History), which is the first archives compilation in Chinese history. Ruyu Zhao, a member of royal clan of the Southern Song Dynasty (1127-1129), compiled the petitions of famous officials to emperors of the Song Dynasty; Lianqi Jiang, an official in the Shiguang (the national 
wrote history. ${ }^{29}$ These individuals were usually themselves deeply influenced by Confucianism. ${ }^{30}$

Many ideas about archives compilations, for example, that archives compilations should be true to the original archives, should indicate the source of archives by adding notes, and should collect archives comprehensively ${ }^{31}$ - all tenets that would reassure the reader of the trustworthiness of the compilations when the original documents could not be viewed - were generated through this process and became the main components of ancient Chinese archival ideas. ${ }^{32}$ These ideas still influence how today's archives are compiled. Although the main purpose of compilations was political, like the histories that were written, they served to disseminate the content of the archives to people who could not access the archives for themselves. They also preserved knowledge about the contents of many ancient archives, especially those created on paper that did not survive for various social or natural reasons.

The records and compilations that were generated through these processes captured the official actions and discourse of the élite, but little about the rest of the people, particularly women (even high status women). To maintain feudal patriarchy, Confucianism proposed Three Obediences for women: obey your father as a daughter, obey your husband as a wife and obey your sons in widowhood; and Four Virtues: morality, proper speech, modest manner and diligent work. Women were thus dependent on men, so their names were not allowed to be recorded in the family tree and they were excluded from both histories and archives. The exceptions were the few who were held up as models for women because, in accordance with the Three Obediences and Four Virtues, they refused to remarry after their husbands died in order to show their loyalty to them or they chose to kill themselves in order to preserve their reputations.

history institution of the Qing Dynasty), compiled Donghualu, which brought together archives and other important historical materials about the history of the Qing Dynasty from 1616 to 1735 .

29 For example, QianSima, a Shiguan of the Western Han Dynasty, compiled Shiji (a 2500-year comprehensive history of ancient China up to the 2nd century BCE), and Gu Ban, a Shiguan of the Eastern Han Dynasty compiled Hanshuor Book of Han (a classical history of China under the Western Han from $206 \mathrm{BCE}$ to $25 \mathrm{CE}$ ).

30 For example, when writing Shiji, QianSiman selected historical documents using Confucian classics as his criteria.

31 Cao, Danganwenxianbianzhuangxue, 25-32.

32 This is because compared with Li, Shiguan were well-educated, they could write down their ideas about archives compilations, and then these ideas could be passed down. 
In ancient China, therefore, records and archives were regarded as political tools that could be used to justify and maintain dynastic rule, their creation, management and compilation were influenced by Confucian culture and served the imperial rule.

\section{Modern Chinese Archival Tradition and the Social Transformation through Modernisation}

The Opium Wars of 1840-42 and 1856-60 forced China onto the path to modernisation. Qichao Liang, the famous thinker and historian of modern China, articulated in his 1923 article Wushinianzhongguojinhuagailun (Evolution of China in the Past Fifty Years) that the past 50 years had been a process towards modernisation. He mentioned three areas for modernisation in particular: the first was to build implements such as weapons and ships employing western science and technology, since these had failed China in the Opium Wars; the second was to modernise institutions. Many people realised that the failure in the wars was not simply caused by poor military equipment, it was also caused by bad structures such as the Keju (the imperial examination) of the centralised imperial rule. The third was to modernise the culture through cultural and ideological reform. ${ }^{33}$ The latter started with the anti-imperialist May Fourth Movement that grew out of student protests in 1919. During the process of modernisation, Chinese nationalism grew, traditional Confucian culture was rejected, democracy and selective scientific and technological development were advocated and western ideas were imported. The Qing Dynasty was finally overthrown in 1921 and the National Government (1925-1948) was founded in 1925. These major social changes necessarily influenced archival practice and ideas during this period.

Many scholars responding to the New Culture Movement's promotion of the idea of 'sorting out national cultural heritage' around the time of the May Fourth Movement needed to access comprehensive materials about national cultural heritage, including those held in dynastic archives. Because of the scandal of ' 8000 Gunnysacks, ${ }^{34}$ scholars at Peking University

33 Liang Qichao, Wushinianzhongguojinhuagailun [Evolution of China in the Past Fifty Years], http://www.my285.com/xdmj/lqc/057.htm

34 In 1921, the Baiyang government selected some of the archives kept in the Cabinet's storage building and then put the rest of these archives into 8,000 gunnysacks and sold them to a paper-making shop for the price of 4,000 silver dollars. Many Chinese people, especially scholars, were outraged at this scandal. In 1922, an academic organisation researching national cultural heritage was founded in Peking University. When some of its best-known professors, including Xizu Zhu, Jianshi Shen, and Heng Ma found out 
took over the Ming-Qing dynastic archives that had been kept by the government and then arranged, compiled and published them. After that, other organisations, including the Archives of the Palace Museum and the history department of Tsinghua University also began to arrange, compile and publish the Ming-Qing dynastic archives that they held. Their activities imply to some degree the breaking of the mode of government control over archives and archives management. The principles of arrangement and the classification of ancient archives were discussed and, although Muller, Feith and Fruin's Dutch Manual ${ }^{\beta 5}$ was not translated into Chinese until 1959, some ideas such as respecting original order and classifying the archives according to their creating agencies were introduced. ${ }^{36}$

In the 1930s, because of its own poor administrative efficiency as well as the influence of western (especially American) theories of administration that were introduced by scholars and officials who had studied in western countries, the National Government initiated the Administrative Efficiency Movement. The main purpose of records and archives management in the government was to improve administrative efficiency, and a new kind of archival entity, Danganshi, was established to manage archives collectively in the agencies of national government. ${ }^{37}$ In 1933, the National Government initiated the Reform of Records and Archives Movement, ${ }^{38}$ the core of which was the Chain Method of Records and Archives. Similar to a registry system, it was proposed by Naiguang Gan, the deputy minister of the Ministry of

that some of the archives of the Cabinet were still preserved in the History Museum of the Baiyang government, they asked Yuanpei Cai, the president of Peking University to write to the Ministry of Education, asking the History Museum to transfer these archives to Peking University for preservation and arrangement. At that time, the Deputy Minister of the Ministry of Education was the famous historian, Heng Chen, and he supported Peking University's request. Because of the scandal over the gunnysacks, the Baiyang government was under the public pressure and had to accept the request. 62 boxes and 1520 gunnysacks of archives were, therefore, transferred to Peking University, whose faculty and some students established a committee for the arrangement of the archives. See Shouyi Li, "Qingneigedanganpoqianjilie [The Transfer History of the Archives Kept in the Warehouse of the Cabinet of the Qing Government]," Forbidden City 2 (2012): 44-51 and Zhou, ZhongguoDanganshiyeshi, 419.

35 Samuel Muller, Johan A. Feith and Robert Fruin. Manual for the Arrangement and Description of Archives, Eric Ketelaar, Theo Thomassen and Peter Horsman, trans. of second edition (Chicago: Society of American Archivists Classic Series, 2003) .

36 See Huichao Zhang, Mingguoshiqi Mingqingdanganzhengliyanjiu, 132-133.

37 This is the origin of contemporary archives rooms and reflects the idea of fonds.

38 See Naiguang Gan, "Wenshudanganliansuanfazhishiyan [Testing the Chain Method of Records and Archives]". This article discusses putting the Chain Method of records and archives into practice to see if it is feasible or if there are any problems, Administrative Efficiency 10 (1934): 423-429. 
Interior, who believed that records and archives could not be separated since archives were filed records and records were unfiled archives. Based on this belief, he proposed that records management and archives management should be integrated and that the mailroom, which was previously only in charge of receiving and dispatching documents, should classify, register and catalogue all the documents according to a classification scheme, and then dispatch them to the related units. After the records were handled, the units should file and transfer them to the Danganshi of the agency where they should be kept according to the classification designated to them by the mailroom. By doing this it would be also convenient to find related archives and attachments to incoming or outgoing documents. The Chain Method of Records and Archives is based on the recognition of the relationship between records and archives. It reflects the idea that the process from the creation, current use, filing and keeping of records is a continuous one. It guided the practice of integrating records and archives management at that time, and also had a great impact on the development of the idea of the life cycle of records in contemporary China.

With the development of this movement and of the archival practice of Danganshi in agencies, it became necessary to train professional archivists. Many schools and universities were established as a way to cultivate the ability to realise modernisation. A major in archives management was established in the Hubei Private Wuchang Wenhua Library School (Boone Library School), which had been founded in 1929. In 1942, the Ministry of Education stipulated that the school should train archivists for the National Government. In 1946, the private Chongshi Archives School was founded in Chongqing by Zhongqi Ying, also to train archivists.

All of these developments stimulated archivists, archival educators, historians and administrators to publish a number of archival works including the thirteen classic modern archival publications. ${ }^{39}$ Meanwhile, influenced

39 The 13 archival works are: "Gongdutonglun [Introduction to Records]" (WangzhiXu, 1931); "Xianzhengfudanganguanlifa [Archives Management of County Government]" (Changyuan Chen, 1936); "Danganguanliyuzhengli [Management and Arrangement of Archives]" (Lucheng He, 1938); "Danganguanlifa [Archives Management]" (Zaofu Long, 1940); "Xianzhengfugongwenchuliyudanganchuli [Records and Archives Management of County Government]" (Shangyan Liang, 1942); "Dangankexueguanlifa [Scientific Methods of Archives Management]" (Hancai Qin, 1942); "Gongwenchulifa [Methods of Records Management]" (Liankuan Zhou, 1945); "Wenshuzhijianhuayuguanli [Simplification and Management of Records]" (Guochen Chen, 1946); "Gongrwendanganguanlifa [Records and Archives Management]" (Zhenlun Fu, 1947); "Danganguanlizhililunyushiji [Theory and Practice of Archives Management]” (Yizhong Huang, 1947); “Gongduxueshi [History on Records 
by western ideas of dividing disciplines according to the object of study, the term 'archival science' was first proposed by Jianshi Shen in January 1935, and a few months later Teng Gu introduced the term in his article Danganzhenglichuderenwujiqijibengongzuo (The Mission and the Preliminary Work of the Archives Arrangement Office): ${ }^{40}$

Using scientific methods to manage archives is the work of Archival Science, which developed in Europe in the last 100 years. ${ }^{41}$

The published archival works, especially the thirteen publications, are regarded as symbolising the formation of Chinese modern archival science. These works were mainly based on the archival practice of Danganshi in agencies and the arrangement of Ming-Qing dynastic archives, encompassing the core issues of archival science such as the definition of archives, the values of archives, archival arrangement, archival appraisal and destruction, archival filing, archival cataloguing, archival preservation and the training of professional archivists. The ideas contained in these works still have a great impact on today's Chinese archival ideas and practices. For example, Lucheng He, in his book Management and Arrangement of Archives, defined 'archives' as records that have been processed and filed for reference. ${ }^{42}$ The three conditions for records to become archives that were discussed in the introduction to this chapter stem from Lucheng He's definition. Another example is the principle of archival arrangement. During this period western library classification systems - the Dewey Decimal Classification system and Library of Congress Classification system - were introduced and adopted by agencies, but in his book Danganzhenglidelilunyushiji (Theory and Practice of Archives Management), Yuzhong Huang contended that:

The difference between archival classification and library classification is that archival classification shall respect the history of creation, and shall

Science]" (TongxinXu, 1947); "Danganguanlifa [Methods of Archives Management]" (Liankuan Zhou, 1947); and "Zhongguodanganguanlixinlun [New Discussions on Chinese Archives Management]" (Zhongqi Ying, 1949). All of these 13 books have been reprinted and republished by the World Publishing Corporation in 2013.

40 Jihong Liang, Zhongguojindai "Danganxue"ciyuanxinkao [New Discovery on the Origin of the Term of Archival Science in Modern China]. Archives Bulletin 5 (2010): 21-24.

41 Baokan Wu, Danganxuegailun [Introduction to Arcbival Science] (Beijing: Renmin University Press, 1998), 318.

42 Lucheng He, Danganzhengliyuguanlifa [Management and Arrangement of Archives] in Archives Bulletin, eds.Danganxuejingdianzhuzuo (Dierquan) [Classic Works of Archival Science Volume 2] (Shanghai: World Publishing Corporation, 2013), 124. 
not disorder the original system. This is the basic and unique principle of archives classification which is not applicable in library classification. ${ }^{43}$

His idea is identical with the principle of provenance. It reflects the historicism implicit in the arrangement of archives and also provides reference for contemporary archival arrangement.

With the eastward dissemination of western learning, some western archival works, including Hilary Jenkinson's Manual of Archive Administration, were translated and many articles introducing foreign archival practice and ideas were published. For example, Chongmin Wang gave a detailed introduction on the archives preservation skills adopted by the U.S. National Archives in his article Meiguoguojiadanganguanyinxiang (Impression of the National Archives of the United States). ${ }^{44}$ These western archival ideas and practices provided references for the archives work at that time, and many archival researchers such as Lucheng He and Zhonglin Yin formed their archival ideas by studying western archival ideas and practice. For example, when Lucheng He gave his definition of the term 'archives', he stated that:

Dangan ("archives" in Chinese) is equivalent to the term 'archives' in English. If we integrate the Chinese and the English, we can define the term Dangan as: records that have been processed and filed for reference. $^{45}$

In modern China, the strong urge for modernisation stimulated the reform of records and archives management, which was a part of managing administrative efficiency. Modern archival education emerged and trained a new class of professional archivists for agencies. These professional archivists were no longer the passive keepers and guardians of government archives. They actively promoted the reform of records and archives management to improve the administrative efficiency. They were also very open-minded and, actively seeking to learn from western ideas and practices, generated and published a series of modern archival ideas. These ideas were based on the archival practices of Danganshi in the agencies, but they still provide significant references for the development of current Chinese archival science. Their efforts, together with the contributions of those historians committed to the arrangement of Ming-Qing dynastic archives, and archival educators and some administrators, brought about the birth of modern

43 Baokan Wu, Danganxuegailun, 329.

44 Baokan Wu, Danganxuegailun, 322.

45 Lucheng He, Danganzhengliyuguanlifa, 124. 
Chinese archival science as a disciplinary area and of capacity building for specialist knowledge and skills in China.

\section{Developing Contemporary Chinese Archival Practice and Ideas}

The foundation of the People's Republic of China (PRC) in 1949 marked a new phase of Chinese archival ideas and practices. Strongly influenced by those of the Soviet Union, Marxism-Leninism became and still is regarded to be the supreme guidance for the development of archival ideas and practice in China. At the core of Marxism-Leninism is the idea of the dictatorship of the proletariat - that the working class controls the political power. After the Russian October Revolution, several decrees relating to archives were issued including an April 1919 decree on the procedure for the storage and destruction of archival files. ${ }^{46}$ This was quickly followed in July by a decree on the abolition of private property rights regarding the archives (i.e., records) of dead Russian writers, composers, artists and scientists held in libraries and museums. ${ }^{47}$ The latter decree abolished private ownership of archives and proposed the notion of State Archival Fonds, meaning that all archives should be owned by the state. ${ }^{48}$ Under this framework, a centralised archives management system was established in the Soviet Union. This idea was to become the most important principle and regulation for archives management in many socialist nations.

Accordingly, after the foundation of the PRC, the notion of a State Archival Fonds was introduced and in 1954, the State Archives Administration of the People's Republic of China (SAAC) was established to guide national archives management. In 1956, a State Department decision stipulated that all archives created before or after the foundation of the PRC by any kind of organisation should be owned by the state and should be managed by the national archives, ${ }^{49}$ thus establishing the centralised archives management system in China. The decision also stated that SAAC should plan to establish national archives in the capital and in all of the regions in China.

46 http://opentextnn.ru/censorship/russia/sov/law/snk/1917/?id=565.

47 http://opentextnn.ru/censorship/russia/sov/law/snk/1917/?id=566.

48 Baokan Wu, "Jianchihefazhangguojiadanganquanzong [Insisting on and Developing the Notion of National Archives Fonds]," in Nerwshiqidanganxueyudangangongzuo [Archival Science and Archival Work in the New Era], ed. BaokanWu (Beijing: China Archives Press, 1997), 105-108. 
The Central Archives was established in 1959 to manage the archives created by the May Fourth Movement of 1919 up until to the foundation of PRC by the Communist Party as well as the archives created after the foundation of the PRC. By March 1963, 19 provincial state archives and 1590 county state archives had been set up in China. ${ }^{50}$

The development of national archives management in the PRC required new professional archivists who were knowledgeable about MarxismLeninism as well as archives management. In 1952, with the help of the archival professionals from the Soviet Union, the teaching and research office in archival science at Renmin University was established. This subsequently developed in 1955 into the department of historical archives to train professional archivists. The archival ideas and archival practices of the Soviet Union, such as the centralised archives management system, ${ }^{51}$ the criteria for archival appraisal (historicism, party spirit, comprehensiveness and systematicity), ${ }^{52}$ theories about the fonds group, ${ }^{53}$ and the concept of State Archival Fonds ${ }^{54}$ were introduced in classes and became the basis of the earliest textbooks in archival science in the PRC.

The development of archival practice and education was interrupted by the Cultural Revolution, which began in 1966 and lasted for 10 years. SAAC was shut down and national archives management was almost forced to stop; universities were closed, thus shutting down archival education also. People "were encouraged to destroy the 'Four Olds' - old customs, old habits, old culture, and old thinking," resulting in tremendous loss of Chinese heritage, ${ }^{55}$ including the loss of many valuable archives.

50 See The Office of the State Archives Administration of China (SAAC), Dangangongzuowenjianhuibian [ Compilations of the Regulations on Archival Work] (Beijing: Archives Press, 1986), 74.

51 Wu, Danganxuegailun, 129-135.

52 'Historicism' means that every record is the product of a specific historical moment, so archival appraisal should consider the historical context of the creation of the records; 'Party spirit' means that archival appraisal should be based on what would benefit the proletariat; 'comprehensiveness' means that the value of archives should be evaluated from different perspectives; 'systematicity' means that the relationship between the records being appraised and other related records should be considered. All of these principles were proposed by the Soviet Union, and then introduced into China. See Zhaowu Chen, Danganguanlixue [Archives Management] (Beijing: Renmin University Press, 1962), pp.88-89.

53 Fonds group refers to a group of fonds related to each other. See Chen, Danganguanlixue, 52.

54 See Wu, Danganxuegailun, 68-85.

55 Jonathan Spence, The Search for Modern China (New York: Norton and Co, 2001). 
The end of the Cultural Revolution and the opening of China to the world as well as the economic reform of 1978 brought new vitality to the Chinese archival community. Universities and schools gradually began to reopen, and today 37 universities offer majors in archival science - a larger education base than in any other country in the world - training professional archivists for governments, enterprises and other organisations. Many textbooks, papers and other works on records and archives management have been published, and prominent archivists and scholars such as Baokan Wu, San Ceng, Tong Pei, Zhaowu Cheng, Huiling Feng, Jiasun He and Rongxiao Fu have been heavily engaged in the development of Chinese archival practice and ideas. They have further enriched and developed such archival ideas as the Chinese life cycle of records and the 'big fonds.'

After it was introduced, the life cycle concept received much attention from some Chinese archival scholars, including professors Zhaowu Cheng, Jiasun $\mathrm{He}$ and Rongxiao Fu (especially the latter two). Influenced by Professor Manual Vazpuez of Argentina, they developed it into a major theory to guide records management in China. ${ }^{56}$ The main components of this theory are that:

1. It is a complete process model that covers records from the point of creation until they are destroyed or designated for permanent retention. That is, the movement of records through these phases has the characteristic of completeness.

2. The life cycle of records can be divided into different stages because of the different values that records take on at each point.

3. Appropriate methods of keeping and managing records should be employed according to their different characteristics at each stage because these characteristics are closely related to the factors involved with their movement. ${ }^{57}$

As for the stages in the life cycle of records, Zhaowu Chen, in keeping with American life cycle ideas, proposed four stages of records movement:

56 Jiasun He and Xuren Shi, "Wenjianlianyutililunyuwenjianshengmingzhouqililun Wenjianyundonglilunzhiyi [Records Continuum and Life Cycle of Records-Research on Records Movement Theory (the First)]," Archives Bulletin 5 (2003): 60-64.

57 See $\mathrm{He}$ and Fu,Wenjianyundongyanjiu - Xinshijiaoxiaduidanganxuejichulilundeshenshi; Jiasun He, "Wenjianshengmingzhouqililunduiwomengdeqishi [The Inspirations of Life Cycle of Records]," Archives Bulletin 6 (1991) and 1 (1992) (this article is relatively long, so a part of it was published on the volume 6, 1991, the rest was published on the volume 1, 1992.); Rongxiao Fu, "Wenjianshengmingzhouqililunyanjiu [Research on the Life Cycle of Records]," Archives 1 (1994) and 4 (1994). 
creation, current use, temporary retention, and permanent retention, ${ }^{58}$ with the responsible agent being, respectively, records creators, records managers, archivists of archives rooms, ${ }^{59}$ and archivists of national archives. In these two latter aspects, Chen's model differs somewhat from the American conceptualisation of the life cycle.

After being discussed for many years, the concept of the life cycle of records was widely accepted in China - until the records continuum was introduced in 2001. With the influx of electronic records, the introduction of post-custodial thinking and the influence of memory studies, the records continuum has attracted increasing attention and is now challenging the theory of the life cycle. There has been a lot of discussion about the relationship between the life cycle of records and the records continuum and which one is more suitable for the electronic records management in China. ${ }^{60}$ However, most researchers think that the life cycle of records is still applicable to electronic

58 Chen, "Zailundangandedingyi - Jianlunwenjiandedingyiheyundongzhouqiwenti.

59 There are several kinds of archives rooms in China today: those in government agencies; those in some corporations or companies; and those in some public institutions such as hospitals, schools or other organisations. All of these archives rooms are under the supervision of the archives administration of their district and also under the supervision of the State Archives of China in accordance with the Archives Act of China. In theory the archivists of archives rooms work for their institutions. But usually this is only the case in the first context - archives rooms established in government agencies have the legal obligation to transfer the archives having long-term value to the government archives of their district.

60 Some archival researchers think that the records continuum will replace the life cycle of records since the records continuum model has advantages in the electronic records management that the life cycle of records has not. See Xiaomi An, "Wenjianlianxutimoshiduidianziwenjianzuiyouhuaguanlideqishi [The Inspirations of the Records Continuum Model for Electronic Records]," Archives Bulletin 3 (2002): 52-55; and Rongxiao Fu and Ying Ji, "Wenjianzhengtiyundongmiaoshu: Shiwenjianshengmingzhouqimoshi, Haishiwenjianlianshutimoshi [Description of the Movement of Records: Life Cycle of Records or Records Continuum]," Archives Management 3 (2008): 7-12.). Some argue that the records continuum is the development of and supplement to life cycle of records in the era of electronic records, but that the life cycle of records is still applicable to electronic records management and we need to adjust and develop it to the new environment. See Xiaoyu Huang, "Dianziwenjianshidaiwenjianshengmingzhouqililundexiugaiyuwanshan [Adjustment and Development of the Life Cycle of Records in the Era of Electronic Records]," Archives Science Study 1 (2003): 6-9. Some contend that the records continuum enriches the law of records movement, and that its essence is not contradictory with the life cycle of records, but that the life cycle of records does not describe the complete law of records movement, so we can research on the records movement more comprehensively based on the records continuum. See Jiasun He, "Wenjianlianxutiyuwenjianshengmingzhouqililun [Records Continuum and Life Cycle of Records]," Archives Bulletin 5 (2003): 60-64; Jiasun $\mathrm{He}$ and Xuren Shi, "Zhidaoyubeizhidaoguanxi-Guanyuwenjianlianxutililun, 
records, although, just as with the principle of respect des fonds, it needs to be updated. ${ }^{61}$ For electronic records, moving from creation to destruction or permanent retention is still a complete process, but there are no obvious boundaries between the stages and electronic records may move forward or backward through the stages with archivists participating at all stages (including systems design), and the whole process of management and upfront control should be reinforced through electronic records management. ${ }^{62}$ This signifies a change in the role of archivists. Traditionally, with the life cycle approach, archivists are only in charge of archives and they do not intervene in the creation of records or the management of current records. Current records management and archives management are totally separated and independent. However, this separation has resulted in many problems, such as inefficiency, which is the main reason why the integration of records management and archives management has been proposed. Actually, the integration had been tested and implemented in some places such as Jilin and Panzhihua before the influx of electronic records, but electronic records and information technology has helped to make it more feasible.

When the term 'fonds' was introduced to China in the 1950s from the Soviet Union, it was defined as all of the archives (records) created by an administration or prominent person. ${ }^{63}$ This definition was based on the traditional opinion that provenance referred to the agency that created those

Wenjianyundonglilunhewenjianshengmingzhouqililunzhisibian [Guiding or Being Guided - Analysis of the Nature of the Records Continuum, the Life Cycle of Records and the Movement of Records]," Zhejiang Archives 9 (2006): 6-9.

61 Maybe there are two main reasons why most Chinese archival researchers persist in the applicability of the life cycle of records: Firstly, the life cycle of records has become a very important theory of records movement and records management after being developed by many Chinese archival researchers for so many years, and it has been widely accepted by Chinese archival community. It is, therefore, more acceptable to modify rather than to discard the concept and actually it has been and still is being used to guide records management and the integration of current records management and archives management in China. Secondly, the Chinese archival community is not very familiar with the records continuum because of the language barrier between its expression in English and explication in Chinese, and not understanding the background to the concept or to recordkeeping practice in Australia very well. Compared with the research that has been conducted on the life cycle of records, research on records continuum has not received sufficient attention in China.

62 See HuilingFeng, Dianziwenjianguanlijiaocheng [Textbook of Electronic Records Management] (Beijing: Renmin University Press, 2001), 5-7, 13-15; and Xiaoyu Huang, "Dianziwenjianshidaiwenjianshengmingzhouqililundexiugaiyuwanshan [Adjustment and Development of the Life Cycle of Records in the Era of Electronic Records]," Archives Science Study 1 (2003): 6-9. 
archives. In 1988, professors Huiling Feng and Jiasun He published a series of papers discussing the definition of the fonds. After teasing out the development of the principle of respect des fonds and analysing the problems with using the traditional definition of fonds in Chinese archival practices, Feng and $\mathrm{He}$ argued that provenance should refer to the process associated with a social activity, ${ }^{64}$ a development of the concept of provenance that precedes the idea of functional provenance underpinning macroappraisal that was first developed in 1989-90 by Terry Cook as a new strategy of the National Archives of Canada. ${ }^{65}$ The fonds, therefore, would comprise all of the related archives created in the course of that activity. From an epistemological perspective, the social activity of human beings consists of both subject and object and the complex interactions between them. The subject is the actor of social activity; the object is the target of social activity. All the archives created by an independent subject when carrying out its function, therefore, form 'subject fonds', while all the archives created around an object such as a project, a product or particular equipment form 'object fonds.' These two kinds of fonds, when taken together, are regarded as 'big fonds'. ${ }^{66}$ In 1998, Feng reiterated that "provenance refers to the creation process and context of a record, that is, the record is created by whom, under what conditions, for what purpose and using what kind of construct?" 67 In 1999, He again discussed the essence of and trend in the development of thinking about respect des fonds and emphasised that it remained an important guiding principle in the age of electronic records. ${ }^{68}$ Today the Chinese archival field is in agreement that while respect des fonds is still applicable in electronic records management, the term "provenance" refers to the creation process and context of a record rather than to a single creator or creating agency.

To reinforce national archives management, the Archives Law of China was enacted in 1987, again stipulating a centralised system, which has become the fundamental principle of archives management. In accordance with this principle, a national archives network has been created, consisting

64 Huiling Feng and Jiasun He, "Quanzonglilundeshizhi [The Essence of Respect des Fonds]," Archives Bulletin 5 (1988): 8-11.

65 Terry Cook, "Macro-appraisal and Functional Analysis: Documenting Governance RatherThan Government," Journal of the Society of Archivists 25 no.1 (2004): 5-18.

66 He and Feng, "Guanyugengxinquanzonggainiandeshexian."

67 Huiling Feng, "Dianziwenjianshidaixinsiwei [New Ideas in the Age of Electronic Records]," Archives Bulletin 6 (1998): 45-49.

68 Jiasun He and Rongxiao Fu, Wenjianyundongguilvyanjiu: Congxinjiaodushenshidanganxuejichulilun [Research on the Movement of Records: Review of the Basic Archival Theories from a New Perspectives] (Beijing: China Archives Press, 1999). 
of the Central Archives and SAAC. ${ }^{69}$ SAAC guides and supervises all archives of any type at any level and the national archives at all levels in charge of the acquisition, arrangement, appraisal, cataloguing, compilation and use of local archives which are mostly the official records created by the local administrations. A series of rules, regulations and standards on archives management have been issued by SAAC to guide national archives management.

'Official archives' kept in national archives have been the focus of the Chinese archival community, therefore. However, since the 1990s the 'societal approach' has emerged and 'memory' is becoming the new focus. This is partly due to historical research trends and the work of members of the international archival community. Influenced by postmodernism and international research on collective memory, some Chinese researchers in history began to study social memory. Historian Mingke Wang argued that researchers should regard historical documents as the residues of social memory, and, when consulting them, should keep in mind: "whose memory this is", "how the documents are created and used" and "how they are kept and forgotten." According to Wang, "Complete or true social memory should consist of both typical history and marginalised history."70 Guangyao Jin, another renowned historian, contended that archives are mainly about the activities of government and élites, and that there are very few records about the lives of grassroots communities, and yet such records are an important part of a city's memory. ${ }^{71}$ Furthermore, with the increase of international communication in the archival field, ideas about the societal approach, and about archives and memory expressed by international archival researchers such

69 In 1993, the Central Archives and the State Archives Administration were merged into a single agency through the Scheme on the Allocation of Function, Internal Organisation and Personnel Arrangement of the Central Archives and the State Archives Administration that was enacted jointly by the General Office of the Central Committee of the Communist Party of China and the General Office of the State Council. Since then, archives and archives administration at all levels have been merged (originally the national archives and the archives administration were two separate agencies, national archives are charged with the management and keeping of archives, while archives administrations are in charge of such administrative management as planning, guiding and supervising the development of archives work by stipulating regulations and standards. But after 1993 they merged into a single agency, for example, Shanghai Municipal Archives merged with Shanghai Archives Administration, and now they are a single agency).

70 Mingke Wang, "Lishishishi, Lishijiyiyulishixinxing [History Fact, History Memory and History Mood]," History Research 5 (2001): 136-191.

71 Guangyao Jin, "Koushulishiyuchengshijiyi [Oral History and City Memory]," Eastday Network. http://sh.eastday.com/qtmt/20111110/u1a937145.html. 
as Terry $\mathrm{Cook}^{72}$ inspired the Chinese archival community. Some Chinese archival researchers began to rethink the nature and role of archives, archivists and archives management from the perspective of memory or from the broader societal context. In 1997, Huiling Feng referred to electronic records as a new kind of memory in her doctoral dissertation. ${ }^{73}$ In 1999, Professor Huadong Ding analysed the reasons why the notion of archival memory was being proposed and its influences. ${ }^{74}$ In 2012, Huiling Feng asserted that archives are an important factor in the construction of collective memory. She argued that archivists should actively participate in the construction, maintenance and transmission of collective memory. She also argued that the ideas, working principles and methods of archivists can have a negative or positive influence on the authenticity and integrity of collective memory. ${ }^{75}$ Meanwhile, beginning in 2002, some national archives in such cities as Shanghai, Guangzhou and Qingdao initiated 'City Memory' projects to preserve their city's memory. The City Memory projects, however, mainly involve documenting old and ruined buildings and neighbourhoods through photography or videography. They have not focused much on the people's memory of the city. On the other hand, people's awareness of civil rights has become stronger, and China has been promoting the transformation of the government role to a more service-oriented government. This necessitates that the Chinese national archives must not only collect the records created by government and serve the government, but also must collect the records created during interactions between citizens and the government, as well as archives about the people. They must also serve the people. In 2008, SAAC proposed developing two systems - one a system of archives resources about the people and another a system supporting ease of use of archives by the people. Some specialised archives documenting communities such as 'the only child,' or educated urban youth going to work in the countryside and mountains during the Cultural Revolution have been collected and kept in national archives.

72 Terry Cook's speeches, "Archives in the Post-Custodial World: Interaction of Archival theory and Practice since the Publication of the Dutch Manual in 1898" to the $13^{\text {th }}$ International Congress on Archives in Beijing, and "Archives and Social Memory" at the Sawyer Seminar held at the University of Michigan in 2001.

73 Huiling Feng, Yongyouxinjiyi: Dianziwenjianyanjiu [Owning New Memory: Research on Electronic Records], doctoral dissertation (Beijing: Renmin University, 1997).

74 Huandong Ding, "Danganjiyiguandexingqijiqiyingxiang [The Rise of the Viewpoint of Archival Memory and its Influences]," Archives Management 1 (2009): 16-20.

75 Huiling Feng, "Danganjiyiguan, Ziyuanguanyuzhongguojiyishuziziyuanjianshe [The Archival Memory View, the Resources View and the Construction of Digital Resources of the Memory of China]," Archives Bulletin 3 (2012): 4-8. 
Today's China is still on the road to modernisation and is undergoing major digital and social transition. The digital transition results in more and more digital archival resources being created not only by government, but also by various organisations, communities and ordinary people. The social transition involves the reform of social structures including cultural, political and economic reform, and inevitably brings about various social contradictions. The current Chinese archival community need to deal with how to develop archival ideas and practice to adapt to these transitions and to help to alleviate some of the social contradictions brought about by social transition.

To sum up, in terms of the shift of Chinese archival paradigm from 'official' to 'societal,' contemporary Chinese archival ideas and practice have gone through several complicated stages: from 1949 to 1966, Chinese archival ideas and practice were influenced by those of the Soviet Union, thereby taking on strong political and class characteristics: records and archives management was a part of the political and administrative system, and mainly served government administration. A centralised control and custodial archival administrative system was built up. After the Cultural Revolution, with the opening of China to the world and subsequent economic and political development, more types of records and archives began to be created by government, organisations, communities and common people. Today, as Xiaomi An notes, "the value of archives as the evidence, memories, identities, knowledge, history and cultural heritage of the people and society is recognized", ${ }^{76}$ and records and archives management serves not only government, but also society and the people of China.

\section{Conclusion}

This chapter provides only a brief description of how, influenced and shaped by social structures, there have been many varieties of archival ideas and practice in the long Chinese history. As has been the trend in other countries, today's Chinese archival community needs to exercise its agency in developing Chinese archival ideas and practices and also adapting them to address these transitions. It needs to rethink current definitions of archives, records and archives management, as well as the roles of archivists, archival education and research within the new societal ethos. It also needs to study such aspects as archives and memory, community archives, and issues of rights, power and accountability that have not been paid much attention in

76 Xiaomi An et al., "Reinventing the Concept of the State Archival Fond in China," Archives and Manuscripts 42 no.2 (2014): 146-150. 
the official paradigm. A careful balancing of the Chinese archival tradition and traditional archival ideas with a receptivity to ideas and practices developed by archival communities outside China will be necessary in order to enhance mutual understanding and learning in the archival multiverse.

\section{Acknowledgements}

My sincere gratitude to Professor Anne Gilliland for her instructive and helpful reviews and edits on this chapter. Many thanks also to the peer reviewers for their comments.

\section{References}

An, Xiaomi. "Wenjianlianxutimoshiduidianziwenjianzuiyouhuaguanlideqishi [The Inspirations of the Records Continuum Model for Electronic Records]," Archives Bulletin 3 (2002): 52-55.

Cao, Xichen. Danganwenxianbianzhuangxue [Archives Compilations]. Beijing: Renmin University Press, 1990.

Chen, Zhaowu. Danganguanlixue [Archives Management] (Beijing: Renmin University Press, 1962)

Chen, Zhaowu. "Wenjian [Records]," Archives Bulletin 4 (1991): 72-73.

Chen, Zhaowu. "Zailundangandedingyi -Jianlunwenjiandedingyiheyingdongzhouqiwenti [Re-visiting the Definition of Archives - and Discussion of the Definition of Records and the Movement Cycle of Records]," Archives Bulletin 2 (1987): 21-25.

Chen, Zhaowu and Baorong He, Danganguanli [Archives Management] (Beijing: Renmin University, 1996), 12-14.

Cook, Terry. "Archives in the Post-Custodial World: Interaction of Archival Theory and Practice since the Publication of the Dutch Manual in 1898," Archivum 43 (1997): 191-214.

Cook, Terry. "The Concept of the Archival Fonds in the Post-custodial Era: Theory, Problems and Solutions", Archivaria 35 (1993): 24-37.

Cook, Terry. "Electronic Records, Paper Minds: The Revolution in Information Management and Archives in the Post-custodial and Post-modern Era", Archives and Manuscripts 22 no.2 (1994): 300-29.

Cook, Terry. "Evidence, Memory, Identity, and Community: Four Shifting Archival Paradigms," Archival Science 13, nos. 2-3 (2013): 95-120.

Cook, Terry. "Macro-appraisal and Functional Analysis: Documenting Governance Rather Than Government," Journal of the Society of Archivists 25 no.1 (2004): 5-18.

Cook, Terry. "What Is Past Is Prologue: A History of Archival Ideas Since 1898, and the Future Paradigm Shift”, Archivaria 43 (1997): 17-63.

Ding, Huandong. "Danganjiyiguandexingqijiqiyingxiang [The Rise of the Viewpoint of Archival Memory and its Influences]," Archives Management 1 (2009): 16-20.

Duarte, Marisa Elena and Miranda Belarde-Lewis, "Imagining: Creating Spaces for Indigenous Ontologies," Cataloging E Classification Quarterly special issue on Indigenous Knowledge Organization, 53 nos.5-6 (2015): 677-702.

Duranti, Luciana and Heather MacNeil. "The Protection of the Integrity of Electronic Records: An Overview of the UBC-MAS Research Project," Archivaria 42 (Fall 1996): 46-67. 
Feng, Huiling. "Danganjiyiguan, Ziyuanguanyuzhongguojiyishuziziyuanjianshe [The Archival Memory View, the Resources View and the Construction of Digital Resources of the Memory of China]," Archives Bulletin 3 (2012): 4-8.

Feng, Huiling. "Dianziwenjianguanliguojiazhanleichuyi [Discussion of the National Strategies for Electronic Records Management]," Archives Bulletin 3 (2006): 4-8.

Feng, Huiling. "Dianziwenjianshidaixinsiwei [New Ideas in the Age of Electronic Records]," Archives Bulletin 6 (1998): 45-49.

Feng, Huiling. Yongyouxinjiyi: Dianziwenjianyanjiu [Owning New Memory: Research on Electronic Records], doctoral dissertation (Beijing: Renmin University, 1997).

Feng, Huiling. Dianziwenjianguanlijiaocheng [Textbook of Electronic Records Management] (Beijing: Renmin University Press, 2001), 5-7, 13-15.

Feng, Huiling and Jiasun He, "Quanzonglilundeshizhi [The Essence of Respect des Fonds]," Archives Bulletin 5 (1988): 8-11.

Flinn, Andrew. "The Impact of Independent and Community Archives on Professional Archival Thinking and Practice." In Hill, J., ed. The Future of Archives and Recordkeeping (London: Facet Publishing, 2011).

Foucault, Michel. The Archaeology of Knowledge (London: Routledge, 1995).

$\mathrm{Fu}$, Rongxiao and Ying Ji. "Wenjianzhengtiyundongmiaoshu:

Shiwenjianshengmingzhouqimoshi, Haishiwenjianlianshutimoshi [Description of the Movement of Records: Life Cycle of Records or Records Continuum]," Archives Management 3 (2008): 7-12.)

Gan, Naiguang "Testing the Chain Method of Records and Archives", Administrative Efficiency 10 (1934): 423-429.

Gilliland, Anne J. and Andrew Flinn. "The Wonderful and Frightening World of Community Archives: What Are We Really Talking About?” Keynote address, Nexus, Confluence, and Difference: Community Archives meets Community Informatics: Prato CIRN Conference Oct 28-30 2013, Larry Stillman, Amalia Sabiescu, Nemanja Memarovic, eds, Centre for Community Networking Research, Centre for Social Informatics, Monash University. http://www.ccnr.infotech.monash.edu.au/assets/ docs/prato2013_papers/gilliland_flinn_keynote.pdf.

He Jiasun. "Wenjianshengmingzhouqililunduiwomengdeqishi [The Inspirations of Life Cycle of Records]," Archives Bulletin 6 (1991).

He Jiasun. "Wenjianshengmingzhouqililunduiwomengdeqishi [The Inspirations of Life Cycle of Records]," Archives Bulletin 1 (1992)

He, Jiasun. "Wenjianlianxutiyuwenjianshengmingzhouqililun [Records Continuum and Life Cycle of Records]," Archives Bulletin 5 (2003): 60-64.

$\mathrm{He}$, Jiasun and Rongxiao Fu, Wenjianyundongguilvyanjiu: Congxinjiaodushenshidanganxuejichulilun [Research on the Movement of Records: Review of the Basic Archival Theories from a Nerw Perspectives] (Beijing: China Archives Press, 1999).

He, Jiasun and Feng Huiling. "Guanyugengxinquanzonggainiandeshexiang [The Idea of Updating the Definition of Fond]," Archives Bulletin 6 (1988): 8-12.

He, Jiasun and Xuren Shi, "Zhidaoyubeizhidaoguanxi - Guanyuwenjianlianxutililun, Wenjianyundonglilunhewenjianshengmingzhouqililunzhisibian [Guiding or Being Guided - Analysis of the Nature of the Records Continuum, the Life Cycle of Records and the Movement of Records]," Zhejiang Archives 9 (2006): 6-9.

$\mathrm{He}$, Jiasun and Xuren Shi, "Wenjianlianyutililunyuwenjianshengmingzhouqililun Wenjianyundonglilunzhiyi [Records Continuum and Life Cycle of Records-Research on Records Movement Theory (the First)]," Archives Bulletin 5 (2003): 60-64.

$\mathrm{He}$, Lucheng, Danganzhengliyuguanlifa (Management and Arrangement of Archives) 


\section{Chapter 3}

in Archives Bulletin, eds. Danganxuejingdianzhuzuo (Dierquan) [Classic Works of Archival Science Volume 2] (Shanghai: World Publishing Corporation, 2013)

Jin, Guangyao. "Koushulishiyuchengshijiyi [Oral History and City Memory]," Eastday

Network. http://sh.eastday.com/qtmt/20111110/u1a937145.html.

Li, Caifu. Zhongguodanganxueshi [History of Chinese Archival Science] (Anhui: Anhui University Press, 2005).

Li, Shouyi. "Qingneigedanganpoqianjilie [The Transfer History of the Archives Kept in the Warehouse of the Cabinet of the Qing Government]," Forbidden City 2 (2012): 44-51.

Liang, Qichao. Evolution of China in the Past Fifty Years, http://www.my285.com/xdmj/ lqc/057.htm

Meng, Hongbo. "A Discussion about the Creation of Confucianism in the Han Dynasty," Tangdu Journal 6 (2008): 39-43.

Muller, Samuel, Johan A. Feith and Robert Fruin. Manual for the Arrangement and Description of Archives, Eric Ketelaar, Theo Thomassen and Peter Horsman, trans. of second edition (Chicago: Society of American Archivists Classic Series, 2003).

Noh, Meung-Hoan. "Confucian Community Construction and Records/Archives

Management of Joseon Dynasty - focused on the meaning of the Uigwe Production, Preservation, and Use," paper presented at AERI 2012, University of California Los Angeles, July 2012, https://aeri2012.wordpress.com/conference-schedule/paperpresentations/community-archiving/.

Pan, Liangen and Dongbing Liu, "Guanyudawenjiangainiandebanxi [Analysing the Concept of Big Records]," Archives Management 5 (2008): 4-10.

The Second History Archives of China, Mingguoshiqiwenjiyudanganguanlicailiao [Materials on the Records and Archives Management of the National Government] (Beijing: Archives Press, 1987).

Spence, Jonathan. The Search for Modern China (New York: Norton and Co, 2001).

State Archives Administration of China (SAAC), Dangangongzuowenjianhuibian [Compilations of the Regulations on Archival Work] (Beijing: Archives Press, 1986).

Wang, Mingke. "Lishishishi, Lishijiyiyulishixinxing [History Fact, History Memory and History Mood]," History Research 5 (2001): 136-191.

Wang, Xin. "Zhongguo Guodaibihuizhiduyanjiu [Research on the Taboo System of Ancient Chinese Records]," Yunnan Archives 5 (2011): 23-24.

Wu, Baokan. Danganxuegailun [Introduction to Archival Science]. (Beijing: Renmin University Press, 1988).

Wu, Pingcai. "Dawenjianguan: Dandaizhongguodanganxuedebirangxuanzhe [The Perspective of Big Records: The Inevitable Choice of Contemporary Chinese Archival Science]," Archives Management 5 (2008): 34-37.

Yang, Xia. "Lunwenjiandegainian [Discussion of the Definition of Records]," Archives Bulletin 1 (2000): 24-26.

Zen, San. Zensanwenji [Collection of San Cen's Works on Archives Management] (Beijing: China Archives Press, 1990).

Zhang, Huichao. MingguoshiqiMingqingdanganzhengliyanjiu [Research on the Arrangement of the Ming-Qing Dynastic Archives during the National Government] (Shanghai:

World Publication Corporation, 2011), 132-133.

Zhao, Yanchan and Na Huang, "Gudaizhongguodanganjiandingyanjiu [Study on Archival Appraisal in Ancient China]," Zhejiang Archives 1 (2011): 63-65.

Zhou, Xueheng. Zhongguo Danganshiyeshi [History of the Development of Chinese Archives]. Beijing: Renmin University Press, 1994. 
Chapter 4

\title{
RECORDKEEPING IN THE CONTINUUM
}

\author{
An Australian Tradition
}

Sue McKemmish

\begin{abstract}
This chapter analyses and reflects on the evolution of records continuum theory and practice in Australia with reference to the historical, societal, juridical and geo-political contexts, philosophies, theories and cultural influences that have shaped them. It extensively references the work of records continuum theorists, researchers, educators and practitioners and explores their distinctive worldview as reflected in the records continuum metanarrative with its evolving theory, concepts, models and applications. An overview of Australian records and archives traditions from pre-colonial times leads into consideration of the formative years of records continuum theory and practice, the emergence of a self-styled recordkeeping community of practice and the significant contribution of records continuum theory and the Records Continuum Model to archives and records discourse and practice nationally and internationally. The chapter concludes with a reflection on the records continuum and the archival multiverse, highlighting the transformative nature of recent records continuum research and writing.
\end{abstract}

\section{Introduction}

The term recordkeeping as used throughout this chapter refers to a broadly defined, inclusive and pluralist concept of recordkeeping as theorised and practised in the continuum:

It encompasses a range of intertwined recordkeeping and archiving processes and activities carried out by records managers and archivists 
for current, regulatory and historical recordkeeping purposes. These purposes include the roles that recordkeeping plays in and through space and time in governance and accountability, remembering and forgetting, shaping identity and providing value-added sources of information. In classificatory terms 'recordkeeping' in this usage subsumes records management and archival administration. It also encompasses the personal and corporate recordkeeping activities undertaken by individuals in their everyday lives, in families, work or community groups, and in organisations of all kinds. ${ }^{1}$

Continuum definitions of recordkeeping reference the Archive in the very broadest sense, "encompassing oral and written records, literature, landscape, dance, art, the built environment and artefacts" insofar as they provide traces of social, cultural and organisational activity that evidence and memorialise individual and collective lives. ${ }^{2}$ These definitions resonate with the idea of an archival multiverse as defined in Chapter 1 of this book, referring to:

the pluralism of evidentiary texts, memory-keeping practices and institutions, bureaucratic and personal motivations, community perspectives and needs, and cultural and legal constructs with which archival professionals and academics must be prepared, through graduate education and through research and development, to engage. ${ }^{3}$

The analysis presented here is distilled from research undertaken over the past 25 years that relates to the evolution of records continuum theory and practice in Australia from its formative years in the mid- $20^{\text {th }}$ century. This research is reported in McKemmish's doctoral thesis, ${ }^{4}$ and in sole and

1 Sue McKemmish, Franklyn Herbert Upward and Barbara Reed. "The Records Continuum Model," in Marcia J. Bates and Mary Niles-Maack, eds. Encyclopedia of Library and Information Sciences, Third Edition (New York: Taylor and Francis, 2009), 4447-59; quote p. 4448. The term, also rendered as 'record keeping' and 'record-keeping', is used in other communities, particularly in North America, to refer to narrower concepts - limited to current or active records in corporate or other organisational contexts, or more narrowly to records management systems (see for example http://www2.archivists.org/glossary/terms/r/recordkeeping).

2 Sue McKemmish. "Traces: Document, Record, Archive, Archives," in Sue McKemmish, Michael Piggott, Barbara Reed and Frank Upward, eds. Archives: Recordkeeping in Society (Wagga Wagga: Centre for Information Studies, Charles Sturt University, 2005), 1-20, quote p. 1.

3 AERI Pluralizing the Archival Curriculum Group (PACG). "Educating for the Archival Multiverse," American Archivist 74 no.1 (Spring/Summer 2011): 69-102; quote p. 73.

4 Sue McKemmish. Constantly Evolving, Ever Mutating: An Australian Contribution to the Metatext (PhD thesis, Monash University Faculty of IT, 2001). 
co-authored work by McKemmish and key Australian records continuum writers, Upward, Reed, Piggott, Iacovino, Hurley, Cunningham and Acland. ${ }^{5}$ The referenced research employed a mix of methods, including theory building and modelling, discourse analysis, case studies, analysis of literary warrant, and historiography. Discourse analysis was used to study trends in the evolution of records continuum thinking, and the parallel manuscript tradition in Australia. Case studies analysed the development and implementation of records continuum best practice and standards. Literary warrant and historical analysis identified the social, national, intellectual and professional frames of reference for Australian thinking and practice.

The chapter itself is based on ongoing observations and reflections spanning my forty years as a recordkeeping practitioner, educator and researcher. ${ }^{6}$ It employs auto-ethnographic methods in the subjective sense of reflecting on my own work and experience of being immersed in, and interacting with, the Australian recordkeeping community of practice, as well as engaging in the development of records continuum theory, the national and international archival discourse, and collaborative initiatives relating to standards development, research and education in Australia and internationally.

\section{Australian Records and Archives Traditions}

Australian records and archives traditions are amongst the most ancient and the most recent in the world. ${ }^{7}$ From a continuum perspective, they

5 The records continuum writings of Upward, Piggott, Reed, Iacovino, Hurley, Cunningham and Acland are cited throughout this chapter. Key texts include two edited collections: Sue McKemmish and Michael Piggott, eds. The Records Continuum; Australian Archives first fifty years (Melbourne: Ancora Press,1994) and McKemmish et al. Archives: Recordkeeping in Society; and two more recent reflexive papers which reflect on the past and look towards the future: Sue McKemmish and Michael Piggott. "Toward the Archival Multiverse," Archivaria 76 (Fall 2013): 111144; Frank Upward, Sue McKemmish and Barbara Reed. "Archivists and Changing Social and Information Spaces: A Continuum Approach to Recordkeeping and Archiving in Online Cultures," Archivaria, 72 (Fall 2011): 197-237.

6 My work in the archival and recordkeeping field began at the Commonwealth Archives (now the National Archives of Australia) in 1975, and continued at the Public Record Office Victoria from 1983 to 1990, followed by over 25 years as an educator and researcher at Monash University, Melbourne.

7 For a collection of old and new writing that explores Australian Indigenous, institutional, corporate and personal recordkeeping traditions from historical, social and cultural perspectives, and a lifetime of reflexive practice, see the seminal work by Michael Piggott. Archives and Societal Provenance: Australian Essays (Oxford: Chandos Publishing, 2012) 
encompass pre-colonial, colonial, and national/neo-colonial phases, ${ }^{8}$ clashing, co-existing, sometimes intersecting, and occasionally reconciling in spacetime. Within Australia today and historically, there is and has been a plurality of traditions within different communities of records, some privileged and some marginalised. Records continuum theory and continuum-based practices take their place amongst these traditions - hence the sub-title of this chapter refers to an, not the, Australian tradition. At the same time, records continuum theorists and scholarly practitioners have been amongst the most prolific writers on Australian archival and recordkeeping traditions, interpreting the recordkeeping landscape in Australia through the lens of the continuum. Most recently records continuum theory has framed consideration of disruptive and radical recordkeeping and archival processes linked to the possibility of a decolonised Archive in a post-colonial Australia. Decolonisation would involve transforming current practices and refiguring archival spaces to be representative of multiple voices and perspectives, thus unsettling the power imbalances embedded in the current records and archives landscape. ${ }^{9}$

With invasion/first settlement in 1788 the closest neighbours of colonial Australia and, from 1901, the new nation state were part of the vast colonial empires of Britain, France and the Netherlands. In the aftermath of the Second World War, when its immediate Southeast Asian and Pacific neighbours were no longer European colonial powers but independent or emerging nations, Australia remained what Labor Prime Minister Paul Keating (1991-1996) famously referred to as a "paid-up member of the Anglosphere, with our dues paid in military commitments in World War I, World War II, in Korea, in Vietnam, in Iraq and in Afghanistan."10

8 From a chronological perspective, referencing the period before European colonisation, which began in 1788 for some Indigenous communities, for others during the $19^{\text {th }}$ century, and for some remote communities persisted well into the $20^{\text {th }}$ century; the period of colonisation until 1901 with the establishment of the nation of Australia; and the period of the nation state, experienced by many Indigenous people as neocolonialism. From a spacetime perspective, the states of being represented by the terms pre-colonial, colonial and national/neo-colonial can be seen as co-existing, clashing, sometimes interacting, sometimes reconciling since the early days of European settlement/invasion.

9 For example, Joanne Evans, Sue McKemmish, Elizabeth Daniels and Gavan McCarthy. "Self-determination and Archival Autonomy: Advocating Activism," Archival Science Special Issue: Archiving Activism and Activist Archiving, 15 no. 4 (2015): 337-368; McKemmish and Piggott, "Toward the Archival Multiverse"; Upward, McKemmish and Reed, "Archivists and Changing Social and Information Spaces".

10 Paul Keating. "Asia in the New Order", recording and transcript of the Keith Murdoch Oration, 14 November 2012, State Library of Victoria, available at 
In spite of its increasingly multicultural and multi-racial population, and economic reliance on trade with China and other Asian nations, its main geo-political alignment remains Anglo-American, with its foreign policy in particular increasingly aligned with the USA. These alignments are reflected in its dominant colonial and national government, business, and religious recordkeeping traditions, and the nature of its public and private libraries and collecting institutions.

Recordkeeping traditions in Aboriginal Australia are many, many thousands of years old and take many forms, including records embodied in people or contained within country, transmitted and accessed through storytelling and performance using speech, dance, art, music and song (for example the songlines in which a wealth of Indigenous knowledge is embedded in country-centric ribbons of song, narrative and performance); rock paintings and carvings; markings on message sticks; and stories of country etched into the linings of possum skin cloaks. While many precolonial recordkeeping traditions, particularly in Aboriginal countries located in pre-colonial times in South Eastern Australia, were disrupted and lost, others continued on in a parallel universe to the Western records and archives traditions that came to Australia with the colonists.

Records and archives were a vital part of the infrastructure that enabled Britain and the other colonising nations of Europe and Asia, including China, to control their far-flung empires and subjugate Indigenous peoples. ${ }^{11}$ The disruption of oral traditions and transmission lines, and loss of language that occurred from the early days of colonisation mirrored the displacement and dispersion of Aboriginal peoples in all but the remotest communities. Thereafter the written record of a coloniser was privileged over the oral record of a colonised people, at first by the colonial registries, and later by government and business recordkeeping systems, as well as public and private collecting practices in colonial, state and national libraries and other collecting institutions, modelled on the great collecting institutions of the UK and Europe. As a result, Indigenous ways of knowing and recordkeeping traditions were marginalised or lost. In colonial and neo-colonial societies, orality and the notion of the "pre-literate society" are "construct ed as embodying inferior forms of evidence, memory and knowledge

http://exhibitions.slv.vic.gov.au/dome100/multimedia/paul-keating-asia-new-order; accessed 22 August 2015.

11 Ann Laura Stoler. "Colonial Archives and the Arts of Governance", Archival Science 2 (2002): 87-109. 
transmission". ${ }^{12}$ Faulkhead's thesis has explored how the binary opposition of orality and literacy, that impacts on Australian legal systems today, and is manifest in much of the writing of Australian history, is perpetuated in mainstream historical and current recordkeeping and archiving practices. ${ }^{13}$

From the late $18^{\text {th }}$ century, colonial registries were modelled on the preaction registry systems of the home offices of government departments and commercial organisations of the colonising nations with their increasingly sophisticated processes of registration, aggregation, classification, indexing and tracking at document level.

While often discussed in the archival literature only in terms of their recordkeeping functions, pre-action registry systems combine workflow and recordkeeping. As Reed highlights, in the registry tradition, centralised work units manage all ingoing, outgoing and circulating correspondence as part of the control, allocation, tracking and monitoring of work - "recordkeeping is embedded in work and the management of records is integral to the way work gets done". ${ }^{14}$ Post-federation, Australian national and state governments and companies with headquarters in Britain and Europe continued the registry tradition into the 1980s when it began to break down with the advent of managerialism and office automation technologies, including US electronic records and document management software modelled on the US post-action records management tradition. In Australia by the 1980s paper registry systems were mostly file- rather than document-based, and were increasingly resource intensive with the exponential growth of records that occurred in the second half of the $20^{\text {th }}$ century. At the same time records management practices, particularly in the business sector, but also increasingly in government agencies, came to be modelled on American practice and framed by life cycle concepts. ${ }^{15}$

Nevertheless the registry tradition continued to be a major influence on the evolution of recordkeeping in the continuum in Australia, in particular in the development of the Australian Series System, Australian electronic records management software, records continuum theory and practice, and Australian contributions to international standards and metadata schemas. As Reed notes:

12 Sue McKemmish, Shannon Faulkhead and Lynette Russell. "Dis-trust in the Archive: Reconciling Records”, Archival Science 11, no.3 (2011): 211-239, quote p. 218.

13 Shannon Faulkhead. Narratives of Koorie Victoria (PhD thesis, Faculty of Arts, Monash University, 2008).

14 Barbara Reed. "Records," in McKemmish et al. Archives: Recordkeeping in Society (2005): 101-130, quote p.114.

15 See Chapter 1 for more on American traditions based on the records life cycle. 
[T] he essential characteristics of the registry system provided the basic recordkeeping influence on generations of Australian recordkeepers. Analysis of its essential features shows great relevance to recordkeeping thinking being translated into the electronic world. Indeed, technological advances are enabling us to return to many of the basic precepts of these systems. ${ }^{16}$

The increasing sophistication of registry systems into the $19^{\text {th }}$ century was in large part an artefact of the recordkeeping requirements of empire. "Terra nullius" (a land belonging to no-one) is a legal fiction of 200 years standing. It asserted that Australia was an uninhabited continent that could be claimed as British crown land by the settlers/invaders, in marked contrast to other former British colonies where Indigenous title to land was recognised (e.g. the US and New Zealand). It justified the dispossession of Indigenous inhabitants, laying the foundation for British sovereignty over the Australian colonies and eventually the establishment of modern Australia. "Terra nullius" was first overturned by the Australian courts in the Mabo land claim case in $1992 .{ }^{17}$ The archival equivalent of "terra nullius" has also prevailed in colonial and national/neo-colonial records and archives history until recent times. Since the latter part of the $20^{\text {th }}$ century there has been growing recognition of Indigenous ways of knowing and the remarkable resilience and sustainability of many Indigenous oral and other recordkeeping traditions has begun to influence mainstream thinking, particularly through exploration of their implications for recordkeeping in the continuum. These developments occurred against the backdrop of Indigenous civil and land rights movements, calls for a national apology to the Stolen Generations who had been removed from their families as children, and a push towards reconciliation. ${ }^{18}$ The engagement of records continuum researchers and writers with Indigenous researchers and com-

16 Reed, "Records", 114-15.

17 C.L. Ogleby. "Terra Nullius, the High Court and Surveyors", in The Australian Surveyor, 38 no. 3 (1993): 171-89.

18 As described by McKemmish, Faulkhead and Russell, "Dis-Trust in the Archive", 217218: "The social movement known as reconciliation within Australia began as a ground swell as settler Australians reflected on the dispossession of Aboriginal Australians. The reconciliation movement aimed to end the conflict between Indigenous and settler Australians that has existed within Australian society since the British colonisation of Australia in $1788 \ldots$ While the reconciliation movement is slowly creating ground roots changes within Australian society, many Aboriginal people are cynical or at best ambivalent about it, in part because, as a group, Indigenous Australians still remain disadvantaged on every social indicator available... Moreover it is often felt that the 
munities is discussed below in the context of the archival multiverse (see also Chapter 16 by Shannon Faulkhead and Chapter 30 by Kirsten Thorpe).

\section{The Formative Years}

The incubators of continuum theory and continuum-based practices in Australia were the forerunners to the National Archives of Australia (the Archival Division of the National Library of Australia during the 1950s, the newly independent Commonwealth Archives Office from 1961, re-badged as the Australian Archives in the 1970s and 1980s), with their dual roles in current and historical government recordkeeping. Government archives programs in Australia emerged in the second half of the $20^{\text {th }}$ century, largely as a result of advocacy by Australian historians that also impacted on library manuscript collection policies. The government archives programs were initially located in the National and State Libraries. Cunningham traces the development of Australian manuscript collections from the establishment of the National Library of Australia in 1901 to the opening of the Mitchell Library in New South Wales, to the establishment of libraries in the other States. ${ }^{19}$ At first their collecting efforts "grew out of the antiquarian work of private collectors and historical societies and, until the 1940s they were characterised by a desire to preserve documents relating to the origins of European settlement in Australia." ${ }^{20}$ From the 1950s, with the establishment and growth of teaching and research programs in Australian history, the collecting activities of the National and State libraries expanded rapidly and new programs were established at the University of Melbourne and the Australian National University focusing on business and trade union archives:

The emergence of new collecting programs and the simultaneous expansion and shift in collecting emphasis by the existing programs stemmed from a desire to serve the needs of this expanding researcher clientele. Both phenomena reflected a more self-confident, nationalistic and prosperous Australia, a nation that was keen to apply the methods

reconciliation process is one where much of the responsibility is upon Indigenous peoples to educate the wider community. It remains to be seen whether recent events have indeed engendered a renewed energy for reconciliation."

19 Adrian Cunningham, "Archival Institutions," in McKemmish et al. Archives: Recordkeeping in Society, 21-50; Graeme Powell. "The Collecting of Personal and Private Papers in Australia", Archives and Manuscripts, 24, no.1 (May 1996): 62-64.

20 Cunningham, “Archival Institutions”, 37. 
of scientific history to the task of understanding and articulating a national identity. ${ }^{21}$

Cunningham traces the subsequent refiguring of collecting policies with the emergence of social history to include documentation relating to the lives of "ordinary people" and social history themes, as well as oral history programs, film and sound archives and, most recently, data archives. ${ }^{22}$

In parallel with these developments, government archival programs emerged from national and state libraries to become independent archival authorities over a 25-year period from 1961, with their dual role as cultural institutions and regulators of government recordkeeping for good governance and democratic accountability purposes enshrined in archival law..$^{23}$ Early Commonwealth government archivists, led by the first Commonwealth Archivist, Ian Maclean, were working in the broader context of the continuing strong, but increasingly file- rather than document-based registry tradition in government agencies. At the same time they were strongly influenced by the legal/administrative archival tradition of the UK as epitomised in the writings of Sir Hilary Jenkinson. ${ }^{24}$ They campaigned for the independence of the Archives Division, formed in the National Library after World War II, realising their goal with the establishment of the Commonwealth Archives Office in 1961. By the 1970s and early 1980s, a strong recordkeeping culture had emerged in the Commonwealth Archives of Australia (later styled the Australian Archives) focused on managing the record as evidence throughout its lifespan. Upward has described how this culture was built on Maclean's legacy, Jenkinsonian views on the nature of records and archives, a concomitant rejection of Schellenbergian and North American life cycle approaches, and the application of European registry traditions to archival description systems. ${ }^{25}$

21 Cunningham, "Archival Institutions", 38.

22 See sections on History and Institutions in Piggott, Archives and Societal Provenance for detailed and insightful explorations of these formative years, including the impact of the 1954 visit of Schellenberg on the formation of library and government archival institutions.

23 Chris Hurley. "From Dustbins to Disk-Drives: A Survey of Archives Legislation in Australia", Appendix 2 in McKemmish and Piggott, The Records Continuum, 206-32.

24 The standard references on the reception of Jenkinson's ideas in Australia are Ian Maclean, "Australian Experience in Record and Archives Management" (1959) and "An Analysis of Jenkinson's 'Manual of Archive Administration' in the Light of Australian Experience" (1962), both reproduced in Peter Biskup, ed. Debates and Discourses: Selected Australian Writings on Archival Theory, 1951-1990 (Canberra, Australian Society of Archivists, 1995), 30-52 and 53-78.

25 Frank Upward. "In Search of the Continuum: Ian Maclean's 'Australian Experience' Essays on Recordkeeping”, in Sue McKemmish and Michael Piggott, 
During the 1970s and 1980s, the Commonwealth Archives Office/ Australian Archives developed and implemented Peter Scott's revolutionary series system with its rich understandings of the multiple and dynamic layers of contextuality that surround records, and their loci in a web of multiple social, functional, provenancial, and documentary relationships. ${ }^{26}$ Although Maclean was an early user of the term continuum to characterise his pioneering approach to integrated recordkeeping and archiving thinking and practice, the term "records continuum" was only used to label such approaches after Atherton ${ }^{27}$ published his groundbreaking article which "showed how the life cycle stages that records supposedly underwent were in fact a series of recurring and reverberating activities within both archives and records management."28

Piggott has chronicled how in parallel with the movement to separate government archives from library control, a distinctive archival professional identity slowly emerged, its beginnings marked in 1951 by the creation of an Archives Section of the Library Association of Australia, and in 1955 by the publication of the first issue of Archives and Manuscripts. ${ }^{29}$ As McKemmish and Piggott explain, the visit in 1954 of Fulbright lecturer and senior member of the US National Archives, T. R. Schellenberg, reinforced the view of government archivists that personal archives and historical manuscripts managed in library systems were not archives:

Archives arose from a 'regular functional activity' and were thus created in a systematic manner, while historical manuscripts were usually the product of 'a spontaneous expression of thought or feeling', and were thus created in a haphazard manner. ${ }^{30}$

While embracing Jenkinsonian views on the nature of records and archives, government archivists took a contradictory view in aligning their thinking

The Records Continuum, 110-30.

26 Chris Hurley. "The Australian (Series) System: An Exposition" in McKemmish and Piggott, The Records Continuum, 150-72.

27 Jay Atherton. "From Life Cycle to Continuum: Some Thoughts on the Records Management-Archives Relationship", Archivaria 21 (1985-86): 43-51.

28 Frank Upward. "Modelling the Continuum as Paradigm Shift in Archiving and Recordkeeping Processes, and Beyond: A Personal Reflection”, Records Management Journal 10:3 (December 2000): 115-39; quote p. 118.

29 Piggott, Societal Provenance, particularly Chapter 3 "Schellenberg in Australia: Meaning and Precedent".

30 McKemmish and Piggott, “Toward the Archival Multiverse”, 118; referencing T. R. Schellenberg. Modern Archives: Principles and Techniques (Chicago: University of Chicago Press, 1956). 
with Schellenberg on the issue of personal archives and historical manuscripts. This can in part be explained by the bifurcated system in both US federal and Australian federal (and most state) jurisdictions in which the so-called national (and in Australia most state) archival institutions are not in fact national or state archives, but the archives of government. As in the US, and unlike in many of the older jurisdictions in Europe, the much more powerful and truly national (and state) libraries (Library of Congress, National Library of Australia and Australian State Libraries) already existed in some cases long before the establishment of independent government archives. Libraries holding personal papers and historical manuscripts managed them as information objects in library catalogues and systems, not according to archival principles, and still do.

An independent professional society, the Australian Society of Archivists (ASA), was finally formed in 1975, with its membership dominated by government archivists, "beginning a pattern of limited professional engagement by manuscript librarians in the archives sector which sadly has typified the local archival landscape ever since" ${ }^{31}$ From the mid-1970s, this fledgling professionalism was nurtured by the university education and research programs being established in a number of leading universities in Australia.

Outside the areas covered by government archival authorities pursuing their dual role of regulating current recordkeeping in their respective jurisdictions, and preserving the archives of the national and state governments, and the collecting activities of the manuscript sections of the national and state libraries, records and archives programs were not well developed and, where they did exist, were very poorly resourced. A few large corporate archives, including bank, company and church archives, were established, and two major collecting archives focusing on records of business and labour at the University of Melbourne and the Australian National University. Major collections were built in the Australian War Memorial, the Film and Sound Archive, and state museums. In more recent times some significant research collections and data archives have emerged, including the Social Science Data Archives at the Australian National University, and the associated Aboriginal and Torres Strait Islander Data Archive. Legacies of the two most dominant records and archives traditions in Australia - the government recordkeeping and the collecting traditions - are the many gaps and silences in the mainstream archival record today, particularly relating to organisations, communities, and individuals "that

31 McKemmish and Piggott, "Toward the Archival Multiverse”, 120. 
fall outside the jurisdiction of government archives and beyond the scope of the collecting policies of major manuscript collections". ${ }^{32}$

\section{The Australian Recordkeeping Community of Practice ${ }^{33}$}

Ironically, at the same time as evidence-based recordkeeping and continuum-based thinking gained traction in terms of professional identity and education, they lost ground for a time in the Australian Archives itself to the information management approaches of the 1980s. However there was a resurgence of evidence-based, continuum approaches during the 1990s to address the findings of a number of Royal Commissions and major government inquiries into a series of accountability crises and corruption cases that rocked the government and corporate sector in the 1980s. This return to evidence and registry-based approaches also shaped the strategic directions of a newly styled National Archives, primarily driven by the challenge of electronic recordkeeping.

The self-defining recordkeeping community of practice that emerged in Australia during these years was made up of records managers and archivists, consultants, educators and researchers, government archival institutions, corporate records and archives programs, and professional associations. From the late 1980s new continuum-based pedagogical, curriculum, and research models were developed at Monash University by educators and researchers recruited from this community, ${ }^{34}$ pioneering a new approach to recordkeeping and archiving education in Australia, and to collaborative research partnerships. The recordkeeping community of practice consciously worked within an evolving records continuum framework. Many of its members had spent their formative years during the 1970s and/or early 1980s in what they subsequently dubbed "the national archives gene pool". The "binding force" operating in this community can be articulated in the following terms:

32 McKemmish and Piggott, "Toward the Archival Multiverse”, 118.

33 Analysis of the Australian recordkeeping community of practice of the 1990s in this section draws heavily on Sue McKemmish. Constantly Evolving, Ever Mutating, and Sue McKemmish. "Placing Records Continuum Theory and Practice," Archival Science, 1 (2001): 333-59, as did subsequent jointly authored works by McKemmish, Upward and Reed, see in particular "The Records Continuum Model”.

34 Frank Upward joined Monash in 1988; Sue McKemmish and Livia Iacovino in 1990; during the early 1990s Chris Hurley, Barbara Reed and Anne Picot were also part of the Monash team, playing crucial roles in research, curriculum development and teaching. 
At this time records continuum thinking focused on the unifying purposes shared by all recordkeeping professionals, defined as to do with the delivery of frameworks for accountable recordkeeping regimes that enable access to essential, useable evidence of social and business activity in the business, social and cultural domains. ${ }^{35}$

A key concern of the recordkeeping community in the 1990s was the recordkeeping-accountability nexus. There was a preoccupation with the findings and recommendations of the Royal Commissions and other inquiries into the role of recordkeeping in good governance and democratic accountability, the role of public archival authorities as agents of accountability in democratic societies, and the part played by poor or negligent recordkeeping in failures in public and corporate accountability and corruption. ${ }^{36} \mathrm{~A}$ growing consciousness of the responsibilities of archivists and records managers in relation to corporate accountability in the public and private sector was in part a reflection of the maturing of the profession. Within this context, the synergies with Bearman's evidence-centred strategies for electronic recordkeeping were significant drivers in the development of standards for accountable recordkeeping regimes, and guidelines for the design and implementation of recordkeeping systems. The community's collaborative efforts involving the professional associations, archival institutions, practitioners, researchers and educators eventually resulted in AS 4390, the Australian Records Management Standard. ${ }^{37}$ It embodied continuum approaches to managing records and archives, was the first such national standard in the world, and formed the basis for the first international standard in this area (ISO15489). ${ }^{38}$ Australians, including Barbara Reed, David Roberts and Judith Ellis, have subsequently played a major role in the ISO's suite of standards in the records management field, including the recordkeeping metadata standard (ISO23081). ${ }^{39}$ The latter

35 Sue McKemmish. "Yesterday, Today and Tomorrow: A Continuum of Responsibility", in Peter J. Horsman, F. C. J. Ketelaar, and Theo H. P. M. Thomassen, eds. Naar een Nieuw Paradigma in de Archivistiek ('s-Gravenhage: Stichting Archiefpublicaties, 1999): 195-210, quote p. 196.

36 Sue McKemmish and Frank Upward, eds., Archival Documents: Providing Accountability Through Recordkeeping (Melbourne: Ancora Press, 1993).

37 Standards Australia. AS4390: Records Management, Standards Australia, 1996.

38 International Standards Organisation. ISO 15489 Information and documentation Records management - Part 1: General; Part 2: Implementation, 2001.

39 International Standards Organisation. ISO 23081 Information and documentation Records Management Processes-Metadata for Records Part 1: Principles, 2006; ISO 23081 Information and Documentation - Managing Metadata for Records Part 2: Conceptual and Implementation Issues, 2009. 
drew heavily on continuum-based collaborative research partnerships led by Monash University in partnership with the National Archives of Australia, State Records New South Wales, Queensland State Archives and the professional associations, and Australian Series System approaches to the description of archival records.

The writings and reports of the Australian recordkeeping community of practice ${ }^{40}$ became part of a larger international discourse that was reconceptualising traditional theory and 'reinventing' records and archives practice in response to the challenges of electronic recordkeeping. ${ }^{41}$ Evolving Australian practice built on the synergies between this discourse and Australian understandings of the nature of records and archives as evidence, the recordkeeping and workflow processes associated with registry systems, rich understandings of multiple provenance and the dynamic contexts of records present in the Australian Series System, as well as records continuum approaches to building holistic, multi-dimensional recordkeeping and archival frameworks. The University of Pittsburgh's Functional Requirements for Evidence in Recordkeeping Project, and Bearman's mix of policy, standards, system design, and implementation tactics, significantly influenced the Australian development of national and institutional standards, and policy and strategic frameworks for the management of electronic records. ${ }^{42}$ The

40 For example: Glenda Acland. "Archivist - Keeper, Undertaker or Auditor?” Archives and Manuscripts 19 no.1 (1991): 9-15, and "Managing the Record Rather than the Relic”, Archives and Manuscripts 20 no.1 (1992): 57-63; Australian Society of Archivists and Australian Council of Archives. Managing Electronic Records (Canberra: ASA and ACA, 1993); Information Exchange Steering Committee. Management of Electronic Documents in the Australian Public Service (Canberra: IESC, 1993); McKemmish and Piggott. The Records Continuum; Greg O'Shea and David Roberts. "Living in a Digital World: Recognizing the Electronic and Poscustodial Realities”, Archives and Manuscripts 24 no.2 (1996): 286-311; Barbara Reed, "Electronic Records Management in Transition”, Archives and Manuscripts 22 no.1 (1994): 164-71; David Roberts. "Defining Electronic Records, Documents and Data", Archives and Manuscripts 22 no.1 (1994): 14-27; Steve Stuckey. "Keepers of the Flame? The Custodial Role of Australian Archives - Its History and Its Future", in McKemmish and Piggott, The Records Continuum, 35-48; Frank Upward and Sue McKemmish. "Somewhere Beyond Custody," Archives and Manuscripts 22 no.1 (1994): 138-49.

41 Bearman and Hedstrom were key proponents of 'reinventing archives' strategies. See, for example David Bearman and Margaret Hedstrom. "Reinventing Archives for Electronic Records: Alternative Service Delivery Options”, in Margaret Hedstrom, ed., Electronic Records Management Program Strategies (Pittsburgh: Archives and Museum Informatics, 1993), 82-98. For a detailed analysis of the international discourse see Terry Cook. "What Is Past Is Prologue: A History of Archival Ideas Since 1898, and the Future Paradigm Shift", Archivaria 43 (1997): 17-63.

42 Bearman first put forward this formulation in: David Bearman. "Electronic Records Guidelines: A Manual for Policy Development and Implementation”, in David 
writings of Hedstrom, ${ }^{43} \mathrm{McDonald},{ }^{44}$ and Hofman ${ }^{45}$ about early implementation of electronic recordkeeping policies and strategic approaches in the New York State Archives and Records Administration, the National Archives of Canada, and the National Archives of the Netherlands respectively, also impacted on the development of these frameworks for Australian practice. Cook's writings on electronic records, postcustodialism, and functional appraisal, closely linked to Canadian re-conceptualisations of the nature of records and of provenance, also influenced the development of Australian records continuum practice. ${ }^{46}$ The development of macroappraisal concepts and methodologies at the National Archives of Canada, and the Dutch view of functional appraisal, especially as developed in the PIVOT project, also had an impact upon evolving appraisal frameworks in Australian government archival institutions. ${ }^{47}$

Bearman. Electronic Evidence: Strategies for Managing Records in Contemporary Organizations (Pittsburgh: Archives and Museum Informatics, 1994), 72-116; see also David Bearman, ed. Archival Management of Electronic Records (Pittsburgh: Archives and Museum Informatics, 1991). In later articles, he elaborated on and refined this formulation. David Bearman. "Record-Keeping Systems", Archivaria 36 (1993), 16-37; David Bearman. "Archival Data Management to Achieve Organizational Accountability for Electronic Records", Archives and Manuscripts 21 no.1 (1993): 14-28.

43 New York, State Archives and Records Administration et al. Strategic Plan for Managing and Preserving Electronic Records in New York State Government: Final Report of the Special Media Records Project (Albany, New York: SARA, 1988); New York, State Archives and Records Administration, Status Report and Evaluation (Albany New York: SARA, 1992); Margaret Hedstrom, ed. Electronic Records Management Program Strategies (Pittsburgh: Archives and Museum Informatics, 1993); Margaret Hedstrom. "Closing Address", in Playing For Keeps: The Proceedings of an Electronic Records Management Conference Hosted by the Australian Archives, Canberra, Australia, 8-10 November 1994 (Canberra: Australian Archives, 1995): 331-6.

44 John McDonald. "Managing Records in the Modern Office: Taming the Wild Frontier”, Archivaria 39 (1995): 70-9.

45 Hans Hofman. "Off the BeatenTrack: The Archivist Exploring the Outback of Electronic Records", in Playing For Keeps: The Proceedings of an Electronic Records Management Conference hosted by the Australian Archives, Canberra, Australia, 8-10 November 1994 (Canberra: Australian Archives, 1995): 68-83.

46 Terry Cook. "Electronic Records, Paper Minds: The Revolution in Information Management and Archives in the Post-custodial and Post-modern Era", Archives and Manuscripts 22 no.2 (1994): 300-29.

47 On macro-appraisal in the National Archives of Canada, see Catherine Bailey. "From the Top Down: The Practice of Macro-Appraisal”, Archivaria 43 (1997): 89-128. On PIVOT, R. C. Hol and A. G. de Vries. "PIVOT Down Under: A Report", Archives and Manuscripts 26 no.1 (1998): 78-101. 


\section{Continuum Theory and the Records Continuum Model}

From the early 1990s Upward, working with colleagues McKemmish and Iacovino at Monash University, was exploring continuum theories linked to an inclusive and pluralist concept of records which embraced archives, defined as records of continuing value, and challenged the binary definitions and demarcated roles of records creators, records managers and archivists embodied in life cycle traditions. All three drew on their immersion in the recordkeeping community of practice in the 1980s and 1990s, including their formative years in the Australian Archives "gene pool" in the 1970s and their in-depth exposure to registry systems. While acknowledging the many and varied meanings of the terms record, archive and archives in different national and cultural contexts, and "the more precise and applied meanings used by recordkeeping professional communities," continuum definitions of the record and recordkeeping focus on the Archive in the very broadest sense, "encompassing oral and written records, literature, landscape, dance, art, the built environment and artefacts" insofar as they provide traces of social and organisational activity, that evidence and memorialise individual and collective lives. ${ }^{48}$ Integrated recordkeeping and archiving processes are characterised as forms of witnessing and memory-making with a critical role in governance, accountability, identity, individual and collective memory, social justice and cultural heritage.

Upward began to theorise about the records continuum and to develop a conceptual model that captured its key features. ${ }^{49}$ His initial theorising on the records continuum drew heavily on postmodern philosophical and historiographical thinking, including the work of Foucault on the archaeology of knowledge and Derrida on the "archive", as well as contemporary social theory, in particular Giddens' exploration of spacetime and structuration theory. ${ }^{50}$ Meanwhile Iacovino began to apply records

48 McKemmish, “Traces: Document, Record, Archive, Archives,” 1.

49 Frank Upward. "Structuring the Records Continuum: Part Two Structuration Theory and Recordkeeping", Archives and Manuscripts 25 no.1 (1997): 10-35; "Structuring the Records Continuum: Part One”, Archives and Manuscripts 24 no.2 (1996): 268-85; and "In Search of the Continuum".

50 Frank Upward, “The Records Continuum," in McKemmish et al, Archives: Recordkeeping in Society, 197-222; Jacques Derrida. Archives Fever (Chicago: University of Chicago Press, 1996); Michel Foucault. The Archaeology of Knowledge (London: Routledge, 1995); Anthony Giddens. The Constitution of Society: Outline of the Theory of Structuration (Cambridge: Polity Press, 1984). 
Figure 4.1: The Records Continuum Model

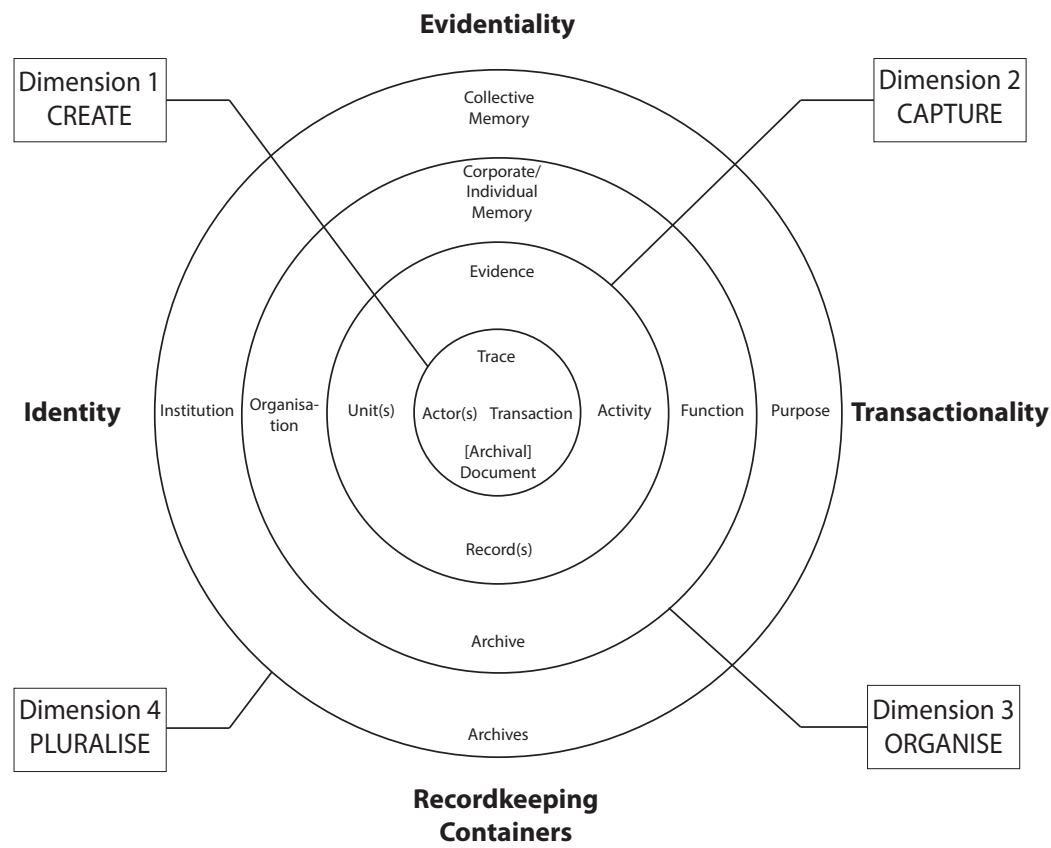

continuum thinking and the model to the nexus between recordkeeping and the law. ${ }^{51}$

From the beginning, Upward claimed that the Records Continuum Model represents "a fully-fledged paradigm shift in which a worldview is being replaced". ${ }^{52} \mathrm{He}$ characterises the pre-existing records life cycle worldview as a way of patterning knowledge and structures for managing records based on the separation of space and time. Whereas the records continuum worldview recognises the unity of space and time, and re-patterns knowledge and structures to manage records and archives in spacetime. He sees the great strength of the Records Continuum Model as a tool for perceiving and analysing complex realities, providing multi-dimensional and multilayered views of recordkeeping and archiving in different spacetimes. ${ }^{53}$ While

51 For example Livia Iacovino. "The Nature of the Nexus Between Recordkeeping and the Law”, Archives and Manuscripts 26 no. 2 (Nov 1998): 216-247; Recordkeeping, Ethics and Law: Regulatory Models, Participant Relationships and Rights and Responsibilities in the Online World (Springer: 2006)

52 Upward, "Modelling the Continuum", 117.

53 Upward, "Modelling the Continuum”, 117-119. 
supporting Ketelaar's view that practice is cultural, and that the detailed knowledge of a profession is applied in particular contexts in different ways, ${ }^{54}$ Upward claims for the model the status of a perduring worldview, persisting through constant renewal. He argues that it can therefore provide a way of patterning and re-patterning professional knowledge and practice across cultures and over long periods of time, and deal with the increasing complexity and plurality of recorded information in the digital world. In this regard, in Chapter 6 of this book, he explores the emergence of continuum theorising by Anglo-American and Australian philosophers from the last years of the $19^{\text {th }}$ century when philosopher and psychologist William James first coined the term "multiverse." He characterises it as a collective academic effort to understand expanding complexity, and discusses the relevance of this collective work to the further evolution of records continuum theory, particularly in relation to the "expanding continuum of recorded information that is the archival multiverse," increasingly complex online environments and pluralistic societies, introducing the emerging field of recordkeeping informatics.

The Records Continuum Model ${ }^{55}$ provides a multidimensional view of recordkeeping in spacetime. It maps the creation of records as traces of actions, events and participants, their capture into systems (broadly defined to incorporate formal and informal recordkeeping processes that manage them as evidence, memory and reliable sources of information), their organisation into the archive of an organisation, group, family or individual, and their pluralisation beyond the boundaries of an organisation, family or group, or an individual life. Pluralisation involves disembedding the record from its original multiple organisational and/or personal contexts and carrying it through spacetime. Thus recordkeeping processes fix the content and structure of records, preserve their integrity by ensuring they are tamperproof, and link them to ever-widening layers of rich metadata about their multiple contexts of creation and use. This enables them to be accessed, used and interpreted in other spacetimes. In continuum terms, while a record's content and structure can be seen as fixed, in terms of its contextualisation, a record is "always in a process of becoming." ${ }^{56}$ Records "can never be set aside from spacetime" as they exist in and through different spacetimes. ${ }^{57}$ This

54 Explored at length in Ketelaar, “The Difference Best Postponed?”.

55 This explanation of the Records Continuum Model draws heavily on Upward, McKemmish and Reed, "The Records Continuum Model", 4450-53.

56 Sue McKemmish. "Are Records Ever Actual?” in McKemmish and Piggott, The Records Continuum, 200.

57 Upward, "Modelling the Continuum”, 119. 
perduring and enduring duality is critical to an understanding of the nature of records in records continuum theory. Records have:

multiple lives in spacetime as the contexts that surround their use and control alter and open up new threads of action. ${ }^{58}$

Ultimately records are definable only in terms of their multiple and dynamic documentary and contextual relationships. They are configured and refigured through spacetime - hence the relevance of Richard Holmes' wave motion analogy of biographical subjects as sub-atomic particles: "never existing in all their complexity in any one place or time ... you cannot freeze them, you cannot pinpoint them, at any particular turn in the road, bend of the river, view from the window." ${ }^{59}$ Like the biographical subject, a record can never be experienced in all its dynamic complexity by a witness at any one point. It is not wholly present at any given moment, a particularly apt way of thinking about digital documents compared with the artefactual view of records as end products.

In the Records Continuum Model, integrated recordkeeping and archiving processes:

- Create [archival] documents-as-trace of the act in which they participate (Dimension 1);

- Capture records-as-evidence by linking documents-as-trace to the transactions, acts, decisions or communications they document, related records, participating agents, and their immediate business or social contexts (Dimension 2);

- Organise records-as-evidence by "placing" them in the contexts of the corporate and/or individual archive, and managing them in frameworks that enable them to function as individual, group, or corporate memory (Dimension 3); and

- Pluralise records-as-individual/group/corporate memory by "placing" them into all-encompassing frameworks that enable them to function as accessible collective memory (Dimension 4). ${ }^{60}$

The Records Continuum Model highlights the evidential, transactional and contextual nature of the archival document, the record, the archive and the archives. In continuum theory, their evidential qualities are seen

58 Upward, "Modelling the Continuum", 119.

59 Richard Holmes, Footsteps: Adventures of a Romantic Biographer (London, 1995), 27.

60 Upward, McKemmish and Reed, “The Records Continuum Model”, 4452. 
as integral to their "recordness" which enables them to serve multiple purposes in support of governance and accountability, the formation of individual, group, corporate, and collective memory, the shaping of identity, and provision of authoritative sources of information. The concept of transactionality "encompasses individual acts of communication, and social and business transactions of all kinds, the social and business activities or processes of which they are a part, the social and business functions they fulfil, and the social purposes they serve." ${ }^{.11}$ As discussed above, the concept of contextuality in the continuum is concerned with the record's rich, complex, dynamic and multi-layered social, functional and documentary contexts of creation, management, and use in and through spacetime.

A unique feature of the records continuum tradition is its emphasis on designing integrated recordkeeping and archival frameworks in the third and fourth dimensions of the continuum before records are created and captured in the first and second dimensions. As described by McKemmish, Upward and Reed, this approach has been a motivating force in the engagement of Australian recordkeeping professionals in the reform of archival laws in Australia to include a role for archival authorities in regulating current recordkeeping, the development of national and international records management standards, the development of guidelines for the design and implementation of recordkeeping systems, and a refiguring of traditional archival appraisal and description. ${ }^{62}$ They trace how continuum thinking and practice are informed by the insights of Hurley and Cook about provenance and the nature of functions, and their implications for archival appraisal and description. Hurley's extensive writings on the Australian Series System, and Bearman's ideas on archival strategies, the evidential nature of transactional records, recordkeeping systems and metadata, and his critique of traditional archival methods have also been critical influences. ${ }^{63}$

61 Upward, McKemmish and Reed, "The Records Continuum Model”, 4450-51, 4455-56.

62 Upward, McKemmish and Reed, "The Records Continuum Model”, 4449.

63 Upward, McKemmish and Reed, "The Records Continuum Model”, 4449. See also Chris Hurley. "Ambient Functions: Abandoned Children to Zoos", Archivaria 40 (1995): 21-39, "Problems with Provenance", Archives and Manuscripts 23 no.2 (1995):

234-59, and "What, If Anything, Is A Function?" Archives and Manuscripts 21 no.2 (1993): 208-20; “The Australian (Series) System: An Exposition" in McKemmish and Piggott, The Records Continuum, 150-72; Terry Cook. "The Concept of the Archival Fonds in the Post-custodial Era: Theory, Problems and Solutions", Archivaria 35 (1993): 24-37, and "Mind Over Matter: Towards a New Theory of Archival Appraisal", in Barbara Craig, ed. The Archival Imagination: Essays in Honour of Hugh A. Taylor (Ottawa: Association of Canadian Archivists, 1992): 38-70; David Bearman. Electronic Evidence: Strategies for Managing Records in Contemporary Organizations (Pittsburgh: Archives and 
Figure 4.2: The RKMS Entity Relationship Model

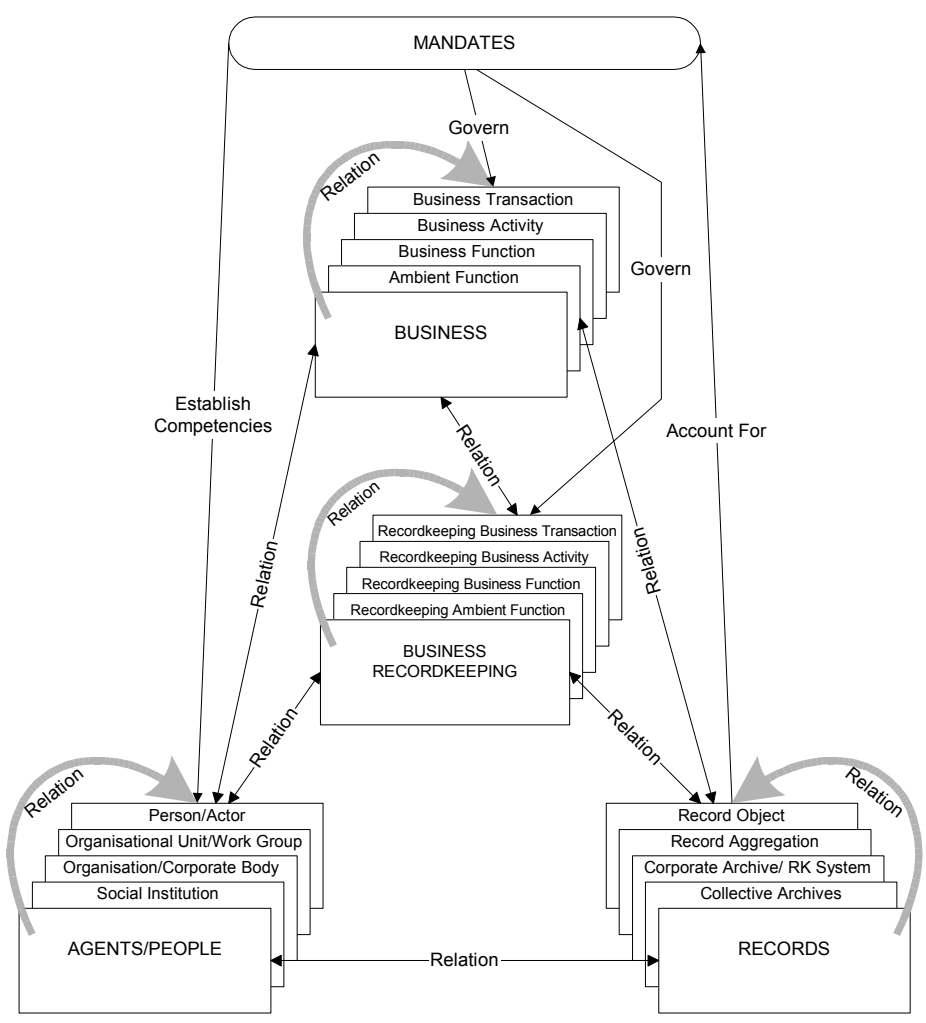

In the continuum's integrated recordkeeping and archival frameworks, appraisal "becomes a multi-faceted, recursive process which begins with defining what should be created (Dimension 1), what should be captured and managed as record (Dimension 2), what should be managed as a part of individual or organisational memory (Dimension 3) and what should be pluralised beyond organisational or individual memory (Dimension 4)." ${ }^{\prime 64}$ Description is also no longer a post hoc archival process, but an integral component of "a series of iterative recordkeeping processes that capture and inextricably link authoritative metadata to documents created in the context

Museum Informatics, 1994); “Documenting Documentation”, Archivaria 34 (1992):

33-49; Archival Methods (Pittsburgh: Archives and Museum Informatics, 1989); David Bearman and Richard Lytle. "The Power of the Principle of Provenance", Archivaria 21 (1985-86): 14-27.

64 Upward, McKemmish and Reed, “The Records Continuum Model”, 4455. 
of social and business activity from creation throughout all dimensions of the continuum.” ${ }^{65}$

In the mid-1990s, continuum-based approaches to description were used in an Australian Research Council funded, Monash-led collaborative research project to develop the Australian Recordkeeping Metadata Schema (see Wendy Duff and Kate Cumming's Chapter 15 for an explanation of the use of literary warrant analysis in this work). The RKMS metadata model was used in the subsequent development of metadata standards by national and state archival authorities in Australia, and became the conceptual framework for Part 1 of the international recordkeeping metadata standard, ISO 23081. The implementation strategies in Part 2 of the standard were based on work undertaken by Standards Australia informed by another Australian Research Council funded, Monash-led collaborative research initiative, the Clever Recordkeeping Project. ${ }^{66}$

Cook has highlighted the rich contextuality represented in the Records Continuum Model and the related metadata model:

The continuum model ... encompasses movement across space and time, recognizing that archival records and their metadata are continually shifting, transforming, and gaining new meanings, rather than remaining fixed, static objects, and that this also occurs in the purely archival fourth dimension ... The most inclusive metadata model anywhere from Monash University, that not coincidentally incorporates continuum thinking, ... focuses not just on authenticity and reliability in dimensions one and two for records, but recognizes that all sixteen key elements of the continuum - four dimensions across four axes - reflect "value added" research knowledge of the archivist, that is continually changing, both collapsing and expanding time and space across the four dimensions, to create a series of rich interconnections and contextual relationships ... ${ }^{67}$

In the recordkeeping community of practice, the Records Continuum Model is widely used as metaphor, and an implementation model, as

65 Sue McKemmish, Glenda Acland, Nigel Ward and Barbara Reed. "Describing Records in Context in the Continuum: The Australian Recordkeeping Metadata Schema”, Archivaria 48 (1999): 3-43; quote p. 6.

66 Joanne Evans, Sue McKemmish and Karina Bhoday. "Create Once, Use Many Times: The Clever Use of Recordkeeping Metadata for Multiple Archival Purposes," Archival Science 5 no.1 (2005): 17-42.

67 Terry Cook. "Beyond the Screen: The Records Continuum and Archival Cultural Heritage", in Lucy Burrows ed. Beyond the Screen: Capturing Corporate and Social Memory, (Melbourne: Australian Society of Archivists, 2000): 8-21, quote pp. 19-20. 
exemplified through its use in the development of standards, metadata entity-relationship models and schemas, and best practice guidelines for the design of recordkeeping systems and appraisal programs. It has also been used extensively as a teaching tool, as a conceptual framework for research, and as a research instrument. ${ }^{68}$

\section{The Records Continuum and the Archival Multiverse}

Records continuum theory and practice emerged in an Australian records and archives landscape dominated by two opposing traditions, a government recordkeeping tradition (strongly influenced by Jenkinson's ideas about the nature of records and archives, and the colonial registry tradition), and a library-based collecting tradition. During the 1990s, it was closely associated with the government recordkeeping tradition and many continuum writers and practitioners focused on the recordkeeping-accountability nexus and the role of archival authorities in regulating public sector recordkeeping to support good governance and democratic accountability. Records continuum theory and collaborative research, particularly on recordkeeping metadata, underpinned their efforts to develop recordkeeping standards at institutional, national and international levels, thus contributing to global efforts to develop a singular, unifying "archival paradigm" as discussed in Chapter 1.

Critics of records continuum theory and practice during the 1990s highlighted the preoccupation with government and corporate recordkeeping. ${ }^{69}$ Although some continuum writers, e.g. McKemmish, Piggott and Cunningham, were engaged from this time in their ongoing exploration of personal recordkeeping in the continuum, ${ }^{70}$ most of the continuum literature

68 Upward, McKemmish and Reed, "Records Continuum Model”, 4454-56.

69 Foremost among them Verne Harris. "Law, Evidence and Electronic Records:

A Strategic Perspective from the Global Periphery”, paper presented to the ICA Conference, Seville, September 2000.

70 Piggott's career, begun at the National Library Manuscripts Department in the 1970s, included processing large collections of politicians' papers. There followed a decade at the Australian War Memorial during which he published $A$ Guide to the Personal Family and Official Papers of C E W Bean (Australian War Memorial, Canberra, 1983). Interests in the papers of academics, composers and diaries and diary keeping nourished by appointments at the National Archives of Australia and University of Melbourne resulted in 'Towards a History of Australian Diary Keeping', Archivaria 60 (Fall 2005): 145-166; "Grainger the Autoarchivist," in David Pear, ed. Facing Percy Grainger, (Canberra: National Library of Australia, 2006), 39-45; and "On First Looking into Loewe's papers," University of Melbourne Collections 3 (December 2008): 18-23. 
during the 1990s related to the government recordkeeping tradition. The power of inclusive continuum concepts of archiving and recordkeeping, and fourth dimension perspectives on plurality were not as yet being harnessed to address the needs of the people, communities and organisations being marginalised by the mainstream traditions.

At the Australian Society of Archivists Conference in 2000, Terry Cook spoke about the inclusiveness of the Records Continuum Model in these terms:

... the continuum may serve as the unifying bond to heal the threatened schism in archives. After all, it includes both evidence and memory on one of its axis, those two uneasy sides of the archival coin. And its pluralizing of memory through societal archives in the fourth dimension of the continuum model, beyond the purview of the institutional or corporate archive in the third dimension, indicates societal-based appraisal at work as I have described it. In short, it does not deny cultural, historical, or heritage roles to archivists, but on the contrary sees these as a critically important part of record-keeping, while respecting as well the vital records management role of creating and maintaining current records in rich context. ${ }^{71}$

At that time, Cook proposed a number of enhancements to the model so that it could better fulfil its unifying potential, including building on and extending then current understandings of evidence and memory in the archival literature (for example in the writings of Brothman, Brown, Cook, Harris, Ketelaar, Nesmith, and $\mathrm{Upward}^{72}$ ), bringing into play new

McKemmish's key continuum-based writings on personal archiving and recordkeeping include "Evidence of me...", Archives and Manuscripts, 24 no. 1 (May 1996): 28-45; a defence with Frank Upward of the 1996 piece and response to its critique by Verne Harris in "In Search of the Lost Tiger, by Way of Sainte-Beuve: Re-constructing the Possibilities in 'Evidence of Me'", Archives and Manuscripts, 29 no. 1 (May 2001): 22-42; and a revisiting of the 1996 piece in the context of new technologies in "Evidence of $\mathrm{Me}$ ... in a Digital World" in Christopher A Lee, ed. I, Digital; Personal Collections in the Digital Era (Chicago: Society of American Archivists, 2011), 115-150. See also Adrian Cunningham. "Beyond the Pale? The 'flinty' relationship between archivists who collect the private records of individuals and the rest of the archival profession", Archives and Manuscripts 24 no. 1 (May 1996): 20-26; "From Here to Eternity: Collecting Archives and the Need for a National Documentation Strategy", LASIE 21 no. 1 (1998): 32-45; "Eternity Revisited: In Pursuit of a National Documentation Strategy and a National Archival System", Archives and Manuscripts 42 no. 2 (Nov 2014): 165-170.

71 Terry Cook, "Beyond the Screen”, 19-20.

72 For example: Brien Brothman. "Declining Derrida: Integrity, Tensegrity, and the Preservation of Archives from Deconstruction”, Archivaria 48 (1999): 64-89; Richard 
insights from other disciplines, further exploration of the continuum's fourth dimensional understandings of the pluralised nature of the archive beyond spatial and temporal boundaries, and fuller realisation of the model's potential in relation to "the citizen's impact on, interaction with, and variance from the state"; its sensitivity to the marginalised, and the ways in which organisational records complement or supplement personal and family records; its cross-institutional, and cross-jurisdictional perspectives; and its embracing of user needs.

He concluded:

These suggestions aside, the critical importance of the continuum rests on five factors: 1) its conceptual interaction of the sixteen levels and axes too often viewed in archival circles as separate and static; 2) its insight that these complex relationships are fluid, multiple, and simultaneous across time and space, not sequential and fixed; 3) its reconciliation (with some adjustments as suggested) of evidence and memory; 4) its potential for imaginatively incorporating private-sector manuscripts with institutional archives; and 5) its assertion through pluralization that societal and thus cultural values will influence appraisal and, indeed, all aspects of record-keeping. ${ }^{73}$

Records continuum research and writing have increasingly explored the fuller realisation of the model's potential as envisioned by Cook. In so doing, they are increasingly drawing in particular on critical theory, postcolonialist views on the decolonisation of the Archive, Indigenous ways of knowing, the concept of the archival multiverse, and insights from the radical archives movement as discussed below. More recent records continuumbased research and writing are challenging the way in which mainstream recordkeeping traditions in Australia and elsewhere sustain particular power configurations and contribute to the marginalisation of many groups. And records continuum researchers and writers are reflecting critically on their

Brown. "The Value of 'Narrativity' in the Appraisal of Historical Documents:

Foundation for a Theory of Archival Hermeneutics”, Archivaria 32 (1991): 152-6; Terry Cook, "What Is Past Is Prologue"; Verne Harris. "Claiming Less, Delivering More: A Critique of Positivist Formulations on Archives in South Africa", Archivaria 44 (1997): 132-41; Eric Ketelaar. "Archivalisation and Archiving”, Archives and Manuscripts 27 no.1 (1999): 54-61, and “The Difference Best Postponed?”; Tom Nesmith. "Still Fuzzy, But More Accurate: Some Thoughts on the 'Ghosts' of Archival Theory," Archivaria 47 (1997): 136-150.

73 Cook, "Beyond the Screen”, 21. 
own engagement in constructing and enabling the construction of a singular, unifying, global "archival paradigm", as discussed in Chapter 1.

In the last 15 years, records continuum theorists and researchers have further explored the continuum's fourth dimensional understandings of the pluralised nature of the Archive. They have demonstrated how public recordkeeping traditions in Australia (and elsewhere) do not necessarily serve the needs of marginalised and vulnerable communities and how poor recordkeeping is failing to play its part in corporate accountability and transparency. They have researched how government and mainstream institutional perspectives are privileged in government recordkeeping and library manuscript traditions and others are marginalised, their voices muted or absent. For example, Hurley has highlighted how modern archival theory and practice are complicit in privileging the "single records creator", defined as the organisation or person who "sets the record aside", and treat individual participants in the transactions and interactions documented in the records as subjects of the records with a very limited range of rights (mostly relating to privacy and access). ${ }^{74}$ In Australia decisions about what records to make and keep as archives are taken by "records creators" (as defined above), records managers and archivists with little or no consultation with the other parties involved or consideration of their recordkeeping needs. Descriptive systems capture the context of the singular creator, and not the contexts of others who participated in the activities or events documented in the record as parties to the transactions. The singular records creator exercises a much more extensive set of rights in the records than those of other participants.

In response Hurley ${ }^{75}$ has developed concepts of co-creation, multiple simultaneous provenance and parallel provenance, complementing Eric Ketelaar's writing on multiple, mutually negotiated rights in communities of records. ${ }^{76}$ Their theoretical perspectives radically redefine agency in records in a way that supports a more extensive suite of rights for all those who participate in or are directly impacted by the events or actions documented in the records creation process. Adopting this approach repositions "records subjects" as well as those who are directly impacted by the record as "participatory records agents". It supports a broader spectrum of rights, responsibilities and obligations relating to appraisal, description, management, accessibility, and

74 Hurley, "Parallel Provenance: (1)" and "Parallel Provenance: (2)".

75 Hurley, "Parallel Provenance: (1)" and "Parallel Provenance: (2)".

76 Eric Ketelaar. "Access: The Democratic Imperative," Archives and Manuscripts 34 no. 2 (2006): 62-81, and "Sharing: Collected Memories in Communities of Records," Archives and Manuscripts 33 no.1 (2005): 44-61. 
use of records in and through spacetime. And it brings the multiple contexts and perspectives of the repositioned subjects and those directly impacted by the records into play in decision making - about what records to create and keep; what records are of continuing value; what metadata needs to be captured to document their multiple contexts; whose rights need to be taken into account in determining disclosure, access, and use policies; and what perspectives need to be addressed in access pathways.

Hurley's ideas about multiple simultaneous provenance and parallel provenance evolved in part from his immersion in issues relating to Indigenous peoples when he was Keeper of Public Records in Victoria and the Chief Archivist in New Zealand and were influenced by Indigenous ways of knowing. The idea of parallel provenance in particular is closely associated with understandings of the incommensurability or irreconcilability of aspects of the different ways of knowing in Indigenous and European traditions, and the diverse perspectives of those involved in the events documented in the records. In this regard, Hurley uses the telling example of how the descriptive systems in government archives link the records of the Stolen Generations ${ }^{77}$ to the government function of "protection of Aboriginal peoples" - whereas Aboriginal families experienced this as child stealing and abuse of human rights. Current government archival descriptive frameworks are designed in a way that privileges and perpetuates the perspective of the government agencies involved in removing the children, and cannot accommodate the multiple views of those involved in or impacted by these actions - they exist in a parallel universe. Hurley's notion of multiple simultaneous provenance challenges archival system designers to build meta-frameworks that can accommodate multiple, potentially contested, or even irreconcilable realities.

In the manuscript tradition records of Indigenous Australians are most likely to be found in the collections of anthropologists and non-Indigenous organisations, including the orphanages and missions where the Stolen Generations were taken after removal from their families. Such collections may contain records and artefacts that were originally created by Indigenous Australians (audio and visual recordings of Indigenous language, ceremonies, dance and stories about country), as well as documents created by the donor person or organisation. They are described in library manuscript systems that focus on describing and indexing content, and their access and use are mostly managed according to agreements with the donor.

77 The Stolen Generations are made up of up to 50,000 Indigenous children who were forcibly removed from their families from 1909 to 1969 under policies aimed at the assimilation of 'half-caste' children into white society and 'breeding out' Aboriginality. 
Within the framework provided by the 2007 UN Declaration on the Human Rights of Indigenous Peoples, there are calls for Indigenous people to become active, participating agents in recordkeeping and archiving practice for all records relating to them, rather than passive, disempowered subjects of records created and maintained by non-Indigenous institutions and organisations. ${ }^{78}$ As discussed and referenced below, Indigenous Australians are also increasingly challenging curatorial practices in cultural institutions that support what they see as a misappropriation of their knowledge and heritage. Other marginalised communities are also pushing for more extensive rights in records, and for recordkeeping systems in public and private sector organisations that better meet their needs. Beyond access and discovery rights, they want to play a part in decision making about the management and accessibility of their records, and to be able to add their own stories to the institutional records relating to them.

The Trust and Technology Project, funded by the Australian Research Council, involved a multidisciplinary team made up of Indigenous and nonIndigenous researchers working in partnership with Indigenous communities, the Public Record Office of Victoria, the Koorie Heritage Trust, the Koorie Records Taskforce, and the Indigenous Special Interest Group of the Australian Society of Archivists. A hoped-for outcome was that the findings of the Project would underpin the development of archival frameworks, strategies and tools driven by the needs of Indigenous communities and individuals, and the building of relationships of trust between the archival community (encompassing archival institutions, organisational recordkeeping programs, the profession, and individual recordkeeping professionals/archivists) and Indigenous communities. It aimed to address the "growing recognition that western archival science and practice reflect and reinforce a privileging of settler/invader/colonist voices and narratives over Indigenous ones, of written over oral records", and the "disempowering effect on Indigenous peoples whose lives have been so extensively documented in archives for the purposes of surveillance, control and dispossession", which is a consequence of their treatment as passive subjects of the archives with very limited rights in records. ${ }^{79}$ The Project produced a Position Statement on Human Rights, Indigenous Communities in Australia and the Archives,

78 Mick Gooda. "The Practical Power of Human Rights: How International Human Rights Standards Can Inform Archival and Recordkeeping Practices," Archival Science 12 no.2 (2012), Special Issue: Keeping Cultures Alive: Archives and Indigenous Human Rights, 141-150.

79 McKemmish, Faulkhead and Russell, "Dis-trust in the Archive", 218. 
a Statement of Principles relating to Australian Indigenous Knowledge and the Archives, and a related Action Agenda for Archival Reconciliation. ${ }^{80}$ If implemented the Action Agenda would reposition Indigenous Australians as participatory agents in recordkeeping and archiving with an extensive suite of rights in records that would support their self-determination and the exercise of their cultural rights as human rights. The Trust and Technology findings provide a case study in how pluralisation continuum-style might contribute to a decolonising of the archive, freeing Indigenous peoples who have hitherto been the "captives of the archives" ${ }^{81}$ Continuum concepts of co-creatorship, multiple simultaneous provenance and parallel provenance, and participatory models of the archive are of particular relevance in this context. Co-designing recordkeeping frameworks, strategies and processes within this conceptual frame in partnership with Indigenous communities could play a reconciling role as part of a broader re-setting of relationships between Indigenous and non-Indigenous Australia.

Linked to an increasing focus on researching the recordkeeping and archiving needs of communities, continuum researchers and writers are also contributing to the development of the emergent concepts of the archival multiverse and the participatory archive, and most recently the concept of archival autonomy. Upward, McKemmish and Reed have explored the role of "continuum consciousness" in transforming recordkeeping and archiving practice to better engage with complexity and plurality in online environments. ${ }^{82}$ Drawing upon Upward's theory-building work and continuum discourse analysis, their paper argues that the continuum as a meta-view of reality helps us to imagine recordkeeping and archival functionality in the archival multiverse, with particular reference to archival purposes associated with identity, social justice, transparency, and accountability. McKemmish and Piggott have explored how the archival multiverse's view of "the pluralism of evidentiary texts, memory-keeping practices and

80 McKemmish, Faulkhead and Russell, "Dis-trust in the Archive”, 218; Sue McKemmish, Livia Iacovino, Eric Ketelaar, Melissa Castan and Lynette Russell. "Resetting Relationships: Archives and Indigenous Human Rights in Australia", Archives and Manuscripts, 39 no.1 (May 2011): 107-44; Livia Iacovino. "Rethinking Archival, Ethical and Legal Frameworks for Records of Indigenous Australian Communities: A Participant Relationship Model of Rights and Responsibilities," Archival Science 10 no. 4 (2010): 353-72.

81 Henrietta Fourmile. "Who Owns the Past? Aborigines as Captives of the Archives," Aboriginal History 13 (1989): 1-2.

82 Frank Upward, Sue McKemmish and Barbara Reed, "Archivists in Changing Information and Social Spaces". 
institutions, bureaucratic and personal motivations, community perspectives and needs, and cultural and legal constructs" ${ }^{183}$ resonates with continuumbased views of the archive as encompassing personal and corporate records that exist "in a complex socio-technical ecosystem" as "a source of legal and cultural evidence and community memory and identity". ${ }^{84}$ Drawing on writings about pluralising the archive and the archival multiverse, ${ }^{85}$ and continuum consciousness, they define the personal archive in the broadest sense to include "all forms, genres and media of records relating to that person, whether captured in personal or corporate recordkeeping systems; remembered, transmitted orally or performed; held in manuscript collections, archival and other cultural institutions, community archives or other keeping places; or stored in shared digital spaces" ${ }^{86}$

Gilliland and McKemmish argue for a new participatory model to be applied in archives that are central to the promotion of social justice, human rights, reconciliation and recovery. They offer a new concept of the participatory archive, "created by, for and with multiple communities, according to and respectful of community values, practices, beliefs and needs" and based on acknowledgement of the rights, responsibilities, perspectives and needs of the multiple parties involved. ${ }^{87}$ They discuss principles and approaches for the participatory archive with reference to how the continuum-based re-definition of agency in records supports participatory processes by vesting participatory agents with a suite of negotiated legal and moral rights and responsibilities. Their ongoing work on rights in records linked to social justice and human rights agendas draws extensively on records continuum thinking and scholarship. ${ }^{88}$ Their

83 AERI Pluralizing the Archival Curriculum Group (PACG), "Educating for the Archival Multiverse", 73.

84 McKemmish and Piggott, "Toward the Archival Multiverse”, 113.

85 For example, Anne J. Gilliland, Andrew J Lau, and Sue McKemmish. "Pluralizing the Archive," chapter 7 in Keiji Fujiyoshi, ed., Archives for Maintaining Community and Society in the Digital Age (Koyasan University, Japan, 2013): 65-74; and PACG, "Educating for the Archival Multiverse".

86 McKemmish and Piggott, "Toward the Archival Multiverse", 113; see also McKemmish, "Evidence of Me ... in a Digital World".

87 Gilliland, Anne J. and Sue McKemmish. "The Role of Participatory Archives in Furthering Human Rights, Reconciliation and Recovery," Atlanti: Review for Modern Archival Theory and Practice 24 (2014): 79-88, quote p. 82.

88 Anne Gilliland and Sue McKemmish. "Rights in Records as a Platform for Participative Archiving”, in Richard Cox, Alison Langmead and Nora Mattern, eds., Studies in Archival Education and Research: Selected Papers from the 2014 AERI Conference (Sacramento: Litwin Books, 2015): 355-86. 
work is complemented by Iacovino's recent research on maximising human rights through participatory archiving. ${ }^{89}$

Using the so-called Forgotten Australians ${ }^{90}$ and the Stolen Generations of Aboriginal children as case studies, research by Evans, McKemmish, Daniels and McCarthy has explored the role of archival activism in supporting social movements linked to human rights and social justice agendas. ${ }^{91}$ They also explore how an extended suite of rights in records and participatory archival approaches might support community selfdetermination and human rights with particular reference to the rights of the child. The lack of holistic, pluralising recordkeeping frameworks, the significant gaps in the Archive in Australia, and the fragmentation and dispersal of records (where they exist or have survived) relating to marginalised communities have an acute impact on communities like care leavers who experienced neglect and abuse in childcare institutions. They need access to records to establish their identity, recover memory and bring perpetrators and the institutions that sheltered them to account. They are also concerned about current and future generations of children in care and the need for cross-jurisdictional, cross-sectoral, pluralising recordkeeping frameworks and infrastructure to support governance and accountability in the organisations and institutions responsible for child care, preventative strategies to reduce the levels of abuse, early detection, reporting, investigation and remedial action, and future archival services that can deliver discoverable, accessible evidence.

Evans, McKemmish, Daniels and McCarthy propose a new concept of archival autonomy, tentatively defined as "the ability for individuals and communities to participate in societal memory, to have their own voice, to become participatory agents in recordkeeping and archiving for identity, memory and accountability purposes. ${ }^{92}$ They identify archival autonomy as a grand societal challenge, and present the case for archival activism to

89 Livia Iacovino. "Shaping and Reshaping Cultural identity and Memory: Maximising Human Rights through a Participatory Archive", Archives and Manuscripts, 43 no. 1 (May 2015): 29-41.

90 'Forgotten Australians' is the name given to the many generations of children who have suffered abuse and neglect in out-of-home care, in government, church and charitable orphanages and homes around Australia. It is a contested term with many so-called Forgotten Australians preferring the term care leavers. In 2013 a Royal Commission into systemic issues relating to the sexual abuse of children in institutional care was launched after 25 years of lobbying by care leavers and their advocacy groups.

91 Evans et al., "Self-determination and Archival Autonomy".

92 Evans et al., "Self-determination and Archival Autonomy", 337. 
become an integral part of social movements on a local and global scale, to "extend beyond encouraging greater diversity in archival collections or supporting the social justice work of others to transform the way that archival and recordkeeping systems connect and communicate, and are threaded into community, organisational and social fabrics. ${ }^{.93}$

\section{Conclusion}

In this chapter the focus has been on the development of an Australian recordkeeping tradition linked to evolving records continuum theory and practice, and the Records Continuum Model. The chapter has explored the historical, societal, juridical and geo-political contexts, and the philosophies, theories and cultural influences that have shaped them. The emergence of the records continuum worldview is presented as a paradigm shift in that it recognises the unity of space and time, and re-patterns knowledge and structures to manage records and archives in spacetime. The Records Continuum Model is explored as a tool for perceiving and analysing complex realities, capable of providing multi-dimensional and multi-layered views of recordkeeping and archiving in different spacetimes, and particularly relevant to understanding the complexities and pluralities of the archival multiverse in a digital age. The chapter has also reflected on the nature of the contributions that the Australian records continuum community has made to archival and recordkeeping theory, the international discourse, the development of international standards, and, more recently, to human rights and social justice agendas.

Today, a new generation of records continuum theorists, researchers and practitioners is taking on the grand archival challenges in Cook's paper. The work of the older generation of continuum theorists is being challenged and extended by a new generation of researchers. For example, a major outcome of Gibbons' doctoral thesis is the five dimensional Mediated Recordkeeping: Culture-as-evidence continuum model as a contribution to the development of conceptual and practical foundations for participatory digital archives. The model introduces a re-named and re-figured first dimension, $\mathrm{Co}-$ Create, and a new fourth dimension, Curate, which recognises the complex role of curation in acts of re-creation. A re-imagined Pluralise dimension (morphed into the fifth dimension) extends the mapping of features of the continuum to include representation of the role of facilitation, networking

93 Evans et al., "Self-determination and Archival Autonomy", 358-9. 
and the use of technologies as memory-making systems (see also Chapter 25 where Leisa Gibbons explores how continuum theory and modelling can be applied to research design itself to explore complexity and pluralism in researching the archival multiverse). ${ }^{94}$ Joanne Evan's Australian Research Council four-year Future Fellowship (2015-2018) enables exploration of the co-design of archival systems, employing participatory design methods. She is working with care leavers and other communities to design rights in records, inclusive appraisal and inclusive metadata into participatory archival systems, and model the activist use of technologies to enable and empower. Her work brings together the understandings and expertise gathered through long engagement in collaborative recordkeeping metadata research by Australian continuum researchers, with the more recent focus on community recordkeeping needs in the multiverse and harnesses the power of continuum ideas on co-creation, pluralisation and complexity to address a radical archival agenda (see also Chapter 22 for Joanne Evans' reflection on participatory design methodologies for archival and recordkeeping research).

Increasingly records continuum researchers are engaging in critical archiving and recordkeeping approaches, questioning the social constructs, values and power differentials embedded in current frameworks, processes, systems and technologies, exploring archival and recordkeeping agency, autonomy and activism, and moving beyond insight and critique with the aim of bringing about transformative outcomes. They are part of a vibrant international community of archival and recordkeeping scholars, researchers and educators who come together annually at the Archival Education and Research Institute (AERI) meetings. The AERI research and education community of practice provides compelling evidence of the maturing of the archival and recordkeeping field, and the multiple traditions that make up the archival multiverse. The research and writings of a critical mass of records continuum researchers and doctoral students in Australia and elsewhere are continuing to evolve continuum theory and re-imagine continuum modelling to better represent the archival multiverse in all its complexity and diversity.

94 Leisa Gibbons, Culture in the Continuum: YouTube, Small Stories and Memory-making (PhD, Faculty of IT, Monash University, 2015). 


\section{Acknowledgements}

I acknowledge my partners and co-authors in the records continuum research and writings extensively referenced in this chapter, in particular those I have collaborated with most closely over the past 25 years, my fellow records continuum theorists Frank Upward, Michael Piggott, Barbara Reed, Livia Iacovino, Chris Hurley, Glenda Acland and Adrian Cunningham.

\section{References}

Acland, Glenda. "Archivist - Keeper, Undertaker or Auditor?" Archives and Manuscripts 19 no.1 (1991): 9-15, and "Managing the Record Rather than the Relic", Archives and Manuscripts 20 no.1 (1992): 57-63

Australian Society of Archivists and Australian Council of Archives, Managing Electronic Records (Canberra: ASA and ACA, 1993)

Archival Education and Research Institute (AERI) Pluralizing the Archival Curriculum Group (PACG). "Educating for the Archival Multiverse," American Archivist 74 no.1 (Spring/Summer 2011): 69-102

Atherton, Jay. "From Life Cycle to Continuum: Some Thoughts on the Records Management-Archives Relationship", Archivaria 21 (1985-86): 43-51

Bailey, "From the Top Down: The Practice of Macro-Appraisal", Archivaria 43 (1997): 89-128

Bearman, David. Electronic Evidence: Strategies for Managing Records in Contemporary Organizations (Pittsburgh: Archives and Museum Informatics, 1994)

Bearman, David. "Electronic Records Guidelines: A Manual for Policy Development and Implementation", in David Bearman, Electronic Evidence: Strategies for Managing Records in Contemporary Organizations (Pittsburgh: Archives and Museum Informatics, 1994): 72-116

Bearman, David. "Documenting Documentation”, Archivaria 34 (1992): 33-49

Bearman, David. Archival Methods (Pittsburgh: Archives and Museum Informatics, 1989)

Bearman, David. "Archival Data Management to Achieve Organizational Accountability for Electronic Records”, Archives and Manuscripts 21 no.1 (1993): 14-28

Bearman, David. "Record-Keeping Systems”, Archivaria 36 (1993): 16-37

Bearman, David. (ed.) Archival Management of Electronic Records (Pittsburgh: Archives and Museum Informatics, 1991)

Bearman, David, and Margaret Hedstrom. "Reinventing Archives for Electronic Records: Alternative Service Delivery Options”, in Margaret Hedstrom, ed. Electronic Records Management Program Strategies (Pittsburgh: Archives and Museum Informatics, 1993), 82-98

Bearman, David, and Richard Lyttle. "The Power of the Principle of Provenance", Archivaria 21 (1985-86): 14-27

Brothman, Brien. "Declining Derrida: Integrity, Tensegrity, and the Preservation of Archives from Deconstruction”, Archivaria 48 (1999): 64-89

Brown, Richard. "The Value of 'Narrativity' in the Appraisal of Historical Documents: Foundation for a Theory of Archival Hermeneutics", Archivaria 32 (1991): 152-6

Cook, Terry. "Beyond the Screen: The Records Continuum and Archival Cultural Heritage", in Lucy Burrows, ed., Beyond the Screen: Capturing Corporate and Social Memory, (Melbourne: Australian Society of Archivists, 2000): 8-21 
Cook, Terry. "What Is Past Is Prologue: A History of Archival Ideas Since 1898, and the Future Paradigm Shift”, Archivaria 43 (1997): 17-63

Cook, Terry. "Electronic Records, Paper Minds: The Revolution in Information Management and Archives in the Post-custodial and Post-modern Era", Archives and Manuscripts 22 no.2 (1994): 300-28

Cook, Terry. "The Concept of the Archival Fonds in the Post-custodial Era: Theory, Problems and Solutions", Archivaria 35 (1993): 24-37

Cook, Terry. "Mind Over Matter: Towards a New Theory of Archival Appraisal”, in Barbara Craig, ed. The Archival Imagination: Essays in Honour of Hugh A. Taylor (Ottawa: Association of Canadian Archivists, 1992), 38-70

Cunningham, A. "Eternity Revisited: In Pursuit of a National Documentation Strategy and a National Archival System", Archives and Manuscripts 42 no. 2 (Nov 2014): 165-170

Cunningham, Adrian. "Archival Institutions," in Sue McKemmish, Michael Piggott, Barbara Reed and Frank Upward, ed. Archives: Recordkeeping in Society (Centre for Information Studies, Charles Sturt University: Wagga Wagga, Australia, 2005), 21-50

Cunningham, A. "From Here to Eternity: Collecting Archives and the Need for a National Documentation Strategy", LASIE 21 no. 1 (1998): 32-45

Cunningham, Adrian. "Beyond the Pale? The 'flinty' relationship between archivists who collect the private records of individuals and the rest of the archival profession", Archives and Manuscripts 24 no. 1 (May 1996): 20-26

Derrida, Jacques. Archives Fever (Chicago: University of Chicago Press, 1996)

Evans, Joanne, Sue McKemmish, Elizabeth Daniels and Gavan McCarthy. "Selfdetermination and Archival Autonomy: Advocating Activism," Archival Science Special Issue: Archiving Activism and Activist Archiving 15 no. 4 (2015): 337-368

Evans, Joanne, Sue McKemmish and Karina Bhoday. "Create Once, Use Many Times: The Clever Use of Recordkeeping Metadata for Multiple Archival Purposes," Archival Science 5 no.1 (2005): 17-42

Faulkhead, Shannon. Narratives of Koorie Victoria, Ph.D. Thesis (Faculty of Arts, Monash University, 2008)

Foucault, Michael. The Archaeology of Knowledge (London: Routledge, 1995)

Fourmile, Henrietta. "Who Owns the Past? Aborigines as Captives of the Archives," Aboriginal History 13 (1989): 1-2

Gibbons, Leisa. Culture in the Continuum: YouTube, Small Stories and Memory-making (PhD, Faculty of IT, Monash University, 2015)

Giddens, Anthony. The Constitution of Society: Outline of the Theory of Structuration (Cambridge: Polity Press, 1984)

Gilliland, Anne and Sue McKemmish. "Rights in Records as a Platform for Participative Archiving”, in Richard Cox, Alison Langmead and Nora Mattern, eds. Studies in Archival Education and Research: Selected Papers from the 2014 AERI Conference (Sacramento: Litwin Books, 2015): 355-86.

Gilliland, Anne J., and Sue McKemmish. "The Role of Participatory Archives in Furthering Human Rights, Reconciliation and Recovery," Atlanti: Review for Modern Archival Theory and Practice 24 (2014): 79-88

Gilliland, Anne J., Andrew J Lau, and Sue McKemmish. "Pluralizing the Archive," chapter 7 in Keiji Fujiyoshi, ed., Archives for Maintaining Community and Society in the Digital Age (Koyasan University, Japan, 2013), 65-74

Gooda, Mick. "The Practical Power of Human Rights: How International Human Rights Standards Can Inform Archival and Recordkeeping Practices," Archival Science 12 no.2 (2012), Special Issue: Keeping Cultures Alive: Archives and Indigenous Human Rights, 141-150 


\section{Chapter 4}

Harris, Verne. "Law, Evidence and Electronic Records: A Strategic Perspective from the Global Periphery", paper presented to the ICA Conference, Seville, September 2000

Harris, Verne. "Claiming Less, Delivering More: A Critique of Positivist Formulations on Archives in South Africa”, Archivaria 44 (1997): 132-41

Hedstrom, Margaret. "Closing Address", in Playing For Keeps: The Proceedings of an Electronic Records Management Conference Hosted by the Australian Archives, Canberra, Australia, 8-10 November 1994 (Canberra: Australian Archives, 1995), 331-6

Hedstrom, Margaret. ed. Electronic Records Management Program Strategies (Pittsburgh: Archives and Museum Informatics, 1993)

Hofman, Hans. "Off the Beaten Track: The Archivist Exploring the Outback of Electronic Records", in Playing For Keeps: The Proceedings of an Electronic Records Management Conference hosted by the Australian Archives, Canberra, Australia, 8-10 November 1994 (Canberra: Australian Archives, 1995): 68-83

Hol, R.C., and A. G. de Vries. "PIVOT Down Under: A Report", Archives and Manuscripts 26 no.1 (1998): 78-101

Holmes, Richard. Footsteps: Adventures of a Romantic Biographer (London, 1995)

Hurley, Chris. "Ambient Functions: Abandoned Children to Zoos", Archivaria 40 (1995): 21-39

Hurley, Chris. "Problems with Provenance", Archives and Manuscripts 23 no.2 (1995): 234-59

Hurley, Chris. "The Australian (Series) System: An Exposition" and "From Dustbins to Disk-Drives: A Survey of Archives Legislation in Australia”, Appendix 2 in Sue McKemmish and Michael Piggott, eds. The Records Continuum; Australian Archives first fifty years (Ancora Press, Melbourne, 1994), 150-72 and 206-32 respectively

Hurley, Chris. "What, If Anything, Is A Function?" Archives and Manuscripts 21 no.2 (1993): 208-20

Iacovino, Livia. "Shaping and Reshaping Cultural Identity and Memory: Maximising Human Rights through a Participatory Archive”, Archives and Manuscripts 43 no. 1 (May 2015): 29-41

Iacovino, Livia. "Rethinking Archival, Ethical and Legal Frameworks for Records of Indigenous Australian Communities: A Participant Relationship Model of Rights and Responsibilities," Archival Science 10 no. 4 (2010): 353-72

Iacovino, Livia. Recordkeeping, Ethics and Law: Regulatory Models, Participant Relationships and Rights and Responsibilities in the Online World (Springer: 2006)

Iacovino, Livia. "The Nature of the Nexus Between Recordkeeping and the Law", Archives and Manuscripts 26 no. 2 (Nov 1998): 216-247

Information Exchange Steering Committee. Management of Electronic Documents in the Australian Public Service (Canberra: IESC, 1993)

International Standards Organisation. ISO 15489 Information and documentation - Records management - Part 1: General; Part 2: Implementation, 2001

International Standards Organisation. ISO 23081 Information and documentation - Records management Processes - Metadata for Records Part 1: Principles, 2006; ISO 23081 Information and documentation - Managing Metadata for Records Part 2: Conceptual and Implementation Issues, 2009

Ketelaar, Eric. “Access: The Democratic Imperative," Archives and Manuscripts 34 no. 2 (2006): 62-81

Ketelaar, Eric. "Archivalisation and Archiving”, Archives and Manuscripts 27 no.1 (1999): 54-61

Ketelaar, Eric. "The Difference Best Postponed? Cultures and Comparative Archival Science," Archivaria 44 (1997): 142-148 
McDonald, John. "Managing Records in the Modern Office: Taming the Wild Frontier", Archivaria 39 (1995): 70-9

McKemmish, Sue. "Evidence of Me ... in a Digital World" in Christopher A Lee, ed. I, Digital; Personal Collections in the Digital Era (Chicago, Society of American Archivists, 2011): 115-150

McKemmish, Sue. "Traces: Document, Record, Archive, Archives," in Sue McKemmish, Michael Piggott, Barbara Reed and Frank Upward, eds. Archives: Recordkeeping in Society (Wagga Wagga: Centre for Information Studies, Charles Sturt University, 2005), 1-20

McKemmish, Sue. Constantly Evolving, Ever Mutating: An Australian Contribution to the Metatext. (PhD thesis, Monash University Faculty of IT, 2001)

McKemmish, Sue. "Placing Records Continuum Theory and Practice," Archival Science, 1 no. 4 (2001): 333-59

McKemmish, Sue. "Yesterday, Today and Tomorrow: A Continuum of Responsibility", in Peter J. Horsman, F. C. J. Ketelaar, and Theo H. P. M. Thomassen, eds. Naar een Nieuw Paradigma in de Archivistiek ('s-Gravenhage: Stichting Archiefpublicaties, 1999), 195210 http://arrow.monash.edu.au/vital/access/services/Download/monash:155404/ DOC

McKemmish Sue. "Evidence of me ...”, Archives and Manuscripts, 24 no.1 (May 1996): 2845 http://arrow.monash.edu.au/vital/access/services/Download/monash:155367/DOC

McKemmish, Sue. "Are Records Ever Actual?” in Sue McKemmish and Michael Piggott, eds. The Records Continuum: Ian Maclean and Australian Archives first fifty years (Clayton: Ancora Press in association with Australian Archives, 1994): 187-203 http://arrow.monash.edu.au/vital/access/services/Download/monash:155356/DOC

McKemmish, Sue, Glenda Acland, Nigel Ward and Barbara Reed, "Describing Records in Context in the Continuum: The Australian Recordkeeping Metadata Schema". Archivaria 48 (1999): 3-43 http://arrow.monash.edu.au/vital/access/services/ Download/monash:155359/DOC

McKemmish, Sue, Shannon Faulkhead and Lynette Russell. "Dis-trust in the Archive: Reconciling Records”, Archival Science 11, no.3 (2011): 211-239

McKemmish, Sue and Anne J. Gilliland. "Archival and Recordkeeping Research: Past, Present and Future," in Research Methods: Information Management, Systems, and Contexts, Kirsty Williamson and Graeme Johanson, eds. (Prahran, Vic: Tilde University Press, 2012), 80-112

McKemmish, Sue, Anne Gilliland-Swetland, and Eric Ketelaar. "Communities of Memory': Pluralising Archival Research and Education Agendas," Archives and Manuscripts 33 no. 2 (Nov 2005): 146-175

McKemmish, Sue, Livia Iacovino, Eric Ketelaar, Melissa Castan and Lynette Russell. Resetting Relationships: Archives and Indigenous Human Rights in Australia, Archives and Manuscripts, 39 no. 1 (May 2011): 107-144

McKemmish, Sue and Michael Piggott. "Toward the Archival Multiverse: Challenging the Binary Opposition of the Personal and Corporate Archive in Modern Archival Theory and Practice," Archivaria 76 (2013): 111-144

McKemmish, Sue and Michael Piggott, eds. The Records Continuum; Australian Archives first fifty years (Melbourne: Ancora Press, 1994)

McKemmish, Sue, Michael Piggott, Barbara Reed and Frank Upward, eds. Archives: Recordkeeping in Society (Wagga Wagga: Centre for Information Studies, Charles Sturt University, 2005)

McKemmish, Sue and Frank Upward, eds. Archival Documents: Providing Accountability Through Recordkeeping (Melbourne: Ancora Press, 1993) 


\section{Chapter 4}

McKemmish, Sue, Franklyn Herbert Upward, and Barbara Reed. "The Records Continuum Model," in Marcia J. Bates and Mary Niles-Maack, eds. Encyclopedia of Library and Information Sciences, Third Edition (New York: Taylor and Francis, 2009): 4447-59

Maclean, Ian. "Australian Experience in Record and Archives Management" (1959) and "An Analysis of Jenkinson's 'Manual of Archive Administration' in the Light of Australian Experience" (1962), both reproduced in Peter Biskup, ed. Debates and Discourses: Selected Australian Writings on Archival Theory, 1951-1990 (Canberra: Australian Society of Archivists, 1995), 30-52 and 53-78 respectively

Nesmith, Tom. "Still Fuzzy, But More Accurate: Some Thoughts on the 'Ghosts' of Archival Theory," Archivaria 47 (1997): 136-150

New York, State Archives and Records Administration et al. Strategic Plan for Managing and Preserving Electronic Records in New York State Government: Final Report of the Special Media Records Project (Albany, New York: SARA, 1988)

New York, State Archives and Records Administration. Status Report and Evaluation (Albany New York: SARA, 1992)

Ogleby, C.L. "Terra Nullius, the High Court and Surveyors", in The Australian Surveyor, 38 no.3 (1993): 171-89

O'Shea, Greg, and David Roberts. "Living in a Digital World: Recognizing the Electronic and Poscustodial Realities", Archives and Manuscripts 24 no.2 (Nov 1996): 286-311

Piggott, Michael. Archives and Societal Provenance: Australian Essays (Oxford: Chandos Publishing, 2012)

Piggott, Michael. "On first looking into Loewe's papers," University of Melbourne Collections 3 (Dec 2008): 18-23

Piggott, Michael. "Grainger the Autoarchivist," in David Pear, ed., Facing Percy Grainger (Canberra: National Library of Australia, 2006), 39-45

Piggott, Michael. “Towards a History of Australian Diary Keeping”, Archivaria 60 (Fall 2005): 145-166

Piggott, Michael. A Guide to the Personal Family and Official Papers of C E W Bean (Australian War Memorial, Canberra, 1983)

Powell, Graeme. "The Collecting of Personal and Private Papers in Australia", Archives and Manuscripts, 24, no.1 (May 1996): 62-64

Reed, Barbara. "Records," in Sue McKemmish, Michael Piggott, Barbara Reed and Frank Upward, eds. Archives: Recordkeeping in Society (Wagga Wagga: Centre for Information Studies, Charles Sturt University, 2005), 101-130

Reed, Barbara. "Electronic Records Management in Transition", Archives and Manuscripts 22 no.1 (May 1994): 164-71

Roberts, David. "Defining Electronic Records, Documents and Data", Archives and Manuscripts 22 no.1 (May 1994): 14-27

Schellenberg, T.R. Modern Archives: Principles and Techniques (Chicago: University of Chicago Press, 1956)

Standards Australia. AS4390: Records Management, Standards Australia, 1996.

Stoler, Ann Laura. "Colonial Archives and the Arts of Governance", Archival Science 2 (2002): 87-109

Stuckey, Steve. "Keepers of the Flame? The Custodial Role of Australian Archives - Its History and Its Future", in Sue McKemmish and Michael Piggott, eds. The Records Continuum; Australian Archives first fifty years (Ancora Press, Melbourne, 1994), 35-48

Upward, Frank. "The Records Continuum," in Sue McKemmish, Michael Piggott, Barbara Reed and Frank Upward, eds. Archives: Recordkeeping in Society (Centre for Information Studies, Charles Sturt University: Wagga Wagga, Australia, 2005): 197-222 
Upward, Frank. "Modelling the Continuum as Paradigm Shift in Archiving and Recordkeeping Processes, and Beyond: A Personal Reflection”, Records Management Journal 10 no. 3 (December 2000): 115-39 http://arrow.monash.edu.au/vital/access/ services/Download/monash:155375/DOC

Upward, Frank. "Structuring the Records Continuum: Part Two Structuration Theory and Recordkeeping”, Archives and Manuscripts 25 no.1 (May 1997): 10-35 http://arrow. monash.edu.au/vital/access/services/Download/monash:155387/DOC; "Structuring the Records Continuum: Part One”, Archives and Manuscripts 24 no.2 (Nov 1996): 268-85 http://arrow.monash.edu.au/vital/access/services/Download/monash:155386/ DOC

Upward, Frank. "In Search of the Continuum: Ian Maclean's 'Australian Experience' Essays on Recordkeeping”, in Sue McKemmish and Michael Piggott, eds. The Records Continuum: Ian Maclean and Australian Archives First Fifty Years (Melbourne: Ancora Press, 1994), 110-30 http://arrow.monash.edu.au/vital/access/services/Download/ monash:155371/DOC

Upward, Frank, and Sue McKemmish. "In Search of the Lost Tiger, by Way of SainteBeuve: Re-constructing the Possibilities in 'Evidence of Me'," Archives and Manuscripts, 29 no.1 (May 2001): 22-42 http://arrow.monash.edu.au/vital/access/ services/Download/monash:155353/DOC

Upward, Frank and Sue McKemmish. "Somewhere Beyond Custody," Archives and Manuscripts 22 no.1 (May 1994): 138-49 http://arrow.monash.edu.au/vital/access/ services/Download/monash:155384/DOC

Upward, Franklyn Herbert, Sue McKemmish and Barbara Reed. "Archivists and Changing Social and Information Spaces: A Continuum Approach to Recordkeeping and Archiving in Online Cultures," Archivaria 72 (Fall 2011): 197-237 
Chapter 5

\title{
DECIPHERING AND INTERPRETING AN ARCHIVAL FONDS AND ITS PARTS
}

\author{
A Comparative Analysis of Textual Criticism \\ and the Theory of Archival Arrangement
}

\author{
Heather MacNeil
}

\begin{abstract}
This chapter examines the conceptual and methodological alliances between the Anglo-American tradition of textual criticism and the European theory of archival arrangement. It argues that the textual critic's efforts to restore a literary text as closely as possible to its original, "ideal" form mirrors the archivist's efforts to preserve the original order of a creator's fonds - meaning the final order in which the records were actively maintained by that creator. Similarly, the immateriality of the literary work as conceived by textual critics shares some affinity with the abstract dimension of the fonds articulated by traditional archival theorists. A comparative analysis of textual criticism and archival arrangement invites us to see the theory of archival arrangement in a different light, throwing into sharp relief the assumptions underlying it and the socio-historical forces that have shaped those assumptions. It also offers new ways of interpreting and revitalising that theory.
\end{abstract}

\section{Introduction}

In "The Ideology of the Text", the literary critic and Marxist theorist Frederic Jameson describes textuality as "a methodological hypothesis whereby the objects of study of the human sciences are considered to constitute so many texts that we decipher and interpret, as distinguished from older views of 
these objects as realities or existents or substances that we in one way or another attempt to know." For Jameson, the advantages of this description are greatest in non-literary disciplines where it serves three useful purposes:

it suggests that the items under consideration should not be taken as given and that one should consider how they come to be produced, isolated, presented to attention;

it marks the meaning of these objects as a problem that needs to be explored; and

it posits that the analyst's methods need to be considered, not just prior to the inquiry to decide what steps will be carried out, but in the process of treating the objects of study themselves. ${ }^{2}$

In this chapter I will decipher and interpret an archival fonds and its parts by means of a comparative analysis of textual criticism and the theory of archival arrangement. For my purposes, theory of archival arrangement refers specifically to the traditional European theory of arrangement as constituted by the principles of respect des fonds and respect for original order; while textual criticism refers to "the reconstruction of an author's intended text and/or the production of a critical edition displaying this intention or some other version of the text."3

Both modern textual criticism ${ }^{4}$ and the theory of arrangement have their roots in the historical discipline of philology, an offshoot of Renaissance

1 Fredric Jameson, "The Ideology of the Text," in The Ideologies of Theory: Essays 1971-1986 (Minneapolis: University of Minnesota Press, 1989), 18.

2 Jonathan Culler [paraphrasing Jameson, 18-19], “Text; Its Vicissitudes,” in The Literary in Theory, eds. Mieke Bal and Hent de Vries (Stanford, Calif.: Stanford University Press, 2007), 104.

3 D.C. Greetham, Textual Scholarship: An Introduction (New York: Garland, 1994), 8. Textual criticism situates itself within the general field of textual scholarship, which Greetham defines as "the general term for all the activities associated with the discovery, description, transcription, editing, glossing, annotating, and commenting upon texts." Greetham, Textual Scholarship, 1.

4 Of course, a tradition of textual scholarship can be traced back to the ancient world. Greetham finds evidence of textual scholarship in the western world in the Athenian attempts in the sixth century BCE "to arrest the decline of the Homeric texts" and by the formation in the third century BCE "of the two rival scholarly libraries in Alexandria and Pergamum." See D.C. Greetham, "Textual Scholarship," in Introduction to Scholarship in Modern Languages and Literatures, 2nd ed., ed. Joseph Gibaldi (New York: Modern Language Association, 1992), 105-106. It is generally agreed, however, that modern textual scholarship began in the Renaissance, "when scholars rediscovered numerous texts from the Roman and Greek past; attempted to edit them; and, beginning in the late 
humanism. Philology focused scholarly attention on the restoration of the past through the language, form and historical context of texts and, according to Donald Kelley, "established a new logos upon the assumption that language reproduced, if it did not actually create, the configurations of reality." In the seventeenth century, textual criticism and diplomatics - which would later form part of the foundation of archival science ${ }^{6}-$ constituted different branches of philological criticism. The former focused on classical and biblical texts and concerned itself with "the study of the text and the editorial restoration of authorial intentions"7 while the latter focused on medieval legal documents and concerned itself with establishing "certain and accurate terms and rules by which authentic [medieval] instruments [could] be distinguished from spurious, and certain and genuine ones from uncertain and suspect ones." Both textual criticism and diplomatics were founded on "a mistrust of texts," and both invoked the language of evidence and proof in describing their objects of analysis: documents became "witnesses" who might be "sincere" or "untrustworthy", and who might offer "testimony" as to the "probity" of a text's transmission. ${ }^{10}$

fifteenth century, made them widely available internationally through a flood of printed editions that used the newly invented technology of movable type." Leah S. Marcus, "Textual Scholarship," in Introduction to Scholarship in Modern Languages and Literatures, $3^{\text {rd }}$ ed., ed. David G. Nicholls (New York: Modern Language Association, 2007), 144.

5 Donald R. Kelley, Foundations of Modern Historical Scholarship: Language, Law and History in the French Renaissance (New York: Columbia University Press, 1970), 24. See also Geert Lernout, "The Angel of Philology," in Editing the Nation's Memory: Textual Scholarship and Nation-Building in Nineteenth-Century Europe, ed. Dirk Van Hulle and Joep Leerssen (New York: Rodolphi, 2008), 46-61.

6 For a detailed description see Heather MacNeil, Trusting Records (Dordrecht: Kluwer, 2000), 1-31.

7 Greetham, Textual Scholarship, 314.

8 De re diplomatica (Paris, 1681), 1, quoted by C.R. Cheney, The Papacy and England 12th14th Centuries (London: Variorum Reprints, 1982), 8.

9 The phrase comes from the textual critic Eugène Vinavar who states "[ $t]$ he term 'textual criticism' implies a mistrust of texts. It presupposes that in any copied text errors are inevitable and that the critic's main function is to correct them." See Eugène Vinavar, "Principles of Textual Emendation," in Studies in French Language and Mediaeval Literature, presented to Professor Mildred K. Pope, by pupils, colleagues, and friends (Freeport, NY: Books for Libraries Press, [1939], rpt. 1969), 352. The historian Marc Bloch makes a similar statement about diplomatics. According to Bloch, the publication of Mabillon's treatise on diplomatics, De re diplomatica, in 1681 marked "the decisive moment in the history of the critical method" because from that day forward, methodological doubt became the starting point of historical inquiry. See Marc Bloch, The Historian's Craft, trans. Peter Putnam (New York: Alfred A. Knopf, 1963), 81.

10 David Greetham, "Facts, Truefacts, Factoids; or, Why Are They Still Saying Those Things about Epistemology?" in The Pleasures of Contamination: Evidence, Text, and Voice in Textual Studies (Bloomington: Indiana University Press, 2010), 97. 
Some of these conceptual and methodological alliances endure in the Anglo-American tradition of textual criticism, as embodied in the eclectic theory of critical editions, and the theory of archival arrangement. The eclectic critic's efforts to restore a literary text as closely as possible to its original, "ideal" form mirrors the archivist's efforts to preserve the original order of a creator's fonds - meaning the final order in which the records were actively maintained by that creator. In both fields preservation and restoration are closely tied to the identification and stabilisation of authorial intentions. In similar fashion, the immateriality of the literary work as conceived by eclectic critics shares some affinity with the abstract dimension of the fonds articulated by traditional archival theorists.

It is these conceptual and methodological alliances that provide the focus for the comparative analysis which is organised along the following parallel lines: the evolution of Anglo-American textual criticism and the evolution of European principles of arrangement; the eclectic theory underpinning critical editions and the theory of archival arrangement; the re-thinking of the eclectic theory of critical editions and the rethinking of the theory of archival arrangement. Juxtaposing and historicising the concepts and methods that have shaped textual criticism and archival arrangement allows us to see the theory of archival arrangement from a different perspective and throws into sharp relief the assumptions underlying it and the sociohistorical forces that have shaped those assumptions. It also opens the theory up to new ways of interpreting and revitalising it.

\section{The Evolution of Anglo-American Textual Criticism}

The rise of philology in general and textual criticism in particular between the seventeenth and nineteenth centuries was attributable, at least in part, to "a slow but accelerating process of textual availability and anamnesis."11 The French Revolution and Napoleonic conquests played a key role in this acceleration. As Joep Leerssen explains, with "the break-up of the ancien régime and its reconstitution, following the bulldozings of Napoleon, into a system of national states", private and monastic manuscript collections were "siphoned off into the institutional, public sphere." ${ }^{2}$ In France alone,

11 Joep Leerssen, "Introduction: Philology and the European Construction of National Literatures," in Editing the Nation's Memory: Textual Scholarship and Nation-Building in Nineteenth-Century Europe, ed. Dirk Van Hulle and Joep Leerssen (New York: Rodolphi, 2008), 18.

12 Joep Leerssen, "Introduction”, 22, 20. 
between 1789 and 1793, the revolutionary government acquired, either through "confiscation" or "absorption," the major book and document collections of the ancien régime. ${ }^{13}$ The massive and chaotic accumulations of books and manuscripts piling up in newly nationalised libraries and archives underscored the need for textual critics "to work out a valid method of sorting the grain from the chaff." ${ }^{14}$

Karl Lachmann's "stemmatic theory of rescension" in the mid-nineteenth century provided that method and transformed the editing of classical texts. ${ }^{15}$ Because no autograph manuscripts of the classical authors existed, the classical texts that had survived through the centuries lay at an unknown number of removes from the originals. ${ }^{16}$ Such texts, Lachmann asserted, "needed to be studied (and recovered) through analysis of the historical accretions that altered them over time." ${ }^{17}$ According to the theory of stemmatics, the recovery of the original text or, more precisely, the archetype ${ }^{18}$ is a two-stage process:

The first stage is rescension (rescensio). The object of rescension is to reconstruct from the evidence of the surviving manuscripts the earliest recoverable form of the text that lies behind them.... When the most primitive state of the text that is recoverable from the manuscripts has been reconstructed, the second main stage of the critical process begins. The transmitted text must be examined and the critic must decide whether it is authentic or not (examinatio); if not, his duty is to emend

13 Lara Jennifer Moore, Restoring Order: The Ecole des Chartes and the Organization of Archives and Libraries in France, 1820-1870 (Duluth, Minn: Litwin, 2001), 4.

14 L.D. Reynolds and N.G. Wilson, Scribes and Scholars: A Guide to the Transmission of Greek and Latin Literature, $3^{\text {rd }}$ ed. (Oxford: Clarendon Press, 1991), 209. This description of the textual critic's task is very similar to Olivier Guyotjeannin's description of the work of the diplomatist, which consisted of "revealing falsifications, separating the wheat from the chaff, and also the straw (the formulary) from the grain (indisputable facts.)" Olivier Guyotjeannin, "The Expansion of Diplomatics as a Discipline,"” American Archivist 59 (Fall 1996): 416.

15 Reynolds and Wilson, Scribes and Scholars, 210. As the authors observe, although it is usually associated with Karl Lachmann, many scholars contributed to the elaboration and refinement of stemmatic theory.

16 Joep Leerssen, "Introduction", 207.

17 Marcus, "Textual Scholarship," 145.

18 The archetype is "the earliest stage of transmission recoverable by recension on the evidence of the surviving documents." D.C. Greetham, foreword to A Critique of Modern Textual Criticism, by Jerome J. McGann (Charlottesville: University Press of Virginia 1992), xiv. 
it (emendatio), if this can be done with a reasonable degree of certainty, or to isolate the corruption. ${ }^{19}$

According to Jerome McGann, "the attempt by classical philologists to recover, or approximate by historical reconstruction, the lost original works of ancient authors produced a 'theory of the critical edition." 20 The systematic collation of all the relevant texts associated with a given work and development of a textual stemma enabled editors to choose a copy-text, i.e., an "early text of a work which the editor selected as the basis of his own.".1 That copy-text would then be emended "to bring the critical text into as close an approximation as possible with the author's no longer extant work."22 The critical text was accompanied by a critical apparatus which displayed the "history" of the text, i.e., a record of the textual variants that had emerged from the collation of the documents. ${ }^{23}$

In the second half of the nineteenth century, the genealogical methods of classical historical philology that had been applied primarily to the study of biblical and classical texts, were increasingly applied to the editing of early modern literatures. By the middle of the twentieth century, however, it was clear some adjustment to these methods was required to account for differences in the way classical scribal texts were produced and transmitted and the way early modern printed texts were published and disseminated. The problems associated with editing Elizabethan texts in particular led the English editor, bibliographer and textual critic W. W. Greg to propose an "English theory" of copy-text. Drawing a distinction between what he called substantives (the actual words, or the "meaning" of a text articulated by its author) and accidentals (the spelling, punctuation and so on, or the "surface features" of a text), Greg contended that:

... the copy-text should govern (generally) in the matter of accidentals, but that the choice between substantive readings belongs to the general theory of textual criticism and lies altogether beyond the narrow principle of the copy-text. Thus it may happen that in a critical edition the text rightly chosen as copy may not by any means be the one that supplies most substantive readings in cases of variation. The failure to

19 Reynolds and Wilson, Scribes and Scholars, 207-208.

20 Jerome J. McGann, A Critique of Modern Textual Criticism (Charlottesville: University Press of Virginia 1992), 23.

21 W.W. Greg, "The Rationale of Copy-Text," Studies in Bibliography 3 (1950-1951): 19.

22 McGann, Critique, 24.

23 McGann, Critique, 24. 
make this distinction and to apply this principle has naturally led to too close and too general a reliance upon the text chosen as basis for an edition, and there has arisen what may be called the tyranny of the copy-text, a tyranny that has, in my opinion, vitiated much of the best editorial work of the past generation. ${ }^{24}$

As Greetham explains,

this suggestion of a 'divided' authority in copy-text (one text for accidentals and possibly several others for imported substantives) then resulted in the production of 'eclectic' editions bearing features from various witnesses, in what became known as the 'text that never was. ${ }^{25}$

Although Greg viewed his proposed rationale simply as a "practical editorial response to the documented conditions of English printing in the sixteenth and seventeenth centuries," ${ }^{26}$ the American bibliographer and textual scholar Fredson Bowers elevated Greg's theory of copy-text to the level of a general principle and applied it to the editing of nineteenth century American literatures. ${ }^{27}$ Bowers also made explicit what had only been implicit in Greg's rationale, i.e., that the goal of a critical edition was to "produce the nearest approximation in every respect of the author's final intentions. ${ }^{28}$ The "Greg-Bowers School" as it became known exercised a powerful influence on the editing of literary texts and by the third quarter of the twentieth century, it constituted the dominant mode of Anglo-American textual criticism. Although subsequent proponents of the "eclectic" or "authorial" or "intentionalist" school of editing have refined Greg's and Bowers' original formulations, they continue to adhere to its main objective, i.e.,

to produce a reading clear-text whose features [are] a fulfillment of authorial intentions by the selection of authorially sanctioned substantive variants from different states of the text, and whose copy-text [is] selected on the basis of its accidentals being as close as possible to authorial usage..$^{29}$

24 Greg, "Rationale of Copy-Text," 26.

25 Greetham, Textual Scholarship, 333-334.

26 Greetham, Textual Scholarship, 335.

27 Fredson Bowers, "Some Principles for Scholarly Editions of Nineteenth-Century American Authors," Studies in Bibliography 17 (1964): 223-228.

28 Bowers, "Some Principles for Scholarly Editions of Nineteenth-Century American Authors," 227.

29 Greetham, Textual Scholarship, 335. 


\section{The Evolution of European Principles of Archival Arrangement}

By the middle of the nineteenth century, diplomatics had evolved into an auxiliary discipline of history with different branches focusing on systems of dating documents, the study of seals, various types of copies and documentary forms, specific chancery procedures, as well as the criticism of forgeries, which had provided its original focus. Its object of analysis, however, continued to be instrumental documents from the medieval period. For the first generation of archivists charged with making the holdings of archival institutions available to the public, knowledge of diplomatics may have equipped them for the task of identifying, analysing, and describing the individual documents in their custody, tracing their origins, and detecting any forgeries; it did not equip them, however, for the task of arranging those documents into meaningful aggregations - a prerequisite to making them available for use.

The elaboration of principles for the arrangement of archival documents in western European jurisdictions over the course of the nineteenth century was prompted by historical circumstances similar to those that precipitated the elaboration of the stemmatic theory of textual criticism in the same period, i.e., the breakdown of old regimes, the surrender of the documents created by those now defunct regimes to the new regimes and their subsequent deposit in newly nationalised archival repositories. The transfer of massive numbers of registers, bundles, cartons, and loose documents from private to public hands and the consequent need to make them accessible to a broader audience prompted the archivists employed in these new repositories to devise methods of organising them for retrieval purposes. Early efforts at classifying archival holdings by topic, geography, or chronology were increasingly seen as inadequate and even disastrous and so the search began for an alternative approach to organising archival documents for retrieval. Over the course of the nineteenth century, that search resulted in the identification and elaboration of two principles of archival arrangement: respect des fonds and respect for original order. ${ }^{30}$

30 Throughout this section I follow Peter Horsman in treating "respect des fonds" and "respect for original order" as the external and internal dimensions of the overarching principle of provenance in order to maintain a clear focus on the specific characteristics of each of these dimensions. See Peter Horsman, "Taming the Elephant: An Orthodox Approach to the Principle of Provenance," in The Principle of Provenance: Report from the First Stockholm Conference on Archival Theory and the Principle of Provenance 2-3 September 1993 (Sweden: Swedish National Archives, 1994), 51. 
Although the principle of respect des fonds was enunciated and implemented in different ways in a number of European jurisdictions during the early part of the nineteenth century, ${ }^{31}$ it is generally conceded that the French articulation of the principle in a circular issued in 1841 by the Minister of the Interior, Tanneguy Duchâtel, and authored by Natalis De Wailly and others constituted its first full expression. "Circular 14", as it was known, rejected classification by topic and by time period, both of which were features of earlier archival classification schemes in the French royal archives and the departmental archives. Instead, the basic unit of archival classification was to be the fonds, i.e., "all the titles that derive from a body, an establishment, a family, or an individual." ${ }^{32}$ The circular's authors took the view that archival fonds "are not the product of a particular period, much less a particular political regime, but the product of people and institutions; and like people and institutions they develop organically and continuously. The only 'natural' organizational scheme, then, is one reflective of the continuity of people and institutions; classification by fonds." 33 The first detailed articulation of the principle of respect for original order for its part was laid out in 1881 in regulations drafted by Max Lehmann and authorised by Heinrich von Sybel for the arrangement of records held in the Prussian Privy State Archives. The regulations "prescribed 'respect for every original order, for every original designation.”34 The historian Friedrich Meinecke, who began working as an archivist in the State Archives in 1887, declared that,

the idea of arranging the records according to their provenance ... injected ... an incredible amount of vitality and individuality into the entire Archives. For the registry of every single agency ... now became a living organism of its own with its peculiar principle of life. ${ }^{35}$

In 1898, a Dutch archival commission formed by Samuel Muller, Johan Feith and Robert Fruin codified these principles and provided them with "a

31 Peter Horsman, "The Last Dance of the Phoenix, or the De-discovery of the Archival Fonds," Archivaria 54 (Fall 2002): 6; see also Luciana Duranti, "Origin and Development of the Concept of Archival Description,” Archivaria 35 (Spring 1993): 50.

32 Moore, Restoring Order, 118.

33 Moore, Restoring Order, 119.

34 Ernst Posner, "Max Lehmann and the Genesis of the Principle of Provenance," in Archives and the Public Interest: Selected Essays by Ernst Posner, ed. Ken Munden (Chicago: Society of American Archivists, 2006), 37.

35 Posner, "Max Lehmann and the Genesis of the Principle of Provenance," 41-42. 
theoretical justification." ${ }^{36}$ Their Manual for the Arrangement and Description of Archives laid out 100 rules to be followed by archivists when arranging and describing the fond ${ }^{37}$ of a given creator. The understanding of the fonds "as an organic whole" outlined in the second rule of the Manual established the overarching rationale for the rest of the rules:

... an archival collection [i.e., a fonds] comes into being as the result of the activities of an administrative body ... and it is always the reflection of the functions of that body. ... an archival collection is an organic whole, a living organism, which grows, takes shape, and undergoes changes in accordance with fixed rules. If the functions of the body change, the nature of the collection changes likewise. The rules which govern the composition, the arrangement and the formation of a [fonds], therefore, cannot be fixed by the archivist in advance; he can only study the organism and ascertain the rules under which it was formed. Every archival collection has, therefore, as it were, its own personality, its individuality, which the archivist must become acquainted with before he can proceed to its arrangement. ${ }^{38}$

While the authors never used the specific terms "respect des fonds" or "respect for original order," both principles were implicit in the rules and viewed by the authors of the Manual as logical outgrowths of the understanding of the fonds as an organic whole. Rule 8 stipulated that, "the various archival collections placed in a depository must be kept carefully separate"; while Rule 16 stated that, "the system of arrangement must be based on the original organization of the archival collection, which in the main corresponds to the organization of the administrative body that produced it." ${ }^{39}$

By 1910, the year the first International Congress of Archivists and Librarians met in Brussels, the Manual had been translated into German, Italian

36 T.R. Schellenberg, Modern Archives: Principles and Techniques (Chicago: University of Chicago Press, 1956, rpt 1975), 175.

37 The Dutch term is "archief." In the English translation of the Manual the term became "archival collection" but its meaning is synonymous with "fonds." See S. Muller, J.A. Feith, and R. Fruin, "The Arrangement of Archival Documents," Manual for the Arrangement and Description of Archives, 2nd ed., trans. Arthur H. Leavitt, with new introductions by Peter Horsman, Eric Ketelaar, Theo Thomasson and Marjorie Rabe Barritt (Chicago: Society of American Archivists, 2003), 13, fn.1.

38 Muller et al., "The Arrangement of Archival Documents," 19.

39 Muller et al., "The Arrangement of Archival Documents," 33, 52. 
and French and its influence was already considerable. Delegates at the Congress ${ }^{40}$ unanimously endorsed the principle of provenance, defined as:

the method of archive organization by which each archival document has to be brought to the archive [fonds] to which it belongs, and within that archive to the series to which it belonged at the time the archive was still a living organism. ${ }^{41}$

According to Peter Horsman, this definition, which integrated the Dutch interpretation of respect des fonds and respect for original order, was "essentially a condensed summary of the most important sections of the Dutch Manual." The principles were elaborated more fully in the writings of later archival theorists such as Hilary Jenkinson, Eugenio Casanova, and Giorgio Cencetti ${ }^{42}$ among others and, by the middle of the twentieth century, they effectively constituted the European theory of archival arrangement. The principles also provided a substantial part of the foundation on which standards for fonds-based archival description were subsequently built. ${ }^{43}$

40 Plans were made to hold subsequent international meetings but the outbreak of war put an end to these plans. The next International Congress on Archives took place in Paris in 1950 during which the International Council on Archives was organised. Archival delegates attending the 1910 Congress were mainly from Western Europe, although representatives from the American Historical Association were also present. The Public Records Office of Great Britain was invited but declined to send a representative. See Frank B. Evans, "Promoting Archives and Research: A Study in International Cooperation," American Archivist 50 (Winter 1987): 49; Lawrence D. Geller, "Joseph Cuvelier, Belgian Archival Education, and the First International Congress of Archivists, Brussels, 1910," Archivaria 16 (Summer 1983): 26-34; Michael Roper, "The International Role of the Public Record Office," The Records of a Nation: The Public Record Office 1838-1988, The British Record Society 1888-1988, ed. G.H. Martin and Peter Spufford (Woodbridge, UK: Boydell Press and the British Record Society, 1990), 15.

41 Peter Horsman, "The Last Dance of the Phoenix, or the De-discovery of the Archival Fonds," Archivaria 54 (Fall 2002): 10.

42 Hilary Jenkinson, A Manual of Archive Administration (Oxford: Clarendon Press, 1922); Eugenio Casanova, Archivistica (Siena: Stab. arti grafiche Lazzeri, 1928); Giorgio Cencetti, Scritti archivistici (Roma: Il Centro di Ricerca editore, 1970).

43 Such standards include Bureau of Canadian Archivists Planning Committee on Descriptive Standards, Rules for Archival Description July 2008 rev. (Ottawa: Bureau of Canadian Archivists, 1990-); International Council on Archives, ISAD(G): General International Standard Archival Description, $2^{\text {nd }}$ ed. (Paris, France: ICA, 2000); and Society of American Archivists, Describing Archives: A Content Standard (Chicago: Society of American Archivists, 2004). 


\section{The Eclectic Theory Underpinning Critical Editions}

The eclectic theory of critical editions posits that the final intentions of the author, inevitably, are corrupted by transmission: copy editors alter the author's punctuation and spelling; friends and relatives revise typescripts and page proofs; publishers subtract and add material to new editions, with or without the author's permission; authors themselves obsessively correct and revise their texts, sometimes in contradictory ways, sometimes in response to external pressures, and so forth. The task of the textual critic then is to reconstruct, from among the many "corrupt" variants of a literary text that have existed over time, the authentic or "ideal" text, namely, the one that best embodies the final intentions of the author. For eclectic critics, a literary work is physically non-specific, existing in multiple artefacts but not located in any single instantiation; thus, the ideal or intended text is a material artefact that seeks to approximate the abstract work. ${ }^{44}$ Thomas Tanselle maintains that, even if the author's intended text never physically existed prior to its reconstitution in an eclectic edition, the inferred authorial intentions that shape it have as legitimate a claim to historical reality as do the texts that were finally published. As he puts it:

If we grant that authors have intentions and therefore that the intentions of past authors are historical facts, we require no further justification for the attempt to recover those intentions and to reconstruct texts reflecting them, whatever our chances of success may be. ${ }^{45}$

His comments emphasise that for textual critics working in the tradition of eclectic editions, the ideal or intended text is the one that reveals the single, creative mind that provided the impetus for the literary work. From their perspective, restoring the text to an imagined historical moment before the onset of corruption is the way to reveal that mind.

Once the text has been decided upon, it is then contextualised through the preparation of a "critical' or "editorial" apparatus. The apparatus contains notes and commentary on the text, the history of its variants, as well as an essay outlining "the editorial principles followed in the edition, the textual history of the individual work, and any special problems emerging from the application of those principles to that particular historical

44 G. Thomas Tanselle, A Rationale of Textual Criticism (Philadelphia: University of Pennsylvania Press, 1989), 76.

45 Tanselle, Rationale, 25-30. 
situation." ${ }^{\prime 46}$ The edition might also contain a historical essay describing "the background, composition, publication, and reception of the work. ${ }^{37} \mathrm{In}$ eclectic critical editions, the apparatus is typically relegated to appendices in order to produce a "clear reading text," i.e., a text uninterrupted by editorial intrusions. Such practice is favoured, Tanselle argues, because:

an editor's primary responsibility is to establish a text; whether his goal is to reconstruct that form of the text which represents the author's final intention or some other form of the text, his essential task is to produce a reliable text according to some set of principles. Relegating all editorial matter to an appendix and allowing the text to stand by itself serves to emphasize the primacy of the text and permits the reader to confront the literary work without the distraction of editorial comment and to read the work with ease. ${ }^{48}$

In clear text editions, then, "all sign of editorial handiwork [are] removed so that the reader is confronted with 'the text itself,' shorn of both historical accretions and of the evidence of the editorial intervention that had recreated this text." ${ }^{\prime 9}$

The eclectic theory of critical editions has been linked to the New Bibliography movement that began in the first half of the twentieth century and which spawned two new types of textual scholarship: analytical bibliography, "the study of the technical history of the printed book" and descriptive bibliography, "the formulaic listing of the technical attributes of each 'ideal copy' of a book." According to Greetham, "this combination of technical sophistication and critical reconstruction of authorial intention gave eclectic editing an understandably forcible role in early and midtwentieth century textual scholarship." ${ }^{50}$ Textual scholars have also noted a methodological alliance between and among eclectic editing, the New Bibliography, and the roughly contemporaneous literary movement of New Criticism. Both the New Bibliography and New Criticism, Greetham explains:

46 G. Thomas Tanselle, "Some Principles for Editorial Apparatus," Studies in Bibliography 25 (1972): 55.

47 Tanselle, "Some Principles for Editorial Apparatus," 56.

48 Tanselle, "Some Principles for Editorial Apparatus," 45.

49 David Greetham, "What is Textual Scholarship?" in A Companion to the History of the Book, ed. Simon Eliot and Jonathan Rose (West Sussex: Wiley-Blackwell, 2009), 29.

50 Greetham, "Textual Scholarship," 110-111. For a more detailed examination of the new bibliography see Laurie Maguire, "The Rise of the New Bibliography," in Shakespearean Suspect Texts: The 'Bad' Quartos and their Contexts (Cambridge, UK: Cambridge University Press, 1996), 21-72. 
required an organicist, artefactual view of literature and a single unitary consciousness for their critical analysis. Both concentrated on an idealized, closed text and thus both rejected the discontinuous operations of literary history, despite New Biblography's apparent reliance on the evidence of history. Both employed positivist methods in a systematic attempt to define their fields comprehensively. And both sought access to a core 'text itself' and sought to formulate strict and objective procedures for textual analysis through the imposition of this technical method ... ${ }^{51}$

Though they differed in a number of important respects, ${ }^{52}$ eclectic editing, the New Bibliography, and New Criticism shared a view of the literary work as a self-contained, "well-wrought urn" and, consequently, tended to idealise and de-historicise literary texts.

\section{The Theory of Archival Arrangement}

Like the eclectic theory of critical editions, the theory of archival arrangement as it is understood today is posited on a presumed affinity between abstract wholes and material parts. Muller, Feith and Fruin compared the work of the archivist in reconstructing the original order of a body of records to that of the paleontologist who seeks to recreate a living organism on the basis of fossil remains; subsequent archival theorists have compared it to the work of the archaeologist who attempts to recreate a civilisation on the basis of stone remains. ${ }^{53}$ The concept of a fonds as the totality of a creator's records communicates a sense of wholeness to something that physically exists only in fragments. The distinction between the abstract fonds and its material manifestation in archival holdings is analogous to the distinction Tanselle and other eclectic editors make between a literary work and its embodiment in material texts. Like a literary work, the fonds is physically non-specific, existing in multiple aggregations and physical places but not (in its purest or "ideal" form) locatable in any single aggregation, place, or even time. The records preserved in archival institutions thus function as a

51 D.C. Greetham, Theories of the Text (Oxford: Oxford University Press, 1999), 141.

52 New Criticism, for example, rejected authorial intention as a relevant consideration in the critical interpretation of a literary work. The emblematic statement of the New Critical view of authorial intention is William K. Wimsatt and Monroe Beardsley, "The Intentional Fallacy," Serwanee Review 54 (1946): 468-488.

53 See, for example, Elio Lodolini, “The War of Independence of Archivists," Archivaria 28 (Summer 1989): 36-47. 
kind of synecdoche in which the part (the material remains of the fonds) stands in for the whole (the entirety of the fonds).

The principle of original order is posited specifically on a presumed affinity between records and their creator in which the arrangement of the records acts as a kind of mirror, or personification, of the entity that produced them. The Italian archival theorist Giorgio Cencetti, for example, believed that an archival fonds, "is the creator itself, in the sense that the original order of the archive, the order given to the archive by its creator, is the manifestation of the administrative structure, the history and, in some way, the very "essence" of the records creator." 54 That presumed affinity suggests an implicit connection with final authorial intentions. ${ }^{55}$ If we understand intention to be synonymous with a creator's purpose, aim, or design, then the records of that creator constitute the tangible remains of its intentions and the original order of the records, the final shape of those remains. Preserving that final shape is thus a means of preserving the creator's final intentions.

Muller, Feith and Fruin acknowledged that, like any living organism, a body of records will change its state many times while under the control of its creator. At the same time, however, they insisted that the archivist could restore "only one particular state of the reconstructed organism," suggesting that the archivists' reconstruction of the creator's original order necessarily entailed a certain degree of normalisation and idealisation. The Dutch trio also recognised that any aggregation of records that survives over time may be subjected to a range of interventions by subsequent custodial authorities. In such cases, they insisted, the task of the archivist was to undo the damage wrought by previous custodians and restore the records to the order given them by their creator. The acts of reconstruction and normalisation involved in the archival arrangement of a fonds bear more than a passing resemblance to the reconstruction and normalisation involved in the preparation of an eclectic edition.

54 Maurizio Savoja and Stefano Vitali, "Authority Control for Creators in Italy: Theory and Practice," Journal of Archival Organization 5 (2007): 123. The personification of the fonds appears in the writing of another Italian archival theorist, Letterio Briguglio, "who defined the archive as an 'historic person'. The concept of person, that is to say an individual to be respected, borrowed from Kant, adds a moral duty to the respect of original order, that becomes the respect for a living being, of an historical organism.” Donato Tamblé, "Archival Theory in Italy Today," Archival Science 1 (2001): 88-89.

55 Authorial is used here in the figurative sense of the originator or creator of something.

56 Muller, Feith and Fruin, 71. 
Reconstruction and normalisation are codified in criteria for determining the boundaries of a fonds - based on considerations of the relative autonomy and functional unity of the fonds' creator $^{57}$ - and the identification of the levels of arrangement - fonds, series, file, item - that are thought to embody the "original order" of the fonds and its parts. The results are then presented in an archival description. In a standardised fonds-based description, the records creator is named in the title and provides the focal point for the administrative/biographical sketch; the documentary aggregations are identified in the scope and content, and part-to-whole relationships are represented through the description's linear and hierarchical multi-level structure. The physical remains of a fonds held within a given archival institution are thus frozen in time ${ }^{58}$ and re-presented as a self-contained totality, i.e., a stabilised text.

In the same way the eclectic theory of critical editions wears the influence of contemporaneous literary movements, the theory of archival arrangement elaborated between the mid-nineteenth and mid-twentieth century carries the influence of the historical thinking of that period. ${ }^{59}$ The "organic" and evolutionary conception of the fonds in the French articulation of respect des fonds, for example, aligned with the notion of history itself as organic, evolutionary, and progressive and was animated, in part, by the historical vision of France's political regime of the time, the July Monarchy. According to Moore, the July Monarchy, which governed France from 1830 to 1848 , was committed to an organicist conception of history and continually compared the French nation to "a living being that had slowly grown into full maturity." Viewed from this angle, the principle of respect des fonds was simply "an extension of [the July Monarchy's] historical vision into the realm of classification. The newly 'restored' archival fonds would offer documentary proof of the slow and inexorable progress of the French 'nation' from the struggles of the medieval period to the triumph of the

57 These are summarised in Michel Duchein, "Theoretical Principles and Practical Problems of Respect des fonds in Archival Science”, Archivaria 16 (Summer 1983): 64-82.

58 Sue McKemmish, "Are Records Ever Actual?" in The Records Continuum: Ian Maclean and Australian Archives First Fifty Years, ed. Sue McKemmish and Michael Piggott (Clayton: Ancora, 1994), 189.

59 This is not surprising given the close relationship between historians and archivists during this time period. Many of the most prominent historians - Michelet and Langlois in France, Galbraith and Hall in England, Meinecke and Lehmann in Germany - spent some time working as archivists over the course of their careers. For an examination of how that relationship has changed since the nineteenth century, see Terry Cook, "The Archive(s) Is a Foreign Country: Historians, Archivists, and the Changing Archival Landscape," American Archivist 74 (Fall/Winter 2011): 600-632. 
July regime." ${ }^{60}$ The principle of respect for original order, for its part, was underpinned by a historiographical ideal pervasive in the late nineteenth century, i.e., a belief in the possibility of entering into direct contact with a past reality. ${ }^{1}$ Such belief manifested itself most palpably in the aspiration of the scientific historian Leopold Ranke to show "how [the past] essentially was," by which, according to Richard Evans, "Ranke meant that he wanted to penetrate by a kind of intuitive understanding to the inner being of the past." ${ }^{\prime 2}$ The articulation of the principle of original order by Cencetti in the post Second World War period was influenced specifically by the thinking of the idealist historian and philosopher Benedetto Croce who believed that the past is re-created in the present through an act of imagination on the part of the historian. ${ }^{63}$

\section{Rethinking the Eclectic Theory of Critical Editions}

Since the 1980s, the eclectic theory of critical editions in general, and the intertwined concepts of the work and final authorial intentions in particular, have been interrogated and found wanting by a number of textual scholars, including Jerome McGann who argues that the eclectic theory of critical editions "hypothesizes two related phenomena that do not exist: i.e., an autonomous author, and an ideal text." ${ }^{64}$ The ideology underlying the theory of final intentions, he contends, is a Romantic one that imagines a solitary, autonomous author "creating a work in an 'originary moment' of

60 Moore, Restoring Order, 122.

61 Jo Tollebeek, “'Turn'd to Dust and Tears': Revisiting the Archive," review of Dust: The Archive and Cultural History, by Carolyn Steedman, History and Theory 43 (2004): 244. See also Tollebeek, "Seeing the Past with the Mind's Eye: The Consecration of the Romantic Historian," Clio 29 (Winter 2000): 167-191; and Ann Rigney, Imperfect Histories: The Elusive Past and the Legacy of Romantic Historicism (Ithaca and London: Cornell University Press, 2001).

62 Richard J. Evans, In Defense of History (New York: W.W. Norton, 1999), 14. Evans maintains that "how it essentially was" is a more accurate English translation of Ranke's famous phrase "wie es eigentlich gewesen" than "what actually happened" which is the common translation. This point has also been made by Peter Novick in That Noble Dream: The "Objectivity Question" and the American Historical Profession (Cambridge: Cambridge University Press, 1988), 28.

63 "Contemporary history comes into being immediately after the act which is being accomplished, as consciousness of that act. ...The condition of its existence is that the deed of which the history is told must vibrate in the soul of the historian." Benedetto Croce, Theory and History of Historiography, trans. Douglas Ainslie (London: G.C. Harrap, 1921), 11-12. The connection between Cencetti and Croce is made by Savoja and Vitali in "Authority Control for Creators in Italy," 123.

64 McGann, Critique, 56. 
composition." ${ }^{65}$ It is, at the same time Platonic, inasmuch as it "locate[s] the ultimate reality of the literary text outside its material embodiment, usually in the mind of the author. ${ }^{166} \mathrm{McGann}$ points to numerous examples of authors whose work is inextricably linked with a variety of collaborators editors, publishers, friends and relations - making it impossible to determine the authors' final intentions or to separate their intentions from those of their collaborators. ${ }^{67}$ Moreover, by focusing all their attention on revealing the mind of the author who created the literary work, the procedures of eclectic editing isolate the authorial text from its subsequent social distribution, that is, from the institutions that transmitted that text to the public; in so doing, they deprive the literary work of much of its meaning. To counteract that tendency, McGann and others argue for an alternative "social" theory of textual criticism in which the entire history of a literary work - from composition to reception and beyond - falls within the scope of textual scholarship. ${ }^{68}$

Building on McGann, the Renaissance textual scholar Leah Marcus proposes the term "new philology" to describe the shift in editorial attention from idealised to historicised literary texts. For Marcus, the term is apt because "it represents a return to some of the historical concerns of nineteenth-century philologists, although ... [it] may arrive at very different conclusions than the old." ${ }^{69}$ The "dominant textual paradigm" of this new philology is a "network," within which, she explains, "the text loses its privileged separateness and is conceptualized as part of a much wider vectoring of forces and objects"70 including the silent shaping hand of editors themselves. Marcus looks closely at some of the ways modern textual critics have emended Renaissance literary texts in order to illustrate this "wider vectoring of forces and objects" and to show, specifically, how the "valorization of textual stability" at the heart of Anglo-American textual criticism has acted in the past to obscure a given text's ideological assumptions. ${ }^{71}$ She cites, as one example, the way in which

65 McGann, Critique, xiii. McGann's association of modern textual criticism with Romantic ideology has not gone unchallenged. See, for example, Tilottama Rajan, "Is there a Romantic Ideology? Some Thoughts on Schleiermacher's Hermeneutic and Textual Criticism," in Text: Transactions of the Society for Textual Scholarship 4, ed. D.C. Greetham and W. Speed Hill (New York: AMS Press, 1988), 59-77.

66 Leah S. Marcus, Unediting the Renaissance: Shakespeare, Marlow, Milton (London and New York: Routledge, 1996), 29-30.

67 For McGann, the prototypical example is Byron. See Critique, 51-54.

68 McGann, Critique, 8-9.

69 Marcus, Unediting the Renaissance, 22-23.

70 Marcus, Unediting the Renaissance, 22-23.

71 Marcus, “Textual Scholarship," 148, 151. 
editors of Shakespeare's The Tempest from the middle of the nineteenth century on have regularly emended Shakespeare's description of Sycorax, the Algerian witch who is Caliban's mother, as a "blue-eyed hag" to mean "bleary-eyed" or "blue around the eyes" notwithstanding the fact that the Renaissance range of meanings for "blue-eyed" was considerably broader than these emendations suggest. As Marcus explains:

In nineteenth-century literature and culture, blue eyes were commonly associated with beauty, innocence, and transcendence, as in Keats' "beauteous woman's large blue eyes" ... or Shelley's eyes "like the deep, blue, boundless heaven,” ... or Arnold's “eyes, so blue, so kind.” Blue eyes were also associated, at a time of expanding colonization and racial consciousness, with British culture and national heritage, with the "white man's burden," and with the superior moral elevation attained by English-speaking peoples. To imagine Sycorax as "blue-eyed" in any positive sense of the term was to violate deeply engrained cultural assumptions. ... Most twentieth century editions have frozen earlier editorial speculation into dogma. ... The witch cannot have blue eyes, because the cultural image of blue eyes is overwhelming positive and Sycorax has to be understood as negative. ${ }^{72}$

In both these examples, Marcus asserts, "editorial constructions of textual stability have aligned with nineteenth- and early-twentieth-century colonial assumptions, which is to say that textual critics, like everyone else, are creatures of their times." ${ }^{73}$ By exposing the complicity between editorial efforts to 'stabilize the text' and colonial assumptions "about what was acceptably English as opposed to unacceptably alien and threatening," Marcus makes a compelling case for the need for textual critics to "destabilize' literary texts by declaring "a preference for variability over fixity of meaning" 74 in the editing of those texts. In making that case she reinforces McGann's call for a social theory of textual criticism.

The theory of eclectic editing was influenced by the New Bibliography and New Criticism. The new textual scholarship has also been influenced, directly or indirectly, by contemporaneous literary theories. McGann's social textual criticism, for example, has been described by John Sutherland as "an unattributed gloss of [Marxist literary theorist] Pierre Macherey's edict

72 Marcus, Unediting the Renaissance, 8-10.

73 Marcus, “Textual Scholarship”, 152.

74 Marcus, Unediting the Renaissance, 16-17. 
... [that] the work is not created by an intention (objective or subjective); it is produced under determinate conditions." ${ }^{75}$ Greetham sees it as an exemplification of Stanley Fish's "historical continuum of interpretive communities [in which] there is a shifting of focus from the nature of auctorial consciousness through the nature of the text to the nature of the reading and reconstruction of books." 76

Considered schematically, the preference for variability over fixity of meaning in the editing of literary texts also parallels the more general shift in critical theory from a reliance on an author's ascribed meaning to the unstable, multiple, and indeterminate meanings of texts associated with the poststructuralist linguistic turn ${ }^{77}$ and the writings of Barthes, Foucault, and Derrida, among others. ${ }^{78}$ In describing the literary text as a "network," Marcus plays deliberately on Barthes's construal of text as "a methodological field," "a network," "a weave of signifiers" and of work as "the object of a literal science," "a fragment of substance," a closed "sign." She comments:

The idea of the text as network or field of force is common in poststructuralist theory - we may think immediately of Barthes' famous

75 John Sutherland, "Publishing History: A Hole at the Centre of Literary Sociology," Critical Inquiry 14 (Spring 1988): 581.

76 D.C. Greetham, "Textual and Literary Theory: Redrawing the Matrix," Studies in Bibliography 42 (1989): 11-12.

77 According to the intellectual historian Martin Jay, the linguistic turn associated with semiotics, structuralism, and poststructuralism is one of three related but distinct linguistic turns taken by mainstream Western philosophy in the twentieth century. The second turn is associated with "ordinary language" philosophy and the work of Ludwig Wittgenstein, J.L. Austin and others, while the third turn is associated with modern hermeneutics and the work of Hans-George Gadamer, Jurgen Habermas, and Paul Ricoeur among others. See Martin Jay, "Should Intellectual History Take a Linguistic Turn? Reflections on the HabermasGadamer Debate," in Modern European Intellectual History: Reappraisals and New Perspectives, ed. Dominic LaCapra and Steven L. Kaplan (Ithaca and London: Cornell University Press, 1982), 86-110.

78 For Barthes, see, for example, "The Death of the Author," and "From Work to Text," in Image, Music, Text, trans. Stephen Heath (New York: Hill and Wang, 1977), 142-148, 155-164; S/Z, trans. Richard Miller (Oxford, UK: Blackwell, 1990). For Derrida, see "Signature, Event, Context," trans. Samuel Weber and Jeffrey Mehlman and "Afterword: Toward an Ethic of Discussion," trans. Samuel Weber in Limited Inc, ed. Gerald Graff (Evanston, Ill.: Northwestern University Press, 1988), 1-24, 111-154. For Foucault, see "What is an Author," trans. Josué V. Harari, in Textual Strategies: Perspectives in Post-Structuralist Criticism, ed. Josué V. Harari (Ithaca, N.Y.: Cornell University Press, 1979), reprinted in The Foucault Reader, ed. Paul Rabinow (New York: Pantheon, 1984), 101-120.

Barthes, "From Work to Text," passim. 
essay, "From Work to Text", and its irreverent rejection of an older philological conceptualization of the literary "Work" along with all of the ponderous nineteenth-century interpretative baggage traditionally carried by the term, for a fresh conceptualization of the "Text" as free of the "Work's" inhibiting ancestry, turgid solidity, and tyrannical domination by the presence of its author. ${ }^{80}$

At the same time, while proponents of the new textual scholarship might embrace (in varying degrees) the free play of meaning associated with Barthes's definition of text and implicitly endorse (again, in varying degrees) his inversion of the distinction Tanselle and other eclectic editors make between "work" and "text," they would also qualify the ahistorical orientation of Barthes' definition of text and his disparaging definition of work by insisting on "a wider historical and cultural matrix as constitutive, an integral part of [the text's] network." ${ }^{81}$ As Marcus puts it:

... history and culture are reimaged [sic] in poststructural terms so that what Barthes would call the "Work" can carry some of the same liberating instability he associates with the "Text". Scholars as methodologically disparate as McGann, Edward Said, Darnton, and Chartier have all called for reinvestigation of what McGann would term the "Work" - the literary artifact as reinvested with the enriching welter of historical circumstances that helped to determine its shape at its inception, and with the shifting material forms in which it was made available to early and later readers. ${ }^{82}$

Marcus would replace Tanselle's Platonic ideal of the work with a "conception of the work as culturally constructed and altering over time." A literary work then would be defined simply as "all the related texts that have been conventionally grouped together under a single title and author's name. Such conventions can (and should) change over time, just as titles and attributions of authorship sometimes change. ${ }^{183}$ Marcus speculates, moreover, that if textual critics were to pay closer attention to "microinvestigation[s] of literary texts in their local and historically contingent

80 Marcus, Unediting the Renaissance, 23.

81 Marcus, Unediting the Renaissance, 23.

82 Marcus, Unediting the Renaissance, 24.

83 Marcus, Unediting the Renaissance, 31. 
forms," ${ }^{84}$ they might discover that it is not "at all clear ... whether a given group of texts is better conceptualized as one work or as several." 85

Textual scholars have also turned their attention to the ideologies embedded in the structure of critical editions. As Greetham observes, "the editor of a traditional critical text is, in the very layout of the edition, enshrining a hierarchy of variants: those which make it onto the textual page are somehow in a different class from those which are printed in the apparatus and collation. ${ }^{86}$ For many proponents of the new textual scholarship, multiple, historically situated and open-ended texts offer a corrective to this rigorous separation of a favoured "clear text" and rejected variants. From their perspective, the World Wide Web is an ideal vehicle for implementing such a corrective and electronic scholarly editions a potentially powerful means of transcending, more generally, the artificial limits imposed by printed, bounded editions. ${ }^{87}$ Bethany Nowviskie elaborates on this potential in her comparison of traditional and electronic scholarly editions:

A scholarly edition contains an editorial essay, which makes an argument about a text or set of texts, and is then followed by an arranged document that constitutes a frozen version of that argument. ... Electronic editions permit us to do things with documents that we've never been able to do before. We can analyze them computationally, and allow the user of the edition to do so for himself, in the terms of his own research queries, in real time. We can provide, for that user, more and better representations of our texts - some of them not possible in any other medium. We can offer multiple views, multiple arguments -embodied arguments made by editors and users alike. We can craft a site for action on and interaction with documents. ${ }^{88}$

84 Marcus, Unediting the Renaissance, 25.

85 Marcus, Unediting the Renaissance, 31.

86 Greetham, Textual Scholarship, 355.

87 For a sampling of these discussions see, for example, Text Editing, Print and the Digital World, ed. Marilyn Deegan and Kathryn Sutherland (Surrey, UK: Ashgate, 2009); Jerome McGann, Radiant Textuality: Literature After the World Wide Web (New York: Palgrave, 2001); Electronic Text: Investigations in Method and Theory, ed. Kathryn Sutherland (Oxford: Clarendon Press, 1997).

88 Bethany Nowviskie, "Interfacing the Edition," (invited paper presented at Millennial Histories and Prophecies: Literary Truth and Scientific Method, University of Virginia Interdisciplinary Graduate Conference, Charlottesville, VA, April 7-8, 2000), available at http://www2.iath.virginia.edu/bpn2f/1866/interface.html. See also Greetham, Textual Scholarship, 357. 
At the same time, it remains to be seen whether users will welcome the erosion of editorial authority implicit in Nowviskie's vision of electronic editions. As Kathryn Sutherland warns,

[i]t does not follow that, because the stabilized text of traditional scholarly editing provoked diverse interpretations and engaged debates around its composite form, a decentered or unstable text will lead to anything richer or more persuasive than solipsistic self-communing ... Similarly, the editor's exercise of a proper expertise may be more liberating for more readers than seemingly total freedom of choice. ${ }^{89}$

Moreover, electronic scholarly editions are not immune from what Marcus calls "the silent editorial shaping of the literary text." As she cautions:

Electronic textual scholarship is, perforce, a creature of its time, and with standardization and stabilization we are sure to create ideological effects as yet unknown ... Setting aside the more visible forms of ideological intervention represented by site design and organization, hypertext markup languages have rhetorics of their own; they can premap and therefore constrict the ways in which we use electronic editions just as predigital editorial practice did. ${ }^{90}$

Notwithstanding these cautionary remarks, it is clear that, in the wake of the new textual scholarship, textual editors are coming to understand that:

No single version of a literary work ... can offer us the fond dream of unmediated access to an author or to his or her era; the more aware we are of the processes of mediation to which a given edition has been subject, the less likely we are to be caught up in a constricting hermeneutic knot by which the shaping hand of the editor is mistaken for the intent of the author, or for some lost, "perfect" version of the author's creation. ${ }^{91}$

This does not mean that the "autonomous author" and the "ideal text" disappear entirely in a socialised conception of the text; they simply lose "their dominating centrality" 92 as the emphasis shifts to the possibility of a

89 Kathryn Sutherland, "Being Critical: Paper-based Editing and the Digital Environment," in Text Editing, Print and the Digital World, ed. Marilyn Deegan and Kathryn Sutherland (Aldershot: Ashgate, 2009), 24.

90 Marcus, "Textual Scholarship," 157.

91 Marcus, "Textual Scholarship," 3.

92 Marcus, "Textual Scholarship," 25. 
multiplicity of authors and texts. To put it in Barthean terms, "[i]t is not that the Author may not 'come back' in the Text, in his text, but he then does so as a 'guest'." ${ }^{93}$ Nor does it mean that textual editors have abandoned eclectic, intentionalist editions. It does mean that there is a considerably more diverse range of editions being produced today, some more radically destabilising than others which is why, for Greetham, "the characteristic feature of textual scholarship [as it is practised today] ... is its democratic pluralism". ${ }^{94}$

\section{Rethinking the Theory of Archival Arrangement}

The theory of archival arrangement is underpinned by a historiographical ideal that has its own Romantic overtones, i.e., a belief in the possibility of entering into direct contact with a past reality. Such belief is embedded in a number of interlocking assumptions: that the surviving remains of the past can stand in for those that have disappeared; that those remains are, in some sense, a personification of the records creator; and that it is possible to enter into the consciousness of that creator and the past itself through its documentary remains..$^{95}$ And just as contemporary textual scholars have challenged the belief that an eclectic edition can reveal the mind of the author, a number of contemporary archival scholars have questioned the assumption that original order can reveal "the inner being" of the past. Brien Brothman, for example, maintains that, "it is as problematical for an archives to maintain that it is remaining faithful to original order, at least strictly so ... as it is for historians to claim that their work somehow captures and represents the past, that is, makes it present once more. ${ }^{196}$ For Brothman, the "order" that emerges from the process of archival arrangement, far from being "organic" and "natural", is more akin to "the imposed socio-historical order of a tended garden."

The notion of original order as something that is constructed rather than found by archivists has been picked up and explored by a number of archival

93 Barthes, "From Work to Text," 161.

94 Greetham, "Textual Scholarship," 112.

95 The comparison is even more direct in the specific case of literary archives. See Jennifer Douglas and Heather MacNeil, "Arranging the Self: Literary and Archival Perspectives on Writers' Archives," Archivaria 67 (Spring 2009): 25-40.

96 Brien Brothman, "Orders of Value: Probing the Terms of Archival Value," Archivaria 32 (Summer 1991): 83.

97 Brothman, "Orders of Value", 85. 
writers in recent years. ${ }^{98}$ Andrew Prescott, for example, observes that the "original order" elaborated by the English archival theorist Sir Hilary Jenkinson in the first half of the twentieth century did not "reflect" the historical vision of evolutionary growth so much as "impose" it:

V.H. Galbraith [the British historian and onetime assistant keeper of records at the Public Record Office of Great Britain] dreamed of an archivist's history in which the past appeared as a vast collection of 'original documents', falling into types, classes and series. The progress of history would appear as a 'slow pageant of slowly changing records, marked from time to time by the occasional disappearance of one class and the gradual emergence of another'. It was this kind of archival Darwinian fantasy that Jenkinson sought to impose. ${ }^{99}$

Stefano Vitali makes a similar observation in connection to the elaboration of original order in the mid-nineteenth century by Francesco Bonaini, the first Director of the Florence State Archive. Bonaini's notion of original order was inextricably linked to his conviction that the order of the records the State Archive had inherited should reflect the political and administrative history of Florence and Tuscany. As Vitali explains, this meant that, "the republican archives would be followed by the Medici and Lorraine archives [and] ordered according to a pattern that was to convey immediately the idea of a systematic development of the history which had created them and which they represented." ${ }^{100}$ If the previous orders of the records did not align with that history they were dismantled and reorganised in order to make them align with it. ${ }^{101}$ These historical examples of the archival construction

98 See, for example, Terry Cook, "Fashionable Nonsense or Professional Rebirth: Postmodernism and the Practice of Archives," Archivaria 51 (Spring 2001): esp. 32-35; Jennifer Douglas, "Archiving Authors: Rethinking the Analysis and Representation of Personal Archives," PhD dissertation, University of Toronto, 2013; Douglas and MacNeil, "Arranging the Self," 25-39; Wendy Duff and Verne Harris, "Stories and Names: Archival Description as Narrating Records and Constructing Meanings," Archival Science 2 (2002): 263-85; Tom Nesmith, "Reopening Archives: Bringing New Contextualities into Archival Theory and Practice," Archivaria 60 (Fall 2005): esp. 269-274; Tom Nesmith, "Seeing Archives: Postmodernism and the Changing Intellectual Place of Archives," American Archivist 65 (Spring/Summer 2002): 24-41; Elizabeth Yakel, “Archival Representation,” Archival Science 3 (2003): 1-25.

99 Andrew Prescott, "The Textuality of the Archive," in What Are Archives? Cultural and Theoretical Perspectives: A Reader, ed. Louise Craven (Aldershot: Ashgate, 2008), 43-44.

100 Stefano Vitali, "The Archive at the Time of Its Institution: The Central Archive of Francesco Bonaini," in The Florence State Archive: Thirteen Centuries of Historical Records, ed. Rosalia Manno Tolu and Anna Bellinazzi (Florence: Nardini Editore, 2002), 20.

101 Vitali, "The Archive at the Time of Its Institution". 
of original order underline the point that archivists are "creatures of their time" and can no more escape their ideological present than textual critics can. They also illustrate how archival scholars and practitioners are rethinking the theory of archival arrangement along lines that are analogous to some of the lines being pursued in the new textual scholarship.

Over the last two decades, the valorisation of a unitary original order, i.e., one bounded by the lifespan of the creator, and the idealised model of the fonds as a totality of records that revolves around a single creator have been challenged on the grounds that each has acted, in its own way, to obscure the complex history of a fonds and its parts, thereby effacing much of its meaning. There is a growing body of archival literature acknowledging that the physical and intellectual orders of records within a fonds are not stabilised at any single point in time; they are continually being shaped and reshaped as those records are re-situated in different environments and re-territorialised by different authorities. Like variant versions of literary texts, these different orders are viewed increasingly by archivists not as a contamination to be eradicated but simply part of the history and evolving meaning of those records and worth studying in their own right. ${ }^{102}$ The recognition that multiple agents and authorities participate in the creation and ongoing history of a fonds and its parts has also thrown into question the current boundaries of the fonds which effectively subordinate these many agents and authorities to a single primary creator and shrink the totality of records to the physical aggregations of records that have survived and are held in a given archival institution. In the wake of that questioning, archivists have called for a more flexible and nuanced model of a fonds that places less emphasis on fixing physical boundaries and more on accommodating complexity. ${ }^{103}$

102 See, for example, Horsman, "Last Dance of the Phoenix," esp. 17-21; Horsman, "Dirty Hands: A New Perspective on the Original Order," Archives and Manuscripts 27 (May 1999): 42-53; Laura Millar, "The Death of the Fonds and the Resurrection of Provenance: Archival Context in Space and Time," Archivaria 53 (Spring 2002): 1-15; Tom Nesmith, "Still Fuzzy But More Accurate: The Ghosts of Archival Theory," Archivaria 47 (Spring 1999): 136-150; Heather MacNeil, "Archivalterity: Rethinking Original Order," Archivaria 66 (Fall 2008): 1-24.

103 See, for example, Debra Barr, "The Fonds Concept in the Working Group on Archival Descriptive Standards Report," Archivaria 25 (Winter 1987-88): 163-70; Terry Cook, "The Concept of the Archival Fonds: Theory, Description, and Provenance in the PostCustodial Era," in The Archival Fonds: From Theory to Practice, ed. T. Eastwood (Ottawa: Bureau of Canadian Archivists, 1992), 31-85; Terry Eastwood, "Putting the Parts of the Whole Together: Systematic Arrangement of Archives," Archivaria 50 (Fall 2000): 93-116; Geoffrey Yeo, "The Conceptual Fonds and the Physical Collection," Archivaria 73 (Spring 2012): 43-80. 
Around these challenges there has emerged what might be described as a social theory of archival arrangement, one that takes into better account the "wider vectoring of forces and objects" that have shaped the creation and ongoing history of a fonds and its parts, including the silent archival shaping of a fonds. That wider vectoring is neatly encapsulated in Tom Nesmith's expansive definition of provenance as:

the societal and intellectual contexts shaping the action of the people and institutions who made and maintained the records, the functions the records perform, the capacities of information technologies to capture and preserve information at a given time, and the custodial history of the records (which may result in many reorderings, winnowings, and even doctorings of them). ${ }^{104}$

Building on this expanded understanding of provenance, a number of archival writers have begun to explore, through case studies, the manifold ways in which a body of records is shaped over time, initially by its creator(s) and subsequently by its custodians. ${ }^{105}$ Other writers have begun to conceptualise more pluralistic and diffuse models of "creation" that move beyond a single records creator to a community of creators. Jeannette Bastian, for example, introduces the concept of a "community of records" which encompasses "the community both as a record-creating entity and as a memory frame that contextualizes the records it creates." ${ }^{\text {106 }}$ Reflecting on the archives of colonial and post-colonial U.S. Virgin Islands specifically, she argues that, within such a community of records, traditional "subjects" of records - the marginalised and dispossessed - would be repositioned as co-creators of records since "without the enslaved, there would have been

104 Nesmith, "Seeing Archives," 35.

105 See, for example, Donna Holmes, "Passive Keepers or Active Shapers: A Comparative Case Study of Four Archival Practitioners at the End of the Nineteenth Century," Archival Science 6 (2007): 285-298; Peter Horsman, "Documenting the Family: Jacob Vriesendorp and his Family Archive," Third International Conference on the History of Records and Archives (I-CHORA) Conference Program and Participants' Papers (Boston: Massachusetts Historical Society, 2007): 133-138; MacNeil, "Archivalterity, 1-24; Geoffrey Yeo, "Custodial History, Provenance, and the Description of Personal Records," Libraries and the Cultural Record 44 (2009): 50-64; Christine Faunch, "Archives of Written Lives: A Case Study of Daphne Du Maurier and her Biographer, Margaret Forster," Archives 35 (April 2010): 28-34.

106 Jeannette Allis Bastian, Owning Memory: How a Caribbean Community Lost its Archives and Found its History (Westport, Conn.: Libraries Unlimited, 2003), 3-4. 
no need for slave lists, without a population, there would be no need for a census." ${ }^{107}$

Numerous means of accounting for the wider vectoring of forces and objects that impinge on a fonds and its parts over time in archival description have been proposed: these include the addition of colophons and various kinds of supplementary and parallel texts that provide additional layers of contextualisation and highlight archival agency in shaping the fonds and its representation. ${ }^{108}$ Duff and Harris have called for a "liberatory standard" for archival description that discloses its own biases and is "hospitable" to multiple and conflicting narratives. ${ }^{109}$ The theme of hospitality is picked up by Geoffrey Yeo who advocates for the accommodation of both the "conceptual fonds" and the "physical collection" - traditionally the fonds' slightly déclassé other in archival descriptive architectures. His reinterpretation of fonds and collection offers a more nuanced and inclusive way of understanding and exploiting the relationship between abstract wholes and material parts. ${ }^{110}$ The relational network model embodied in the Australian series system - which echoes the

107 Jeannette Allis Bastian, "Reading Colonial Records Through an Archival Lens: The Provenance of Place, Space and Creation," Archival Science 6 (2006): 282-283. For variations on the same theme see also Bastian, "In a 'House of Memory': Discovering the Provenance of Place," Archival Issues 28 (2003-2004): 9-19; Anne Gilliland, "Contemplating Co-creator Rights in Archival Description," Knowledge Organization 39 (2012): 340-346; Chris Hurley, "Parallel Provenance (1): What if Anything is Archival Description?," Archives and Manuscripts 33 (May 2005): 110-145; Eric Ketelaar, "Sharing: Collected Memories in Communities of Records," Archives and Manuscripts 33 (2005): 44-61; Sue McKemmish, Shannon Faulkhead and Lynette Russell. "Distrust in the Archive: Reconciling Records." Archival Science 11.3-4 (2011): 211-239; Joel Wurl, "Ethnicity as Provenance: In Search of Values and Principles for Documenting the Immigrant Experience," Archival Issues 29 (2005): 65-76.

108 See, for example, Terry Cook, "Fashionable Nonsense or Professional Rebirth: Postmodernism and the Practice of Archives, Archivaria 51 (Spring 2001): 34-35; Gabrielle Dean, "The Archeology of Archival Practice: Disciplinarity and Disorder," Archive Journal 1 (April 2011), accessed December 19, 2012, http://www.archivejournal. net/issue/1/archives-remixed/the-archeology-of-archival-practice/; Jennifer Douglas, "Archiving Authors," esp. 209-252; Michele Light and Tom Hyry, "Colophons and Annotations: New Directions for the Finding Aid," American Archivist 65 (Fall/Winter 2002): 216-30; Heather MacNeil, "Trusting Description: Authenticity, Accountability, and Archival Description Standards," Journal of Archival Organization 7 (2009): 99-101; Tom Nesmith, "Reopening Archives," 271-72; Athanasios Velios, "Creative Archiving: A Case Study from the John Latham Archive," Journal of the Society of Archivists 32 (2011): 255-271.

109 Duff and Harris, "Stories and Names," 279-285. See also Chris Hurley, "Parallel Provenance (1)," 110-145; and Chris Hurley, "Parallel Provenance (2): When Something is Not Related to Everything Else," Archives and Manuscripts 33 (November 2005): 52-91.

110 Yeo, "Conceptual Fonds and Physical Collection." 
network paradigm of the new textual scholarship - offers yet another approach to representing that relationship. ${ }^{111}$ As Jenny Bunn suggests in her analysis of archival description as a form of systems thinking, the series system replaces "the traditional difference between whole and part with that between system [the records] and environment [the records' contexts]."112

Many of these alternative means and models are premised on the possibilities of online and interactive environments. In the same way that proponents of the new textual scholarship see electronic scholarly editions as an effective means of presenting users with multiple and open-ended representations of literary texts with which they can interact, a number of archival writers see online archival descriptions as a means of transcending the linearity and univocality of traditional finding aids and a potentially powerful communication vehicle through which archivists and users can co-create multiple and open-ended representations of record aggregations. ${ }^{113}$ While this is undoubtedly true and the possibilities eminently worth exploring, the cautionary notes sounded by Sutherland and Marcus previously in regard to electronic scholarly editions suggest the need also to investigate and critique the possible unintended consequences of the erosion of archival authority and the kinds of ideologies that may be insinuating themselves, however inadvertently, into the emergent genre of online archival description.

All of these discussions reflect, albeit in different ways, a shift from an idealised to a socialised model of archival arrangement, one that takes into explicit account "the enriching welter of historical circumstances" that have shaped a body of records over time. As Laura Millar puts it, "the question we need to ask is not 'how did the records come to be?' The question, rather, is 'how did these records come to be here?"'114 Such a model also implies a

111 See, for example, Mark Wagland and Russell Kelly, "The Series System: A Revolution in Archival Control," in The Records Continuum: Ian Maclean and the Australian Archives First Fifty Years, ed. Sue McKemmish and Michael Piggott (Clayton, Australia: Ancora Press, 1994), 131-149; Chris Hurley, "Problems with Provenance," Archives and Manuscripts 23 (1995): 234-259; Bob Krawczyk, "Cross Reference Heaven: The Abandonment of the Fonds as the Primary Level of Arrangement for Ontario Government Records," Archivaria 48 (Fall 1999): 131-53.

112 Jenny Bunn, "Questioning Autonomy: an Alternative Perspective on the Principles which govern Archival Description," Archival Science (published online 12 Mar. 2013): [6].

113 See, for example, the case studies in A Different Kind of Web: Nerw Connections Between Archives and our Users, ed. Kate Theimer (Chicago: Society of American Archivists, 2011); see also Elizabeth Yakel, "Who Represents the Past? Archives, Records, and the Social Web," in Controlling the Past: Documenting Society and Institutions, ed. Terry Cook (Chicago: Society of American Archivists, 2011), 257-278.

114 Millar, "The Death of the Fonds," 12. 
de-centring and reconceptualising of the archival fonds along the lines of the new textual scholarship's re-conceptualisation of the literary work "as culturally constructed and altering over time." In that reconceptualisation, the fonds could carry with it some of the same "liberating instability" of the reimagined literary work. ${ }^{115}$ This does not mean that the dual notions of a single creator and an original order vanish completely; they simply lose "their dominating centrality" as emphasis shifts to the possibility of a multiplicity of creators and orders. Taking some licence with Barthes' earlier quip about the return of the author, it is not to say that these notions may not be brought back into the theory of arrangement, but that they do so as guests.

The allusion to Barthes suggests one final point of comparison between textual criticism and the theory of archival arrangement. Like the new textual scholarship, the new archival scholarship has been influenced, both directly, and indirectly, by postmodern schools of thought. Brothman's and Prescott's critiques of original order, for example, draw on the poststructuralist linguistic turn in history with its "conception of the inaccessibility of a past reality in any other than a mediated, textualized form." 116 Bastian frames the notion of a "community of records" within post-colonial and subaltern discourse to draw attention to the complex cultural identities of "colonizer" and "colonized" and the possibilities for discovering the voices of the "other" through the cracks and silences in colonial records. Duff and Harris's "liberatory standard" for description acknowledges a debt to Derridean deconstructionism; and postmodern thinking in general provides the backdrop for Nesmith's reflections on provenance which emphasise the ambiguity of origins and endings and the contingency of meaning.

Taken altogether, the various strands of this new archival scholarship weave a persuasive argument for a social theory of arrangement in which the entire history of a given body of records falls within the scope of the theory of archival arrangement. While there are several points of common

115 For a variation on this idea see Yeo, "The Conceptual Fonds and the Physical Collection," 43-80.

116 Gabrielle M. Spiegel, "History, Historicism, and the Social Logic of the Text," in The Past as Text: The Theory and Practice of Medieval Historiography (Baltimore: Johns Hopkins University Press, 1999), 22. As Spiegel elaborates, "there is no sound epistemological reason, within a poststructuralist universe, to distinguish between literary and other uses of language. If the literary text is denied the ability to represent reality so also are all texts, and the distinction traditionally drawn between literature and "document" becomes meaningless since both participate equally in the unconscious play and intertextuality of language itself. If we cannot reach "life" through literature, we cannot reach "the past" through documents." Spiegel, "History, Historicism, and the Social Logic of the Text," 8. 
interest and concern among the contributors to this new scholarship, there are also varying levels of agreement among them and no single orthodoxy. This suggests, perhaps, that the defining characteristic of the new archival scholarship, like the new textual scholarship, is its democratic pluralism.

\section{Conclusion}

A comparative analysis of textual criticism and the theory of archival arrangement teaches that, as objects of study, archival aggregations are not realities or substances that we somehow attempt to know; they are texts that we decipher and interpret. Although "text" is not a term archivists typically use in referring to an archival fonds, ${ }^{117}$ it is, nevertheless, fitting, given that the term derives in part from the Latin textere, "to weave," and, as D.F. McKenzie observes, "refers, not to any specific materials as such, but to their woven state, the web or texture of the materials." 118 What defines a text, therefore, "is not the presence of linguistic elements but the act of construction." ${ }^{119}$ To describe an archival fonds as a text is to foreground its constructed nature as well as the process of its construction, i.e, the ways in which a web of records and their relationships is shaped and reshaped over time.

This returns us to the beginning of this chapter and Jameson's enumeration of the purposes served by treating the subjects of non-literary disciplines as texts. To re-cast those purposes in the light of the comparative analysis: conceptualising an archival fonds as text serves three useful purposes: first, it suggests that the nature of fonds should not be taken as given and that archivists and archival institutions should consider how they came to be produced, isolated, and presented to attention. Second, it marks the meaning (or rather meanings) of a given fonds as a problem worth exploring. Finally, it posits that the archivist's own methods need to be considered as part of the problem worth exploring; in other words, archivists and archival institutions cannot stand outside that web of records and their relationships as they investigate it; they need to locate themselves within it.

117 An exception is Brien Brothman. See his "Declining Derrida: Integrity, Tensegrity, and the Preservation of Records from Deconstruction," Archivaria 48 (Fall 1999): 64-88.

118 D.F. McKenzie, Bibliography and the Sociology of Texts (Cambridge UK: Cambridge University Press, 1999), 13.

119 McKenzie, Bibliography and the Sociology of Texts, 43. 


\section{References}

Barr, Debra. "The Fonds Concept in the Working Group on Archival Descriptive Standards Report." Archivaria 25 (Winter 1987-88): 163-170.

Barthes, Roland. "The Death of the Author." In Image, Music, Text, edited and translated by Stephen Heath, 142-148. New York: Hill and Wang, 1977.

Barthes, Roland. "From Work to Text." In Image, Music, Text, edited and translated by Stephen Heath, 155-164. New York: Hill and Wang, 1977.

Barthes, Roland. S/Z. Translated by Richard Miller. Oxford: Blackwell, 1990.

Bastian, Jeannette Allis. "In a 'House of Memory': Discovering the Provenance of Place.” Archival Issues 28 (2003-2004): 9-19.

Bastian, Jeannette Allis. "Reading Colonial Records Through an Archival Lens: The Provenance of Place, Space and Creation." Archival Science 6 (2006): 267-284.

Bastian, Jeannette Allis. Owning Memory: How a Carribean Community Lost its Archives and Found its History. Westport, Conn.: Libraries Unlimited, 2003.

Bloch, Marc. The Historian's Craft, translated by Peter Putnam. New York: Alfred A. Knopf, 1963.

Bowers, Fredson. "Some Principles for Scholarly Editions of Nineteenth-Century American Authors.” Studies in Bibliography 17 (1964): 223-228.

Brothman, Brien. "Declining Derrida: Integrity, Tensegrity, and the Preservation of Records from Deconstruction." Archivaria 48 (Fall 1999): 64-88.

Brothman, Brien. "Orders of Value: Probing the Terms of Archival Value." Archivaria 32 (Summer 1991): 78-100.

Bunn, Jenny. "Questioning Autonomy: an Alternative Perspective on the Principles which govern Archival Description.” Archival Science (published online 12 Mar. 2013): n.p.

Bureau of Canadian Archivists Planning Committee on Descriptive Standards. Rules for Archival Description, July 2008 rev. Ottawa: Bureau of Canadian Archivists, 1990-.

Casanova, Eugenio. Archivistica. Siena: Stab. arti grafiche Lazzeri, 1928.

Cencetti, Giorgio. Scritti archivistici. Roma: Il Centro di Ricerca editore, 1970.

Cheney, C.R. The Papacy and England 12th - 14th Centuries. London: Variorum Reprints, 1982.

Croce, Benedetto. Theory and History of Historiography. Translated by Douglas Ainslie. London: G.C. Harrap, 1921.

Cook, Terry. "The Archive(s) Is a Foreign Country: Historians, Archivists, and the Changing Archival Landscape." American Archivist 74 (Fall/Winter 2011): 600632.

Cook, Terry. "The Concept of the Archival Fonds: Theory, Description, and Provenance in the Post-Custodial Era." In The Archival Fonds: From Theory to Practice, edited by T. Eastwood, 31-85. Ottawa: Bureau of Canadian Archivists, 1992.

Cook, Terry. "Fashionable Nonsense or Professional Rebirth: Postmodernism and the Practice of Archives." Archivaria 51 (Spring 2001): 14-35.

Culler, Jonathan. “Text; Its Vicissitudes." In The Literary in Theory, edited by Mieke Bal and Hent de Vries, 99-116. Stanford, Calif.: Stanford University Press, 2007.

Dean, Gabrielle. "The Archeology of Archival Practice: Disciplinarity and Disorder." Archive Journal 1 (April 2011). Accessed December 19, 2012. http://www. archivejournal.net/issue/1/archives-remixed/the-archeology-of-archival-practice/.

Deegan, Marilyn and Kathryn Sutherland, eds. Text Editing, Print and the Digital World. Surrey, UK: Ashgate, 2009. 


\section{Chapter 5}

Derrida, Jacques. “Afterword: Toward an Ethic of Discussion.”Translated by Samuel Weber. In Limited Inc, edited by Gerald Graff, 111-154. Evanston, Ill: Northwestern University Press, 1988.

Derrida, Jacques. “Signature, Event, Context.” Translated by Samuel Weber and Jeffrey Mehlman. In Limited Inc, edited by Gerald Graff, 1-24. Evanston, Ill: Northwestern University Press, 1988.

Douglas, Jennifer. "Archiving Authors: Rethinking the Analysis and Representation of Personal Archives.” PhD diss., University of Toronto, 2013.

Douglas, Jennifer. "Origins: Evolving Ideas about the Principle of Provenance." In Currents of Archival Thinking, edited by Terry Eastwood and Heather MacNeil, 23-43. Santa Barbara: Libraries Unlimited, 2010.

Douglas, Jennifer and Heather MacNeil. "Arranging the Self: Literary and Archival Perspectives on Writers' Archives.” Archivaria 67 (Spring 2009): 25-40.

Duff, Wendy and Verne Harris. "Stories and Names: Archival Description as Narrating Records and Constructing Meanings." Archival Science 2 (2002): 263-85.

Duranti, Luciana. "Origin and Development of the Concept of Archival Description." Archivaria 35 (Spring 1993): 47-54.

Eastwood, Terry. "Putting the Parts of the Whole Together: Systematic Arrangement of Archives." Archivaria 50 (Fall 2000): 93-116.

Evans, Frank B. "Promoting Archives and Research: A Study in International Cooperation." American Archivist 50 (Winter 1987): 48-65.

Evans, Richard J. In Defense of History. New York: W.W. Norton, 1999.

Faunch, Christine. "Archives of Written Lives: A Case Study of Daphne Du Maurier and her Biographer, Margaret Forster.” Archives 35 (April 2010): 28-34.

Foucault, Michel. "What Is an Author." In Textual Strategies: Perspectives in PostStructuralist Criticism. Translated and edited by Josué V Harari. Ithaca, NY: Cornell University Press, 1979. Reprinted in The Foucault Reader, edited by Paul Rabinow, 101-120. New York: Pantheon, 1984.

Geller, Lawrence D. "Joseph Cuvelier, Belgian Archival Education, and the First International Congress of Archivists, Brussels, 1910.” Archivaria 16 (Summer 1983): 26-34.

Gilliland, Anne. "Contemplating Co-creator Rights in Archival Description." Knowledge Organization 39 (2012): 340-346.

Greetham, D. C. Foreword to A Critique of Modern Textual Criticism by Jerome J. McGann, ix-xix. Charlottesville: University Press of Virginia, 1992.

Greetham, D.C. “Textual and Literary Theory: Redrawing the Matrix.” Studies in Bibliography 42 (1989): 1-24.

Greetham, D. C. Textual Scholarship: An Introduction. New York: Garland, 1994.

Greetham, D. C. “Textual Scholarship.” In Introduction to Scholarship in Modern Languages and Literatures, $2^{\text {nd }}$ ed., edited by Joseph Gibaldi, 103-137. New York: Modern Languages Association, 1992.

Greetham, D.C. Theories of the Text. Oxford: Oxford University Press, 1999.141.

Greetham, David [D.C.]. "Facts, Truefacts, Factoids; or, Why Are They Still Saying Those Things about Epistemology?” In The Pleasures of Contamination: Evidence, Text, and Voice in Textual Studies, 87-115. Bloomington: Indiana University Press, 2010.

Greetham, David. "What Is Textual Scholarship?" In A Companion to the History of the Book, edited by Simon Eliot and Jonathan Rose, 21-32. West Sussex: Wiley-Blackwell, 2009.

Greg, W.W. “The Rationale of Copy-Text.” Studies in Bibliography 3 (1950-1951): 19-36. 
Guyotjeannin, Olivier. “The Expansion of Diplomatics as a Discipline." American Archivist 59 (Fall 1996): 414-421.

Holmes, Donna. "Passive Keepers or Active Shapers: A Comparative Case Study of Four Archival Practitioners at the End of the Nineteenth Century." Archival Science 6: 3-4 (2007): 285-298.

Horsman, Peter. "Dirty Hands: A New Perspective on the Original Order." Archives and Manuscripts 27 (May 1999): 42-53.

Horsman, Peter. "Documenting the Family: Jacob Vriesendorp and his Family Archive." In Third International Conference on the History of Records and Archives (I-CHORA) Conference Program and Participants' Papers, 133-138. Boston: Massachusetts Historical Society, 2007.

Horsman, Peter. "The Last Dance of the Phoenix, or the De-discovery of the Archival Fonds." Archivaria 54 (Fall 2002): 1-23.

Horsman, Peter. "Taming the Elephant: An Orthodox Approach to the Principle of Provenance." In The Principle of Provenance: Report from the First Stockholm Conference on Archival Theory and the Principle of Provenance 2-3 September 1993, 51-63. Sweden: Swedish National Archives, 1994.

Hurley, Chris. "Parallel Provenance (1): What if Anything is Archival Description?" Archives and Manuscripts 33 (May 2005): 110-145.

Hurley, Chris. "Parallel Provenance (2): When Something is Not Related to Everything Else." Archives and Manuscripts 33 (November 2005): 52-91.

Hurley, Chris. "Problems with Provenance." Archives and Manuscripts 23 (November 1995): 234-259.

International Council on Archives. ISAD $(G)$ : General International Standard Archival Description, $2^{\text {nd }}$ ed. Paris, France: ICA, 2000.

Jameson, Fredric. “The Ideology of the Text.” In The Ideologies of Theory: Essays 1971-1986, vol. 1, 17-71. Minneapolis: University of Minnesota Press, 1989.

Jay, Martin. "Should Intellectual History Take a Linguistic Turn? Reflections on the Habermas-Gadamer Debate." In Modern European Intellectual History: Reappraisals and New Perspectives, edited by Dominick LaCapra and Steven L. Kaplan, 86-110. Ithaca and London: Cornell University Press, 1982.

Jenkinson, Hilary. A Manual of Archive Administration. Oxford: Clarendon Press, 1922.

Kelley, Donald R. Foundations of Modern Historical Scholarship: Language, Law and History in the French Renaissance. New York: Columbia University Press, 1970.

Ketelaar, Eric. "Sharing: Collected Memories in Communities of Records." Archives and Manuscripts 33 (2005): 44-61.

Krawczyk, Bob. "Cross Reference Heaven: The Abandonment of the Fonds as the Primary Level of Arrangement for Ontario Government Records." Archivaria 48 (Fall 1999): 131-53.

LaCapra, Dominick. "Rethinking Intellectual History and Reading Texts.” In Rethinking Intellectual History: Texts, Contexts, Language, 23-71. Ithaca and London: Cornell University Press, 1983.

Leerssen, Joep. "Introduction: Philology and the European Construction of National Literatures." In Editing the Nation's Memory: Textual Scholarship and Nation-Building. in Nineteenth-Century Europe, edited by Dirk Van Hulle and Joep Leerssen, 13-27. New York: Rodolphi, 2008.

Lernout, Geert. "The Angel of Philology." In Editing the Nation's Memory: Textual Scholarship and Nation-Building in Nineteenth-Century Europe, edited by Dirk Van Hulle and Joep Leerssen, 46-61. New York: Rodolphi, 2008.

Light, Michelle and Tom Hyry. "Colophons and Annotations: New Directions for the Finding Aid.” American Archivist 65 (Fall/Winter 2002): 216-30. 


\section{Chapter 5}

Lodolini, Elio. “The War of Independence of Archivists.” Archivaria 28 (Summer 1989): 36-47.

MacNeil, Heather. "Archivalterity: Rethinking Original Order." Archivaria 66 (Fall 2008): $1-24$.

MacNeil, Heather. "Picking Our Text': Archival Description, Authenticity and the Archivist as Editor." American Archivist 68 (Fall/Winter 2005): 264-78.

MacNeil, Heather. "Trusting Description: Authenticity, Accountability, and Archival Description Standards." Journal of Archival Organization 7 (2009): 89-107.

MacNeil, Heather. Trusting Records. Dordrecht: Kluwer, 2000.

Maguire, Laura. "The Rise of the New Bibliography." In Shakespearean Suspect Texts: The 'Bad' Quartos and their Contexts, 21-72. Cambridge: Cambridge University Press, 1996.

Marcus, Leah S. "Textual Scholarship." In Introduction to Scholarship in Modern Languages and Literatures, $3^{\text {rd }}$ ed., edited by David G. Nicholls, 143-159. New York: Modern Languages Association, 2007.

Marcus, Leah S. Unediting the Renaissance: Shakespeare, Marlow, Milton. London and New York: Routledge, 1996.

McGann, Jerome J. A Critique of Modern Textual Criticism. Charlottesville: University Press of Virginia, 1992.

McGann, Jerome J. Radiant Textuality: Literature After the World Wide Web. New York: Palgrave, 2001.

McKemmish, Sue. “Are Records Ever Actual?” In The Records Continuum: Ian Maclean and Australian Archives First Fifty Years, edited by Sue McKemmish and Michael Piggott. Clayton: Ancora, 1994.

McKemmish, Sue, Shannon Faulkhead and Lynnette Russell. "Distrust in the Archive: Reconciling Records," Archival Science 11 (2011): 211-239.

McKenzie, D.F. Bibliography and the Sociology of Texts. Cambridge, UK: Cambridge University Press, 1999.

Millar, Laura. "The Death of the Fonds and the Resurrection of Provenance: Archival Context in Space and Time." Archivaria 53 (Spring 2002): 1-15.

Moore, Lara Jennifer. Restoring Order: The Ecole des Chartes and the Organization of Archives and Libraries in France, 1820-1870. Duluth, Minn: Litwin, 2001.

Muller, S., J.A. Feith and R. Fruin. Manual for the Arrangement and Description of Archives, $2^{\text {nd }}$ ed. Translated by Arthur H. Leavitt. Chicago: Society of American Archivists, 2003.

Nesmith, Tom. "Reopening Archives: Bringing New Contextualities into Archival Theory and Practice." Archivaria 60 (Fall 2005): 259-274.

Nesmith, Tom. "Seeing Archives: Postmodernism and the Changing Intellectual Place of Archives." American Archivist 65 (Spring/Summer 2002): 24-41.

Nesmith, Tom. "Still Fuzzy But More Accurate: The Ghosts of Archival Theory." Archivaria 47 (Spring 1999): 136-150.

Novick, Peter. That Noble Dream: The "Objectivity Question" and the American Historical Profession. Cambridge: Cambridge University Press, 1988.

Nowviskie, Bethany. “Interfacing the Edition.” Invited paper presented at Millenial Histories and Prophecies: Literary Truth and Scientific Method, University of Virginia Interdisciplinary Graduate Conference, Charlottesville, VA, April 7-8, 2000.

Posner, Ernst. "Max Lehmann and the Genesis of the Principle of Provenance." In Archives and the Public Interest, edited by Ken Munden, 36-44. Chicago: Society of American Archivists, 2006.

Prescott, Andrew. "The Textuality of the Archive." In What Are Archives? Cultural and Theoretical Perspectives: A Reader, edited by Louise Craven, 31-51. Aldershot: Ashgate, 2008. 
Rajan, Tilottama. "Is there a Romantic Ideology? Some Thoughts on Schleiermacher's Hermeneutic and Textual Criticism." In Text: Transactions of the Society for Textual Scholarhip 4, edited by D. C. Greetham and W Speed Hill, 59-77. New York: AMS Press, 1988.

Reynolds, L.D. and N.G. Wilson. Scribes and Scholars: A Guide to the Transmission of Greek and Latin Literature, $3^{\text {rd }}$ ed. Oxford: Clarendon Press, 1991.

Rigney, Ann. Imperfect Histories: The Elusive Past and the Legacy of Romantic Historicism. Ithaca and London: Cornell University Press, 2001.

Roper, Michael. "The International Role of the Public Record Office." In The Records of a Nation: The Public Record Office 1838-1988, The British Record Society 1888-1988, edited by G.H. Martin and Peter Spufford, 9-16. Woodbridge, UK: Boydell Press and the British Record Society, 1990.

Savoja, Maurizio and Stefano Vitali. "Authority Control for Creators in Italy: Theory and Practice." Journal of Archival Organization 5 (2007): 121-148.

Schellenberg, T.R. Modern Archives: Principles and Techniques. Chicago: University of Chicago Press, 1956, reprint 1975.

[Society of American Archivists]. Describing Archives: A Content Standard. Chicago: Society of American Archivists, 2004.

Spiegel, Gabrielle M. "History, Historicism, and the Social Logic of the Text." In The Past as Text: The Theory and Practice of Medieval Historiography, 3-28. Baltimore: Johns Hopkins University Press, 1999.

Sutherland, Kathryn. "Being Critical: Paper-based Editing and the Digital Environment." In Text Editing, Print and the Digital World, edited by Marilyn Deegan and Kathryn Sutherland, 13-25. Aldershot: Ashgate, 2009.

Sutherland, Kathryn, ed. Electronic Text: Investigations in Method and Theory. Oxford: Clarendon Press, 1997.

Sutherland, John. "Publishing History: A Hole at the Centre of Literary Sociology." Critical Inquiry 14 (Spring 1988): 574-589.

Tamblé, Donato. “Archival Theory in Italy Today." Archival Science 1 (2001): 83-100.

Tanselle, G. Thomas. A Rationale of Textual Criticism. Philadelphia: University of Pennsylvania Press, 1989.

Tanselle, G. Thomas. "Some Principles for Editorial Apparatus.” Studies in Bibliography 25 (1972): 41-88.

Theimer, Kate, ed. A Different Kind of Web: New Connections Between Archives and our Users. Chicago: Society of American Archivists, 2011.

Tollebeek, Jo. "Seeing the Past with the Mind's Eye: The Consecration of the Romantic Historian.” Clio 29 (Winter 2000): 167-191.

Tollebeek, Jo. “Turn'd to Dust and Tears': Revisiting the Archive." Review of Dust: The Archive and Cultural History, by Carolyn Steedman. History and Theory 43 (2004): 237-248.

Velios, Athanasios. "Creative Archiving: A Case Study from the John Latham Archive." Journal of the Society of Archivists 32 (2011): 255-271.

Vinavar, Eugène. "Principles of Textual Emendation." In Studies in French Language and Mediaeval Literature, presented to Professor Mildred K. Pope, by pupils, colleagues, and friends, 351-70. Freeport, NY: Books for Libraries Press, [1939], rpt. 1969.

Vitali, Stefano. "The Archive at the Time of its Institution: The Central Archive of Francisco Bonaini.” In The Florence State Archive: Thirteen Centuries of Historical Records, edited by Rosalia Manno Tolu and Anna Bellinazzi, 19-21. Florence: Nardine Editore, 2002. 


\section{Chapter 5}

Vitali, Stefano. "What Are the Boundaries of Archival Context? The SIASFI Project and the Online Guide to the Florence State Archives, Italy." Journal of Archival Organization 3 (2005): 243-260.

Wagland, Mark and Russell Kelly. "The Series System: A Revolution in Archival Control." In The Records Continuum: Ian Maclean and the Australian Archives First Fifty Years, edited by Sue McKemmish and Michael Piggott, 131-149. Clayton, Australia: Ancora Press, 1994.

Wimsatt, William K. and Monroe Beardsley. "The Intentional Fallacy.” Serwanee Review 54 (1946): 468-488.

Wurl, Joel. "Ethnicity as Provenance: In Search of Values and Principles for Documenting the Immigrant Experience." Archival Issues 29 (2005): 65-76.

Yakel, Elizabeth. "Archival Representation." Archival Science 3 (2003): 1-25.

Yakel, Elizabeth. "Who Represents the Past? Archives, Records, and the Social Web." In Controlling the Past: Documenting Society and Institutions, edited by Terry Cook, 257-278. Chicago: Society of American Archivists, 2011.

Yeo, Geoffrey. "The Conceptual Fonds and the Physical Collection." Archivaria 73 (Spring 2012): 43-80.

Yeo, Geoffrey. "Custodial History, Provenance, and the Description of Personal Records." Libraries and the Cultural Record 44 (2009): 50-64. 
Chapter 6

\title{
THE ARCHIVAL MULTIVERSE AND EDDIES IN THE SPACETIME CONTINUUM
}

\author{
Frank Upward
}

\begin{abstract}
The foundation of this contribution is its account of philosophies of emergence in the era that the philosopher William James coined the term "multiverse." This was when continuum thinking first emerged as a collective academic effort to understand expanding complexity. The same style of thinking emerged in Anglo-American archival practice in the 1930s (in a less discursive form) and was carried through within the development of fully fledged records continuum practices in Australia in the 1950s and 1960s. This account of that emergence will be largely controlled by the concept of spacetime eddies expressed initially in research on social media conducted by Leisa Gibbons at Monash University, which also appears in this volume. As a model for knowledge formation it suggests chaos not order and the purpose of this contribution is to encourage researchers to think about the role of archival and recordkeeping practices in attempting to bring some order back into the expanding continuum of recorded information that is the archival multiverse.
\end{abstract}

\section{Introduction: Spacetime Eddies and the Multiverse Conundrum}

The philosopher Tim Wilkinson began a recent article on "the multiverse conundrum" with the following words:

In his 1895 essay Is Life Worth Living? the American philosopher William James wrote, "Truly, all we know of good and duty proceeds from nature ... [which] is all plasticity and indifference - a moral 
multiverse, as one might call it" and a new word was born. A century later, and James's neologism has been commandeered by physicists and pressed into service in a somewhat different context. These days 'multiverse' refers to the literal existence of multiple universes. But is such a thing even possible? ${ }^{1}$

In philosophy, the conundrum is a new way of expressing an old problem, such as in continuum mathematics, theistic debate, and more recently political discussion via the concept of known unknowns. How can one prove hypotheses that depend upon the notion of some things being unknown and perhaps even unknowable?

In pressing the concept of the multiverse into archival service, archivists and archival educators (present and budding) have recently presented the world with a monistic entity, the archival multiverse, defined by the pluralism of "evidentiary texts, memory-keeping practices and institutions, bureaucratic and personal motivations, community perspectives and needs, and cultural legal constructs" in and through time. ${ }^{2}$ This present volume which discusses the archival multiverse will help articulate how that term is used within archival research across archival paradigms. In contributing to that discussion, this piece involves going back to the intellectual currents surrounding James' original usage of the term multiverse. In continuum theories, the multiverse's chaos-generating capacity is derived in part from the plasticity and indifference of the way it eddies around in spacetime, producing unexpected links as different ideas and practices connect and affect each other both spatially (across locations) and temporally (across time). It is this eddying in archival time which I want to try to portray in this chapter. The points that remake the future are multiple, interactively generating new ideas and features. They often occur independently of each other and then unexpectedly meet again and generate more new ideas and features. The eddies described here will come from mathematics, philosophy, sociology, and archival practices, which will make the account and its implications seem wide-ranging and far-reaching. It is an overview, however, and can never be wide enough. The multiverse involves eddies from more directions than any of us individually can ever hope to comprehend.

1 Tim Wilkinson, “The Multiverse Conundrum,” Philosophy Now 89 (July/August 2013), http://philosophynow.org/issues/89/.

2 Pluralizing the Archival Curriculum Group, "Educating for the archival multiverse" American Archivist 74 no. 1 (Spring/Summer (2011): 69-101. 
More practically (and records continuum thinking involves AngloAmerican forms of pragmatism, as I will demonstrate below), the concept of spacetime eddies can give us a much firmer grip on how records, as Sue McKemmish has argued, are always "in a state of becoming". ${ }^{3}$ The concept of spacetime eddies is a mechanism for viewing this process of becoming across plurality, creating narratives of emergence (what can possibly happen) and immanence (what is about to happen). Emergence and immanence are relevant to both the formation of archives and the use of those archives to achieve what the Australian continuum thinking archivist David Roberts described as "future proofing" within programs he led at The State Authority of New South Wales (State Records NSW), programs that are still currently in operation. ${ }^{4}$ The aim in records continuum approaches is to use the processes of recordkeeping to "future proof" ourselves and our communities.

\section{Philosophies of Emergence}

My first encounter with the term "spacetime eddy" was in a draft of work by Leisa Gibbons where it was used as a metaphor to describe the swirling nature of social media. ${ }^{5}$ To me, it means what it describes: Ideas and events eddy around and meet in unpredictable ways. It provides an interesting method for examining how connections in the spaces between ideas and our actions fuel many philosophies of emergence, some of which will be discussed in this piece. In reading what follows, it is worth using Wikipedia to get a feel for how online summary sources are invigorating the study of broad sweeps of ideas. We cannot individually hope to read major works by all the people discussed here and few of us can understand many of the mathematical elements in spacetime continuum theory. Most of us can, however, view things across wide surfaces of knowledge looking for patterns and possible connections within the eddying of spacetime and in doing so increase our understanding of processes that contribute to the emerging expansion of complexity, refashioning our simplifications as we learn more.

3 Sue McKemmish, "Are Records Ever Actual?" in McKemmish, S., and Piggott, M., The Records Continuum, Ian Maclean and Australian Archives First Fifty Years (Melbourne: Ancora Press, 1994), pp.187-203.

4 The State Records Authority of New South Wales, Australia: http://www.records.nsw. gov.au/.

5 Leisa Gibbons, Engaging complexity: social media as cultural heritage, $\mathrm{PhD}$ Thesis being undertaken at Caulfield School of Information Technology, Monash University. 
As a major eddy in philosophy, the emergence of complexity became a significant area of study early in the twentieth century. It shaped continuum thinking of the time, encouraging intellectuals to think about process, about motion, and about pluralism. For some it encouraged them to do so in non-linear fashion, drawing on ideas originating from many places and many times. The next two sections will present a selective overview of that style of thinking in mainland Europe, using three figures from the late nineteenth and first half of the twentieth century: Gabriel Tarde, Albert Einstein, and Henri Bergson. Two of the figures, Einstein and Bergson, are iconic continuum thinkers and the third, Tarde, is being rediscovered within modern sociology-based theories about complex social interactions.

\section{Sociological and Scientific Relativism}

\section{Gabriel Tarde (1843-1904) ${ }^{6}$}

In a demonstration of the non-linearity of developments in discourse, Gabriel Tarde, Bergson's predecessor at the Collège De France, has in recent years emerged from his successor's shadow. French sociologist Bruno Latour recently described Tarde's work in exploring complexity, addressing the "confusing plasma composed of myriads of monads, a chaos, a brew that social scientists will do anything to avoid staring in the eyes." He brought this chaos together within a slightly mystical version of a monistic whole [a continuum], arguing that "in the bosom of each thing there resides every other thing real and possible." There are no borders between things, and the study of the complexity of one thing takes you into the study of everything else. The part is always more complex than the whole, and Tarde was interested in explaining the big through the small. In Tarde's work, the smallest examples are always richer in difference and complexity than their aggregates. ${ }^{7}$

Tarde was one of a number of philosophers in his era who was as much sociologist as philosopher. As such, he can be aligned with Georg Simmel as one of the grandfathers of the sociology of emergence. This sociology studies how social functioning evolves out of complex interactions. Both

6 Barbara Reed made me aware of the work of Gabriel Tarde. His thinking helps tie individual creation processes to the expanding whole that is the archival multiverse and to the complexity of the part within what is described later using the ideas of Samuel Alexander as the theatre of perpetual movement.

7 This paragraph is heavily based on Bruno Latour, "The Whole is Always Smaller than Its Parts: A Digital Test of Gabriel Tarde's Monad," accessed via Latour's website http:// www.bruno-latour.fr/node/330 on 31 July 2012. 
Tarde and Simmel are often said to stand squarely opposed to the orderly positivism of Auguste Comte, another philosopher/sociologist, but one that Tarde would presumably have regarded as focused on ironing out the messiness of difference. The sociology of emergence was relativist and has had clear successors expanding and complexifying its ideas, including more recent exponents such as Erving Goffman (the dynamic effects of faceto-face interaction), Zygmunt Bauman (the influence of strangeness and otherness), Pierre Bourdieu (genetic structuralism), Bruno Latour (actornetwork theory), and Anthony Giddens (structuration theory).

The modern archivist can hope to find in the sociologists of emergence ways of staring down the complexity of today's expanding archival multiverse, along with ways of thinking about how to manage the whole (the archival multiverse as a continuum) and the massive complexity of its parts. In research programs, however, we should not be misled into thinking that the more recent the work the better in touch with modernity it will be. It is difficult not to read Latour's account of Tarde without being convinced that he was in touch with modern complexity theory and the need to simplify things while acknowledging the difficulties involved in producing simplifications that will need constant adjustment.

\section{Albert Einstein (1879-1955)}

Einstein, perhaps the best known of all continuum thinkers, was a product of his times. He, like many of his era, was interested in ways of connecting time to ideas about emergent phenomenon. In one example of spacetime eddying, Einstein drew upon the work of Hermann Minkowski, the Polish mathematician whose ideas provided the base for what is sometimes known as Einstein-Minkowski spacetime. ${ }^{8}$ Minkowski, who in turn drew upon Einstein's work, posited that spacetime was a continuum of movement involving four dimensions. Time was one of those dimensions but was inseparable from three spatial dimensions commonly described as length, breadth and height. Far from being a dominant dimension (as it was in Comte's positivism), Minkowski observed that time was starting to be seen as a shadow of itself, always tucked away behind the immediacy of past, present, and future moments. ${ }^{9}$

8 http://en.wikipedia.org/wiki/Minkowski_space accessed on 3 Nov 2013. This entry gives a clear account of Einstein-Minkowski spacetime as an eddying construct built upon the exchange of ideas.

9 Frank Upward, "Modelling the Continuum as Paradigm Shift in Recordkeeping and Archiving Processes and Beyond: A Personal Reflection," Records Management Journal 
Movement through time is a constant shadow in Einstein's theories. His special theory states that the speed of light, while constant when thought of as being independent of its source, only has meaning when observed travelling between two physical systems moving relative to each other. His later general theory of relativity extended that motion based argument to gravity, the movement of heavenly bodies, and our infinite universe.

Apart from Minkowski's continuum, Einstein was undoubtedly influenced by developments in chemistry where the atomic structure of matter was being explored. Tables of elements were being developed and far from leading to certainties of identity, the research began to focus on the pragmatics of what emerges when atoms combine. What started out as a positivist form of study began to point to emergence and chaos theories. Chemists had yet to discover the chaos that happens when atoms are split but Einstein was beginning to set out the primary level tools for thinking about exactly that within his theories of relativity. Moreover, one can identify in his work an interest in emergence and the variability of motion, the positioning of the observer, and the exponential expansion of things, all of which can lead to the questioning of Newtonian laws.

By the 1930s, Einstein had become a global celebrity of sorts, suggesting the far-reaching appeal and accessibility of his ideas. Yet it took almost a century for science teachers in Australia to overcome the anxieties of teaching such a seemingly complex set of ideas, and to explore how to teach Einstein's spacetime thinking and quantum mechanics at primary school level. They found that it is not only feasible, but that the students enjoyed the stimulation. ${ }^{10}$

Predating Einstein was Georg Cantor's continuum hypothesis (1878) dealing with set theory. He pointed to the nature of infinity and continuum mathematicians have since expanded and complexified spacetime thinking and quantum mechanics. Some of these figures include:

- Kurt Gödel, who argued that the continuum hypothesis cannot be disproved;

- Imre Lakatos, who posited that the fragmentation of mathematics into specialisations restricted the cross-pollination of mathematics as a multiverse (although he did not use that word); and

10, no.3 (2000): 115-139. This article presents a summary based on Rob Salgado http:// www.phy.syr.edu/courses/modules/LIGHTCONE/minkowski.html accessed January 2000.

10 A colleague Joanne Evans pointed out the research project teaching Einstein in Western Australian Primary schools - http://theconversation.edu.au/testing-the-theory-takingeinstein-to-primary-schools-9710 accessed on 9 Nov 2012. 
- Benoit Mandelbrot, whose fractal theorising in a world of infinitely expanding complexity demonstrated the possibility of identifying recursive patterns. ${ }^{11}$

Accepting Einstein's quantum mechanics and spacetime thinking in elementary form means facing up to the reality of systems in motion and accepting time as a shadow of itself. That might sound mystical, but research over the last few decades has shown that archivists have a motion-based tool in recordkeeping metadata. Rather than being beaten down by archives as things in motion, archivists can develop their own form of quantum mechanics by applying recordkeeping metadata within our computation processes (not simply metadata about the record as a thing, but data about its source, transmission and the web of relationships in which it exists). It is a way of managing the greater complexity of the parts. As Upward, Reed, Oliver, and Evans have recently argued using Tarde style chaos theory:

... Metadata schemes are essential to control the chaos of the parts but again we tend to shy away from the relativity of expanding complexity. All information professionals understand the simpler whole. Metadata is the quantum mechanics of information management, harnessing and manipulating the atomic structure of recorded information. Information specialists within the diversity of continuum informatics have found it easy to formulate their own metadata schemes relating to the things they used to manage separately within their specializations. The result has been the production of a host of competing schemes that might or might not be relevant within particular applications. Archivists and records managers, for example, have produced records metadata schemes which deal with individual information objects, the sort of things that were once held in paper form on shelves and our now held in digital form on servers, or are accessible using them. ${ }^{12}$ Records metadata helps us produce and place individual objects and is useful, but in the multiverse of metadata schemes, it struggles to gain attention. ${ }^{13}$

11 My knowledge of Cantor, Mandelbrot and Gödel does not extend far past Wikipedia (and Youtube for Mandelbrot) but Lakatos's best-known book is accessible to a nonmathematician such as myself (Imre Lakatos. Proofs and Refutations, Cambridge University Press, 1976).

12 See for example the technical report, ISO 23081-3, Information and Documentation: Managing Metadata for Records, Part 3, Self-Assessment Method (2011).

13 Frank Upward, Barbara Reed, Gillian Oliver and Joanne Evans, "Recordkeeping Informatics: Re-figuring a Discipline in Crisis with a Single Minded Approach" Records Management Journal 1(2013). 
Metadata might be a complex soup as different professions find their own use for it, but for archivists and records managers the continuum goal is clear enough. How can recordkeeping metadata as an archival form of quantum mechanics contribute to revealing time as shadow of itself, clinging to moments in our past, present and future?

\section{Postmodern Relativism}

The explorations of motion found a natural home in sociologies of emergence and Einstein's scientific relativism, but they also found a home in French philosophies during the twentieth century, philosophies often labelled in Anglo-American discourse as postmodern. One starting point for exploring this form of relativism is Henri Bergson's book Creative Evolution. ${ }^{14}$

\section{Henri Bergson (1859-1941)}

In exploring time as "a shadow of itself," archivists have been exposed to the influence of French philosopher Henri Bergson, perhaps unwittingly. Before spacetime thinking became "rocket science," Bergson advanced ideas about creative evolution processes that influenced many other thinkers. Whereas Einstein's contribution came to be locked up within specialisations such as cosmology, nuclear physics, and quantum mechanics, the strand of continuum thinking represented by Henri Bergson came to be associated with discussions of postmodernity.

Bergson can be seen as the father of the idea that "all is archive."15 Though many of his contemporaries were thinking along similar lines of emergence, the concept of creation was central to his theory of evolution, an idea that has survived within the history of ideas. As Gilles Deleuze described, “... at each moment, everything tends to be spread out into an instantaneous, indefinitely divisible continuum, which will not prolong itself into the next instant, but will pass away, only to be reborn in the following instant in a flicker or shiver that constantly begins again." ${ }^{16}$ Archival time is the past, the present and the future combined in that shiver of emergence, a moment when time is a shadow of itself.

14 Henri Bergson, Creative Evolution (New York: Henry Holt and Company, 1911).

15 This idea is explored throughout Frank Upward, Sue McKemmish, and Barbara Reed. "Archivists and Changing Social and Information Spaces: A Continuum Approach to Recordkeeping and Archiving in Online Cultures." Archivaria 72 no. 72 (February 12, 2011). http://journals.sfu.ca/archivar/index.php/archivaria/article/view/13364.

16 Gilles Deleuze, Bergsonism, translated by H. Tomlinson (New York: Zone Books, 1988): 86-87. 
In his book on Bergsonism, Deleuze included a drawing of the flicker, which Stillman and Upward have re-presented as an information continuum model (Figure 6.1). ${ }^{17}$

Figure 6.1: All is Archiving (Stillman and Upward, after Bergson, after Deleuze)

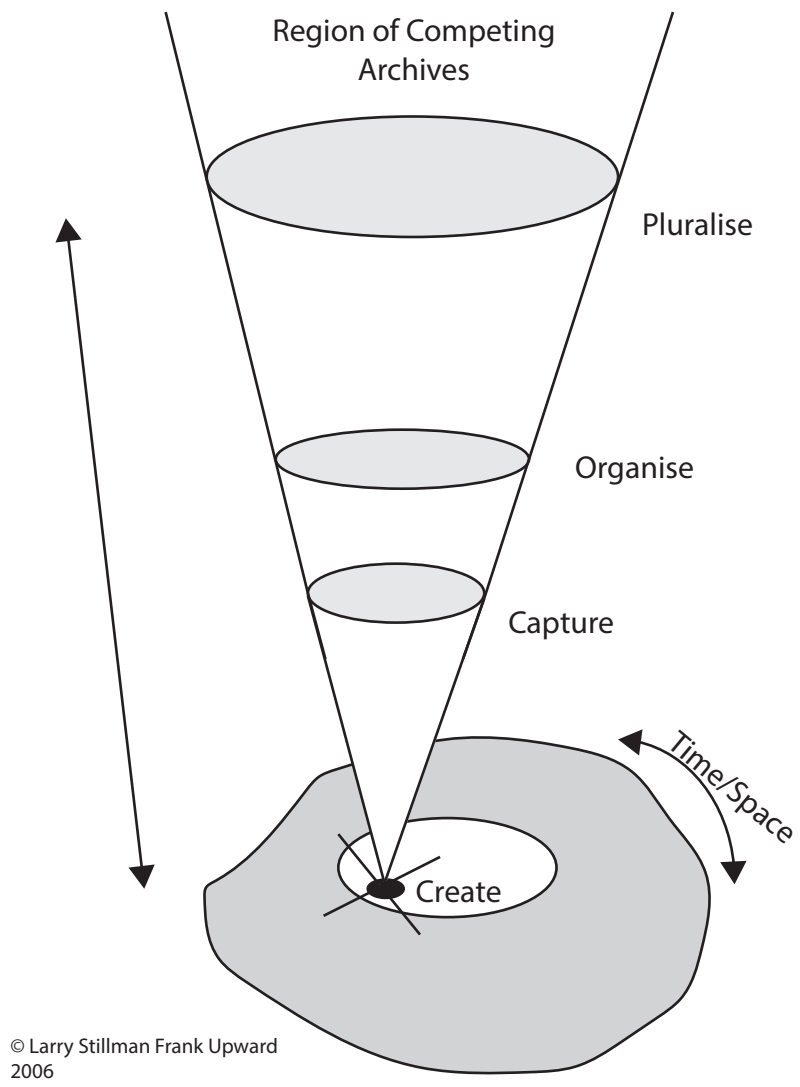

The figure incorporates the Deleuzian (née Bergson) plateau of immanence with a processing continuum. The information continuum element of the cone (the capture, organisation, and pluralisation elements) for archivists should point to the way archival action affects the amplification of a moment that might otherwise be unnoticed amidst billions of other moments. Such

17 The original platonic cone model is present in Deleuze's book on Bergsonism on page 60 and was represented in this new form in Frank Upward and Larry Stillman, "Community Informatics and the Information Processing Continuum" in Larry Stillman and Graeme Johanson (eds), Constructing and Sharing Memory (Newcastle: Cambridge Scholars Publishing, 2007). 
amplification is important to the "flicker" of reconstruction, but for us today, the complexity of managing the exponentially expanding number of such moments cannot be denied.

Bergsonism as a way of thinking has regularly resurfaced in new forms over the years and has entered archival discourse via a host of his genetic descendants who have taken relativist positions in understanding knowledge formation. This link might be identified as the recognition that the archive, in bringing about order through its control mechanisms (often a totalitarian function of the archive), paradoxically produces unexpected outcomes because of its plasticity. Variations of themes relating the archive to chaos and order can be found running across the work of philosophers, including:

- Michel Foucault and the heterotopian archive as a site of liberation and resistance. On the other hand, the restrictive nature of the archiving processes in discourse is regularly used to repress ideas. To understand a discourse and open up the dynamic aspect of its plurality we need to subject its archive to careful archaeological and genealogical scrutiny over long periods of time [archival time?] to identify logical patterns.

- Jacques Derrida and the repressive archive, the impact of otherness upon it, and the need to deconstruct the Nietzschean edifices we construct.

- And Gilles Deleuze, who described what is being called spacetime eddying here as machinic connectivity - the way ideas combine and produce ideas that are dissimilar to their parents, sometimes monstrously so. ${ }^{18}$

Bergson, then, is already represented in archival research frameworks via the ideas of such intellectual descendants who, in North American and Australasian academic literature, were seen as postmodern when postmodernity was at the height of academic fashion in the last few decades of the twentieth century. The trend is not as strong now. Postmodernity topographically (i.e., in particular spacetime) has become a devalued term; it has been used in so many contradictory ways that it has lost its power as

18 Michel Foucault insisted students and colleagues of his read the work of Henri Bergson. Deleuze wrote a book on Bergsonism. Derrida was once asked whether he had a large library to which he joked that, yes, it had many books in it, and that he had even read three of them. One of those properly read books was certain to have been Bergson's Creative Evolution [the Derrida anecdote is from a guest lecture at Monash University in 2011 by Eric Ketelaar using a clip from the documentary film about the philosopher, Derrida, 2002]. 
a label. Topologically, however, as Lyotard points out, postmodernity refers to the post modo, the future anterior. ${ }^{19}$ In a Bergsonian interpretation, the postmodern is the archive as the vanguard of change. It exists creatively in the flicker of archival time, and will always be with us.

\section{Anglo-American Transcendental Pragmatism}

How does one approach the complexity of the multiverse? Anglo-American continuum thinking attempts to address this question pragmatically as we will see in this section, describing ideas "fathered" by three American pragmatists, William James, Charles Peirce, John Dewey, along with two British philosophers, Alfred Whitehead and Samuel Alexander.

Pragmatism can go hand-in-hand with continuum thinking, given that it is by definition an approach based on spacetime realities. The Macquarie Dictionary for example explains that pragmatics treats phenomenon by referring to "their causes, antecedents, conditions and results." ${ }^{20}$ In the most fundamental fashion, it is grounded theory, working at a level that only becomes transcendental when we try to create meta-realities that make sense of chaos, plasticity and indifference across time and in different spaces.

\section{William James (1842-1910)}

William James and two of his colleagues, John Dewey and Charles Peirce, can collectively be seen as the fathers of American pragmatism and all of them acknowledged their debt to Henri Bergson. Pragmatism takes account of the flicker of time and James, as we have seen, gave us the term multiverse to describe the plasticity and moral indifference of its wave motion. James, like many continuum philosophers of his era, was deeply religious and was trying to find ways of thinking about evolution that accepted the role of a supreme deity. We live in an information age that has to deal with the moral multiverse's plasticity and indifference at levels of intensity that James could not even have begun to imagine. How might one establish the integrity and authenticity of those components in that information that can help us use time to conquer time? The archival profession might in its daily life see itself as the provider of information objects to others but what lies beyond that?

19 Jean-François Lyotard, "What is Postmodernity?" in The Postmodern Condition: A Report on Knowledge, trans. by Geoff Bennington and Brian Massumi, (Manchester: Manchester University Press, 1984): 81. There is a thorough contemporary account of the academic diversification of the term postmodern in Margaret Rose, The Post-Modern and the Post-Industrial (Cambridge: Cambridge University Press, 1991): 1-39. 
For James' colleagues, there was a continuum of meaning to be managed using information management principles (Peirce) and a world of expanding transactionality to be democratically governed (Dewey).

\section{Charles Peirce (1839-1914)}

Of the Anglo-American figures being discussed, Peirce has the strongest continuing presence, aided by the way he constantly re-expressed his ideas from different vantage points. This turns reading his work into a mind game that many people still seem to enjoy playing. ${ }^{21}$ At the heart of the game is his semiotic continuum of firstness, secondness and thirdness. There can be no agreed meaning for these terms - Peirce was constantly adjusting his explanations - but at a logical level they can, for archivists, correspond with:

- The firstness of registration processes, which can refer to a control mechanism for managing the way direct information spaces are transferred into indirect information spaces and are documented in ways that can be revivified (not revived, but given new life) at a later time,

- The secondness of indexicality which refers to the signs we use when recording actions and processing information including bringing it back to mind,

- And the thirdness of our interpretative schemes used to fit our ideas into views of the world around us.

Anyone interested in the meanings for the three dimensions of the semiotic continuum will find many different explanations of Peirce's trilogy on the Internet, but the above three elements recur in those explanations.

More formally, I would argue that in relation to the aforementioned Bergsonian view of creation, registration processes help stop the archive from unravelling as it is carried forward, indexicality is part of the recursivity of recall and dissemination, and interpretative schemes control our ways of organising the archive. This sounds simple, but it is complex in operation. The three dimensions are involved with each other and in application can all be very different and have changed over time with the exponential expansion

21 See Charles Peirce http://en.wikipedia.org/wiki/Semiotic_elements_and_classes_of_ signs. The information-based interpretation of his concepts of firstness, secondness, and thirdness was prompted by Frances Morrissey, "Introduction to a Semiotic of Scientific Meaning, and Its Implications for Access to Scientific Works on the Web," Cataloguing E Classification Quarterly, 33 nos. 3/4 (2002): 67-97. The difficulty of accessing Peirce's work is discussed in the Stanford Encyclopedia of Philosophy, http://plato.stanford.edu/ entries/peirce/\#access (The websites were accessed on 25 July 2011). 
in human transactionality and of our information and communication technologies. In other words, the applications of the semiotic continuum are individually far more complex than the whole. That Peirce's continuum is still worth researching, however, is testimony to how well he observed the relationship between the simpler monistic whole and the much greater complexity of its parts and how much continuing logic there is in his intellectually hard won concepts of firstness, secondness and thirdness.

\section{John Dewey (1859-1952)}

The third member of this pragmatic trilogy, John Dewey, researched and wrote across many areas that these days we would regard as separate specialisations. Like many others who were thinking about the evolutionary impact of spacetime, he believed that knowledge was both provisional and could be constructed using scientific methods. Dewey multiplied complexity by focusing on the parts in action; transactionality was an evolutionary motor, driving on change and the expansion of all things. He was an activity-based theorist, whether he was discussing philosophy, politics, the importance of learning through doing (a major contribution of his to the philosophy of education), or any of the other topics that attracted his generalist mind.

Dewey's diverse interests were brought together as a monism not by the Deity (i.e., as understood by James) or the observation point (i.e., as Peirce would have it), but by transactionality. Transactionality is a formative process crucial to the management of the part within the whole/part relationship. In Dewey's hands, it was all about "becoming as becoming." Dewey was an acute observer of the phenomenon of expanding transactionality in the first half of the twentieth century and saw clearly that transactions were both spacetime-specific and could be seen in terms of recursive patterns, patterns that would vary over time but patterns nevertheless.

Dewey set out a complex categorisation of transactions and a simpler tripartite classification of actions. ${ }^{22}$ At the simpler level there is continuum of action involving self-action, interaction, and the transaction. In particular times and places, however, this simple construct allows for expanding complexity as the three elements interact with the expanding nature of, and apparatus for, human transactions. For recordkeepers, Dewey's approach to transactionality can lead to studies of what documentation to create, how robustly to capture that transaction as a record, how to organise it as part of an archive and how to make it part of the plurality of the archival

22 A basic explanation of Dewey's tripartite classification of action is provided in http:// www.newworldencyclopedia.org/entry/John_Dewey accessed 10 November 2012. 
multiverse, a records continuum model that while simple is also capable of great complexity. ${ }^{23}$

\section{Alfred Whitehead (1861-1947)}

While American pragmatists were influenced by Bergson, one of England's best known continuum thinkers, Alfred North Whitehead, was influenced by Tarde. This influence at times seemed to resemble direct duplication, such as when he argued that the universe was an organism in the sense that "each of the vast numbers of actual entities of which it is composed enters in one way or another into the constitution of every other." 24 This is a proposition that one still hears today in arguments about why we need to protect the full diversity of our ecosystems, and in those degrees of separation games by which one can move from something or someone to something or someone else in a relatively few moves.

Whitehead, like Dewey, was an archetype of the wide-ranging thinker of his era. He possessed a breadth of view that enabled him to examine the multiverse. Whitehead thought outside the box more scientifically than Tarde or Dewey as befitted a member of one of England's most famous family of scientists. If Tarde's basic theme was chaos, and Dewey's the expanding nature of transactionality, Whitehead's theme was that the chaos could be managed using scientific method. He advocated applying scientific method across the diversity of studies which might differ in their subject matter but did not need to differ in their methods.

In a modern sterile reading, Whitehead can be allowed to pass into footnote territory as a loser. He was writing before science comprehensively divided itself into a multiplicity of specialisations using a diverse array of methodologies. By the 1970s, according to cogent arguments by Jean François Lyotard and many others, faith in the scientific method had been replaced in knowledge formation by "informationism," a term suggested recently in an article addressing the manner in which the notion that information is power

23 There is a clear connection between Dewey's thinking and the records continuum model published in Frank Upward, "Structuring the Records Continuum, Part One: Postcustodial Principles and Properties," Archives and Manuscripts 24 no. 2, (1996), 268-285. Upward was trained as a teacher in Australia in 1966 at Melbourne University when Dewey's continuum based approach to learning-by-doing was a major source of inspiration.

24 Quentin Gibson, Facing Philosophical Problems (Melbourne: F.W.Cheshire 1961): 37-38. As Gibson noted: "Both writers [Samuel Alexander and A.N. Whitehead] take time seriously. For both of them, the world is a process, and nothing actually falls outside of that process." 
has been taking attention away from how poorly the information object might have been formed as evidence and memory. ${ }^{25}$

The prior references to the similarity of Whitehead's philosophical positioning with the pragmatics of modern approaches to ecology are a useful reminder that ideas can recommence in different frames of reference. As we grapple with digital recordkeeping in modern information ecologies, we should keep a transcendental eye on the whole and a pragmatic eye on the expanding complexity of the parts. Now that faith in Whitehead-style scientific method has receded, perhaps revamped archival methods using time to conquer time can give some scientific coherence to studying the diversity of the archival multiverse?

\section{Samuel Alexander (1859-1938)}

Probably the best known continuum philosopher in England writing about the significance of studying the way time conquers time (incidentally also the theme of T.S. Eliot's poem Burnt Norton) was Samuel Alexander, an Australian expatriate who taught at Manchester University. Einstein published his theory of general relativity at the same time that Samuel Alexander's guest lectures at Glasgow University were being published as Space, Time and Deity. The synchronicity meant that Alexander managed to make cross references to Einstein in the introduction to his book and each author's status presumably benefited by this coincidence. Together, their works constituted a major change in the ways we can think about motion. Einstein helped overthrow major elements of the Newtonian universe, while Alexander helped turn the Aristotelian study of "being as being" (the study of the characteristics of things) into a pragmatic continuum variant, the study of "becoming as becoming."

At the time of the publication of these works, metaphysical philosophy was a popular pursuit and an expected part of a classical education in some countries. The pursuit had yet to be driven back by academic fragmentation into a specialisation within a specialisation, and its practitioners were public celebrities. This was particularly the case in the United Kingdom. Possibly it was their colonial and industrial expansion that made the British educated classes more prone to think about expansion, complexity, and the convergence of spacetime. Indeed when Samuel Alexander died in 1938 a

25 Lyotard gives an impressive list of thinkers who had moved to emphasising information within knowledge formation at the beginning of The Postmodern Condition. The term "informationism" comes from Upward, Reed, Oliver and Evans, "Recordkeeping Informatics: Re-figuring a Discipline in Crisis with a Single Minded Approach.” 
newspaper in his hometown Manchester compared him favourably with Immanuel Kant. ${ }^{26}$

Alexander contributed to the Bergsonian "post modo" continuum eddy which argued that everything was in a state of creative evolution. $\mathrm{He}$ prioritised the need to study things as they form and change over spacetime. An example from his book: if you are going to study the ant, do not forget to study what happens when the ant's nest is poked with a stick. His analogies, however, were more typically drawn from chemistry and Alexander turned to metaphors like the need to mimic the study of "molecules of gas that dash against the sides [of a vessel] and each other in all manner of lines of advance. The gas is not considered as it is at any moment but as it exists over a lapse of time." 27

Alexander provides us with some of the clearest examples for explaining the need to study things in motion. In a rippling example of spacetime eddying and the "few degrees of separation" argument, the main home for modern scholarly records continuum thinking in recent times has been Monash University in Australia, which, when it was founded in the 1960s, named its main lecture theatre after Alexander in recognition of his seminal emphasis upon studying the universe as a theatre of perpetual movement. ${ }^{28}$

Alexander's contribution to philosophy can be subject to a more sterile reading. One can pin him down to his place and time and leave him there as a philosopher of historical interest, as if history has no continuing interest in the use made of Alexander's ideas. In a sterile view, he had a particular desire, common in his age, to associate continuum thinking with the notion of a deity. Alexander brought together Bergson's flicker with the singular complexity of the spacetime continuum to argue a concept of immanence. Science was taking us to a more God-like view of the universe. Like William James, he was trying to impart a spiritual perspective upon the plasticity and indifference of the multiverse. In philosophy, from a narrow linear perspective continuum thinking has moved on but as we will see in the rest of this article, Alexander's ideas continued to eddy around, particularly in Australia.

26 Australian Dictionary of Biography, Volume 7, 1891-1939 (Melbourne: Melbourne University Press, 1979): 33-34.

27 Samuel Alexander, Space-Time and Deity, The Gifford Lectures at Glasgow 1916-1918, (London: Macmillan \& Co., 1920): 63.

28 In naming "The Samuel Alexander Theatre," those doing the naming were probably influenced by John Passmore, One Hundred Years of Philosophy, (London: Hammersworth Penguin, 1994) in which Alexander's once considerable reputation in British philosophy was emphasised and explained in terms of the theatre of perpetual movement. 


\section{Records Continuum Practices}

In 1988, in Hobart at a conference of the Australian Society of Archivists, archival scholar Terry Eastwood argued that ideas about archives reflect intellectual trends, a spacetime perspective that should regularly be included in our research frameworks. ${ }^{29} \mathrm{It}$ is difficult to do so, however, as connections between ideas and archival functions can be difficult to prove. One operates as discursive practice, and the other has to be pinned down non-discursively to what is being done at particular points in spacetime. The connections are always likely to be unknown. The following exposition of the connection between spacetime and records continuum thinking should be read as something like a preliminary archaeological dig, looking speculatively for sites that might require further attention.

\section{Northern Eddies}

So far this essay has focused on North Atlantic views of spacetime, setting up a possible discussion of the divide between Northern and Southern theory, a recent and influential theme in sociology pitting Western European and North American ideas against those from peripheral and colonial societies. ${ }^{30}$ However, before looking at the periphery (continuum practices in Australia) can a case be established that the spacetime explorations from the 1890s to 1930s had much impact on "northern" archival practice in the 1930s?

If we look at Great Britain and Sir Hilary Jenkinson's Manual of Archives Administration, the answer to that question would seem to be yes. The first edition of the manual was produced in 1921 at the height of discussions about the work of Einstein and Alexander in Britain, and the second edition was produced in 1937 when Europe was plunging into chaos in the years leading up to World War II. Jenkinson offered the world the order of the archive. He was interested in source, transmission, and the study of things as they formed. The transcendental pragmatism of the spacetime thinkers with their emphasis upon causes, antecedents, conditions, and results would have, at the very least, confirmed his commitment to what he called "the moral defence of archives." In his concepts of moral and physical preservation, for example, he gave

29 Terry Eastwood, "Reflections on the Development of Archives in Canada and Australia," in Archival Documents: Providing Accountability through Recordkeeping, Sue McKemmish and Frank Upward (eds.) (Melbourne: Ancora Press, 1993): 27-40.

30 See for example Raewyn Connell, Southern Theory: The Global Dynamics of Knowledge in Social Sciences (Sydney and Melbourne: Allen and Unwin, 2007). 
priority to documenting the processes of formation within archival descriptive processes and maintaining the chain of custody so no unauthorised changes to the order of archives could be made. If moral defence was in place it then becomes more meaningful to consider access issues and physical preservation which involve documenting archival formation processes and protecting the physical material against the ravages of movement through time. Wherever Jenkinson's inspirations came from, this is classic Alexandrine continuum thinking, studying archives as they form, re-form, and move across the stage of the theatre of perpetual movement.

In terms of the drive to use time to conquer time, however, a most remarkable development in the 1930s was the formation of the USA's national archival authority, remarkable because of the decade in which it occurred. Today, we have grown accustomed to archival institutions losing funding at times of fiscal crisis, but it would seem that the First World War, followed a decade later by an economic depression, demonstrated the need to tie our archival sources of law, administration, and history together in ways that improved spacetime management. A new national archival institution with links to government business of the day was formed at a time when funding was in short supply, government business was trying to find new directions, and some economists were beginning to suggest the need for a bit of judicious economic pump-priming. What could be more judicious than to allocate some resources to a "future proofing" institution?

In the 1930s and 1940s, notable American federal and state archivists like Phillip Brooks and Margaret Cross Norton wrote about and helped establish management structures for the full lifespan of records and did so in ways that, under the definition of the archival multiverse given at the beginning of this article, were pluralistic. Norton, a librarian, archivist, and administrator, used the term "archives" to refer to official records of government agencies, separating them out from historical manuscripts. However, that did not mean that in her mind the collecting tradition of libraries disappeared from our archival understandings. In accordance with the unpredictable nature of the multiverse and the operation of the eddies in the spacetime continuum, that distinction resurfaced two decades later in the still current but now archaic title given to the Australian archival journal, Archives and Manuscripts. Norton and others might look as if they took the United States down a European path in which the background of archivists as literary scholars, historians and civil servants working in government institutions tended to dominate views of the archival profession, but in both the United States and Australia, the library-based manuscript tradition could not be left 
out of any vision even if at times this more multiverse-based approach has created tensions. ${ }^{31}$

However, the records continuum approach historically became connected in many minds to the dominant government stream of archival practice because that is where people were looking to find ways of future proofing themselves from economic depressions and wars. Ernst Posner, a refugee from Germany, acknowledged the strength of this continuumbased administrative strand of archival thought in the United States when he ended his first article as an American academic archivist with the following passage:

If all the public records of a nation are one sole undivided fonds, the agencies that are destined to receive and keep them ultimately will be justified in claiming the right to give their advice as to how the files of government offices should be organized and kept from the beginning so as to insure a satisfactory original arrangement that will also be suitable for retention by the archives agencies. We may assume that gradually the archivists will become the nation's experts who must be consulted in all questions of public record making and record keeping and likewise become the trustees who will safeguard the written monuments of the past, of the present day, and of the future..$^{32}$

If the Great Depression of the 1930s and the need for a New Deal had stimulated thought about how to use time to conquer time or implement what is now called a records continuum approach, the stimulation did not dissipate in the next few decades, re-enforced by a World War and a general desire in the post-war years not to repeat the mistakes of the past. Posner's dream, in essence, was what began to be implemented as a records continuum approach in Australia during the 1950s and 1960s but in Australia it has existed within a more pluralistic American form than the administrative form imagined by Posner and some of its more recent forms will be discussed at the end of this piece.

31 The archives and manuscripts tradition in Australia and its American connections are examined in Frank Upward, "Association Amongst Archivists in the 1950s" in Frank Upward and Jean Whyte (eds.), Peopling a Profession. (Melbourne: Ancora Press: 1991): 93-106.

32 Ernst Posner, "Some Aspects of Archival Developments since the French Revolution" in A Modern Archives Reader, Maygene Daniels and Timothy Walch (eds.), (Washington D.C.: National Archives and Records Administration, 1984): 14. 


\section{Southern Theory and Future Proofing}

There has been a tendency for Australians to see records continuum practices as Southern theory (theory from a peripheral society) and ask the rest of the world to take into account our discoveries. Such an approach orients the account of Australian continuum practitioner Michael Piggott in his essay, Two Cheers for the Records Continuum. Certainly, Australians have dominated the international literature on the topic as his essay demonstrates. ${ }^{33}$ Australia's national archival institution, however, owed much to the thinking and practices of Great Britain and the United States in the 1930s. ${ }^{34}$ The idea of establishing a national archival authority in Australia had been given massive impetus in that decade through the influence of Sir Hilary Jenkinson at the UK Public Records Office in publicly promoting the role of archives, and by the formation of the United States' National Archives and Records Administration. Australia's Deputy Commonwealth Librarian, Harold White, was dispatched to the United States to study their approach to government archives and he produced a report recommending a similar approach in Australia. ${ }^{35}$ The outbreak of war interrupted developments but some of the things that pleased White included the fact that in the United States the archival profession had strong links to librarianship and NARA had been formed in ways that carefully picked out a distinctive but complementary role focusing on a government National Archives at cultural levels and government transactionality at records services level. When the issue of establishing an archival institution was resumed in the post-war years, White had become the National Librarian and had the chance to implement many aspects of his own report.

Records continuum practices emerged in Australia thanks to White's influence and the work of two archivists, Ian Maclean and Peter Scott. It is also easy to imagine (but harder to prove) that Australian approaches to emergence owe much to Samuel Alexander, a Northern theorist even though he was born in Australia. Alexander departed its shores as a young man undertaking postgraduate studies in England and did not return home.

33 Chapter 12 in Michael Piggott, Archives and Societal Provenance, Australian Essays (Oxford: Chandos Publishing, 2012): 175-196.

34 For example, see Michael Piggott, "Beginnings" in Sue McKemmish and Michael Piggott, The Records Continuum, (Melbourne: Ancora Press, 1994): 1-17, and Upward, "Association Amongst Archivists in the 1950s" (1991).

35 White's paper is reprinted in Australian Institute of Librarians, Symposium on Archives in Australia, [Conference] Proceedings, Canberra, 1942, and discussed in Upward, "Association Amongst Archivists in the 1950s (1991). 
His influence on Australia, however, was vast. By the 1930s, chairs of philosophy along the East Coast of Australia were dominated by Alexander's recommended appointees. Thousands of Australian students over several generations absorbed versions of his process-based spacetime interpretations as interpreted by his disciples, including the author of this article who was influenced by the Gibson brothers, Quentin and Boyce. ${ }^{36}$

For whatever reason, Australians have always produced leading academics at home with philosophies of emergence. That is the sort of boast that needs research-based justification but a few examples will have to suffice here. Some of these scholars include:

- The historian Graeme Davison (who, in an Australian academic joke which crystallises both the strengths and weakness of Michel Foucault, has been said to write the sort of histories that Foucault might have written if he had known any history), ${ }^{37}$

- The political scientist John Keane whose landmark history of democracy advises us to "always regard the languages, characters, events, institutions and effects of democracy as thoroughly historical" (a typically pragmatic Australian continuum approach) ${ }^{38}$

- And before them, the philosopher John Passmore, whose book One Hundred Years of Philosophy is an unsurpassed general history of philosophy from the 1850 s to the 1950 s written very much in a "theatre of perpetual movement" style of someone influenced by Alexander. ${ }^{39}$

In the archival profession, there is Ian Maclean, Australia's chief archivist, who completed a classics degree at Melbourne University towards the end of the 1930s. A decade and a half later while working with the Commonwealth

36 Key enthusiasts for Alexander's philosophy included John Anderson, Chair of the Department of Philosophy at Sydney University, 1927-1953, and the Gibson Family at Melbourne University (across an even longer time span) including father William Ralph Boyce Gibson and his son Alexander Boyce Gibson. An introduction to philosophy written by another son, Quentin Gibson was read (and enjoyed for its pragmatic approach) by thousands of first year university students over the years. Frank Upward's first subject in philosophy at Melbourne University was delivered by A.B. Gibson (at the end of his career) and the required preliminary reading for it was his brother's book.

37 See for example Graeme Davison, The Use and Abuse of Australian History, (Sydney and Melbourne: Allen and Unwin, 2000) and The Unforgiving Minute: How Australia Learned to Tell the Time, (Oxford: Oxford University Press, 1993). In the introduction to the latter, author Ken Inglis makes the Foucault joke.

38 John Keane, The Life and Death of Democracy (New York: W.W. Norton, 2010): 875-876.

39 Passmore, One Hundred Years of Philosophy (1957). 
Public Service Board, he stared hard at the expanding complexity of recordkeeping processes within what was known as the Registrar's Project. Collectively the organisations had analysed patterns of transactions identifying different file types within the government's administrative registries which registered and filed administrative correspondence as it was received and circulated it on files for action. Registries also dispatched all outgoing correspondence, continuing the filing work as they did so. Ian Maclean estimated that only about ten percent of correspondence was registry controlled - although he considered it to be the crucial complex components of office business. Outside of the registries, agency recordkeeping processes relied on the skills and knowledge of in-house operational specialists who drew on their professional backgrounds to establish systems that suited their tasks. ${ }^{40}$

Registrars were appointed to government departments to set up registry systems and to manage the disposition of the series of operational records held outside the registry. Sets of records from both the registry and the operational series could then be transferred to the archival authority whenever it was deemed to be convenient to do. This, crucially from a records continuum perspective, included what was deemed to be temporary records. Within a continuum approach, archival institutions should not lose contact with the management of temporary records. Whereas in Europe and North America, archival institutions offered services for permanent records, the Commonwealth Archives Office also offered services for temporary records on the grounds that the archive was a monistic whole, and even after destruction one should still be able to have some knowledge of the place records once occupied in that whole. In the 1980s, the new organisation, the Australian Archives, was advised by one of those simplifying management review teams of that decade to stay with permanent records as core business and leave temporary records to agencies and in the process lost contact with recordkeeping, ultimately passing Australian leadership in this area to State Records NSW, that state's Recordkeeping Roundtable, and the Records Continuum Research Group at Monash University. ${ }^{41}$

Perhaps co-incidentally, perhaps because of the spacetime eddying of intellectual currents, or perhaps simply as a consequence of applying.

40 See Frank Upward "In Search of the Records Continuum: Ian Maclean's Australian Experience - Essays on Recordkeeping" in Sue McKemmish and Michael Piggott (eds.), The Records Continuum (Melbourne: Ancora Press, 1994): 110-130.

41 See: Recordkeeping Roundtable, http://rkroundtable.org/; State Records, New South Wales, www.records.nsw.gov.au/staterecords; and the Monash University Records Continuum Research Group website, www.infotech.monash.edu.au/research/groups/rcrg. 
continuum-based thinking to information management, the registrar's project echoed Dewey's emphasis upon transactionality and Peirce's secondness and thirdness within the application of its analysis of American records management techniques for indexing and classification schemes. There was a significant difference, however. In the United States, registries had disappeared from the federal government in 1915 after a Congressional report had argued they were too labour intensive for a time of increasing transactionality. ${ }^{42}$ They were replaced by emerging business records management techniques where mail went straight to action officers who then controlled what was sent to records units for filing. Firstness (i.e., registration) disappeared from federal administrative recordkeeping processes.

On the other hand, firstness as a concept temporarily grew stronger in Australia. By the mid-1960s, Peter Scott, the other Australian architect of a continuum approach, had taken the series approach of the registrar's project and had devised a system of control for the transfer of permanent and temporary sets of records to the archives which Ian Maclean quickly approved for implementation. Maclean later noted that Scott's system was the last building block to what has become known as a records continuum approach. The essence of the system was registration. Agencies were registered, as well as the series they produced, and one could establish a matrix of relationships between the agency and the series as they moved through spacetime. Scott and Maclean had unlocked the secrets for a system that managed paperbased records in motion using the series to identify and control the recurring fractals (including fragments transferred to the archives). Scott provided the simpler whole that could sit above the greater complexity and fluidity of the parts, while Maclean had studied the complexity.

To those not used to thinking of archival systems as something that can apply to things in motion, the Maclean/Scott nexus undoubtedly sounds complex unless one has worked in the environment they created before it was emasculated in the $1980 \mathrm{~s} .{ }^{43}$ In that decade within the newly formed

42 The reference to the report came from a note I took in 1993 when reading through microfiche copies of The American Archivist. The note was used in writing an article but has long since disappeared. I am no longer sure if it was a Senate or House of Representatives report but assume it was the Senate. The reason the fact sticks in my mind is the date, 1915, the year I erroneously thought the USA entered the First World War, the sort of cause of increasing transactionality that could lead to registries being abandoned in federal government recordkeeping in the USA in the second decade of the twentieth century replaced by business records management techniques of that era.

43 This is discussed in Frank Upward, Managing the Flicker: Continuum Concepts and the Formation of Archives, Ph.D. Thesis, Monash University (2009), in Chapter Three: The First Generation - Discovering and Losing the Records Continuum. 
Australian Archives, the system was divorced from temporary records and used as a purely custodial system for the transfer and internal management of "permanent" government records. From then on, the other way of disputing the claim that Scott was the deviser of a system for the archival multiverse is to assume that Scott's system was as narrow as the application of it became in its home base. The system, however, was born within both a continuum tradition and an Australian archives and manuscripts tradition, much like the one that Margaret Cross Norton described in the United States in the 1930s. In its design, it was grand in its conceptualisation of the "public record". Rather than merely covering government records and dividing them up into a multiplicity of record groups as most government archival institutions were doing at the time, Scott produced an over-arching archival control system for Commonwealth records that:

- could be applied to series of documents as government records, publications, business records, personal papers, and archives of community groups, other governments, and non-government agencies,

- could be applied at any point in the lifespan of the series wherever it was being formed or held,

- and could be applied to temporary and permanent sets of records from that series which could then be transferred regularly from the agency to external repositories. ${ }^{44}$

If the records continuum approach was as good as some Australians have obviously thought it to be, why was it not immediately accepted as global archival theory rather than seen to be Southern theory, an antipodean curiosity that might have some elements worth grafting on to more mature approaches? That in itself is a research project of worth. We had a system for a multiverse in Australia fifty years ago and it failed to be widely accepted. Why did it fail to be recognised for its continuum-based daring, and what if anything can be learned from its failure?

It did not help globally that while the approach was based on Northern intellectual currents, it became Southern theory. Ian Maclean happened to be visiting the United States in 1958 as part of a global tour when the

44 Adrian Cunningham, The Arrangement and Description of Archives amid Administrative and Technological Change: Essays and Reflections By and About Peter Scott (Brisbane: Australian Society of Archivists, 2010). It includes a copy of Scott's 1966 article on the abandonment of respect des fonds, by which he meant abandoning the principle that dictated that archivists arrange material within records groups in their repositories. 
National Archives and Records Administration formally rejected the continuum's recordkeeping single mind approach (in Posner's terms, a mind which considered both the making of records and their keeping) that had been extended in Australia. ${ }^{45}$ Although no parties at that time were using the term "continuum," its rejection came in the form of the completeness of the recognition of the role of records managers in the "life-cycle" of records and the acceptance of a time-dictated role for the archives as an institution whose core business became centred on looking after records after their administrative use had died. That idea was dominant in Europe where archives might be seen as arsenals of law and history, but the focus on current administrative procedures was weaker than it had been in the United States. Maclean had derived his approach from reports on American practice and, not surprisingly, was disappointed. As he wrote to Dr. Wayne Grover of the United States' National Archives and Records Administration, the "majority of archivists [in Australia] have ceased to be concerned primarily with collecting the records of the past for use by the present generation and are now concerned with organizing the records of the present for use in the immediate future." The Commonwealth Archives Office had completed a large amount of salvage work on Commonwealth records and was turning its attention to current recordkeeping processes. It must have been disconcerting to Maclean to see the institutional model for so much Australian archival thinking turning its back on what Maclean regarded as the future of his organisation. ${ }^{46}$ It left Australia professionally isolated and turned a northern intellectual current into Southern theory.

\section{Conclusion}

Within archival education programs, it is possible to use the archival multiverse as a way of expressing plurality and teach elements of that plurality while successfully ignoring the chaos inducing aspect of the archival multiverse itself. In research, however, the effects of plurality need to

45 For a modern approach to the recordkeeping single mind, see Upward, Reed, Oliver and Evans, "Recordkeeping Informatics: Re-figuring a Discipline in Crisis with a Single Minded Approach."

46 See Ian Maclean, "Comments for Dr. Grover Following Visit to the National Archives and Records Services (Based in discussions with Dr Bahmer)", First Report on Overseas Scholarship Programme Containing a General Description of Scholarship Activities and Comments on Archives Institutions Visited, (Canberra, 1959), Appendix 1. See also Upward, "In Search of the Records Continuum, Ian Maclean's Australian Experience Essays on Recordkeeping” (1994). 
be looked at closely if we are to manage Tarde's "confusing plasma composed of myriads of monads, a chaos, a brew." In other words, from a research perspective, expanding complexity should not be ignored and in another piece in this volume, Leisa Gibbons looks at social media from that perspective. Other developments in digital recordkeeping such as cloud computing also need understandings of how complexity multiplies and take us in to the need to identify new patterns as they emerge.

Adding to the complexity, William James' theories about the multiverse cannot be ignored by archivists. The quarrel he and Bergson had with Darwinian theories as they and Samuel Alexander tried to cement God's place in evolutionary thinking no longer carries that much weight in philosophy, but archivists cannot dodge their own ethical multiverse conundrum.

This piece began by referencing the problem of determining what we can know about something which is not only unknown but possibly unknowable. The eddying of spacetime occurs in the spaces in between ideas and in between events and does not leave clear traces of many of the connections with one obvious exception: archival documents as a record of interaction. This means that while the evolution of our information and communication technologies is often plastic and indifferent, archivists still need to strive to fashion ethical approaches to forming archives. We might no longer be as willing to see archival institutions as the major arsenals of law, administration, and history as we once did, but how we form and maintain archives remains a major way of judging the integrity of our actions as individuals, groups, or organisations.

It might be old-fashioned (neo-Jenkinsonian even) but the formation of archives is still a major ethical issue. Can we re-invent archival practices to take account of the much more complex information ecologies of this century without losing contact with archival ethics? Former second generation Australian continuum archivists at Monash University, Sue McKemmish, Livia Iacovino and myself, have tried to adjust to the archival multiverse in such fashion by engaging with an informatics approach. Informatics as a term covers studies of the computational, cognitive and social aspects of the way we represent, process, and communicate information. The term can be applied to managing the multiverse as a whole. When it comes to the parts, three major strands have emerged which have some broad correlation with the older archival trilogy of history, administration, and the law.

One predominantly historical strand (in the all is archive sense) is community informatics, which is not just an Australian development of course. In the United States and Great Britain, it is increasingly present in archival 
studies that address community archiving processes and more recently, in research on the archival multiverse. In Australia, one focus has been on indigenous archives while, as Leisa Gibbons contribution elsewhere indicates, the Scott tradition of description is receiving recommencement within explorations of multiple provenance and co-creatorship emphasising the cross community significance of archival formation in modern information ecologies. ${ }^{47}$

A second seemingly administrative strand, recordkeeping informatics, is under construction. It is emerging from the collaboration of a few Australasian archivists and educators and aims to re-establish recordkeeping as a specialisation based on using time to conquer time while making this role clearer to other information management specialists. It combines recordkeeping metadata techniques and continuum thinking with project based analyses of information cultures, business processes and access. ${ }^{48} \mathrm{While}$ on the surface this seems like a records services approach most applicable to in-house business and government operations, in a continuum approach the archive is all the one whole (a multiverse) and recordkeeping processes have major ethical implications for all forms of archival formation and access.

The third law related strand is juridical informatics. It is necessarily international and is the most complex. As Livia Iacovino has written in the abstract for an article published in Spanish:

Adherents to the records continuum recognise that the authenticity of the record can be protected in multiple ways indefinitely, including outside the walls of an archival institution. However, the sheer quantity and dispersal of digital information in personal and public domains make it difficult to evaluate its authenticity. Archivists will need to think creatively about the nature of a record, the impact of social media on user expectations, trusted digital repositories that protect the authenticity of government and non-government records for accountability, restorative justice and identity, and greater user participation in the management of a self-authenticating archive. ${ }^{49}$

47 See Sue McKemmish's contributions relating to Indigenous archives in Upward, McKemmish, and Reed, "Changing Spaces" (2011).

48 Upward, Reed, Oliver and Evans, "Recordkeeping Informatics: Re-figuring a Discipline in Crisis with a Single Minded Approach."

49 Livia Iacovino, "User Participation and Archival Activism: Cornerstones of Accountability, Restorative Justice and Identity in the Self-authenticating Digital Archive," Tabula 16 (2013): 103-121. 
In short, informatics in this triple approach is a monism which can then be developed in specialised ways to match the expanding complexity of the parts. Perhaps digital recordkeeping and the way it is exposing the archival multiverse will give us the possibility of pushing archival activity back to the forefront of efforts to develop more ethical information resource management techniques based on the significance of archives to history, administration and the law.

\section{Acknowledgements}

Leisa Gibbons contributed the concept of spacetime eddying and played a role in developing the ideas in this piece and I am grateful for her assistance as I am for Andrew Lau's editorial work.

\section{References}

Alexander, Samuel. Space, Time, and Deity: The Gifford Lectures at Glasgow, 1916-1918. London: Macmillan and Company, 1920.

Bergson, Henri. Creative Evolution. Translated by Arthur Mitchell. New York: Henry Holt and Company, 1911. https://www.gutenberg.org/files/26163/26163-h/26163-h.htm.

Blair, David. “Testing the Theory: Taking Einstein to Primary Schools.” The Conversation. Accessed April 27, 2015. http://theconversation.com/testing-the-theory-takingeinstein-to-primary-schools-9710.

Connell, Raewyn W. Southern Theory: Social Science and the Global Dynamics Of Knowledge. Cambridge: Polity, 2007.

Cunningham, Adrian, ed. The Arrangement and Description of Archives amid Administrative and Technological Chang : Essays and Reflections by and about Peter J. Scott. Brisbane: Australian Society of Archivists, 2010.

Davison, Graeme. The Unforgiving Minute: How Australia Learned to Tell the Time. Melbourne ; New York: Oxford University Press Australia and New Zealand, 1993.

Davison, Graeme. The Use and Abuse of Australian History. Sydney: Allen \& Unwin, 2000.

Eastwood, Terry. "Reflections on the Development of Archives in Canada and Australia." In Archival Documents: Providing Accountability through Recordkeeping, edited by S. McKemmish and Frank Upward (Melbourne: Ancora Press, 1993), 27-40.

Gibbons, Leisa. "Engaging Complexity: Social Media as Cultural Heritage.” Doctoral thesis, Monash University, 2014.

Gibson, Quentin. Facing Philosophical Problems. Melbourne and London: F.W. Cheshire, 1948.

Iacovino, Livia. "User Participation and Archival Activism: Cornerstones of Accountability, Restorative Justice and Identity in the Self-Authenticating Digital Archive." Tabula 16 (2013): 103-21.

International Standards Organization. "Information and Documentation: Managing Metadata for Records, Part 3, Self-Assessment Method," 2011. http:/www.iso.org/ iso/catalogue_detail.htm?csnumber=57121.

“John Dewey." New World Encyclopedia. Paragon House Publishers, 2013. http://www. newworldencyclopedia.org/entry/John_Dewey. 
Keane, John. The Life and Death of Democracy. New York: W. W. Norton \& Company, 2009.

Lakatos, Imre, John Worrall, and Elie Zahar, eds. Proofs and Refutations: The Logic of Mathematical Discovery. Cambridge: Cambridge University Press, 1976.

Latour, Bruno, Pablo Jensen, Tommaso Venturini, Sébastian Grauwin, and Dominique Boullier. "'The Whole Is Always Smaller than Its Parts' - a Digital Test of Gabriel Tardes' Monads." The British Journal of Sociology 63, no. 4 (December 1, 2012): 590-615.

Lyotard, Jean-Francois, and Fredric Jameson. The Postmodern Condition: A Report on Knowledge. Translated by Geoff Bennington and Brian Massumi. 1st edition. Minneapolis: University Of Minnesota Press, 1984.

Maclean, Ian. First Report on Overseas Scholarship Programme Containing a General Description of Scholarship Activities and Comments on Archives Institutions Visited. Canberra: National Archives of Australia, 1959.

Macquarie Dictionary: Australia's National Dictionary. 5th ed. Sydney: Macmillan, 2009.

McKemmish, Sue. “Are Records Ever Actual?” In The Records Continuum: Ian MacLean and Australia Archives First Fifty Years, edited by S. McKemmish and Michael Piggott (Canberra, Australia: Ancora Press, 1994), 187-203.

McKemmish, Sue, and Michael Piggott, eds. The Records Continuum: Ian Maclean and Australian Archives - First Fifty Years. Canberra, Australia: Ancora Press, 1994.

"Minkowski Space." Wikipedia, the Free Encyclopedia, March 21, 2015. http://en.wikipedia. org/w/index.php?title=Minkowski_space\&oldid=652864205.

Morrissey, Frances. "Introduction to a Semiotic of Scientific Meaning, and Its Implications for Access to Scientific Works on the Web.” Cataloging E Classification Quarterly 33 no. 3-4 (August 1, 2002): 67-97. doi:10.1300/J104v33n03_05.

Nairn, Bede, and Geoffrey Serle, eds. Australian Dictionary of Biography, Volume 7, 18911939, A-CH. Carlton South, Victoria: Melbourne University Press, 1979.

Passmore, John. A Hundred Years of Philosophy. Harmondsworth, Middlesex, England: Penguin Books Ltd, 1994.

Piggott, Michael. Archives and Societal Provenance: Australian Essays. 1 edition. Oxford: Chandos Publishing, 2012.

Pluralizing the Archival Curriculum Group (PACG). "Educating for the Archival Multiverse.” The American Archivist 74 no. 1 (April 1, 2011): 69-101.

Posner, Ernst. "Some Aspects of Archival Development since the French Revolution.” In A Modern Archives Reader: Basic Readings on Archival Theory and Practice, edited by Maygene F. Daniels and Timothy Walch. Washington, D.C: National Archives and Records Administration, 1984.

Rose, Margaret A. The Post-Modern and the Post-Industrial: A Critical Analysis. Cambridge England ; New York: Cambridge University Press, 1991.

"Semiotic Elements and Classes of Signs." Wikipedia, the Free Encyclopedia, February 24, 2015.http://en.wikipedia.org/w/index.php?title=Semiotic_elements_and_classes_of_ signs\&oldid=648705036.

Upward, Frank. "Association amongst Archivists in the 1950s." In Peopling a Profession: Papers from the Fourth Forum on Australian Library History, Monash University, 25 and 26 September 1989, edited by Frank Upward and Whyte (Melbourne: Ancora Press, 1991), 93-106.

Upward, Frank. "In Search of the Continuum: Ian MacLean's 'Australian Experience' Essays on Recordkeeping." In The Records Continuum: Ian MacLean and Australia Archives First Fifty Years, edited by Sue McKemmish and Michael Piggott (Canberra, Australia: Ancora Press, 1994), 110-30. 


\section{Chapter 6}

Upward, Frank. "Managing the Flicker: Continuum Concepts and the Formation of Archives.” Doctoral thesis, Monash University, 2009.

Upward, Frank. "Modelling the Continuum as Paradigm Shift in Recordkeeping and Archiving Processes, and beyond: A Personal Reflection." Records Management Journal 10 no. 3 (December 1, 2000): 115-39.

Upward, Frank. "Structuring the Records Continuum (Part One): Postcustodial Principles and Properties." Archives and Manuscripts 24 no. 2 (1996).

Upward, Frank, Sue McKemmish, and Barbara Reed. "Archivists and Changing Social and Information Spaces: A Continuum Approach to Recordkeeping and Archiving in Online Cultures." Archivaria 72 no. 72 (February 12, 2011). http://journals.sfu.ca/ archivar/index.php/archivaria/article/view/13364.

Upward, Frank, Barbara Reed, Gillian Oliver, and Joanne Evans. "Recordkeeping Informatics: Re-Figuring a Discipline in Crisis with a Single Minded Approach.” Records Management Journal 23 no. 1 (2013): 37-50.

Upward, Frank and Stillman, Larry. "Community Informatics and the Information Processing Continuum.” In Constructing and Sharing Memory: Community Informatics, Identity and Empowerment, edited by Larry Stillman and Graeme Johanson (Newcastle, UK: Cambridge Scholars Publishing, 2007), 262-74.

Wilkinson, Tim. "The Multiverse Conundrum." Philosophy Now 89 (2012): 35-38. 
Chapter 7

\title{
ARCHIVAL TURNS AND RETURNS
}

\author{
Studies of the Archive
}

\author{
Eric Ketelaar
}

\begin{abstract}
The past fifteen years have witnessed a growing multidisciplinary interest in different aspects of archives and archival phenomena. The most notable movement was the "archival turn" in various disciplines. Outside the traditional boundaries of archival science (archivistics) a new concept of "the archive" has been embraced by anthropologists, sociologists, psychologists, philosophers, cultural and literary theorists, and artists. This paper charts the paradigms of various "archiviologies" as caused by the "archival turn", and suggests how these could yield "archival returns" or new perspectives enriching archival theory, methodology, and practice.
\end{abstract}

\section{Introduction}

The past fifteen years have witnessed a growing multidisciplinary interest in different aspects of archives and archival phenomena. The most notable movement was the "archival turn" in various disciplines. One of the archival turns entailed a move from archives as sources to archives as epistemological sites and the outcome of cultural practices (see section 2). Another archival turn engages with the archive as a metaphor, often leading to ontological reframing of the archive (see section 3). To the extent that archivistics adopts these reconceptualisations one can speak of an "archival return" in exchange for the use of archivistics' concepts by other disciplines (see section 4). Archivistics is inspired by the way other disciplines approach the archive(s). One example is the archive viewed as art (and art viewed as archive) (see section 5). Some of these extra-archivistics approaches have, through 
the adoption of methods (in particular research methodologies, as other chapters of this book attest), a great but more formal effect on archivistics (see section 6). On the other hand, "turns" in other disciplines may have an intrinsic or material effect on the ontology of the archive(s), inevitably leading to the adoption of concepts. I will deal in section 7 with this influence from respectively a linguistic turn, a social turn, a performative turn, and a representational turn. In the final section, I will conclude that archival turns and returns challenge archival practice and archival theory to understand and apply a multiform approach of archive(s) "as it is" in conjunction with treating phenomena "as archives." The impact of these turns and returns on the archivistics multiverse will hopefully increase as more students and scholars coming from various disciplines engage with the archivistics domain. But equally important is the engagement by archivists with disciplines who have made the archival turn but who rarely recognise what archival practice can contribute to their discourse.

The main method used for this study is content and discourse analysis in the framework of an interpretivist research paradigm and a research methodology inspired by records continuum thinking, allowing "for broad definitions of what is a record and for postcustodial, postcolonial notions of the Archive." ${ }^{2}$

\section{What is the Archive?}

Let us not begin at the beginning, nor even at the archive.

But rather at the word "archive"... ${ }^{3}$

Archief - archive(s) as a whole of documents were defined in the Manual for the Arrangement of Description of Archives (1898) right "at the beginning as the foundation upon which everything must rest." The "everything"

1 Luciana Duranti and Giovanni Michetti, in their chapter "The Archival Method" in this volume, acknowledge that archivistics research "focuses on objects, methods and activities that are already recognized as part of the archival field, while other research aims at increasing archival knowledge through the investigation of different domains and/or the use of different methodologies."

2 Sue McKemmish and Anne Gilliland, "Archival and Recordkeeping Research: Past, Present and Future," in Research Methods: Information, Systems and Contexts, ed. Kirsty Williamson and Graeme Johanson (Prahran: Tilde, 2013): 93.

3 Jacques Derrida, Archive Fever: A Freudian Impression, trans. Eric Prenowitz (Chicago and London: University of Chicago Press, 1996), 1.

4 Samuel Muller, Johan A. Feith and Robert Fruin. Manual for the arrangement and description of archives, trans. Arthur H. Leavitt (Chicago: Society of American 
that the Dutch Manual treated was the origin and composition of archival repositories, followed by a methodology of and practical guidelines for the arrangement and description of archival documents. The definition of the Manual did cover written documents, drawings, and printed matter, but not photographs, nor the archives of families and individuals. ${ }^{5}$ These limitations have led to redefinition over the course of the years. Still, the core of the 1898 definition has been kept intact: archives are an organic whole of documents received, produced, and set aside in the transaction of public or private business, as instruments and by-products of such activity. Archives differ from collections in that they are provenance-bound and have originated not artificially but organically. ${ }^{6}$ However, archives are like collections in that (in multiverse thinking) they are constructed by the creator and any subsequent curator and user. Throughout this chapter the terms archive(s) and record(s) are used to denote "information created, received, and maintained as evidence and/or as an asset by an organization or person, in pursuance of legal obligations or in the transaction of business or for its purposes, regardless of medium, form or format."7

As in archival methodology and practice, the theory of archivistics has archive(s) as its object. ${ }^{8}$ Archivistics focuses on the archive(s) as an end, not as means, like the historian or administrator or any other user would consider archives. Archivists have been emancipated from being a subclass of the historian's profession and with this, archivistics has claimed its place as an autonomous discipline. ${ }^{9}$ However, archivistics is not a historical stranger

Archivists, 2003): Section 1. In Dutch archival terminology, as in a number of other languages, "archival documents" include records.

5 Terry Eastwood, "A Contested Realm: The Nature of Archives and the Orientation of Archival Science," in Currents of Archival Thinking, ed. Terry Eastwood and Heather MacNeil (Santa Barbara: Libraries Unlimited, 2010), 7.

6 According to the Canadian Rules for Archival Description a collection is "An artificial accumulation of documents of any provenance brought together on the basis of some common characteristic, e.g. way of acquisition, subject, language, medium, type of document, name of collector, which may be treated for descriptive purposes as a unit under a common title." James Currall, Michael Moss and Susan Stuart, "What is a Collection?" Archivaria 58 (2004): 138.

7 ISO 30300. Information and Documentation-Records Management-Part 2: Guidelines (Geneva, International Standards Organization, 2011): 3.1.7.

8 Eric Ketelaar, "Archivistics: Science or Art?" in The Future of Archives and Recordkeeping: A Reader, ed. Jennie Hill (London: Facet, 2011): 89-100. See also in this volume Luciana Duranti and Giovanni Michetti, "The Archival Method."

9 Luciana Duranti, "Archival Science," in Encyclopedia of Library and Information Science, vol. 59 suppl. 22, ed. A. Kent (New York/Basel: Marcel Dekker, 1997): 1-19; Theo Thomassen, "The Development of Archival Science and its European Dimension," in 
because the discipline includes historical scholarship dealing with the history of archives, archiving, and the archival profession..$^{10}$

New concepts of "the archive" have been embraced by anthropologists, sociologists, psychologists, philosophers, cultural and literary theorists, artists, and others. Their different approaches of the archive contribute to a Derridean "archiviology":

a word that does not exist but that could designate a general and interdisciplinary science of the archive ... of everything that can happen to the economy of memory and to its substrates, traces, documents ... ${ }^{11}$

Derrida's influence on the archivistics discourse cannot be overestimated: no scholar in our field can fail to engage with Derrida's work (not just with the first few pages of Archive Fever but, as Brothman and Harris did, with much more of his oeuvre). And this is precisely because "when Derrida writes about archives, the discussion stretches beyond and beneath our profession's conception of archives. ${ }^{12}$ A discipline that sticks to the canonical terminology ("the archive' is not an archive(s) according to the profession's conception of archives") can never renew itself. ${ }^{13}$

\section{Archival Turn I}

The use of archives as such is of interest to scholars of archivistics and archivists. But just as reading books is not part of librarianship, doing history or whatever else with archival documents does not solely belong to archivistics. Anthropologist Ann Stoler called this move from archives-assources to archives-as-subject the archival turn. This turn - towards archiving

The Archivist and the Archival Science: Seminar for Anna Christina Ulfsparre... (Lund: Landsarkivet, 1999), 67-74; Theo Thomassen, "A First Introduction to Archival Science," Archival Science 1 (2001): 373-85; Francis X. Blouin and William Rosenberg, Processing the Past: Contesting Authority in History and the Archives (Oxford: Oxford University Press, 2011).

10 Terry Cook, "The Archive(s) is a Foreign Country: Historians, Archivists, and the Changing Archival Landscape," American Archivist 74 (2011): 600-32. The history of archives and archiving has recently attracted the interest of archivists and historians, each group risking to reach only fellows in their own domain. See also footnote 36.

11 Derrida, Archive Fever, 34.

12 Brien Brothman, "Declining Derrida: Integrity, Tensegrity, and the Preservation of Archives from Deconstruction," Archivaria 48 (1999): 66.

13 Alexandrina Buchanan, "Strangely Unfamiliar: Ideas of the Archive from Outside the Discipline," in The Future of Archives and Recordkeeping. A Reader, ed. Jennie Hill (London: Facet, 2011), 55. 
as a process rather than archives as things - which was signalled by Nicholas Dirks as early as 1997 at a Bellagio conference ${ }^{14}$ - has led her and her colleagues to critically reflect:

On the making of documents and how we choose to use them, on archives not as sites of knowledge retrieval but knowledge production, as monuments of states as well as sites of state ethnography. This is not a rejection of colonial archives as sources of the past. Rather, it signals a more sustained engagement with those archives as cultural artifacts of fact production, of taxonomies in the making, and of disparate notions of what made up colonial authority. ${ }^{15}$

Stoler presented this view of archives as epistemological sites rather than as sources on a number of occasions, three of them events of extraordinary importance for the discipline of archivistics: the Sawyer seminar Archives, Documentation, and Institutions of Social Memory, the journal Archival Science, and the book Refiguring the Archive. In 2000-2001, the Sawyer seminar brought some 70 scholars from fifteen countries to the University of Michigan in Ann Arbor (USA) in twenty-eight sessions. ${ }^{16}$ The participants came from various disciplines in the humanities and social sciences, less than one-third being scholars and practitioners in archivistics. The point of departure of the seminar's organisers, Fran Blouin and Bill Rosenberg, was "a conception of archives not simply as historical repositories but as a complex of structures, processes, and epistemologies." ${ }^{\prime 7}$ Ann Stoler's two papers convincingly argued for the archival turn, and in several other presentations the archival turn was visible. Stoler's paper "Colonial Archives and the Arts of Governance" was subsequently published in the second volume (2002) of Archival Science. The journal (founded in 2001) marked

14 Nicholas B. Dirks, "Annals of the Archive: Ethnographic Notes on the Sources of History," in From the Margins. Historical Anthropology and Its Future, ed. Brian Keith Axel (Durham/London: Duke University Press, 2002): 47-65.

15 Ann L. Stoler, "Colonial Archives and the Arts of Governance," Archival Science 2 (2002): 90-91; Ann L. Stoler, "Colonial Archives and the Arts of Governance. On the Content in the Form," in Refiguring the Archive, ed. Carolyn Hamilton et al. (Dordrecht/Boston/London: Kluwer Academic Publishers, 2002): 85.

16 Francis X. Blouin and William G. Rosenberg, eds. Archives, Documentation, and Institutions of Social Memory. Essays from the Sawyer Seminar. (Ann Arbor: University of Michigan Press, 2006). Ann Stoler's two papers (she was one of the few participants with a double bill) were later published and evolved into a chapter "The Pulse of the Archive" of Ann L. Stoler, Along the Archival Grain. Epistemic Anxieties and Colonial Common Sense. (Princeton and Oxford: Princeton University Press, 2009). 
the emancipation of archivistics as an autonomous scholarly discipline. ${ }^{18}$ Its second volume consisted of two issues on the theme of "Archives, Records, and Power." The guest editors, Terry Cook and Joan Schwartz, argued in their introduction that archives "are not passive storehouses of old stuff, but active sites where social power is negotiated, contested, confirmed." ${ }^{19}$ This argument was at the heart of the book Refiguring the Archive, published in the same year and by the same publisher as the two issues of "Archives, Records, and Power" in Archival Science. ${ }^{20}$ The book Refiguring the Archive was a reflection of and an extension of the seminar with the same title, hosted in 1998 by the South-African University of Witwatersrand in conjunction with four archival institutions. The thirteen sessions of the seminar attracted speakers and discussants from a wide range of disciplines and professions. In their introduction to Refiguring the Archive, editors Carolyn Hamilton, Verne Harris and Graeme Reid stressed the constructedness of archives, not simply as sources but as sites of contested knowledge:

Today scholars pay greater attention to the particular processes by which the record was produced and subsequently shaped, both before its entry into the archive, and increasingly as part of the archival record. ${ }^{21}$

One of the seminar's sessions was conducted by Jacques Derrida, speaking not only about (and elaborating on) his Archive Fever but also (and primarily) on the current situation of the archive in South Africa and the challenge of the archive of testimonies gathered by the Truth and Reconciliation Commission. ${ }^{22}$ As I argued, Derrida's influence on the archivistics discourse cannot be overestimated. However, here one has to ask to what extent Derrida has been instrumental in making the archival turn. Ann Stoler contends, "Derrida's splash came only after the archival turn had already been made." ${ }^{23}$ Her witnesses are, among others, Thomas Richards' The Imperial Archive and Roberto Gonzalez Echevarria's Myth

18 Buchanan, "Strangely unfamiliar," 39.

19 Joan M. Schwartz and Terry Cook, "Archives, Records, and Power: The Making of Modern Memory," Archival Science 2 (2002): 1-19.

20 Carolyn Hamilton, et al., eds. Refiguring the Archive (Dordrecht/Boston/London: Kluwer Academic Publishers, 2002).

21 Hamilton, Refiguring the Archive, 9.

22 Derrida, Archive Fever; Jacques Derrida, "Archive fever. A seminar..," in Refiguring the Archive, ed. Carolyn Hamilton et al. (Dordrecht/Boston/London: Kluwer, 2002): 38-80.

23 Stoler, "Colonial Archives," 92; Stoler, "On the Content in the Form," 86. 
and Archive. ${ }^{24}$ Whether or not these scholars can be regarded as champions of the archival turn, many other writers and other disciplines "discovered" the archives-as-subject rather than archives-as-sources only at the behest of Derrida.

Ann Stoler's move from extraction from the archive to an ethnography of the archive has been followed in studies of documentary practices by anthropologists, psychologists, and sociologists. One of Stoler's students, Penelope Papailias, published ethnographies of archival construction, exploring personal rather than government archives as social worlds. ${ }^{25}$ She demonstrates how a textual anthropology enables and is enabled by a theory of the archive as an end rather than as a means. She deconstructs the archive "as monument (or antimonument) projecting a particular group's power, cohesion, and claim on place. ${ }^{26}$ Ilana Feldman, another student of Stoler, studied filing as a regime of ruling practices, arguing that files "have interests which govern their content and their form." ${ }^{27}$ Gregory Rawlings studied "documentary aesthetics" in the practices of annotating files that effectively reshape their political and documentary forms. ${ }^{28}$

Ethnography of documentary practices and documentary practices in ethnography were brought together by anthropologist Annelise Riles and her colleagues, claiming that:

to study documents, then, is by definition also to study how ethnographers themselves know. The document becomes at once an ethnographic object, an analytical category, and a methodological orientation. ${ }^{29}$

24 Thomas Richards, The Imperial Archive: Knowledge and the Fantasy of Empire (London: Verso, 1993); Roberto G. Echevarria, Myth and Archive: A Theory of Latin American Narrative (Durham/ London: Duke University Press, 1998).

25 Penelope Papailias, Genres of Recollection: Archival Poetics and Modern Greece (New York: Palgrave MacMillan, 2005); Penelope Papailias, "Writing Home in the Archive: 'Refuge Memory' and the Ethnography of Documentation," in Archives, Documentation, and Institutions of Social Memory. Essays from the Sawyer Seminar, ed. Francis X. Blouin and William G. Rosenberg (Ann Arbor: University of Michigan Press, 2006): 402-16.

26 Papailias, Genres, 227.

27 Ilana Feldman, Governing Gaza. Bureaucracy, Authority, and the Work of Rule, 1917-1967 (Durham/London: Duke University Press, 2008): 45.

28 Gregory Rawlings, "Statelessness, Citizenship and Annotated Discriminations: Meta Documents and the Aesthetics of the Subtle at the United Nations," History and Anthropology 22 (2011): 461-79.

29 Annelise Riles, ed. Documents. Artifacts of Modern Knowledge (Ann Arbor: University of Michigan Press, 2006): 7. 
Their research confirmed (what proponents of the records continuum model had already accepted) that "moments of document creation anticipate future moments in which documents will be received, circulated, instrumentalized, and taken apart again., ${ }^{30}$ Riles and her colleagues are

interested in how diverse types of agency are produced, stretched, or abbreviated through the medium of the document; in short, in the responses, human and nonhuman, that documents demand or offer up. ${ }^{31}$

This echoes Bruno Latour's discussion of documentary practices of scientists, engineers, and lawyers ${ }^{32}$ and Sellen and Harper's ethnographic research of the use of paper in the mythical paperless office. ${ }^{33}$

Scholars of literary and media studies have made the archival turn too, in their engagement with "archival poetics", or the creation and construction of the archive. In 1999, a whole issue of Studies in the Literary Imagination was devoted to the poetics of the archive. ${ }^{34}$ Three years later, media theorist Nanna Verhoeff reflected on archival poetics - as making, forging underpinning the relationship between the real archive and the conceptual metaphor of "the archive" as a cultural model. ${ }^{35}$

Lastly and at last, historians have begun an archival turn by turning their attention to the agency of the archive. ${ }^{36}$ This has taken the form of historical

30 Riles, Documents, 18.

31 Riles, Documents, 21.

32 Bruno Latour, Science in action. How to follow scientists and engineers through society (Cambridge Mass.: Harvard University Press, 1987); Bruno Latour, La fabrique du droit, transl.: The Making of Law: An Ethnography of the Conseil d'Etat (Cambridge: Polity, 2002). See also Geoffrey C. Bowker, Memory Practices in the Sciences (Cambridge Mass./London: MIT Press, 2005); Eric Ketelaar, "Exploration of the archived world: from De Vlamingh's Plate to digital realities," Archives and manuscripts 36 (2) (2008): 13-33.

33 Abigail Sellen and Richard Harper, The Myth of the Paperless Office (Cambridge Mass./ London: MIT Press, 2002).

34 Paul J. Voss and Marta L. Werner, "Toward a Poetics of the Archive: Introduction," Studies in the Literary Imagination 32 (1999): i-viii.

35 Nanna Verhoeff. "Archival Poetics," Screening the Past: An International Electronic Journal of Visual Media and History 14 (2002), https://web.archive.org/ web/20021124213805/http://www.latrobe.edu.au/screeningthepast/firstrelease/ fr0902/nvfr14d.htm, accessed March 23, 2015, repr. in Narrative Theory. Critical Concepts in Literary and Cultural Studies, vol. 2, Mieke Bal, ed. (New York: Routledge, 2004): 388-410.

36 Ann Blair, ed., "Toward a Cultural History of Archives," special issue of Archival Science 7, no. 4 (2007); and Randolph C. Head, ed., "Archival Knowledge Cultures in Europe, 1400-1900," special issue of Archival Science, 10, no. 3 (2010); Blouin and Rosenberg, Processing the Past; Filippo de Vivo, Andrea Guidi, and Alessandro Silvestri, "Archival 
studies of archival systems or studies reflecting on the historian's engagement with the archive. Several papers presented at the Sawyer seminar testify to these concerns. ${ }^{37}$ Two of these may be mentioned as fine examples: Lara Moore's history of the École des chartes and the development of libraries and archives in France between 1820-1870 and Jennifer Milligan's research on the archives of Second Empire France. ${ }^{38}$ Antoinette Burton collected "archive stories" about the encounters of researchers with and within the archives. ${ }^{39}$ An early example of this "reflexive turn" ${ }^{\prime 40}$ is Arlette Farge's The Taste of the Archive. ${ }^{41}$

We may conclude that the archival turn and "the centrality of the archive to contemporary scholarship and criticism" ${ }^{\prime 2}$ have led scholars in various disciplines to archival research, looking up from (the content of) the archival document to and through the archive, looking beyond - and questioning - its boundaries. ${ }^{43}$ This archival turn coincided with (and sometimes influenced) the shift in archivistics from the actual archival document to its functional process or context of creation; ${ }^{44}$ from the archive as a product to archive as

Transformations in Early Modern European History," European History Quarterly, 46 (2016): 421-34. See also chapter 14 in this volume, Randolph C. Head, "Historical Case Studies of Pre-Modern European Archives: A Comparative Approach.”

37 Blouin and Rosenberg, Archives.

38 Lara J. Moore, Restoring Order. The Ecole des Chartes and the Organization of Archives and Libraries in France, 1821-1870 (Litwin Books, Duluth, Minn., 2008); Jennifer Milligan, "What is an Archive?' in the History of Modern France," in Archive Stories: Facts, Fictions, and the Writing of History, ed. Antoinette Burton (Durham/ London: Duke University Press, 2005): 159-83; Jennifer Milligan, "The Problem of Publicité in the Archives of the Second Empire France," in Archives, Documentation, and Institutions of Social Memory: Essays from the Sawyer Seminar, ed. Francis X. Blouin and William G. Rosenberg (Ann Arbor: University of Michigan Press, 2006): 20-35.

39 Antoinette Burton, ed. Archive Stories: Facts, Fictions, and the Writing of History (Durham/London: Duke University Press, 2005); Carolyn Steedman, Dust (Manchester: Manchester University Press, 2002); Alexis E. Ramsey et al., Working in the archives: practical research methods for rhetoric and composition (Carbondale: Southern Illinois University Press, 2009).

40 Buchanan, "Strangely unfamiliar," 51.

41 Arlette Farge, The Allure of the Archives, transl. Thomas Scott-Railton (New Haven, CT: Yale University Pres, 2013). [Original edition, Arlette Farge, Le goût de l'archive (Paris: Seuil, 1989)].

42 Michael O'Driscoll and Edward Bishop, “Archiving 'Archiving'," English Studies in Canada 30 (2004): 10.

43 See also Luciana Duranti and Giovanni Michetti, in "The Archival Method" (chapter 2).

44 Elizabeth Yakel, "The Way Things Work: Procedures, Processes, and Institutional Records," American Archivist 59 (1996): 454-64. 
a process; ${ }^{45}$ from the physical artefact to the "very act and deed" which first caused that artefact to be created; ${ }^{46}$ shifting the

emphasis from the analysis of the properties and characteristics of individual documents to an analysis of the functions, processes, and transactions which cause documents to be created. ${ }^{47}$

In this new approach, an archive and archiving are much more than the storage of a document. Archiving is the process and product of continuous record formation: ${ }^{48}$ to and fro from creation and capture of documents into the recordkeeping system, to their management, disposal, use, and pluralisation into society - and all this in ever changing and ever constructed contexts. Verne Harris calls this "an ever-unfolding horizon of context(s) ... an incessant movement of continual recontextualisation." ${ }^{\text {"49 }}$ These contexts are

shaping the action of the people and institutions who made and maintained the records, the functions the records perform, the capacities of information technologies to capture and preserve information at a given time, and the custodial history of the records. ${ }^{50}$

45 Eric Ketelaar, "Writing on Archiving Machines," in Sign Here! Handwriting in the Age of New Media, ed. Sonja Neef, José van Dijck, and Eric Ketelaar (Amsterdam: Amsterdam University Press, 2006): 183-95.

46 Hugh Taylor, "My Very Act and Deed': Some Reflections on the Role of Textual Records in the Conduct of Affairs," American Archivist 51 (1988): 456-69; repr. in Imagining Archives: Essays and Reflections by Hugh A. Taylor, ed. Terry Cook and Gordon Dodds (Lanham/Oxford: Scarecrow Press, 2003), 131-48.

47 Terry Cook, "What Is Past Is Prologue: A History of Archival Ideas since 1898, and the Future Paradigm Shift," Archivaria 43 (1997): 47.

48 Frank Upward, "The records continuum" in Archives: Recordkeeping in Society, ed. Sue McKemmish et al., (Wagga-Wagga: Charles Sturt University, 2005): 197-222; Brien Brothman, "Archives, Life Cycles, and Death Wishes: A Helical Model of Record Formation," Archivaria 61 (2006): 235-69.

49 Verne Harris, "Ethics and the Archive: 'An Incessant Movement of Recontextualisation," in Controlling the Past: Documenting Society and Institutions - Essays in Honor of Helen Willa Samuels, ed. Terry Cook (Chicago Society of American Archivists, 2011): 360.

50 Tom Nesmith, "Seeing Archives: Postmodernism and the Changing Intellectual Place of Archives," American Archivist 65 (2002): 35. See also Nesmith's earlier definition of provenance: "the social and technical processes of the records' inscription, transmission, contextualization, and interpretation which account for it [the record's] existence, characteristics, and continuing history" Tom Nesmith, "Still Fuzzy, But More Accurate: Some Thoughts on the 'Ghosts' of Archival Theory," Archivaria 47 (1999): 146. 


\section{Archival Turn II}

Thus far we were concerned with the ontology of the archive(s). But there is a second archival turn, which has led to using the archive(s) as a methodological lens to analyse entities and processes as archive(s). In this perspective, instead of viewing archive(s) "as it is," something is viewed "as archive." ${ }^{51}$ Or, as Terry Cook put it, the latter is symbolic, metaphoric, and discursive, while the former is institutional, cultural, and curatorial. ${ }^{52}$

Using archive(s) as a metaphor is not something recent. The Oxford English Dictionary quotes a 1603 source "These curious meddlers ... make of their memorie a most unpleasant Archive or Register." ${ }^{53}$ Hundreds of journals since the $18^{\text {th }}$ century and modern websites have "archive(s)" in their title, because they are a storehouse of knowledge "as archive." ${ }^{54}$ In many cases the figurative use of archive(s) is not sustained and developed comprehensively. This is apparent in most publications which inflate the archive to become the Foucauldian archive: "the general system of the formation and transformation of statements, ${ }^{55}$ or in other words, the rules governing a meaningful discourse in a particular culture. To give only one example of such stretching of the meaning of archives: Thomas Richards' "imperial archive" was "not a building, nor even a collection of texts, but the collectively imagined junction of all that was known or knowable ... a fantasy of knowledge collected and united in the service of state and Empire."

It seems that anything can be considered "as archive(s)": bookmarks and bovid teeth, caves, forests, glaciers, peat bogs and sand dunes, molluscs and mosses, the human genome, Youtube - and several hundreds more. Often it is not clear if an analogy or an actual equivalence is meant, or a fiction: "as

51 I borrow this distinction from Diana Taylor, The Archive and the Repertoire: Performing Cultural Memory in the Americas (Durham/London: Duke University Press, 2003): 3.

52 Cook, "The archive(s) is a Foreign Country," 622.

53 The philosophie, commonly called, the Morals, written by the learned philosopher, Plutarch of Cheronea, trans. Philemon Holland (London: A. Hatfield, 1603), 140.

54 Between 1771 and 1830, 66 journals with "Archiv" in their title were established by the German Archiv: Willy Flach, "Goethes Literarisches Archiv" in Archivar und Historiker. Studien zur Archiv- und Geschichtswissenschaft. Zum 65. Geburtstag von Heinrich Otto Meisner (Berlin: Rütten \& Loening, 1956), note 38a.

55 Michel Foucault, Archaeology of Knowledge (London/New York: Routledge, 2002): 145-146. See also Knut Ebeling and Stephan Günzel, Archivologie. Theorien des Archivs in Wissenschaft, Medien und Künsten (Berlin: Kulturverlag Kadmos, 2009).

56 Richards, The Imperial Archive, 11; Marlene Manoff, "Theories of the Archive from Across the Disciplines," Libraries and the Academy 4 (2004): 9-25. 
if it were an archive." ${ }^{57}$ Some people see a danger in equating the archive "as archive" with the archive "as it is" (or rather, as they believe it to be). Kate Eichhorn warns:

Since the archival turn in the early 1990s, researchers have reconfigured everything from collections of graffiti under highway overpasses to the human genome as types of archives. The plasticity of the concept has opened up new avenues through which to question the authority of the archive while simultaneously legitimizing non-institutional collections as important sites of research and inquiry. However, there is also a danger in the term's over-application. If any collection can be an archive, we risk losing sight of an important distinction between carefully constructed and highly regulated collections that produce "official" narratives about the past and shape people's lives in the present and random collections of objects and documents that bring pleasure to the collector but have little or no impact on the larger order of things. ${ }^{58}$

I see this differently. Let anything be "as archive" and let everyone be an archivist. ${ }^{59}$ The important question is not "what is an archive," but how does this particular individual or group perceive and understand an archive? ${ }^{20}$ This allows "locating cultural expressions beneath a wide and all-encompassing umbrella of records and archives." ${ }^{61}$ Whether these have little or no impact on the larger order of things is not an essential characteristic of archive(s) "as it is." The creator, the user, and the archivist find meaning and make meaning in an archive and those meanings help in structuring and restructuring the relationship between the self and "the larger order of things." ${ }^{2}$ Archival

57 H. Vaihinger, The Philosophy of "As if", transl. C.K. Ogden (Routledge \& Kenan Paul, London 1935, $2^{\text {nd }}$ ed).

58 Kate Eichhorn, "Archival Genres: Gathering Texts and Reading Spaces," Invisible Culture 12 (2008): 1-10, http://rochester.edu/in_visible_culture/Issue_12/eichhorn/ eichhorn.pdf, accessed March 25, 2015.

59 Eric Ketelaar, "Everyone an Archivist," in Managing and Archiving Records in the Digital Era: Changing Professional Orientations, ed. Niklaus Bütikofer, Hans Hofman, and Seamus Ross (Baden, hier + jetzt, Verlag für Kultur und Geschichte, Baden, 2006): 9-14.

60 Adapted from Victoria L. Lemieux, "Let the Ghosts Speak: An Empirical Exploration of the "Nature" of the Record," Archivaria 51 (2001): 91.

61 Jeannette A. Bastian, "The Records of Memory, the Archives of Identity: Celebrations, Texts and Archival Sensibilities," Archival Science 13 (2013): 123.

62 Eric Ketelaar, “Cultivating Archives," Archival Science 12 (2012): 19-33. 
turns stretch the traditional boundaries of archivistics. Its object remains (as I will discuss later, at the end of section 7) records and archives as provenance-bound instruments for and by-products of transactions of public or private business.

\section{Archival Returns}

Moreover, engagement with a metaphoric archive(s) can lead into treating the archive(s) in more ontological and epistemological modes. Of such moving beyond a mere metaphorical archive, I give three examples. They show how new understandings of archives can be obtained or old understandings be enhanced by reconceptualising them in parallel with phenomena in other domains. I call this 'archival return' because archivistics may consider these reconceptualisations both as a coming back and as a return in exchange for the use of some of its own concepts by other disciplines.

\section{The City as Archive}

Vyjayanthi Rao, an anthropologist at the New School for Social Research in New York and thus a colleague of Ann Stoler, uses the concept of the city-as-archive as a tool with which to explore both the complexities of contemporary cities as well as the processes by which archives are constituted. ${ }^{63}$ Instead of reading the city as an archive preserving an alreadyknown space, she proposes to understand cities and archives as processes, to encompass a dynamic sense of ordering and interpretation. This understanding of the archive as a process and the analogical relationship between city and archive allows us to conceive the city-as-archive, as

a way of navigating the voids of the present, as a practice of intervening into and reading the urban fabrics created by these voids, not for reading the urban fabric as a quilt or a palimpsest of historical forms preserved within the archive. ${ }^{64}$

Beyond its role as a repository of evidence about the past, the archive and the city are always directed toward a putative future. The 'archival return' benefitting archivistics lies in Rao's multifarious conceptualisation of the archive-as-process and in using the anthropological understanding

63 Vyjayanthi Rao, "Embracing Urbanism: The City as Archive," New Literary History 40 (2009): 371-83.

64 Rao, “Embracing Urbanism,” 381. 
of 'urban voids' to suggest that the archive (as Derrida wrote) is shaped by the anticipation of the future - the future anterior. ${ }^{65}$

\section{The Body as Archive}

A second example is provided by André Lepecki, a scholar of performance studies. He proposes in The Body as Archive that the "will to archive" in contemporary dance leads to the will to re-enact dances. ${ }^{66}$ This "means that each 'will' acts upon the other to re-define what is understood by 'archiving' and what is understood by 're-enacting."'67 This redefining is carried out through the dancer's body. The re-enactment does not serve

to fix a work in its singular (originating) possibilization but to unlock, release, and actualize a work's many (virtual) com- and incompossibilities, which the originating instantiation of the work kept in reserve, virtually. ${ }^{68}$

After having discussed three of such re-enactments, Lepecki concludes that recent dance re-enactments could be seen not as melancholic compulsions to repeat but as "the choreographic activation of the dancer's body as an endlessly creative, transformational archive. ${ }^{169}$ Here I see the "archival return" in the discussion of the archive as keeping former instantiations (of a dance, of a record) "in reserve," to be released in later re-enactments. The choreographic activation of the dancer's body is analogous to the activation of a record. Archiving implies interactions, interventions, interrogations, and interpretations by creator, user, and archivist; these are activations that co-determine the archive's meaning. ${ }^{70}$ Activating the archive affects retrospectively all earlier meanings: we can no longer read the archive as our predecessors have read that archive. ${ }^{71}$ Also vice-versa: just as "the originating instantiation" of a dance keeps possibilities for later re-enactment in reserve, so gets each activation of a record along the records continuum extra significance in the light of

65 Derrida, Archive Fever, 18, 68; Derrida, "Archive Fever: A Seminar," 40.

66 Allan Sekula, "The Body and the Archive," October 39 (1986): 3-64. This text is about $19^{\text {th }}$ century photographic archives capturing the bodies of criminals.

67 André Lepecki, "The Body as Archive: Will to Re-Enact and the Afterlives of Dances," Dance Research Journal, 42 (2) (2010): 31.

68 Lepecki, "The Body as Archive," 31.

69 Lepecki, "The Body as Archive," 46.

70 Eric Ketelaar, “Tacit Narratives: The Meanings of Archives," Archival Science 1 (2001): 143-55.

71 Ketelaar, “Tacit Narratives," 138. 
subsequent activations. We call this retrospective causality (Freud's Nacbträglichkeit): events that occur later may change not just the significance, but even the nature of prior events. ${ }^{72}$ Archives "have not a single past, but an unbroken sequence of past times leading backward from the present moment." ${ }^{73}$

\section{The Plantation as Archive}

The third example comes from a researcher of postcolonial literature. Alfredo Lopez suggests that

A reading of the plantation as an archive, rather than as a single unified, stable signifier, offers possibilities for understanding the plantation image's continued efficacy as a signifier for today's postcolonial, postglobal South. ${ }^{74}$

The visual archive of "the" plantation

... is really a composite consisting of all the photographs and portraits of plantations produced and circulated for the past two centuries or so. The "original" can be no more than a fleeting glimpse and a hypothetical construction. ${ }^{75}$

That archive is appropriated in the service of ideological, nationalist, or regionalist imperatives:

Yet because the very act of defining the archive's limits (or borders) is necessarily an act of exclusion, it is also always vulnerable to the other's breach. The other is always already there as an irreducible condition of the very establishment of the archive, and its presence inside is nowhere more clearly acknowledged than in the desire for its exclusion - a desire that cannot be gratified except by the destruction of the archive itself. ${ }^{76}$

72 Susan van Zyl, "Psychoanalysis and the Archive: Derrida's Archive Fever," in Refiguring the Archive, ed. Carolyn Hamilton et al. (Dordrecht/Boston/London: Kluwer, 2002), 53-55.

73 Kenneth L. Ames, "Introduction", in The Colonial Revival in America, ed. Alan Axelrod (Wilmington Del: Henry Francis du Pont Winterthur Museum, 1975): 6, quoted by Spencer R. Crews and James E. Smis, "Locating Authenticity: Fragments of a Dialogue," in: The Poetics and Politics of Museum Display, ed. Ivan Karp and Steven D. Lavine (Washington and London: Smithsonian Institution Press, 1991): 160.

74 Alfred J. López, "The Plantation as Archive: Images of 'the South' in the Postcolonial World," Comparative Literature 63 (2011): 402.

75 López, "The Plantation as Archive," 405.

76 López, "The Plantation as Archive," 408. 
The "archival return" offered by Lopez is the insight of the original (of an image of a plantation, or of a digital record) as a hypothetical construction signifying a world outside the record, and his perception of the archive's borders, the deconstruction of inside and outside, inclusion and exclusion.

\section{Archive as Art, Art as Archive}

In a growing number of disciplines the archive is:

a strong metaphor for any corpus of selective forgettings and collections - and, as importantly, for the seductions and longings that such quests for, and accumulations of, the primary, originary, and untouched entail. ${ }^{77}$

These seductions and longings are the subject of 'romances of the archive", so masterfully analysed by Suzanne Keen: novels which celebrate seeking and finding well-preserved but concealed documents as memories of a usable past. ${ }^{78}$ The genre - of which A.S. Byatt's Possession is a model became popular in Britain after the Falklands War, as authors and readers went searching for representations of Britishness (or rather, Englishness) in a postcolonial and postimperial world. Keen construes "archives literally, as collections of documents and the places housing, protecting and concealing them," excluding metaphoric archives such as the Foucauldian archive. ${ }^{79}$ Romances of the archive deal with issues that are quintessential for "real life" archive and archivistics too. The protagonists in these novels, for example,

quarrel about who deserves to have custody of the past. They often offer revisionist or frankly counterfactual versions of history. They disagree about whether secrets must be kept for our own protection or revealed to bring the culpable to justice. ${ }^{80}$

77 Stoler, "Colonial Archives," 94; Stoler, "On the Content in the Form," 87.

78 Suzanne Keen, Romances of the Archive in Contemporary British Fiction (Toronto/Buffalo/ London: University of Toronto Press, 2001); Manoff, "Theories of the Archive." See also Arlene B. Schmuland, "The Archival Image in Fiction: An Analysis with an Annotated Bibliography," American Archivist 62 (1999): 24-73; The Fictional World of Archives, Art Galleries \& Museums, http://fictionalarchives.blogspot.com, accessed March 25, 2015.

79 Keen, Romances of the Archive, 12; Knut Ove Eliassen, "The Archives of Michel Foucault," in The Archive in Motion. New Conceptions of the Archive in Contemporary Thought and Nerw Media Practices, ed. Eivind Røssaak (Oslo: Novus Press, 2010), $29-51$.

80 Keen, Romances of the Archive, 230. 
Keen does not deal with novels consisting entirely of a wholly imaginary archive or collection, which are the subject of Marco Codebò's Narrating from the Archive ${ }^{81} \mathrm{He}$ analyses - drawing quite substantially from archivistics theory and methodology - "archival novels" where archival objects (records, catalogues, lists, calendars) are inserted into the narrative. ${ }^{82}$ An early example of such "archivization of the narrative" is the Journal of the Plague Year ... in $1665 \ldots$ written by a citizen ... but from the pen of Daniel Defoe and published in 1722. However, according to Codebò, "it is in postmodernism that archival novels meant to question the archive's epistemology achieve true formal definition," ${ }^{83}$ precisely because Codebò's archival novels, just as Keen's romances of the archive, "engage the reader in a discussion concerning the truth-value of records and/or the reliability of the archive's procedures." 84

Romances of the archive occur not only in literature, but in the visual arts, too. Archivistics should learn from the arts, for, according to Charles Merewether:

it is in the spheres of art and cultural production that some of the most searching questions have been asked concerning what constitutes an archive and what authority it holds in relation to its subject. ${ }^{85}$

Merewether is exaggerating, I think, but nevertheless it is worthwhile to observe how artists have engaged with the archive(s), if only because that can show how archives are situated in society. Artists romance the archive in referencing memory, history, and personal recollection. ${ }^{86}$ As Matthias Winzen writes, "the artistic gesture behind such works is to hold on to, unfold, document, reveal, enumerate, or to sort its own material content." ${ }^{87}$ Their work demonstrates:

81 Marco Codebò, Narrating from the Archive: Novels, Records, and Bureaucrats in the Modern Age. (Danvers Mass.: Rosemont, 2010).

82 See also Echevarria, Myth and Archive.

83 Codebò, Narrating from the Archive, 54.

84 Codebò, Narrating from the Archive, 15.

85 Charles Merewether, "Introduction/Art and the Archive," in The Archive, ed. Charles Merewether, 10-17 (London: Whitechapel Gallery, 2006), 10.

86 Sven Spieker, ed., The Big Archive. Art From Bureaucracy (Cambridge Mass./London: MIT Press, 2008); Monika Rieger, "Anarchie im Archiv: Vom Künstler als Dammler," in Archivologie. Theorien des Archivs in Wissenschaft, Medien und Künsten, ed. Knut Ebeling and Stephan Günzel (Berlin: Kulturverlag Kadmos, 2009), 253-69; Buchanan, "Strangely Unfamiliar."

87 Ingrid Schaffner and Matthias Winzen, eds., Deep Storage. Collecting, Storing, and Archiving in Art. (Munich/New York: Prestel, 1998), 25. See also Okwui Enwezor, Archive Fever - Uses of the Document in Contemporary Art (New York: International 
one of the paradoxical effects of archiving, because at a certain point the individual components are deemed to be only another expression of those objects that surround it. Uniqueness, specificity, and individuality are destroyed within the process of archiving. ${ }^{88}$

Moreover, this archival mode of representation applied by an artist like Christian Boltanski "withdraws objects from the contexts in which they were originally present." ${ }^{89}$ Artists seek in what Hal Foster calls their "archival impulse":

to make historical information, often lost or displaced, physically present ... Sometimes archival samplings push the postmodernist complications of originality and authorship to an extreme..$^{90}$

Other archival artists play with the category of the collection, ranging their materials according to what Foster terms a quasi-archival logic, and presenting them in a quasi-archival architecture. Arnold Dreyblatt is one such artist. ${ }^{91}$ With his $T$ Projects, he follows the movement of records and their meaning inside and outside organisations, using a duplicate archive of over 4000 documents that he created from state archives in Europe and North America. These "T documents" are also used in Dreyblatt's reading projects, which simulate the living environment in which records are created, stored, and used. For each reading project several hundred people are invited to take part in a functioning yet temporal "archival installation system."

Center of Photography, 2008); Ernst van Alphen, "Archival Obsessions and Obsessive Archives," in What is Research in the Visual Arts? Obsession, Archive, Encounter, ed.

Michael Ann Holly and Marquard Smith (Williamstown Mass.: Sterling and Francine Clark Art Institute, 2008), 65-84; Ernst van Alphen, "Visual Archives and the Holocaust: Christian Boltanski, Ydessa Hendeles and Peter Forgacs," in Intercultural Aesthetics. A Worldview Perspective, ed. Antoon Van den Braembussche, Heinz Kimmerle and Nicole Note (S.1.: Springer, 2009), 137-55; Buchanan, "Strangely unfamiliar"; Manoff, "Theories of the Archive."

88 Van Alphen "Archival Obsessions," 66.

89 Van Alphen, "Visual Archives," 143.

90 Hal Foster, "An Archival Impulse," October 110 (2004): 4. Foster's Archival Impulse is discussed by Susanne Østby Sæther, "Archival Art: Negotiating the Role of New Media," in The Archive in Motion. New Conceptions of the Archive in Contemporary Thought and New Media Practices, ed. Eivind Røssaak (Oslo: Novus Press, 2010), 77-108.

91 Ernest W. Uthemann, ed., Arnold Dreyblatt. Aus den Archiven/From the Archives (Heidelberg: Kehrer Verlag, 2003); Arnold Dreyblatt and Jeffrey Wallen, "Hands on the Document: Arnold Dreyblatt's T-Archive," in Sign Here! Handwriting in the Age of New Media, ed. Sonja Neef, José van Dijck, and Eric Ketelaar (Amsterdam: Amsterdam University Press, 2006), 134-49. 
In immersive installations and performances like the ones created by Boltanski, Dreyblatt, and others, people and documents alike become an "immersive archive." ${ }^{92}$ The viewer/reader/user is involved as a member of the "creative audience." ${ }^{\text {"3 }}$ The artworks demonstrate in their own way that records are created and used by people who themselves are components of and controlled by recordkeeping systems. We may call this the duality of archival structures, in Giddens' sense: "the structured properties of social systems are simultaneously the medium and outcome of social acts." Archival artists also problematise concepts as originality and authenticity and thereby provide an archival return.

\section{Adopting and Adapting}

In the analysis of both archive(s) "as it is" and "archive as," (aspects of) theory, methodology, and practice of other disciplines can have an effect on archivistics. This goes further than just drawing analogies between archival studies and other disciplines that are grappling with similar issues and concerns. Cross-disciplinary comparisons, as Elisabeth Kaplan writes, can

help us to view our field in a larger context, shedding new light on familiar thought and practice, reorienting us toward the broader intellectual climate in which we work. ${ }^{95}$

However, from comparison we should take one step further and consider the possibility of a real influence. This may be distinguished in what one may call (a) an intrinsic or material effect on the ontology of the archive(s) and (b) a formal effect on the methodology of archivistics. The latter occurs when archivistics adopts methods (in particular research methodologies) from another discipline. This may lead to an effect on an ontological level, but not necessarily so. Such methodological adopting and adapting by archivistics has come from various quarters:

92 Sæther, "Archival Art," 84-90.

93 Manuel Castells, Communication Power (Oxford: Oxford University Press, 2009).

94 Anthony Giddens, A Contemporary Critique of Historical Materialism. (London/ Basingstoke: Macmillan Press, 1981), 19.

95 Elisabeth Kaplan, "Many Paths to Partial Truths: Archives, Anthropology, and the Power of Representation," Archival Science 2 (2002): 211. 
- $\quad$ Ethnic studies ${ }^{96}$

- Human-computer interaction and information retrieval ${ }^{97}$

- Modelling methods and techniques ${ }^{98}$

- Critical theory ${ }^{99}$

- Systems development ${ }^{100}$

- Actor-network theory ${ }^{101}$

- Grounded theory ${ }^{102}$

- Ethnography ${ }^{103}$

- Speech act theory ${ }^{104}$

The impact of each of these on the archival method (and vice versa the reception of archivistics into these domains) will be dealt with later in this book.

\section{Turning}

The other influence (intrinsic or material, as opposed to formal) occurs whenever a concept in archivistics theory is approached from one or more other disciplines that employ a similar concept. This may lead the archivistics domain to adopt and adapt respectively (a) influenced concepts, (b) concepts with

96 See in this volume, Kelvin White, "Ethnic Studies as a Theoretical Framework for Archival and Recordkeeping Research."

97 In this volume: Anneli Sundqvist, “Archival Mediation: Studying Users' Interaction with Access Systems”; Anne Gilliland and Jonathan Furner, "Archival IR: Applying and Adapting Information Retrieval Approaches in Archives and Recordkeeping Research."

98 Hans Hofman, “The Use of Models and Modelling Techniques from a Recordkeeping Research Perspective," in this volume.

99 Anthony W. Dunbar, "Introducing Critical race Theory to Archival Discourse: Getting the Conversation Started," in this volume.

100 Joanne Evans, "Capacities and Complexities: A Reflection on design Methodologies for Archival and Recordkeeping Research," in this volume.

101 Michael Wartenbe, "ANTs in the Archive: Actor-Network Theory as a Research Methodology for Archival Science," forthcoming in Archival Science.

102 In this volume: Jenny Bunn, "Grounded Theory: A Straightforward and Complicated Approach to Research"; Sarah Ramdeen and Alex H. Poole, "Using Grounded Theory to Understand the Archival Needs of Geologists."

103 Karen Gracy, "Documenting Communities of Practice: Making the Case for Archival Ethnography," in this volume.

104 Pekka Henttonen, "Looking at Archival Concepts and Practice in the Light of Speech Act Theory," in this volume. 
newly acquired relevance, and (c) totally new concepts. ${ }^{105}$ Let me illustrate this with some examples of these effects on archivistics from different disciplines. I will deal with respectively a linguistic turn, a social turn, a performative turn, and a representational turn. It will become clear that these rubrics are not mutually exclusive.

\section{Linguistic Turn}

Since every author seems to have his or her own definition of linguistic turn, let me explain what I consider under this rubric. The linguistic turn in archivistics leads to re-viewing a text not only in terms what it means, but primarily how the text works. ${ }^{106}$ Documents consist of words; they are texts. Textuality is a concept in linguistics, in literary theory and - even before the literary turn in many disciplines - in anthropology. ${ }^{107}$ Textuality gives radical precedence to the reader over the author or creator of a text (as Barthes notes: "the unity of a text is not in its origin, it is in its destination"), ${ }^{108}$ while acknowledging that any text stays open through intertextuality. A text is not enclosed within the margins of a document, but is "a differential network, a fabric of traces referring endlessly to something other than itself, to other differential traces. ${ }^{109}$ As Brien Brothman writes:

Authorial lordship over documentary meaning diminishes significantly once, actually well before the work is released into the public domain of textuality. Because such notions vitiate the concepts of "author", "origins", and "uniqueness" they obviously complicate the belief that records possess a single definable provenance. ${ }^{110}$

105 I borrow this distinction from Sherry L. Xie, "Building Foundations for Digital Records Forensics: A Comparative Study of the Concept of Reproduction in Digital Records Management and Digital Forensics," American Archivist 74 (2011): 581.

106 Lynn Hunt, "Introduction: History, Culture, and Text," in The New Cultural History, ed. Lynn Hunt (University of California Press, Berkeley, Los Angeles, London, 1989), 15.

107 James Clifford and George E. Marcus, eds. Writing Culture: The Poetics and Politics of Ethnography (Berkeley/Los Angeles/London: University of California Press, 1986);

Steven Lubar, "Information Culture and the Archival Record," American Archivist 62 (1999): 10-22.

108 Roland Barthes, The Death of the Author (1967), quoted by Sue Breakell, "Encounters with the Self: Archives and Research," in The Future of Archives and Recordkeeping: A Reader, ed. Jennie Hill (London: Facet, 2011), 29.

109 Jacques Derrida, "Living On," in Deconstruction and Criticism, ed. Harold Bloom et al. (New York: Continuum, 2004), 69.

110 Brothman, "Declining Derrida," 77. 
Brothman wrote this some fifteen years ago, at a time when, as he stated, few if any archivists would be willing to abide the characterisation of records or archives as simply one order of text. Since then, archivistics has witnessed a linguistic turn comparable to the one that has enriched (some would say, haunted) other disciplines in the humanities and social sciences. ${ }^{111}$

Heather MacNeil, for example, has explored how authenticity, originality, and intentionality have been discussed in the context of textual criticism. ${ }^{112}$ Drawing on the insights of the new textual scholarship, MacNeil argues that

the authenticity and meaning of a body of records is shaped not only by its archival bond but, also, by what we might term a custodial bond, meaning the relations that exist between a body of records and the various custodial authorities that interact with the records over time, including archivists and archival institutions. ${ }^{113}$

As the archival approximation of the notion of textualterity, MacNeil proposes archivalterity, which "refers to the acts of continuous and discontinuous change that transform the meaning and authenticity of a fonds as it is transmitted over time and space." ${ }^{114}$ An exploration of archivalterity yields several key insights, among them the insight that "original order" is not found, but is constructed and reconstructed by archivists. ${ }^{115}$

Literary critics, historians and others have discovered the poetics of records, which may be read as rhetorical texts. ${ }^{116}$ Historian David Sabean, for example, looks at protocols and other records:

111 Andrew Prescott, "The Textuality of the Archive," in What are Archives? Cultural and Theoretical Perspectives: A Reader, ed. Louise Craven (Aldershot: Ashgate, 2008), 31-51.

112 Heather MacNeil, "Picking Our Text: Archival Description, Authenticity and the Archivist as Editor," American Archivist 68 (2005): 264-78; Heather MacNeil, "Archivalterity: Rethinking Original Order," Archivaria 66 (2008): 1-24. See elsewhere in this volume: Heather MacNeil, "Deciphering and Interpreting an Archival Fonds and its Parts: A Comparative Analysis of Textual Criticism and the Theory of Archival Arrangement." See also Geoffrey Yeo, "Nothing is the same as something else': significant properties and notions of identity and originality," Archival Science 10 (2010): 85-116; Papailias, Genres; Breakell, "Encounters".

113 MacNeil, "Archivalterity," 14.

114 MacNeil, "Archivalterity," 14.

115 More on the agency of archivists in section 7 under "Representational Turn".

116 Peter Becker and William Clark, "Introduction," in Little Tools of Knowledge: Historical Essays on Academic and Bureaucratic Practices, eds. Peter Becker and William Clark (Ann Arbor: University of Michigan Press, 2011), 1-34; Miles Ogborn, Indian ink. Script and Print in the Making of the English East India Company 
for issues of emplotment and story line, styles of narrative, strategies for shaping discourses and memories, the hierarchies of reportorial [sic] context, conspirational alliances, strategies of revenge and aggrandizement, and ways of imposing silence and channelling discourse. ${ }^{117}$

Studies of rhetoric have confirmed (and expanded) archivists' notions of the constructedness of records. ${ }^{118}$ As two scholars of organisational behaviour attest:

Records are not factual, neutral, technical documents alone, although while serving legitimate ends they must appear this way, and while serving illegitimate ones even more so. They are designed - implicitly or explicitly - to produce an effect in some kind of audience, which itself actively uses records to interpret events. This is not to suggest conscious deceit or cynicism on the part of either record keepers or users (although ... this is certainly possible). Rather it is simply to acknowledge and open up for analysis the conditions under which organizational records are produced and used. ${ }^{119}$

Van Maanen and Pentland's findings and comparable research by others have been superbly woven in a general recordkeeping context by Ciaran Trace. ${ }^{120}$ But that archival return has not yet been acknowledged as a return in other domains: Trace's paper is cited almost exclusively by other archivistics scholars.

(Chicago/London: University of Chicago Press, 2007); Voss and Werner, "Toward a Poetics of the Archive"; Verhoeff, "Archival Poetics"; Verhoeff, The West; Ramsey, Working in the archives; Christoph Reinfandt, "Reading texts after the linguistic turn: approaches from literary studies and their implications," in Reading primary sources: The interpretation of texts from nineteenth-and twentieth-century history, ed. Miriam Dobson and Benjamin Zimmerman (London/New York: Routledge, 2009), 37-54. See also in this volume Randolph C. Head, "Historical Case Studies of PreModern European Archives: A Comparative Approach.”

117 David W. Sabean, "Peasant Voices and Bureaucratic Texts: Narrative Structure in Early Modern German Protocols," in Little Tools of Knowledge: Historical Essays on Academic and Bureaucratic Practices, ed. Peter Becker and William Clark (Ann Arbor: University of Michigan Press, 2001), 67.

118 Charles E. Morris, "The Archival Turn in Rhetorical Studies: Or, the Archive's Rhetorical (Re)turn," Rhetoric E Public Affairs 9 (2006): 113-15.

119 John van Maanen and Brian T. Pentland, "Cops and Auditors: The Rhetoric of Records," in The Legalistic Organization, ed. Sim B. Sitkin and Robert J. Bies (Thousand Oaks: Sage Publications, 1994), 53.

120 Ciaran B. Trace, "What is Recorded is Never Simply 'What Happened': Record Keeping in Modern Organizational Culture," Archival Science 2 (2002): 137-59. 


\section{Social Turn}

Apart (but not wholly separate) from the linguistic turn, ${ }^{121}$ archivistics has experienced a social turn, influenced by or linked to social studies as sociology, anthropology, organisational behaviour, and ethnomethodology. We have come to understand that an archive is not just an agency of storage, but a process, a mediated social and cultural practice. Archivistics, therefore, turned to study

the characteristics of records in their social and cultural contexts and how they are created, used, selected and transferred through time. We strive at a better understanding of the way people in organizations create and maintain records. ${ }^{122}$

A record is not a thing in itself; it is an active constituent of social relations, an insight gained by ethnomethodology (the study of the methods used for producing recognisable social orders) as early as in the 1960s. Elizabeth Yakel was the first in archival science to use results of ethnomethodological research, in her thesis Recordkeeping in Radiology: The Relationships between Activities and Records in Radiological Processes. ${ }^{123} \mathrm{~A}$ recent project Expanding the Options: Strategies for Preserving Electronic Records of Collaborative Processes (sponsored by the National Historical Publications and Records Commission and the University of Michigan School of Information) reconceptualised the basic principle of provenance as it applies to network organisations, by focusing

not on information technology or record-keeping systems per se, but rather on the social process of translation whereby organisational memory gives rise to records that can be appraised and preserved by an archivist. ${ }^{124}$

121 Sometimes the linguistic turn and the social turn are merged and labelled as "the cultural turn": Victoria E. Bonnell and Lynn Hunt, "Introduction," in Beyond the Cultural Turn. New Directions in the Study of Society and Culture, ed. Victoria E. Bonnell and Lynn Hunt (Berkeley/Los Angeles/London: University of California Press, 1999), 1-32.

122 Eric Ketelaar, "Archives in the Digital Age: New Uses for an Old Science. Archives E Social Studies: A Journal of Interdisciplinary Research 1 (2007): 168, available at http:// archivo.cartagena.es/files/36-168-DOC_FICHERO1/10-ketelaar_archives.pdf, accessed March 25, 2015.

123 Yakel, "The Way Things Work"; Elizabeth Yakel, "The Social Construction of Accountability: Radiologists and Their Record-Keeping Practices," The Information Society: An International Journal 17 (2001): 233-45.

124 Peter Botticelli, "Records Appraisal in Network Organizations," Archivaria 49 (2000): 164. 
The linguistic turn and the social turn in archivistics converge in the study of genres as socially recognised communicative transactions. JoAnne Yates' Control through Communication: The Rise of System in American Management was right from its publication in 1989 recognised by archivists and scholars as an essential study of documentary genres. ${ }^{125}$ Yates and her colleague at MIT Wanda Orlikowski have since embarked on various projects researching how members of teams and organisations shape use of electronic communication media over time. Their contributions have been picked up by other scholars in archivistics. ${ }^{126}$

I referred earlier to ethnographic studies of documentary practices by anthropologists, psychologists, and sociologists. They cross the boundaries of traditional archivistics by dealing with record formation in paper or in "sociodigital systems." In these systems records are socially constructed artefacts, ${ }^{127}$ constituted mutually by people (Wanda Orlikowski's duality of technology), they are "actants" (Bruno Latour's action-network theory) with moral agency (Peter-Paul Verbeek's morality of things). ${ }^{128}$ As all texts, records

determine social spaces, both as products of the social world of authors and as textual agents at work in that world, with which they entertain often complex and contestatory relations. ${ }^{129}$

This constructivist view of the mutual interplay between records and the social world (reminding of Giddens' double hermeneutics) is important, but

125 JoAnne Yates, Control through Communication. The Rise of System in American Management (Baltimore/London: Johns Hopkins University Press, 1989).

126 Gillian Oliver, Yunhyong Kim and Seamus Ross, "Documentary genre and digital recordkeeping: red herring or a way forward?" Archival Science 8 (2008): 295-305; Eichhorn, "Archival Genres"; Gillian Oliver and Wendy M. Duff, ed. "Genre Studies in Archives," special issue of Archival Science 12, no. 4 (2012); Fiorella Foscarini, "Organizational Records as Genres. An Analysis of the 'Documentary Reality' of Organizations from the Perspectives of Diplomatics, Records Management and Rhetorical Genre Studies," in Genre Theory in Information Studies ed. Jack Andersen (London: Emerald, 2015), 115-32.

127 Lynn M. Olson, "Record Keeping Practices: Consequences of Accounting Demands in a Public Clinic," Qualitative Sociology 18 (1995): 45-70.

128 Wanda J. Orlikowski, "The Duality of Technology: Rethinking the Concept of Technology in Organizations," Organization Science 3 (1992): 398-427; Latour, Science in action; Peter P. Verbeek, What Things Do: Philosophical Reflections on Technology, Agency, and Design (University Park, Pa: Pennsylvania State University Press, 2005).

129 Gabrielle M. Spiegel, "History, Historicism, and the Social Logic of the Text in the Middle Ages," Speculum 65 (1990): 77, repr. in Gabrielle M. Spiegel, The Past as Text. The Theory and Practice of Medieval Historiography (Baltimore/London: Johns Hopkins University Pres, 1997): 3-43. 
should not be an excuse to play down the agency of the archivist (as classical archivists used to do themselves!), in favour of the agency of records.

\section{Performative Turn}

Records have agency because they can accomplish something, make a difference in status before and after. François Cooren, scholar of communication studies and sociology of organisations, put it in this way: "texts, such as reports, contracts, memos, signs, or work orders, perform something". ${ }^{130}$ This insight - influenced by genre studies and speech act theory - may be termed the performative turn in archivistics. Consider replacing "performances" in the following quote from Diana Taylor by "records":

Performances function as vital acts of transfer, transmitting social knowledge, memory, and a sense of identity through reiterated, or ... twice-behaved behaviour. ${ }^{131}$

As historical anthropologist Penelope Papailias writes, genre "might be defined less by what it is than what it does, and brought into dialogue with theories of the performative." 132 "Records could be said to perform when they are used, and there is a possibility for them to be reinvented as new performance events." ${ }^{133}$ A green paper by the National Archives of Australia on the preservation of digital records argues that each viewing of a digital record:

is a new "original copy" of itself - two people can view the same record on their computers at the same time and will experience equivalent "performances" of that record. ${ }^{134}$

The illocutionary force of the text of a play is, according to Paul Thom, like the illocutionary force of a recipe for baking a cake: "It is a set of instructions

130 François Cooren, "Textual Agency: How Texts Do Things in Organizational Settings," Organization 11 (2004): 374-76.

131 Taylor, The Archive and the Repertoire, 2-3.

132 Papailias, Genres, 23.

133 Sarah Jones, Daisy Abbott and Seamus Ross, "Redefining the performing arts archive," Archival Science 9 (2009): 165-171, quote p.169. See also Heather MacNeil, "Trust and professional identity: narratives, counter-narratives and lingering ambiguities," Archival Science 11 (2011): 175-92.

134 Helen Heslop, Simon Davis and Andrew Wilson. An Approach to the Preservation of Digital Records (Canberra: National Archives of Australia, 2002), 8, http://www.naa. gov.au/Images/An-approach-Green-Paper_tcm16-47161.pdf, accessed March 25, 2015. 
for how to do something, namely, how to perform the play." ${ }^{35}$ Could one say the same of a digital record, being a set of instructions on how to perform that record?

Just as the digital record seems to disappear without a trace from the screen - leading to the ontological question, where and what is the record? so the performance seems to die when the curtain falls. Diana Taylor refutes this view:

The way I see it, performance makes visible (for an instant, live, now) that which is always already there: the ghost, the tropes, the scenarios that structure our individual and collective life. These specters, made manifest through performance, alter future phantoms, future fantasies. ${ }^{136}$

She subsequently asks, "What conditions of visibility are needed to conjure up the ghost?" This is a relevant question for archivistics too. InterPARES 2 included case studies in the creative and performing arts to understand how these disciplines conceptualise authenticity, reliability and accuracy in interactive and dynamic systems in music, dance, theatre, moving images, and interactive media installation. The researchers found that "methods for ensuring authenticity and reliability of multiple artworks can stand as a model for how those qualities can be preserved in digital record systems outside of the arts." ${ }^{137}$ Reversely, "performance scholars are looking to archival theory to address the challenge of representing performance."138

\section{Representational Turn}

The linguistic turn "sharpened our awareness of the epistemological significance of presentation and representation." ${ }^{139}$ For an historian, representation

135 Paul Thom, For an Audience: A Philosophy of the Performing Arts (Philadelphia: Temple University Press, 1993), 39.

136 Taylor, The Archive and the Repertoire, 143. See also Esther Peeren, The Spectral Metaphor: Living Ghosts and the Agency of Invisibility (Basingstoke/New York: Palgrave Macmillan, 2014).

137 John Roeder et al., "Part Three - Authenticity, Reliability and Accuracy of Digital Records in the Artistic, Scientific and Governmental Sectors: Domain 2 Task Force Report," [electronic version] in International Research on Permanent Authentic Records in Electronic Systems (InterPARES) 2: Experiential, Interactive and Dynamic Records, ed. Luciana Duranti and Randy Preston (Padova: Associazione Nazionale Archivistica Italiana, 2008), 32, http://www.interpares.org/display_file.cfm?doc=ip2_book_part_3_ domain2_task_force.pdf, accessed March 25, 2015.

138 Jones, Abbott and Ross, "Redefining," 170.

139 Johannes Fabian, Memory Against Culture: Arguments and Reminders (Durham/London: Duke University Press, 2007): 13-14. 
is both an object of study and the "terminal phase of the historiographical operation itself." 140 The concept of representation is important in studies of collective (or social or cultural) memories. ${ }^{141}$ Collective memory is "the representation of the past, both that shared by a group and that which is collectively commemorated, that enacts and gives substance to the group's identity, its present conditions and its vision of the future." ${ }^{142}$ Collective memory studies is a booming field cultivated by a host of disciplines. References to the archive(s) have been swelling since 2008. ${ }^{143}$ Most studies conflate archives and collective memory ${ }^{144}$ or equate representations with archives. We have, however, to realise that representations - or cultural practices of historical remembrance - are not a substitute for archival memory, but rather a complement to the archive. ${ }^{145}$ Archives are not the cultural or social memory of a community. They are "among countless different devices used in the process of transforming individual memories into collective remembering." ${ }^{146}$

Archivistics practitioners and theorists are now taking a representational turn by venturing into collective memory studies. ${ }^{147}$ Unfortunately the return from archivistics into other disciplinary fields is still rather marginal. Not much has changed since Confino warned:

140 Paul Ricoeur, Memory, History, Forgetting, trans. Kathleen Blamey and David Pellauer (Chicago: University of Chicago Press, 2004): 228.

141 Alon Confino, "Collective Memory and Cultural History: Problems of Method," The American Historical Review 102 (1997): 1386-1403.

142 Barbara A. Misztal, Theories of Social Remembering (Maidenhead/Philadelphia: Open University Press, 2003): 7.

143 See in this volume Jeannette Allis Bastian, "Collective Memory and Archival Research." Much of the text in this section is based on Eric Ketelaar, "Archives, Memories and Identities," in Archives and Recordkeeping: Theory into Practice, ed. Caroline Brown (London: Facet, 2013): 131-70.

144 Margaret Hedstrom, "Archives and Collective Memory: More Than a Metaphor Less Than an Analogy," in Currents of Archival Thinking, ed. Terry Eastwood and Heather MacNeil (Santa Barbara: Libraries Unlimited, 2010): 163-79.

145 Jay Winter, "Historians and Sites of Memory," in Memory in Mind and Culture, ed. Pascal Boyer, and James V. Wertsch (Cambridge: Cambridge University Press, 2009): 252-68.

146 Laura Millar, "Touchstones: Considering the Relationship between Memory and Archives," Archivaria 61 (2006): 119.

147 See Jeanette Bastian, "Collective Memory and Archival Research," in this volume; Trond Jacobsen, Ricardo L. Punzalan, and Margaret L. Hedstrom, "Invoking collective memory': mapping the emergence of a concept in archival science", Archival Science 13, no. 2/3 (2013): 241. Caroline Brown, "Memory, Identity and the Archival Paradigm," special issue of Archival Science 13, no. 2/3 (2013). 
Many studies of memory are content to describe the representation of the past without bothering to explore the transmission, diffusion and, ultimately, the meaning of this representation. ${ }^{148}$

In Aleida Assmann's schema, cultural memory consists of what she used to call functional and storage memory, but recently termed the "canon" and the "archive." The archival memory, she posits, lingers in a state of latency and is accessible only to specialists, it is not "transformed into a living memory supported by public awareness and validation by cultural institutions and the public media."149 Both Assmann's assumption that archival memory can be made dynamic only through permeating the borderline with functional memory, and her neglect of the role of the "specialists" (are they Derrida's archons?) can be challenged. On the other hand, her schema has more merit than I formerly thought. In the first place, the archive is not conflated with cultural memory. Neither is the archive seen as something outside cultural memory, but it is conceptualised as one of the components of cultural memory.

Also, I now see in her distinction between canon and archive a solution to the problem of how to define the unique qualities of archives as "touchstones" for memory. ${ }^{150} \mathrm{I}$ am guided here by James O'Toole, who reviewed four aspects of the uniqueness of an archival document: (1) the physical document itself is unique, or (2) the information it contains is unique, or (3) the process which the record produced is unique, or (4) the uniqueness is derived from the way individual items have been assembled into files, that is the document in the context of other documents. ${ }^{151}$ I would argue that of the four parameters the last two are the most important to define the unique qualities of archives as distinct from other memory tools. Archives are unique because of their "contextual envelope" 152 constituted by the uniqueness of the processes and

148 Confino, "Collective Memory," 1395.

149 Aleida Assmann, "Memory, Individual and Collective," in The Oxford Handbook of Contextual Political Analysis, ed. R.E. Goodin, and C. Tilly (Oxford: University of Oxford Press, 2006): 221; Aleida Assmann, "Re-framing Memory: Between Individual and Collective Forms of Constructing the Past," in Performing the Past. Memory, History and Identity in Modern Europe, ed. K. Tilmans, F. van Vree, and J. Winter (Amsterdam: Amsterdam University Press, 2010): 43. See also Régine Robin, La Mémoire Saturée. (Paris: Éditions Stock, 2003): 407-8.

150 Millar,"Touchstones”; Ketelaar, "Archives, Memories and Identities".

151 James M. O’Toole, “On the Idea of Uniqueness," American Archivist 57 (1994): 632-58, repr. in American Archival Studies: Readings in Theory and Practice, Randall C. Jimerson, ed. (Chicago: Society of American Archivists, 2000): 245-77.

152 Barbara L. Craig, "Selected Themes in the Literature on Memory and their Pertinence to Archives," American Archivist 65 (2002): 287. 
functions that produced records and by the context of other documents created by the same activity over time. Archives are not unique because the information they contain is unique, and only rarely because the document as artefact has an intrinsic uniqueness. ${ }^{153}$

The historian's representation of the past has been likened to the painter's:

Like the painter, the historian represents (historical) reality by giving it a meaning, through the meaning of his text, that reality does not have of itself. ${ }^{154}$

Isn't the archivist doing the same when (s)he is processing (appraising, ordering, describing, etc.) records and archives? But

the archivist is bound to have an ambiguous relationship to representation since he or she is the locus of mediation between the information artefact, the description of it, the digitized simulacrum of it, and the user of it. ${ }^{155}$

Elizabeth Yakel, in a groundbreaking essay, has demonstrated that archival practices are representational practices. ${ }^{156}$ She argues for studying archival representations because they "speak not only about the collections for which they act as surrogates, but also about archival practice and archivists." ${ }^{157}$ Such studies, by focusing on representation, as an object of study and as the practice itself, are essential for understanding what happens when analogue records are digitised: what do the digital surrogates represent? ${ }^{158}$ Studying archival representations would also add to our understanding of the agency of the archivist in the construction and transmission of cultural memories.

153 The latter is close to the symbolic significance of a document: James M. O'Toole, "The Symbolic Significance of Archives," American Archivist 56 (1993): 234-255, repr. in American Archival Studies. Readings in Theory and Practice, Randall C. Jimerson, ed. (Chicago: Society of American Archivists, 2000), 47-72; Ketelaar, "Cultivating Archives."

154 Frank R. Ankersmit, "Historical Representation," History and Theory 27 (1988): 214.

155 Bernadine Dodge, "Across the Great Divide: Archival Discourse and the (Re) presentations of the Past in Late-Modern Society," Archivaria 53 (2002): 20.

156 Elizabeth Yakel, “Archival Representation," Archival Science 3 (2003): 1-25.

157 Yakel, “Archival Representation," 25.

158 Alicia Rekrut, Reconnecting Mind and Matter: Materiality in Archival Theory and Practice. Master's thesis (University of Winnipeg, 2009), http://hdl.handle.net/1993/3161 accessed March 25, 2015; Charles Jeurgens, "The Scent of the Digital Archive. Dilemmas with Archive Digitisation," bmgn - Low Countries Historical Review, 128-4 (2013): 30-54, http://www.bmgn-lchr.nl/index.php/bmgn/article/view/9348 accessed March 25, 2015. 
The latter is a subject that is frequently overlooked in memory studies, not taking into account (probably not knowing) the growing number of studies by archivistics scholars. ${ }^{159}$

But what is the archive(s) representing? Bernadine Dodge writes:

Archivists perform a variety of tasks which engage with the past in one way or another. And while we likely agree that a reconstruction of an authentic past is unachievable, most of us still adhere to the notion that our professional practices lend themselves to the preservation of authentic fragments and textual artefacts which indicate something of real events, real deeds, real decisions, real administrative structures, real lives. ${ }^{160}$

Do archivists represent authentic records and archives to represent an authentic past? Could they learn from other disciplines? "Authenticity will be the buzzword of the twenty-first century." ${ }^{\text {"161 }}$ However, the InterPARES 2 research team was puzzled in finding that in many sciences the concept of authenticity is not well developed, in contrast with accuracy. On the other hand,

questions about the accuracy of the data maintained over time are not dissimilar to questions relating to the authenticity of records maintained over time ... Concerns for data lineage can be seen as analogous to archival concerns over provenance and the chain of custody, and the recognition that reliable datasets are connected to authoritative data collectors has echoes of archival concerns for the authority of records creators. ${ }^{162}$

Another important finding of InterPARES that may lead to a return in archivistics:

is the relative importance that scientists place on the content of a record in terms of its data quality (i.e., the accuracy of its content) when appraising its long-term value, something that archivists have hitherto generally considered irrelevant when conducting appraisals. ${ }^{163}$

159 Schwartz and Cook, "Archives, Records, and Power"; Harris, "Ethics”; Randall C. Jimerson, Archives Power. Memory, Accountability, and Social Justice (Chicago: Society of American Archivists, 2009); Susan Tucker, The Most Public of All History: Family History and Heritage Albums in the Transmission of Records (Doctoral thesis, University of Amsterdam, 2009), http://dare.uva.nl/record/325290, accessed March 25, 2015; Cook, "The Archive(s) is a Foreign Country".

160 Dodge, "Across the Great Divide," 17 (my italics).

161 Dodge, "Across the Great Divide," 16.

162 Roeder, et al., "Part Three - Authenticity," 22.

163 Roeder, et al., "Part Three - Authenticity," 22. 
Authenticity is a central concept in many disciplines, among them archivistics and diplomatics, as well as in the arts. In musical performance practice, for example, philosopher Peter Kivy distinguishes four notions of authenticity: (1) authenticity as authorial intention (faithfulness to the composer's intentions), (2) authenticity as sound (faithfulness to the sound of a performance during the composer's lifetime), (3) authenticity as practice (faithfulness to the practice of a performance during the composer's lifetime), and (4) the "personal authenticity" of the performer: faithfulness to the performer's own self. ${ }^{164}$ These notions of authenticity could reverberate in archivistics, comparable to the reception of legal and historical notions of authentic evidence in archivistics - analysed by Heather MacNeil in her book Trusting Records ${ }^{165}$ - and from there into museology, copyright law, digital forensics, etc. - as shown by the citations to MacNeil's book. The InterPARES experiments in the performative arts confirmed the need for expansions to the traditional conceptions of authenticity, reliability and accuracy. ${ }^{166}$

\section{Conclusion}

Archival turns and returns stretch the traditional boundaries of archivistics. Its object remains: records and archives as provenance-bound instruments for and by-products of transactions of public or private business. However, we now approach that object in different modes: archival turns and returns allow - or force - to view the archive(s) in a different light in archival practice and in archival theory.

In archival practice a turn from archives-as-sources to archives-assubject would seem to be taken for granted. But sometimes archivists and archival managers in their ambition to exploit the archives seem to forget that one cannot provide users with information from the archives, without having studied the archive(s). Archivists are scholars of recordkeeping who have knowledge "about records creation, its surrounding personal and organizational cultures, types of records, record-keeping systems, and custodial and archival histories." 167 That knowledge has to be shared with

\footnotetext{
164 Peter Kivy, Authenticities: Philosophical Reflections on Musical Performance (Ithaca/ London: Cornell University Press, 1995).

165 Heather MacNeil, Trusting Records: Legal, Historical and Diplomatic Perspectives (Dordrecht/ Boston/London: Kluwer, 2000); MacNeil, "Trust and Professional Identity."

166 Roeder et al., "Part Three - Authenticity," 39.

167 Tom Nesmith, "What's History Got to Do with It?: Reconsidering the Place of Historical Knowledge in Archival Work," Arcbivaria, 57 (2004), 27.
} 
and promoted to society, and it can be enhanced by interaction with the disciplines who have made the archival turn. Archivists should endeavour more engagement (in conferences, publications and other outreach activities) with disciplines who have made the archival turn but who rarely recognise what archival practice can contribute to their discourse.

New understandings of archives - anything "as archive" - could enrich archival practice. In community archiving, for example, professional and "amateur" archivists are dealing with an archival multiverse that crosses the borders of traditional archival practice. So, for example, could the archival impulse in installation art lead to acknowledging the user as "produser" participating in immersive (digital) environments. Consequently, the use of archives can be embedded in or at least framed as an experience. This can also be regarded as a consequence of the social turn, which emphasises that a record is an interface with and constituent of the social world. This is reinforced by the performative turn which may cause archival practice to refocus on what a record does in social relations, rather than what a record is. Such reconceptualisations of what archives are - and for whom, for what, when and where - will benefit from the turns in archivistics of which some examples were given in section 7 .

For archival theory, the archival turns and returns entail the need to understand and apply the multiform approach of archives "as it is" in conjunction with treating phenomena "as archives." Both views can be framed in an understanding of the archive as a process and as a mediated social and cultural practice. Time and space condition the archive (archivalterity). The "will to archive" is a will to re-enact performative instantiations of the archive, which are activations of the ever-changing archive. This leads to questioning traditional concepts of originality, uniqueness, authenticity and authority. In dealing with these questions archivistics researchers should communicate with other disciplines. The same is true for further research of the duality (or double hermeneutics) of archival structures and contexts (with respect to archives "as it is" and "as archives"). In researching the ontological and epistemological archive(s) archivistics applies the archival method that is specific for the discipline, ${ }^{168}$ but also adopts methods from other disciplines, as evidenced by various chapters in this book.

Archivistics is increasingly profiting from different turns (linguistic, social, performative, and representational). The impact of these turns on the

168 See the chapter "The Archival Method" by Luciana Duranti and Giovanni Michetti, in this volume. 
research agenda of the archivistics multiverse will hopefully increase as more students and scholars coming from elsewhere engage with the archivistics domain. But for that influx and influence to materialise, the outcome of archivistics research should be promoted among other disciplines more prominently as a beneficial archival return.

\section{References}

Alphen, Ernst van. "Archival Obsessions and Obsessive Archives," in What is Research in the Visual Arts? Obsession, Archive, Encounter, ed. Michael Ann Holly, and Marquard Smith (Williamstown Mass.: Sterling and Francine Clark Art Institute, 2008), 65-84.

Alphen, Ernst van. "Visual Archives and the Holocaust: Christian Boltanski, Ydessa Hendeles and Peter Forgacs," in Intercultural Aesthetics. A Worldview Perspective, ed. Antoon Van den Braembussche, Heinz Kimmerle, and Nicole Note (S.1.: Springer, 2009), 137-155.

Ames, Kenneth L. "Introduction", in The colonial revival in America, ed. Alan Axelrod (Wilmington Del: Henry Francis du Pont Winterthur Museum, 1975), 10-13.

Ankersmit, Frank R. "Historical Representation," History and Theory 27 (1988): 205-28.

Assmann, Aleida. "Memory, Individual and Collective," in The Oxford Handbook of Contextual Political Analysis, ed. R.E. Goodin, and C. Tilly (Oxford: University of Oxford Press, 2006), 210-24.

Assmann, Aleida. "Re-framing memory. Between individual and collective forms of constructing the past," in Performing the Past. Memory, History and Identity in Modern Europe, ed. K. Tilmans, F. van Vree, and J. Winter (Amsterdam: Amsterdam University Press, 2010), 35-50.

Bastian, Jeannette A. "The records of memory, the archives of identity: celebrations, texts and archival sensibilities," Archival Science 13 (2013): 121-31.

Becker, Peter and William Clark. "Introduction," in Little Tools of Knowledge. Historical Essays on Academic and Bureaucratic Practices, ed. Peter Becker and William Clark (Ann Arbor: University of Michigan Press, 2011), 1-34.

Blair, Ann, ed. "Toward a Cultural History of Archives," special issue of Archival Science 7, 4 (2007).

Blouin, Francis X. and William G. Rosenberg, eds. Archives, Documentation, and Institutions of Social Memory. Essays from the Sawyer Seminar (Ann Arbor: University of Michigan Press, 2006).

Blouin, Francis X. and William Rosenberg. Processing the Past: Contesting Authority in History and the Archives (Oxford: Oxford University Press, 2011).

Bonnell, Victoria E., and Lynn Hunt. "Introduction," in Beyond the Cultural Turn. New Directions in the Study of Society and Culture, ed. Victoria E. Bonnell and Lynn Hunt (Berkeley/Los Angeles/London: University of California Press, 1999), 1-32.

Botticelli, Peter. "Records Appraisal in Network Organizations," Archivaria 49 (2000): 161-91.

Bowker, Geoffrey C. Memory Practices in the Sciences (Cambridge Mass./London: MIT Press, 2005).

Breakell, Sue. "Encounters with the self: archives and research," in The Future of Archives and Recordkeeping. A reader, ed. Jennie Hill (London: Facet, 2011), 23-36.

Brothman, Brien. "Declining Derrida: Integrity, Tensegrity, and the Preservation of Archives from Deconstruction," Archivaria 48 (1999): 64-88. 
Brothman, Brien. "Archives, life cycles, and death wishes: a helical model of record formation," Archivaria 61 (2006): 235-69.

Brown, Caroline, ed. "Memory, identity and the archival paradigm," special issue of Archival Science 13, 2/3 (2013).

Buchanan, Alexandrina. "Strangely unfamiliar: ideas of the archive from outside the discipline," in The Future of Archives and Recordkeeping. A reader, ed. Jennie Hill (London: Facet, 2011), 37-62.

Burton, Antoinette, ed. Archive Stories. Facts, Fictions, and the Writing of History (Durham/ London: Duke University Press, 2005).

Castells, Manuel. Communication power (Oxford: Oxford University Press, 2009).

Clifford, James and George E. Marcus, ed. Writing Culture. The Poetics and Politics of Ethnography (Berkeley/Los Angeles/London: University of California Press, 1986).

Codebò, Marco. Narrating from the Archive: Novels, Records, and Bureaucrats in the Modern Age (Danvers Mass.: Rosemont, 2010).

Confino, Alon. "Collective Memory and Cultural History: Problems of Method," The American Historical Review 102 (1997): 1386-1403.

Conway, Paul. "Digital transformations and the archival nature of surrogates," Archival Science 15 (2015): 51-69.

Cook, Terry. "What Is Past Is Prologue: A History of Archival Ideas since 1898, and the Future Paradigm Shift," Archivaria 43 (1997): 17-63.

Cook, Terry. "The Archive(s) is a Foreign Country: Historians, Archivists, and the Changing Archival Landscape," American Archivist 74 (2011): 600-32.

Cooren, François. "Textual agency: How texts do things in organizational settings". Organization 11 (2004): 373-93.

Craig, Barbara L. "Selected Themes in the Literature on Memory and their Pertinence to Archives," American Archivist 65 (2002): 276-89.

Crews, Spencer R. and James E. Smis, "Locating authenticity: fragments of a dialogue, in: The poetics and politics of museum display, ed. Ivan Karp and Steven D. Lavine (Washington and London: Smithsonian Institution Press, 1991): 109-75.

Currall, James, Michael Moss, and Susan Stuart, "What is a Collection?" Archivaria 58 (2004): 131-46.

Derrida, Jacques. “Archive fever. A seminar..," in Refiguring the archive, ed. Carolyn Hamilton et al. (Dordrecht/Boston/London: Kluwer, 2002), 38-80.

Derrida, Jacques. Archive Fever, trans. Eric Prenowitz (Chicago and London: University of Chicago Press, 1996).

Derrida, Jacques. "Living On," in Deconstruction and Criticism, ed. Harold Bloom et al. (New York: Continuum, 2004), 62-142.

Dirks, Nicholas B. "Annals of the Archive: Ethnographic Notes on the Sources of History," in From the Margins. Historical Anthropology and Its Future, ed. Brian Keith Axel (Durham/London: Duke University Press, 2002), 47-65.

Dodge, Bernadine. "Across the Great Divide: Archival Discourse and the (Re)presentations of the Past in Late-Modern Society," Archivaria 53 (2002): 16-30.

Dreyblatt, Arnold and Jeffrey Wallen, "Hands on the Document: Arnold Dreyblatt's T-Archive," in Sign Here! Handwriting in the Age of New Media, ed. Sonja Neef, José van Dijck, and Eric Ketelaar (Amsterdam: Amsterdam University Press, 2006), 134-49.

Duranti, Luciana. "Archival science," in Encyclopedia of library and information science, vol. 59 suppl. 22, ed. A. Kent (New York/Basel: Marcel Dekker, 1997), 1-19.

Eastwood, Terry. "A Contested Realm: The Nature of Archives and the Orientation of Archival Science," in Currents of archival thinking, ed. Terry Eastwood and Heather MacNeil (Santa Barbara: Libraries Unlimited, 2010), 3-21. 
Ebeling, Knut and Stephan Günzel, Archivologie. Theorien des Archivs in Wissenschaft, Medien und Künsten (Berlin: Kulturverlag Kadmos, 2009).

Echevarria, Roberto G. Myth and Archive: A Theory of Latin American Narrative (Durham/ London: Duke University Press, 1998).

Eichhorn, Kate. "Archival Genres: Gathering Texts and Reading Spaces," Invisible Culture 12 (2008): 1-10: http://rochester.edu/in_visible_culture/Issue_12/eichhorn/eichhorn. pdf, accessed March 25, 2015.

Eliassen, Knut Ove. "The Archives of Michel Foucault,"in The Archive in Motion. New Conceptions of the Archive in Contemporary Thought and New Media Practices, ed. Eivind Røssaak (Oslo: Novus Press, 2010), 29-51

Enwezor, Okwui. Archive Fever - Uses of the Document in Contemporary Art (New York: International Center of Photography, 2008).

Fabian, Johannes. Memory against Culture. Arguments and reminders (Durham/London: Duke University Press, 2007).

Farge, Arlette. The Allure of the Archives, transl. Thomas Scott-Railton (New Haven, CT: Yale University Pres, 2013) (orig edn Arlette Farge, Le goût de l'archive (Paris: Seuil, 1989).

Feldman, Ilana. Governing Gaza. Bureaucracy, Authority, and the Work of Rule, 1917-1967 (Durham/London: Duke University Press, 2008).

Flach, Willy. "Goethes Literarisches Archiv" in Archivar und Historiker. Studien zur Archivund Geschichtswissenschaft. Zum 65. Geburtstag von Heinrich Otto Meisner (Berlin: Rütten \& Loening, 1956), 44-71.

Foscarini, Fiorella. "Organizational Records as Genres. An Analysis of the 'Documentary Reality' of Organizations from the Perspectives of Diplomatics, Records Management and Rhetorical Genre Studies" in Genre Theory in Information Studies ed. Jack Andersen (London: Emerald, 2015), 115-32.

Foster, Hal. "An Archival Impulse," October 110 (2004): 3-22.

Foucault, Michel. Archaeology of Knowledge (London/New York: Routledge, 2002).

Giddens, Anthony. A Contemporary Critique of Historical Materialism. Vol. 1 Power, property and the state (London/Basingstoke: Macmillan Press, 1981).

Hamilton, Carolyn, Verne Harris, Jane Taylor, Michele Pickover, Graeme Reid, and Razia Saleh, ed. Refiguring the Archive (Dordrecht/Boston/London: Kluwer Academic Publishers, 2002).

Harris, Verne. "Ethics and the archive: 'An incessant movement of recontextualisation", in Controlling the past. Documenting society and institutions. Essays in honor of Helen Willa Samuels, ed. Terry Cook (Chicago: Society of American archivists, 2011), 345-62.

Head, Randolph, ed. "Archival Knowledge Cultures in Europe, 1400-1900," special issue of Archival Science, 10, 3 (2010).

Hedstrom, Margaret. "Archives and collective memory: More than a metaphor less than an analogy," in Currents of archival thinking, ed. Terry Eastwood and Heather MacNeil (Santa Barbara: Libraries Unlimited, 2010), 163-79.

Heslop, Helen, Simon Davis and Andrew Wilson. An Approach to the Preservation of Digital Records (Canberra: National Archives of Australia, 2002), 8 http://www.naa. gov.au/Images/An-approach-Green-Paper_tcm16-47161.pdf, accessed March 25, 2015.

Hunt, Lynn. "Introduction: History, Culture, and Text," in The new cultural history, ed. Lynn Hunt (University of California Press, Berkeley, Los Angeles, London, 1989), 1-22.

ISO 30300. Information and Documentation - Records Management - Part 2: Guidelines (Geneva: International Standards Organization, 2011). 
Jacobsen, Trond, Ricardo L. Punzalan, and Margaret L. Hedstrom. "Invoking 'collective memory': mapping the emergence of a concept in archival science”, Archival Science 13 (2013): 217-51.

Jeurgens, Charles. "The Scent of the Digital Archive. Dilemmas with Archive Digitisation," bmgn - Low Countries Historical Review 128-4 (2013): 30-54, http://www.bmgn-lchr. nl/index.php/bmgn/article/view/9348 accessed March 25, 2015.

Jimerson, Randall C. Archives Power. Memory, Accountability, and Social Justice (Chicago: Society of American Archivists, 2009).

Jones, Sarah, Daisy Abbott, and Seamus Ross. “Redefining the performing arts archive," Archival Science 9 (2009): 165-71.

Kaplan, Elisabeth. "Many Paths to Partial Truths: Archives, Anthropology, and the Power of Representation," Archival Science 2 (2002): 209-20.

Keen, Suzanne. Romances of the Archive in Contemporary British Fiction (Toronto/Buffalo/ London: University of Toronto Press, 2001).

Ketelaar, Eric. “Tacit Narratives: The Meanings of Archives," Archival Science 1 (2001): 143-55.

Ketelaar, Eric. "Writing on Archiving Machines," in Sign Here! Handwriting in the Age of Nerw Media, ed. Sonja Neef, José van Dijck, and Eric Ketelaar (Amsterdam: Amsterdam University Press, 2006), 183-95.

Ketelaar, Eric. "Everyone an archivist", in: Managing and Archiving Records in the Digital Era. Changing Professional Orientations, ed. Niklaus Bütikofer, Hans Hofman, and Seamus Ross (Baden, hier + jetzt, Verlag für Kultur und Geschichte, Baden, 2006) 9-14.

Ketelaar, Eric. "Archives in the Digital Age: New Uses for an Old Science. Archives \&" Social Studies: A Journal of Interdisciplinary Research, 1 (2007): 167-91, available at http://archivo.cartagena.es/files/36-168-DOC_FICHERO1/10-ketelaar_archives. pdf, accessed March 25, 2015.

Ketelaar, Eric. "Exploration of the archived world: from De Vlamingh's Plate to digital realities," Archives and manuscripts 36 (2) (2008): 13-33.

Ketelaar, Eric. "Archivistics: science or art?" in The future of archives and recordkeeping. A reader, ed. Jennie Hill (London: Facet, 2011), 89-100.

Ketelaar, Eric. "Cultivating Archives," Archival Science 12 (2012): 19-33.

Ketelaar, Eric. "Archives, memories and identities," in Archives and Recordkeeping: Theory into Practice, ed. Caroline Brown (London: Facet, 2013), 131-70.

Kivy, Peter. Authenticities. Philosophical Reflections on Musical Performance (Ithaca/London: Cornell University Press, 1995).

Latour, Bruno. Science in action. How to follow scientists and engineers through society (Cambridge Mass.: Harvard University Press, 1987).

Latour, Bruno. La fabrique du droit, transl.: The Making of Law: An Ethnography of the Conseil d'Etat (Cambridge: Polity, 2002).

Lemieux, Victoria L. "Let the Ghosts Speak: An Empirical Exploration of the "Nature" of the Record," Archivaria 51 (2001): 81-111.

Lepecki, André. "The Body as Archive: Will to Re-Enact and the Afterlives of Dances," Dance Research Journal, 42 (2) (2010): 28-48.

López, Alfred J. “The Plantation as Archive: Images of the 'South' in the Postcolonial World”. Comparative Literature 63 (2011): 402-22.

Lubar, Steven. "Information Culture and the Archival Record," American Archivist 62 (1999): 10-22.

Maanen, John van, and Brian T. Pentland. "Cops and Auditors: The Rhetoric of Records," in The Legalistic Organization, ed. Sim B. Sitkin, and Robert J. Bies (Thousand Oaks: Sage Publications, 1994), 53-90. 
MacNeil, Heather. Trusting Records. Legal, Historical and Diplomatic Perspectives (Dordrecht/ Boston/London: Kluwer, 2000).

MacNeil, Heather. "Picking Our Text: Archival Description, Authenticity and the Archivist as Editor," American Archivist 68 (2005): 264-78.

MacNeil, Heather. “Archivalterity: Rethinking Original Order," Archivaria 66 (2008): $1-24$.

MacNeil, Heather. "Trust and professional identity: narratives, counter-narratives and lingering ambiguities," Archival Science 11 (2011): 175-92.

Manoff, Marlene. "Theories of the Archive from Across the Disciplines," Libraries and the Academy 4 (2004): 9-25.

McKemmish, Sue, and Anne Gilliland. "Archival and recordkeeping research. Past, present and future," in Research Methods. Information, Systems and Contexts, ed. Kirsty Williamson and Graeme Johanson (Prahran: Tilde, 2013), 79-112.

Merewether, Charles. "Introduction/Art and the Archive," in The Archive, ed. Charles Merewether (London: Whitechapel Gallery, 2006), 10-17.

Millar, Laura. "Touchstones: Considering the Relationship between Memory and Archives," Archivaria 61 (2006): 105-26.

Milligan, Jennifer. "What is an Archive?' in the History of Modern France," in Archive Stories. Facts, Fictions, and the Writing of History, ed. Antoinette Burton (Durham/ London: Duke University Press, 2005), 159-83.

Milligan, Jennifer. "The Problem of Publicité in the Archives of the Second Empire France," in Archives, Documentation, and Institutions of Social Memory. Essays from the Sawyer Seminar, ed. Francis X. Blouin, and William G. Rosenberg (Ann Arbor: University of Michigan Press, 2006), 20-35.

Misztal, Barbara A. Theories of Social Remembering (Maidenhead/Philadelphia: Open University Press, 2003).

Moore, Lara J. Restoring Order. The Ecole des Chartes and the Organization of Archives and Libraries in France, 1821-1870 (Duluth, Minn.: Litwin Books, 2008).

Morris, Charles E. "The Archival Turn in Rhetorical Studies; Or, the Archive's Rhetorical (Re)turn," Rhetoric \& Public Affairs 9 (2006): 113-15.

Muller, Samuel, Johan A. Feith, and Robert Fruin. Manual for the arrangement and description of archives, trans. Arthur H. Leavitt, with new introd. (Chicago: Society of American Archivists, 2003).

Nesmith, Tom. "Still Fuzzy, But More Accurate: Some Thoughts on the 'Ghosts' of Archival Theory," Archivaria 47 (1999): 136-50.

Nesmith, Tom. "Seeing Archives: Postmodernism and the Changing Intellectual Place of Archives," American Archivist 65 (2002): 24-41.

Nesmith, Tom. "What's History Got to Do with It?: Reconsidering the Place of Historical Knowledge in Archival Work," Archivaria 57 (2004): 1-27.

O'Driscoll, Michael, and Edward Bishop. "Archiving 'Archiving”'. English Studies in Canada 30 (2004): 1-16.

O’Toole, James M. “On the idea of uniqueness,” American Archivist 57 (1994): 632-58, repr. in American Archival Studies. Readings in Theory and Practice, Randall C. Jimerson, ed. (Chicago: Society of American archivists, 2000), 245-77.

O’Toole, James M. “The symbolic significance of archives," American Archivist 56 (1993): 234-255, repr. in American Archival Studies. Readings in Theory and Practice, Randall C. Jimerson, ed. (Chicago: Society of American archivists, 2000), 47-72.

Ogborn, Miles. Indian ink. Script and Print in the Making of the English East India Company (Chicago/London: University of Chicago Press, 2007).

Oliver, Gillian, and Wendy M. Duff, ed. "Genre Studies in Archives," special issue of Archival Science 12, 4 (2012). 
Oliver, Gillian, Yunhyong Kim, and Seamus Ross. "Documentary genre and digital recordkeeping: red herring or a way forward?” Archival Science 8 (2008): 295-305.

Olson, Lynn M. "Record Keeping Practices: Consequences of Accounting Demands in a Public Clinic,” Qualitative Sociology 18 (1995): 45-70.

Orlikowski, Wanda J. "The Duality of Technology: Rethinking the Concept of Technology in Organizations," Organization Science 3 (1992): 398-427.

Papailias, Penelope. Genres of Recollection: Archival Poetics and Modern Greece (New York: Palgrave MacMillan, 2005).

Papailias, Penelope. "Writing Home in the Archive: 'Refuge Memory' and the Ethnograpohy of Documentation," in Archives, Documentation, and Institutions of Social Memory. Essays from the Sawyer Seminar, ed. Francis X. Blouin, and William G. Rosenberg (Ann Arbor: University of Michigan Press, 2006), 402-16.

Peeren, Esther. The Spectral Metaphor: Living Ghosts and the Agency of Invisibility (Basingstoke/New York: Palgrave Macmillan, 2014).

Prescott, Andrew. "The Textuality of the Archive," in What are Archives? Cultural and Theoretical Perspectives: A Reader, ed. Louise Craven (Aldershot: Ashgate, 2008), 31-51.

Ramsey, Alexis E., et al. Working in the archives: practical research methods for rhetoric and composition (Carbondale: Southern Illinois University Press, 2009).

Rao, Vyjayanthi. "Embracing Urbanism: The City as Archive," New Literary History 40 (2009): 371-83.

Rawlings, Gregory. "Statelessness, Citizenship and Annotated Discriminations: Meta Documents and the Aesthetics of the Subtle at the United Nations," History and Anthropology 22 (2011): 461-79.

Reinfandt, Christoph. "Reading texts after the linguistic turn: approaches from literary studies and their implications," in Reading primary sources. The interpretation of texts from nineteenth- and twentieth-century history, ed. Miriam Dobson and Benjamin Zimmerman (London/New York: Routledge, 2009), 37-54.

Rekrut, Alicia. Reconnecting Mind and Matter: Materiality in Archival Theory and Practice. (Master's thesis University of Winnipeg, 2009), http://hdl.handle.net/1993/3161 accessed March 25, 2015.

Richards, Thomas. The Imperial Archive: Knowledge and the Fantasy of Empire (London: Verso, 1993).

Ricoeur, Paul. Memory, History, Forgetting, trans. Kathleen Blamey, and David Pellauer. (Chicago: University of Chicago Press, 2004).

Rieger, Monika. "Anarchie im Archiv. Vom Künstler als Dammler," in Archivologie. Theorien des Archivs in Wissenschaft, Medien und Künsten, ed. Knut Ebeling, and Stephan Günzel (Berlin: Kulturverlag Kadmos, 2009), 253-69.

Riles, Annelise, ed. Documents. Artifacts of Modern Knowledge (Ann Arbor: University of Michigan Press, 2006).

Robin, Régine. La mémoire saturée (Paris: Éditions Stock, 2003).

Roeder, John, Philip Eppard, William Underwood, and Tracey P. Lauriault, "Part Three - Authenticity, Reliability and Accuracy of Digital Records in the Artistic, Scientific and Governmental Sectors: Domain 2 Task Force Report," [electronic version] in International Research on Permanent Authentic Records in Electronic Systems (InterPARES) 2: Experiential, Interactive and Dynamic Records, ed. Luciana Duranti and Randy Preston (Padova: Associazione Nazionale Archivistica Italiana, 2008) http://www.interpares.org/display_file.cfm?doc=ip2_book_part_3_domain2_task_ force.pdf, accessed March 25, 2015.

Sabean, David W. "Peasant Voices and Bureaucratic Texts: Narrative Structure in Early Modern German Protocols," in Little Tools of Knowledge. Historical Essays 
on Academic and Bureaucratic Practices, ed. Peter Becker, and William Clark (Ann Arbor: University of Michigan Press, 2001), 67-93.

Sæther, Susanne Østby. "Archival Art: Negotiating the Role of New Media," in The Archive in Motion. New Conceptions of the Archive in Contemporary Thought and New Media Practices, ed. Eivind Røssaak (Oslo: Novus Press, 2010), 77-108.

Schaffner, Ingrid, and Matthias Winzen, ed. Deep Storage. Collecting, Storing, and Archiving in Art (Munich/New York: Prestel, 1998).

Schmuland, Arlene B. “The Archival Image in Fiction: An Analysis with an Annotated Bibliography," American Archivist 62 (1999): 24-73.

Schwartz, Joan M. and Terry Cook. "Archives, records, and power: the making of modern memory," Archival Science 2 (2002): 1-19.

Sekula, Allan. "The Body and the Archive," October 39 (1986): 3-64.

Sellen, Abigail, and Richard Harper. The Myth of the Paperless Office (Cambridge Mass./ London: MIT Press, 2002).

Spiegel, Gabrielle M. "History, Historicism, and the Social Logic of the Text in the Middle Ages," Speculum 65 (1990): 59-86, repr. in Gabrielle M. Spiegel, The Past as Text. The Theory and Practice of Medieval Historiography (Baltimore/London: Johns Hopkins University Press, 1997), 3-43.

Spieker, Sven, ed. The Big Archive. Art From Bureaucracy (Cambridge Mass./London: MIT Press, 2008).

Steedman, Carolyn. Dust (Manchester: Manchester University Press, 2002).

Stoler, Ann L. "Colonial Archives and the Arts of Governance," Archival Science 2 (2002): 87-109.

Stoler, Ann L. "Colonial Archives and the Arts of Governance. On the Content in the Form," in: Refiguring the Archive, ed. Carolyn Hamilton, et al. (Dordrecht/Boston/ London: Kluwer Academic Publishers, 2002), 83-100.

Stoler, Ann L. Along the Archival Grain. Epistemic Anxieties and Colonial Common Sense. (Princeton and Oxford: Princeton University Press, 2009).

Taylor, Diana. The Archive and the Repertoire. Performing Cultural Memory in the Americas. (Durham/London: Duke University Press, 2003).

Taylor, Hugh. "'My Very Act and Deed': Some Reflections on the Role of Textual Records in the Conduct of Affairs," American Archivist 51 (1988): 456-69; repr. in Imagining Archives. Essays and Reflections by High A. Taylor, ed. Terry Cook, and Gordon Dodds (Lanham/Oxford: Scarecrow Press, 2003), 131-48.

The Fictional World of Archives, Art Galleries \& Museums: http://fictionalarchives.blogspot. com, accessed March 25, 2015.

The philosophie, commonly called, the Morals, written by the learned philosopher, Plutarch of Charonea, trans. Philemon Holland (London: A. Hatfield, 1603).

Thom, Paul. For an Audience. A Philosophy of the Performing Arts (Philadelphia: Temple University Press, 1993).

Thomassen, Theo. "The Development of Archival Science and its European Dimension," in The Archivist and the Archival Science. Seminar for Anna Christina Ulfsparre ... (Lund: Landsarkivet, 1999), 67-74.

Thomassen, Theo. "A First Introduction to Archival Science," Archival Science 1 (2001): 373-85.

Trace, Ciaran B. "What is Recorded is Never Simply 'What Happened': Record Keeping in Modern Organizational Culture," Archival Science 2 (2002): 137-59.

Tucker, Susan. The Most Public of All History: Family History and Heritage Albums in the Transmission of Records (Doctoral thesis, University of Amsterdam, 2009), http://dare. uva.n1/record/325290, accessed March 25, 2015. 
Upward, Frank. "The records continuum" in Archives: Recordkeeping in Society, ed. Sue McKemmish, Michael Piggott, Barbara Reed, and Frank Upward (Wagga-Wagga: Charles Sturt University, 2005), 197-222.

Uthemann, Ernest W., ed. Arnold Dreyblatt. Aus den Archiven/From the Archives (Heidelberg: Kehrer Verlag, 2003).

Vaihinger, H. The Philosophy of "As if", transl. C.K. Ogden (London: Routledge \& Kenan Paul, 1935, $2^{\text {nd }}$ ed).

Verbeek, Peter P. What Things Do: Philosophical Reflections on Technology, Agency, and Design (University Park, Pa: Pennsylvania State University Press, 2005).

Verhoeff, Nanna. "Archival Poetics," Screening the Past: An International Electronic Journal of Visual Media and History 14 (2002), https://web.archive.org/web/20021124213805/ http://www.latrobe.edu.au/screeningthepast/firstrelease/fr0902/nvfr14d.htm, accessed March 25, 2015, repr. in Narrative Theory. Critical Concepts in Literary and Cultural Studies, vol. 2, Mieke Bal, ed. (New York: Routledge, 2004), 388-410.

Vivo, Filippo de, Andrea Guidi, and Alessandro Silvestri. "Archival Transformations in Early Modern European History," European History Quarterly, 46 (2016): 421-34.

Voss, Paul J., and Marta L. Werner. "Toward a Poetics of the Archive: Introduction," Studies in the Literary Imagination 32 (1999): i-viii.

Wartenbe, Michael. "ANTs in the Archive: Actor-Network Theory as a Research Methodology for Archival and Recordkeeping Research," forthcoming in Archival Science.

Winter, Jay. "Historians and Sites of Memory," in Memory in Mind and Culture, ed. Pascal Boyer, and James V. Wertsch (Cambridge: Cambridge University Press, 2009), 252-68.

Xie, Sherry L. "Building Foundations for Digital Records Forensics: A Comparative Study of the Concept of Reproduction in Digital Records Management and Digital Forensics," American Archivist 74 (2011): 576-99.

Yakel, Elizabeth. "The Way Things Work: Procedures, Processes, and Institutional Records," American Archivist 59 (1996): 454-64.

Yakel, Elizabeth. "The Social Construction of Accountability: Radiologists and Their Record-Keeping Practices," The Information Society: An International Journal 17 (2001): 233-45.

Yakel, Elizabeth. "Archival Representation," Archival Science 3 (2003): 1-25.

Yates, JoAnne. Control through Communication. The Rise of System in American Management (Baltimore/London: Johns Hopkins University Press, 1989).

Yeo, Geoffrey. "Nothing is the same as something else': significant properties and notions of identity and originality," Archival Science 10 (2010): 85-116.

Zyl, Susan van. "Psychoanalysis and the Archive: Derrida's Archive Fever," in Refiguring the Archive, ed. Carolyn Hamilton et al. (Dordrecht/Boston/London: Kluwer, 2002), 39-59. 
Chapter 8

\title{
MEMORY RESEARCH / ARCHIVAL RESEARCH
}

\author{
Jeannette A. Bastian
}

\begin{abstract}
Memory studies are an increasing focus of academic attention in many disciplines, particularly in the social sciences. Archivists, while long concerned with issues of memory, have not traditionally focused on memory as an area of research. This chapter approaches memory studies as an emerging research area for archivists, offering a rationale for archival engagement in memory studies, exploring previous research, suggesting new avenues and methods of research, and giving examples of research approaches. The object is to construct a base for memory research by archivists, suggesting new approaches while acknowledging existing ones.
\end{abstract}

\section{Introduction}

In his twentieth century classic, The Great War and American Memory, literary scholar Paul Fussell utilises poetry, prose and literary criticism to analyse, evoke and encapsulate collective memories of World War I. ${ }^{1}$ In The Politics of Regret, sociologist Jeffrey Olick examines the role that memory plays in social structures arguing that "collective memory ... is not identical to the memories of a certain percentage of the population but constitutes a social fact in and of itself." ${ }^{2}$ Considering "whole new orders of documentation" and focusing on society's collective cultural recognition, historian Raphael Samuel identifies "the growing importance of 'memory places' in ideas of

1 Paul Fussell, The Great War and Modern Memory. London: Oxford University Press, 1975.

2 Jeffrey Olick, The Politics of Regret: On Collective Memory and Historical Responsibility. New York: Routledge, 2007, p.7. 
the historical past." ${ }^{3}$ Merlin Donald, a cognitive neuroscientist, makes the distinction between "biological memory - memory contained within the individual, and external memory - memory as part of a collective external storage system." ${ }^{\text {G }}$ Gillian Straker, a psychoanalyst grounded in the theory that the past shapes the future, examines traumatic memories within the archival narratives of apartheid victims. ${ }^{5}$

As these examples suggest, while memory studies have become ubiquitous over the past four decades, each discipline grapples with memory through the prism of its own critical structures. As a consequence of these varied lenses, memory studies and memory research closely ally to disciplinespecific perspectives while at the same time, as demonstrated through the recently published Collective Memory Reader, they are gradually coalescing around common themes and issues. ${ }^{6}$ Collective memory - also referred to as social or cultural memory - is the primary focus of this scholarship, although personal memory - closely related to the collective - is inevitably part of the equation. Both personal and collective memory draw core principles from the writings of sociologist Maurice Halbwachs. Halbwachs, considered the father of collective memory, maintained that "while the collective memory endures and draws strength from its base in a coherent body of people, it is individuals as group members who remember ... every collective memory requires the support of a group delimited in time and space." Although Halbwachs referred to the relationships of individuals within social frameworks, his concept also applies more broadly to the study of collective memory itself where scholars are supported by the frameworks of their own disciplines and tend to define collective memory through those frames.

For archivists, a discipline-specific memory lens might include provenance, evidence or authenticity, but equally might be applied through activities associated with appraisal, access or preservation. What constitutes memory research seems more complicated and difficult to delineate and define for archivists than for sociologists, historians or literary scholars because in

3 Raphael Samuel, Theatres of Memory. London: Verso, 1999, pp.25, 39.

4 Merlin Donald, excerpt from Origins of the Modern Mind: Three Stages in the Evolution of Culture and Cognition, in J. Olick, ed. The Collective Memory Reader. London: Oxford University Press, 2011, p.325.

5 Gillian Straker, "Shaping Subjectivities, Private Memories, Public Archives," Psychoanalytic Dialogues 21 (2011): 643.

6 The Collective Memory Reader brings together over 90 essays on memory that span eras and disciplines but are organised into general common themes.

7 Maurice Halbwachs. On Collective Memory. Lewis A. Coser, ed. and trans., University of Chicago Press, p.22. 
many ways, archivists have always claimed a special relationship to memory. From Jean-Pierre Wallot's "houses of memory" to Verne Harris's "memory archivist," from Eric Ketelaar's "tacit memory"10 to McKemmish, Gilliland and Ketelaar's "communities of memory,"11 memory and archives have been linked together and seen as sharing an implicit and significant relationship. Not only do archivists collect, manage and preserve the "stuff" of memory, but through appraisal, they also participate to a great extent in determining its continuity and perpetuation. What gets remembered and what gets forgotten may not be completely in the hands of archivists, but certainly archivists through society's reliance on evidence and sources - have a prominent role to play in those decisions. Australian archivist Michael Piggott points out that since memory is "a fundamental relationship in [Hilary] Jenkinson's notion of a record," 12 a firm connection between records and memory was established early on in the minds of archival practitioners.

In digital environments in particular, where memory seems at once all-pervasive and infinitely fragile, long-term stability and survival may be dependent upon archival and preservation skills. Recent archival preoccupations with the power of archives identify memory as an important sphere of archival influence. Terry Cook and Joan Schwartz note that "Archives - as records - wield power over the shape and direction of historical scholarship, collective memory, and national identity, over how we know ourselves as individuals, groups and societies." ${ }^{13}$

With all these points of symbiosis between archives and memory, it might seem natural to assume that memory would be a conspicuous focus of archival research and scholarship, taking full advantage not only of the ubiquity and familiarity of the "stuff" but also of the many entry points into memory that

8 Jean-Pierre Wallot, "Building a Living Memory For the History of Our Present: New Perspectives on Archival Appraisal," Journal of the Canadian Historical Association 2 (1991): 282.

9 Verne Harris, "The Archival Sliver, Power, Memory and Archives in South Africa," Archival Science 2 (2002): 85.

10 Eric Ketelaar, “Tacit Narratives: The Meaning of Archives," Archival Science 1 (2001): 131-141.

11 Sue McKemmish, Anne Gilliland-Swetland and Eric Ketelaar, "Communities of Memory': Pluralising Archival Research and Education Agendas," Archives \& Manuscripts, 33 no.1 (2005): 146-174.

12 Michael Piggott, "Archives and Memory," in Sue McKemmish, Michael Piggott, Barbara Reed and Frank Upward, eds. Archives: Recordkeeping in Society. Wagga Wagga, New South Wales: Charles Sturt University, 2005, p.300.

13 Joan M. Schwartz and Terry Cook, "Archives, Records, and Power: The Making of Modern Memory” Archival Science 2 (2002): 2. 
archival processes suggest. And yet this is not the case. While aspects of memory are often part of the research equation, archivists' direct scholarly engagement with memory has tended to be theoretical, speculative and descriptive rather than empirical. Even in their own historical research, archivists rarely address memory directly although memory and memories also have a history. And as Richard Cox indicates in his study of the Secretary's Office (formerly a Public Records office) at Colonial Williamsburg in the United States, archivists have also neglected to remember themselves and their own field of endeavour. ${ }^{14}$ Questions of how archivists understand and engage memory in ways that illuminate, enhance and add new meaning to what they do, how they do it and the outcomes for which they strive have yet to be fully addressed in ways that add new dimensions to the principles and practices of the archival profession.

Why should archivists study memory? What are the archival issues around memory and through which lenses could those issues be explored to the benefit of both archival theory and practice? This essay probes these questions by offering a rationale for archival engagement in memory studies, exploring previous research, suggesting new avenues and methods of research, and giving examples of research approaches.

\section{Why Should Archivists Study Memory?}

A recent study by researchers at the University of Michigan examined the archives/memory relationship as expressed in the archival literature. Focusing on 165 articles published between 1980 and 2010 in four leading English-language archives journals, the authors analysed ways in which archivists engaged with memory. They identified major theoretical trends that largely revolved around the relationships between archives and memory. These relationships included cultural heritage, power dynamics and archives as surrogates for memory. An extensive citation analysis identified the archival theorists who were most influential in the profession and also queried whether archivists were influenced by writings on memory from outside the profession..$^{15}$ The authors concluded that heavy reliance on the same sources, a lack of references to scholarship in other fields and a lack of references

14 Richard J. Cox, "Public Memory Meets Archival Memory: The Interpretation of Williamsburg's Secretary's Office," American Archivist 68 (Fall/Winter 2005): 279-296.

15 Trond Jacobsen, Ricardo Punzalan and Margaret Hedstrom, "Invoking Collective Memory: Mapping the Emergence of a Concept in Archival Studies," Archival Science, 13 (June 2013): 217-251. 
by authors outside the archival field to archival writings suggested that "to a significant degree English language archival scholarship on collective memory remains insular and self-referential. ${ }^{16}$ Interestingly, this perceived insularity is echoed in a similar observation by Olick and his co-editors in the introduction to the Collective Memory Reader. There, the editors find that while the past decade in memory studies has seen advances in theoretical, conceptual and empirical scholarship, "unfortunately, this progress has been hampered by continued lack of cross-disciplinary and cross-case reading, as well as by redundancy and lack of systematicity in the enterprise."17

Turning these observations around, however, also suggests a more positive interpretation of this siloed thinking. Rather than embracing an interdisciplinary approach, each scholar may identify memory as a tool or strategy to be embedded within his or her own field of study. Instead of seeing memory studies either as a separate discipline in its own right or even as a shared concept, scholars may be concentrating on incorporating memory into their own fields in ways that augment and extend those disciplines, enabling responses to contemporary concerns in ways that were not previously possible. Seen in this light, the enthusiasm of archivists for theoretical engagements with memory but their lack of complex engagement at the research level may be simply a process of evolution. As archivists come to terms with the theoretical issues, they may be ready to move on to describing or testing these theories through empirical and historical research. There are indications in the literature that this testing has already begun, with the Michigan study itself representing a significant effort in establishing a foundation for that research.

But the archival diffidence around memory research may have other causes as well. To a large extent the spectre of memory hovers over all archival endeavours. Memory - often under the rubric of 'corporate memory' - is just one of a laundry list of core values associated with records that also includes evidence, authenticity, trust and access. ${ }^{18}$ Memory seems so obviously embedded in archival concerns that the memory aspect emerges as only one component within a much more intricate complex of archival issues.

The lack of focus on memory as a research subject in itself may also be because memory, considered as an isolated factor, is inevitably subjective, not directly a part of the deliberate work of archivists although a distinct

16 Jacobsen, Punzalan and Hedstrom, "Invoking Collective Memory", 226.

17 Olick et. al., The Collective Memory Reader, 38.

18 The Society of American Archivists' website lists seven core values including 'history and memory.' http://www2.archivists.org/statements/saa-core-values-statement-andcode-of-ethics. 
by-product. And, of course, the lingering accoutrements of the traditional and positivist archival stance of neutrality are not easily discarded, despite a plethora of new reflections and interpretations on this central motif in the archival literature. The values embedded in memory are not neutral. While archival thinking on neutrality has significantly advanced over the past decade, such a non-objective subject as memory is still to be approached gingerly and with caution (for further discussion on the subject of archival neutrality, see the chapter by Novak in this volume). Despite several decades of diminishing faith in the possibility of professional neutrality, archivists, while generally recognising the inevitable biases of records- creation and keeping nonetheless continue to strive for impartiality if not neutrality itself. Considering memory as a strategy or integral component of archival processes invites a dangerous but seductively slippery slide into interpretation.

While archivists might generally agree that the records in their care have a relationship to memory, they might also agree with German archivist Angelika Menne-Haritz that "archives do not store memory. But they offer the possibility to create memory." ${ }^{\prime 19}$ In other words, it is up to non-archivist researchers to interrogate, probe and interpret the records and make the memory connections. A more nuanced approach is suggested by literary historian Aleida Assman who, in "Canon and Archive," makes a distinction between two types of collective memory, active circulating memory (the canon) and passive historical memory (the archive). She describes both a tension and a continuing interchange between these two types of memory as society forgets, remembers and forgets again. Assman argues that although:

the function of the archive, the reference memory of a society, provides a kind of counterbalance against the necessarily reductive and restrictive drive of our working memory, [it also] creates a meta-memory, a second order memory that preserves what has been forgotten. ${ }^{20}$

As researchers probe the forgotten memory, it moves over into active memory.

Yet another perspective is offered by Piggott, who takes issue with the assumption of connections between archives and memory. He cautions that "through archivists' carefree use of memory several critical questions are ignored," and he questions whether "archivists should have an exclusive or

19 Angelika Menne-Haritz, "Access - The Reformulation of an Archival Paradigm," Archival Science 1 (March 2001): 59.

20 Aleida Assman, "Canon and Archive," in Astrid Erll and Ansgar Nunning, eds. A Companion to Cultural Memory Studies. New York: de Gruyter, 2010, p.106. 
central role in memory-making within institutions and society."21 Through a series of examples he suggests that, "sometimes group or individual memory is supported by archives; nothing more." 22

If archivists recognise and accept that their implicit connections to the business of memory is highly complex, then it is appropriate, in fact, highly desirable, for them to understand that connection much better, to experiment with modes of memory as part of their collections, and to test viewpoints such as those offered by Menne-Haritz, Assman and Piggott. Making sense of the relationships between memory and records would not only aid in all aspects of archival work but might also inspire innovation and new directions, particularly in the areas of description, access and appraisal. Margaret Hedstrom argues that "the current state of archival science recognizes a relationship between archives and memory, but the terms and conditions of that relationship are not well understood." ${ }^{23}$ Defining that relationship might not only lead to reconsiderations of the place of memory within the archives, but re-assessments of the nature of archival documentation.

Expressions of memory come in many forms and formats that may not necessarily be those traditional ones with which archivists are most comfortable. Memory expressions and traces are often found in oral testimony, monuments, artefacts and commemorations. Sociologist Barry Schwartz suggests that at least one of the advantages of studying collective memory is the ability to better understand under-documented communities. He writes:

fuller inclusion of minorities into the social mainstream and lessened tolerance of inequalities induce criticism of conventional interpretations of history, recognition of past immorality, and greater sensitivity to the way historians have written the powerless out of the historical record. ${ }^{24}$

The difficulties that archivists encounter in engaging with memory are also the major benefits. Incorporating and accepting the often non-traditional traces and expressions that carry memory into the archives also expands archivists' abilities to document communities that might otherwise be inaccessible to them.

21 Piggott, "Archives and Memory," 306.

22 Piggott, "Archives and Memory," 315.

23 Margaret Hedstrom, "Archives and Collective Memory: More than a Metaphor, Less Than an Analogy," in Terry Eastwood and Heather MacNeil, eds., Currents of Archival Thinking. Libraries Unlimited, 2009, p.163.

24 Olick et. al., The Collective Memory Reader, 13. 
There are many ways in which archivists can engage memory not only through the records they collect, but also through the ways in which they manage and conceptualise the records in their care. Hedstrom, arguing against the conventional notion of archives as storehouses of memory, suggests that "archives are the sources for the potential recovery of memories that have been lost." She points out that "archives may be of most value not when collective memory persists, but when they provide the only sources for insight into events and ideas that are long forgotten, rumored but not evidenced, or repressed and secreted away." ${ }^{25}$ In a similar vein and in a comprehensive review on archival writings on memory, Anthea Josias points out that in the archival discipline:

Two key dimensions surface in writings that make connections between archivists and collective memory. The archival process is being interrogated for its role as a contributor to collective memory, ... and collective memory is being viewed as a framework that might shed light on a conception of archives that is more broadly encompassing of multiple voices. ${ }^{26}$

Both writers, positioning archivists potentially on the cusp of memory research, encourage involvement, emphasising that archivists are uniquely positioned to uncover, recover and bring to light that which has been either deliberately or accidentally forgotten.

\section{How Can Archivists Study Memory?}

Both the familiar everyday core work of archivists - appraisal, preservation, description, access - as well as expanded visions of the ways in which the memory lens can extend the archival boundaries to include records in a variety of oral, material, performative and textual formats, offer archivists fruitful opportunities for memory work. Considering the collective memory of the Vietnam War and the AIDS epidemic, communications scholar Marita Sturken refers to the vast array of cultural products and texts including photographs, films, artefacts, oral testimonies and documents - all the "stuff" of archives - as "technologies of memory ... in that they embody and generate memory and are thus implicated in the power dynamic's of

25 Hedstrom, "Archives and Collective Memory," 176.

26 Anthea Josias, "Towards an Understanding of Archives as a Feature of Collective Memory," Archival Science, 11 (2011): 95-112. 
memory's production." ${ }^{\text {27 }}$ Considering "technologies of memory" from archival perspectives, how do they contribute to an understanding of memory and how do archival activities in relation to these formats provide deeper understandings of the events, people, places and evidence to which they pertain?

This section of the essay briefly examines some of the ways in which archivists have married core archival concepts to collective memory issues in more traditional ways. The paucity of these studies suggests that this is fertile ground for deeper explorations. The last section suggests some innovative approaches.

\section{Organisational Culture}

Corporate memory, long a familiar component, together with evidence and authenticity, of the traditional three-legged stool of archival value is probably the memory trope most familiar to archivists. Defined in the Society of American Archivists' Archival Glossary as, "the information in records and in individuals' personal knowledge that provides an understanding of an organization's or group's history and culture, especially the stories that explain the reasons behind certain decisions or procedures, ${ }^{28}$ the construct of corporate memory carries overtones of cultural memory as it provides a memory lens for understanding organisations and their records. The investigation of corporate memory, aspects of its formation, its durability, its reliability and its relationship to corporate culture have not been deeply probed although the potential for understanding corporate memory as a microcosm of institutional, even national memory suggests productive areas for research. A very few research studies beginning in the 1990s have explored the creation of organisational records in terms of organisational culture. Ciaran Trace and Elizabeth Yakel respectively examined police records and radiology. ${ }^{29}$ The Pittsburgh Project of the 1990s established literary warrant as a base for studying corporate memory and also paved the

27 Marita Sturken, Tangled Memories: The Vietnam War, the AIDS Epidemic, and the Politics of Remembering. University of California Press, 1997, p.10.

28 Richard Pearce-Moses, A Glossary of Archives and Records Terminology, Chicago, IL: Society of American Archivists, 2005, http://www2.archivists.org/glossary.

29 Ciaran Trace, "What Is Recorded Is Never Simply 'What Happened': Record Keeping in Modern Organizational Culture," Archival Science 2 no. 1-2 (January 1, 2002): 137-159; Elizabeth Yakel, "Recordkeeping in Radiology: The Relationships Between Activities and Records in Radiological Processes." Ph.D. dissertation, University of Michigan, 1997. 
way for a close examination of electronic recordkeeping systems and their relationships to the organisations that create them. This research avenue has not been well exploited but could be a unique archival lens on understanding organisational culture as broadly conceived.

Extending the understanding of organisational cultural to analyse the impact of specific archival practices offers another conduit into institutional memory practices. Joanna Sassoon explores this path through examining the collecting and selection decisions over time of two archival institutions in Western Australia and how these decisions affected understandings of a contested past. Sassoon uses theories of collective memory as tools to interrogate these decisions. As she explains:

the concept of collective memory can be used both to explain the active role played by archival institutions in framing memory and therefore history and as an analytical tool to investigate why archival institutions can be seen in the present as sites of contest. ${ }^{30}$

\section{Trust and Evidence}

Memory is often fragile, changeable and contested but by focusing on archival values of trust and evidence, the archives can not only ground a memory within the event itself, but can also facilitate connections between the event, the ways in which it is remembered and the memories it engenders. In "The Grandmother's Story": Oral Tradition, Family Memory, and a Mysterious Manuscript," Robert Fisher uses archival tools to authenticate a manuscript based on an oral transmission and in doing so, not only uncovers a complex family history but also confirms and solidifies a family memory. Fisher advocates for the critical importance of memory research in archival practice, arguing that "such research is essential for us to maintain our status as a knowledge-creating profession and fulfil our obligation to society as the keepers of memory." ${ }^{31}$

Archives can authenticate a narrative and track its continuity as it moves from event to memory, and archivists can also document the ways in which specific events interface with memory to create a broader collective remembrance. In "The Great War, Archives, and Modern Memory," Robert McIntosh

30 Joanna Sassoon, "Phantoms of Remembrance: libraries and archives as 'the collective memory," Public History Review, 10 (2003): 45.

31 Robert C. Fisher, “The Grandmother's Stor”: Oral Tradition, Family Memory, anda Mysterious Manuscript," Archivaria 57 (2004): 127-128. 
examines this type of documentation and its interface with memory through Canadian military records from World War I. Responding to Terry Cook's challenge that, "we need to understand better our own politics of memory, the very ideas and assumptions that have shaped us, if we want our 'memory houses' to reflect more accurately all components of the complex societies they allegedly serve, ${ }^{, 32}$ McIntosh illustrates the role that archivists play in shaping a documentary narrative of war and its subsequent memory. He concludes that:

our memory of the past is embedded in a vast array of documents whose contents and meaning have been constituted and shaped along a long continuum of records manufacture astride which archivists are crucially poised. To be prepared to explain our archives-making across the spectrum of our work is to accept our accountability for these actions. It is also fully to acknowledge our authorship, our vital place in the creation of society's memory. ${ }^{33}$

Although archivists have tended to be wary of oral testimony as evidence, recent research is demonstrating that the interplay between oral testimony and archival sources is not only a powerful combination in the formation of collective memory but also a path to a rich and nuanced understanding of events and actions. Patricia Galloway examines the "construction and reproduction of orally preserved tradition in the presence of a surrounding literate culture" 34 by analysing two examples: the culture of mnemonics among medical students in the context of standard texts, and "traditional memory preservation in the context of modern record keeping and archiving currently practiced by the Mississippi Band of Choctaw Indians." 35 Similarly, Joanna Sassoon demonstrates relationships between oral history and social justice in her discussion of the Forgotten Australians and Former Child Migrants history project. ${ }^{36}$

Pointing to a more history-focused approach to archives/memory research, Israeli historian Kobi Peled re-examines the 1949 Arab-Israeli War by

32 Terry Cook, "What is Past is Prologue," Archivaria, 43 (1997): 19.

33 Robert McIntosh, "The Great War, Archives, and Modern Memory," Archivaria 46 (Fall 1998): 20.

34 Patricia Galloway, "Oral Tradition in Living Cultures," in Jeannette A. Bastian and Ben Alexander eds., Community Archives: The Shaping of Memory. London: Facet Publishing, 2009, p.68.

35 Galloway, "Oral Tradition in Living Cultures," 68.

36 Joanna Sassoon, “'Memory for Justice' or 'Justice for Memories'; Remembering Forgotten Australians and Former Child Migrants," Archifacts (October 2010): 25 -34. 
seeking to "foster a dialogue between archival sources and oral testimonies; between the written word and the spoken (and recorded) one. ${ }^{37}$ He hopes to understand the perspectives from both sides of the conflict and notes that he conceives of:

oral history ... as a way to examine and modify the historical picture as portrayed via the sources of the Jewish side alone ... oral history can fill a void by accommodating the point of view of the 'other side,' the voices of those who barely wrote anything, yet preserved memories of the events in their minds. ${ }^{38}$

In this case, evidence is reified through conflating oral and archival testimonies.

\section{Accountability and Social Justice}

In "Khmer Rouge Archives: Accountability, Truth and Memory in Cambodia," Michelle Caswell explores the potential for archives in fostering accountability and, through accountability, promoting social justice. She demonstrates that while records both facilitate collective memory and also hold it historically accountable, collective memory in turn elucidates and adds value to the records. Caswell suggests that while the archives can be effective in creating public memory their role may be limited. At the same time, however, she notes that "perhaps the most possible form of justice on this earth is to give voice to the victims of the Khmer Rouge through accountability, truth, and memory ... archives are doing just that." ${ }^{39}$

Social justice and the power of records to recover hidden or forgotten memories have become important memory lenses for archivists in the first decades of the twenty-first century. Like Caswell, Anthea Josias and Ricardo Punzalan focus on the memory potential of archives to foreground the records of previously disenfranchised communities. Josias points to the District Six Museum in South Africa where "a central concern has been about the making of memory as a way of countering deeply held institutionalized

37 Kobi Peled, "Oral Testimonies, Archival Sources, and the 1948 Arab-Israeli War: A Close Look at the Occupation of a Galilean Village," Journal of Israeli History, 33 no.1 (2014): 41.

38 Peled, "Oral Testimonies, Archival Sources, and the 1948 Arab-Israeli War", 42.

39 Michele Caswell, "Khmer Rouge Archives: Accountability, Truth, and Memory in Cambodia," Archival Science 10 (2010): 42. 
and apartheid-shaped historical frameworks." ${ }^{\text {40 }}$ Punzalan, describing the establishment of the Culion Leprosy Museum and Archive on an island in the Philippines, provides a case study that "illustrates how records and the establishment of archives figure at a moment of remembrance and commemoration ... how archives assume a particular meaning in the process." ${ }^{31}$ All three are examples of the ways in which records support and become crucial memories in the fight for social justice.

Examination of institutional context, analysis of authenticity and trustworthiness, shaping narratives through documentation, supporting social justice and creating public access - are all ways in which archivists utilise the lenses of their own discipline to study memory, its potential, its effects and its impacts. The very definition of archives as evidence - of preserved records created as the by-products of activities - implies that archives facilitate transmission of the memory of the events that they document. As the surrogates for the actual activities, archives can both lend integrity to memory and function as memory touchstones.

\section{New Memory Research Directions}

Sturken suggests that "memory is often embodied in objects - memorials, texts, talismans, images." She writes that "though one could argue that such artifacts operate to prompt remembrance, they are often perceived actually to contain memory within them or indeed to be synonymous with memory." ${ }^{\prime 2}$ This construct, a counter to those theories that conceptualise archives as touchstones or surrogates of memory, offers a forceful role for archives in making memory connections, opening up the potential of going beyond documenting or representing memory to embodying memory. Joan Schwartz takes this position in "The Archival Garden, Photographic Plantings, Interpretive Choices, and Alternative Narratives," a close reading of a nineteenth century photograph of the suspension bridge at Niagara Falls. Through rich, detailed descriptions of the context of the photograph, its technologies, its preservation over time and its placement within the National Archives of Canada, Schwartz demonstrates that:

40 Anthea Josias, "Toward an Understanding of Archives as a Feature of Collective Memory," Archival Science (2011): 98.

41 Ricardo Punzalan, “'All the Things We Cannot Articulate': Colonial Leprosy Archives and Community Commemoration," in Jeannette A. Bastian and Ben Alexander, eds. Community Archives: The Shaping of Memory. London: Facet, 2009, p.199.

42 Sturken, Tangled Memories, 19. 
initially embraced as a surrogate for first-hand information, 'The Niagara Suspension Bridge' has subsequently been recast as a work of art, its meaning variously shaped by historical circumstances, geographical imaginings, disciplinary perspectives, institutional practices and market forces. $^{43}$

Through a series of actions - many by archivists themselves - the photograph goes beyond representing the bridge to being a particular memory of it. The photograph becomes the thing itself.

Yet another relatively unexplored area of memory research in the archival field considers an event and its memory on a continuum, where both should be documented in the archives. Brien Brothman points towards this continuum as he theorises a distinction between history's past and memory's past. He suggests that:

Memory's archivist is interested in the past's residue as material for promoting integrated knowledge, social identity, and the formation of group consciousness; history's archivist is interested in finding records and, in them, uncovering evidence to develop a linear narrative about a past that is ours, yet different from us. ${ }^{44}$

Through the documenting of both the event and its memories over time, the event continues to be dynamic. And as memory brings the event forward it also establishes its societal accountability. Seen as a continuum, both the event and the memory enrich one another, forming a more complete and complex historical understanding.

Implementing such a continuum approach to the intersection of archives and memory is one assignment in a graduate class I teach on collective memory. Students work in groups on semester-long projects that help them work through the connections between memory and archives. Each group selects a topic - an historical event, social movement, cultural movement, historical figure or issue - and considers how it has been remembered over time and how it is currently being remembered and made accessible to the public. In examining the collective memory of this event, person or

43 Joan M. Schwartz, "The Archival Garden, Photographic Plantings, Interpretive Choices, and Alternative Narratives," in Terry Cook, ed. in Controlling the Past: Documenting Society and Institutions: Essays in Honor of Helen Willa Samuels. Chicago: Society of American Archivists, 2011, p.104.

44 Brien Brothman, "The Past that Archives Keep: Memory, History, and the Preservation of Archival Records," Archivaria 51 (2000): 62. 
movement, students examine all aspects of how it has been remembered including its archives and artefacts, web sites/physical sites, primary and secondary sources. In addition to presenting the various perspectives on the subject, students analyse the ways in which it has been remembered, and draw conclusions about its collective memory and the impact of different types of remembrance. They present their analysis within a theoretical framework, often one that has been discussed in class. The groups are asked to consider the following questions:

- Does the historical meaning and/or significance of the event change over time?

- What is the relationship between the archives and records of the event and the memory of the event?

- How do different media shape the memory of the event?

- Who is the intended audience for each representation of the event? What is the anticipated reception?

- Do political demands or needs shape a historical or memorial narrative?

- What is the role of the community and/or individual on the shaping of historical meaning?

- Do different types of documents help to shape different historical interpretations? In turn, how do they shape public understanding of the past?

- Are there competing claims to history or memory? If so, how does this contestation of memory continue to shape an overall memory?

- Does the introduction of new "texts" and/or new interpretations alter or reconfigure the meaning of the past?

- How do individual memories interact with constructed "collective" memories?

Following an event, person, object or even era through its many memory permutations and re-uses offers intriguing avenues of memory research within the archival field. Each of the questions above, particularly those that deal directly with the relationships between the documentation and collective memory, suggest research opportunities. Interrogating a variety of documentary sources including film, photographs, texts and oral histories as well as a variety of non-textual sources such as oral interviews, artefacts and cultural expressions, facilitates holistic understandings of events and people that have a significant impact upon the documentary process. Both the event 
and the memory enhance and support one another while providing a broad and longitudinal context. ${ }^{45}$

Community archives present yet another promising area of memory research. Pioneered by Andrew Flinn and his colleagues at University College London, research in community archives embraces a broad diversity of communities and groups who may share common beliefs, geographies, ethnicities, lifestyles and ideologies. These groups are not waiting for archivists to document them but are documenting themselves. Flinn defines community archives as:

collections of material gathered primarily by members of a given community and over whose use community members exercise some level of control ... the defining characteristic of community archives is the active participation of a community in documenting and making accessible the history of their particular group and/or locality on their own terms. ${ }^{46}$

The grassroots and often spontaneous nature of community archives also makes them critical sites for archivists wishing to understand and embrace social and cultural activism, as illustrated by recent research on archivists' involvement with the Occupy Movement. John Erde concludes that "Wide participation in archives should also be pursued as a political strategy, a means of distributing 'archives power' as widely as possible. Archivists are active agents in archives construction., ${ }^{, 47}$

\section{Conclusion}

Although archivists engage implicitly with memory through their traditional principles and procedures, they have been slow to recognise memory and memory studies as theoretical lenses through which to enhance and add meaning to archival practice. The increased emphasis on memory as an active

45 Topics of these student projects have included The Titanic, Boston monuments-Public art, Ellis Island and immigration, and Rosie the Riveter. Examples of articles researching case studies of the memory continuum are: Amy Adamczyk, "On Thanksgiving and Collective Memory: Constructing the American. Tradition," Journal of Historical Sociology 15 (2002): 343-356, and Jeannette A. Bastian, "Flowers for Homestead: A Case Study in Archives and Collective. Memory," American Archivist 72 (Spring/ Summer 2009): 113-132.

46 Andrew Flinn, Mary Stevens and Elizabeth Shepherd, "Whose Memories, Whose Archives? Independent Community Archives, Autonomy and the Mainstream," Archival Science 9 (2009): 73.

47 John Erde, "Constructing Archives of the Occupy Movement," Archives and Records 35 (August 2014): 88. 
component of many humanities and social science disciplines suggests that expressions of memory - be they oral, textual, material or performative - are today as vital as are traditional documents to understanding and analysing the many diverse social and cultural manifestations of our global community. The tools of memory have acquired a legitimacy and an evidential gravity of their own. If archivists are to continue to be relevant to the documenting of society, then it is critical that memory and the expressions and traces of memory be incorporated into archival practice as well as theory.

Memory research by archivists not only helps to frame archives-specific memory constructs, but also affirms the role of the archivist in the memory process. As archivists move beyond memory theory and into empirical memory research, they expand the breadth and significance of the records in their care and discover broader contexts for the archival mission.

\section{References}

Adamczyk, Amy. "On Thanksgiving and Collective Memory: Constructing the American. Tradition," Journal of Historical Sociology 15 (2002): 343-356.

Assmann, Aleida. "Canon and Archive," in Astrid Erll and Ansgar Nunning, eds. A Companion to Cultural Memory Studies. New York: de Gruyter, 2010, pp.97-107.

Bastian, Jeannette A. "Flowers for Homestead: A Case Study in Archives and Collective

Memory," American Archivist 72 (Spring/ Summer 2009): 113-132.

Brothman, Brien. "The Past that Archives Keep: Memory, History, and the Preservation of Archival Records," Archivaria 51 (2000): 48-80.

Caswell, Michelle. "Khmer Rouge Archives: Accountability, Truth, and Memory in

Cambodia," Archival Science 10 (2010): 25-44.

Cook, Terry. "What is Past is Prologue," Archivaria, 43, (1997): 17-63.

Cox, Richard J. "Public Memory Meets Archival Memory: The Interpretation of

Williamsburg's Secretary's Office," American Archivist 68 (Fall/Winter 2005): 279-296.

Erde, John. "Constructing Archives of the Occupy Movement," Archives and Records 35 (August 2014): 77-92

Fisher, Robert C. “'The Grandmother's Story': Oral Tradition, Family Memory, and a Mysterious Manuscript," Archivaria 57 (2004): 127-128.

Flinn, Andrew, Mary Stevens and Elizabeth Shepherd, "Whose Memories, Whose

Archives? Independent Community Archives, Autonomy and the Mainstream," Archival Science 9 (2009): 71-86

Fussell, Paul. The Great War and Modern Memory. London: Oxford University Press, 1975.

Galloway, Patricia. "Oral Tradition in Living Cultures," in Jeannette A. Bastian and Ben Alexander eds., Community Archives: The Shaping of Memory. London: Facet Publishing, 2009, pp. 65-86.

Halbwachs, Maurice. On Collective Memory, Lewis A. Coser, trans. and ed. Chicago: University of Chicago Press, 1992.

Harris, Verne. "The Archival Sliver, Power, Memory and Archives in South Africa," Archival Science 2 (2002): 63-86. 
Hedstrom, Margaret. "Archives and Collective Memory; More than a Metaphor, Less Than an Analogy," in Terry Eastwood and Heather MacNeil, eds., Currents of Archival Thinking. Libraries Unlimited, 2009, 163-179.

Jacobsen, Trond, Ricardo Punzalan and Margaret Hedstrom, "Invoking Collective Memory: Mapping the Emergence of a Concept in Archival Studies," Archival Science 13 nos. 2-3 (2013): 217-251.

Josias, Anthea. "Towards an Understanding of Archives as a Feature of Collective Memory," Archival Science 11 (2011): 95-112.

Ketelaar, Eric. “Tacit Narratives: The Meaning of Archives," Archival Science 1 (2001): 131-141.

McIntosh, Robert. “The Great War, Archives, and Modern Memory," Archivaria 46 (Fall 1998): 2-31.

McKemmish, Sue, Anne Gilliland-Swetland and Eric Ketelaar "Communities of Memory': Pluralising Archival Research and Education Agendas," Archives E̋ Manuscripts, 33 (May 2005): 146-174.

Menne-Haritz, Angelika. "Access - The Reformulation of an Archival Paradigm," Archival Science 1 (March 2001): 57-82.

Olick, Jeffrey. The Politics of Regret: On Collective Memory and Historical Responsibility. New York: Routledge, 2007.

Pearce Moses, Richard. A Glossary of Archival and Records Terminology Chicago: Society of American Archivists, 2005, http://www2.archivists.org/glossary/.

Peled, Kobi. "Oral Testimonies, Archival Sources, and the 1948 Arab-Israeli War: A Close Look at the Occupation of a Galilean Village," Journal of Israeli History, 33 no.1 (2014): 41-61.

Piggott, Michael. "Archives and Memory," in Sue McKemmish, Michael Piggott, Barbara Reed and Frank Upward, eds. Archives: Recordkeeping in Society. Wagga Wagga, New South Wales: Charles Sturt University, 2005, pp. 299-328.

Punzalan, Ricardo. "All the Things We Cannot Articulate': Colonial Leprosy, Archives and Community Commemoration," in Jeannette Bastian and Ben Alexander, eds., Community Archives, the Shaping of Memory. London: Facet, 2009, 197-219.

Samuel, Raphael. Theatres of Memory. London: Verso, 1999, pp.25, 39.

Sassoon, Joanna. "Phantoms of Remembrance: Libraries and Archives as 'the Collective Memory," Public History Review, 10 (2003): 40-70.

Schwartz, Joan M. "The Archival Garden, Photographic Plantings, Interpretive Choices, and Alternative Narratives," in Terry Cook, ed., Controlling the Past: Documenting Society and Institutions: Essays in Honor of Helen Willa Samuels. Chicago: Society of American Archivists, 2011, 69-110.

Schwartz, Joan M. and Terry Cook. "Archives, Records, and Power: The Making of Modern Memory," Archival Science 2 (2002): 1-19.

Society of American Archivists, Core Values Statement. http://www2.archivists.org/ statements/saa-core-values-statement-and-code-of-ethics.

Straker, Gillian. "Shaping Subjectivities, Private Memories, Public Archives," Psychoanalytic Dialogues 21 (2011): 643-657.

Sturken, Marita. Tangled Memories; The Vietnam War, the AIDS Epidemic, and the Politics of Remembering. Berkeley, CA: University of California Press, 1997.

Trace, Ciaran. "What Is Recorded Is Never Simply 'What Happened': Record Keeping in Modern Organizational Culture.” Archival Science 2, no. 1-2 (January 1, 2002): 137-159. 


\section{Chapter 8}

Wallot, Jean-Pierre. "Building a Living Memory For the History of Our Present: New Perspectives on Archival Appraisal," Journal of the Canadian Historical Association 2 (1991): 263-282.

Winter, Jay. Remembering War, The Great War Between Memory and History in the Twentieth Century. New Haven: Yale University Press, 2006.

Yakel, Elizabeth. Recordkeeping in Radiology: the Relationships Between Activities and Records in Radiological Processes. Ph.D. dissertation, University of Michigan, 1997. 
Chapter 9

\title{
WHEN IS A RECORD?
}

\author{
A Research Framework for Locating \\ Electronic Records in Infrastructure
}

\author{
Amelia Acker
}

\begin{abstract}
This chapter presents a framework for archival researchers to account for the transmission and materiality of electronic records research by locating them within the infrastructures of contemporary, networked communication. The first section contextualises the research need by presenting the importance of layers of infrastructure to the creation and circulation of born-digital records transmitted across wireless networks. The second section presents a research framework for studying new information communication technologies and emerging electronic records contexts, and reflects on why such a framework is necessary and how it has been constructed. The framework, which builds upon records continuum theory and the concept of spacetime, takes up the logical and physical aspects of Thibodeau's digital object model and applies Trace's microethnographic approach to networked communication, has three elements: Layers of Infrastructure and Context, Examining Networked Recordkeeping, and Engaging with Information Retrieval. The third section presents the case study of Kurt Mix and the British Petroleum Oil Spill in order to illustrate the possibilities of how this framework might be applied in archival research.
\end{abstract}

\section{I}

On a recent trip to Australia, I took a twelve-hour train ride from Melbourne to Sydney. Within a few stops we entered the countryside and I began to count kangaroos as the train passed through open fields. After seeing well over twenty kangaroos, I started sending text messages to acquaintances back in the United States. The kangaroos were not a surprise to my fellow 
Australian passengers on the train, but I wanted to share my excitement with friends and family back home at how many kangaroos I had counted in the countryside. I had travelled to Australia to present preliminary research upon the infrastructure of mobile communication. In my research I examine how technologists, recordkeepers, archivists, and information scientists are confronting issues of digital materiality and preservation with emerging formats and the information systems that create, delete and store digital traces created with mobile devices.

Texting while riding on the train gives us the opportunity to think about how we organise ourselves around networks. Infrastructural networks, or large scale technical networks and incumbent standards, protocols and the social institutions that enforce them engender the creation and reception of records, which are also comprised of the digital traces of transmission that produce such records. When I arrived in Australia, I had bought a Vodafone prepaid subscriber identification module (SIM) card at the airport to send messages, access the internet and make a few calls while travelling. This SIM card allowed me to use my phone to make calls and send text messages via an Australian service provider's mobile network infrastructure. Sporadically throughout the rest of the train trip I would receive a flurry of text messages in my inbox responding to messages I had sent earlier in the day. After receiving a few batches of new messages all at the same time, it became clear to me that the mobile data network coverage that my new SIM card afforded followed the station stops of the train. The network would go dark as the train travelled between stations, and I would lose the network connection at the places along the way where the kangaroos actually were.

My inbox, with five or six new text messages, pointed to a fleeting mobile data connection and eventually, I was able to predict when we would approach the next station. I would reply as quickly as I could once the train was within network range of a station with the hopes of receiving a text before the next train stop, usually forty minutes away. It was in this fevered pace of playing catch-up and responding to texts at each train stop that I realised I was experiencing the limits of two types of network coverage. First, I was playing with the clock, aware that as soon as a few more people got on or off the train I would lose the data connection. And second, just as the train would continue speeding towards my next destination, I was experiencing the possibilities of connection and disconnection through the affordances of infrastructure, in one sense from industrialisation by way of the train network and in another, due to the information age as manifested by my mobile phone and its access to network coverage. My experience of 
"boundedness" from both networks was producing an information landscape as I moved through time over the course of the daylong trip, and through space along the several hundred miles of train tracks. ${ }^{1}$ As I travelled through this landscape I was creating and receiving records along the way.

\section{Information Landscapes and the Postcustodial Era}

Paying attention to networks across geographical landscapes allows us to consider how infrastructure influences the possibilities of records that can be produced, and furthermore, how they are circulated and transmitted in digital networks. As mobile computing begins to influence the fabric of social life, important research questions arise: How do we locate and identify records within infrastructure? How does infrastructure shape the records that are preserved, identified, and the ways archival scholars evaluate them?

We experience the infrastructure of digital information networks in our everyday lives in the same ways that we experience other industrial infrastructures, such as trains, indoor plumbing, central heating, or copperwire telephone landlines. The absence or presence of certain nodes in a technical network, whether that be a train station, a cellular tower or a $\mathrm{Wi}-\mathrm{Fi}$ hotspot characterises modern living, and the feeling of being infrastructured is something that we all experience at different junctures in everyday life. ${ }^{2}$ Network connections (and disconnections) reconfigure the spaces around us, just as they influence the ways in which we move ourselves around these networks because they enable a multiplicity of experience. ${ }^{3}$ Train networks and mobile phone networks create mobility; both networks move things through time and space in material ways. At first glance, it may not be obvious why archivists should focus upon infrastructures that create records (by which I mean information with content, context and structure recorded on a fixed media). Increasingly however, records of our time are created, transmitted

1 For more on the experience of being bound by infrastructure, see Silvia Lindtner, Ken Anderson and Paul Dourish, "Cultural Appropriation: Information Technologies as Sites of Transnational Imagination," in Proceedings of the ACM 2012 Conference on Computer Supported Cooperative Work (2012): 77-86.

2 Paul Dourish and Genevieve Bell address the relationship between experience and information in "The Infrastructure of Experience and the Experience of Infrastructure: Meaning and Structure in Everyday Encounters with Space," Environment and Planning B: Planning and Design 34 no.3 (2007): 414-430.

3 For a fuller description of how mobile networks enact space has been theorised, see Jason Farman, Mobile Interface Theory: Embodied Space and Locative Media, 1st ed. Routledge, 2011. 
and stored in distributed infrastructures and large-scale technical networks of digital information communication technologies (ICTs) ranging from mobile phones to cloud storage services. Archival scholars need proactively to locate records in infrastructure because in digital information infrastructure the threshold between active and inactive records is dissolving and the process of transmission is not well theorised. ${ }^{4}$

Locating such a threshold, or even discrete stages in the circulation of electronic records, has proven to be a challenge for traditional archival theories and practice, and in particular to the life cycle approach, as electronic record systems have become increasingly networked and complex. The life cycle model for records management, as described by Theodore Schellenberg, was the primary model for records management in North America from the 1960s to the 1990s, and in many cases its influence can still be seen, especially as it relates to the archivist's custody over records. ${ }^{5}$ The life cycle model prescribes stages of recordkeeping in addition to who will manage records and when, offloading specific management roles to records managers and archivists at different stages. ${ }^{6}$ Perhaps most importantly, it is not until records become inactive in the life cycle model that archivists take responsibility for their management. ${ }^{7}$

In the 1990s, scholars such as David Bearman, Terry Cook, Sue McKemmish and Frank Upward argued for a new paradigm in archives. They argued that this paradigm should account for the new realities of electronic record environments that moved beyond the discrete stages of the life cycle approach and the limited archival oversight of inactive records only. ${ }^{8}$ It supports a postcustodial era where the role of the archivist shifts from being a custodian over inactive records in a centralised repository, to the management of records over time, beginning where and when records are created and including all the practices of use and circulation. Postcustodial theory, as Frank Upward has written, is not the opposite of custodial, but

4 For more on the archival threshold in the digital era see Richard Pearce-Moses, "Janus in Cyberspace: Archives on the Threshold of the Digital Era," The American Archivist 70 no.1 (2007): 13-22.

5 Philip C. Bantin, "Strategies for Managing Electronic Records: A New Archival Paradigm? An Affirmation of Our Archival Traditions?” Archival Issues, 23 (1998): 23.

6 Bantin, "Strategies for Managing Electronic Records," pp. 23-25. For more on the life cycle model see Ira A. Penn, Gail Pennix and Jim Coulson, Records Management Handbook, Hampshire, England: Gower Publishing Limited, 1994, pp.12-17.

7 Luciana Duranti, “Archives as a Place," Archives and Manuscripts, 24 no.2 (1996): 252.

8 Linda J. Henry, "Schellenberg in Cyberspace," American Archivist 61 no.2 (Fall 1998): 309 . 
instead de-emphasises the physical custody of records and focuses upon the transactionality of electronic records. ${ }^{9}$

Despite the attention to process that comes with postcustodial approaches, many archivists are trained to think that whenever electronic records move across space (conceptually or physically), they are at risk of having elements of record-ness stripped by another system, thus rendering them non-records. ${ }^{10}$ These moments of risk for electronic records are largely located at transfer points - processes of transmission that move information through space and time - because there exists at those moments a possibility to violate authenticity and change a record's fundamental attributes. The condition of becoming a record also threatens that existence. When a digital entity passes from one system to another, the control over the authenticity and integrity of that record follows. Such a "systematic vulnerability" has been identified by some historians as a condition of high modernity that separates technology from the social and is not just limited to archival spaces of enactment and custody. ${ }^{11}$

Archival theorists have written extensively about what the era of postcustodial recordkeeping signifies for archivists as professionals. ${ }^{12}$ Many archival scholars believe that it is at these junctures of transition that we should "reinsert" ourselves into digital preservation planning efforts, especially through description and metadata. ${ }^{13}$ It is interesting to consider the power of custody in approaching the problem of transition because it is the archivist's shifting custody over the recordkeeping system and the description

9 A note on terminology, in this article "transactionality" refers to physical and conceptual transactions, where "transmission" refers to the cultural logic of transmission in the digital age, including practices and social institutions such as standards built up around transactions. See Frank Upward, "Structuring the Records Continuum - Part One: Postcustodial Principles and Properties," Archives and Manuscripts 24 no.2 (1996): 4.

10 Heather MacNeil, "Providing Grounds for Trust II: The Findings of the Authenticity Task Force of InterPARES," Archivaria 1 no.54 (2002): 28.

11 Paul Edwards argues that systematic vulnerability is a result of "modernist settlement" or that of separating the social from the technological as theorised by Bruno Latour. For more on this connection, see Paul N. Edwards, "Infrastructure and Modernity: Force, Time, and Social Organization in the History of Sociotechnical Systems," Modernity and Technology (2003): 195-196.

12 For more see Terry Cook, "Electronic Records, Paper Minds, The Revolution in Information Management and Archives in the Post-Custodial and Post-Modernist Era," Archives and Manuscripts, 22 no.2 (1994): 300-328, and "What is Past is Prologue: A History of Archival Ideas Since 1898, and the Future Paradigm Shift," Archivaria 43 (1997): 17-63.

13 David Bearman, "Moments of Risk: Identifying Threats to Electronic Records," Archivaria, 62 (2007): 15-46. 
of the processes of archiving that is radically transferred in the postcustodial era. ${ }^{14}$ Yet there remains a dearth of work that theorises or even describes the archivist's role in what Manuel Castells has called, "the material organisation of time-sharing social practices that work through flows". ${ }^{15}$

In the space of flows, the time between active and inactive records has become disjointed and indistinguishable: as records are created and distributed across memory sites that range from mobile phones to USB flash drives to gaming systems, they instantly become subject to backup or digital storage (the so-called "archive" created by copying stored information across file structures on storage media), even when deleted or deaccessioned. This rupture of time with digital ICTs forces archivists to reconsider how records emerge in current networks and how they are kept, transmitted and received across space and also where archival callings shifts in the space of flows. Locating records as they move through infrastructure relies on identifying the material culture of mobile communication and recognising change: from devices, to cell towers, undersea cables, wires and server farms, even to phone bills. Furthermore, archivists have an imperative to account for recorded information as it moves through infrastructure, because the mobility of electronic records through spacetime is an indelible aspect of their enduring value in the postcustodial era. ${ }^{16}$

Even with the postcustodial emphasis on the processes of archiving and understanding of complex transactionality, the transmission of records across wireless networks has often been overlooked in archival studies and not well understood. ${ }^{17}$ This is possibly due to the fact that infrastructure can be "mundane to the point of boredom" and that wirelessness remains ripe for examination. ${ }^{18}$ It may also be that most postcustodial approaches privilege

14 See: Cook, "What is Past is Prologue," p. 47; Greg O'Shea and David Roberts, "Living in a Digital World: Reorganizing the Electronic and Post-Custodial Realities," Archives and Manuscripts, 24 no.2 (November 1996): 286-311; and Frank Upward and Sue McKemmish, "Somewhere Beyond Custody," Archives and Manuscripts, 22 no.1 (May 1994): 136-149.

15 Manuel Castells, "An Introduction to the Information Age." In The Information Society Reader, Frank Webster, Raimo Blom, Erkki Karvonen, Harri Melin, Kaarle Nordenstreng and Ensio Puoskari eds. London and New York: Routledge, 2004, p.147.

16 For more on the concept of spacetime, see Frank Upward "Modeling the Continuum as Paradigm Shift in Recordkeeping and Archiving Processes, and Beyond a Personal Reflection," Records Management Journal 10 no.3 (2000): 115-139, and Sue McKemmish "Placing Records Continuum Theory and Practice," Archival Science 1 no.4 (2001): 346.

17 Frank Upward, "Structuring the Records Continuum Part One: Post-custodial Principles and Properties," Archives and Manuscripts 24 no.2 (2009): 268-285.

18 Susan Leigh Star, "The Ethnography of Infrastructure," American Behavioral Scientist 43 no.3 (November 1, 1999): 377-391. The significance of wirelessness on the network 
logical aspects of records over the physical, and lean towards a tendency to treat electronic information as immaterial. The ways in which information infrastructure moves records from one system to another is conspicuously missing from the study of electronic records in archival scholarship. ${ }^{19}$ To address this gap, the framework I propose here looks carefully at the historical ontology of records, or how records move through infrastructure at the point of creation, transmission and reception. The following discussion addresses how and why this framework was developed to address the ways in which records that are being generated today challenge all archival functions as we know them.

\section{Examining Electronic Incunabula in the Twenty-First Century}

Margaret Hedstrom's framework for analysing electronic incunabula urges archivists to examine evolving forms of documentation and to "think big enough." ${ }^{20}$ Her framework draws heavily upon concepts and techniques from the history of technology that place electronic recordkeeping into historical, social and cultural contexts. The framework was, however, developed before microprocessors and the rise of personal computing and perhaps most importantly, before the internet revolutionised networked communication. Twenty years later, mobile communication devices, search engines and social media platforms present radically new kinds of formats, or networked electronic incunabula, for archival scholars to consider. Hedstrom's framework for studying electronic records begins with the consideration of specific contexts of information technology, starting with the origins of its development and looking at the evolution of documentation and organisational change. ${ }^{21} \mathrm{I}$ argue that by examining the infrastructure of records, archivists can think big enough about the "black box" and all the

culture has been theorised at length by Adrian Mackenzie. See Adrian Mackenzie, Wirelessness: Radical Empiricism in Network Cultures. Cambridge, MA: The MIT Press, 2010.

19 Margaret Hedstrom, "Understanding Electronic Incunabula: A Framework for Research on Electronic Records," American Archivist 54 no.3 (July 1, 1991): 334-354.

20 Hedstrom, "Understanding Electronic Incunabula". As in the first outputs of the printing press prior to 1501 , incunabula refers to "the earliest stages or first traces in the development of anything." Oxford English Dictionary, 1933, I:188.

21 A close reading of the framework reveals that Hedstrom describes infrastructure (through stabilisation and co-constitution of the social) but does not specifically identify it as such. 
layers of construction behind digital records and emerging documentation practices. ${ }^{22}$

In the same year that Hedstrom published her framework for electronic records research, Terry Cook commented on the problems posed by the transmission of emerging formats through telecommunication:

Combining computerized information in these new formats with a telecommunications revolution affecting the transmission of electronic records threatens decision-making accountability and corporate memory: if an electronic document has no physical existence, but rather is a "virtual" composite of disparate information appearing but fleetingly on a terminal, how does the institution let alone the archivist preserve evidence $[\ldots]$ ? Where is the evidence of accountability of the transaction? Where is the context? What is the provenance? ${ }^{23}$

If we update our approach to electronic incunabula to account for the infrastructure of large-scale networks, the realities of instant back-up and distributed storage, and the processes of producing and transmitting such digital traces, archivists may be able to answer, with confidence, the questions Cook posed. In the space of flows of the network society, "transactionality is rampant. The distinctions that archivists make between hierarchy and functionality, chatter and business, organizational structure and authority, become less easy to make." ${ }^{24}$ As wireless transmission becomes more complex and pervasive in the twenty-first century, the need to focus upon the processes of transactionality, and their means through infrastructure, has increasing material consequences and archival relevancy.

\section{Digital Materiality and the Physicality of Information}

The materiality of digital communication in infrastructure is essential to understanding the context, content and provenance of new electronic formats. ${ }^{25}$ In her microethnography of the creation of a digital document on

22 Bruno Latour, Science in Action: How to Follow Scientists and Engineers Through Society. Cambridge, MA: Harvard University Press, 1987. For more on the significance of opening "black boxes" see Langdon Winner, "Upon Opening the Black Box and Finding it Empty: Social Constructivism and the Philosophy of Technology," Science, Technology, E̋ Human Values 18 no.3 (1993): 362-378.

23 Terry Cook, "Easy to Byte, Harder to Chew: The Second Generation of Electronic Records Archives," Archivaria 1 no.33 (1991): 206.

24 Upward. "Structuring the Records Continuum, Part Two," p.18.

25 Materiality, as a concept, refers to the medium or physical format used to store information or a text. For more on digital materiality and archiving, see: Matthew 
a personal computer, Ciaran Trace encourages archivists to go inside the black box of the computer in order to understand the nature of born-digital records and to adequately serve the communities that they document. ${ }^{26}$ This is what Richard Pearce-Moses has identified as "under-the-hood IT skills" for archivsts. ${ }^{27}$ The digital materiality of records is an underdeveloped area of scholarship in archival studies, in part because electronic records are often seen as being without physicality:

[T] he record remains a problematic construct even within the archival community. Within the U.S., there is insufficient common understanding of the nature of the record and how the record as a construct might be operationalized in digital environments, such as distributed and multiprovenancial databases where there is often not a readily discernible physical information object that corresponds to paper notions of a record. ${ }^{28}$

Over the past two decades, the "problematic construct" of the record has persisted in archival research, in part because how records exist across distributed environments is not well-documented (through microethnography or otherwise) or understood in material ways. While recent work examines the nature of the record in digital environments as a conceptual construct, there is little research that examines records as material things that move through systems with physical constraints such as storage memory or devices. ${ }^{29}$

The 1990s saw two influential research projects that sought to identify the functional requirements for preserving electronic evidence: the University of Pittsburgh Electronics Records Project (hereafter, the Pittsburgh Project) and the UBC-MAS Research Project. ${ }^{30}$ Both projects revealed conceptions of evidence that overlooked the unique materiality of electronic records.

Kirschenbaum, Erika L. Farr, Kari M. Kraus, Naomi Nelson, Catherine Stollar Peters and Gabriela Redwine, Digital Materiality: Preserving Access to Computers as Complete Environments. UC Office of the President: California Digital Library, 2009, http:// escholarship.org/uc/item/7d3465vg.

26 Ciaran B. Trace, "Beyond the Magic to the Mechanism: Computers, Materiality, and What It Means for Records to Be 'Born Digital', Archivaria 72 no.72 (February 12, 2011): 5-27.

27 Pearce-Moses, "Janus in Cyberspace."

28 Anne J. Gilliland, "Management of Electronic Records," Annual Review of Information Science and Technology (ARIST) 39 (2005): 219-253.

29 In this case, I am referring to both primary (i.e., Random Access Memory) and secondary (i.e., magnetic or optical disc media) memory in computing.

30 The University of Pittsburgh Electronic Records Project Functional Requirements for Evidence in Recordkeeping. School of Information Sciences, University of Pittsburgh, 
While the Pittsburgh Project advocated a new postcustodial paradigm shift, the UBC-MAS Research Project tested the validity of traditional diplomatic and archival concepts in electronic records systems. The UBCMAS project identified an archival threshold for electronic recordkeeping systems as a "space beyond which no alteration or permutation is possible, and where every written act can be treated as evidence and memory."31 The Pittsburgh Project identified a new postcustodial paradigm to preserve evidence and develop strategies for archivists' distributed governance over electronic records corpora. Instead of applying tests of validity to recordkeeping systems or requiring application models as UBC-MAS advocated, the Pittsburgh Project developed best practices for distributed custody informed by the latest research in the legal, records management and information technology professions. Or as Philip C. Bantin described, "a distributed strategy for custody [that] necessitates the creation of legally binding agreements with offices, of reliable means of auditing records, of an extensive network of training programs, and of other mechanisms designed to ensure that custodians of records understand their responsibilities and are living up to those expectations." ${ }^{32}$ In both projects the materiality of electronic records is understated, this is largely due to a focus on evidence as a logical concept.

Herein lies a deep fold in archival theories of electronic records. The materiality of electronic records - the processes of their production, transmission and storage - has been at once overlooked and overtaken by an unrelenting commitment to definitions. Whether these definitions are tied to early eras of traditional archives and analogue recordkeeping, or to the distributed model of professional responsibility and functional requirements of best practices in a new era, both subsume the nature of electronic records in part, (and perhaps in favour) of the archivists' ability to ensure evidence and authenticity through governance or distributed custody. Moreover, since Schellenberg's landmark appraisal framework, many North American archivists have focused on the informational content of transmission (how a record informs) as well as a record's capacity as evidence. ${ }^{33}$ If we understood

1996; and Luciana Duranti and Heather MacNeil, "The Protection of the Integrity of Electronic Records: An Overview of the UBC-MAS Research Project," Archivaria 42 (Fall 1996): 46-67.

31 Duranti, "Archives as a Place," 252.

32 Bantin, "Strategies for Managing Electronic Records," 23.

33 Theodore R. Schellenberg, Modern Archives: Principles and Techniques. Chicago: Society of American Archivists, 1996. 
the electronic record to be an aggregate of physical, conceptual and logical entities, then we could trust diffused control of electronic records to many actors (records creators, managers, and archivists), and even more it if such entities reside across many platforms and are supported by networked infrastructure. Materiality includes the physical form (however atomic the bits), but also extends to the systems, practice, and social institutions that are built up around artefacts.

\section{Records Continuum Theory and Relationships Between Record Concepts}

Increasingly, the transmission and distributed custody of digital collections rely upon the infrastructure of many platforms. ${ }^{34}$ One significant way to approach the processes involved in the transmission of electronic records is through the lens of continuum thinking. Historically attributed to the work of Australian archivist Ian McLean, records continuum theory has gained increasing traction in archival debates about electronic records since the 1990s. ${ }^{35}$ The continuum approach stresses the processes of transactions and the overlapping character of evidentiality, which is significant for theorising electronic recordkeeping environments. Sue McKemmish explains that as records acquire "ever-broadening layers of contextual knowledge in order to carry their meanings through time," conceptualising a records continuum allows us to consider the significance of archival interventions as part of a process. ${ }^{36}$

For continuum theorists, such as Frank Upward and McKemmish, recordkeeping is a continuum of processes that encapsulates the life cycles of records and their attendant activities. These activities range from the creation of records, to capture, organisation of recordkeeping processes, and pluralisation of records. Taken together, these four dimensions of recordkeeping establish the evidentiality of context as records move across

34 For the purposes here, platform means hardware architecture and software framework. For more on records in digital environments, see Amelia Abreu, Amelia Acker and Carolyn Hank, "New Directions for 21st Century Digital Collections," Proceedings of the American Society for Information Science and Technology 49 no.1 (2012): 1-3.

35 Frank Upward, “In Search of the Continuum: Ian Maclean's 'Australian Experience' Essays on Recordkeeping." In The Records Continuum. Ian Maclean and Australian Archives First Fifty Years, Sue McKemmish and Michael Piggott, eds. Clayton: Ancora Press in association with Australian Archives, 1994.

36 McKemmish, "Placing Records Continuum Theory and Practice," Archival Science 1 no.4 (2001): 354. 
spacetime, just as they may serve differently in multiple contexts. ${ }^{37}$ The continuum approach allows archival scholars to move beyond artificial distinctions from the records life cycle model by deemphasising static stages of records. Continuum thinking thereby enables theorising of multiple possibilities and future layers of meaning (pluralisation) (for more on continuum theory, see the chapters by Upward and Gibbons in this volume).

Continuum scholars have also argued for understanding records as having both logical and physical properties, while focusing on the logic of processural realities. ${ }^{38}$ The shifting landscape of digital preservation has given rise to approaches that complement the continuum approach. ${ }^{39}$ For example, Kenneth Thibodeau has proposed a model of preserving digital objects that moves beyond the traditional understanding of records as physical or conceptual things. ${ }^{40} \mathrm{He}$ argues for understanding records as logical, physical and conceptual objects. ${ }^{41}$ If we approach records as physical objects with a material history that is represented and moves through information systems, then we can understand their creation and preservation from the inside out - from abstract to concrete networked communication realities. For Thibodeau, this is illustrated through relationships among the three properties of digital objects, however "the relationships between levels must be known or knowable." ${ }^{2}$ These relationships between logical, physical and conceptual levels may be one-to-one or one-to-many. In any case, understanding and describing the relationships between concepts must be process-oriented and understood across a continuum. They must also be described at the platform level, as well as by the scales of infrastructures that are enacted as records are created, used, and preserved.

37 Kate Cumming, "Ways of Seeing: Contextualizing the Continuum," Records Management Journal 20 no.1 (2010): 42. I employ the concept of spacetime in the same way as it is employed in the records continuum model developed by Frank Upward. As Upward has argued, no records can be outside of spacetime, but are subject to it, and an archivist's actions upon records reverberates through it.

38 Upward, "Structuring the Records Continuum - Part One," 5.

39 New areas include the emerging fields of personal digital archiving, digital duration, and scientific workflow and provenance documentation.

40 A digital object is an object of any type of information or any format that is expressed in digital forms, and includes a range of electronic records and their digital traces of transmission.

41 Kenneth Thibodeau, Overview of Technological Approaches to Digital Preservation and Challenges in Coming Years. Washington, D.C: Council on Library and Information Resources, 1997. For more on the logical object of the record as a processable unit see especially pp.7-9.

42 Thibodeau, Overview of Technological Approaches to Digital Preservation and Challenges in Coming Years, 11 (emphasis added). 


\section{II}

Based upon my review and contemplation of the approaches described above, I propose a framework that takes into consideration infrastructure, technological practice and information retrieval aspects, updating Hedstrom's research agenda by expanding it to include broadening layers of infrastructure in ICTs moving between scales of analysis. ${ }^{43}$ This framework takes up the logical and physical aspects of Thibodeau's digital object model and applies the methodological approach that Trace has developed in her microethnography of computerised document creation. The framework relies on the records continuum to map these infrastructures and illustrate dimensions of recordkeeping in spacetime.

Employing a framework that examines infrastructure, especially at the level of transmission, points to conditions of modernity that include the fluency of infrastructure and the vulnerability of transactions across electronic recordkeeping environments. ${ }^{44}$ This proposed framework enables archival scholars to move between registers of analysis and develop a mutual orientation towards infrastructural analysis and continuum thinking. The framework aims to illustrate the significance of what can be learned by going into the black box, following records through infrastructure, and knowing what happens at moments of transmission.

\section{First Element: Layers of Infrastructure and Context}

The first element of the framework is concerned with describing the multiple levels of infrastructure that make records possible in different contexts. Understanding layers of infrastructure also reveals information about the digital materiality of electronic records in logical and physical ways.

According to Geoffrey Bowker and Susan Leigh Star, there are four elements of infrastructure: when technologies become a part of established routines they soon come to be understood commonly across different communities of practice (as boundary objects); these technologies become transparent through use, and they become embedded into socio-technical systems. ${ }^{45}$ By examining what lies beneath these processes of such

43 Scales provide the opportunity to move between registers of analysis and levels of infrastructure.

44 Edwards has examined such infrastructural fluencies and vulnerabilities in "Infrastructure and Modernity."

45 Geoffrey C. Bowker and Susan Leigh Star, Sorting Things Out: Classification and Its Consequences, 1st ed. Cambridge, MA: The MIT Press, 1999. 
stabilisation - the code, standards, network architecture, public policy and market forces that make pushing an electronic record from a recorded event to an information object, archivists can see the layers of context that shape records as they move through spacetime. This approach also allows us to consider how records are continuously active - always in the process of becoming entities in a continuum. ${ }^{46}$

Peter Botticelli argues that incorporating such an active view of the record into organisational contexts gives archival scholars the opportunity to observe the process of networked recordkeeping. Instead of understanding records as by-products of organisational transactions (that is, traditional Schellenbergian and Jenkinsonian understandings of records), Botticelli suggests that archivists should appraise records as a "class of technological artifacts in a socio-technical system." ${ }^{\text {77 }}$ Applying the study of infrastructure to records in systems is a way of examining how the historical context and development of distributed activities are technologically and socially coconstituted because of the focus on mobility and processing of information across networks. ${ }^{48}$ As the nature of knowledge work changes with twentyfirst century ICTs, it is incumbent upon archivists to understand the shifting contexts and layers of infrastructure. The method of infrastructure studies explores new forms of sociality that are being enabled by ICTs and the social, ethical and political values of self-perpetuating infrastructures. ${ }^{49} \mathrm{I}$ argue that there is a mutual orientation between infrastructure studies and the records continuum model: both are attuned to the layers of technologies, practices and events in different contexts.

In digital environments, records are more malleable and, many argue, more accessible than they are in analogue formats. This raises questions such as: what are the layers of infrastructure that make the use, access and re-use of electronic records possible? And how do layers of infrastructure affect the enduring value, authenticity and integrity of electronic records? Numerous contemporary archival scholars have pointed to the fact that "the digital" destabilises inherited concepts such as original record or the authentic first

46 Upward, "Structuring the Records Continuum - Part One."

47 Peter Botticelli, "Records Appraisal in Network Organizations," Archivaria 1 no.49 (January 1, 2000): 174.

48 Geoffrey C. Bowker, "Toward Information Infrastructure Studies: Ways of Knowing in a Networked Environment." In International Handbook of Internet Research, Jeremy Hunsinger, Lisbeth Klastrup and Matthew Allen, eds. New York: Springer, 2010, pp. $97-117$.

49 Bowker, "Toward Information Infrastructure Studies," 105. 
copy. ${ }^{50}$ However few of these scholars go beyond this conceptual bind and look at the material history and the social choices such as standards development or the design of platforms that lie behind that destabilisation. Historians of technology know that many of these givens are the result of a series of choices that have been made. Each is filled with values that must be located, and the influences of those values must be taken seriously. ${ }^{51}$ For example, consider systems that delete records after certain storage limits are reached (e.g., mobile phones and computer operating systems). This is a design choice of which archivists, lawyers and historians should be aware because it changes ideas about the history and evidence values of records as well as their data structure and context.

Sue McKemmish, writing about capturing documentary context in information systems, has touched upon the problem of complexity and context in electronic environments:

The loss of physicality that occurs when records are captured electronically is forcing archivists to reassess basic understandings about the nature of the records of social and organizational activity, and their qualities as evidence. Even when they are captured in a medium that can be felt and touched, records as conceptual constructs do not coincide with records as physical objects. Physical ordering and placement of such records captures a view of their contextual and documentary relationships, but cannot present multiple views of what is a complex reality. The traditional custodial role takes on another dimension when it is accepted that the record is only partly manifest in what is in the boxes on the repository shelves. The purpose of archival systems is to ensure that records are preserved in the context of their creation and use, and retain their qualities as evidence so that when retrieved for future use their meaning and significance can be understood..$^{52}$

50 David Bearman, "Moments of Risk: Identifying Threats to Electronic Records," Archivaria 62 (2007: 15-46); Luciana Duranti, Diplomatics: New Uses for an Old Science. Lanham MD: Scarecrow Press, 1998; Luciana Duranti, Terry Eastwood, and Heather MacNeil, Preservation of the Integrity of Electronic Records (Dorderecht: Springer, 2002); Anne J. Gilliland-Swetland, Enduring Paradigm, New Opportunities: The Value of the Archival Perspective in the Digital Environment. Washington D.C.: Council on Library and Information Resources, 2000.

51 Paul Dourish and Genevieve Bell, Divining a Digital Future: Mess and Mythology in Ubiquitous Computing. Cambridge, MA: MIT Press, 2011.

52 Sue McKemmish, “Are Records Ever Actual?” In The Records Continuum: Ian Maclean and Australian Archives First Fifty Years, Sue McKemmish and Michael Piggott, eds. Clayton: Ancora Press in association with Australian Archives, 1994, 187-203. 
McKemmish uses the physicality of records (or loss of it) as an entry point to reiterate the importance of data structure on context of creation, whether that be on the shelf or in the file structure of an operating system, and how that is experienced in practice by the creator or user. In addition to platform design decisions and local memory storage limits, the history of object-oriented data structures, relational databases, even NoSQL (not only structured query language) are approaches that do not adhere to relational approaches and affect the realities of archival objects in different digital environments and their contexts of creation. Further, data structures can heavily influence the flavours of database populism that may arise and the recordkeeping possibilities when employing systems such as those used in social media. ${ }^{53}$

The data structures that we employ are not only conscious design choices - they also affect the possibilities for description, retrieval and access in the future. Data structures "infrastructure" the representation of records in existing systems as well as shape the contexts of their reception or access. In some cases, the design of the system limits the possibilities of creating traces at the same time as it institutes values. Archivists should examine multiple and different contexts of record creation, use and interpretation in addition to the creation of metadata during transmission, but they should also consider how the data infrastructures create and shape evidence. This includes considering system design, user experience and the limits of creating records with a device that involves (to name but a few) an external battery, an operating system, a Wi-Fi or mobile data connection, limited local storage and possible cloud back up. In each of these cases (and beyond) these objects involve layers of infrastructure that have significant archival implications for preservation, authenticity, and evidence.

\section{Second Element: Examining Networked Recordkeeping}

Contemporary recordkeeping practices rely on distributed, networked systems, yet there are very few descriptions of records transitioning across systems as a process, or of how their history of stabilisation (as formats) affects their circulation. This raises a key research question: What do these processes look like, conceptually, logically and physically (i.e., "under-thehood")? Archival scholars and practitioners who have worked with electronic records since the 1980s agree that there are some crucial "moments of

53 Kevin Driscoll, "From Punched Cards to 'Big Data': A Social History of Database Populism," Communication +1, 1 no.1 (August 29, 2012). 
risk" for electronic records throughout their creation and existence. ${ }^{54}$ The moments that present the greatest risks are, paradoxically, those transactional points when entities cross systems and result in the creation of records. Furthermore, this is how such transactions, from web publishing, to retrieving electronic records off hard disks, to accessing online information work. Moments of risk are points where control over a record is ceded or diminishes at the levels of capture, ingest, and accession. ${ }^{55}$ In other words, these are moments of transition across information systems, or when the record itself is transformed, copied from one user to another or from system to system, or accessed by one platform from another. ${ }^{56}$

Conceptually, these threats are understood as areas for archival concern and logically, they have been theorised for several decades. Physically, however, they are not well documented. Records are seen as fragile and at risk during transmission, instead of being understood as a fundamental part of how electronic records physically and logically function in contemporary modes of communication. According to Bearman, the greatest risks are at the level of capture and access because these two moments are "outside the scope" of most models of archival preservation (see Hofman's chapter in this volume for discussion of archival preservation models). The nature and effects of these risks are critical to the archival perspective, although they are still seen as beyond the threshold of the archivist's responsibility in the archival life cycle model. While traditional analogue archives ingest records after they become inactive, electronic records are always active, even as they are accessed as archives. Instead of assuming that these are innate moments of vulnerability, my proposed framework allows us to ask what if archivists approached transmission as moments of authority - and to locate, understand and describe them as archival objects of interest? Bearman and other postcustodial scholars argued for examining process through continuum thinking but what does that mean for networked recordkeeping environments that include multiple platforms and distributed storage?

54 Bearman, "Moments of Risk"; McKemmish, "Are Records Ever Actual?”

55 If a draft, $\mathrm{mp} 3$ or a digital photograph is sent, whether through email or transfer from one machine to another with a USB flash drive, it goes through a series of junctures to move from one (physical) place to another (at the levels of copy, capture, ingest, access). Users who use networked ICTs to communicate understand the distributed nature of transmission and failure in a variety of conceptual and logical ways. Further, they create practices and improvise around these junctures.

56 Matthew Kirschenbaum, Mechanisms: New Media and the Forensic Imagination. Cambridge MA: MIT Press, 2008. 
Despite its supposed riskiness, Bearman focuses on the relationships that records have across systems because these relationships transform the nature of the information: "[ICTs] are not just providing a new method for transmission of information but changing the social character of the communication. ${ }^{57}$ Understanding the nature of electronic records as a process of creation and transmission is a useful conceptual move and can be seen in Bearman's commitment to the materiality of electronic records. Bearman was one of the first archival thinkers to raise the importance of "the conceptual" to electronic records theory in the early 1990s, and a decade later he began to publish about the "material conditions for communication" in twenty-first century computing environments.$^{58} \mathrm{He}$ argued that the most problematic part of working with electronic records is in their identification, which then poses some serious, practical concerns for managing them:

A record is any communication between one person and another, between a person and a store of information available to others, back from the store of information to a person or between two computers programmed to exchange data in the course of business. What is excluded in this definition is any information that remains within the computer workspace of a single individual, inaccessible to others, for private information or under editing and development. When the information is shared with another person or a machine accessible to others, it becomes a record.

The virtue of this definition is the ease with which individuals can understand it and the simplicity of instructing computing and communications systems to capture it. ${ }^{59}$

For Bearman, the nature of the electronic record is "the when" of its accessibility and sharing as it is transmitted between systems. It is this that signifies a transaction. Or, as McKemmish has argued, when a record crosses boundaries is what defines it. ${ }^{60}$

Crossing boundaries, moving between systems, sharing information, and sending drafts that become records are all ways of conceiving of records for postcustodial archival scholars. Yet it remains unclear why transmission is

57 Bearman, Electronic Evidence, 14.

58 Bearman, Electronic Evidence, 14.

59 David Bearman, "Electronic Mail. Is it a Record? How Can it be Managed?" presentation to the Society of Canadian Office Automation Professionals, Ottawa, March 31, 1993.

60 McKemmish, “Are Records Ever Actual,” 187-203. 
not examined as a part of the process of becoming a record. I argue that even postcustodial definitions of electronic records becoming in spacetime overlook material constraints and employ tropes of immateriality. ${ }^{61}$ Frequently, these tropes rely on a loss of physicality or a reliance on understanding electronic environments as "purely logical" constructs without material constraints. ${ }^{62}$ Nevertheless, despite a reliance on immateriality to explain transmission, we can credit Bearman for drawing attention to the material conditions of electronic communication, the physical constructs of recordkeeping systems, and the logical boundaries of archival principles such as fonds or provenance.

Archival systems produce as much as they record and document, and this is dependent on the context and materiality of the records they hold. As new information technologies shift the ways of communicating, exchanging, organising and preserving knowledge, they also transform the very terms of that knowledge. This second element assumes that the description and deep understanding of the processes of transmission are viable and essential parts of arriving at archival understandings regarding distributed records, which also has stakes for our evaluation of the materiality of recordkeeping. It prompts archivists to examine networked recordkeeping in order to locate new and improvised forms and practices of record creators and users.

\section{Third Element: Engaging with Information Retrieval}

The rise of digitally-born records and the inexpensive cost of digital storage together present a pressing and challenging area of information management, but also provide an opportunity for archivists to apply traditional principles to new possibilities for retrieval and access in an era of big data collections and inquiry. Increasingly it is cheaper (and arguably easier) to save swaths of electronic records through backup instead of weeding, appraisal, and deletion (see Gilliland's chapter in this volume, "Designing Expert Systems for Archival Evaluation and Processing of Computer Mediated Communications," for a more detailed discussion of appraisal of massive quantities of digitally-born materials). Claims of the "big data revolution" have generated significant amounts of response in

61 Jean-François Blanchette, “A Material History of Bits," Journal of the American Society for Information Science and Technology 62 no.6 (2011): 1042-1057.

62 Bearman frequently uses metaphors of immateriality when describing the future of archival principles in electronic systems, often diametrically opposing them to the past of analogue (paper-based) recordkeeping systems, e.g., "The 'fonds' as a physical construct will completely disappear because the boundaries of recordkeeping systems will be purely logical ones," Electronic Evidence, 254. 
virtually every area of society. ${ }^{63} \mathrm{Big}$ data is positioned as a potential resource in business, government, academic research and military applications, and many information professions are developing analytic tools for retrieval and enterprise.$^{64}$ Likewise, academic big data initiatives in disciplines such as physics and astronomy have promised new levels of analytics and discovery. The ability to capture more data has grown as sensors for collecting data become smaller and more ubiquitous. Moreover, the cost effectiveness of saving and the ability to re-use recorded information has led to a paradigm of data intensive science. ${ }^{65} \mathrm{In}$ an age of big data, there is potential for archivists to leverage traditional concepts such as provenance and the archival bond to enhance information retrieval and access. ${ }^{66}$

Historically, information retrieval (IR) has been understood as retrieving documents and sometimes portions of text in order to satisfy a user's need. ${ }^{67}$ Among the most common is the retrieval of single documents that can be accomplished in two ways, through indexing by subject or searching by keyword or phrase. Where indexing is concerned with representations of documents (or information objects), searching refers to the ways in which documents are matched to specific queries by unique markers within the body of a document's text or internal structure. A great preponderance of early-automated information retrieval systems that were designed and built in the twentieth century were intended for retrieving references to surrogates of documents through indexing and abstracting (for example, union catalogues). Then, with the rise of institutional and personal computing and the decrease in storage and processing costs there was a shift to fulltext retrieval of documents by searching with key words. Some IR systems, including search engines combine both indexing and document structure of

63 For critiques of the promises of big data see, Danah Boyd and Kate Crawford, "Critical Questions for Big Data: Provocations for a Cultural, Technological, and Scholarly Phenomenon," Information, Communication \& Society 15 no.5 (2012): 662-679.

64 See, for example, Christine L. Borgman, Jillian Wallis, and Noel Enyedy, "Little Science Confronts the Data Deluge: Habitat Ecology, Embedded Sensor Networks, and Digital Libraries," International Journal on Digital Libraries 7 no.1 (2007): 17-30; Clifford Lynch, “Big Data: How do Your Data Grow?” Nature 455 (2008): 28-29; Steve LaValle, Eric Lesser, Rebecca Shockley, Michael S. Hopkins and Nina Kruschwitz, "Big Data, Analytics and the Path from Insights to Value," MIT Sloan Management Review 52 no. 2 (2011): 21-32.

65 Christine L. Borgman, Scholarship in the Digital Age: Information, Infrastructure, and the Internet. Cambridge, MA: MIT Press, 2007.

66 David A. Bearman and Richard H. Lytle, "The Power of the Principle of Provenance," Archivaria 1 no.21 (1985): 14-27.

67 Karen Spärck-Jones and Peter Willett, Readings in Information Retrieval. San Francisco, CA: Morgan Kaufmann, 1997. 
web-based objects (such as web pages) to leverage both approaches. In both forms of retrieval, the design and approach to indexing and searching are obliged to use the document as the base unit of measure - words, phrases and structures within a given document were and are the guiding principles of searching for relevant information with automated retrieval systems. By virtue of privileging the internal structure of the document as the guiding measure, both forms of IR neglect relationships between documents, known as "intra-document structure" and their context of creation as meaningful information or possible access points (see chapter by Furner and Gilliland in this volume for more detailed description of IR approaches and discussion of their potential in archival and recordkeeping applications).

While most IR approaches are concerned with retrieving records as single entities, archivists and records managers are concerned with how records should be collectively managed and viewed in the aggregate. Records have the most power in aggregate, according to Schellenberg, because "records have a collective rather than a unitary significance. ${ }^{168}$ Accordingly, for some diplomatic scholars such as Luciana Duranti, it is only after a document is set aside and designated to be "put into relation to other records" that it becomes a record and acquires an archival bond. ${ }^{69}$ For Duranti, the record and its archival bond are dependent upon a formal method of setting records aside after transmission for capture, description and preservation. Such an orthodox border of designated capture that is dependent on a recordkeeping system may leave some types of records on the sidelines, however. Some scholars have noted that transitory records may escape system safeguards and further, that systems often intentionally do not capture transitory or secondary records, which may also function as the most important metadata in networked contexts. ${ }^{70}$ Although escaping such a system safeguard and thereby the archival bond may mean that some stray records fail to meet diplomatic tests for "recordness", many archivists agree that such records may be no less important to the wider historical record.

As already discussed, from a continuum perspective, the archival bond is a relationship that perdures through the dimensions of creation, capture,

68 Theodore R. Schellenberg, Modern Archives; Principles and Techniques. Chicago, IL: Society of American Archivists, 1996, p.67.

69 Luciana Duranti, “The Archival Bond," Archives and Museum Informatics 11 (1997): 216.

70 Steve Stuckey, "The Australian Archives Policy on Electronic Records.” In Playing for Keeps: The Proceedings of an Electronic Records Management Conference Hosted by the Australian Archives (Australian Archives, 1995), 121; Elizabeth Shepherd and Geoffrey Yeo, Managing Records: A Handbook of Principles and Practice, Facet Publishing, 2003, pp.108-109. 
organising and pluralisation. Continuum theorists have argued that the principle of provenance (often co-terminous with a single records creator or fonds) should be expanded to include the interactions between multiple fonds or creators. Such an expansion can be seen in Chris Hurley's critique of provenance. ${ }^{71}$ Classic archival principles hold that provenance has two distinctions, "records of the same provenance should not be mixed with those of a different provenance, and the archivist should maintain the original order in which the records were created and kept." ${ }^{\text {72 }}$ Hurley argues that a concept of provenance that only recognises a single context denies the possibilities of records crossing and interacting with multiple fonds and bodies of records (e.g., networked social platforms or Web 2.0). The concepts of simultaneous multiple provenance and parallel provenance, for Hurley and others, is a means by which archivists might capture the recordkeeping realities of distributed digital environments such as social networks. As opposed to traditional understandings of provenance, parallel provenance allows archival description to represent multiple contexts of creation, records creators and individual fonds that might exist as a result of the co-creation or convergence of records or archival collections. When applied to archival description, parallel provenance offers a way to account for the networked nature of platforms by expanding the possibilities for description. This in turn enables dynamic retrieval that traditional IR approaches cannot accomplish. ${ }^{73}$

By leveraging the principles of multiple simultaneous and parallel provenance and the archival bond to expand description, archivists are in a unique position to locate and interpret evidence of records in the aggregate and as collections of value in information retrieval approaches. Retrieval through provenancial relationships that exploit the power of connective tissue across records in data structures, enables archivists to locate and thus support emerging recordkeeping contexts in ways that traditional and most current IR approaches overlook. In other words, the potential for online retrieval for archivists lies in the circumstances of a record's formation, interaction between fonds, and parallel provenancial relationships in aggregations. Each can be used as access points in retrieval. ${ }^{74}$

71 Chris Hurley, "Parallel Provenance: (1) What if Anything is Archival Description?" Archives and Manuscripts 33 no.1 (2005): 110, and "Parallel Provenance: (2) When Something is Not Related to Everything Else," Archives and Manuscripts 33 no.2 (2005): 52.

72 Gilliland-Swetland, "Enduring Paradigm, New Opportunities."

73 For more on IR applications in archives and recordkeeping, see Furner and Gilliland's chapter on the subject in this volume.

74 Richard Lytle, "Intellectual Access to Archives," American Archivist 43 no.2 (April 1, 1980): 191-207. 
Despite such significance, little is still understood about the implications for archival retrieval with large-scale digital record corpora. In 1985, Richard Lytle and David Bearman claimed that archivists' "unique perspective" as provided by the principle of provenance would be their contribution to the future of information management in the digital records era. ${ }^{75}$ Lytle and Bearman predicted that automated information systems would greatly benefit from provenance-based retrieval and identified design factors and guiding principles for empirical research. Retrieving archival evidence through description and access by searching across relationships between networked records, however, remains under-explored for professional archivists and the communities that they serve. Increasingly, the ways in which "provenance" is incorporated into contemporary system architecture do not employ provenance as it is understood by archivists. Instead, the term is used to refer to workflow processes in an organisation or distributed work group. ${ }^{76}$

Information retrieval systems are designed according to the standards and conventions of the institutional networks in which they operate. As archives increasingly ingest electronic records they will need to implement new ways of organising and providing access to their collections. There is evidence that data scientists grappling with databases and massive amounts of data are on the forefront of the conceptual and technical retrieval problems of document structure, workflow, and what many scientists who work with big data are also increasingly calling "provenance." 77 The dearth of work in archival scholarship that is looking ahead to these retrieval issues is both a poison and a cure. Identifying places outside archives where provenance and multiple contexts are seriously considered is one step towards building a meaningful hermeneutic, or guiding interpretative principle, for the future of automated retrieval systems that embed archival infrastructure into system design and implementation.

This third element in the framework, therefore, emphasises the archival approach to groups of records, interaction between fonds, and evidence that the archival bond affords and that many current information retrieval approaches neglect. In the next section I present the case study of Kurt Mix

75 Bearman and Lytle, "The Power of the Principle of Provenance."

76 Luc Moreau, "Usage of 'Provenance': A Tower of Babel Towards a Concept Map," presented at the Life Cycle Seminar, Mountain View, CA, 2006.

77 Luc Moreau et al., "The Provenance of Electronic Data," Communications of the $A C M$ (2007), http://citeseerx.ist.psu.edu/viewdoc/summary?doi=10.1.1.97.5546, and Luc Moreau, "Usage of 'Provenance': A Tower of Babel Towards a Concept Map." 
to illustrate the applicability of the framework for encouraging archival scholars to expand their inquiry into scales infrastructure and electronic records' boundary-crossing through the continuum. The case points to the problems that arise as information is shared and distributed between people across systems as well as the multiplicity of archival conflicts that can arise in connection with emerging records practices using mobile, networked communication.

\section{III}

On 20 April 2010, the Deepwater Horizon mobile offshore drilling rig exploded off the southeast coast of Louisiana in the Gulf of Mexico. The explosion killed 11 people and injured 17 more rig workers. The Deepwater Horizon rig burned and sank causing the Macondo oil well to blow out. The Deepwater Horizon Oil Spill, also known as the "BP Oil Disaster," is the largest marine oil spill in the history of the petroleum industry. The oil well leaked unabated for three months as British Petroleum (BP) attempted several times to cap off the blown well using different methods. BP and the U.S. government initially underestimated how much oil spilled during the crisis (now estimated at 205.8 million gallons). ${ }^{78}$

A month after trying to stop the spill, on 26 May 2010 BP announced a project called "Top Kill" to drill mud at a high pressure into the broken well. Kurt Mix, a BP drilling project engineer was on a team that used models to approximate the flow of the blowout as part of preparation for Top Kill. For two days Mix reported progress back to his supervisor regarding the progress of Top Kill using his mobile phone. He exchanged approximately 200 text messages with his supervisor in a text thread (a thread is a series of text messages exchanged between users) sharing estimates of the flow rate. Later Mix used text messaging to discuss the project with another BP contractor. Three days later, on 29 May 2010, BP announced that Top Kill had failed and that they were exploring other contingency options. In October 2010, Mix deleted the thread of text messages with his supervisor. In August of the following year, he deleted another thread of communications between him and another contractor. Mix deleted approximately 300 text messages related to Top Kill from his iPhone in total, comprising two text threads between him and two other BP employees.

78 As investigations and civil lawsuits are ongoing, see "Deepwater Horizon Oil Spill," 2013 http://en.wikipedia.org/wiki/Deepwater_Horizon_oil_spill. 
Two years after the Deepwater Horizon explosion, in April 2012, the first criminal charges associated with the BP oil disaster were filed by the U.S. government against Kurt Mix for obstructing justice by destroying evidence related to the actual amount of oil leaking from the blowout. ${ }^{79}$ Throughout the spill, BP released flow estimates that were later found to be several times lower than the actual amount spilling from the Macondo well. The U.S. Justice Department alleges that the text messages that Mix sent and received contain information related to the actual flow rate of oil during the spill, contrary to the information that $\mathrm{BP}$ reported to the government, media and the public during the time of the disaster. Court documents allege that Mix deleted the text threads after learning that a vendor working for BP's outside counsel would be imaging his mobile phone. According to these charges:

there is probable cause to believe that (1) on or about October 4, 2010, and (2) again between on or about August 19, 2011 and August 20, 2011, Mix did knowingly and corruptly alter, destroy, mutilate, and conceal a record, document, or other object, and attempted to do so, with the intent to impair the object's integrity and availability for use in an official proceeding, in violation of 18 U.S.C. $§ 1512(\mathrm{c})(1){ }^{80}$

Digital forensic analysis was able to recover some of the texts deleted from Mix's phone but not all of the texts he received. The complete exchanges were recovered from other devices through forensic discovery. ${ }^{81}$

The threads of text messages that Mix allegedly deleted present a case study through which to read records moving through infrastructure, to examine emerging records practice with material constraints, and to consider the implications for information retrieval in archival contexts. Mix is not being charged for the content of the text messages, but instead for deleting threads of texts from the local storage on his mobile phone with the intent to hide or obscure information. The evidence of obstruction is located in the metadata and transmission data about the texts and in the practice of

79 Later these charges would expand to include deleting hundreds of voicemail messages. Walter Pavlo, "Former BP Engineer Kurt Mix Still Deleting 'Stuff,' Government Alleges," Forbes Blog, March 28, 2013, http://www.forbes.com/sites/ walterpavlo/2013/03/28/fmr-bp-engineer-kurt-mix-still-deleting-stuff-govt-alledges/.

80 Barbara O'Donnell, "Affidavit in Support of a Criminal Complaint and Arrest Warrant for Kurt Mix," United States Department of Justice, April 23, 2012.

81 In legal investigations, forensic discovery is used to recover electronic evidence to support or disprove claims in a court of law or in civil proceedings. Luciana Duranti has written about the future of digital forensics to archival practice in "From Digital Diplomatics to Digital Records Forensics," Archivaria 68 (2010): 39-66. 
managing them, or in this case deleting text threads. The text thread itself is consequential because it is both evidence of a read receipt from his coworker's devices, and also confirmation of multiple text transmissions over a given period of time. The thread also indicates that there are digital traces of the conversation on multiple telecommunication servers, message centres that store and forward text messages, possibly hosted by multiple network providers, as well as on the devices belonging to the people who texted with Mix. The text thread as a physical, logical and conceptual entity exists in many different places and times, and there are records of transmission that prove this (e.g., timestamps, billing statements, messaging application logs). Indeed, such charges of obstruction stand against Mix because these traces do exist in many places - on the supervisor's phone, the vendor's image backup service, BP's discovery files as part of legal proceedings, AT\&T's servers (for a time the only U.S. network provider with iPhone devices and coverage), and perhaps even on Mix's MobileMe account. ${ }^{82}$

The charges against Mix speak to each element of the framework that I have proposed. We can consider the infrastructure that makes the text messages possible - the largescale technical telecommunication networks that send and transmit the text messages, as well as the metadata, or the records of transmission that prove such texts were sent and received between the BP employees. The mobile phone practices of Mix himself also have archival consequences: What is at stake when we use personal mobile devices to send business messages to supervisors or collaborators? Who is responsible for documenting and preserving business transactions that occur with (almost) real-time text messages during federal emergencies or ecological disasters like the BP Oil Spill? And further, as the U.S. Justice Department charges against Mix illustrate, the evidence of obstruction is not the content of the texts themselves, but instead the intent to obscure or destroy the evidence between communications - the texts in aggregate as a thread. The retrieval implications are not in the words used within the text messages, but instead in the structure of the records as aggregates, or in the thread as a collection of records. The transmission processes are crucial in addition to their timeliness. Consider, for example, if Mix and his supervisor has been in limited service ranges and texts were delayed or lost during the

82 MobileMe is a subscription-based suite of online services and mobile software used by iOS users, including the iPhone 4. Since June 30, 2012 the service has ceased to exist and replaced by iCloud. John D. Sutter, "iCloud: Revolution of the next MobileMe?" CNN Tech Blog, June 9, 2011, http://www.cnn.com/2011/TECH/web/06/07/icloud. reaction/index.html. 
disaster. The original order and provenancial information in the case of Mix is as important to the first criminal charges filed against $\mathrm{BP}$ as it is to the archivists who will document the largest spill in the history of oil disasters.

\section{When is the Future? Or, How Soon is Now?}

Given that text messaging has increasingly become a part of the modality of documentation in modern life, archivists need mechanisms to understand how mobile communication like text messages exist physically and conceptually, and how the technology will reach closure and stabilisation through social construction. ${ }^{83}$ Most archival scholars would approach this documentary quagmire by asking what is a record in terms of text messaging as a records creation practice. The digital traces of the thread's transmissions may well be more consequential than the content of the texts themselves, however, as, for example, at the time of Mix's trial. What is a record on Mix's mobile device now is different from what it was in Spring 2011, and it is still becoming, acquiring layers of context as it moves through infrastructure both technologically and socially. Moreover, the infrastructure that makes mobile communication possible will change over time.

Historically, archival scholars have been concerned with materiality and have developed highly faceted ways for interpreting context in and through the materiality of records (e.g., conservation science, diplomatics, and descriptive bibliography). By reinserting the concern for materiality into electronic records discourse as it relates to the digital preservation and description of layered digital traces that are networked and wirelessly transmitted, archivists have the opportunity to revisit the importance of the materiality of information to the field of information science as wider discipline.

Digital traces such as text messages evade definitions of electronic records with enduring value that are predicated upon the degree to which they are stable and fixed. ${ }^{84}$ However, digital traces should be important to archivists because archival theories about evidence can intervene in ontological questions of how these traces will be enrolled in regimes of evidence in forensics, digital preservation and jurisprudence in the twenty-first century, as we see

83 Trevor J. Pinch and Wiebe E. Bijker, "The Social Construction of Facts and Artifacts: Or how the Sociology of Science and the Sociology of Technology Might Benefit Each Other." In The Social Construction of Technological Systems: New Directions in the Sociology and History of Technology, Wiebe E. Bijker, Thomas P. Hughes and Trevor J. Pinch, eds. Cambridge, MA: MIT Press, 1987, pp. 17-50.

84 Bruno Latour, "Beware, Your Imagination Leaves Digital Traces," Times Higher Literary Supplement 6 no.4 (2007). 
in the unfolding story of Kurt Mix, or in other high profile litigation and civil suites that involve electronic communication, or in the U.S. presidential records act that has expanded to include emails and phone.

There is a pervasive commitment to defining the record in electronic environments that has existed in archival scholarship since the 1980s. A remnant of positivist orthodoxy, the commitment dictates we must redefine, "What is a record?" each time a new electronic record, platform, or information object is encountered. As I have written earlier, Gilliland argues archival scholarship in the 1990s on electronic records management focused upon the nature of the record and concepts were defined and redefined as archivists confronted the impacts of computing on ICTs. ${ }^{85}$ In 1997, Peter Marsden identified this same trend in terms of archival scholarship relating to electronic networked environments, with commentators proffering predictions for how archivists should prepare for future technology and information systems:

One witnesses a constant redefinition of terms and the appropriation of others, all of which leaves a sense that whatever archival footings remain, they are on very unstable ground. For most archivists, however, the need is not to attack the past nor to resist the future. ${ }^{86}$

Although practitioners, as Marsden points out, simply need some guidance that does not create disjunction between now and the future, I argue that many archival scholars are fixated on coming to universal understandings and applications of "what a record is" in the diplomatic sense and have been slow to find universal, catholic approaches that include all information objects of evidential value, as well as multiple contexts of records-becoming. We must open the scope of our inquiry to the layers of infrastructure, networked recordkeeping practice and the possibilities for archival retrieval within the records continuum. By incorporating spacetime, and scales of infrastructure to our analysis we can arrive at a fuller understanding of electronic records, and also an expansion of archival callings. ${ }^{87}$

85 Anne J. Gilliland, Conceptualizing Twenty-first-century Archives, Chicago: Society of American Archivists, 2014.

86 Paul Marsden, "When Is the Future? Comparative Notes on the Electronic RecordKeeping Projects of the University of Pittsburgh and the University of British Columbia," Archivaria 1 no.43 (1997): 158.

87 Richard J. Cox has described part of these new archival callings as educating and informing "citizen archivists" in Personal Archives and a New Archival Calling: Readings, Reflections and Ruminations, Duluth, MN: Litwin Books, 2008, pp. 23-24. 


\section{Always Becoming, or, When is a Record?}

I would argue that the project of defining a record should be abandoned in the age of networked records. Instead we must expand our focus to the ever-broadening layers of infrastructure and context through which digital records move. As we have seen with text messages, in the digital age the ontological purity and drive for the true nature of the record is over. It cannot be exclusively or definitively located because it is not in a single place, it never ends, and is "always in a process of becoming" in the continuum. ${ }^{88}$ Instead, a record's mediation through time and space, its mobility and its perdurance as archival infrastructure are the ultimate factors in determining enduring value. Analysing how a record moves through networks, and asking when a record is becoming, and how and where it persists is a more productive method for understanding the nature and effects of infrastructure and ultimately how electronic evidence is socially constituted with technologies. The nature of the electronic record that is wirelessly transmitted across networks, platforms, and information systems cuts through the inertia of these tired defining exercises: the very basis of the digital is its flexibility, unfixity, combinations and re-combinations.

Instead of asking, "what is a record?" when we encounter new information communication technologies, it behooves archival scholars instead to ask, "when is a record in infrastructure?" How can we account for the processes of creation, transmission, storage and retrieval that occur throughout the continuum? Tracing evidence through infrastructure proves to be a way to get at record-ness without getting caught up in the rigmarole of asking what a record should be or how complete it is every time archivists and records managers encounter "new" digital traces and unknown systems, from new platforms, to emergent ways of communicating with new formats. Marsden pointed to a sense of "unstable ground" that kept archival scholars from resisting the future. Fifteen years later, we are still encountering unstable ground with digital landscapes. But what has been overlooked are the layers of infrastructure and large-scale technical networks that really have hardened and stabilised around emerging recordkeeping practices in networks that create a constant sense of instability for archivists.

An updated framework of archival scholarship that accounts for this unsteady terrain and the multiple ontologies of recordkeeping practice is needed to support archival scholarship relating to digital records. Continuum scholars have begun this work in modelling spacetime, parallel provenance 
and recordkeeping in the continuum, still, infrastructure often remains invisible. Moreover, such a framework must account for infrastructure from an archival perspective. Archivists can also locate new possibilities where such a framework can be applied. The framework I have proposed pushes back upon conceptions of information and electronic records as abstract and immaterial. Furthermore, it prompts a critical challenge to recover the materialness of things like digital traces, by examining the infrastructure, technical architecture, code and standards that shape our ability to migrate the digital landscape in addition to continuum thinking about recordkeeping.

Archivists also face a pervasive trend in information science that deemphasises the genre and form of electronic records. Matthew Kirschenbaum calls this the "tactile fallacy," arguing that electronic information's lack of tangibility does not negate form or physical qualities. In addition to overcoming a reliance on immateriality to explain digital forms, we need to describe new ways of being in the world that are supported by electronic records. Many archival scholars take contemporary recordkeeping practices as objects of study but emerging social practices around records in networks and social platforms such as the law, digital forensics and telecommunication policy remain ripe for analysis. Presently, archivists do not have a method for understanding the stabilisation of emerging formats over time, how they are created or used. ${ }^{89}$ Studying the conditions of possibility for things to exist, at moments of creation and then over time, and examining the historical ontology of records within infrastructure together provide a means of analysing epistemological transformations, emerging categories of knowledge, and techniques of inscription. ${ }^{90}$

\section{The Future of Digital Communication}

There are whole swaths of records created with personal communication devices (ranging from digital cameras, to media players, to tablets and so on) that are not and will not be documented by historical collecting institutions such as libraries, archives and museums. Instead emerging documentation will be fixed by individuals and survive on networks in what

89 For example, we can trace the historical ontology of blogs (and their comments), from personal weblogs or commentaries published online to established, structured forms that are now seen as primary sources.

90 David Ribes and Jessica Beth Polk, "Historical Ontology and Infrastructure." In Proceedings of the 2012 iConference, iConference '12, 2012, pp. 254-262. For more on epistemological transformations and techniques of inscription, see Michel Foucault, The Archaeology of Knowledge, New York, NY: Vintage, 2012. 
we are now calling personal digital archives. Presently we use our mobile phones as primary communication devices. There are more than 7 billion mobile phone subscriptions worldwide, and texting with mobile phones is now the most popular form of daily communication. ${ }^{91}$ We use these mobile devices to communicate in a variety of ways beyond voice traffic - we send messages, access the Internet, contact friends, family and co-workers, take photographs, and use them for location services. In a relatively short time, mobile computing with handsets has become the primary way of communicating information in terms of volume, frequency and penetration throughout the world.

Clearly, archivists must account for emerging electronic formats such as mobile records, however it is uncertain that existing approaches to electronic records can account for the complexities of today's digital infrastructure. Consequently, archivists face a challenge to update and expand their methods for both understanding and managing such records. This is a daunting task as multiple devices, new formats, operating systems, service providers, local storage and cloud backup, mobile applications, and shifting legal landscapes keep many records managers and archival scholars from seriously considering the future of mobile communication or personal archives in archival institutions. Each aspect adds layers of context, links in multiple chains of custody, and distributed provenancial information to the continuum. The significance of this framework addresses the challenge of complex systems, the invisibility of infrastructure and the shifting responsibilities of the archivist in the digital age.

Heather MacNeil writes that there needs to be a "more equitable balance between the product and the process of documentary creation and transmission." ${ }^{92}$ Given the possibilities of emerging digital traces in contemporary society, it is time for archivists to consider how infrastructure supports and shapes record creation and transmission and is mutually oriented towards continuum models. In order to understand the archival realities more fully we need to understand the processes of communication in the continuum, the practices of communicating as well as the traces that are generated, produced and exchanged as part of a trail of evidence that bonds

91 Sherna Noah, "Texting Overtakes Talking as Most Popular Form of Communication in UK," The Independent (July 18, 2012), http:/www.independent.co.uk/news/uk/ home-news/texting-overtakes-talking-as-most-popular-form-of-communication-inuk-7956016.html.

92 International Telecommunications Union, "World Telecommunication/ICT Indicators database 2015 (19th Edition/December 2015)”, ITU, Switzerland, 2015. 
the data, texts, pictures and tweets that have been sent and transmitted across systems. Studying how and when records move along infrastructure, in networked practice, and through retrieval contexts will give archivists a renewed opportunity to examine contemporary physical realities of our work with material traces in new forms and new spaces in the postcustodial era. It may be a daunting task, but if I am right that locating records within complex scales of infrastructure provides archivists with a renewed calling to examine the possibilities of transmission within the continuum, then we must attempt to try.

\section{References}

Abreu, Amelia, Amelia Acker and Carolyn Hank. "New Directions for 21st Century Digital Collections," Proceedings of the American Society for Information Science and Technology, 49 no.1 (2012): 1-3.

Bantin, Philip C. "Strategies for Managing Electronic Records: A New Archival

Paradigm? An Affirmation of our Archival Traditions?” Archival Issues 23 no.1 (1998): 17-34.

Bearman, David. "Electronic Mail. Is it a Record? How can it be Managed?" presentation to the Society of Canadian Office Automation Professionals, Ottawa, March 31, 1993.

Bearman, David. Electronic Evidence: Strategies for Managing Records in Contemporary Organizations. Pittsburgh, PA: Archives \& Museum Informatics, 1994.

Bearman, David A. "Moments of Risk: Identifying Threats to Electronic Records," Archivaria 62 (2006): 15-46.

Bearman, David A., and Richard H. Lytle. "The Power of the Principle of Provenance," Archivaria 21 (1985): 14-27.

Blanchette, Jean-François. "A Material History of Bits," Journal of the American Society for Information Science and Technology 62 no.6 (2011): 1042-1057.

Borgman, Christine L. Scholarship in the Digital Age: Information, Infrastructure, and the Internet. Cambridge, MA: MIT Press, 2007.

Borgman, Christine L., Jillian C. Wallis, and Noel Enyedy. "Little Science Confronts the Data Deluge: Habitat elEcology, Embedded Sensor Networks, and Digital Libraries," International Journal on Digital Libraries 7 nos.1-2 (2007): 17-30.

Botticelli, Peter. "Records Appraisal in Network Organizations," Archivaria 49 (2000): 161-191.

Bowker, Geoffrey C. "Biodiversity Datadiversity," Social Studies of Science 30 no.5 (2000): 643-683.

Bowker, Geoffrey C., Karen Baker, Florence Millerand and David Ribes. “Toward Information Infrastructure Studies: Ways of Knowing in a Networked Environment." In International Handbook of Internet Research. Dordrecht, The Netherlands: Springer Netherlands, 2010, pp. 97-117.

Bowker, Geoffrey C., and Susan Leigh Star. Sorting Things Out: Classification and its Consequences. Cambridge, MA: The MIT Press, 2000.

Boyd, Danah, and Kate Crawford. "Critical Questions for Big Data: Provocations for a Cultural, Technological, and Scholarly Phenomenon," Information, Communication E Society 15 no.5 (2012): 662-679. 
Castells, Manuel. "An Introduction to the Information Age." In The Information Society Reader, Frank Webster, Raimo Blom, Erkki Karvonen, Harri Melin, Kaarle Nordenstreng, and Ensio Puoskari, eds. London and New York: Routledge, 2004, pp. 138-149.

Clifford, Ben, Ian Foster, Jens-S. Voeckler, Michael Wilde and Yong Zhao. "Tracking Provenance in a Virtual Data Grid," Concurrency and Computation: Practice and Experience 20 no.5 (2008): 565-575.

Cook, Terry. "Easy to Byte, Harder to Chew: The Second Generation of Electronic Records Archives," Archivaria, 33 (1991): 202-216.

Cook, Terry. "Electronic Records, Paper Minds, The Revolution in Information Management and Archives in the Post-Custodial and Post-Modernist Era," Archives and Manuscripts 22 no.2 (1994): 300-328.

Cook, Terry. "What is Past is Prologue: A History of Archival Ideas Since 1898, and the Future Paradigm Shift," Archivaria, 43 (1997): 17-63.

Cox, Richard J. Personal Archives and a Nerw Archival Calling: Readings, Reflections and Ruminations. Duluth, MN: Litwin Books, LLC, 2008.

Craven, Louise. What are Archives?: Cultural and Theoretical Perspectives: A Reader. Farnham: Ashgate, 2008.

Cumming, Kate. "Ways of Seeing: Contextualizing the Continuum," Records Management Journal 20 no.1 (2010): 41-52.

Dourish, Paul and Genevieve Bell, "The Infrastructure of Experience and the Experience of Infrastructure: Meaning and Structure in Everyday Encounters with Space," Environment and Planning B: Planning and Design 34 no.3 (2007): 414-430.

Dourish, Paul and Genevieve Bell. Divining a Digital Future: Mess and Mythology in Ubiquitous Computing. Cambridge, MA: MIT Press, 2011.

Driscoll, Kevin. "From Punched Cards to "Big Data": A Social History of Database Populism." Communication +1, 1 (2012): 1-33.

Duranti, Luciana. "Archives as a Place," Archives and Manuscripts 24 no.2 (November 1996): 242-255.

Duranti, Luciana. "The Archival Bond," Archives and Museum Informatics 11 nos.3-4 (1997): 213-218.

Duranti, Luciana. Diplomatics: Nerw Uses for an Old Science. Lanham, MD: Scarecrow Press, 1998.

Duranti, Luciana. “From Digital Diplomatics to Digital Records Forensics," Archivaria 68 (2010): 1-12.

Duranti, Luciana, Terry Eastwood and Heather MacNeil. Preservation of the Integrity of Electronic Records (Dordrecht: Springer, 2002).

Duranti, Luciana and Heather MacNeil. "The Protection of the Integrity of Electronic Records: An Overview of the UBC-MAS Research Project," Archivaria 42 (1996): 46-67.

Edwards, Paul N. "Infrastructure and Modernity: Force, Time, and Social Organization in the History of Sociotechnical Systems." In Modernity and Technology, Thomas J. Misa, Philip Brey and Andrew Feenberg, eds. Cambridge, MA: MIT Press, 2003, pp.185-225.

Farman, Jason. Mobile Interface Theory. New York: Routledge, 2012.

Foucault, Michel. The Archaeology of Knowledge Reprint edition (September 12, 1982). New York, NY: Vintage, 2012.

Functional Requirements for Evidence in Recordkeeping. School of Information Sciences, University of Pittsburgh, 1996. Last modified February 16, 2004, http://www. archimuse.com/papers/nhpre/. 


\section{Chapter 9}

Gilliland, Anne J. Conceptualizing Twenty-first-century Archives, Chicago: Society of American Archivists, 2014.

Gilliland, Anne J. "Management of Electronic Records," Annual Review of Information Science and Technology (ARIST) 39 (2005): 219-253.

Gilliland-Swetland, Anne J. Enduring Paradigm, New Opportunities: The Value of the Archival Perspective in the Digital Environment. Washington, D.C.: Council on Library and Information Resources. 2000.

Hedstrom, Margaret. "Understanding Electronic Incunabula: A Framework for Research on Electronic Records,” American Archivist 54 no.3 (1991): 334-354.

Henry, Linda J. “Schellenberg in Cyberspace,” American Archivist 61 no. 2 (1998): 309-327.

Hurley, Chris. "Parallel Provenance: (1) What if Anything is Archival Description?" Archives and Manuscripts 33 no.1 (2005): 110-145.

Hurley, Chris. "Parallel Provenance: (2) When Something is not Related to Everything Else," Archives and Manuscripts 33 no.2 (2005): 52-91.

International Telecommunications Union, "World Telecommunication/ICT Indicators database 2015 (19th Edition/December 2015)”, ITU, Switzerland, 2015.

Kirschenbaum, Matthew G. Mechanisms: New Media and the Forensic Imagination. Cambridge, MA: MIT Press, 2008.

Kirschenbaum, Matthew, Erika L. Farr, Kari M. Kraus, Naomi Nelson, Catherine Stollar Peters, and Gabriela Redwine. Digital Materiality: Preserving Access to Computers as Complete Environments. UC Office of the President: California Digital Library, 2009, http://escholarship.org/uc/item/7d3465vg.

Latour, Bruno. "Beware, Your Imagination Leaves Digital Traces," Times Higher Literary Supplement 6 no.4 (2007): 1-3.

Latour, Bruno. Science in Action: How to Follow Scientists and Engineers through Society. New York: Harvard University Press, 1987.

Latour, Bruno, and Steve Woolgar. Laboratory Life: The Construction of Scientific Facts. Princeton, NJ: Princeton University Press, 1979.

LaValle, Steve, Eric Lesser, Rebecca Shockley, Michael S. Hopkins and Nina Kruschwitz. "Big Data, Analytics and the Path from Insights to Value," MIT Sloan Management Review 52 no.2 (2011): 21-32.

Lindtner, Silvia, Ken Anderson, and Paul Dourish. "Cultural Appropriation: Information Technologies as Sites of Transnational Imagination," Proceedings of the ACM 2012 Conference on Computer Supported Cooperative Work (2012): 77-86.

Lynch, Clifford. “Big Data: How do your Data Grow?” Nature 455, no.7209 (2008): 28-29.

Lytle, Richard H. "Intellectual Access to Archives," American Archivist 43, no.2 (1980): 191-207.

Mackenzie, Adrian. Wirelessness: Radical Empiricism in Network Cultures. Cambridge, MA: MIT Press, 2010.

MacNeil, Heather. "Trusting Records in a Postmodern World," Archivaria, 51 (2001): 36-47.

MacNeil, Heather. "Providing Grounds for Trust II: The Findings of the Authenticity Task Force of InterPARES," Archivaria 54 (2002): 24-58.

Marsden, Paul. "When is the Future? Comparative Notes on the Electronic Recordkeeping Projects of the University of Pittsburgh and the University of British Columbia," Archivaria 43 (1997): 158-173.

McKemmish, Sue. "Are Records Ever Actual?” In The Records Continuum. Ian Maclean and Australian Archives First Fifty Years, Sue McKemmish and Michael Piggott, eds. Clayton, Vic: Ancora Press in association with Australian Archives, 1994, pp.187-203. 
McKemmish, Sue. "Placing Records Continuum Theory and Practice," Archival Science 1 no.4 (2001): 333-359.

Moreau, Luc. "Usage of Provenance: A Tower of Babel Towards a Concept Map." Position paper for the Microsoft Life Cycle Seminar, Mountain View, California, July 10, 2006.

Moreau, Luc, Paul Groth, Simon Miles, Javier Vazquez-Salceda, John Ibbotson, Sheng Jiang and Steve Munroe. "The Provenance of Electronic Data," Communications of the ACM 51 no.4 (2008): 52-58.

Noah, Sherna. "Texting Overtakes Talking as Most Popular Form of Communication in UK," The Independent (July 18, 2012), http://www.independent.co.uk/news/uk/ home-news/texting-overtakes-talking-as-most-popular-form-of-communicationin-uk-7956016.html.

O'Donnell, Barbara. "Affidavit in Support of a Criminal Complaint and Arrest Warrant for Kurt Mix," Department of Justice, United States of America, April 23, 2012.

O'Shea, Greg, and David Roberts, "Living in a Digital World: Reorganizing the Electronic and Post-Custodial Realities," Archives and Manuscripts 24 no.2 (1996): 286-311.

Pavlo, Walter. "Former BP Engineer Kurt Mix Still Deleting 'Stuff,' Government Alleges," Forbes Blog, March 28, 2013, http://www.forbes.com/sites/ walterpavlo/2013/03/28/fmr-bp-engineer-kurt-mix-still-deleting-stuff-govtalledges/.

Pearce-Moses, Richard. "Janus in Cyberspace: Archives on the Threshold of the Digital Era," American Archivist 70 no.1 (2007): 13-22.

Penn, Ira A., Gail Pennix, and Jim Coulson. Records Management Handbook. Aldershot: Gower, 1994.

Pinch, Trevor J., and Wiebe E. Bijker. “The Social Construction of Facts and Artifacts: Or how the Sociology of Science and the Sociology of Technology Might Benefit Each Other." In The Social Construction of Technological Systems: Nerw Directions in the Sociology and History of Technology, Wiebe E. Bijker, Thomas P. Hughes, and Trevor J. Pinch eds. Cambridge, MA: MIT Press, 1987, pp.17-50.

Ribes, David, and Jessica Beth Polk. "Historical Ontology and Infrastructure," Proceedings of the 2012 iConference, (2012): 254-262.

Schellenberg, Theodore R. Modern Archives: Principles and Techniques. Chicago, IL: Society of American Archivists, 1996.

Shepherd, Elizabeth, and Geoffrey Yeo. Managing Records: A Handbook of Principles and Practice. London: Facet Publishing, 2003.

Star, Susan Leigh. "The Ethnography of Infrastructure," American Behavioral Scientist 43 no.3 (1999): 377-391.

Stuckey, Steve. "The Australian Archives Policy on Electronic Records.” In Playing for Keeps: The Proceedings of an Electronic Records Management Conference Hosted by the Australian Archives, Canberra, Australia: Australian Archives, 1995.

Sutter, John D. “iCloud: Revolution of the next MobileMe?," CNN Tech Blog, June 9, 2011, http:/www.cnn.com/2011/TECH/web/06/07/icloud.reaction/index.html.

Thibodeau, Kenneth. "Overview of Technological Approaches to Digital Preservation and Challenges in Coming Years." In Meg Bellinger, Laura Campbell, Margaret Hedstrom, Deanna Marcum, Kenneth Thibodeau, Donald Waters, Titia van der Werf and Colin Webb. The State of Digital Preservation: An International Perspective. Washington, D.C: Council on Library and Information Resources, 2002, pp.4-31.

Trace, Ciaran B. "Beyond the Magic to the Mechanism: Computers, Materiality, and What It Means for Records to Be 'Born Digital'," Archivaria 72 (2011): 5-27. 


\section{Chapter 9}

Upward, Frank. "In Search of the Continuum: Ian Maclean's 'Australian Experience' Essays on Recordkeeping." In The Records Continuum. Ian Maclean and Australian Archives First Fifty Years, Sue McKemmish and Michael Piggott, eds. Clayton, Vic: Ancora Press in association with Australian Archives, 1994, pp.110-130.

Upward, Frank. "Modelling the Continuum as Paradigm Shift in Recordkeeping and Archiving Processes, and Beyond a Personal Reflection," Records Management Journal 10 no.3 (2000): 115-139.

Upward, Frank. "Structuring the Records Continuum Part One: Post-custodial Principles and Properties," Archives and Manuscripts 24 no.2 (2009): 268-285.

Upward, Frank. "Structuring the Records Continuum Part Two: Structuration Theory and Recordkeeping," Archives and Manuscripts 25 no.1 (2009): 10-35.

Upward, Frank and Sue McKemmish, "Somewhere Beyond Custody," Archives and Manuscripts, 22 no.1 (1994): 136-149.

Wikipedia. “Deepwater Horizon Oil Spill," http://en.wikipedia.org/wiki/Deepwater_ Horizon_oil_spill.

Willett, Peter, and Karen Spärck-Jones, eds. Readings in Information Retrieval. San Francisco: Morgan Kaufmann, 1997.

Winner, Langdon. "Upon Opening the Black Box and Finding it Empty: Social Constructivism and the Philosophy of Technology," Science, Technology, \& Human Values 18 no.3 (1993): 362-3. 
Chapter 10

\title{
BEYOND PILLARS OF EVIDENCE
}

\section{Exploring the Shaky Ground of Queer/ed Archives and their Methodologies}

\author{
Jamie A. Lee
}

\begin{abstract}
In this chapter, I critically consider the ways in which heteronormativity, homonormativity, and the politics of respectability come together to both haunt and produce the digital narratives that constitute the Arizona Lesbian, Gay, Bisexual, Transgender, and Queer (LGBTQ) Storytelling Project, in order to discover how memories are sometimes disciplined to re-produce normative narratives about queer pasts. I look and listen for the queering potentials in shared stories and in the digital and participatory technologies that record them. While conversations about "queering the archive" are not new and are, in fact, taking place transnationally, these conversations are extended here to explore the ways in which conformity to archival norms can be treacherous. I ask whether an archive can be a space of radical intervention or if it must always and only be a repository for stories that reproduce normative iterations of histories that inform powerful and normativising national imaginaries. For those of us committed to intervening in traditional archival constructs and related practices of collecting and documenting, we can see that such practices run the risk of reproducing sexual normativities and social divisions. We should, therefore, understand the queer/ed archive as always in motion - forming and re-forming itself as we constitute and remember its collections. Ultimately, this chapter argues for the need to develop a Queer/ed Archival Methodology (Q/M), to help ensure that complex, nonnormative, and even contradictory histories have their places in society's record.
\end{abstract}


"Archives are always in a state of becoming; they are always in transition." (Gilliland, 2010) ${ }^{1}$

"Interest in notions of a single past, an unattainable but real sense of historical truth, has been displaced by a sense of past plural and of past imperfect, a past that emphasizes the 'becoming' rather than the 'became.' History, then, is a series of spaces where each individual is free to determine a past - some based on archives and some not." (Blouin, 1999) ${ }^{2}$

Over the past five years while meeting and conducting oral history interviews with lesbian, gay, bisexual, transgender and queer (LGBTQ) peoples for the Arizona LGBTQ Storytelling Project, the first oral history project and collection of its kind in the state of Arizona, I have had to take a closer and critical look at how I, too, am implicated in what I have come to understand and experience as a shared storytelling process with archival repercussions. As I work towards developing a Queer/ed Archival Methodology ${ }^{3}$ while attending to my greater project to develop the Arizona Queer Archives through the Institute for LGBT Studies at the University of Arizona, I am wrestling with three overarching questions: (1) How can archives hold normative and non-normative stories, materials, and practices together as both complementary and also contradictory without subordinating or otherwise invalidating either and so that each can still be considered worthy of our archival attention? (2) Can a Queer/ed Archival Methodology be a radical intervention into normative archival practices and structures and to what ends? And (3) What does it mean and what does it look like for queer/ed archives to be radically open spaces?

To answer these questions, I bring together a number of key terms and concepts including radical intervention, multiply-situated subjects, queer, politics of respectability, haunting, disidentification, third space, and also

1 Anne J. Gilliland, “Afterword: In and Out of the Archives," Archival Science 10 (2010), 339.

2 Francis. X. Blouin, Jr. "Archivists, Meditation, and Constructs of Social Memory," Archival Issues 24,no. 2 (1999), 110.

3 I write in sincere gratitude to Professor Ken McAllister who challenged me with new ways of thinking about archives, archival methodologies, and their unique relationship to qualitative research methods. I have learned so much through our conversations about popular/mass culture archives and the "GONZO" gut instincts that drive each of us in our own archival trajectories. Listening to our guts is certainly the force behind how we know and how we question what we know. Thank you. 
archives. I see these terms as guides to better understand the queer and presumably radical endeavour that I am pursuing and proposing. Whether it is community-based and/or institutionally-driven by professional and nonprofessional archivists, a queer intervention can be accomplished in the collection, preservation, and sharing of LGBTQ-identified communities and their collections. A radical intervention, however, goes one step further to re-imagine traditional archival principles and practices in order to better understand the particular epistemology of those archives developed for and with non-normative multiply-situated subjects. Here, I use Adela C. Licona's concept of multiply-situated subjects ${ }^{4}$ together with her third-space both/and approach to acknowledge the ways in which subjects - and their stories - can be situated as both dominant and non-dominant, normative and non-normative, proper and improper, legitimate and illegitimate. By third space, I understand her to mean not just a location, but a relational space of contestation as well as a space of shared understanding and meaningmaking. ${ }^{5}$ Third space moves me beyond the either/or to the both/and understanding of lived histories in order to see such demarcations as porous and productive. I have come to understand archives as always potentially third spaces that are contested and ambiguous through their connections to both community and institution, through their collection of contested stories and practices, as well as through the ongoing challenge to notions of "proper" and "legitimate" archival norms and practices.

Since the 1898 publication of The Manual for the Arrangement and Description of Archives (a.k.a. the Dutch Manual), the first internationally accepted articulation of the codification, standardisation, and professionalisation of archival practices, many in the archival field have been taught to see archives

4 In the past I have used the term "marginalised," but realise that its use automatically positions a subject as inside or outside while Licona's term helps me to understand the different spaces and places where we each may carry varying weight of privilege(s) based on our assembling and overlapping identity categories. Adela C. Licona, Zines in Third Space: Radical Cooperation and Borderlands Rhetoric. Albany, NY: State University New York Press, 2012, p.12.

5 Licona's work on third space theory, and specifically that on borderlands rhetorics, highlights the ways in which borders have come to mark the division between two parts; however, at this division is the overlap of the both/and. For example, in Zines in Third Space, she discusses third space theory as the generative space in which, as a young girl, her Mexican-American family made meaning of the Dick and Jane books taught in their U.S. grade schools with the naming of their dog 'Lunares,' which is translated in English as 'Spot' or 'mole.' She explores the liminality of the border and binaries in order to inquire into the politics of articulation and practices of coalition building in these spaces that are intended to separate, but, in reality, become creative and productive spaces of coming together. Licona, Zines in Third Space, 131. 
as pillars of historical evidence and the professional archivist as 'the keeper' of society's record. ${ }^{6}$ As I reviewed how the terms archive and archives were being defined in the Society of American Archivists' Glossary of Archival and Records Terminology, ${ }^{7}$ I recognised the tension between "professional" and "amateur" archivists. The Glossary in this defining instance appears to support a limiting dichotomy between the professional archivist as legitimate and the amateur or non-professional archivist as illegitimate. This dichotomy is further realised through suggestions that "the nonprofessional appropriation of the term 'archives' appears to be part of an attempt by the scholar or database builder to lend panache or cachet and an air of respectability to what otherwise might be a little more than a personal hobby or collecting fetish" (emphasis my own). However, following other academic disciplines as well as critical archival theorists - including Bastian, Cook, Flinn, Gilliland, Jimerson, Ketelaar, and McKemmish - through the postmodern and the cultural turn, archives can be understood as not static, but rather dynamic sites within a spectrum of pasts, presents, and futures. In "What is Past is Prologue: A History of Archival Ideas Since 1898, and the Future Paradigm Shift," Canadian archival theorist Terry Cook analyses the history of archival thought since the publication of the Dutch Manual, focusing on those theorists who recognised and expressed "radical changes in the nature of records, record-creating organisations, record-keeping systems, record uses, and in the wider cultural, legal, technological, social, and philosophical trends in society," as well as the impact of these changes on the collective discourses and practices of the archival profession. ${ }^{9}$ The trends that emerged

6 The concept of "society's record" comes from Jesse Shera, an influential theorist in the field of Library and Information Science. His 1972 book, The Foundations of Education for Librarianship, develops his theories based on an historical and sociological understanding of society and its variable relationship to its recorded knowledge. Shera argued that, "... the role of the librarian in society is that of a mediator between man and his graphic records." See Jesse Hauk Shera, The Foundations of Education for Librarianship. New York: Becker and Hayes, 1972, p. 193. In an earlier paper, I have argued that the librarian, archivist, and information professional are more than a mediator. Rather than a single focus on mediation, I have considered a cartographic representation to highlight the multiple pathways to information while also emphasising the active reciprocal and collaborative connections in the production and consumption of knowledges.

7 Richard Pearce-Moses, A Glossary of Archival and Records Terminology. Chicago, IL: Society of American Archivists, 2005. http://www2.archivists.org/glossary.

8 Society of American Archivists (SAA), http://www2.archivists.org/; William J. Maher, "Archives, Archivists, and Society," American Archivist 6 no.2 (Fall 1998): 252-265. Incoming presidential address delivered 30 August 1997.

9 Terry Cook, "What is Past is Prologue: A History of Archival Ideas Since 1898, and the Future Paradigm Shift," Archivaria 43 (1997): 17. 
since 1898 suggest a shift from a product- or records-focused activity to a process-oriented activity, to preserve the collective memory of nations and peoples. This shift to process exposes a liminal third space in which archivists themselves might be considered dynamic, embodied, multiply-situated subjects who move between and beyond this limiting dichotomy of professional/amateur. ${ }^{10}$ Re-thinking the constraints of a dichotomy that divides the professional and the amateur is important to queer/ed archival practices because it moves archivists and their collections beyond the proper, the legitimate, and the normative. This is especially important to those archivists who are interested in the mundane, the ordinary, and the non-normative.

As a methodology, queer must relate to the epistemological and worldmaking endeavour of archives being built specifically to represent voices and peoples that are often excluded from what is considered "proper," professional, and traditional archives. Conversations about queering the archive are not new and are, in fact, taking place transnationally. For those of us committed to critically intervening in the traditional archival constructs and practices of collecting and documenting, we can see how traditional practices can run the risk of reproducing sexual normativities and social divisions. Because consistency is important in archival practices, I argue that developing a Queer/ed Archival Methodology, Q/M, can help to ensure that even complex, contradictory, and non-normative histories have their places in society's record. Here, I explore those spaces that hold the potential for archives to house queer/ed histories, as well as the potential for visitors to the archives to engage in a queer/ed reading or listening of the digital video oral histories that constitute the Arizona Lesbian, Gay, Bisexual, Transgender, Queer (LGBTQ) Storytelling Project. I critically engage in the ways that heteronormativity, homonormativity, and the politics of respectability come together in these digital narratives to consider how memories are sometimes disciplined to produce normative narratives about queer pasts. I look and listen for the queering potentials in these stories while also being alert to the queering potentials of digital participatory technologies. In this chapter, I am considering how dominant ideology haunts LGBTQpeoples and their archival collections. Understanding the queer/ed archives, then, as always in

10 Andrew Flinn highlights the ways that a multitude of archives and archival formations might, in fact, look more alike than we might think. He suggests viewing them "as diverse expressions of a similar activity rather than emphasize the differences." Andrew Flinn, "The Impact of Independent and Community Archives on Professional Archival Thinking and Practice" in The Future of Archives and Recordkeeping: a Reader, Jennie Hill, ed. London: Facet Publishing, 2011, p.148 
motion - forming and re-forming itself as we constitute and re-member its collections - can and should be a part of the queering process, practice, and methodology.

\section{Queer Terminologies}

Conversations taking place about the social justice potential of archives, especially when archives are conceived of as fertile spaces where dominant, normative, and colonising power structures are being analysed and critiqued, can be related to queer/ed and queer/ing archival practices. I use the term "queer" as a practice of mis/recognising, critiquing, and challenging the stable notions of identity and belonging as they have become normativised. For me, queer is a way of life - an approach that pushes against socially constructed boundaries and taken-for-granted assumptions. A queer practice helps me to recognise and reside in the generative spaces between what is known and what is unknown and what is still becoming. "Queer," in its resignification and reclamation, holds the potential for radical openness and new configurations of archival practices that value change, contestation, and ambiguity as parts of an archival process of becoming. Queer is not just about sex, sexuality, and gender; it is more about the practice and politics of radically open spaces with queer/ed potentials that we have yet to know or recognise. Queer is an active part of the imagined, unstable, improper, illegitimate, ambiguous, contradictory, and unknown - all important parts of becoming. Such a conceptualisation of queer may begin with constructions of identity but moves also toward notions of un/belonging.

\section{Queer as Identity Marker}

As I use the term, the concept and theory of "queer" must first relate to the non-normative identities of lesbian, gay, bisexual, transgender. It must also relate to my project, which is situated in the development of the Arizona Queer Archives and making accessible the Arizona LGBTQ Storytelling Project, a digital video oral history collection with one hundred hours of interviews collected since 2008. I consciously steer away, however, from using the term as a catchall category or umbrella term that subsequently subsumes and assimilates all difference across contexts.

In an oral history interview that I conducted for the Arizona LGBTQ Storytelling Project, Peter, an 83-year-old gay man who grew up in Ireland in the 1930s, expressed his own transformation in coming to understand 
his same-sex desires without having the words to name it. Years after his first same-sex experience he learned of the term "homosexual," but because he had been offended by the derogatory name-calling of "queer" and "fag," he expressed disappointment in the naming of the archives. In a letter to the Institute for LGBT Studies dated 14 November 2012, he explained his disappointment in the use of the word "queer":

I have read extensive academic and intelligent essays on its origin and usage in the homosexual world; I'm aware that it surfaced in the ACT-UP era and that it was a defiant attempt to appropriate and use extensively in our own descriptions and discourses a commonly used slur and thereby to drown it or 'de-fang' it. However successful it has been, the success has come in my opinion only in already enlightened circles, and nothing has convinced me that it ever will be other than one of the many degrading descriptions used by bigoted and ignorant members of society, to separate and 'ghettoize' us as outcasts.

Peter's letter is a reminder of the history of "queer" as complex. It has denoted, and for some continues to denote the abnormal, perverse, and sick. "Queer" has impacted, harmed, and marked non-normative individuals; it has, however, as Peter notes above, been re-signified in meaningful ways. Like the bodies and the heterogeneous communities that they marked, these terminologies are still today becoming, shifting, and changing through space and time.

I move forward in my efforts to develop a $\mathrm{Q} / \mathrm{M}$ aware of the history of "queer" as pejorative labelling for peoples who did not fit normative ideals. This sort of labelling established rigid social categories with acceptable norms and helped further develop biopolitical state control. However, "queer" for me holds the promise of the not-yet-known or knowable and, therefore, of an unrealised potential. I use "queer" as my own self-identifier as I consider it to hold the fluidity and dynamic potential of my own desires, sexuality, and gender as a self-aware archivist interested in non-normative collecting and collections. Q/M recognises the potential of archives not only to house his/ stories, counter-histories, and artefacts but also to become sites of radically re-configured histories, social subjects, relations, and understandings.

Through reading accounts of LGBTQ history outside of and beyond the monolithic gay-rights narrative which often starts with Stonewall in 1969, I have come to better understand the ways in which race, class, gender, and sexuality are always already implicated and contingent in what and who are queer/ed and in which categories. I have come to question specifically 
how and whose histories are (partially) told and (partially) visible. Elizabeth Lapovsky Kennedy and Madeline D. Davis in their ground-breaking book Boots of Leather, Slippers of Gold: The History of a Lesbian Community significantly highlight for me the ways in which the label of "queer" impacted the newly visible lesbian community in Buffalo, NY from the mid-1930s to the early 1960s as they worked to find the language to talk about their everyday experiences:

A key element in the new forms of visibility was the explicit discussion of lesbianism with the heterosexual world, including the appropriation and transformation of derogatory words like 'dyke' and 'queer.' Narrators of the 1950s asserted their lesbianism through appearance alone ... The topics of lesbianism and homosexuality, and the words themselves, did not become part of common conversations until gay liberation. ${ }^{11}$

Working-class and predominantly white women, who faced derogatory and repressed understandings of non-normative gender and sexuality categories, searched for and built communities in which they could be with others like them. Throughout their book, Kennedy and Davis use quotation marks around the word queer to denote how their oral history participants working-class butch and femme lesbians - were using "queer" as a way to refer to themselves to indicate the social stigma with which they were regularly dealing and had possibly internalised. ${ }^{12}$ The language and discourse about non-normative, multiply-situated lives from within these communities continue to evolve and are temporally and spatially contingent. Moreover, naming practices are connected to power dynamics that call for critical interrogation. Gloria Anzaldúa describes her self-identification and personal experiences of living in and along a borderlands context and especially in relation to the dominant structures of white middle-class lesbians and gay men creating the language to frame the conversations about same-sex love, desires, relationships, and identities:

I think of lesbians as predominantly white and middle-class women and a segment of women of color who acquired the term through osmosis, much the same as Chicanas and Latinas assimilated the word "Hispanic." When a "lesbian" names me the same as her, she subsumes me under her category. I am of her group but not as an equal, not as a

11 Elizabeth Lapovsky Kennedy and Madeline D. Davis, Boots of Leather, Slippers of Gold: The History of a Lesbian Community, PLACE: Penguin Books, 1993, p.188.

12 Kennedy and Davis, Boots of Leather, Slippers of Gold, 7. 
whole person - my color erased, my class ignored ... "Lesbian" doesn't name anything in my homeland ... If I have to pick an identity label in the English language I pick "dyke" or "queer" ... Even as we seek shelter under "queer" we must not forget that it homogenizes, erases our difference. ${ }^{13}$

Anzaldúa illuminates the power and potential of naming and how that power can visibilise and invisibilise distinct dimensions of multiply-situated subjects.

\section{Queer as Politics and Practice}

"To queer" as a verb working on and within the archives is an act of intervention, an upsetting of the normative archival structures that continue to uphold and reproduce the hegemonic power dynamics that work to exclude rather than include. Power works through the hands of the archivist doing "proper" work to collect, name, organise, preserve, and share. As an archivist, I am called to the inner workings of the archives to look closely at the mechanisms in place to make the work of the archivist something to manage again and again. According to queer rhetorical scholar, K.J. Rawson, a queer function, especially in relation to the histories of archival development for and with those who hold power in society, helps to elicit an engaged mode of critical inquiry ${ }^{14}$ as one that challenges interconnected mechanisms of state power. ${ }^{15}$ Using Licona's third-space approach, this queer intervention in archival notions of the "proper" and the recognition and inclusion of the "improper" as legitimate is key to further expand nonnormative access to, and legibility of, archives through considering archival characteristics and their performativity. This space between the proper/ improper, the legible/illegible, is a generative space within the spectrum of professional/non-professional in which archives and their collections can be read and understood as "real" or "official" archives.

With the Arizona LGBTQ Storytelling Project in mind, queer/ed approaches may also bring into question notions of truth, evidence, and

13 Gloria Anzaldúa, “To(o) Queer the Writer - Loca, escritora y chicana.” In Living Chicana Theory, Carla Trujillo, ed. Berkeley, CA: Third Woman Press, 1998, pp.263-264.

14 K.J. Rawson, "Archiving Transgender: Affects, Logics, and the Power of Queer History," PhD diss. Syracuse, 2010, p.3.

15 Rawson, “Archiving Transgender", 70. 
authenticity. ${ }^{16}$ This makes developing a queer/ed methodology particularly vexing. Challenging assumptions and questioning what has been taken for granted as "natural" or "normal" and "truthful" must necessarily carry into archival methodologies. The archives, then, is not interested in deterministic temporality as a positivist progress narrative moving through histories of discrimination and civil rights movements, but more focused on the cyclical movements in which some interconnected parts are visible and others are hidden while still always working and at play. In her presentation titled "Jumping Generations: Back to the LGBT Futures in the Feminist Archive, ${ }^{17}$ feminist theorist and Media and Culture scholar, Iris van der Tuin, highlighted the ways that a feminist epistemology helps to shift away from a waves metaphor with its focus only on the waves above the water. She suggests looking at the entire process of wave development to see its cyclical movement within a larger assemblage where many waves all perform together without an end result in mind. ${ }^{18}$ With a critical eye towards eliminating the tendency for essentialism and the reflection of universality, ${ }^{19}$ a $\mathrm{Q} / \mathrm{M}$ can offer creative ways to consider how personal collections might relate and refer to one another without the need for assimilation into one tidy narrative. Cook suggests that arrangement within the archives must be understood as "not so much as a method for organizing records, but as an intellectual construct created through the archivist's analysis of the numerous relationships that exist between records, creators, and functions." ${ }^{20}$ I would add "users" to Cook's list of relational players. It is through the everyday experts and the

16 Gayatri Spivak raises concerns about the archives being considered a repository of facts and the urgency to enter the archives with the intention to "read" them as representations and constructed histories. She argues "this is 'literature' in the general sense - the archives selectively preserving the changeover of the episteme - as its condition' with 'literature in the narrow sense - all the genres - as its effect." Gayatri Spivak, A Critique of Postcolonial Reason: Toward a History of the Vanishing Present. Cambridge, MA: Harvard University Press, 1999, p.203.

17 Iris van der Tuin, "Jumping Generations: Back to the LGBT Futures in the Feminist Archive.” Presentation given as part of the Queer Connections Speaker Series through the Institute of LGBT Studies at the University of Arizona, 21 March 2013.

18 For an of-colour critique of the waves metaphor, see Krista Jacob and Adela C. Licona, "Writing the Waves: A Dialogue on the Tools, Tactics, and Tensions of Feminisms and Feminist Practices over Time and Place," NWSA Journal, 17 no. 1 (Spring, 2005): 197-205.

19 For further reading on the elevation of the white male experience (gay or straight) as that which is considered universal and a generality when compared to bodies of colour, see also Judith Halberstam, In a Queer Time E Place: Transgender Bodies, Subcultural Lives. New York \& London: New York University Press, 2005, p.4.

20 Terry Cook, "What is Past is Prologue: A History of Archival Ideas Since 1898, and the Future Paradigm Shift," Archivaria 43 (1997): 31. 
participation of the community members that these relationships come alive in the archives. The archives developed through $\mathrm{Q} / \mathrm{M}$ are not necessarily interested in unity, but spaces for many voices, many knowledges and are committed to what rhetorical game scholar Ken McAllister would consider preservation through use. ${ }^{21}$ In other words, the ongoing interactions in and with archives and their collections holds a queering potential.

\section{Queering the Archive}

As an archivist and steward of queer and LGBTQ-identified individual and collective memories, I am aware that conformity to archival norms may be necessary, but I am always aware that these norms can also be treacherous. Archival narratives may be or become dominant narratives. Such dominant narratives can function to sustain what Lauren Berlant, in The Queen of America Goes to Washington City: Essays on Sex and Citizenship, considers the "National Symbolic" and further defines as "the archive" of stereotypical images ${ }^{22}$ such as those aesthetic and discursive 'national' objects that work to provide an affective intimacy among citizens. Berlant suggests that, "the collective possession of these official texts ... creates a national 'public' that constantly renounces political knowledge where it exceeds intimate mythic national codes." 23 Belonging to and longing for this national symbolic, therefore, illuminates what it means to be good citizens while archival practices, namely selection and appraisal, might quietly extend the normativising power of liberalism on the archives through holding those materials which tell of a normative life while dismissing or subordinating non-normative materials. Liberalism and this sedimentation of the national symbolic into identities - individual and collective - often works against non-normative, multiply-situated subjects by offering a specific hope for inclusion in society and along with it, society's

21 Visit the website for the Learning Games Initiative Research Archive (LGIRA) to learn more about McAllister and Ruggill's concept of preservation through use as they offer the only circulating video game archive in the world: http://lgira.mesmernet.org/.

22 Berlant calls on Homi Bhabha's argument from "The Other Question" that "the stereotype is an essential mental ligament of modern national culture, as the common possession of aesthetic and discursive 'national' objects provides an affective intimacy among citizens that no commonly memorised political genealogy or mass experience of democracy has yet successfully effected." The national stereotype is a hybrid form, a form of feeling, of alienation, and of sociality while also becoming varying degrees of control between the normative and non-normative subjects as well as between nonnormative multiply-situated subjects themselves working much like the politics of respectability. Lauren Berlant, The Queen of America Goes to Washington City: Essays on Sex and Citizenship. Durham \& London: Duke University Press, 1997, p.103. 
archives, but requiring continued subjugation in the injured states. ${ }^{24}$ The national symbolic offers one dominant narrative that continues to tell the story of power and domination in order to uphold this biopolitical state control.

With these interests and concerns in mind, I have been developing a $\mathrm{Q} / \mathrm{M}$, which I see as a framework that ought to be read as a queer, feminist, participatory, and, potentially decolonial guide for archivists - professional and non-professional - to be self-aware, self-reflective, as well as critical throughout the process of developing archives for, with, and about multiplysituated non-normative subjects. As a critical practice, archivists moving through queer/ed archival development are encouraged to take notes and document their own struggles, processes, and successes since even day-to-day archival labor has a place in later theorising the hands-on methods that may make up each new iteration of a $\mathrm{Q} / \mathrm{M}$. As a framework, this methodology assists archivists in creating general knowledge about how an archive works with and for the communities it portends to represent.

With a participatory ethic as its core value, this methodology is designed to focus on cultural archives that are delicately entangled with peoples and peoples' lives. Following Eve Tuck's work, a participatory ethic is "best described as a set of beliefs about knowledge, where it comes from and how knowledge is validated and strengthened ... to return to the people the legitimacy of the knowledge they are capable of producing through their own verification systems ... as a guide to their own action. ${ }^{25}$ In other words, everyday stories - and perhaps everyday archivists - have a place in queer/ed archives and in their productions.

\section{Haunting the Archives and the Ghostly Matter Therein}

Now I turn to the issues to which archivists must attend when developing archives with and for non-normative multiply-situated subjects. Considering a $\mathrm{Q} / \mathrm{M}$, not only should archivists look at their archival collections, they should also look into the entrenched archival norms and the ways that these norms might be re-imagined. A Q/M should question the spaces that are privileged and also those that are subordinated, obscured, or effectively

24 See Wendy Brown's States of Injury: Power and Freedom in Late Modernity. Princeton, NJ: Princeton University Press, 1995.

25 Eve Tuck, Urban Youth and School Pushout: Gaterways, Get-aways, and the GED. New York: Routledge, 2011, p.4. 
silenced. As LGBTQ historians Bronski, Eaklor, and Stryker ${ }^{26}$ have noted, controlling bodies through cultivating good traits while eliminating the bad is part of the state project to normalise populations. Through bio-political strategies, bodies and their non-normative desires and identities continue to exist, but they are disciplined and managed, and such disciplining is implicated in the histories that we do and do not tell, do and, therefore, do not preserve for their enduring value. Shame, guilt, fear, and other self-effacing emotions have also permeated the process of telling by those in LGBTQ communities.

In order to help me understand the production and methodologies of queer archives and the queering of archival practices, I use the concept of politics of respectability ${ }^{27}$ that Deborah Gould sets out in Moving Politics: Emotion and ACT UP's Fight Against AIDS. Gould defines politics of respectability as "concerned above all with social acceptance, while it entails efforts of some members of a marginalized group both to disprove dominant stereotypes about the group and to regulate and 'improve' the behaviour of its members in line with socially approved norms." ${ }^{\text {28 }}$ Generally, these socially approved norms are heteronormative, which Lauren Berlant and Michael Warner define as:

the institutions, structures of understanding, and practical orientations that make heterosexuality seem not only coherent - that is, organized as a sexuality - but also privileged. Its coherence is always provisional, and its privilege can take several (sometimes contradictory) forms: unmarked, as the basic idiom of the personal and the social; or marked, as a natural state; or projected as an ideal or moral accomplishment. ${ }^{29}$

26 Michael Bronski, A Queer History of the United States. Boston: Beacon Press, 2011; Vicki L. Eaklor, Queer America: A People's GLBT History of the United States. New York: The New Press, 2008; Susan Stryker, Transgender History. Berkeley, CA: Seal Press, 2008.

27 Evelyn Brooks Higginbotham first coined the term "politics of respectability" to describe the work of the Women's Convention of the Black Baptist Church during the Progressive Era. Higginbotham specifically referred to African American's promotion of temperance, cleanliness of person and property, thrift, polite manners, and sexual purity. Righteous Discontent: The Women's Movement in the Black Baptist Church, 1880-1920. Cambridge, MA: Harvard University Press, 1994.

28 Deborah B. Gould, Moving Politics: Emotion and ACT UP's Fight Against AIDS. Chicago: University of Chicago Press, 2009, p.89.

29 Lauren Berlant and Michael Warner, “Sex in Public," Queer Studies: An Interdisciplinary Reader, Robert J. Corber and Stephen Valocchi, eds. Malden, MA: Blackwell Publishing, 2003, pp.179-80. 
Queer/ed archives, then, must address and complicate the enduring force of such politics ${ }^{30}$ through practices that open the archives to the potentiality of new narratives and re-imagined ways of living, being and knowing.

As an archivist interested in queer/ed approaches, I need consciously to recognise the force and function of the politics of respectability that are at play in selective remembering and forgetting. I draw from Avery F. Gordon's book, Ghostly Matters: Haunting and the Sociological Imagination, and her concept of haunting to engage critically with select oral history interviews from the Arizona LGBTQ Storytelling Project. I do this in order to consider those in-between spaces where ghostly matter linger within remembered (re)constructions, as each of us, when narrating our lives, selects and deselects what we feel needs to be remembered and what needs to be forgotten. Aspirations to normalcy are normativising strategies that regulate stories, bodies, desires, and the spaces through which we make meaning. One strategy for such a recognition is to be attuned to complicated ways of being, knowing and living in order to create a space of access that can be creative, ambivalent, fearful, hopeful, and even incongruent.

Archives have many categories that bodies are intended to neatly fall into. Following Gilliland's notion that "archives are always in a state of becoming,"31 I suggest that, like Licona's idea of "bodies so far," ${ }^{32}$ the bodies

30 During the 1950s and 1960s, the politics of respectability and of heteronormativity were at play in the development and growth of early homophile organisations such as The Mattachine Society and the Daughters of Bilitis. As Boyd highlights, a tension existed between bar owners/bar goers and the homophile activists because the former were concerned with securing the right to public space for lesbian and gay people while the latter were concerned with acceptance and especially assimilation. Although they shared the public space of bars and found mutual interests empowering, the homophile activist organisations in San Francisco were working towards an "assimilationist project of social uplift, using language of integration and often time expressing disdain for queer and gender-transgressive qualities of bar-based communities." Boyd, Nan Alamilla, Wide Open Town: a History of Queer San Francisco to 1965, University of California Press, 2003, p.162. Gay and lesbian visibility at this time produced high levels of fear and stress for those who were visible as well as those who were not. One of the main concerns for the Daughters of Bilitis was how they each would build their own self-esteem and self-worth while trying to build a community. These homophile activists worked to project respectable public images of lesbians and gay men and at times turned their backs on the diversity of the lesbian and gay community in order to be accepted into the mainstream.

31 Anne Gilliland, “Afterword: In and Out of the Archives," Archival Science 10 (2010): 339.

32 Adela C. Licona's keynote address titled " $M i$ ' ja, just say you're a feminist like you used to...": Paltrolling E Performing Queer Rhetorics in the Everyday at the Queering Spaces/ Queering Borders Queer Studies Conference at University of North Carolina-Asheville, April 2013. 
constituting the archives are also always in states of becoming. Bodies too have markings and categories that hold, contain and constrain them. In “To(o) Queer the Writer, Gloria Anzaldúa argues that:

Marking is always 'marking down.' While I advocate putting Chicana, tejana, working-class, dyke-feminist poet, writer-theorist in front of my name, I do so for reasons different than those of the dominant culture. Their reasons are to marginalize, confine, and contain. My labeling of myself is so that the Chicana and lesbian and all the other persons in me don't get erased, omitted, or killed. Naming is how I make my presence known, how I assert who and what I am and want to be known as. Naming myself is a survival tactic. ${ }^{33}$

The available critical vocabularies and categories fail to hold each of us while also adequately communicate the complexity and intricacies of the tension between oppression and subjectivity. Multiply-situated subjects are implicated in many ways. In "Moving Locations: The Politics of Identities in Motion," Aimee Carrillo Rowe and Adela C. Licona express the urgency to see identities as non-static in order to open up the potential for "motion, expansiveness, and the sensation of being compelled forward." ${ }^{4}$ Their urgency targets the feminist scholars and activists whose static identities have served as the unexamined foundation of the feminist alliance base that, as I posited earlier when discussing utilising entrenched traditional archival practices without critical interrogation, risks reproducing the same exclusions that I seek to remedy. Identity is constructed again and again over time and across space. Relational practices are re-envisioned and re-constructed revealing spaces of the in-between where coalitional subjectivity can flourish and where ghostly matter may haunt and linger, thus challenging the ways in which meaning is made in and from these spaces.

Haunting, according to Gordon, "is an animated state in which a repressed or unresolved social violence is making itself known, sometimes very directly, sometimes more obliquely." ${ }^{35}$ Haunting can exist in the archives. Utilising the oral history interviews as primary sites of inquiry, I work to identify such hauntings in the narratives, cadences, pauses, and gaps in our storytelling.

33 Gloria Anzaldúa, “To(o) Queer the Writer - Loca, escritora y chicana.” In Living Chicana Theory, Carla Trujillo, ed. Berkeley, CA: Third Woman Press, 1998, p.264.

34 Aimee Carrillo Rowe and Adela C. Licona, "Moving Locations: The Politics of Identities in Motion," NWSA Journal 17, no. 2 (2005): 11.

35 Avery F. Gordon, Ghostly Matters: Haunting and the Sociological Imagination. Mankato: University of Minnesota Press, 1997, p.xvi. 
Focusing on the dynamic potential is important in queer archival production, and, therefore, I have come to understand haunting as a kind of relationship that we enter into - an experience, a tentative connection perhaps. Jasbir Puar draws from the concept of Gordon's haunting throughout Terrorist Assemblages: Homonationalism in Queer Times, relating it to the smoothing out of the binary between past and present and arguing that "the becomingfuture is haunting us." ${ }^{36}$

I use Puar's concepts of snapshots, flashpoints, and hauntings to consider critically the hauntings of normativities and the politics of respectability as they come together in stories, collections, and archives. I am most interested in how memories can be disciplined to produce normative narratives about queer pasts. Puar asks, "What does it mean to be examining, absorbing, feeling, reflecting on, and writing about the archives as it (the archives) is being produced, rushing at us - literally, to entertain an unfolding archive?"37 Considering Puar's concepts of queer temporalities in which snapshots, flashpoints, and hauntings exist, her collapsible frames might upend the constructs of time. At the same time, they speak to Licona's elimination of binaries as a more generative both/and approach capable of re-imagining archival constructs as they relate to greater historical contexts and the play between individual and collective memories.

Within the Storytelling Project and from a third-space approach, the oral history interview is the snapshot - the glimpse of that moment - in which history is both being made and vanishing. The archive is a collection of snapshots still always becoming. To interrogate the temporality of the instant and the image, I pull from the film/video discourse of fastforwarding, rewinding, pausing, and slow motion to move frame-by-frame without the moment being strictly tied to a past, present, and future. For me, it's the freezing of the frame, the racking of the focus, and the zooming out in order to make visible what is obscured within the frame - it is what is in the margins, the background, slightly out of focus in the foreground, and even on the cutting room floor. The frame is a snapshot - a seemingly static yet fleeting image of investigation open for that moment's meaning making. Throughout this becoming-time, stories, peoples and archives are shaped and re-shaped. Individual and collective identities and memories are always in motion, development, process and negotiation.

\footnotetext{
36 Jasbir Puar, Terrorist Assemblages: Homonationalism in Queer Times. Durham, NC: Duke University Press, 2007, p.xx.
}

37 Puar, Terrorist Assemblages, xix. 
The flashpoint is in motion. Many frames are moving and building to a critical mass. Here is the place where change can occur and can be understood as a relational opportunity between the narrator, the archivist and the visitor to the archive. When one exists within the collapsed frames, one is open to this potential transformation. Theorist David Kazanjian builds on Walter Benjamin's thoughts on memory and history:

In relation to flashes, aufblitz, "flashpoints," what he defines as "burst[ing] into action and being, not out of nothing, but transformed from one form to another, and ... the powerful effects of that transformation or emergence." Flashpoints signal a procedural becoming-time, a centripetal turbulence of illumination so powerful that it may blind the past even as it spotlights the present and lights up the future..$^{38}$

As a relational, inter-relational, and intra-relational focus of how identities and archives are in motion within dynamic categories, I use Puar's deliberate move from intersectionality ${ }^{39}$ - which "presumes that components such as race, class, gender, sexuality, nation, age, and religion are separable analytics" ${ }^{\prime 40}$ and can thus be pulled apart - to assemblage - an affective hodgepodge that recognises other contingencies of belonging that might not fall so easily into what is sometimes denoted as identity politics. The assemblage, as a series of dispersed but mutually implicated and messy networks, draws together enunciation and dissolution, causality and effect, and organic and nonorganic forces. ${ }^{41}$ The archive, then, has the potential to hold many pasts, many futures, and transformations. Considering assemblage as adapted to entangled forces that merge and dissipate time, space and body against linearity, coherency and longevity, a $\mathrm{Q} / \mathrm{M}$ can presume that categories played out in the everyday are always dynamic and can be understood similarly to our shifting identities. As Carrillo Rowe and Licona argue:

We may not be the "same" person in different geographical contexts; what an "identity" means may shift from place to place, and the communities that define us are apt to shift over time. What it means, for instance, to be "queer" varies from bar to bedroom to workplace; what it means to be

38 Puar, Terrorist Assemblages, xviii.

39 Kimberlé Crenshaw, "Demarginalizing the Intersection of Race and Sex: A Black Feminist Critique of Antidiscrimination Doctrine, Feminist Theory and Antiracist Politics," University of Chicago Legal Forum (1989): 139-167.

40 Puar, Terrorist Assemblages, p. 212.

41 Puar, Terrorist Assemblages, p. 211. 
"American" shifts from India to the United States to Mexico; racialized, gendered, and classed identities may shift from ghetto to boardroom to gated community to classroom. Such locational meanings are contingent upon the communities to which we belong. The meanings we assign to any given identity category emerge through the relational practices in which those categories get played out in our daily lives. ${ }^{42}$

If bodies can be considered stories so far, always becoming and multiplysituated, queer/ed archives and archival practices might offer important sites and ways to build alliances across power differentials as well as strategies that "empower all of us to recognize, but not be incapacitated by, our own sets of privilege." ${ }^{\text {"3 }}$ This dynamic potential makes it urgent to keep the archives flexible and transformable.

Haunting is the lingering presence. These temporal expressions of becoming help to indicate how archivists might frame and re-frame community stories and artefacts in order to understand better human complexities. As Puar notes, "Haunting is a very particular way of knowing what has happened or is happening ... a transformative recognition." ${ }^{.44}$ Utilising the principles of social justice media, oral history methods and a participatory ethic as a core value, in my efforts to first imagine and then develop a $\mathrm{Q} / \mathrm{M}, \mathrm{I}$ am moved by this notion of transformative recognition. The realisation of haunting and being haunted, like affect, can lead one to an ongoing bodily inventory-taking of coming into contact and interacting with the world. Can these important connections and intimate entanglements produce new knowledges that the archives can capture and make visible and knowable? If so, how?

\section{What Haunts the Archives?}

"Since variants desire to be accepted by society, it behooves them to assume community responsibility ... For only as they make positive contributions to the general welfare can they expect acceptance and full assimilation into the communities in which they live." (Mattachine Society, 1956$)^{45}$

42 Aimee Carrillo Rowe and Adela C. Licona, "Moving Locations: The Politics of Identities in Motion,” NWSA Journal 17, no. 2 (2005): 12.

43 Rowe and Licona, "Moving Locations", 12.

44 Puar, Terrorist Assemblages, p. xx.

45 Mattachine Society, "Mattachine Review" 2, no. 1 (January 1956); Nan Alamilla Boyd, Wide Open Town, University of California Press, 2003, p. 159. 
"Respectability, on a straight society's terms, was the price for admission." (Gould, 2009) ${ }^{46}$

Some contributors to the Arizona LGBTQ Storytelling Project appear to want to demonstrate how normal they are and even speak about being "the best they can be." I have come to understand this storytelling practice better through the politics of respectability together with Lisa Duggan's concept of the new homonormativity - "a politics that does not contest dominant heteronormative assumptions and institutions, but upholds and sustains them, while promising the possibility of a demobilized gay constituency and a privatized, depoliticized gay culture anchored in domesticity and consumption"47 - which goes hand-in-hand with invitations to "good citizenship" and the US nation-state formation. In this push for normalcy, there becomes an even narrower threshold for alternative non-dominant national ideals. These narrow spaces and what's inside them haunt LGBTQ communities through practices of self-regulation, which further challenge the notions of authenticity and truth, but still highlight for me the potential of a $\mathrm{Q} / \mathrm{M}$ to hold contested and contradictory stories as truths. Below, Eve and Les both mention "marriage" in their oral history interviews, but express very different perspectives on relational practices.

"We have not had a ceremony. We have talked about having a ceremony once we have a child and doing kind of a family ceremony. We're - we've both been married. Neither of us are interested necessarily in getting married again, although I think we've changed a little over time. At this point, we're not interested in having a ceremony in the state of Arizona. If we want to get married, then - and we're also not interested in going to a state, getting married there, and coming back to Arizona. I think that if we, um, I think that if things don't change in the state of Arizona within the next three to five years, then we probably will leave and move to a place that recognizes samesex couples as real human beings."

Eve (age 39) interview Arizona LGBTQ Storytelling Project 30th September, 2009

46 Gould. Moving Politics, p. 89.

47 Lisa Duggan, The Twilight of Equality? Neoliberalism, Cultural Politics, and the Attack on Democracy. Boston, MA: Beacon Press, 2003, p.50. 
"Gordon and I have been together twenty years. I mean, how more married can you be? Juan has been with us now for four years. Um, and the same would be true for Juan. He is a very kind, gentle, loving person. It works for us and it works very well. If you saw our master bedroom, I mean, we have probably the biggest bed you've ever seen because it's a bed for three."

Les (age 60-something) interview Arizona LGBTQ Storytelling Project 10th April, 2010

As you can see in Eve's oral history interview excerpt, she and her partner have been contemplating marriage, but feel thwarted by the anti-gay legislation in the state of Arizona. She runs through a number of options since some states offer legal same-sex marriage. Her final sentence spells out the haunting of the dehumanising bio-political strategies that have been regulating and, as Scott Lauria Morgensen argues, "terrorizing" LGBTQ bodies since the late $19^{\text {th }}$ century. ${ }^{48}$ Being recognised as a "real human being" is important to Eve as she feels like a second-class citizen without the legal right to marry. The haunting here shows traces of the liberal respectability and rights-based movement that started in the 1970s but also has roots in the earlier ideas of assimilation occurring during the development of the homophile movement of the 1950s and 1960s. Today, this aspiration for normalised civil rights has become deeply embedded in our LGBTQ lives and these desires for acceptance have been inculcated so that many from these communities embody homonormativity. In creating this desire for "gay equality" and the subsequent LGBTQ communities' investment into this civil rights agenda, the gay public sphere is contained and becomes, therefore, manageable by the dominant mainstream publics and capitalist enterprise. ${ }^{49}$ Through exercising a gay moralism, our own LGBTQ communities selfregulate and attack the non-normativities that exist and are visible within our own groups.

In his interview, Les explains his three-man relational configuration and asks, "how more married can we be?" The Arizona law stating that marriage

48 Scott Lauria Morgensen, "Settler Homonationalism: Theorizing Settler Colonialism within Queer Modernities," in GLQ: A Journal of Lesbian and Gay Studies special edition on Sexuality, Nationality, Indigeneity, Daniel Heath Justice, Mark Rifkin, and Bethany Schneider, eds. 16 nos.1-2 (2010): 105.

49 See Jasbir Puar, "Circuits of Queer Mobility: Tourism, Travel, and Globalization," GLQ: A Journal of Lesbian and Gay Studies 8 nos.1-2 (2002): 101-137; and Rosemary Hennessy, Profit and Pleasure: Sexual Identities in Late Capitalism. New York: Routledge, 2000, and "Queer Visibility in Commodity Culture," Cultural Critique, 29 (1994): 31-76. 
is defined as only between one man and one woman hangs in the air. During the full interview the interviewer asks Les how he negotiates a three-man relationship in terms of "jealousy." Here, the interviewer's questions and the exchange are also informed and haunted by heteronormativity and its focus on monogamy. What is non-normative to the interviewer is revealed as normative to the interviewee. This haunting can only be recovered because the recorded dialogue and its verbatim transcript in its entirety is part of the queer/ed archives. At times, there seems to be a missing public narrative within which to frame how this non-normative relational knowledge is further understood, interpreted and translated. For the archives, this story of queer/ed relational practices may open up some visitors to recognising that, as Berlant and Warner assert, "queer culture constitutes itself in many ways other than through the official publics of opinion culture and the state, or through the privatized forms normally associated with sexuality." ${ }^{\prime 0}$ Les, Gordon and Juan and how they together define their relationship require a space in the queer/ed archives alongside Eve and her partner who await legal marriage opportunities to symbolise their belonging. The queer/ed archives benefits from such differing desires and experiences.

\section{Conjuring the Queer Archives}

The role of the archivist can no longer be thought of as that of the neutral mediator, but instead as one that is subjective in the shaping of archival collections. Being conscious of our roles as shapers, there must be a system of accountability as "an ethical mandate that urges people to act responsibly by being in constant conversation and contact with others (including otherthan-human ancestors and land)." ${ }^{51}$ In contrast with the Jenkinsonian notions of "official" being the "truth," Gilliland points to re-imagining lived knowledges as truth and argues, with reference to community archives that, "the defining characteristic [of community archives] is the active participation of a community in documenting and making accessible the history of their particular group and/or locality on their own terms." ${ }^{2}$ This is much like the decolonising research methodologies laid out by

50 Lauren Berlant and Michael Warner, “Sex in Public." In Queer Studies: An Interdisciplinary Reader, Robert J. Corber and Stephen Valocchi, eds. Malden, MA: Blackwell Publishing, 2003, p.175.

51 Kimberly Christen, "Access and Accountability: The Ecology of Information Sharing in the Digital Age," Anthropology News (2009): 4.

52 Gilliland, “Afterword," 339. 
feminist scholars such as Linda Tuhiwai Smith and Mary Louise Pratt. As an archivist committed to a $\mathrm{Q} / \mathrm{M}$, therefore, I consider the queer/ed archives as spaces of counter publics - many voices and lived knowledges that inform and envision adopting a queerness that "transcends particular identity categories like gay and lesbian and instead analyzes struggles over categories of sexual normativity and dissidence, to produce a history that encompasses a variety of historical actors. ${ }^{53}$ These complex, contested and contradictory LGBTQ histories serve as narratives and counter narratives that may fall into Berlant's "National Symbolic." Alternately, they might subvert the nationalist ideologies altogether by insisting on re-imagining the non-normative spaces as generative and coalition-building in order to explore further and produce creative spaces for new collective and social imaginaries, or else land somewhere in between or shift over time. There is value in how stories and counter stories produce counter publics and new ways to understand communities and selves as lived histories might congeal and adhere meaningfully in multiple ways in archival contexts.

Horacio Roque Ramírez's article, "Gay Latino Histories/Dying to Be Remembered: AIDS Obituaries, Public Memory, and the Queer Latino Archive," informs our ability to see how bodies are further regulated in and out of the archives. To find and imagine an archives or collection of historical traces in spaces that have often been overlooked ties into Gordon's use of the verb to conjure as a "particular form of calling up and calling out the forces that make things what they are in order to fix and transform a troubling situation. ${ }^{54}$ As a method of uncovering these lost and missing pieces of history, Roque Ramírez carries on this verb usage to explain the potentials within the queer archives:

To conjure the practice of queer archives opens up exciting epistemological possibilities, such as queering the Latina archive or racializing the queer archive. Also, however, queer archiving practices stir a host of theoretical debates, with empirical claims for historical knowledge production receiving postmodern critiques of the hegemonic, essentialist, and exclusionary practices in history writing, museum collections, and archival repositories. Simply put, some bodies and their representations - white, male, middle-class, heterosexual, and

53 Kevin P. Murphy, "Gay Was Good: Progress, Homonormativity, and Oral History." In Queer Twin Cities: Twin Cities GLBT Oral History Project. Mankato: University of Minnesota Press, 2010, p.307.

54 Gordon, Ghostly Matters, p.22. 
Anglo - have been much more present than all others in the official halls, drawers, and pages of 'evidence.' Yet despite these critiques of what counts as history, evidence, and archival importance, there have also been activists and academic movements for recognizing precisely the missing, neglected, and largely undocumented cultures, bodies, and histories of entire communities, usually within the same logics of historical rendition and archival practices. ${ }^{55}$

Even within a queer paradigm and the embodied push towards difference or sameness, this regulation of bodies-of-colour is problematic, leading me to question the possibilities and practices of developing an archive that is queer/ ed and radically open. Andrea Smith suggests that "queer culture and queer politics do not obey the member/nonmember logics of race and gender." Moreover, in his introduction to Fear of a Queer Planet: Queer Politics and Social Theory, Michael Warner further critiques the push for queer to be the umbrella term that holds all non-normative subjects by suggesting that "if queerness is dominated by whiteness, then it follows a logic of belonging and non-belonging. It also relies on a shared culture - one based on white supremacy." 57 As Hiram Perez notes, "Queer theory, when it privileges difference over sameness absolutely, colludes with institutional racism in vanishing, hence retrenching, white privilege. It serves as the magician's assistant to whiteness's disappearing act." ${ }^{15}$ Perez is arguing that - much like with multiculturalism - when we conflate all difference it becomes a sameness that is still entrenched within the hierarchies that are societally in place. Considering how working to prevent re-entrenching hierarchies has an impact upon the archives through selection, appraisal, classification and making accessible, the queer/ed archives then must deliberately create spaces for more stories along with their silences and hauntings.

55 Horacio N. Roque Ramírez, "Gay Latino Histories/Dying to be Remembered: AIDS Obituaries, Public Memory, and the Queer Latino Archive." In Beyond El Barrio: Everyday Life in Latinalo America, Gina M. Pérez, Frank Andre Guridy and Adrian Burgos, eds. New York: New York University Press, 2010, p.105.

56 Andrea Smith, "Queer Theory and Native Studies: The Heteronormativity of Settler Colonialism," in GLQ: A Journal of Lesbian and Gay Studies special edition on Sexuality, Nationality, Indigeneity, Daniel Heath Justice, Mark Rifkin, and Bethany Schneider, eds. 16 nos. 1-2 (2010): 45.

57 Michael Warner, Fear of a Queer Planet: Queer Politics and Social Theory, Michael Warner, ed. Minneapolis: University of Minnesota Press, 1993, p.xvii.

58 Hiram Perez, "You Can Have My Brown Body and Eat It, Too!" Social Text, nos. 84-85 (2005): 187. 
"I identify as gender-queer. I identify as queer. And maybe even more than those things, as a poet and a teacher. So, in terms of my gender-queer identity, for me, that's really about feeling very happy to have been born in the body that I was born in, which is female, and incredible grateful for my experience as a woman - growing up as a woman, living as a woman. And really feeling like my transition, which has just been taking testosterone, has just allowed me to foreground another part of myself without, hopefully without erasing what came before. And so, for me, I feel pretty comfortably situated in both genders, even though I know the world sees me as a guy. To me, that's a little bit funny because even when I look in the mirror, I am still a little bit surprised that folks see just a guy because of the facial hair and things like that. So, yeah, gender-queer and queer in terms of my sexuality because it has not been stable. [LAUGHTER.] And, um, I like that. It feels fluid. And that fluidity, I think, has also been a result of my transition and becoming and accepting that my own comfort in my body changes and moves in and out as well."

TC (age 30-something) interview Arizona LGBTQ Storytelling Project 29th April, 2010

"I just feel that every day when I look in the mirror, [LONG PAUSE. CRYING.] I think about when my father committed suicide, they didn't call me for three weeks. No one entered that home because they knew he had AIDS. His pets ate him. And I had to scrape him from the floor. That is not the way society should be. [CRYING.] And every day I look in the mirror, I'm like 'I'm gonna be the best damn person I can."

Jim (age 50-something) interview Arizona LGBTQ Storytelling Project 21 March 2012

The oral history interview excerpts from TC and Jim highlight for me the complexities of individual histories. More importantly for the greater queer/ed archives, they highlight distinctive stories at that moment and the nature of collective memory as always becoming. Through his transition, TC continues to return to the woman he has been without erasing this part of his identity and his history. Jim also returns to his father's death as a way to understand his present and future as a gay man filled with the pain of 
remembering. Both stories instantiate the collapsible frames of space and time as well as the necessity for adequate room within the queer/ed archives to preserve and share their stories. A $\mathrm{Q} / \mathrm{M}$ will guide the archivist in the cradling of such complex stories while also identifying, along with the users of the archive, the moments of haunting and the ghostly matter that animate these spaces. In Disidentifications: Queers of Color and the Performance of Politics, José Esteban Muñoz argues that disidentification "is the third mode of dealing with dominant ideology, one that neither opts to assimilate within such a structure, nor strictly opposes it; rather, disidentification is a strategy that works on and against dominant ideology." ${ }^{159}$ Disidentification is a tactic that recognises the shifting terrain of resistance. Importantly, the queer/ed archive can become an embodied strategy to recognise the hauntings in our memories, as well as in our telling, so that we can then look more closely at the silences to not necessarily fill them with something else, but to find the ghosts that already reside there to make meaning in these spaces.

\section{Conclusion}

A Queer/ed Archival Methodology focuses on the concept of participation to include the multiple roles of the archivist - both professional and everyday experts. A Q/M acknowledges that archivists work with their hands, head and heart to develop, collect and make meaning of heterogeneous collections from diverse perspectives of non-normative, multiply-situated peoples. Without the constraining dichotomies instructing the archivist on what is proper and improper, there is indeed mobility and, I might add, momentum within these emergent third spaces that support the messy, contradictory, overlapping, and complex lives. Carrillo Rowe and Licona suggest that because the interstitial cannot be fixed within the gaze of the white Western imaginary, it constitutes a "world that cannot, will not, be colonized." ${ }^{\prime 0}$ For queer/ed archives to become radical interventions, I follow Gordon's desire to reanimate the utopian because "radical political change will come about only when new forms of subjectivity and sociality can be forged by thinking beyond the limits of what is already comprehensible ... and that will be possible when a sense of what has been lost or of what we never had can be brought back from exile and articulated fully as a form of longing in this

59 José Esteban Muñoz, Disidentifications: Queers of Color and the Performance of Politics. Minneapolis: University of Minnesota Press, 1999, p.8.

60 Emma Pérez, The Decolonial Imaginary: Writing Chicanas into History. Bloomington, Indiana University Press, 1999, p.5. 
world." ${ }^{61}$ Here is where and when the archives can be mobile, animated, and performative while also radically open to its third space potential for both contested and contradictory histories; fears and tensions; creative and fertile explorations; and certainly a messy, generative spirit. Through the work to develop the queer/ed archives' mission and vision statements; the asset-driven community-focused collection policy; categorical keywords and processes; community and institutional relationship priorities; fundraising goals and strategies; as well as virtual and physical accessibility, attention to these hauntings and the ghostly matter gets at the potential for queerness and queer politics. In the queer/ed archives, we will find those who are, are not, or may not want to be respectable - a bed for three and a body that is both/and and so much more. Imagine what we can learn, document, remember and forget outside of the normativising impetus of the politics of respectability!

\section{References}

Anzaldúa, Gloria. “To(o) Queer the Writer - Loca, escritora y chicana.” In Living Chicana Theory, edited by Carla Trujillo (Berkeley, CA: Third Woman Press, 1998), 263-276.

Berlant, Lauren. The Queen of America Goes to Washington City: Essays on Sex and Citizenship. Durham, NC: Duke University Press, 1997.

Berlant, Lauren, and Michael Warner. “Sex in Public.” In Queer Studies: An Interdisciplinary Reader, Robert J. Corber and Stephen Valocchi, eds. Malden, MA: Blackwell Publishing, 2003, pp. 547-566.

Bhabha, Homi. "The Other Question: Stereotype, Discrimination, and the Discourse of Colonialism." The Location of Culture, 66-84. New York: Routledge, 1994.

Blouin, Francis. X., Jr. "Archivists, Meditation, and Constructs of Social Memory." Archival Issues 24, no. 2 (1999): 101-12.

Boyd, Nan Alamilla. Wide Open Town: a History of Queer San Francisco to 1965. University of California Press, 2003.

Bronski, Michael. A Queer History of the United States. Boston: Beacon Press, 2011.

Brown, Wendy. States of Injury: Power and Freedom in Late Modernity. Princeton, NJ: Princeton University Press, 1995.

Canaday, Margot. The Straight State: Sexuality and Citizenship in the Twentieth-Century America. Princeton, NJ: Princeton University Press, 2009.

Carrillo Rowe, Aimee and Adela C. Licona. "Moving Locations: The Politics of Identities in Motion," NWSA Journal 17 no. 2 (2005): 11-14.

Christen, Kimberly. "Access and Accountability: The Ecology of Information Sharing in the Digital Age." Anthropology Nerws, 2009.

Cook, Terry. "Electronic Records, Paper Minds: The Revolution in Information Management and Archives in the Postcustodial and Postmodernist Era." Archives and Manuscripts 22 (1994): 315-20.

61 Gordon, Ghostly Matters, p. xiii. 
Cook, Terry. "What is Past is Prologue: A History of Archival Ideas since 1898, and the Future Paradigm Shift." Archivaria 43 (1997): 17-63.

Cook, Terry. "Archival Science and Postmodernism: New Formulations for Old Concepts." Archival Science 1, no. 1 (2001): 3-24.

Crenshaw, Kimberlé. "Demarginalizing the Intersection of Race and Sex: A Black Feminist Critique of Antidiscrimination Doctrine, Feminist Theory and Antiracist Politics." University of Chicago Legal Forum, (1989): 139-167.

Cvetkovich, Ann. "Queer Archival Futures: Case Study Los Angeles.” In E-misférica 9.1-9.2 Marianne Hirsch and Diana Taylor, eds. On the Subject of Archives, (Summer 2012).

Duggan, Lisa. The Twilight of Equality? Neoliberalism, Cultural Politics, and the Attack on Democracy. Boston: Beacon Press, 2003.

Eaklor, Vicki L. Queer America: A People's GLBT History of the United States. New York: The New Press, 2008.

Eastwood, Terry and Heather MacNeil, eds. Currents of Archival Thinking. Santa Barbara, CA: Libraries Unlimited, 2010.

Flinn, Andrew. “The Impact of Independent and Community Archives on Professional Archival Thinking and Practice." In The Future of Archives and Recordkeeping: a Reader. Jennie Hill, ed. (London: Facet Publishing, 2011), 145-170.

Freeman, Elizabeth. Time Binds: Queer Temporalities, Queer Histories. Durham, NC: Duke University Press, 2010.

Gilliland, Anne. "Afterword: In and Out of the Archives." Archival Science 10 (2010): 333-343.

Gordon, Avery F. Ghostly Matters: Haunting and the Sociological Imagination. Mankato: University of Minnesota Press, 1997.

Gould, Deborah B. Moving Politics: Emotion and ACT UP's Fight Against AIDS. Chicago: University of Chicago Press, 2009.

Halberstam, Judith. In a Queer Time \& Place: Transgender Bodies, Subcultural Lives. New York \& London: New York University Press, 2005.

Hennessy, Rosemary. "Queer Visibility in Commodity Culture." Cultural Critique, 29 (1994): 31-76.

Hennessy, Rosemary. Profit and Pleasure: Sexual Identities in Late Capitalism. New York: Routledge, 2000.

Higginbotham, Evelyn Brooks. Righteous Discontent: The Women's Movement in the Black Baptist Church, 1880-1920. Cambridge, MA: Harvard University Press, 1994.

Jacob, Krista and Adela C. Licona. "Writing the Waves: A Dialogue on the Tools, Tactics, and Tensions of Feminisms and Feminist Practices over Time and Place." NWSA Journal, 17 no. 1 (Spring, 2005): 197-205.

Jimerson, Randall C. Archives Power: Memory, Accountability, and Social Justice. Chicago: Society of American Archivists, 2009.

Kennedy, Elizabeth Lapovsky and Madeline D. Davis. Boots of Leather, Slippers of Gold: The History of a Lesbian Community. New York: Penguin Books, 1993.

Licona, Adela C. Zines in Third Space: Radical Cooperation and Borderlands Rhetoric. Albany, NY: SUNY Press, 2012.

Morgensen, Scott Lauria, "Settler Homonationalism: Theorizing Settler Colonialism within Queer Modernities." GLQ: A Journal of Lesbian and Gay Studies, special edition on Sexuality, Nationality, Indigeneity, Daniel Heath Justice, Mark Rifkin, and Bethany Schneider, eds. 16 nos. 1-2, (2010): 105-132.

Muñoz, José Esteban. Disidentifications: Queers of Color and the Performance of Politics. Minneapolis: University of Minnesota Press, 1999. 


\section{Chapter io}

Murphy, Kevin P. "Gay Was Good: Progress, Homonormativity, and Oral History." In Queer Twin Cities: Twin Cities GLBT Oral History Project, 305-318. Mankato: University of Minnesota Press, 2010.

Pérez, Emma. The Decolonial Imaginary: Writing Chicanas into History. Bloomington, IN: Indiana University Press, 1999.

Perez, Hiram. "You Can Have My Brown Body and Eat It, Too!" Social Text, nos. 84-85, (2005): 171-91.

Puar, Jasbir K. "Circuits of Queer Mobility: Tourism, Travel, and Globalization.” GLQ: A Journal of Lesbian and Gay Studies, 8 no. 1-2, (2002): 101-137.

Puar, Jasbir K. Terrorist Assemblages: Homonationalism in Queer Times. Durham, NC: Duke University Press, 2007.

Rawson, K.J. “Archiving Transgender: Affects, Logics, and the Power of Queer History.” PhD diss. Syracuse, 2010.

Roque Ramírez, Horacio N. "Gay Latino Histories/Dying to be Remembered: AIDS Obituaries, Public Memory, and the Queer Latino Archive." In Beyond El Barrio: Everyday Life in Latinalo America, edited by Gina M. Pérez, Frank Andre Guridy, and Adrian Burgos (New York: New York University Press, 2010), 103-128.

Sandoval, Chela. Methodology of the Oppressed. Mankato: University of Minnesota Press, 2000.

Shera, Jesse Hauk. The Foundations of Education for Librarianship. New York: Becker and Hayes, 1972.

Smith, Andrea. "Queer Theory and Native Studies: The Heteronormativity of Settler Colonialism." In GLQ: A Journal of Lesbian and Gay Studies 16 no. 1-2 (2010): 42-68.

Society of American Archivists (SAA) website: http://www2.archivists.org/.

Somerville, Siobhan B. Queering The Color Line: Race and the Invention of Homosexuality in American Culture. Durham, NC: Duke University Press, 2000.

Spivak, Gayatri. A Critique of Postcolonial Reason: Toward a History of the Vanishing Present. Cambridge, MA: Harvard University Press, 1999.

Stryker, Susan. Transgender History. Berkeley, CA: Seal Press, 2008.

Tuck, Eve. Urban Youth and School Pushout: Gaterways, Get-aways, and the GED. New York: Routledge, 2011.

Tuhiwai Smith, Linda. Decolonizing Methodologies: Research and Indigenous Peoples. London: Zed Books, 1999.

Michael Warner, introduction to Fear of a Queer Planet: Queer Politics and Social Theory, Michael Warner, ed. Minneapolis, MN: University of Minnesota Press, 1993. 
Chapter 11

\title{
RACE AND CULTURE
}

\section{An Ethnic Studies Approach to Archival and Recordkeeping Research in the United States}

\author{
Kelvin L. White
}

\begin{abstract}
This paper focuses on conceptual frameworks developed in ethnic studies and contemplates what these might contribute in terms of approaching archival and recordkeeping research through an African American lens. The paper explores the epistemological lineage of ethnic studies, its emergence and evolution in the US context; defines and discusses key concepts and contexts salient to ethnic studies, including cultural environments; and comments on the relationships between ethnic studies ideas and those drawn from both traditional and postmodern archival thinking. Lastly, the paper discusses some of the considerations in using conceptual frameworks from ethnic studies in research related to archival practice and recordkeeping in the United States.
\end{abstract}

... no partial aspect of social life and no isolated phenomenon may be comprehended unless it is related to the historical whole, to the social structure conceived as global entity. ${ }^{1}$

\section{Introduction: Of Culture, Race and Racism}

One case that has been frequently used to illustrate the complexities of racial identity in the United States is that of Susie Guillory Phipps. ${ }^{2}$ Phipps was

1 Paul Connerton, How Societies Remember. New York: Cambridge University Press, 1989, p.12.

2 See, for example, Frances Frank Marcus, "Louisiana Repeals Black Blood Law," New York Times (July 5, 1983), http://www.mixedracestudies.org/wordpress/?tag=susie- 
born in 1934 and grew up on a farm in Acadia Parish, Louisiana. As a teenager, she eventually left home, married, and started a family. By 1977 she had married her second husband, Andy Phipps, who was known as an affluent shrimper and owner of a wholesale shrimping company. One day in 1977, Susie Phipps needed a copy of her birth certificate in order to apply for her passport. When she arrived at the Division of Vital Records in New Orleans, the clerk approached Phipps about an apparent discrepancy. Phipps had always identified herself and her family as being white. The clerk summoned Phipps into her office to show Phipps that she was the daughter of two black parents and that according to Louisiana law at the time of her birth, she was, in fact, black ("coloured" was the description used back then). According to various print sources, Phipps reportedly responded that she was in shock and "... was sick for three days." ${ }^{3}$ Phipps consulted with state officials to have her birth certificate changed because, according to her, the racial categorisation stated on the birth record was an obvious mistake since she had been raised and lived as a "white" woman, had given birth to several "white" children, married two white men, and most significantly, she looked white. State officials informed her that while there are laws and regulations that (with the appropriate documentation) allowed someone to annotate name changes and spellings, correct birth dates, change and/or add a parent's name, and change gender designation, there were no stipulations for changing one's race on the birth certificate unless the individual could provide evidence to the state that left no doubt that the record had been tampered with, changed, or contained erroneous information.

Phipps insisted that her racial designation shown in the birth record was a mistake. Five year later she filed a lawsuit to change the designation primarily because, despite what the record indicated, she did not identify as a black woman. After a trial involving considerable publicity and personal expense (roughly US\$40,000 in research and legal fees), the courts ruled in favour of the state. Louisiana law had historically defined a black person as one who had any traceable amount of black ancestors. In 1970 (after Jim

guillory-phipps; Michael Omi, "Racial Identity and the State: The Dilemmas of Classification," Law E Inequality XV no.1 (Winter 1997); Rachel E. Moran and Devon W. Carbado. Race Law Stories. Foundation Press, 2008; Jonathan Tilove discusses the case in the context of the Ramapough Mountain People of New Jersey, and their claims for federal recognition as a Native American tribe although they had long been described as predominantly black people of mixed race: "Of Susan Guillory Phipps and Chief Redbone: The Mutability of Race," (July 9, 1992), Newhouse News service, http://jonathantilove.com/mutability-of-race/.

3 Calvin Trillin, “American Chronicles: Black or White," New Yorker, April 14, 1986, p.62. 
$\left.\mathrm{Crow}^{4}\right)$ the law was changed and in effect, quantifiably defined a black person as anyone with 1/32 or more of black ancestry. State officials argued that there was no evidence that Phipps' records had been either tampered with or changed and that the state had not violated the law. In fact, the state demonstrated that she was $5 / 32$ black by researching her family's birth and marriage certificates, ecclesiastical records and other historical documents such as slave inventories dating as far back as the $18^{\text {th }}$ century. These records indicated that both of her parents were black (albeit light-skinned) and that her great-great-great-great grandmother, Margarita, was a slave of a French landowner's wife. After his wife had died, the latter had fathered children with Margarita. Moreover, the state informed Phipps that her first husband was also a black man. In the end, Phipps' birth certificate was not changed. She appealed to the Louisiana Supreme Court, but the court refused to hear her case. According to the State Health Department lawyer who represented the state of Louisiana in the case:

Mrs. Phipps had lost her case against the state because most of the older records attesting her racial background and other evidence indicating race had corroborated the information on her birth certificate, placed there by a midwife.

He said publicity generated by the case had brought "a surge" of requests from applicants wishing to change the description of their race on old birth records. In 1980, he said, the state stopped mentioning race on birth records except in the state's confidential files, kept for statistical purposes. ${ }^{5}$

Louisiana subsequently repealed the law, replacing it with legislation requiring a "preponderance of evidence" to change racial designation on birth records, although without specifying how factors such as self-identity, lived experience, appearance or personal/family documentation might be weighed against the designations contained in accumulated official documentation of different generations of family members.

If race is merely a social construct that has no real meaning, as some scholars suggest, then why did Phipps insist that she was a white woman? ${ }^{6}$

4 The phrase "Jim Crow" refers to the segregation laws enacted in former US Confederate States (i.e., "the South") after Reconstruction (1877). These laws, while primarily targeted at African Americans, mandated racial segregation in public places between whites and non-whites from 1877 until 1965. The Civil Rights Act of 1964 and the Voting Rights Act of 1965 overruled Jim Crow laws.

5 Marcus, "Louisiana Repeals Black Blood Law."

6 For more detailed discussion on the meanings of race, see Kwame Anthony Appiah, In My Father's House: Africa in the Philosophy of Culture. New York: Oxford University 
Why did she spend nearly US $\$ 40,000$ (equivalent to nearly US\$150,000 in today's economy) to disprove her legal blackness? Would she have responded in the same way if her birth certificate showed that she was a descendant of white European immigrants? Obviously, she was bothered by the revelation and wanted the legal and historical record to reflect her lived experiences. Phipps' example is one that illustrates both ends of the spectrum when defining race. At one end of the spectrum is the essentialist formulation. It suggests that race is something that is fixed - just as certain physiological aspects of the body or the official record, themselves often used to establish not only race but also gender, are considered by certain schools of thought to be fixed rather than flexible or constantly "becoming." it demonstrates that race is not merely a social construct or an illusion that should be void of meaning and might beneficially be eliminated. Rather, it illustrates the reality of race - it exists and it matters! It also illustrates how records can become tools that can construct an official identity that might be quite different from that of one's personal or self-identity. Moreover, that official identity can trump personal or self-identity in legal or official contexts and there can be important legal and bureaucratic as well as affective consequences for the individuals in question if their juridical and personal identities fail to match up.

There are other archival and recordkeeping implications of Phipps' ordeal. First, there is the issue of power. Specific communities construct and use records to produce social power in order to increase their ability to influence others for personal advantage. In Phipps' case, a racist society defined and recorded blackness in ways that depicted it as inferior to whiteness.

Press, 1992; and Cornel West, Race Matters, Boston: Beacon Press, 2001.

7 Much recent critical work in race, ethnicity, gender and archival studies rejects such claims of fixity as they relate to the nature and pluralisation of the record as well as the rigidity of traditional physiological categorisations and binaries, e.g., of gender. See, for example, Dean Spade, Normal Life: Administrative Violence, Critical trans Politics, and the Limits of the Law. Brooklyn, NY: South End Press, 2011. Similarly, records continuum theorists such as McKemmish argue that rather than becoming intellectually and physically fixed when they cross the archival threshhold and are entered into the archival bond, records continuously take on new meanings as they move across space, time and all the different contexts in which they are situated or perform. See Sue McKemmish, "Are Records Ever Actual," in Sue McKemmish and Michael Piggott, eds. The Records Continuum: Ian Maclean and Australian Archives: The First Fifty Years. Clayton: Ancora Press in association with Australian Archives, 1994, pp.187-203, http://www.infotech.monash.edu.au/research/groups/rcrg/publications/smcktrc.html. For an exposition of classic ideas on the nature of the record, archives, and the archival bond, see Luciana Duranti, "The Archival Bond," Archives and Museum Informatics 11 nos.3-4 (1997): 213-218 and "Archives as a Place," Archives E Manuscripts 24 no.2 (1996): 242-255. 
This stigma became fixed and immutable through highly interdependent recordkeeping and legal structures despite Phipps' lived experience. Second, there is the issue of ideology, which includes control over how people know and what they know. Dominant groups demonstrate the power of their ideas when they materialise them or make physical objects such as records that then serve to reinforce all sorts of power differentials that are also tied up in race and ethnicity issues. These records and their effects can be located not only in archives but also in other public spheres such as mass media, law, economics and education. Third, Phipps' case begs many questions about the very nature of archives as classically understood. How is the archive defined and by whom? More specifically, who and what shaped or influenced the concepts of the archive and archives as we understand them today, and for what purposes? More generally, how do societal norms (culture) influence recordkeeping activities and regulations? What was the purpose of keeping records on race in the United States and how were these records used apart from documenting a birth, marriage, or death? Whose values are reflected in the official record? How are subjugated groups described in the record? Who, if anyone is allowed to "correct" the record in an attempt to "set it straight" so that it reflect the realities of what actually happened and responds to the "right" values? Who developed and established the "rules" pertaining to such decisions, and for what purposes? Who or what provides meaning and assigns values to records when it comes to deciding their long-term fate?

The Phipps case reminds us that the concepts of race and ethnicity are, at best, messy. Historically, race has been bureaucratically and physiologically defined by categorising groups of people according to physical traits that might result from their genetic ancestry such as skin colour, body shape, facial features, hair texture, and so forth. These, as well as other social and political constructions of race, can also draw upon meanings and characteristics (perceived or otherwise) associated with particular races and racial stereotypes. ${ }^{8}$ For example, African Americans are often associated with an overall lack of intelligence; Native Americans are depicted as loyal sidekicks or aggressive alcoholics; and stereotypes of Asian Americans suggest a group that is passive and politically inactive. Well into the twentieth

8 For examples and explanations of how race and racism developed in a Latin American context, see Peter Wade, Race and Ethnicity in Latin America. Chicago: Pluto Press, 1997, and Blackness and Race Mixture: The Dynamics of Racial Identity in Colombia. Baltimore: The Johns Hopkins University Press, 1993; and Edward E. Telles, "Racial Ambiguity among the Brazilian Population,” Ethnic and Racial Studies 25 (1998): 415; and Race in Another America: The Significance of Skin Color in Brazil. Princeton: Princeton University Press, 2004. 
century, negative traits were believed to be passed on genetically and this belief was the primary basis of the Eugenics Movement (discussed later in the chapter), which aimed to reduce the reproduction of groups having less desirable traits. Racial paradigms and categorisations are also significant because they can be the basis of pejorative stereotypes between racial groups. By contrast, ethnicity is traditionally viewed in terms of a population of people who identify with each other on the basis of race. ${ }^{9}$ At the risk of oversimplification, the significance of ethnicity lies in shared cultures and group history. ${ }^{10}$

The aim of this chapter is to discuss conceptual frameworks used in ethnic studies and to contemplate their possible value for approaching archival and recordkeeping research through an African American lens. The chapter includes a discussion on culture, what it means, how it functions, and how mankind uses it to create social power in relation to one another. It argues that the role of culture is key to understanding the interacting dynamics of race, archives, and power. The chapter also looks at how the ethnicity paradigm became the dominant racial paradigm in the United States and how dominant groups constructed race and racism as tools to strengthen and maintain power. Lastly, the chapter contemplates how conceptual frameworks from ethnic studies might be useful in archival and recordkeeping research. While there certainly also needs to be more, and more in-depth, contemplation of how multiple critical frameworks might be drawn, for example, from critical race and feminist studies, and applied together with those from ethnic studies, to examine the complexities of intersectionality (i.e., the intersections and interactions between different forms of oppression, discrimination or marginalisation that might be at work) and double marginalisation, such an in-depth discussion is outside the scope of this paper. ${ }^{11}$ The chapter by Dunbar in this volume will provide the reader with more detail on the nature and application of relevant conceptual framings in Critical Race Theory, and the chapter by Lee provides an excellent

9 William Peterson, "Concepts of Ethnicity." In Concepts of Ethnicity: Selections from the Harvard Encyclopedia of American Ethnic Groups. Cambridge: Harvard University Press, 1982; and Nathan Glazer, "Blacks and Ethnic Groups: The Difference, and the Political Difference it Makes," Social Problems 18 no.4 (1971): 444-461.

10 These definitions are an attempt to simplify a complicated discussion within the parameters of limited space.

11 For an extensive in-depth discussion on critical race theory and its relationship with continuum theory, see Chapter 12, Anthony W. Dunbar, "Prologue; Introducing Critical Race Theory to Archival Discourse: Getting the Conversation Started" (reprint from Archival Science 6 (2006): 109-129). 
example of how multiple conceptual frameworks taken from different fields (e.g., queer theory, somatechnics) might be used within the same study.

\section{My Standpoint}

Much of my work is informed by Berger and Luckmann's notion of the social construction of reality, ${ }^{12}$ which posits that individuals and groups interact with one another in order to create social systems that have meanings. ${ }^{13}$ This process occurs in four stages defined by Berger and Luckmann as routinisation, institutionalisation, legitimation, and annihilation. To write this chapter, I used Berger and Luckmann's stages of reality creation to explore both the constructs and meanings of race, culture and the archive in relation to one another.

As an African American scholar who happens to be a social constructivist, I approach the archive as a sociocultural construct. By this I mean that the archive (not limited to physical places or buildings and potentially including bodily entities and intangible memory-keeping practices) is where source knowledge (regardless of whether or not it is deemed valid or reliable) is preserved for future use. Societies assign value to and employ the archive much as they do with other constructs such as race, gender and identity. As such, I believe that these entities develop subjective meanings as a result of the world in which they exist and function. ${ }^{14}$

\section{Background: A Note on Race, Ethnicity and Culture}

A discussion on race and ethnicity is both beneficial and necessary in a US context not because there is any significant scientific merit in either concept, but because it is the context in which the United States as a federal entity legally functioned from its origin until 1965, and even later in states such as

12 "Reality," as I use and understand it, is not indicative of something that is true or false, or right or wrong. Rather, it refers to a group's perception of what it perceives to be reality. Simply because something is perceived as "real" (or unreal) does not automatically make it so. As such, one's reality could be totally wrong and false. It is possible, therefore, for someone to be sincerely wrong.

13 For a more extended discussion of the social construction of reality, see Peter Berger and Thomas Luckmann, The Social Construction of Reality. Garden City, NY: Anchor Books, 1966.

14 See, for example, Anne Gilliland and Kelvin White, "Perpetuating and Extending the Archival Paradigm: The Historical and Contemporary Roles of Professional Education and Pedagogy," InterActions 5 no.1 (2009), https://escholarship.org/uc/ item/7wp1q908\#page-3. 
Louisiana. This point will become clearer later in the chapter. While there is no biological basis for racial distinction, however, there are socio-bistorical bases, which explains why race should be seen as unstable (this instability will also be discussed later in the chapter). This is not to suggest that because of its inherent instability race does not exist or that it should be ignored, but rather that its social meanings are constantly being changed and shaped by political struggles that vary in different contexts. Race and racial dynamics are social and historical processes that will vary depending on locations. For example, understandings of race in the northern and western parts of the United States during the 1930s varied significantly from those in the Deep South; and racial dynamics in the United States are not the same as they are in Latin American countries since race evolved along different trajectories and within different social (including cultural) and historical contexts.

To understand the dynamics between the concepts of culture and race better, it is first necessary to understand the concept of culture, its function, and how it is transmitted. Anthropologists commonly define culture as the complex whole that includes knowledge, belief, art, law, morals, values, customs, and other capabilities and habits acquired by mankind. ${ }^{15}$ This includes social things that people do, make and think. Culture is not biological, but rather it is behavioural. Another way of defining culture is to see it as the primary tool that individuals use to pursue actions that they perceive to be in their self-interest; it is used by humanity to produce social power to achieve its goals in relation to other people and the natural environment.

Archives are cultural artefacts. Someone created them, defined their meanings, established criteria for what goes into them, and defined what role they were intended to play in society. In other words, they are created for specific purposes. What shapes and defines how records and the repositories that contain those records are manifested is culture - e.g., the prevailing set of values, customs, and knowledge - and by the same measure they are also vital in perpetuating culture. They look differently in different contexts, but all function in similar ways in that they are the repositories of what is considered important and worthy of transmitting to the next generation. An archive, therefore might be a brick and mortar building with a controlled environment, but it might equally take the form of an intangible body of

15 Leslie A. White, The Science of Culture. New York: Grove Press, 1949; and Pouwell Slurink, "Causes of Our Complete Dependence on Culture" in The Ethological Roots of Culture, R.A. Gardner, ed. Dordrecht. Boston and London: Kluwer Academic Press, 1994, p.461. 
narratives, songs, dances or rituals created and performed by the community as memory and recordkeeping acts in ways that ensure and demonstrate their trustworthiness and authenticity to the community (see the chapter by Faulkhead in this volume for a discussion of the nature of Australian Indigenous narratives as family and community oral records and cultural heritage).

What shapes archival processes and concepts? Culture. What is remembered or recorded is shaped by culture. The archive's form is shaped by culture, as are the processes of creating, using, sharing and discarding records before they make it into the archive. Hence archives, together with their constituent parts, are fragments of sociocultural systems and are in constant motion - merging, splitting, diversifying and moving apart. They are not static, neutral or immutable sites of "sacred" historical knowledge. They are, rather, sites of knowledge and cultural production and re-production - places where decisions are made about whose story is worth remembering, in which ways and from which perspectives.

To help make this point clearer, the model below is a basic anatomy of culture used by anthropologist Ralph Linton who defines culture as the "configuration of learned behaviour and results of behaviour whose component elements are shared and transmitted by members of a particular society." ${ }^{16}$

Culture is intangible; however, it is manifested through the activities of humankind - e.g., practice, whether that be professional practice, everyday life, sacred, legal system, or forms of government. From an archival perspective, the stories and heroes that are remembered or forgotten and the modes through which societies choose to do so are all manifested in some type of record, whether in oral, written, digital or kinetic form. In any case, the function and intent is to record. Culture cannot be archived, per $s e$, but manifestations or evidence of culture can. The record in its various manifestations is what should be the focus of the archivist.

Figure 11.1 provides a model for visualising culture at four different levels:

- Symbols

- Heroes and heroines

- Rituals and norms

- Values

16 Ralph Linton, The Cultural Background of Personality. New York: Appleton-Century Crofts, 1945. 
Figure 11.1. Onionskin Example of Culture ${ }^{17}$

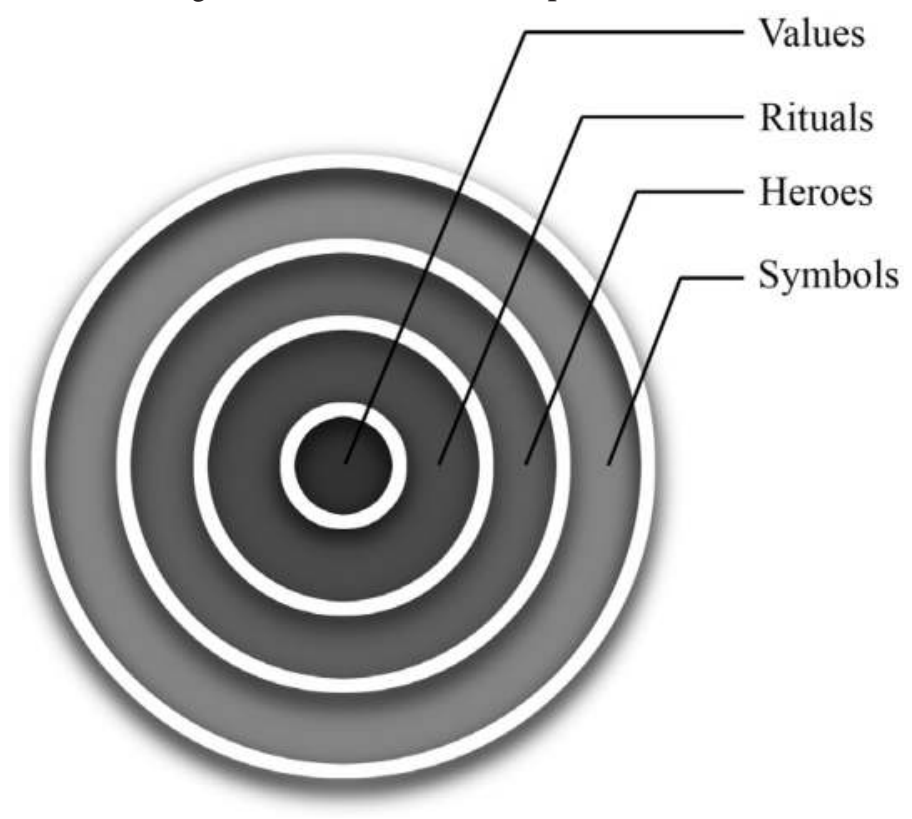

Symbols represent the outermost layer of culture. Symbols refer to the objects, words, languages, gestures, and so forth that have a particular meaning for members of a culture. Heroes and heroines represent a deeper level of culture that is associated with both mythical and real men and women who epitomise certain traits that are highly esteemed or respected in a culture. Heroes and heroines are ideas of model citizens. The third layer of culture is ritual and norms. This layer is where collective activities are located (for example, ways of greeting, ceremonies commemorating life, death, or other significant events). These activities are often considered socially essential in cultures. The final and deepest level of culture is values and this level forms the core of culture. A simple definition of values is the absolute principles or standards that determine what is of worth and important in life (for example, what is good and evil, right and wrong, natural and supernatural or unnatural). As the core of culture, values permeate and influence all cultural layers, thus shaping what becomes a tangible and visual manifestation of culture. These values can be hard to recognise without a deep analysis and thorough understanding of each of these layers and their relationships with each other.

17 This model is based on the work of Geert Hofstede. See Geert Hofstede, "Culture and Organizations,” International Studies of Management \& Organization no. 4 (1980): 15-41. 
The interaction between layers is influenced by core values through the realm of cultural practices. This realm should be of particular interest to the archival community because it is where recordkeeping practices, which I define as the activities that define the act of recording, take place. These practices include intent (motive), the act of creating a record (broadly defined), using it, preserving it, accessing it, and (re)-defining it. Yet this realm has been one of the most problematic for archival practice, research and education primarily because the field has done little to understand the role of cultural values (with the exception of explorations of organisational culture in business and government settings) in recordkeeping activities. Furthermore, the field has much work to do in terms of broadening understandings and raising awareness of the groups or communities whose recordkeeping practices fall outside of the traditional archival paradigm that informs so much of professional best practices and standards. With the exception of continuum thinking as conceptualised by the Australian recordkeeping field, the traditional paradigm is often disseminated as the paradigm tout court - the only, universal, paradigm that informs any "legitimate" form of recordkeeping (for a fuller discussion of the different paradigmatic approaches and archival traditions at work around the globe, see the chapters by Gilliland, McKemmish and Lian in this volume). One of the major objectives in socio-cultural analysis is to understand the different ways in which people use culture as a source of social power to achieve their goals in relation to other people and the natural environment.

To illustrate how culture influences recordkeeping activities in a specific context, the next section explains the culture of race and ethnicity and how these developed conceptually and practically in the United States. African American views on race and ethnicity were constructed within a specific context in response to certain parameters that were imposed by those outside the African American community. The following section is important for understanding the context of how racism (a cultural manifestation) shaped archives, their stories, and the perspective from which their stories are told.

\section{The Culture of Race and Ethnicity in the United States}

In essence, ethnic studies centres on the study of race and race dynamics from the perspective of ethnicity. As a field, it is interdisciplinary in nature and focuses on racialised people in the world. The term "racialised" is tricky, however. To help keep the concept clear, it is important to understand that 
its ultimate objective and effect is to dehumanise specific groups. How this is done depends on spatial and temporal elements. For example, the basic concept of "race" as scholars think of it today (on the basis of various shades of skin colour) did not become prevalent until it was authenticated by bad "science" - i.e., pseudo-sciences such as Spencerism, anthropometry and social Darwinism. During this time, race was primarily attached to skin colour. The darker the skin, the more inferior and "non-human" one was considered. After scientific racism was debunked as a fraud, the idea of race still persisted, but skin colour was no longer a criterion for racialising a group. ${ }^{18}$ For example, in the United States, African Americans are racialised based on skin colour, but nearly every non-black immigrant group that migrated to the United States during the $20^{\text {th }}$ Century was racialised despite skin colour. This happened to Italian Americans, Irish Americans, Chinese Americans, and Mexican Americans. Native Americans were also racialised - as evidenced by the attempted ethnic genocide of Indians by early settlers. ${ }^{19}$ Thus, these groups, many of which would not typically be considered a "race", were grouped as a race for the purpose of dehumanising them and pejoratively differentiating them from the dominant groups who exercised their power to racialise.

Likewise, many groups have been racialised outside the United States. For example, the Roma (commonly and derogatorily referred to as "gypsies") are racialised throughout much of Europe.$^{20}$ Intraracial racialisation occurred in the United Kingdom between the English and Scottish settlers and their

18 See, for example, Nancy Stephan,'The Hour of Eugenics,' Race, Gender and Nation in Latin America. Ithaca: Cornell University Press, 1991.

19 Here, I refer to nigrescence theory, which explains one process of changing from one racial/ethnicity identity to another. For example, a "nigger" changes into "negro" who then changes into "colored" and "black." I do not think that this process is limited to African Americans. It is the author's opinion that this process has occurred in relation to nearly every major newly arrived immigrant group entering the United States in the early $20^{\text {th }}$ Century. For example, during this time, Italian immigrants were primarily thought of pejoratively (as were Mexicans, Chinese, and Irish immigrants) when they began to arrive in the United States on a mass scale. Nigrescence theory was developed by William Cross and can be explored in Cross' works. See William E. Cross, "The Negro-to-Black Conversion Experience," Black World (1971): 13-27, and Shades of Black: Diversity in African-American Identity, Philadelphia, PA: Temple University Press, 1991.

20 See, for example, Belinda Cooper, “'We Have No Martin Luther King:' Eastern Europe's Roma Minority," World Policy Journal no. 4 (2002): 69. See also Tamara Štefanac and Kelvin L. White, "The Representation, Rights and Identity of Croatia's Roma Community: Exploring the Archival Implications," in Willer, Mirna, Anne J. Gilliland, and Marijana Tomic, eds, Records, Archives and Memory: Selected Papers from the Conference and School on Records, Archives and Memory Studies, University of Zadar, Croatia, May 2013, (Zadar: University of Zadar Press, 2015, in press). 
descendants, and the native Irish. ${ }^{21}$ Another example of intraracial racism is Cyprus, which has a long history of conflict between Turkish Cypriot and Greek Cypriot citizens. In all of these cases, skin colour has little, if anything, to do with the racialisation process. ${ }^{22}$

Historically ethnicity, nation and class have been the three main paradigms used to study race in the United States. ${ }^{23}$ These paradigms have particular core assumptions that serve as guides for research about the culture of race and race relations. As such, contemporary aspects of race and racial dynamics in the United States are understood by relying on those two paradigms of which ethnicity, since the last half of the $20^{\text {th }}$ century, has been the most dominant and relied upon.

Ethnicity theory has its origins in 1920s America where it challenged the then prevalent ideas of social Darwinist, Spencerist, and the eugenicist thinking ${ }^{24}$ which emanated out of England and the United States during the late 1870 s as a way of scientifically explaining racial inferiority. Whites and those with light skin were considered superior to blacks and those with darker skin since it was believed that white skin was part of humanity's natural order. During this time, hereditary characteristics such as sexuality and intelligence were also associated with race. After the end of the Second World War in 1945 and the exposure of the atrocities associated with it (e.g., the genocides of Jews, Slavs and Roma), biologism took on more negative connotations and generally became seen as a pejorative construct. Furthermore, attacks against biological determinism were launched by advocates of the Progressive Era in the United States. Horace M. Kallen ${ }^{25}$ was one such leader. He coined the concept of cultural pluralism by arguing that cultural diversity was compatible with national pride and that respect for ethnic diversity and racial difference actually strengthened the United States. He focused on the acceptance of different immigrant-based cultures.

Another prominent figure in the early development of the ethnicity paradigm was Robert Ezra Park. Park was an American sociologist of the

21 See, for example, Peter Berresford Ellis, Eyewitness to Irish History. Hoboken, NJ: John Wiley \& Sons, Inc., 2004.

22 See, for example, Loucas Kakoulli, The Left and the Turkish Cypriots: The Cyprus Problem from a Different Perspective. Nicosia: Cassoulides Press, 1990.

23 For a more thorough discussion on nation and class-based paradigms, see Michael Omi and Howard Winant, Racial Formation in the United States. New York: Routledge, 1994.

24 For a more detailed discussion of racial movements such as the Eugenics Movement, see Stephan, The Hour of Eugenics.

25 Horace Kallen, Cultural Pluralism and the American Idea: An Essay in Social Philosophy. Philadelphia: University of Pennsylvania Press, 1956. 
"Chicago School" who is noted for developing the theory of assimilation which became a major current in the ethnicity paradigm. This theory divided racial dynamics between incoming immigrants to the United States and the dominant (existing) race into 4 distinct stages: contact, conflict, accommodation and assimilation. Initial contact occurs when immigrants are first introduced to the mainstream culture. Conflict caused by competition for resources (for example, employment, socioeconomic status and education) follows contact. Once this occurs, a hierarchical relationship forms between the immigrant group and the existing dominant group through which one group is dominated. The last stage of the cycle is when the immigrant group becomes fully assimilated into the dominant culture. Park based his theory of assimilation on 4 assumptions:

Racial/ethnic groups are not central or persistent elements of modern societies;

Racism and racial oppression are caused by other things such as economics and psychology;

The most important aspects of racism are rooted in American attitudes and prejudices; and

Immigrants from the developing world are essentially the same as white European ethnic groups. In other words, racial minorities of the 3rd world will assimilate just as easily as those white European ethnic groups did. This is often referred to as the "immigrant analogy."

Hence, both Kallen's and Park's perspectives were based on a conceptual model of white immigrants who had come to the United States from across the Atlantic ocean that did not consider immigrant groups from other parts of the world, descendants of former slaves brought to the United States by force, or peoples indigenous to these lands who were identified as racial minorities such as Asian Americans, Latin Americans, African Americans and Native Americans. This omission is significant because it became the main reason why, during the 1970s and 1980s, ethnicity theory was challenged by class and nation-based conceptualisations of race. These challenges were initiated by black and other minority movements primarily because they rejected the following underlying assumptions:

26 Omi and Winant, Racial Formation in the United States, 16-20. 
The United States was committed to racial equality (this was challenged especially because the country had used race as the central axis of social organisation since it came into existence);

Ethnic groups of colour could be assimilated into American life in the same ways that white ethnic groups had been in the past (Park's assumption \#4); and (probably most significant)

Racial minorities desired to assimilate (there was voluminous evidence of enclaves of minority groups who maintained their own identities, values and cultures and did not aspire to become anglicised). ${ }^{27}$

Omi and Winant, describing the United States as a racial dictatorship, emphasise that the United States is far from being a colour-blind society and that race has been at the very core of the United States' development:

From the very inception of the Republic [United States] to the present moment, race has been a profound determinant of one's political rights, one's location in the labor market, and indeed one's sense of "identity". The hallmark of this history has been racism, not the abstract ethos of equality, and while racial minority groups have been treated differently, all can bear witness to the tragic consequences of racial oppression. The U.S. has confronted each racially defined minority with a unique form of despotism and degradation. The examples are familiar: Native Americans faced genocide, blacks were subjected to racial slavery, Mexicans were invaded and colonized, and Asians faced exclusion. ${ }^{28}$

They continue:

For most of its existence both as European colony and as an independent nation, the U.S. was a racial dictatorship. From 1607 to $1865-258$ years - most non-whites were firmly eliminated from the spheres of politics. After the Civil War there was the brief egalitarian experiment of Reconstruction which terminated ignominiously in 1877. In its wake followed almost a century of legally sanctioned segregation and denial of the vote, nearly absolute in the South and much of the Southwest, less effective in the North and far West, but formidable in any case. These barriers fell only in the mid-1960s, a mere quarter century ago ... Patterns of racial inequality have proven, unfortunately to be quite

27 For more examples, see Omi and Winant, Racial Formation in the United States, 21.

28 Omi and Winant, Racial Formation in the United States, 1. 
stubborn and persistent ... It is important, therefore, to recognize that in many respects, racial dictatorship is the norm against which all U.S. politics must be measured [emphasis added]. ${ }^{29}$

The key here in the development of ethnic conceptual frameworks is that race and racial dynamics needed to be addressed because they were the very fibre of how the United States organised members of its society; race (as opposed to the individual) determined almost everything. Race and racism had systematically permeated nearly, if not all, of America's institutions, including education, marriage, law, religion, history, science, sports and the arts. One of the general weaknesses of ethnicity theory at the time is that it did not effectively address the institutional and ideological nature of race in America. As a result, ethnicity theory found itself under increasing attack from many blacks and other racial minority groups who demanded more emphasis on group rights and recognition. During the 1970s and 1980s, therefore, ethnicity theorists had to reformulate their ideas, which led to neo-conservatism - championing individual over group rights and emphasising the danger of state activities that promoted antidiscrimination policies. Hence, they argued, the state should be colourblind. Despite subsequent challenges and reformulations of ethnicity theory, this paradigm has dominated and shaped academic thinking well into contemporary times.

The nation-based paradigm, coming into fruition in the mid-1960s, ${ }^{30}$ might appear on the surface to be the most radical of the three racial paradigms. Its proponents, growing weary of the call for moderate, nonviolent integration, demanded that the colonisers allow all members of those "colonised" through subjugation (racially oppressed peoples who were generally forced to enter into the United States) to be nationally liberated for the purpose of developing their own cultural autonomy and deracinating colonialism in order to restructure society along non-racial lines. Hence, the nation-based paradigm has its roots in capitalism as colonialism (unlike previous imperial systems that were not phenomena that operated globally, but rather were geographically determined) and argues that Europe carved up the world's existing nations and territories primarily for economic reasons

29 Omi and Winant, Racial Formation in the United States, 65-66.

30 Its antecedents date back as early as the 1920s with contributions from civil rights activists such as W.E.B. Du Bois, Marcus Garvey, George Padmore, Malcolm X, Nkrhumah, Franz Fanon, and others. For a broader discussion on the nation-based paradigm see Omi and Winant, Racial Formation in the United States. 
and assigned to each one power, privilege, misery and exploitation according to the needs and desires of Europe's ruling powers. Assignments were usually made along racial distinctions in order to maintain colonial dominance. The nation-based approach emphasised a broader range of racial oppression political, cultural, economic, spatial segregation, and so forth - than did the ethnicity and class-based paradigms.

\section{Cultural Worlds, Ethnic Studies, and Archival and Recordkeeping Research}

How does the previous discussion fit in with archival and recordkeeping research? I posit that archival and recordkeeping research, if approached through an ethnic studies lens, can shed a brighter light on more detailed, "hidden" parts of society that otherwise would be missed or purposively forgotten. A key space in which culture (symbols, heroes, rituals, and values), race and ethnicity intersect is that of recordkeeping and archival practices. Pederson reminds the archival community that humans are "the only species on earth that systematically documents its thoughts and activities by making and keeping records." ${ }^{31}$ Other scholars have demonstrated that although all humans create some form of records, it is usually the records of those with the need, capability, and power to preserve them that find their way into the official archive, and that much of what comprises contemporary archival theory - for example, conceptualisations of the record, evidence, ownership, appraisal, authenticity, respect des fonds and so forth - has been developed to support bureaucratic, administrative and commercial activities in order to sustain and perpetuate those types of institutions and environments from their particular perspectives. ${ }^{32}$

Since the 1970s, there have been increasing calls within the archival community to take a wider, more nuanced and more systematic interest in the socio-cultural aspects of archival practice. Key examples of approaches that became prominent in the discourse of the 1980s and 1990s are the socalled "documentation strategy," ${ }^{33}$ and the Canadian concept of the total archive, both of which emphasised collecting a wider range of materials

31 McKemmish et al., "Professing Archives," 51.

32 See Gilliland and White, "Perpetuating and Extending the Archival Paradigm."

33 See Elizabeth Snowden Johnson for a recent reflection: "Our Archives, Our Selves: Documentation Strategy and the Re-appraisal of Professional Identity," American Archivist 71 (Spring/Summer 2008): 190-202. 
including maps and other forms of documentary materials. ${ }^{34}$ By the start of the twenty-first century, a discernable movement toward self-documenting by identity-based communities (a.k.a. archives from the bottom up; grassroots archives; community-centred archives; oppositional archives) who felt themselves to be excluded from, misrepresented in, or oppressed or marginalised by records and archives, began to gain the attention of the archival field..$^{35}$

Over the past decade, a growing cadre of archival scholars and educators have not only questioned and challenged contemporary and traditional archival theory and practice, but have also begun to address other sociocultural dynamics of the archival discourse and to build infrastructures that support alternate epistemologies and recordkeeping activities. For example researchers at Monash University collaborated with practitioners and Koorie community members to develop and implement an information system that was sensitive to the archival needs of Indigenous communities. ${ }^{36}$ Other examples include collaborative studies undertaken by Gilliland and McKemmish on the conditions and circumstances of archival education in Pacific Rim countries. ${ }^{37}$ Data from these studies indicated that both archival educators and professionals were not educated or being educated to address the ways of remembering that are traditional in ethnic or Indigenous communities and that archival education curricula tend to highlight national, political and enterprise priorities and internationallydeveloped best practices. Emergent pluralistic archival education plays

34 See Laura Millar, "Discharging our Debt: The Evolution of the Total Archives Concept in English Canada," Archivaria 46 (1998): 104-146.

35 See Jeannette A. Bastian and Ben Alexander, eds. Community Archives: The Shaping of Memory (London: Facet, 2009); Andrew Flinn, "Archival Activism: Independent and Community-led Archives, Radical Public History and the Heritage Professions," InterActions: UCLA Journal of Education and Information Studies 7 no.2 (2011), http:// escholarship.org/uc/item/9pt2490x; and Anne J. Gilliland and Andrew Flinn, "Community Archives: What are We Really Talking About?" Keynote address, Nexus, Confluence, and Difference: Community Archives Meets Community Informatics: Prato CIRN Conference Oct 28-30 2013, Larry Stillman, Amalia Sabiescu, Nemanja Memarovic, eds. Centre for Community Networking Research, Centre for Social Informatics, Monash University, 2013. ISBN 978-0-9874652-1-4, http://www.ccnr. infotech.monash.edu.au/assets/docs/prato2013_papers/gilliland_flinn_keynote.pdf.

36 See Fiona Ross, Sue McKemmish, and Shannon Faulkhead, "Indigenous Knowledge and the Archives: Designing Trusted Archival Systems for Koorie Communities," Archives and Manuscripts 34 no.2 (November 2006): 112-151.

37 Anne Gilliland, Andrew Lau, Yang Lu, Sue McKemmish, Shilpa Rele, and Kelvin White, "Pluralizing the Archival Paradigm: Critical Discussions Around the Pacific Rim," Archives \& Manuscripts 35 no. 2 (November 2007): 10, and Anne Gilliland, Sue McKemmish, Zhang Bin, Kelvin White, Yang Lu, and Andrew Lau, "Pluralizing the Archival Paradigm: Can Archival Education in Pacific Rim Communities Address the Challenge?" American Archivist 71 no. 1 (Spring/Summer 2008): 87. 
a significant role in developing a more diverse pool of archival practitioners and future educators who are able to respond to the information needs of various ethnic communities in culturally sensitive ways. ${ }^{38}$ The archival education program at the University of Oklahoma is an exemplar in that it emphasises and prepares students to be both socio-culturally aware and sensitive to diverse recordkeeping environments and activities, and the implications these have for marginalised or underrepresented communities. This is done by introducing students to archival concepts as they apply across three communicative modes (i.e., orality, literacy, and digitality) in their first archives class and ensuring that concept-based teaching that is centred on these communicative modes is integrated throughout the archival curriculum. Assignments require students to apply these concepts to both mainstream and under-represented communities. This has proven to be one effective way of cultivating cultural sensitivity in students.

While the archival field has made some progress towards making archives more relevant to society, there is still much work to be done, especially in the area of race and racial dynamics. It is in this vein that ethnic studies frameworks may be useful to recordkeeping, archival research and practice. The problem is twofold, and in order to illustrate the problem, it is necessary to understand how humanity has organised itself over time and how archival theory has adapted to mankind's self-organisation.

According to American anthropologist John Bodley, there are three worlds in which cultures operate: the tribal, imperial, and the commercial. ${ }^{39}$ Within these worlds, since culture is not static, I posit the existence of multiple cultural spheres within which culture is created, manifested, preserved, transformed, transmitted and diminished. Bodley uses basic concepts of cultural anthropology and a culture-scale perspective to compare cultures of increasing scales as he focuses on universal human concerns. He is primarily concerned with how people use culture to get what they want in relation to other people (i.e., how do they create and organise social power). His categories

38 See the Archival Education and Research Institute (AERI) Pluralizing the Archival Curriculum Group (PACG), "Educating for the Archival Multiverse," American Archivist (Spring/Summer 2011): 68-102; Anne Gilliland, "Neutrality, Social Justice and the Obligations of Archival Educators and Education in the Twenty-first Century," Archival Science 11 nos. 3-4 (2011): 193-209, and "Pluralizing Archival Education: A Non-Zero-Sum Proposition," Chapter 10 in Through the Archival Looking Glass: A Reader on Diversity and Inclusion, Mary Caldera and Kathy Neal, eds. (Chicago, IL: Society of American Archivists, 2014): 231-268.

39 John H. Bodley, Cultural Anthropology: Tribes, States and the Global System, $5^{\text {th }}$ ed. New York: Rowman \& Littlefield, 2011, p. 11. 
are based on the history of mankind's population growth. Early humans organised themselves in small groups - tribes - and there was no need of large, bureaucratic governments, as we know them today. Everyone in the tribe was guaranteed access to everything needed (e.g., food, land, and sociocultural knowledge) to sustain life and be human. As the world's population grew, humans began to organise themselves into chiefdoms and states. Bodley refers to this as the imperial world. Increased populations led to increased competition for limited resources. As the global population grew and natural resources became increasingly limited, humanity began organising itself into modern nation-state governments. He calls this sphere the commercial world.

What is culture's role, according to Bodley? He states: "people are unique animals in our almost total reliance on culture as our primary means of survival. Culture is socially transmitted information that shapes our behavior" [emphasis his]. ${ }^{40}$ Thus, culture plays a fundamental role in creating social power for survival purposes and for passing this knowledge to direct offspring. Examining the main cultural processes occurring in each sphere illustrates this. According to Bodley, humanisation is the main cultural process occurring in the tribal world (see Figure 11.2). It is also the most crucial because, according to him, it is "centered on the household and involves the maintenance and reproduction of individual humans, human society, and human culture. ${ }^{.41}$ Humanisation is defined as the "production, maintenance, and reproduction of human beings and culture;" ${ }^{\prime 2}$ politicisation, which is defined as the "production and maintenance of centralized political power by co-opting the humanization process." ${ }^{" 3}$ This is the primary cultural process occurring in the imperial world. Lastly, there is the commercial world in which commercialisation is the primary cultural process. He defines commercialisation as the "production and maintenance of private profitmaking business enterprise as the means of accumulating capital, by co-opting the humanization and politicisation processes." ${ }^{\prime 4}$ What is striking here is the collective and gradual drive to increase social power for a particular group at another group's expense, and the major processes - namely politicisation and commercialisation - are used to co-opt the most crucial process - the humanisation process! Dehumanisation (racialisation is only one of many ways to dehumanise someone) plays a fundamental role in this process.

\footnotetext{
40 Bodley, Cultural Anthropology, 10.

41 Bodley, Cultural Anthropology, 21.

42 Bodley, Cultural Anthropology, 11.

43 Bodley, Cultural Anthropology, 10.

44 Bodley, Cultural Anthropology, 11.
} 
Figure 11.2. Bodley's Cultural Processes and Subprocesses by Cultural World ${ }^{45}$

\section{Tribal World}

Major cultural process

- Humanisation: the production, maintenance, and reproduction of human beings and culture Subprocesses

- Conceptualisation: producing abstract concepts and symbols that shape behaviour

- Materialisation: giving physical form to concepts

- Verbalisation: producing speech

- Socialisation: producing human societies by exogamy

- Cultural Transmission: reproducing culture

\section{Imperial World}

Major cultural process

- Politicisation: the production and maintenance of centralised political power by attempting Subprocesses to co-opt the humanisation process

- Taxation: extracting surplus production to support government

- Conquest: extracting booty, slaves, and tribute

- Specialisation: government employment

- Militarisation: development of professional military

- Bureaucratisation: hierarchical command structure

- Urbanisation: development of cities

Commercial World

Major cultural process

- Commercialisation: the production and maintenance of private profit-making business enterprise as a means of accumulating capital, by attempting to co-opt the humanisation Subprocesses and politicisation process

- Commodification: market for land, labour, money, basic goods and services

- Industrialisation: mass production, distribution, and consumption of goods and services

- Capitalisation: ownership of means of production separated from labour

- Corporatisation: business enterprise becomes suprahuman

- Externalisation: cost of commercial growth are socialised

- Supralocalisation: business enterprise is detached from community

- Financialisation: finance institutionalised, separated from production

What roles do the archive and the archivist play in this process? The archivist is the processor and the archives are the "vaults" (broadly defined to be sensitive to the various modalities and forms in which they exist) in which selected elements of each process are preserved for the sake of maintaining culture as well as remembering it. This is telling in the Phipps case where race was recorded by parts of society that benefitted from doing so. Those in power (i.e., whites) recorded race to fix and perpetuate the dehumanisation

45 To see the original table, see Bodley, Cultural Anthropology, 11. 
of non-whites - not only through their decisions about what to record, but also through the development of the rules by which the record was created and could be changed. As such, archives become tools for the creation and maintenance of social power until change occurs outside the archive.

It is important to point out that most contemporary archival theory and practice developed in and in response to bureaucratic recordkeeping practices (located in the cultural sphere of politicisation in Figure 11.2). As more commercialisation - an offshoot of politicisation - occurs, such theory and practices slowly adapts to the archival needs located within the cultural spheres of politicisation and commercialisation, but still at a much faster pace than it addresses the archival needs located in the sphere of humanisation. Thus, contemporary theory is more responsive to records predominantly created and used in the sphere of politicisation and commercialisation.

Figure 11.3: Archival Processes and Subprocesses

Realm of archival activity

- Record creation and recordkeeping practices:

○ What, why, and how records were created, represented, used, and trusted?

- What, why, and how were records transmitted for cultural knowledge and recordand memory-keeping purposes across time and cultures?

- Evidence of major and subprocesses captured by and/or about a specific ethnic/racial group

- Traces of racial dynamics between groups

From the archival perspective, the realm of creating and maintaining records is where one would find evidence of the major processes and subprocesses. For each of Bodley's worlds, one could ask how each of the processes are captured and become part of the records multiverse. The above framework (see Figure 11.3) could be useful in elucidating the obscure, yet rich realm of recordkeeping and archival activities that, when collectively examined, more deeply explains not only society's cultural values, but also the rationale, purpose and meanings behind its activities.

The key thing to understand is that such evidence is defined, interpreted, and understood by the cultural group producing the evidence rather than by an outsider. Bates' notion of the pervasiveness of information is also applicable to archives and their content. ${ }^{46}$ All societies document, but not all groups within a society document in the same way because culture, history, politics, economics, race, gender, class, and so forth influence recordkeeping activities.

46 Marcia J. Bates, "The Invisible Substrate of Information Science," Journal of the American Society for Information Science, 50 no. 12 (October 1999): 1043. 
These spheres are not exhaustive, but they can serve as examples that should be of particular importance to the archival community because they are where recordkeeping and archival practices are located. By not acknowledging the multiplicity of cultural spheres and the recordkeeping and archival activity that exists in each sphere, archival theory and practice continue to function as hegemonic tools that facilitate the reproduction of dominant culture. $^{47}$

An example that illustrates this point is the concept of archival appraisal. In 1999, archival scholar Terry Cook spoke at the University of Maryland..$^{48}$ In his presentation, he defines appraisal theory as the "concepts that determine 'value' and enunciates the generic attributes of those concepts that apply to the selection of records for enduring preservation." He states:

Appraisal imposes a heavy social responsibility on archivists. In the stirring words of Pam Wernich, a South African archivist writing in 1988, archivists are doing nothing less than "moulding the future of our documentary heritage." Archivists determine "which elements of social life are imparted to future generations ..." As a profession, we archivists need to realize continually the gravity of this task ... We are deciding what is remembered and what is forgotten, who in society is visible and who remains invisible, who has a voice and who does not ... In many societies ... certain classes, regions, ethnic groups, or races, women as a gender and non-heterosexual people, have been de-legitimized by their relative or absolute exclusion from archives, and thus from history and mythology - sometimes unconsciously and carelessly, sometimes consciously and deliberately. Why?

To answer Cook's question, it is useful to situate archival appraisal in a racial, political, historical context. Thus, a better way for archivists undertaking appraisal to phrase Cook's question might be "Whose knowledge is most worthy?" instead of "what knowledge is most worthy?" The decision to

47 See, for example, Society of American Archivists, “*A* Census”; Kelvin L. White and Anne J. Gilliland, "Promoting Reflexivity and Inclusivity in Archival Education, Research and Practice," Library Quarterly 80 no.3 (July 2010): 231-248; Gilliland et al., "Pluralizing the Archival Paradigm: Critical Discussions Around the Pacific Rim," 10; Gilliland et al., "Pluralizing the Archival Paradigm: Can Archival Education in Pacific Rim Communities Address the Challenge?” 87; and Gilliland and White, "Perpetuating and Extending the Archival Paradigm."

48 Terry Cook, "Archival Appraisal and Collection: Issues, Challenges, New Approaches," Special Lecture Series at the University of Maryland and to NARA Staff, College Park, MD, April 1999. http://www.mybestdocs.com/cookt-nara-990421-2.htm. 
define one group's knowledge, culture and history as worthwhile to pass along to others while those of others are erased from the historical record says something about who has power in society. What many archivists fail to reflect upon is that during the process of appraisal, it is in their attempts to be impartial in preserving what is believed to have most value for society, that they perpetuate and privilege the values of the dominant group.

Underlying these issues is a particular set of questions: What is the relationship between archival functions such as appraisal and knowledge production? What roles do ideology and hegemony play in this relationship? What is the relationship between the archive and knowledge production? What type of knowledge is produced? Whose knowledge is reproduced? As already noted, race remains a hegemonic force in American society (and it will be for some time to come). It is hegemonic in the sense that it's truly total, as the Phipps example illustrated. In her case, race and racism, which are sociocultural constructs, dictated codifications of race as well as its immutability once recorded, regardless of one's phenotype. Williams provides an excellent summary of the Gramscian concept of hegemony. $\mathrm{He}$ states:

For hegemony supposes the existence of something which is truly total, which is not merely secondary or superstructural, like the weak sense of ideology, but which is lived at such a depth, which saturates the society to such an extent [that it] ... even constitutes the limit of common sense for most people under its sway ... corresponds to the reality of social experience very much more clearly than any notions derived from the formula of base and superstructure. For if ideology were merely some abstract imposed notion, if our social and political and cultural ideas and assumptions and habits were merely the result of specific manipulations, ... [or] a kind of overt training which might be simply ended or withdrawn, then society would be very much easier to move and to change than in practice ... . This notion of hegemony ... emphasizes the facts of domination. ${ }^{49}$

Williams' statement shows how hegemony acts to saturate society's very consciousness so that the educational, professional, and social world within which society interacts, along with interpretations placed on its actions,

49 R. Williams, "Base and Superstructure in Marxist Cultural Theory." In Schooling and Capitalism, Roger Dale, Geoff Esland, and Madeleine MacDonald, eds. London:

Routledge and Kegan Paul with Open University Press, 1976, p.202. 
becomes the only world. Thus, information institutions such as archives could be viewed as one of the main agencies that transmit dominant culture. Williams also describes a process that he refers to as the "selective tradition," which he defines as:

that which, within the terms of an effective dominant culture, is always passed off as 'the tradition,' the significant past. But always the selectivity is the point; the way in which from a whole possible area of past and present, certain meanings and practices are chosen for emphasis, certain other meanings and practices are neglected and excluded. ${ }^{50}$

For the archivist, archival appraisal is the space in which the selective tradition is manifest.

The second part of the problem is related to local socio-historical contexts: if one does not understand race and its dynamics in its local context, then one cannot fully understand American history and its records universe. An ethnic studies framework serves as a lens through which one can examine and understand racial phenomena and the records associated with them. Like culture, race is not tangible. However, manifestations or evidence of race and racial conceptualisations exist in the record. The argument is not that there is a scarcity of historical records about racial experiences, but rather that the records that exist are usually one-sided in that they were created by and from the perspectives of the dominant group and rarely by or from the perspectives of the subjects themselves. For example, when the origins and formation of the African American community in the United States is examined, slave records, which primarily function as evidence of commercial and legal transactions, are referenced. From a plantation's birth registers, historians have been able to piece together an interpretation of the social and cultural practices of slaves living on the South Carolina Good Hope plantation..$^{51}$ The first recorded birth in the register occurred in Africa in 1760. The last birth recorded was in 1857 - 97 years later. The register included over 200 slaves and covered some of the most formative years of the African American experience. However, its history was recorded by the plantation whites who recorded slave births for the purpose of keeping an accurate inventory of the slave owner's property. Little evidence has been found that sheds light on the social life of slaves from the slave's perspective. This does not mean that slaves did not document their

50 Raymond Williams, "Base and Superstructure in Marxist Cultural Theory," 203.

51 John W. Blassingame, The Slave Community: Plantation Life in the Antebellum South. Revised and enlarged edition. New York: Oxford University Press, 1979, p.174. 
life on the plantation, but rather that they created different types of records for different reasons. One such record is the slave spiritual. The slave song functions as a counternarrative to the official plantation record, since these songs originated from the slave community. The use of spirituals, Blassingame argues, shows that preliterate, pre-modern Africans:

were so imperfectly acculturated into the secular American society into which they were thrust ... that they were forced to fall back upon the only cultural frames of reference that made any sense to them and gave them a feeling of security ... the slaves' oral tradition ... ${ }^{52}$

The spiritual functioned as a record of a people who found through it the status, harmony, and values they needed in order for them and their offspring to survive life during enslavement. Furthermore, what if communities were limited in their ways of communicating at a mass scale, both among themselves and among others, and found it more effective to do so through a medium that the dominant culture deemed insignificant and transitory? From this perspective, could a song be considered part of the historical record since it functioned as an alternative source of evidence of social, cultural, political and identity discourses that not only sought to provide entertainment, but also presented nonjudgmental prescriptions and blueprints for what life should and could be like for a particular community?

The black press is another example of how a subaltern community documented and provided commentary on culture in the United States, particularly during the first half of the twentieth-century. During the 1930s and 40s, the black press served a unique role not only for the African American community, but also for the nation in terms of redefining class, race and nationhood. It also played a significant role in resetting the terms of public conversation. The difficulties African American writers experienced in getting published forced them to work creatively to broadcast their ideas in a variety of formats. Writers who wanted to address their position in society had to provide information that made sense of a country torn by economic and social systems. Editors had to find fresh ways to address sensitive issues like discrimination, economic policy, and racial and gender roles. Therefore, it is not uncommon to see writers forming and reforming ideologies, creating and recreating a public sphere, and crafting and re-crafting nationhood through formats such as narratives, photographs, poetry, jeremiads, and comic strips. Although each format proved equally important and sufficient as creative 
negotiations, they were also a means through which proposals for alternative paths were presented to the nation..$^{53}$

The previous examples show that what might be significant to a subculture might be insignificant to the dominant culture. What happens when the medium of the record itself does not fit the generally accepted definition of a record, as defined by the mainstream? Frameworks drawn from ethnic studies can shed light on such issues and provide space to examine how records functioned within their socio-historical contexts. Whether the mode of records creation was through narrative, poem, songs or comic strips, it nevertheless amounted to exchanges between community members over crucial questions, roles and expectations. These exchanges dealt with issues that had deep, long lasting effects on the African American community and actively created positive change. In these instances, these modes of communication became evidence of social acts. Furthermore, in a society in which African American writers/artists struggled to get anything into print, how might the particular forms in which they wrote have an affect upon understandings of a record?

\section{Conclusion}

This chapter raises questions about the nature of creating and keeping records in various societal contexts. It argues that culture plays a significant role in documenting the processes of humanisation, politicisation, and commercialisation. In other words, skin colour does nothing in a real sense unless something else informs one how to interpret skin colour or ethnicity. Culture is what shapes one's meaning of skin colour, not vice versa. Furthermore, the chapter uses the development of racial culture in the United States to show how societal power is created by one group in order to dominate other groups by dehumanising them. It illustrates how this process is captured and supported by recordkeeping and archiving activities, not only by decision-making about what becomes the archival record, but also by constructing the rules of archivy and the criteria for which materials can be legitimately considered worthy of being preserved. The chapter uses the Phipps case as an illustration not only of the implications of racism on the lived experiences of those affected by it, but also of how the values of

53 For more discussion on the role of the Black Press, see Lee Finkle, Forum for Protest: The Black Press During World War II. Cranbury, NJ: Associated University Presses, Inc., 1975; and Todd Vogel, ed., The Black Press: New Literary and Historical Essays. New Brunswick: Rutgers University Press, 2001. 
a racialised society, implicate records and recordkeeping, in an African American context. The extent to which the values exhibited by the records resonate with the value judgment brought to bear during the archival appraisal process has tremendous implications for the role and conduct of archival appraisal and indeed, all subsequent archival activities with those records. Finally, the chapter shows that the very nature of recordkeeping and archiving speaks volumes about what a society values and with whom it is competing for the goal of survival. This is the driving force behind recordkeeping, archiving, and the rules associated with such activities.

\section{References}

Appiah, Kwame Anthony. In My Father's House: Africa in the Philosophy of Culture. New York: Oxford University Press, 1992.

Archival Education and Research Institute (AERI) Pluralizing the Archival Curriculum Group (PACG). "Educating for the Archival Multiverse," American Archivist (Spring/ Summer 2011): 68-102

Bastian, Jeannette A., and Ben Alexander, eds. Community Archives: the Shaping of Memory. Principles and Practice in Records Management and Archives. London: Facet Publishing, 2009.

Bates, Marcia J. “The Invisible Substrate of Information Science.” Journal of the American Society for Information Science 50 no.12 (October 1999): 1043-1050.

Berger, Peter and Thomas Luckmann. The Social Construction of Reality. Garden City, NY: Anchor Books, 1966.

Blassingame, John W. The Slave Community: Plantation Life in the Antebellum South. Revised and enlarged edition. New York: Oxford University Press, 1979.

Bodley, John. Cultural Anthropology: Tribes, States, and the Global System. $5^{\text {th }}$ ed. New York: Rowman \& Littlefield, 2011.

Connerton, Paul. How Societies Remember. New York: Cambridge University Press, 1989.

Cook, Terry. "Archival Appraisal and Collection: Issues, Challenges, New Approaches." Special Lecture Series at the University of Maryland and to NARA Staff, College Park, MD, April 1999. http://www.mybestdocs.com/cookt-nara-990421-2.htm.

Cooper, Belinda. “We Have No Martin Luther King:' Eastern Europe’s Roma Minority.” World Policy Journal no. 4 (2002): 69-77.

Cross, William E. “The Negro-to-Black Conversion Experience.” Black World (1971): 13-27.

Cross, William E. Shades of Black: Diversity in African-American Identity, Philadelphia, PA: Temple University Press, 1991.

Drake, St. Clair. Black Folk Here and There. Afro-American Culture and Society, vol. 1. Los Angeles: The Regents of the University of California, 1987.

Drake, St. Clair. Black Folk Here and There: An Essay in History and Anthropology, Volume 2 (Afro-American Culture and Society). Los Angeles: University of California Center for Afro-American Studies, 1991.

Dunbar, Anthony W. "Introducing Critical Race Theory to Archival Discourse: Getting the Conversation Started.” Archival Science 6 no.1 (March 2006): 109-129.

Duranti, Luciana. "The Archival Bond," Archives and Museum Informatics 11 nos.3-4 (1997): 213-218. 
Duranti, Luciana. "Archives as a Place." Archives E Manuscripts 24 no.2 (1996): 242-255.

Ellis, Peter Berresford. Eyewitness to Irish History. Hoboken, NJ: John Wiley \& Sons, Inc., 2004.

Finkle, Lee. Forum for Protest: The Black Press During World War II. Cranbury, NJ: Associated University Presses, Inc., 1975.

Flinn, Andrew. "Archival Activism: Independent and Community-led Archives, Radical Public History and the Heritage Professions." InterActions: UCLA Journal of Education and Information Studies 7 no.2 (2011), http://escholarship.org/uc/item/9pt2490x.

Franklin, John Hope. Racial Equality in America. Columbia, MO: University of Missouri Press, 1993.

Gilliland, Anne J. "Pluralizing Archival Education: A Non-Zero-Sum Proposition.” Chapter 10 in Through the Archival Looking Glass: A Reader on Diversity and Inclusion, Mary Caldera and Kathy Neal, eds. (Chicago, IL: Society of American Archivists, 2014): 231-268.

Gilliland, Anne J. "Neutrality, Social Justice and the Obligations of Archival Educators and Education in the Twenty-first Century." Archival Science 11 nos. 3-4 (2011): 193-209.

Gilliland, Anne and Andrew Flinn, "Community Archives: What are We Really Talking About?" Keynote address, Nexus, Confluence, and Difference: Community Archives Meets Community Informatics: Prato CIRN Conference Oct 28-30 2013, Larry Stillman, Amalia Sabiescu, Nemanja Memarovic, eds. Centre for Community Networking Research, Centre for Social Informatics, Monash University, 2013. ISBN 978-09874652-1-4, http://www.ccnr.infotech.monash.edu.au/assets/docs/prato2013_ papers/gilliland_flinn_keynote.pdf.

Gilliland, Anne, Andrew Lau, Yang Lu, Sue McKemmish, Shilpa Rele, and Kelvin White. "Pluralizing the Archival Paradigm: Critical Discussions Around the Pacific Rim." Archives E̋ Manuscripts 35 no.2 (November 2007): 10-39.

Gilliland, Anne, Sue McKemmish, Zhang Bin, Kelvin White, Yang Lu, and Andrew Lau. "Pluralizing the Archival Paradigm: Can Archival Education in Pacific Rim Communities Address the Challenge?” American Archivist 71 no. 1 (Spring/Summer 2008): 87-117.

Gilliland, Anne and Kelvin White. "Perpetuating and Extending the Archival Paradigm: The Historical and Contemporary Roles of Professional Education and Pedagogy." InterActions 5 no.1 (2009), https://escholarship.org/uc/item/7wp1q908\#page-3.

Glazer, Nathan. "Blacks and Ethnic Groups: The Difference, and the Political Difference it Makes.” Social Problems 18 no.4 (1971): 444-461.

Johnson, Elizabeth Snowden. "Our Archives, Our Selves: Documentation Strategy and the Re-appraisal of Professional Identity.” American Archivist 71 (Spring/Summer 2008): 190-202.

Kallen, Horace. Cultural Pluralism and the American Idea: An Essay in Social Philosophy. Philadelphia: University of Pennsylvania Press, 1956.

Kakoulli, Loucas. The Left and the Turkish Cypriots: The Cyprus Problem from a Different Perspective. Nicosia: Cassoulides Press, 1990.

Linton, Ralph. The Cultural Background of Personality. New York: Appleton-Century Crofts, 1945.

McKemmish, Sue. “Are Records Ever Actual.” In Sue McKemmish and Michael Piggott, eds. The Records Continuum: Ian Maclean and Australian Archives: The First Fifty Year (Clayton: Ancora Press in association with Australian Archives, 1994): 187-203, http://www.infotech.monash.edu.au/research/groups/rcrg/publications/smcktrc.html

McKemmish Sue, Michael Piggott, Barbara Reed and Frank Upward. "Professing Archives: A Very Human Enterprise." In Archives: Recordkeeping in Society. Cambridge: Woodhead Publishing, 2005, pp.51-74. 


\section{Chapter it}

Millar, Laura. "Discharging our Debt: The Evolution of the Total Archives Concept in English Canada." Archivaria 46 (1998): 104-146.

Moran, Rachel E., and Devon W. Carbado. Race Law Stories. Foundation Press, 2008.

Omi, Michael. "Racial Identity and the State: The Dilemmas of Classification." Law \& Inequality XV no.1 (Winter 1997).

Omi, Michael and Howard Winant. Racial Formation in the United States. New York: Routledge, 1994.

Peterson, William. “Concepts of Ethnicity." In Concepts of Ethnicity: Selections from the Harvard Encyclopedia of American Ethnic Groups. Cambridge: Harvard University Press, 1982.

Raboteau, Albert J. Slave Religion: The "Invisible Institution" in the Antebellum South. Oxford: Oxford University Press, 1980.

Ross, Fiona, Sue McKemmish, and Shannon Faulkhead. "Indigenous Knowledge and the Archives: Designing Trusted Archival Systems for Koorie Communities." Archives and Manuscripts 34 no.2 (November 2006): 112-151.

Society of American Archivists. “*A* Census: Diversity Report,” 2005. http://www. archivists.org/a-census/reports/Banks-ACENSUS.pdf.

Slurink, Pouwell. "Causes of Our Complete Dependence on Culture." In The Ethological Roots of Culture, R.A. Gardner, ed. Dordrecht. Boston and London: Kluwer Academic Press, 1994.

Spade, Dean. Normal Life: Administrative Violence, Critical trans Politics, and the Limits of the Law. Brooklyn, NY: South End Press, 2011.

Štefanac, Tamara and Kelvin L. White, "The Representation, Rights and Identity of Croatia's Roma Community: Exploring the Archival Implications.” In Willer, Mirna, Anne J. Gilliland, and Marijana Tomic, eds, Records, Archives and Memory: Selected Papers from the Conference and School on Records, Archives and Memory Studies, University of Zadar, Croatia, May 2013, (Zadar: University of Zadar Press, 2015, in press).

Stephan, Nancy. 'The Hour of Eugenics,' Race, Gender and Nation in Latin America. Ithaca: Cornell University Press, 1991.

Telles, Edward E. "Racial Ambiguity Among the Brazilian Population." Ethnic and Racial Studies 25 (1998): 415-441.

Telles, Edward E. Race in Another America: The Significance of Skin Color in Brazil. Princeton: Princeton University Press, 2004.

Tilove, Jonathan. "Of Susan Guillory Phipps and Chief Redbone: The Mutability of Race." (July 9, 1992), Newhouse News service, http://jonathantilove.com/mutability-of-race/.

Trillin, Calvin. "American Chronicles: Black or White." New Yorker (April 14, 1986): 62-78.

Vogel, Todd, ed. The Black Press: New Literary and Historical Essays. New Brunswick: Rutgers University Press, 2001.

Wade, Peter. Blackness and Race Mixture: The Dynamics of Racial Identity in Colombia. Baltimore: The Johns Hopkins University Press, 1993.

Wade, Peter. Race and Ethnicity in Latin America. Chicago: Pluto Press, 1997.

West, Cornel. Race Matters. Boston: Beacon Press, 2001.

White, Kelvin L. and Anne J. Gilliland. "Promoting Reflexivity and Inclusivity in Archival Education, Research and Practice.” Library Quarterly 80 no.3 (July 2010): 231-248.

White, Leslie A. The Science of Culture. New York: Grove Press, 1949.

Williams, Raymond. "Base and Superstructure in Marxist Cultural Theory." In Schooling and Capitalism, Roger Dale, Geoff Esland, and Madeleine MacDonald, eds. London: Routledge and Kegan Paul with Open University Press, 1976. 
Chapter 12

\title{
INTRODUCING CRITICAL RACE THEORY TO ARCHIVAL DISCOURSE
}

\author{
Getting the Conversation Started
}

Anthony W. Dunbar

\section{(C) Springer Science+Business Media B.V. 2006}

This chapter was originally published under the same title in Archival Science 2006, 6 (1): 109-129. The digital rights to the chapter are owned or controlled by Springer, and unfortunately it was not possible to include this chapter in the digital edition of Research in the Archival Multiverse.

The chapter can be obtained through Springer at http://link.springer.com/article/10.1007/s10502-006-9022-6 or through purchasing a print copy of Research in the Archival Multiverse. 


\title{
Chapter 13
}

\section{WITHOUT A TRACE}

\section{Sexuality and the Colonial Archive ${ }^{1}$}

\author{
Anjali Arondekar
}

There were no papers, the ostensible reason for my visit, and of course, no trace of the Rani. Again, a reaching and an un-grasping.

- Gayatri Chakravorty Spivak, A Critique of Postcolonial Reason ${ }^{2}$

The past few decades of scholarship have witnessed a rich outpouring of critical thought on the colonial archive and its varied instantiations. For better or for worse, the turn to the archive is no longer the sacrosanct domain of the discipline of history. The archive has emerged as the register of epistemic arrangements, recording in its proliferating avatars the shifting tenor of academic debates about the production and institutionalisation of knowledge. As Foucault observed, the idea of the archive animates all

1 Reprinted from the Journal of the History of Sexuality "Methods, History, Praxis" special issue, 14 nos.1-2 (Winter/Spring 2005): 10-27.

2 This essay would not have been possible without many timely and productive conversations with Geeta Patel, Lucy Mae San Pablo Burns, Indrani Chatterjee and Gina Dent. Gayatri Chakravorty Spivak, "History" in A Critique of Postcolonial Reason: Toward a History of the Vanishing Present (Cambridge: Harvard University Press, 1999), 242. It is worth noting here that this chapter on "History" extends the arguments of an earlier piece, "The Rani of Sirmur: An Essay in Reading the Archives," History and Theory, 24 no.3 (October 1985), 247-72. In the earlier piece Spivak ends with the promise that she will "look a little further, of course. As the archivist assured me with archivistic glee: it will be a search" (270). The quotation cited at the beginning of this article illustrates the message of the earlier study, cautioning scholars once again about the dangers of reading the colonial archives as verifiable documents/signs of historical subjectivity. 
knowledge formations, and is the structure that makes meaning manifest. ${ }^{3}$ Jacques Derrida has termed the quest for such a meaning-making network, "le mal d'archive," or "archive fever." The literal and figural site of the archive both permits the "commencement" of and provides the "commandment" for intellectual labour. "Archive fever" expresses the craving for this archive, the desire to enter it and to procure it, even unto death. ${ }^{4}$ Such a deconstructive reading of the archive as a necessary and precarious repository of meaning has been embraced as well as resisted by historians and anthropologists. Social historian Carolyn Steedman reminds that the material deposits of the past (dust, in her case), whose affective reach exceeds all forms of theorisations, are the "real" drama of archive fever: "You think, in the delirium: it was their dust that I breathed in."

Even as the concept of a fixed and finite archive has come under siege, there has been an explosion of multiple/alternate archives that seek to remedy the erasures of the past. Scholarship in South Asia, in particular, has recast the colonial archive as a site of endless promise, where new records emerge daily and where accepted wisdom is both entrenched and challenged. In some ways, these archival expansions resemble the contours of the earlier canon wars in literary studies, as they question received notions of proof, evidence, and argumentation, particularly in fields involving historical inquiry.

Like other fields of inquiry, sexuality studies have turned to the colonial archive for legitimacy. Queer texts, topics, and themes have been discovered in the archive and examined exuberantly. The process of "queering" pasts has been realised through corrective reformulations of "suppressed" and misread colonial materials. ${ }^{6}$ These reformulations have intervened decisively in colonial historiography, not only decentring the idea of a coherent and desirable imperial archive but also forcing us to rethink colonial methodologies. Implicit in this rethinking, however, is the assumption that the archive, in all its multiple articulations, is still the source of knowledge about

3 Michel Foucault, The Order of Things: An Archaelogy of the Human Sciences (New York: Vintage, 1973), 15.

4 Jacques Derrida, Archive Fever: A Freudian Impression, trans. Eric Prenowitz (Chicago: University of Chicago Press, 1995), 1-6, 7-23.

5 Carolyn Steedman, Dust: The Archive and Cultural History (New Brunswick: Rutgers University Press, 2002), 19. There is much more to be said about Steedman's ambitious claims to reimagine cultural history through such readings of the archive. For one trenchant critique, see Jo Tollebeek, "'Turn'd to Dust and Tears': Revisiting the Archive," History and Theory 43 (May 2004), 237-248.

6 Ruth Vanita, ed., Queering India: Same-Sex Love and Eroticism in Indian Culture and Society (New York: Routledge, 2002), 1-14. 
the colonial past. The inclusion of oral histories, ethnographic data, popular culture, and performances may have fractured traditional definitions of the archive (and for the better), but the telos of knowledge production is still deemed more approachable through what one finds, if only one can think of more capacious ways to look.

I am not suggesting here that such archival modes are facilely flawed or merely enact a different order of archival truth claims. The new material on homosexuality does not purport simply to "correct" and/or reveal the truth about the history of sexuality in the colonial period. While there might be a certain evangelical flavour to some of the scholarship, most of the work indicates that the authors are keenly aware of the shifting parameters of space, time, and knowledge and of the role of the archive in such entanglements. David Halperin, for example, has often made a case for historicism in the study of sexuality, a historicism that would acknowledge the alterity of the past as well as the irreducible cultural and historical particularities of the present. The recent turn to geopolitics in sexuality studies has also highlighted historical differences across geopolitical sites, emphasising the uneasy and sometimes impossible portability of sexual categories. ${ }^{7}$ As a result of such deliberations, and as historical sources extend to include materials hitherto considered inappropriate and/or unreliable evidentiary paradigms are being reinvented.

Of interest is the fact that such archival turns still cohere around a temporally ordered seduction of access, which stretches from the evidentiary promise of the past into the narrative possibilities of the future. That is, even though scholars have foregrounded the analytical limits of the archive, they continue to privilege the reading practices of recovery over all others. Does this mean that the logic of the positivist archive is becoming the new dogmatism of our scholarship, unremitting and total in its analytical hold? And if so, how can we, as readers who continue to access and inhabit archives, formulate new reading practices that rupture such a logic? The intellectual challenge here is to juxtapose productively the archive's fiction-effects (the archive as a system of representation) alongside its truth-effects (the archive

7 See, for example, Lee Wallace, Sexual Encounters: Pacific Texts, Modern Sexualities (Ithaca: Cornell University Press, 2003); Licia Fiol-Matta, A Queer Mother for the Nation: the state and Gabriela Mistral (Minneapolis: University of Minnesota Press, 2003). There is, of course, a rich body of scholarship on sexuality and diaspora/ globalisation studies, but such work overwhelmingly focuses on analysis of contemporary issues, with colonialism appearing more as a referent than a sustained period of study. See, for example, Arnaldo Cruz-Malavé and Martin F. Manalansan IV, eds., Queer Globalizations: Citizenship and the Afterlife of Colonialism, ed.. (New York: New York University Press, 2003). 
as material with "real" consequences), as both agonistic and co-constitutive. These (new) reading practices, I suggest, must emerge not against the grain of archival work but from within it, except the imperative here is not about founding presence but more about confounding our understanding of how and why we do archival work. ${ }^{8}$

In this essay, I approach the possibility of a more differentiated archival logic through a consideration of the following questions: If the imperial archive is the sign of colonialism's reach, then what does the record show? How is the history of sexuality recorded in the colonial moment, and how can we return to that moment to produce, as it were, a counterrecord of that history? How does one think through the current privileged lexicon of erasure, silence, and recovery within a colonial context, such as that of nineteenth-century India, whose archival instantiations emphasise the centrality rather than liminality of the race/sex nexus? Or alternately, what epistemological imperatives undergird current scholarship? Is out critical history really distinct from the methods and fields of argumentation of the past? How can one accept sexuality studies' claims for innovative interdisciplinarity if the very turn to interdisciplinarity is an epistemological restaging of the colonial state? If the current turn in sexuality studies to divergent temporalities and spatialities assume "race" is an a priori marker of such divergence, and how is such a turn related to the racial logics of the colonial state? To explore these questions we must begin by examining the archival imperatives of recent scholarship.

\section{Lost and Found: The Archive as Open Secret}

We must always have a place

to store the darkness

- Agha Shahid Ali, A Nostalgist's Map of America9

The archive industry is booming, and especially so in studies of colonialism. Inspired in part by the intellectual provocations of the Subaltern Studies

8 In a related context Philippa Levine argues for an archival logic that offers "creative means to see past a dominant creed, not to uncover an impossible truth but to identify the very operations of power, both when it succeeds and, as interestingly, when it fails." See "Discipline and Pleasure: Response", Victorian Studies (Winter 2004): 325.

9 Agha Shahid Ali, A Nostalgist's Map of America: Poems (New York: W.W. Norton and Co. 1991), 49. 
group, the question of the archive and its formations has become a lively source of contention in South Asian historiography. The recovery of subaltern consciousness mandated a reassessment of the idea of what constituted the national archive, a site that had hitherto systematically erased the labour of subaltern groups in independence struggles. In many ways, to cite Ranajit Guha in a slightly different context, the failure of the Indian nation lay in its own historical amnesia. However, this condition could be mitigated by a new historiography, one that would make subalternity the focal point of narration. ${ }^{10}$ Guha's ecall was echoed in much of the early work of the Subaltern Studies group and later expanded beyond modes of recovery to include wider discussions of how myriad ways that colonial power had been mediated through structures such as the colonial archive. ${ }^{11}$

The recovery model of archival research was first criticised by Gayatri Spivak, who argued for a more self-reflexive analysis of the instrumentality of this new "subaltern" consciousness. ${ }^{12}$ Spivak's early critique made way for more capacious readings of the archive, as evidenced in the inclusion of such issues as gender, race, and culture in the more recent volumes of the Subaltern Studies group. ${ }^{13}$ While shifts in critical modes have occurred, the additive model of subalternity still persists, where even as the impossibility of recovery is articulated the desire to add, to fill in the gaps with voices of other unvoiced "subalterns," remains. One recalls here Bernard Cohn's playful warnings about the seduction of gaps in the record, incarnated in Philias Fillagap and Lucy Lacuna, a pair of anthropologists who attempt diligently to find the missing record, the unvoiced voices of the subalterns, without paying much heed to the epistemic questions at hand. ${ }^{14}$

10 Ranajit Guha, Elementary Aspects of Peasant Insurgency in Colonial India (Delhi: Oxford University Press, 1983).

11 See Dipesh Chakrabarty, Habitations of Modernity (Chicago: Univeristy of Chicago Press, 2002), Gyan Prakash, "The Impossibility of Subaltern History," Nepantla: Views from the South, 1 no.2 (2000): 287-294, and Tony Ballantyne, "Archive, Discipline, State: Power and Knowledge in South Asian Historiography," New Zealand Journal of Asian Studies 3 no.1 (June 2001): 87-105.

12 Gayatri Chakravorty Spivak, "Can the Subaltern Speak?" in C. Nelson and L. Grossberg, eds., Marxism and the Interpretation of Culture (Chicago: University of Illinois Press, 1988), 271-311.

13 There is clearly much more to be said about the debates and difference within the Subaltern Studies collective. For more detailed readings of the early shifts in the Subaltern Studies group see Saloni Mathur, "History and Anthropology in South Asia: Rethinking the Archive," Annual Review of Anthropology 29 (2000), 89-106.

14 Bernard Cohn, "History and Anthropology: The State of Play," Comparative Studies in Social History 22 (1980) 198-221. 
Several scholars of colonialism have questioned these archival assumptions and predilections. Thomas Richards has argued that the colonial archive (especially in South Asia) was based upon the belief that imperial knowledge was both "positive and comprehensive,"15 and Nicholas Dirks has contended that the colonial archive registers the state's increasing reliance on ethnography as form of knowledge. ${ }^{16}$ Feminist historians, such as Antoinette Burton and Betty Joseph, share some of Dirks' re-imagining of the colonial archive as both participant in and observer of the past (i.e., an agent of policy and a source of ethnography); however, they caution against "panoptical" readings of the archive. ${ }^{17}$ That is, to recognise the archive as the total (albeit precarious) site of colonial knowledge is still to succumb to a certain dangerous territoriality. Burton wonders why it is still so difficult for scholars of colonialism to detach themselves from the claims of an official archive. Such claims, Burton writes, sediment the contours of the archive as the standard through which disciplinary models are measured: "In this sense, guardians of the official archive - however delusional they may be remain as convinced of its panoptical possibilities as they do of its capacity to legitimate those who submit to its feverish gaze."18 Within such a policed state of knowledge, texts that fall outside the purview of official archives are read as flimsy evidence and historically specious - largely the conjectures of those engaged in too much cultural thinking. It is, Burton points out, no coincidence that such texts are usually gendered (as in the case of the writings of the three female colonial subjects she speaks of), and moored (or dwelling, to use her metaphor) in archives of their own making. While still holding on to the idea of an archive that will "surrender female subjects," both Burton and Joseph have initiated a much-needed critique and compel a wider and gendered understanding of the colonial archive.

Despite such a rise in archival consciousness, some scholars have observed that the turn to archival research remains largely "extractive," particularly in studies of colonialism. In the words of historical anthropologist Ann Stoler, students of "the colonial experience 'mine' the content of government

15 Thomas Richards, Imperial Archive: Knowledge and the Fantasy of Empire (London, New York: Verso, 1993), 7.

16 Nicholas Dirks, "Annals of the Archive: Ethnographic Notes on the Sources of History" in Brian Keith Axel, ed., Historical Anthropology and Its Futures: From the Margins (Durham: Duke University Press, 2002), 47-65.

17 Betty Joseph, Reading the East India Company, 1720-1840: Colonial Currencies of Gender (Chicago and London: University of Chicago Press, 2004), 1-32.

18 Antoinette Burton, Dwelling in the Archive: Women Writing House, Home, and History in Late Colonial India (New York: Oxford University Press, 2003), 137-45. 
commissions and reports, but rarely attend to their peculiar form and context." Hence, the need she writes, for scholars to move "from archive-as-source to archive-as-subject," to pay attention to the process of archiving, not just to the archive as a repository of facts and objects. ${ }^{19}$

While Stoler clearly articulates the limits of the archival imperative in colonial historiography, she is silent about, or rather detached from, similar questions in sexuality studies. This is especially curious given her remarkable readings of Foucault's oeuvre within the context of empire. While she speaks of sex, intimacy, and affect, she does not engage substantively with these issues as they are understood in sexuality studies. While I am not interested here in suggesting a corrective to Stoler's scholarship, I do wish to initiate a conversation between the archival imperatives of colonial historiography and those of sexuality studies. What can sexuality studies learn from the archival debates in colonial studies, and vice versa? Even as we ask, What kind of history the colonial archive have, can we not, following David Halperin, similarly ask, What kind of history sexuality has? ${ }^{20}$ Let me turn now to that question.

The historiography of sexuality (at least as practiced in the EuroAmerican academy) has often turned to the colonial archive. In many ways, as Philip Holden argues, there is a "profound connection" between colonial historiography and sexuality studies, one that derives less from a theoretical than a historical context. Both, Holden rightly suggests, "find the latter part of the nineteenth century a period of radical historical discontinuity." The late nineteenth century is the period that marks the intensification of imperial domains, territorial redistributions, and the rise of nationalist movements. ${ }^{21} \mathrm{It}$ is also the period, to follow Foucault's pronouncements, when homosexuality emerged as a set of identifications that articulated and differentiated sexuality's relationship to knowledge and power. Scholars in disciplines ranging from literature and anthropology (the more favoured locations), to law and science have held up the colonial archive as a storehouse of historical information about the secrets of sexuality's pasts.

19 Ann Laura Stoler, "Colonial Archive and the Arts of Governance," Archival Science 2 (2002): 87-109.

20 David Halperin writes: "Once upon a time, the very phrase 'the history of sexuality' sounded like a contradiction in terms: how, after all, could sexuality have a history? Nowadays, by contrast, we are so accustomed to the notion that sexuality does indeed have a history that we do not often ask ourselves what kind of history sexuality has" (How to Do the History of Homosexuality [Chicago: University of Chicago Press, 2002], 105).

21 Philip Holden, “Coda: Rethinking Colonial Discourse Analysis and Queer Studies," in Philip Holden and Richard Ruppel, eds., Imperial Desire: Dissident Sexualities and Colonial Literature (Minneapolis: University of Minnesota Press, 2003), 304. 
In a recent study, Colonialism and Homosexuality, Robert Aldrich identifies the perils of such efforts, writing that it is crucial to bear in mind that "colonial homosexuality did not proclaim itself openly." 22 Aldrich's scholarly efforts are largely aimed at revealing the secret lives of a range of male homosexuals across colonial sites, from E.M. Forster in Sri Lanka to lesser known figures such as Jean Sénac in Algeria. Aldrich's overall argument relies upon narratives of recovery (letters, memoirs) that operate, I would argue, through the logic of the "open secret." Homosexuality emerges as the structural secret of the archive, without whose concealment the archive ceases to exist. Alternately, the recovery of the hidden documents of homosexuality surrenders presence, but only to reinstate its archival liminality. To take some liberties with D.A. Miller's original formulations, writing the history of colonial homosexuality is ruled by the paradoxical proposition that the homosexual is most himself when he is most secret, most absent from writing - with the equally paradoxical consequence that such self-fashioning is most successful when it has been recovered for history. ${ }^{23}$ This movement from archival secrecy to disclosure echoes what Eve Kosofsky Sedgwick has famously called the "epistemology of the closet." ${ }^{24}$ Such a movement then relies on the maintenance within the epistemological system of the hidden, secret term, keeping all binaries intact.

While Aldrich focuses primarily on European sources, other writings on the relationship between history and homosexuality in non-European locations employ similar analytical models of recovery. Nayan Shah's muchcited early essay on sexuality and the uses of history in South Asia warns against an unmediated recovery of the past. He is still one of the few scholars of sexuality who question the dependence on a recovered history to sanction our surviving present: "We may trap ourselves in the need of a history to sanction our existence. South Asian lesbians and gay men are present now. On that alone we demand acknowledgement and acceptance." However, while maintaining that "the past is not a thing waiting to be discovered and recovered," Shah advocates strategies of historical research that derive from a differentiated language of loss and discovery. Shah must rely on the coming-out materials of his contemporaries (classic models of the logic of the secret) to think critically about the archives of the past. He grafts a lexicon

22 Robert Aldrich, Colonialism and Homosexuality (London and New York: Routledge, 2003), 404.

23 D.A. Miller. The Novel and the Police (Berkeley, 1988), 199-200.

24 Eve Kosofsky Sedgwick, Epistemology of the Closet (Berkeley: University of California Press, 1990), 1-64. 
of "resisting silences," "liberation," onto the project of archival research. In all fairness, it is important to note here that Shah's essay appeared in a now classic collection, A Lotus of Another Color (1993), which was the first of its kind to bring South Asian queer materials together. Its appearance in a collection of largely literary materials - fiction, poetry and personal memoirs - makes Shah's historiographical efforts all the more noteworthy. ${ }^{25}$

On the other hand, while the literary turn continues to produce innovative readings of sexuality and the colonial archive, it has been lambasted for its elision of extraliterary sources and its preoccupation with discursive tropes of representation. The privileging of literary materials yields too much discourse analysis, it seems, and too little engagement with historical documents. While multidisciplinary research on sexuality and colonialism has done much to overcome the backlash against the overdetermination of literary sources in sexuality studies, its success has been limited. Anne McClintock's much acclaimed Imperial Leather (1995), for instance, is heralded for deploying a range of cultural texts - advertisements, maps, and treaties, as well as fiction - and for invoking literature as only one of many sources. It is not that literatury sources are redeemed in such scholarly formats, but rather that they are placed in commensurate relationship to other sources.

Indrani Chatterjee warns against the pitfalls of disciplinary thinking, an analytic retreat that she characterises as one of the "more pernicious aspects" of colonial educational establishments in India. In nineteenthcentury colonial India, Chatterjee explains, the demarcation of the separate domains of "Literature" and "History" was created to stabilise the writing of history within a fixed form and method. Such a division masked the colonial establishment's inability to understand that precolonial history in South Asia, for example, was written primarily "in the dominant literary genre of a particular community, located in space, at a given moment in time." The slippages between history and literature became impossible to discern because through time, communities changed modes of literary production, and "when such a shift occurred, the earlier genre lost patronage as well as historicity, and became more "literary" (or was meant to be read that way). ${ }^{26}$ Since today's history becomes tomorrow's

25 Nayan Shah, "Sexuality, Identity and the Uses of History" in Rakesh Ratti, ed., $A$ Lotus of Another Color: An Unfolding of the South Asian Gay and Lesbian Experience (Boston: Alyson Publications, 1993), 122-24. See also Ruth Vanita and Saleem Kidwai, eds., Same-Sex Love in India: Readings from Literature and History (New York: St. Martin's Press, 2000).

26 Indrani Chatterjee, "Introduction" in Indrani Chatterjee, ed., Unfamiliar Relations: Family and History in South Asia (New Brunswick and London: Rutgers University 
literature, multidisciplinarity is a methodological requirement rather than a hermeneutical choice.

In anthropological writings on homosexuality and the colonial archive the archival turn has mandated a rethinking of the narrative of progress that left some disciplines as belatedly interested in theoretical questions of sexuality. As Kath Weston has demonstrated, the "classic debates which moulded social sciences into a distinctive set of disciplines relied, often as not, on illustrative examples drawn from sexuality." Colonial ethnographers such as Evans-Pritchard, John Shortt, and Malinowski used what Weston calls a "flora and fauna approach," producing scattered references to homosexuality in their varied writings on different geopolitical sites. Such references, Weston argues, have been viewed mistakenly as sources of empirical facts rather than as hermeneutic sign-posts for anthropology's early reliance on the instrumentality of sexuality to construct narratives of culture and power. ${ }^{27}$ Weston's complications notwithstanding, current scholarship still functions as a vexed, theoretical antidote to earlier models of a flawed, colonial geography of perversions. Rudi Bleys's ambitious study, The Geography of Perversion: Male-to-male Sexual Behaviour outside the West and the Ethnographic Imagination (1995), is one such example that interprets "male-to-male sexual behaviour among non-western populations in European texts between approximately 1750 and 1918.” Covering a dizzying and often haphazard array of colonial ethnographic materials drawn from Latin America, Africa, Asia, and Oceania, Bleys goes on an old-fashioned global hunt for the homosexual, with the occasional apology for not having enough materials by non-European subjects. ${ }^{28}$ The archival mode here shifts from savage-to-salvage, Bleys revisits colonial ethnographic/anthropological materials and mines them for their endorsements and descriptions of homosexuality in all its cross-cultural forms. Repeatedly in these crosscultural forays one finds a reliance on colonial ideas of alterity for the form and content of largely Western models of male homosexuality.

Elizabeth Povinelli is one of the few scholars who complicate such a reliance on the colonial archive by referring to the importance of what she terms "modal ethics." Through her work on Aboriginal communities

Press, 2004), 6-9.

27 Kath Weston, Long Slow Burn: Sexuality and the Social Science (New York: Routledge, 1998), 1-28.

28 Rudi Bleys, The Geography of Perversion: Male-to-male Sexual Behaviour Outside the West and the Ethnographic Imagination, 1750-1918 (New York: New York University Press, 1995), 1-16. 
in Australia Povinelli pushes the question of how and why we recover lost materials in the colonial archive. She believes that "who and what are being recuperated from the breach and shadow of the settler archive and colonial history" merit careful attention. Translating into text a ritual practice that functions through orality, for example, risks a return to the very knowledge technologies of colonial liberalism. Focusing on rituals that lift "sex out of corporeal practices," coheres sexuality to structures of knowledge. In such cases, Povinelli argues, scholars have an "obligation" to engage in what she calls a project of "radical interpretation." 29

In the remaining sections of this article I examine two archival traces drawn from the foundational sites of the colonial archive - law and anthropology - that require such interpretation.

\section{Habeas Corpus: Show Me the Body}

It cannot be doubted that such atrocities are frequent in the present day. A gentleman of the highest veracity assured me that a late Judge of Hooghly once mentioned to him that when about to sentence a native to imprisonment on proof of his having committed this crime in corpore capellae, he intimated his decision to the native jury, who hinted that, if so much severity was to be employed against so prevalent a crime, the prisons of Bengal would not large enough to hold the culprits. Convictions for this crime are however rare; I only find one in the Records - of Unnatural Crime with a Cow - at Dinagepore. Police Report, L.P., 1845, p.23

- Dr. Norman Chevers, A Manual of Medical Jurisprudence for India $(1870)^{30}$

For Norman Chevers, one of the leading colonial experts on medical jurisprudence, the discourse on unnatural sexual conduct in colonial India appears embedded in an evidentiary paradox: the known prevalence of the crime, and the equally known rarity of its documentation. That sexual perversion (e.g., homosexuality) was a condition of the colonial subject was

29 Elizabeth Povinelli, The Cunning of Recognition: Indigenous Alterities and the Making of Australian Multiculturalism (Durham: Duke University Press, 2002), 71-5.

30 Dr. Norman Chevers, A Manual of Medical Jurisprudence for India: Including the Outline of a History of Crime Against The Person in India (Calcutta: Thacker, Spink \& Co., Publishers to the University, 1870), 706. 
one of the familiar claims underwriting the project of colonial difference in India. Unlike representations of homosexuality in the metropole, in colonial India homosexuality was naturalised. It was a "frequent" phenomenon, though sparsely documented in the official archive - a "fact" corroborated, as Chevers noted, by a "native jury." Chevers's observations rendered native perversity intelligible through a foundational everywhere/nowhere model of colonial governance. Such a model scripted native perversity as ontological excess by employing the language of "proof," "veracity," and certainty even while bemoaning the colonial state's lack of official documentation. "Such atrocities" may indeed be everywhere, but "convictions are ... rare."

Chevers's description of an official archive denuded of all traces of a "crime" that must surely exist is uncannily echoed in contemporary scholars' analytical models of colonialism. In discussing homosexuality Ann Stoler and others reiterate the colonial dynamic they are attempting to overcome: homosexuality remains obvious and elusive - undeniable anecdotally (in colonial travelogues, ethnopornography, etc.), yet rarely substantiated in any official archival form. ${ }^{31}$ Is this indeed what the record shows, or do sexuality studies in the colonial period mandate a different order of archival reasoning?

The Indian Penal Code contains numerous citations to successful sodomy convictions. These appear in legal tables and case records compiled between 1860 and 1861, when the Penal Code and the Code of Criminal Procedure, respectively, were established, and 1920. The Judicial Statements (Criminal) for the North-West Provinces record that in 1879 forty-one persons were convicted for unnatural offenses, and seventy-two were still on trial. ${ }^{32}$ Similarly, the imperial returns for offenses reported and persons tried and either committed or acquitted in Punjab record that in 1874 sixty-two persons were convicted under Section 377 (the antisodomy statute). These same returns record that in 1880, thirty-eight persons were convicted, and fourteen remained on trial. ${ }^{33}$ However, the number of actual transcripts of cases and judgments available between 1860 and 1920 in the various colonial

31 Despite her claims, Stoler still stumbles over the "absent presence of the dangers of homosexuality" in Dutch archives. She speaks of the threat of homosexuality as a "deflected discourse, one about sodomitical Chinese plantation coolies, about degenerate subaltern European soldiers, never about respectable Dutch men," only to withdraw and admit that "my silence on this issue ... reflects my long-term and failed efforts to identify any sources that do more than assume or obliquely allude to this 'evil"' (Race and the Education of Desire: Foucault's History of Sexuality and the Colonial Order of Things [Durham: Duke University Press, 1995], 129, n. 96).

32 Oriental and India Office Collections (hereafter OIOC), L/PJ/6/26/1616 (1880).

33 Criminal Justice in the Punjab and its Dependencies,1869-81, 3 vols. (Lahore, 1892), Apps. 2 and 9. 
Presidencies, for the decades between 1860 and 1920 is much smaller. ${ }^{34} \mathrm{I}$ was able to find only five case records and judgments under Section 377 for the period: Queen Empress v. Naiada (Allahabad, 1875-78), Jiwan v. Empress (Punjab, 1884), Queen Empress v. Khairati (Allahabad, 1884), Sardar Abmed v. Emperor (Lahore, 1914), and Ganpat v. Emperor (Lahore, 1918). ${ }^{35}$

Of these cases, only one, Queen Empress v. Khairati, serves as the precedent and illustration of Section 377 in the various legal commentaries, digests and reports that are available from the period 1885 to $1920 .{ }^{36}$ Its use as precedent is perplexing: it is not the earliest of the five cases, it lacks important details, and it is the only one that ended with an acquittal (the other four cases were all successfully prosecuted). ${ }^{37}$ The particulars of the conviction as disclosed in an excerpt from the judgment of the sessions judge reveal that Khairati was initially arrested for "singing in women's clothes among the women of a certain family" of his village and was thereafter subjected to a physical examination by the civil surgeon. Upon examination, Khairati was shown "to have the characteristic mark of a habitual catamite - the distortion of

34 Worth considering here is the easier availability of sodomy cases in the records of the Nizamut Adawlut, and the Sudder Foujdaree Adawlut prior to the establishment of the Penal Code in 1860. For instance, I was able to locate over fifteen judgments between 1829 and 1859 in the Reports of Cases Determined in the Court of Nizamut Adawlut, 182750 (Calcutta: Thacker and Spink, 1851-59).

35 Queen Empress v. Naiada I.L.R (Indian Law Reports) 1 (Allahabad, 1875-78), 43-7; Jiwan v. Empress P.R.(Punjab Reports) (Punjab, 1884), 4; Queen Empress vs. Khairati, ILR (Indian Law Reports) 6 (Allahabad, 1884), 204-206; Sardar Ahmed vs. Emperor A.I.R. (All India Reporter) (Lahore, 1914), 565; and Ganpat v. Emperor A.I.R. (All India Reporter) (Lahore, 1918), 322.

36 The Khairati case continued to be cited past 1920, and, in fact, is still routinely referenced in current legal commentaries on Section 377. However, the post-1920 period in colonial India requires a more sustained discussion of Indian nationalism and its efforts at legal reform, which is beyond the parameters of this study. For more on contemporary debates on Section 377 in India see Suparna Bhaskaran, Detours of Decolonization (forthcoming).

37 While there has been a rich outpouring of scholarship on nineteenth-century homosexuality and criminality, most of it has focused on sites in the metropole. The critical difference of location makes the claims of that scholarship less applicable to colonial sites like India. For instance, Ed Cohen has written extensively about the Wilde trials, and William Cohen has provided deft readings of the failures of the Boulton-Parks sex scandals. However, both studies assume that in Victorian England homosexuality was regarded as aberrant and marginal, even though their own readings suggest the centrality of its presence. As I have previously mentioned, such a claim to "secrecy" and/or abnormalcy is untenable within the colonial context, where native sexual excess is assumed, even if archival evidence of that excess is ostensibly unavailable. See Ed Cohen, Talk on the Wilde Side: Towards a Genealogy of a Discourse on Male Sexualities (New York: Routledge, 1993) and William Cohen, Sex Scandal: The Private Parts of Victorian Fiction (Durham: Duke University Press, 1996). 
the orifice of the anus into a shape of a trumpet - and also to be affected with syphilis in the same region in a manner which distinctly points to unnatural intercourse within the last few months." When asked about his physical condition, Khairati denied all charges of sodomy and argued that he had suffered a serious case of dysentry, which caused the extension in his anus. His explanation was dismissed as insufficient, for it did not account for the presence of syphilis in the same region. The sessions judge, Mr. J. L. Denniston, concluded that while none of the three circumstances (wearing women's clothes, subtended anus, and the presence of syphilis) was sufficient evidence of criminality, taken together they left no "doubt that the accused had recently been the subject of sodomy."

However, when the case was later brought before the Allahabad High Court, Judge Straight (seriously!) quickly dismissed Khairati's earlier convictions for lack of precise detail about the particulars of the offense: the "exact time, place, and persons with whom these offences were committed" were not fully discovered. Judge Straight concluded his remarks on the case by declaring that while the "accused is clearly a habitual sodomite," and while he could "fully appreciate the desire of the authorities at Moradabad to check these disgusting practices, neither they nor he can set law and procedure at defiance in order to obtain an object, however laudable." ${ }^{38}$

How does one read the presence of the Khairati case within a historiography of sexuality and colonialism? How does a case that stumbles over critical issues of evidence, criminality, and legal codification become the colonial sign for crimes against nature? Such an archival turn, I would suggest, requires a theory of reading that moves away from the notion that discovering an object will somehow lead to a formulation of subjectivity from the presumption that if one finds a body, one can recover a person. Even as the discourse of law becomes the space of reform (e.g., current efforts to repeal Section 377), the very sign of the law as evidence needs to be examined. Such a reading practice would undo the current practice in contemporary sexuality studies of excavating in order to posit a history of presence. It would also complicate the additive model posited by the Subaltern Studies group, which attempts to mitigate or amend the failures/negations of nationalist historiography can be amended through an engagement with the voices of women, Dalits, and others.

One way to conceive of this shift to the object as subject-effect is to think of it as a trace, both beyond and within the Derridean spectrality 
model, and to consider, as it were, both the forensics and the metaphysics of that trace. ${ }^{39}$ One must work with the empirical status of the materials, even as that empirical status is being rendered fictive. With regard to the case of Queen Empress v. Khairati, every reading of its archival imprint requires a repetition of Khairati's forensic embodiments (a subtended anus), even as Khairati as subject cannot be found. The theoretical and historical provocation is to engage with the material imprint of archival evidence as "recalcitrant event" (to borrow Shahid Amin's term), "to move beyond the territory of the contested fact, the unseen record, from the history of evidence and into the realm of narration." ${ }^{40}$ Here, the "recalcitrant event" as trace eludes the historian/scholar's attempts at discovery, but offers new ways of both mining and undermining the evidence of the archive. I would push Amin's formulations further and suggest that to view archival evidence as recalcitrant event reads the notion of the object against a fiction of access, where the object eschews and solicits interpretative seduction.

\section{A Secret Report}

In the final pages of his famous translation of The Arabian Nights Richard Burton turned his attention to pederasty - "le vice contre nature." ${ }^{41}$ It is here that he first provided his readers with the scant but calculatedly sensational details of a secret government "report" on Kárachi's "three lupanars or bordels, in which not women but boys and eunuchs, the former demanding nearly a double price, lay for hire.” Having recently "annexed Sind," Gen. Charles Napier (the "Devil's Brother") authorised the report in 1845, specifically requesting Burton, "the only officer who could speak Sindhi," to "indirectly make enquiries and to report upon the subject." We are told that Kárachi was "not more than a mile from camp" and that Burton agreed to undertake the project "on express condition that the report should not be forwarded to the Bombay Government.” Disguised as a travelling merchant, Abdullah the Bushiri, Burton then proceeded to infiltrate Kárachi's multiple sites of

39 For more on the theory of the subject-effect see Gayatri Chakravorty Spivak, "Subaltern Studies: Deconstructing Historiography," in Ranajit Guha, ed., Subaltern Studies IV: Writings on South Asian History and Society (New Delhi: Oxford University Press, 1985), 330-63.

40 Shahid Amin, "Writing the Recalcitrant Event," edited and abridged transcript of talk given on 5 July 2001 at Remembering/Forgetting: Writing Histories in Asia, Australia and the Pacific (http://www/iisg.nl/ sephis/).

41 At this time, pederasty signified the larger terrain of sexual relations between men and did not rigidly denote intergenerational sex. 
"porneia" and to procure the "fullest details, which were duly dispatched to the Government House." However, Napier's departure from Sindh soon after resulted in Burton's report (along with two other "sundry reports" on Sindh that he had authored) being sent to Bombay by Napier's rivals. So scandalous were the contents of the report that its exposure resulted in Burton's "summary dismissal from the service." Burton provided no further details, either on the report's contents or on its current location. Or so the story goes. ${ }^{42}$

The mystery surrounding this lost report inaugurated a tale of archival losses that haunted Burton's entire career. Just as his career in India began (and failed) with the composition of an alleged report on male homosexuality, so was his death forty-five years later embroiled in controversies over lost records on the same subject. Burton, the story continues, became obsessed with translating the missing twenty-first chapter of The Perfumed Garden, reputed to be 500 pages of Arabic, which was to appear unexpurgated as The Scented Garden, a staggering treatise on homosexuality with " 882 pages of text and footnotes and a 100-page preface." Announcements of Burton's death in 1890 were accompanied with indignant accusations against his widow, Isabel Burton, the prime executor of his estate. The public consensus was that Isabel Burton had burnt the copious and much-awaited "Oriental" manuscripts in an effort to safeguard her husband's reputation against further criticism. In her own letter to the Morning Post in 1891 Isabel Burton fuelled public ire, acknowledging that the burnt materials were related to the same "certain passion" as was the Kárachi report: "His last volume of The Supplemental Nights had been finished and out on November 13, 1888. He then gave himself up entirely to the writing of this book, which was called The Scented Garden, a translation from the Arabic. It treated of a certain passion." ${ }^{43}$ In 1923, Norman Penzer, Burton's first bibliographer, chronicled the difficulty of finding suitable library space for Burton's writings and personal collections, a difficulty made more painful by the fact that many of Burton's original "Oriental" manuscripts had been destroyed previously at a fire in Grindley’s depository. ${ }^{44}$

42 Richard Burton, "Terminal Essay," in The Book of the Thousand Nights and a Night (London, 1886) 178-79. In this article I follow Burton's spelling of Kárachi.

43 Morning Post, 19 June 1891.

44 Norman M. Penzer, An Annotated Bibliography of Sir Richard Francis Burton K.C.M.G. (London: A. M. Philpot Ltd., 1923), 291-97. Penzer describes the difficulties he had in even procuring Burton's collections for libraries after the death of Isabel Burton. One of Burton's executors, Mrs. Fitzgerald, "started to cause endless trouble, and actually wanted to burn all the MSS. and books." 
That the archival myth surrounding the Kárachi report takes centre stage in the iconography surrounding Burton's lost works is abundantly clear. The report, as archival object, came into existence after all only through being lost. Its presence was sustained only through additional stories about its vanishing. The mystery surrounding its disappearance and/or existence has spawned endless speculation and debate. Several biographers of Burton concur that in 1845 Napier sought Burton's linguistic and spying skills for a singularly important report, but they provide different theories regarding its existence and circulation. Fawn Brodie contends that it was burnt by Isabel Burton along with all the other "peculiar" Burton manuscripts. Edward Rice and Glenn Burn suggest that the report, if there was one, was delivered orally and never existed as a written document. ${ }^{45}$ Christopher Ondaatje's hagiographical account, Sindh Revisited: A Journey in the Footsteps of Captain Sir Richard Burton (1990), zealously retraces and relives, as it were, Burton's formative years in India in the hope of finding the infamous report. ${ }^{46}$ Jonathan Bishop's article goes so far as to conclude that speculations about Burton's particular brand of participant-observation (a skill that earned him the title of "Dirty Dick") must be laid to rest, as he was clearly "uncircumcised" when he visited the Kárachi brothels, and thus could not risk participation for fear of exposure! ${ }^{47}$ In other words, the report's contents may well have

45 Fawn Brodie, The Devil Drives: A life of Sir Richard Burton (New York: Norton, 1967), 347. See also Edward Rice, Captain Sir Richard Francis Burton: The Secret Agent Who Made the Pilgrimage to Mecca, Discovered the Kama Sutra, and Brought the Arabian Nights to the West (New York: Harper Perennial, 1990), 128-30.

46 Christopher Ondaatje, Sindh Revisited: A Journey in the Footsteps of Captain Sir Richard Burton: 1842-49, the Indian Years (Harper Collins: Toronto, 1990). Ondaatje's efforts exemplify the celebratory fervour with which the life of Burton has been resurrected in the past few decades. As one reviewer says of this book, "Richard Burton and Christopher Ondaatje were bound to join up one day. The Intrepid, restless adventurer and the intrepid, restless entrepreneur are soul mates, and only the divide of time separated them. Now Christopher Ondaatje has solved that problem with his fascinating, sometimes moving, and often gripping account of the great Victorian explorer. Sindh Revisited is as intriguing in its exploration of Burton's obsessive need to push out into the 'unknown' world as it is in delineating Ondaatje's own need to push out beyond the restrictions of his own known world" (John Fraser, Master, Massey College, University of Toronto, as reported on www. ondaatje.com).

47 Jonathan Bishop, "The Identities of Sir Richard Burton: The Explorer as Actor," Victorian Studies, 1 no.2 (1957). Bishop's conclusions are drawn from a review of Burton's medical reports, which show no record of a circumcision in his annual medical examination, conducted in 1845. While Bishop's research is clearly thorough, his conclusions reveal a rather limited understanding of male-to-male sexual encounters, where the scene of "uncircumcision" functions as the definitive marker of Burton's anthropological innocence. 
been scandalous, but stories of Burton's own participation in the brothel's activities must be drastically revisited. James Casada, on the other hand, is less generous and caustically concludes that the details of the report were "nothing more than figments of Burton's fertile imagination." ${ }^{48}$

The available official records tell an equally perplexing tale of the report and Burton's relationship to its existence, and its deleterious effects on his army career. Richard Burton spent seven years in India, from mid-1842 to mid-1849, serving variously as an army field surveyor and intelligence officer. In 1843 he was appointed regimental interpreter and sent to the Eighteenth Bombay Native Infantry, stationed in Sindh, which had recently and most brutally been acquired as a British possession. There he served under Sir Charles Napier, who was the governor of the province until 1849. Burton's last year in India was spent recovering from sickness in the mountains of Goa, after which he was forced to return to England.

Burton's service record indicates that he was a model officer, and contains no mention of any scandal or unbecoming behaviour on his part. On the contrary, he is lauded for his fine efforts as a linguist and surveyor for the Bombay army. ${ }^{49}$ Burton may well have regarded his entire India career as a professional failure, but that story is not corroborated by the official records of the colonial state. ${ }^{50}$ Casada suggests that Burton may simply have "forfeited his commission for overstaying his leave" in Mecca (he was asked to return to India no later than March 1854), and Burton acknowledged as much in $A$ Pilgrimage to Mecca. ${ }^{51}$

My interest in recounting the story of the Karachi report and its dissemination lies not so much in debunking the articulated theories of its absence/presence but rather in identifying what is at stake in continuing the debate. The alleged "report," I suggest, is a dense textual palimpsest, less a record of native pederasty in India than evidence of a clash among the multiple colonial epistemes undergirding its evocation. One can argue that the reference to an Indian intelligence report within the translation of a foundational Arabic text simply renarrates that text, interpreting the mystical

48 James A. Casada, Sir Richard F. Burton: A Bibliographical Study (London: Mansell Publishing Ltd., 1990), 9.

49 Dane Kennedy, "Orientalist" in The Highly Civilized Man: Richard Burton and the Victorian World (Cambridge: Harvard University Press, 2005). I am grateful to Professor Kennedy for his informal comments on Burton in India and for sharing excerpts from his forthcoming book.

50 Z/L/MIL/5/21-22, 35, OIOC, L/MIL/12/73 (1842-51) OIOC.

51 Richard F. Burton, Personal Narrative of a Pilgrimage to Al-Madinab \& Meccah, ed. Isabel Burton, with an introduction by Stanley Lane-Poole (London, 1898), 29. 
world of the Arabian Nights through recourse to colonial empiricism. ${ }^{52}$ The extensive representations of pederasty in ancient erotic texts are overlaid by a "report" that proves that native pederasty is real and lives outside the pages of Burton's translation. Yet, while such a gesture corroborates the presence of native vice, it equally, or perhaps more stridently, invokes the scandal of British participation in such activities. After all, Burton tells us, Napier ordered the report because the brothels are a mere "mile from the camp."

Napier himself articulated related concerns around the widespread presence of "infamous beasts" in a memoir recorded for Sir John Hobhouse in 1846: There is public morality supported by putting down the infamous beasts who, dressed as women, plied their trade in the Meers' time openly; and there is this fact to record, that the chief of them were recipients of stipends from the Ameers, as the government records I became possessed of as collector testified." 53

Napier's reference here to "government records" indicates clear and official fore-knowledge of such "immoral activities" amongst native subjects and rulers. Not only was the native populace prone to le vice, but native rulers lent it state support. The Kárachi report became a mediating form through which the excesses of the primitive cover over any excesses of the civilised. But while the focus remains on the "infamous beasts," the fear of moral contamination was never far behind.

Indeed, what the report does is underscore the grids of intelligibility within which claims of both presence and absence have been asserted and questioned. But what would happen if we were to shift archival attention from the ultimate discovery of this report to understanding the compacted role its evocation plays? What if we were to consider the report less as a lost archival object and more as an embedded sign whose evidentiary status (as an official product of state intelligence) decisively links sexuality, colonial anthropology, and governance? The salacious detail, after all, is lodged not in a marginal footnote, but in the body of the text, in an official form that mandates legitimacy and attention. What would it mean then to abandon our fascination with the contents of the report, and to turn our attention, as it were, to the secrets that are encrypted in the form itself? And finally, what would it mean to resituate this historiographical metalepsis and to read the

52 See Colette Colligan, “'A Race of Born Pederasts': Sir Richard Burton, Homosexuality, and the Arabs," Nineteenth-Century Contexts 25 no.1 (2003): 1-20.

53 Lt. Gen. Sir W. Napier, The Life and Opinions of General Sir Charles James Napier (London: John Murray, 1857), 28. 
report instead as an archival trace that resurfaced in muted terms in Burton's later writings, as the haunting sight of the male nautch?

\section{Coda: Limits and Politics}

The traveler wandering from town to town forgot the path to his house. What was mine, what was yours, both of the self and of the other, lost, then, to memory.

- Miraji, Tin rang ${ }^{54}$

If it is by now evident that the colonial archive has emerged as the centre of interpretation and contestation in the historiography of sexuality, it is equally clear that the structure of the archive is necessarily inchoate. There is always a politics of the archive, as Thomas Osborne suggests, because rarely is it a simple matter of revealing secrets that are waiting to be found. The continued efforts of the Hindu right to mobilise the idea of the "archive" toward sectarian ends (most aggressively through the rewriting of history text-books) is a dangerous instantiation of the very logic to which I am referring. ${ }^{55}$ As I have suggested, archives are untenable without readers, and "across the gap between the archives and its motivating interests there is a perpetual agonism. ${ }^{16}$ What are the political stakes embedded in this relentless consumption of the idea of the archive? Is the relationship between the colonial state and the archive undone or merely refurbished through our intellectual labour? Achille Mbembe notes that despite all efforts to democratise and widen the arc of the archive, as it were, the archive still survives as a talisman, as a sort of "pagan cult" where the powers of the

54 "Nagari nagari phira musafir ghar ka rasta bhul gaya/ ... kya hai mera kya hai tera apna paraya bhul gaya," in Miraji, Tin rang (Pindi: Kitab Numa, 1968), 151. The cited translation of Miraji's poem is provided by Geeta Patel in her wonderful book, Lyrical Movements, Historical Hauntings: On Gender, Colonialism, and Desire in Miraji's Urdu Poetry (Stanford: Stanford University Press, 2001), 32. Patel writes: "Miraji was an acclaimed Muslim male poet, who wrote under a Hindu woman's name, and whom contemporary critics described as mad, sexually perverse, and a voyeur. Miraji's short life (1912-49) spanned the final period of British colonialism in South Asia, and his work played a part in the nationalist struggle" (3-15).

55 For more details on the text-book controversy see Romila Thapar, "The Future of the Indian Past," Outlook India, April 1, 2004.

56 Thomas Osborne, "The Ordinariness of the Archive," History of the Human Sciences 12, no.2 (1999): 51-64. 
archive recreate through a inventive but uncannily similar logic the original act of creation. The debt of the colonial state is paid off through its archival debris, where deaths of the past are breathed into life through the archives of the present. Mbembe speaks specifically of the case of South Africa, where the artifactualisation of memory through the idea of the archive as talisman "softens the anger, shame which the archive tends" because of its function of recall. ${ }^{57}$ Sexuality studies are an equal accomplice in such archival mythmaking and must remain alert to its own methodological and analytical foibles. Not to do so would be to forgo the histories of colonisation, to brush aside the possibilities and impossibilities accorded by the idea of an archive.

57 Achille Mbembe, "The Archives and the Political Imaginary," in Carolyn Hamilton, Verne Harris, eds., Refiguring the Archive (Cape Town: David Philip, 2002), 20-37. 


\section{Part III}

Overviews of the Application of Research Methods in Archival Studies 


\title{
HISTORICAL CASE STUDIES OF PRE-MODERN EUROPEAN ARCHIVES
}

\section{A Comparative Approach}

\author{
Randolph C. Head
}

\begin{abstract}
Despite an abundance of descriptive studies, very little comparative research on archival practice in pre-modern Europe has been carried out. Spurred by this observation, the author carried out a comparative study of early modern archives in Switzerland, and is currently carrying out a larger comparative study of cases from across Europe between 1400 and 1800. Initial questions about what can be learned from the ordering methods used by pre-modern archival personnel were stimulated by the research of medievalists and the Marburg school. The Swiss case revealed a seeming progression from listing to mapping to taxonomy as primary approaches to organising repositories. Expanding this approach to cases across Europe required narrowing the phenomena considered specifically to finding aids, since otherwise the amount of material would have become overwhelming. When carried out, comparative evidence showed that the pattern emerging from the Swiss case represented only one pathway within a heterogeneous European terrain. Comparative analysis thus improves our ability to understand both individual cases and larger contexts. It enables clearer perception of the features of particular archives or repositories, while helping the researcher avoid pitfalls such as reading too much into the features of a particular accumulation or assuming that local peculiarities are representative of a larger pattern.
\end{abstract}




\section{Introduction}

Comparison is a powerful method of historical investigation that offers valuable results when applied to the history of archives. By comparing multiple cases from an appropriately selected universe, historians can discern phenomena not visible through the investigation of a single records repository, such as the transfer of methods and approaches from one centre or region to another and the constraints and opportunities all archival personnel in a given period and region experienced. In addition, a comparative approach helps us avoid pitfalls such as reading too much into the features of a particular accumulation or assuming that local peculiarities represent a larger pattern. This essay will illustrate these points by weaving together three levels of analysis: first, a discussion of past research on the history of archives in Europe and the scope (or more often lack) of comparison in such work; second, the general methodological issues that comparison raises, as well as the particular challenges and constraints that the comparative study of premodern archives involves; and third, a description of a current project (carried out by the author) investigating the tools, or finding aids, created and used in a selection of European archives from the late fifteenth to the early eighteenth century. The genealogy of the specific project illustrates both the opportunities and the risks of the comparative approach for academic historians as well as for archival scientists and practicing archivists.

\section{The Research Landscape: Phenomena and Approaches in Historiography}

\section{The European Archival Landscape, 1400-1800}

European political units, from the papacy and the Holy Roman Empire to kingdoms, cities, monasteries, and even villages, have been accumulating stored records in various ways since the High Middle Ages. The quantity and scope of document creation and accumulation varied tremendously, however, while hair-raising encounters with fires, theft, transfers and simple abandonment to water, insects, and mould led to very different degrees of preservation. Not only were medieval document collections unstable, but record-making and record-use also changed over time, and always included practices well beyond the forensic uses of documents as evidence. Issuing charters often involved claims to status, and displaying old documents could 
dramatise the antiquity and respectability of claims to dominion. ${ }^{1}$ No matter the purposes, the end result by the early fifteenth century was a landscape of documents gathered into collections ranging from a few charters in a chest to extensive records facilities containing not only annotated original documents but finding aids such as indexed copy-books and chronological registers that allowed for facilitas inveniendi (ease of finding) when a particular document was needed. ${ }^{2}$

In addition to the larger cultural shifts that characterised the end of the European Middle Ages - the Renaissance movement, the European discovery of the Americas and the growth of maritime trade to Asia - the fifteenth century also experienced major changes in how documents were produced and preserved in Western Europe. The introduction and rapid spread of paper manufacturing made the production of documents less costly for a wide variety of users, leading to more rapid accumulation as well. ${ }^{3}$ Among those users were political entities of all kinds, which eagerly deployed the newly affordable medium to record a wide range of administrative information that had previously been handled orally, or recorded in evanescent media not suitable for longer-term preservation. Indeed, the rapid expansion of bureaucratic administration in this period rested on the coevolution between new media available for record-keeping and new methods of governance; the result was much greater production of governmental records. Many of these survived for shorter or longer periods, if only because of the durability of paper and its tendency to pile up unless action or neglect leads to its destruction. In the fifteenth century, many polities thus found themselves in possession not only of a growing volume of documents in organised form (whether in boxes and bags or bound or copied into record books of various kinds), but also of additional "archives" that were little more than haphazard accumulations of

1 The literature on medieval record-making, -keeping, and -using is extensive and sophisticated. A foundational work is M. T. Clanchy, From Memory to Written Record: England 1066-1307, 2 ${ }^{\text {nd }}$ ed. (Oxford: Blackwell, 1993). A lovely illustration of what could lie behind the creation of charters appears in Peter Brun, Schrift und politisches Handeln. Eine 'zugeschriebene' Geschichte des Aargaus 1415-1425 (Zurich: Chronos, 2006). See more generally the collection edited by Karl Heidecker, Charters and the Use of the Written Word in Medieval Society (Tournhout: Brepols, 2000).

2 The term taken from Helmut Zedelmeier's essay on alphabetisation in the late Middle Ages, "Facilitas inveniendi: The Alphabetical Index as a Knowledge-management Tool," Indexer 25 no. 4 (2007): 235-242.

3 See the observations in Ann Blair, "Introduction," in Archival Knowledge Cultures in Europe, special issue of Archival Science, 10 no. 3 (2010): 195-200, and more generally, R. J. Lyall, "Materials: the Paper Revolution," in Book Production and Publishing in Britain 1375-1475, ed. J. Griffiths J and D. Pearsall (Cambridge: Cambridge University Press, 1989), pp.11-29. 
material deposited in basements, towers and other storerooms. ${ }^{4}$ Sooner or later, magistrates and their officers had to decide what to do about such material and their responses form a perfect opportunity for comparative analysis. The project under discussion here carries out such a comparison, with particular focus on the evolution of finding aids for a period stretching from the late fifteenth to early eighteenth century.

\section{Archives and Scholarship from the Early Modern Period to the Present}

Changes in archival accumulation and organisation in the early modern period were accompanied by changes in history-writing, which increasingly included stored administrative records among the sources it drew upon. ${ }^{5}$ Before the nineteenth century, however, archival history was confined largely to internal documentation in particular archives and to brief and quite general discussions of the value of building effective archives that were published in the context of early guides to and legitimations of archival practice. Early practitioners such as von Ramingen, Bonifacio, Aebbtlin, and Mabillon discussed past record-keeping practices in their writings, but any comparisons remained largely abstract or implicit. ${ }^{6}$ Theoretical discussions by such authors as Seckendorff and Leibniz also evoked archives' and libraries' pasts without studying these systematically. ${ }^{7}$ More ambitious late eighteenth-

4 Nicholas Popper provides a vivid description of such haphazard accumulation in the Tower of London as late as 1600 in "From Abbey to Archive: Managing Texts and Records in Early Modern England," in Archival Knowledge Cultures in Europe, special issue of Archival Science, 10 no.3 (2010): 249-266, esp. 259-60.

5 See Markus Friedrich, Die Geburt des Archivs: Eine Wissensgeschichte (Munich: Oldenbourg Verlag, 2013), esp. 231-276.

6 Early published works on the theme are rare. They include: Jacob von Ramingen, Von der Registratur/Und ihen Gebäwen und Regimenten / deßgleichen von ibren Bawmeistern und Verwaltern / und jrer qualifcationen und habitibus (Heidelberg: Mattheus Harnisch, 1571) and other works; Baldassare Bonifacius, "Liber de Archivis" (ed. and tr. Lester K. Born), American Arcbivist 4 no.4 (1941): 221-237; Georg Aebbtlin, Anfübrung Zu der Registratur=Kunst /Vorderist denen Hoch=Loblichen Herrschafften und Obrigkeiten zu underthänigen Bericht und Erinnerung (Ulm: Christian Balthasar Kühnen, 1669); and parts of Jean Mabillon, De re diplomatica libri VI (Paris: Billane, 1681), especially Book I, Chapter II.

7 Veit Ludwig von Seckendorff's influential manual of administrative practice, Teutscher Fürsten-Stat / Oder: Gründliche und kurtze Beschreibung / Welcher Gestalt Fürstenthumer / Graf= und Herrschafften im Heil. Röm. Reich Teutscher Nation / ... regieret...zu werden pflegen (Frankfurt: Thomas Matthias Götz, 1660), discusses record-keeping in Part I, Ch. 6 and Part II, Ch. 6; Gottfried Wilhelm Leibniz drafted several essays on the theme, published in his Politische Schriften: Band 3, 1677-1689 (Berlin: Akademie Verlag, 1986). 
century projects for the publication of charters also required some comparative consideration of the archival history that had resulted in their contemporary dispersion, although their authors rarely considered archives per se a subject of historical investigation. ${ }^{8}$

In the nineteenth century, the emergence of new approaches to historical research and thinking - above all, the document-centred "scientific" history promoted by Leopold von Ranke and the German seminar model - made archival research a central and necessary element in serious scholarship by valorising documents (Urkunden und Akten) above chronicles and other forms of narrative sources. In the same era, the post-Napoleonic nation-states increasingly began identifying national history, pursued in the archives, as a primary site for legitimation and cultural unification. ${ }^{9}$ One result of this confluence was the emergence of a new discipline of archivistics, generally conceived as an "auxiliary science" of history as well as a type of service to the state. Archivists were increasingly trained in historical methods, and expected to carry out historical research of their own in addition to their duties in managing and preserving existing records. ${ }^{10}$ The complex fabric of intellectual and personal relations between academic historians and historically-trained archivists, analysed recently by Francis X. Blouin and William G. Rosenberg, shaped the writing about archives undertaken by both. ${ }^{11}$

Since archivists typically worked on their own collections and had been trained in the positivist and particularist tradition of nineteenth- and early twentieth-century historiography, they rarely took comparative approaches directed toward larger historical questions. Rather, the most common form of publication consisted of histories of specific archival accumulations by their own curators, either as freestanding publications or as introductions to published guides for using the collection in question. A second important genre that emerged mostly in the twentieth century consisted of synoptic

8 See the discussion in A. Hessel, "Zur Geschichte der Regesten," Archiv für Urkundenforschung 10 (1928): 217-225.

9 Work on nineteenth-century archival formation is burgeoning. See for example Benjamin Kafka, The Demon of Writing: Powers and Failures of Paperwork (New York: Zone Books, 2012); Lara J. Moore, Restoring Order: The Écôle des Chartes and the Organization of Archives (Duluth: Litwin Books, 2008); and Jennifer Milligan, Making a Modern Archive: The Archives Nationales of France, 1850-1887 (Dissertation, Rutgers, 2002) (Ann Arbor: UMI, 2002).

10 A nice example of an archivist explaining why he and his peers should carry out historical research is contained in Paul Schweizer, "Geschichte des Zürcher Staatsarchivs," Neujahrsblatt zum Besten des Waisenhauses in Zürich 116 (1894): 2-40.

11 Francis X. Blouin and William G. Rosenberg, Processing the Past: Contesting Authority in History and the Archives (Oxford: Oxford University Press, 2011). 
works that surveyed archival history as part of larger overviews of the field of archivistics. Whether oriented more to theoretical questions, like Casanova and Bautier, or oriented more towards training archivists in a particular national context, like Brennecke and Meisner, such studies relied on knowledge about many archives to generalise about historical developments, and thus contained an implicit comparative element. ${ }^{12}$

Historians - in the new disciplinary sense that emerged in the nineteenth century - also wrote little about the history of the archives they used for their research. One may speculate that historians' dependence on archives as the foundation for their work, and their training in methods of source criticism that privileged the individual document as the object of critical reflection, tended to silence critical approaches to archives, or to "the archive" as whole. ${ }^{13}$ Only after French critical theory, most notably in Michel Foucault's work, appropriated the concept of the archive as a key term for postmodern theories of knowledge did some historians - especially those facing archives that silenced their subjects, such as feminist and postcolonial historians - begin reflecting on archives as historical subjects, rather than as sites of research. This development represented part of the so-called cultural turn, in which historians took a more self-conscious approach to their own research practices in various ways. ${ }^{14}$

This was the situation in the late 1990s when the fortuitous discovery of a fascinating source motivated me to begin studying early modern archives

12 Eugenio Casanova, Archivistica (Siena: Stab. Arti Grafiche Lazzeri, 1928); Adolf Brennecke, Archivkunde: Ein Beitrag zur Theorie und Geschichte des europäischen Archivwesens (Leipzig: Kohler \& Amelang, 1953); Heinrich Otto Meisner, Urkundenund Aktenlehre der Neuzeit (Leipzig: Kohler \& Amelang, 1950); Robert-Henri Bautier, "La phase cruciale de l'histoire des archives: la constitution des dêpots d'archives et la naissance de l'archivistique (XVIe - XIXe siècle)," Archivum: Revue Internationale des Archives 18 (1968): 139-149.

13 On the emergence of diplomatics and its initial relationship to historiography, see Alfred Hiatt, "Diplomatic Arts: Hickes against Mabillon in the Republic of Letters," Journal of the History of Ideas 70 no.3 (2009): 351-373; and Randolph Head, "Documents, Archives and Proof Around 1700,” Historical Journal 56 no. 4 (2013): 909-930.

14 See e.g., Carolyn Steedman, Dust: The Archive and Cultural History (New Brunswick: Rutgers University Press, 2002); Antoinette Burton, ed., Archive Stories: Facts, Fictions and the Writing of History (Durham and London: Duke University Press, 2006); and Randolph Starn, Varieties of Cultural History (Goldbach: Keip Verlag, 2002), which contains two essays specifically interrogating the relationship between history-writing and archival history. Feminist and post-colonial scholarship has been especially important in putting 'the archive' in question: e.g., Ann Laura Stoler, Along the Archival Grain: Epistemic Anxieties and Colonial Common Sense (Princeton: Princeton University Press, 2009); Thomas Richards, The Imperial Archive: Knowledge and the Fantasy of Empire (London: Verso, 1993); and Miles Ogborn, India Ink: Script and Print in the Making of the East India Company (Chicago: University of Chicago Press, 2007). 
and their inventories. A review of the historical and archivistical literature at the time revealed that much of it was oriented towards particular documents or archives, and that most work with a broader focus pertained to the Middle Ages. Only a smattering of work addressed early modern archives as phenomena that could cast light on broad issues of historical debate especially in the context of the new cultural history - including early modern state-formation, the history of memory, the history of legal and political discourses, or the political and institutional cultures of the early modern era. Since the 1990s, however, a flood of valuable work has emerged, and research is only accelerating. Focused research groups in German-speaking Europe have been particularly active, notably at Münster, Zurich, Munich, and Bielefeld, and historians' work on archives is now far more extensive than it was even a decade ago. ${ }^{15}$

\section{Comparative Methods in Archival History: A Case Study}

\section{Challenges for Comparison in History-Writing}

Comparative methods are applicable in a wide range of disciplines in the human sciences, and cover a wide range of approaches. In historical studies, comparison is often an implicit methodological element even in studies that are not explicitly comparative: explanations anchored in a particular time and place often function in part by reference to similar phenomena in other contexts, even if the comparative method is not deployed as a primary research strategy. Explicit comparison in historical research most often takes the form of case studies, which preserve the historical discipline's concern for context and particularity while allowing comparisons among properly contextualised phenomena across cases. Because historians typically compare their cases in context, rather than comparing measurements of particular quantifiable variables taken across contexts, specifying the evidence to be compared and its level of abstraction is critical for successful comparison: useful comparison requires comparing things that are similar in significant ways. Because of

15 The developments are illustrated by two special issues of Archival Science, in 2007 and 2010 respectively, which contain a broad range of papers by historians addressing the theoretical challenges of archival history and demonstrating how they can be addressed. See Ann Blair, ed., Toward a Cultural History of Archives, special issue of Archival Science 7 no. 4 (2007); and Randolph Head, ed., Archival Knowledge Cultures in Europe, 1400-1900, special issue of Archival Science 10 no.4 (2010). 
historians' attention to context as well as direct evidence historical comparisons are also particularly vulnerable to the "apples-and-oranges" critique: are the cases under consideration sufficiently similar that we can usefully compare their differences? These considerations take concrete form when considering the development of my current project to compare archival organisation and archival finding aids in early modern Europe.

In the mid-1990s, while pursuing a different project on institutional culture in early modern Switzerland, I encountered a singular and fascinating source document, the Index Archivorum Generalis that Zurich's city secretary (and future Burgermeister), Johan Heinrich Waser, completed in 1646. ${ }^{16}$ This substantial quarto volume contains not only an overview of the 475 boxes that Waser established for his chancellery in the course of reorganising the city's documents, but also a synoptic catalogue of the material found in all of the city's offices, and a ten-page manuscript discussion of the issues he confronted in carrying out this work. ${ }^{17}$ Out of this remarkable source grew first a project to compare archival organisation across multiple Swiss cases, then a larger one to extend similar comparisons across Western Europe.

\section{Theoretical Approaches to the Zurich Inventory}

Having decided to analyse this document's significance for the political and institutional culture of early modern Switzerland, I immediately sought to contextualise it by finding historical literature on archival organisation in the same region. This led me to the work of Peter Rück, a major figure in German archival science and author of numerous works about the production, preservation, and organisation of documents in Savoy and Switzerland. ${ }^{18}$ Rück brought a powerful theoretical as well as empirical approach to his work, with roots in East German archival theory of the 1950s and 1960s. Drawing on Marxist theories of base and superstructure, East German scholars such as Ingo Rösler had proposed that the order found in archival

16 Staatsarchiv Zürich, Kataloge 11.

17 As my later research has revealed, such reflective commentary by working archive professionals in the early modern period is extremely rare. The text is analysed in the context of other archives in the region and period in Randolph Head, "Knowing Like a State: The Transformation of Political Knowledge in Swiss Archives, 1470-1770," Journal of Modern History 75 no.4 (2003): 745-782.

18 Peter Rück, "Zur Diskussion um die Archivgeschichte: Die Anfaenge des Archivwesens in der Schweiz (800-1400)," Mitteilungen der Vereinigung Schweizerischer Archivare 26 (1975): 3-40; idem, ed., Mabillons Spur: Zweiundzwanzig Miszellen aus dem Fachgebiet für Historische Hilfswissenschaften (Marburg an der Lahn: Institut für Historische Hilfswissenschaften, 1992). 
accumulations necessarily constituted a reflection (Wiederspiegelung) of class relations and power structures in the society that produced the records involved. ${ }^{19}$ Rück idealised this concept by arguing that "archived sources are a self-interpretation, a self-mirroring of the dominant powers" in a society, and that one goal of archival history was therefore to determine "according to what order and selection dominant powers transmitted the totality of their selfrepresentation." ${ }^{20}$ The "ideal-topographical" system that Rück postulated for Savoy was also visible in Waser's description of the arrangement of archival boxes in Zurich. Such a system of organising archival material turns each archive into a miniature representation of larger political structures. Rück's crucial insight was that the historian could use inventories and even the arrangement of archival material as sources, in and of themselves.

Rück's theoretical claims, and his brilliant analysis of a major reorganisation and inventory of the archives of the Dukes of Savoy in the 1440s known as the Clairvaux Register, thus provided guidance about how to read the 1646 Zurich inventory as a map of political concepts in its maker's mind. At this point, any comparisons my project made between the Zurich inventory and other accumulations were still implicit, however. I was using other cases - in particular, Savoy - primarily to reveal salient features of the Zurich inventory, rather than building set of phenomena for comparison or mapping variation among multiple cases.

\section{From Context to Comparison: Multiple Swiss Cases}

The next step, clearly, was to make comparison an explicit method for further research by bringing in additional cases from the same region. After consultation with informed colleagues and review of the available material, I carried out archival investigations of inventories from the fifteenth through the early eighteenth century across German-speaking Switzerland, including in Aarau, Chur, Lucerne, and in a number of family archives. For each case, research was restricted to a few genres of documents, with a primary focus on old inventories and the papers of early modern archivists and their work, if these existed. I was able to contextualise this evidence because of my familiarity with the politics and political culture of early modern Switzerland, and

19 See for example Ingo Rösler, " $Z u$ einigen Fragen der marxistischen Archivwissenschaft," Archivmitteilungen 17 no.6 (1967): 222-229; and Helmut Lötzke, "Methodologische Probleme der marxistischen Archivwissenschaft," Archivmitteilungen 17 no. 6 (1967): 216-22.

20 Rück, "Die Ordnung der herzoglich savoyischen Archive unter Amadeus VIII. (1398-1451)," Archivalische Zeitschrift 67 (1971): 11-101, here p. 11, p. 12. 
by reading in secondary literature about the institutions and political conjunctures surrounding each inventory I examined. Such restrictions on evidence are an integral part of comparative research for two reasons. First, limiting research to specific document types ensures that the results will be comparable: for this project, inventories represented an easily identifiable genre with shared formal characteristics, which could be compared with confidence that the creators of these texts had similar intentions and goals. Second, expanding the geographic and temporal scope of any project without simultaneously limiting its view to fewer, carefully chosen phenomena for each case, will inevitably produce unmanageable amounts of information.

The collection of evidence from a larger universe of Swiss cases, in turn, allowed for a more precise definition of the phenomena I was interested in comparing, and more expansive insights into the particular cases. The development of historical comparative case studies almost always involves such inductive discoveries, as the project moves past preliminary definitions based on a single case. My initial work with the Waser inventory had immediately revealed that Waser's work was rich in patterns, but without either a theoretical or comparative context, it was not possible to fully recognise these patterns, much less assess their significance. Only contextualisation through the theoretical perspective found in Rück's work made it apparent the Waser had drawn on a shared conceptual approach - the 'ideal-topographical' as defined by Rück - in laying out the 475 boxes in his system. Many other inventories I examined showed signs of an ideal-topographical approach, in which conceptual categories, archival spaces, and inventory organisation all mirrored one another. Only after comparing multiple cases did it become possible to analyse the differences in how the ideal-topographical approach was implemented in the various cases, and to correlate such differences in a systematic way with the political authority that the magistrates who created each inventory exercised. Comparison thus revealed the flexibility of ideal-topographical inventorisation, and how it could be adapted to different political contexts. ${ }^{21}$

However, not all Swiss inventories I examined were ideal-topographical. Rather, a clear temporal pattern in the use of the ideal-topographical method emerged. Before the early sixteenth century, the inventories I saw did not use this method, relying instead on the simple listing of records, sometimes in chronological order, but also according to diverse ordering principles or to no principles at all. Such early inventories also covered much less material, concentrating primarily on sealed parchment charters, without including the administrative material (Akten) that Waser's and other ideal-topographical

21 Full discussion in Head, "Knowing." 
inventories encompassed. By the late seventeenth-century, another distinct approach appeared, as exemplified by the 1698 archival reorganisation that took place in Lucerne. ${ }^{22}$ The Lucerne project broke radically from idealtopographical mapping by creating a set of inventory categories based on the actions of a coherent state. An abstract taxonomy of action replaced the mapping of physical spaces against archival spaces that lay at the core of ideal-topographical organisation.

Comparison of Swiss cases thus revealed a second level of analysis, one more abstract than that derived from comparing one ideal-topographical inventory with another. My research had revealed that the ideal-topographical system was only one possibility for early modern Swiss archivists. One can therefore also compare the trajectory of its use - early adoption, widespread use, eventual abandonment - to the deployment of other systems, such as listing and taxonomy. As a historian, I could then fruitfully correlate the results of this comparison with the changing self-understanding of Swiss magistrates as their polities evolved from a loose Confederation of autonomous privileged corporations within the Holy Roman Empire to a sovereign state recognised by the European concert of nations. ${ }^{23}$ The final result of this project - beginning with a single source, contextualising it with relevant historical and theoretical literature, then expanding the range of analysis inductively by adding comparisons to further cases - was a multilevel comparative study that illuminated Swiss institutional culture from the fifteenth to early eighteenth centuries.

\section{From Comparison to Narrative: The Dynamics of an Expanded Comparative Project}

\section{Designing a European Comparative Project}

The project's next step was to broaden the range of cases across a substantial portion of Western Europe. This promised to increase the salience of any

22 Analysed in Head, "Knowing," and in greater detail in Randolph C. Head, "Mirroring Governance: Archives, Inventories and Political Knowledge in Early Modern Switzerland and Europe," Archival Science 7 no.4 (2008): 317-329.

23 The historical context is analysed most comprehensively in Bernhard Stettler, Die Eidgenossenschaft im 15. Jahrhundert: Die Suche nach einem gemeinsamen Nenner (Zurich: Verlag Markus Widmer-Dean, 2004); and Thomas Maissen, Die Geburt der Republik: Staatsverständnis und Repräsentation in der frühneuzeitliche Eidgenossenschaft (Göttingen: Vandenhoek \& Ruprecht, 2006). 
comparative results about state building, archivists' conceptions of order, and the influence of developments in other fields of knowledge. Switzerland, after all, is both small and politically atypical for the early modern period. Such broadening again required narrowing the scope of the evidence that I considered from each case. An enormous literature exists on the political structures and administrative history of each European region: full consideration of such material for a wide range of cases would swamp even the most dedicated reader. Moreover, the range of variation in how European states kept their records is considerable, leading to greater and greater challenges to comparability. Additionally, the preservation of and access to inventories is also very uneven, and as a pragmatic matter, my familiarity with various languages, hands, and historical backgrounds became an additional limitation.

Ultimately, I chose to limit myself narrowly to three genres of sources: (1) inventories and finding aids, (2) papers from the internal operation of an archive where available, and (3) published contemporary guides to the organisation and operation of archives. Additionally, I concentrated on moments when a new archival order was established in a given institutional context, since these were most likely to illustrate contemporary approaches to the challenge of managing stored records. Finally, I chose my cases from a variety of political entities, from cities to monarchies to trading companies, but all from regions with connections to the Habsburg dynasty, including Iberia, Switzerland, Germany, and the Netherlands. The final study will thus be necessarily incomplete: Italy, France, and England each possess a rich archival history of their own, and future scholars will test any conclusions I reach against what they find in these traditions. Every comparative case study faces similar challenges in selecting cases and defining the range of evidence from each case to consider: the number of personnel available and their skills, the time available, and the goals of the project as a whole all shape the resulting decisions.

\section{Evidence Reshapes the Comparative Framework}

The course of several years' research in multiple archives across Europe once again reshaped the comparative project. As had been the case for the Swiss study, further theoretical reading and inductive conclusions led me to refine the scope of my comparisons, the specific phenomena under study, and the range of conclusions I will later argue. Ultimately, the greater range of cases transformed the narrative that emerged from a comparison of Swiss archives. 
The evolution from lists to maps to taxonomies found in Switzerland did not hold up for Europe as a whole; rather, the inclusion of more cases led to a narrative at a higher level of abstraction, in which different combinations of organising tools appeared, which early modern record-keeping personnel deployed in flexible and heterogeneous ways. The expansion of my universe of cases thus transformed my narrative: a story about lists, topography, and taxonomies in Switzerland turned into a story about a European archival landscape characterised by multiple entangled paths of evolution in the sixteenth and seventeenth centuries, even though well-established confluences existed at the beginning and end of the narrative.

The confluence at the beginning of the narrative was a shared stock of "little tools of knowledge"24 available to most European document managers by the fifteenth century. At the end of the narrative in the eighteenth century, the emergence of archival professionals with a specialist literature and standard methods represented a second moment of confluence. In between, however, European archives were characterised by considerable heterogeneity in their organisation. This heterogeneity, I argue, resulted primarily from the growing volume of documents to manage and from the declining circulation of knowledge about how to manage them - a result of increased concern for secrecy and less movement of chancellery staff after the mid-fifteenth century. To illustrate this early modern diversity, I will briefly discuss three specific cases that show how early modern records managers approached their work using different combinations of inventories, indexes, and registers. Early efforts to bring order to existing collections in Innsbruck and Würzburg reveal how differently inventories and indexing could work together, thus highlighting the flexibility that agents in the early sixteenth century enjoyed. I will then summarise a more ambitious effort to create a recordkeeping system, the Hofregistratur established in Innsbruck after 1560, which built on and transcended older registry techniques.

\section{Two Early Sixteenth-Century Cases: Wilhelm Putsch and Lorenz Fries}

Early modern chancery secretaries and archivists faced enormous challenges but they did not invent their methods from scratch. Medieval practices for managing charters and letters through registers and cartularies, often in

24 The term is defined and introduced in Peter Becker and William Clark, eds., Little Tools of Knowledge: Historical Essays on Academic and Bureaucratic Practices (Ann Arbor: University of Michigan Press, 2000). 
tabular form or with indexes, provided serviceable tools for the new challenge of organising administrative documents on paper. Scholastic efforts to systematise knowledge produced another set of tools initially designed to organise knowledge in books, such as tables of contents, foliation, systematic excerpting, and alphabetised cross-references. ${ }^{25}$ The basic methods involved seem to have been widely distributed across Europe by the late fifteenth century, setting a shared baseline for early modern divergence. While they may seem mundane, such "little tools" can have important consequences.

Major efforts to organise existing documents and make them accessible through inventories (taking the term broadly) took place across Europe during the fifteenth and sixteenth centuries, often in tandem with administrative reforms and expanded chancelleries. ${ }^{26}$ Two examples from the early sixteenth century illustrate how existing techniques could be applied to this task in quite different ways.

From the 1510s to 1550 s, Wilhelm Putsch served the Austrian Habsburgs in the Habsburg depositories in Innsbruck and Vienna by creating monumental inventories with indexes for the administrative documents that had accumulated in the Schatzgewölbe (arched vaults) beneath the chancellery offices. In Innsbruck, Putsch inventoried approximately 30,000 separate items stored in 177 boxes, filling five volumes. ${ }^{27}$ His inventory mirrored the collection he worked with, with each inventory volume (colour coded by its binding) corresponding to one storage case, and listing that case's contents box by box. ${ }^{28}$ Since the material itself was arranged rather haphazardly, Putsch's

25 Simon Teuscher has connected techniques found in medieval law books to archival practice. See especially his "Document Collections, Mobilized Regulations, and the Making of Customary Law at the End of the Middle Ages," Archival Science 10 no.3 (2011): 210-230. On alphabetisation, see Lloyd W. Daly, Contributions to a History of Alphabetization in Antiquity and the Middle Ages (Brussels: Latomus, 1967); and Zedelmaier, "Facilitas inveniendi." On Scholastic tools, see Richard Rouse and Mary Rouse, "Statim Invenire: Schools, Preachers, and New Attitudes to the Page," in Renaissance and Renewal in the Twelfth Century, ed. Robert L. Benson and Giles Constable (Oxford: Clarendon Press, 1982), pp.201-225.

26 Otto Stolz, "Archiv- und Registraturwesen der oberösterreichischen (tirolischschwäbischen) Regierung im 16. Jahrhundert," Archivalische Zeitschrift $42 / 43$ (1934): 81-136, here p. 87, points to the wave of organising accumulated charters and papers in the German lands in the early sixteenth century.

27 Tiroler Landesarchiv [TLA], Repertorium B 368-373. The calculation in Stolz, "Archiv- und Registraturwesen," p. 92. Oskar Stowasser documents Putsch's career, including his missions from Innsbruck to Vienna and the condition of the Vienna accumulations before he began his work: "Das Archiv der Herzöge von Österreich,” Mitteilungen des Archivrates 3 no.1 (1919): 15-62.

28 TLA, Repertorium B368 (the index volume for Innsbruck), fol. 1r describes the colour-coding. Although the armoires corresponding to each inventory volume gathered 
inventory volumes were not particularly useful by themselves. He therefore also produced a comprehensive index to his inventory volumes. Indexing, rather than listing, represented a major step in controlling the information in a collection, but it also raised challenges. How did one choose the headings in an index and how did one arrange these? Putsch followed scholastic best practice by giving documents multiple entries when appropriate, with each index entry placed after the appropriate multi-letter stub, usually of three phonemes, which had been inked on blank index sheets before he began. ${ }^{29}$ For domains and people, he used proper names as the basis for indexing, instead of descriptors such as first names, "castle of," etc., which he placed on the left of the main term. The result was a powerful tool that allowed both searching and browsing of massive document accumulations, one that drew on the medieval tradition of chancellery registers while extending it to new kinds of materials and new medial forms.

The case of Würzburg in the same period offers an alternative approach to bringing order to an existing collection. The challenge was similar: the prince-bishops of Würzburg had a secular administration that had produced a heterogeneous mass of documents by the early 1500s, which defied intuitive access. Many important records appeared in or were copied into books held in the chancellery, known as the Libri Diversarum Formarum, but others lay loose in boxes stored in the inaccessible castle strong room across the river on top of a hill. From the 1520s through the 1550s, the bishops invested heavily in their administrative apparatus, creating a number of separate secretariats. The task of making accessible the information in the Libri and in the castle fell to Lorenz Fries, a chancellery secretary with a knack for organisation.

In contrast to Putsch, Fries chose not to assemble a comprehensive set of inventories or shelf-lists that mirrored the documents in their locations. Rather, he created only a multidimensional index for the prince-bishops' collection, with no central inventory. The resulting three-volume finding

documents belonging to a single action-category, at least in principle, actual documents stayed in haphazard subgroups according to the vicissitudes of their accumulation. See in general, Otto Stolz, Geschichte und Bestände des Staatlichen Archives zu Innsbruck (Vienna: Adolf Holzhausens Nachfolger, 1938), which gives considerably more detail than Wilfried Beimrohr, Das Tiroler Landesarchiv und seine Bestände (Innsbruck: Tiroler Landesarchiv, 2002).

29 Alphabetisation by one to three letters was well established in the high Middle Ages: see Daly, Contributions, p. 69; Zedelmaier, "Facilitas." Putsch's use of three-letter stubs is most clearly visible in his index for the Görz archive inventory, but the same principle seems to have functioned in his Innsbruck and Vienna indexes. See the illustration in Stowasser, "Archive," p. 47. 
aid, known as the Hohe Registratur, consisted of an extensive set of headings, with entries for each heading pointing both to documents located in the castle and to relevant material recorded in the Libri. ${ }^{30}$ While many of the headings referred to places - domains, villages, and farms - they also included people, topics such as "apothecaries," and even general categories of action such as "treaties." Fries' finding aid was thus organised around genres of information, not around pieces of paper. Moreover, the Hohe Registratur made no effort to mirror the physical location of the documents in its own structure; rather, the three volumes simply covered the alphabet from A to $Z$. Both the table of contents and the body of each volume contained crossreferences to other headings. For some headings, Fries also provided a brief historical overview before listing the relevant documents in the bishops' collection, turning the Hohe Registratur into a kind of historical gazetteer and encyclopaedia, as well. ${ }^{31}$ Fries' work thus had antecedents in medieval concordances, though he too had to adapt and extend these methods to respond to the particular circumstances of Würzburg. ${ }^{32}$

The result of Fries' efforts was an information-management tool so effective that it remained in constant use until late in the eighteenth century. However, its very efficiency in providing quick documentary references for every domain and village and for many general topics meant that it also limited knowledge, especially about the inconvenient boxes in the bishop's castle, which had no comprehensive inventory. The bishop's council usually relied on Fries' index, only occasionally ordering a "full search" in the castle. ${ }^{33}$ By concentrating attention on those documents that Fries indexed, the Hobe Registratur thus effectively decreased the number of easily accessible records in the prince-bishop's archive. After all, the user disappointed from the results of using Putsch's Innsbruck index volumes could still browse in the inventories, but Fries' decision to skip the inventory phase left no such option.

When we compare these two cataloguing and organising efforts - the comprehensive inventory with supplementary index undertaken by Putsch in Innsbruck, and the index without inventory that Lorenz Fries created in Würzburg - what stands out are the differences in how they deployed the same

30 Staatsarchiv Würzburg, Standbücher 1011, 1012, 1014.

31 For further detail, see the discussion in Thomas Heiler, Die Würzburger Bischofschronik des Lorenz Fries (Gest. 1550): Studien zum historiographischen Werk eines fürstbischöflichen Sekretärs und Archivars (Würzburg: Schöningh, 2001).

32 I thank Paul Saenger for this insight. See on concordances, Rouse and Rouse, "Statim Invenire," 211-12; and Bella Hass Weinberg, "Book Indexes in France: Medieval Specimens and Modern Practices," The Indexer 22 no.1 (2000): 2-13.

33 Dr. Jens Martin, Würzburg archive staff, personal communication, February 2008. 
standard techniques. Each confronted document storage in books, armoires and boxes, and each created book-format guides to the collections' contents that combined subject categories and alphabetisation. Neither Putsch nor Fries rearranged the actual collections involved by some master plan. ${ }^{34}$ Putsch made inventories that mirrored the messy physical arrangement of the documents, making an index essential. This index - an index to the inventory, not an index to the collection itself - represented a significant step in separating knowledge in documents from knowledge about documents. In Würzburg, in contrast, Lorenz Fries' Hohe Registratur made no effort to mirror the heterogeneous collection, resting instead on a comprehensive system of categories about the political world - places, people, actions - which it organised alphabetically. Here, too, an index became the essential finding aid, but in a different way. Fries also abstracted the process of information finding differently from Putsch, and made no inventory to accompany his index. ${ }^{35}$ This made the Hohe Registratur highly effective for finding the specific loci he indexed, but at the cost of obscuring other documents.

\section{New Archival Approaches from the Late Sixteenth Century: Innsbruck, 1564}

Each of the cases above involved the retrospective organisation of accumulated charters and other documents. What these approaches did not do, however, was to manage the burgeoning output of the new sixteenthcentury bureaucracies. A further case shows how medieval techniques of recordkeeping could be reconfigured to this end: the so-called Hofregistratur that began operating in Innsbruck in the 1560 s. $^{36}$ The German term for early modern systems that managed documents in this way is Registratur, or registry; this echoes the older term Register from which registries drew many techniques - but a registry fulfilled a fundamentally different function, and indeed created new challenges for archivists.

34 In Savoy in the 1440s and in Zurich in 1646, however, reorganisations of materials did accompany the creation of new finding aids.

35 Fries and his assistants created volume indexes for the Libri Diversorum Formarum, and a summary index and box indexes to the castle collection. See August Schäfller, "Die Urkunden und Archivalbände des hochstiftlich wirzburgischen Archives im 16. Jahrhundert," Archivalische Zeitschrift (1885): 141-57 and (1886): $19-52$.

36 The term "Hofregistratur" was applied by Otto Stolz in his 1934 and 1938 discussions; in the Innsbruck archives this rubric has been re-entitled the "Ober-österreichsche Hofrat”; see Beimrohr, Das Tiroler Landesarchiv, pp. 87-88. 
The newly appointed Archduke Ferdinand II in Innsbruck anticipated an ongoing flow of documents once he took up his new post in $1564 .{ }^{37} \mathrm{In}$ response, the administrative chancelleries in Innsbruck deployed a new system that broke decisively from the practices observable in Putsch's inventories. ${ }^{38}$ As soon as Ferdinand acceded as ruler of the Tyrol, his secretaries began operating the new document registry, which became the foundation for a system that continued without major changes until $1667 .{ }^{39}$ Three steps defined the management of every incoming or outgoing document from the Archduke's chancery: storage in a chronological series, registration in a running registry volume, and indexing in the registry volume's index. The simplicity of the storage scheme is striking, and drove the other aspects of the system. Most incoming material - including letters from petitioners, reports from various agents, correspondence with other chanceries, orders to subordinates - was directed into only two document series, one fiscal and one political, which in turn were divided only into incoming and outgoing documents. ${ }^{40}$ Documents from the four resulting streams were bundled together monthly and stored in annual fascicles, with each document placed by the date of its issue - which meant that adjacent documents usually had no connection to one another. The formal dimension of chronology largely determined the actual storage of documents in this system.

Consequently, an indexing process was essential for making documents available, since a document's location and contents were entirely unrelated.

37 Ferdinand was the younger son of Emperor Ferdinand I, and brother to Emperor Maximilian II. He administered Bohemia on his father's behalf from 1547-1564, and became Archduke of Tirol on his father's death. He remained in Prague until 1567, however, although his registry in Innsbruck began operation in 1564. The registry continued to operate after his death in 1595.

38 The genealogy of the Hofregistratur is not yet clear: it may draw on earlier Tyrolean practices, especially in fiscal administration, but also shows interesting similarities to Venetian practice. See esp. Otto Stolz, Geschichte der Verwaltung Tirols (Innsbruck: Universitätsverlag Wagner, 1998), 28-32, who suggests that strictly chronological deposition of documents with concurrent registration arose earlier in the $16^{\text {th }}$ century.

39 The system is described in great detail in Stolz, "Archiv- und Registraturwesen," 107-113. I have worked carefully with the early volumes in the series to examine the indexing principles. TLA, Hofrat, Journale/Protokolle, Einkommene Schriften, Series R, vol. 1; Einkommene Schriften, Series K, vol. 25; Konzeptbücher, Series R, vol. 49; Konzeptbücher, Series K, vol. 73 (all from 1564 to 1566).

40 I am simplifying here, since from the outset, material on certain important topics went into special series by subject, enabling the chancery staff to browse, rather than having to go through the considerable trouble of pulling documents one-by-one from multiple locations. Stolz, "Archiv- und Registraturwesen," discusses the limitations of purely chronological storage of documents. There were some 186 special rubrics. Beimrohr, Tiroler Landesarchiv, p. 100. 
On its arrival or before its issuance, each document received an entry in one of four running registry volumes, which corresponded to the four main document series. To make the registry itself usable, each document simultaneously received multiple entries in an alphabetical index at the beginning of each volume. To find documents about a particular person, domain or subject, therefore, one first searched the index, which led to the summary in the registry, which in turn gave the date, which ultimately located the document. The Hofregistratur's operations thus absolutely depended on systematic indexing - that is, on the production of information about information, or metadata - to allow its users to move from a category to a summary to a document.

Innsbruck possessed a particularly well-articulated example of a technique that emerged, mutatis mutandis, in various parts of Continental Western Europe in the sixteenth century. ${ }^{41}$ Early modern registry was rooted in medieval indexed registers for the emission of charters or letters, but radically expanded these to manage multiple parallel flows of documents as part of a comprehensive system. It is worth noting how labour-intensive such expansion was. In Innsbruck, every document had to be analysed, registered and placed in sequence, and the index had to be constantly updated. Ferdinand's personal chancery alone employed fourteen men by 1600, including a chancellor and vice-chancellor, four secretaries, and eight scribes. ${ }^{42}$ Even so, disorder crept in constantly. The tendency of later archivists to extract documents also steadily sapped the underlying coherence of the system, while the proliferating registry volumes themselves became unwieldy.

\section{Conclusion}

Comparative examination of the finding aids from pre-modern document collections reveals the power of the tools available to contemporary archivists while also revealing how diversely they could be deployed. Considerable additional research is needed before we will fully understand how changes in archival practice were connected to changes in political institutions and

41 Stolz, "Archiv- und Registraturwesen," 113, laments the lack of comparative studies, and few have emerged since then. Most recent is the extremely useful analysis by Thea Miller, "The German Registry: Evolution of a Recordkeeping Model," Archival Science 3 (2003), pp. 43-63, which focuses on the Prussian variant, which has important differences. On early registry in Brandenburg, Joachim Lehmann, "Registraturgeschichtliche und quellenkundliche Aspekte älterer Kanzleiregister," Archivmitteilungen 26 no.1 (1976): 13-18.

42 Stolz, Geschichte der Verwaltung, 31. 
culture, and to broader cultural transformations. The following comments represent only a first attempt to recharacterise the overall trajectory. In contrast to the relative homogeneity of fourteenth and fifteenth century practice in most Western European political chancelleries, we find increasing structural variation emerging in the sixteenth and seventeenth centuries. While the scattered evidence on their intentions suggests that archivists always sought to create finding aids that reflected their collections, we find little consistency in how they used the suite of approaches available to them, and little commitment to any single way of connecting the political world, their collections, and their finding tools. Only when archiving began to be professionalised in the second half of the eighteenth century did convergence of both methods and conceptions slowly return, encouraged by the publication of guides and other professional literature. The richness and variability of early modern practices (which could easily be illustrated through further cases) also points toward changing conceptions of law and the emergence of a published literature of chancery practice, especially in the Holy Roman Empire, as spurs for the convergence on more standard techniques that began in the eighteenth century. ${ }^{43}$

In my own research, it was expanding the scope of my comparisons from Switzerland to Habsburg Europe that forced me to generate this more abstract narrative of historical change in archival inventories in early modern Europe. As my research continues, I will continue to connect the choices that archivists in specific situations - not just Putch in Innsbruck and Fries in Würzburg, but three generations of royal secretaries in Lisbon, the Ayala family in Simancas, and many more - to their institutional and cultural environment. Expanding comparisons, as we have seen, both enriches our understanding of each case compared and helps us build larger historical narratives. Equally, however, further comparison may require further revision of my comparative framework itself, or may lead to revisions of the larger narrative as further evidence emerges.

More generally, this essay argues that comparison through case studies is an effective tool for archival science and archival history, expanding what we can learn from examination of archival inventories and other finding aids across multiple cases. Archival inventories are particularly suited to comparison, precisely because they share both formal and contextual similarities that make them comparable: despite the many differences that also become visible, they are more oranges than apples, at least within a 
single cultural tradition. ${ }^{44}$ Nevertheless, we must not forget that comparisons also benefit from inductive discoveries made during the examination of each case in context, which refine the definitions of the things being compared. Comparative research is thus always also recursive. Further comparative studies of archives and their organisation should thus expect to follow different paths, each one shaped by the initial starting point of their authors, the cases chosen (for both theoretically-justified and pragmatic reasons), and the discoveries made during the course of research. Like other humanistic methods, therefore, comparative historical studies of archives are openended contributions to an ongoing conversation among scholars that allows for new perspectives and moves on to different topics as our own intellectual context changes.

\section{References}

Aebbtlin, Georg. Anfübrung Zu der Registratur=Kunst / Vorderist denen Hocb=Loblichen Herrschaften und Obrigkeiten zu underthänigen Bericht und Erinnerung. Ulm: Christian Balthasar Kühnen, 1669.

Bautier, Robert Henri. "La Phase Cruciale de L'histoire Des Archives: La Constitution Des Dêpots D'archives et La Naissance de L'archivistique (XVle - XIXe Siècle).” Revue Internationale Des Archives 18 (1968): 139-49.

Becker, Peter, and William Clark, eds. Little Tools of Knowledge: Historical Essays on Academic and Bureaucratic Practices. Ann Arbor, MI: University of Michigan Press, 2001.

Beimrohr, Wilfried. Das Tiroler Landesarchiv Und Seine Bestände. 47. Innsbruck: Tiroler Landesarchiv, 2002.

Benson, Robert Louis, Giles Constable, and Carol Dana Lanham. "Statim Invenire: Schools, Preachers, and New Attitudes to the Page." In Renaissance and Renewal in the Twelfth Century, 201-25. Oxford: Clarendon Press, 1982.

Blair, Ann. "Introduction." Archival Science 10, no. 3 (December 8, 2010): 195-200.

Born, Lester. "Baldassare Bonifacio and His Essay De Archivis." The American Archivist 4, no. 4 (October 1, 1941): 221-37. doi:10.17723/aarc.4.4.36u35457n6g45825.

Brenneke, Adolf. Archivkunde: Ein Beitrag zur Theorie und Geschichte des europäischen Archivwesen. Leipzig: Kohler \& Amelang, 1953.

Brun, Peter. Schrift Und Politisches Handeln: Eine "Zugeschriebene" Geschichte Des Aargaus 1415-1425. Zurich, Chronos Verlag, 2006.

Burke, Peter. Varieties of Cultural History. Goldbach: Keip Verlag, 2002.

Burton, Antoinette. Archive Stories: Facts, Fictions, and the Writing of History. Durham, NC: Duke University Press, 2006.

Casanova, Eugenio. Archivistica. Siena: Stab. Arti Grafiche Lazzeri, 1928.

Clanchy, Michael T. From Memory to Written Record: England 1066-1307. Oxford: Blackwell, 1993.

44 Whether finding aids can be compared similarly across largely separate cultural traditions is an open question I hope to address in the future. If, as seems likely, archiving served different agendas and took place through divergent methods and subject matter, then finding aids might be comparable only at a relatively high level of abstraction. 
Daly, Lloyd William. Contributions to a History of Alphabetization in Antiquity and the Middle Ages. Brussels: Latomus, 1967.

Friedrich, Markus. Die Geburt Des Archivs: Eine Wissensgeschichte. Munich: Odenbourg Verlag, 2013.

Head, Randolph. "Knowing Like a State: The Transformation of Political Knowledge in Swiss Archives, 1450-1770." The Journal of Modern History 75, no. 4 (2003): $745-82$.

Head, Randolph C. "Documents, Archives, and Proof Around 1700.” The Historical Journal 56 no. 4 (2013): 909-30.

Head, Randolph C. "Mirroring Governance: Archives, Inventories and Political Knowledge in Early Modern Switzerland and Europe." Archival Science 7, no. 4 (2007): 317-29.

Heidecker, Karl. Charters and the Use of the Written Word in Medieval Society. Tournhout: Brepols, 2000

Heiler, Thomas. Die Würzburger Bischofschronik Des Lorenz Fries (gest. 1550): Studien Zum Historiographischen Werk Eines Fürstbischöflichen Sekretärs Und Archivars. Würzburg: Verlag F. Schöningh, 2001.

Hessel, A. “Zur Geschichte der Regesten.” In Archiv für urkundenforschung. Berlin: Verlag Walter de Gruyter \& Co., 1928.

Hiatt, Alfred. "Diplomatic Arts: Hickes against Mabillon in the Republic of Letters." Journal of the History of Ideas 70 no. 3 (2009): 351-73.

Kafka, Ben. The Demon of Writing: Powers and Failures of Paperwork. New York: Zone Books, 2012.

Leibniz, Gottfried Wilhelm. Politische Schriften: Band 3, 1677-1689. Berlin: Akademie Verlag, 1986.

Lötzke, Helmut. "Methodologische Probleme Der Marxistischen Archivwissenschaft." Archivmitteilungen 17 no. 6 (1967): 216-22.

Lyall, R. J. "Materials: The Paper Revolution." In Book Production and Publishing in Britain 1375-1475, edited by Jeremy Griffiths and Pearsall, Derek, 11-29. Cambridge, UK: Cambridge University Press, 1989.

Mabillon, Jean. De re diplomatica libri VI. Paris: Billane, 1681.

Maissen, Thomas. Die Geburt Der Republic: Staatsverständnis und Repräsentation in Der Frühneuzeitlichen Eidgenossenschaft. Vol. 4. Göttingen: Vandenhoeck \& Ruprecht, 2006.

Meisner, Heinrich Otto. Urkunden- und Aktenlehre der Neuzeit. Leipzig: Koehler \& Amelang, 1950.

Miller, Thea. "The German Registry: The Evolution of a Recordkeeping Model." Archival Science 3 no. 1 (2003): 43-63. http://link.springer.com/article/10.1007/BF02438928.

Milligan, Jennifer Susan. "Making a Modern Archive: The Archives Nationales of France, 1850-1887.” Dissertation, Rutgers University, 2002.

Moore, Lara Jennifer. Restoring Order: The Ecole Des Chartes and the Organization of Archives and Libraries in France, 1820-1870. Duluth, MN: Litwin Books Llc, 2008.

Popper, Nicholas. "From Abbey to Archive: Managing Texts and Records in Early Modern England.” Archival Science 10 no. 3 (2010): 249-66. http://link.springer.com/ article/10.1007/s10502-010-9128-8.

Rösler, Ingo. "Zu Einigen Fragen Der Marxistischen Archivwissenschaft." Archivmitteilungen 17 no. 6 (1967): 222-29.

Rück, Peter. "Die Ordnung Der Herzoglich Savoyischen Archive Unter Amadeus VIII.” Archivalische Zeitschrift 67 no. 1 (1971): 11-101. 


\section{Chapter I 4}

Rück, Peter., ed. Mabillons Spur: Zweiundzwanzig Miszellen Aus Dem Fachgebiet Für Historische Hilfswissenschaften. Marburg: Institut für Historische Hilfswissenschaften, 1992.

Rück, Peter. "Zur Diskussion um die Archivgeschichte: Die Anfaenge des Archivwesens in der Schweiz (800-1400).” Mitteilungen Der Vereinigung Schweizerischer Archivare 26 (1975): 3-40.

Schweizer, Paul. "Geschichte Des Zürcher Staatsarchives." Neujabrsblatt Zum Besten Des Waisenhauses in Zürich 116 (1894): 2-40.

Seckendorff, Veit Ludwig von. Teutscher Fürsten-Stat / Oder: Gründliche und kurtze Beschreibung / Welcher Gestalt Fürstenthumer / Graf= und Herrschafften im Heil. Röm. Reich Teutscher Nation / ... regieret ... zu werden pflegen. Frankfurt: Thomas Matthias Götz, 1656.

Steedman, Carolyn. Dust: The Archive and Cultural History. New Brunswick, NJ: Rutgers University Press, 2002.

Stettler, Bernhard. Die Eidgenossenschaft Im 15. Jahrhundert: Die Suche Nach Einem Gemeinsamen Nenner. Zurich: Widmer-Dean, 2004.

Stoler, Ann Laura. Along the Archival Grain: Epistemic Anxieties and Colonial Common Sense. Princeton, NJ: Princeton University Press, 2008.

Stolz, Otto. "Archiv- und Registraturwesen der Oberösterreichischen (tirolisch-

Schwäbischen) Regierung im 16. Jahrhundert.” Archivalische Zeitschrift 42/43 (1934): 81-136.

Stolz, Otto. Geschichte Der Verwaltung Tirols: Teilstück Des 2. Bandes Der Geschichte Des Landes Tirol. Innsbruck: Wagner, 1998.

Stolz, Otto. Geschichte Und Bestände Des Staatlichen Archives Zu Innsbruck. Vol. 6. Vienna: Hof-und Staatsdruckerei, 1938.

Stowasser, O. "Das Archiv Der Herzöge von Österreich." Mitteilungen Des Archivrates 3 no. 1 (1919): 15-62.

Teuscher, Simon. "Document Collections, Mobilized Regulations, and the Making of Customary Law at the End of the Middle Ages." Archival Science 10 no. 3 (2010): 211-29. http://link.springer.com/article/10.1007/s10502-010-9127-9.

Von Ramingen, Jacob. Von der Registratur, Und jren Gebäwen ond Regimenten, deßgleichen von ibren Bawmeistern und Verwaltern, und jrer qualifcationen und habitibus. Heidelberg: Mattheus Harnisch, 1571.

Weinberg, Bella Hass. "Book Indexes in France: Medieval Specimens and Modern Practices.” The Indexer 22 no. 1 (2000): 2-13.

Zedelmaier, Helmut. "Facilitas Inveniendi: The Alphabetical Index as a Knowledge Management Tool." The Indexer 25 no. 4 (2007): 235-42. 
Chapter 15

\title{
RESPECT MY AUTHORITY
}

\author{
Literary Warrant Assessment \\ as an Archival Research Method
}

\author{
Wendy Duff and Kate Cumming
}

\begin{abstract}
Literary warrant assessment is an emerging method in archival research. It offers opportunities to explain, interpret, communicate, test, or benchmark research findings, and its flexibility means this method can be scaled for application in many research projects. In this chapter, Duff and Cumming explore the evolution of literary warrant assessment as an archival research method and analyse case studies of its use in archival research to demonstrate the strengths and limitations of this method. They conclude that literary warrant assessment has tremendous potential as an archival research method through its capacity to facilitate multidisciplinary research, its potential to enable diverse sources and multiple perspectives to be incorporated into research and its capacity to represent voices that might otherwise not be heard.
\end{abstract}

\section{Introduction}

According to the Online Oxford English Dictionary, the many meanings of the word warrant include "One who or something which protects or authorises" or "One who is answerable for a fact or statement; an authoritative witness" or "Justification or authority for an action, belief or feeling." In bibliographic literature, researchers have proposed warrant as an authority for determining and/or verifying decisions about requirements for a system. ${ }^{2}$

1 Online Oxford English Dictionary, http://www.oed.com/.

2 Clare Beghtol, "Semantic Validity: Concepts of Warrant in Bibliographic Classification Systems," Library Resources and Technical Services, 30 no.2 (1986): 110. 
Literary warrant was first proposed in 1911 by Wyndham Hulme as the basis of classification systems. ${ }^{3}$ Hulme argued that instead of building classification systems from abstract theories of classification or generalised ontologies, systems should be derived from the most authoritative and appropriate "warrant," the physical materials actually requiring management within the library's own collections. ${ }^{4}$ Hulme stated that, "A class heading is warranted only when a literature in book form has shown to exist, and the test of the validity of a heading is the degree of accuracy with which it describes the area of subject-matter to the class." 5

In the bibliographic community, along with literary warrant, classification theorists have proposed that scientific/philosophical or educational warrant, user warrant, and cultural warrant can also serve as authority for the development of a system. ${ }^{6}$ Bliss suggested that when scientific principles or educational warrant have become stable and consensual, they should be the basis of classification. ${ }^{7}$ Lee has observed that classes we develop are a reflection of our culture and are not "natural" and noted, "the belief that the culture in which we live is the source of our values implies that the literature produced by our society, along with classification of concepts, reflects the values, needs and accumulated knowledge of our culture." ${ }^{8}$ Therefore, cultural warrant suggests "that any kind of knowledge representation and/or organization system can be maximally appropriate and useful for individuals in some culture only if it is based on the assumptions, values and predispositions in some culture. Conversely, if a system is not based on those assumptions, it will be appropriate and useful to some lesser extent for individuals in that culture."

While literary warrant assessment and related forms of warrant assessment have had longstanding use in classification theory, the recordkeeping community first utilised literary warrant assessment in 1994 in the foundational

3 RK Olding, Reading in Library Cataloging (Melbourne: FW Cheshire, 1966), 105-106.

4 Anne Gilliland and Sue McKemmish, "Building an Infrastructure for Archival Research," Archival Science 4 (2004): 191

5 E. Wyndham Hulme, "Principles of Book Classification," The Library Association Record 13 (15 November 1911): 447.

6 Beghtol, “Semantic Validity,” 109-125.

7 Beghtol, "Semantic Validity".

8 Joel M. Lee, "E. Wyndham Hulme: A Reconsideration," in A Variety of Librarianship: Essays in Honour of John Wallace Metcalfe, ed. W. Boyd Rayward (Sydney: Library Association of Australia, 1976), 111.

9 Clare Beghtol, "A Proposed Ethical Warrant for Global Knowledge Representation and Organization Systems," Journal of Documentation, 5 (2002): 511. 
research project at the University of Pittsburgh, the Functional Requirements for Evidence in Recordkeeping..$^{10}$ The project methodology involved extracting statements from formal warrant sources including legislation, standards and codes of best practice that delineated recordkeeping requirements. These sources of warrant were used by the project team to identify, refine and validate the functional requirements, which specified the organisational and system requirements for good corporate recordkeeping. Further, when identifying strategies to promote the Project's functional requirements statements, the researchers suggested that " $[t]$ he requirements themselves may lack authority, but the sources upon which they are based are viewed as being extremely credible by professions that archivists need to influence."11 The research team, therefore, decided to use statements as "literary warrant," as "proof or justification that organisations and individuals must adhere to the requirements because these are based on practices established by their own profession or industry." ${ }^{12}$ Subsequent research by Wendy Duff suggested that use of warrant in this way might serve as an effective tool to improve the acceptance and dissemination of the Project's research findings. ${ }^{13}$

Since then, the frequent use of warrant by the recordkeeping community has seen its assessment evolve from its library roots into a distinctly archival method. This chapter will explore the key characteristics of archival literary warrant assessment and identify research design issues that need to be considered when deploying this method. The chapter provides three illustrative case studies that demonstrate use of archival warrant assessment. Between them, the authors of this chapter were involved in each of the research projects from which these case studies were derived. Much of the chapter's analysis is therefore based on the authors' personal reflections and experiences. In addition the authors use analysis of published research reports and relevant literature to answer questions such as: What is literary warrant assessment? What are the strengths and weaknesses of this method? What research purposes can it serve?

10 School of Information Sciences, University of Pittsburgh, "Functional Requirements for Evidence in Recordkeeping," (1996), http://www.sis.pitt.edu/ bcallery/pgh/ MainPage.htm.

11 Wendy Duff, "Harnessing the Power of Warrant," The American Archivist, 61 no. 1 (Spring 1998): 92.

12 Duff, "Harnessing the Power of Warrant," 92.

13 Wendy Duff, "Increasing the Acceptance of the Functional Requirements for Electronic Evidence," Archives and Museum Informatics 10 no. 4 (1996): 326-351. 


\section{Characteristics of Literary Warrant Assessment and Its Evolution as an Archival Research Method}

As Beghtol has argued, in its original bibliographic context literary warrant assessment and related forms of warrant, such as cultural and user warrant, have provided an authority for determining and/or verifying decisions about requirements for a system. ${ }^{14}$ Formative early use of warrant assessment in archival research focused on its capacity to articulate and define system requirements but even in these early projects, warrant assessment was not simply limited to specifying system requirements. In its use in archival research, warrant assessment is a flexible, scalable, powerful method that can be used to collect, analyse and compare data, as well as to test, validate, communicate and revise research. Researchers utilise this method to assist with explaining, interpreting, translating and communicating research findings, and also as a validation or benchmarking measure, to test the comprehensiveness of research findings and to identify gaps in preliminary research. The case studies explored in this chapter examine warrant in the form of formal recordkeeping standards, legislation, best practice requirements, professional literature and other authoritative sources and use the results of warrant analysis to develop new means of articulating, defining, communicating and deploying tools that reflect recordkeeping best practice. Broader forms of warrant assessment have, however, been used to explore social mandates for recordkeeping and recordkeeping behaviour using novels, biography and memoir as key sources of warrant. ${ }^{15}$ Recent research with Indigenous communities in Australia has used literary warrant assessment to establish community archiving requirements. ${ }^{16}$ Literary warrant analysis could be seen as privileging written over oral transmission of knowledge and this potential bias needs to be considered in research design. ${ }^{17}$ In their recent work, Gilliland and McKemmish have extended the definition of literary warrant to include authoritative oral communications

14 Beghtol, "Semantic Validity," 110.

15 Sue McKemmish, "Evidence of Me," Archives and Manuscripts, 24 no. 1 (May 1996): 28-45, http://infotech.monash.edu/research/groups/rcrg/publications/ recordscontinuum-smckp1.html

16 Kirsten Thorpe, "Aboriginal Community Archives: A Case Study in Ethical Community Research," (in this volume).

17 Shannon Faulkhead, "Negotiated Methodologies: Designing Research Respectful of Academic and Indigenous Traditions," (in this volume). 
from community Elders as well as experiential knowledge of domain experts as core sources of warrant. ${ }^{18}$

Warrant assessment operates by aligning research to specific warrant relating to a particular time, theory, practice, jurisdiction or space. Warrant assessment does not present generalised, universal, or timeless perspectives on an issue. Instead, it provides groundedness, detail, relevancy, and specificity to research analysis and a direct connection to contemporary practice, specific knowledge, or current societal attitudes and values. Warrant analysis can be defined very broadly and is open to an exploration of all forms of authoritative cultural, community, practical, legal, social, technical and theoretical warrant. Warrant sources can be existing, recognised sources of authority or, considering the concept of cultural warrant, can be requirements from emerging voices or insights that are based on the assumptions, values, and predispositions of a culture.

Iacovino, in her examination of recordkeeping and the law, argued for a very broad understanding of warrant, emphasising that, in addition to regulations and standards, "literary warrant also refers to the authority of the concepts, literature, research and practice of a profession which provides a form of empirical or accepted evidence from which the profession's knowledge domain builds its theory and models." ${ }^{19}$ In contrast and while not rejecting broader notions, Hedstrom sees value in the interpretation of warrant as "not a universal set of requirements but specific mandates and rules for record-keeping which vary in different national, business, and professional environments." ${ }^{20}$ Within archival research then, sources as varied as a corporate business rule, a theoretical concept, the voice of a community Elder or the insights of a user community have all been used as valid warrant sources. Warrant sources can therefore be selected to represent research objectives of any scale and they can include practical, theoretical, or cultural constructs. The defining characteristic of warrant sources is not their scale or breadth of impact, but their authority, importance and resonance within the culture, jurisdiction, location, country, environment, community, profession, or society where they have applicability or relevance.

18 Sue McKemmish and Anne Gilliland, "Archival and Recordkeeping Research: Past, Present and Future," in Kirsty Williamson \& Graeme Johanson (eds), Research Methods: Information, Systems and Contexts (Prahan, Vic.: Tilde University Press, 2012), 80-112.

19 Livia Iacovino, "Multi-Method, Interdisciplinary Research in Archival Science: The Case of Recordkeeping, Ethics and Law," Archival Science 4 (2004): 272.

20 Margaret Hedstrom, "Building Record-Keeping Systems: Archivists Are Not Alone on the Wild Frontier," Archivaria 44 (1997): 51. 
Early archival research, including the case studies referenced in this chapter, have used literary warrant assessment to address questions related to best practice in corporate settings but, as shown, this method has the scope for much more expansive use. Future research utilising warrant assessment could identify the societal dimensions of recordkeeping and archiving using a very broad range of warrant sources. Archival literary warrant assessment could also be combined with other methods developed by the library community, for example, user, scientific, and cultural warrant as methods that have great potential for understanding specific community and societal needs and requirements for archives. ${ }^{21}$ The results of this type of research would impact on all areas of recordkeeping practice and would ultimately, in themselves, form a rich source of professional warrant for archivists.

\section{Issues with Warrant Assessment}

Despite the widespread applicability and usability of its findings, challenges for literary warrant assessment do arise. Issues raised by the use of warrant as a research method include authority, biases, and comprehensiveness.

Bias is a key issue. The sources used in any research using warrant will bias the results of that research. The results of warrant assessments will always reflect the nature, domain, extent, and theoretical framework of the profession, society, culture, community, or regulators that devised or promulgated the warrant sources. Depending on the required research outcomes, recordkeeping projects may need to ensure that the warrant sources they use have authority within a profession or society. For example, in research for her dissertation carried out in the United States, Duff found that statements drawn from legal sources had a greater ability to influence than other sources. She also found that sources written by archivists had limited impact on evaluations made by the lawyers, auditors and information technologists she interviewed. ${ }^{22}$ In this example, the authority of individual warrant sources had significant impact and the capacity of warrant to serve required research objectives was dependent on the value that individuals placed on the sources of the warrant statements. However, while authoritative sources such as standards, laws and best practices usually represent accepted and well-established knowledge and beliefs, it is important to be aware that other less well established views or practices or new innovative approaches

21 Beghtol, "Semantic Validity," 109-125.

22 Duff, "Harnessing the Power of Warrant," 88-105. 
are often missing from these sources. These challenges may need to be considered and balanced in research design when using literary warrant assessment as a method.

Researchers also need to endeavour to understand any conceptual or theoretical frames of reference that have influenced their warrant sources, as well as any commercial interests that may have shaped them. Warrant sources such as standards, laws, regulations and best practices can seem to be authoritative but can have in-built biases, justifications, faults, or dependencies that researchers may not identify or acknowledge.

Furthermore, researchers will also have their own biases and these biases will affect their selection of warrant sources, their identification of the warrant statements they deem important and their interpretation of the meaning of a statement. Although researchers can use a combination of authoritative sources to gather a broader understanding of the requirements for recordkeeping as delineated in laws, community requirements, standards, best practices, and other relevant sources, their own background and beliefs will affect the meaning they take from these sources. It is also likely that researchers will foreground some sources and exclude others, depending on the theoretical framework of their research project. For example, traditional knowledge constitutes powerful warrant in Australian Indigenous communities but its impact may be understated or unrecognised as warrant by non-Indigenous researchers. Determining what constitutes warrant is therefore a very important consideration, particularly in less empowered or structured communities.

Thus researchers undertaking warrant research should critically examine the biases that underlie the warrant sources they use and the biases inherent in their own perspectives and then interrogate the warrant sources with full awareness of these issues. Researchers also need to mitigate bias in their research design and warrant selection, or explicitly reference it in discussions contextualising research methods.

Finally, in terms of comprehensiveness, it is important to be aware that the results of warrant analysis can never be universal. Researchers associated with the Pittsburgh Project asked of their findings, "Can we collect universal warrant and pool our resources or is warrant naturally dependent upon a state, country or other political or cultural division?"23 Theories of cultural warrant suggest that we cannot collect universal warrant, a conclusion supported by

23 Richard J Cox and Wendy Duff, "Warrant and the Definition of Electronic Records: Questions Arising from the Pittsburgh Project," Archives and Museum Informatics 11 nos. 3-4 (1997): 224. 
archival researcher Margaret Hedstrom. ${ }^{24}$ If striving for comprehensiveness, researchers must consider a great diversity of warrant sources, question their research findings and examine the breadth of their research scope.

The remainder of this chapter will use case study assessments to explore specific implementations of warrant assessment. Case studies assessing use of warrant analysis in the Functional Requirements for Evidence in Recordkeeping Project conducted at the University of Pittsburgh, ${ }^{25}$ Monash University's Australian Recordkeeping Metadata Project ${ }^{26}$ and the International Research on Permanent Authentic Records in Electronic Systems (InterPARES) 2: Experiential, Interactive and Dynamic Records project ${ }^{27}$ will outline ways in which this method has been applied and the research purposes it has served.

\section{Method in Action: Literary Warrant and the Pittsburgh Project}

Researchers in The Pittsburgh Project believed that the requirements for electronic recordkeeping should derive from authoritative sources such as the law, customs, standards, and professional best practices accepted by society and codified in the literature of different professions concerned with records and recordkeeping. Based on this view they framed its use of warrant to support a set of functional requirements for recordkeeping. ${ }^{28}$ The project's researchers and its advisory board, consisting of a small group of professionals and consultants working with electronic records, had developed a draft set of functional requirements for electronic recordkeeping systems and the team sought warrant to support and refine the requirements. The researchers conducted an extensive search to locate sources possibly relevant to recordkeeping from the field of law, auditing, information technology, and standards that related to specific industries. Experts practicing or teaching

24 Hedstrom, "Building Record-Keeping Systems," 51.

25 School of Information Sciences, "Functional Requirements for Evidence in Recordkeeping."

26 Records Continuum Research Group, "SPIRT Recordkeeping Metadata Project," (2000), http://www.infotech.monash.edu.au/research/groups/rcrg/projects/spirt/.

27 Luciana Duranti and Randy Preston (eds), International Research on Permanent Authentic Records in Electronic Systems (InterPARES) 2: Experiential, Interactive and Dynamic Records, [electronic version] (Padova, Italy: Associazione Nazionale Archivistica Italiana, 2008). http://www.interpares.org/ip2/ip2_index.cfm.

28 For a description of the functional requirements and a history of the project, see Wendy Duff, "Preserving Electronic Evidence: A Research Study Funded by the NHPRC," Archivaria 42 (Fall 1996): 28-45. 
in law, auditing, or information science reviewed the list and evaluated the authority of each source identified by the researchers. The researchers then searched (using the indexes and skimming) each authoritative source for statements that related to the requirements. They limited their search for statements that related directly to the draft requirements and did not seek out statements that related to records in general. The team extracted authoritative statements that supported the functional requirements from laws, regulations, case law, auditing standards, and information technology standards, which they used to populate a warrant database. The researchers coded each statement and linked them to the relevant functional requirement. They then compared the warrant statements to the appropriate draft functional requirement and refined the requirements where necessary. In describing the process, research team members David Bearman and Ken Sochats stated:

We have systematically reviewed hundreds of sources considered authoritative by lawyers, auditors, information technology specialists and archivists and records managers. In these sources we have identified statements that pertain explicitly to the characteristics or attributes of evidence or records. ${ }^{29}$

The research team used literary warrant to validate, interrogate or provide 'warrant' for the functional requirements for recordkeeping they had developed and used warrant to refine the final formal iteration of the Functional Requirements.

Following its use in revising the Functional Requirements, the Project team then used warrant as a tool to assess the credibility of the requirements and as a mechanism to promote their incorporation into organisational recordkeeping systems. Duff has noted that "[p]ersuasion research suggests that when cognitive effort is too great to interpret a message, people will use simple decision rules that depend on persuasion cues, e.g., source credibility with message validity." Consequently, within the Pittsburgh Project the products of literary warrant analysis also became a means to explain the relevance of the Functional Requirements to persons beyond the recordkeeping profession. ${ }^{30}$

29 David Bearman and Ken Sochats, “Metadata Requirements for Evidence," (1994), http://www.archimuse.com/papers/nhprc/BACartic.html.

30 Wendy Duff, "Increasing the Acceptance of the Functional Requirements for Evidence,” 326-351. 
Duff studied the ability of warrant statements to increase professionals' judgments of the importance of a functional requirement. To do this, she employed a quasi-experimental design involving sixty participants (twenty lawyers, twenty auditors and twenty information professionals). Duff compiled four lists, L1-L4, each containing the twenty functional requirements promulgated by the University of Pittsburgh Project and containing different types of warrant statements (e.g., from legal or auditing literature drawn from the project's warrant database). ${ }^{31}$ She then randomly selected functional requirements and their accompanying warrant from these lists to create four research instruments.

For example, she randomly selected a functional requirement statement from LI and assigned it to the first research instrument (RI), and then randomly selected a functional requirement and its accompanying auditing warrant from L2 and assigned it to RI. If the functional requirement already existed in RI (having been taken from another list), she selected a new requirement from $\mathrm{L} 2$. She then randomly selected a functional requirement and its accompanying legal warrant from L3 and L4 and assigned it to R1 following the procedure described above. This process continued until RI had a complete set of functional requirements (1-20), with five functional requirements (FR) being presented on their own, five being accompanied by auditing warrant (FRA), five being accompanied by legal warrant (FRL), and five being accompanied by information technology warrant (FRIT). She created the second set of functional requirements accompanied by literary warrant (R2) following the same procedure, except that she excluded any warrant statements used in RI. She followed the same procedure to create research instruments R3 and R4. Each research instrument contained the same functional requirements but no warrant statement appeared in more than one research instrument.

Table 15.1 shows the statements of warrant used to support each functional requirement in each of the four research instruments.

Duff then randomly assigned a research instrument to each interview with one of the participants as shown in table 15.2.

31 L1 contained the twenty functional requirements developed by the University of Pittsburgh; L2 contained the functional requirements augmented with statements of auditing warrant; L3 had the functional requirements augmented with statements of legal warrant; and L4 had the functional requirements augmented with statements of information technology warrant. 
Table 15.1. The Research Instruments

\begin{tabular}{|l|l|l|l|l|}
\hline Functional Requirement Number & RI1 & RI2 & RI3 & RI4 \\
\hline 1 & FR & FRA & FRIT & FRL \\
\hline 2 & FRA & FRL & FR & FRIT \\
\hline 3 & FRIT & FRA & FRL & FR \\
\hline 4 & FRL & FRIT & FRA & FR \\
\hline 5 & FRA & FR & FRIT & FRL \\
\hline 6 & FRIT & FR & FRL & FRA \\
\hline 7 & FR & FRIT & FRA & FRL \\
\hline 8 & FRA & FR & FRIT & FRL \\
\hline 9 & FRL & FRA & FR & FRIT \\
\hline 10 & FR & FRL & FRIT & FRA \\
\hline 11 & FR & FRA & FRL & FRIT \\
\hline 12 & FRIT & FRL & FRA & FR \\
\hline 13 & FRA & FRIT & FR & FRL \\
\hline 14 & FRL & FRIT & FRA & FR \\
\hline 15 & FRIT & FRA & FRL & FR \\
\hline 16 & FRL & FRIT & FR & FRA \\
\hline 17 & FRA & FR & FRL & FRIT \\
\hline 18 & FR & FRL & FRIT & FRA \\
\hline 19 & FRL & FR & FRA & FRIT \\
\hline 20 & FRIT & FRL & FR & FRA \\
\hline
\end{tabular}

Table 15.2. Assignment of Research Instruments to Groups

\begin{tabular}{|c|c|}
\hline Groups of Subjects & Research Instruments \\
\hline Group A & RI1 \\
\hline Group B & RI2 \\
\hline Group C & RI3 \\
\hline Group D & RI4 \\
\hline
\end{tabular}

During the interview, Duff proceeded in the following manner:

- She presented a participant with a piece of paper with the first functional requirement statement and any accompanying warrant from their randomly assigned research instrument. 
- Using a script, she briefly described the functional requirement. Where warrant existed, Duff stated that the research team had based the functional requirement on the warrant statement and noted, but did not emphasise, the source of the warrant.

- She asked the participants to rate, on a scale of one to nine (with one being not important at all, five being of average importance, and nine being extremely important) the importance of designing systems that meet this requirement.

- The participants recorded the answers on a worksheet.

Duff recorded the number of questions the participants asked during the interview, but did not record the actual questions or answers given. She kept all answers very brief to minimise the variability of the information given to the participants.

After the completion of all interviews, Duff performed analysis of variance tests on the data from the interviews to identify any statistical differences among the participants' rating of importance. The data indicated that warrant influenced the importance rating of only two of the twenty functional requirements. Legal warrant had the greatest effect on the rating of importance provided by the participants, and warrant had the greatest effect on the rating provided by the lawyers in her study.

\section{Method in Action 2: Monash Recordkeeping Metadata Project}

Researchers associated with Monash University undertook the eighteen month-long Australian research project, Recordkeeping Metadata Standards for Managing and Accessing Information Resources in Networked Environments Over Time for Government, Commerce, Social, and Cultural Purposes. ${ }^{32}$ The research team believed that, in an increasingly electronic and decentralised world, a comprehensive specification of recordkeeping metadata is necessary to support business, social and cultural needs for the creation and maintenance of evidence through time. The main deliverable of the project was therefore the Australian Recordkeeping Metadata Schema (RKMS). The

32 Funding for the development of the Australian Recordkeeping Metadata Schema was provided by the Australian Research Council and a consortium of industry partners (National Archives of Australia, State Records Authority of New South Wales, Queensland State Archives, Records Management Association of Australia, and Australian Council of Archives). For a full description of the project see Records Continuum Research Group, "SPIRT Recordkeeping Metadata Project." 
RKMS was not designed as an implementation schema but rather was intended as a metadata framework "within which metadata standards can be developed for targeted application in different sectors or domains, for example in the government sector, in a particular corporate context or in archival institutions." ${ }^{33}$ In order to make the RKMS as comprehensive as possible, an objective of the project's research was to gain very broad and diverse understandings of the requirements that recordkeeping metadata must serve and the functionality it must possess in all possible implementation environments through space and time.

The project team determined that literary warrant assessment would be an appropriate research method to gain an understanding of multiple business, social and organisational contexts for recordkeeping and the requirements that apply to, or that are derived from these contexts. Other research methods, including conceptual modelling, metadata mapping, meta-modelling and empirical instantiation were also used to conceptualise, define, test and refine the evolving RKMS, but warrant assessment played a key role in testing and defining the alpha version of the RKMS. ${ }^{34}$ The team decided that the warrant sources used must be authoritative, capable of testing, and expanding the evolving RKMS and representative of diverse forms of recordkeeping practice. The selection of warrant sources was also informed by the Project's conceptual frame of reference. The Project's research was explicitly based in continuum theory and a required research deliverable was that the RKMS should be able to represent recordkeeping action across any dimension of the continuum model. Therefore the warrant sources selected had to represent the breadth of recordkeeping capacity in the continuum model, from the first (create) dimension through to the fourth (pluralise) dimension.

Warrant sources include the Commonwealth of Australia's Electronic Transactions Act (2001), selected as a source that could help to define the purposes that recordkeeping metadata must serve in the first recordkeeping dimension of the continuum model. The ISO 9000 suite, particularly AS/ NZS ISO 9001:2000, Quality Management Systems - Requirements, was selected as a source providing warrant for the second and third, the capture

33 Sue McKemmish, Glenda Acland, Nigel Ward and Barbara Reed, "Describing Records in Context in the Continuum: the Australian Recordkeeping Metadata Schema", Archivaria 48 (1999), http://www.infotech.monash.edu.au/research/groups/rcrg/ publications/archiv01.html.

34 Records Continuum Research Group, "SPIRT Recordkeeping Metadata Project Methodology," (2000), 3, http://www.infotech.monash.edu.au/research/groups/rcrg/ projects/spirt/methodology.html. 
and organise, dimensions of the continuum and General International Standard on Archival Description (ISAD[G]) was selected as a source providing warrant for the fourth pluralising dimension of the continuum.

Given that the research project had a specific and explicit grounding in continuum theory, the selection of warrant sources also consciously sought to mitigate bias; project researchers included warrant sources representing other conceptual viewpoints into their warrant assessments. An overarching concern, however, was that the warrant sources in combination should represent the breadth of continuum practice. ${ }^{35}$

The project team defined a diverse range of sources as warrant, including the theoretical model of the records continuum. In justifying the selection of a theoretical framework as a warrant source, the project team argued that conceptions of how records are to be seen or understood directly affect their management and maintenance requirements. With specific reference to metadata, Australian recordkeeping professional Barbara Reed has explained further by saying:

How we conceptualise our recordkeeping practice defines how we specify metadata. If records are passive objects to be described retrospectively, we will take a different view of what metadata is appropriate than if we see record metadata as accumulating from a set of recordkeeping processes which can recur a number of times over the record's existence. $^{36}$

35 In addition to the works of Chris Hurley (see note 38), the full set of warrant sources used was: AS 4390.1-1996 Australian Standard: Records Management, (Homebush: Standards Australia), 1996; US Department of Defense DOD 5015.2-STD, Design Criteria Standard for Electronic Records Management Software Applications (November 1997); University of Pittsburgh, School of Information Sciences, "Functional Requirements for Evidence in Recordkeeping"; University of British Columbia, School of Library, Archival and Information Studies, "Protection of the Integrity of Electronic Records Project”, (1999), http://www.slais.ubc.ca/users/ duranti/; ISAD (G) General International Standard Archival Description, Committee on Descriptive Standards, International Council on Archives (1999), http://www.icacds. org.uk/eng/ISAD(G).pdf; Commonwealth of Australia, Electronic Transactions Act (1999), http://www.comlaw.gov.au/Details/C2011C00445; International Standards Organization, ISO 9001:2000: Quality Management (2000), http://www.iso.org/iso/ home/standards/management-standards/iso_9000.htm; Frank Upward, "Structuring the Records Continuum - Part 1: Post Custodial Principles and Properties," Archives and Manuscripts, Volume 24 no. 2 (1996): 268-285 and Frank Upward, "Structuring the Records Continuum - Part 2: "Structuration Theory and Recordkeeping," Archives and Manuscripts, 25 no. 1, (1997): 10-35.

36 Barbara Reed, "Metadata: Core Record or Core Business," Archives and Manuscripts, 25 no. 2 (November 1997): 238. 
Therefore, by studying the continuum model in detail, researching examples of its implementation and by understanding how recordkeeping operates in its framework, it became a warrant source to identify the recordkeeping requirements that metadata must be able to fulfil through the continuum. For example, a dimensional reading of the model clearly shows the interrelationships between actors, acts, and documents that combine to constitute document creation in the first dimension. In the warrant assessment exercises these interrelationships were iteratively decomposed by researchers into warrant statements. These ultimately showed that the warrant source of the first dimension of the continuum model requires, in part, metadata that can identify actors, acts, and documents and also identify and contextualise the relationships that exist between these entities. These decompositions were extended to cover all dimensions of the continuum model, to identify the recordkeeping requirements that metadata should fulfil through the continuum.

The team also used the body of published literature by Australian recordkeeper Chris Hurley as another warrant source. Hurley's writings on descriptive theory and practice have been very influential, both in Australia and internationally. ${ }^{37}$ The Project's warrant assessment exercise examined over twenty years worth of Hurley's research papers, each of which covered issues broadly associated with aspects of record description. ${ }^{38}$ Hurley's works were selected as warrant because of Hurley's own authoritative status in Australian national and international recordkeeping communities. His writing also provides a unique perspective on descriptive theory and practice that is unmatched by any other source and explores complex and evolving understandings of recordkeeping context, such as ambience and simultaneous multiple provenance and the complexities of defining and documenting

37 Hurley has published extensively in Australian and international journals, including Archives and Manuscripts, Archivaria and New Zealand Archivist. He is a Research Associate in the Faculty of Information Technology at Monash University in Melbourne and an Affiliate with the Department of Information Studies at the University of Southern California. His research on record description has contributed significantly to the national systems of archival control used in both Australia and New Zealand and to the archival control system used in the Australian state of Australia.

38 The works were: "What, if Anything, is a Function?" (1993), "The Australian ('Series') System: An Exposition" (1994), "Ambient Functions - Abandoned Children to Zoos" (1995), "The Problem with Provenance" (1995), "The Making and Keeping of Records: What are Finding Aids For?” (1998), "The Making and Keeping of Records: The Tyranny of Listing” (2000), "Relationships in records" (2001-2004). These are among Hurley's consolidated works available on his website http://www.descriptionguy.com/ description.html. 
entity relationships through time. These valuable components of descriptive theory have not yet been translated in their entirety into other sources, nor codified to their full extent in other official sources and so Hurley's original research papers remain the definitive source for these complex but valuable descriptive statements that relate directly to metadata research. As the project team sought complex warrant sources that would test, improve and expand the capacities of the evolving RKMS, Hurley's published works provided a very appropriate measure and gave valuable perspectives on description that were not contained in other warrant sources. ${ }^{39}$

When it came to assessing sources, a standard assessment process was applied to each warrant source. First, a project researcher read the source, followed by any supporting literature around the source, such as published articles, commentaries, implementation guides or other supporting manuals. This literature provided further definition of the role and operation of the source, confirmed its place in the continuum model and helped to identify how metadata requirements within the warrant source may be expected to operate. Next the researcher re-examined the warrant source and manually extracted recordkeeping requirements from it that could be fulfilled by metadata implementation. In conducting the warrant assessments of Hurley's work, project researchers iteratively decomposed the arguments in his research papers until his descriptive ideas and recommendations were able to be converted into a series of simple warrant statements relating to metadata and description.

Each recordkeeping requirement extracted from a source was documented as a specific warrant statement. These were captured in a series of spreadsheets, one for each source. The team generated hundreds of warrant statements through this analysis process with, on average, 20-30 warrant statements derived for each warrant source. Each of the warrant statements was coded according to the type of metadata functionality it represented. The research team had developed a hypothesised set of eight metadata functionality statements at the start of the research project. By using these values to code each of the warrant statements, the researchers were able to aggregate and compare warrant statements while simultaneously testing

39 In terms of research outcomes, the key value in Hurley's works came from the warrant statements they provided about fourth dimensional recordkeeping. For example, his article "Ambient Functions - Abandoned Children to Zoos," explores the potential offered by the description of function entities in archival descriptive strategies, but also investigates notions of ambience, or the capacity to provide further context to archival provenance statements and how this can be used to create broader descriptive frameworks for contextual or interoperability purposes. 
the comprehensiveness of their hypothesised set of metadata functionality statements.

When completed, the full set of warrant statements extracted from the warrant sources combined to provide a very broad perspective on the purposes that recordkeeping metadata should serve through time and through recordkeeping environments. Iteratively mapping the alpha version of the RKMS to these statements contributed to its evolution and also ultimately served as a final benchmarking test to ensure the broad scope and capacity of the RKMS. Warrant analysis was, therefore, a valuable method for ensuring the comprehensiveness of this significant research product.

\section{Method in Action 3: \\ The Development of a Metadata Schema Registry - The Work of the InterPARES 2 Project}

The International Research on Permanent Authentic Records in Electronic Systems (InterPARES) 2: Experiential, Interactive and Dynamic Records was a multi-year, multi-national research initiative examining issues associated with the creation, management and maintenance of dynamic digital records. ${ }^{40}$ The Description Cross-Domain Task Force, a specific project team in the broader research project, investigated "the roles and requirements, manifestations and management of metadata in the creation of reliable and preservation of authentic digital entities." ${ }^{\prime 11}$ The primary product from the Task Force's research was the Metadata and Archival Description Registry and Analysis System (MADRAS). During the course of the Task Force's research, MADRAS evolved into a complex and powerful metadata schema assessment tool that, by the project conclusion, incorporated and described a broad range of multi-national, multi-purpose and multi-jurisdictional metadata schemas.

The purpose of MADRAS was to register metadata schemas from across the world, describe their functionality and assess their capacity to meet specific recordkeeping requirements. The researchers used literary warrant

40 Full details of the project including its final report are available on the InterPARES website, www.interpares.org.

41 Anne Gilliland et al, "Part Six - Investigating the Roles and Requirements, Manifestations and Management of Metadata in the Creation of Reliable and Preservation of Authentic Digital Entities: Description Cross-domain Task Force Report," [electronic version] in Luciana Duranti and Randy Preston (eds) International Research on Permanent Authentic Records in Electronic Systems (InterPARES) 2. 
assessment as one of the research methods to develop the capacities of the MADRAS registry. To determine effective criteria for schema assessments and to provide uniform points of reference, researchers benchmarked the schema they intended to register against warrant sources. The benchmarks, or warrants, against which all registered metadata schema were assessed were the Benchmark Requirements Supporting the Presumption of Authenticity of Electronic Records and Baseline Requirements Supporting the Production of Authentic Copies of Electronic Records produced by the InterPARES 1 research initiative $^{42}$ and ISO 23081-1:2006 - Information and Documentation Records Management Processes - Metadata for Records - Part 1: Principles. ${ }^{43}$

By selecting the Benchmark and Baseline requirements and ISO 23081 as warrant sources, Task Force researchers ensured MADRAS was able to accommodate multiple conceptual models for managing records in its structure. The Benchmark and Baseline requirements reflect a management view where different parties have responsibilities for different aspects of record and archival preservation. ISO 23081 takes a more end-to-end or continuumbased perspective and places traditional 'archival' responsibilities into the contemporary recordkeeping domain. By being alert to conceptual differences and accommodating all relevant perspectives in their warrant selection, the Task Force made MADRAS a more robust and broadly applicable tool that could be used to register and evaluate a very wide range of metadata schemas and application profiles and determine ways in which these schemas should be augmented or modified in order to better meet relevant requirements.

In performing the warrant assessments, the InterPARES Benchmark and Baseline Requirements and ISO 23081 were examined to identify warrant statements that would enable judgments to be made about the recordkeeping and archival capacities of the metadata schema under assessment. This was a complex and iterative process involving analysis and the decomposition of extracted warrant source statements into statements that could be applied to metadata. ${ }^{44}$ In addition, to ensure that they could serve as functional benchmarks in the MADRAS database, researchers had to

42 See Authenticity Task Force, "Appendix 2: Requirements for Assessing and Maintaining the Authenticity of Electronic Records," in Luciana Duranti and Randy Preston (eds) International Research on Permanent Authentic Records in Electronic Systems (InterPARES) 2.

43 International Organization for Standardization, ISO 23081-1:2006 - Information and Documentation - Records Management Processes - Metadata for Records - Part 1: Principles.

44 This assessment methodology is further explained in Gilliland, "Part Six - Investigating the Roles and Requirements, Manifestations and Management of Metadata," 13. 
further operationalise all the warrant statements extracted from the warrant sources. This required converting the text based statements extracted or decomposed from the warrant sources into a series of questions that could then be used by researchers in their schema assessment. For example, in this operationalisation process, clause 9.3.1d from ISO 23081, which states "capture the business rules or other system controls that regulate records management operations" was translated into Question 507 in the Metadata Schema Analysis Questions: "Does the schema contain elements to identify rules or controls for records management operations? If so, indicate the applicable element(s). ${ }^{\prime 4}$ In this way a very comprehensive set of questions concerning metadata functionality was assembled by the project team and used to structure their assessments.

The extensive iterative prototyping and complex warrant analysis that went into the construction of MADRAS' analytical framework occurred over a three-year period. ${ }^{46}$ This detailed warrant analysis work resulted in a very rigorous metadata testing framework that has successfully registered and assessed metadata schema from a range of different countries, jurisdictions and management environments. MADRAS provides a very comprehensive mechanism to assess, critique and compare complex metadata frameworks and serves as an effective aid to help improve metadata implementation.

Drawing from the work of Duff and her colleagues from the Pittsburgh Project, the Authenticity Task Force also used warrant analysis to construct a set of definitive statements about the importance of metadata for communication and promotional purposes. ${ }^{47}$ The Task Force constructed a Warrant Database to reference literature and other sources that required the creation and maintenance of metadata to support the management, maintenance and preservation of records. ${ }^{48}$ The database was designed to capture specific warrant references ( 177 warrant records were captured in all) that identify different perceptions of metadata from a range of community, theoretical and business perspectives. Within the InterPARES framework, these warrant statements have been used in communication strategies and educational settings to promote the role and impact of the InterPARES

45 The full set of Metadata Schema Analysis Questions is available at Appendix 17 of Duranti and Preston, International Research on Permanent Authentic Records in Electronic Systems.

46 Gilliland, "Part Six - Investigating the Roles and Requirements, Manifestations and Management of Metadata," 12.

47 Duff, "Harnessing the Power of Warrant," and "Increasing the Acceptance of the Functional Requirements for Electronic Evidence."

48 This database is available at http://www.interpares.org/ip2/ip2_warrant_db.cfm. 
research. The Warrant Database has also assisted project researchers to communicate with the metadata schema developers whose schemas have been registered and assessed within MADRAS. The warrant database has also been used to contextualise and explain any gaps in a given schema that were identified in the MADRAS assessment process back to the original schema owners or creators, thereby grounding the feedback provided in legitimate statements of warrant. ${ }^{49}$

\section{Conclusions}

In looking back over the Pittsburgh Project a year after its completion, project researchers Richard Cox and Wendy Duff concluded that,

Warrant may turn out to be the most important outcome from the project. Warrant drives how we define records, why we manage records in the ways we do, and how we develop functional requirements for records systems. Warrant is certainly the most important aspect of the original research calling for additional research. ${ }^{50}$

The authors believe that literary warrant is indeed the enduring legacy of the Pittsburgh research. The Pittsburgh Project and its reconceptualisation of literary warrant assessment marked the point at which warrant assessment became a distinctly archival method.

Each of the projects discussed in this chapter relate to metadata research with a focus on best practice recordkeeping and archival requirements but warrant assessment as defined in archival research has an ever-broadening scope and mandate. This research method has incredible potential for future use in archival research through its ability to allow multiple perspectives to be incorporated into research and its capacity to represent voices that might otherwise not be heard. It is a method that allows innovation, allows diverse sources to be incorporated into research and can represent emerging voices and perspectives alongside traditional best practice requirements. Emerging examples of this type of research using literary warrant assessment range from defining social mandates for recordkeeping and recordkeeping behaviour to determining community archiving requirements.

49 Gilliland, "Part Six - Investigating the Roles and Requirements, Manifestations and Management of Metadata," 25-26.

50 Cox and Duff, "Warrant and the Definition of Electronic Records," 224. 
In the current era of complex research questions and interdisciplinary and collaborative projects, another key value of literary warrant as a research method lies in its ability to capture, represent and compare the views of diverse disciplines and communities. It is truly a multidisciplinary method. Iacovino has commented on this aspect of warrant assessment, arguing that it is "an essential tool for interdisciplinary research." ${ }^{51}$ Duff has asserted that expansive, multidisciplinary perspectives on warrant are necessary to confront contemporary archival research challenges. "If archivists are to take their rightful place as regulators of an organisation's documentary requirements, they will have to reach beyond their own professional literature, and understand the requirements for recordkeeping imposed by other professions and society in general." ${ }^{2}$ The time is therefore ripe for further exploration and application of the method of literary warrant assessment, and for the application of the valuable research findings it produces.

\section{References}

Authenticity Task Force, InterPARES Project. "Appendix 2: Requirements for Assessing and Maintaining the Authenticity of Electronic Records," in Luciana Duranti (ed) The Long Term Preservation of Authentic Electronic Records: Findings of the InterPARES Project. (San Miniato, Italy: Archilab, 2005). http://www.interpares.org/book/ interpares_book_k_app02.pdf.

Bearman, David and Ken Sochats. "Metadata Requirements for Evidence." (1994). http:// www.archimuse.com/papers/nhprc/BACartic.html.

Beghtol, Clare. "Semantic Validity: Concepts of Warrant in Bibliographic Classification Systems," Library Resources and Technical Services, 30 no. 2 (1986): 109-125.

Beghtol, Clare. "A Proposed Ethical Warrant for Global Knowledge Representation and Organization Systems," Journal of Documentation, 5 (2002): 507-532.

Cox, Richard J. "Re-discovering the Archival Mission: The Recordkeeping Functional Requirements Project at the University of Pittsburgh, A Progress Report." Archives and Museum Informatics, 8 no. 4 (1994): 279-300.

Cox, Richard J and Wendy Duff. "Warrant and the Definition of Electronic Records: Questions Arising from the Pittsburgh Project." Archives and Museum Informatics 11 nos. 3-4 (1997): 223-31.

Duff, Wendy. "Increasing the Acceptance of the Functional Requirements for Electronic Evidence." Archives and Museum Informatics 10 no. 4 (1996): 326-51.

Duff, Wendy. "Preserving Electronic Evidence: A Research Study Funded by the NHPRC." Archivaria 42 (Fall 1996): 28-45.

Duff, Wendy. "Harnessing the Power of Warrant." The American Archivist, 61 no. 1 (Spring 1998): 88-105.

Duff, Wendy. "The Influence of Literary Warrant on the Acceptance and Credibility of the Functional Requirements for Recordkeeping: A Dissertation Proposal.” Dec. 151995.

51 Iacovino, "Multi-Method, Interdisciplinary Research in Archival Science," 272.

52 Duff, "Harnessing the Power of Warrant," 105. 
Duff, Wendy. "Compiling Warrant in Support of the Functional Requirements for Recordkeeping." Bulletin for the American Society for Information Science and Technology, 23, no.5: 12-13.

Duranti, Lucinda and Randy Preston (eds), International Research on Permanent Authentic Records in Electronic Systems (InterPARES) 2: Experiential, Interactive and Dynamic Records, [electronic version] (Padova, Italy: Associazione Nazionale Archivistica Italiana, 2008). http://www.interpares.org/ip2/ip2_index.cfm.

Evans, Joanne and Lori Lindberg. "Describing and Analyzing the Recordkeeping Capabilities of Metadata Sets." Paper presented at the Dublin Core 2004 Conference Shanghai. www.dcpapers.dublincore.org/index.php/pubs/article/view/772/0.

Evans, Joanne and Nadav Rouche. "Utilising Systems Development Methods in Archival Systems Research: Building a Metadata Schema Registry." Archival Science (2004) 4: 315-334.

Faulkhead, Shannon. "Negotiated Methodologies: Designing Research Respectful of Academic and Indigenous Traditions," in Anne J. Gilliland, Sue McKemmish and Andrew Lau (eds), Research in the Archival Multiverse (Monash University Publishing: 2016).

Gilliland, Anne et al. "Part Six - Investigating the Roles and Requirements, Manifestations and Management of Metadata in the Creation of Reliable and Preservation of Authentic Digital Entities: Description Cross-domain Task Force Report," [electronic version] in International Research on Permanent Authentic Records in Electronic Systems (InterPARES) 2: Experiential, Interactive and Dynamic Records, Luciana Duranti and Randy Preston (eds) (Padova, Italy: Associazione Nazionale Archivistica Italiana, 2008). http://www.interpares.org/display_file_.cfm?doc+ip2_book_part_6_ description_task_force.pdf

Gilliland, Anne and Sue McKemmish. "Building an Infrastructure for Archival Research", Archival Science 4 (2004): 149-197.

Hedstrom, Margaret. "Building Record-Keeping Systems: Archivists Are Not Alone on the Wild Frontier", Archivaria 44 (1997): 44-71.

Hulme, E. Wyndham. "Principles of Book Classification," The Library Association Record 13 (15 November 1911): 444-449.

International Standards Organization, ISO 15489-1: 2001 Information and Documentation - Records Management - Part 1: General.

International Organization for Standardization. ISO 23081-1:2006 - Information and Documentation - Records Management Processes - Metadata for Records - Part 1: Principles.

Lee, Joel M. "E. Wyndham Hulme: A Reconsideration," in A Variety of Librarianship: Essays in Honour of John Wallace Metcalfe, ed. W. Boyd Rayward (Sydney: Library Association of Australia, 1976).

Olding, RK, Reading in Library Cataloguing, (Melbourne: F. W Cheshire, 1966).

McKemmish, Sue. "Evidence of Me," Archives and Manuscripts, 24 no.1 (May 1996): 2845. http://infotech.monash.edu/research/groups/rcrg/publications/recordscontinuumsmckp1.html.

McKemmish, Sue, Glenda Acland, Nigel Ward and Barbara Reed. "Describing Records in Context in the Continuum: the Australian Recordkeeping Metadata Schema”, Archivaria 48 (1999). http://www.infotech.monash.edu.au/research/groups/rcrg/ publications/archiv01.html.

McKemmish, Sue and Anne J. Gilliland. "Archival and Recordkeeping Research: Past, Present and Future", Kirsty Williamson \& Graeme Johanson (eds), Research Methods: Information, Systems and Contexts (Prahan, Vic.: Tilde University Press, 2012): 80-112. 
Records Continuum Research Group. SPIRT Recordkeeping Metadata Project (2000). http://www.infotech.monash.edu.au/research/groups/rcrg/projects/spirt/.

Reed, Barbara. "Metadata: Core Record or Core Business." Archives and Manuscripts, 25 no.2 (1997): 218-241.

School of Information Sciences, University of Pittsburgh, "Functional Requirements for Evidence in Recordkeeping," (1996). http://www.sis.pitt.edu/ bcallery/pgh/ MainPage.htm.

School of Information Sciences, University of Pittsburgh, "Literary Warrant Supporting Functional Requirements”, (1996). http://www.sis.pitt.edu/ bcallery/pgh/ LiteraryWarrant.htm.

Standards Australia. Australian Standard: Records Management: AS4390 - 1996. (Standards Australia, Homebush,1996).

State Records Authority of NSW. Standard on Digital Recordkeeping. Sydney, 2008.

Thorpe, Kirsten. "Aboriginal Community Archives: A Case Study in Ethical Community Research," in Anne J. Gilliland, Sue McKemmish and Andrew J Lau (eds), Research in the Archival Multiverse (Monash University Publishing: 2016). 


\title{
Chapter 16
}

\section{NEGOTIATED METHODOLOGIES}

\author{
Designing Research \\ Respectful of Academic and Indigenous Traditions
}

\author{
Shannon Faulkhead
}

\begin{abstract}
When working with minority or underrepresented groups and communities, one of the first thoughts of a researcher, as well as of those groups and communities, is sourcing a 'culturally safe' research design - a concern for both researchers and communities. During research with Indigenous peoples in Australia, this thought must be embedded into the research agenda and any guidelines for culturally and ethically based research. Certainly it was at the forefront of my thinking when commencing my own doctoral research, but there was more to it - I wanted a research design that was respectful of both Koorie community and academic traditions. It required constant negotiations to find similarities and differences that could and would work together to achieve this underlying aim. This chapter provides a review of that research -'Narratives of Koorie Victoria'. It describes and discusses my journey of negotiating the methodological approach I used, including the development of my research question, the paradigm that shaped and directed the scope, design, and method, and my personal reflections on this research six years on.
\end{abstract}

\section{Introduction}

Within Indigenous Australian communities introducing oneself can often result in lengthy conversations connecting through family and community. Introducing oneself in writing is more difficult. I can introduce myself, but there is little mechanism for you to introduce yourself to me. Yet I am willing 
to give it a go. I am from Australia. I am a Koorie ${ }^{1}$ woman from Sunraysia, in northwest Victoria, Australia. I am of Indigenous Australian, English, German, Spanish, and African descent. Much of my identity, and the knowledge that influences who I am, originates from family and community oral records. I identify as Koorie or Indigenous Australian.

When working with minority or underrepresented groups and communities, one of the first thoughts for both the researchers and communities involved is developing a 'culturally safe' research design. This thought was at the forefront of my thinking when commencing my own doctoral research, but there was more to it. I wanted a research design that was respectful of both Koorie community and academic traditions. It required constant negotiations to find similarities and differences that could and would work together to achieve this underlying aim. These negotiations never seemed to be 'straightforward' and I did not have a 'rock solid' plan that was followed through from the beginning. In fact, in some ways the methodology was developed retrospectively through reflection. This chapter describes and discusses my research journey, including the development of my research question, the paradigm that shaped and directed the scope, design, and method used, and my personal reflections on this research six years on. However, first I should explain what my research was investigating.

\section{The Research Question - ‘Narratives of Koorie Victoria'}

I have observed three understandings of narratives of Koorie people, culture and history. The first is of the continuing destructive power of narratives about Indigenous Australians - especially those originating from colonialinvasion. ${ }^{2}$ The second is of the positive power that narratives can have - such

1 Koorie is a term of self-identification used by some Indigenous Australian people from Victoria and southern parts of New South Wales, meaning 'our people', 'man' or 'person'. Indigenous Australian people have various terms of self-reference from tribal and language group names through to regional terms such as Koorie or Koori (south-eastern Australia), Palawa (Tasmania), Nunga (South Australia) and Murri (Queensland). While using this term, it should be recognised and respected that this is not a blanket term adopted by all Indigenous Australian people from this region. Many prefer their own clan, nation, or state title, or the generic terms 'Indigenous Australian' or 'Australian Aboriginal and Torres Strait Islander'. When referring to Australia as a whole, the term 'Indigenous Australian' will be used.

2 One person's colonisation is another's invasion. This is the situation within Australia. Although the term 'colonisation' is the more commonly used term, I do not feel that it is the correct term in reference to what occurred in Australia, 
as in combating negative stereotypes and in creating community pride. And thirdly that there are narratives that require a shared space that acknowledges both Koorie and non-Indigenous knowledge and enables the narratives to present a comprehensive and holistic cultural heritage in perpetuity. These understandings morphed into my research question: How do the methods of narrative creation and transmission of and about Koorie people, culture and history have an impact on the collective knowledge and peoples of Victoria?

I defined 'narratives' as any story or narrative created through the use of Koorie and/or government records. It referenced all the narratives of Koorie Victoria $^{3}$ drawn from Koorie and non-Indigenous sources, as well as the interactions between Koorie cultural discourses and the dominant cultural discourse of Australia involving various individuals or groups with diverse aims, objectives and purposes of creation. These narratives may be culturally informed or uninformed, positive or negative in nature, racially impartial or biased; all tell of some aspect of the people, culture and history of Koorie Victoria. They tell of people and how they interact with the world around them as well as the lessons learnt from people who previously walked the land and their actions; in other words, they are stories of people, culture and history or some combination of these three.

My research examined the ways in which the Koorie and Victorian communities relate to and are affected by these narratives - how people interact with the narratives and what the emotional and physical responses to them are. It originated from the supposition that the dominant cultural discourse (the current foundation for the legal, government and academic structures within Australia, originating from Great Britain) has damaged the ability of Koorie communities to share and create their own narratives. If true, this inability not only has an impact upon Koorie peoples, but also upon the broader Victorian community to which Koorie peoples also belong.

especially since there were no treaties made in recognition of the nations of people already in possession of the land now known as Australia. Some may view this as an attempt to wring guilt or remorse, however I use it in an attempt to reflect an Indigenous perspective of the past. Also, I am not convinced that all people who came to Australia can be defined as invaders - a suggested division is given by Conner: "Invasion" conveys the fact that the British arrived on Aboriginal land uninvited and then took it for their own use, while the term "settlement" is needed [...] to convey the fact that once the land had been taken, the British settled the land with their farms and towns." John Connor, The Australian Frontier Wars 17881838 (Sydney: University of New South Wales Press, 2002), p. xi.

3 'Koorie Victoria' is an abbreviated term for 'Koorie people, culture and history of Victoria'. 
Orality continues to be the predominant method of transmitting knowledge ${ }^{4}$ within Indigenous Australian communities. Orality includes the spoken word, images and performance. ${ }^{5}$ The orality of images is the combination of the image and the oral account or story/ies that explains or adds to the image. Indigenous knowledge is also transmitted via text. ${ }^{6}$ Both orality and text were used prior to colonial-invasion. Indigenous knowledge is not just within the people and land; it is also contained within books, records and multimedia formats, and stored in libraries and archives. Records about Indigenous people resulting from records of colonial-invasion are also Indigenous knowledge. These records contain knowledge about/or belonging to Indigenous people. As such Indigenous peoples are owners of the knowledge contained within those records, and possibly the records themselves. Although the legal frameworks and legislation governing many archival institutions do not recognise this ownership of records as Indigenous knowledge, as Russell notes, the "material can become Indigenous through reclamation processes ..."7

Australia has two primary forms of narratives to describe the period when Indigenous and non-Indigenous peoples came to co-habit this continent. The first is that of colonisation and settlement - involving hardships, but typically suggesting a peaceful occupation by the newcomers. The second is that of invasion, where wars and battles were fought, and the people and land were treated as spoils of a war.

\section{Working within a Paradigm}

This research postulated that all narratives are created with a cultural agenda or bias. Cultural agendas are not always conscious, yet they can direct the method and manner in which narratives are created in covenant with an individual's cultural framework. Everyone has a framework, often built

4 Both records and narratives.

5 Wayne Atkinson, "Oral History and Cultural Heritage [Conference Paper]," in Aboriginal Cultural Heritage Seminar. Aborigines Advancement League, 1984, p. 2.

6 Muecke has suggested that Indigenous people had a written language, but that it was iconographic not alphabetical. In: Stephen Muecke, Textual Spaces: Aboriginality and Cultural Studies, ed., Gunther Kress, Communication and Culture (Kensington, NSW: New South Wales University Press, 1992).

7 Lynette Russell, "Indigenous Knowledge and Archives: Accessing Hidden History and Understandings.," in Australian Indigenous Knowledge and Libraries, Martin Nakata and Marcia Langton, eds., Kingston, ACT: Australian Academic and Research Libraries (AARL), 2005, p. 170. 
upon the morals and social structures of their community/family beliefs and world understandings. While there is nothing inherently wrong about such agendas, they can be damaging if not recognised or acknowledged when applied to researching or creating narratives of another's culture and history. When producing narratives about a different culture or peoples, a cultural agenda becomes a cultural bias; a cultural lens ${ }^{8}$ that focuses on different peoples and cultures as 'other.' As Brady has observed:

Currently we are operating within a climate where difference is regarded as dangerous. When I look out at you, I look at you with a face that actually represents a history of colonisation. Loss of ownership of land, loss of human rights, and for some of us, the loss of colour but not the loss of our culture. ${ }^{9}$

Bias becomes problematic when it manifests itself in beliefs and suggestions that difference constitutes wrongness - that the 'other' is inferior or flawed when compared to someone else's worldview. Cultural bias is recognised within all aspects of society today. Strategies, frameworks and ethics regimes have been established to acknowledge and counteract such bias, to create the illusion of culturally unbiased research and narratives; an illusion to the point that it is doubtful that a narrative can ever be unbiased. An author' ${ }^{10}$ choice of language and method of transmission automatically places his or her narrative within a cultural and social context. This is something openly acknowledged in this chapter - that the methodology and research reflects my cultural agenda or bias. I do not regard this as good or bad; it is just as it is.

Unacknowledged cultural agendas are evident in relation to research practices of the past. This has been a particular concern for Indigenous researchers and communities who have been scrutinised by researchers as 'other' since the introduction of non-Indigenous peoples to their countries, as voiced by Linda Tuhiwai Smith:

8 As discussed in: Ian Anderson, "Black Bit, White Bit," in Blacklines: Contemporary Critical Writing by Indigenous Australians, Michele Grossman, ed., Carlton, Victoria: Melbourne University Press, 2003; Genevieve Cashman, "[Koorie Oral History and Health]" Minor Thesis, University of Melbourne, 2002; Marcia Langton, "Anthropologists Must Change," Identity 4 no. 4 (1981); Joan Winch and Ken Hayward, "Doing It Our Way': Can [Aboriginal] Cultural Traditions Survive in Universities," Nerw Doctor, no. 70 (1999).

9 W W Brady, "Observing the Other," Eureka Street 9, no. 1 (1999): 28.

10 "Author" is used in terms of being the creator of a narrative regardless of form, not necessarily of a written piece. 
From the vantage point of the colonized, a position from which I write, and choose to privilege, the term "research" is inextricably linked to European imperialism and colonialism. The word itself, "research", is probably one of the dirtiest words in the indigenous world's vocabulary. When mentioned in many indigenous contexts, it stirs up silence, it conjures up bad memories, it raises a smile that is knowing and distrustful. It is so powerful that indigenous people even write poetry about research. The ways in which scientific research is implicated in the worst excesses of colonialism remains a powerful remembered history for many of the world's colonized peoples. ${ }^{11}$

Creating a culturally safe method of research has been a priority for Indigenous peoples in response to negative experiences with researchers and culturally biased research practices. When researching the transgenerational effects of trauma in Indigenous Australia, this was of particular concern to Judy Atkinson - an Indigenous researcher researching within her own community:

My design of an ethical and safe process for the study reflected my knowledge of the deep pain this country and its Indigenous peoples held from their colonial history.

The issues of greatest concern to me were the need to understand and to be responsive to the implications of hierarchical structures, and the potential for the intentional or unintentional misuse of power in the relationship between the researcher and the researched; the obligations of relating within an Aboriginal community environment, which necessitates reciprocity; and the ethical obligations and responsibilities of the researcher to ensure the participants' safety and confidentiality. ${ }^{12}$

As already noted, cultural bias is not necessarily a fault in narratives, unless it is abused, unacknowledged, or deliberately hidden. Interacting with cultures other than one's own can change preconceptions, ${ }^{13}$ and guide or redirect the

11 Linda Tuhiwai Smith, Decolonizing Methodologies: Research and Indigenous Peoples. London: Zed Books, 1999, p. 1.

12 J. Atkinson, Trauma Trails, Recreating Song Lines: The Transgenerational Effects of Trauma in Indigenous Australia, North Melbourne, Australia: Spinifex Press, 2002, p. 14.

13 Jeremy Beckett, "Autobiography and Testimonial Discourse in Myles Lalor's 'Oral History'," in Telling Stories: Indigenous History and Memory in Australia and New Zealand, Bain Attwood and Fiona Magowan, eds., Crows Nest, N.S.W.: Allen \& Unwin, 2001, p. 123 . 
focus or aim of the project and/or narrative/s, but it cannot prevent cultural bias. Comparisons of people and societies happen regularly through human interaction and are rarely devoid of cultural interpretations. 'Subjects' often have no control over how their knowledge is understood or transmitted to others. Cultural bias is well recognised within various ethical and cultural processes. Although guidelines have been created to prevent harm to others, they are often created from within the same cultural framework as the bias, meaning that it may not be possible to consistently prevent harm occurring.

In relation to research of Koorie people, the application of a cultural lens was originally linked to anthropology, ${ }^{14}$ but over time it has been applied to other research disciplines such as medicine, archaeology and history in varying degrees. The result was culturally biased research, which became problematic when it was presented as the 'truth' about a culture, instead of culturally based observations of another culture:

Anthropologists described reality from their own perspectives and history which was then classified by university standards as "the truth". The only people who are able to describe within context are the ones who have the lived experience of those being researched. ${ }^{15}$

The misrepresentation of cultural perspectives is also linked to the past inability of anthropologists, ${ }^{16}$ and of other researchers, to recognise that Indigenous people are Australians who have retained their Indigenous cultural identity. While the difficulties of researchers, researching cultures different from their own within Australia, date back to colonial-invasion, Indigenous Australians and researchers are concerned with the impact upon the Indigenous community today. Although understanding the context of the development of knowledge systems, as described by Turnbull and Farrell, can assist in 'cross cultural comparisons', contextualism would need to be applied to both cultures involved in the comparison. ${ }^{17}$

14 For example: Anderson, "Black Bit, White Bit"; Langton, "Anthropologists Must Change"; Winch and Hayward, "Doing It Our Way': Can [Aboriginal] Cultural Traditions Survive in Universities."

15 “'Doing It Our Way': Can [Aboriginal] Cultural Traditions Survive in Universities," 25.

16 Marcia Langton, "Urbanizing Aborigines: The Social Scientists Great Deception," Social Alternatives 2, no. 2 (1981).

17 "Let us turn now to one anthropologist's account of how to make cross cultural comparisons between knowledge systems. F. Allan Hanson argues that that most appropriate method for making such comparisons is based on what he calls "contextualism". Hanson argues that knowledge systems depend on two basic factors - the nature of external reality and the nature of what we commonly call the human 
Cultural agendas and bias are increasingly well recognised, but the effects of cultural domination are still being unravelled within Australia. The Western cultural structure is so ingrained within Australia that authorship from within this structure is still seen as culturally neutral whereas Indigenous authorship is labelled biased and emotive if it does not adhere to these structures. There are so many dichotomies existing within Australia's discourse that it has been a challenge for me to avoid falling into some of them. Dichotomies are created through the cultural identification of the 'other'. Within Australia many cultures that do not adhere to the 'white' British Anglo-Australian Christian structure are often viewed as 'other'. Illustrations of this include past Australian government policies of 'Assimilation' and 'White Australia', where 'other' cultures were pressured to conform to idealised 'white' standards of living - standards that not even all 'white' people could or would embrace. While these policies are no longer officially in effect, representations of the 'other' in the media and government actions still create this division, thus continuing historic attempts to create fear of the 'other'. This is evidenced in media reportage, policy and actions on issues such as the 'war on terrorism', the referencing of 'Boat people' instead of immigration, and race riots, where 'other' races and cultures are presented as wrong or bad. This is a form of racism that is often not recognised by the broader community but is particularly felt by those identified as being of 'other' cultures. It is an insidious form of racism in that it presents a particular standard of what it is to be an Australian, creating an illusion that all Australians should want to aspire to this cultural 'standard'. When some people, whilst proud to be Australian, wish to retain the cultural or religious beliefs of their heritage, there is an intolerance within the mainstream discourse of this position, suggesting a wrongness. This not only separates them from mainstream Australia, but also creates fear or shame and the pressure to change and adhere to an idealised version of what it is to be Australian.

The three dichotomies examined in terms of Koorie Victoria - oralityliteracy, memory-history, and tradition-modernity - are the key ones that consistently needed to be addressed throughout my research.

mind. Thus even if external reality remains constant, knowledge systems will vary because the human mind develops differently in different cultures. Hanson maintains that there is "a single, independently existing world" but that the basic assumptions, categories, and rules of thought may vary from culture to culture. In order to understand any system of thought it is necessary to study in the context of these assumptions, categories, and rules. Hence the term "contextualism"." David Turnbull and Lyndsay Farrall, Interpreting the Australian Environment: The Aboriginal Experience and the Colonial Experience, 2nd pilot ed. Geelong, Vic.: Deakin University, 1983, p. 9. 


\section{Orality-Literacy}

The development of literacy has been used as an indicator of social evolution and of intelligence or societal positioning. The application of this measurement to Indigenous Australians evolved from English society where lack of literacy was linked to lower intelligence or social class, despite the lack of education tolerated in a large part of the population. It was also believed that the development of literacy reflected the development of man - gestures, speech, images to record events and maps, then iconography, and finally the formation of writing. Grossman writes that:

Social-anthropological and liberal humanist perspectives on the relationship between orality and literacy have thus tended to locate orality along a progressivist axis, as an early phase of sociocultural development that once confronted by technologies of alphabetic writing, inevitably yields to the efficacy of literacy-based communication and cognition. ${ }^{18}$

The societal construction of status and development indicated by a link between literacy and intelligence was then applied to non- and pre-literate cultures with whom the British came into contact - the orality-literacy dichotomy became a division of 'other'. Ginibi points to the consequences of this:

And because we Aboriginal people came from an oral tradition it is we who always had to conform to the standard of the invaders, and learn the Queen's English so you mob out there can understand what the hell we are on about. ${ }^{19}$

Indigenous Australian cultures have often been viewed as pre- or nonliterate,${ }^{20}$ with their lack of literacy used to indicate primitiveness by colonialinvaders and others. In order to communicate with the colonial-invaders, Indigenous Australian people adopted the English language and Western-

18 Michele Grossman, "Beyond Orality and Literacy: Textuality, Modernity and Representation in Gularabulu: Stories from the West Kimberley," in Boundary Writing: An Exploration of Race, Culture, and Gender Binaries in Contemporary Australia, Lynette Russell, ed., Honolulu: University of Hawaii Press, 2006, p. 151.

19 Ruby Langford Ginibi, "Voicing the Land: Asal Armidale," Notes and Furphies 39, no. Oct (1997), p. 19.

20 There is some debate as to whether the art created prior to colonial-invasion of Australia is technically 'art' and therefore a form of oral culture, or iconography and therefore a step towards literacy. See: Muecke, Textual Spaces: Aboriginality and Cultural Studies. 
style literacy ${ }^{21}$ which developed their ability to document their concerns and needs within an alien system "as a mode of political and cultural selfrepresentation from quite early in colonial history ..."22

Despite Indigenous Australians adopting Western-style literacy ${ }^{23}$ as a form of communication, the orality-literacy dichotomy is still being employed, with the implication that Indigenous Australian culture and people are still being compared to Western standards of class and intelligence. ${ }^{24}$ This belief sets orality and literacy in opposition to each other, instead of as parts of something bigger - human interaction. Both text and oral narratives are methods of storage and transmission that enable narratives to travel and be preserved from one generation to the next. Literacy and orality are not in opposition to each other; instead they interact constantly throughout everyday life. Oral narratives are written down, and written narratives are read aloud. The primary difference between the two is that a person can often survive without being able to read, but would find it difficult to survive without being able to communicate with others through speech, touch, facial expressions, hand signals, and/or gestures. Orality is interaction whilst text is a tool of human interaction.

\section{Memory vs History}

The lack of cultural terminology in reference to narratives by Koorie Victoria is reflected by the 'memory' versus 'history' dichotomy; a dichotomy created

21 Ginibi, "Voicing the Land: Asal Armidale"; Ian Anderson, "Introduction: The Aboriginal Critique of Colonial Knowing," in Blacklines: Contemporary Critical Writing by Indigenous Australians, Michele Grossman, ed., Carlton, Victoria: Melbourne University Press, 2003; Penny van Toorn, "Indigenous Australian Life Writing: Tactics and Transformations," in Telling Stories: Indigenous History and Memory in Australia and New Zealand, Bain Attwood and Fiona Magowan, eds., Crows Nest, NSW: Allen \& Unwin, 2001.

22 Anderson, "Introduction: The Aboriginal Critique of Colonial Knowing," 18.

23 "Indigenous Australian life writing is often viewed as a recent phenomenon, a new literary and historiographical form that emerged initially in the mid twentieth century, expanded gradually through the 1960s and 1970s, and eventually proliferated spectacularly in the 1980s and 1990s. However, today's indigenous life writings are part of an older discursive formation that dates back to early colonial times, and incorporates traditional indigenous paradigms and protocols of oral communication. In the discipline of literary studies, this older intercultural body of life writing has remained largely invisible because literary criticism and scholarship have focused exclusively on long narratives published in book form," van Toorn, "Indigenous Australian Life Writing: Tactics and Transformations."

24 In reference to anthropology: Grossman, "Beyond Orality and Literacy," 150. 
within Western academic debate, as explained by Nora and Kitzman. ${ }^{25}$ So often the lack of written records linked to 'memory' and 'collective memory' creates the perception of them as flawed, subject to forgetting, ${ }^{26}$ emotional, impressionistic, and easily adapted in response to current concerns or events. ${ }^{27}$ 'History' is linked to writing and written records, and seen as permanent, rational, objective and factual. The difficulty with this division is that records located in oral memory are not simply memories; they are often narratives that are passed on from one generation to the next, taught to children using methods of rote and other forms of learning that have been tried and tested over time. Oral cultures have narratives that have been created by experts in their field based upon experience, research and discussion. Memory making and transmission are part of the human condition, existing in everyone. Oral methods of learning are similar, in many ways, to written methods of learning - both systems involve remembering and forgetting, ${ }^{28}$ facts and events. Similarly all narratives of the past, be they sourced from written history or oral memory, have strengths and weaknesses. ${ }^{29}$ Nevertheless the term 'oral memory' still indicates weakness within a paradigm that privileges the written word. ${ }^{30}$

25 Pierre Nora and Lawrence D. Kritzman, Realms of Memory: Rethinking the French Past, European Perspectives, New York: Columbia University Press, 1996, p. 3.

26 Forgetting is actually an important part of memory and collective memory - remembering that which is important. For example: Susan Crane, "Ahr Forum: Writing the Individual Back into Collective Memory," American Historical Review 102 no. 5 (1997): 1380.

27 Faye's review of Peter Novick's book, "The Holocaust in American Life", suggests that Novick views Holocaust narratives as being current constructs of the past and not memories of the actual events. Esther Faye, "Collective Memory and the Holocaust," Australian Historical Association Bulletin, no. 91 (2000): 74.

28 Anne Brewster, Rosemary Van den Berg, and Angeline O'Neill, Those Who Remain Will Always Remember: An Anthology of Aboriginal Writing. Fremantle, W.A.: Fremantle Arts Centre Press, 2000, p. 13.

29 Various authors have suggested that collective memory and history are the same thing in different forms, with other suggesting that the division between history and collective memory is a recent one: Peter Burke, "History as Social Memory," in Memory: History, Culture and the Mind, Thomas Butler, ed., Oxford: Basil Blackwell, 1989; Crane, "Ahr Forum: Writing the Individual Back into Collective Memory"; Paula Hamilton, "The Knife Edge: Debates About Memory and History," in Memory and History in Twentieth-Century Australia, Kate Darian-Smith and Paula Hamilton, eds., Melbourne: Oxford University Press, 1994. Others dismiss this suggestion: Maurice Halbwachs and Lewis A. Coser, On Collective Memory, The Heritage of Sociology, Chicago: University of Chicago Press, 1992; Nora and Kritzman, Realms of Memory: Rethinking the French Past.

30 Halbwachs and Coser, On Collective Memory; Nora and Kritzman, Realms of Memory: Rethinking the French Past. 


\section{Tradition vs Modernity}

Culture is a continuous link through the past, present and future - Indigenous peoples and culture have developed and changed over time, and with colonialinvasion the changes occurred more rapidly. Constructing a 'traditional' and 'modern' dichotomy has served as a method of identifying the 'real' Indigenous people through terms such as "tradition' and 'traditionally-oriented', and excluding those who have changed, using terms such as "non-traditional' or 'urban' etc." ${ }^{1}$ These terms divide people by suggesting that 'real' Indigenous Australians are traditional, and 'modern' or 'urban' Indigenous Australians are not 'real' due to their changing way of life and adaptations to their constantly changing environment since colonial-invasion. It is a strange perception when you consider the pride that Western civilisation places on its development over time and its adoption and adaptation of knowledge and technology developed by others. So while the survival of Indigenous peoples and culture in the face of invasion and wars is celebrated, Indigenous Australians are disapproved of for employing similar methods of survival.

While the ways in which some of these dichotomies are applied are changing, the overarching dichotomy of oral versus written is still very much an issue within narratives of Koorie Victoria, supporting the view of the research reported here - that narratives are created with a cultural agenda or bias. Hopefully this explanation of the dichotomies will not be construed as meaning that I subscribe to a dichotomy of Koorie versus Australian settler. Rather, this section attempts to explain where Koorie cultural discourse has been situated within the mainstream and the dichotomies in which the discourse has been cast - dichotomies, it should be noted, that have been associated with a lot of hurt and pain. It is hoped that through better understanding of these dichotomies and their damaging effects, current and future Koorie cultural discourses can be relocated in frameworks which transcend them, recognising that we are dealing not with dichotomies, but with continua of orality and text; remembering and forgetting; past, present and future.

\section{Putting the Research Together}

Any illumination of past, present, and future First Nations conditions demands a complete deconstruction of the history and application of

31 David Hollinsworth, "Guidelines for Non-Racist Language Use in Aboriginal Studies," Indigenous College of Education and Research, The Unaipon School, UniSA, http://www.unisa.edu.au/Unaipon/current/non-racist.asp. 
ideology and, most importantly, of the impact (personal and political) of racism. That is, we need to know how we got into the mess we're in. "Colonialism means that we must always rethink everything". We need to have an analysis of the colonisation and our cultural past to decolonise our mind, heart, body and spirit. Without this critical knowledge, we are operating in a vacuum. Colonization of Aboriginal peoples could not have been perpetuated and maintained without the role of knowledge extraction and propagation of false consciousness. Henderson claims that if the context of a person's reality does not allow one to move in their world and to discover as much about themselves as they can, then such a context is artificial. These false images and misrepresentations that hinder Aboriginal people from seeing themselves as they really are have disconnected them from their natural contexts and have created 'artificial contexts'. Thus, re-contextualising Aboriginal experiences, events and history can help us make sense of our reality. ${ }^{32}$

Many of the issues that were highlighted in the course of my research were evident from the start. Although there might seem to be circularity, this is really more of a positive feedback loop that adds to both the findings and the analysis. The research narrative tells the story of the physical and emotional effects of the relationships between two cultural discourses - those of the dominant ${ }^{33}$ Australian community and those of the Koorie community whilst the research informing it is situated within and straddles these same discourses. This dual-occupancy is illustrated through the multi-disciplinary location of the research and researcher. In other words, the origins of the research question are located in both the community and academia and the focus of the research is on the use of Koorie oral records and institutional archival records in the creation of narratives. This shared place is ideal for

32 Kathy Absolon and Cam Willet, "Aboriginal Research: Berry Picking and Hunting in the 21st Century," First Peoples Child and Family Review. A Journal on Innovation and Best Practices in Aboriginal Child Welfare 1, no. 1 (2004): 9-10. In this quote, Absolon \& Willett references J. Y. Henderson's “Challenges of Respecting Indigenous World Views in Eurocentric Education," in Voice of the Drum: Indigenous Education and Culture. R. Neil, ed., Brandon, Manitoba: Kingfisher Publications, 2000; "The Context of the State of Nature," in Reclaiming Indigenous Voice and Vision, Marie Battiste, ed., Vancouver, BC: UBC Press, 2000; Bell Hooks, Black Looks: Race and Representation, Boston, MA: South End Press, 1992; Smith, Decolonizing Methodologies: Research and Indigenous Peoples.

33 Dominant culture refers to the culture that dominates a community's systems of communication, law, governance and social structure. Within Australia, this culture originated in Britain and is based on the Anglo-Saxon male. This dominant culture is based within Western knowledge system. 
this research, but it is still a new place, and, therefore, one that is still being created and negotiated.

\section{Research Design and Methodology}

Research which has been imposed on Aboriginal peoples to define socalled Aboriginal problems has generally evolved from cultural and social engineering theories, which continue to influence current policy, research, government debates and social perceptions. [ ... ] Therefore it was vital to find a culturally safe way to research sensitive issues such as intra-cultural violence. ${ }^{34}$

Sourcing a 'culturally safe' research design when undertaking research with Indigenous peoples is a constant concern for researchers and communities alike, resulting in various research guidelines for culturally and ethically based research (particularly in health research). ${ }^{35}$ Although cultural safety was a concern for this research, I also wanted to design my research in a way that was respectful of both Koorie community and academic traditions.

My research question originated from personal experience, and anecdotal reports from other Indigenous Australians regarding experiences with research and narratives about them and their communities. These reports not only highlighted a range of research issues, but also demonstrated how Koorie communities are attempting to share their narratives beyond their immediate community - moving beyond the Koorie discourse into the wider mainstream Australian discourse. It was a conscious decision to take this community experience and develop it as a research project that would be conducted from within the academy - a bastion of mainstream Australian

34 Atkinson, Trauma Trails, Recreating Song Lines: The Transgenerational Effects of Trauma in Indigenous Australia, 13. This quote also references: Smith, Decolonizing Methodologies: Research and Indigenous Peoples.

35 For example: National Aboriginal Health Strategy Working Party, A National Aboriginal Health Strategy [Canberra]: [National Aboriginal Health Strategy Working Party], 1989; National Health and Medical Research Council (Australia), "Values and Ethics: Guidelines on Ethical Conduct in Aboriginal and Torres Strait Islander Health Research," Canberra: National Health and Medical Research Council, 2003; Paul Stewart and Priscilla Pyett, "Victorian Aboriginal Ethics Project Report: A Community Report from Onemda VicHealth Koori Health Unit," Melbourne: Onemda VicHealth Koori Health Unit, 2005; VicHealth Koori Health Research and Community Development Unit, "Research: Understanding Ethics." Melbourne: VicHealth Koori Health Research and Community Development Unit, 2001. 
discourse. It was a conscious decision in that minority communities are only too aware that for different cultural narratives to be heard and understood within the broader Australian and international discourses, they need to be recreated in a format recognised by that discourse. To balance the community base of this research with academic rigour, I felt the need to create a research design and methodology that neither subsumed nor created a dichotomy between the discourses.

The analytical technique employed is one that I learnt from Elders at the Koorie Heritage Trust Inc. and have been using for many years. It involves gathering various forms of records, regardless of their origin or cultural bias, and reinterpreting them to create narratives from a Koorie perspective that can then be added to (or integrated into) the cultural discourse of the wider community of Victoria and beyond. This process has rewards for the whole community in that it preserves, informs and repatriates to the Koorie community knowledge that may have been unknown or believed lost, while at the same time educating the wider community about the past from a different cultural perspective than the widely accepted dominant one.

This research technique was the basis of my data collection, and underpinned my analysis and presentation. Although this is a method developed as a process of decolonisation by the Koorie community, it is also located within academic research methods. In particular it has elements in common with grounded theory: 'theory which is built from the ground upwards, that is from data observed and collected in the field ${ }^{36}$, and more specifically, the constructivist form of grounded theory. Gilliland and McKemmish define grounded theory building further:

this method is concerned with discovering concepts and hypotheses and developing theory directly from data that is collected from the field and provides relevant and interesting cases for analysis. Tends to be associated with interpretivist research ... [used] where little is known about a particular situation or phenomenon. ${ }^{37}$

The method used to gather the data is a form of interpretative research 'favouring' naturalistic inquiry:

36 Kirsty Williamson, Research Methods for Students, Academics and Professionals: Information Management and Systems, Ross Harvey and Stuart Ferguson, eds., 2nd ed., Topics in Australasian Library and Information Studies, Wagga Wagga: Charles Sturt University, Centre for Information Studies, 2002, p. 332.

37 Anne Gilliland and Sue McKemmish, "Building an Infrastructure for Archival Research,” Archival Science 4 (2004): 178. 
Researchers who are interpretivists favour naturalistic inquiry (where field work usually takes place in the "natural setting") are concerned with "meaning". They believe that the social world is interpreted or constructed by people and is therefore different from the world of nature. ${ }^{38}$

(See Bunn's chapter in this volume for a more detailed discussion of grounded theory). In line with the community approach to research described above, a combination of these forms was used, including content, discourse, narrative and conceptual analysis. ${ }^{39}$

The data collection method and forms of data analysis applied to this research respect the existing methods of inquiry used within the Koorie community, where there are many time-honoured processes of knowledge transmission. The basic underlying premise is that orality is still very important, and there are many who still use the forms and structures of storytelling. The principles of Koorie orality are a sense of trust in the transmission of knowledge and the worldview that everything is connected even when you are unable to see the connection:

Our fore-fathers worked in harmony with the universe communicating with all the surrounds within a symbiotic understanding of honour, respect, reciprocity. The world and universe is a whole with each to play a part in that relationship. There is no separation of parts. This is a perfect understanding of naturalistic inquiry. ${ }^{40}$

\section{Challenging the Design}

For knowledge to be shared, trust needs to be developed between the parties. Even with the acceptance of this worldview, and the development of trust, the interview process may take a longer time than it might in more researcher-controlled research - where the researcher is the 'expert' instead of a participant. Some interviews may require more than one visit to gain trust and to access the knowledge required, and while some stories may appear to be going off the topic, they eventually flow back to the subject at hand. The researcher must allow the knowledge holder to present his

38 Williamson, Research Methods for Students, Academics and Professionals: Information Management and Systems.

39 Gilliland and McKemmish, "Building an Infrastructure for Archival Research," 179.

40 Winch and Hayward, "Doing It Our Way': Can [Aboriginal] Cultural Traditions Survive in Universities," 25. 
or her knowledge in their own way - this may involve one person talking the whole time, a discussion, or questions and answers - it differs for each person. Stopping the interview, and/or recording, because the knowledge holder may have gone 'off topic' or is 'repeating' something already said could dissolve the existence of trust, ${ }^{41}$ and prevent the knowledge required being conveyed. Also, by stopping the knowledge holder, one might miss out on relevant aspects of the story.

It was difficult for me to undertake this type of research as a sole practitioner. In my practice, group or collective research is a more natural process when the research involves people. A group provides input and advice, with expertise in different areas, allowing for discussion and exchange of ideas. Another issue experienced in undertaking this research is that the knowledge being presented originates from others. This research is a conduit for others to present their knowledge; in my view, being the author does not equate to being the knowledge owner. To combat these issues I created the collective I yearned for through colleagues, friends and family. As for knowledge ownership, I rejected the concept that the interviewer is the author or copyright owner of interviews. In this research, each of the knowledge holders was the author of his or her interview; it is their knowledge and this was acknowledged as such by the use of their name when the research was published, unless anonymity was requested. It is hoped that this concept of authorship is a step towards better practice in regards to recognising knowledge ownership within research and narrative creation.

For this research design to work, both the researcher and knowledge holder had to understand the nature and focus of the project. If the knowledge holder is a storyteller, then there is usually little control of the interview by the researcher once the interview commences. It is important, especially in this situation, that the pre-interview discussion is used to explain the project, discuss the preferred method of interview, the backgrounds of both parties, and any issues. This method can create difficulties for the analysis process, as each of the interviews can be very different and this makes comparisons and identifying themes very difficult at times. Yet it is also a positive in that the data are very rich, with much 'meaning' to be explored and analysed.

Research involving human interaction is emotionally draining. The research design requires the researcher to be a part of the interview process thus creating a two-way relationship. This relationship can be demonstrated

41 'Trust' in the sense that one is respectful of the knowledge holder, the knowledge that person holds, and that one wants to learn what he or she has to share. 
through the interview verbally as a discussion or questions and comments, but it can also be in the form of emotions and body language - a smile, a tear, expression of shock, a nod of understanding, a head shake or sound of acknowledgement, laughter, or a touch; recognition that the knowledge being shared is understood and appreciated - an emotional link between the researcher and knowledge holder. I had the advantage of knowing many of my knowledge holders, thus allowing for an open and comfortable interviewing relationship. It also, however, heightened emotional responses that I experienced - emotions that continued throughout the analysis and write-up of the data.

The emotions that lingered throughout the research process were often in response to the memory of the interview, be it a giggle at remembering a facial expression from a particular part of the interview, or a tear at a sad story, or anger at the unfairness within our society. These provided cues for painful or sad memories. Although this has been an obstacle, it has also provided opportunities to relive positive stories, and to laugh at humour included within the recorded words. I suppose I am saying that listening and reading these transcripts did not solely provide me with data. It is also constantly linking me to people and community on an emotional and physical level. However, some emotions are not even connected to the interview, but to events that occurred around or after the interview, to either the researcher or knowledge holder. The hardest of these to deal with were the deaths.

Two very strong and amazing women from the Victorian Koorie community died unexpectedly during this research process - one within weeks of conducting our interview, and the other a year to the day after our interview. Listening or reading these interviews was difficult due to linked emotions. It was especially hard as both referred to actions they had planned, yet were left unfinished.

The death of knowledge holders also raises a number of concerns regarding the use of the interviews: Should family members be consulted about the use of the interviews? Would the knowledge holders want their interviews used? Should they be named? Even with consent forms, these questions and others constantly need to be addressed and revised on an individual basis. Developing and applying this research design was a learning experience, with benefits and problems, but because it was continually responsive to the needs and experiences of those included, it was a design appropriate for this research.

Deciding on appropriate research methods is a complicated process. It first required me to overcome my personal preconceived notions against 
'mainstream' research methods. Most of my life I have been told stories of bad and/or culturally inappropriate research practices ${ }^{42}$ undertaken with Koorie communities. To combat this prejudice, I needed to discover or develop a research method that met the requirements of both the academic strictures of doctoral and community research. This research has constantly faced the difficulty of researching impacts upon Koorie communities within a Western research paradigm. Undertaking research in a way that was not going to have an adverse impact upon the participants was a major concern. To address this concern, the underlying principle of the data collection became that the participants were to be partners in the research process. This approach has been developed in response to situations in which research has been used to control and discriminate against Indigenous peoples. Indigenous peoples worldwide have been fighting for involvement in research in the form of control, consultation, recognition, and partnership in all forms of research ${ }^{43}$ - they are battles to have Indigenous voices heard. It

42 Onemda VicHealth Koori Health Unit, University of Melbourne has workshopped similar stories to produce a series of community reports on the distrust of mainstream research in health: VicHealth Koori Health Research and Community Development Unit, "We Don't Like Research ...: But in Koori Hands It Could Make a Difference," Melbourne: VicHealth Koori Health Research and Community Development Unit, 2000; "Research: Understanding Ethics"; Onemda VicHealth Koori Health Unit, "We Can Like Research ... In Koori Hands: A Community Report on Onemda Vichealth Koori Health Unit's Research Workshops in 2007," Melbourne: Onemda VicHealth Koori Health Unit, 2008.

43 Examples of guidelines and discussions of Indigenous peoples involvement in research: Absolon and Willet, "Aboriginal Research: Berry Picking and Hunting in the 21st Century"; David R. Biggins, "Research in Aboriginal Health: Priorities, Ethics and Philosophy," New Doctor 70 (1998); E Bourke, "Dilemmas of Integrity and Knowledge: Protocol in Aboriginal Research" (paper presented at the 1st National Rural Health Workshop, Whyalla, July 13-15 1995); M, Brady, "Some Problems of Method and Theory in Aboriginal Research," in Research and the Delivery of Legal Services, P. K. Cashman and Law Foundation of New South Wales, eds., Sydney: Law Foundation of New South Wales, 1981; Joanne Brown, Ernest Hunter, and Mary Whiteside, "Talking Back: The Changing Nature of Indigenous Health Research Feedback," Health Promotion Journal of Australia 13, no. 2 (2002); Rick Henderson et al., "Development of Guideline for NonIndigenous People Undertaking Research among the Indigenous Population of North-East Victoria," The Medical Journal of Australia 176, no. 10 (2002); Kim Humphery, "Dirty Questions: Indigenous Health and 'Western' Research," Australian and New Zealand Journal of Public Health 25 no. 3 (2001); Margaret Kovach, "Indigenous Knowledge(s) and Research: Creating Space for Different Ways of Knowing within the Academy," in First Nations, First Thoughts Conference (Edinburgh: Centre of Canadian Studies, University of Edinburgh, 2005); Ruby Langford, “Our Heritage - Your Playground,” Australian Archaeology 16(1983); Langton, "Anthropologists Must Change"; Winch and Hayward, "Doing It Our Way': Can [Aboriginal] Cultural Traditions Survive in Universities.” 
is from these principles that the following protocols relating to data collection were developed: the participants were fully informed about the research, they retained authorship/ownership of their words, they had control of the ways in which their interviews were used and what happened to them after the research, and they could at any time prior to the completion of the research withdraw their interview. This was done through the Explanatory Statement and the pre-interview process. Colleagues flagged some of these protocols as potentially risky in terms of difficulties such as follow-up time, contacting knowledge holders if they were to move, and possible problems during the write-up due to differences in the data gathered from each participant. In my opinion however, that the participants had control over their involvement in the interview process and the research outweighed such considerations.

\section{Data Collection}

This research had four data sources: people who participated in the interviews and focus groups; documentary sources, mainly represented in the literature; my own experiences and experiential knowledge; and the Trust and Technology project (T\&T project), its data, findings and the experiences of working in the team.

\section{Interviews and Focus Groups}

\section{The Participants and Their Selection}

I knew or had prior knowledge of many of the people and organisations that I wanted to approach to be participants in my research. Initially I thought this would be a problem in terms of bias and objectivity, but it became apparent that the benefits of knowing some of the participants helped in applying the research design as described above.

I approached twenty-six authors for interviews and six organisations for focus groups. Of the twenty-six potential interviews, fourteen authors were interviewed, two declined, three did not respond, four showed interest but did not respond to follow-up correspondence, and three are still willing to be interviewed but we were unsuccessful in finding suitable times during the period of this project. Of the six organisations approached, four focus groups were conducted. The period of time spent on negotiating these interviews 
and focus groups was lengthy and with negotiated priorities, often due to the busy nature of life. This meant that some interviews took weeks and months to organise. A number of the interviews were re-scheduled up to five times before they actually occurred, or eventually did not occur.

All the participants were given the option to be identified by name in the research. Of the fourteen interviews, one transcript was removed and the remaining thirteen chose to be identified by name. Of the sixteen participants across four focus groups, one transcript (of two participants) was removed due to technical problems and one participant requested to be de-identified.

The selection of individuals and institutions for the data collection originally followed a more quantitative approach, where I had decided that there would need to be equal participant numbers in regards to the type of authors - i.e., equal numbers of Koorie and non-Indigenous participants from the areas of oral, written, performance and art narratives. It quickly became apparent that this was not only impossible it was also unnecessary. It was unnecessary as the research was investigating the impact of narratives of Koorie Victoria upon the people of Victoria, and therefore methods and impact were of more importance than was comparing Koorie and nonKoorie authors. It was not possible in that it was difficult to locate certain types of non-Indigenous authors of narratives of Koorie Victoria (for example storytellers). Although I was still aiming for equal representation of Koorie and non-Indigenous knowledge holders, the non-Indigenous participants were predominantly academics whilst the Koorie participants were academics and artists, with many being both.

Although statewide representation was initially envisaged, after the first five interviews it seemed unnecessary to pursue this approach. Firstly, there did not appear to be any obvious geographical differences, and also many of the participants who had been identified in the metropolitan area had moved to Melbourne due to the nature of their work and still had strong ties with their country or community of origin.

\section{Pre-interview Process}

Pre-interview discussions were conducted with the participants to outline the project and the interview process, complete the consent form, discuss the broad subject topics, and answer any questions. Pre-interviews were not recorded. The purposes of the pre-interview discussions were to provide an opportunity for the participants: 
- To understand and feel comfortable with the research process;

- To think about and discuss the ideas they wanted to present in the interview (sometimes it is difficult to provide answers on the spur of the moment, so discussing the process and the reasoning behind the research may help the participant to think through, or discuss with others, what he or she would like to say);

- To allow the participants to decide if they would like someone else to be present at the interview to support them (this is particularly relevant when the research area is of a sensitive nature).

With regard to the organisational groups, the heads of the organisations were contacted and a process suitable to them was negotiated. I did not provide individual pre-interviews to the focus group participants, but I did provide copies of the topic areas, and allowed time prior to the recording of the session for questions and discussion.

Pre-interviews can be risky in that the participants may start the interview before the recording begins, but they can also provide the interviewer with background on the participant that can be used as prompts during the interview. The pre-interview discussion also allows for an agreement on the participant's preferred interview process. Every individual has a preferred way in which $\mathrm{s} /$ he transmits knowledge. Some are storytellers and once they have grasped the purpose of the discussion are happy to talk on it. Some are conversationalists and prefer interaction with another in the form of a discussion. Yet others prefer to answer questions and receive feedback that the information being provided is what is required for the research.

\section{Interview Process}

If the preferred interview method was story telling, questions were only asked when clarification was required, or if questions were the preferred method. The interviewee, or knowledge holder, was not stopped if the discussion or answers seemed to be veering off, as this is often part of a thought process and almost always will veer back to the topic. The focus groups were similar in process to the interviews, with questions asked when discussion stalled or if the group members were unsure what the next topic was. All the interviews and focus groups were audio recorded and transcribed with consent forms completed.

I felt that the standard research method option of destroying the researchers' copies of the recordings and transcripts after a five-year retention 
period $^{44}$ was in opposition to the spirit of archiving. The interviewees were given the option of having the research copy destroyed, returned to them, or lodged at a nominated Indigenous repository. The focus group participants were given the options of the recordings and transcripts being destroyed or lodged at a nominated Indigenous repository (as there was only one research copy and numerous participants). All of the participants opted for the research copy being lodged with the Indigenous repository. While originally the option was to be that the participant could nominate a repository, this would have meant that individual interviews would be scattered across institutions instead of being accessible in one place. The participants retain the option of lodging their own copies with other repositories.

Three of the interview participants did not receive their transcripts for comment, although consent forms for the use of the interviews had been completed. One of these was due to the participant requesting not to have the transcript or recording sent out - he stood by everything said in the interview. The other two were due to the deaths of two of the participants.

\section{The Literature as Data Source}

This research was consciously conducted in a shared epistemological and intellectual space - multi-discourse and multi-disciplinary. So while there was a conventional literature review, the literature was also analysed as a data source for the key concepts, constructs, and features that form the framework for the cultural discourses of Koorie and non-Indigenous Victoria, and their interaction.

Identifying the literature for this area of research was complicated; there was too much material as opposed to not enough. Within a month it became clear that many of the concepts that existed within this area of research, as reflected in the literature, are culturally challenged and biased, archaic in terms of a modern world, had numerous and varying definitions and conceptual frameworks relating to various disciplines and/or, were inadequate for the purposes of this research. One of the first areas discussed with one of my supervisors was the inadequacy and confusion surrounding the concepts and terms 'oral history' and 'Indigenous oral history', resulting in a paper titled 'What is Australian Indigenous Oral History?' cowritten with Professor Lynette Russell for the International Oral History

44 The retention period specified by Monash University. 
Conference. ${ }^{45}$ Other areas of confusion and contention were definitions of identity, culture and history in discourses that had differing cultural control and responsibility frameworks.

Suffice it to say, the literature around oral histories and orality, Indigenous research methodologies, narrative (processes, general use, role in healing and identity, counter-narratives, and the importance of and differences in Indigenous narratives), archives and minority groups, and Indigenous writings (writings by Indigenous peoples, and analyses of Indigenous literature and use of literacy), and subsequent discussions relating to it, were a significant source of data for exploring concepts, informing the conceptual structure of the research, and restructuring my personal experiences. Analysing and reflecting on the literature as a data source became a continual part of conceptualising and contextualising my way of thinking, particularly with regard to the relationships between the dominant and Koorie discourses reflected in the literature.

\section{Personal Experience}

I worked for the Koorie Heritage Trust Inc. (KHT) ${ }^{46}$ for nine years prior to undertaking this research, and held various positions during that time. When I first started working for the KHT I still felt I was a 'newbie' to the Koorie community, as I had only found out that I had Indigenous ancestry five or maybe six years before; I still knew very little about Indigenous Australian history or culture, let alone Koorie history or culture. It was a culture shock. After fifteen years in Australia's education system with very little indication that Indigenous people even existed today, just the permanent exhibition Koorie $^{47}$ opened my eyes and ears to a new world of amazing narratives - narratives that made me proud, that made me laugh, that made me cry, that made me ME. I grew as the Trust grew. It was a good and occasionally a scary experience.

My time with the Trust combined with my continuing place within the Koorie community form my personal experience. This experience has become a data source that has been drawn upon throughout the research process. It

45 Shannon Faulkhead and Lynette Russell, "What Is Australian Indigenous Oral History?" in International Oral History Association Conference, Sydney: Unpublished, 2006.

46 For more information on this organisation see: http://www.koorieheritagetrust.com/.

47 Koorie was an exhibition created by the Koorie Heritage Trust Inc. and was housed in the old home of the Museum of Victoria at 325 Swanston Street, Melbourne, Victoria until its closure. The book resulting from this exhibition is Koorie Heritage Trust Inc., Koorie. Melbourne: Koorie Heritage Trust Inc, 1991. 
has been used to shape the research design, it helped with my interaction with the participants and the analysis of the data, it has provided additional knowledge, and has filled gaps in other data. My personal experience has also been a filter. All of the knowledge gathered for this story has gone through me.

Including my personal experience as a data source was not easy. As I have already intimated, I have had to work through my personal biases to undertake this research. My earlier versions of this narrative were culturally biased in a negative or 'us versus them' way. It is difficult not to reflect the transgenerational hurt that the Koorie community continues to experience from actions of colonial-invasion in my writings.

\section{Trust and Technology (T\&T) Project Data}

The 'Trust and Technology: building an archival system for Indigenous oral memory' (T\&T Project) ${ }^{48}$, was a joint project between Monash University, Public Record Office Victoria, the Koorie Heritage Trust Inc., the Koorie Records Taskforce, and the Indigenous Issues Special Interest Group of the Australian Society of Archivists, and was undertaken from 2004 to 2008. The project aimed to develop an archival system for Koorie oral knowledge and was premised on the development of trust of archival systems and technology to achieve an oral memory archive that respects the needs and concerns of the Koorie community particularly in regards to access. An important underpinning of the project has been the recognition of the impact of past archival practices on the Indigenous community and the development of an understanding of the current impact of those practices.

I found my interaction with the T\&T project and the project team to be vital to my own research. Since the data from the T\&T project was collected for different purposes from those of this research ${ }^{49} \mathrm{I}$ used it as a data source in much the same way as I did the literature. In particular it provided information regarding Koorie experiences of, and interactions with, archival institutions. Similarly to my experience with reviewing literature related to my topic, discussions regarding the re-conceptualisation of various terms and constructs were a constant process, in which the $T \& T$ project team was a vital part. This process was often a two-way conversation and involved interaction between

48 For more detailed information on the T\&T project, see the website at: http://www. infotech.monash.edu.au/research/centres/cosi/projects/trust/ (accessed 25 April 2015).

49 As a member of the T\&T project team, my access and use of the data was in accordance with the Monash University Standing Committee on Ethics in Research Involving Humans applications. 
the two projects, where knowledge and understandings were exchanged and used within the respective projects and joint publications. As the T\&T project and my research have progressed, they have shared definitions and addressed issues together allowing for a self-reflectivity that would not have been possible without the support of the T\&T research team. It was, however, difficult to engage with the raw data of this project. This difficulty was because I was not involved in the data gathering process. The research design for T\&T also changed and developed over the period of the project, and adopted and addressed many of the research design features listed above - it has been a positive and self-reflective project. ${ }^{50}$ Experiences with the T\&T project, and lessons learnt from it, therefore, contributed to the research design and method discussed here.

The process of combining the data sources was an instinctive and intuitive process, with the data working together to create this narrative and my personal experience acting as a filter deciding on what fit where and how. Self-reflectivity played a large part in this process, with each area of the narrative being reflected upon and discussed and reworked.

\section{Data Analysis}

A combination of four forms of data analysis was applied to the data content, narrative, discourse and concept analysis. These four forms were derived from my experience with Koorie research methods. Self-reflection and deep thought within and across these forms of analysis brought the data described above together to form the narrative.

\section{Content Analysis}

Content analysis is described by Gilliland and McKemmish as:

Examination of both the visible and the underlying, latent or symbolic content of a document based upon a system of coding content. ${ }^{51}$

50 For more details about the self-reflective process in the research design and methods of the T\&T project, see Shannon Faulkhead et al., "Is Community Research Possible within the Western Academic Tradition?" in Researching with Communities: Grounded Perspectives on Engaging Communities in Research, Andy Williamson and Ruth DeSouza, eds., Auckland: Muddy Creek Press, 2007.

51 Gilliland and McKemmish, "Building an Infrastructure for Archival Research," 179. See also: E. Babbie, The Practice of Social Research 10th ed., Belmost, CA: Wadsworth, 2004; Kimberly A. Neuendorf, The Content Analysis Guidebook, Thousand Oaks, CA: Sage, 2002; Carl W. Roberts, ed., Text Analysis for the Social Sciences: Methods for 
Content analysis was applied to this research to draw the topics and issues relating to this narrative from the interviews and focus groups, and to identify the main themes. From early on in this project, I viewed the process of narrative creation as involving research, creating the actual narratives, sharing them with others and making decisions as to whether they should be transmitted to future generations. To understand how narratives impact upon the community, it was necessary to develop an understanding of how they interacted with the community. Through this need for understanding combined with reading through the transcripts, and/or listening to them, four overarching themes evolved relating to the research question emerged:

1. Stories and narratives - What are stories and narratives? Who creates them and why? What are their purposes? How do they interact with individuals and communities? How do they become part of a community's knowledge?

2. Connecting through records - Both Koorie and dominant narratives of Koorie Victoria are created through the collection and analysis of the records available. How and where are records stored? How are they accessed? What methods and disciplines are used in the interpretation of these records?

3. Community research - Community research can be viewed in two ways -1 . Research undertaken by community, and 2 . Research that is respectful of community.

4. Transmission through generations - Within Koorie communities the ability to transmit knowledge through the generations is an important aspect of community and cultural responsibility. This is the area in which narrative creation can, will or has had an impact upon communities.

I then revisited the interview transcripts to draw out examples that discuss these themes and to assist me in answering my research question, while also drawing on my personal experience and the literature as a filter and locating these findings within the broader context of narrative creation and Koorie Victoria.

Drawing Inferences from Texts and Transcripts, Mahwah, NJ: Lawrence Erlbaum, 1997;

Mark D. West, ed. Applications of Computer Content Analysis, Westport, CT: Ablex, 2001; Klaus Krippendorf, "Some Principles of Information Storage and Retrieval in Society," General Systems 20 (1975); Mark D. West, ed., Theory, Method, and Practice in Computer Content Analysis, Westport, CT: Ablex, 2001. 


\section{Discourse Analysis}

Discourse analysis is defined by Gilliland and McKemmish as:

Use of analytical constructs for the historical analysis of ideas, looking at the principal aspects of the discourse in terms of the context of the time and using the literature of the discipline and related disciplines, the major sources for theory building. This method assumes a constant evolution of ideas and identifies when radical changes occur and their impact on existing concepts. It often focuses on discourse generated by influential parties to examine what it reveals about ideologies and power differentials. ${ }^{52}$

Discourse analysis is an interesting analytical approach as it applies to numerous types of analysis of actual discourse (e.g., speech patterns, writing styles, linguistics and language itself) used by various social science disciplines. As indicated by Gilliland and McKemmish, the term is also used within archival science in relation to how discourse changes over time and how this can reflect power disparities. It is in this latter sense that the term is used here. I have not used discourse analysis to analyse the elements of discourse, but instead to analyse the historical power struggle existing within Australia as reflected through research and professional literature. It has taken the form of tracing the development and evolution of concepts and their contexts, and tracking the emergence of differing conceptual frameworks, particularly in the literature. This has been undertaken through the analysis of the data to produce an in-depth discussion and interpretation of the literature as it links to the main concepts and themes of research. The self-reflective process discussed throughout this chapter complemented this analysis since some of the historical perspectives presented in the literature were difficult and at times emotionally exhausting to analyse in a non-adversarial style.

\section{Narrative Analysis}

Narrative analysis is defined by Gilliland and McKemmish as:

52 Gilliland and McKemmish, "Building an Infrastructure for Archival Research," 179.

See also: N. Fairclough, Language and Power (Essex, England: Longman, 1989); Bernd Frohmann, "Discourse Analysis as a Research Method in Library and Information Science," Library and Information Science Research 16 (1989); Livia Iacovino, "MultiMethod Interdisciplinary Research in Archival Science: The Case of Recordkeeping, Ethics and Law," Archival Science 4, no. 3-4 (2004). 
Body of related unobtrusive techniques for examining how narrative or rhetorical tropes are used in documents to "tell stories", or advance specific perspectives or arguments. ${ }^{53}$

Power is often maintained through controlling which narratives are told and by whom on a particular topic. This control can be achieved through denigrating the author and his or her work. With Indigenous Australians, the people themselves were denigrated and their narratives, often located within the Australian Indigenous community, were regarded as having less authority than did works about Indigenous peoples. Although this denigration is obvious within the terms and style of the discourse (discourse analysis), it is through comparing narratives by Indigenous and non-Indigenous authors that the methods can be documented (narrative analysis).

The narratives I encountered while working at the KHT were often in opposition to those that existed within the mainstream discourse. Many of the mainstream narratives either indicated or stated outright that there were no Indigenous people left in Victoria - that they had died out (which I knew to be untrue) - leaving us with only the pre-history context of their existence. The narratives shared through my time at the KHT were ones that placed Koorie people within the context of Victoria and Australia's past and present. One of the first things I learned at the KHT was that Koorie people have a continuous cultural existence on this land. I experienced narratives via artworks, artefacts, and books, but the most powerful narratives (for me) were those where Koorie perspectives of written records were presented as an oral narrative; oral and text - different perspectives - presented together to provide a holistic view. Aunties and Uncles would share with us events of the past and how they affected Koorie peoples; they would tell of survival and sharing the pride of their Ancestors. We would then share records and documents we found to see what else there was to know. It was the most amazing way to learn - and teach - as we would then share the narratives with others.

Narrative analysis was the main method used in this research, particularly when analysing the interview and focus group recordings and transcripts. It was also used to complement the analysis of the literature, and in reflecting on my narrative of self and on the discourse of the T\&T project.

53 Anne Gilliland and Sue McKemmish, "Building an Infrastructure for Archival Research," 179. See also Andrew Abbott, Methods of Discovery: Heuristics for the Social Sciences. New York, NY: Norton, 2003. 


\section{Conceptual Analysis}

Conceptual analysis is described by Jonathan Furner as:

A technique that treats concepts as classes of objects, events, properties, or relationships. The technique involves precisely defining the meaning of a given concept by identifying and specifying the conditions under which any entity or phenomenon is (or could be) classified. ${ }^{54}$

One of the major difficulties in this research has been defining and explaining my choices with regard to concepts and terminology. When I started the research, I had very clear definitions of terms, but these changed. I have always found the nuances of words and the conceptual understandings attached to them to be interesting, in particular how and why they change. While discourse analysis tracks the power differential and narrative analysis the methods of narrative creation used, conceptual analysis is more about the complexities, interrelationships, and differences that can be applied to a single word. Take, for example, 'research'. What does this mean? Does the understanding of this word and its application differ between cultures? Is there history associated with the word, making it offensive to some groups? While there is a fine line between the three forms of analysis, each has a different purpose in this research.

Although I have presented these forms of analysis separately here, in the research process a combination of these occurred simultaneously. Bringing these analyses together to form this narrative was an instinctive process involving self-reflection and deep thought. It involved building the theoretical and practical understandings of this research area from the ground up, and balancing a holistic view with the understanding that the narrative emerging is only a segment or beginning of a much larger story. This narrative may be the broader context of the $T \& T$ project and the relationship between Koorie oral memory and archives, but there is an even broader context - or contexts - still where this narrative sits.

54 Quote from Jonathan Furner, "Conceptual Analysis: A Method for Understanding

Information as Evidence, and Evidence as Information," Archival Science 4, no. 3-4 (2004): 179. See also Frank Jackson, From Metaphysics to Ethics: A Defence of Conceptual Analysis. Oxford: Oxford Scholarship Online, 1998; Brian Leiter, "Introduction," in The Future for Philosophy, Brian Leiter, ed. Oxford: Oxford University Press, 2004. 


\section{On Reflection}

Although the connection between people, culture, community, and history has always interested me, when I began this research I did not realise how much of a connection there would be, and how much of myself would become part of the research process. The exploration of these connections and my personal journey of discovery have made a positive and important contribution to my research, but I also found this process confronting. I was constantly analysing every aspect of my beliefs and attitudes - consistently analysing my own biases and how and what dichotomies I applied to those that I did not even consciously acknowledge as 'other' - for example, 'non-Indigenous'. Whilst I dislike the use of the prefix 'non' to describe Australians who are not Indigenous, there is no term of self-identification other than Australian. And as Indigenous Australian peoples are also 'Australian' this is not a term that can be used to differentiate between the original people of this land and the newcomers. It was difficult not to create a 'us' and 'them' dichotomy.

It has been a few years since I undertook this research, and it is interesting to go back and revisit the research process instead of the findings of the research. There were certainly some aspects that were difficult both emotionally and physically, but I am unsure if I would do it any differently today. Each research undertaking is unique and as such has its own quirks and knots. With this research, parts of the research methodology were developed as I progressed. This was not by choice, but because I was entering the research from a different perspective, it was difficult to visualise how it should work. Moreover, there was little similar research to compare it to at the time. Although it would have been nice to have a clear plan at the start, I would have missed all the messiness that made this research what it became.

With the data collection it was always clear that the data being used would be from conducting interviews and focus groups, existing literature, and T\&T Project data. It was not until the write-up that it was pointed out to me that I was missing a major data source that I had used, but had failed to identify. This was 'personal experience'. It did not occur to me that my life experiences not only led to this research, but also were a data source that needed to be identified and analysed. I found this difficult, as it required a deep reflection on 'how' and 'what' I did in terms of the research. I found the 'interviews and focus groups' and the 'literature' a lot more fun. Despite the ethical and emotional issues around ownership and analysis, I liked being able to include other voices in my research $-\mathrm{I}$ was no longer alone in my writings. 
The literature was also interesting. I was set the task on a number of occasions to write a literature review of what I was reading, but it was quickly identified that I am 'literature review challenged'. I kept engaging with the literature and producing original pieces of research instead of 'reviewing' the literature and research that had gone before me, although it did work out in the end. The T\&T Project data entailed a different situation again. Although I had ethics clearance to use the T\&T data, I found that I preferred to use the project as a whole instead of the individual interviews. This was for a number of ethical and logistical reasons. For example, I did not conduct the interviews, so I could never quite get the feel of what the data were - I did not have a personal connection; while my research was connected to the Project, the researchers had very different aims and ambitions; and, the Project was complicated in that it also evolved as it progressed.

With the analysis, I did not realise I was conducting the various forms of analysis until I started writing up my research methodology chapter. I analysed the data according to what I thought it required - I was responding to the data, not making the data fit the style of analysis. Once the forms of analysis were identified I was able to analyse the process on a different level. The content analysis was vital for the research, as each interview and focus group was very different. Without topics and issues identified it would have been difficult to use them effectively. The discourse analysis was emotionally exhausting, whilst the narrative analysis was complex. Indigenous and nonIndigenous narratives are very different in style and purpose and at times it felt wrong to compare them. This has been a constant ethical issue - does comparing one thing against another detract from the importance of each individually? I still have no answer for this. Conceptual analysis is in many ways a constant process for researchers. Every time you learn something new you reassess all that came before - you build upon and update your own personal archive.

\section{Conclusion}

One of the most natural processes is to tell stories that share our experiences and knowledge. One of the hardest processes has been to share how the information and knowledge contained within a narrative is developed. The processes of data collection and analysis were intense (particularly the analysis), but I never considered that I was undertaking a process - I was creating a narrative. The relationship between Koorie and academic research methods were balanced through commonplace processes of discussion and reflection. 
Identifying the correlations between academia and community research, though, required being hit over the head with a sledgehammer to remind me that I wanted to be able to balance these two constructs, and therefore I needed to be able to present both - especially within my own research. This chapter is not just about explaining how I went about researching this narrative, but also to locate this research within my own narrative.

The need for a research philosophy that considers and incorporates the research design and methods of more than one cultural paradigm is important. As an example, action research includes research principles that aim to protect the research participants, but within its frame of reference, researchers can at times forget that they are also participants. In this research it was evident that research principles do not (always) take into consideration the wellbeing of the researchers. Although Indigenous research principles often include the Indigenous researcher as a participant, nonIndigenous researchers are often perceived as exerting coercive power and therefore the principle does not extend to them. This research posited that research that co-exists within more than one cultural paradigm requires a respectful partnership between researchers and community; the sharing of control; that all voices are enabled to contribute to the overall outcomes; self-reflection; open discussion of methods and issues specific to the research being undertaken; and consideration of the emotional and physical wellbeing of all participants, including all members of the research team.

Unconsciously I began this research with a generalised negative view of non-Indigenous narratives and research of Koorie Victoria. As this view changed so did my approach to and understanding of research. This has resulted in this research not only being about the research, but also a case study in itself of respectful research that negotiates the location of a community-based research topic within an academic research structure. This negotiation has been difficult given my constant need to reflect community, and to address the academic requirements of a doctoral thesis. Despite the difficulties involved, I believe that research that embraces community and academic research principles is possible if the researchers involved are committed to doing so. I would like to state, though, that this research philosophy, principles and method would not be appropriate for all research involving community and that each research project needs to be based on a carefully negotiated partnership between the researchers and community. 


\section{Final Word}

The connection between people, culture, community, and history has always interested me, however when I began this work, I never realised how much of a connection there would be, and how much of myself would become part of the narrative. Although the exploration of these connections and my personal journey of discovery have made a positive and important contribution to my research, I also found this process confronting.

This research asked the question: How do the methods of narrative creation and transmission of and about Koorie people, culture and history impact on the collective knowledge and peoples of Victoria? I wrote extensively addressing this question, but the final sentence I wrote in my thesis sums up my thoughts and feelings. This research has been a personally public journey that is far from conclusion. Australia is a shared space, this research is asking for its discourse and collective knowledge to be so too.

\section{References}

Abbott, Andrew. Methods of Discovery: Heuristics for the Social Sciences. New York, NY: Norton, 2003.

Absolon, Kathy, and Cam Willet. "Aboriginal Research: Berry Picking and Hunting in the 21st Century." First Peoples Child and Family Review. A Journal on Innovation and Best Practices in Aboriginal Child Welfare 1 no. 1 (2004): 5-17.

Anderson, Ian. "Black Bit, White Bit." In Blacklines: Contemporary Critical Writing by Indigenous Australians, edited by Michele Grossman, 43- 51. Carlton, Victoria: Melbourne University Press, 2003.

Anderson, Ian. "Introduction: The Aboriginal Critique of Colonial Knowing." In Blacklines: Contemporary Critical Writing by Indigenous Australians, Michele Grossman, ed. Carlton, Victoria: Melbourne University Press, 2003, pp.17-24.

Atkinson, J. Trauma Trails, Recreating Song Lines: The Transgenerational Effects of Trauma in Indigenous Australia. North Melbourne, Australia: Spinifex Press, 2002.

Atkinson, Wayne. "Oral History and Cultural Heritage” [Conference Paper]. In Aboriginal Cultural Heritage Seminar. Aborigines Advancement League, 1984.

Babbie, E. The Practice of Social Research 10th ed. Belmost, CA: Wadsworth, 2004.

Beckett, Jeremy. "Autobiography and Testimonial Discourse in Myles Lalor's 'Oral History.” In Telling Stories: Indigenous History and Memory in Australia and New Zealand, Bain Attwood and Fiona Magowan, eds. Crows Nest, N.S.W.: Allen \& Unwin, 2001, pp. 123-42.

Biggins, David R. "Research in Aboriginal Health: Priorities, Ethics and Philosophy." New Doctor 70 (Summer 98/99 1998): 28-30.

Bourke, E. "Dilemmas of Integrity and Knowledge: Protocol in Aboriginal Research." Paper presented at the 1st National Rural Health Workshop, Whyalla, July 13-15 1995.

Brady, M. "Some Problems of Method and Theory in Aboriginal Research." In Research and the Delivery of Legal Services, P. K. Cashman and Law Foundation of New South Wales, eds. (Sydney: Law Foundation of New South Wales, 1981), 281-5.

Brady, W. "Observing the Other." Eureka Street 9, no. 1 (1999): 28-30. 


\section{Chapter i6}

Brewster, Anne, Rosemary Van den Berg, and Angeline O'Neill. Those Who Remain Will Always Remember: An Anthology of Aboriginal Writing. Fremantle, W.A.: Fremantle Arts Centre Press, 2000.

Brown, Joanne, Ernest Hunter, and Mary Whiteside. "Talking Back: The Changing Nature of Indigenous Health Research Feedback." Health Promotion Journal of Australia 13, no. 2 (2002): 34-39.

Burke, Peter. 'History as Social Memory." In Memory: History, Culture and the Mind, edited by Thomas Butler, 97-111. Oxford: Basil Blackwell, 1989.

Cashman, Genevieve. "[Koorie Oral History and Health].” Minor Thesis, University of Melbourne, 2002.

Connor, John. The Australian Frontier Wars 1788-1838. Sydney: University of New South Wales Press, 2002.

Crane, Susan. "Ahr Forum: Writing the Individual Back into Collective Memory." American Historical Review 102, no. 5 (1997): 1372-412.

Fairclough, N. Language and Power. Essex, England: Longman, 1989.

Faulkhead, Shannon. "Narratives of Koorie Victoria." Ph.D. thesis, Monash University, 2008.

Faulkhead, Shannon, and Lynette Russell. "What Is Australian Indigenous Oral History?" In International Oral History Association Conference. Sydney: Unpublished, 2006.

Faulkhead, Shannon, Lynette Russell, Diane Singh, and Sue McKemmish. "Is Community Research Possible within the Western Academic Tradition?” In Researching with Communities: Grounded Perspectives on Engaging Communities in Research, Andy Williamson and Ruth DeSouza, eds. (Auckland: Muddy Creek Press, 2007), 39-56.

Faye, Esther. "Collective Memory and the Holocaust." Australian Historical Association Bulletin, no. 91 (2000): 72-78.

Frohmann, Bernd. "Discourse Analysis as a Research Method in Library and Information Science.” Library and Information Science Research 16 (1989): 119-38.

Furner, Jonathan. "Conceptual Analysis: A Method for Understanding Information as Evidence, and Evidence as Information." Archival Science 4, no. 3-4 (2004): 233-65.

Gilliland, Anne, and Sue McKemmish. "Building an Infrastructure for Archival Research." Archival Science 4 (2004): 149-97.

Ginibi, Ruby Langford. "Voicing the Land: Asal Armidale." Notes and Furphies 39, Oct (1997): 19-21.

Grossman, Michele. "Beyond Orality and Literacy: Textuality, Modernity and Representation in Gularabulu: Stories from the West Kimberley.' In Boundary Writing: An Exploration of Race, Culture, and Gender Binaries in Contemporary Australia, Lynette Russell, ed. (Honolulu: University of Hawaii Press, 2006), 149-69.

Halbwachs, Maurice, and Lewis A. Coser. On Collective Memory. The Heritage of Sociology. Chicago: University of Chicago Press, 1992.

Hamilton, Paula. “The Knife Edge: Debates About Memory and History.” In Memory and History in Twentieth-Century Australia, Kate Darian-Smith and Paula Hamilton, eds. Melbourne: Oxford University Press, 1994, p.viii.

Henderson, J. Y. "Challenges of Respecting Indigenous World Views in Eurocentric Education." In Voice of the Drum: Indigenous Education and Culture, R. Neil, ed. (Brandon, Manitoba: Kingfisher Publications, 2000), 59-80.

Henderson, J.Y.. “The Context of the State of Nature." In Reclaiming Indigenous Voice and Vision, Marie Battiste, ed. (Vancouver, BC: UBC Press, 2000), 11-38.

Henderson, Rick, David S. Simmons, Lisa Bourke, and Janice Muir. "Development of Guideline for Non-Indigenous People Undertaking Research among the Indigenous Population of North-East Victoria." The Medical Journal of Australia 176, no. 10 (20 May 2002 2002): 482-85. 
Hollinsworth, David. "Guidelines for Non-Racist Language Use in Aboriginal Studies.” Indigenous College of Education and Research. The Unaipon School. UniSA, http:// www.unisa.edu.au/Unaipon/current/non-racist.asp.

Hooks, Bell. Black Looks: Race and Representation. Boston, MA: South End Press, 1992.

Humphery, Kim. “Dirty Questions: Indigenous Health and 'Western' Research." Australian and New Zealand Journal of Public Health 25 no. 3 (2001): 197-202.

Iacovino, Livia. "Multi-Method Interdisciplinary Research in Archival Science: The Case of Recordkeeping, Ethics and Law.” Archival Science 4 no. 3-4 (2004): 267-86.

Jackson, Frank. From Metaphysics to Ethics: A Defence of Conceptual Analysis. Oxford: Oxford Scholarship Online, 1998.

Koorie Heritage Trust Inc. Koorie. Melbourne: Koorie Heritage Trust Inc., 1991.

Kovach, Margaret. "Indigenous Knowledge(s) and Research: Creating Space for Different Ways of Knowing within the Academy." In First Nations, First Thoughts Conference. Edinburgh: Centre of Canadian Studies, University of Edinburgh, 2005.

Krippendorf, Klaus. "Some Principles of Information Storage and Retrieval in Society." General Systems 20 (1975): 15-35.

Langford, Ruby. “Our Heritage - Your Playground.” Australian Archaeology 16 (1983): 1-6. Langton, Marcia. “Anthropologists Must Change.” Identity 4 no. 4 (1981): 11.

Langton, Marcia. "Urbanizing Aborigines: The Social Scientists Great Deception.” Social Alternatives 2 no. 2 (1981): 16-22.

Leiter, Brian. "Introduction." In The Future for Philosophy, Brian Leiter, ed. Oxford: Oxford University Press, 2004.

Muecke, Stephen. Textual Spaces: Aboriginality and Cultural Studies. Gunther Kress, ed. Kensington, N.S.W.: New South Wales University Press, Communication and Culture Series,1992.

National Aboriginal Health Strategy Working Party. A National Aboriginal Health Strategy. [Canberra]: [National Aboriginal Health Strategy Working Party], 1989.

National Health and Medical Research Council (Australia). "Values and Ethics: Guidelines on Ethical Conduct in Aboriginal and Torres Strait Islander Health Research." Canberra: National Health and Medical Research Council, 2003.

Neuendorf, Kimberly A. The Content Analysis Guidebook. Thousand Oaks, CA: Sage, 2002.

Nora, Pierre, and Lawrence D. Kritzman. Realms of Memory: Rethinking the French Past. European Perspectives. New York: Columbia University Press, 1996.

Onemda VicHealth Koori Health Unit. "We Can Like Research ... In Koori Hands: A Community Report on Onemda VicHealth Koori Health Unit's Research Workshops in 2007.” Melbourne: Onemda VicHealth Koori Health Unit, 2008.

Roberts, Carl W., ed. Text Analysis for the Social Sciences: Methods for Drawing Inferences from Texts and Transcripts. Mahwah, NJ: Lawrence Erlbaum, 1997.

Russell, Lynette. "Indigenous Knowledge and Archives: Accessing Hidden History and Understandings.” In Australian Indigenous Knowledge and Libraries, Martin Nakata and Marcia Langton, eds. Kingston, ACT: Australian Academic and Research Libraries (AARL), 2005.

Smith, Linda Tuhiwai. Decolonizing Methodologies: Research and Indigenous Peoples. London: Zed Books, 1999.

Stewart, Paul, and Priscilla Pyett. "Victorian Aboriginal Ethics Project Report: A Community Report from Onemda Vichealth Koori Health Unit.” Melbourne: Onemda VicHealth Koori Health Unit, 2005.

Turnbull, David, and Lyndsay Farrall. Interpreting the Australian Environment: The Aboriginal Experience and the Colonial Experience. 2nd pilot ed. Geelong, Vic.: Deakin University, 1983. 


\section{Chapter i6}

van Toorn, Penny. "Indigenous Australian Life Writing: Tactics and Transformations.” In Telling Stories: Indigenous History and Memory in Australia and New Zealand, Bain Attwood and Fiona Magowan, eds. (Crows Nest, N.S.W.: Allen \& Unwin, 2001), $1-20$.

VicHealth Koori Health Research and Community Development Unit. "Research: Understanding Ethics.” Melbourne: VicHealth Koori Health Research and Community Development Unit, 2001.

VicHealth Koori Health Research and Community Development Unit. "We Don't Like Research ... : But in Koori Hands It Could Make a Difference.” Melbourne: VicHealth Koori Health Research and Community Development Unit, 2000.

West, Mark D., ed. Theory, Method, and Practice in Computer Content Analysis. Westport, CT: Ablex, 2001.

West, Mark D., ed. Applications of Computer Content Analysis. Westport, CT: Ablex, 2001.

Williamson, Kirsty. Research Methods for Students, Academics and Professionals: Information Management and Systems. Topics in Australasian Library and Information Studies., edited by Ross Harvey and Stuart Ferguson. 2nd ed. Wagga Wagga: Charles Sturt University, Centre for Information Studies, 2002.

Winch, Joan, and Ken Hayward. "Doing It Our Way': Can [Aboriginal] Cultural Traditions Survive in Universities." New Doctor, no. 70 (Summer 1998/99 1999): 25-27. 
Chapter 17

\title{
GROUNDED THEORY
}

\section{A Straightforward and Complicated Exposition}

\author{
Jenny Bunn
}

\begin{abstract}
Grounded Theory is a popular and yet much-contested approach to research. Attempting to steer a course through the many shades of meaning which employ the label Grounded Theory, this narrative outlines Grounded Theory's origin, subsequent development, and techniques, highlights what the author finds it useful to see as its defining distinctions and tenets, and explores the philosophical and epistemological implications of those distinctions and tenets. In so doing it seeks to communicate a better sense of Grounded Theory, both within and without the context of Archival Science.
\end{abstract}

\section{Introduction}

Grounded Theory was first formulated by the sociologists Barney Glaser and Anselm Strauss in 1967, but it has long defied clear definition and has engendered ongoing debate and disagreement. This chapter seeks to steer a course through the many shades of meaning which employ the label Grounded Theory. It also seeks to give those interested in employing it themselves a better sense of what 'it' is, and what embracing all the necessarily concomitant confusion and complexity might mean for them in their research.

To this end, Grounded Theory will be presented in two different ways; firstly a straightforward one, outlining the story that is conventionally told of its evolution and development, as well as discussing the activities involved in following a Grounded Theory approach to research; and secondly a complicated one, highlighting what I have found to be the defining distinctions and tenets within Grounded Theory that allow me to establish 
a philosophical and epistemological position in respect of my research. Later sections will therefore draw on my own experience of undertaking Grounded Theory and will be more auto-ethnographic in tone. To conclude, some observations will also be made on the subject of Grounded Theory in Archival Science.

\section{A Straightforward Exposition of Grounded Theory - Evolution}

Despite the multiplicity of narratives concerning the origins of Grounded Theory, there is a fixed inception point in the 1967 publication of Glaser and Strauss' volume entitled The Discovery of Grounded Theory: Strategies for Qualitative Research. This work was the original formulation of what was thenceforth known as Grounded Theory, but it was also only the opening move in an ongoing quest for a definitive formulation of the same. It was followed in 1978 by Glaser's book Theoretical Sensitivity: Advances in the Methodology of Grounded Theory; and then in 1990 by the publication of a work by Strauss and a new collaborator, Juliet Corbin, entitled Basics of Qualitative Research: Grounded Theory Procedure and Techniques. This last work marked the beginning of a difference of opinion between Strauss and Glaser, with Glaser publishing his own volume, Basics of Grounded Theory Analysis: Emergence vs. Forcing, in 1992, in which he sought to distinguish his view from that of Strauss and Corbin in the following way; "Anselm's methodology is one full of conceptual description and mine is Grounded Theory. They are very different, the first focusing on forcing and the second on emergence". ${ }^{1}$

The debate between Strauss and Glaser was curtailed by the death of Anselm Strauss in 1996, but the debate about Grounded Theory has been continued by a second generation of Grounded Theory proponents, alongside the continuing work of Barney Glaser. One of the most prominent of these is Kathy Charmaz, whose works on Grounded Theory include Constructing Grounded Theory: a Practical Guide Through Qualitative Analysis and (with Janice Morse, Phyllis Stern, Juliet Corbin, Barbara Bowers and Adele Clarke) Developing Grounded Theory: The Second Generation. ${ }^{2}$ The distinction between first and second generation Grounded Theory is often made in terms of an epistemological difference between positivist or objectivist perspectives

1 Barney Glaser, Basics of Grounded Theory Analysis: Emergence versus Forcing. Mill Valley: Sociology Press, 1992, p.122.

2 Kathy Charmaz. Constructing Grounded Theory: A Practical Guide Through Qualitative Analysis. Thousand Oaks: Sage Publications, 2006; Janice Morse, Phyllis Noerager 
and constructivist ones, such that, as Charmaz has put it, the difference is between "Objectivist and Constructivist Methods". ${ }^{3}$ As a result and, as stated by Bryant and Charmaz in The Sage Handbook of Grounded Theory "Most scholars would agree that GTM [the Grounded Theory Method] has three versions", these being "the Glaserian school of GTM, the Strauss and Corbin school, and the Constructivist". ${ }^{4}$

Within the frame of this general agreement, however, the differences between the Glaserian and Strauss and Corbin schools tend to receive less attention than those between both these schools (first generation Grounded Theory) and that of the Constructivist school (second generation Grounded Theory.) Where it is specifically discussed, the difference between the Glaserian and the Strauss and Corbin schools tends to be couched in terms similar to those employed by Heath and Cowley, who speak of a different emphasis on induction, deduction and verification, with Glaser being seen as more purely inductive, in contrast to Strauss and Corbin for whom "deduction and verification dominate". ${ }^{5}$ According to Heath and Cowley, "it is methodological rather than ontological and epistemological aspects that have been cited as the main source of divergence" between Glaser and Strauss and Corbin and they themselves assume the two schools have a shared ontology, albeit with "slight epistemological differences". ${ }^{6}$

Slight differences tend not to make as dramatic a narrative as large ones, and this is perhaps one of the reasons for the greater attention paid to the difference between first and second generation Grounded Theory, a difference which, as has already been stated, is seen very much in the epistemological terms of an opposition between positivist and constructivist outlooks. It is in these terms that a story can be constructed that places Grounded Theory against the backdrop of the vital questions about knowledge and its acquisition that underpin all research activity. We shall return to this backdrop later in

Stern, Juliet Corbin, Barbara Bowers, Kathy Charmaz and Adele Clarke. Developing Grounded Theory: The Second Generation. Walnut Creek, California: Left Coast Press, 2009.

3 Kathy Charmaz, "Grounded Theory Objectivist and Constructivist Methods," in Strategies of Qualitative Inquiry, Norman Denzin and Yvonna Lincoln, eds. $2^{\text {nd }} \mathrm{ed}$. (Thousand Oaks: Sage Publications, 2000), pp.509-35.

4 Anthony Bryant and Kathy Charmaz, "Introduction. Grounded Theory Research: Methods and Practices" in The Sage Handbook of Grounded Theory, Anthony Bryant and Kathy Charmaz, eds. (Thousand Oaks: Sage Publications, 2007), p.10.

5 Helen Heath and Sarah Cowley, "Developing a Grounded Theory Approach: A Comparison of Glaser and Strauss." International Journal of Nursing Studies 41 (2004): 144.

6 Heath and Cowley, "Developing a Grounded Theory Approach", 142. 
this chapter, since an examination of these questions is perhaps better suited to a complicated, rather than a straightforward exposition. For now then, we return to the story, a story which is also seen as the history of Grounded Theory and which can be found in greater detail in many texts including those of Bryant and Charmaz, and Thomas and James. ${ }^{7}$

In this story Grounded Theory is seen as a child of its time in that it was a challenge to the dominant and predominantly quantitative research orthodoxy of 1960s social science academic circles in the United States of America. As such a challenge, the feeling seems to be that it has been both phenomenally successful and fundamentally contradictory. For example, Thomas and James state that: "there can be little doubt that it has been a major - perhaps the major - contributor to the acceptance of the legitimacy of qualitative methods in applied social research" (original emphasis). ${ }^{8}$ However, both they and Bryant and Charmaz assert that this legitimacy arose, at least initially, by "imposing a positivist mantle on that process". And so, Charmaz for one, characterises Glaser's position as coming; "close to traditional positivism, with its assumptions of an objective, external reality, a neutral observer who discovers data [...] and objectivist rendering of data". ${ }^{10}$ Strauss, on the other hand, she characterises as post-positivist, in keeping with the earlier assertion by Heath and Cowley that the two first generation schools had only "slight epistemological differences". ${ }^{11}$

Looking back at Grounded Theory as a product of its time allows its perceived positivist position to be excused to an extent. As Byrant and Charmaz put it:

The reputability and quest for legitimate academic status of qualitative research demanded that it should claim some basis of validity equal to that of quantitative practices, so why not try to establish a 'scientific' basis for applying and validating qualitative research ${ }^{12}$

7 Anthony Bryant and Kathy Charmaz, "Grounded Theory in Historical Perspective: An Epistemological Account" in The Sage Handbook of Grounded Theory, Anthony Bryant and Kathy Charmaz, eds. (Thousand Oaks: Sage Publications, 2007), pp.3157. Gary Thomas and David James, "Reinventing Grounded Theory: Some Questions about Theory, Ground and Discovery," British Educational Research Journal 32 no. 6 (2006): 767-795.

8 Thomas and James, "Reinventing Grounded Theory", 767.

9 Bryant and Charmaz, "Grounded Theory in Historical Perspective", 48.

10 Charmaz, "Grounded Theory," 510.

11 Charmz, "Grounded Theory," 510. Heath and Cowley, "Developing a Grounded Theory Approach", 142.

12 Bryant and Charmaz, "Grounded Theory in Historical Perspective", 35. 
In the current context however, such a position can no longer be excused. As Preben Mortensen points out in his article discussing archival theory, "philosophers and historians of science have discredited the positivist idea of science, according to which science is beyond and above historical, social, cultural and political contexts. ${ }^{13}$ Times and thinking about epistemology have changed and the idea of the researcher as a neutral, objective observer distinct from an external reality is more or less untenable, with constructivism becoming instead the dominant paradigm of the day. Some researchers, therefore, such as Bryant and Charmaz, choose to reformulate Grounded Theory in a more constructivist vein, repositioning it "in the light of the current philosophical and epistemological landscape". ${ }^{14}$ Others, such as Thomas and James, choose to reject it outright, arguing that: "Continued allegiance to grounded theory procedures - or strangely, loyalty simply to the term "grounded theory," unstitched from its procedures or putative ends - stunts and distorts the growth of qualitative inquiry". ${ }^{15}$

Those who choose to continue the tradition of Grounded Theory have a difficult job to, as Byrant and Charmaz put it, "distinguish between the core aspects of the method without which it wouldn't be GTM, and the aspects which can be traced back to the historical context within which GTM developed, and which can therefore be dispensed with." ${ }^{16}$ This job is made even harder since the epistemological claims made within the founding texts of Grounded Theory are, as noticed by Bryant and Charmaz, "often couched in ambiguous terms. ${ }^{17} \mathrm{I}$ therefore choose not to take this task on at the current time, and will turn instead to outlining my personal and present understanding of what it is to undertake Grounded Theory.

\section{A Straightforward Exposition of Grounded Theory - Procedures}

On the surface doing Grounded Theory appears to be a fairly straightforward process involving the following stages, as characterised on the Glaserianleaning Grounded Theory Online site:

13 Preben Mortensen, "The Place of Theory in Archival Practice," Archivaria 47 (1999), 1.

14 Bryant and Charmaz, "Grounded Theory in Historical Perspective", 50.

15 Thomas and James, "Reinventing Grounded Theory”, 790.

16 Bryant and Charmaz, "Grounded Theory in Historical Perspective", 50.

17 Bryant and Charmaz, "Grounded Theory in Historical Perspective", 32. 
1. Identify your substantive area - your area of interest. [ ... ]

2. Collect data pertaining to the substantive area. $[\ldots]$

3. Open code your data as you collect it. [ ... ]

4. Write memos throughout the entire process $[\ldots]$

5. Conduct selective coding and theoretical sampling $[\ldots]$

6. Sort your memos and find the theoretical code $[\ldots]$

7. Read the literature and integrate with your theory through selective coding $[\ldots]$

8. Write up your theory. Job done! ${ }^{18}$

The complexity underlying this overview is not difficult to find and it is clear that there is a degree of overlap and fuzziness concealed within the simple linear model. For example, stages 2 and 3 are acknowledged to occur simultaneously, 4 seems to run through 1-8 and both 5 and 7 involve something called selective coding.

Many explanations of how to do Grounded Theory therefore eschew linear narratives and instead consist of more discursive narratives that cohere around outlining a number of specific activities or techniques that permeate the process, such as coding, theoretical sampling and memo-writing. For example, Charmaz writes as follows:

On coding -

Analysis begins early. We grounded theorists code our emerging data as we collect it. Through coding, we start to define and categorize our data. [ ... ] Coding starts the chain of theory development. ${ }^{19}$

On theoretical sampling -

As we grounded theorists refine our categories and develop them as theoretical constructs, we likely find gaps in our data and holes in our theories. Then we go back to the field and collect delimited data to fill those conceptual gaps and holes - we conduct theoretical sampling. ${ }^{20}$

18 "What is Grounded Theory," www.groundedtheoryonline.com/what-is-grounded-theory.

19 Charmaz, "Grounded Theory," 515.

20 Charmaz, "Grounded Theory," 519. 


\section{On memo-writing -}

This step helps to spark our thinking and encourages us to look at our data and codes in new ways. [ ... ] Through memo writing, we elaborate processes, assumptions, and actions that are subsumed under our codes. $^{21}$

In this way, accounts of the Grounded Theory process tend to be more descriptive than prescriptive and clear instruction is not easy to find. For example, even Strauss and Corbin, who, of all the proponents of Grounded Theory, are generally held to have placed "much stronger emphasis on its [Grounded Theory's] proceduralisation and formalisation into a series of techniques" 22 , write that its techniques and procedures "are not meant to be used rigidly in a step-by-fashion" and that the characteristics of a grounded theorist "will never develop if researchers focus solely on the procedures presented in this text and apply them in a rote manner". ${ }^{23}$

This sentiment is echoed by Yazdan Mansourian who, writing in the context of highlighting a number of "common questions that arise for researchers who want to use GT [Grounded Theory] for the first time," notes that "GT should not be considered as a step-by-step manual for researchers", and that "researchers should interpret the nature of GT in the way that suits their research context and then clarify the reasons for each step in the research". ${ }^{24} \mathrm{I}$ too am in sympathy with this view and consider that, just as Grounded Theory was discovered by Glaser and Strauss in 1967, so too it must be re-discovered anew by each researcher attempting it.

Nevertheless, I will attempt to relate how I currently choose to explain the process of Grounded Theory to others. It is an account that is structured around what might be seen as Grounded Theory's central tenets or principles: open-ness, emergence and integration. These can also be seen as temporal stages.

21 Charmaz, “Grounded Theory," 517.

22 Inaam Idrees, Ana Cristina Vasconcelos and Andrew Cox, "The Use of Grounded Theory in PhD Research in Knowledge Management A Model Four-stage Research Design," Aslib Proceedings: New Information Perspectives 63 no. 2/3 (2011): 190.

23 Anselm Strauss and Juliet Corbin, Basics of Qualitative Research: Grounded Theory Procedures and Techniques. $2^{\text {nd }}$ ed. Thousand Oaks: Sage Publications, 1998, pp. 8, 14.

24 Yazdan Mansourian, “Adoption of Grounded Theory in LIS Research," Nerw Library World 107 no. 9/10 (2006): 397. 


\section{Open-ness}

Open-ness relates to the beginning of the process (roughly equivalent to stages 1-4 of the framework previously outlined) and is an idea that sees expression in both the concept of theoretical sensitivity and the dictum that Grounded Theory does not start with a literature review, or a preconceived problem, question or hypothesis. Theoretical sensitivity is dealt with extensively in a book of the same name published by Glaser in 1978 and it may be thought of as a state of mind as well as "the ability to conceptualize and organize, make abstract connections, visualize and think multivariately". ${ }^{25}$ Those embracing the ideas of theoretical sensitivity and of open-ness start from no other point than the selection of an area of interest and a desire to work out the main concern of the participants in that area and how they (the participants) resolve it.

Data collection in Grounded Theory starts early and "all is data" because "the researcher does not need to buy into any particular data as sanctified, objective or valid". ${ }^{26}$ As Charmaz notes, "Grounded theorists have been accused, with some justification, of slighting data collection." I would agree that Grounded Theory is not strong in its consideration of data and how to acquire it. ${ }^{27}$ For my own study, much of the data I deliberately collected came from interviews.

Data analysis also starts early, at the same time as data collection, through the initiation of open coding. Open coding, or "running the data open" involves looking at the data line-by-line and creating as many codes and categories as necessary to represent and delineate what is going on within it. ${ }^{28}$ In undertaking this coding, researchers also employ the constant comparative method, which involves constantly questioning how the codes and categories being created are the same or different, how they relate or do not relate. This questioning is captured in the writing of memos which detail the ideas inherent in the codes and categories as well as any others triggered by the process or the critical reflection of the researcher. Ultimately it is envisaged that as data collection, data analysis and memo-writing continue,

25 Barney Glaser, Theoretical Sensitivity. Mill Valley: Sociology Press, 1978; and Barney Glaser, "Remodeling Grounded Theory," Forum: Qualitative Social Research 5 no. 2 (2004): 43, http://www.qualitative-research.net/index.php/fqs/article/view/607/1315.

26 Barney Glaser, "All is data," Grounded Theory Review 6 no.2 (2007), http:// groundedtheoryreview.com/2007/03/30/1194/; and Barney Glaser, Doing Grounded Theory: Issues and Discussions. Mill Valley: Sociology Press, 1998, p. 8.

27 Charmaz, "Grounded Theory," 514.

28 Glaser, "Remodeling Grounded Theory," 48. 
the researcher will start to gain a sense of what it is that lies at the heart of the matter.

\section{Emergence}

As we have already seen, emergence (in opposition to forcing) is one of the definitional distinctions employed in Grounded Theory. This aspect will be addressed in more detail at a later stage, but for now emergence is presented as that stage in the process (roughly equivalent to stages 4-6 of the framework previously outlined) when the researcher starts to formulate what it is that is important within the substantive area in question. It is at this point that the techniques being used move away from open coding and more towards theoretical sampling and selective coding. Theoretical sampling is the process whereby further data collection is driven by the questions arising from the analysis of the data already collected. Selective coding is the process of distinguishing the so-called core category (that lies at the heart of the matter) and concentrating on exploring that core and its related categories until the point of theoretical saturation. The core category is identifiable through its being "central, relating to as many other categories and their properties as possible and accounting for a large portion of the variation in a pattern of behavior". ${ }^{29}$ Theoretical saturation is defined as the point when, "no additional data are found whereby the sociologist can develop properties of the category". 30

During this phase, the limits of the data under consideration will probably expand beyond that being explicitly collected as such, to include the memos written alongside the previous data collection and analysis process. The process of memo-writing nonetheless continues throughout this phase as well. At its end, the researcher will have ceased data collection and will start to look towards writing up.

\section{Integration}

Integration is the final phase of the process of Grounded Theory (roughly equivalent to stages 7-8 of the framework previously outlined). It starts with the researcher having achieved a sense of what seems to lie at the heart of

29 Glaser, "Remodeling Grounded Theory," 54.

30 Barney Glaser and Anselm Strauss, The Discovery of Grounded Theory: Strategies for Qualitative Research. New York: Aldine De Gruyter, 1967, p 61. 
the matter under examination and ends with the production of something (ordinarily a written text) that crystallises what that is and places it in a context. To this end the data under consideration expands again at this point to include the sort of literature that would normally have been read earlier as part of a traditional literature review.

In this way the literature also becomes subject to the kind of questioning and comparison applied to all the previous data and must earn its place within what has already started to take shape, rather than shaping it from the outside. Equally, this point means that the selection of which literature to read should not be driven by the idea of its being a comprehensive representation of a specific field, area or discipline, but rather, by a process akin to that of theoretical sampling, whereby the questions that arise during the formulation of the end product, drive what should be read next.

Given the contested nature of Grounded Theory, the above account would almost certainly not meet with universal agreement from all those who consider themselves grounded theorists, but it is offered in the spirit of providing a starting point for those considering undertaking Grounded Theory for themselves. That starting point however, comes with the proviso that embarking on Grounded Theory may, and in my opinion should, lead them to explore the more complicated questions this raises.

\section{A Complicated Exposition of Grounded Theory - Defining Its Own Terms}

In what has gone before I have spoken of Grounded Theory in terms of it being a process in the context of undertaking research. I have not referred to it as either a method or a methodology and this has been deliberate because I do not feel comfortable using these terms in the context of Grounded Theory without considerable preamble. Further, I am aware that I have deliberately side-stepped the question of where I position myself in relation to the interconnecting debates about a) the three different schools of Grounded Theory and b) their underlying epistemologies. In the rest of this chapter I will seek to explain and explore how my own experience of Grounded Theory has brought me to this position of ambiguity, since it is still a position and one I do not take lightly or unthinkingly.

When I started doing formal research (in the form of doing a Ph.D. on the subject of archival description), I was returning to academia after a break of over ten years, during which time I had worked as an archivist in a number of institutions in the UK. As such, I had developed a degree of suspicion 
about the value of such research, beyond it being just an academic exercise, and a lack of consideration of or experience with what were presented to me as research methods and methodology. I started to read around the subject so that I would be able to choose a way to proceed and my suspicions were immediately further raised by the way it seemed that I had to first make another choice, between the research paradigm in which I wished to operate: positivism, post-positivism or interpretivism. Moreover this choice seemed to be about "philosophical positions and theoretical frameworks relating to how knowledge and knowledge systems are defined" - things about which I felt in no position at the time to make a choice. ${ }^{31}$ Grounded Theory caught my attention because it seemed to me, at the time, to offer a way to avoid having to make this decision. For example, in the article "Naturalist Inquiry and Grounded Theory", Glaser is not impressed by the axioms ("the set of undemonstrated (and undemonstrable) 'basic beliefs' accepted by convention or established by practice as the building blocks of some conceptual or theoretical structure or system") of Lincoln and Guba's own naturalist paradigm of research, and writes "GT's axiom is simple: let's see what is going on and it's 'whatever emerges". ${ }^{32}$ For the same reason, I instinctively retreated from the debate of second generation proponents which seemed to be forcing me to make a choice against an objectivist and positivist position and for an interpretivist and constructivist one, rather than allowing me to retain an open mind on the more epistemological and philosophical questions until I felt more qualified to make a choice.

Looking back, this decision was naive and could potentially have led to me failing to ever fully understand the implications of the way in which, simply by undertaking research, I was implicitly making epistemological claims. That it did not have this outcome, I put down to the way in which Grounded Theory's complicated and contested nature forced me to puzzle it, and hence the nature of research more widely, out for myself. Indeed, I also came to see this as a vital part of the open-ness that I had established as a central tenet of Grounded Theory. For could this idea not mean that just as starting out with a hypothesis about what the problem was could limit researchers, so too could framing their work from the outset with a fixed hypothesis about the nature of knowledge and how it was possible

31 Anne Gilliland and Sue McKemmish, "Building an Infrastructure for Archival Research,” Archival Science 4 no. 3 (2004): 165.

32 Barney Glaser, "Naturalist Inquiry and Grounded Theory," Forum: Qualitative Social Research 5 no.1 (2004): 7, 36, http://www.qualitative-research.net/index.php/fqs/article/ view/652/1412. 
to research, investigate, and understand our world? I therefore came to feel that in looking for Grounded Theory I should not distinguish it by the distinction between objectivist and constructivist paradigms, since that distinction was not about Grounded Theory per se, but rather about a much wider context of changes in thinking about epistemology and the nature of research. On account of that context, it did eventually become clear to me that I would, at some point, have to justify and explain my research process in epistemological terms in order to gain academic legitimacy, but I still saw no reason why that justification and explanation could not come out of that research, rather than in advance of and apart from it.

Similarly another distinction associated with research in which I could not find Grounded Theory was that between method and methodology. Jin Tan states that one of the problems for new researchers approaching Grounded Theory is to decide "whether GT is a methodology or a method". ${ }^{33}$ However, even when Tan addresses the question directly, no answer one way or the other would seem to be forthcoming. Instead Tan states, "The author does not suggest that Strauss offers a more practical method than Glaser's or vice versa, nor does she say that GT can be adopted either as a methodology or a method". ${ }^{34}$ Which is it then? Or perhaps, more relevantly to the current discussion, why does it matter?

The distinction between method and methodology often comes down to a method being something one does, e.g., techniques such as interviewing and coding which one applies as part of a research design, and methodology being the study of method, or thinking about what you are doing/your epistemological approach. Methodology can (and often is) therefore, conflated with method (and to an extent also with what is termed here the research paradigm) to mean an explanation of the undertaking of research that explicitly discusses associated ontological, philosophical and epistemological perspectives and how the method being pursued fits with those perspectives. Or, put another way, methodology often acts as an expression of a bridge or connection between theory and practice, in particular the theory and practice of research. In this respect, however, it is not exclusive to research, but is used in other contexts as well. For example, Heather MacNeil has written, in the context of archival science that; "There are many ways of exploring the relationship between theory and practice. The model I have

33 Jin Tan, "Grounded Theory in Practice: Issues and Discussion for New Qualitative Researchers," Journal of Documentation 66 no.1 (2010): 93.

34 Tan, "Grounded Theory in Practice," 100. 
chosen to use places theory and practice along a continuum; between the two, and bridging them, is methodology". 35

Given the above, I came to believe that the distinction between method and methodology tended to come into being only when you were trying to justify and explain your method in epistemological terms, or at least in whatever terms were currently used to assert the legitimacy and validity of what you had done in your research. As we have seen above, I had decided that the process of fixing this frame should run throughout my research process and as such, I made the decision to try not to define Grounded Theory in any of the above terms.

This decision however, left me in a dilemma. If I was not to define Grounded Theory in conventional terms such as in terms of the distinction between objectivist and constructivist paradigms or method and methodology, how was I to define it? One way was to just get on and do it, following the sorts of explanation of the process mentioned earlier, but another way was to look at it in its own terms. In which terms, the critical distinctions seemed to be those between Grounded Theory and conceptual description, and emergence and forcing. It is to these that we now turn.

\section{A Complicated Exposition of Grounded Theory - Defining It in Its Own Terms}

As we have seen above, Glaser has used both these distinctions to distinguish his own idea of Grounded Theory from those of others, including his fellow discoverer, Anselm Strauss, but the distinctions are also important to the others from whom he distinguishes himself. For example, with regards to the distinction between Grounded Theory and conceptual description, Strauss and Corbin take time within their work to distinguish between, what they term, "description", "conceptual ordering" and "theory"; ${ }^{36}$ and Charmaz feels the need to ask the question "If grounded theorists have the methods to construct theory, why do many studies remain descriptive?"37 Before we deal with this distinction in more detail however, we turn to that between emergence and forcing, because it was my experience of this distinction that first

35 Heather MacNeil, "Archival Theory and Practice: Between Two Paradigms," Archivaria 37 (1994): 7, http://journals.sfu.ca/archivar/index.php/archivaria/article/ view/11982/12941.

36 Strauss and Corbin, Basics of Qualitative Research, 15-25.

37 Charmaz, Constructing Grounded Theory, 137. 
allowed me to formulate what Grounded Theory meant to me, and thereby to consciously make the decision that that was what I was doing.

It would be easy to argue that, at least initially, my project was not a Grounded Theory one. For example, despite the emphasis placed on simultaneous data collection and analysis, my first batch of interviewing took place in a concentrated period of just over a month, followed by a further two months of transcription. It was only then that data analysis began and this analysis was initially undertaken using coding software, in this case NVivo, something about which many grounded theorists, including both Glaser and Charmaz, have expressed reservations on the grounds, for example, that "the programs may unintentionally foster an illusion that interpretive work can be reduced to a set of procedures". ${ }^{38}$

Nevertheless, despite these deviations, I still sought to approach my coding in what I thought of as a Grounded Theory way, by starting with open coding. Within NVivo, it is possible to create nodes, which are roughly equivalent to codes. A node is a label to which you can attach all apparently related text. Initially, after my first pass over the interview transcripts, I had created over 100 free nodes, that is over 100 themes or ideas had suggested themselves as a result of reading the transcripts. In NVivo, such free nodes differ from so called tree nodes, in that tree nodes can be hierarchically related to each other in branching trees and free nodes cannot. Faced with this plethora of nodes, the next logical step seemed to be to organise them into structures of tree nodes, but it soon became apparent that this approach was both futile and, more importantly, forcing.

It was futile because, despite spending inordinate amounts of time attempting to fit all the initial codes into a schema, many just would not fit. It was forcing because it became clear to me that such efforts had more to do with my desire to impose order (and control) over the data, rather than with discovering what it signified. I therefore made the decision to break up the trees of nodes and to make no further attempts to impose a structure or structures. Instead I paid greater attention to the relationships between nodes, since NVivo also allows for the creation of so-called relationship nodes. The idea was to find evidence in the data for relationships, such that a structure could emerge, rather than be imposed. It was also from this point onwards that much more use was made of the ability to interrogate the data using NVivo's various query features, such that the voices in the data continued to be in conversation with me.

38 Charmaz, "Grounded Theory," 520-21. Glaser, Doing Grounded Theory, 185-6. 
This strategy did work up to a point, but eventually the use of NVivo was completely abandoned. Not only did I find myself falling into the trap of what Lyn Richards has termed "coding fetishism", whereby "the act of coding becomes an end in itself", I was also seeing my codes as some kind of physical manifestation of my emerging thinking. ${ }^{39}$ I was trying to create direct correlation, rather than work in the connection between the two. Once more then, my strategy changed and from this point onwards, I saw coding not as a matter of attaching pieces of text to particular labels (although I did continue this practice up to a point, cutting and pasting phrases into a Word document); rather I saw it in the creation of a number of extended memos, entitled "random thoughts on x", that is in the capture of my attempts to make sense of $x$. Looking back, I would now characterise this part of the process as my version of selective coding, since I was choosing to concentrate on a specific number of codes (represented by $\mathrm{x}$ ), which I was starting to see as the core, in the sense of lying at the heart of the matter.

As a result of the above experience I now see emergence versus forcing as an injunction to resist for as long as possible the urge to make things make sense, to make them fit. This is very difficult, because all your instincts tell you that the whole point of your research is to do exactly that. In my opinion however, it is often easy, when moving towards making things fit as your end point, to forget that in so doing, you are also employing a frame (the thing into which they fit). Grounded Theory urges you to keep that frame in flux and in question for as long as possible. Eventually, however, it is necessary (certainly in the context of doing a Ph.D.) to reach an end point and the temporal stage moves from emergence to integration. It is in the phase of integration that the distinction between conceptual description and Grounded Theory starts to become more relevant. It is at this point that the researcher must tackle head on the question of fit, of the mutual dependence or interconnectedness of frame and findings, process and product.

It is now nine years since I started my first foray into Grounded Theory, five since its completion, and so perhaps it is time to be less ambiguous about my own epistemological position. For the avoidance of doubt, my outlook is broadly constructivist and, arguably because of that, I am still quite confused as to how it is that we can know anything at all. In this position, I am influenced by the thinking of the second order cyberneticians Maturana and Varela, who discuss at length how we must "walk on the razor's edge [ ... ] to understand the regularity of the world we are experiencing at

39 Lyn Richards, "Qualitative Computing: A Methods Revolution?” International Journal of Social Research Methodology 5 no. 3 (2002): 269. 
every moment, but without any point of reference independent of ourselves that would give certainty to our descriptions and cognitive assertions". ${ }^{40}$ As Bryant and Charmaz point out in the discussion of the rise of social constructionist challenges in sociology, it is all too easy when following this path to end up either at "the extreme of arguing that in fact no external reality" exists or "to end in complete relativism, according equal status to all and any representations of reality". ${ }^{41}$ Thus, whereas Thomas and James condemn Grounded Theory for "sleight of hand in reasoning about inquiry", I cannot. ${ }^{42}$ They may find themselves annoyed with its "desire to have it both ways", but I can see only a practical compromise. ${ }^{43}$

Thomas and James further problematise this sleight of hand and desire to have it both ways in the following terms:

They [grounded theorists] want the comfortable feeling that comes from a denial of the arrogance of foundationalism and essentialism [... ] while clinging on to an epistemological security blanket - one woven from the associated notions that (a) some clearer distillation of truth can be established about the particulars and generalities of social behavior, and (b) that this can be established using the cogs and levers of structured inquiry. ${ }^{44}$

Personally I feel such a statement might apply more generally. Who (grounded theorist or not) really wants to throw away completely the epistemological security blanket that they can, and do, have knowledge of themselves and the 'real' world around them? Inquiry in a constructivist paradigm is underpinned by difficulty and apparent paradox; in many ways that is the point of the paradigm. For me, making a distinction between Grounded Theory and conceptual description helps me to maintain my awareness of this fact. Grounded Theory is not conceptual description because the product of the process does not exist simply as an idea or theory apart from any or all of; the process of its generation, the researcher undertaking it and that which it seeks to describe. Rather it exists as a complex integration of sense making and sense made, defined only by that which it defines and grounded in this seemingly paradoxical circularity. Grounded Theory does not play to

40 Humberto Maturana and Francisco Varela, The Tree of Knowledge: The Biological Roots of Human Understanding. Boston and London: Shambhala, 1998, p.241.

41 Bryant and Charmaz, "Grounded Theory in Historical Perspective", 37.

42 Thomas and James, "Reinventing Grounded Theory," 790.

43 Thomas and James, "Reinventing Grounded Theory," 784.

44 Thomas and James, "Reinventing Grounded Theory," 780. 
an easy separation or connection between thinking and being and becomes in itself, its terms and tenets, the realisation of an essential circularity within all attempts at inquiry in a constructivist paradigm.

Again, given the contested nature of Grounded Theory, the above account would almost certainly not meet with universal agreement from all those who consider themselves grounded theorists, but it is a demonstration of the way in which, as Mansourian puts it "GT [Grounded Theory] should not be considered as a step-by-step manual for researchers", and that "researchers should interpret the nature of GT in the way that suits their research context and then clarify the reasons for each step in the research". ${ }^{45}$ The same is true whatever form your research takes and I will not enter into any attempt to assert Grounded Theory's innate superiority as an approach to research. For me, as a novice experiencing for the first time what it meant to do 'research', Grounded Theory offered a way to explore that meaning in a very open way for which I will always be grateful. Moreover, and although I did not know it when I started, it did prove a good fit with the subject of my inquiry, leading me to see that subject (nominally archival description) not just as the practice of describing archives, but also as the practice of trying to deal with the epistemological questions inherent in all our descriptions (and attempts to understand the world around us). Would I have reached the same conclusion if I had not taken a Grounded Theory approach? I suspect not, but we can never know.

\section{Grounded Theory in Archival Science}

Grounded Theory would appear to have gained some traction within Archival Science, and is reported as having been adopted by a number of studies as follows:

- $\quad$ Research by Paul Conway into the use of digitised photographic archives at the Library of Congress involved "the qualitative analysis of interview transcripts using the grounded theory method". 46

- The InterPARES 1 research project in part "adopted a grounded theory approach in which case studies of electronic systems were

45 Yazdan Mansourian, “Adoption of Grounded Theory”, 397.

46 Paul Conway, "Modes of Seeing: Digitized Photographic Archives and the Experienced User," American Archivist 73, no. 2 (2010): 436, http://archivists.metapress.com/content/ mp275470663n5907/?p=7f2bf0f784714e6fa8e78230187f72ac\&pi=1. 
examined in order to identify and describe phenomena associated with the records and their contexts". ${ }^{47}$

- Victoria Lemieux's study into the recordkeeping practices of failed Jamaican banks was based on data which was "coded and analysed using an approach informed by the Grounded Theory methodology with the assistance of qualitative data analysis software". ${ }^{4}$

- Karen Gracy's "research on competing definitions of value in the world of film preservation", whilst seeking to make the case for archival ethnography, also discussed grounded theory in sections headed "Analysis", "Coding" and "Memo writing". ${ }^{49}$

Even amongst this brief sample though, individuals appear to have seen Grounded Theory differently; as a method, an approach, a methodology, and a series of specific techniques. This highlights what I mean by the problems caused by seeking to define Grounded Theory in these terms, because it causes a need to engage with the argument of which is correct. I choose not to engage with that argument in these terms, since that would be unhelpful. There is a useful argument to be had (and an ever evolving consensus) about what constitutes good research practice, but it cannot be conducted purely in terms of Grounded Theory.

In attempting to define Grounded Theory in the context of Archival Science then, I would rather point the reader towards discussion within the field about research paradigms, methods and methodologies, and the relationship between theory and practice. This discussion is ongoing and has been articulated over the years by numerous individuals, including; Burke, Roberts, Eastwood, Livelton, Mortensen and Williams. ${ }^{50}$ This volume also takes its

47 Anne Gilliland-Swetland, "Testing Our Truths: Delineating the Parameters of the Authentic Archival Electronic Record," American Archivist 65 no. 2 (2002): 202, http:// archivists.metapress.com/content/f036wp74710g1483/?p=5eed5ec4cc3946e48407b3539c $14868 \mathrm{~d} \& \mathrm{pi}=3$.

48 Victoria Lemieux, "Let the Ghosts Speak: An Empirical Exploration of the "Nature" of the Record," Archivaria 51 (2001): 83, http://journals.sfu.ca/archivar/index.php/ archivaria/article/view/12795/13995.

49 Karen Gracy, "Documenting Communities of Practice: Making the Case for Archival Ethnography," Archival Science 4 nos. 3/4 (2004): 336, $352-55$ [reprinted in this monograph].

50 Frank Burke, "The Future Course of Archival Theory in the United States," American Archivist 44 no.1 (1981): 40-46; Terry Eastwood, "What is Archival Theory and Why is it Important?" Archivaria 37 (1994): 122-30, http://journals.sfu.ca/archivar/index. php/archivaria/article/viewArticle/11991; Trevor Livelton, Archival Theory, Records, and the Public (Lanham: The Society of American Archivists and The Scarecrow Press, 1996); Mortensen, "The Place of Theory," 1-26; John Roberts, "Practice Makes Perfect, 
part in that discussion, as does earlier work of two of its editors, Gilliland and McKemmish. ${ }^{51}$ In this earlier work, Gilliland and McKemmish discuss what a research paradigm for Archival Science might look like and they write that:

In emergent archival research, liberation may well lie in the challenge of applying the apparent opposites of interpretive and positivist approaches to studying archival phenomena [...] In part it may lead to understandings that some phenomena in our world behave in ways which are susceptible to being seen from a positivist perspective, while others are more readily understood from an interpretivist viewpoint. And perhaps the creative tension generated will lead us to yet other ways of seeing. ${ }^{52}$

It is in this creative tension that I see Grounded Theory as operating and it is this operating in creative tension, rather than just acknowledging it, that, for me, distinguishes Grounded Theory. Then again, another place in which I would see Grounded Theory within Archival Science is the attempts to define archival theory and comments such as the following from Terry Eastwood:

However much an applied discipline might rely on knowledge of other disciplines to build its theoretical picture of the nature of the things on which it acts, it cannot adopt that knowledge directly for its theory, because the grounds of its theory must suit its perspective and purposes. ${ }^{53}$

The same is true of Grounded Theory, its grounds (tenets) must fit with its perspectives and purposes and this is why it can only be defined in its own terms. For it is only when viewed in those terms that it becomes a consistent and coherent whole, and it is a sense of that whole that I have sought to communicate here.

Theory Makes Theorists," Archivaria 37 (1994): 111-121, http://journals.sfu.ca/archivar/ index.php/archivaria/article/view/11990/12953; John Roberts, "Response to Terry Eastwood's Paper," Archivaria 37 (1994): 131-133, http://journals.sfu.ca/archivar/index. php/archivaria/article/view/11992/12955; Caroline Williams, "Studying Reality: The Application of Theory in an Aspect of UK Practice," Archivaria 62 (2006): 77-101, http://journals.sfu.ca/archivar/index.php/archivaria/article/view/12889/14120.

51 Gilliland and McKemmish, "Building an Infrastructure for Archival Research."

52 Gilliland and McKemmish, "Building an Infrastructure for Archival Research.” 170.

53 Eastwood, "What is Archival Theory," 125. 


\section{Acknowledgements}

I would like to thank Alexandra Eveleigh, Sarah Ramdeen, Elizabeth Shepherd and the anonymous reviewers for their comments on earlier drafts of this piece.

\section{References}

Bryant, Anthony and Kathy Charmaz. "Introduction. Grounded Theory Research: Methods and Practices." In The Sage Handbook of Grounded Theory, Anthony Bryant and Kathy Charmaz, eds. (Thousand Oaks: Sage Publications, 2007), 1-28.

Bryant, Anthony and Kathy Charmaz. "Grounded Theory in Historical Perspective: An Epistemological Account." In The Sage Handbook of Grounded Theory, Anthony Bryant and Kathy Charmaz, eds. (Thousand Oaks: Sage Publications, 2007), 31-57.

Burke, Frank. "The Future Course of Archival Theory in the United States." American Archivist 44 no. 1 (1981): 40-46.

Charmaz, Kathy. Constructing Grounded Theory: A Practical Guide Through Qualitative Analysis. Thousand Oaks: Sage Publications, 2006.

Charmaz, Kathy. “Grounded Theory Objectivist and Constructivist Methods.” In Strategies of Qualitative Inquiry, Norman Denzin and Yvonna Lincoln, eds. $2^{\text {nd }}$ ed. (Thousand Oaks: Sage Publications, 2000), 509-35.

Conway, Paul. "Modes of Seeing: Digitized Photographic Archives and the Experienced User” American Archivist 73 no. 2 (2010): 425-463. http://archivists.metapress.com/ content/mp275470663n5907/?p=7f2bf0f784714e6fa8e78230187f72ac\&pi=1.

Eastwood, Terry. "What is Archival Theory and Why is it Important?" Archivaria 37 (1994): 122-30. http://journals.sfu.ca/archivar/index.php/archivaria/article/ viewArticle/11991.

Gilliland, Anne and Sue McKemmish. "Building an Infrastructure for Archival Research." Archival Science 4 no.3 (2004): 149-67.

Gilliland-Swetland, Anne. "Testing Our Truths: Delineating the Parameters of the Authentic Archival Electronic Record." American Archivist 65 no. 2 (2002): 196-215. http://archivists.metapress.com/content/f036wp74710g1483/?p=5eed5ec4cc3946e48 407b3539c14868d\&pi=3.

Glaser, Barney. Basics of Grounded Theory Analysis: Emergence versus Forcing. Mill Valley: Sociology Press, 1992.

Glaser, Barney. "Constructivist Grounded Theory?” Forum: Qualitative Social Research 3 no. 3 (2002). http://www.qualitative-research.net/index.php/fqs/article/view/825

Glaser, Barney. Doing Grounded Theory: Issues and Discussions. Mill Valley: Sociology Press, 1998.

Glaser, Barney. "Naturalist Inquiry and Grounded Theory." Forum: Qualitative Social Research 5 no.1 (2004).

http://www.qualitative-research.net/index.php/fqs/article/view/652/1412

Glaser, Barney. "Remodeling Grounded Theory." Forum: Qualitative Social Research 5 no. 2 (2004). http://www.qualitative-research.net/index.php/fqs/article/view/607/1315.

Glaser, Barney. The Grounded Theory Perspective: Conceptualisation Contrasted with Description. Mill Valley: Sociology Press, 2001.

Glaser, Barney. Theoretical Sensitivity. Mill Valley: Sociology Press, 1978.

Glaser, Barney and Anselm Strauss. The Discovery of Grounded Theory: Strategies for Qualitative Research. New York: Aldine De Gruyter, 1967. 
Gracy, Karen. "Documenting Communities of Practice: Making the Case for Archival Ethnography." Archival Science 4 no. 3 (2004): 335-65.

"Grounded Theory ... Is it for me?" Accessed 17 April 2013. http://www. groundedtheoryonline.com/what-is-grounded-theory/classic-grounded-theory

“The Grounded Theory Institute." http://www.groundedtheory.com/.

Heath, Helen and Sarah Cowley. "Developing a Grounded Theory Approach: A Comparison of Glaser and Strauss." International Journal of Nursing Studies 41 (2004): 141-150.

Idrees, Inaam, Ana Cristina Vasconcelos and Andrew Cox. "The Use of Grounded Theory in PhD Research in Knowledge Management A Model Four-stage Research Design.” Aslib Proceedings: New Information Perspectives 63 no. 2/3 (2011): 188-203.

Lemieux, Victoria. "Let the Ghosts Speak: An Empirical Exploration of the "Nature" of the Record." Archivaria 51 (2001): 81-111. http://journals.sfu.ca/archivar/index.php/ archivaria/article/view/12795/13995.

Livelton, Trevor. Archival Theory, Records, and the Public. Lanham: The Society of American Archivists and The Scarecrow Press, 1996.

MacNeil, Heather. "Archival Theory and Practice: Between Two Paradigms." Archivaria 37 (1994): 6-20. http://journals.sfu.ca/archivar/index.php/archivaria/article/ view/11982/12941.

Mansourian, Yaxdan. “Adoption of Grounded Theory in LIS Research.” New Library World 107 no. 9/10 (2006): 386-402.

Maturana, Humberto and Francisco Varela. The Tree of Knowledge: The Biological Roots of Human Understanding. Boston and London: Shambhala, 1998.

Morse, Janice, Phyllis Noerager Stern, Juliet Corbin, Barbara Bowers, Kathy Charmaz and Adele Clarke. Developing Grounded Theory: The Second Generation. Walnut Creek, California: Left Coast Press, 2009.

Mortensen, Preben. "The Place of Theory in Archival Practice." Archivaria 47 (1999): 1-26. http://journals.sfu.ca/archivar/index.php/archivaria/article/view/12695/13867.

Richards, Lyn. "Qualitative Computing: A Methods Revolution?” International Journal of Social Research Methodology 5 no. 3 (2002): 263-276.

Roberts, John. "Practice Makes Perfect, Theory Makes Theorists." Archivaria 37 (1994): 111-121. http://journals.sfu.ca/archivar/index.php/archivaria/article/ view/11990/12953.

Roberts, John. “Response to Terry Eastwood's Paper.” Archivaria 37 (1994): 131-133. http://journals.sfu.ca/archivar/index.php/archivaria/article/view/11992/12955.

Strauss, Anselm and Juliet Corbin. Basics of Qualitative Research: Grounded Theory Procedures and Techniques. $2^{\text {nd }}$ ed. Thousand Oaks: Sage Publications, 1998.

Tan, Jin. "Grounded Theory in Practice: Issues and Discussion for New Qualitative Researchers.” Journal of Documentation 66 no. 1 (2010): 93-112.

Thomas, Gary and James, David. "Reinventing Grounded Theory: Some Questions about Theory, Ground and Discovery.” British Educational Research Journal 32 no. 6 (2006): 767-795.

Williams, Caroline. "Studying Reality: The Application of Theory in an Aspect of UK Practice." Archivaria 62 (2006): 77-101. http://journals.sfu.ca/archivar/index.php/ archivaria/article/view/12889/14120.

"What is Grounded Theory?" www.groundedtheoryonline.com/what-is-grounded-theory 


\title{
LOOKING AT ARCHIVAL CONCEPTS AND PRACTICE IN THE LIGHT OF SPEECH ACT THEORY
}

\author{
Pekka Henttonen
}

\begin{abstract}
The chapter describes how speech act theory was used through a process of theory derivation to develop a theoretical framework explicating how records (and archival documents) differ from other kinds of information and why following archival methodology and preserving contextual information is important for records. In general, the chosen interdisciplinary approach worked well: it could give answers to research questions and also build a bridge between positivist and interpretivist paradigms in archival science. One weakness of the theoretical framework is its abstractness. The framework also does not cover all the ways in which a fonds might contribute to understanding and interpreting documents.
\end{abstract}

In this chapter I examine and reflect on how speech act theory was used to examine archival concepts and practice. ${ }^{1}$ Speech act theory is a theory describing what we do in or by speaking. The purpose of the chapter is to explicate the research process behind the study and to focus on its methodological aspects. It should be noted, therefore, that this does not give a full picture of speech act theory or related argumentation and sources.

1 Pekka Henttonen, Records, Rules and Speech Acts: Archival Principles and Preservation of Speech Acts. Tampere: University of Tampere, 2007. 


\section{Research Questions}

The study was inspired by my experience as a professional archivist. In my professional education I had been acquainted with ideas in archival theory, which in the study was understood broadly as the whole of ideas in professional literature about what archival material (records and archives) is. ${ }^{2}$ Although these ideas served well as a basis for professional activities, I felt that they were lacking from a theoretical perspective. I was baffled by three questions:

Firstly, there was no theory explaining "recordness", that is, the distinction between records and other types of information. In archival literature the distinction seemed to be taken for granted, but what was said about records was mostly descriptive: one described the activity of creating records (e.g., "transactions"), the context of records creation (e.g., "organisations"), attributes that records have (e.g., "interrelated with other records"), or consequences of having records (e.g., "evidence"). For instance, according to Bruce Dearstyne, "records are extensions of human memory, purposefully created to record information, document transactions, communicate thoughts, substantiate claims, advance explanations, offer justifications, and provide lasting evidence of events." ${ }^{3}$ For me this and similar definitions begged new questions. What information (or trace about the past) would not be evidence? Does not everything take place in some context? Why are concepts of "rights" and "obligations" so often associated with records?

Secondly, the theoretical basis of archival methodology was obscure. By "archival methodology" I mean the reliance of archival professionals on specific principles - first and foremost the principle of provenance as applied when records are processed (i.e., arranged, catalogued and described). The principle of provenance states that records and archives of the same provenance must not be intermingled with those of any other provenance and that the archives of a single provenance should retain the arrangement established by the original agency. ${ }^{4}$ In the archival literature one could find reasons for following the principle in archival work. Still, it was to some

2 See Luciana Duranti, "The Concept of Appraisal and Archival Theory," The American Archivist 57 (1994): 328-344.

3 Cited in Richard J. Cox, “The Record: Is It Evolving?” Records Retrieval Report 10 (1994): 1-16.

4 Peter Walne, Frank B. Evans and François J. Himly, Dictionary of Archival Terminology. Dictionnaire de terminologie archivistique. English and French. With Equivalents in Dutch, German, Italian, Russian and Spanish, New York: K. G. Saur, 1984, pp.130; 134; 143. 
extent unclear what takes place when we follow the principle of provenance. What do we achieve by it?

Thirdly, what links the answers to the previous questions together: how does the need to follow archival principles follow from what records are? Several writers have argued that there is a linkage: that archival methods are a consequence of the characteristics of records ${ }^{5}$ (see for example, the chapter by Duranti and Michetti in this volume). However, there was no theory explicitly connecting the "nature" of records and the ways in which records are treated in archives.

\section{Selecting a Meta-theoretical Interdisciplinary Approach}

The above questions pertained to the relationship and essence of recordness and archival principles. It seemed obvious that it would not be possible to answer them by using archival concepts themselves. This would only lead to repeating what had been said in archival literature. Instead, one had to look at archival theory from outside: in a word, to create a "meta-theory" for archival theory - a theory that would be independent from archival theory, but at the same time that would yield a new perspective on what archival theory says. The study attempted, therefore, to find an alternative conceptualisation that is compatible with the way in which archival theory sees archival phenomena and that also bridges the gap between recordness and archival principles. In a way, this alternative conceptualisation would "explain" what archival researchers are saying and give a new perspective to archival phenomena.

The meta-theoretical approach was inspired by lack of theory construction in archival science. Anneli Sundqvist notes that the concept of archival science is largely used as a general term referring to knowledge in the archival field regardless of its scientific level. ${ }^{6}$ Ideas that are elevated to the position of theory are often not theories. For instance, the principle of provenance

5 Luciana Duranti, “Archival Science," in Encyclopedia of Library and Information Science,. Volume 59, Supplement 22, (New York: Marcel Dekker, 1997), pp.1-19; Terry Eastwood, "Nailing a Little Jelly to the Wall of Archival Studies," Archivaria 35 (1993): 232-252; James O'Toole, "On the Idea of Uniqueness," The American Archivist 57 (1994): 632-658.

6 Anneli Sundqvist, "Arkiv- och informationsvetenskap [Archival science and information studies]," in Information, förvaltning och arkiv: En antologi [Information, management and archives: an anthology] (Härnösand: Landsarkivet i Härnösand, 2005), pp.8-31. 
is sometimes described as a theory, but it is more a method or a description of how a fonds is formed than a theory. ${ }^{7}$ The best-known works that have had the most influence on both archival theory and practice -for instance, books by the Dutch trio of Muller, Feith, and Fruin, and Jenkinson, or by Schellenberg or Casanova - are above all textbooks, not theoretical treatises. ${ }^{8}$

The meta-theoretical approach also led to an interdisciplinary approach. Any new meta-theoretical conceptualisation could not "hang in the air": there had to be research to support it and it seemed likely that there might be research approaches for that. The explosion of research in the 20th century - especially in the social sciences - had relatively little effect on archival science until recently. This made it probable that there might be theories and research in other disciplines that could be used in the study. Thus, the goal was to find approaches from other disciplines that might be used to answer research questions. This would not only contribute to theory building in archival science, but would also potentially create fruitful connections between research areas.

\section{Solving Conceptual Issues}

The research questions also invoked conceptual issues that had to be solved. The research questions were about or closely related to archival theory, but there is no single archival theory. There are only ideas that have been expressed by archival thinkers in different times and contexts employing different concepts that are to some extent local to a particular linguistic, cultural or administrative area (for a more detailed discussion of the various archival theories and traditions at work in the field, see the chapters by Gilliland, McKemmish and Lian in this volume). For instance, in the Finnish language there is no clear linguistic or conceptual separation between records and archives management. The Finnish paradigm seemingly follows the Australian-born records continuum model, although with the lack of theoretical discussion it is almost impossible to say what might be the theoretical background behind the Finnish records and archives management practices. ${ }^{9}$ There is only one identifiable Finnish concept (asiakirja), which covers "private papers", "records", and "archival documents".

7 Claes Gränström, "Arkivteori [Archival theory]," in Arkivvetenskap [Archival science], Anna Christina Ulfsparre, ed. (Lund: Studentlitteratur, 1995), pp.3-28.

8 Sundqvist, "Arkiv- och informationsvetenskap", 8-31.

9 Jaana Kilkki, "Bearmania. Frosting Finnish Archival Practice with Imported Archival Theory," Comma 1 (2004): 43-53. 
So if the research is about "archival ideas" and "records," then what is it really about? What is understood by records? Is records management part of the archival sphere? How should we deal with the heterogeneity of ideas and concepts? An additional problem was that there is very little archival literature in Finnish. Thus, although my own thinking was formed by the Finnish environment, the sources were for the most part written in English and I was also writing in English. Thus, linguistic and conceptual borders had to be crossed multiple times in both directions in the research process.

Resolving the conceptual problems required taking a stance regarding the relationship between language and reality. If concepts are seen either as existing independently from reality or as elements that define what is the reality, then an attempt to find common ground behind different conceptualisations is clearly futile. However, my scientific worldview is inclined to critical realism: concepts are more than mere social constructions or instruments used to discuss and interpret the world around us: ${ }^{10}$ there is an independent reality with which concepts have a relationship, although we may find it hard to say what that relationship actually is. Because of this stance I could assume that in the end archival thinkers are fundamentally talking about the same phenomena even when doing it in different languages and with different concepts. Because my thinking is formed by the Finnish records and archives management environment, I used the terms in their Finnish meaning, and this had to be made explicit in the study.

\section{Creating a New Theoretical Framework by Theory Derivation}

The goals of the meta-theoretical interdisciplinary approach were, thus, to create a new conceptualisation that would open up concepts in archival theory, to give new insight into what records and archives are, and to provide new concepts for analysing them. This conceptualisation was referred in the study as a "theoretical framework." The framework was created through the process of theory derivation:

Theory derivation is the process of using analogy to obtain explanations or predictions about phenomena in one field from the explanations or predictions in another field. Thus, a theory (T1) from one field of

10 Pertti Töttö, Syvällistä ja pinnallista: teoria, empiria ja kausaalisuus sosiaalitutkimuksessa [Deep and superficial: theories, empirical evidence and causality in social scientific research], Tampere: Vastapaino, 2005. 
interest (F1) offers some new insights to a theorist who then moves certain content or structural features into his own field of interest (F2) to form a new theory (T2). ${ }^{11}$

Lorraine Olszewski Walker and Kay Coalson Avant see five basic steps in theory derivation. The process is iterative and some or all steps are repeated until the level of sophistication of the theory is acceptable: ${ }^{12}$

- Becoming familiar with the literature on the topic of interest

- Reading widely in other fields for ideas

- Selecting a parent theory for use in derivation

- Identifying what content and/or structure from the parent theory is to be used

- Developing or redefining new statements from the content or structure of the parent theory in terms of the phenomenon of interest to the theorist.

Walker and Avant observe that theory derivation is "an easy way to develop theory rapidly in a new field," but that finding a suitable parent theory can be difficult. ${ }^{13}$ In my case, I had pondered "recordness" in archival literature many times without finding a satisfactory solution that would put different pieces together. To find new ideas, I read broadly in literatures that I believed might prove useful. Finally, John Fiske's "Introduction to Communication Studies" put the study on the right track by referring to Harold D. Lasswell's early verbal communication model. ${ }^{14}$ Lasswell states that it is essential to think "who says what, in which channel, to whom, with what effect." ${ }^{15}$ It immediately occurred to me that this model could be applied to records, even though this was far from what Lasswell originally had in mind. It seemed intuitively clear that Lasswell's model revealed something essential about the differences between records and other types of information.

However, although Lasswell's model seemed to point to the right direction, it did not give a foundation for my research. Merely pointing out possible differences did not seem to be enough. There should be more

11 Lorraine Olszewski Walker and Kay Coalson Avant, Strategies for Theory Construction in Nursing, Norwalk Connecticut: Appleton-Century-Crofts, 1983, p.163.

12 Walker and Avant, Strategies for Theory Construction, 165-66.

13 Walker and Avant, Strategies for Theory Construction, 165-66.

14 John Fiske, Introduction to Communication Studies. London: Routledge, 1990.

15 Harold D. Lasswell, "The Structure of Function of Communication in Society," in The Communication of Ideas, ed. Lymon Bryson (New York: Institute for Religious and Social Studies, 1948), pp.37-51. 
than just individual idea(s) or a list of record characteristics. Thus, more was needed to achieve the goals of the research. A central element proved to be classical speech act theory - which was first introduced by John L. Austin and was subsequently complemented by John R. Searle - together with other ideas, most notably Searle's theory about the construction of social reality and Lee Thayer's description of communication in organisations. ${ }^{16}$ Speech act theory had already been noted by some archival writers, ${ }^{17}$ but it had not been explored in detail.

\section{Parent Theories for the Theoretical Framework}

Before describing what took place in the study, it is necessary to state briefly the main ideas of the parent theory and the derived theoretical framework. Most components of the parent theory come from classical speech act theory and Searle's theory of the construction of social reality. Both fall into the category of philosophy of language. In addition, Lee Thayer's study ${ }^{18}$ from communication research proved useful.

When people use language - either in speech or in writing - they "do things with words," as Austin put it: they command, persuade, express feelings, promise, and so forth. ${ }^{19}$ In almost any speech act one can identify four levels that are also (somewhat confusingly) known as "acts." These take place when a speech act is made: there is an utterance act (e.g., uttering words, sentences, morphemes), a propositional act (e.g., referring and predicating), an illocutionary act (e.g., stating, questioning, commanding, promising) and a perlocutionary act (e.g., persuading, scaring, or alarming someone by making the illocutionary act). ${ }^{20}$

It is possible to categorise speech acts in different ways. Both Austin and Searle suggested a categorisation. Some categories of speech acts have

16 John L. Austin, How To Do Things With Words: The William James Lectures delivered at Harvard University in 1955, 2nd ed., J. O. Urmson and Marina Sbisà, eds. Cambridge MA: Harvard University Press, 1975; John R. Searle, The Construction of Social Reality. New York: The Free Press, 1995; Lee Thayer, Communication and Communication Systems in Organization, Management, and Interpersonal Relations. Lanham: University Press of America, 1986.

17 E.g. Brien Brothman, "Afterglow: Conceptions of Record and Evidence in Archival Discourse," Archival Science 2 nos.3-4 (2002): 311-42; Heather MacNeil, Trusting Records: Legal, Historical and Diplomatic Perspectives. Dordrecht: Kluwer, 2000, pp.69-70.

18 Thayer, Communication and Communication Systems.

19 Austin, How To Do Things With Words.

20 Searle, Speech Acts, 24-6. 
been previously associated with records in literature. ${ }^{21}$ They are known as "declarations" (in Searle's terminology) or "performatives" (a category suggested by Austin). A successful performance of a declaration guarantees that the speech act corresponds to the world. For example, "I hereby declare war" is a declaration. If a war is declared correctly, there is a war; what is said becomes the state of affairs. Other prime examples of declarations are excommunicating, christening and firing from employment. ${ }^{22}$ Linguistic capabilities are not enough to make declarations - extra-linguistic institutions, such as the church, the law, private property and the state are also required and usually both the speaker and the hearer must have a special position within the institution. ${ }^{23}$

Declarations play an important role in society because they can change institutional facts. "Institutional facts" are the second fundamental category of facts in the reality in which we live. First there are "brute facts," which are facts of the physical world. Then there are institutional facts, which are not physical. Institutional facts exist because people believe them to exist, but they are still real and objective in the sense that they are not a matter of one's preferences, evaluations or moral attitudes. For instance, the height of Mount Everest is a brute fact, but an institutional fact is that it belongs to the state of Nepal. Ownership of land, being married, having money in one's pocket, and mortgaging a house are also examples of institutional facts. ${ }^{24}$

Behind institutional facts lie status-functions. These impose functions upon entities where there is no function prior to imposition: because of statusfunctions, a piece of paper, for example, may give someone the right to drive a car, a pole in the land may serve as a landmark between two countries, and a person can be a judge. Status-functions are basically deontic, that is,

21 Brothman, "Afterglow”; Matti Pulkkinen, "Sähköisen asiakirjan arkistointi [Archiving Electronic Records]," in Arkistot ajassa ja ikuisuudessa. Suomen viidennet arkistopäivät 2.-3.6.1998 Oulussa [Archives in Time and Space. The Proceedings of the 5th Finnish Archival Conference, Oulu, 2 - 3 June 1998, Vuokko Joki and Samuli Onnela, eds. Oulu: Oulun maakunta-arkisto, 1998, pp.79-81; Matti Pulkkinen, "Sähköisen asiakirjan semantiikka [The Semantics of Electronic records]," in Arkisto. Arkistoyhdistyksen julkaisuja 6 [The Archive. Publications of the Society of Finnish Archivists 6], Markku Mäenpää, ed. Helsinki: Arkistoyhdistys, 1999, pp.83-96. See also Geoffrey Yeo, "Representing the Act: Records and Speech Act Theory," Journal of the Society of Archivists 31 (2010): 95-117. doi 10.1080/00379816.2010.50678; Peter Monteith, "Can Records Speak for Themselves?” Journal of the Society of Archivists 31 (2010): 119-133. doi 10.1080/00379816.2010.506783.

22 Stephen C. Levinson, Pragmatics. London: Cambridge University Press, 1983, p.240.

23 John R. Searle, Expression and Meaning: Studies in the Theory of Speech Acts. Cambridge: Cambridge University Press, 1979, pp.5-7; 16-20.

24 Searle, Speech Acts, 51; Searle, The Construction of Social Reality, 1-2. 
they are about what is right, wrong, obligatory or permissible. For instance, passing a landmark on the border without a valid passport is wrong, and persons with the status of border guards have permission and an obligation to arrest rulebreakers. Status-functions are often imposed and new institutional facts created by performing explicit speech acts. According to Searle this is possible because of "constitutive rules," which unlike "regulative rules" do not regulate actions but rather make activity possible. ${ }^{25}$

Searle was looking at society at large. Thayer suggests that task performance in formal organisations is guided by rules that Thayer calls "constraints." A constraint is a rule in the following form: under these circumstances, do $\mathrm{X}$, under those constraints, do $\mathrm{Y}$, and so on. The function of constraints is to limit the degrees of freedom, relative to any particular task-function, which an individual might otherwise exercise. A manager's task is to make changes in the system of constraints when needed. ${ }^{26}$ In other words, inside an organisation there is an "institution" (the organisation itself) and participants in the speech act have a position within the institution (e.g., "boss", "worker"). The rules allow for some people in higher positions to make speech acts that change the deontic status of others in the organisation (e.g., the boss instructs on what to do next or how to react in a situation). Thus, although neither Thayer nor Searle made this connection, the environment for speech acts in an organisation is similar to that in the society at large when the social reality is constructed: in both there are positions in which persons can change deontic status by making speech acts.

\section{Theoretical Framework: Preservation of Rule-bound Speech Acts}

When these ideas were adapted to the archival field, the conclusion was that records are speech acts of a certain kind. Archival writers had already noted that genuine record making is, in the best cases, "performative - acts of speech in which 'saying makes it so." ${ }^{27}$ In the study, however, this idea was developed further. In short, I argued that records are speech acts that change the deontic status of the world, either by altering institutional facts or changing constraints in organisations. This can happen because of the rules governing the speech act.

25 Searle, The Construction of Social Reality, 13-29.

26 Thayer, Communication and Communication Systems, 94-9.

27 Brothman, "Afterglow". 
To capture the essence of this, a new concept, "rule-bound speech act," was coined in the study. A rule-bound speech act is an ideal-type of recordgenerating speech act. Like Max Weber's ideal-type this is a methodological concept, designed to assist a social science researcher in understanding and explaining social phenomena. Because a rule-bound speech act is an ideal-type, it is not a description of reality, but instead gives a coherent and unambiguous meaning to elements, which would otherwise seem incoherent and chaotic. An ideal-type is "ideal" in the sense that it is never, or is very rarely, encountered in all its purity in real life. ${ }^{28}$ When rule-bound speech acts take place there are rules: ${ }^{29}$

Defining what roles there are and who is in which role (e.g., president, citizen, salesman);

Stating what actions are possible, permissible, obligatory, or forbidden in those roles (e.g., signing a treaty, reporting taxable income, making an offer to a customer);

Defining how to act in a situation (e.g., take action against the reported threat of terrorist activity, send report of taxable income, deliver ordered goods);

Making actions possible (e.g., declare a war, apply for status as a war veteran, make a contract with the customer); and,

Defining the deontic consequences of the act (e.g., the state or company is bound by new obligations; the administration has to take the next step in the handling of the matter).

In real life, record-generating speech acts may differ from the ideal type in more than one way. This makes the line between rule-bound and nonrule-bound speech acts (records and non-records) fuzzy. Firstly, there are not always rules belonging to all five categories or, at least, it is not clear whether there are rules and if there are, what they might be. For instance, when a tsunami hit the coast of the Indian Ocean in 2004, a local Finnish embassy made a report about it to the Foreign Ministry, but it was not immediately clear what should happen when the report was received. Secondly, the explicitness and strength of the rules can vary. Sometimes rules governing a speech act are very explicit, public and enforced in society as laws. Sometimes, to the contrary, they are informal, shared only by a small

28 Julien Freund, The Sociology of Max Weber. London: The Penguin Press, 1968, p.63.

29 Henttonen, Records, Rules and Speech Acts, 128. 
group (e.g., members of the organisation), breaking them is not rigorously sanctioned, and it may even be unclear what the rules are. Thirdly, the degree of deontic consequences created by a speech act is not the same in terms of scope and duration. Sometimes deontic consequences are long-lasting and are honoured by a large group of people for generations (e.g., when a new state border is defined in a peace treaty). But deontic consequences can also be minimal, local and short-lived (e.g., my boss tells me what I should do next). Long-lasting rule-bound speech acts that are binding for a large group of people are naturally easier to recognise and are also socially more important.

Despite these variations, the mechanism is always the same: there is a speech act that changes the deontic status of the world. "Archetypal" records, like wills and court verdicts, are archetypal precisely because they correspond most clearly and closely to the ideal type. In real life, drawing the line between records and non-records is usually much more difficult, as discussion in the archival literature shows. ${ }^{30}$ An example of a borderline case is a Master's thesis. As a thesis it is a rigorously controlled rule-bound speech (e.g., possible only for a university student). On the other hand it is a study that does not require an institutional position to be accomplished. Accordingly, a thesis can easily be viewed either as a part of a library or of an archival collection.

\section{Contribution of the Theoretical Framework: Putting Things Together}

From an archival perspective, the framework allows many things in archival thinking to be "put together." It explains the difficulties the field has in defining records, and makes it obvious why concepts like "rights," "obligations," "law" and "accountability" are so often used in connection with them. Records are typically created in organisations and the organisational context is a natural environment for making rule-bound speech acts, because in organisations rules and relationships between persons (such as between fellow workers on an assembly line) are formalised or standardised, whether explicitly or implicitly. ${ }^{31}$

30 E.g., Cox, "The Record: Is It Evolving?" 1-16. Zawiyah M. Yusof and Robert W. Chell, "The Eluding Definitions of Records and Records Management: Is a Universally Acceptable Definition Possible? Part 1. Defining the Record," Records Management Journal 8 no.2 (1998): 95-112.

31 Thayer, Communication and Communication Systems, 94-5. 
This framework also answers the research questions: it explains why having information about context is so important for the interpretation of records. Deontic consequences depend on the context of the speech act: just producing the right words is not enough. For a successful speech act, you need a speaker who has the right to execute the act, and who performs it correctly so that it can have the appropriate consequences. These features are external, they are not "in" the sounds or marks on a medium that are produced to make the act happen. From a record you can see that a speech act has been made, but the context is not included in the document. To evaluate consequences and understand the meaning of a speech act you have to know what the context was. The act may give hints about its context - for instance, the issuer of a passport is stated in the passport - but this information can be missing or needs to be confirmed from other sources. In addition, the same information may bring about dissimilar deontic changes in different professionals reading the document. This may not be obvious by looking at the document and, therefore, it can be understood later only if the context can be identified. For instance, more than one category of personnel uses information in a hospital information system for the administration of medical care. For physicians the system provides quick information about the patient's condition. Pharmacists have a different set of interests. They are less concerned with the overall medical treatment of each individual patient and more concerned with ensuring proper administration of medication. The physicians' primary use of information is retrospective; they want to know what has happened over the last 24-hour period. In contrast, the nurses, who must arrange their activities in such a way as to ensure that each patient receives appropriate attention at relevant points in the shift, look to the same system to provide prospective information about the activities that will need to be carried out in order to effect the prescribed regime of care. ${ }^{32}$ The information that a patient already received his medication may necessitate that a physician changes the medication (because the patient is not getting any better) or a stricture for a nurse not to give any more medication (because the patient already received it for the day). To understand what deontic consequences there were and to whom, we need more than just the information that was transmitted: we need information about the speech act context. What the document tells about the context may need to be complemented, either by adding metadata, making archival

32 Madhu C. Reddy, Paul Dourish and Wanda Pratt, Coordinating Heterogeneous Work: Information and Representation in Medical Care, http://citeseer.nj.nec.com/ correct/444159. 
descriptions, or by applying the principle of provenance, for instance, when the document alone does not reveal the participants of the speech act or its organisational context. Sometimes the information given in an act about its context can be entirely false. This is not acceptable, especially when the deontic consequences are socially important. Hence, there is a need for authentic records - which explains the interest in authenticity in archival literature. ${ }^{33}$

Record-creating speech acts require extra-linguistic institutions: and they require those institutions, such as private property (otherwise it would not be possible to sell it by deed of sale), church, government or organisational structure to succeed. If participants of the speech act do not have appropriate positions defined by the institutions (e.g., somebody is selling the property without being its owner), the acts are meaningless and void. By contrast, non-record creating speech acts do not require these kind of extra-linguistic institutions, and only the capability to use language is needed to succeed. Linguistic competence, which comes from obeying the rules of language, is enough, for instance, to make a statement that it is raining, or to promise to come for a visit. A newspaper article is a good example of a non-recordgenerating speech act. A newspaper article informs you. The writer's position as a professional journalist may add credibility to the article's statements. Still, making the speech act in an article is possible for anyone, including for persons who are not members of the editorial staff. The speech act is meaningful, therefore, regardless of who is behind it, unlike in a deed of sale, for instance. A newspaper article also cannot create new obligations that must be followed (although it can inform one about obligations made by speech acts elsewhere). Thus, rules belonging to the five above-mentioned categories are missing.

\section{Building and Testing the Framework}

Building the framework was the fifth step in the process of theory derivation: developing or redefining new statements from the content or structure of the parent theory in terms of the phenomenon of interest to the theorist. In this step, philosophical methods played a major role since speech act theory

33 E.g., Laura Millar, Authenticity of Electronic Records: A Report Prepared for UNESCO and the International Council on Archives. Paris: International Council on Archives, 2004; Luciana Duranti, "Reliability and Authenticity: The Concepts and Their Implications," Archivaria 35 (1993): 5-10. 
originates in the philosophy of language. Also, getting from parent theories to derived theory was a matter of formulating questions and enunciating answers. Thus, acceptability of the answers depends on the acceptability of the premises and the soundness of the argumentation. This approach was complemented by analysing archival literature to see what features are associated with recordness in archival theory. Excerpts for analysis (from 69 writers) were collected from textbooks, articles, glossaries, and other publications in English, Swedish, and Finnish languages. The data was categorised to identify what was regarded as the context of record creation, how the activities creating records were described, and what was understood as the intention or purpose of record creation. ${ }^{34}$

The applicability of speech act theory was tested empirically by speculating that archives actually "preserve speech acts" in that they store information that helps a user to understand the speech act in a document. To convert this to a factual statement, it was further speculated that archival methodology - which in the Finnish environment was interpreted to mean the practice of keeping registries (using a book, cards or other media to list the receipt and dispatch of the records in organisation) and following the principles of respect des fonds (not intermingling records / archives of the same provenance with those of any other provenance) and the registry principle (retaining the arrangement established by the creating agency) - stores information about levels in a speech act on the one hand (that is, utterance acts, propositional acts, illocutionary acts, and perlocutionary acts), and on the other hand, about the context of the speech act. Studies that I consulted in sociolinguistics, discourse analysis and semantics ${ }^{35}$ suggested that there are four features of speech act context that are most relevant for understanding it: addressor, addressee, topic, and setting. Thus, the working theory was that if we look at "documentary context" - which consists of 1) the archival fonds to which a record or manuscript belongs (i.e., its provenance), 2) the place of the record or manuscript inside the fonds in a file, box, or bound volume, and 3) the entry in the register (if the record was registered) - we will find information about the speech act. That information will be about the relevant propositional, illocutionary and perlocutionary acts as well as

34 Henttonen, Records, Rules and Speech Acts, 75-100.

35 Gillian Brown and George Yule, Discourse Analysis. Cambridge: Cambridge University Press, 1983, p.41; Dell Hymes, Foundations in Sociolinguistics. An Ethnographic Approach. Philadelphia: University of Pennsylvania Press, 1974, pp.53-62; David Lewis, "General Semantics," in Semantics of Natural Language, D. Davidson and G. Harman, eds. (Dordrecht: Reidel, 1972), p.173. 
about the addressor, the addressee, the topic, and the setting (time and place) of the act.

Next a sample of eight archival documents was examined to see whether the working theory was supported. For every document, the results of the analysis were summarised in a table (for an example, see Table 18.1) and discussed in the text. The table shows what information the documentary context gave about the speech act and indicates whether the same information could be also found by looking at the document. Information that was only in the document was ignored in the analysis. For instance, one of the records analysed was a list of Finnish names. The letter filed next to it revealed that the list was about Finns who had perished in the sinking of RMS Titanic and that the list had been created in the Russian Consulate in London. Thus, it was interpreted that in this case both the propositional act (what was "spoken about") and the "speaker" could be seen only from the place of the record in the fonds.

Table 18.1. Example of the table used in the analysis

\begin{tabular}{|l|c|c|c|c|c|c|c|c|}
\hline \multirow{2}{*}{$\begin{array}{l}\text { The source of } \\
\text { information }\end{array}$} & \multicolumn{3}{|c|}{ Functional levels } & \multicolumn{5}{c|}{ Components } \\
\cline { 2 - 9 } & Pr. & Il. & Per. & A-s & A-e & Topic & Time & Pl. \\
\hline The fonds & & & & & (x) & & & \\
\hline $\begin{array}{l}\text { The place in } \\
\text { the fonds }\end{array}$ & & $\mathrm{X}$ & ( $)$ & & & & & \\
\hline The register & & & -1 & & & & & \\
\hline
\end{tabular}

Legend: Pr. $=$ propositional act. Il. = illocutionary act. Per. $=$ perlocutionary act. $\mathrm{A}-\mathrm{s}=$ addressor. $\mathrm{A}-\mathrm{e}=$ addressee. $\mathrm{Pl}$. = place. $\mathrm{X}=$ the information is missing from the document or cannot be with certainty deduced from it. $(\mathrm{x})=$ the information is also in the document. $\dashv=$ shows what the act is a perlocutionary act of (the information is not in the document). $(F)=$ shows the perlocutionary act resulting from the act (the information can be also found in the document).

The method used in the selection of the sample of eight records or manuscripts was theoretical sampling. In qualitative research, when theoretical sampling is used, the cases studied are selected on the basis of the research questions being investigated, the theoretical positioning of the research and, most importantly, the explanation or account being developed. ${ }^{36}$ The examples were selected by introspection. After formulating the working theory, I reflected upon archival documents in the light of my personal

36 David Silverman, Doing Qualitative Research. A Practical Handbook, London: Sage, 2000, pp.102-12. 
professional experience of records and archives, asking myself whether there are archival documents in which this or that aspect of a speech act or context might be identified because archival principles had been applied. For instance, I asked myself whether one might discover a perlocutionary act by looking at the documentary context. From my experience I could right away say that one might sometimes see the perlocutionary act of a speech act from the other records in the same file. This happens, for instance, when a proposal is made about how to fill a vacancy. In this case there are two records in the file: the letter containing the proposal and the resulting letter of appointment, which is the perlocutionary act of the former. The next step was to find an example of this and analyse it using the table described above. The analysis often revealed that some other information about the speech act context and the act was also conveyed in the documentary context. ${ }^{37}$

\section{Discussion}

Overall, the chosen meta-theoretical interdisciplinary approach worked quite well. The theoretical framework that was created in the study allowed detailed questions to be posed about the information that was conveyed by means of the principle of provenance and registries. It offered a theoretical explanation for recordness and it explained the need to follow archival principles in archival work. It also linked central questions of archival theory to a field of research that has been widely studied in other disciplines.

At the same time, the study had limitations. Some are related to its basic tenets, some to the methodology, and still others to issues that could not be resolved. Firstly, not everyone is probably ready to accept the idea that it is possible to discern a common reality underlying archival theoretical discourse and that it can be analysed via speech act theory and the theoretical framework that was suggested in the study. Some may argue that archival thinkers may make individual or group interpretations that differ from the suggested "independent reality." This may be true, but the argument cannot be answered if the interpretations are not first defined. However, one should note that the theoretical framework in the study does not require that we have shared interpretations and agree upon the meaning of records. Speech act theory is flexible and allows different interpretations for the acts. According to Sandy Petrey: 
... speech act analysis stands opposed to every vision of the text as an object, as a given and permanent entity that is what it's always been and will always remain so. Considered as acts, literary and non-literary utterances alike change in conjunction with the conventions they invoke and by which they are assessed..$^{38}$

Hence, the suggested framework positions itself between the positivist and intepretivist paradigms in archival science. ${ }^{39}$ The interpretivist research paradigm states that there is no one objective reality, but rather multiple realities which are socially and individually constructed, and positivist notions see existence of an objective reality and universal principles that go beyond any particular historical, legal or cultural context. ${ }^{40}$ The suggested framework simultaneously allows both views. Changing social reality by speech acts is universal: it is not restricted to any particular time, place or social setting. Institutional facts created by speech acts have "objective" existence: they exist, because people believe them to exist, but they are not a matter of one's preferences, evaluations or moral attitudes. However, at the same time, speech act theory does not suggest that there is no one truth or message. For example, the words contained in a will: "I give and bequeath my watch to my brother" do not produce a legacy at the moment of enunciation. The testator's death must occur for his or her property to be transferred, which means that the words will "do things" in a context different from that in which they are produced. Whether at that time there are institutions enabling the transference of private property through a will, and whether the words are read as an expression of brotherly love, is not for the writer to decide. Petrey also suggests that speech act theory and Jacques Derrida's deconstructionism are not incompatible, but only lead to a different emphasis. ${ }^{41}$ If archival principles help a user to reconstruct and understand the speech act this might be an expression of the immutable and universal qualities of archival work that are suggested by Luciana Duranti, ${ }^{42}$ because archival principles may contribute to understanding of speech acts regardless of the specific historical and cultural environment (although it is not yet proven that this actually takes place).

38 Sandy Petrey, Speech Acts and Literary Theory. New York: Routledge, 1990, p.131.

39 Anne Gilliland and Sue McKemmish, "Building an Infrastructure for Archival Research," Archival Science 4 (2004): 149-97.

40 Gilliland and McKemmish, "Building an Infrastructure."

41 Petrey, Speech Acts and Literary Theory, 78.

42 Duranti, "Archival Science", 1-19. See also the chapter by Duranti and Michetti in this volume. 
A limitation of the study is the abstractness of the suggested theoretical framework. In this respect it has the same weakness that has been found in Searle's theory of social construction. Stanley B. Barnes compared Searle's theory to how a sociologist sees social reality. He concluded that there are no fundamental contradictions, although Searle "achieves clarity and perspicuity at the cost of simplifications - [whereas] sociologists - are prepared to sacrifice simplicity to empirical adequacy." ${ }^{\prime 3}$ In a similar manner, the theoretical framework in the study is a simplification, not a description of empirical reality. A further limitation is that the empirical part of the study - in which documents were examined to see what information the principle of provenance and registries convey - was limited by the small sample and the method of selection. This was enough to show that speech act theory can be used to analyse the significance of documentary context. It also showed that in some cases at least some information about speech act levels and their context is carried within documentary context. However, this does not show that it happens often or that the information carried is important for archival users. For such conclusions one needs more studies.

Speech act theory is not applicable to some archival areas. Although speech act theory is not limited to oral - or even to linguistic - communication, ${ }^{44}$ studies of speech acts have been either orally-oriented or have paid no attention to the medium used in communication. Therefore, the theory has limited applicability when documents are looked as evidence of something that has taken place in another place or time or independently from the act of record making. It still has value when we want to understand the moment of record creation or how archival principles contribute to the understanding of documents, but it is possible to look at records and archives also from other perspectives and in such situations speech act theory is less useful.

This can be seen in the conceptualisation of records in the light of speech act theory which is different from how records have been previously conceptualised in archival literature. A "transaction" in archival literature is not identical to the creation of a record, nor is an "act" ("activity whose purpose is to produce the result produced") in contemporary diplomatics equivalent to a record documenting it—although in the case of dispositive

43 Stanley B. Barnes, "Searle on Social Reality: Process is Prior to Product," in Speech Acts, Mind, and Social Reality: Discussions with John R. Searle, Günther Grewendorf and Georg Meggle, eds. (Dordrecht: Kluwer, 2002), pp.247-58.

44 Jens Allwood, "A Critical Look at the Speech Act Theory," in Logic, Pragmatics and Grammar, Östen Dahl, ed. (Lund: Studentlitteratur, 1977), pp.53-69; Sándor Hervey, Semiotic Perspectives. London: George Allen Unwin, 1982, pp.93-94. 
documents (e.g., wills and contracts where the purpose of the document is to put into existence a juridically relevant act) they come quite close. In diplomatics, action and documentation of that action are two separate things. ${ }^{45}$ Speech act theory, however, does not allow for such distinctions: it knows only the act of using language when the record is created. Another complication arises from the ways in which written speech acts can be "re-used" and replicated in different contexts. For instance, a letter can be forwarded to a new addressee, introduced as evidence in divorce proceedings, and its contents copied to a new document. Speech act theory does not offer ready tools for analysing this kind of communication.

In Finnish terminology there is no conceptual difference between records and personal papers. However, the suggested theoretical framework fits poorly into the personal papers. Firstly, when we communicate with our friends, relatives, and family members the rule and institutional environment suggested by the concept of rule-bound speech acts is largely missing. ${ }^{46}$ Secondly, the sample that was analysed in the study did not find any evidence suggesting that information about the speech act context or its levels is carried in the documentary context in the archives where personal papers are concerned. Although the sample was so small that a more thorough study might perhaps produce different results, in light of the theoretical framework, personal papers are a different category from "proper" records.

It is obvious that how a fonds may contribute to the understanding and interpretation of documents in it is not limited to information about the levels and context of speech acts. Our understanding and knowledge of this is still quite limited. To cite an example given by Debra Barr: a person who is a member of a Trotskyist organisation has kept all the letters from his fellow Trotskyists in one place. Barr notes that some information would be lost if the letters were to be intermingled with others. ${ }^{47}$ Thus, one quickly finds oneself in a sphere where concepts taken from speech act theory and linguistics are not applicable. However, speech act theory is a good tool when we want to understand some aspects of record creation and the connection between recordness and archival principles. It may also have practical value. The concept of rule-bound speech acts could be operationalised by creating a checklist of questions to measure the degree to which a speech act

45 Luciana Duranti, Diplomatics: Nerw Uses for an Old Science. Lanham, MD: Society of American Archivists and The Scarecrow Press, 1998.

46 Henttonen, Records, Rules and Speech Acts, 132-33.

47 Debra Barr, "Protecting Provenance: Response to the Report of the Working Group on Description at the Fonds Level," Archivaria 28 (1989): 141-45. 
corresponds to the ideal type of rule-bound speech. This tool might be then used to evaluate "recordness," perhaps to identify acts that are important for accountability process, and to test how the ideal type corresponds to popular or professional conception of records.

\section{References}

Allwood, Jens. "A Critical Look at the Speech Act Theory." In Logic, Pragmatics and Grammar, Östen Dahl, ed. Lund: Studentlitteratur, 1977, pp. 53-69.

Austin, John L. How To Do Things With Words: The William James Lectures Delivered at Harvard University in 1955, 2nd ed., J. O. Urmson and Marina Sbisà, eds. Cambridge, MA: Harvard University Press, 1975.

Barr, Debra. "Protecting Provenance: Response to the Report of the Working Group on Description at the Fonds Level." Archivaria 28 (1989): 141-145.

Barnes, Stanley B. "Searle on Social Reality: Process is Prior to Product," in Speech Acts, Mind, and Social Reality: Discussions with John R. Searle, Günther Grewendorf and Georg Meggle, eds. (Dordrecht: Kluwer, 2002), 247-58.

Brothman, Brien. "Afterglow: Conceptions of Record and Evidence in Archival Discourse." Archival Science 2 nos.3-4 (2002): 311-42.

Brown, Gillian, and George Yule. Discourse Analysis. Cambridge: Cambridge University Press, 1983.

Cox, Richard J. “The Record: Is It Evolving?” Records Retrieval Report, 10 (1994): 1-16.

Duranti, Luciana. "Archival Science," in Encyclopedia of Library and Information Science, Vol. 59, Supplement 22 (New York: Marcel Dekker, 1997), 1-19.

Duranti, Luciana. Diplomatics: New Uses for an Old Science. Lanham: Society of American Archivists and The Scarecrow Press, 1998.

Duranti, Luciana. "Reliability and Authenticity: The Concepts and Their Implications." Archivaria 35 (1993): 5-10.

Duranti, Luciana. “The Concept of Appraisal and Archival Theory," The American Archivist, 57 (1994): 328-344.

Eastwood, Terry. "Nailing a Little Jelly to the Wall of Archival Studies." Archivaria 35 (1993): 232-52.

Fiske, John. Introduction to Communication Studies. London: Routledge, 1990.

Freund, Julien. The Sociology of Max Weber. London: The Penguin Press, 1968.

Gilliland, Anne, and Sue McKemmish. "Building an Infrastructure for Archival Research.” Archival Science 4 (2004): 149-197. doi:10.1007/s10502-006-6742-6.

Gränström, Claes. "Arkivteori [Archival theory]," in Arkivvetenskap [Archival science], Anna Christina Ulfsparre, ed. (Lund: Studentlitteratur, 1995), 3-28.

Henttonen, Pekka. Records, Rules and Speech Acts: Archival Principles and Preservation of Speech Acts. Tampere: University of Tampere, 2007.

Hervey, Sándor. Semiotic Perspectives. London: George Allen Unwin, 1982.

Hymes, Dell. Foundations in Sociolinguistics: An Ethnographic Approach. Philadelphia: University of Pennsylvania Press, 1974.

Kilkki, Jaana. "Bearmania. Frosting Finnish Archival Practice with Imported Archival Theory." Comma 1 (2004): 43-53.

Lasswell, Harold D. "The Structure of Function of Communication in Society," in The Communication of Ideas, Lymon Bryson, ed. (New York: Institute for Religious and Social Studies, 1948), 37-51. 


\section{Chapter i 8}

Levinson, Stephen C. Pragmatics. London: Cambridge University Press, 1983.

Lewis, David. "General Semantics," in Semantics of Natural Language, Donald Davidson and Gilbert Harman, eds. (Dordrecht: Reidel, 1972), 169-218.

MacNeil, Heather. Trusting Records: Legal, Historical and Diplomatic Perspectives. Dordrecht: Kluwer, 2000.

Millar, Laura. Authenticity of Electronic Records: A Report Prepared for UNESCO and the International Council on Archives. Paris: International Council on Archives, 2004.

Monteith, Peter. "Can Records Speak for Themselves?" Journal of the Society of Archivists 31 (2010): 119-133. doi: 10.1080/00379816.2010.506783.

O'Toole, James. "On the Idea of Uniqueness." The American Archivist 57 (1994): 632-58.

Pulkkinen, Matti. "Sähköisen asiakirjan arkistointi [Archiving Electronic Records]." In Arkistot ajassa ja ikuisuudessa. Suomen viidennet arkistopäivät 2.-3.6.1998 Oulussa [Archives in Time and Space. The Proceedings of the 5th Finnish Archival Conference, Oulu, 2 - 3 June 1998, Vuokko Joki and Samuli Onnela, eds. (Oulu: Oulun maakuntaarkisto, 1998), 79-81.

Pulkkinen, Matti. "Sähköisen asiakirjan semantiikka [The Semantics of Electronic Records]," in Arkisto. Arkistoyhdistyksen julkaisuja 6 [The Archive. Publications of the Society of Finnish Archivists 6], Markku Mäenpää, ed. (Helsinki: Arkistoyhdistys, 1999), 83-96.

Petrey, Sandy. Speech Acts and Literary Theory. New York: Routledge, 1990.

Reddy, Madhu C., Paul Dourish and Wanda Pratt. Coordinating Heterogeneous Work: Information and Representation in Medical Care, http://citeseer.nj.nec.com/ correct/444159.

Searle, John R. The Construction of Social Reality. New York: The Free Press, 1995.

Searle, John R. Expression and Meaning: Studies in the Theory of Speech Acts. Cambridge: Cambridge University Press, 1979.

Searle, John R. Speech Acts: An Essay in the Philosophy of Language. Cambridge: Cambridge University Press, 1980. Original edition, 1969.

Silverman, David. Doing Qualitative Research: A Practical Handbook. London: Sage, 2000.

Sundqvist, Anneli. "Arkiv- och informationsvetenskap [Archival science and information studies]," in Information, forvaltning och arkiv: En antologi [Information, management, and archives: an anthology] (Härnösand: Landsarkivet i Härnösand, 2005), 8-31.

Thayer, Lee. Communication and Communication Systems in Organization, Management, and Interpersonal Relations. Lanham, MD: University Press of America, 1986.

Töttö, Pertti. Syvällistä ja pinnallista: Teoria, empiria ja kausaalisuus sosiaalitutkimuksessa [Deep and superficial: theories, empirical evidence and causality in social scientific research]. Tampere: Vastapaino, 2005.

Wagner David G., and Joseph Berger. "Do Sociological Theories Grow?” American Journal of Sociology 90 no.4 (1985): 697-728.

Walne, Peter, Frank B. Evans, and François J. Himly. Dictionary of Archival Terminology. Dictionnaire de terminologie archivistique. English and French. With Equivalents in Dutch, German, Italian, Russian and Spanish. New York: K. G. Saur, 1984.

Walker, Lorraine Olszewski, and Kay Coalson Avant. Strategies for Theory Construction in Nursing. Norwalk, Connecticut: Appleton-Century-Crofts, 1983.

Yeo, Geoffrey. "Representing the Act: Records and Speech Act Theory." Journal of the Society of Archivists 31 (2010): 95-117. doi 10.1080/00379816.2010.50678.

Yusof, Zawiyah M. and Robert W. Chell. “The Eluding Definitions of Records and Records Management: Is a Universally Acceptable Definition Possible? Part 1. Defining the Record," Records Management Journal 8 no.2 (1998): 95-112. 
Chapter 19

\title{
ARCHIVAL MEDIATION
}

\section{Studying Users' Interaction with Access Systems}

\author{
Anneli Sundqvist
}

\begin{abstract}
Records are complex materials because of their origin in complex social processes. Records held in archival repositories are usually not directly accessible, only through an intermediary. Thus, accessing records can also be a rather complex process, involving several instances of mediation, both personal and artefactual. Traditional means of mediation like finding aids and inventories remain valuable tools, but in recent years new ways of making records accessible have emerged, based, for instance, on Internet technology and social media. The purposes of this chapter are to explore research studies of users' interaction with artefactual intermediaries, to survey what methods are used and their strengths and limitations, and to analyse the extent to which methods used in other fields, for instance $\mathrm{HCl}$ research and media and communication studies, can be appropriated by archival science.
\end{abstract}

\section{Introduction}

Records ${ }^{1}$ present distinctive challenges for access and use for several reasons. They are generated through complex social processes, and are used and reused as parts of other processes. They are created in one context, but may be used in totally different contexts by persons other than those who originally created them, often to answer questions not related to their original purpose.

1 The aim of this article is not to analyse concepts of archival theory. The concept of records is used in a rather inclusive sense that is consistent with established definitions: recorded information generated in activities performed by organisations or persons. 
The ways in which records are conceptualised and described in the original situation do not necessarily correspond to the ways they might be queried later. ${ }^{2}$ When records are held by archival repositories, accessing them can also be a rather complex procedure, involving several forms of mediation, both human and artefactual (e.g., an automated system or a finding aid). Traditional means of mediation such as finding aids and inventories remain valuable tools, but in recent years new ways of making records accessible have emerged, based on, for example, web technology and social media. There is reason to assume that these developments have also generated increasing interest from the research community, especially since there is a contemporaneous growth in archival science research in general. If so, this calls for the study of archival science ${ }^{3}$ research as such. Self-reflection and the making of a historiography are important elements in the establishment and consolidation of a discipline.

This chapter examines research about users' interaction with artefactual intermediaries to define its state of the art in archival science. ${ }^{4}$ It aims to survey what research methods are used, and for what purposes; to analyse the epistemological and disciplinary origins of the methods; and, to discuss their applicability in archival science research. The study is based on a review of articles in archival science journals, reports and theses that deal with use, users, access, information seeking, and archival description. It emphasises the methods used and the performance of the studies examined rather than their results, although it includes a commentary on the strengths and weaknesses of the methods. The analysis is guided by a conceptual framework emerging from basic social science methodology.

\section{Studying Use and Users}

Academic research in archival science is still of limited proportions, but in recent years an increase in research activity and publishing can be observed.

2 E.g., Anneli Sundqvist, "Search Processes, User Behaviour and Archival Representational Systems.” Ph.D. diss., Mid Sweden University, 2009.

3 Archival science is here referred to as academic education, research and scholarly publishing that together define themselves as archival science. Different institutions and research traditions delimit the discipline in slightly different ways and the argument of this article is not that a uniform definition is necessary, but that intradisciplinary discussion and awareness is desirable. The analysis in this chapter also refers to some research with other disciplinary backgrounds that addresses concepts and issues of significance for archival theory and practice, and therefore can be considered to contribute to archival science.

4 The analysis was undertaken in 2011. 
This is to a large extent influenced by the challenges presented by information technology for archival practices. Contemporary research has particularly focused on applied research about electronic recordkeeping, but there is a growing interest in socio-cultural aspects of records and archives. ${ }^{5}$ However, there is also an overlap between these two strands of research when it comes to the study of the need for, the use of, and the access to records.

The value of studying use and users in an archival context was hardly recognised until the 1980s. A special issue of The Midwestern Archivist raised the theme "the use of user studies", and several limited empirical studies were published in that issue and elsewhere during that decade. ${ }^{6}$ Paul Conway in particular articulated methodological issues and developed elaborated survey templates. ${ }^{7}$ Despite these promising developments, interest in use and user studies seemed to fade until the early 2000s, when much had changed about archival use environments and questions concerning use, users and access gained renewed attention. ${ }^{8}$ The concept of user studies is, however, not unambiguous, since it relates to a variety of topics including the composition of user groups, quantitative metrics, accessed material, information needs, information-seeking behaviour, and channels of communication. ${ }^{9}$ Sundqvist has identified five overriding themes in archival science research related to users, namely: use of records, users and user groups; the search process and use of intermediaries; interaction with search tools; and user queries. ${ }^{10}$ Given the

5 Sue McKemmish and Anne Gilliland, "Archival and Recordkeeping Research: Past, Present and Future," in Research Methods: Information, Systems, and Contexts, Kirsty Williamson and Graeme Johanson, eds. (Prahran, Victoria: Tilde Publishing and distribution, 2013), pp. 79-112.

6 William J. Maher, "The Use of User Studies and Archival Mission and User Studies," The Midwestern Archivist 11 no. 1 (1986): 15-26; Roy C. Turnbaugh, "Archival Mission and User Studies," The Midwestern Archivist 11 no. 2 (1986): 15-34; Diane L. Beattie, "An Archival User Study: Researchers in the Field of Women's History," Archivaria 29 (1989/1990): 33-55; Paul Conway, "Research in Presidential Libraries: A User Survey," Midwestern Archivist 11 (1986): 35-56; Jacqueline Goggin, "The Indirect Approach: A Study of Scholarly Users of Black and Women's Organizational Records in the Library of Congress Manuscript Division," Midwestern Archivist 11 (1986): 57-67; Lucia S.

Principe, "Everyman and Archives," Archivum 29 (1982): 135-141; Michael Roper, "The Academic Use of Archives," Archivum 29 (1982): 27-45.

7 Conway, "Research in Presidential Libraries," 35-56; Paul Conway, "Facts and Frameworks: An Approach to Studying the Users of Archives," The American Archivist 49 (1986): 393-407.

8 For overviews see Carolyn Harris, "Archives Users in the Digital Era: A Review of Current Research Trends," Dalhousie Journal of Information \&' Management 1 (2005), http://hdl.handle.net/10222/13360; Anneli Sundqvist, "The Use of Records - A Literature Review," Archives E Social Studies 1 (2007): 623-653; Sundqvist, "Search Processes."

9 E.g., Rania Siatri, “The Evolution of User Studies,” Libri 49 (1999): 132-141.

10 Sundqvist, "Search processes." 
diversity of possible topics of investigation and the paucity of coverage up to the last decade, recent user studies from an archival science perspective could be expected to be rather heterogeneous, with different purposes and different subjects of study, and thus requiring different methodological approaches.

\section{Archival Mediation and Representation}

As already intimated, searching for records in archival repositories is often a complex and highly mediated process. Records are usually not directly accessible; first the relevant records have to be identified and located, then the relevant items or traces within those records have to be identified and located, and finally, the content must be understood and interpreted. This process may involve several iterations and several instances of mediation, both human and artefactual. ${ }^{11}$ When the mediating process involves human intermediaries, it often takes form of a question negotiation where the intermediary (for instance an archivist) has to intervene and interpret the requests of the user, make them concrete and translate them into relevant access points. ${ }^{12}$ When users interact with human intermediaries, artefacts such as information systems, finding aids, or classification systems still play an important role in the search process and can function as a means of communication between users and human intermediaries. Indeed, artefactual intermediaries are often surrogates for records, representing them in a summarised and often abstracted form. ${ }^{13}$ Through time various forms of representations have been created, but their primary functions have been as means for managing and controlling records, even if they also have functioned as search tools. In some cases, however, the primary purpose has in fact been to enhance access, as indicated by the American term "finding aid". A finding aid can thus be manifested in various forms. Today many finding aids are created digitally or digitised, often as part of more comprehensive access systems, and published on websites. To an increasing extent these systems also provide access to the records themselves or to digitised copies of archival materials. This means that human mediation is

11 Sundqvist, "Search processes."

12 Margaret Hedstrom, "Archives, Memory, and Interfaces with the Past." Archival Science 2 (2002): 21-43; Helen R. Tibbo, "Primarily History in America: How U.S. Historians Search for Primary Materials at the Dawn of the Digital Age," American Archivist, 66 (2003): 9-50; Sundqvist, "Search Processes."

13 Yakel refers to "representational systems that contain those surrogates to stand in for or represent actual archival materials". Elizabeth Yakel, "Archival Representation," Archival Science 3 (2003): 2. 
supplemented or supplanted by computer-mediated devices, and that the accessibility of records is becoming more and more dependent upon the effective construction and performance of these devices. ${ }^{14}$ Thus, the design of artefactual intermediaries becomes crucial. As Yakel says:

It is in finding aids that users' representations of archives meet archivists' representations of collections. If these two cognitive representations intersect enough, the user is able to locate and utilise the archives and to identify primary sources that may hold the answer to his or her inquiry. If these representations diverge, the access tools are useless for the researcher. Creating finding aids that are true boundary objects is key. ${ }^{15}$

To promote access to records and to provide adequate services, it is necessary to understand how users approach the records, how they interact with intermediaries, and to what extent these facilitate access to records. Knowledge about user behaviour and use of intermediaries is thus required.

\section{Research about Users' Interaction with Artefactual Intermediaries}

This section presents an overview of studies of users' interaction with artefactual intermediaries, i.e. access systems, using different methodological approaches. As was stated above, research on this topic is limited and only a minor part of archival science research actually deals with user-intermediary interaction. The subject is to some extent addressed in studies of the general information-seeking behaviour of users of archival materials, and in studies of research practices. These studies are of various kinds: larger quantitative surveys ${ }^{16}$, or small qualitative interview studies. ${ }^{17}$ Some researchers have

14 Wendy M. Duff, "Understanding the Information-seeking Behaviour of Archival Researchers in a Digital Age: Paths, Processes and Preferences." Proceedings of the DLM-Forum: @ccess and Preservation of Electronic Information, Best Practices and Solutions, Barcelona, 6-8 May 2002, 331-339; Hedstrom, "Archives, Memory, and Interfaces with the past," 21-43.

15 Yakel, "Listening to Users," 122.

16 E.g., Michael Stevens, "The Historian and Archival Finding Aids," Georgia Archive 5 (1977): 64-74; Tibbo, "Primarily History in America", 9-50; Ian G. Anderson, "Are You Being Served? Historians and the Search for Primary Sources," Archivaria, 58 (2004): 81-129; Wendy M. Duff, Barbara Craig, and Joan Cherry, "Finding and Using Archival Resources: A Cross-Canada Survey of Historians Studying Canadian History," Archivaria 58 (2004): 51-80.

17 E.g. Elizabeth Yakel and Laura L. Bost, "Understanding Administrative Use and Users in University Archives," American Archivist 57 (1994): 596-615; Elizabeth Yakel, 
carried out more comprehensive studies of user behaviour in natural settings, using a multi-method design with various sources and different techniques of data collection, ${ }^{18}$ that is, studies of real life situations such as actual searches for records at archival repositories and workplaces. In these cases, research has touched upon issues like users' apprehension of different kind of intermediaries, to what extent certain intermediaries are used, and user preferences. However, the focus is not primarily the interaction between users and the intermediaries. Other studies emphasise the search process and users' search behaviour more closely, i.e. with an explicit focus on users' interaction with search tools such as traditional finding aids, databases, or online applications. These kinds of studies are the subject of the present chapter. The majority of the studies dates from the 2000s, but a few were conducted earlier.

The advent of computer-mediated intermediaries appears to have sparked an interest of experimental research in archival science. Some research has been modelled on studies in Library and Information Science (LIS), but has been adapted to the specific circumstances of using archival collections, or making comparisons between traditional ways of describing archival materials and more library-oriented content-based approaches like subject indexing. Some researchers argue that searching for archival materials differs from information seeking in general. ${ }^{19}$ This is partly due to the fact that records are often required for reasons other than their information content, e.g., as evidence or because of their intrinsic value, and partly due to the collective character of archival materials that also has an impact upon the ways records are represented and described. Therefore, content-oriented search strategies are not always considered appropriate and LIS methods not directly applicable in an archival context, but require some modification if

"Listening to Users," Archival Issues 26 (2002): 111-127; Wendy M. Duff, and Catherine A. Johnson, "Accidentally Found on Purpose: Information-Seeking Behavior of Historians in Archives," Library Quarterly 72 (2002): 472-496; Wendy M. Duff, and Catherine A. Johnson, "Where is the List with All the Names? Information-Seeking Behavior of Genealogists," American Archivist 61 (2003): 79-95; Elizabeth Yakel and Deborah A. Torres, "AI: Archival Intelligence and User Expertise," American Archivist, 66 (2003): 51-78.

18 E.g., Conway, "Research in Presidential Libraries," 35-56; Paul Conway, Partners in Research. Improving Access to the Nation's Archive. User Studies at the National Archives and Records Administration. Pittsburgh: Archives \& Museum Informatics, 1994; Sundqvist, "Search Processes."

19 Lokman I. Meho and Helen R. Tibbo, "Modeling the Information-seeking Behavior of Social Scientists: Ellis's Study Revisited," Journal of the American Society for Information Science and Technology 54 (2003): 570-587; Duff and Johnson, "Accidentally Found on Purpose," 472-496; Yakel and Torres, "AI: Archival Intelligence and User Expertise," 51-78; Sundqvist, "Search Processes." 
they are adopted. However, evaluations of different search strategies and the efficiency of subject-based versus contextually-based search tools, i.e. a "library" versus an "archival" approach, have been the focus of some research. A notable early example is Lytle's comparisons of the efficiency of provenance-based and content-based information retrieval respectively, based on a small-scale "real-world" experiment testing search performance in authentic descriptions of a particular archival collection and a subject index based on a thesaurus. ${ }^{20}$

An underlying theme in Lytle's study, as well as in several others, is how the content and structure of the intermediaries affect user performance. Duff and Stoyanova conducted a user evaluation of archival description interfaces, employing a focus group, in order to identify user preferences about the content and format displayed in archival access systems, including four displays modelled on real-life archival systems, and two that were specially constructed for the project. ${ }^{21}$ Daniels and Yakel studied the success rates of different search strategies through a series of information retrieval experiments using two archival databases. ${ }^{22}$ Scheir performed a study of nonhistorian, non-academic novice users' interaction with finding aids online in order to assess how certain features of the finding aids - terminology, navigation, display and structure - facilitated or constrained their search performance. ${ }^{23}$ The usability of an authentic EAD interface was tested by Yakel using a controlled laboratory experiment. ${ }^{24}$ An entirely artificial setting was constructed for the LEADERS Project in order to test the functionality of the application with the help of user feedback. ${ }^{25}$ Bringing this a step further is research that is studying how archival description and traditional finding aids could be enhanced and complemented through additional search options. Fachry et al. sought to evaluate EAD finding aids, examining whether direct access to archival material linked to the relevant

20 Lytle, "Intellectual access to Archives," 191-208.

21 Wendy M. Duff and Penka Stoyanova, "Transforming the Crazy Quilt: Archival Displays from a Users Point of View," Archivaria 45 (1998): 44-79.

22 Morgan G. Daniels and Elizabeth Yakel, "Seek and You May Find: Successful Search in Online Finding Aid Systems," American Archivist 73 (2010): 535-568.

23 Wendy Scheir, "First Entry: Report on a Qualitative Exploratory Study of Novice User Experience with Online Finding Aids," Journal of Archival Organization 3 (2005): 49-85.

24 Elizabeth Yakel, "Encoded Archival Description: Are Finding Aids Boundary Spanners or Barriers for Users?” Journal of Archival Organization 2 (2004): 63-77.

25 Anna Sexton, Geoffrey Yeo, Chris Turner and Susan Hockey, "User Feedback: Testing the LEADERS Demonstrator Application," Journal of the Society of Archivists 25 (2004): 189-208. 
descriptive information within digital finding aids was deemed more useful when compared to using a system that provided access to the whole fonds; if the use of structural presentation can improve users' satisfaction in locating relevant information, or help them in deciding if a collection is relevant; if individual items can be used as entry points for accessing an archival description; and if so, if it affects the users' satisfaction in accessing relevant information. ${ }^{26}$ Zhang and Kamps evaluated the use of web transaction logs as a means to study user behaviour and evaluation of retrieval systems. ${ }^{27} \mathrm{~A}$ further development of Internet dissemination of finding aids is to promote user interaction, for instance through social media, such as wikis, commenting systems, book-marking and social tagging. The Next Generation Finding Aid Project at the University of Michigan, for example, inspired by human-computer interaction (HCI) research, aimed to study whether social navigation tools could enhance access to archival materials. ${ }^{28}$

Another, partly overlapping strand of research has aimed to analyse search strategies and user behaviour of different categories of users, primarily those with various degrees of experience. Prom conducted an experimental study of interaction with electronic finding aids in a controlled setting, testing the hypothesis that expert users and novices undertook different search strategies and achieved different results. ${ }^{29}$ Chapman conducted a smaller usability test of the online finding of an historical collection. ${ }^{30}$ The purpose of the study was to gain a better understanding of how different user categories - novices and experts - interact with online finding aids, and to evaluate to which extent the properties of the finding aids contribute to search performance. Ethnographic studies might provide deeper insights into such aspects as users' experiences, perceptions and motivations and in her dissertation, Sweeney addressed the experiences of first-time archival

26 Khairun N. Fachry, Jaap Kamps and Junte Zhang, "Access to Archival Material in Context." II XX '08 Proceedings of the Second International Symposium on Information Interaction in Context 2008, pp. 102-109.

27 Junte Zhang, and Jaap Kamps, "Search Log Analysis of User Stereotypes, Information Seeking Behavior, and Contextual Evaluation." IIiX'10 Proceeding of the Third Symposium on Information Interaction in Context, August 18-21, 2010, New Brunswick, New Jersey, USA, pp. 245-254.

28 Magia G. Krause and Elizabeth Yakel, "Interaction in Virtual Archives: The Polar Bear Expedition Digital Collections Next Generation Finding Aid," American Archivist 70 (2007): 282-314.

29 Christopher J. Prom, "User Interactions with Electronic Finding Aids in a Controlled Setting," Journal of Archival Organization 7 (2004): 234-268.

30 Joyce C. Chapman, "Observing Users: An Empirical Analysis of User Interaction with Online Finding Aids," Journal of Archival Organization 8 (2010): 4-30. 
researchers when seeking archival sources. ${ }^{31}$ This included their interaction with finding aids, which appeared to be an obstacle in the research process. The above-mentioned LEADERS Project also included a comparison between different user categories concerning search behaviour, performance and opinions of the application.

The result of this overview shows that even if the sample is small, research about users' interaction with artefactual intermediaries could be performed in various ways, using different designs and different sources. An analysis of the different approaches follows below.

\section{Methodological Approaches}

\section{a. General Research Designs}

Social science research embraces a plethora of methods. Archival science research has been informed by several other disciplines and, as a demarcated discipline, can be said to represent methodological pluralism. ${ }^{32}$ Even the small sample analysed in the present study shows a significant variety. Depending on considerations such as the research problem, the epistemological standpoints and the maturity of the research field, different research designs are suitable. A research design has been defined as the "logical sequence that connects the empirical data to a study's initial research questions and, ultimately, to its conclusions. ${ }^{33}$ Simply put, it is a plan that guides the research process. Even if research designs differ considerably from each other, they usually involve the formulation of research questions, the selection of relevant data, the selection of data collection techniques and methods for the analysis of data. ${ }^{34}$ The overall designs used when studying user-intermediary interaction in archival science research can be roughly divided into two approaches: experimental or experimental-like research and non-experimental field research, with experimental studies predominating over the past decade (see Table 19.1).

31 Shelley. T. Sweeney, "The Source-Seeking Cognitive Processes and Behavior of the InPerson Archival Researcher.” Ph.D. diss. University of Texas, Austin, 2002.

32 McKemmish and Gilliland, "Archival and Recordkeeping Research: Past, Present and Future," 79-112.

33 Robert. K. Yin, Case Study Research, Design and Methods. 4 ed., Vol. 5, London: SAGE, 2009.

34 Kirsty Williamson, Frada Burstein and Sue McKemmish, "Introduction to Research in Relation to Professional Practice," in Research Methods for Students, Academics and Professionals. Information Management and Systems, Kirsty Williamson, ed. (Wagga Wagga: Centre for Information Studies, Charles Sturt University, 2002), pp. 5-13. 
Table 19.1. Research designs

\begin{tabular}{|l|l|}
\hline Experimental research & Non-experimental research \\
\hline Lytle & Krause \& Yakel $^{*}$ \\
\hline Prom & Duff \& Stoyanova* \\
\hline Sexton et al & Sweeney \\
\hline Yakel & Zhang \& Kamps \\
\hline Scheir & \\
\hline Fachry et al & \\
\hline Chapman & \\
\hline Daniels \& Yakel & \\
\hline
\end{tabular}

* These studies can be regarded as hybrid designs. They are experimental in the sense that the settings are constructed for testing and evaluation, but use qualitative designs for data collection and analysis.

Experimental research is supposed to be undertaken in order to establish a relationship between two or more variables, especially cause-effect relationships, i.e. what impact does one phenomenon (the independent variable) have upon another phenomenon (the dependent variable). A formal experiment or "true experiment" is based on the statistical testing of hypotheses, that is an assumption about a particular case deductively derived from general principles. ${ }^{35}$ The testing is accomplished by the comparison between two groups of randomly assigned subjects, of which one is exposed to the phenomenon in question, and another, the control group, is not. An alternative to control groups is to expose the experimental group to several instances of testing under different conditions, where one is considered as the baseline to which the others are compared. ${ }^{36}$ Experimental research design can be said to rely on a classic "scientific" methodology, in the sense that it originated within the natural sciences and with a positivist approach. Experimental research thus usually deals with quantitative data and methods of analysis. The fundamental idea behind experimental research is that the researcher creates a closed system, where the independent variable can be isolated, controlled and manipulated so that its impact on the dependent variable can be measured. These conditions are best met in a laboratory setting,

35 E.g., Kerry Tanner, "Experimental Research Design," in Research Methods for Students, Academics and Professionals. Information Management and Systems, Kirsty Williamson, ed. (Wagga Wagga: Centre for Information Studies, Charles Sturt University, 2002), pp. 125-146.

36 E.g., Tanner, "Experimental Research Design,” 125-146. 
with artificially created interactions between the subjects and the conditions to which they are exposed. In environments outside laboratories it is much more difficult to isolate the variables and avoid the impact of confounding and sometimes unknown variables. A limitation of laboratory experiments, however, is that generalising the results and drawing more far-reaching conclusions may be difficult. Human activity and behaviour are complex phenomena, and isolated actions performed out of context may give little advice about real-life settings. Experimental studies in real-life settings (field experiments) are therefore not unusual in social science. According to Lytle “... many laboratory experiments are so far removed from real information systems that what can be concluded has no practical value." ${ }^{37}$ Nevertheless, some researchers consider that naturalistic experiments involving human behaviour and relationships also are difficult to evaluate, since there are too many uncontrollable and unknown variables that might interfere. ${ }^{38}$

Most of the experimental studies discussed above follow a standard procedure: an initial questionnaire to capture participants' demographics; the actual experiment, often performed as a retrieval test; and, a final interview or questionnaire to sum up the participants' more qualitative opinions. The most salient feature of studies, however, is that none actually qualifies as a true experiment. The closest approach is presented by Prom, who is the only one that tests a formal hypothesis. ${ }^{39}$ The others studies are guided by a principal research question, but no explicit hypothesis. Prom conducted an experimental study in a controlled setting to test the hypothesis that expert users and novices undertake different search strategies and achieve different results. Comparisons were made between subjects who were computer experts, archival experts, and novices. In all, 89 subjects participated, recruited by means of flyers put up at a university campus, listservs for students at the university's history and library science departments, and a listserv for professional practitioners (in order to reach expert users). Demographic data and the subjects' previous experiences were collected from subjects through a questionnaire, and the respondents were then categorised as experts or novices. The experiment was performed as a controlled test that included a set of nine tasks where the subjects were using nine different web interfaces to find collections and folders containing records within the collections. The results and time to complete the tasks

37 Lytle, "Intellectual Access to Archives," 192.

38 Alison J. Pickard, Research Methods in Information. London: Facet, 2007.

39 Prom, "User Interactions," 234-268. 
were automatically recorded in a database and analysed quantitatively. Thirty-five participants performed the test on-site under observation, the rest off-site. During the observations, search patterns, key strokes and mouse movements were manually recorded and coded according to the search strategy being employed, and entered into the database. Reactions and comments were also noted, and short interviews were performed after the sessions. As a substitute for these data, however limited, off-site participants were provided with the opportunity to make comments on each task during their performance. The study thus included both quantitative and qualitative data. For each task, descriptive statistics were calculated and an analysis of variance was undertaken to compare the groups, i.e. testing the actual hypothesis. However, his study still lacks some of the fundamental elements of a true experiment, namely randomly assigned participants and control groups. In all of the studies, the participants consist either of a purposive sample, or voluntarily admitted subjects from a limited population. In general, there is a bias towards students and academics among the subjects, which more or less means that the studied populations are not representative samples of the general public or even of users of records in general. The generalisability of the results could thus be questioned. In several studies the samples are also very small, which makes a quantitative analysis meaningless.

The concept of non-experimental field research is here basically used as a generic term for research that aims to gather primary data in natural settings. ${ }^{40}$ This includes naturalistic studies where the researcher observes and records a phenomenon in its natural context during a period of time, while interfering as little as possible, but also other kinds of research purposing to study authentic behaviour in a real-life social context irrespective of the methods the researchers use to obtain knowledge. A range of different designs can be used, including surveys, case studies, interview studies and ethnographic studies. In the overview above, interview studies can be identified, as well as ethnographic observations and analysis of documentation generated in authentic search and retrieval activities. The common denominator is to capture real-world experiences, phenomena and behaviour in a natural setting, and to reach a deeper understanding of a particular problem. The most salient example is Sweeney's ethnographic study of users' experiences of archival settings. The research design combined a case study approach and grounded theory, a methodological approach involving the generation of theory by the collection and analysis of data (i.e., grounded theory starts 
with data, not with a theory or hypothesis that is applied to empirical data.) A "multi-method, multi-site exploratory examination"41 was employed. The sites consisted of three separate repositories: an academic archives, a government archives, and a private archives. A purposive selection of first-time visitors was undertaken, resulting in fourteen research subjects. Each individual subject was observed during one research episode. The data collection was performed by means of a brief introductory interview, non-participant observation including note-taking with a detailed contextual description and audio taping of reference interviews, and tape recordings of subjects' interaction with finding aids using think-aloud protocols. In the think-aloud component, the informants were asked to perform a search task and to describe what they were doing and thinking, while their comments and actions were observed and recorded. The interviews were mostly unstructured, and they evolved during the research period. The data collection also included a brief exit interview and videos of the physical premises.

Research designs did consequently vary from laboratory-like experimental studies to comprehensive ethnographical studies.

\section{b. Data, Data Collection Techniques and Data Analysis}

An important part of a research design is to identify the data sources for the intended study, that is to decide what kind of data will provide answers to the research questions, and to select data collection techniques and methods of analysis accordingly. These decisions are closely interrelated and interdependent. At the same time, they are each distinct components of the research process and the combination of data sources, data collection and analysis can vary. Nevertheless, the research process has a certain inherent logic due to the underlying ontological and epistemological premises, which put some restrictions on the choices a researcher might make.

The social sciences traditionally distinguish between quantitative and qualitative methods, which originally stem from different philosophical paradigms with different ontological and epistemological foundation positivism and interpretivism. ${ }^{42}$ However, it is a simplified view to regard all quantitative research as positivist and all qualitative research as interpretivist. First, the paradigms as such are not monolithic, but wide-ranging ideational constructs encompassing various schools of thought and even inherent contradictions; second, research can be related to other paradigms, such as

41 Sweeney, "The Source-Seeking Cognitive Processes," 41.

42 E.g. Pickard, Research Methods in Information. 
critical realism and pragmatism, with a more pluralistic view on appropriate research methods. From such a perspective both qualitative and quantitative methods could be justified; the choice is dependent on the research problem, and a combination of qualitative and quantitative methodologies are feasible and sometimes even desirable in order to elucidate a problem thoroughly. To explicate complex phenomena, multifaceted approaches are considered to be fruitful. ${ }^{43}$ The line between the two methodological approaches can also be somewhat blurred. A pure qualitative study is based on qualitative data and analysed or interpreted with qualitative methods. A pure quantitative study is based on quantitative data and analysed by statistical methods. However, combinations between the two are possible, as several of the studies discussed above illustrate (see Table 19.2).

\section{Table 19.2. Data and data analysis}

\begin{tabular}{|l|l|l|}
\hline Method of analysis & Qualitative & Quantitative \\
\cline { 1 - 2 } Data & $\begin{array}{l}\text { Scheir, Duff \& Stoyanova, } \\
\text { Sweeney, Yakel, Krause \& Yakel }\end{array}$ & $\begin{array}{l}\text { Sexton et al, Fachry et al, Chapman, } \\
\text { Daniels \& Yakel, Krause \& Yakel, } \\
\text { Prom }\end{array}$ \\
\hline Quantitative & - & Lytle, Prom, Zhang \& Kamps \\
\hline
\end{tabular}

This overview does not provide any particular examples, but quantitative data are also possible to interpret qualitatively. Even if the data is discreet and measurable, the purpose of the research could be to identify patterns or themes, rather than to show representativeness, or establish causality and correlations. The combination of data and methods of analysis has, however, certain limits. Qualitative data can, for instance, only be statistically processed to a certain degree. ${ }^{44}$

As table 19.2 shows, it is also possible to combine different kinds of data and different kinds of analyses in one study, for instance in Krause \& Yakel's study of the Next Generation Finding Aid Project at the University of Michigan. ${ }^{45}$ As part of the project, an experimental website was launched, where the digitised collections from the U.S. military intervention in northern

43 Catherine Marshall and Gretchen B. Rossman, Designing Qualitative Research. 2 ed. Thousand Oaks CA: Sage Publications, 1995.

44 E.g., Liwen Vaughan, Statistical Methods for the Information Professional: A Practical, Painless Approach to Understanding, Using, and Interpreting Statistics. Medford, NJ: Information Today, 2001.

45 Krause and Yakel, "Interaction in Virtual Archives," 282-314. 
Russia at the end of World War I were accessible through EADbased finding aids combined with structured entry points and free-text search. The site also included several interactive functions such as bookmarking, comments, link paths and the ability to add user profiles. Data were collected through web transaction logs; a survey questionnaire posted on the website concerning the users' assessment of the features of the site, and about their use of the collections; a semi-structured interview with three of the respondents concerning accessibility, expectations, features and interaction with other users; and, an analysis of user comments on the website. The web logs were analysed and described as frequency distributions, and the survey data (from only six respondents, however) was analysed both quantitatively and qualitatively. The interviews were transcribed and coded inductively along with the user comments according to the themes that emerged.

The studies presented above used distinctively different types of data. Several of the studies were performed as retrieval experiments where the participants were supposed to solve sets of pre-defined tasks. However, the task performance set-ups generated several kinds of data: query matches, transaction logs, recordings of search patterns, written statements on the task performance, and interview data. These kinds of data were also used in the non-experimental studies. As the researchers have used various data sources, it follows that they also have used different data collection techniques: questionnaires, interviews, extraction of transaction logs from the internet and retrieval systems, observations, and gathering written statements from the participating subjects. Two of the studies above claimed to use survey techniques, but they were actually referring to questionnaires, i.e. a form designed to gather data. ${ }^{46}$ Surveys are often performed with the help of questionnaires, but the concepts are not synonymous. Surveys are rather a research design than a data collection technique, comprising the gathering of primary data for quantitative analysis. Questionnaires are instruments that are used in several of the studies, either to gather demographic data about the participants or to capture their opinions, experiences and so forth. Fachry et al. used questionnaires as the primary data collection technique, while others primarily used them as a complement to other forms of data collection.

Another common method according to the research overview above is interviews. Often a semi-structured interview protocol was used. A distinction can be made between standardised and structured interviews: a 
standardised interview is one where the interview questions are the same and are articulated the same way in all interviews, while a structured interview means that it has a thematic disposition. ${ }^{47}$ In both cases the questions can be open or closed-ended, but a standardised interview does not allow for individual follow-up questions or refinement of the questions during the research process. According to this distinction, the interview protocols used in these studies were structured, but not standardised. Another approach to interviews is the use of focus groups. Such use is appropriate when a group rather than individual behaviour is the object of study, and when actions and motivations are of interest. ${ }^{48}$ Focus groups were employed in the LEADERS Project and by Duff and Stoyanova. ${ }^{49}$

Observation is yet another technique used in the studies. Wang identifies two basic methods of observations in studying information behaviour: field observation (non-participant) and participant observation. ${ }^{50}$ The first is a common method of studying human behaviour in real-life situations, applying a non-interventionist strategy without intruding on the observed situation. This research strategy has been employed by Sweeney, making field notes and audio taping reference interviews. ${ }^{51}$ However, if a researcher's presence is apparent in some way, it could be argued that he or she might influence the behaviour of the subjects. The other method requires the researcher's active participation, for instance by commenting or asking questions during the period of observation, or at least by the researcher's close presence. Such observations could be performed either in an experimental set-up or when studying the subjects' "natural" behaviour in an authentic situation. This interventionist strategy has also been undertaken by Sweeney as part of her "multi-method, multi-site exploratory examination", and by Daniels and Yakel. In both cases a so-called "think-aloud" protocol was used.

Of the data used in the studies, query matches and transaction logs could be defined as pure quantitative data. The studies using this kind of data applied ordinary statistical methods of analysis like frequency distribution, measures of tendency and variability, and correlations. The studies in the

47 Jan Trost, Kvalitativa intervjuer. Lund: Studentlitteratur, 1997.

48 Victoria Wibeck, Fokusgrupper: Om fokuserade gruppintervjuer som undersökningsmetod. Lund: Studentlitteratur 2000.

49 Duff and Stoyanova, "Transforming the Crazy Quilt."

50 Peiling Wang, "Methodologies and Methods for User Behavioral Research," Annual Review of Information Science and Technology (ARIST) 34 (1999): 53-99.

51 Sweeney, "The Source-Seeking Cognitive Processes." 
overview that could be considered as "pure" qualitative studies, using both qualitative data and qualitative methods of analysis, followed consistent, established techniques of analysing qualitative data: transcription and datadriven coding into categories, or applying pre-defined categories in the coding process.

\section{Discussion}

As has already been discussed, the studies in this overview can be roughly divided into experimental research and non-experimental field study research, with the former being the predominant approach. However, the experimental research could rather be said to use quasi-experimental research designs instead of true experimental designs. A field where true experiments can be an option is information retrieval, where large amounts of data can be obtained from stable systems. Quasi-experimental research designs share some of the elements of experimental research, but not all of them. Quasi-experiments usually lack random assignments and some of the control instances required in true experimental research. Quasi-experiments can establish relationships between phenomena, but cannot establish causality since there are too many uncontrollable variables that might intervene. This should not be regarded as that quasi-experimental research is "bad" or trivial, but rather that it cannot conclude that a fact is ultimately proven (if anything is said to be actually proven - this becomes a question of epistemological concern). Carefully designed quasi-experimental research can generate valuable knowledge about certain phenomena that otherwise cannot be obtained, and it can give plausible indications of a possible finding. As Tanner has noted, "In field research [ ... ] were it is not possible to use a true experimental design, a quasi-experiment is an option which does permit some hints of causality to be made - but not conclusively proven." ${ }^{52}$ Some of the studies, however, cannot even be defined as quasi-experimental, but rather as pre-experimental research. They include some kind of testing, but lack experimental and control conditions as well as randomisation. The samples are very small, and no meaningful comparisons can be made. This means that no conclusive results can be drawn and that the explanatory value is weak since there are many potential causes behind any outcome.

Non-experimental research makes up a small, but heterogeneous collection of studies, discernible in the present chapter as a negation to ex-

52 Kerry Tanner, "Experimental Research Design,” 125-146. 
perimental studies, including both quantitative and qualitative data and methods of analysis. The small number, however, gives reason to consider those as a single category even if it to some extent is out of convenience. The fact that there is little research done in this area is in itself an argument for field research. There is a need for exploratory studies and identification of analytical concepts that could be further tested, iterated and built-upon. Experimental studies require a linear design, where analytical categories are clearly defined and the study is guided by a hypothesis or at least a general idea that can be tested. This means that they are less suited for exploratory or tentative research, which is how most of the studies above could be defined.

Both quantitative and qualitative data and methods of analysis are thus to be found among the studies, sometimes in combination. What can be questioned is sample size. In Lytle's quantitative study, the small sample undermined the reliability and generalisability of the results. The same applies to some of the studies that used qualitative data, but to some extent analysed them quantitatively (at least by providing some descriptive statistics). The question is what can be concluded from such metrics other than the identification of categories and patterns, which probably could be described without providing exact figures that might give a false impression of exactitude and reliability. The $\mathrm{chi}^{2}$ and ANOVA tests are analytical instruments that have been used in several of the studies to establish correlations and make comparisons between qualitative variables, but those tests require a rather comprehensive sample to draw conclusions with any certainty. Yakel has argued that it is customary in usability testing to rely on small samples and still consider the results meaningful. ${ }^{53}$ However, when used as a research method this may not be enough to fulfil research standards. Even if the aim is to establish patterns and possibilities, rather than to explain, predict or prove relationships between variables, it might be valuable to build on a larger sample or to replicate the studies to help identify those patterns.

The overview provided above demonstrates how the same basic research designs can employ multiple data sources and thus methods of gathering data, and that different designs can also employ the same type of data. The selection of methodological approach, data and methods of analysis is, however, not random. Irrespective of the paradigmatic point of view, it is necessary to base research on a sound methodological framework. The ontological and

53 Yakel, “Encoded Archival Description,” 63-77. 
epistemological underpinning defines which phenomena are relevant or even possible to study, and how knowledge about them could be obtained (if at all). In other words, it frames the potential research questions and lays the foundation for the methodological approaches and the research design. If the philosophical standpoint for instance is that objective knowledge does not exist, it is pointless to measure and prove something statistically. At the other extreme, if the standpoint is that an objective reality exists and that the intention of research is to gain objective and universal knowledge about real-world phenomena, interpretivist approaches and subjective conceptions are irrelevant. With the possible exception of Sweeney, whose work is a doctoral thesis where a more elaborated methodological discussion can be expected, very little is said in most of the studies about the epistemological premises for the research, the choice of research design, or the robustness of the results. The underlying ontological and epistemological conceptions and their connection to methodological approach can, however, be implicit and made clear through a consistent research design. This is often the case with journal publications, where there is little room for more comprehensive methodological reasoning. However, the absence can also be a sign of lack of awareness, or, worse, sloppy research evidenced by an eclectic and inconsistent mode of procedure.

None of the studies discussed here can be considered to use a particular archival method or theoretical standpoint. They rely on standard social science methods, but also borrow from two particular fields of research: human-computer interaction (HCI), of which usability studies can be considered as a sub-field, and information retrieval (IR). Again with the exception of Sweeney, who is a somewhat of an outlier in this overview and is using ethnographic methods, all studies could be said to have employed forms of either usability testing or retrieval experiments, even if they are not always referred to as such. Those that explicitly refer to HCI/usability testing are Yakel, Krause and Yakel, Fachry et al, and Chapman, while Lytle, Fachry et al and Daniels and Yakel employ an IR framework. Archival science research relating to user interaction with artefactual intermediaries can thus be said to approach information systems and library and information sciences, and draw upon methods developed in those disciplines.

What then is the motivation behind this kind of research? As well as demonstrating diversity in methodological issues, the studies exhibit diversity in aims. The purposes of undertaking research about users' interaction with finding aids and similar intermediaries are to study user behaviour, to evaluate 
search strategies, or to assess the performance and usability of an artefactual intermediary. A fourth purpose can be identified in Zhang and Kamps' study - to evaluate a research method - but the authors of this study also studied user behaviour. The focus can either be on humans, i.e. the users and their behaviour, or on the artefacts, i.e. the finding aids and access systems, but these themes are not clearly-defined, discreet categories, but are overlapping and to some extent dependent areas. Search behaviour, for instance, depends upon the capabilities of the systems being examined, and system functionality and the efficiency of search strategies is dependent upon the intended target groups. The emphasis is put on one or more specific aspects, systems or human behaviour, but the intended outcome is basically the same: to contribute to the development of more appropriate services or devices in order to enhance access to archival materials. This kind of research has thus a rather instrumental or pragmatic approach, even when it is of an exploratory character.

\section{Concluding Remarks}

The purpose of this chapter was to explore research relating to users' interaction with artefactual intermediaries. The aims of the studies discussed diverge, but they still range in the same direction. The ultimate objective seem to be to gain knowledge about how users approach archival materials with different aids, and the results are presumed to contribute to enhanced access. This may be the most evident demarcation of the research field. A deficiency, however, is that most of the subjects partaking in the studies in this overview fit a rather narrow profile. To what extent the results could be transferable to other environments and other potential user categories, or contribute to outreach to users other than those who currently use archival materials is uncertain.

Overall, the present study indicates that even if this might appear to be a narrow and clearly-defined field of study, it is in fact neither uniform nor unambiguous. A quasi-experimental research design is most common, but the set-up, the data, the data collection techniques, and the methods of analysis are diverse. This might seem to be a paradox since the research field is so small, but the sprawling result is doubtless a consequence of the fact that the research field is immature and still seeking its form. To draw upon Thomas Kuhn's framework, the field is still in its pre-paradigmatic phase. ${ }^{54}$

54 Thomas S. Kuhn, The Structure of Scientific Revolutions. Chicago: University of Chicago Press, 1962. 
While this might reflect the state of archival science in general, which can be considered as multi-methodological, multi-disciplinary and so forth, this is not the subject of this discussion. Diversity should furthermore not be discarded as an inferior state. On the contrary, it can open up more pluralistic avenues of research and contribute to new and unexpected knowledge. What can be questioned is the quality and methodological soundness of some of the studies, even those that are published in peer-reviewed journals. With a few exceptions, most take the form of small, tentative or exploratory studies, yet the limits and methodological implications of this are not always recognised. A better understanding of the characteristics of data and the methods of analysis and what problems they can address - indispensable elements of a research design - is required. A further issue to explore is whether there is a need for research methods to be devised that are specific to archival science. In the area subject to the present study, the appropriation of HCI and IR methods seems fruitful, but the characteristics of archival science and its methodological implications need to be better understood. Increased methodological awareness within the archival science research community is, therefore, a necessity, as is increased discussion of the implications for research design of different ontological and epistemological standpoints.

\section{References}

Anderson, Ian G. "Are You Being Served? Historians and the Search for Primary Sources." Archivaria, 58 (2004): 81-129.

Beattie, Diane L. “An Archival User Study: Researchers in the Field of Women's History.” Archivaria 29 (1989/1990): 33-55.

Chapman, Joyce C. "Observing Users: An Empirical Analysis of User Interaction with Online Finding Aids.” Journal of Archival Organization 8 (2010): 4-30.

Conway, Paul. "Facts and Frameworks: An Approach to Studying the Users of Archives." The American Archivist 49 (1986): 393-407.

Conway, Paul. Partners in Research. Improving Access to the Nation's Archive. User Studies at the National Archives and Records Administration. Pittsburgh: Archives \& Museum Informatics, 1994.

Conway, Paul. "Research in Presidential Libraries: A User Survey.” Midwestern Archivist 11 (1986): 35-56.

Daniels, Morgan G. and Elizabeth Yakel. "Seek and You May Find: Successful Search In Online Finding Aid Systems." American Archivist 73 (2010): 535-568.

Duff, Wendy M. "Understanding the Information-seeking Behaviour of Archival Researchers in a Digital Age: Paths, Processes and Preferences." Proceedings of the DLM-Forum: @ccess and Preservation of Electronic Information, Best Practices and Solutions, Barcelona, 6-8 May 2002, 331-339.

Duff, Wendy M., Barbara Craig, and Joan Cherry, "Finding and Using Archival Resources: A Cross-Canada Survey of Historians Studying Canadian History." Archivaria 58 (2004): 51-80. 


\section{Chapter i9}

Duff, Wendy M. and Catherine A. Johnson, "Accidentally Found on Purpose: Information-Seeking Behavior of Historians in Archives.” Library Quarterly 72 (2002): 472-496.

Duff, Wendy M. and Catherine A. Johnson, "Where is the List with All the Names? Information-Seeking Behavior of Genealogists." American Archivist 61 (2003): 79-95.

Duff, Wendy M. and Penka Stoyanova. "Transforming the Crazy Quilt: Archival Displays from a Users Point of View." Archivaria 45 (1998): 44-79.

Fachry, Khairun N., Jaap Kamps and Junte Zhang, "Access to Archival Material in Context." II $X X$ '08 Proceedings of the Second International Symposium on Information Interaction in Context, 2008, pp. 102-109.

Goggin, Jacqueline. "The Indirect Approach: A Study of Scholarly Users of Black and Women's Organizational Records in the Library of Congress Manuscript Division.” Midwestern Archivist 11 (1986): 57-67.

Harris, Carolyn. "Archives Users in the Digital Era: A Review of Current Research Trends." Dalhousie Journal of Information E Management 1 (2005), http://hdl.handle. net/10222/13360.

Hedstrom, Margaret. "Archives, Memory, and Interfaces with the Past." Archival Science 2 (2002): 21-43.

Krause, Magia G. and Elizabeth Yakel, "Interaction in Virtual Archives: The Polar Bear Expedition Digital Collections Next Generation Finding Aid.” American Archivist 70 (2007): 282-314.

Kuhn, Thomas S. The Structure of Scientific Revolutions. Chicago: University of Chicago Press, 1962.

Maher, William J. "The Use of User Studies and Archival Mission and User Studies.” The Midwestern Archivist 11 no. 1 (1986): 15-26.

McKemmish, Sue and Anne Gilliland, "Archival and Recordkeeping Research: Past, Present and Future." In Research Methods: Information, Systems, and Contexts, Kirsty Williamson and Graeme Johanson, eds. Prahran, Victoria: Tilde Publishing and distribution, 2013, pp. 79-112.

Meho, Lokman I. and Helen R. Tibbo. "Modeling the Information-seeking Behavior of Social Scientists: Ellis's Study Revisited." Journal of the American Society for Information Science and Technology 54 (2003): 570-587.

Principe, Lucia S. "Everyman and Archives." Archivum 29 (1982): 135-141.

Prom, Christopher J. "User Interactions with Electronic Finding Aids in a Controlled Setting." Journal of Archival Organization 7 (2004): 234-268.

Roper, Michael. "The Academic Use of Archives." Archivum 29 (1982): 27-45.

Scheir, Wendy. "First Entry: Report on a Qualitative Exploratory Study of Novice User Experience with Online Finding Aids." Journal of Archival Organization 3 (2005): 49-85.

Sexton, Anna, Chris Turner, Geoffrey Yeo and Susan Hockey, "Understanding Users: A Prerequisite for Developing New Technologies." Journal of the Society of Archivists 25 (2004): 33-49.

Sexton, Anne, Geoffrey Yeo, Chris Turner and Susan Hockey, "User Feedback: Testing the LEADERS Demonstrator Application." Journal of the Society of Archivists 25 (2004): 189-208.

Siatri, Rania. “The Evolution of User Studies.” Libri 49 (1999): 132-141.

Stevens, Michael. “The Historian and Archival Finding Aids.” Georgia Archive 5 (1977): 64-74.

Sundqvist, Anneli. "Search Processes, User Behaviour and Archival Representational Systems.” Ph.D. diss., Mid Sweden University, 2009. 
Sundqvist, Anneli. "The Use of Records - A Literature Review." Archives E Social Studies 1 (2007): 623-653.

Sweeney, Shelley. T. "The Source-Seeking Cognitive Processes and Behavior of the InPerson Archival Researcher." Ph.D. diss. University of Texas, Austin, 2002.

Tanner, Kerry. “Experimental Research Design.” In Research Methods for Students, Academics and Professionals. Information Management and Systems, Kirsty Williamson, ed.

(Wagga Wagga: Centre for Information Studies, Charles Sturt University, 2002), pp. 125-146.

Tibbo, Helen R. "Primarily History in America: How U.S. Historians Search for Primary Materials at the Dawn of the Digital Age." American Archivist, 66 (2003): 9-50.

Trost, Jan. Kvalitativa intervjuer. Lund: Studentlitteratur, 1997.

Turnbaugh, Roy C. "Archival Mission and User Studies." The Midwestern Archivist 11 no. 2 (1986): 15-34.

Vaughan, Liwen. Statistical Methods for the Information Professional: A Practical, Painless Approach to Understanding, Using, and Interpreting Statistics. Medford, NJ: Information Today, 2001.

Wang, Peiling. "Methodologies and Methods for User Behavioral Research." Annual Review of Information Science and Technology (ARIST) 34 (1999): 53-99.

Wibeck, Victoria. Fokusgrupper: Om fokuserade gruppintervjuer som undersökningsmetod. Lund: Studentlitteratur 2000.

Williamson, Kirsty, Frada Burstein and Sue McKemmish, "Introduction to Research in Relation to Professional Practice." In Research Methods for Students, Academics and Professionals. Information Management and Systems, Kirsty Williamson, ed. (Wagga Wagga: Centre for Information Studies, Charles Sturt University, 2002), pp. 5-13.

Yakel, Elizabeth. "Archival Representation." Archival Science 3 (2003): 1-25.

Yakel, Elizabeth. "Encoded Archival Description: Are Finding Aids Boundary Spanners or Barriers for Users?” Journal of Archival Organization 2 (2004): 63-77.

Yakel, Elizabeth. "Listening to Users." Archival Issues 26 (2002): 111-127.

Yakel, Elizabeth and Laura L. Bost, "Understanding Administrative Use and Users in University Archives." American Archivist 57 (1994): 596-615.

Yakel, Elizabeth and Deborah A. Torres, "AI: Archival Intelligence and User Expertise." American Archivist, 66 (2003): 51-78.

Yin, Robert. K. Case Study Research, Design and Methods. 4th ed., Vol. 5. London: SAGE, 2009.

Zhang, Junte and Jaap Kamps. "Search Log Analysis of User Stereotypes, Information Seeking Behavior, and Contextual Evaluation." II $X$ X'10 Proceeding of the Third Symposium on Information Interaction in Context, August 18-21, 2010, New Brunswick, New Jersey, USA, pp. 245-254. 
Chapter 20

\title{
ARCHIVAL IR
}

\section{Applying and Adapting Information Retrieval Approaches in Archives and Recordkeeping Research}

\author{
Jonathan Furner and Anne J. Gilliland
}

\begin{abstract}
In this chapter, the prospects for archival information retrieval (IR) as a research area within the archives and recordkeeping domain are reviewed with the aim of encouraging its application. IR is characterised as a body of techniques with wide applicability, but with relatively little influence, historically, on the design of systems offering intellectual access to archives and records. Significant terminological differences (and overlaps) are noted between the IR and data archiving fields, and archives and recordkeeping domains. The principal concepts and objectives of IR are summarised, and the trajectory of archival IR outlined, with a focus on myths, challenges, and recent developments. XML retrieval is identified as a primary locus for researchers in archival studies to participate in the design and development of the next generation of IR systems. It is suggested that potential advances in archival IR - such as helping users to find previously unknown and possibly "smoking gun"-type documents; establishing the meaningful absence (as opposed to the presence) of documents or their contents; and exploiting multiple types and sources of metadata - may find wider application in other domains such as litigation support systems, news retrieval, audiovisual archives, data mining, and digital asset management.
\end{abstract}




\section{Introduction}

Information retrieval (IR) is the name that has been used since the $1950 \mathrm{~s}$ to refer to an interdisciplinary field of inquiry that draws its methods from computer science, library and information science, linguistics, statistics, and psychology. ${ }^{1}$ Researchers in IR seek to improve our understanding of the ways in which people can find, among large quantities of resources that contain information (broadly defined), resources of the particular kinds that they want. Over the course of the past seven decades, the scope of the field has been extensively and variously delineated by researchers with wide-ranging interests, but definitions have frequently included, as objects of study, the beliefs, goals, values, intentions, actions, and products of the following groups:

- information seekers (a.k.a. searchers), i.e., those who are looking for information;

- IR systems designers, i.e., those who devise and build systems and services (manual or automated, analogue or digital, stand-alone or networked) that provide assistance to information seekers; and,

- intermediaries such as indexers, cataloguers, and processors, who preprocess information resources in such ways as to make them more accessible to seekers - for example, by identifying terms, headings, codes, or descriptors of some kind, to use as descriptive labels for resources (or for classes of resources), and by creating more- or less-complete representations of, or surrogates for, resources.

Often IR is conceived rather more narrowly as the art and science of producing and improving upon computerised retrieval systems (a.k.a. search engines) that help information seekers both to find more of the information that is wanted, and to avoid more of the information that is not. ${ }^{2}$ Some IR research is dedicated to the creation and/or implementation of such systems, or of particular system components such as user interfaces; ${ }^{3}$ other research involves the measurement and evaluation of the performance (a.k.a. retrieval

1 See, e.g., Christopher D. Manning, Prabhakar Raghavan, and Hinrich Schütze, Introduction to Information Retrieval (New York, NY: Cambridge University Press, 2008); Ricardo Baeza-Yates and Berthier Ribeiro-Neto, Modern Information Retrieval: The Concepts and Technology behind Search, 2nd ed. Harlow, UK: Addison-Wesley, 2011.

2 See, e.g., W. Bruce Croft, Donald Metzler, and Trevor Strohman, Search Engines: Information Retrieval in Practice (Boston, MA: Addison-Wesley, 2010).

3 See, e.g., Max L. Wilson, Search User Interface Design (San Rafael, CA: Morgan \& Claypool, 2012). 
effectiveness) of such systems; ${ }^{4}$ and yet other research constructs theory that seeks to explain why systems of one kind perform to a higher standard than those of another. ${ }^{5}$

IR techniques have been widely applied in diverse settings. At the time of writing, the world's most widely used IR system is the Web search engine Google. The Microsoft Windows and Apple Mac operating systems both incorporate search engines that allow computer users to find relevant resources in their own personal collections. In libraries both physical and digital, patrons use OPACs (online public access catalogues) to identify desired materials. Each of these kinds of automated IR system has a long and more-or-less illustrious history.

In contrast, IR techniques have not been so widely applied in the provision of access to records and archives. Prior to the 1980s and the widespread implementation of electronic recordkeeping, archivists in countries and sectors with strong registry traditions relied upon a centralised registry office or system that structured workflow and identified, classified, controlled, and sometimes eliminated records generated by bureaucratic activity prior to those records being received by the institutional archives. These registry systems thus provided the fundamental infrastructure for manual information retrieval for both active and archival records. In the absence of registry systems - for example in the United States where they were never widely adopted, or where archival resources resulted from personal activity - archivists relied upon their own knowledge of archival holdings, and of their associated filing schemes and finding aids, in order to meet users' expressed needs. As automated recordkeeping was increasingly implemented and hierarchical information flows and centralisation of recordkeeping activities were replaced by network structures, registry systems increasingly broke down, as did many other forms of systematised bureaucratic filing systems. A need arose, therefore, for records creators and archivists to implement robust IR mechanisms for the increasingly voluminous products of institutional recordkeeping. Commercial developers addressed this need by designing electronic records management (ERM) systems, electronic document management (EDM) systems, digital asset management (DAM) systems, and other forms of resource management systems for use within and across bureaucratic settings. Developments of this kind were accompanied by bursts of enthusiasm in the 1980s and 1990s for

4 See, e.g., Donna Harman, Information Retrieval Evaluation (San Rafael, CA: Morgan \& Claypool, 2011).

5 See, e.g., Thomas Roelleke, Information Retrieval Models: Foundations and Relationships (San Rafael, CA: Morgan \& Claypool, 2013). 
"archival informatics" in general, and for subject indexing of archival holdings in particular. ${ }^{6}$ However, the notion of or need for "archival IR" - i.e., the adoption and adaptation of IR concepts and techniques to address specific archival and recordkeeping needs and problems - remained substantively unaddressed within archival studies. ${ }^{7}$

Historically, when those in the archives and recordkeeping domain did talk about archival IR, one or more myths, or (at best) only partial truths - typically about what is "classic IR," and how it is not applicable in archival contexts were frequently perpetuated. A list of these might include the following:

- IR is all about the provision of access to information - whereas archives are all about the preservation of the products (e.g., records) of bureaucratic and personal activity as evidence of that activity.

- IR is a post hoc set of activities, conducted after resources have been acquired by a library or other repository - whereas long-term access considerations for records need to begin from the moment a recordkeeping system is being designed by or for a records creator, and to continue throughout the life of those records.

- IR is primarily about helping information seekers gain item-level access to resources - whereas archival processing is primarily about describing, explaining, and presenting the products of active and archived recordkeeping in context, in order to facilitate primary and secondary use, and re-use. That context comprises the various agents, activities, mandates and functions associated with those products and the relationships between them in and through time. ${ }^{8}$

6 David Bearman was probably the first to use the term "informatics" in an archival context. Bearman's consulting firm, Archives \& Museum Informatics, began publishing the Archival Informatics Nerwsletter in 1987; this journal was itself retitled Archives and Museum Informatics in 1989, and Archival Science in 2000. The principal catalyst for debates about the value of subject indexing in archival settings was Richard H. Lytle's doctoral dissertation of 1979, "Subject Retrieval in Archives: A Comparison of the Provenance and Content Indexing Methods" (University of Maryland).

7 We use the term "archival studies" here because it encompasses "the fullest range of archival practice, ideas, and research from multiple professional, community, and disciplinary perspectives"; see Archival Education and Research Institute (AERI) Pluralizing the Archival Curriculum Group (PACG), "Educating for the Archival Multiverse," American Archivist 74 no. 1 (2011): 72. See also Kelvin L. White and Anne J. Gilliland, "Promoting Reflexivity and Inclusivity in Archival Education, Research, and Practice," Library Quarterly 80 no. 3 (2010): 231-248.

8 See, e.g., Sue McKemmish, Glenda Acland, Nigel Ward, and Barbara Reed, "Describing Records in Context in the Continuum: The Australian Recordkeeping Metadata Schema," Archivaria 48 (Fall 1999): 3-37; and Dharma Akmon, Ann Zimmerman, 
- IR places high value on improvements in the quality of itemlevel subject indexing - whereas archivists focus on collection-level description to ensure that items are always retrieved in context, and eschew subject description and retrieval based on corporate, personal, or place names because of the high incidence of inconsistencies and historical and cultural variations in the choice and form of such names. At any rate, archivists could never costeffectively become involved in detailed, item-level subject indexing of their holdings, simply because of the magnitude of the manual effort apparently required.

- IR is good only for searchers whose information needs can be expressed as topical subjects - whereas archival holdings are typically described provenancially, usually by personal and organisational names that are also subject to historical and cultural variation, and that are notoriously inconsistently applied by records creators, archival processors, and end-users.

- IR relies on resource descriptions made up of statements of certain observable characteristics of bibliographic materials, such as title, author's name, and publication date, and hence is primarily the domain of libraries of such materials - whereas records and other archival materials usually lack such bibliographic characteristics. Moreover, archivists are wary about what they would have to "give up," "shoehorn," or add on to their descriptive processes in order to be able to take advantage of classic IR techniques.

- IR is primarily about making advances in the design of algorithms to be followed by machines - whereas archival retrieval invariably relies on the unique talents, specialised knowledge, and prodigious memories of the bumans who take care of archival holdings, especially those who have been closely engaged with processing and providing reference services to particular holdings.

- IR is good only for digital resources - whereas the majority of archival holdings are not yet in digital form, and some holdings may never be.

- IR is good only for textual resources - whereas archival holdings often include non-textual materials such as photographs and audiovisual recordings. 
Clearly, many of these claims about the lack of applicability of IR to archival settings are based upon outdated notions about contemporary IR techniques, as well as outmoded conceptualisations of archives and their descriptive practices today. Several are demonstrably being debunked not only by advances in contemporary recordkeeping systems, but also by broad-based archival developments. These latter include the following: mass digitisation of holdings; creation of metadata for each digitised item; generation of searchable full-text versions of digitised textual documents; creation of linked data; development of standards for the structure of authority files; construction and sharing of standardised authority files; and increasing reliance on full-text search engines to provide enhanced searching of online finding aids generated by "slimline" processing procedures such as More Product, Less Process (MPLP). ${ }^{9}$ The end result - networks of largescale online archives, implemented at intra- and inter-institutional levels and in centralised and federated forms, that are making available born-digital as well as digitised content, together with collection- and item-level metadata - present a compelling case for developing a robust agenda for archival IR research that will support and enhance use of these archives. ${ }^{10}$

In this chapter, through an exposition of classic IR ideas and approaches and contemplation of the conditions and needs of twenty-first century archives and recordkeeping, we argue that IR provides a key set of concepts and methods for those who seek to enhance archival access and use. Furthermore, because of its conceptual and temporal complexities, the archives and recordkeeping domain offers IR researchers opportunities to probe some of these complexities further, and provides a rich vein for nuanced development of the IR field as a whole. Our aim with this chapter, then, is to review both the actuality of, and the potential for, the application of IR

9 See, e.g., Lina Bountouri and Manolis Gergatsoulis, "The Semantic Mapping of Archival Metadata to the CIDOC CRM Ontology," Journal of Archival Organization 9 nos. 3-4 (2011): 174-207; Sally H. McCallum, "An Introduction to the Metadata Object Description Schema (MODS)," Library Hi-Tech 22 no. 1 (2004): 82-88; Michelle Mascaro, "Controlled Access Headings in EAD Finding Aids: Current Practices in Number of and Types of Headings Assigned," Journal of Archival Organization 9 nos.3-4 (2011): 208-225; Thomas J. Frusciano, "Online Finding Aids, Catalog Records, and Access - Revisited," Journal of Archival Organization 9 no. 1 (2011): 1-3; Jane Zhang, "Archival Representation in the Digital Age," Journal of Archival Organization 10 no. 1 (2012): 45-68; Shannon Bowen Maier, "MPLP and the Catalog Record as a Finding Aid," Journal of Archival Organization 9 no. 1 (2011): 32-44 ; and Mark A. Greene, "MPLP: It's Not Just for Processing Anymore," American Archivist 73 no. 1 (2010): 175-203.

10 Anne J. Gilliland, "Reconceptualizing Records, the Archive and Archival Roles and Requirements in a Networked Society," Knygotyra [Book Science] 63 (2014): 17-34. 
approaches in archival studies research - revisiting the above myths in the process, as appropriate. After a brief note about how potentially confusing terminological overlaps and differences between and within the IR field and the archives and recordkeeping domain might be addressed, we present a broad outline of the conceptual framework that we shall be using to situate archival IR, simultaneously in the field of IR as classically understood and in archival studies. We then review a selection of prior and recent approaches to archival IR, and speculate about the future prospects for archival IR, before drawing some final conclusions.

\section{A Note on Terminology}

That there has been almost no historical interaction between the archival and IR fields ${ }^{11}$ is immediately evident from the terminology used in each context. Some terms are used in both fields, but denote different concepts in each; some concepts are shared by both fields, but are denoted by different terms in each. Such conflict importantly reflects deeper conceptual differences between the roles, procedures, and points of engagement of those who have historically developed the IR field and those in the archives and recordkeeping domain (and, more recently, in the related field of data archiving). Unlike other fields associated with the "information sciences" whose scholars characteristically have looked to the early 20th-century documentation movement for inspiration, ${ }^{12}$ archival studies is concerned specifically with aligning records, their users, and their uses from the moment they are imagined in the design of a records (a.k.a. recordkeeping) system, and for as long as the resources generated by those systems continue to exist, whether in their original setting or after transfer to a physical or digital archives. As a result, IR considerations must begin at the point of the creation of the original records system, and must continuously be attuned to and aligned with the needs, behaviours, and practices of the various kinds of users who wish to access the records system and its content over time. In

11 In 1937, the National Archives was a founding member of the American Documentation Institute (ADI). The forerunner of today's Association for Information Science and Technology (ASIS\&T), ADI was concerned with the classification of, and access to, scientific and social scientific documentation. This involvement ceased when the archives was subsumed into the General Services Administration in 1941, and the archival field in the US did not continue to maintain any kind of close relationship with the information science field that was so instrumental in the development of IR.

12 See, e.g., Michael K. Buckland, "What is a 'Document'?" Journal of the American Society for Information Science 48 no. 9 (1998): 804-809. 
this respect, archives and recordkeeping as a domain has close ties to the emerging field of data curation, as well as to institutionally-based professions, such as librarianship and museum collections management, that still largely rely upon post hoc information processing to support information retrieval.

Much of the canonical IR terminology can be traced at least to the Cranfield tests - a series of influential experiments, conducted by the British librarian Cyril Cleverdon in the 1960s, in which the impact on retrieval effectiveness of several different methods of indexing was measured in a controlled setting ${ }^{13}$ - and, further back, to Calvin Mooers' first use of the term "information retrieval" in 1950. ${ }^{14}$ Meanwhile, archives and recordkeeping terminology has evolved according to the field's own historical and cultural trajectories over centuries, and arguably with rather less agreement than can be found in IR. However, certain archival terminology is now too embedded in national and international standards for archivists to contemplate change. For archival IR to gain traction, the meanings of terms must be clarified, distinguished, and mapped, so that confusion (both internal and external) may be avoided. This is certainly not a problem that is unique to this context, but rather is illustrative of the processes that have to occur when any method is adopted, adapted, and internalised within a new domain, and especially if it is hoped that outcomes will be fed back into the parent field.

The terminological difficulties that plague any discussion at the intersection of archival studies and information studies may be summarised as follows:

1. The term "information," notoriously, is used in a large number of different ways, to the extent that there is seldom much agreement on a preferred sense even within relatively small user groups, let alone across entire disciplines, professions, or national traditions.

(a) Some find it possible to distinguish objective senses of information-as-signifier (e.g., marks on a page) from subjective senses of information-as-signified (e.g., meanings ascribed to marks). (b) At the same time, some commonly see a benefit in distinguishing between information that has ultimate value (e.g.,

13 Cyril W. Cleverdon, "The Cranfield Tests on Index Language Devices," Aslib Proceedings 19 no. 6 (1967): 173-194; F. Wilfred Lancaster, Information Retrieval Systems: Characteristics, Testing, and Evaluation (New York: Wiley, 1968).

14 See, e.g., Calvin N. Mooers, "Coding, Information Retrieval, and the Rapid Selector," American Documentation 1 no. 4 (1950): 225-229; see also Mark Sanderson and W. Bruce Croft, "A History of Information Retrieval Research," Proceedings of the IEEE 100 (May 13, 2012): 1444-1451. 
the content of a document that precisely meets an information seeker's needs) and information that has merely instrumental value (e.g., the metadata that comprise a description of the document sought). (c) Third, some distinguish between the information supplied by a document in virtue of its content (e.g., information about subject matter) and that supplied in virtue of its existence, form, and/or structure (e.g., information about provenance or context). Indeed, some of those in the last camp will assess the informational value and the evidentiary value of a document separately, implying that information and evidence are different things - or, at least, that information-as-evidence is a discrete species of the information genus.

2. In the field of information retrieval, it is typically assumed that, even if the information sought is essentially subjective, it is valid to base retrieval on inferences drawn from observation and measurement of objective information; and that metadata are of clear utility in serving as surrogates for documents in the collections searched. The information/evidence distinction, however, is not one that routinely impinges on IR systems design. In archival studies, on the other hand, it is this third distinction that historically has been treated as by far the most important, to the extent that external debates about the nature of information have remained tangential to the interests of archivists and archival theorists alike. All else being equal, the evidentiariness of a given archival record is likely to be valued more highly than its informativeness, and it is the nature of evidence (not information) that usually exercises philosophically-inclined minds in the archival field.

3. Another term in ordinary usage has several remarkably different technical senses, not just in archival studies and IR, but also in the related field of library science. The English term "records" has been in common use since the fourteenth century in referring to documents of a particular kind - viz., those that serve as archival evidence. ${ }^{15}$ Only since the late 1950 s has the term "record" been used also to mean a unit of information (superseding the slightly

15 In some archival contexts, "records" is reserved specifically to refer to organisational, business, governmental, public, or legal records, to be contrasted with the "papers" or "manuscripts" that provide evidence of the activities of individual persons and families. 
earlier use of "item" for the same purpose). The use of "record" in place of "entry," e.g., in the phrase "catalogue entry," is more recent still, dating from the mid-1960s (whereas its precursor can be traced back at least to the sixteenth century). The present situation, then, is one of no little confusion, in which a single term does triple duty as the name for, (a) in archives and records management, and in recordkeeping more broadly, a class of documents, ${ }^{16}$ (b) in computer science, a class of descriptions (of objects in general), ${ }^{17}$ and (c) in librarianship, a different class of descriptions (of documents in particular). ${ }^{18}$

16 ISO 30300:2011 Information and Documentation-Management Systems for Records - Fundamentals and Vocabulary is the most recent of a sequence of ISO standards defining "record(s)" as "information created, received, and maintained as evidence and information by an organization or person, in pursuit of legal obligations or in the transaction of business" - a definition with clear echoes both of that supplied by Frank B. Evans, Donald F. Harrison, Edwin A. Thompson, and William L. Rofes in "A Basic Glossary for Archivists, Manuscript Curators, and Records Managers," American Archivist 37 no. 3 (1974): 415-433 ("all recorded information, regardless of media or characteristics, made or received and maintained by an organization or institution in pursuance of its legal obligations or in the transaction of its business"), and of the one given in the U.S. Government's Records Disposal Act of 1943 ("all ... documentary materials, regardless of physical form or characteristics, made or received ... in pursuance of Federal law or in connection with the transaction of public business, and preserved ... as evidence ... or because of the informational value of data contained therein").

17 ISO/IEC/IEEE 24765:2010 Systems and Software Engineering - Vocabulary defines "record" as "a set of related data items treated as a unit"; ISO/IEC 2382-4:1999 Information Technology - Vocabulary - Part 4: Organization of Data talks of "elements" instead of "items." One of the earliest dictionary definitions of this sense of "records" appears in Glossary of Terms Used in Automatic Data Processing, ed. Erroll de Burgh Wilmot (London: Business Publications Limited, 1960): "items of information constituting a complete file." Since at least 1954, "item" had been used for this purpose: see, e.g., Grace M. Hopper, "A Glossary of Computer Terminology," Computers and Automation 3 no. 5 (1954): 14-18, 20, 22, where "item" is defined as "a set of one or more fields containing related information."

18 Although ISO 8459:2009 Information and Documentation-Bibliographic Data Element Directory for Use in Data Exchange and Inquiry defines "record" similarly to ISO/IEC/IEEE 24765:2010, as "group of data elements usually treated as a unit and often organized into sub-units called fields, which identifies, describes, and facilitates retrieval of an entity," it also defines "catalogue record" as "record in a cataloguing system that describes, analyses, and controls bibliographic, authority, or holdings data." The use of "entry" with this sense may be found in Antonio Panizzi's "Rules for the Compilation of the Catalogue," in Catalogue of Printed Books in the British Museum, Vol. 1 (London: Trustees of the British Museum, 1841), v-ix. The term "catalog record" gained currency with the first reports of the Library of Congress's Machine-Readable Cataloging (MARC) Project in the mid-1960s - see, e.g., Henriette D. Avram, Ruth S. Freitag, and Kay D. Guiles, A Proposed Format for a Standardized Machine-readable Catalog Record 
4. "Information" and "record" are not the only sites of contesting claims on semantic resources. For example: In IR, "collection" is normally used as it is in library science, to refer generally to any "gathering of documents assembled on the basis of some common characteristic," ${ }^{19}$ regardless of whether that shared characteristic is provenance. In archival and records terminology, on the other hand, "collection" is sometimes used to refer specifically to any thematically-based or other purposive gathering of documents assembled without regard to provenance (e.g., by collectors or by collecting archives), ${ }^{20}$ other times to refer specifically to any gathering of documents with shared provenance (e.g., by institutional archives), ${ }^{21}$ and yet other times (often in the plural form "collections") to denote the holdings of a given repository (e.g., archives, historical collections, and library special collections). ${ }^{22}$

5. Similarly little consensus, cross- or intra-disciplinary, exists on the appropriate generic term to use in referring to the kind of things that may potentially form the contents of archival repositories - i.e., what we (until this point in the present paper) have been calling "documents." In the most recent glossary published under the auspices of the Society of American Archivists, Richard Pearce-Moses settles on "material" as "an encompassing, generic term to describe the broad variety of items that an archives might collect, regardless of medium, format, or type," noting that this is done in order to "avoid connotations carried by terms such as record, document, or object," and that in this sense "'material' is

(Washington, DC: Library of Congress, 1965) - but the "Glossary" appearing as Appendix D in the Anglo-American Cataloguing Rules, 2nd ed (Chicago: American Library Association, 1978), 563-572, still defines "entry" as "a record of an item in a catalogue," without also defining "record." The widespread use of "record" in preference to "entry" in the library context dates rather from the 1980s, with the sharp acceleration during that period in a shift from card catalogues to OPACs (online public access catalogues).

19 Sense 2 of "collection" in ISO 5127:2001 Information and Documentation - Vocabulary.

20 See, e.g., Describing Archives: A Content Standard, 2nd ed (Chicago: Society of American Archivists, 2013), 21.

21 Synonymous, in other words, with the terms "fonds" and "record group": see, e.g., the "Notes" to the entry for "Collection" in Richard Pearce-Moses, A Glossary of Archival \&" Records Terminology (Chicago: Society of American Archivists, 2005), 76.

22 See, e.g., sense 3 of "collection" in ISO 5127:2001 Information and DocumentationVocabulary. 
roughly synonymous with 'resource."'23 This compromise, however, tends to reflect the somewhat anomalous situation in the United States - where, unlike in many other regions of the world, the archival domain encompasses both institutional archives and historical manuscript and library special collections.

At the same time, the candidacy of "document" (let alone the briefly popular "document-like object") as the appropriately generic term is impugned by tendencies in some quarters to interpret it as denotative only of textual, non-official, or even non-evidentiary things, or only of specific instances of records, such as medieval charters. In recent years, the term "resource" has emerged from the digital library community as a contender for naming this top-level category of things - including both records and (some) nonrecords - that may be collected, described, sought, and discovered. ${ }^{24}$ In the digital-library domain, however, a sharp distinction is often drawn between (on the one hand) resources and (on the other) metadata. It should be noted that the archival community makes such a distinction only between records (or materials) and descriptive metadata. In other words, archivists are among those who are careful to acknowledge that, depending upon the context,

23 Richard Pearce-Moses, A Glossary of Archival \& Records Terminology (Chicago: Society of American Archivists, 2005), 244.

24 One of the pioneer users of "resource" in this way was Michael F. Schwartz - see, e.g., his "The Networked Resource Discovery Project," in Proceedings of the IFIP XI World Congress (1989), 827-832. In the mid-1990s, the Online Computer Library Center (OCLC) embarked on an Internet Resource Cataloging Project, leading to a Metadata Workshop in Dublin, OH, in March 1995, convened in collaboration with the National Center for Supercomputing Applications - see, e.g., Stuart Weibel, "Metadata: The Foundations of Resource Description," D-Lib Magazine 1 no. 1 (1995), http://www.dlib.org/dlib/July95/07weibel.html. The outcome of this work, critical to the coalescence of the digital library field, was the Dublin Core Metadata Element Set, a specification of "a core set of metadata elements to describe networked resources" originally codified in RFC 2413:1998 Dublin Core Metadata for Resource Discovery and most recently standardised as ISO 15836:2009 Information and Documentation - The Dublin Core Metadata Element Set. "Resource" is defined in ISO 15836 as "anything that may be identified," and in the World Wide Web Consortium's specification of the Resource Description Framework (RDF) as "any object that is uniquely identifiable by a Uniform Resource Identifier (URI)" see, e.g., Eric Miller, "An Introduction to the Resource Description Framework," D-Lib Magazine 4 no. 5 (1998), http://www.dlib.org/dlib/may98/miller/05miller. html. The library community's RDA: Resource Description and Access, published in 2010 as a replacement for the Anglo-American Cataloging Rules, 2nd ed. (AACR2), defines "resource" more narrowly as "a work, expression, manifestation, or item," thus excluding entities associated with those resources such as persons, corporate bodies, families, concepts, objects, events, and places; see http://www.rdatoolkit.org/ for more on RDA. 
metadata of non-descriptive types may themselves also be considered as records.

In contemplating various possible strategies for tackling the terminological problem for the purposes of writing this chapter, we felt that it was important to resist the temptation of presenting it as one of a simple dichotomy between IR on the one hand, and archives and recordkeeping on the other, since even within-field consensus about the meanings of terms is not complete. We considered three alternative strategies, as follows:

One strategy would be to undertake a mapping between the canonical terminology used in IR and that used in archives and recordkeeping, but this approach runs the risk of inadequately representing the nuances and historical shifts that have taken place within each context. In the archives and recordkeeping domain, for example, where one is dealing with differing professional formations in different jurisdictions, arriving at terminological consensus has been notoriously difficult. There have been extensive debates about the definitions of and relationships between such fundamental terms as "record" and "archive," and even about the scope of the term "records management," in the technical committees that oversee the development and revision of ISO records management standards. ${ }^{25}$ Similarly, the International Council on Archives (ICA), which promulgates the ISAD suite of standards for archival description, ${ }^{26}$ has been unable since 1988 to bring a dictionary or glossary to publication. ${ }^{27}$ We pondered whether it might be possible to identify multiple discrete positions or perspectives in each area (e.g., in IR, traditional and progressive, objectivist and subjectivist; and in archives and recordkeeping, life cycle, records continuum, and digital curation) that are each characterisable by more-or-less stable definitions of how each term is being used within that perspective. Such an approach would certainly make for interesting research in its own right, but we felt that it was too large and complex an endeavour for what we were attempting to achieve with this chapter. A variant approach might have been to construct, for each term, a list of the

25 International Organization for Standardization, Technical Committee 46, Subcommittee 11 (ISO/TC 46/SC 11): Archives/Records Management, Relationship between the ISO 30300 Series of Standards and Other Products of ISO/TC 46/SC 11:2. Vocabulary (2012), http://www.niso.org/apps/group_public/download.php/9745/White_ paper-Rel.ship_30300_standards-VOCABULARY-v5.pdf.

26 See http://www.icacds.org.uk/eng/standards.htm for more on ISAD.

27 The most recent ICA-approved source is Dictionary of Archival Terminology: English and French; with Equivalents in Dutch, German, Italian, Russian and Spanish, ed. Peter Walne, 2nd rev. ed. (München: K. G. Saur, 1988). 
properties that a given entity must have if it is to be denoted by that term in a given domain, but again, given the distinct differences in perspectives identified above, that was also deemed to be too complex an approach for this chapter.

A second strategy would be to use purposively disambiguated and nonaligned language to present our exposition. We attempted this strategy in our initial drafts of this chapter, but felt that it ended up diminishing the canonical aspects of traditional IR, and engaged us in all sorts of terminological contortions that only added confusion into an already complex discussion.

A third strategy, and the one that we ultimately pursued, would fall in the space between the previous two: retaining canonical IR terminology as refracted through the lenses of the DCMI Glossary, ${ }^{28}$ ISO 5127:2001 Information and Documentation - Vocabulary and ISO 25964-1:2011 Information and Documentation - Thesauri and Interoperability with Other Vocabularies - Part 1: Thesauri for Information Retrieval but also employing terms that are central to archival studies, including some of the terms defined in two standards that are now being widely adopted in digital archives and recordkeeping, and in data curation. ISO 30300:2011 Information and Documentation - Management Systems for Records - Fundamentals and Vocabulary, seeks to update and reconcile terminology used in various prior ISO standards for records management (RM). ${ }^{29}$ While nominally labelled RM, it has been strongly influenced by records-continuum conceptualisations of recordkeeping that encompass archival activities, and currently represents the most expansive (albeit incomplete) consensus of different records management and archival constituencies. ISO 14721:2012 Space Data and Information Transfer Systems - Open Archival Information System (OAIS) - Reference Model, developed by the Consultative Committee for Space Data Systems (CCSDS) and initially adopted in 2003, is directed toward data archiving and is intended for use across diverse domains. It is being implemented by many digital archives, preservation, and curation initiatives as the underlying framework supporting the ingest, management, and retrieval of a diversity of digital content, and provides something of a bridge between the archives and recordkeeping domain and broader

28 Mary Woodley, DCMI Glossary (2005), http://dublincore.org/documents/usageguide/ glossary.shtml.

29 ISO/TC 46/SC 11, Relationship between the ISO 30300 Series of Standards and Other Products. 
constituencies concerned with retrieval of "archived" digital information objects. $^{30}$

Table 20.1 lists some of the key terms used in the five standards noted above. Beyond providing the reader with the meanings of terms in their different contexts, the table clearly illustrates the different preoccupations and perspectives of the areas that need to be aligned, or at least understood, if archival IR is to become more widely pursued.

\section{Table 20.1. Definitions of selected terms in five standard glossaries.}

Note: Term/definition pairs marked with an asterisk $\left(^{*}\right)$ are those used in this chapter.

\section{Resources}

\begin{tabular}{|l|l|}
\hline \multirow{1}{*}{ DCMI Glossary } & Document \\
\hline & $\begin{array}{l}\text { resource: anything that has identity ... } \\
\text { *information resource: any entity, electronic or otherwise, capable } \\
\text { of conveying or supporting intelligence or knowledge; e.g., a book, } \\
\text { a letter, a picture, a sculpture, a database, a person }\end{array}$ \\
\cline { 2 - 2 } & $\begin{array}{l}\text { document-like object: any discrete information resource that is } \\
\text { characterised by being fixed (i.e., having identical content for each } \\
\text { user); ... includes text, images, movies, and performances }\end{array}$ \\
\hline ISO 25964-1:2011 & $\begin{array}{l}\text { document: any resource that can be classified or indexed in order } \\
\text { that the data or information in it can be retrieved }\end{array}$ \\
\hline ISO 5127:2001 & $\begin{array}{l}\text { document: recorded information or material object which can be } \\
\text { treated as a unit in a documentation process }\end{array}$ \\
\cline { 2 - 2 } & $\begin{array}{l}\text { unit of description: document and its parts or aggregations treated } \\
\text { as an entity }\end{array}$ \\
\hline ISO 30300:2011 & $\begin{array}{l}\text { *document: recorded information or object which can be treated as } \\
\text { a unit }\end{array}$ \\
\hline ISO 14721:2012 & - \\
\hline
\end{tabular}

30 The choice of terminology in ISO 14721:2012 is justified as follows: "As this reference model is applicable to all disciplines and organizations that do, or expect to, preserve and provide information in digital form, these terms cannot match all of those familiar to any particular discipline (e.g., traditional Archives, digital libraries, science data centres). Rather, the approach taken is to use terms that are not already overloaded with meaning so as to reduce conveying unintended meanings. Therefore it is expected that all disciplines and organizations will find that they need to map some of their more familiar terms to those of the OAIS Reference Model. This should not be difficult and is viewed as a contribution, rather than a deterrent, to the success of the reference model. For example, archival science focuses on preservation of the 'record'. This term is not used in the OAIS Reference Model, but one mapping might approximately equate it with 'Content Information within an Archival Information Package'." See Consultative Committee for Space Data Systems, Reference Model for an Open Archival Information System (OAIS), Recommended Practice CCSDS 650.0-M-2 (2012), http://public.ccsds.org/publications/archive/650x0m2.pdf. 


\begin{tabular}{|l|l|}
\hline 1.2 & Records \\
\hline DCMI Glossary & - \\
\hline ISO 25964-1:2011 & - \\
\hline ISO 5127:2001 & $\begin{array}{l}\text { record[2]: document created or received and maintained by } \\
\text { an agency, organisation, or individual, in pursuance of legal } \\
\text { obligations or in the transaction of business }\end{array}$ \\
\hline ISO 30300:2011 & $\begin{array}{l}\text { सrecord(s): information created, received, and maintained as } \\
\text { evidence and as an asset by an organisation or person, in pursuit of } \\
\text { legal obligations or in the transaction of business }\end{array}$ \\
\hline ISO 14721:2012 & $\begin{array}{l}\text { content information: a set of information that is the original target } \\
\text { of preservation or that includes part or all of that information ... }\end{array}$ \\
\hline & $\begin{array}{l}\text { archival information package (AIP): an information package, } \\
\text { consisting of the content information and the associated } \\
\text { preservation description information (PDI), that is preserved } \\
\text { within an OAIS }\end{array}$ \\
\hline
\end{tabular}

\begin{tabular}{|l|l|}
\hline 1.3 & Archives \\
\hline DCMI Glossary & - \\
\hline ISO 25964-1:2011 & - \\
\hline ISO 5127:2001 & $\begin{array}{l}\text { archives[1]: records[2] of the same provenance accumulated by an } \\
\text { organisation or person in the course of the conduct of affairs, and } \\
\text { preserved because of their enduring value }\end{array}$ \\
\hline ISO 30300:2011 & *archives[1]: records maintained for continuing use \\
\hline ISO 14721:2012 & $-{ }^{\dagger}$ \\
\hline
\end{tabular}

† ISO 14721:2012 uses the term "archive" not to refer to resources, but to a kind of organisation; see "7. Resource-management organizations and systems," below in this table

2.

Collections of resources

\begin{tabular}{|l|l|}
\hline DCMI Glossary & - \\
\hline ISO 25964-1:2011 & - \\
\hline ISO 5127:2001 & $\begin{array}{l}\text { *collection[2]: gathering of documents assembled on the basis of } \\
\text { some common characteristic }\end{array}$ \\
\hline ISO 30300:2011 & - \\
\hline ISO 14721:2012 & $\begin{array}{l}\text { archival information collection (AIC): an archival information } \\
\text { package whose content information is an aggregation of archival } \\
\text { information packages }\end{array}$ \\
\hline
\end{tabular}

3.

\begin{tabular}{|l|l|}
\hline DCMI Glossary & $\begin{array}{l}\text { metadata: in general, data about data; functionally, structured data } \\
\text { about data; ... includes data associated with either an information } \\
\text { system or an information object for purposes of description, } \\
\text { administration, legal requirements, technical functionality, use and } \\
\text { usage, and preservation ... }\end{array}$ \\
\hline $\begin{array}{l}\text { record: some structured metadata about a resource, comprising one } \\
\text { or more properties and their associated values }\end{array}$ \\
\hline
\end{tabular}




\begin{tabular}{|c|c|}
\hline & $\begin{array}{l}\text { metadata record: a syntactically correct representation of the } \\
\text { descriptive information (metadata) for an information resource ... }\end{array}$ \\
\hline ISO 25964-1:2011 & $\begin{array}{l}\text { metadata: data that identify attributes of a document, } \\
\text { typically used to support functions such as location, discovery, } \\
\text { documentation, evaluation, and/or selection }\end{array}$ \\
\hline \multirow[t]{2}{*}{ ISO 5127:2001 } & $\begin{array}{l}\text { record[1]: set of data on one person or object, selected and } \\
\text { presented for a predefined specific purpose }\end{array}$ \\
\hline & $\begin{array}{l}\text { description[1]: ... results ... [of operations] including capturing, } \\
\text { analysing, organising and recording of data on documents in order } \\
\text { to ensure their identification and control }\end{array}$ \\
\hline ISO 30300:2011 & $\begin{array}{l}\text { "metadata: data describing context, content, and structure of } \\
\text { records and their management through time }\end{array}$ \\
\hline \multirow[t]{2}{*}{ ISO 14721:2012 } & metadata: data about other data \\
\hline & $\begin{array}{l}\text { preservation description information (PDI): the information } \\
\text { which is necessary for adequate preservation of the content } \\
\text { information ... }\end{array}$ \\
\hline
\end{tabular}

4.

Users of resources

\begin{tabular}{|l|l|}
\hline DCMI Glossary & - \\
\hline ISO 25964-1:2011 & - \\
\hline ISO 5127:2001 & $\begin{array}{l}\text { *information user: utiliser of infrastructures, services, or material } \\
\text { offered by information centres }\end{array}$ \\
\hline ISO 30300:2011 & - \\
\hline ISO 14721:2012 & $\begin{array}{l}\text { consumer: the role played by those persons, or client systems, } \\
\text { who interact with OAIS services to find preserved information of } \\
\text { interest and to access that information in detail }\end{array}$ \\
\hline & $\begin{array}{l}\text { designated community: an identified group of potential consumers } \\
\text { who should be able to understand a particular set of information ... }\end{array}$ \\
\hline
\end{tabular}

\section{Resource-description processes}

\begin{tabular}{|l|l|}
\hline DCMI Glossary & $\begin{array}{l}\text { *indexing: the process of evaluating information entities and } \\
\text { creating terms that aid in finding and accessing the entity ... }\end{array}$ \\
\hline ISO 25964-1:2011 & $\begin{array}{l}\text { indexing: intellectual analysis of the subject matter of a document } \\
\text { to identify the concepts represented in and allocation of the } \\
\text { corresponding index terms to allow the information to be retrieved }\end{array}$ \\
\hline ISO 5127:2001 & $\begin{array}{l}\text { *description[1]: operations ... including capturing, analysing, } \\
\text { organising and recording of data on documents in order to ensure } \\
\text { their identification and control }\end{array}$ \\
\hline & $\begin{array}{l}\text { indexing: denotation of the content or form of a document by } \\
\text { means of words[1], phrases, or notations[2], according to the rules } \\
\text { of an indexing language }\end{array}$ \\
\hline ISO 30300:2011 & indexing: establishing access points to facilitate retrieval \\
\hline ISO 14721:2012 & - \\
\hline
\end{tabular}

6. Resource-discovery processes

\begin{tabular}{|l|l|}
\hline DCMI Glossary & $\begin{array}{l}\text { *resource discovery: the process through which one searches and } \\
\text { retrieves an information resource }\end{array}$ \\
\hline ISO 25964-1:2011 & $\begin{array}{l}\text { *information retrieval: all the techniques and processes used } \\
\text { to identify documents relevant to an information need, from a } \\
\text { collection or network of information resources }\end{array}$ \\
\hline
\end{tabular}




\begin{tabular}{|l|l|}
\hline ISO 5127:2001 & $\begin{array}{l}\text { information retrieval: process of recovering specific information[1] } \\
\text { or information[2] from a store }\end{array}$ \\
\hline & $\begin{array}{l}\text { document retrieval: process of recovering specific documents from } \\
\text { a store }\end{array}$ \\
\hline ISO 30300:2011 & - \\
\hline ISO 14721:2012 & - \\
\hline
\end{tabular}

\section{Resource-management organisations and systems}

\begin{tabular}{|l|l|}
\hline DCMI Glossary & - \\
\hline ISO 25964-1:2011 & - \\
\hline ISO 5127:2001 & $\begin{array}{l}\text { archives[2]: organisation or part of an organisation responsible for } \\
\text { selection, acquisition, preservation, and availability of one or more } \\
\text { archives[1] }\end{array}$ \\
\hline ISO 30300:2011 & $\begin{array}{l}\text { *archives[3]: an organisation, agency, or programme responsible for } \\
\text { selecting, acquiring, preserving, and making available archives[1] }\end{array}$ \\
\hline ISO 14721:2012 & $\begin{array}{l}\text { 'records system: information system which captures, manages, and } \\
\text { provides access to records over time }\end{array}$ \\
\hline & $\begin{array}{l}\text { archive: an organisation that intends to preserve information for } \\
\text { access and use by a designated community }\end{array}$ \\
\hline $\begin{array}{l}\text { open archival information system (OAIS): an archive, consisting } \\
\text { of an organisation ... of people and systems, that has accepted } \\
\text { responsibility to preserve information and make it available for a } \\
\text { designated community }\end{array}$ \\
\hline
\end{tabular}

\section{Resource-discovery systems}

\begin{tabular}{|l|l|}
\hline 8.1 & Retrieval system \\
\hline DCMI Glossary & $\begin{array}{l}\text { discovery software: a computer application designed to simplify, } \\
\text { assist, and expedite the process of finding information resources }\end{array}$ \\
\hline $\begin{array}{l}\text { search engine: a utility capable of returning references to relevant } \\
\text { information resources in response to a query }\end{array}$ \\
\hline ISO 25964-1:2011 & - \\
\hline ISO 5127:2001 & $\begin{array}{l}\text { 'retrieval system: system allowing access to representations of } \\
\text { documents, their addresses in a collection[2], and the documents } \\
\text { themselves }\end{array}$ \\
\hline ISO 30300:2011 & - \\
\hline ISO 14721:2012 & $\begin{array}{l}\text { access aid: a software program or document that allows consumers } \\
\text { to locate, analyse, order, or retrieve information from an OAIS }\end{array}$ \\
\hline
\end{tabular}

\begin{tabular}{|l|l|}
\hline 8.2 & Finding aid \\
\hline DCMI Glossary & - \\
\hline ISO 25964-1:2011 & - \\
\hline ISO 5127:2001 & $\begin{array}{l}\text { *finding aid: retrieval system produced to establish control over } \\
\text { records[2] ... }\end{array}$ \\
\hline ISO 30300:2011 & - \\
\hline ISO 14721:2012 & $\begin{array}{l}\text { finding aid: a type of access aid that allows a user to search for and } \\
\text { identify archival information packages of interest }\end{array}$ \\
\hline
\end{tabular}




\section{IR: A Conceptual Framework}

\section{Intellectual Access and Resource Discovery}

By implementing an IR system, giving people the opportunity to use it, and providing them with tools to understand the resources they are accessing, the stewards of a given collection of resources are said to provide intellectual access to the resources in that collection. Intellectual access may be distinguished from at least two other kinds of access to resources:

- the physical access that is given to those who are able to interact physically with the resources themselves (or, according to some conceptions, with the content of the resources); and

- the legal access that is given to those who are permitted, under the laws or regulations of the relevant jurisdiction or stipulations of a donor or institution, to make use of the resources in certain prescribed ways.

Intellectual access is access of the kind that is enjoyed by those who are successful in finding the resources that they want. IR systems help to provide such access to the extent that they assist the user in the process of resource discovery - i.e., in the process by which the searcher identifies resources that, to a greater or lesser degree, match whatever criteria the searcher uses, at any given time, to judge resources' desirability (or "relevance").

Conventionally, the primary means by which IR systems support resource discovery has been through the effective operation of, among others, two system components: one that generates more- or less-detailed descriptions of resources, and one that generates rankings of resources on the basis of the degree to which their descriptions match the criteria specified in users' queries (a.k.a. search statements; expressions of users' information needs). In other words, an IR system typically includes an indexing mechanism, which takes care of resource description, and a relevance ranking mechanism, which ranks resources in order of their descriptions' degree of similarity, or probability of relevance, to individual queries.

\section{Resource Description, Metadata, and Authority Control}

In library science, the process of resource description has been known historically either as cataloguing or as indexing, in rough accordance with 
the nature of the resources being described: typically, book-length resources are catalogued, while resources such as journal articles, which may be conceived as parts of larger wholes, are indexed. From the 1990s onwards, the term metadata has come to be used more frequently to denote the content of the products of resource description; and cataloguers and indexers are now often said to be in the business of assigning metadata to resources. ${ }^{31}$ In the archives and recordkeeping domain, in contrast, the library terminology of cataloguing and indexing is less frequently used: in recordkeeping, bureaucratic records would typically be classified and/or filed according to a preestablished scheme; and in archives, content would be collectively and hierarchically arranged and described in the course of archival processing, and the descriptive product would be a finding aid. Moreover, to the extent that the terminology of metadata has come to be used in archives and recordkeeping, the term refers generally to all data relating to an information resource, its creation, management and use that are generated over the course of its life, not just descriptive data intended specifically to facilitate discovery (a.k.a. descriptive metadata). Much of this metadata, even if it is not created expressly for descriptive purposes, can nevertheless be exploited in IR.

Metadata may be created or assigned either manually or automatically. ${ }^{32}$ Since the 1960s, the comparative quality of manual and automatic metadata creation in terms of its utility for IR has been debated frequently and at length, usually with a shared understanding of metadata "quality" that gives most weight to the utility with which assigned metadata allow searchers to discriminate between more-relevant and less-relevant resources. ${ }^{33}$ Assigning metadata to represent the topical subjects of the contents of resources known variously as subject description, subject cataloguing, subject indexing, or sometimes simply indexing - is often considered independently as an especially problematic case. The influential Cranfield tests in the 1960s appeared to show that the products of simple methods of automatic subject indexing, relying only on the extraction of meaningful words from preexisting titles and abstracts of resources, are at least as valuable as those of some more-complex (and therefore costlier) methods of automatic subject

31 See, e.g., Murtha Baca, ed., Introduction to Metadata, 2nd ed. (Los Angeles: Getty Research Institute, 2008), http://www.getty.edu/research/publications/electronic_ publications/intrometadata/index.html.

32 See, e.g., Anne J. Gilliland, “Setting the Stage," in Introduction to Metadata, 2nd ed., ed. Murtha Baca (Los Angeles: Getty Research Institute, 2008), 1-19, http://www.getty. edu/research/publications/electronic_publications/intrometadata/setting.html.

33 See, e.g., Gerard Salton, "A Comparison Between Manual and Automatic Indexing Methods," American Documentation 20 no. 1 (1969): 61-71. 
indexing, and as those of manual subject indexing. ${ }^{34}$ Later studies appeared to demonstrate the relatively high quality of sets of index terms extracted automatically from the full-texts (instead of the titles and abstracts) of resources, and of sets of index terms obtained by carrying out certain kinds of statistical analyses of the frequency of occurrence of term-types, both in individual resources and in whole collections. ${ }^{35}$

Notwithstanding the empirical evidence that current methods of automatic subject indexing are highly effective, libraries have persisted in dedicating non-trivial amounts of time and money to the manual assignment of index terms selected from predefined lists (i.e., authority files), such as the list of subject headings authorised by the Library of Congress (LCSH). ${ }^{36}$ The justifications given for doing so typically invoke a rationalist argument to the effect that, when index terms are "controlled" in the way that LC subject headings are, (a) the chances are increased that a searcher will choose a search term which matches an index term assigned to a wanted resource, and/or (b) the searcher is able more easily (and ultimately more effectively) to browse among the classes of resources represented by individual index terms. With the adoption of national and international standards for describing archival content such as DACS, EAC-CPF, and ISAAR(CPF), archives are investing more than they have in the past in assigning subject headings within their finding aids. ${ }^{37}$ However, wary of the costs involved and also the problems of negotiating the idiosyncratic, archaic, or technical language used in many archival resources, many archivists remain unconvinced that vocabulary control of subject terms is effective in supporting user access. Instead, they

34 See Cyril W. Cleverdon, “The Cranfield Tests on Index Language Devices," Aslib Proceedings 19 no. 6 (1967): 173-194. See also Karen Spärck Jones, "The Cranfield Tests," in Information Retrieval Experiment, ed. Karen Spärck Jones (London: Butterworths, 1981), 256-284; Cyril W. Cleverdon, "The Significance of the Cranfield Tests on Index Languages," in SIGIR '91: Proceedings of the 14th Annual International ACM SIGIR Conference on Research and Development in Information Retrieval (New York: ACM Press, 1991), 3-12.

35 See, e.g., Karen Spärck Jones, “Retrieval System Tests 1958-1978," in Information Retrieval Experiment, ed. Karen Spärck Jones (London: Butterworths, 1981), 213-255; and Gerard Salton and Christopher Buckley, "Term Weighting Approaches in Automatic Text Retrieval," Information Processing E Management 24 no. 5 (1988): 513-523. See also Karen Spärck Jones, "Reflections on TREC," Information Processing E Management 31 no. 3 (1995): 291-314; "Further Reflections on TREC," Information Processing E Management 36 no. 1 (2000): 37-85; and "What's the Value of TREC?" ACM SIGIR Forum 40 no. 1 (2006): 10-20.

36 See http://id.loc.gov/authorities/subjects.html for more on LCSH.

37 See http://www2.archivists.org/standards/describing-archives-a-content-standardsecond-edition-dacs for more on DACS; http://www.ica.org/?lid=10203 for ISAAR(CPF); and http://eac.staatsbibliothek-berlin.de/ for EAC-CPF. 
believe that keyword searching of full-texts of finding aids and digital or digitised documents, when combined with searches of (a) provenancial access points (i.e., creator or collector), and (b) indicators of collection structure or arrangement, has a greater likelihood of producing a match between the terminology used by the searcher and that used in relevant resources.

Establishing control over vocabularies of index terms involves activities of several kinds: ${ }^{38}$

- identifying the semantic relationships that exist among terms: equivalence relationships, between terms that have similar meanings; hierarchical relationships, between terms that are broader and narrower in scope; and associative relationships, between terms whose meanings are related in some way;

- identifying a set of entity-classes (such as "Agent," "Action," "Object," "Concept," "Event," "Place" ... ), each of which is made up of a discrete set of paradigmatically related terms; and

- creating a set of authority data for each term, in which representations of semantic term-term relationships and (potentially) further metadata about the term are recorded.

Each of the resulting sets of authority records for terms in a given entity-class forms an authority file. Since provenance is traditionally the primary access point for archival resources, the recent development of the ICA standards for corporate, personal and family name authorities (ISAAR(CPF)) and for recordkeeping functions (ISDF) has led to new efforts to create and share archival and recordkeeping authority data. ${ }^{39}$ This is occurring most notably in Europe where historically dynamic national boundaries and the movement of unique records during and after conflicts and conquests has often resulted in records of the same provenance or relating to the same region being distributed across multiple repositories, frequently within different national jurisdictions. Records pertaining to the same region, population, or bureaucratic function may also be created using different languages and terminology, depending upon the ruling administration.

The primary benefits of maintaining authority files may be summarised as (a) the potential for sharing the files among distributed users, as is done on a global scale in the case of the Library of Congress's name and subject

38 See, e.g., Patricia Harpring, Introduction to Controlled Vocabularies: Terminology for Art, Architecture, and Other Cultural Works (Los Angeles: Getty Research Institute, 2010), http://www.getty.edu/research/publications/electronic_publications/intro_controlled_ vocab/.

39 See http://www.ica.org/?lid=10208 for ISDF. 
authorities, for example; (b) if the files are co-constructed by multiple collaborating institutions, a reduction in the average amount of authority work to be done in any single institution, and potentially an increase in the scope of the information included in the authority file because each contributor may be in possession of different information; (c) the elimination of redundancy in resource descriptions, with the data about a given term recorded only once instead of in every metadata statement in which it is included as an access point; and, in the archival context in particular, (d) support for the disambiguation of similar entries relating to different names or functions, and for the collocation of variant entries relating to the same name or function, thus assisting the user in identifying relevant archival resources.

More recently, system designers have explored the idea that metadata might usefully be supplied by "the crowd" of end-users, in addition to, or even rather than by professional cataloguers. ${ }^{40}$ Some systems allow for the direct "tagging" or annotation of resources by end-users; others log searchers' queries, treat their "click-throughs" as implicit relevance judgments, and assign as index terms the search terms that are most frequently used by those searchers who click through to view the resource in question. "Recommender" systems - which log the decisions made by end-users for example to cite, view, download, or purchase particular resources, and treat as metadata the resulting user profiles, and/or the features of resources that are closely related in co-occurrence networks - may similarly be conceived as implementing a variant of indirect crowdsourcing. ${ }^{41}$

\section{3. “Relevance” Ranking}

The "scare quotes" around the first word in the subheading, above, are intended to highlight the nature of the conceit at the heart of the IR process, which is that it is possible for IR systems accurately to determine the degree of relevance of any given resource to any given searcher at any given time. We should rather say that IR systems vary in the mean effectiveness with which they are capable of distinguishing resources that are more likely to

40 See, e.g., Jonathan Furner, "Folksonomies," in Encyclopedia of Library and Information Sciences, 3rd ed., ed. Marcia J. Bates and Mary Niles Maack (Boca Raton, FL: CRC Press, 2010), 1858-1866.

41 See, e.g., Jonathan Furner, “On Recommending," Journal of the American Society for Information Science and Technology 53 no. 9 (2002): 747-763. For those who are uncomfortable with the suggestion that recommender systems are based, even if "indirectly," on manual indexing, a slightly more attractive alternative might be to view such systems as "semi-automatic," reflecting the sense in which people, rather than machines, are the ultimate executors of the acts, representations of which are used to characterise individual resources. 
be relevant from those that are less likely to be - on the basis of statistical analyses of the frequency of occurrence of certain features (for example, terms or links) in individual resources, in whole collections, in assigned metadata, and in queries. In other words, at best, we can be optimistic that systems can estimate probabilities of relevance; divining the actual relevance of a resource to a searcher at time $t$ - which is an entirely subjective matter is (currently, at least) beyond the capability of any machine.

Nevertheless, once we have persuaded ourselves that even if this poses a problem for the theorist, it does not for the practitioner, two important ideas - (a) that resources may be ranked in order of the likelihood that they are relevant under prevailing conditions, and (b) that systems may be evaluated by determining how well, on average, they can predict the preference orderings of users - will be grasped straightforwardly enough. Two complementary measures of retrieval effectiveness are especially well known: Recall is the proportion of relevant records that are retrieved; precision is the proportion of retrieved records that are relevant. One fairly conventional way of summarising the effectiveness of a given ranking mechanism is to plot the mean precision scores obtained at a series of incrementally increasing levels of recall (e.g., $0.1,0.2,0.3, \ldots, 1.0$ ).

\section{Resources and Representations}

As we discussed in "A Note on Terminology" above, a distinction has often been drawn in the digital-library community, as well as in others that overlap similarly with the IR domain, between resources - i.e., the materials whose informational and/or evidentiary content is what is sought by searchers - and metadata, i.e., the descriptions or representations of resources with which searchers' queries are compared (descriptive metadata, in archival terms). ${ }^{42}$

Both across and, to a lesser extent, within individual collections, resources may vary in medium, form, and structure, as well as in many independent aspects of content (such as subject and genre) and context (such as place and date of production and identity of creator). In particular, some resources exist only in analogue form, e.g., as handwritten or typewritten manuscripts, as printed publications, or as photographic prints. Others are born-digital, and remain accessible primarily in that form. Yet other resources that were

42 It should be obvious that, here, we are choosing not to use "record" in the sense of "catalogue record," "bibliographic record," or even "metadata record," in order (a) to avoid confusion with the senses in which it is used in archives and recordkeeping, and (b) to reflect the increasing tendency, even within library contexts, to emphasise data and metadata rather than records. 
originally created in analogue form owe their physical accessibility (and, if described and/or available as searchable full-text, their intellectual findability) largely to their having been reproduced digitally.

Similarly, descriptive metadata may vary on several dimensions, although efforts to standardise and thus to reduce variation have had some success. Widely used national and international standards exist for:

- data models (sometimes also referred to as metadata models) that specify the kinds of entity that may be represented by metadata (viz., not only "document-like" resources, but also specific agents, events, places, etc., as well as kinds of agent, event, place, etc.), and, in the case of entity-relationship models, the relationships between those entity-types. The design of relational databases and other metadata standards may be based around such models;

- metadata element sets that specify the attributes of the resources of any given kind, or in any given collection, for which values may be determined and recorded;

- sets of rules (e.g., rules for description, or for cataloguing) that provide consistent guidance, for the person who assigns metadata, in determining, for each attribute of each resource, the appropriate value, and the appropriate form in which that value is recorded;

- controlled vocabularies that specify the "preferred" value-types available to metadata specialists and, perhaps, to searchers; and

- the encoding, format, and exchange of metadata, whether as an integral component of individual resources, or in separate, independently-managed authority files. ${ }^{43}$

Just like resources, descriptive metadata may also be made available in analogue form (e.g., as catalogue cards) and/or in digital form (e.g., as database records). In principle, therefore, we might expect to see instances of four kinds of resource/metadata systems: (1) analogue resources, analogue metadata; (2) analogue resources, digital metadata; (3) digital resources, analogue metadata; and (4) digital resources, digital metadata. In practice, examples of systems in the third category are rare (printed directories of websites come to mind). Nonetheless, the history of the development of databases of cultural resources, such as the materials found in libraries, archives, and museums, has been one of movement, mainly in the last few

43 See, e.g., Mary W. Elings and Günter Waibel, "Metadata for All: Descriptive Standards and Metadata Sharing Across Libraries, Archives, and Museums," First Monday 12 no. 3 (2007), http://firstmonday.org/ojs/index.php/fm/article/viewArticle/1628. 
decades of the twentieth century and the first few of the twenty-first, from the first of these four categories to the last. In libraries, for example, the transition from (what we might call) Phase 1 to Phase 2 began in the late 1960s, with the introduction of the MARC (Machine-Readable Cataloguing) format for bibliographic records, and continued with the wholesale replacement, over the following quarter-century, of card catalogues by OPACs (online public-access catalogues). ${ }^{44}$ The move from Phase 2 to Phase 4 began in earnest with the rapid expansion of the Web in the 1990s, and latterly has been spearheaded by projects such as Google Books, the Hathi Trust Digital Library, and the Digital Public Library of America, in which massive quantities of library resources, previously available only in analogue form, have been digitised and uploaded to the network for remote access online. ${ }^{45}$

\section{Content, Context, and Structure}

We alluded above to an important distinction between the content of a resource and the multiple contexts in which that resource is produced, interpreted, and used. The textual content of a published book, for example, may be distinguished from the context of its production; likewise, attributes of its content, such as subject, may be distinguished from attributes of its context, such as place of production. Furthermore, we may isolate attributes of the structure of a resource, such as the extent to which the component parts of its content are differentiated from one another. One textual resource may be viewed as being highly structured, in the sense that a hierarchical structure of discrete chapters, sections, and paragraphs, is clearly indicated by conventional devices in the resource itself; another may be viewed as being quite unstructured, in the sense that no such devices are used to break up a lengthy stream of text.

While they may seem facile, these distinctions are significant for discussions of IR, since the history of methods of description can be interpreted as a sequence of changes of emphasis, in each phase of which one or other of the general attribute-types - content, context, or structure - is newly highlighted (see Table 20.2).

Overlaying this classification are several other notable distinctions and dimensions of difference (see Table 20.3).

44 See http://www.loc.gov/marc/ for more on MARC.

45 See http://books.google.com/ for Google Books, http://www.hathitrust.org/ for the HathiTrust Digital Library, and http://dp.la/ for the Digital Public Library of America. 


\section{Table 20.2. Classification of IR systems according to the kinds of attributes whose values serve as the sources of resource descriptors.}

1950s- The early development of IR systems, from the 1950s on, involved methods of automatic indexing that were based on statistical analysis, initially of the content of machine-readable surrogates for resources, and subsequently of the content (i.e., the "full texts") of the resources themselves.

1960s- Later phases of development have turned the spotlight onto context-based approaches in which the content of resources (or records) that are related in some way to the original (e.g., by co-citation) is automatically analysed in order to identify suitably discriminating descriptors.

1990s- Link-based approaches - such as Google's assignment to every web page of a score ("PageRank"), derived from analysis of the web's structure of hypertext links among pages, that serves as an indicator of the page's relative importance within that structure - may be conceived as emphasising the macro-structural attributes of collections considered as wholes.

1990s- Yet other approaches - those developed in the burgeoning subfield of XML retrieval, for example - are based on the micro-structural analysis of the relationships among the component parts of the content of individual resources.

Table 20.3. Classification of IR systems on selected dimensions.

\begin{tabular}{|c|c|c|}
\hline & A & B \\
\hline State of resources? & analogue & digital \\
\hline State of metadata? & analogue & digital \\
\hline \multirow{2}{*}{$\begin{array}{l}\text { Method of metadata- } \\
\text { creation? }\end{array}$} & manual & automatic \\
\hline & $\begin{array}{l}\text { by assignment (potentially } \\
\text { from other sources) }\end{array}$ & $\begin{array}{l}\text { by extraction or inference } \\
\text { (from the resource in } \\
\text { question) }\end{array}$ \\
\hline $\begin{array}{l}\text { [If metadata-creation is } \\
\text { manual:] Selectors of index } \\
\text { terms? }\end{array}$ & professionals & "the crowd" \\
\hline $\begin{array}{l}\text { [If index terms are } \\
\text { crowdsourced:] } \\
\text { Method of crowdsourcing? }\end{array}$ & direct & indirect \\
\hline State of authority data? & analogue & digital \\
\hline Authority control? & controlled & uncontrolled \\
\hline \multirow{2}{*}{$\begin{array}{l}\text { Method of authority file- } \\
\text { creation? }\end{array}$} & manual & automatic \\
\hline & top-down & bottom-up \\
\hline Interface features? & $\begin{array}{l}\text { little automated search } \\
\text { assistance }\end{array}$ & $\begin{array}{l}\text { much automated search } \\
\text { assistance }\end{array}$ \\
\hline Meta-search capability? & single repository only & multiple repositories \\
\hline
\end{tabular}


From this unpromisingly complex proliferation of dimensions on which IR systems may be classified (and there are surely many more), we can salvage the following simplification - the observation of a general trend from (A) analogue resources; manual metadata-creation only, by assignment, by professionals; analogue controlled vocabularies, constructed manually, top-down; no automated search assistance; single-repository search; to (B) digital resources; multiple methods of manual and automatic metadatacreation in combination; digital uncontrolled "folksonomies", constructed semi-automatically, bottom-up; automated search assistance of multiple kinds; multi-repository search.

We might well be prompted to ask: How has this trajectory played out in archives? What are the determinants of the similarities and differences between the provision of access to archival resources and to resources of other kinds? What does the future hold? An essential preliminary to answering such questions is a characterisation of the archival and recordkeeping information environment, and this is the subject of the following section.

\section{The Archival Information Environment}

Recordkeeping environments and associated archival traditions and practices vary considerably from country to country and from sector to sector. The archival information environment, therefore, can be either tightly or loosely bounded depending upon the conceptualisation of recordkeeping and records systems that is being applied. Suffice it to say here that in some environments, archival considerations for IR will be threaded across the life of any given records system or resource and will pertain to a diversity of users, both primary (i.e., the creators and other users of active records systems) and secondary (i.e., those such as scholars, lawyers, human rights activists, hobbyists, and other members of the general public who need or wish to use resources generated by those systems, regardless of whether or not they are under archival control). In other environments, however, archival IR will encompass only activities relating to searching, retrieval, and use of resources in archival custody. In either case, resources and their associated metadata exist in a complex of ever-changing, ever-accumulating, temporally and contextually bound relationships with various other entities. ${ }^{46}$

46 The existence of this complex is an underlying assumption of the ISO Records Management standards and is specifically delineated in the records continuum model and the entity-relationship (ER) conceptual model for the Australian Recordkeeping Metadata Schema (RKMS) that has been the basis for the ER conceptual model 


\section{Characteristics of Archives and Archival Resources}

Archives today, whether physical or digital, may serve one or more roles. Firstly, they may serve a recordkeeping role as mechanisms for accountability, transparency, and institutional memory within governance, business, and other bureaucratic settings. It should be noted that in some institutional settings, especially those engaged in classified or otherwise sensitive or competitive activities, the ability to search is available only within the institution that created the records. Secondly, they may perform a societal or community role as cultural or memory institutions, which frequently places them in proximity to libraries and museums. Thirdly, and most recently, they may be engaged in data or media archiving, especially in fields such as certain sciences that generate large quantities of digital data, or in film production and preservation. What is common among almost all archives is that they typically provide access to voluminous and often unique resources whose distinctive materiality and circumstances are considered to be evidential in themselves. As already discussed, it is this evidential value of archival resources, rather than their informational value, that tends to take priority in all archival activities. The quality of evidentiariness, and the need to locate and retrieve relevant evidence (as opposed to discrete pieces of information or data), are also what provide the most interesting challenges to traditional IR applications. There are also preservation and policy considerations that can have significant impact on the ways in which retrieval of archival content is automated. The fragility, uniqueness, and sometimes high economic value and complex ownership or confidentiality status of many archival resources can complicate the procedures by which legal, physical, and intellectual access is granted to individual items. Because of legal and security requirements, not every user is necessarily permitted the same level of access to archival resources. Data compilation and mining across online archival resources held in different archives is also increasingly of concern to personal privacy experts, and data protection laws in some countries prohibit users from compiling enough resources from different places to be able to profile subjects mentioned in those resources.

Again, as already alluded to, although digitisation of archival resources is increasing the possibility of item-level or even within-item retrieval, the primary principle of organisation in archives remains the aggregation - i.e., either accumulations of records generated by a single recordkeeping activity (a.k.a. a

currently being developed for ICA archival descriptive standards. RKMS specifies multiple entities, including agents, mandates and functions. See McKemmish et al., "Describing Records in Context in the Continuum." 
record series), or quantities of documentation created or collected by an individual or other entity (a.k.a. an archival collection). Just as the holdings of archives around the world vary in many different respects, the materials that make up a single aggregation are often highly heterogeneous in subject matter, genre, medium, form, structure, and even provenance (the principle of respect des fonds notwithstanding). Likewise, the component parts of a single archival item (e.g., a scrapbook containing press clippings, photographs, annotations, and greeting cards; or an electronic mail message with various kinds of attachments) can be highly heterogeneous in the same respects. These characteristics pose problems for the standardisation of descriptive techniques, and reinforce the frequently expressed idea that, in general, archival resources are typically less likely to be useful in respect of their supplying information about a particular subject than they are in respect of their being evidence of a particular event or activity.

In recognition of the hierarchical structure formed (so the dominant view suggests and the descriptive standards assert ${ }^{47}$ ) by the relationships obtaining among archival materials within a single aggregation, much of the description of those resources has been undertaken at levels of aggregation of broader or narrower scope (e.g., record groups, collections, fonds, series, files), rather than at the level of individual items that is the norm in libraries, or at the level of the documentary inter-relationships between fonds that the archival bond and a conceptual model such as the records continuum model would suggest. ${ }^{48}$ Unlike library catalogue records, individual archival descriptions are often arranged hierarchically in the form of a finding aid or inventory, and the structure of such a description - just like the structure of any complex textual resource - can be captured in machine-readable form most consistently and effectively by encoding it using an XML-based markup standard like EAD (Encoded Archival Description)..$^{49}$ Different kinds of metadata may be similarly hierarchically related to each other, depending upon the level of granularity and the unit of analysis (e.g., repository,

47 See International Council on Archives, General International Standard Archival Description, 2nd ed. (Paris: International Council on Archives, 1999).

48 See Luciana Duranti, "The Archival Bond," Archives and Museum Informatics 11 nos. 3-4 (1997): 213-218; Frank Upward, Sue McKemmish and Barbara Reed, "Archivists and Changing Social and Information Spaces: A Continuum Approach to Recordkeeping and Archiving in Online Cultures," Archivaria 72 (Fall 2011): 197-238; Sue McKemmish, "Traces: Document, Record, Archive, Archives," in Archives: Recordkeeping in Society, Sue McKemmish, Michael Piggott, Barbara Reed, and Frank Upward, eds. (Wagga Wagga, Australia: Centre for Information Studies, Charles Sturt University, 2005); McKemmish et al., "Describing Records in Context in the Continuum." 
fonds, series, item, digital component). In fact, archival descriptions and recordkeeping metadata of other kinds are dynamic artefacts to which changes and additions are continually being made, as the resources that they describe become subject to new kinds of processes, interpretations, and uses, and as their physical condition deteriorates or otherwise shifts from the moment they were first conceived or created (i.e., not just after they were received by an archives and described). This expansion of the scope of metadata beyond merely the descriptive provides an exceptionally rich, but generally under-exploited infrastructure for IR.

Traditionally, the resource-discovery process in archives has been a very physically-based one. It involves first contemplating in which archives one might potentially find materials of interest; then going to any published descriptions of the holdings of those archives (e.g., finding aids); and finally, if any description appears to be promising, contacting or visiting the relevant archives to consult with a reference archivist prior to gaining physical access to the materials themselves in order to ascertain whether or not they are indeed what one wants. This situation is changing, however, as mass digitisation efforts, as well as several decades of digital recordkeeping that has been generating born-digital records, are resulting in increasing quantities of archival resources being made available online. The previous lack of large quantities of digitised resources meant that techniques for automatic indexing were rarely applied systematically in the creation of metadata for archival materials. In those cases in which automatic indexing has been used to enhance retrieval of archival materials (e.g., when a search engine indexes the web pages its crawlers find), the indexing (and thus any subsequent searching) is of the content of pre-existing, machine-readable, web-accessible finding aids and/or catalogue records, manually constructed as descriptions of archival resources. Reference models such as OAIS hold out the promise, not only of automated searching and retrieval of archival resources, but also of the ability to order and deliver a customised retrieval set (or dissemination information package, DIP) from a digital archive. The explicit recognition in OAIS of the role of such a customisation capability validates archival concerns that IR mechanisms need to take into account particular user and resource contexts and restrictions.

Reflecting archivists' commitment to "the power of the principle of provenance, ${ }^{, 50}$ the relatively small amount of subject indexing that has been done in

50 This is the title of an influential paper by David A. Bearman and Richard H. Lytle, Archivaria 21 (Winter 1985-86): 14-27. 
archives (notwithstanding a concerted push in this direction in the late 1980s and early $\left.1990 \mathrm{~s}^{51}\right)$ is primarily based on analysis of data drawn from existing descriptions of the contexts in which archival materials were produced, interpreted, and used, rather than from the content of those resources. While an understanding of the provenance or resources is likely to remain stable over time, a limitation to subject indexing is that it is usually undertaken only at one particular moment, and thus reflects the perspectives of the person or institution assigning the descriptors, as well as the cultural context of that time. Archival resources, however, accumulate multiple layers of meaning and are subject to different interpretive frames over time, and these may not be supported by previously assigned descriptors. ${ }^{52}$ Moreover, the very few inter-processor overlap studies that have been undertaken of archival description suggest that different archivists are in any case highly unlikely to describe the same archival holdings using the same subject terms. This may partly be a result of the small amount of training in assigning subject access points that most archivists have received, but is more probably an indication of the heterogeneity of subjects covered in many archival holdings, the different historical and contemporary expressions of and perspectives on those subjects, and difficulties in placing reasonable limits on the amount of subject indexing that an archives is able to do, to the most benefit of users. ${ }^{53}$

\section{Characteristics of Users}

Users and uses of archives remain understudied in comparison (for example) to users and uses of libraries, and it is unclear to what extent the findings of library research might be transferable into the archival domain. Whereas

51 Earlier studies and position papers relating to enhancing subject access to archives include Mary Jo Pugh, "The Illusion of Omniscience: Subject Access and the Reference Archivist," American Archivist 45 no. 1 (1982): 35-36; Avra Michelson, "Description and Reference in the Age of Automation," American Archivist 50 no. 2 (1987): 192-208; David Bearman, "Authority Control Issues and Prospects," American Archivist 52 no. 3 (1989): 286-299; Jackie M. Dooley and Helena Zinkham, "The Object as 'Subject': Providing Access to Genres, Forms of Material, and Physical Characteristics," in Beyond the Book: Extending MARC for Subject Access, ed. Toni Peterson and Pat Molholt (Boston: G. K. Hall, 1990), 43-80; Harriet Ostroff, "Subject Access to Archival and Manuscript Material," American Archivist 53 no. 1 (1990): 100-105; Richard Smiraglia, "Subject Access to Archival Materials Using LCSH," Cataloging E Classification Quarterly 11 nos. 3-4 (1990): 63-90; and Jackie M. Dooley, "Subject Indexing in Context," American Archivist 55 no. 2 (1992): 344-354.

52 In some contexts, social tagging might offer mitigation of this concern.

53 See Michelson, "Description and Reference in the Age of Automation." 
current conceptions of the archival multiverse posit a multiplicity of user groups, each with unique clusters of interests and motivations, ${ }^{54}$ it has been common for traditional archival user studies to draw (a) a fourfold distinction between creators, archives staff, scholar-researchers, and "the public"; (b) among scholar-researchers, a binary distinction between "serious researchers" - e.g., professional historians, biographers, and historic preservationists - and others; and (c) among members of the public, distinctions between those who need to access documents for personal purposes (e.g., property records, citizenship status, veteran benefits), lawyers, journalists, amateur genealogists, other avocational users, college students, and K-12 teachers and students. User studies have uncovered patterns in the ways in which archival users search for desired resources, suggesting that personal, organisational, geographical, and historical names of particulars (i.e., proper nouns) tend to be more popular and/or more useful as search terms than are words or phrases denoting general concepts or universals. The explanation usually runs as follows: Names are commonly used to identify the particular corporate bodies, persons, families, places, events, etc., that are participants in the provenance, or context of production, of archival resources; whereas concepts are commonly used to identify the kinds of things that are aspects of the subjects of archival resources. And, as already stated, archival users tend to be more interested in the evidentiary qualities of archival resources than they are in the informational qualities. So, for those who subscribe to this understanding of the primary utility of archival resources, the creation of, and provision of access to, descriptions of the context in which resources are produced are more important than are those of resources' content.

The benefits that searchers are presumed to derive from the representation, provided in finding aids, of the hierarchical, multi-level structure of aggregations of archival resources are, somewhat surprisingly, less well understood. The prescription of multi-level description has long been a cornerstone of archival principles and practices, and hierarchical structure is a core feature of XML-based encoding standards such as EAD and its siblings. But interface designers have struggled to translate the structural data embedded in EAD-encoded finding aids into visual displays that consistently meet users' requirements for ease of navigation. Further study of

54 Anne J. Gilliland and Sue McKemmish, "Rights in Records as a Platform for Participative Archiving," in Studies in Archival Education and Research: Selected Papers from the 2014 AERI Conference, ed. Richard J. Cox, Alison Langmead, and Eleanor Mattern (Sacramento, CA: Litwin Press, forthcoming). 
searchers' goals and preferences when navigating within and between finding aids (and between finding aids and authority data) is necessary. ${ }^{55}$

\section{Development Trajectory of Archival IR Systems}

We earlier suggested that it might be useful to think of the development of IR systems in libraries as a sequence of transitions between certain phases defined in retrospect, and noted that a transition from Phase 1 (analogue resources, analogue metadata) to Phase 2 (analogue resources, digital metadata) can be observed to have taken place largely in the 1970s and 1980s. In archives, the situation is complicated by the fact that finding aids can be considered simultaneously both as metadata (describing archival resources) and as resources; i.e., they are formulated as intellectual products, but also serve as records and thus as evidence of archives' own activities. The transition in archives from Phase 1 to Phase 2 has taken place over a longer period, and remains far from complete. ${ }^{56}$ At this point, however, we can begin to perceive a rough timeline of archival IR systems development that draws attention not only to recent successes but perhaps also to the kinds of advances that we might expect to be made in the near future. In particular, reference to our previous proto-classification (depicted in Table 20.3) leads us to the summary presented in Table 20.4.

55 See, e.g., J. Gordon Daines, III, and Cory L. Nimer, "Re-imagining Archival Display: Creating User-friendly Finding Aids," Journal of Archival Organization 9 no. 1 (2011): 4-31.

56 However compellingly it is presented as being exemplary of the state of the art, OCLC's ArchiveGrid, for instance, is essentially a union catalogue of digital records - both single-level catalogue records and multi-level finding aids - describing the overwhelmingly analogue contents of primarily North American archival repositories. See http://archivegrid.org/. 
Table 20.4. Evolution of archival IR systems.

\begin{tabular}{|c|c|c|c|}
\hline & Up to early 1980 s & Early 1980 s to present & Future \\
\hline \multirow[t]{2}{*}{$\begin{array}{l}\text { State of } \\
\text { resources? }\end{array}$} & analogue resources & $\begin{array}{l}\text { analogue and growing } \\
\text { volume of digitised and } \\
\text { born-digital resources }\end{array}$ & digital resources \\
\hline & $\begin{array}{l}\text { arranged in accordance } \\
\text { with classification, filing, } \\
\text { and registration schemes } \\
\text { manually applied by } \\
\text { records creators, filing } \\
\text { clerks, and registrars; } \\
\text { sometimes arrangement } \\
\text { is imposed by archivists }\end{array}$ & $\begin{array}{l}\text { arranged in accordance } \\
\text { with schemes manually } \\
\text { or automatically applied } \\
\text { by records creators, } \\
\text { administrators, or } \\
\text { software; sometimes } \\
\text { arrangement is imposed } \\
\text { by archivists }\end{array}$ & $\begin{array}{l}\text { arranged automatically } \\
\text { and in multiple ways }\end{array}$ \\
\hline \multirow[t]{2}{*}{$\begin{array}{l}\text { State of } \\
\text { metadata? }\end{array}$} & $\begin{array}{l}\text { analogue descriptions: } \\
\text { single-level catalogue } \\
\text { records (collection or } \\
\text { item) and multi-level } \\
\text { finding aids }\end{array}$ & $\begin{array}{l}\text { analogue and digital } \\
\text { descriptions: registry } \\
\text { metadata, creator } \\
\text { filing schemes, file } \\
\text { transfer lists, single- } \\
\text { level catalogue records, } \\
\text { multi-level finding aids, } \\
\text { item-level descriptive } \\
\text { metadata for individual } \\
\text { digital resources }\end{array}$ & $\begin{array}{l}\text { digital descriptions: } \\
\text { registry metadata, } \\
\text { creator filing schemes, } \\
\text { single-level catalogue } \\
\text { records, multi-level } \\
\text { finding aids, item-level } \\
\text { descriptive metadata } \\
\text { for individual digital } \\
\text { resources }\end{array}$ \\
\hline & $\begin{array}{l}\text { analogue indexes to } \\
\text { descriptions }\end{array}$ & $\begin{array}{l}\text { analogue and digital } \\
\text { indexes to descriptions }\end{array}$ & $\begin{array}{l}\text { digital indexes to } \\
\text { descriptions }\end{array}$ \\
\hline $\begin{array}{l}\text { Method of } \\
\text { metadata- } \\
\text { creation? }\end{array}$ & manual assignment & $\begin{array}{l}\text { manual assignment, and } \\
\text { automatic inference from } \\
\text { digital resources; end- } \\
\text { user online tagging }\end{array}$ & $\begin{array}{l}\text { primarily automatic } \\
\text { inference; some manual } \\
\text { assignment by creators } \\
\text { and archivists; end-user } \\
\text { online tagging }\end{array}$ \\
\hline $\begin{array}{l}\text { [If metadata- } \\
\text { creation is } \\
\text { manual:] } \\
\text { Selectors of } \\
\text { index terms? }\end{array}$ & $\begin{array}{l}\text { creators, records } \\
\text { administrators, } \\
\text { archivists, and volunteers }\end{array}$ & $\begin{array}{l}\text { creators, records } \\
\text { administrators, } \\
\text { archivists, volunteers, } \\
\text { and end-users }\end{array}$ & $\begin{array}{l}\text { creators, records } \\
\text { administrators, } \\
\text { archivists, volunteers, } \\
\text { and end-users }\end{array}$ \\
\hline $\begin{array}{l}\text { [If index } \\
\text { terms are } \\
\text { crowdsourced:] } \\
\text { Method of } \\
\text { crowdsourcing? }\end{array}$ & $\mathrm{n} / \mathrm{a}$ & direct and indirect & primarily indirect \\
\hline
\end{tabular}




\begin{tabular}{|c|c|c|c|}
\hline & Up to early 1980 s & Early 1980 s to present & Future \\
\hline \multirow{2}{*}{$\begin{array}{l}\text { State of } \\
\text { authority data? }\end{array}$} & analogue & analogue and digital & digital \\
\hline & $\begin{array}{l}\text { data accessible to local } \\
\text { institution only }\end{array}$ & $\begin{array}{l}\text { some open data; most } \\
\text { data accessible to local } \\
\text { institution only }\end{array}$ & $\begin{array}{l}\text { linked open data; some } \\
\text { data accessible to local } \\
\text { institution only }\end{array}$ \\
\hline $\begin{array}{l}\text { Authority } \\
\text { control? }\end{array}$ & $\begin{array}{l}\text { uncontrolled and } \\
\text { controlled; emphasis } \\
\text { on name authorities; } \\
\text { authority forms } \\
\text { applicable to local } \\
\text { institution only or to } \\
\text { sector or discipline }\end{array}$ & $\begin{array}{l}\text { controlled; increased } \\
\text { emphasis on subject } \\
\text { authorities; national } \\
\text { and international } \\
\text { standardisation of } \\
\text { conceptual and data } \\
\text { models, metadata } \\
\text { element sets, rules for } \\
\text { description, controlled } \\
\text { vocabularies, encoding } \\
\text { formats; authority } \\
\text { forms applicable to local } \\
\text { institution only or to } \\
\text { sector or discipline }\end{array}$ & $\begin{array}{l}\text { multi-tiered (local, } \\
\text { regional, national, } \\
\text { global) authority } \\
\text { control; authority forms } \\
\text { applicable to local } \\
\text { institution only or to } \\
\text { sector or discipline }\end{array}$ \\
\hline \multirow{3}{*}{$\begin{array}{l}\text { Method of } \\
\text { authority file- } \\
\text { creation? }\end{array}$} & manual & manual & $\begin{array}{l}\text { manual and semi- } \\
\text { automatic }\end{array}$ \\
\hline & $\begin{array}{l}\text { top-down and bottom- } \\
\text { up (creators) }\end{array}$ & $\begin{array}{l}\text { top-down and bottom- } \\
\text { up (creators) }\end{array}$ & $\begin{array}{l}\text { top-down and bottom- } \\
\text { up (creators and end- } \\
\text { users) }\end{array}$ \\
\hline & local collaboration only & $\begin{array}{l}\text { local, intra- and inter- } \\
\text { institution collaboration }\end{array}$ & $\begin{array}{l}\text { local, intra- and inter- } \\
\text { institution collaboration, } \\
\text { global collaboration }\end{array}$ \\
\hline $\begin{array}{l}\text { Interface } \\
\text { features? }\end{array}$ & $\begin{array}{l}\text { no automated search } \\
\text { assistance }\end{array}$ & $\begin{array}{l}\text { EDM filing systems and } \\
\text { records classification } \\
\text { schemes; computer- } \\
\text { assisted content-based } \\
\text { keyword searching } \\
\text { of descriptions and } \\
\text { authority files }\end{array}$ & $\begin{array}{l}\text { EDM filing systems and } \\
\text { records classification } \\
\text { schemes; computer- } \\
\text { assisted content/ } \\
\text { context/structure- } \\
\text { based search of digital } \\
\text { resources, descriptions, } \\
\text { and authority files; } \\
\text { object- and pattern- } \\
\text { matching techniques; } \\
\text { specifications of } \\
\text { best practices for the } \\
\text { provision of computer- } \\
\text { assisted access }\end{array}$ \\
\hline $\begin{array}{l}\text { Meta-search } \\
\text { capability? }\end{array}$ & within-repository search & $\begin{array}{l}\text { within- and cross- } \\
\text { repository search }\end{array}$ & $\begin{array}{l}\text { within-repository and } \\
\text { universal search }\end{array}$ \\
\hline
\end{tabular}




\section{Quasi-IR Developments}

Since the 1980s, progress in developing infrastructure to support archival access can be perceived as being made on five interrelated fronts that, as critical as their results are for the provision of high-performance access systems, might not strictly be considered as "IR" because they do not involve direct attention being paid to key aspects such as automated methods of indexing or relevance ranking.

In the first place, the development of national and international records management metadata and archival descriptive standards together with widespread adoption of item-level metadata standards such as METS and Dublin Core for digitised resources have brought the field closer to the goal of universal standardisation or at least interoperability of metadata content and structure. ${ }^{57}$

Second, cross-repository searching is becoming increasingly easy as union databases of archival finding aids and other metadata grow larger and involve greater proportions of the institutional base in the geographical areas that they cover. OCLC's ArchiveGrid and the European Commission's Archives Portal Europe are paving the way to the construction of true archival equivalents of the library community's WorldCat, while initiatives such as the California Digital Library's Online Archive of California (OAC) and JISC's Archives Hub in the UK demonstrate what can be achieved at regional and national levels. ${ }^{58}$

Third, the magnitude of the effects on access of differences in the user interfaces to databases of archival collection descriptions is becoming increasingly obvious as growth is seen both in the literature on users' difficulties with interpreting online displays of finding aids, and in the number of more-or-less ad hoc trials of newly-designed interfaces being undertaken by system developers. It remains unclear to what extent, and in what respects, interface designers are taking into account the findings of user studies - partly because those findings are not always especially conclusive. There is certainly room in the future for further rigorous testing of the relationships between the presence or absence of particular features of user interfaces, and levels of different kinds of users' satisfaction with the quality of access that they experience.

57 See Anne J. Gilliland, Conceptualizing Twenty-first-century Archives (Chicago: Society of American Archivists, 2014).

58 See http://archivegrid.org/ for ArchiveGrid; http://www.archivesportaleurope.net/ for Archives Portal Europe; http://worldcat.org/ for WorldCat; http://www.oac.cdlib.org/ for OAC; and http://archiveshub.ac.uk/ for Archives Hub. 
Fourth, digitisation and (where feasible) conversion to machine-readable text, of the content both of archival descriptions and of the resources-beingdescribed themselves, are being conducted on an increasingly wide scale. Digitised materials are being linked to digital finding aids in descriptive systems and also contributed to web portals and multi-repository library, archives, and museum systems such as Europeana. ${ }^{59}$

Finally, growing effort is being expended in the construction of files of archival authority data, and in making sure that these authority files are used to archival information-seekers' best advantage. For example, interactive interfaces that allow users to make their own suggestions of "tags," i.e., words or phrases that are descriptive of some aspect of their experience while viewing a record or resource, have been the object of investigations into the comparative value of crowdsourcing, not only as a method of manually indexing finding aids, but also as a method of improving the richness of the lead-in (i.e., non-preferred) vocabulary in an authority file. Meanwhile, and perhaps more significantly, several groups continue to pursue collaborative initiatives, with the aim of building shared authority files that provide national or even international control of the terms, especially of names (which are notoriously historically, culturally and politically contingent) used as access points in finding aids. In the US, the major efforts in this direction are being coordinated as components of a National Archival Authorities Infrastructure (NAAI), envisaged by Daniel Pitti (University of Virginia) and colleagues. ${ }^{60}$ As well as a National Archival Authorities Cooperative (NAAC), modelled on the Library of Congress's Name and Subject Authority Cooperative Programs (NACO and SACO), the NAAI vision includes global access to the authority file produced by participants in the Social Networks and Archival Context (SNAC) project. ${ }^{61}$ The NAAC will allow archivists from multiple participating institutions to contribute, to a shared file, authority records that comply with content and encoding standards such as ISAAR(CPF) and EAC-CPF. Meanwhile, the SNAC project is jump-starting the creation of this shared authority file through the development and use of innovative methods for the automatic extraction, from participating institutions' finding aids, of contextual metadata about

59 See http://www.europeana.eu/.

60 See Daniel V. Pitti, "National Archival Authorities Infrastructure," http://ecommons. cornell.edu/bitstream/1813/28718/7/Pitti_SNAC-NAAC_Cornell.pdf.

61 See http://socialarchive.iath.virginia.edu/NAAC_index.html for NAAC; see http://socialarchive.iath.virginia.edu/ for SNAC. 
the persons, corporate bodies, etc., whose names are controlled in the authority records.

Just as the library authority data in the Virtual International Authority File (VIAF) are exposed on the Semantic Web as linked open data (LOD) structured in accordance with the Resource Description Framework (RDF) data model, each name identified by its own unique Uniform Resource Identifier (URI) and discoverable by queries written in the SPARQL Protocol and RDF Query Language, the intention is for archival authority data to be accessible, readable, and actionable not only by human users, but also by web services that automate the process of establishing links among multiple, distributed sets of archival descriptions and authority records. ${ }^{62}$ With the development of the Semantic Web, the promise of authority control in the archival context that was identified by David Bearman and others as early as the 1970 s is finally being realised. ${ }^{63}$

\section{XML Retrieval}

As already noted, there has been surprisingly little research in archival studies that could readily be categorised as "true" IR. Some pioneering work was undertaken in the late 1970s by Richard H. Lytle at the University of Maryland. In a limited experiment that was never replicated, Lytle compared the effectiveness of subject- and provenance-based retrieval. ${ }^{64}$ The primary body of work on archival IR, strictly defined, has been much more recent, and has focused on XML retrieval.

XML (eXtensible Markup Language) is a standard encoding format used to represent the internal structure of textual documents. ${ }^{65}$ Any resource that is "marked up" using XML takes the form of a hierarchy of nested statements about that resource's structure. Each statement at the lowest level of the

62 See http://viaf.org/ for VIAF; see, e.g., http://lodlam.net/ for more on LOD, http:// semanticweb.org/ for more on the Semantic Web, http://www.w3.org/RDF/ for more on RDF, http://www.w3.org/Addressing/ for more on URIs, and http://www.w3.org/ TR/sparql11-overview/ for more on SPARQL.

63 See, e.g., David A. Bearman, "Automated Access to Archival Information: Assessing Systems," American Archivist 42 no. 2 (1979): 179-190.

64 Lytle, "Subject Retrieval in Archives"; "Intellectual Access to Archives: 1. Provenance and Content Indexing Methods of Subject Retrieval," American Archivist 43 no. 1 (1980): 64-75; and "Intellectual Access to Archives: II. Report of an Experiment Comparing Provenance and Content Indexing Methods of Subject Retrieval," American Archivist 43 no. 2 (1980): 191-207. See also Bearman and Lytle, "The Power of the Principle of Provenance."

65 See, e.g., http://www.w3.org/XML/ for more on XML. 
hierarchy consists of a component, or element, of the text of the resource - a section, perhaps, or a paragraph, heading, or subheading, enclosed by a pair of labels, or tags, that indicate the element's type, and sometimes indicate the values of certain attributes of that element, too.

When the textual content of each resource in a collection is marked up in XML, a number of benefits accrue. For example, since structural markup is independent of presentational markup, the marked-up document can be rendered on screen in any of a number of different styles or layouts, defined in stylesheets designed for this purpose. The information architect can then design, build, and evaluate web interfaces to collections of XML documents by tinkering with presentation styles, without necessarily having to worry about making permanent changes to documents' content or structure.

For the designers of IR systems, in particular, the prospect of having large resource collections marked up using XML is exciting because it appears to offer the opportunity to enable users, not only to identify relevant documents, but also to identify, as precisely as possible, the elementary components of each document that are most relevant to the user. Searchers might use a specialised query language like XPath or XQuery to access databases of XML-encoded resources, and to retrieve the contents only of elements that satisfy certain specified criteria. ${ }^{66}$ These criteria might include not only the presence of a particular combination of keywords in an element's content (a "content-only" search), but also contextual criteria, such as the position of the element in the path that may be taken to it from the root of the tree (a "content-and-structure" search). Since queries of this type are similar to those typical of traditional database searches (cf. SQL), research in what has come to be known as XML retrieval straddles the IR and database management fields, focusing as it does on the retrieval of "semi-structured" content rather than structured (databases) or unstructured (IR) ${ }^{67}$

Overlapping elements are not allowed in XML: in other words, the structure of an XML document must be tree-like. Given this constraint, retrieval from collections of XML-encoded resources is often viewed partly as a matter of matching (or determining the degree of similarity between) paths. The result of a search may be a list of elements ranked in order of their probability of relevance to the query, just as in traditional IR, but that query may well include a specification of a desired path-type, as well as of desired

66 See http://www.w3.org/TR/xpath/ for more on XPath; http://www.w3.org/TR/xquery/ for more on XQuery.

67 See, e.g., Mounia Lalmas, XML Retrieval (San Rafael, CA: Morgan \& Claypool, 2009). 
term-types. On the other hand, recursive structure, in which one instance of a particular element-type may be nested in another instance of the same type, is supported by XML. While this support for recursion provides great flexibility for the designer of XML schemas or document type descriptions (i.e., domain-specific statements of the structural requirements that XML documents must meet if they are to be validated as "well-formed" in their domain), it requires designers and users of search languages like XQuery to take account of a potentially bewildering set of possible scenarios.

Users of IR systems are especially likely to find an XML-search capability useful when their interests take them to areas in which lengthy documents, like most books and many archival finding aids, are the norm. Given that the archival informatics community has dedicated much time and effort to the development of an XML-based encoding standard for finding aids (i.e., EAD), it would be remarkable if archival retrieval system designers had not explored the potential for leveraging this existing base of ready-encoded, tree-structured resources by experimenting with XML retrieval. ${ }^{68}$ Yet, it does indeed appear that the range of options available to would-be exploiters of EAD structure remains far from exhausted. The archival literature is still replete with accounts of cases in which users are seen to have difficulties of various kinds when attempting to use online finding aids to locate resources that they want, and relatively few papers have described implementations or (better yet) evaluations of innovative search engines in archival contexts.

One recent project that may yet inspire the further work that is much needed in this area is README (Retrieving Encoded Archival Descriptions More Effectively), carried out by a team led by Jaap Kamps at the University of Amsterdam, and reported most comprehensively in Junte Zhang's dissertation. ${ }^{69}$ Zhang's methods exemplify one of the conventional designs in IR research: construct a test collection of documents, queries, and associated relevance judgments (in this case, a set of EAD finding aids and search logs from the Dutch Nationaal Archief); build an IR system (in this case, one that is tailored for retrieval of EAD elements); and conduct ad hoc experiments with the aim of evaluating the impact, on standardised measures of recall and precision, of controlled variations in certain of the conditions under which searches take place.

68 See http://www.loc.gov/ead/ for more on EAD.

69 See http://staff.science.uva.nl/ kamps/readme/ for more on the README project; see Junte Zhang, "System Evaluation of Archival Description and Access" (Ph.D. diss., University of Amsterdam, 2011, http://www.illc.uva.nl/Research/Dissertations/DS2011-04.text.pdf, for the dissertation). 
Zhang's study is particularly important because it appears to be the first to test two hypotheses that previously have instead been treated in the archival literature as assumptions: (1) that the grouping of archival resources according to their provenance is beneficial for those seeking intellectual access to the materials; and (2) that arrangement by original order is similarly beneficial. These assumptions derive, of course, from two principles that have been considered central to the archival enterprise since the nineteenth century; together, they amount to the widespread conviction that it is of vital importance for an access system to take account of contextual description when determining the relevance of archival resources.

In one of his tests, Zhang compared (a) the effectiveness of retrieval when searchers used an interface that displayed retrieved EAD elements in the context of their finding aids (and that thus grouped together those elements that shared similar contexts) with (b) the retrieval effectiveness obtained when searchers used an interface that displayed retrieved elements out of context and in order of the elements' probability of relevance. His findings were that "element + provenance" ranking did indeed outperform simple "element" ranking, but (in a potentially damning result that is rather glossed over in the report) that a standard full-text retrieval system taking no account of any EAD encoding markedly outperformed both. ${ }^{70}$ In another test, Zhang again compared the retrieval effectiveness of two systems: (a) in one, retrieved elements were ranked by probability of relevance, and (b) in the other, retrieved elements were listed in the original order in which they appear in each finding aid. Results indicated that relevance ranking is usually the better option.

Zhang also examined users' search behaviours, finding significant differences between inexperienced and experienced searchers of EAD finding aids, in several aspects of search activity. Despite these differences, however, the same type of system was found to work best for both user groups, indicating that efforts to personalise the archival search experience for members of different groups may not be worthwhile.

\section{Conclusion}

Research conducted by the computer scientists at the San Diego Supercomputer Center and the US National Archives at the end of the 1990s developed the Persistent Archives Technology (PAT) as an XML-based 
method for preserving electronic records. PAT separated document content and structure so that each could be stored separately in software-independent form in a way that the document could subsequently be reconstructed. To do this, the document structures of electronic records were computationally inferred from commonalities in structure across similar types of documents, and an XML DTD created on the fly for those structures. ${ }^{71}$ This process had two interesting implications for IR. First, it facilitated searching and retrieval according to document structure, rather than by content or language. Second, it allowed researchers to identify documents with anomalous structures, i.e., documents that for some reason were not similar to others within the same aggregation.

The latter is a particularly interesting approach for archival IR that bears further investigation, since it suggests a potential strategy for helping users such as historians and lawyers who may be hoping to find previously unknown, and possibly "smoking gun"-type documents. It also suggests, conversely, the application of archival IR in efforts to establish the absence (as opposed to the presence) of documents or of aspects of those documents' contents, and thus to meet the archivist's goal of ensuring that records-creators are seen to be held accountable for inappropriate or undesirable actions, e.g., failing to create documents, or unduly withholding or redacting documents for security, privacy, or other purposes. ${ }^{72}$ In general, ideas such as these point to ways in which advances in archival IR that exploits multiple types and sources of metadata may find wider application in other domains where similarly rich contextual metadata exists, e.g., litigation support systems, news retrieval, audiovisual archives, data mining, and digital asset management.

It seems highly likely that, at least in the short term, XML retrieval will continue as the most productive source of inspiration for archival IR system design. The Initiative for the Evaluation of XML Retrieval (INEX) was

71 Bertram Ludaescher, Richard Marciano, and Reagan Moore, "Towards Selfvalidating Knowledge-based Archives," in 11th Workshop on Research Issues in Data Engineering (Heidelberg, Germany: IEEE Computer Society, 2001, http://www.sdsc. edu/ ludaesch/Paper/ride01.html); Reagan Moore, Chaitan Baru, Arcot Rajasekar, Bertram Ludaescher, Richard Marciano, Michael Wan, Wayne Schroeder, and Amarnath Gupta, "Collection-based Persistent Digital Archives: Part 1," D-Lib Magazine 6 no. 3 (2000), http://www.dlib.org/dlib/march00/moore/03moore-pt1. html; Reagan Moore, Chaitan Baru, Arcot Rajasekar, Bertram Ludaescher, Richard Marciano, Michael Wan, Wayne Schroeder, and Amarnath Gupta, "Collection-based Persistent Digital Archives: Part 2,” D-Lib Magazine 6 no. 4 (2000), http://www.dlib. org/dlib/april00/moore/04moore-pt2.html.

72 See, e.g., The Declassification Engine: Computational Analysis of Official Secrecy, http://www.declassification-engine.org/. 
established in 2002 as a venue for researchers to compare their IR systems' performance in a variety of controlled environments. ${ }^{73}$ Recent tracks at INEX have focused on linked data, tweet contextualisation, snippet retrieval, and social book search. Finding-aid element search may not (yet?) be the highest of INEX participants' priorities, but those interested in contributing to the development of the next generation of archival IR systems could do worse than engage in a concentrated study of the results presented annually at INEX workshops.

Notwithstanding the emphasis that has been placed in this paper on intellectual access and resource discovery, it should also be clear that these are not the only archival processes that may benefit from application of IR techniques. Any task that relies on the classification, clustering, filtering, description, ranking, etc., of documents automatically, with minimal manual intervention, can be supported more or less effectively using IR-like methods. For example: Garcia-Fernandez et al. have tested an IR-like method of automatically determining the publication dates of documents. ${ }^{74}$ Essentially, this task can be conceived equally well as a classification problem, where new documents are to be classed by publication date, or as a retrieval problem, where new documents are queries to which publication dates are retrieved in response, in order of likelihood. The general procedure is as follows: ${ }^{75}$

1. Create a training corpus of documents whose publication dates are already known, and whose textual content is machine-readable (i.e., digitised using optical character recognition [OCR] software, if necessary).

2. Classify the documents in the training corpus by publication date: i.e., partition the training corpus into subsets, where each subset comprises the documents published in a specified date range (e.g., a year, if new documents are to be dated to the nearest year).

3. For each subset of documents, analyse the textual content of the members of the subset to produce a feature vector consisting of a

73 See https://inex.mmci.uni-saarland.de/ for more on INEX. See also, e.g., Mounia Lalmas and Anastasios Tombros, "Evaluating XML Retrieval Effectiveness at INEX," ACM SIGIR Forum 41 no. 1 (2007): 40-57.

74 Anne Garcia-Fernandez, Anne-Laure Ligozat, Marco Dinarelli, and Delphine Bernhard, "When Was It Written? Automatically Determining Publication Dates," in String Processing and Information Retrieval, ed. Roberto Grossi, Fabrizio Sebastiani, and Fabrizio Silvestri (Berlin: Springer, 2011), 221-236.

75 See, e.g., Thorsten Joachims, "Text Categorization with Support Vector Machines: Learning with Many Relevant Features," in Machine Learning: ECML-98, ed. Claire Nédellec and Céline Rouveirol (Berlin: Springer, 1998), 137-142. 
set of values, each value representing a different feature. This may be done using simple text-processing software, so that each value of the feature vector represents the mean normalised frequency of occurrence of a word or term used in the text. Alternatively, a linear classifier such as a support vector machine (SVM) algorithm may be used to first construct, for each subset, a binary model capable of generating a feature vector that consists of both positive and negative values, thus characterising not only those features that are present in the given subset and absent elsewhere, but also those that are absent in the given subset and present elsewhere.

4. For each new machine-readable "query"-which, in this case, would be each new document for which a date is sought:

a) compare the feature vector of the query to the feature vector of each subset of the training set; and

b) assign to each query the date associated with the subset of documents whose feature vector is most similar to the query's, where the degree of similarity between vectors is calculated using a standard measure such as the cosine coefficient.

c) [Optional] Add the query to the subset with which it is now associated, so that that subset's feature vector may be recalculated.

Garcia-Fernandez et al. applied this procedure to a corpus of a few thousand extracts from articles published in seven French newspapers over the period 1801-1944, combining it with various non-statistical methods that rely on the automatic identification of within-text "clues," such as named entities (which are likely to have birth/creation and possibly also death dates), neologisms (birth dates), archaisms (death dates), and instances of French spelling reforms (birth and death dates).

Garcia-Fernandez et al's results were not especially promising: in their best-performing setting, they were able to identify correctly only $14 \%$ of years and $42 \%$ of decades. Work in this area is ongoing, however, and temporal information retrieval (T-IR) is presently (in the mid-2010s) on the cusp of becoming a recognised research specialisation. ${ }^{76}$ "In general, T-IR aims to satisfy search needs by combining the traditional notion of document

76 See Ricardo Campos, Gaël Dias, Alípio M. Jorge, and Adam Jatowt, "Survey of Temporal Information Retrieval and Related Applications," ACM Computing Surveys 47 no. 2 (2015): article no. 15. 
relevance with temporal relevance." 77 Typically, T-IR projects address the problem of satisfying the searcher's needs for documents that share not only a certain topical content, but also some specified temporal quality, whether absolute (such as date or period of production, or of coverage) or relative to the searcher (such as timeliness or currency). Web T-IR, for example, focuses on techniques for extracting, from very large collections such as the Internet Archive, particular past versions of web pages. In some contexts, however, it can be useful to define the scope of T-IR more expansively, so that it includes studies of data-, event-, and entity-retrieval as well as document-retrieval systems. Such a broader conception would encompass, for example, the design of services like Google Books Ngram Viewer that respond to queries with data plotted on a timeline, ${ }^{78}$ database query languages that allow for the specification of times and intervals as regular expressions in queries, and tools for automatically extracting temporal information from unstructured and semi-structured datasets.

In many corpora of archival materials, especially those made up of official records, documents' dates of creation (as distinct from the dates of publication sought by Garcia-Fernandez et al.) are already authoritatively established, and automated methods of determining dates will not be needed. In manuscript collections, on the other hand, it is more usual for creation dates to be unknown or merely estimated. In these latter cases, it is not inconceivable that, along with similar applications of machine learning techniques such as automatic authorship attribution, ${ }^{79}$ T-IR techniques may be useful more generally in supporting (if not fully automating) identification of the provenance of orphan materials. ${ }^{80}$ Indeed, if archival IR researchers were able to take the lead on the development of a T-IR that engages directly, not only with the temporal, but also with the evidentiary qualities of archival materials - for example, by using automatically-identified provenance data to establish the evidentiariness of particular records - we might even come to know an IR that borrows and learns from archival science, rather than vice versa.

77 Campos et al., "Survey of Temporal Information Retrieval," 15:2.

78 See https://books.google.com/ngrams/.

79 See, e.g., Patrick Juola, "Authorship Attribution," Foundations and Trends in Information Retrieval 1 no. 3 (2006): 233-334.

80 See also http://www.w3.org/TR/prov-overview/ for details of a data model and related RDF-based standards that support the interchange of provenance information on the Web. 


\section{Acknowledgements}

The authors would like to thank two anonymous referees for their very helpful comments on an earlier draft of this paper.

\section{References}

Akmon, Dharma, Ann Zimmerman, Morgan Daniels, and Margaret Hedstrom. "The Application of Archival Concepts to a Data-intensive Environment: Working with Scientists to Understand Data Management and Preservation Needs." Archival Science 11 nos. 3-4 (2011): 329-348.

Archival Education and Research Institute (AERI) Pluralizing the Archival Curriculum Group (PACG). "Educating for the Archival Multiverse.” American Archivist 74 no. 1 (2011): 69-101.

Avram, Henriette D., Ruth S. Freitag, and Kay D. Guiles. A Proposed Format for a Standardized Machine-readable Catalog Record. Washington, DC: Library of Congress, 1965.

Baca, Murtha, ed. Introduction to Metadata. 2nd ed. Los Angeles: Getty Research Institute, 2008. http://www.getty.edu/research/publications/electronic_publications/ intrometadata/index.html.

Baeza-Yates, Ricardo, and Berthier Ribeiro-Neto. Modern Information Retrieval: The Concepts and Technology behind Search. 2nd ed. Harlow, UK: Addison-Wesley, 2011.

Bearman, David A. "Authority Control Issues and Prospects." American Archivist 52 no. 3 (1989): 286-299.

Bearman, David A. "Automated Access to Archival Information: Assessing Systems." American Archivist 42 no. 2 (1979): 179-190.

Bearman, David A., and Richard H. Lytle. "The Power of the Principle of Provenance." Archivaria 21 (Winter 1985-86): 14-27.

Bountouri, Lina, and Manolis Gergatsoulis. "The Semantic Mapping of Archival Metadata to the CIDOC CRM Ontology." Journal of Archival Organization 9 nos. 3-4 (2011): 174-207.

Buckland, Michael K. "What is a 'Document'?" Journal of the American Society for Information Science 48 no. 9 (1998): 804-809.

Campos, Ricardo, Gaël Dias, Alípio M. Jorge, and Adam Jatowt. "Survey of Temporal Information Retrieval and Related Applications." ACM Computing Surveys 47 no. 2 (2015): article no. 15.

Cleverdon, Cyril W. "The Cranfield Tests on Index Language Devices." Aslib Proceedings 19 no. 6 (1967): 173-194.

Cleverdon, Cyril W. "The Significance of the Cranfield Tests on Index Languages." In SIGIR '91: Proceedings of the 14th Annual International ACM SIGIR Conference on Research and Development in Information Retrieval, 3-12. New York: ACM Press, 1991.

Consultative Committee for Space Data Systems. Reference Model for an Open Archival Information System (OAIS). Recommended Practice CCSDS 650.0-M-2.2012. http://public.ccsds.org/publications/archive/650x0m2.pdf.

Croft, W. Bruce, Donald Metzler, and Trevor Strohman. Search Engines: Information Retrieval in Practice. Boston, MA: Addison-Wesley, 2010.

Daines, J. Gordon, III, and Cory L. Nimer. "Re-imagining Archival Display: Creating User-friendly Finding Aids.” Journal of Archival Organization 9 no. 1 (2011): 4-31.

The Declassification Engine: Computational Analysis of Official Secrecy. http://www. declassification-engine.org/. 
Dooley, Jackie M. "Subject Indexing in Context." American Archivist 55 no. 2 (1992): 344-354.

Dooley, Jackie M., and Helena Zinkham. "The Object as 'Subject': Providing Access to Genres, Forms of Material, and Physical Characteristics." In Beyond the Book: Extending MARC for Subject Access, edited by Toni Peterson and Pat Molholt, 43-80. Boston: G. K. Hall, 1990.

Duranti, Luciana. "The Archival Bond." Archives and Museum Informatics 11 nos. 3-4 (1997): 213-218.

Elings, Mary W., and Günter Waibel. "Metadata for All: Descriptive Standards and Metadata Sharing Across Libraries, Archives, and Museums." First Monday 12 no. 3 (2007). http://firstmonday.org/ojs/index.php/fm/article/viewArticle/1628.

Evans, Frank B., Donald F. Harrison, Edwin A. Thompson, and William L. Rofes. "A Basic Glossary for Archivists, Manuscript Curators, and Records Managers.” American Archivist 37 no. 3 (1974): 415-433.

Frusciano, Thomas J. "Online Finding Aids, Catalog Records, and Access - Revisited.” Journal of Archival Organization 9 no. 1 (2011): 1-3.

Furner, Jonathan. “On Recommending." Journal of the American Society for Information Science and Technology 53 no. 9 (2002): 747-763.

Furner, Jonathan. "Folksonomies." In Encyclopedia of Library and Information Sciences. 3rd ed. Edited by Marcia J. Bates and Mary Niles Maack, 1858-1866. Boca Raton, FL: CRC Press, 2010.

Garcia-Fernandez, Anne, Anne-Laure Ligozat, Marco Dinarelli, and Delphine Bernhard. "When Was It Written? Automatically Determining Publication Dates." In String Processing and Information Retrieval, edited by Roberto Grossi, Fabrizio Sebastiani, and Fabrizio Silvestri, 221-236. Berlin: Springer, 2011.

Gilliland, Anne J. Conceptualizing Twenty-first-century Archives. Chicago, IL: Society of American Archivists, 2014.

Gilliland, Anne J. "Reconceptualizing Records, the Archive and Archival Roles and Requirements in a Networked Society." Knygotyra [Book Science] 63 (2014): 17-34.

Gilliland, Anne J. “Setting the Stage." In Introduction to Metadata. 2nd ed. Edited by Murtha Baca (Los Angeles: Getty Research Institute, 2008), 1-19. http://www.getty. edu/research/publications/electronic_publications/intrometadata/setting.html.

Gilliland, Anne J., and Sue McKemmish. "Rights in Records as a Platform for Participative Archiving." In Studies in Archival Education and Research: Selected Papers from the 2014 AERI Conference, edited by Richard J. Cox, Alison Langmead and Eleanor Mattern. Sacramento, CA: Litwin Press, forthcoming.

Greene, Mark A. "MPLP: It's Not Just for Processing Any More," American Archivist 73 no. 1 (2010): 175-203.

Harman, Donna. Information Retrieval Evaluation. San Rafael, CA: Morgan \& Claypool, 2011.

Harpring, Patricia. Introduction to Controlled Vocabularies: Terminology for Art, Architecture, and Other Cultural Works. Los Angeles: Getty Research Institute, 2010. http://www. getty.edu/research/publications/electronic_publications/intro_controlled_vocab/.

Hopper, Grace M. "A Glossary of Computer Terminology." Computers and Automation 3 no. 5 (1954): 14-18, 20, 22.

International Council on Archives. General International Standard Archival Description. 2nd ed. Paris: International Council on Archives, 1999.

International Council on Archives. International Standard Archival Authority Record for Corporate Bodies, Persons, and Families. 2nd ed. Paris: International Council on Archives, 2004. 
International Organization for Standardization. ISO/IEC 2382-4:1999 Information Technology - Vocabulary - Part 4: Organization of Data. Geneva, Switzerland: ISO, 1999.

International Organization for Standardization. ISO 5127:2001 Information and Documentation - Vocabulary. Geneva, Switzerland: ISO, 2001.

International Organization for Standardization. ISO 8459:2009 Information and Documentation - Bibliographic Data Element Directory for Use in Data Exchange and Inquiry. Geneva, Switzerland: ISO, 2009.

International Organization for Standardization. ISO 14721:2012 Space Data and Information Transfer Systems - Open Archival Information System (OAIS) - Reference Model. Geneva, Switzerland: ISO, 2012.

International Organization for Standardization. ISO 15836:2009 Information and Documentation - The Dublin Core Metadata Element Set. Geneva, Switzerland: ISO, 2009.

International Organization for Standardization. ISO/IEC/IEEE 24765:2010 Systems and Software Engineering - Vocabulary. Geneva, Switzerland: ISO, 2010.

International Organization for Standardization. ISO 25964-1:2011 Information and Documentation - Thesauri and Interoperability with Other Vocabularies - Part 1: Thesauri for Information Retrieval. Geneva, Switzerland: ISO, 2011.

International Organization for Standardization. ISO 30300:2011 Information and Documentation - Management Systems for Records - Fundamentals and Vocabulary. Geneva, Switzerland: ISO, 2011.

International Organization for Standardization, Technical Committee 46, Subcommittee 11 (ISO/TC 46/SC 11): Archives/Records Management. Relationship between the ISO 30300 Series of Standards and Other Products of ISO/TC 46/SC 11:2. Vocabulary. 2012. http://www.niso.org/apps/group_public/download.php/9745/White_paperRel.ship_30300_standards-VOCABULARY-v5.pdf.

Joachims, Thorsten. "Text Categorization with Support Vector Machines: Learning with Many Relevant Features.” In Machine Learning: ECML-98, edited by Claire Nédellec and Céline Rouveirol (Berlin: Springer, 1998), 137-142.

Juola, Patrick. "Authorship Attribution." Foundations and Trends in Information Retrieval 1 no. 3 (2006): 233-334.

Lalmas, Mounia. XML Retrieval. San Rafael, CA: Morgan \& Claypool, 2009.

Lalmas, Mounia, and Anastasios Tombros. "Evaluating XML Retrieval Effectiveness at INEX." ACM SIGIR Forum 4 no. 1 (2007): 40-57.

Lancaster, F. Wilfred. Information Retrieval Systems; Characteristics, Testing, and Evaluation. New York: Wiley, 1968.

Ludaescher, Bertram, Richard Marciano, and Reagan Moore. “Towards Self-validating Knowledge-based Archives." In 11th Workshop on Research Issues in Data Engineering. Heidelberg, Germany: IEEE Computer Society, 2001. http://www.sdsc. edu/ ludaesch/Paper/ride01.html.

Lytle, Richard H. "Subject Retrieval in Archives: A Comparison of the Provenance and Content Indexing Methods." PhD diss., University of Maryland, 1979.

Lytle, Richard H. "Intellectual Access to Archives: 1. Provenance and Content Indexing Methods of Subject Retrieval." American Archivist 43, no. 1 (1980): 64-75.

Lytle, Richard H. "Intellectual Access to Archives: II. Report of an Experiment Comparing Provenance and Content Indexing Methods of Subject Retrieval." American Archivist 43 no. 2 (1980): 191-207.

Maier, Shannon Bowen. "MPLP and the Catalog Record as a Finding Aid." Journal of Archival Organization 9 no. 1 (2011): 32-44. 
Manning, Christopher D., Prabhakar Raghavan, and Hinrich Schütze. Introduction to Information Retrieval. New York, NY: Cambridge University Press, 2008.

Mascaro, Michelle. "Controlled Access Headings in EAD Finding Aids: Current Practices in Number of and Types of Headings Assigned." Journal of Archival Organization 9 nos. 3-4 (2011): 208-225.

McCallum, Sally H. "An Introduction to the Metadata Object Description Schema (MODS).” Library Hi-Tech 22 no. 1 (2004): 82-88.

McKemmish, Sue. “Traces: Document, Record, Archive, Archives.” In Archives: Recordkeeping in Society, edited by Sue McKemmish, Michael, Piggott, Barbara Reed, and Frank Upward. Wagga Wagga, Australia: Centre for Information Studies, Charles Sturt University, 2005.

McKemmish, Sue, Glenda Acland, Nigel Ward, and Barbara Reed. "Describing Records in Context in the Continuum: The Australian Recordkeeping Metadata Schema." Archivaria 48 (Fall 1999): 3-42.

Michelson, Avra. "Description and Reference in the Age of Automation." American Archivist 50 no. 2 (1987): 192-208.

Miller, Eric. "An Introduction to the Resource Description Framework." D-Lib Magazine 4 no. 5 (1998). http://www.dlib.org/dlib/may98/miller/05miller.html.

Mooers, Calvin N. “Coding, Information Retrieval, and the Rapid Selector." American Documentation 1 no. 4 (1950): 225-229.

Moore, Reagan, Chaitan Baru, Arcot Rajasekar, Bertram Ludaescher, Richard Marciano, Michael Wan, Wayne Schroeder, and Amarnath Gupta. "Collection-based Persistent Digital Archives: Part 1.” D-Lib Magazine 6 no. 3 (2000). http://www.dlib.org/dlib/ march00/moore/03moore-pt1.html.

Moore, Reagan, Chaitan Baru, Arcot Rajasekar, Bertram Ludaescher, Richard Marciano, Michael Wan, Wayne Schroeder, and Amarnath Gupta. "Collection-based Persistent Digital Archives: Part 2.” D-Lib Magazine 6 no. 4 (2000). http://www.dlib.org/dlib/ april00/moore/04moore-pt2.html.

Ostroff, Harriet. "Subject Access to Archival and Manuscript Material." American Archivist 53 no. 1 (1990): 100-105.

Panizzi, Antonio. "Rules for the Compilation of the Catalogue." In Catalogue of Printed Books in the British Museum, Vol. 1, v-ix. London: Trustees of the British Museum, 1841.

Pearce-Moses, Richard. A Glossary of Archival \& Records Terminology. Chicago: Society of American Archivists, 2005.

Pitti, Daniel V. “National Archival Authorities Infrastructure.” http://ecommons.cornell. edu/bitstream/1813/28718/7/Pitti_SNAC-NAAC_Cornell.pdf.

Pugh, Mary Jo. "The Illusion of Omniscience: Subject Access and the Reference Archivist." American Archivist 45 no. 1 (1982): 35-36.

Roelleke, Thomas. Information Retrieval Models: Foundations and Relationships. San Rafael, CA: Morgan \& Claypool, 2013.

Salton, Gerard. "A Comparison Between Manual and Automatic Indexing Methods." American Documentation 20 no. 1 (1969): 61-71.

Salton, Gerard, and Christopher Buckley. "Term Weighting Approaches in Automatic Text Retrieval.” Information Processing E Management 24 no. 5 (1988): 513-523.

Sanderson, Mark, and W. Bruce Croft. "A History of Information Retrieval Research." Proceedings of the IEEE 100 (May 13, 2012): 1444-1451.

Schwartz, Michael F. “The Networked Resource Discovery Project." In Proceedings of the IFIP XI World Congress, 827-832.1989.

Smiraglia, Richard. "Subject Access to Archival Materials Using LCSH." Cataloging \& Classification Quarterly 11 nos. 3-4 (1990): 63-90. 
Society of American Archivists. Describing Archives: A Content Standard. 2nd ed. Chicago: Society of American Archivists, 2013.

Spärck Jones, Karen. “The Cranfield Tests.” In Information Retrieval Experiment, edited by Karen Spärck Jones, 256-284. London: Butterworths, 1981.

Spärck Jones, Karen. “Retrieval System Tests 1958-1978.” In Information Retrieval Experiment, edited by Karen Spärck Jones, 213-255. London: Butterworths, 1981.

Spärck Jones, Karen. "Reflections on TREC." Information Processing E' Management 31 no. 3 (1995): 291-314.

Spärck Jones, Karen. “Further Reflections on TREC.” Information Processing E Management 36 no. 1 (2000): 37-85.

Spärck Jones, Karen. "What's the Value of TREC?” ACM SIGIR Forum 40 no. 1 (2006): 10-20.

Upward, Frank, Sue McKemmish, and Barbara Reed. "Archivists and Changing Social and Information Spaces: A Continuum Approach to Recordkeeping and Archiving in Online Cultures." Archivaria 72 (Fall 2011): 197-238.

Walne, Peter, ed. Dictionary of Archival Terminology: English and French; with Equivalents in Dutch, German, Italian, Russian and Spanish. 2nd rev. ed. München: K. G. Saur, 1988.

Weibel, Stuart. "Metadata: The Foundations of Resource Description." D-Lib Magazine 1 no. 1 (1995). http://www.dlib.org/dlib/July95/07weibel.html.

White, Kelvin L., and Anne J. Gilliland. "Promoting Reflexivity and Inclusivity in Archival Education, Research, and Practice.” Library Quarterly 80 no. 3 (2010): 231-248.

Wilmot, Erroll de Burgh, ed. Glossary of Terms Used in Automatic Data Processing. London: Business Publications Limited, 1960.

Wilson, Max L. Search User Interface Design. San Rafael, CA: Morgan \& Claypool, 2012.

Woodley, Mary. DCMI Glossary. 2005. http://dublincore.org/documents/usageguide/ glossary.shtml.

Zhang, Jane. "Archival Representation in the Digital Age." Journal of Archival Organization 10 no. 1 (2012): 45-68.

Zhang, Junte. "System Evaluation of Archival Description and Access." PhD diss., University of Amsterdam, 2011. http://www.illc.uva.nl/Research/Dissertations/ DS-2011-04.text.pdf. 


\title{
THE USE OF MODELS AND MODELLING IN RECORDKEEPING RESEARCH AND DEVELOPMENT
}

\author{
Hans Hofman
}

\begin{abstract}
Until a few decades ago, modelling methods and techniques were rarely a part of the archivist's toolkit. With the advent of computers and the development of information systems, this situation has changed. Initially mainly used for developing archival systems (e.g., for information analysis or system design), they began to be applied to building theoretical models relevant to the recordkeeping ${ }^{1}$ domain. Examples are the activity models produced in the InterPARES projects, the records continuum model, and the OAIS reference model. So far, there has been little evaluation of modelling as a research method in an archival context or of the application of these models, e.g., how useful they are in different contexts, what pitfalls there might be in applying them, or what limitations models might have. This chapter discusses these issues and places modelling as a research method in relation to other research methods.
\end{abstract}

\section{Introduction}

With the increasing complexity of the world in which we are living, there is a growing need to have both overviews and deep understandings of how phenomena are interrelated and fit together. Humans build models of all sorts to do just that. They could be mental (i.e., modelling cognitive processes, behaviours and perceptions), conceptual (i.e., identifying different concepts

1 The term "recordkeeping" is used throughout to refer to all of the activities, processes, functions, agents, and contexts (including those that are archival) involved in the creation, management and use of records throughout their lifetime. 
and subconcepts in a particular domain and how these are related to each other when viewed from a particular perspective), physical (e.g., constructing smaller or larger physical or virtual representations of objects), or theoretical (e.g., an explanatory or predictive model of a situation, behaviour or theory). While in research we explicitly construct and apply models, in our daily lives we rely considerably on models that are implicit, in that we are not aware of them because we develop them over time by building on experiences, thoughts, and discussions.

The result is an ongoing process of creating, refining and shaping the models we have conceived about how we view/look at phenomena or the world around us. Explicit models come in different forms. One might be a three-dimensional miniature world where we can find small-scale replicas of well-known buildings and places, including roads with people and cars, a railway system with trains, perhaps a port with ships. Another might be twodimensional, such as an architectural drawing or road map that uses lines and symbols to show us selected pieces of reality. Models can also be very abstract and theoretical. An excellent example of these characteristics, and also of the value of models, is the Standard Model of particle physics. This is a very theoretical model developed to understand the basics of our existence. It reflects "an internally consistent theory that describes the interactions between all known particles in terms of quantum field theory." Until recently there was no concrete evidence of some of the theorised particles, the most crucial one being the so-called Higgs-Boson particle that is supposed to glue all the other particles together in a unified model. However, in July 2012 the discovery of this particle was announced worldwide. ${ }^{3}$ Such an example illustrates how the very existence of a model helps to stimulate research and discussions about and around it and to move theorising forward.

It is no different in the recordkeeping domain. Over the last two decades the recordkeeping profession has experienced almost a 'quantum leap' in theoretical thinking. Many different models have been developed, including the Records Continuum Model and the InterPARES activity models. These examples show that developing and applying models has become part of our theoretical thinking and conceptual evolution. It is therefore important to discuss in more detail what modelling as a recordkeeping research method

2 See http://en.wikipedia.org/wiki/Standard_Model.

3 It raised a lot of excitement in the scientific world, and the press and other media selected it as the breakthrough of the year 2012, see for instance: http://www. sciencemag.org/careers/2012/12/sciences-breakthrough-2012. The theoretical model had proven to be true. 
is and how it can be used. In a special thematic issue of Archival Science on research methods, editors Anne Gilliland and Sue McKemmish stated that there is:

a challenging from within and without of the assertions, assumptions and even the vocabularies that have sustained the field of archival science for most of its modern life. Such challenges are not only theoretical, they also come from the need to deconstruct and disambiguate that is an essential part of designing automated systems and tools, developing metadata schemas, delineating best practices, gathering benchmark data, and, quite simply, talking across community boundaries. ${ }^{4}$

Not only do models help in getting a better understanding of the different aspects of recordkeeping, but also they help in developing strategies for addressing records issues in increasingly complex records-creating environments. ${ }^{5}$ Models can also be very effective tools for cross-disciplinary communication. It is in all of these contexts that modelling as a research method can be of enormous help in recordkeeping research and development.

The topic of models and modelling is such a broad domain in different sciences that it is impossible to cover all aspects. In this chapter, therefore, I will underline and explain the need for models and modelling as a research and development method in the recordkeeping domain. I will first discuss what models are and why modelling is useful. Subsequently I will give a brief overview of how modelling in general is done. Then I will discuss the role of modelling in recordkeeping research in recent years. Finally, I will use a case study of the InterPARES projects in the period $1998-2006^{6}$ as an illustrative example of the way modelling can be used in research and its value as a research method.

For the most part, the chapter is based on in-depth reflection on my own experience with models and model building, particularly with reference to the InterPARES projects. One of the main areas of interest in these projects was modelling as a method for the investigation as well as an expression

4 Anne Gilliland and Sue McKemmish, "Introduction," Archival Science 4 nos. 3-4 (2004): 144.

5 I would like to thank John McDonald for his valuable comments and suggestions while writing this article.

6 Led by Luciana Duranti, the three InterPARES Projects (International research on Permanent Authentic Records in Electronic Systems) and now the InterPARES Trust, have convened an international and cross-domain group of academic researchers and practitioners to investigate questions relating to the preservation of authentic records generated out of and managed within a wide range of technological environments. 
of various digital preservation strategies (specifically preserving authentic, reliable records). Additionally, the chapter builds on my experience in (information) system development in the field of archives management at the Nationaal Archief of the Netherlands. ${ }^{7}$

\section{What is Modelling?}

Models and the processes of constructing them, as discussed in this chapter, are abstract reflections of what people do in a certain context. Plainly speaking, modelling may be described as a way of capturing a defined piece of real world activity into one or more diagrams, using boxes, circles, arrows, lines or other symbols that have a specific meaning in that context. The intent is to get a shared understanding of who is involved, how things are related, and what is happening. ${ }^{8}$

7 In this respect I am referring to my education as a system analyst /information manager, and the information systems that have been developed for the Dutch National Archives under my responsibility between 1984 and 1992.

8 When one looks for a definition of a model, it turns out that there are many. An example of a generic definition of model is: "Graphical, mathematical (symbolic), physical, or verbal representation or simplified version of a concept, phenomenon, relationship, structure, system, or an aspect of the real world," http://www.

businessdictionary.com/definition/model.html). It covers both 2- and 3-dimensional models. The same source also states that: "The objectives of a model include (1) to facilitate understanding by eliminating unnecessary components, (2) to aid in decision making by simulating 'what if' scenarios, (3) to explain, control, and predict events on the basis of past observations. Since most objects and phenomenon are very complicated (have numerous parts) and much too complex (parts have dense interconnections) to be comprehended in their entirety, a model contains only those features that are of primary importance to the model maker's purpose." It also makes a distinction between "(1) Iconic model: least abstract, physical, 'look-alike' model, such as a model airplane or train. (2) Analogous model: more abstract but having some resemblance to what it represents, such as a chart, graph, map, network diagram. (3) Symbolic model: mostabstract model with no resemblance but only an approximation to what it represents, such as a mathematical equation or formula, financial statement, language, and set of accounts. There are more specific definitions. For example, when searching for a definition of scientific modelling, Wikipedia offers a more process-oriented definition: "Scientific modelling is the process of generating abstract, conceptual, graphical and/ or mathematical models," http://en.wikipedia.org/wiki/Scientific_modelling).

McKemmish and Gilliland define "model building" as the use of formal activity, entity, relationship, role, and data modelling techniques (UML, DFD, IDEF(0), ORM, $\mathrm{RDF}$ ) in iterative, exploratory processes, enabling precise description and structuring functions, processes, information flows, etc. This definition is rather technical and identifies, among others, functions, processes and information flows as objects to be described and structured. See Anne Gilliland and Sue McKemmish, "Building an Infrastructure for Archival Research," in Archival Science, 4 nos. 3-4 (2004): 190. More about models can be found at: http://plato.stanford.edu/archives/sum2009/ntries/ models-science/\#RepModIModPhe. 
A fundamental question, however, is what are we actually modelling reality or just an interpretation of it? The main characteristic of modelling is to identify what should be modelled and then to focus on the essential aspects of the subject matter that is being analysed, leaving out irrelevant details. This process is called abstraction. Models are not, and can never be, a comprehensive reflection of reality. They are always a simplification. Moreover, they can only represent one specific view on that reality, that is, our understanding of that reality. Part of the art of modelling is to leave out details and exceptions, and not to aim for 100\% coverage. The $80-20$ rule should be the main rule of thumb. A map is a good example of the principle of abstraction. Apart from being two-dimensional, it usually focuses on one aspect, for instance, the roads, topography, or history of the same geographical space. When different views, therefore, are taken of the same object, the results will be different.

To extend this argument to the recordkeeping domain, it makes a difference if you take records management as the main perspective or start from the business process. In the first case, the business process is part of the context of managing records, while in the second one, managing records is more or less integrated into the business view. It does not necessarily mean that either one is better, but it helps to position the object of the research more clearly and thus will provide better insight.

In an organisational context, modelling focuses on the processes and data produced and used in those processes. It is the domain of system development and business process modelling. Within the context of developing and building information systems, modelling deals with functional aspects or processes in order to identify what functionality is required. It also deals with data in order to identify what data/information will be captured and/or processed. Information analysts try to capture the business logic with these two types of models and then to translate them into the software application being used by an information system. ${ }^{9}$

If you look at definitions of data modelling they are closer to how modelling as a method is currently being used in the recordkeeping domain:

Data modeling is a process used to define and analyze data requirements needed to support the business processes within the scope of corresponding information systems in organizations. ${ }^{10}$

9 "Information system" is defined here as "the whole of people, procedures, rules, resources for managing information." It is not only the software application. 
A data model is usually developed as part of an information system. It starts with a high-level, conceptual schema that describes the semantics of (the entities within) a domain. It ends with a physical model that will be implemented in the information system. It does not always mean, however, that one has to go through all stages. This method could very well be used for analysis and to promote discussion leading to the development of a conceptual data model. The way in which process and data models will be implemented is also very much dependent on the available technology. For an example of a simple data model see Figure 21.1 below.

Figure 21.1. Simple Data Model ${ }^{11}$

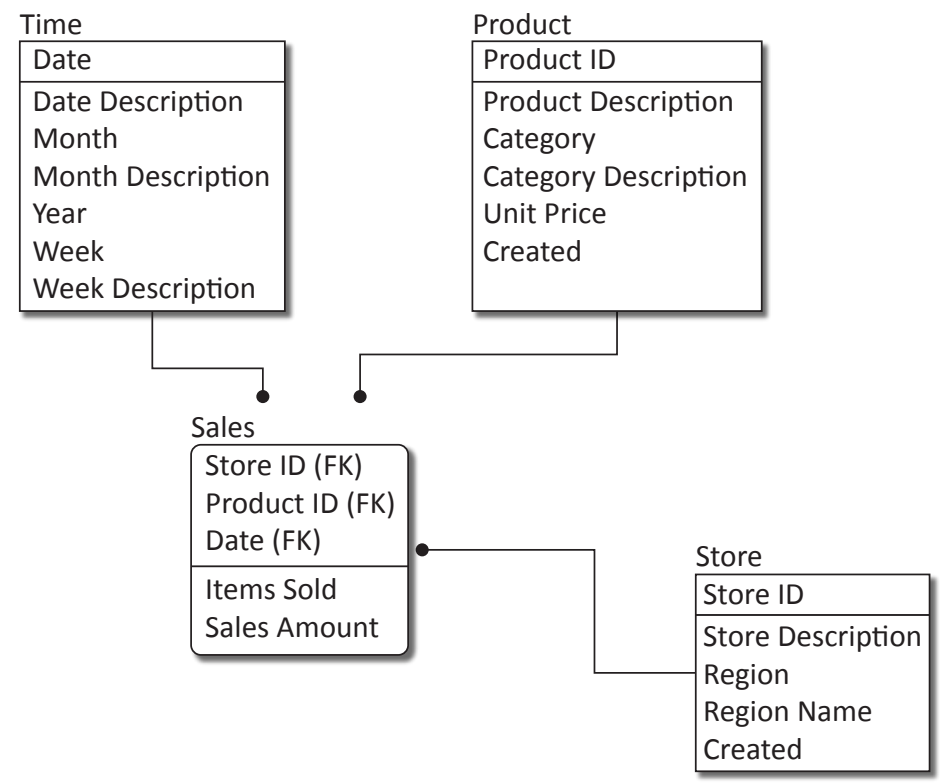

Business process modelling methods are intended to analyse and improve current processes and, until recently, focused on activities and procedural aspects. However, it turns out that in a competitive business environment this is insufficient if a company wants to be "operationally innovative." Therefore an interesting new method has been invented where the approach is no longer activity-centric, but "artefact-centric."12 Business artefacts are "data objects whose manipulations define in an important way the underlying processes in a

11 See http://www.1keydata.com/datawarehousing/data-modeling-levels.html

12 See http://en.wikipedia.org/wiki/Artifact-centric_business_process_model. 
business model." ${ }^{13}$ The result is a model that "represents an operational model of business processes in which the changes and evolutions of business data, or business entities, are considered as the main driver of the processes." ${ }^{.14} \mathrm{It}$ "focuses on describing how business data is changed/updated, by a particular action or task, throughout the process. ${ }^{15}$ In a traditional business approach information is treated within the context of individual business activities, rather than looking at the whole information context. In artefact-centric approaches, data is a driving force for the business process. Additionally:

An important distinction between artifact centric models and traditional data flow (computational) models is that the notion of the life cycle of the data objects [emphasis mine] is prominent in the former, while not existing in the latter. ${ }^{16}$

Figure 21.2 shows an example of a simple business process.

Figure 21.2. Simple Business Process Model ${ }^{17}$

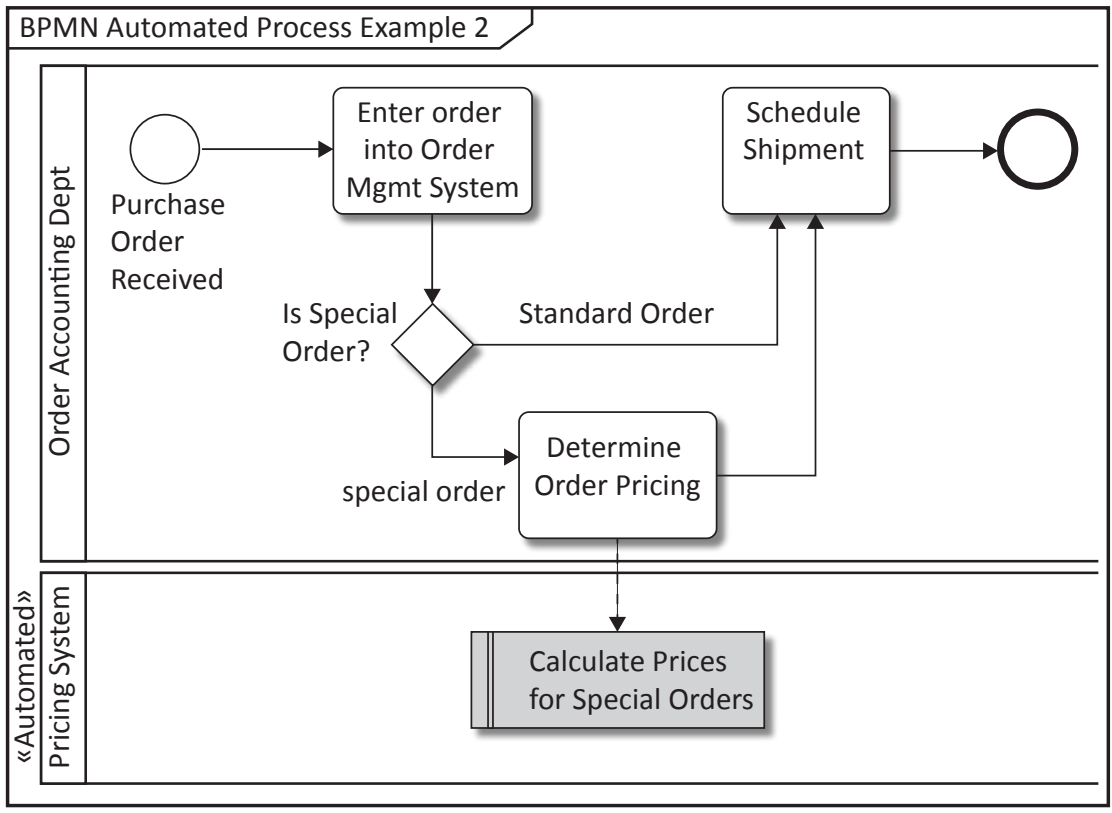

13 See http://en.wikipedia.org/wiki/Artifact-centric_business_process_model.

14 See http://en.wikipedia.org/wiki/Artifact-centric_business_process_model.

15 See http://en.wikipedia.org/wiki/Artifact-centric_business_process_model.

16 See http://en.wikipedia.org/wiki/Artifact-centric_business_process_model.

17 See https://blogs.msdn.microsoft.com/nickmalik/2008/06/26/blame-the-computera-business-process-modeling-anti-pattern/. 
The perspective that is taken in the case of recordkeeping is related to business processes and the creation and management of records, including the context in which this all takes place. It makes a difference, however, whether you take the business (process) or the records (data) perspective, as each will result in a different model, with each model useful for its own purpose. When one tries, for example, to model a business process together with the related recordkeeping processes it will be based on several inputs: a set of collected and interpreted data about one or more case studies together with relevant concepts and notions; and the assumptions of those who are conducting the modelling exercise. It is essential to be clear about those assumptions and to make them explicit. The process of modelling can thus be described as a continuous process of appraising what is relevant and what is not, given the perspective taken and the purpose of the model.

\section{Why Modelling?}

Models can serve as powerful research tools for many different purposes. Models:

- help you to understand how things interact or have interacted based on an analysis and interpretation of collected empirical data (case studies). They can represent the current situation or reconstruct what once existed. They often highlight specific aspects of a defined situation while omitting irrelevant aspects.

- visualise and reflect ideas and theories or theoretical concepts and help to facilitate understanding within or across domains. They are mostly deductively constructed, but could also be based on a generalisation of empirical data, and thus represent an idealised situation or reference model. A reference model enables comparison with a given situation to see what is missing or could be improved.

- help you to design something that does not yet exist with the intent to develop or build it. This is what usually happens in information system development where models will guide the development of systems.

- are important because they support and stimulate cross-disciplinary communication. The modelling process forces you, for instance, to be explicit about things. 
Records managers and archivists have a long history in which they have been at the tail-end of managing information and records, and that position has shaped their "world view" (often referred to as a life cycle approach). Archivists in particular were used to looking at archives as physical remainders of personal or organisational activities that have to be organised and described according to a set of defined principles and rules. The description of the (archival) records provided a retrospective account of the activities of a person or organisation and how those activities were "performed." Such an approach, which deals with individual organisations, fixed in time, is rather simple and conducted within clear boundaries. It is a two-dimensional perspective: an archive of a record creator is described as a relic, an historical artefact. Nevertheless, it provides a view on the archive and its creator that is reflected in the structure of the description, albeit looking at the record in hindsight. The question must be asked, however, as to whether this "traditional" view is still adequate to meet the challenges coming from new and more complex digital and networked environments?

New information technologies open up completely new ways of communicating and connecting people as well as of doing business. The number of communication channels is increasing with no respect for formerly accepted procedures and protocols. Information in a web 2.0 environment is abundant and ubiquitous, created, used and shared by many and flowing everywhere. Organisations are slowly sucked into this new world as well, but need more time to adapt themselves as they are often bound to formal and bureaucratic structures. Their transformation needs to follow proper procedures, and will involve major cultural change within and across organisations. At the same time existing boundaries are no longer clear, but rather diffuse and blurred. New organisational structures are emerging and information is created, used, and shared by multiple channels. Processes are being organised in different ways and tend to be more and more connected in chains or in networks. There are more and more complex distributed environments that in many cases lack effective control in terms of information management, privacy, and security, not to mention recordkeeping.

Modelling serves as a rigorous method for understanding those emerging complex environments and for analysing this complex digital world, e.g., how things are interrelated and what is needed to build new governance structures and policies that might establish innovative and more effective ways for managing information and records. In a research context, modelling also builds shared understandings to support concept development and theory building. It is a particularly powerful tool in testing and 
validating concepts, and conceptualising research problems. Modelling provides, therefore, an important instrument whereby recordkeeping research can inform theory and practice.

\section{The Process of Modelling}

The process of modelling is as important as the model itself. It determines, for instance, the quality of the model. In the following section therefore the main aspects of the modelling process are discussed.

As already mentioned, models serve as an alternate mode of communication to written explanations. It is said that "a picture tells more than a 1000 words," however, although models could be to some extent selfexplanatory, they always need a structured and more detailed description, defining what the components of the diagrams mean. They are usually the result of a collaborative effort, building on the knowledge and expertise of the participants. Modelling as a structured process is an excellent method to stimulate people to make their thoughts and underlying assumptions explicit. It is important, therefore, to bring the "right people", with relevant and different backgrounds, to the table. This will foster active and productive discussion and is crucial for a successful modelling exercise. Modelling is particularly useful in multidisciplinary contexts, and in collaborations that bring together academic, industry and professional partners, but it should be noted that it cannot be done effectively in such situations without good project management.

Modelling requires a process with strict rules and procedures. Most of the existing methods are formal, structured and rigorous in their application. Examples of methods used in the projects referenced in this chapter are Unified Modelling Language (UML), Data Flow Diagrams (DFD), and the Integrated Definition Method (IDEF(0)). ${ }^{18}$ The elements, such as boxes, circles, arrows or lines used in these methods have specific meanings and differ from method to method. Following these conventions and rules is mandatory, otherwise others may misunderstand the results. Modelling in the above-mentioned methods is a process with a structured set of steps often starting from a high-level viewpoint and subsequently drilling down to increasingly more detailed levels, until a model has been constructed that reflects in a sufficiently granular way what has to be analysed for a specific

18 For further information on IDEF(0) see http://en.wikipedia.org/wiki/IDEF and Robert P. Hanrahan, "The IDEF Process Modeling Methodology," 1995, http://www. sba.oakland.edu/faculty/mathieson/mis524/resources/readings/idef/idef.html. 
purpose. Of course, you could use more informal approaches and make sketches or diagrams that do not follow one of these methods. In these cases it is even more essential to explain and define precisely the meaning of the different components. The advantage of using a systematic and structured approach, however, is that it forces people to follow all required steps systematically and therefore helps to be consistent and comprehensive.

As indicated above, modelling methods can be process-oriented (what is or should be actually done) or data-oriented (what data are or should be created and used). The more recent methods are often object-oriented, where the data structures are combined with the methods or services that can interact with other objects. Objects are entities which have attributes and services. Attributes describe the state of an entity. They can be grouped into classes that have similar attributes or services. Entities (or objects) could be for instance "records" or "agents" or "activities." Object classes can be related to each other in different ways, such as through aggregation, generalisation or specialisation. ${ }^{19}$ Next to the objects that need to be known to an information system it is necessary to analyse and identify the interaction of users with the information system. This is done by developing so-called use cases that help in understanding what the system is supposed to do (i.e., its functionality). Different complementary views may therefore be necessary to get insight into the defined object area.

Central to the modelling exercise is identifying the object area or domain to be modelled and especially identifying what the boundaries are. What is the focus (object) of the modelling exercise? The more precise the boundaries that can be established, the more helpful it will be when analysing the object area. Next to this delineation it is crucial to identify what perspective will be taken on the identified object area. In the case study of how modelling was used as a research method in the InterPARES 1 and 2 projects that is presented below, I discuss two different perspectives taken on managing records. One perspective focused on the records and their preservation over time (the Chain of Preservation Model) and was informed by records life cycle thinking. The other focused on business activities and their related records (the Business-driven Recordkeeping Model) with reference to records continuum theory and the related records continuum model. These different perspectives and theoretical frames of reference led to very different models, each having its own benefits, depending on the object or purpose

19 For more information on UML, see for instance Wikipedia, http://en.wikipedia.org/ wiki/Unified_Modeling_Language, or Michael Jesse Chonoles and James A. Schardt, UML 2.0 for Dummies, Hoboken, NJ: Wiley, 2003. 
of examination, and the purpose of the model. ${ }^{20}$ It is obvious that the perspective determines what is relevant and what not. The case study also provides more detail of the modelling process in a research context.

Before presenting the case study, however, the next section discusses the role that modelling has played so far in recordkeeping research.

\section{The Role of Modelling in Recordkeeping Research}

As touched on earlier in the chapter, in the emerging, rapidly developing, and complex digital environment there is a feeling amongst records professionals that they are losing control because of the increasing number of communication channels. These seem to fragment more and more our known world of managing records, and to blur existing organisational boundaries as well as the distinction between personal and public spaces. The world has turned into a more complex networked society with information flows requiring new kinds of control mechanisms. It is of utmost importance for the recordkeeping community to understand this new world, and be able to develop the innovative recordkeeping strategies they need. This section will go into more depth on the relevance and the implications of modelling as a recordkeeping research method to support theory building and transformed practise.

Although records-related models have been developed, modelling as such is still not a widely used method in recordkeeping practise. So far, the use has been limited to mainly theoretical research initiatives trying to address the challenges of the digital age. In this context a few models were produced, such as those published by the $\mathrm{UBC}^{21}$ and InterPARES ${ }^{22}$ Projects, the Australian Records Continuum Model $^{23}$ and the related recordkeeping metadata models developed in the SPIRT Recordkeeping Metadata and Clever Recordkeeping Metadata (CRKM) Projects. ${ }^{24}$ They were developed to support theory building, proof of concept and analyses of a situation or

20 See http://www.interpares.org/ip2/ip2_models.cfm.

21 The official title of the so-called UBC Project was "The Preservation of the Integrity of Electronic Records," and it was led by Luciana Duranti and a team of researchers from the University of British Columbia between 1994 and 1997. See http://interpares.org/ UBCProject/intro.htm.

22 See http://www.interpares.org, InterPARES 1, 1999-2001 and InterPARES 2, 20022006.

23 Frank Upward, "Structuring the Records Continuum - Part One: Postcustodial Principles and Properties," Archives E̋ Manuscripts 24 no.2 (1996). See also: http://www. infotech.monash.edu.au/research/groups/rcrg/publications/recordscontinuum-fupp1.html.

24 See http://infotech.monash.edu/research/groups/rcrg/crkm/outcomes.html. The project ran from 2003-2006. 
case study. For these purposes their particular strength relates to the rigour and systematic approach they bring to visualising core components and their interrelationships, as well as the power of the modelling process as a communication and development tool in collaborative multidisciplinary projects and university-industry partnerships.

In the case of the UBC and InterPARES Projects, modelling was used within the broader context of research on the long-term preservation of authentic electronic records, using $\operatorname{IDEF}(0)$ as the specific modelling technique. The resulting models reflect processes such as to "manage an archival fonds" (UBC) or to perform the "preservation function" and the "appraisal function" (InterPARES1). The first built more on a consolidated view of what happens in practice in managing archival records, while the latter two provided a more theoretical view on what is needed to appraise or preserve authentic electronic records. The modelling process and its outcomes in the second stages of InterPARES are discussed in the case study below.

The Records Continuum Model represents a paradigm shift from the records life cycle perspective (see also Chapter 1 by Gilliland, Chapter 3 by Lian, Chapter 4 by McKemmish, Chapter 6 by Upward, and Chapter 25 by Gibbons). In a way it could be seen as a parallel to the Standard Model of particle physics mentioned in the introduction, since it tries to glue the world of records together and show the fundamental entities and their relationships. The Records Continuum Model, since it was first published in 1996, has served as a focal point of lively and stimulating discussions, and has helped to broaden the scope of archival and recordkeeping thinking, offering multiple perspectives and dimensions. It is an abstract semantic model representing and structuring the core entities of the recordkeeping domain and their relationships in a new paradigm. The model itself shows the structure (how the entities are related) and the spaces in which entities reside. The temporal dimension is not really reflected in the model, but lies in the interpretation of the model. ${ }^{25}$ It is difficult to represent $3-$ or more dimensional models in a two-dimensional drawing. Nonetheless, the model allows you to take multiple viewpoints, unlike the traditional records-centric view. In this sense it has immensely enriched the theoretical basis of the field and brought much more depth to its thinking. ${ }^{26}$ Moreover

25 The interpretation of the model in practice is therefore not that easy. It often leads to simplified explanations of the model (sounding more like life-cycle or sequential approaches), and thus missing the multidimensionality and the dynamics of the model.

26 The shift in thinking about records is also summarised by Terry Cook: “.... archival theoretical discourse is shifting from product to process, from structure to function, from archives to archiving, from the record to the recording context, from the 'natural' 
the broader scope and the multiple dimensions of the model provide new ways for analysing the impact of the new virtual web environment on recordkeeping.

The Records Continuum Model provided the conceptual frame of reference for the metadata modelling work undertaken in the Monash University SPIRT Recordkeeping Metadata Project. It was instrumental in developing a model Recordkeeping Metadata Schema (RKMS) which comprised a standardised set of structured recordkeeping metadata elements, a framework for developing and specifying recordkeeping metadata standards and a framework for reading or mapping metadata sets in ways which can enable semantic interoperability. The RKMS provided the metadata model for the international recordkeeping metadata standard ISO 23081 and was implemented in a range of Australian national and state government metadata standards. Modelling of metadata entities, relationships and elements was undertaken using two formal modelling techniques, the Resource Description Framework RDF and Object Role Modelling ${ }^{27}$. The modelling highlighted inconsistencies and gaps in the RKMS, enabled precise description and rigorous structuring of the Schema, provided for better specification of relationships than in other schemas, and served as a graphical means of communicating the RKMS. ${ }^{28}$

The Clever Recordkeeping Metadata (CRKM) Project focused on the interoperability between information systems and the feasibility of a "create metadata once and use many times" approach. ${ }^{29}$ In the increasingly

residue or passive by-product of administrative activity to the consciously constructed and actively mediated "archivalisation" of social memory." Terry Cook, "Archival Science and Postmodernism: New Formulations for Old Concepts," Archival Science 1 no.1 (2001): 4.

27 Resource Description Framework (RDF) and ORM or Object Role Modelling are both methods to conceptually describe information (data) and relationships. RDF is used especially in the web environment. ORM is a method to model 'the semantics of a universe of discourse'. It describes 'elementary facts' expressed in diagrams and by using natural language. Unlike entity-relationship diagrams there are no attributes. The 'elementary facts' are expressing relationships. The method is based on the Natural language Information Analysis Method (NIAM) that was initially developed in the Netherlands by G.M. Nijssen. For more information see: http://en.wikipedia.org/wiki/ Resource_Description_Framework and http://en.wikipedia.org/wiki/Object-role_ modeling.

28 Sue McKemmish, Glenda Acland, Nigel Ward and Barbara Reed, "Describing Records in Context in the Continuum: The Australian Recordkeeping Metadata Schema," Archivaria 48 (2000): 3-43.

29 For a full report on this project see: http://infotech.monash.edu/research/groups/rcrg/ crkm/outcomes.html and http://infotech.monash.edu/research/groups/rcrg/crkm/ outcomes.html\#finalreport. 
networked digital environment interoperability is a core issue. In order to enable better interoperability, different versions of a "metadata broker model" were developed to identify the issues, for example with respect to mapping metadata schemas from different domains. The models developed helped to analyse the relationships between these information systems (environments) and what would be needed to move records and their metadata as smoothly as possible from one environment to another, following the principle of "create once, use many times," 30 The project resulted in much better insights into the issues related to recordkeeping functionality and recordkeeping metadata interoperability, and identification of areas that need further research. ${ }^{31}$

Another model that is often referred to in the recordkeeping community is the Open Archival Information System (OAIS) reference model, which was developed by the space data community in collaboration with the library community and a few archival institutions. It aims to define what processes are necessary to store and preserve digital information, and is accompanied by an information model. ${ }^{32}$ It has attracted a lot of attention from all over the

30 Models that were developed include the CRKM Metadata Broker models that show how metadata could be exchanged between different environments. They helped for instance to identify interoperability issues from a recordkeeping perspective.

31 In science a distinction is made between the logic of science, which is the logic of 'what is' (or was), and the logic of engineering, which is the logic of 'what is possible' and of 'how to make it happen'. See for instance Callaos, who states: 'Scientific thinking, especially in the empirical sciences, for example, mainly (but no uniquely) proceeds from the concrete to the general, from concrete observations to the formulation of general hypothesis and general laws. Engineering thinking proceeds mainly (but not uniquely) from the general to the concrete, from scientific abstractions to concrete designs, artifacts, tools and technologies.' See Nagib Callaos, "The Essence of Engineering and Meta-engineering: A Work in Progress," p.9, http://www.iiis.org/Nagib-Callaos/Engineering-and-Meta-Engineering/. One could add that a thorough understanding of the why and what is necessary before new things can be developed or made. In this context the Records Continuum Model can be seen as a scientific model, while the SPIRT RKMS and CRKM models are engineering models. For more information about the CRKM project see http:// www.infotech.monash.edu.au/research/groups/rcrg/crkm/outcomes.html (reports), and Joanne Evans, Sue McKemmish and Karuna Bhoday, "Create Once, Use Many Times: The Clever Use of Recordkeeping Metadata for Multiple Archival Purposes," Archival Science 5 no.1 (March 2005): 17-42; Joanne Evans, Barbara Reed and Sue McKemmish, "Interoperable Data: Sustainable Frameworks for Creating and Managing Recordkeeping Metadata," Records Management Journal 18 no.2 (2008): 115-129. For others, see http://www.infotech.monash.edu.au/research/groups/rcrg/ crkm/publications.html.

32 Consultative Committee for Space Data Systems. Open Archival Information System Reference Model (ISO 14721:2012), http://public.ccsds.org/publications/BlueBooks.as px?RootFolder $=$ http $\% 3 a \% 2 f \% 2$ fpublic $\% 2$ eccsds $\% 2$ eorg $\% 2$ fpublications $\% 2$ farchive $\% 2$ fForms. 
world since its first publication in 1997 and has become the main reference model in the field of digital preservation. The model itself is not particularly records-oriented, but it is used by e.g. libraries, archives, data centres as the model to be followed. However, the perspective taken is based upon a life cycle approach and is rather traditional and retrospective, in that the model begins with the assumption that there is some pre-existing digital material that needs to be preserved. The model does not directly reference the fact that in a digital environment, preservation must start with the creation of digital information. ${ }^{33}$ Still, when used in that broader context it is a useful reference model and helps to identify the main processes involved in preserving digital information. It is not an implementation model, although it is often seen as such. The model has to be customised for different domains. For the records domain, it forms the basis for the already mentioned InterPARES Preservation Function Model. There is, however, little evidence that this last model is being used widely, if at all.

All these models provide a rigorous means of deconstructing, decomposing, analysing and describing complex phenomena, taking different perspectives, positioning recordkeeping in different business contexts and thus identifying the relationships within specific contexts, and zooming in on particular areas without losing the broader context. At a lower level, modelling as a method can help you to obtain a better understanding of recordkeeping processes themselves, and of the entities involved and their relationships. ${ }^{34}$ Different viewpoints can be taken, including viewpoints that go beyond the traditional archival approaches reflected in models such as those developed in InterPARES1. Those models focused mainly on records as artefacts - the residue of an appraisal process - coming from the back-end of the lifecycle of information and created in the clearly defined organisations and hierarchical governance structures that were typical of much of the $20^{\text {th }}$ century. For the $21^{\text {st }}$ century, new models that include the record creating environments and take into account the changing nature of modern organisations and how they may operate in the virtual world of the web are essential. This is an example of how, in a research context, modelling can reverse

33 More recent projects in digital preservation, such as the Pericles Project (http:// pericles-project.eu), however, already recognise this problem and have tried to develop other approaches (such as "sheer curation") that are getting close to recordkeeping.

34 See also the conceptual and relationship recordkeeping metadata models as developed by the SPIRT project, 1998-1999, http://www.infotech.monash.edu.au/research/ groups/rcrg/projects/spirt/deliverables/conrelmod.html. The high level model has been included in the ISO 23081 metadata for records standard (Part 1, 2006). 
the perspective of past and much current practise, and look first of all at the business environment to understand why and how records are created and used. The examples also show why the characteristics of modelling, as mentioned at the beginning of the section about 'Why Modelling', make it such a powerful instrument in research. Building models enables visualisation of ideas and concepts, analysis of interrelationships, and design of something that does not yet exist. It also provides a means of interdisciplinary communication.

In the last two decades modelling has become part of the toolkit of recordkeeping researchers, albeit so far still in a limited sense (and perhaps more at a theoretical rather than practical implementation level). Records practitioners have argued that models to date have been, for the most part, too abstract to be useful. A possible exception might be metadata modelling, which has become more heavily used across different metadata development communities, including by those engaged in developing archival descriptive standards. However, practitioners have not historically been trained in such approaches - a lack that could be remedied through education. So doing would align with the idea that more multidisciplinary approaches are needed in addressing recordkeeping requirements. In a similar vein, both recordkeeping practitioners and researchers also need to understand and engage with models used by those other disciplines, e.g., in developing information systems. That may, however, require time to achieve. It should also be noted that practitioners are not the only intended audience for these models - they have great potential to serve as alternative communication tools with systems developers or members of other communities who may not have a very nuanced understanding of archival and recordkeeping concepts and considerations (and vice versa).

It would be interesting to undertake more in-depth research to explore to what extent, under what circumstances, and for what purposes models are being used in the records community in research and in practice, and additionally to identify where modelling within the recordkeeping domain would be most useful. However, it would be even more beneficial to look beyond the boundaries of the recordkeeping community and to analyse what ongoing research and development $(\mathrm{R} \& \mathrm{D})$ modelling initiatives exist that are relevant to recordkeeping, in order to connect with these activities, thereby contributing recordkeeping principles and perspectives and seeking better integration of recordkeeping with business processes. At the same time, the use of modelling and models in other domains could usefully inform the role and application of models in recordkeeping research and practice. 


\section{The InterPARES Experience: An Example of a Research Modelling Process}

The sequential InterPares Projects brought together researchers from different backgrounds and countries to discuss issues and do research on how to preserve the authenticity of electronic records over time. The main goal of InterPARES 1 was to "develop the theoretical and methodological knowledge essential to the permanent preservation of electronically generated records and on the basis of that knowledge to formulate model strategies, policies and standards capable of ensuring their preservation". ${ }^{35}$ One of the core research methodologies was the "ancient" discipline of diplomatics, applying it in a modern, digital context (see Chapter 24 by MacNeil on contemporary archival diplomatics in this volume for more details). The project was operating with a clear identification of requirements and research questions relevant to the different domains of appraisal, authenticity, and preservation, and these provided good guidance for the research to be undertaken. A fourth domain was devoted to developing a framework for policies, strategies and standards for the long-term preservation of electronic records. Each of the domains was assigned to a task force.

The task force in InterPARES 1 that was charged with investigating the preservation aspect used modelling as a method to get an understanding of which processes are required for the preservation of authentic electronic records over time, their purpose and how they interrelate. ${ }^{36}$ The modelling technique chosen was $\operatorname{IDEF}(0)$, which allows for the identification of the different processes; the input and output of these processes; the policies, frameworks, constraints, and the (archival and institutional) requirements that control the processes; and finally the mechanisms and resources that are necessary to perform the processes. One should be aware that the very choice of the modelling technique already brings with it some limitations, in this case, its process-oriented view. It is necessary to identify these limitations in order to assess whether the technique is suitable or not. ${ }^{37}$ In the InterPARES 1

35 Luciana Duranti, ed., The Long-term Preservation of Authentic Records: Findings of the InterPARES Project, Archilab, Saggistica/Strumenti no. 5 (2005), p.14, http://www. interpares.org/book/index.cfm.

36 For a full report on the work of the Preservation Task Force, see Duranti, The Long-term Preservation of Authentic Records, pp.99-116 and Appendix 6, pp.293-328.

37 It would have been useful to employ an additional modelling technique that focuses more on relationships between the processes and the (meta)data, such as Dataflow Diagrams (DFD), but due to time constraints that was not possible. Such an exercise, however, would be complementary and beneficial, not only because it would highlight 
project, an analysis of potential modelling tools was conducted to determine which would be the most suitable for the purposes of the research.

The modelling process consisted of the following main steps:

- Definition of the boundaries of the activity area to be analysed and its relationship with the environment as well as the focus or perspective to be taken. The resulting diagram is called a "contextdiagram."

- Decomposition of the activity area into more detailed levels of processes.

- Naming and definition of all boxes and arrows, thereby documenting the model.

- Validation of the developed model.

The process of constructing the diagrams and developing the model consisted of many sessions in which the task force engaged in lively and prolonged discussions. The participants were practitioners from archival institutions and business companies, academic researchers and computer scientists, each of whom brought different perspectives to the table but were not necessarily familiar with each other's areas of expertise. The interdisciplinary character was very useful in the exchange. For example, the computer scientists could offer their domain knowledge, where the archivists might not be that familiar with computer science. On the other hand the archivists had the recordkeeping domain knowledge required to delineate the preservation function, which was not well known to the computer scientists. These discussions turned out to be very fruitful and opened up new insights and different perspectives and led to a richer picture of what the preservation function actually should look like. ${ }^{38}$

The benefit of this systematic process is that the discussion on what lower level activities are necessary in order to accomplish a given upper level activity provides a means of checking whether the upper level diagram is correct. The decomposition into further levels of detail forces the group to be more precise about what is meant by the identified activities at the higher levels. As such it also works as a consistency check. Because of this iterative process of decomposition it is possible to get a clear idea of what activities

the (meta)data perspective, but also because it would be a validity check of the process-based model.

38 The report can be found in http://www.interpares.org/book/interpares_book_f_part3. pdf. The final result is a model consisting of 17 diagrams ( 69 activities in total). 
and processes are needed in order to process the inputs and to produce and deliver the outputs at the highest level. By identifying shortcomings, the model was improved continuously. It was also very important that all changes and the underlying rationales were documented, because previously resolved issues sometimes resurfaced in later sessions and it helped to know how and why something was changed earlier.

Developing a model is one thing, but knowing whether the model is valid in different (business) contexts, is another. Since the model was developed based on the combined knowledge of a group of people, it resulted in a rather theoretical model. How does one know if it will be applicable in practice and if so, in what situations it might be best applicable? Also, how does one know whether other people can understand the model? The best way to find out is to identify one or more suitable cases, collect the necessary information and do a walkthrough of either a part of the model or of the whole model. Walkthroughs help to identify what possible gaps, errors or inconsistencies there might be. The model can then be improved accordingly. The author of one of the system development methodologies, Edward Yourdon, developed the concept of structured walkthroughs based on the work of others. ${ }^{39}$ It is itself a research method used to test system requirements for the development of information systems.

In the case of the InterPARES 1 example the objectives of a walkthrough were: ${ }^{40}$

- To test the $\operatorname{IDEF}(0)$ model for the preservation of electronic records (version 5.1) with data from a specific case - in other words, to demonstrate that there is a real-world interpretation of the model.

- To create examples of a preservation strategy, preservation action plan(s), and targeted preservation methods, linked to the body of records in an actual case.

- To specify more precisely the definition of an activity as a transformation of inputs to outputs.

39 E. Yourdon. Structured Walkthroughs, 4th ed. Englewood Cliffs, NJ: Yourdon Press, 1989. The state of the art is described in D. Freedman and G. Weinberg. Handbook of Walkthroughs, Inspections and Technical Reviews, 3rd ed. New York: Dorsett Home Publishing, 1990.

40 The version of the Preservation Function model that was tested here was version 5.1 (slightly different from the published version 6.0). For the report on the walkthrough see Duranti, Long-term Preservation of Authentic Records, Appendix 7 of InterPARES 1 report, pp.329-355 and http://www.interpares.org/book/interpares_book_p_app07.pdf. 
- To identify the data elements of the inputs and outputs with reference to activities represented in the model. ${ }^{41}$

In InterPARES 2 the focus of research shifted to developing an understanding of records generated by experiential, interactive and dynamic systems, including their process of creation and their present and potential use in different contexts. The research team was of a highly interdisciplinary nature and consisted of representatives from record creating organisations, IT and computer experts, recordkeeping professionals and information science specialists as well as scholars in the arts. Three research domains were established, respectively on records creation, authenticity and methods of appraisal and preservation, each of them in three different focus areas artistic, scientific and government activities. Alongside the work in these domains and focus areas, four cross-domains addressed terminology, policy, description and modelling, respectively. For each of the focus areas research data were collected through case studies. The results of the domain and focus areas became the basis for the work done in the cross-domain groups. The modelling cross-domain also used the appraisal and preservation models developed by InterPARES 1 in an attempt to build a single, unified model. In the end this research in fact resulted in two distinct views that were expressed in two different models, the Business-driven Recordkeeping Model (Figure 21.3), which focused on the business activity and related records, and the Chain of Preservation Model (Figure 21.4), which focused on the record and its management. ${ }^{42}$ Both of these models also served as important inputs for the description cross-domain, which sought to identify what metadata is needed to ensure the creation and preservation of authentic electronic records. The activities identified in the models helped to specify what metadata needed to be created, when, how, where and by whom. One of the results was an initial metadata specification model for the Chain of Preservation Model..$^{43}$

To illustrate the two different perspectives represented in the Businessdriven Recordkeeping Model and the Chain of Preservation Model, I have selected the high level process diagrams for each of the two models in $\operatorname{IDEF}(0)$. Figure 21.3 presents the three identified main processes of the Business-driven Recordkeeping Model: Manage Business Framework, Carry

41 See Duranti, Long-term Preservation of Authentic Records, Appendix 7 of InterPARES 1 report, pp.329-355 and http://www.interpares.org/book/interpares_book_p_app07.pdf.

42 See http://www.interpares.org/ip2/ip2_models.cfm.

43 More on this topic, see Duranti and Preston, International Research on Permanent Authentic Records in Electronic Systems (InterPARES2), 340-341. 
out Business Activity, and Manage Records. In the Chain of Preservation Model (Figure 21.4), the main identified processes are: Manage Framework for Chain of Preservation, Manage Records in a Record-making System, Manage Records in a Recordkeeping System, and Manage Records in a Permanent Preservation System.

In the first model, the focus is on the role of records in business activities, including managing records over time. In the second model the focus is the management of the records over time, while business activities are represented as part of the context of this recordkeeping activity. Because the view is different, the high level functions are different, namely "manage business" and "manage chain of preservation." This difference in focus, therefore, leads to a different decomposition. Does this mean that either of the models is, so to speak, "better" than the other? No, not necessarily, because that depends on what one wants to achieve with a model. The models could very well be complementary. However, because the worldviews behind the two models are very different and in fact incommensurate, the application of each model will lead to different recordkeeping approaches or solutions. Conversely, modelling also exposes how different worldviews exist, or might exist. In order to make the results of applying these models comparable it would be necessary to explore how they could be reconciled. One approach could be to assess ways of relating them to each other. For example one could say the Chain of Preservation model focuses on the 'Manage Records'-activity in the BDR-model. From this perspective it would be possible to compare the application outcomes. Another example is the difference between metadata models with 1 or more entities, such as flat models like Dublin Core or the ISAD/G with basically one entity, and the SPIRT Recordkeeping metadata model with in principle 5 entities. ${ }^{44}$ Flattening the SPIRT model and reducing the number of entities could be one approach to reconciling it with Dublin Core or $\operatorname{ISAD}(G){ }^{45}$

These examples illustrate how important it is to be clear about what the objectives are for developing the model as well as what exactly it is that one wants to analyse. This will help to identify what perspective should be taken and what the most suitable modelling method would be. It will also determine what the resulting model will look like.

44 For Dublin Core see http://dublincore.org/documents/abstract-model/. For ISAD (G) see http://www.icacds.org.uk/eng/ISAD(G).pdf . For the SPIRT Recordkeeping metadata model, see http://www.ica.org/sites/default/files/CBPS_2000_Guidelines_ ISAD\%28G\%29_Second-edition_EN.pdf.

45 This is for instance done in ISO 23081 Part 2 (2009), clause 6.3 by providing some guidelines on this issue. 
Figure 21.3. The Business-driven Recordkeeping Model

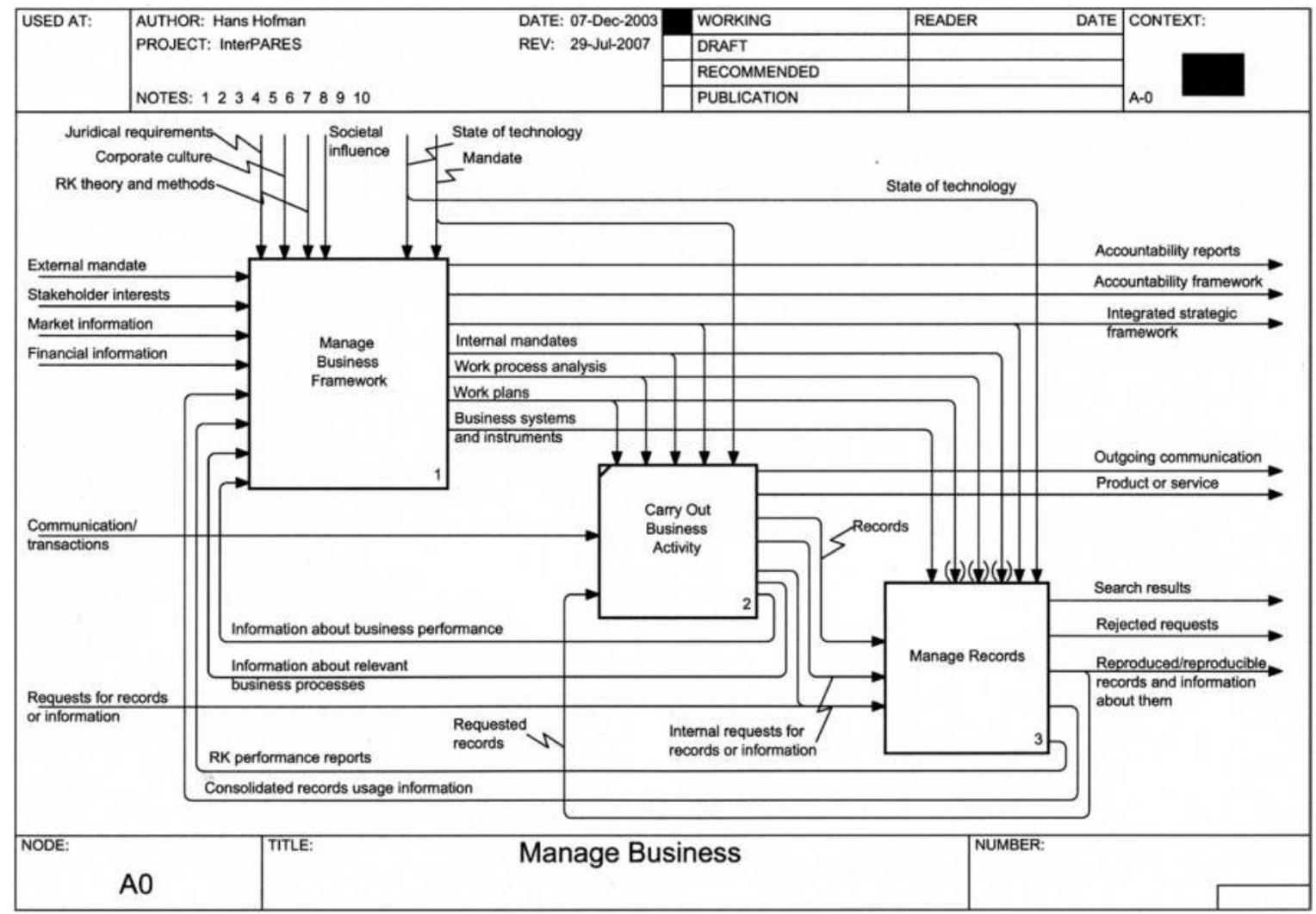


Figure 21.4: The Chain of Preservation Model

The many arrows make the diagram rather complex and difficult to understand. The focus here, however, is on the boxes as the identified activities.

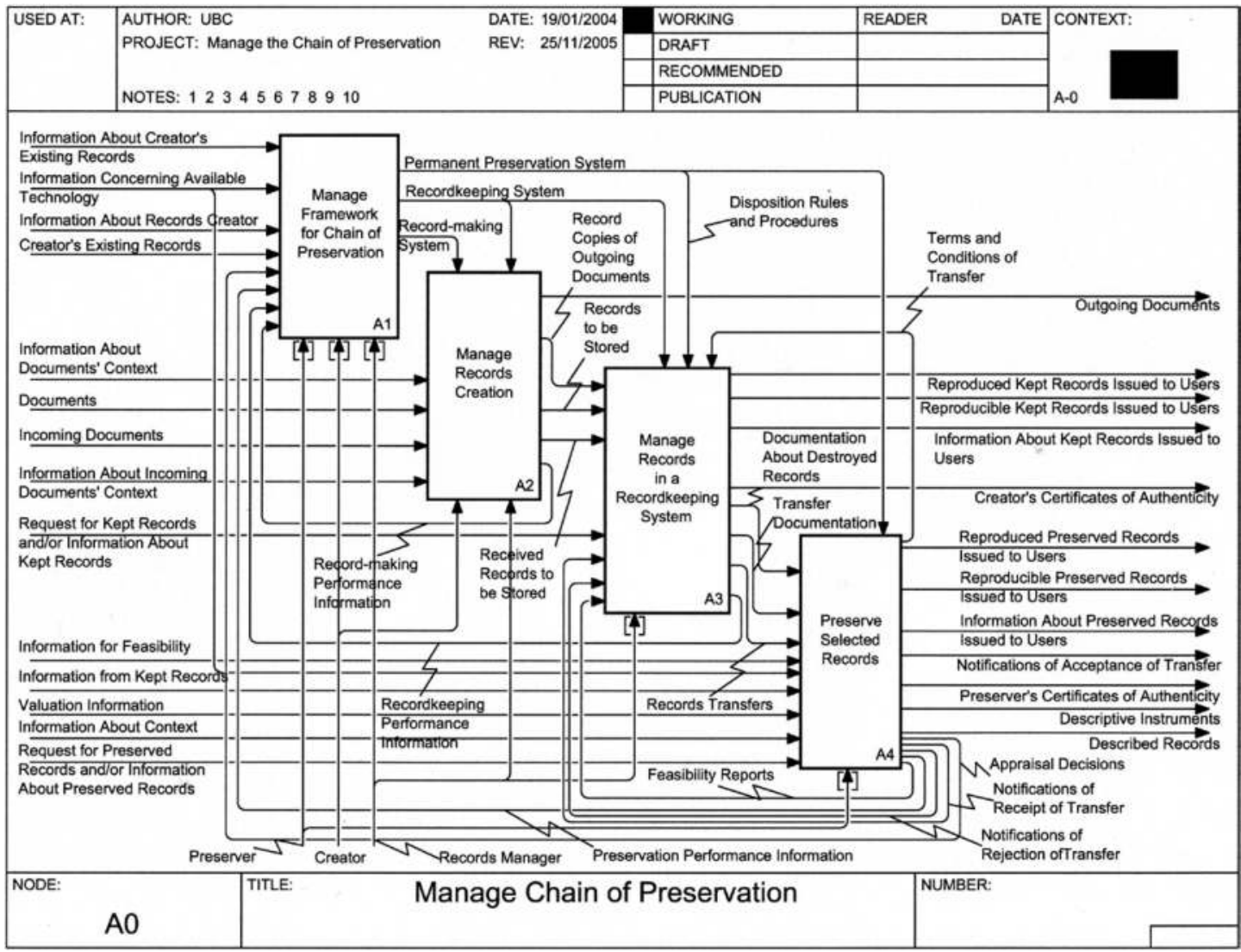


The figures also illustrate the way in which decomposition was used in the developments of the models. From the high level of defining the domain in its context, you proceed from each activity into a lower level of decomposition, like opening up a Russian Matruschka nesting doll, until an activity cannot be decomposed any further. Most modelling methods (e.g., UML, $\mathrm{DFD}, \operatorname{IDEF}(0)$ ) support decomposition of components (i.e., processes, data objects) into lower levels without losing the higher level context. Each of the boxes in the diagrams above can be further decomposed, thus explaining every time again in more detail what is needed to carry out/achieve the higher level activity.

Increasingly, modelling methods are supported by specific software tools. Although the modelling exercise can be done on paper, the advantage of these software tools is that they have integrated mechanisms for checking the integrity and consistency of what has been done. The sophistication of the tools, however, means that they are not always easy for the non-expert to work with, although their useability may be improved over time. Another important consideration in selecting a modelling tool is the possibility of migrating from one version of the modelling software to the next, as well as of exporting models to other information systems. The latter is a problem in the sense that one can lose the functionality or behaviour of the model. In the case of the InterPARES project, the $\operatorname{IDEF}(0)$ models were exported by transforming them into a fixed format (PDF) in order to be published and available to a broader audience. Only within the originating context can they be adapted and maintained if required. ${ }^{46}$

Models are not cast in stone. They will have to evolve over time as thinking and knowledge progress, and also in response to developments in technology and/or society. The more abstract the model, the longer it potentially will last. However, conceptual models are also situated in time/space, and are reflections of what people know, what technologies and/or infrastructures enable, and what juridical requirements allow at any specified moment. ${ }^{47}$ So the question when using a model always has to be, is it still relevant or suitable? Models, therefore, need to be maintained and updated. In order to

46 There are so many modelling tools that it would require an article in itself to discuss them in sufficient detail. Examples of specialist tools are System Architect and Enterprise Architect which support several modelling techniques, and BPWIN for BPM or ERWIN for data modelling, but there are many more. A simplified tool is MS Visio.

47 See for instance Frank Upward, Sue McKemmish, and Barbara Reed, "Archivists and Changing Social and Information Spaces: A Continuum Approach to Recordkeeping and Archiving in Online Cultures," Archivaria 72 (2011): 203. They state for instance that "an archival document exists differently in spacetime and can never be experienced in all complexity by a witness at any one point." This will be equally true for any model. 
achieve this, models need to be documented properly, which will usually be the case if specific modelling methods have been used, because they require certain types of documentation and must abide by particular rules and conventions for it.

\section{Conclusion}

This article provides a first introduction to the "wonderful world" of modelling as a recordkeeping research method. It has barely scratched the surface of this broad domain, identifying only a few types of models and modelling techniques, but there are many more out there. ${ }^{48}$ To what extent they may be useful in recordkeeping contexts in research and in practice is still to be further explored. The modelling methods and efforts discussed here provide, however, an idea of how modelling has been and could be used for researching and analysing recordkeeping, both at theoretical and practical levels.

In summary, there are important reasons to use modelling more widely in a recordkeeping context, ranging from analysing different recordkeeping dimensions in order to get a better understanding of theoretical principles, to the development and testing of concepts and practical instruments that will support implementation of proper recordkeeping functionality in a continuously evolving digital environment. It is also a powerful way of communicating across disciplines in both the academic and business world.

\section{References}

Business Dictionary.com, http://www.businessdictionary.com/definition/model.html. Callaos, Nagib. "The Essence of Engineering and Meta-engineering: A Work in Progress," http://www.iiis.org/Nagib-Callaos/Engineering-and-Meta-Engineering/.

Chonoles, Michael J. \& James A. Schardt. UML 2.0 for Dummies, Hoboken, NJ: Wiley, 2003.

The Clever Recordkeeping Metadata Project, http://infotech.monash.edu/research/groups/ $\mathrm{rcrg} / \mathrm{crkm} /$ outcomes.html.

Consultative Committee for Space Data Systems. Open Archival Information System Reference Model (ISO 14721:2012), http://public.ccsds.org/publications/BlueBooks. aspx?RootFolder=http\%3a\%2f\%2fpublic\%2eccsds\%2eorg\%2fpublications $\% 2$ farchiv e\%2fForms.

48 An example is the work of the Object Management Group (OMG), which published and maintains the UML, but also other standards such as Records Management Services (RMS, v.1.0, published April 2011, http://www.omg.org/spec/RMS/1.0), case management, agent metadata model, and Business Process Model and Notation (BPMN). See for more information and specifications http://www.omg.org/spec/. 
Cook, Terry. "Archival Science and Postmodernism: New Formulations for Old Concepts." Archival Science, 1 no.1 (2001): 3-24.

Duranti, Luciana, ed. The Long-term Preservation of Authentic Records: Findings of the InterPARES Project, Archilab, Saggistica/Strumenti no 5, 2005, pp.99-116 and Appendix 6, pp.293-328, http://www.interpares.org/book/index.cfm.

Duranti, Luciana, and Randy Preston, eds. International Research on Permanent Authentic Records in Electronic Systems (InterPARES2). Experiential, Interactive and Dynamic Records, Padova 2008, pp.225-304, Appendix14, pp. 498-590, and Appendix 15, pp. 591-653, http://www.interpares.org/ip2/book.cfm.

Evans, Joanne, Sue McKemmish and Karuna Bhoday, "Create Once, Use Many Times: The Clever Use of Recordkeeping Metadata for Multiple Archival Purposes." Archival Science 5 no.1 (2005): 17-42.

Evans, Joanne, Barbara Reed and Sue McKemmish. "Interoperable Data: Sustainable Frameworks for Creating and Managing Recordkeeping Metadata." Records Management Journal 18 no.2, (2008): 115-129.

Freedman, D. and G. Weinberg. Handbook of Walkthroughs, Inspections and Technical Reviews, 3rd Ed. New York: Dorsett Home Publishing, 1990.

Gilliland, Anne and Sue McKemmish. "Building an Infrastructure for Archival Research,” Archival Science 4, nos.3-4 (2004): 149-199.

Gilliland, Anne and Sue McKemmish. "Introduction." Archival Science 4 nos.3-4 (2004): 143-147.

Hanrahan, Robert P. “The IDEF Process Modeling Methodology,”1995, http://www. sba.oakland.edu/faculty/mathieson/mis524/resources/readings/idef/idef.html.

InterPARES website, www.interpares.org.

McKemmish, Sue, Glenda Acland, Nigel Ward and Barbara Reed. "Describing Records in Context in the Continuum: The Australian Recordkeeping Metadata Schema.” Archivaria 48 (2000): 3-43.

The Object Management Group (OMG), http://www.omg.org.

The Preservation of the Integrity of Electronic Records website, http://interpares.org/ UBCProject/intro.htm.

SPIRT Recordkeeping Metadata Project, http://www.infotech.monash.edu.au/research/ groups/rcrg/projects/spirt/index.html

Stanford Encyclopedia of Philosophy, Summer 2009 edition, http://plato.stanford.edu/ archives/sum2009/ntries/models-science/\#RepModIModPhe.

Upward Frank, Sue McKemmish and Barbara Reed. "Archivists and Changing Social and Information Spaces: A Continuum Approach to Recordkeeping and Archiving in Online Cultures." Archivaria 72 (2011): 197-237.

Upward, "Structuring the Records Continuum - Part One: Postcustodial Principles and Properties." Archives E Manuscripts 24 no.2 (1996): 268-285.

Yourdon, E. Structured Walkthroughs, 4th Ed. (Englewood Cliffs, NJ: Yourdon Press, 1989). 


\title{
CAPACITIES AND COMPLEXITIES
}

\author{
A Reflection on Design Methodologies \\ for Archival and Recordkeeping Research
}

\author{
Joanne Evans
}

\begin{abstract}
Digital and networking technologies bring into question not only traditional theories and practices, but also how we go about formulating and framing our research response to them. Dealing with emergent phenomena calls for us to take a more proactive stance in formulating and addressing our research problems and questions. This brings design methodologies and systems development methods to the forefront of theory - as well as systems-building. Studying how recordkeeping and archiving capabilities are built into new kinds of information systems has the potential to enrich our understanding, as well as progress their instantiation in processes, technologies and tools. Design research methodologies are being embraced in a number of disciplines to explore interplays between theory, practice and technological interventions. The integrated goals of practical problem solving and theory building and explanation have the potential to deliver rigorous and relevant research and practice outcomes, especially when faced with the social and technologically complexity of 'wicked problems'. This paper will explore the evolution and use of design research methodologies, and provide a commentary on their capacity to examine complex and/or emergent phenomena within the archival multiverse in multiple cultural, social and organisational contexts, and disciplinary, professional, and community settings.
\end{abstract}

\section{Introduction}

We live in exciting and confronting times with digital and networking technologies raising many profound challenges for archival practice, theory 
and research. These challenges extend beyond the need for mere technical adjustments as new information technologies transform the way we personally and professionally communicate and interact in our everyday lives, and reshape organisational and societal strictures and structures, including the forms, features and configurations of our archives. Much has been done, and will continually need to be done, in order for us to understand, harness and exploit the capabilities of new information technologies for archival and recordkeeping endeavours in a myriad of contexts - some old, mature and traditional, others new, emergent and transformational. It will not only require rigorous and relentless interrogation, critique, re-imagining and re-figuring of our theories and practices, but also of the formulations and frameworks which we use to investigate them. ${ }^{1}$

A key imperative for the discipline and the profession is to facilitate the development of archival and recordkeeping infrastructure for a digital and networked information era. Our role is to provide frameworks and facilities to enable the transmission of recorded information through space and time as evidence of activities; evidence that forms, shapes and informs personal, organisational, community, institutional and societal memory. ${ }^{2}$ This information infrastructure, a network of 'pervasive enabling resources', ${ }^{3}$ should support recordkeeping and archiving in and across local, global, public, private, micro and macro domains. Enabling complex interconnected networks of technology, data, information, processes, procedures and people to ensure records that serve as evidence of social and business activities are created, maintained and made accessible for as long as required lies at the heart of our professional endeavours. ${ }^{4}$ This infrastructure encompasses not just the tangible and physical components, but also the intangible and

1 Anne Gilliland and Sue McKemmish, "Building an Infrastructure for Archival Research,” Archival Science 4 no. 3 (2004): 149-97; Anne Gilliland and Sue McKemmish, "Archival and Recordkeeping Research: Past, Present and Future," in Research Methods: Information, Systems and Contexts, ed. Kirsty Williamson and Graeme Johanson (Prahran, Victoria, Australia: Tilde University Press, 2013), 79-103.

2 Terry Cook, "Evidence, Memory, Identity, and Community: Four Shifting Archival Paradigms," Archival Science 13 no. 2-3 (2013): 95-120.

3 Geoffrey C. Bowker et al., "Toward Information Infrastructure Studies: Ways of Knowing in a Networked Environment," in International Handbook of Internet Research, ed. Jeremy Hunsinger, Lisbeth Klastrup, and Matthew Allen (Springer, 2010), 97-117, http://interoperability.ucsd.edu/docs/07BowkerBaker_InfraStudies.pdf.

4 David Bearman, "Record-Keeping Systems," Archivaria 36 (Autumn 1993): 16-37;

Sue McKemmish, "Understanding Electronic Recordkeeping Systems: Understanding Ourselves," Archives and Manuscripts 22 no. 1 (May 1994): 150-62. 
intellectual mechanisms through which they are instantiated and connected. We are also now much more conscious of our own hand in shaping that evidence, contributing to what gets remembered and what gets forgotten. ${ }^{5}$

Continuum conceptualisations of recordkeeping offer a way of making sense of all this, particularly with the emergence of increasingly complex digital information ecologies. ${ }^{6}$ From this perspective records creation, preservation and dissemination are intrinsically and intricately entwined in acts of recordkeeping that defy easy separation and segregation. Continuum thinking is thus a driver of substantive transformations of archival and recordkeeping systems and practices. It re-figures archival roles and responsibilities from managing 'dead records' in archival repositories ${ }^{7}$ to providing infrastructures that enable sustainable and scalable recordkeeping and archiving processes in and across personal, organisational, community and societal systems. ${ }^{8}$ It calls for a move from siloed, segregated, and singular object management systems to open interconnected, integrated, integratable, and sustainable networks of evidential and memory management systems. This infrastructure requires a combination of support for embedding archival and recordkeeping capabilities into business and social systems, as well as the development of systems that support the processes for which archivists and other recordkeeping professionals have particular responsibility. ${ }^{9}$ As a consequence the need for knowledge and skills in designing and developing, along with implementing and managing, these kinds of systems within the archival field has come to the fore.

But this is also not just about technological design. The disruptions caused by digital and networking information technologies have played a part in opening up a broader discourse on archives and recordkeeping in

5 Cook, "Evidence, Memory, Identity, and Community".

6 Frank Upward et al., "Recordkeeping Informatics: Re-Figuring a Discipline in Crisis with a Single Minded Approach," Records Management Journal 23 no. 1 (March 2013): 37-50, doi:10.1108/09565691311325013.

7 Chris Hurley, "Parallel Provenance (If These Are Your Records, Where Are Your Stories?),” 2005, http://infotech.monash.edu/research/groups/rcrg/publications/parallelprovenance-combined.pdf.

8 Joanne Evans, Barbara Reed, and Sue McKemmish, "Interoperable Data: Sustainable Frameworks for Creating and Managing Recordkeeping Metadata," Records Management Journal 18 no. 2 (June 13, 2008): 115-29, doi:10.1108/09565690810882977.

9 Hans Hofman, "Rethinking the Archival Function in the Digital Era" (International Council of Archives Congress 2012, Brisbane, 2012), http://www.ica2012.com/files/ data/Full\%20papers\%20upload/ica12final00187.pdf; Barbara Reed, "Service-Oriented Architectures and Recordkeeping," Records Management Journal 18 no. 1 (2008): 7-20, doi:10.1108/09565690810858488. 
society, and a critique of professional structures, roles and responsibilities. As the power of archives to enfranchise, disenfranchise, empower and disempower has been laid bare, a further challenge is to enable archival practices and systems to better reflect and represent a multiplicity of voices and viewpoints. A neat alignment of documents in files in boxes on shelves belies the complexity of the lives and messiness of the actions contained therein. ${ }^{10}$ To acknowledge this complexity, to embrace the disorder in the order, to make the connections and the disconnections, to reveal the presences and the absences, requires frameworks which continually bring pasts into the present, capture rich and varied representations of the here and now into the future, and invite and sustain rather than limit participation.

In the early days of computing we might have been able to get away with a 'fast paper' approach, viewing electronic records as mere digital versions of their paper counterparts and automating paper-based records and archives management practices and processes. Within this discourse on electronic records in the latter part of the 20th century some pointed to a more profound change - a recognition of the ways in which digital information and digital information systems were reshaping processes and structures,${ }^{11}$ and rescaling recordkeeping and archival challenges. ${ }^{12} \mathrm{It}$ is now abundantly clear that the transformations in moving to digital and online activities and cultures are deeper and more profound; they are no longer just gathering pace but are now well and truly upon us. Not only do we have to be able to cope with new documentary forms and formats but that they are also born networked, rather than just born digital, and born outside the bounds of traditional organisational controls and boundaries. We therefore need archival and recordkeeping technologies for the multiverse - integrated (or at the very least integrate-able) systems, processes and tools to create, capture, manage and make accessible a multiplicity of record forms, formats and contexts. And we need them now. Many feel that if we continue to cling to traditional processes and practices configured for a paper paradigm,

10 My colleague Shannon Faulkhead alerted me to the idea of the complexity and messiness in archival arrangement and organisation, when looked at from the perspective of the 'subjects' of records.

11 Terry Cook, "Electronic Records, Paper Minds: The Revolution in Information Management and Archives in the Post-Custodial and Post-Modernist Era," Archives and Manuscripts 22 no. 2 (November 1994): 300-329.

12 David Bearman, Archival Methods, Archives and Museum Informatics Technical Report \#9 (Pittsburgh, 1989), http://www.archimuse.com/publishing/archival_methods/index. html; Bearman, "Record-Keeping Systems." 
we risk becoming increasing irrelevant and may be left facing professional obsolescence. ${ }^{13}$

But with our archival and recordkeeping infrastructures designed and developed around the characteristics and needs of paper recordkeeping systems and institutional memory management frameworks, how do we transform them for a distributed, dynamic, digital and networked world? How do we ensure capacities to create and keep authentic, accurate, reliable evidence of activities in the form of records and archives are adequately incorporated into emerging micro and macro systems, processes and structures? How do we take advantage of new technological capabilities to do this? How do we enable their support of an archival multiverse with multiple ways of knowing and practicing co-existing, intersecting and integrating where appropriate? Diverging and differentiated where not appropriate? How do we design and develop better archival and recordkeeping infrastructure to support individual, community, organisational and societal needs? What do we need to know? What do we need to know how to do?

Social and technological complexity and uncertainty suggests that we are faced with a wicked problem - characterised by indeterminacy, plurality, temporality and interdependencies that defy definitive solution. ${ }^{14}$ While initially used to characterise the complexity facing social policy planners, the concept has been utilised in other areas, particularly to capture degrees of intractability in designing and developing socio-technical systems. The term has been used previously to characterise the problems facing software engineers as they design and build new information systems ${ }^{15}$ and more recently in describing the archival challenges in preserving social media. ${ }^{16}$

13 Cook, "Electronic Records, Paper Minds: The Revolution in Information Management and Archives in the Post-Custodial and Post-Modernist Era"; David Bearman,

Electronic Evidence: Strategies for Managing Records in Contemporary Organizations (Pittsburgh: Archives \& Museum Informatics, 1994); Barbara Reed, "Reinventing Access," Recordkeeping Roundtable, August 1, 2014, http://rkroundtable.org/2014/08/01/ reinventing-access/; Kate Cumming and Anne Picot, "Reinventing Appraisal," Recordkeeping Roundtable, August 1, 2014, http://rkroundtable.org/2014/08/01/ reinventing-appraisal/; Joanne Evans, Sue McKemmish, and Barbara Reed, "Making Metadata Matter: Outcomes from the Clever Recordkeeping Metadata Project.," Archives and Manuscripts 37 no. 1 (May 2009): 28-56; Upward et al., "Recordkeeping Informatics."

14 Horst W. J. Rittel and Melvin M. Webber, "Dilemmas in a General Theory of Planning," Policy Sciences 4 no. 2 (June 1973): 155-69.

15 Peter DeGrace and Leslie Hulet Stahl, Wicked Problems, Righteous Solutions: A Catolog of Modern Software Engineering Paradigms, 1st ed. (Prentice Hall, 1990).

16 Lisa P. Nathan and Elizabeth Shaffer, "Preserving Social Media: Opening a MultiDisciplinary Dialogue" (The Memory of the World in the Digital age: Digitization and 
It is argued that scientific and reductionist thinking, along with linear design process models, are unsuited for tackling these kind of problems. ${ }^{17}$ Addressing the wicked problem of supporting an archival multiverse requires systemic solution-seeking, incorporating abilities to identify and handle multiplicities, contradictions, and contested ideas both in, and through, time and space.

\section{Introductory Reflections}

My archival career has certainly been intimately and intricately shaped by the imperatives and opportunities of digital and networking technologies and the wickedness of recordkeeping and archival infrastructure problems. It began back in 1995 at the Australian Science Archives Project (ASAP), an initiative of the History and Philosophy Department of the University of Melbourne, to foster the better representation of records relating to Australian science, medicine and technology in archival repositories. ${ }^{18}$ It involved the application of the Australian Series System principles and model, using relational database technologies, to the development of an archival documentation management system to manage a large-scale archival processing project as government owned brown coal mines and electricity generators were privatised. ${ }^{19}$ This was followed by subsequent diffusion of the resultant archival database system into other processing contexts and incorporation of web capabilities as the 'information superhighway'20 emerged. This then led to the development of a new archival software system for documenting and publishing contextual knowledge networks as gateways to archival, published, and other kinds of documentary resources. ${ }^{21}$

Preservation, Vancouver, British Columbia, Canada: UNESCO, 2012), http://www. unesco.org/new/fileadmin/MULTIMEDIA/HQ/CI/CI/pdf/mow/VC_Nathan_ Shaffer_27_B_1140.pdf.

17 Richard Buchanan, "Wicked Problems in Design Thinking," Design Issues 8 no. 2 (April 1992): 5-21, doi:10.2307/1511637; DeGrace and Stahl, Wicked Problems, Righteous Solutions.

18 Joanne Evans, "Designing Dynamic Descriptive Frameworks," Archives and Manuscripts 42 no. 1 (March 2014): 5-18, doi:10.1080/01576895.2014.890113.

19 Joanne Evans, "Structure of the ADS," in Archives and Reform - Preparing for Tomorrow, Proceedings of the Australian Society of Archivists Conference, Adelaide, 25-26th July 1997, 1997, http://www.asap.unimelb.edu.au/pubs/articles/asa97/ADSStructure.htm.

20 Information Superhighway was the term used in the 1990s for the Internet. Used by Al Gore it had particular relevance as a moniker as the World Wide Web exploded into being.

21 Joanne Evans, "Exploring Bright Sparcs: Creation of a Navigable Knowledge Space," Cataloguing Australia 24 no. 1-4 (December 1999): 147-57; Gavan McCarthy and Joanne Evans, "The Open Resource Scholarly Network: 
My system design and development work has been extensively shaped by continuum and post-custodial discourses in the Australian and international archival community. ${ }^{22} \mathrm{~A}$ post-custodial perspective places intellectual control over distributed networks of records (i.e. archives), rather than just the caretaking of records in custody, as the focus and scope of archival and recordkeeping endeavours. ${ }^{23}$ These ideas are embodied in what has become known as the Australian Series System, a model of description and control applicable to records throughout their lifespan, rather than just those in the hands of an archival institution. ${ }^{24}$ At its heart lies a relational model that separates, and then interrelates, descriptions of records with descriptions of contexts in which they are created, managed and used. Its potential lies in its capacity to enable ongoing documentation of rich and multi-faceted layers of contexts surrounding records in order to facilitate their management and their meaning as they move through space and time.

In such a climate, ASAP (and its successor incarnations) was a place for exploring the tools and technologies of a post-custodial archival organisation, and part of the Australian community of practice exploring recordkeeping and archiving from a continuum perspective..$^{25}$ ASAP was not an archival repository. Its role was to facilitate the transfer of records relating to Australian science, medicine and technology into archival institutions, and provide integrated discovery services to this distributed archive. We were implementing and testing Australian Series System

New Collaborative Partnerships Between Academics, Libraries, Archives and Museums," in VALA 2002: Evolving Information Futures, 11th Biennial Conference \& Exhibition 6-8 February 2002 (VALA 2002: Evolving Information Futures, Melbourne, 6-8 February 2002, Melbourne, Australia: Victorian Association for Library Automation, 2002), 197-207, http://www.vala.org. au/vala2002/2002pdf/15McCEva.pdf; Gavan McCarthy and Joanne Evans, "Mapping the Socio-Technical Complexity of Australian Science: From Archival Authorities to Networks of Contextual Information," Journal of Archival Organization 5 no. 1-2 (2008): 149-75, doi:10.1300/J201v05n01_08; Gavan J. McCarthy and Joanne Evans, "Principles for Archival Information Services in the Public Domain," Archives and Manuscripts 40 no. 1 (2012): 54-67, doi:10.1080/015 76895.2012.670872.

22 Frank Upward and Sue McKemmish, "Somewhere Beyond Custody," Archives and Manuscripts 22 no. 1 (May 1994): 138-47.

23 Frank Upward, "Structuring the Records Continuum: Part One: Postcustodial Principles and Properties," Archives and Manuscripts 24 no. 2 (November 1996): 268-85.

24 Chris Hurley, "The Australian ('Series') System: An Exposition," in The Records Continuum: Ian Maclean and Australian Archives First Fifty Years, ed. Sue McKemmish and Michael Piggott (Clayton: Ancora Press, 1994), 150-72.

25 Sue McKemmish, "Placing Records Continuum Theory and Practice," Archival Science 1 no. 4 (2001): 333-59, doi:10.1007/BF02438901. 
concepts, demonstrating their applicability outside of government archival institution circles and exploring their practical realisation in a post-custodial framework. We were asking questions as to how the capabilities of the new digital and networking technologies were transforming what we did and what we knew about records and archives. For me, iteratively and reflectively designing, developing and deploying archival systems has become a way of learning, knowing, conceptualising and developing theoretical and practical understanding.

\section{Design Research}

Research approaches based solely on the study of existing recordkeeping technologies, practices and systems are not well suited to dealing with the kinds of questions regarding the needs of new archival infrastructures raised above. Archival and recordkeeping systems are social constructions, manmade and artificial, products of countless contextual contingencies. They are designed and shaped by a multitude of indivisible social and technical practices, which then, in turn, reflect structure. This duality of action and structure, with neither having primacy, is a core principle of Giddens' Structuration Theory, ${ }^{26}$ a theory to make sense of how social systems are created and reproduced, which has been encompassed in continuum conceptualisations of recordkeeping. ${ }^{27}$ While better understanding of extant systems can contribute to conceptualising archival and recordkeeping systems of the future, this alone will not produce the kind of innovation that is needed to transform our practices and systems into the emerging digital and networked information age, and enable them to be reflective of more than just a single or dominant narrative.

Design thinking and development approaches have been identified as a possible way to address wicked problems given that embracing uncertainty and ambiguity is part of their nature, thus providing a framework to enable exploration of the problem space and the development of a dialog with potential solutions. ${ }^{28}$

26 Jon Clark, Celia Modgil, and Sohan Modgil, eds., Anthony Giddens: Consensus and Controversy, 1st ed. (Falmer Press, 1990).

27 Frank Upward, "Structuring the Records Continuum: Part Two: Structuration Theory and Recordkeeping," Archives and Manuscripts 25 no. 1 (May 1997): 10-35.

28 Erik Stolterman, "The Nature of Design Practice and Implications for Interaction Design Research," International Journal of Design 2 no. 1 (2008): 55-65. 
No prescribed linear process will work with a wicked problem. In part because the design effort must start producing robust solutions just to illuminate the hidden issues and flush out the hidden stakeholders. ${ }^{29}$

The multiplicity of stakeholders and perspectives encompassed by wicked problems is also leading to a recognition that this dialog needs to be collaborative and participatory.

The expertise which you need in dealing with a wicked problem is usually distributed over many people. Those people who are the best experts with the best knowledge are usually those who are likely to be affected by your solution. ${ }^{30}$

Better understanding and articulation of a problematic situation and the new interventions that may lead to improvements are the desired mutually constituted outcomes of these kinds of design and development processes. It is this blend of the practical and theoretical, and the need to deal with the yet unknown, which has seen research communities seeking to understand and more effectively manage the impact of new information technologies on processes, practices and systems turn to design and development methodologies. What is it about doing design and development in a research context, rather than just studying it in practice, that has the potential to enrich our understanding? Why might such methods be particularly relevant for examining complex and/or emergent phenomena within the archival multiverse in multiple cultural, social and organisational contexts, and disciplinary, professional, and community settings?

Designing is a process of transforming an idea into some kind of material form. It is a purposeful activity where the intent in the transformation of the idea is generally to make a useful difference to the situation or task at hand. It is a multi-faceted, recursive, iterative and reflexive activity. It involves multiple modes of data/information gathering including observing, conceptualising, analysing, doing, synthesising, evaluating and reflecting, within overarching development cycles. It is a complex, structured, creative problem solving activity applicable not just to how manufactured products

29 Jeff Conklin, Min Basadur, and GK VanPatter, "Rethinking Wicked Problems: Unpacking Paradigms, Bridging Universes," NextD Journal Ten, no. Conversation 10.1 (2007), http://humantific.com/wp-content/uploads/2009/07/NextD_10/ NextD_10_1.pdf.

30 Horst W. J. Rittel, "On the Planning Crisis: Systems Analysis of the 'First and Second Generations,” Bedriftsokonomen 8 (1972): 394. 
look, but how systems and services function, perform and impact. ${ }^{31}$ In design and development processes, prior knowledge is built upon, developed and transformed as conceptualisations are materialised.

The latter half of the 20th century has seen the emergence of design as a distinct academic discipline, based on a "designerly way of knowing" distinguished from cultures of inquiry in science and the humanities. ${ }^{32}$ Design, as a culture of inquiry, studies artificial phenomena, using methods of 'modelling, pattern-formulation, [and] synthesis', with 'practicality, ingenuity, empathy and a concern for appropriateness' being valued. ${ }^{33}$ Design takes on 'ill-defined, ill-structured or 'wicked' problems' for 'which [not] all the necessary information is, or ever can be [made] available to the problem-solver. ${ }^{34}$ It is a solution-focused investigative strategy, often involving re-casting, re-figuring and changing the way of thinking about what is the problem in the quest for its (re)solution. ${ }^{35}$ While designing involves 'a synthesis of knowledge and skills from both the sciences and the humanities, in pursuit of practical tasks' it also goes beyond their mere application. ${ }^{36}$ Designing has the potential to generate new understanding of the design problem, solution, processes and/or materials, with this new knowledge embodied in people, processes and products. ${ }^{37}$

Herbert Simon's The Sciences of the Artificial ${ }^{8}$ has played a significant part in the discourse arguing for recognition of the distinctive nature of the design discipline. While many disagree with his positivist positioning The Sciences of the Artificial is oft cited as a seminal work of design theory. ${ }^{39}$ Educational theorist Donald Schön was a particular critic of Simon's view of design as a rational problem solving process. Schön instead conceptualised design as

31 UK Department of Trade and Industry, Creativity, Design and Business Performance, November 2005, http://www.dti.gov.uk/files/file13654.pdf.

32 Bruce Archer, "Design as a Discipline," Design Studies 1 no. 1 (July 1979): 17-20, doi:10.1016/0142-694X(79)90023-1; Nigel Cross, "Designerly Ways of Knowing," Design Studies 3 no. 4 (October 1982): 221-27, doi:10.1016/0142-694X(82)90040-0; Nigel Cross, Designerly Ways of Knowing (London: Springer London, 2006), http://link. springer.com/chapter/10.1007/1-84628-301-9_1.

33 Cross, Designerly Ways of Knowing, 2.

34 Cross, Designerly Ways of Knowing, 7.

35 Cross, Designerly Ways of Knowing.

36 Cross, Designerly Ways of Knowing, 2.

37 Cross, Designerly Ways of Knowing, 100.

38 The Sciences of the Artificial (Cambridge MA: MIT Press, 1969).

39 Buchanan, "Wicked Problems in Design Thinking"; Nigel Cross, John Naughton, and David Walker, "Design Method and Scientific Method," Design Studies 2 no. 4 (October 1981): 195-201, doi:10.1016/0142-694X(81)90050-8. 
reflective practice to embrace the messiness, uncertainties, instabilities and value conflicts in many design situations. ${ }^{40}$ For Schön 'actionable theory' rather than 'covering laws' is the goal of design inquiry.

Design inquiry consists not only in creating plans but in enacting them.... The kind of generalization I have in mind is not the "covering law" variety - a general perhaps statistical, proposition applicable to all instances in which certain combinations of variables are present. It consists, rather in framing the problem of a problematic situation, and strategies of action appropriate to its solution, in such a way that both the problem and the action strategies can be carried over to new situations perceived as being similar to the first. ${ }^{41}$

Schön's emphasis is on processes of reflection-in-action, not just reflectionon-action, generating the 'practice knowledge' which becomes part of the design repertoire 'available for projection to further situations. ${ }^{42}$

Design is thus increasingly being recognised and accepted as a methodology for systematic research inquiry. As a research approach it can generate new knowledge and understanding about designing processes, the purpose, structure, form and impacts of designed artefacts, and of the situational, environmental and cultural context under investigation. It may involve the deployment of a variety of data collection techniques depending on the nature of the design problem, its situation and the phases of the design process. Design as research must also feature some use of methods (e.g., reflective and/or ethnographic techniques) to explicitly and overtly identify and communicate the research process and its results, both to enable scrutiny and to facilitate re-use. ${ }^{43}$

What might the archival field learn from the uptake of design as a research methodology in allied disciplines such as information systems, education, and library and information science? To structure this discussion I will use my own experience of first coming across design as a research approach in the Information Systems literature, before being introduced to the discourse in the Education and then Library and Information Science disciplines.

40 Kees Dorst and Judith Dijkhuis, "Comparing Paradigms for Describing Design Activity," Design Studies 16 no. 2 (April 1995): 261-74, doi:10.1016/0142694X(94)00012-3; Donald A. Schön, The Reflective Practitioner: How Professionals Think In Action (Basic Books, 1984).

41 Donald A. Schön, "The New Scholarship Requires a New Epistemology," Change, December 1995, 31, Academic OneFile.

42 Schön, "The New Scholarship Requires a New Epistemology."

43 Cross, "Special Issue Design Education." 


\section{Design Science Research in Information Systems}

Design Science Research has received increasing attention in the Information Systems discipline over the past few decades. According to Baskerville, initial discussions of applying systems development methods to Information Systems research in the early nineties, ${ }^{44}$ have matured into a discourse of Design Science Research as its own distinct research paradigm in which a variety of research methodologies, methods and techniques can be applied. ${ }^{45}$ Proponents of the approach argue that it 'directly tackles two of the key issues of the discipline: the central, albeit controversial role of the IT artefact in Information Systems research ... and the perceived lack of professional relevance of Information Systems research'. ${ }^{46}$ It has the potential to put Information Systems research at the forefront of practical innovation (i.e. 'creation of innovative artefacts to solve real-world problems') rather than being consigned to merely studying their aftermath. By taking on 'wicked problems' and embracing/embedding design and development complexities in research processes the approach could also enable greater insight into and understanding of the principles, concepts and constructs of the Information Systems discipline. It is this 'scholarly contribution to the knowledge base ${ }^{247}$ which is one of the markers that distinguish design activities as research rather than as professional design practice, ${ }^{48}$ and which has led to calls within the Information Systems community for Design Science Research to be making greater contributions. ${ }^{49}$ Thus it is both the designed artefact and

44 Jay Nunamaker, Minder Chen, and Titus D. M. Purdin, "Systems Development in Information Systems Research," Journal of Management Information Systems 7 no. 3 (Winter 1991): 89-106.

45 Richard Baskerville, "What Design Science Is Not," European Journal of Information Systems 17, no. 5 (October 2008): 441-43, doi:10.1057/ejis.2008.45; Alan R. Hevner and Samir Chatterjee, Design Research in Information Systems: Theory and Practice, 2010th ed., vol. 22, Integrated Series in Information Systems (Springer, 2010); Alan R. Hevner et al., "Design Science in Information Systems Research," MIS Quarterly 28, no. 1 (March 2004): 75-105; David Jones and Shirley Gregor, "The Anatomy of a Design Theory," Journal of the Association for Information Systems 8, no. 5 (May 1, 2007), http://aisel. aisnet.org/jais/vol8/iss5/1.

46 Hevner and Chatterjee, Design Research in Information Systems: Theory and Practice, 22:9.

47 Robert Wayne Gregory, "Design Science Research and the Grounded Theory Method: Characteristics, Differences, and Complementary Uses," 2010, http://is2.lse.ac.uk/asp/ aspecis/20100012.pdf.

48 Hevner and Chatterjee, Design Research in Information Systems: Theory and Practice, 22:15.

49 Gregory, "Design Science Research and the Grounded Theory Method: Characteristics, Differences, and Complementary Uses"; Hevner et al., "Design Science in Information Systems Research." 
the theory accounting for its purpose, structure, behaviour and functionality that needs to be articulated and communicated. ${ }^{50}$

It is acknowledged that much Design Science Research in Information Systems done to date has been situated within a positivist research tradition. ${ }^{51}$ Critiques of this reductionist positioning suggest that richer scholarly and practical contributions may be made if interpretive and social constructionist perspectives are embraced. This would allow for design complexities, constructs and contexts to be revealed and explored. Discussions of integration of Design Science Research with Action Research, ${ }^{52}$ Soft Systems Methodology, ${ }^{53}$ Grounded Theory, ${ }^{54}$ and participatory research approaches ${ }^{55}$ have resulted. If a premise of Design Science Research is to solve practical, real world problems, then approaches that do not adequately engage with socio-technical relationships in a sophisticated manner may be limited in their application relevance and/or capacity to enable practical and/or theoretical innovation.

50 Jones and Gregor, "The Anatomy of a Design Theory."

51 Gregory, "Design Science Research and the Grounded Theory Method:

Characteristics, Differences, and Complementary Uses”; Judy McKay and Peter Marshall, "Science, Design, and Design Science: Seeking Clarity to Move Design Science Research Forward in Information Systems" (18th Australasian Conference on Information Systems, 5-7 December 2007, Toowomba, 2007), http://www. swinburne.edu.au/ict/research/riso/publications/ACIS07_science_design_design_ science.pdf; Björn Niehaves, "On Epistemological Pluralism in Design Science," Scandinavian Journal of Information Systems 19 no. 2 (January 1, 2007), http://iris. cs.aau.dk/tl_files/volumes/volume19/no2/Niehaves.pdf.

52 Juhani Iivari and John Venable, "Action Research and Design Science Research - Seemingly Similar but Decisively Dissimilar,” 2009, http://www.ecis2009.it/ papers/ecis2009-0424.pdf; Pertti Järvinen, "Action Research Is Similar to Design Science,” Quality \& Quantity 41 no. 1 (February 2007): 37-54, doi:10.1007/s11135005-5427-1.

53 Richard Baskerville, Jan Pries-Heje, and John Venable, "Soft Design Science Methodology," in Proceedings of the 4th International Conference on Design Science Research in Information Systems and Technology, DESRIST '09 (New York, NY, USA: ACM, 2009), doi:10.1145/1555619.1555631.

54 Gregory, "Design Science Research and the Grounded Theory Method: Characteristics, Differences, and Complementary Uses."

55 Mark Bilandzic and John Venable, "Towards Participatory Action Design Research: Adapting Action Research and Design Science Research Methods for Urban Informatics," The Journal of Community Informatics 7 no. 3 (December 15, 2011), http:// ci-journal.net/index.php/ciej/article/view/786/804; Sue McKemmish et al., "Inclusive Research Design," Information, Communication and Society 15 no. 7 (2012): 1106-35, do i:10.1080/1369118X.2012.707225; Maung K. Sein et al., "Action Design Research," MIS Quarterly 35 no. 1 (March 2011): 37-56. 


\section{Design-Based Research in Education}

Education is another discipline where there is growing advocacy for DesignBased Research, particularly to develop 'understanding [of] how, when and why educational innovations work in practice. ${ }^{56}$ The Design-Based Research Collective argues that it enables 'the study of learning in context through the systematic design and study of instructional strategies and tools' and 'can help to create and extend knowledge about developing, enacting, and sustaining learning environments. ${ }^{57}$ Designing as an integral part of the research process allows for emergent behaviour and knowledge to be incorporated into the designed artefact and its understanding, as well as fostering meaningful practitioner and researcher partnerships and collaborations with shared agendas for change. Design-Based Research implies 'a commitment to understanding the relationships amongst theory, designed artefacts and practice', achieving practical outcomes as well as contributions to theories of learning and teaching. ${ }^{58}$ It tackles head on the situated complexity of teaching and learning activities and aims for both integrated practical and theoretical outcomes.

The intention of design-based research in education is to inquire more broadly into the nature of learning in a complex system and to refine generative or predictive theories of learning. Models of successful innovation can be generated through such work - models, rather than particular artefacts or programs, are the goal. ${ }^{59}$

Wider adoption of Design-Based Research approaches are also being urged in order to foster more socially responsible inquiry, particularly with regard to the design and application of new instructional technologies.

Instead of tinkering around the edges of teaching and learning challenges by conducting quasi-experimental studies focused on small changes in learning environments or even conducting oneoff qualitative studies of esoteric cases, instructional technology researchers and their colleagues in other academic disciplines must

56 The Design-Based Research Collective, "Design-Based Research: An Emerging Paradigm for Educational Inquiry," Educational Researcher 32 no. 1 (February 2008): 5 .

57 The Design-Based Research Collective, "Design-Based Research".

58 The Design-Based Research Collective, "Design-Based Research", 6.

59 The Design-Based Research Collective, "Design-Based Research", 7. 
begin to tackle the huge problems we face in the first quarter of the 21 st Century. ${ }^{60}$

The goal of this kind of research goes beyond just understanding how these technologies work to knowing how they contribute to making education better. A review of a decade of Design-Based Research in education suggests cautious optimism that with continued uptake there will be a move from current studies of small-scale innovations to the tackling of grander challenges. ${ }^{61}$

\section{Design-Based Research in Library and Information Science}

As in education, interplays between theory and practice, and researchers and professional practitioners are of continual discussion and debate in the Library and Information Science (LIS) community, especially when it comes to new information technologies and their far-reaching impacts on library processes, practices and structures. The use of design-based research methodologies, particularly when it comes to understanding how people interact with information and fostering the development of user-centred information systems and services, has recently been posited by Bowler and Large:

Design-based research attempts to address the call for user-centred, context-based, evidence-driven practice. It works in the field, not in the artificial confines of the laboratory, and draws upon the authentic knowledge of practitioners and users. It sets out to solve real-life problems with innovative solutions, results in a working product that has been honed to suit the actual needs of its users, and contributes explanatory models or theory. ${ }^{62}$

It is perhaps surprising given the amount of systems design and development work being undertaken by the Library and Information Science

60 Thomas C. Reeves, Jan Herrington, and Ron Oliver, "Design Research: A Socially Responsible Approach to Instructional Technology Research in Higher Education," Journal of Computing in Higher Education 16 no. 2 (2005): 110.

61 Terry Anderson and Julie Shattuck, "Design-Based Research A Decade of Progress in Education Research?,” Educational Researcher 41 no. 1 (February 2012): 16-25, doi:10.3102/0013189X11428813.

62 Leanne Bowler and Andrew Large, "Design-Based Research for LIS," Library \& Information Science Research 30 no. 1 (March 2008): 45, doi:10.1016/j.lisr.2007.06.007. 
community that they note a lack of explicit identification with designbased research methodologies. ${ }^{63}$ A review of Design Science for the 2009 Encyclopedia of Library and Information Science arrived at a similar conclusion:

\begin{abstract}
Although design is an important part of professional and research practice in the library and information sciences, it is at present undertheorized. ... Our social science traditions provide strengths in understanding the use of systems, their integration into work life, and how users evaluate results; these traditions could usefully be expanded to study design as professional practice. ${ }^{64}$
\end{abstract}

\title{
Characteristics, Quality, Methods and Techniques of Design Research
}

Across all these disciplines, questions have been raised regarding how the intellectual and methodological quality of design research is determined. As one would expect, these are reflective of philosophical and epistemological stances as discussed above. Some take a positivist positioning, looking for determinations of objectivity, reliability and validity and seeking generalisable outcomes. Others come from an interpretive position valuing rigour, evidence of researcher reflexivity and contextual sensitivity, and seeking rich understanding of the researched situation for translation to other contexts. Like all good research, good design research should reflect methodological coherence and consistency, as well as mastery and proficiency in the research methods and techniques deployed. It also should reflect good design processes and practices. These tend to be mutually reinforcing rather than mutually exclusive. Good design is iterative and reflexive, deploys known (or designs and investigates new) design principles, and is critical of itself in the search for better interventions into a problematic situation.

Discussion of the distinction between works of design research from works of design practice is a common thread in the discourses across the disciplines. In Information Systems the framing of the design and development work around the research problem and questions is emphasised, ${ }^{65}$ and a key

63 Bowler and Large, "Design-Based Research for LIS," 42.

64 Judith Weedman, "Design Science in the Information Sciences," in Encyclopedia of Library and Information Sciences, Third Edition (Taylor \& Francis, 2009), 1564, http:// www.tandfonline.com/doi/abs/10.1081/E-ELIS3-120043534.

65 Frada Burstein, "Systems Development in Information Systems Research," in Research Methods for Students, Academics and Professionals: Information Management and Systems, 
concern within the discipline has been to formulate evaluation criteria for assessing the quality of design-science research. ${ }^{66}$ In design and educational disciplinary contexts the reflective and communicative aspects are highlighted. ${ }^{67}$ All stress commitment to achieving practical and theoretical outcomes. $^{68}$

Models of design research methodologies across the disciplines all reflect the iterative and recursive nature of design processes. ${ }^{69}$ Cycles of design and development move from defining and redefining the problem and aspects of the potential solution through to the realisation and rendering of the designed artefact and analysis of its impact.

Design-based research is, like all design, a process. The questions one asks depend on where one is situated along the continuum of development. In other words, the method fits the question of the moment. ${ }^{70}$

A variety of research data gathering techniques may be deployed in design studies, providing inputs to the design process and evaluating its outcomes. One of the particular challenges is the way in which data related to the designing is captured - the knowing and reflecting in action rather than the knowing and reflection on action. Schön writes of 'Designing as reflective conversation with the materials of a design situation. ${ }^{71}$ Design research requires deploying techniques to capture that conversation - 'methods that document processes of enactment [to] provide critical evidence to establish

ed. Kirsty Williamson, 2nd edition (Wagga Wagga: Centre for Information Studies, Charles Sturt University, 2002), 147-58.

66 Ron Weber, "Design-Science Research," in Research Methods: Information, Systems and Contexts, ed. Kirsty Williamson and Graeme Johanson (Prahran, Victoria, Australia: Tilde University Press, 2013), 346-266.

67 Cross, Designerly Ways of Knowing, 102; The Design-Based Research Collective, "Design-Based Research: An Emerging Paradigm for Educational Inquiry."

68 Reeves, Herrington, and Oliver, "Design Research"; Burstein, "Systems Development in Information Systems Research"; Bowler and Large, "Design-Based Research for LIS."

69 See for example 'The Systems Development Method' in Burstein, "Systems Development in Information Systems Research. 'The DRM Framework' in Lucienne T. M. Blessing and Amaresh Chakrabarti, DRM, a Design Research Methodology (Springer, 2009), 17, http://www.springer.com/engineering/mechanical+engineering/book/9781-84882-586-4. 'Predictive and Design Research Approaches in Educational Technologies' in Thomas C. Reeves, "Design Research from a Technology Perspective," in Educational Design Research, ed. Jan Van den Akker et al. (Taylor \& Francis, 2006), 59.

70 Bowler and Large, "Design-Based Research for LIS," 41.

71 Donald A. Schön, "Designing as Reflective Conversation with the Materials of a Design Situation," Knowledge-Based Systems 5 no. 1 (March 1992): 3-14, doi:10.1016/0950-7051(92)90020-G. 
warrants for claims about why outcomes occurred. ${ }^{72}$ This would include the representations created through the design process e.g. diagrams, models, versions of prototypes - in essence records of design transactions - and may also incorporate techniques for overt reflection e.g. reflective journaling by participants in the design processes.

This discussion highlights the wealth of material available to inform and guide design as a research methodology and method in disciplines that can be aligned, in varying ways, with archival and recordkeeping studies. All feature systems, informatics and interaction design as a part of professional work. All are grappling with the impacts of digital and networking technologies, and all have discourses questioning the voices and values that are represented in practices, process, systems and structures. What lessons from design research in these disciplines may provide a foundation for utilising design research methodologies in archival and recordkeeping studies, particularly when it comes to our more intractable problems and challenges?

\section{Design Research Reflections}

Given my professional experience, it is perhaps not surprising that I was drawn to design as a research method when I undertook my doctoral research as part of the Clever Recordkeeping Metadata (CRKM) Project, an Australian Research Council Linkage Project undertaken at Monash University in the mid-2000s. This research partnership between academics and practitioners aimed to advance practice by developing a prototype of automated recordkeeping metadata capture and re-use in an integrated systems environment, and to advance the theory by validating and refining the concepts under investigation in light of insights arising from studying their implementation. ${ }^{73}$ As an action research project it utilised a systems development method to create a demonstrator of the 'create once, use many times' concept. The action research framework provided the mechanism for reflecting-on-action, as two iterations of this demonstrator were prototyped. Reflection-in-action was captured through the development and management of the project's documentary record, including, for example, mechanisms for recording and reflecting on designing conversations.

72 The Design-Based Research Collective, "Design-Based Research: An Emerging Paradigm for Educational Inquiry,” 7.

73 Evans, Reed, and McKemmish, "Interoperable Data." 
In concert with the CRKM Project, I also became involved in metadata registry development as part of the InterPARES 2 Project. There I found myself using my database and programming skills to prototype requirements as the specification of the metadata schema for the registry unfolded, turning to design to 'conceive and plan what does not yet exist'. ${ }^{74} \mathrm{My}$ colleague and I wrote of these experiences as an illustration of the use of systems development methods in archival research contexts. ${ }^{75}$ Subsequently I've also had the opportunity to reflect on the participatory nature of designbased research ${ }^{76}$ and the situation of investigations of inclusive information systems design within inclusive research design frameworks. ${ }^{77}$

My philosophical positioning draws me to the way design research is articulated in the educational literature particularly when framed in terms of social responsibility. ${ }^{78}$ From this position research is not just about understanding how technological systems work, but how they impact and contribute to making things - education, recordkeeping, archiving - better. Of particular appeal is the potential for inclusive research designs, where all involved - academics, recordkeeping practitioners, records creators and records users - can be co-creators of research processes. Table 22.1 shows how the characteristics of socially responsible design research in education may be translated, and extended, for inclusive archival and recordkeeping research.

\section{Design Methodologies in Archival and Recordkeeping Research}

The situation in Archival and Recordkeeping Research appears to be similar to Library and Information Science, with a lack of explicit identification with design research methodologies in the literature. While there has been an increasing amount of design and development work as the impacts of new digital and networking technologies and new conceptualisations of archival and recordkeeping endeavours are incorporated into practice, research

74 Buchanan, "Wicked Problems in Design Thinking," 18.

75 Joanne Evans and Nadav Rouche, "Utilizing Systems Development Methods in Archival Systems Research: Building a Metadata Schema Registry," Archival Science 4 no. 3-4 (2004): 315-34, doi:10.1007/s10502-005-2598-4.

76 Joanne Evans et al., "Bridging Communities: Foundations for the Interchange of Ideas," Information, Communication E Society 15 no. 7 (2012): 1055-80, doi:10.1080/136911 8X.2012.704062.

77 Sue McKemmish et al., "Inclusive Research Design," Information, Communication \& Society 15 no. 7 (2012): 1106-35, doi:10.1080/1369118X.2012.707225.

78 Reeves, Herrington, and Oliver, "Design Research.” 


\section{Table 22.1. Translating and extending socially responsible design research in education for inclusive archival and recordkeeping research}

\begin{tabular}{|l|l|}
\hline $\begin{array}{l}\text { Characteristic from Reeves, Herrington } \\
\text { \& Oliver 2005 }\end{array}$ & $\begin{array}{l}\text { Translation to Inclusive Archival and } \\
\text { Recordkeeping Research }\end{array}$ \\
\hline $\begin{array}{l}\text { A focus on broad-based complex problems } \\
\text { critical to higher education }\end{array}$ & $\begin{array}{l}\text { A focus on broad-based complex problems critical } \\
\text { to archival and recordkeeping endeavours }\end{array}$ \\
\hline $\begin{array}{l}\text { The integration of known and hypothetical } \\
\text { design principles with technological } \\
\text { affordances to render plausible solutions to } \\
\text { these complex problems }\end{array}$ & $\begin{array}{l}\text { The integration of known and hypothetical } \\
\text { archival design principles with technological } \\
\text { affordances to render plausible solutions to these } \\
\text { complex problems }\end{array}$ \\
\hline $\begin{array}{l}\text { Rigorous and reflective inquiry to test and } \\
\text { refine innovative learning environments as } \\
\text { well as to reveal new design principles }\end{array}$ & $\begin{array}{l}\text { Rigorous and reflective inquiry to test and refine } \\
\text { innovative archival and recordkeeping environments } \\
\text { as well as to reveal new archival design principles }\end{array}$ \\
\hline $\begin{array}{l}\text { Long term engagement included continual } \\
\text { refinement of protocols and questions }\end{array}$ & $\begin{array}{l}\text { Long term engagement included continual } \\
\text { collaborative refinement of protocols and questions }\end{array}$ \\
\hline $\begin{array}{l}\text { Intensive collaboration among researchers } \\
\text { and practitioners }\end{array}$ & $\begin{array}{l}\text { Intensive collaboration among allparticipants in } \\
\text { the research }\end{array}$ \\
\hline $\begin{array}{l}\text { A commitment to theory construction } \\
\text { and explanation while solving real-world } \\
\text { problems }\end{array}$ & $\begin{array}{l}\text { A commitment to theory construction and } \\
\text { explanation while solving real-world problems }\end{array}$ \\
\hline
\end{tabular}

activities tend to be written up as positioned around designing processes rather than being incorporated within them. The focus is on research around inputs into systems design, for example requirements gathering methods, or the outputs, for example ways in which the designed artefact is evaluated. ${ }^{79}$ By doing so there is a tendency, perhaps unwittingly, to subscribe to closed 'waterfall' models of digital systems development, where requirements are specified and then handed over to programmers to interpret and build, with the result then tested and evaluated. My experience as a programmer is of the myriad of design decisions and revelations that occur in the rendering of requirements. Indeed programming, like design, had been described as a theory building activity:

... Programming properly should be regarded as an activity by which the programmers form or achieve a certain kind of insight, a theory, of the matters at hand. ${ }^{80}$

79 Magia Ghetu Krause and Elizabeth Yakel, "Interaction in Virtual Archives: The Polar Bear Expedition Digital Collections Next Generation Finding Aid," American Archivist 70 no. 1 (2007): 282-314. An illustrative example where the research focus is on the evaluation of the designed artefact rather than on the designing processes.

80 Peter Naur, "Programming as Theory Building," Microprocessing and Microprogramming 15 no. 5 (May 1985): 253, doi:10.1016/0165-6074(85)90032-8. 
I would advocate, therefore, for more stories about designing processes and how archival thinking plays out in them. Ethnographic and autoethnographic research methods may be one way to realise this. ${ }^{81}$ These kinds of methods enable the study of the design and the designing within its constituting context and provide the mechanism for rigorous reflection to reveal, test and refine the archival design principles at play. My argument above is that with the maturing of the discourse on design research methodologies over a variety of allied disciplines that we do not need to be shy of embracing it. I would also go further and argue that we need greater archival and recordkeeping systems design and development capabilities in both research and practice.

Archival design is indeed a wicked problem. It is not just about preserving (or what perhaps at the moment seems more like salvaging) the digital stuff we have now, but securing archival and recordkeeping capabilities in the digital and networked systems of the future. These systems are not just technically different. We are increasingly aware that they will need to be conceptually different, reflective of pluralistic rather than singular perspectives, as we look for our archival and recordkeeping infrastructures to be capable of dealing with multiple ways of knowing and being co-existing in time and space. ${ }^{82}$

\section{Designing Community and Participatory Archives}

In the emerging literature around community and participatory archives we are seeing new archival conceptualisations revealed through systems design and development processes. One example is Huvila's use of two digital archives action research projects to explicate features of a participatory archives model. Through systems design and development work he identifies and explores principles of 'decentralized curation', 'radical user orientation' and 'contextualisation of both records and the entire archival process', to enable the re-figuring of roles and responsibilities. ${ }^{83} \mathrm{He}$ notes that these features are 'lacking support in the traditional non-participatory archival paradigm $^{\text {'84 }}$ signifying that refiguring relationships requires refiguring of archival systems and processes, rather than just adding on social media

81 Evans et al., "Bridging Communities: Foundations for the Interchange of Ideas."

82 Pluralizing the Archival Curriculum Group, "Educating For the Archival Multiverse," American Archivist 74 Spring/Summer 2011 (2011): 69-101.

83 Isto Huvila, "Participatory Archive: Towards Decentralised Curation, Radical User Orientation, and Broader Contextualisation of Records Management," Archival Science 8 no. 1 (March 1, 2008): 25, doi:10.1007/s10502-008-9071-0.

84 Huvila, "Participatory Archive". 
capabilities. While the latter may invite a degree of participation - fast volunteers (à la fast paper) who may add to our descriptive systems on our terms only - it tends to reinforce existing archival power and control.

Another example is Shilton and Srinivasan's participatory archiving methodology. ${ }^{85}$ Drawing on experiences from previous community archive projects, they articulate how archival processes of appraisal, arrangement and description may be refigured around participation. They then point to how this model may be instantiated through a project to develop 'a cooperative communication hub and digital archive for the South Asian diasporic community in Los Angeles' ${ }^{86}$ for its further testing and refinement.

The work of Tim Sherratt, Digital Humanities scholar, is a further example. Through his mashups and widgets he is experimenting with the design of archival services that enable participatory archival spaces. Here, access and description become entwined as they move from frameworks designed around passive consumption of information about records to those in which collaborators, rather than users, can play with content and context, and the connections amongst them. His work points to re-imagined and fundamentally transformed archival services designed around capacities to collaborate and connect. ${ }^{87}$

These examples exhibit the key characteristics of design methodologies for archival research. These researchers are tackling complex problems regarding the social responsibility of archival systems through design approaches. They are investigating the rendering of archival principles in technological systems, in rigorous and reflective cycles of engagement and collaboration. Both practical solutions and development of understanding of the relationship between archives and community, along with exemplars of participatory archival processes and systems are outcomes for future and further instantiation, development and refinement. Through this kind of research the re-imagined archival systems of the future with capabilities to represent complex, multi-layered, multi-voiced and continually evolving relationships between records, people, organisations, communities, functions, activities and events, become more of a reality. ${ }^{88}$

85 Katie Shilton and Ramesh Srinivasan, "Participatory Appraisal and Arrangement for Multicultural Archival Collections," Archivaria 63 Spring 2007 (Spring 2007): 87-101.

86 Shilton and Srinivasan, "Participatory Appraisal and Arrangement for Multicultural Archival Collections," 99.

87 Tim Sherratt, “Archives of Emotion,” Discontents, November 30, 2012, http:// discontents.com.au/archives-of-emotion/.

88 Sue McKemmish, Barbara Reed, and Michael Piggott, "Chapter 7: The Archives," in Archives: Recordkeeping in Society, ed. Sue McKemmish et al., Topics in Australasian 


\section{Conclusion}

Digital and networking technologies have thrown down a gauntlet to the archival and recordkeeping discipline and profession. Heralding an age of information abundance they challenge us to open up our siloed archival systems to better allow for those represented in records to make and manage their own archival spaces, connections and interpretations. They provide us with the chance to overcome the current dysfunctions of fragmented and dispersed archival systems, although harnessing these capabilities is no straightforward task. If we limit ourselves to automating or augmenting what we have done in the paper recordkeeping paradigm then we will fail not just our users, but also ourselves. Our challenge is to continually imagine and create archival and recordkeeping infrastructure for the future.

The quest for ubiquitous, pervasive, inclusive recordkeeping and archival systems for the digital and networked information age is one that must be shared. With traditional recordkeeping and archival management frameworks in crisis from disruptive digital information technologies, co-ordinated theoretical, methodological and technical innovation is urgently needed. Design research methodologies provide a way in which we can explore and exploit innovation through the co-development of practical solutions and theoretical understanding of records, recordkeeping, archives and archival design principles. It has the potential to unite archival and recordkeeping researchers, practitioners and users in the research and development of robust and resilient frameworks and systems that enable communities to capture, preserve, transmit, control and connect their recorded knowledge as evidence and memory.

\section{References}

Anderson, Terry, and Julie Shattuck. "Design-Based Research A Decade of Progress in Education Research?” Educational Researcher 41 no. 1 (February 2012): 16-25. doi:10.3102/0013189X11428813.

Archer, Bruce. "Design as a Discipline.” Design Studies 1 no. 1 (July 1979): 17-20. doi:10.1016/0142-694X(79)90023-1.

Baskerville, Richard. "What Design Science Is Not." European Journal of Information Systems 17 no. 5 (October 2008): 441-43. doi:10.1057/ejis.2008.45.

Baskerville, Richard, Jan Pries-Heje, and John Venable. "Soft Design Science Methodology." In Proceedings of the 4th International Conference on Design Science Research in Information Systems and Technology. DESRIST'09. New York, NY, USA: ACM, 2009. doi:10.1145/1555619.1555631.

Library and Information Studies, Issue 24 (Wagga Wagga: Centre for Information Studies, Charles Sturt University, 2005), 159-95. 
Bearman, David. Archival Methods. Archives and Museum Informatics Technical Report \#9. Pittsburgh, 1989. http://www.archimuse.com/publishing/archival_methods/index. html.

Bearman, David. Electronic Evidence: Strategies for Managing Records in Contemporary Organizations. Pittsburgh: Archives \& Museum Informatics, 1994.

Bearman, David. "Record-Keeping Systems." Archivaria 36 (Autumn 1993): 16-37.

Bilandzic, Mark, and John Venable. "Towards Participatory Action Design Research: Adapting Action Research and Design Science Research Methods for Urban Informatics." The Journal of Community Informatics 7 no. 3 (December 15, 2011). http://ci-journal.net/index.php/ciej/article/view/786/804.

Blessing, Lucienne T. M., and Amaresh Chakrabarti. DRM, a Design Research Methodology. Springer, 2009. http://www.springer.com/engineering/mechanical+engineering/ book/978-1-84882-586-4.

Bowker, Geoffrey C., Karen Baker, Florence Millerand, and David Ribes. “Toward Information Infrastructure Studies: Ways of Knowing in a Networked Environment." In International Handbook of Internet Research, edited by Jeremy Hunsinger, Lisbeth Klastrup, and Matthew Allen (Springer, 2010), 97-117. http://interoperability.ucsd. edu/docs/07BowkerBaker_InfraStudies.pdf.

Bowler, Leanne, and Andrew Large. "Design-Based Research for LIS.” Library E' Information Science Research 30 no. 1 (March 2008): 39-46. doi:10.1016/j. lisr.2007.06.007.

Buchanan, Richard. "Wicked Problems in Design Thinking.” Design Issues 8 no. 2 (April 1992): 5-21. doi:10.2307/1511637.

Burstein, Frada. "Systems Development in Information Systems Research.” In Research Methods for Students, Academics and Professionals: Information Management and Systems, edited by Kirsty Williamson, 2nd edition (Wagga Wagga: Centre for Information Studies, Charles Sturt University, 2002), 147-58.

Clark, Jon, Celia Modgil, and Sohan Modgil, eds. Anthony Giddens: Consensus and Controversy. 1st ed. Falmer Press, 1990.

Conklin, Jeff, Min Basadur, and GK VanPatter. "Rethinking Wicked Problems: Unpacking Paradigms, Bridging Universes.” NextD Journal 10 Conversation 10.1 (2007). http:// humantific.com/wp-content/uploads/2009/07/NextD_10/NextD_10_1.pdf.

Cook, Terry. "Electronic Records, Paper Minds: The Revolution in Information Management and Archives in the Post-Custodial and Post-Modernist Era." Archives and Manuscripts 22 no. 2 (November 1994): 300-329.

Cook, Terry. "Evidence, Memory, Identity, and Community: Four Shifting Archival Paradigms." Archival Science 13 no. 2-3 (2013): 95-120.

Cross, Nigel. "Designerly Ways of Knowing.” Design Studies 3 no. 4 (October 1982): 22127. doi:10.1016/0142-694X(82)90040-0.

Cross, Nigel. Designerly Ways of Knowing. London: Springer London, 2006. http://link. springer.com/chapter/10.1007/1-84628-301-9_1.

Cross, Nigel, John Naughton, and David Walker. "Design Method and Scientific Method.” Design Studies 2 no. 4 (October 1981): 195-201. doi:10.1016/0142694X(81)90050-8.

Cumming, Kate, and Anne Picot. "Reinventing Appraisal." Recordkeeping Roundtable, August 1, 2014. http://rkroundtable.org/2014/08/01/reinventing-appraisal/.

DeGrace, Peter, and Leslie Hulet Stahl. Wicked Problems, Righteous Solutions: A Catolog of Modern Software Engineering Paradigms. 1st ed. Prentice Hall, 1990.

Dorst, Kees, and Judith Dijkhuis. "Comparing Paradigms for Describing Design Activity." Design Studies 16 no. 2 (April 1995): 261-74. doi:10.1016/0142-694X(94)00012-3. 


\section{Chapter 22}

Evans, Joanne. "Designing Dynamic Descriptive Frameworks." Archives and Manuscripts 42 no. 1 (March 2014): 5-18. doi:10.1080/01576895.2014.890113.

Evans, Joanne. "Exploring Bright Sparcs: Creation of a Navigable Knowledge Space." Cataloguing Australia 24 no. 1-4 (December 1999): 147-57.

Evans, Joanne. "Structure of the ADS." In Archives and Reform - Preparing for Tomorrow, Proceedings of the Australian Society of Archivists Conference, Adelaide, 25-26th July 1997, 1997. http://www.asap.unimelb.edu.au/pubs/articles/asa97/ADSStructure.htm

Evans, Joanne, Shannon Faulkhead, Rosetta Manaszewicz, and Kirsten Thorpe. "Bridging Communities: Foundations for the Interchange of Ideas." Information, Communication E' Society 15 no. 7 (2012): 1055-80. doi:10.1080/1369118X.2012.704062.

Evans, Joanne, Sue McKemmish, and Barbara Reed. "Making Metadata Matter: Outcomes from the Clever Recordkeeping Metadata Project." Archives and Manuscripts 37 no. 1 (May 2009): 28-56.

Evans, Joanne, Barbara Reed, and Sue McKemmish. "Interoperable Data: Sustainable Frameworks for Creating and Managing Recordkeeping Metadata.” Records Management Journal 18 no. 2 (June 13, 2008): 115-29. doi:10.1108/09565690810882977.

Evans, Joanne, and Nadav Rouche. "Utilizing Systems Development Methods in Archival Systems Research: Building a Metadata Schema Registry.” Archival Science 4 no. 3-4 (2004): 315-34. doi:10.1007/s10502-005-2598-4.

Gilliland, Anne, and Sue McKemmish. "Archival and Recordkeeping Research: Past, Present and Future." In Research Methods: Information, Systems and Contexts, edited by Kirsty Williamson and Graeme Johanson (Prahran, Victoria, Australia: Tilde University Press, 2013), 79-103.

Gilliland, Anne, and Sue McKemmish. "Building an Infrastructure for Archival Research.” Archival Science 4 no. 3 (2004): 149-97.

Gregory, Robert Wayne. "Design Science Research and the Grounded Theory Method: Characteristics, Differences, and Complementary Uses,” 2010. http://is2.lse.ac.uk/asp/ aspecis/20100012.pdf.

Hevner, Alan R., and Samir Chatterjee. Design Research in Information Systems: Theory and Practice. 2010th ed. Vol. 22. Integrated Series in Information Systems. Springer, 2010.

Hevner, Alan R., Salvatore T. March, Jinsoo Park, and Sudha Ram. "Design Science in Information Systems Research.” MIS Quarterly 28 no. 1 (March 2004): 75-105.

Hofman, Hans. "Rethinking the Archival Function in the Digital Era." Brisbane, 2012. http://www.ica2012.com/files/data/Full\%20papers\%20upload/ica12final00187.pdf.

Hurley, Chris. "Parallel Provenance (If These Are Your Records, Where Are Your Stories?),” 2005. http://infotech.monash.edu/research/groups/rcrg/publications/ parallel-provenance-combined.pdf.

Hurley, Chris. "The Australian ('Series') System: An Exposition." In The Records Continuum: Ian Maclean and Australian Archives First Fifty Years, edited by Sue McKemmish and Michael Piggott (Clayton: Ancora Press, 1994), 150-72.

Huvila, Isto. "Participatory Archive: Towards Decentralised Curation, Radical User Orientation, and Broader Contextualisation of Records Management." Archival Science 8 no. 1 (March 1, 2008): 15-36. doi:10.1007/s10502-008-9071-0.

Iivari, Juhani, and John Venable. "Action Research and Design Science Research Seemingly Similar but Decisively Dissimilar,” 2009. http://www.ecis2009.it/papers/ ecis2009-0424.pdf.

Järvinen, Pertti. “Action Research Is Similar to Design Science.” Quality \& Quantity 41 no. 1 (February 2007): 37-54. doi:10.1007/s11135-005-5427-1.

Jones, David, and Shirley Gregor. "The Anatomy of a Design Theory." Journal of the Association for Information Systems 8 no. 5 (May 1, 2007). http://aisel.aisnet.org/jais/ vol8/iss5/1. 
Krause, Magia Ghetu, and Elizabeth Yakel. "Interaction in Virtual Archives: The Polar Bear Expedition Digital Collections Next Generation Finding Aid.” American Archivist 70 no. 1 (2007): 282-314.

McCarthy, Gavan, and Joanne Evans. "Mapping the Socio-Technical Complexity of Australian Science: From Archival Authorities to Networks of Contextual Information." Journal of Archival Organization 5 no. 1-2 (2008): 149-75. doi:10.1300/ J201v05n01_08.

McCarthy, Gavan, and Joanne Evans. "The Open Resource Scholarly Network: New Collaborative Partnerships Between Academics, Libraries, Archives and Museums." In VALA 2002: Evolving Information Futures, 11th Biennial Conference E Exhibition 6-8 February 2002, Melbourne, Australia: Victorian Association for Library Automation, 2002, 197-207. http://www.vala.org.au/ vala2002/2002pdf/15McCEva.pdf.

McCarthy, Gavan J., and Joanne Evans. "Principles for Archival Information Services in the Public Domain." Archives and Manuscripts 40 no. 1 (2012): 54-67. doi:10.1080/01 576895.2012.670872.

McKay, Judy, and Peter Marshall. "Science, Design, and Design Science: Seeking Clarity to Move Design Science Research Forward in Information Systems.”Toowomba, 2007. http://www.swinburne.edu.au/ict/research/riso/publications/ACIS07_science_ design_design_science.pdf.

McKemmish, Sue. "Placing Records Continuum Theory and Practice." Archival Science 1 no. 4 (2001): 333-59. doi:10.1007/BF02438901.

McKemmish, Sue. "Understanding Electronic Recordkeeping Systems: Understanding Ourselves." Archives and Manuscripts 22 no. 1 (May 1994): 150-62.

McKemmish, Sue, Frada Burstein, Rosetta Manaszewicz, Julie Fisher, and Joanne Evans. "Inclusive Research Design." Information, Communication and Society 15 no. 7 (2012): 1106-35. doi:10.1080/1369118X.2012.707225.

McKemmish, Sue, Barbara Reed, and Michael Piggott. “Chapter 7: The Archives.” In Archives: Recordkeeping in Society, edited by Sue McKemmish, Michael Piggott, Barbara Reed, and Frank Upward. Topics in Australasian Library and Information Studies, Issue 24 (Wagga Wagga: Centre for Information Studies, Charles Sturt University, 2005), 159-95.

Nathan, Lisa P., and Elizabeth Shaffer. "Preserving Social Media: Opening a MultiDisciplinary Dialogue.”Vancouver, British Columbia, Canada: UNESCO, 2012. http://www.unesco.org/new/fileadmin/MULTIMEDIA/HQ/CI/CI/pdf/mow/ VC_Nathan_Shaffer_27_B_1140.pdf.

Naur, Peter. "Programming as Theory Building." Microprocessing and Microprogramming 15 no. 5 (May 1985): 253-61. doi:10.1016/0165-6074(85)90032-8.

Niehaves, Björn. “On Epistemological Pluralism in Design Science.” Scandinavian Journal of Information Systems 19 no. 2 (January 1,2007). http://iris.cs.aau.dk/tl_files/ volumes/volume19/no2/Niehaves.pdf.

Nunamaker, Jay, Minder Chen, and Titus D. M. Purdin. "Systems Development in Information Systems Research.” Journal of Management Information Systems 7 no. 3 (Winter 1991): 89-106.

Pluralizing the Archival Curriculum Group. "Educating For the Archival Multiverse.” American Archivist 74 Spring/Summer 2011 (2011): 69-101.

Reed, Barbara. "Reinventing Access." Recordkeeping Roundtable, August 1, 2014. http:// rkroundtable.org/2014/08/01/reinventing-access/.

Reed, Barbara. "Service-Oriented Architectures and Recordkeeping." Records Management Journal 18 no. 1 (2008): 7-20. doi:10.1108/09565690810858488. 


\section{Chapter 22}

Reeves, Thomas C. "Design Research from a Technology Perspective." In Educational Design Research, edited by Jan Van den Akker, Koeno Gravemeijer, Susan McKenney, and Nienke Nieveen (Taylor \& Francis, 2006), 52-66.

Reeves, Thomas C., Jan Herrington, and Ron Oliver. "Design Research: A Socially Responsible Approach to Instructional Technology Research in Higher Education.” Journal of Computing in Higher Education 16 no. 2 (2005): 96-115.

Rittel, Horst W. J. "On the Planning Crisis: Systems Analysis of the 'First and Second Generations.” Bedriftsokonomen 8 (1972): 390-96.

Rittel, Horst W. J., and Melvin M. Webber. "Dilemmas in a General Theory of Planning." Policy Sciences 4 no. 2 (June 1973): 155-69.

Schön, Donald A. "Designing as Reflective Conversation with the Materials of a Design Situation." Knowledge-Based Systems 5 no. 1 (March 1992): 3-14. doi:10.1016/09507051(92)90020-G.

Schön, Donald A. "The New Scholarship Requires a New Epistemology." Change, December 1995. Academic OneFile.

Schön, Donald A. The Reflective Practitioner: How Professionals Think In Action. Basic Books, 1984.

Sein, Maung K., Ola Henfridsson, Sandeep Purao, Matti Rossi, and Rikard Lindgren. "Action Design Research." MIS Quarterly 35 no. 1 (March 2011): 37-56.

Sherratt, Tim. "Archives of Emotion.” Discontents, November 30, 2012. http://discontents. com.au/archives-of-emotion/.

Shilton, Katie, and Ramesh Srinivasan. "Participatory Appraisal and Arrangement for Multicultural Archival Collections.” Archivaria 63 Spring 2007 (Spring 2007): 87-101.

Simon, Herbert A. The Sciences of the Artificial. Cambridge MA: MIT Press, 1969.

Stolterman, Erik. "The Nature of Design Practice and Implications for Interaction Design Research.” International Journal of Design 2 no. 1 (2008): 55-65.

The Design-Based Research Collective. "Design-Based Research: An Emerging Paradigm for Educational Inquiry." Educational Researcher 32 no. 1 (February 2008): 5-8.

UK Department of Trade and Industry. Creativity, Design and Business Performance, November 2005. http://www.dti.gov.uk/files/file13654.pdf.

Upward, Frank. "Structuring the Records Continuum: Part One: Postcustodial Principles and Properties." Archives and Manuscripts 24 no. 2 (November 1996): 268-85.

Upward, Frank. "Structuring the Records Continuum: Part Two: Structuration Theory and Recordkeeping." Archives and Manuscripts 25 no. 1 (May 1997): 10-35.

Upward, Frank, and Sue McKemmish. "Somewhere Beyond Custody." Archives and Manuscripts 22 no. 1 (May 1994): 138-47.

Upward, Frank, Barbara Reed, Gillian Oliver, and Joanne Evans. "Recordkeeping Informatics: Re-Figuring a Discipline in Crisis with a Single Minded Approach." Records Management Journal 23 no. 1 (March 2013): 37-50. doi:10.1108/09565691311325013.

Weber, Ron. "Design-Science Research." In Research Methods: Information, Systems and Contexts, edited by Kirsty Williamson and Graeme Johanson (Prahran, Victoria, Australia: Tilde University Press, 2013), 346-266.

Weedman, Judith. "Design Science in the Information Sciences." In Encyclopedia of Library and Information Sciences, Third Edition (Taylor \& Francis, 2009), 1493-1506. http:// www.tandfonline.com/doi/abs/10.1081/E-ELIS3-120043534. 


\title{
DESIGNING EXPERT SYSTEMS FOR ARCHIVAL EVALUATION AND PROCESSING OF COMPUTER- MEDIATED COMMUNICATIONS
}

\section{Frameworks and Methods ${ }^{1}$}

\author{
Anne J. Gilliland
}

\begin{abstract}
The third-party identification, evaluation, long-term preservation, and retrieval of networked computer-mediated communications (CMC) such as electronic mail and social media have recently become subjects of much public debate. They also present persistent challenges for archivists. This chapter first offers a retrospective reflection on an applied research study that was conducted almost two decades ago investigating the possibilities of automating how university archivists appraise and acquire electronic mail. It describes the context of the study and the research design and methods that were employed. The latter included using bibliometrics to identify appraisal domain experts, acquiring and codifying knowledge from those experts, and the iterative development and testing of an expert appraisal system. The chapter then reflects upon what was learned from the study in terms of the utility of the methods and the aspects of this research approach that might remain useful for archival processing of documentation generated by social media such as Twitter, and email and cell phone communications today. It concludes by reflecting more broadly on how archival systems development research stands the test of time as technology evolves, institutional roles and conceptual frameworks shift, and methodological approaches gain or lose appeal.
\end{abstract}

1 The author would like to thank Amelia Acker for her close reading and insightful comments on drafts of this paper. 


\section{Introduction}

Which materials created through computer-mediated communications $(\mathrm{CMC})^{2}$ such as tweets, electronic mail, SMS (short message service), Facebook, and blogs might be sufficiently valuable to posterity to preserve and make available in the future? What processes could be used to make that determination and acquire, manage, redact sensitive information, disseminate and retrieve those materials? Given the massive volumes and interrelatedness, the potentially sensitive nature of some of these communications, and the usually very limited human resources of archives, how might aspects of those processes be effectively and appropriately handled automatically?

In January 2015, University of Oregon officials placed the head of special collections and the electronic records archivist on paid administrative leave following what the university claimed was the unlawful release by the archives of 22,000 digital documents that included confidential correspondence of the last four university presidents relating to faculty, staff, and students. The University of Oregon is a public university and is subject to Oregon public records law, although the law does provide for certain types of sensitive information to remain confidential. The University Archives had acquired the documents as an electronic accession as part of its records management program. The documents were released upon the reference request of a University of Oregon economics professor before they had been individually reviewed and potentially redacted by the archivists for any sensitive content. That professor subsequently uploaded one of the presidential memos to an online blog and the university began investigating how he obtained it.

By March 2015, the head of special collections was informed that his contract would not be renewed and the electronic records archivist had resigned her position. In April 2015, the former head of special collections gave an interview to a local newspaper in which he claimed that the university had used him as a scapegoat. He maintained that "the library was being deluged with electronic archival material ... The mass of material was so great that there was no way special collections could vet or even organize it ... Archivists nationally are struggling with the same problem." A 2013 external review had in fact

2 Computer-mediated Communications (CMC) refers to any communicative transaction, such as email, text messaging, and instant messaging, that employs two or more networked computers. In recent years, the term has increasingly been used in the context of research into the social effects and implications of such technologically-mediated communication. See Denis McQuail, McQuail's Mass Communication Theory. 5th ed. London: Sage Publications, 2005 and C. Thurlow, L. Lengel, and A. Tomic, Computer Mediated Communication: Social Interaction and the Internet. London: Sage, 2004. 
found that the library was indeed understaffed and the University Librarian had subsequently attempted to draw the university administration's attention to the insufficiency of the resources of the archives-administered records management program. The university countered the claim of scapegoating in a prepared statement: "Regardless of the Libraries' infrastructure, however, it is the responsibility of the Head of Special Collections and University Archives to supervise the archives and records management unit, and to ensure that documents containing private and confidential information are properly reviewed and not improperly released." ${ }^{3}$

The case provides a sobering opportunity to reflect upon the capacity of current records management and archival appraisal practices, archival processing, and reference services to cope with the exigencies presented by high volumes of digitally created documents, especially when their content is not readily apparent. Indeed, it has led to a flurry of commentary among archivists on social media about where to turn for assistance in addressing the same conditions in their archives and asking where relevant research studies might be found. It also unfortunately underscores how, despite these issues having been flagged to archivists over two decades ago, they continue to be more pressing than ever. In that spirit, therefore, the first part of this chapter provides a retrospective overview of the historical context and methods of a study I conducted between 1993 and 1995 investigating the possibilities of automating how archivists appraise (i.e., arrive at decisions about whether records and other materials have archival value) and acquire electronic mail through the iterative development and testing of an expert system (i.e., a computer system that is designed to emulate the decision-making processes of a human expert). The second part of the chapter contemplates contemporary CMC contexts, values and needs, drawing on the example of the Twitter Archive at the Library of Congress, and discussing how the questions posed above continue to provide a rich vein for ongoing research investigation and not just in the context of university archives and records management. The chapter concludes with some more general thoughts on the role of systems development research ${ }^{4}$ and how it stands the test of time

3 Diane Dietz, "Former UO Archivist Describes 'Humiliating' Dismissal: He Says University Leaders Saw Him as a Scapegoat after a Controversial Records Release," The Register-Guard (Sunday, April 19th, 2015), http://registerguard.com/rg/news/ local/32990068-75/former-uo-archivist-james-fox-tells-his-side-of-his-dismissal. html.csp.

$4 \quad$ Nunamaker and Chen described systems development as both a research methodology and a research domain, especially as used in the field of information systems. As a methodology, it falls under applied science and "belongs to the engineering, 
as technology evolves, institutional priorities and conceptual frameworks shift, and methodological approaches gain or lose appeal.

\section{Developing an Expert System to Appraise University Electronic Mail: Context of the Study}

In the United States, the critical need to conduct empirical research into the most effective ways to manage electronic records was recognised at the Working Meeting on Research Issues in Electronic Records, held in 1991 and sponsored by the U.S. National Historical Publications and Records Commission. ${ }^{5}$ I began my study in 1993 (the year of the first public release of the World Wide Web), in the midst of some major conceptual shifts and research thrusts relating to recordkeeping and electronic recordkeeping more specifically. Alarms were already sounding about the need to assess the "recordness" and long-term values of the growing use of email and grapple with its preservation. For example, in the U.S., what came to be known as the PROFS lawsuit ${ }^{6}$ regarding White House email and the U.S. National Archives' lack of records management oversight over it, had been wending its way through the U.S. courts since 1989 and would continue to do so for several more years; and at the University of Michigan, where I had been working as an archivist and doing my doctoral studies, another prominent lawsuit with archival implications was brought by a former student who sought to access faculty email under state freedom of information legislation. In Canada and the U.S., Terry Cook and David Bearman had recently

developmental and formulative type of research" (p.632). They argued that the systems building process, especially one that includes software development, moves through the following phases: construction of a conceptual framework; development of systems architecture; analysis and design of the system; building the system; and observing and evaluating the system. Jay F. Nunamaker, Jr. and Minder Chen, "Systems Development in Information Systems Research," Proceedings of the Twenty-Third Annual Hawaii International Conference on Systems Sciences, IEEE, 1990, pp. 631-640. More recent developments in systems development, such as those described by Joanne Evans in her chapter in this volume, integrate more reflexive and even qualitative approaches for a less technologically deterministic methodological approach.

5 See Tora Bikson, "Research on Electronic Information Environments: Prospects and Problems" and Margaret Hedstrom, "Understanding Electronic Incunabula: A Framework for Research on Electronic Records," papers presented 24 January 1991 at the Working Meeting on Research Issues in Electronic Records. Washington, D.C.: National Historical Publications and Records Commission, 1991.

6 Armstrong v. Executive Office of the President. See David A. Bearman. "The Implications of Armstrong v. Executive Office of the President for the Archival Management of Electronic Records," The American Archivist 56 (1993): 674-689. 
separately published several "out-of-the-box" treatises on the management of electronic records that promoted evidentiary and systems thinking and ideas about post-custodiality. ${ }^{7}$

At the same time, however, academic research in archival studies was in its infancy, ${ }^{8}$ with little in the way of international collaboration or even ready access to literatures from other countries. Trans-national cross-fertilisation, as it influenced the American context, occurred more at the level of government archives practice, where archivists had occasions to interact with each other through various forums and initiatives, than in other archival settings or in academic programs. The American archival profession is large, diverse, and decentralised. The National Archives had not played the same central role in the framing, standardisation, automation, or integration of professional practices across government or other types of repositories in recent decades as had national archives in many other countries, and national archives staff

7 Both individuals emphasised how electronic records could serve as digital evidence of decision-making and transactions occurring in the course of organisational activity, and looked for ways to identify how and ensure that recordkeeping systems created such evidence. See Terry Cook, "Easier to Byte, Harder to Chew: The Second Generation of Electronic Records Archives," Archivaria 33 (1992): 202-216; "Electronic Records, Paper Minds: The Revolution in Information Management and Archives in the Post-custodial and Post-modern Era," Archives and Manuscripts 22 (1994): 300-329; "It's 10 O'Clock-Do You Know Where Your Data Are?” Technology Review 98 (1995): 48-53; Terry Cook and Eldon Frost, "The Electronic Records Archival Programme at the National Archives of Canada: Evolution and Critical Factors of Success," in Margaret Hedstrom, ed, Electronic Records Management Program Strategies: Archives and Museum Informatics Technical Report No. 18. Pittsburgh, PA: Archives and Museum Informatics, 1993, pp. 38-47; David A. Bearman, Electronic Records Guidelines: A Manual for Policy Development and Implementation. Pittsburgh, PA: Archives and Museum Informatics, 1990; David A. Bearman (ed.) Archival Management of Electronic Records. Pittsburgh, PA: Archives and Museum Informatics Technical Report no. 13, 1991; David A. Bearman, "Archives in the Post-Custodial Age," in Archival Management of Electronic Records, Archives and Museum Informatics Technical Report No. 13. Pittsburgh, PA: Archives and Museum Informatics, 1991; "An Indefensible Bastion: Archives as a Repository in the Electronic Age," in Archival Management," pp.14-24; "Diplomatics, Weberian Bureaucracy, and the Management of Electronic Records in Europe and America," The American Archivist 55 (1992): 168-181; "Record-keeping Systems," Archivaria 36 (1993): 16-37; Electronic Evidence: Strategies for Managing Records in Contemporary Organizations. Pittsburgh, PA: Archives and Museum Informatics, 1994; Sue McKemmish and Frank Upward, "Somewhere Beyond Custody," Archives and Manuscripts 22 no.1 (1994): 138-149; Greg O’Shea and David Roberts, "Living in a Digital World: Recognizing the Electronic and Post-custodial Realities, Archives and Manuscripts 24 no.2 (1996): 286-311.

8 Anne J. Gilliland and Sue McKemmish, "Archival and Recordkeeping Research: Past, Present and Future," in Research Methods: Information Management, Systems, and Contexts Kirsty Williamson, ed. Prahran: Tilde University Press, 2012, pp.80-112. 
tended to be more active in government and data archives associations than in the national and regional professional associations. There wasn't at the time, therefore, a large degree of interaction between government archivists, especially those who worked with electronic records and at the federal level, and the college and university $(\mathrm{C} \& \mathrm{U})$ archivists, who comprised the largest sector of the American archival profession. Moreover, C\&U archives had a strong tradition of autonomy from each other and from government archives.

My epistemological stance at the time was rooted in a belief that the implementation of descriptive standards, automated processes, and archivallyoriented information retrieval could "normalise" what were often poorly articulated and idiosyncratic archival practices. I saw the benefits of such an approach being that it might nudge the U.S. archival profession towards the new behaviours and explicit conceptualisations necessary to address the challenges of born-digital materials and to exploit the networking and computational capabilities of information technology. ${ }^{9}$ This stance was undoubtedly influenced by the optimism prevalent at the time that information technology and standardisation could make sweeping and rapid changes in ideas, institutions, and practices that might have centuries of social and cultural embeddedness. In recent years, however, the limitations of this stance have become increasingly apparent to me and I have incorporated a more anthropological and bottom-up approach toward the a priori analysis of records creation and recordkeeping systems and metadata. I use ethnographic and community analysis methods to try to understand in socio-cultural and political terms why these processes, systems and metadata came about, how they operated, and to whose benefit and whose disadvantage in order to generate a lens for determining feasible and context-appropriate parameters for systems development. ${ }^{10}$ This latter inclination in part informs how I have chosen to put this chapter together. Nevertheless, my grounding in systems analysis and information retrieval based in information science and business information systems taught me the value of rigorously defining concepts that needed to be operationalised in context when working with both human and digital systems, and I find myself constantly returning to that as a reference point in my research.

9 In some ways, it could be argued that the introduction of first the MARC Archival and Manuscripts Control Format and then Encoded Archival Description in the U.S. did indeed have such an effect on the descriptive practices of C\&U archives.

10 Anne J. Gilliland, "Reflections on the Value of Metadata Archaeology for Recordkeeping in a Global, Digital World," Journal of the Society of Archivists 32 no.1 (April 2011): 97-112. 
My professional and research engagement in the early 1990s had both been directed toward addressing how to identify ways to assess the potential archival values of materials created by $C M C$ and for $C \& U$ archives to acquire any deemed to have such value. In 1993, I had just finished working on the Bentley Historical Library Computer Conferencing Appraisal Project, a federally-funded research project at the University of Michigan that developed approaches for appraising, accessioning and automatically describing active and inactive "computer conferences," an early form of social media. ${ }^{11}$ That project was strongly influenced by Helen Samuels' ideas about documenting academic environments. ${ }^{12} \mathrm{I}$ wished to build upon that experience by examining how a university archives, with limited technological and human resources, and a consciously less clearly defined recordkeeping mandate than most government archives would have, could automatically appraise and acquire electronic mail generated in the course of university activities. At the same time, I was frustrated by the number of assertions that I encountered in expositions of archival theories and practices in the professional literature that had never been tested to ascertain their validity or efficacy over time and in different contexts. This frustration drew me toward a method - expert systems development - that required precise conceptual articulations of those assertions and that provided both a feedback mechanism and a real-time accounting of its own efficacy. I was not alone in this impulse for precision and clarity. The Pittsburgh Project, led by Richard Cox and David Bearman, was underway at the same time. It generated a number of "production rules" that were designed to be built into electronic recordkeeping systems to ensure the creation of trustworthy, segregable and preservable records. ${ }^{13}$ Similarly, the Preservation of the Integrity of Electronic Records Project (a.k.a. the UBC Project) led by Luciana Duranti, Terry Eastwood and Heather MacNeil, used IDEF0 modelling to depict graphically and unambiguously the workflow, inputs,

11 Anne J. Gilliland-Swetland and Carol Hughes, "Enhancing Archival Description for Public Computer Conferences of Historical Value: An Exploratory Study," The American Archivist 55 no.2 (Spring 1992): 316-330; Anne J. Gilliland-Swetland and Gregory T. Kinney, "Uses of Electronic Communications to Document an Academic Community: A Research Report," Archivaria 38 (Fall 1994): 79-96.

12 Helen W. Samuels, Varsity Letters: Documenting Modern Colleges and Universities. Chicago: Society of American Archivists and Scarecrow Press, 1992.

13 Richard J. Cox, "Re-discovering the Archival Mission: The Recordkeeping Functional Requirements Project at the University of Pittsburgh, A Progress Report. Archives and Museum Informatics 8 (1994): 279-300; Richard J. Cox and Wendy Duff. "Warrant and the Definitions of Electronic Records: Questions Arising from the Pittsburgh Project," Archives and Museum Informatics 11 (1997): 223-231. 
outputs, constraints and resources involved in creating reliable records in electronic systems. ${ }^{14}$ However, in both cases (which constituted some of the earliest North American collaborative research led by archival studies academics), what were supposed to be clear articulations of archival and recordkeeping ideas still proved to be too arcane for systems designers and even archivists to follow with ease (see the chapter by Hofman in this volume for more on this issue).

The first ARPANET network mail message was transmitted by Ray Tomlinson between two machines side by side in a lab in 1971. By 1993, CMC technologies had been available for over twenty years and electronic mail was by far the most prolific and prominent application. However, even though there was considerable ferment in the field over other forms of electronic records and recordkeeping, the challenges and potential of the materials created through the use of CMC had only obliquely been addressed by both archival practice and the professional literature. Various government agencies published draft guidelines on the retention of electronic mai $1^{15}$ but actual implementation of these guidelines remained a problem. This was largely because of the legal, technical, and political difficulties that these materials presented to archivists. Examples of these difficulties included defining what might legally be considered a record in a given environment; how best and when to capture and appraise such transient materials; and how to avoid violating, or being perceived by email users to violate, their personal privacy.

Until litigation started to occur in the U.S. regarding the possible record status of email, there had also been a pervading sense among administrative users and archivists alike that such electronic communications were employed only by a limited sector of organisations and not used for the important administrative activities traditionally falling under the purview of records management or documented by existing archival programs. Moreover, many administrators and archivists viewed email as a convenient informal and informational way to communicate, similar to the use of the telephone. As they do today, individual emails frequently contained a mixture of official and

14 Luciana Duranti, Terry Eastwood, and Heather MacNeil, Preservation of the Integrity of Electronic Records. Dordrecht, The Netherlands: Kluwer Academic, 2002; Luciana Duranti and Heather MacNeil, "The Protection of the Integrity of Electronic Records: An Overview of the UBC-MAS Research Project," Archivaria 42 (1996): 46-67, and "Protecting Electronic Evidence: A Third Progress Report on a Research Study and its Methodology," Archivi \& Computer 6 no.5 (1996): 343-404.

15 Examples would include the National Archives and Records Administration and the State Archives and Records Administration of New York. 
personal business that in itself posed major problems for archival appraisal, arrangement, and description. ${ }^{16}$ Since in most cases individual emails were created within a system that did not make distinctions between the kind of business or personal function in the context of which the email was sent, it was felt that appraisal, if it was going to take place at all, would need to be conducted not of the system as a whole, but in some way at the level of the individual materials that it transmitted and stored.

At the same time, because of how it had enhanced scholarly communication in the academic setting, computer-mediated communication and its impact on the research community and society in general, had become a subject of study in itself. ${ }^{17}$ The influence of documentation strategists within the archival community was also beginning to be felt in the world of electronic records management. Advocates of documentation strategy approaches ${ }^{18}$ were quick to point out that archival involvement in systems design and analysis could bring with it increased opportunities to document not only official activities, but also issues and movements of social and topical value. Terry Cook had noted in 1991: "Policy files ... suffer from underdocumentation because important decisions are made by telephone, personal conversation, or in other ways." ${ }^{19}$ Electronic mail was, increasingly, one of those "other ways." Helen Samuels argued that the spectrum of college and university activities had been poorly documented by archivists. ${ }^{20}$

16 For further discussion of these and other management issues at the time, see Anne J. Gilliland-Swetland, Policy and Politics: Electronic Communications and Electronic Culture at The University of Michigan, A Case Study in the Management of Electronic Mail, in Society of American Archivists case study series Archival Administration of Electronic Records and the Use of New Technologies in Archives. Chicago: Society of American Archivists, 1996.

17 Communications researchers, especially those who were sociometricians and bibliometricians, were particularly interested in this area.

18 A documentation strategy is an appraisal methodology that, "consists of four activities: 1. choosing and defining the topic to be documented, 2. selecting the advisors and establishing the site for the strategy, 3. structuring the inquiry and examining the form and substance of the available documentation, and 4 . selecting and placing the documentation." Helen W. Samuels, "Who Controls the Past?" The American Archivist 49 no. 2 (Spring 1986): 116.

19 Terry Cook, The Archival Appraisal of Records Containing Personal Information: A RAMP Study with Guidelines. Paris: UNESCO, April 1991: 4.

20 "A modern, complex, information-rich society requires that archivists reexamine their role as selectors. The changing structure of modern institutions and the use of sophisticated technologies have altered the nature of records, and only a small portion of the vast documentation can be kept. Archivists are challenged to select a lasting record, but they lack techniques to support this decision-making. Documentation strategies are proposed to respond to these problems." Samuels, "Who Controls 
She suggested that several core functions, including intellectual life and socialisation, were poorly represented in the administrative records traditionally acquired by C\&U archives and in 1992 published Varsity Letters, a blueprint for documenting academic environments. ${ }^{21}$ The findings of the Bentley Historical Library Conferencing Appraisal Project indicated that these functions were more completely documented within computer conferences, supporting speculation that this would likely also be the case with email.

A major and immediate problem for all archives, and especially for C\&U archives remained how to undertake such an appraisal task, especially with a small staff and often with little or no available technical expertise. Electronic mail not designated for archival retention at, or soon after the point of its creation was unlikely to endure long enough to be appraised within its original context because of system purges and purposeful or accidental deletions by users. However, as the Bentley Project had also found, the problem was complicated by the concern that if archivists were able to identify a mechanism for designating email for archival retention at the point of original transmission, they might somehow violate the privacy of correspondents who legally and ethically would have expectations that their current email would be both confidential and secure from outside viewers except with their explicit knowledge and consent. ${ }^{22}$

This exploratory study, therefore, represented an empirical effort to identify an inexpensive, readily comprehensible automated way to identify documentation of long-term value from the ever-growing mass of electronic mail created, communicated, and received within a major public university.

\section{Research Design}

In line with the research in communications and organisational behaviour on which I was drawing at the time, the underlying philosophy of the research design was to blend qualitative and quantitative techniques in such a way as to strengthen the reliability and generalisability of the resulting

the Past?", 109. In this, Samuels was echoing another university archivist, Nicholas Burckel, who wrote that: "Another collecting focus [of] college and university archives should be the intellectual and cultural atmosphere which the university engenders. This can hardly be determined from a look at transcripts of college catalogs." See Nicholas C. Burckel, "The Expanding Role of A College or University Archives," Midwestern Archivist 1 (1976): 5.

21 Samuels, Varsity Letters.

22 Gilliland and Kinney, "Electronic Communications." 
research outcomes. ${ }^{23}$ Although it built on prior work with the Bentley Conferencing Appraisal Project, I did not model the study directly from any one approach used previously in archival research. I considered and ultimately rejected a number of possible conceptual and systems approaches, chief among them was that being taken by the Pittsburgh Project, which was based around the transactional nature of business communications. ${ }^{24}$ While this transactional approach was derived from standard business practices and regulatory needs, it did not completely correspond to how the law might interpret a record in an electronic mail environment. Instead its value lay in the fact that it afforded archivists and systems developers a way to identify a record and the opportunity to develop archival functional requirements for electronic mail systems that operated at the user interface level. At this level, Bearman argued, users would be empowered to identify their outgoing and incoming mail according to a menu of different genres and document usages, some of which would be scheduled for archival retention based on their form-of-material. ${ }^{25}$ In order to capture email in the electronic environment, therefore, this approach offered two options: establish an institutional information policy that mandated appropriate disposition for specific types of record material being transmitted; and/or build a mechanism into the architecture of the email system for email users to identify their own transactions according to their administrative value (e.g., filing and routing menus), as has been done at the World Bank and the Northern Territory Department of Mines in Australia. The major limitation of this approach in terms of its relevance for this study was that it was directed toward the capture of official materials, the scope of which can be

23 See, in particular, the collection of essays by authors such as Christine Borgman, Leah Lievrouw, Belver Griffith, and William Paisley contained in "Bibliometrics and Scholarly Communications," a special issue of Communication Research (October 1989) edited by Christine L. Borgman.

24 In this context, Bearman defined records as:

at one and the same time the carriers, products, and documentation, of transactions. Transactions are, by definition, communicated from one person to another, from a person to a store of information (filing cabinet, computer database) available to another person at a later time, or from a store of information to a person or another computer. As such, transactions must leave the mind or computer memory in which they are created, or must be used by a person with access to the same computer memory. The transaction record must be conveyed across a software layer, and typically across a number of hardware switches.

David A. Bearman, "Towards a Reference Model for Business Acceptable Communications," unpublished paper, December 6, 1994.

25 David A. Bearman, "Electronic Records Guidelines," in Electronic Evidence: Strategies for Managing Records in Contemporary Organizations. Pittsburgh, PA: Archives and Museum Informatics, 1994, p. 100. 
harder to ascertain in institutions such as universities where records tend to be less defined by legal and regulatory mandates, and where materials created by faculty members and sometimes even prominent administrators are often treated more like personal papers than official records. Moreover, Samuels' research had exhorted C\&U archives to assess a much broader base of university documentation than just administrative records. The Pittsburgh approach would likely miss less formal correspondence, as well as sequences of email interactions between pairs and clusters of individuals. It also left opportunities for email users to misfile documents accidentally or deliberately, possibly leading to incorrect retention and destruction actions.

I decided instead to develop an automatic appraisal mechanism in the form of a prototype expert system that could function as a "front-end" to a specific university email system, but that was potentially extensible to any stored file of undifferentiated CMC, in order to assist institutional archivists in the appraisal process. Such a front-end system would facilitate the appraisal of both official and personal communications. At the same time, it would distance not only the email user, but also the human archivist from the active record and thereby mitigate some of the threats to personal privacy perceived by individual email users, and any risk that archival "monitoring" of electronic communications might affect the nature of the interaction by making the participants self-conscious or feel intimidated. ${ }^{26}$

This approach was suggested by a project that developed in the mid-1980s as an engineering and business application at the Massachusetts Institute of Technology (MIT). MIT's Information Lens Project was a rule-based information management system that emphasised the development of customisable filters or templates that could be used by individual or group end-users of electronic mail to handle their communications and information overload more effectively. These filters were built around what they termed "cognitive," "economic," and "social" factors that I suspected had many analogies to the criteria and values used by archivists during the appraisal process. The expert assistant I envisaged would contain a series of progressively refined and customisable filters and profiles designed to deselect both individual email messages and specific types of email of no archival value. The filters would be based upon a set of rules codifying expert views on appraisal, but defined in the context of the mandate of the individual archives. They would

26 These user concerns had been previously identified by the Bentley Conferencing Appraisal Project. 
act upon analyses of the form, body (i.e., content), and header, routing and signature file information contained in the messages, apparent message trails, and knowledge about the positions and status of email senders and recipients drawn from the university's standardised electronic directory system. ${ }^{27}$

The study assumed that, as with manual appraisal, a high percentage of active email would probably be filtered out automatically as having little or no archival value (e.g., listserv messages, routine memoranda), and the remainder that was identified as potentially having long-term value could be digitally stored for three or more years. After that period, archivists could subject the stored material to a more thorough manual appraisal, which would assure, with the benefit of hindsight, the retention and description of only those materials deemed to exhibit values in line with the university archives' mandate and appraisal policies. ${ }^{28}$ (Of course it should be noted that today, as the University of Oregon case highlights, even if over $90 \%$ or more of email were to be filtered out, the remaining percentage would likely still be too voluminous for manual processing and further computational approaches such as those being attempted with the Twitter Archive and discussed later in this chapter would need to be applied.)

In order to carry out the research, a bibliometric analysis of citations in the archival literature (a quantitative technique) was first conducted in order to identify living individuals who might be considered "experts" in the area of archival appraisal. The choice to use bibliometrics was in part because I had already used the method in other research and therefore was familiar with what it entailed and also its limitations. It was in part also a political choice. I wanted an "objective" way to identify figures whose ideas about appraisal had been influential upon the archival field in North America that would withstand challenges as to why others had not been asked to participate in this study. And I wanted to work with these figures in particular because I was also interested in whether a codification of the range of prominent appraisal ideas was even possible.

27 An X.400 system.

28 This was an approach alluded to by Terry Cook, when he stated that, "Records centre storage can be used to gain distance and perspective in making appraisal decisions, especially for those series of records involving public issues or government functions which are controversial, hotly debated in public forums, and emotion laden for many citizens (including archivists) at the time of their occurrence." Cook, Archival Appraisal, 81. 
Since the North American archival literature was relatively small and inbred, ${ }^{29} \mathrm{I}$ sought to augment the identification of experts from the citation analysis using a snowball sample to ensure that all possible experts would be identified and contacted. In line with accepted thinking regarding knowledge acquisition from experts, I conducted focused written and oral interviews and follow-up discussions with the identified experts (a qualitative technique) to ensure that theoretical expert knowledge was adequately represented in the core and alternative rules included in the expert system rule-base. Evaluation of the various development stages of the prototype expert system were based upon archival practitioners' expert appraisal experience and knowledge (that is, the appropriate university archivists) in the context of the specific mission, needs, and culture of the institution whose communications were being appraised (qualitative), as well as by examination of the statistical data regarding the ratios of filtered and non-filtered electronic communications (quantitative).

A modular approach such as this was valuable when conducting an exploratory study in areas about which little knowledge or data existed, and where consequently there was a danger of developing a study that could have both low validity and low generalisability. Each of the three research phases (that is, the citation analysis, the knowledge acquisition process, and the building of the expert prototype) had considerable research value in itself and was designed to stand independently of the others. This modular approach allowed possible confounding variables to be identified in the research phase where they first appeared, and also ensured that the research was not a wasted effort should subsequent modules fail to produce successful results.

\section{Research Methods and Related Procedures}

\section{a. Bibliometric Citation Analysis}

Bibliometrics is a quantitative research method that belongs to the same family of "metrics" methods as sociometrics. It is used, through mathematical and statistical analysis of citations and sometimes of content, to discern patterns and draw inferences about the influence and dispersion of particular

29 Anne J. Gilliland-Swetland, "Archivy and the Computer: A Citation Analysis of North American Periodical Articles," Archival Issues 17 no.2 (1992): 95-112. 
authors, publications and subjects within a given literature or literatures, and by extension, field or fields. Since its inception, certain bibliometric "norms" or "laws" have been discerned, largely based on scientific and technical literature analyses, for how literatures within fields are expected to behave. Bibliometrics does not, however, address biases inherent in citation practices, and the data analysed is not always complete or accurate. Logistically, its biggest limitation until recent developments in automated citation identification and indexing on the web, was that citations had to be identified and then analysed by hand (as was the case for this study). ${ }^{30}$ This was both time-consuming and did not scale well when large amounts of documentation needed to be analysed. ${ }^{31}$

In bibliometrics, researchers must unambiguously define and then rigidly follow their own rules about how a given literature is selected and analysed. Declaring these rules in writing up one's results is also important since it enables others to replicate the study or the study's approach using with subsequent or similar citation sets, thus allowing for cumulative knowledge development about a field. Since this study was concerned with codifying archival appraisal as it was understood and practiced in North America, only the North American monographic and periodical literature was analysed, and 1972 was selected as the likely first date when authors on appraisal might have begun to be aware of CMC. ${ }^{32}$ There was no benchmark in archival research for developing citation analysis guidelines other than that the guidelines be explicitly stated and consistently followed so that the analysis

30 See Nicola De Bellis, Bibliometrics and Citation Analysis: From the Science Citation Index to Cybermetrics. Chicago: Scarecrow Press, 2009.

31 This facet of bibliometrics is evidenced in the context of this study in that it built on prior applications of bibliometrics, and Kimberly Anderson's study (discussed in her chapter in this volume), in turn, applies some of the rules used in the study discussed in this chapter.

32 The periodicals examined were the American Archivist, Prologue, Archival Issues (formerly the Midwestern Archivist), Provenance (formerly Georgia Archive), Archivaria, and the Public Historian, which at the time, according to Richard Cox: " . . should be, essential reading for any archivist." These journals, with the exception of Prologue, which was an official publication of the National Archives and Records Administration, were published by major archival associations in the U.S. As such, most of the journals were distributed free to members of those organisations and also were sent by subscription to many archival and library repositories. See Richard J. Cox, "American Archival Literature: Expanding Horizons and Continuing Needs, 1901-1987," The American Archivist 50 (1987): 317. 
could be replicated by other researchers. ${ }^{33}$ Rules for analysis, therefore, had to be established for this study. ${ }^{34}$

33 In fact, subsequent bibliometric research conducted by Richard Cox and by Kimberly Anderson both drew upon the procedures laid out in this study. See Richard J. Cox, "Searching for Authority: Archivists and Electronic Records in the New World at the fin-de-siècle," First Monday 5 nos. 1-3 (January 2000). Available: http://firstmonday.org/ htbin/cgiwrap/bin/ojs/index.php/fm/article/view/721/630; and Kimberly Anderson. Appraisal Learning Networks: How University Archivists Learn to Appraise Through Social Interaction, doctoral dissertation. Los Angeles: University of California Los Angeles, 2011.

34 A simple approach to identifying which articles and other texts to analyse would have been to select a well known library and information science or humanities indexing, abstracting or citation resource and then to cull all those items covered in the last 21 years that were indexed under the professional terminology "archival appraisal." This was not feasible, however, since archival science to a certain extent was located on the periphery of each of the coverage areas of such resources and in no case were all of the journals to be used in the citation analysis indexed and/or abstracted by any one resource. Moreover, in the instances where one of the journals was indexed or abstracted, the subject headings used were not sufficiently accurate regarding the archival definition of appraisal to be useful for this citation analysis. Using titles catalogued in OCLC, monographs were selected for analysis, therefore, where relevant word stems (e.g., "apprais", "sampl*" or "select"), terms (e.g., "valuation" or "assessment") or phrases (e.g., "documentation strategy" or "documentation plan") appeared in either the title statement, the index terms assigned by Library of Congress Subject Headings, or both. In the case of periodicals, each issue was physically examined and for an article to be selected for analysis, the word stems "apprais*" and/ or "select";" and/or either of the phrases "documentation strategy" or "documentation plan" had to appear three or more times in any or all of the title, abstract, or first or last paragraphs. These words stems and the frequency of their occurrence were chosen to try to avoid false drops in the form of articles or texts relating to the appraisal of manuscript materials for monetary value, an entirely different area of study. These rules were based on those used by the author for a previous citation analysis of archival literature. See Gilliland-Swetland, "Archivy and the Computer."

35 The analysis of citations and references contained in the literature applied the following rules: a) References contained in footnotes, endnotes, and bibliographies were all analysed for both monographs, and monographic and journal articles. Since the journals varied widely in the type of work that they presented as an article (e.g., review articles, commentaries, case studies, author responses, proceedings of meetings, and news items) a working definition of what constituted an article within the scope of this study excluded news notes, letters to the editor, and book reviews but included all the other types listed above; b) The number of citations made to each individual author was counted; c) Citations to multiple works by the same author were counted; d) Multiple citations within an analysed publication to the same work were counted only once; e) In cases where citations are to works with multiple authors, each author was counted separately; f) Citations to non-North American authors were included under the assumption that they were exerting an influence on American thinking about appraisal. This work was all done by hand, but today more of this can be automated because so much is now in digital form. However, not all the publications are indexed by the leading indexing or citation services and indeed some that used to be have been dropped in recent years. This constitutes a major logistical limitation to using bibliometrics in archival research. 


\section{b. Expert Knowledge Acquisition}

Living authors whose works fell in the top $25 \%$ of citations identified were considered to be experts in the field of archival appraisal and were contacted and asked to participate in this study. ${ }^{36}$ These individuals were sent a letter explaining the nature of the study, asking for their cooperation, and giving a short list of broad questions and issues regarding archival appraisal for their consideration. The questions asked about what the experts regarded as the central tenets of archival appraisal theory, which texts they believed to be particularly valuable, how they personally might approach the appraisal of electronic records, what practical factors should be considered together with archival theory during the appraisal process, including any considerations that they felt might be specific to the college and university environment, and the names of those individuals whom they regarded as experts in the area of archival appraisal. The process of knowledge acquisition involved a telephone or in-person interview (which was recorded in cases where it was both feasible and agreed to by the experts) or written responses structured around the broad questions and issues introduced in advance by the letter to the experts. This knowledge acquisition process was only semi-structured and used open-ended questions in order to encourage candid comments and free-flowing thoughts on the part of the experts as to the rules, guiding principles, and heuristics they used in archival appraisal. It also left me free to follow up on any points that were unclear or insufficiently articulated to be codifiable.

Any individuals identified by these experts but not by the citation analysis as being influential in the area of archival appraisal (perhaps because newly published works had not yet been significantly cited, or because an individual did not publish but held a key position as an appraisal archivist), were also considered to be experts and were contacted with the same letter and questions, and the same interview process was conducted. The knowledge acquired from each expert was codified into a set of unambiguous statements and was sent back to the expert for his or her review, clarification and comment, and then revised again. Sometimes this process was repeated

36 In the initial proposal for this research and consistent with bibliographic citation norms for scientific literature, I had expected that living authors who were included in the top $50 \%$ of citations would be those selected as experts. However, the resulting bibliometric data did not match the expected norms, perhaps because archivists during the time period covered, coming from a strong historical disciplinary approach, might not have used footnotes and references in the same way as did scientific authors; or simply because they tended to publish less and the literature was more scattered. 
several times. This ensured that the knowledge accurately represented the views expressed by the experts as well as giving them an opportunity to add further thoughts they might have on the subject.

\section{c. Building and Testing the Expert System}

The decision to develop an expert appraisal assistant was based on several characteristics exhibited by expert systems that made this technology particularly appealing for a project of this nature. Expert systems were widely employed (and remain so today) in situations where complex reasoning and expert knowledge are involved, for example, in business decision-making, marketing, and scientific and medical diagnostics. In particular, I hoped that the ability of expert systems to make decisions when faced with uncertainty should allow archivists to build a system that not only included any categorical principles that guide archival appraisal theory, but also the kinds of institutional and even personal heuristics that inform archival appraisal practice (although the reasoning abilities and potential to function as useful assistants of expert systems can only be as good as the extracted and coded knowledge of which they are built). There were several additional advantages to taking an expert systems approach that were not necessarily the case in other forms of systems development. These advantages included the facts that expert systems could:

- be developed and used by individuals who are not highly skilled in computer programming (in this case, even by archivists themselves);

- be cost-beneficial in that they require relatively little investment in terms of time, staff, and software, with the potential of a large pay-off in functionality;

- be rapidly prototyped and easily modified, especially small to midsized systems that utilise up to approximately 200 rules;

- be constructed using a variety of off-the-shelf expert "shell" 37 software rather than original programming, if desired, or a mixture of both, allowing for a high degree of customisation;

37 An expert "shell" is a software package that facilitates the development of an expert system. Shells provide a minimum of an inference engine, interface design capabilities, and a mechanism for developing and integrating rules. The actual content of the system is added by the systems developer or knowledge engineer. 
- provide a readily customisable interface in addition to a knowledge base and an inference engine, which can make a system very accessible to archivists who do not have much technical background;

- provide various feedback mechanisms that could elucidate the underlying appraisal decision criteria and processes; and,

- interface as "front ends" to other systems such as databases or telecommunications systems.

The major limitation of expert systems is that they are "brittle" in that they do not know what they do not know. They need to work, therefore, within carefully drawn subject or functional limits. Without such limits, they tend to crash ungracefully when faced with a situation for which they were not programmed. As a result, expert systems technology would not be appropriate for use in a situation where a system would be required to operate alone twenty-four hours per day and where a poor, or simply wrong decision might cause a major accident or incident. In the case of archival appraisal, however, I decided that the needs to have the system running constantly and to make a "correct" decision regarding every single email were not crucial and therefore that the above limitations did not pose sufficient reason not to use expert technology (perhaps in light of the University of Oregon case I was mistaken about that!).

I selected an expert system shell based on the opinions of several individuals knowledgeable in the area of knowledge engineering, reviews of existing expert systems, and necessary technical specifications. I also believed that it was important that the software have explanation facilities, such as understandable rule-tracing, in order that the reasoning behind expert appraisal decisions could be deduced and explained to a court of law, other archivists, or concerned research communities. At the same time, however, one of the points of the study was to look at the extent to which an archivist could articulate archival requirements of an expert appraisal assistant to a systems designer who would then build it, without needing to become intimately involved in the details of coding the system. ${ }^{38}$

38 In terms of expert system capabilities, I had sought a minimum of the following: ability to work with frames (a structure for representing facts or data, procedures and default values that was originally derived from semantic networks and that can be linked together like building blocks) and inheritance; ability to handle a mid-sized rule-base; and ability to function as a front-end to an electronic communications system or file of communications. Whether the system would need to be able to work with semantic networks would depend upon whether rules were developed that might require such a level of natural language processing. 
Aluri and Riggs had outlined several stages involved in building a library expert system (and these are largely in line also with those outlined by Nunamaker and Chen as typical in systems development approaches: 1) identification of a topic or area; 2) conceptualisation (for example, what will be the system's parameters, who will be the experts and engineers, how will the work flow be organised?); 3) formalisation (decisions regarding the tools, concepts, and design to be used); 4) implementation of a prototype; and 5) evaluation of the system. ${ }^{39}$ These stages, which are fairly generic for the development of an expert system in any environment, were largely followed in this study. Today there are software applications that will interview experts and automatically code the knowledge derived thereby. However, this process still needed to be conducted manually at the time when this study was being undertaken.

As already indicated, the prototype developed for this study served as an expert assistant in the appraisal (more properly, an expert assistant in the "meta-selection", because it was making a preliminary selection and not a final appraisal) of electronic mail in a public university setting. Although it could potentially function proactively as an archival front end to an institutional mail system, or its rule-base could be further customised to serve administrative or personal information management purposes, it was never intended to function completely independently of human review. This prototype was customised to address the mandate and appraisal practices of one particular university archives in a major American public research university.

Methodologies advocated in expert systems literature for testing the effectiveness of prototype and operational expert systems were scant at the time and lacking in rigour. On the whole, they were not relevant for the nature and use of this prototype system. ${ }^{40}$ Instead, I devised my own

39 See Aluri Rao and Donald E. Riggs, "Applications of Expert Systems in Libraries," Advances in Library Automation and Networking 2 (1988): 1-43.

40 The most detailed discussion of evaluation criteria found was contained in Vickery and addressed to the library reference environment: System power - does the system exhibit intelligent behaviour in performing complex tasks? How robust was the system with imprecise or incomplete data? How flexible was the system? System response time. Transparency of the system, and effectiveness of the user interface and explanation facility. Does the system perform equal to a human? Hardware and software performance. Ease of use. Completeness and consistency of the knowledge base. Comparison with the same library service when performed manually. Unfortunately, many of these evaluation criteria proved either to be impossible to implement because of system and data limitations, or would have yielded results that would have been hard to interpret because of confounding variables. Instead I used the evaluation mechanisms 
evaluation strategy, employing the assistance of the university archivists who were responsible for traditional appraisal of official institutional correspondence to assess a sample of messages being caught in the filters, and then refining the filters accordingly and retesting them. In line with the iterative development, evaluation, and refinement processes that underlie systems development as a method, this strategy needed to be modified several times as the project progressed. Nevertheless, the university archivists highlighted several interesting difficulties in making manual judgments due to the volume of messages, the difficulties of making accurate judgments at the level of individual item and with what they considered to be technical content. Moreover, because the messages dated from 1989 to 1994 and had not been redacted to reduce their potentially sensitive nature (i.e., I did not have permission myself to view them individually, as discussed further below), I chose to examine the results of the filter combinations only in terms of changes in the ratios of items marked for retention and deletion with different filter combinations.

\section{Reflections on the Methods, Extracted Knowledge, and Test Collection Used}

The citation analysis proved to be an excellent mechanism for providing some insight into the nature of the archival field at the time. It indicated that the North American archival profession had a very small, and rather self-referential corpus of opinion leaders in the area of appraisal, and that there was very little influence from literatures in other fields (including technological fields) or archival traditions. Instead, many authors published only a few thoughtful and thus heavily cited articles or monographs in their career but did not pursue a consistent and rigorous path of research investigation. This would likely be different today, since there are now international research journals with broader coverage, and an identifiable cohort of academics and practitioners doing research in the field in North America and around the world.

Eight of the experts identified agreed to participate in the study. Together they represented a range of ages, backgrounds, and proponents of differing appraisal strategies, something that initially I considered to be optimal. A larger number would have made the codification process considerably

outlined below, and while they drew upon some of Vickery's criteria, they were tailored more closely to the archival environment and the task to be performed in this instance. See Alina Vickery and Helen Brooks, Expert System for Referral. Library and Information Science Research Report No. 66, British Library, 1988. 
more difficult. However, most expert systems only attempt to represent the knowledge acquired from one individual and as it transpired, and as guidance on expert knowledge acquisition cautions, heterogeneity among the experts due to being drawn from different archival traditions (i.e., U.S. and Canadian - see Chapters 1, 3 and 4 by Gilliland, Lian, and McKemmish, respectively, for further discussion of this consideration), different theoretical approaches (e.g., macroappraisal, Schellenbergian) and different institutional backgrounds (e.g., university archives and special collections, government archives, historical society) proved to be a significant limitation in defining a definitive rule base. With hindsight, another way in which this process could have been handled, if the experts had been available to do so or if the system had been available to them remotely, would have been for them to interact with the rule base directly and make corrections "on-the-fly". However, that option was not available at the time. Alternatively, a Delphi process could have been used. Delphi studies are used to reach consensus or predict an outcome or correct answer. They most commonly involve asking experts to respond to a series of rounds of questionnaires. After each round, the facilitator summarises and anonymises the experts' responses and the reasons they gave for those responses and sends them out to the experts, encouraging them to revise their prior responses in light of the summaries and reasoning provided. The benefit of such an approach for developing an expert rule-base would be to obtain a single consensus about rules. While this might have improved the technical outcomes of the study, however, it would not have elucidated the range of opinions on appraisal - something that was a topic of interest for this study. Moreover, similarly to any standard that is developed through a group consensus development process, it would likely be less responsive to individual institutional or local contexts, and this study was trying to find a balance between broad agreements and local mandates and practices regarding appraisal goals, values and processes.

Based on the knowledge acquisition process that took place through interviews and written correspondence, a wide range of thoughtful comments on appraisal emerged that demonstrated the complexity and sophistication of appraisal. This knowledge acquisition process also demonstrated a considerable lack of consensus between experts on why and how appraisal is conducted that was strongly based on their levels of experience and milieus. In fact, when I grouped the acquired knowledge into 45 principles and heuristics, only 2 of these were expressed by all the experts. Most of the experts who participated in this study, as is probably the case with archivists in general, were extremely comfortable expressing their ideas using text and 
drawing upon the richness of language to convey the subtleties of the art, as well as the science, of appraisal. Complex and sophisticated knowledge expressed in this way can be a double-edged sword, however, when it comes to developing a system to emulate human processes such as appraisal. While such knowledge can lead to the development of a very powerful system, a lack of clarity, precision, or consistency in how it is expressed to a designer, as well as inherent system limitations, can result in a system that has to resort to using simpler, less controversial concepts. Today another way to go might be to opt to examine the heuristics of personal file management, beginning with studies that have been published in the growing literature on personal digital archives. When one builds one's own email filters and filing schemes, in some ways that is analogous to building one's own expert system to appraise and classify one's email.

After finding so little commonality among the appraisal experts, I could have ended my research with those findings, but I did not believe that such a finding negated the needs originally outlined for developing automated appraisal front ends for electronic communications, nor that it made it impossible to develop an expert appraisal system tailored to one specific institutional archives. Because of the lack of agreement on appraisal principles and heuristics (i.e., rules of thumb), I modified and considerably simplified the original design and combined the acquired knowledge into several groupings representing the type of appraisal considerations cited, as well as how frequently each consideration was cited.

Unlike the Bentley Conferencing Appraisal Project, which worked with both live and "archived" conferences, the prototype was iteratively tested using an extensive test collection of electronic mail of academic and research provenance associated with one senior administrator of a leading Midwestern university. The email had been downloaded and a certain amount of redaction had taken place to address sensitivity and privacy concerns. I had sought out as extensive, heterogeneous, and organisationally crosssectional source of academic administrative and/or research electronic communications as possible. For political and legal reasons, obtaining a test collection for this study, whether live or offline and "massaged," proved to be possibly the most problematic aspect of the entire project. While far from ideal for the actual systems development and testing, I was very fortunate to have been given the opportunity to work with this particular test collection. This issue, however, points up two very important issues relating to archival research infrastructure as well as generalisability and validity concerns for research outcomes. The first of these is the essential need for test collections 
with which those engaged in archival systems design can work. The second are the benefits and disadvantages of artificial and real-life test collections. The information retrieval (IR) community has a long history of working with artificial test collections (i.e., collections of materials or bibliographic data specifically constructed for conducting retrieval tests and where every item is known). These allow for accurate assessment of such aspects as recall and precision, replication of empirical studies, and subjecting the same test collection to a barrage of different research approaches (see Furner and Gilliland's chapter in this volume for more discussion of IR research). However, such collections can be far removed from the complexities and idiosyncrasies of a real-life system and its contents, where also the content can be surmised but often not accurately delineated. While using a real-life system and its contents can make for more realistic studies, it can be difficult to evaluate the efficacy of the tests being carried out, especially in terms of measurements because its contents, parameters and characteristics might not be completely determinable. It might also be hard to identify variables that could be affecting evaluation results. The test collection to which I had access unfortunately combined the limitations of both artificial and real-life test collections.

A further note on the limitations of my test collection is also warranted here since recognition of these limitations in the context of this study is, in itself, a valuable component of the research. The most obvious limitations in this study (and also considering Samuels' documentary objectives that inspired it) was that the test collection only contained the outgoing messages, and therefore reflected the electronic mail creation patterns of only one key individual within the university. A major characteristic of email, and many forms of materials created by $\mathrm{CMC}$, is the way in which they messages are connected as trails of correspondence, or by clusters of senders and recipients. Any automated archival system needs to be able to comprehend and exploit these relationships because they are an important aspect of the documentary context of those materials. For this reason, testing on a live system would be preferable to that of a limited and massaged test set. Moreover, when the messages in the test collection had been saved as ASCII text, they were saved as they had been seen through the email viewer and were stripped of much of their routing information, again essential evidential detail for assessing records (it should be noted that this would also be a matter of concern for real life acquisitions if the creators had massaged or redacted them significantly before transferring them to the archives). The research data security protocol that I was required to follow also provided an additional challenge that 
would need to be addressed in real-life developments of this sort (where the archivist or systems developer would presumably be employed by the institution) in that it necessitated that the researcher and programmer design the expert system without looking at the format or contents of the messages in the test collection.

\section{Possible Roles for Automated Systems Development in Contemporary Third-party Identification, Evaluation, Long-term Preservation and Retrieval of CMC}

Although in the interim much has changed in the archival field and with $\mathrm{CMC}$ technologies, communications infrastructures, and the ubiquity and nature of their usage (see Acker's chapter in this volume), this study remains one of a very small corpus applying systems development as a research method to address the archival problems associated with long-term management of and access to CMC and indeed to understand the nature of CMC and the associated behaviours and patterns of their users/creators. ${ }^{41}$ This is particularly surprising given the fact that traditional correspondence, and now digital correlates such as electronic mail, text messages and tweets are often viewed as rich sources of both information and evidence by researchers and indeed investigators of all kinds. Moreover, the processes that are or might be applied in the evaluation, capture, and potential secondary uses of CMC by parties other than the creators or authors of those media have been the subject of several public controversies in recent years. Prominent examples include the release by Wikileaks in 2010 of more than a quarter of a million classified State department cables (known as Cablegate); and

41 Researchers at the Massachusetts Institute of Technology (MIT) have been working in this area for many years, and as already noted, some of that research influenced the study discussed here. Most recently, in July 2013, MIT researchers announced the development of Immersion, an application that offers many qualities that archivists might consider when developing digital tools to support how their own users might approach "reading" and analysing archived CMC: "Immersion is an invitation to dive into the history of your email life in a platform that offers you the safety of knowing that you can always delete your data. Just like a cubist painting, Immersion presents users with a number of different perspectives of their email data. It provides a tool for selfreflection at a time where the zeitgeist is one of self-promotion. It provides an artistic representation that exists only in the presence of the visitor. It helps explore privacy by showing users data that they have already shared with others. Finally, it presents users wanting to be more strategic with their professional interactions, with a map to plan more effectively who they connect with." See https://immersion.media.mit.edu/ 
revelations made by Edward Snowden since June 2013 that the U.S. National Security Agency (NSA), as part of its terrorist surveillance program, was provided unsupervised access to all fiber-optic communications, including electronic mail and text messaging conducted using major American telecommunications providers, and associated metadata. ${ }^{42}$ Such cases have not only raised questions about whistleblowing and unsanctioned release of privileged or sensitive communications, but also about how any organisation, including Wikileaks and U.S. intelligence agencies, might be in a position manually or computationally to gain access, review and, when necessary, redact, such a high volume of materials before release, as well as how it might arrive at assessments about what to release and what to redact.

Since 2010, Wikileaks' activities have escalated and so has the volume of materials being released. In July 2012, more than five million leaked electronic mail messages from Stratfor, a geopolitical intelligence firm that provides strategic analysis and forecasting to individuals and organisations around the world, were released. In the same month Wikileaks also released over two million electronic mail messages between Syrian political figures, agencies and companies. Undoubtedly, when faced with such a high volume of materials that individually or in relation to one another may reveal unanticipated information and evidence through their content or communication patterns, there is a role to be played by automated systems that can assist with the appraisal or evaluation and possibly even the redaction of the documentation. However, for those interested in uncovering information and evidence from such documentation, for example, the news media or intelligence operations, there is also a role for developing systems that are able to do more sophisticated forms of evidentiary retrieval.

While these examples have occurred for the most part outside the archival purview (although they have attracted much archival commentary), and presumably also open up venues for the kind of research discussed here in other than strictly archival applications, they also provoke many questions about the archival evaluation and processing of records generated by CMC and indeed what constitutes an archive and what records in the context of

42 It is worth noting, however, that despite what my own research indicates about the evidentiary importance of CMC metadata, and the deep concerns expressed by many archivists, the U.S. National Archives has asserted that "the data being collected by NSA is classified as "raw signal intelligence." Both congressional legislation and administrative order define "raw signal intelligence" as a nonpermanent, federal record. It will be destroyed." Frank Boles, "SAA Should Say Something!" Society of American Archivists press release (February 2015), http://www2.archivists.org/news/2015/saashould-say-something. 
CMC. Some of these are illustrated by the example of the Twitter Archive at the Library of Congress, which I will discuss in some detail in this section.

Foreseeing the rise to prominence of CMC, Canadian archivist Catherine Bailey wrote in 1989 that:

Electronic mail is the nearest written equivalent to the correspondence of the pre-World War II era, when decision makers committed their thoughts, feelings and judgments to discursive prose in official letters. The letters conveyed information for an immediate purpose; they were not written with an eye to history, nor did they serve the purpose of most official letters today - after-the-fact confirmation of decisions already reached. ${ }^{43}$

In 2013, the Library of Congress' justification for its decision to preserve or "archive" the Twitter Archive resonated with the same sentiments:

As society turns to social media as a primary method of communication and creative expression, social media is supplementing and in some cases supplanting letters, journals, serial publications and other sources routinely collected by research libraries.

Archiving and preserving outlets such as Twitter will enable future researchers access to a fuller picture of today's cultural norms, dialogue, trends and events to inform scholarship, the legislative process, new works of authorship, education and other purposes. ${ }^{44}$

Twitter is one of the most prominent examples of how, and how fast, everevolving forms of computer-mediated communications have transformed not only the ways and scale at which humans communicate with each other, but also the milieus in which they do so and the influence they can exert. The future of $\mathrm{CMC}$ and electronic mail appears today to be bound up with social media platforms and mobile operating systems like Android and Apple that support messaging (combining instant messaging, texting and electronic mail) and engaging multiple stakeholders, new pricing and market structures, protocols and standards. While there is debate on just how valuable the Twitter Archive will turn out to be, and also about the wisdom of acquiring social media through such a firehose approach and with little clarity about how, if ever, widespread access might be supported, there is no doubt that

43 Catherine Bailey, "Archival Theory and Electronic Records," Archivaria 29 (Winter 1989-90): 73.

44 Library of Congress, "Update," p.1. 
embedded in other forms of $\mathrm{CMC}$, such as email, interactive web pages, text messaging, Skype sessions, and blogs, is evidence in the form of the traces of and reflections on contemporary decision-making, reporting, conversations and other activities. Previously such traces and reflections would have been contained in the formal and informal correspondence, reports, diaries and notes that today fill the shelves of archives and provide some of our richest insights into these activities. This is not to say that direct equivalencies can be drawn between the communications media of today and those previously in terms of how they are used and by whom, but rather to argue that much of what we are likely to value most as future historical documentation of organisational and personal activities and decision-making is now distributed across a web of interdependent and otherwise linked digital "documents."

In keeping with its mission to "acquire, preserve and provide access to a universal collection of knowledge and the record of America's creativity for Congress and the American people, ${ }^{\prime 45}$ in 2010 the Library of Congress in Washington, D.C. entered into a controversial partnership with Twitter and social data provider Gnip to build and preserve an archive of tweets. This corporate partnership arguably suggests the dawn of a new kind of archival access model. At the time of writing, Gnip is the only third party developer that has access to all the firehose data from Twitter. Other vendors, clients and the growing corpus of researchers investigating social media patterns and implications must pay for access to tweet metadata through their tools and algorithms, or ping the public application programming interface (API) and hope for the best, a situation where access costs and availability of accumulated data not only limit such research, but also the replicability of its results and the testing of findings over time.

The Twitter Archive has received considerable media attention in terms of the strategies that are being employed by the Library of Congress and its partners, as well as the amount of public money that has been used to preserve the content of a form of CMC that is seen to be primarily trivial or over-hyped in terms of its influence by some, and to be a revolutionary tool capable of supporting private speech, citizen journalism, community organising, and tracking world events such as elections, military conflicts, and pandemics by others. ${ }^{46}$ Either way, it may be several years before its impact today can really begin to

45 Library of Congress, "Update on the Twitter Archive at the Library of Congress," January 2013, p.5.

46 See, for example, James Gleick, "Roving Librarians of the Twitterverse," New York Review of Books Blog, January 16, 2013, www.nybooks_com_blogs_nyrblog_2013_ jan_16_librarians_twi.pdf; Evgeny Morozov, "Facebook and Twitter are Just Places Revolutionaries Go," The Guardian, 7 March 2011. 
be understood. Its high profile and the associated debates about value have added to the existing pressure on American archives to justify or re-think the continued relevance and effectiveness of their traditional role and practices, and the values around which their judgments are based. It challenges archives to respond more proactively to the ways in which documentation is being created and might be used in the digital, networked world, lest they cede their role as preservers and providers of essential recorded evidence to others who are more technologically innovative. For example, would a national archival repository, rather than the world's largest bibliographic institution, similarly have determined that public tweets were worthy of acquiring and if so, on what basis, when and according to what processes? How well would their practices hold up to the kind of public scrutiny that the Library of Congress has experienced? Does the Twitter Archives' approach suggest that archival appraisal, ${ }^{47}$ and perhaps even archival description are no longer either relevant or, indeed feasible? However, if appraisal is indeed an obsolete approach, then why has the Library of Congress found that it needs to justify its commitment to preserve and make the Twitter Archive available in terms of future research value and the costs entailed and what evidence can it draw upon to support such justifications?

As of January 2013, the Twitter Archive had completed retrospective accessioning of public tweets from Twitter's founding in 2006 to 2010, and was digitally ingesting ${ }^{48}$ the tweets that had been sent each day subsequent to 2010 (almost half a billion a day and growing in January 2013). ${ }^{49}$ The Twitter feed is acquired in real-time (i.e., without any lag time between tweeting and ingestion), and without going through any appraisal process

47 The process of determining the value and thus the disposition of records based upon their current administrative, legal, and fiscal use; their evidential and informational value; their arrangement and condition, their intrinsic value; and their relationship to other records. A broader definition offered by documentation strategists is that of any selection activity that enables archivists to identify recorded information that has enduring value.

48 The term "ingestion," which is increasingly used instead of the archival term "accession" in relation to the acquisition and initial processing of digital materials, reflects the influence of the language used in the Open Archival Information System (OAIS) Reference Model, first developed in 1997 by the space science community as a high level model for the "archiving" of the massive quantities of digital data being generated in the space science community through such activities as remote sensing via satellite. See the Consultative Committee for Space Data Systems/International Organization for Standardization, Space Data and Information Transfer System: Open Archival Information System: Reference Model. Geneva, Switzerland: International Organization for Standardization, 1999.

49 Library of Congress, "Update," p.1. 
(i.e., no selection mechanism is used, for example, to identify only tweets associated in some way with America). The Library of Congress argues that, "It is clear that technology to allow for scholarship access to large data sets is lagging behind technology for creating and distributing such data. Even the private sector has not yet implemented cost-effective commercial solutions because of the complexity and resource requirements of such a task. ${ }^{{ }^{50}}$ In place of archival description, as already discussed, data mining of the chronologically-organised Twitter Archive is being conducted by Gnip to identify ways in which the current content of over 170 billion tweets and their associated metadata might be automatically discovered and retrieved. The Twitter Archive is also being made available to other commercial parties such as financial services providers, marketing companies, and social monitoring and analytics firms to mine, use for predictive modelling, and extend with additional services and capabilities. As yet, however, there is no public access to the Twitter Archive.

The massive volume, digital format, brevity and high variety (e.g., many tweets have embedded links or pictures) of individual tweets, as well as the high degree of inter-dependency and relatedness that often exists between them (i.e., their documentary context), lend a lot of heft to arguments in favour of abandoning traditional library selection and cataloguing processes, and relying on computational power to perform aspects of those functions as necessary. ${ }^{51}$ One wonders whether, if there were more investment by and expertise within archives to develop their own access tools and algorithms, such commercial fee-based approaches and the limitations to use that can result might be averted.

Even though the Library of Congress sees itself as addressing a set of data rather than evidence management concerns, ${ }^{52}$ keeping everything and relying upon computational processing to manage archived tweets and make

50 Library of Congress, "Update," p.1.

51 For example, Gnip is required to protect deleted tweets from public disclosure. In a paper world, materials that were intended to be deleted would likely not even reach the archives, but if they did, archivists would make a decision about their disposition as part of physically processing the entire accumulation or collection. In a digital world, however, the traces of deleted material, if not the actual deleted material (as in the case of Twitter) often persist, and the approach taken by the Twitter Archive is not to attempt to eliminate them before or after acquiring them, but instead to render them publicly irretrievable through the retrieval mechanisms being developed for use with the Archive.

52 Archivists, in conducting appraisal and description, which might be viewed as the parallel archival activities to library selection and cataloguing, are concerned about collectively assessing and elucidating the value of the materials in hand as evidence of bureaucratic, community and personal activity as well as for their informational value. 
them discoverable or compilable is a strategy that supports the capture and (re)presentation of a record (in the sense used by the Library of Congress) that is not static or isolatable but is, rather, continuously accumulating and evolving. It is, in fact, an approach that was advocated as long ago as 1991 by David Bearman, archives and museum informaticist, as well as some prominent information scientists. It was not taken up by archivists at the time, ${ }^{53}$ but today is very much in keeping with continuum ideas about the dynamic and interactive nature of recordkeeping and use. ${ }^{54}$ In line with methods used in social network analysis, ${ }^{55}$ this computational approach also offers new capabilities to end users that were not possible when working in a physical paradigm, such as following threads, trails and patterns of tweeters with particular personal profiles, tweets and retweets; identifying dispersion rates for events and other phenomena discussed in tweets; and potentially visually mapping these by date, geography or influential tweeters, or correlating them with other digital traces of particular events. ${ }^{56}$

53 David A. Bearman, "Archives in the Post-Custodial Age," Archival Management of Electronic Records, Archives and Museum Informatics Technical Report No. 13. Pittsburgh, PA: Archives and Museum Informatics, 1991. Gerard Salton and Chris Buckley developed a system around the same time that used flexible text matching procedures to retrieve documents from large text collections with unrestricted subject matter. Salton and Buckley's method broke texts into semantically homogeneous excerpts such as paragraphs or sentences; used a standard automatic indexing process to assign natural language weighted content identifiers; and then detected similarities between particular text items, or between text items and search requests, by comparing term vectors at various levels of detail. The authors gave several examples of how the system works, intellectually and mathematically, one of which was testing recall and precision in retrieval from a non-bibliographic collection of electronic mail. See Gerard Salton and Chris Buckley, “Global Text Matching for Information Retrieval," Science 253 (1991): 1012-1015.

54 Frank Upward, Sue McKemmish and Barbara Reed, "Archivists and Changing Social and Information Spaces: A Continuum Approach to Recordkeeping and Archiving in Online Cultures," Archivaria 72 (Fall 2011): 197-238.

55 Social network analysis is primarily a quantitative method that is concerned with social and cultural aspects and effects of virtual social structures. It typically employs two categories of methods to look at relationships between network members such as their positions, strength and clustering and diversity of their connections: network data collection (either socio-centric or ego-centric) that looks at the strength, confirmation and multiplicity of networked relationships of groups or individuals; and network data visualisation, that uses various visualisation methods alone or in combination such as maps and matrices. See Ajith Abraham, Aboul-Ella Hassanien and Václav Suásel, eds., Computational Social Network Analysis: Trends, Tools and Research Advances, London: Springer-Verlag, 2011; Carlos A.R. Pinheiro, Social Network Analysis in Telecommunications, John Wiley and Sons, 2011; and Carlos A.R. Pinheiro and Marcus Helfert, eds., Exploratory Analysis in Dynamic Social Networks, iConcept Press, 2012.

56 In his research relating to the use of Twitter in Egypt, Arnold discusses the development of an API that allows the curation of information on individual users who 


\section{Some Concluding Thoughts on Applying Systems Development Methods in Archival Research}

Since I undertook this research there have been several major shifts in archival thinking, and there are now many academic researchers in archival studies. Jerry $\mathrm{McDonough}$ has commented that academics have space and time to think and reflect. They can also look in places that practitioners cannot. ${ }^{57}$ When I was doing this study, reflexive approaches ${ }^{58}$ to systems analysis and development were not a major consideration and Donald Schön's work was just beginning to become influential in library and information science. However, even though systems design requires definitional precision, it still hardcodes the biases of the developer and the development context into the system, and every researcher in the field should attempt to acknowledge and account for that (see Evans' chapter in this volume). Ideally, conceptual work should come before implementation, but in a fast-paced technological world, there isn't always time for that conceptualisation to occur or to mature, which is one reason why more of it tends to occur in academia than in practice. It should be noted also that methods and frameworks themselves go in and out of fashion. When I started this study, expert knowledge extraction was popular and American archivists still believed that they could use appraisal and life cycle management to cope with the growing volumes of electronic materials. Although they evolve more slowly than the

an archivist might be interested in following (such as "elite" or professional tweeters), using the user's profile page, and allowing the researcher to track such aspects as the user's bio, who s/he is following, and who is following him/her. For search terms, it is possible to use common and niche hashtags, threads. Arnold says that methods can be qualitative, e.g., a researcher setting up his/her own account and embedding him/ herself in conversations, or qualitative, e.g., counting tweets, retweets and mentions, and these can be analysed respectively using content or statistical analysis software. Scraping software is also available. Arnold also notes that a research might examine language issues, e.g., colloquial or formal, vernacular or standardised. See Timothy Jason Arnold, "Digital Curation in the Age of Twitter: Curating a Collection of Tweets from the Egyptian Revolution," paper presented at the Council of State Archives and Society of American Archivists Joint Annual Meeting, New Orleans, August 2013. Another approach developed by George Washington University Libraries that can be used for archival acquisition and analysis purposes is Social Feed Manager, which manages rules and streams from social data sources. See https:// github.com/gwu-libraries/social-feed-manager.

57 Jerome McDonough, comments made at the Digital Preservation Symposium, Ann Arbor, June 25-27, 2011.

58 Reflecting critically upon the interpretative frameworks that are brought to bear in systems development and how they have been constructed, as well as upon the systems developers' own actions. 
current rate of technological development, this study illustrates that archival and recordkeeping frameworks, ideas and methods, and even institutional roles and mandates will also not last forever, but they do have an essential currency in the present, and systems development such as that discussed here is rooted in that present.

Systems development also has a lot of moving parts and someone invested in this kind of research has to resign herself to the fact that it will date quicker than will most theoretical work. It can have a more immediate payoff, however, if experimental systems subsequently go into production, and it can yield insights that can immediately be used in the next project. The study discussed in this chapter ultimately provided some early proofof-concept in support of automating or partially automating functions such as appraisal, accessioning or even potentially description and retrieval. To be taken further, however, it would have needed to have been a part of a series of ongoing, cumulative studies. Experimental prototypes such as the one described here that was developed using a test collection might then be implemented and tested with a real-life system, and subsequently, perhaps with multiple real-life systems with similar or different characteristics in order to isolate what might be effective and what not. Such a trajectory of studies was beyond the scope of work described here and really has yet to substantively occur in archival systems design and development.

Since conducting this study, the fields of knowledge extraction and data mining have advanced considerably although expert systems are still widely used in business, medicine and certain other sectors. The research community has grown impatient with the slow progress in the field of artificial intelligence research out of which this method emerged and now favours other forms of community knowledge extraction such as ontological modelling. ${ }^{59}$ Information communications and recordkeeping technologies have also evolved quite dramatically, with administrative and personal activities being conducted in a highly networked and mobile fashion in a constant accumulation of meanings and metadata (see, again, Acker's chapter in this volume). As a result, there have been serious challenges made to the

59 "Generally an ontology can be defined as a linguistic artifact that defines a shared vocabulary of basic concepts for discourse about a piece of reality (subject domain) and specifies what precisely those concepts mean. As such, ontologies provide the basis for semantic modeling of subject domain, information integration, and communication in the domain.” See Leonid Kalinichenko, Michele Missikoff, Federica Schiappelli, Nikolay Skvortsov, "Ontological Modeling," Proceedings of the 5th Russian Conference on Digital Libraries RCDL2003, St.-Petersburg, Russia, 2003, citeseerx.ist.psu.edu/viewdoc/ download?doi=10.1.1.101.5714.pdf 
continuing necessity and utility of appraisal, a solid part of the archival field for over a century. The notion of the self-describing record and intensified use of information retrieval and data mining techniques, as with the Twitter Archive, similarly challenge the traditional parameters envisaged around description.

At the same time, however, as the University of Oregon case illustrates, there is an increasingly demonstrable need for automated assistance with evaluating, processing and retrieving exceedingly high volumes of documentation generated by computer-mediated communications in both recordkeeping and other documentary contexts. The archival field is in almost the direct inverse of the situation in which information retrieval finds itself: there has been a lot of theorising, a small amount of modelling and rules production, and an even smaller amount of actual systems development. It remains unknown which aspects of IR might transfer into archival science (even bibliometric norms do not appear to fit, and precision and recall have been demonstrated to be not necessarily useful measures), and IR and systems design, which are cumulative by nature, have no track record to build upon. And yet systems development research is going to be essential if archives are to work with $\mathrm{CMC}$ and other forms of digital materials, and if archivists choose not to engage with it, contemporary examples would suggest that there are plenty of other communities who are desirous to do so, and who may not have the same sensibilities as archivists. A robust trajectory of archival systems development needs not only researchers with sound technological and IR expertise, but also models, hypotheses, unambiguous definitions and schematics, experimental and live testbeds and test collections, and a culture of evaluation and refinement.

\section{References}

Abraham, Ajith, Aboul-Ella Hassanien and Václav Suásel, eds. Computational Social Network Analysis: Trends, Tools and Research Advances. London: Springer-Verlag, 2011.

Anderson, Kimberly. Appraisal Learning Networks: How University Archivists Learn to Appraise Through Social Interaction, doctoral dissertation. Los Angeles: University of California Los Angeles, 2011.

Arnold, Timothy Jason. "Digital Curation in the Age of Twitter: Curating a Collection of Tweets from the Egyptian Revolution," paper presented at the Council of State Archives and Society of American Archivists Joint Annual Meeting, New Orleans, August 2013.

Bailey, Catherine. "Archival Theory and Electronic Records," Archivaria 29 (Winter 198990): 180-196.

Bearman, David A. ed. Archival Management of Electronic Records. Pittsburgh, PA: Archives and Museum Informatics Technical Report no. 13, 1991. 
Bearman, David A. "Archives in the Post-Custodial Age," Archival Management of Electronic Records, Archives and Museum Informatics Technical Report No. 13. Pittsburgh, PA: Archives and Museum Informatics, 1991.

Bearman, David A. "Diplomatics, Weberian Bureaucracy, and the Management of Electronic Records in Europe and America," The American Archivist 55 (1992): 168-181.

Bearman, David A. Electronic Evidence: Strategies for Managing Records in Contemporary Organizations. Pittsburgh, PA: Archives and Museum Informatics, 1994.

Bearman, David A. "Electronic Mail," Electronic Evidence: Strategies for Managing Records in Contemporary Organizations. Pittsburgh, PA: Archives and Museum Informatics, 1994.

Bearman, David A. "Electronic Records Guidelines," in Electronic Evidence: Strategies for Managing Records in Contemporary Organizations. Pittsburgh, PA: Archives and Museum Informatics, 1994.

Bearman, David A. Electronic Records Guidelines: A Manual for Policy Development and Implementation. Pittsburgh, PA: Archives and Museum Informatics, 1990.

Bearman, David A. "The Implications of Armstrong v. Executive Office of the President for the Archival Management of Electronic Records," The American Archivist 56 (1993): 674-689.

Bearman, David A. "An Indefensible Bastion: Archives as a Repository in the Electronic Age," in David Bearman, ed. Archival Management of Electronic Records. Pittsburgh: Archives \& Museum Informatics, 1991, pp. 14-24.

Bearman, David A. "Record-keeping Systems," Archivaria 36 (1993): 16-37.

Bearman, David A. "Towards a Reference Model for Business Acceptable Communications," unpublished paper, December 6, 1994.

Bikson, Tora. "Research on Electronic Information Environments: Prospects and Problems," paper presented 24 January 1991 at the Working Meeting on Research Issues in Electronic Records. Washington, D.C.: National Historical Publications and Records Commission, 1991.

Blair, David C. Languages and Representation in Information Retrieval (New York: Elsevier Science, 1990).

Boles, Frank. "SAA Should Say Something!" Society of American Archivists press release (February 2015), http://www2.archivists.org/news/2015/saa-should-say-something.

Borgman, Christine L. ed. "Bibliometrics and Scholarly Communications," special issue of Communication Research (October 1989).

Burckel. Nicholas C. "The Expanding Role of A College or University Archives," Midwestern Archivist 1 (1976): 16-32.

Consultative Committee for Space Data Systems/International Organization for Standardization. Space Data and Information Transfer System: Open Archival Information System: Reference Model. Geneva, Switzerland: International Organization for Standardization, 1999.

Cook, Terry. The Archival Appraisal of Records Containing Personal Information: A RAMP Study with Guidelines. Paris: UNESCO, April 1991.

Cook, Terry. "Easier to Byte, Harder to Chew: The Second Generation of Electronic Records Archives," Archivaria 33 (1992): 202-216.

Cook, Terry. "Electronic Records, Paper Minds: The Revolution in Information Management and Archives in the Post-custodial and Post-modern Era," Archives and Manuscripts 22 (1994): 300-329.

Cook, Terry. “It's 10 O'Clock - Do You Know Where Your Data Are?” Technology Review 98 (1995): 48-53. 


\section{Chapter 23}

Cook, Terry and Eldon Frost. "The Electronic Records Archival Programme at the

National Archives of Canada: Evolution and Critical Factors of Success," in Margaret

Hedstrom, ed. Electronic Records Management Program Strategies: Archives and Museum Informatics Technical Report No. 18 (Pittsburgh, PA: Archives and Museum Informatics, 1993), 38-47.

Cox, Richard J., "American Archival Literature: Expanding Horizons and Continuing Needs, 1901-1987," The American Archivist 50 (1987): 306-323.

Cox, Richard J. "Re-discovering the Archival Mission: The Recordkeeping Functional Requirements Project at the University of Pittsburgh, A Progress Report. Archives and Museum Informatics 8 (1994): 279-300.

Cox, Richard J. "Searching for Authority: Archivists and Electronic Records in the New World at the fin-de-siécle," First Monday 5 nos. 1-3 (January 2000).

Cox, Richard J., and Wendy Duff. "Warrant and the Definitions of Electronic Records: Questions Arising from the Pittsburgh Project. Archives and Museum Informatics 11 (1997): 223-231.

De Bellis, Nicola. Bibliometrics and Citation Analysis: From the Science Citation Index to Cybermetrics. Chicago: Scarecrow Press, 2009.

Dietz, Diane. "Former UO Archivist Describes 'Humiliating' Dismissal: He Says University Leaders Saw Him as a Scapegoat after a Controversial Records Release," The Register-Guard (Sunday, April 19th, 2015), http://registerguard. $\mathrm{com} / \mathrm{rg} /$ news/local/32990068-75/former-uo-archivist-james-fox-tells-his-sideof-his-dismissal.html.csp.

Duranti, Luciana, Terry Eastwood, and Heather MacNeil. Preservation of the Integrity of Electronic Records. Dordrecht, The Netherlands: Kluwer Academic, 2002.

Duranti, Luciana and Heather MacNeil. "The Protection of the Integrity of Electronic Records: An Overview of the UBC-MAS Research Project," Archivaria 42 (1996): $46-67$.

Duranti, Luciana, and Heather MacNeil. "Protecting Electronic Evidence: A Third Progress Report on a Research Study and its Methodology," Archivi E Computer 6 no.5 (1996): 343-404.

Gilliland, Anne J. "Archival Appraisal: Practising on Shifting Sands," in Archives and Recordkeeping: Theory Into Practice, Patricia Whatley and Caroline Brown, eds. London: Facet Press, 2014.

Gilliland, Anne. "Reflections on the Value of Metadata Archaeology for Recordkeeping in a Global, Digital World," Journal of the Society of Archivists 32 no.1 (April 2011): 97-112.

Gilliland, Anne J. and Sue McKemmish. "Archival and Recordkeeping Research: Past, Present and Future," in Research Methods: Information Management, Systems, and Contexts Kirsty Williamson, ed. (Prahran: Tilde University Press, 2012), 80-112.

Gilliland-Swetland, Anne J. "Archivy and the Computer: A Citation Analysis of North American Periodical Articles,” Archival Issues 17 no. 2 (1992): 95-112.

Gilliland-Swetland, Anne J. Policy and Politics: Electronic Communications and Electronic Culture at The University of Michigan, A Case Study in the Management of Electronic Mail, in Society of American Archivists case study series Archival Administration of Electronic Records and the Use of New Technologies in Archives. Chicago, IL: Society of American Archivists, 1996.

Gilliland-Swetland. Anne J. and Carol Hughes. "Enhancing Archival Description for Public Computer Conferences of Historical Value: An Exploratory Study," The American Archivist 55 no.2 (Spring 1992): 316-330. 
Gilliland-Swetland, Anne J. and Gregory T. Kinney. "Uses of Electronic Communications to Document an Academic Community: A Research Report," Archivaria 38 (Fall 1994): 79-96.

Gleick, James. "Roving Librarians of the Twitterverse," New York Review of Books Blog, January 16, 2013, www.nybooks_com_blogs_nyrblog_2013_jan_16_librarians_twi.pdf

Hedstrom, Margaret. "Understanding Electronic Incunabula: A Framework for Research on Electronic Records," paper presented 24 January 1991 at the Working Meeting on Research Issues in Electronic Records. Washington, D.C.: National Historical Publications and Records Commission, 1991.

Kalinichenko, Leonid, Michele Missikoff, Federica Schiappelli, Nikolay Skvortsov. "Ontological Modeling," Proceedings of the 5th Russian Conference on Digital Libraries RCDL2003, St.-Petersburg, Russia, 2003, citeseerx.ist.psu.edu/viewdoc/ download?doi=10.1.1.101.5714.pdf

Library of Congress. "Update on the Twitter Archive at the Library of Congress," January 2013.

McKemmish, Sue and Frank Upward. "Somewhere Beyond Custody," Archives and Manuscripts 22 no.1 (1994): 138-149.

McQuail, Denis. McQuail's Mass Communication Theory. 5th ed. (London: Sage Publications, 2005).

Morozov, Evgeny. "Facebook and Twitter are Just Places Revolutionaries Go," The Guardian (7 March 2011).

Nunamaker, Jay F. Jr. and Minder Chen. "Systems Development in Information Systems Research," Proceedings of the Twenty-Third Annual Hawaii International Conference on Systems Sciences, IEEE, 1990, pp. 631-640

O'Shea, Greg and David Roberts. "Living in a Digital World: Recognizing the Electronic and Post-custodial Realities, Archives and Manuscripts 24 no.2 (1996): 286-311.

Pao, Miranda Lee. Concepts of Information Retrieval. Englewood: Libraries Unlimited, 1989.

Pinheiro, Carlos A.R. Social Network Analysis in Telecommunications. New York: John Wiley and Sons, 2011.

Pinheiro, Carlos A.R. and Marcus Helfert, eds. Exploratory Analysis in Dynamic Social Networks. Hong Kong: iConcept Press, 2012.

Rao, Aluri and Donald E. Riggs. "Applications of Expert Systems in Libraries," Advances in Library Automation and Networking 2 (1988): 1-43.

Salton, Gerard and Chris Buckley. "Global Text Matching for Information Retrieval," Science 253 (1991): 1012-1015.

Samuels, Helen W. "Who Controls the Past?" The American Archivist 49 no.2 (Spring 1986): 109-124.

Samuels, Helen W. Varsity Letters: Documenting Modern Colleges and Universities. Chicago: Society of American Archivists and Scarecrow Press, 1992.

Thurlow, C., L. Lengel, and A. Tomic. Computer Mediated Communication: Social Interaction and the Internet. London: Sage, 2004.

Upward, Frank, Sue McKemmish and Barbara Reed. "Archivists and Changing Social and Information Spaces: A Continuum Approach to Recordkeeping and Archiving in Online Cultures," Archivaria 72 (Fall 2011): 197-238.

Vickery, Alina and Helen Brooks. Expert System for Referral. Library and Information Science Research Report No. 66, British Library, 1988. 


\section{Part IV}

\section{Research Case Studies}


Chapter 24

\title{
CONTEMPORARY ARCHIVAL DIPLOMATICS AS A METHOD OF INQUIRY
}

\section{Lessons Learned from Two Research Projects}

\author{
Heather MacNeil
}

\section{(C) Springer 2006}

This chapter was originally published under the same title in Archival Science 2004, 4 (3-4): 199-232. The digital rights to the chapter are owned or controlled by Springer, and unfortunately it was not possible to include this chapter in the digital edition of Research in the Archival Multiverse.

The chapter can be obtained through Springer at http://link.springer.com/article/10.1007/s10502-005-2592-x or through purchasing a print copy of Research in the Archival Multiverse. 

Chapter 25

\title{
EXPLORING SOCIAL COMPLEXITY
}

\section{Continuum Theory and a Research Design Model for Archival Research}

\author{
Leisa Gibbons
}

\begin{abstract}
Over the past 25 years, archival science as a field has grown in volume, sophistication, and construction. Of particular interest are trends in archival research focusing on communities and their archival needs, as well as the broader social issues that impact these communities. How might a researcher conceptualise and account for their position within the research project? How might the researcher design the project in a way that balances the needs and interests of communities and those of the researcher? In response to such questions, this essay describes a model for reflexive research design based on continuum theory. Utilising a case study exploring the social media platform YouTube, this essay presents a research design model that emphasises continual and iterative reflection on the part of the researcher before, during, and after the processes of research. The purpose of the model is to provide the researcher with a tool for construction, to help design the plan, approach and goals of a research project, to draw out potential research methods, methodologies, tools and community knowledge through an interwoven and non-linear development process. The model addresses complexity in developing research projects across disciplines and stakeholders by providing a means to understand or "see" the mix of contexts brought to and created as a result of research. The model is an outcome of the YouTube case study research I performed, but is also part of it as working with it played a significant role shaping the research as an ongoing process.
\end{abstract}




\section{Introduction}

Archival science as a research discipline has been steadily growing in volume, sophistication, and construction over the past 25 years. Significant developments include formation and advocacy of research agendas; identification of emerging research trends; dedicated international research and educational programs; and more recently, investigation into the emergence of critical thinking and pluralist research methods. ${ }^{1}$ Increasingly archival research has begun to address the multiplicity inherent in the construction, access, use and re-use of records, recordkeeping and archives within communities, including investigation into hidden, alternative or contested narratives and voices. Archival science research is becoming more complex, subjective, multi-faceted and multidimensional.

Scholars have recognised that within this emerging complexity there is a serious need to critically re-examine, test and reshape archival studies discipline and practice. ${ }^{2}$ There have been calls for and a growing concern about reflexivity and transparency, particularly in relation to how research is designed and reported. ${ }^{3}$ In particular, how communities are defined and included in research requires careful consideration and transparency in research design and decision making. Eric Ketelaar discusses the complexities of understanding community as collective identity. The processes of memory contribute to an archival understanding that a community exists as a "community of memory," held together by belonging and commonality and how the past is mediated through "memory texts" in any form. ${ }^{4}$ The implication is that understanding the different roles and values community is vital in order to be able to establish the archival needs and concerns of a community. ${ }^{5}$ Jeanette Bastian refers to community as "a group of people

1 Sue McKemmish and Anne Gilliland, "Archival and Recordkeeping Research: Past, Present and Future", in Research Methods: Information, Systems and Contexts, ed. by Kirsty Williamson and Graeme Johanson (Prahran, VIC: Tilde University Press, 2013): 79-112

2 Kelvin L. White and Anne J. Gilliland, "Promoting Reflexivity and Inclusivity in Archival Education, Research, and Practice," The Library Quarterly 80 no. 3 (July 1, 2010): 231-48, doi:10.1086/652971.

3 Anne Gilliland and Sue McKemmish, "Building an Infrastructure for Archival Research," Archival Science 4 no. 3 (2004): 149-97, doi:10.1007/s10502-006-6742-6.; Cox, 1994.

4 Eric Ketelaar, "Sharing: Collected Memories in Communities of Records," Archives and Manuscripts 33 (2005): 44-61.

5 Sue McKemmish, Anne Gilliland-Swetland, and Eric Ketelaar, "'Communities of Memory': Pluralising Archival Research and Education Agendas," Archives and 
who share common elements or identities and who think of themselves in terms of these common elements" but do not have to actually be in physical or literal contact and can also be part of other communities. ${ }^{6}$ Addressing the multiplicity of communities in archival research unlocks the potential of deeper understandings of diverse and contested contexts and meanings, but at the same time exposes archival scholars to complexity.

In archival discourse, and in particular in the work of records continuum scholars in Australia, elements of social complexity such as time, recurrence and the nature of being continuous have been explored using sociologist Anthony Giddens' structuration theory. ${ }^{7}$ Structuration theory concerns the joining of the action (of a subjective actor) to the objective or structure. The generation of social systems is a recursive process, so what is being created influences what is learned; which in turn has an impact upon what is consequently produced. Structures are the strong, continuous influences that course through history. Structures are a result of human action and knowledge and their existence is scattered across time and space as traces of interactivity, or what those in the archival profession might call records. ${ }^{8}$ Ideas about the recursive replication of social systems, or what Giddens refers to as "duality of structure," were translated by Frank Upward into models and a framework for analysis and articulation of how recorded information is captured and managed over time. ${ }^{9}$ The resulting continuum models, particularly the Information Continuum Model, present a multi-dimensional conception of how recorded information, and resulting knowledge, has continuing value over time, rather than it being part of a linear life cycle where time "ends."10

Manuscripts 33 no. 1 (2005): 146; Jeannette Allis Bastian, "Reading Colonial Records Through an Archival Lens: The Provenance of Place, Space and Creation," Archival Science 6 (2006): 267-84, doi:10.1007/s10502-006-9019-1.

6 Bastian, "Reading Colonial Records through an Archival Lens".

7 Frank Upward, "Structuring the Records Continuum (Part One): Postcustodial Principles and Properties," Archives and Manuscripts 24 no. 2 (1996), http://infotech. monash.edu/research/groups/rcrg/publications/recordscontinuum-fupp1.html; Frank Upward, "Structuring the Records Continuum (Part Two): Structuration Theory and Recordkeeping," Archives and Manuscripts 25 no. 1 (1997), http://infotech.monash.edu/ research/groups/rcrg/publications/recordscontinuum-fupp2.html.

8 Anthony Giddens, The Constitution of Society: Outline of the Theory of Structuration (University of California Press, 1986).

9 A simple explanation of the "duality of structure" states that human activity helps to form objects and then objects help to influence future activities. Giddens, The Constitution of Society; Upward, "Structuring the Records Continuum (Part Two): Structuration Theory and Recordkeeping."

10 Information continuum theory refers to the array of models developed by Frank Upward. The well-known Records Continuum Model (RCM) was presented to 
In my research, I looked towards YouTube to explore how continuum theory and models can help to understand "evidence of culture." In doing so I also sought solutions on how to design and undertake a social research project that featured multiple complexities. The result was a Research Design Model, which I present in this essay, developed as a tool to aid in the design, exploration and communication of the complex and multi-faceted research methodology used in the YouTube case study project. By way of illustration, I present some of the design decisions and implications made in relation to the YouTube case study.

\section{Designing Research}

The purpose of the Research Design Model, shown in Figure 25.1, is to provide the researcher(s) with a tool for construction: to help design the plan, approach, and goals of a research project, and to explore, test, and include potential research methods, methodologies, and tools drawing from multiple knowledge sources through an interwoven and non-linear development process. This process identifies and lays bare the knowledge monuments and influences that direct and drive research. These knowledge monuments are generated over multiple time periods by the varied and diverse stakeholders in a research project, including individuals, community, research groups, organisations and the academy. The goal of the model is not necessarily to guarantee that a certain point of view is represented in the name of inclusion, but rather, to be able to acknowledge that there are multiple points of view and seek to understand how they impact on the research, the researchers, the stakeholders and/or community as well as archival theory and practice.

the archival profession in 1996 via his paper "Structuring the Records Continuum (Part One): Postcustodial Principles and Properties" published in Archives and Manuscripts. After this Upward (sometimes in collaboration with others), developed other continuum models which represent various dimensions across an information continuum encompassing what he refers to as "facets of memory within recorded information" (Frank Upward, "Continuum Mechanics and Memory Banks [Series of Parts]: Part 1: Multi-Polarity”, Archives and Manuscripts, 33 (2005): 87). The models represent dimensions such as information systems and data, publication and access, as well as cultural heritage. The RCM is concerned with recordkeeping within a continuum, however, this model (which much has been written about) provides the primary conceptual foundations shared across all the models which includes notions of transactionality, contextuality and plurality over time and space. The continuum models are based on Giddens' structuration theory, as well as postmodern and postcustodial thinking. 
Figure 25.1: Research Design Model

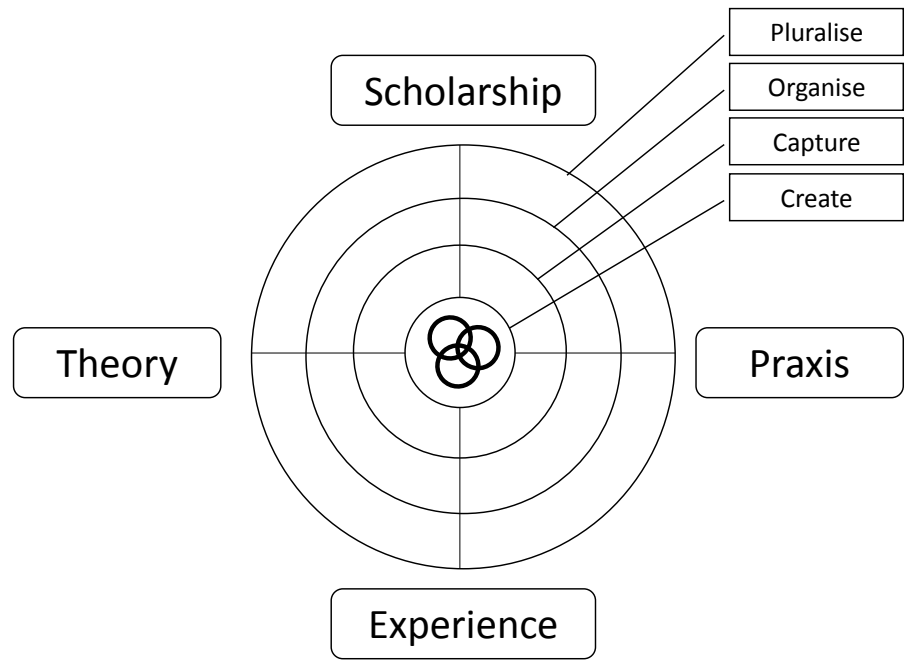

Research design refers to how the research is conceptualised: the structure, the position, the type, the methods and how it all fits together. It should have a logical and cohesive construction that can be defended at the conclusion of the research, and be able to be examined and tested in future research. Some sources refer to research design as being the over-arching structure and presentation of ideas in a research project: the aims, uses, purposes, intentions and plans, taking into account resources available. ${ }^{11}$ Robert K. Yin refers to research design as "the logic that links the data to be collected (and the conclusions to be drawn) to the initial questions of the study." ${ }^{12}$ Strauss and Corbin refer to research design in qualitative research as being a way of thinking and studying that underlies the research. ${ }^{13} \mathrm{McKemmish}$, Burstein, Manaszewicz, Fisher, and Evans describe research design as “... identification of the research problem and the desired outcomes; determining rights in the research data and outcomes; framing of the research with reference to

11 Catherine Hakim, Research Design: Successful Designs for Social and Economic Research, 2nd ed., Social Research Today (London, UK: Routledge, 2000).

12 Robert K Yin, Case Study Research: Design and Methods, 3rd ed. (Thousand Oaks, CA: Sage Publications, 2003), 19.

13 Anselm L Strauss and Juliet M Corbin, Basics of Qualitative Research: Techniques and Procedures for Developing Grounded Theory, 3rd ed. (Thousand Oaks, California: Sage Publications, Inc., 2008), 1. 
appropriate philosophical and theoretical approaches; selection and integration of methodologies and methods; identification of the most appropriate techniques of data collection and analysis; iterative implementation, evaluation and adaptation of the research design as the research unfolds; and dissemination of results. ${ }^{14}$ There are several key words in this definition that suit the nature of complex research design: rights, integration, and iteration. These are critical elements to consider in research that address the potential of co-authored creation across the span of a research project. Effective and inclusive design requires drawing all these threads together in a flexible way. ${ }^{15}$

Inclusive research design is generated in interaction with the Research Design Model through exploration and interaction with Theory, Scholarship, Background, and Praxis. These four axes represent different domains in information and knowledge generation spaces. The axes span the multiple dimensions of the continuum in the same fashion as Upward's other continuum models, referring to information activities over spacetime (Create, Capture, Organise, and Pluralise) and layers of social contexts (individuals, groups, communities, societies, institutions). ${ }^{16}$ Each quarter of the model is called a quadrant and forms a relationship of intersections in which threads are crafted and woven. The four quadrants rely on each other to interact and, perforce, influence each other. At the centre of the model sits what I have called "the knot of creation." The model acts an instrument that helps to explore these domains to uncover the past, present and future actions and decisions made by the researcher(s), including the evolving knowledge being created as it impacts on the research project and the researcher(s) themselves. These elements of the model are discussed and explained in more detail in this essay in reference to the YouTube case study.

14 Sue McKemmish, Frada Burstein, Rosetta Manaszewicz, Julie Fisher, and Joanne Evans, "Inclusive Research Design: Unravelling the Double Hermeneutic Spiral," Information, Communication E Society 15, no. 7 (September 2012): 1107. doi:10.1080/13 69118X.2012.707225.

15 "Drawing together the threads" is the title of a section in Giddens' The Constitution of Society referring to the process of bringing together what appears to be disparate areas. Giddens mentions the phrase in relation to undertaking social research and how to apply structuration theory to explore how research is done (research work). Giddens, The Constitution of Society.

16 The multiple meanings for the dimensions are mentioned in various papers by continuum researchers. Mentioned in this essay are two: Frank Upward, "The records continuum”. In S. McKemmish, M. Piggott, B. Reed, \& F. Upward (Eds.), Archives : Recordkeeping in Society (Wagga Wagga, NSW: Centre for Information Studies, 2005), pp.197 - 222; and Sue McKemmish, "Evidence of me", Archives and Manuscripts, 45 no.3 (1996): 174-187. 


\section{Why YouTube?}

YouTube, as a social technology available online, provides an opportunity to explore intent. Users of YouTube are archiving all the time by creating, collecting, organising, and describing content uploaded or shared on the platform. "Archivalisation", a term created by Eric Ketelaar that references Derrida's "archivization", refers to the "conscious or unconscious choice to consider something worth archiving." ${ }^{\prime 17}$ This concept emphasises the act and process of recordkeeping for multiple purposes, i.e., witnessing and memorialisation, as well as administration and evidence. The act and process of recordkeeping tells a story of the "social and cultural factors" that impacted the actions of the record keepers at the time. The notion of notion of archivalisation highlights the fundamental need for people to engage in memory-making ${ }^{18}$ activities. Memory-making involves the processes of creating, collecting, organising, and describing content; it is a process driven and influenced by interaction with technologies. Memory-making concerns the narratives within which recordkeeping sits for individuals, as well as the groups and community of participants, and even the corporate entities that might provide or own the social platforms themselves. These narratives contain valuable information about the process of developing and bearing a cultural identity and how this process works in an application as well as across linked social media.

Communication and interaction help shape which videos stay up, how people feel when videos are removed, as well as how people react when something happens in the community. ${ }^{19}$ The information that is important to a community might not always exist in the video, but rather, in other spaces within the channel or webpage. ${ }^{20}$ Ultimately the result is that the stories of YouTube are many, multi-layered, and developed recursively in multiple processes of interaction across time and space. Participation in online social culture goes beyond the actions of the individual and occurs in relationship

17 Eric Ketelaar, "Archivalisation and Archiving," Archives and Manuscripts 27 (1999): 57.

18 Memory-making is a concept developed as an outcome of the YouTube research project and references Ketelaar's archivisation. Memory-making is about interaction, interpretation and communication embedded in practices, norms and values that contribute to the continuous dynamism, iteration and progression of cultural heritage.

19 Patricia Lange, "Publicly Private and Privately Public: Social Networking on YouTube," Journal of Computer-Mediated Communication 13 no. 1 (2007): 361-80, doi:10.1111/ j.1083-6101.2007.00400.x.

20 C. Shah, "ContextMiner: Supporting the Mining of Contextual Information for Ephemeral Digital Video Preservation," International Journal of Digital Curation 4 no. 2 (2009): 171-83, doi:http://dx.doi.org/10.2218/ijdc.v4i2.106. 
to how technology is used and what information is being communicated. ${ }^{21}$ Cultural use of technology, including technological systems such as YouTube, play a role in how these technologies evolve as tools for communication, community, networking, and in turn validating, innovating and reinforcing practices. Burgess and Green refer to constant co-creation in the cycle of participation. ${ }^{22}$ These ideas about co-creation as an act of participation by individuals working with each other, knowingly and unknowingly, highlight the role of multiple creators and contexts and their diverse stories as part of cultural production. ${ }^{23}$

Archival and other collecting institutions need to understand and embrace complexity if they want to remain relevant. An understanding of the multiple contexts or narratives that play out in online communities and spaces is critical. The continuous cycle of recursive interaction discussed above also reveals a complexity of action in tandem with technologies that is not effectively captured by traditional methods of selection, description and arrangement. In the YouTube context, the outcomes of culture refer not to the video and its content, but rather the memory-making and recordkeeping processes involved in how the video came to be and what its use achieves. Individuals, groups, communities, and cultural institutions all have roles to play in the transmission of cultural stories and the heritage of practice in these spaces. The site of the cultural institution within this continuum of interactions is part of a "whole narrative," and does not just play a passive role as "container" of cultural heritage.

\section{The Information Continuum and Knowledge Generation}

The major continuum influence on the development of the Research Design Model is the Information Continuum Model (ICM) presented in Figure 25.2. The ICM was created immediately after the Records Continuum Model (RCM) in 1996 by Don Schauder, Frank Upward, Barbara Reed, and Sue McKemmish. ${ }^{24}$ In 2005, Upward claimed that the ICM could poten-

21 Henry Jenkins et al., Confronting the Challenges of Participatory Culture: Media Education for the 21st Century (Chicago, IL: MacArthur Foundation, 2006).

22 Jean Burgess and Joshua Green, YouTube: Online Video and Participatory Culture, Digital Media \& Society (Cambridge, UK: Polity Press, 2009).

23 Burgess and Green, YouTube: Online Video and Participatory Culture.

24 The ICM has been developed further by Larry Stillman and Graeme Johanson: D. Schauder, G. Johanson, and L. Stillman. "Sustaining and Transforming a Community 
tially be used to explore knowledge formation as it presents a conceptual process of how information is "represented, recalled and disseminated." 25 This statement by Upward, read during the initial development of the YouTube case study had a profound impact on the way I began to understand my role as researcher. Additional writings by researchers working with the ICM, as detailed in this section, also contributed to the development of my understanding of the role of communication and memory in undertaking academic research.

\section{Figure 25.2: Information Continuum Model}

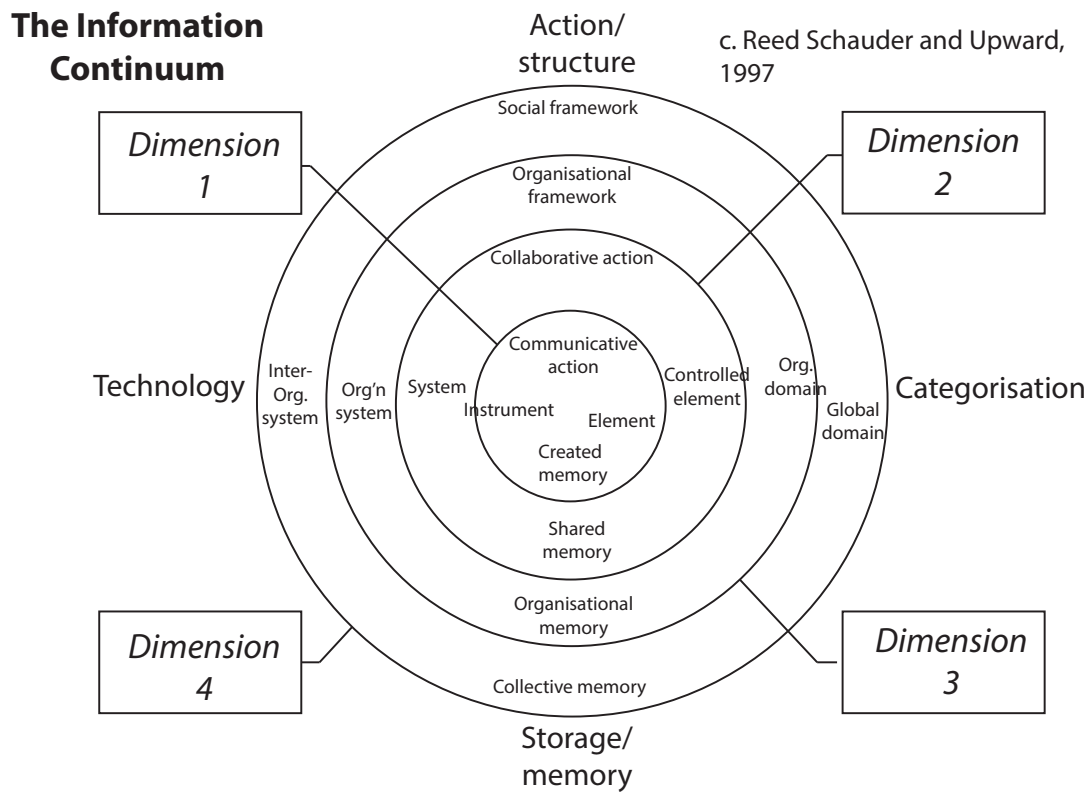

The ICM, similar to the Records Continuum Model, is based on Giddens' structuration theory referencing recursivity of action and structure. It was designed to help identify and analyse "the multiple patterns of interdependence which sustain (and constrain) the lives of people" in relation to the

Network: The Information Continuum Model and the Case of VICNET," The Journal of Community Informatics 1 no. 2 (February 3, 2005). http://ci-journal.net/index.php/ciej/ article/view/239; Frank Upward, "Continuum Mechanics and Memory Banks [Series of Parts]: Part 1: Multi-Polarity," Archives and Manuscripts 33 no. 1 (May 2005): 84-109.

Upward, "Continuum Mechanics and Memory Banks", 95 
"creation, organisation and sharing of information."26 Information activities in the ICM are understood as being part of communicative transactions, drawing from Kaufer and Carley's constructuralist theory. ${ }^{27}$ Kaufer and Carley's communicative transactions represent a reflexive knowledgegeneration process where individuals interact, communicate, adapt and readjust according to their social and cultural informational surroundings, or "sociocultural landscape". ${ }^{28}$ These transformative interactions are represented on the Action/structure axis of the ICM. Communicative transactions are also understood through Giddens' structuration theory where "texts" are a form of transformative action and an allocative resource. ${ }^{29}$

Giddens refers to written texts (such as books) and their machinery (such as the printing press) as the resources humans use to record information and pass on knowledge. ${ }^{30}$ Schauder, Stillman and Johanson define text in the ICM as the techno-social situation or phenomena present in stored memory, metadata and technology. ${ }^{31}$ Text is represented in the axes of Technology (artefacts, objects and systems), Storage/memory (stored memory) and Categorization [sic] (metadata) on the ICM related to dimensions of activity: (1) Create, (2) Capture, (3) Organise and (4) Pluralise. Upward describes the Records Continuum Model and ICM as "different ways of managing memory objects as record items and text respectively," but while the RCM focuses on the relationship between records, evidence and memory, the ICM

26 Upward, “Continuum Mechanics and Memory Banks”, 80

27 Upward, "Continuum Mechanics and Memory Banks"; Kathleen Carley, "On the Persistence of Beliefs." Working Paper, Department of Social and Decision Sciences, Carnegie Mellon University, 1990. http://alliance.casos.cs.cmu.edu/publications/ papers/carley_1991_persistencebeliefs.PDF; David S. Kaufer, and Kathleen M. Carley, Communication at A Distance: The Influence of Print on Sociocultural Organization and Change. Hillsdale, N.J.: Lawrence Erlbaum Associates, 1993.

28 Kaufer and Carley, Communication at A Distance, 143

29 Giddens' allocative resources are the tools by which humans can dominate nature and contribute to individual, systems and societal transformation through their storage and management. Anthony Giddens, The Constitution of Society: Outline of the Theory of Structuration. Cambridgeshire, UK: Polity Press, 1984. Gillian Oliver, "Investigating Information Culture: A Comparative Case Study Research Design and Methods", Archival Science, 4 (2004), 287-314 <http://dx.doi.org/10.1007/s10502-005-2596-6>; D. Schauder, G. Johanson, and L. Stillman. "Sustaining and Transforming a Community Network: The Information Continuum Model and the Case of VICNET," The Journal of Community Informatics 1 no. 2 (February 3, 2005).

30 Schauder, Johanson, and Stillman, "Sustaining and Transforming a Community Network".

31 Schauder, Johanson, and Stillman, "Sustaining and Transforming a Community Network", 83 
highlights "how recorded information is represented, recalled and disseminated" to examine "text as memory." ${ }^{2}$

The ICM provides an instrument to understand the power of information or knowledge and the role of texts as memory-making processes (Created memory, Shared memory, Organisational memory, and Collective memory) when they are found / used / applied / abused / destroyed. These performative actions with text help to understand the power relationships between people and memory as power over information, including recorded information as described in the RCM. The ICM therefore provides a system or instrument enabling the application of Giddens' ideas about retention and control of knowledge through information representation, retrieval and dissemination utilising various modes of storage (Storage/memory), as well as technologies (Technology), such as writing and print, and management including classification and arrangement (Categorisation). ${ }^{33}$

The outcome of information organisation is knowledge (stored memory) that is invested in the actions and outcomes of further social transactions and externalised in texts. Externalised knowledge can be controlled by use of classification, a sense-making process that adds context (Categorisation axis), and by building information systems to help recall and disseminate (Technology axis). People who use knowledge see it as information, but value, absorb and refine it according to their own experiences, expectations and frameworks (Action/structure axis). Therefore, in the interaction with and use of information, we/humans/knowledgeable agents go back to the beginning of the cycle, knowing a little more about "something."

The ICM has been adopted in research related to information culture, and community informatics. Researchers in community informatics use the ICM to identify, audit and assess sustainable community initiatives. ${ }^{34}$

32 Schauder, Johanson, and Stillman, "Sustaining and Transforming a Community Network", 93 \& 95

33 Schauder, Johanson, and Stillman, "Sustaining and Transforming a Community Network”; Giddens, The Constitution of Society, 377; David S. Kaufer, and Kathleen M. Carley, Communication at A Distance: The Influence of Print on Sociocultural Organization and Change. Hillsdale, N.J.: Lawrence Erlbaum Associates, 1993; Frank Upward, "Structuring the Records Continuum" Part 2; Upward, Frank, and Larry Stillman, 'Community Informatics and the Information Processing Continuum', in 3rd Prato International Community Informatics Conference (CIRN 2006) (Monash University, 2006) <http://ccnr.infotech.monash.edu/conferences-workshops/prato2006papers. html>

34 Schauder, Johanson and Stillman. "Sustaining and Transforming a Community Network; Stillman, L., 'Understandings of Technology in Community-Based Organisations: A Structurational Analysis' (Thesis PhD, Monash University, 2007). 
Gillian Oliver's research into organisational information management culture applied the ICM to "distinguish the scope" of the project, and was also used as an analytic instrument to help determine the different ways information could be managed, and deployed. ${ }^{35}$ Additionally, Stillman modelled "the various stages of a qualitative research process" utilising the ICM to develop the "cone" model, which identifies the creation of research data at the interview stage (Create), the capture of interview data as recording and transcription (Capture), organisation and management of the data (Organise) and subsequent development and distribution of academic objects (Pluralise). ${ }^{36}$

The role of the ICM in knowledge generation, particularly in relation to how communicative transactions are influenced by motivation, action and adaption, influenced how I conceived the role of research in a recursive information/knowledge cycle. Reflecting Stillman's cone, a research thesis or report can be understood as a text generated through research processes. It is an "academic object" in the Pluralise dimension. An academic object plays a (potentially) transformative role in the generation of new knowledge. In research design the important lesson of the ICM lies in identification of the monuments of knowledge and the structures that influence how research is conducted, as well as acknowledgement of the subjective, and the impact of the social world on interpretation and communication.

\section{Exploring Complexity: YouTube and Applying the RDM}

The Research Design Model was developed at the same time as I investigated the literature about YouTube and information continuum theory, and each of these contexts played a significant, reflexive role in its progression and use. An annotated version of the model presented in Figure 25.3 presents some of the questions and processes that occurred during the development of the YouTube case study. The model was applied in three key areas of the YouTube research case study:

35 Gillian Oliver, "Investigating Information Culture: A Comparative Case Study Research Design and Methods", Archival Science, 4 (2004), 287-314: 295

36 Frank Upward and Larry Stillman, "Community Informatics and the Information Processing Continuum”, in 3rd Prato International Community Informatics Conference (CIRN 2006) (Monash University, 2006): 49 <http://ccnr.infotech.monash.edu/ conferences-workshops/prato2006papers.html> 
1. In the design of the case study and in particular, the breadth and type of people that formed the communities from which I collected data;

2. As part of the reflection on the social and ethical implications of being part of the community I was studying; and

3. In the process of choosing, developing and interpreting information tools, such as modelling, to reflect on, further develop as well as communicate my findings.

Figure 25.3: YouTube and the Research Design Model

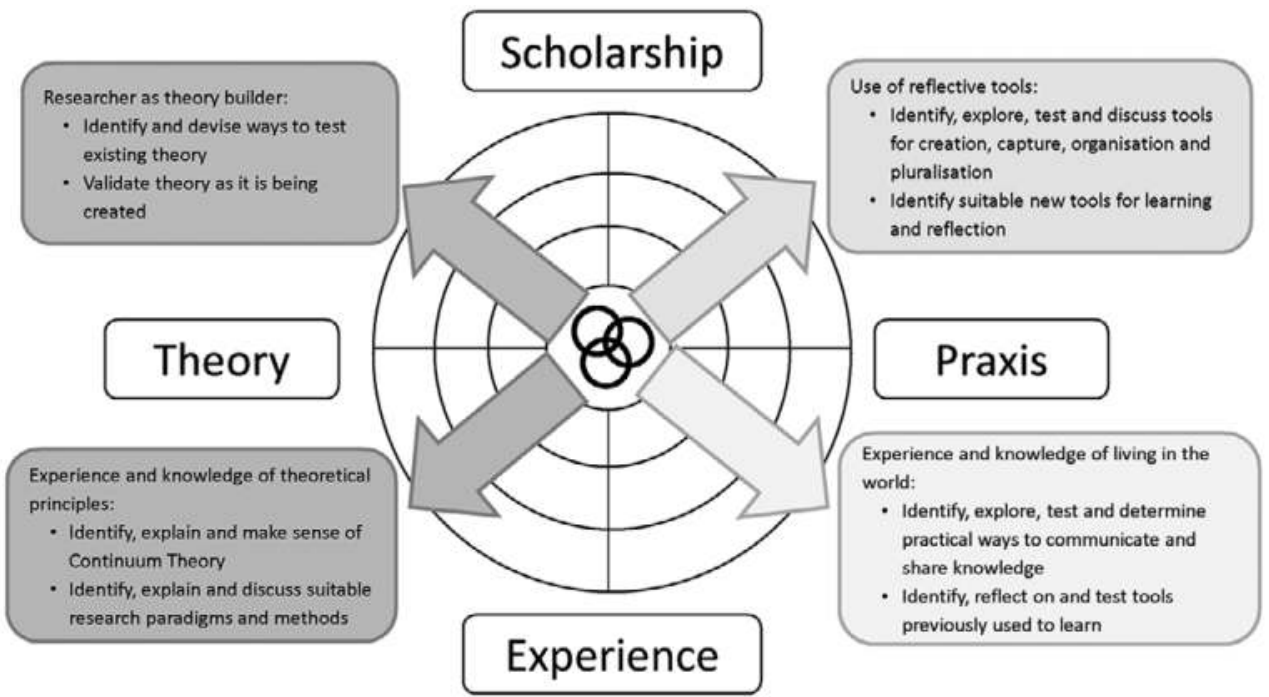

The four axes, Scholarship, Theory, Praxis, and Experience are parts that contribute to a whole picture. Not only do the four axes serve as tools to help ascertain a particular aspect of that picture, but they also provide strong contextual links (threadings).

The axis of Theory presents the opportunity to explore the driving principles of project design. This axis refers to the foundations, concepts, assumptions, authoritative frameworks, and discourse in research. Traditional frameworks contain long-held and established rules of engagement, imbued with conventional authority and language. Theory is concerned also with the creation of tradition through categorisation and control of information and knowledge, providing new opportunities for the growth of knowledge.

The Experience axis concerns skills, knowledge and interests brought to the project by the researcher, but also by the stakeholders including YouTube participants, and domain experts consulted during the research, as well as 
archival practitioners and institutions and the research supervision team. The inclusion of this axis reflects and acknowledges the subjective, interpretive, and active nature of research. Reflection about my own experience and what I brought to the research as an avid technologist and futurist, social media user, YouTube watcher, screen writer and film boffin, archivist and continuum theorist, as well as scholar, interpreter, and participant in the research, helped me to identify and explore these multiple roles and their implications. This process provided a framework and map that enabled relationships and links to be identified and communicated, ultimately helping me realise the vital importance of communicating my position, influences and bias.

The Scholarship axis refers to the recursive relationship that the researcher has in the evolving present of conducting the research. This axis refers to the process of learning via communication, whether it is about discovering the topic, designing a project, collecting data, realising the goals, or developing links with the participants and their community or the discipline and its community. In projects undertaken by a sole researcher, this axis provides an opportunity to explore reflexively assumptions made during the project. One goal of scholarship as a concept is its transferability to other disciplines; however, learning as an action works at much more subtle levels at various times throughout the project where the researcher's communication with her self can provide deeper insight. The multi-dimensional framework of the model helped to reveal and explore the implications of the numerous active identities of Yousers (YouTube users) and their communities in other social media platforms, and across the diverse and abundant spaces on the internet. ${ }^{37} \mathrm{~A}$ Youser is both a records creator for his/her own records as well as online records across various platforms: a YouTube video might be found on YouTube, Facebook, MySpace, blogs, emails. A YouTube identity may also be found on other online communities, such as Twitter, or in the blogosphere, especially with ever-growing integrations between platforms. ${ }^{38} \mathrm{My}$ application of the model to analyse the distribution of online created content

37 Frank Upward, Sue McKemmish, and Barbara Reed, "Archivists and Changing Social and Information Spaces: A Continuum Approach to Recordkeeping and Archiving in Online Cultures," Archivaria 72 no. 72 (February 12, 2011), http://journals.sfu.ca/ archivar/index.php/archivaria/article/view/13364.

38 For example, in late 2013, Google, which owns YouTube, updated the video sharing platform to become more tightly integrated with its own social networking service Google+. Yousers are now required to log in with their Google+ account to comment on YouTube videos. Stuart Dredge, "YouTube Aims to Tame the Trolls with Changes to Its Comments Section,” The Guardian, November 7, 2013, sec. Technology, http:// www.theguardian.com/technology/2013/nov/07/youtube-comments-trolls-moderationgoogle. 
and its related identities, contributed significantly to conceptually limiting the scope of the YouTube case study. It also helped me to construct the links between how to define a Youser and how to identify other structurating influences on the project.

Praxis is the axis that refers to practical engagement with tools that contribute to information and knowledge creation, capture, organisation and communication. Practical tools can be technology-based, or can describe a research technique such as modelling, or a method. Interaction and application of tools and their outcomes provides the opportunity to explore greater complexity. The use of tools over time becomes more complex and starts to include aggregating tools such as databases and repositories. A tool can take on different meanings in different dimensions. For example, a literature review is primarily used to situate a research idea in discourse and time (which correspond with information and analysis in the Capture and Organise dimensions). Over time, multiple interactions with varied disciplinary discourse can also be used to identify opportunities for alternative ways to construct and deliver a literature review as research findings, for example, an adaptive literature review (Pluralise/Create).

Technological tools used in the YouTube case study included qualitative software programs for data organisation and analysis (NVivo), blogs to capture reflections, use of email, Skype video, MSN Messenger (an instant messaging application), and a digital voice recorder to capture interviews. Additionally, my computer plays the role of tool for creation, capture, and organisation of the data, as well as a tool for its pluralisation through the internet and library databases. Other software programs such as mindmapping tools (XMind and Cmap) helped render complex maps of the links and strategies of the research, which were then embedded in posters, papers, blogs and my thesis. Additionally, reflection on the Scholarship and Praxis domains of the model helped to identify a double hermeneutic ${ }^{39}$ at play in the YouTube case study: I need to work with information and information tools in order to develop information and knowledge about information processes.

39 The double hermeneutic is described by Giddens, The Constitution of Society, as the complex relationship between a researcher and the researched (participants), where information is constructed, interpreted and communicated all within the social world the same world that the researcher is attempting to research. Informatics and archival researchers that have explored the implications of the double hermeneutic: Schauder, Johanson, and Stillman, "Sustaining and Transforming a Community Network, 325; Sue McKemmish, Frada Burstein, Rosetta Manaszewicz, Julie Fisher, and Joanne Evans. "Inclusive Research Design: Unravelling the Double Hermeneutic Spiral," Information, Communication E Society 15 no. 7 (September 2012). 
The axes of the model and their relationships to each other as quadrants help to expose these relationships and to develop strategies to make sense of their role and use in (evolving) research design.

\section{The Role of the Quadrants}

The quadrants are the intersections between each axis - these intersections and style of categorising activities are not found explicitly in the continuum models or their writings. The purpose and actions of the quadrants provide another space upon which to structure knowledge generation. The act of "crossing the line" of a quadrant provides the researcher with a tool to develop categories. Actions can be threaded out in what appears to be a linear fashion over a duration of time, up along a singular path of an axis. The following explanations of the quadrants focus on the single-interpreter view as this was how the quadrants were initially articulated and described.

The Theory axis interacts with the Scholarship and Experience axes as quadrants: Theory/Scholarship and Theory/Experience respectively. The Theory axis working alongside others as quadrants plays a potential transformative role by challenging traditions and frameworks through interaction with the other axes. For example, in the first phase of an adaptive literature review ${ }^{40}$ of the YouTube case study, I could not find much information about YouTube from an archival perspective, and so began to explore the rich and diverse conversations emerging about the importance of YouTube in other disciplines. This practical reality was identified through database and Google searches (Praxis/ Scholarship) which resulted in my turning back to the academic literature to find out how I could devise a strategy to accommodate the lack of archival discussion, but also the emerging diversity of knowledge (Theory). Ultimately, these explorations and reflections led to the recognition of emergent literature ${ }^{41}$ and discourse analysis as playing a significant role in the research and generation of knowledge. I legitimised emergent literature as a key concept

40 A literature review can be a dynamic research tool that goes further than simply identifying gaps or summarising previous research and can be used as part of a mixed methods approach. In this sense the literature review has multiple purposes whose findings can inform the research in various ways. For more information see: Kathleen M. Collins, Anthony J. Onwuegbuzie, and Qun G. Jiao, eds., Toward a Broader Understanding of Stress and Coping: Mixed Methods Approaches, Digital (Charlotte, NC: Information Age Publishing Inc., 2010) <http://www.infoagepub.com/products/ Toward-a-Broader-Understanding-of-Stress-and-Coping>

41 Emergent literature is a term I coined to describes the "coming into existence" of a discourse. 
by defining it, and including it in the design of the research, including how I would actively seek emerging conversation from multiple sources.

Experience and Theory are considered to be more or less static; however, there is always an element of the unknown in how they apply to research - and this will come about only through action. One of the strengths of continuum theory is in situating the social actor - the researcher - in a particular research spacetime. The Theory/Experience quadrant however refers to the researcher's experience and knowledge of theoretical principles and acknowledges that subjectivity is part of the research and research actions and learning. This is a dynamic, interpretive space that evolves that asserts influence over the Theory/Scholarship quadrant over time. During this time a researcher is able to contextualise his/her research, develop an understanding of its impact and potential, determine the audience, and consider the ethical implications of the research. Taking into consideration context in an instance or event contributes to the building of knowledge and its transferability into a greater knowledge pool. ${ }^{42}$

Theory/Scholarship quadrant highlights the relationship between the mechanisms of formal academic research and the goal of scholarship. The role of mentors, supervisors and peers within academia, as well as texts and scholars within the discipline and how they impact on the learning culture and development, are highlighted in this quadrant.

In $2009,{ }^{43}$ a practising archivist said to me in response to a conference paper I had presented that YouTube videos were absolutely ephemeral just like postcards, and so could (and should) be collected and arranged in similar ways. In 2010, an archival academic responded to my comments about YouTube being a website experience or a moving image experience, saying that YouTube was quite definitely about videos and was part of the moving image form. These two reactions and ways of understanding YouTube highlighted the different ways in which YouTube is understood. As a result, these experiences influenced the decision to include interviews with domain experts in the research process.

This type of reflective analysis strongly resonated with the ideas I was having about how people use language to explain and explore ideas and it mirrored my interests in language as classification and its power as a tool of construction:

\footnotetext{
42 Gilliland and McKemmish, "Building an Infrastructure for Archival Research."

43 Leisa Gibbons, "Testing the continuum: User-generated cultural heritage on YouTube," Archives and Manuscripts 37 (2009): 89.
} 
- What are people saying about YouTube and how are they classifying it?

- How do people define YouTube?

- What words are used to describe interactions and activity on YouTube?

This led me back to the dimensions of the model and thoughts about who were creating and disseminating stories about YouTube significant and in what ways. I had already started to explore what fields and disciplines I would study as part of the adaptive literature review, and so began thinking that perhaps I could ask experts in these fields.

The Scholarship/Praxis quadrant highlights the role of the double hermeneutic and the consequence of interpreting a social world while existing in it. This quadrant also reflects the complexity of analysing information whilst creating it, and developing models as maps as well as instruments of further interpretation. The question of ethics emerged as an important interest for this project as expressed in the relationship between participant and researcher, especially when undertaking research into online social phenomena. The model's axes work as tools to focus attention of the apparatus used by the individuals, groups and communities in the production of knowledge. For example, one of the primary ethical considerations was developed out of an understanding or learning the uses of technology and the internet as a medium to communicate for research. My interaction with the technologies meant becoming part of the community that I was exploring in my research - I needed to create a YouTube channel and identity. In becoming a registered user of YouTube, I become a part of a community, but in reality, I do not contribute much to the community. Rather, I "harvest" information from the community - both online, as well as in the individual interviews. ${ }^{44}$

The model provides a mechanism to map and thereby explore these multiple contexts in relation to practical research design implications (Praxis), such as my engagement with YouTube as the researcher. These issues include how I engaged with YouTube (Experience/Praxis) prior to the research project as viewer and sharer of material, and the influence of my assumptions about YouTube (Experience/Theory) as part of the construction of knowledge, as well as what it would mean to transform this existing role to that of researcher (Praxis/Scholarship). Figure 25.4 shows this mapping and highlights the need

44 Dag Elgesem, "What Is Special about the Ethical Issues in Online Research?" Ethics and Information Technology 4 no. 3 (2002): 195-203. 
to explore the practical implications of undertaking research across multiple dimensions. How will I conduct myself on YouTube as a researcher? How will I identify myself on YouTube and to potential participants? Do I consider myself a Youser? What tools can I utilise to reflect on, explore and communicate the assumptions I have made as a YouTube user about my experiences as a user and as a researcher?

Figure 25.4: Using the Research Design Model

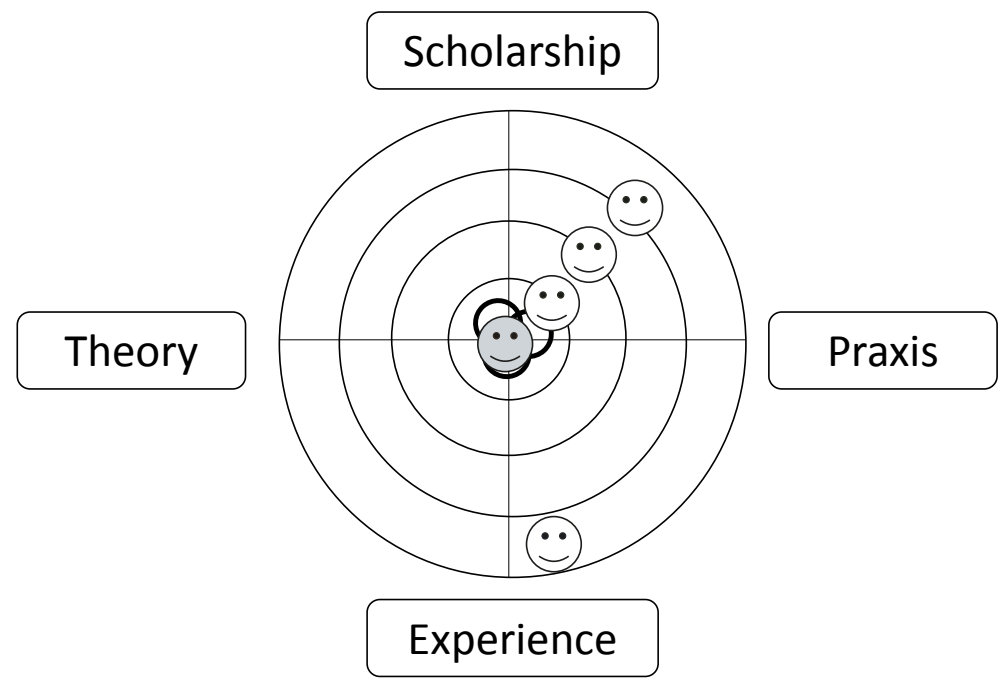

The research ethics application process also helped me to identify potential privacy issues related to only being able to contact YouTube users by being registered as reflected in the Organise dimension of the Theory/Scholarship quadrant. I also needed to use YouTube tools to search, view and track who I was interested in, including subscribing to channels as part of the content analysis which engages with the Capture and Organise dimensions on the Scholarship/Praxis quadrant. In addition, the practical tools needed to undertake the research were also those I was researching, which is represented in the Create dimension of the Praxis/Scholarship. I needed to be able to explore the tools using a researcher identity employing established research methods which spans the Create, Capture, and Organise dimensions on the Theory/Scholarship axis. These multi-dimensional issues highlighted gaps and opportunities. For example, the creation of a YouTube identity 
links across all dimensions, but in my mapping of the expected outcomes, I had not considered how I would situate and communicate these implications and issues to the different communities - the academic community related to ethics permission, as well as the thesis writing and defence, and the YouTube community on the website, as well as when I contacted and interviewed research participants. The former is linked to the Pluralise dimension of the Theory/Scholarship quadrant and the latter to the Pluralise dimension of the Scholarship/Praxis.

The intersection between Praxis and Experience reveals the evolution of the subjective and highlights the role of the interpretative actor within a research project. This quadrant refers also to the process of personal growth and development as part of undertaking a research project. The acceptance of peers and colleagues through the creation of information objects, such as models, posters, papers, and presentations, contributes to the ongoing and evolving self-assurance of the researcher as well as validation and confidence in the research. The use of quadrants to categorise knowledge and influences on knowledge generation provides a tool with which to construct research. The non-linear space that the quadrants provide is also useful when approaching linear structure such as writing a thesis or project report. The model helped to highlight assumptions by deliberately incorporating a personal perspective to identify assumptions about the technologies and language (Praxis/Experience axis). Deliberate design decisions were made to identify, reflect on and craft rigorous processes that complemented the research problems, case study participants, and bias in the analysis over time.

One of the major outcomes of the exploration of the intersections between Experience and Praxis was the development of the method reflective practice. Reflective practice, described in detail in the previous section, refers simply to the process of learning through doing. Reflection refers to deep thinking about issues, implications and influences of the work, as well as cohesion of the ideas and the project as a whole. The combination of the terms "practice" and "reflective" are used here in acknowledgement of the tools that I had already started to use consistently in the research, particularly IT tools and model-making. Ideas about different learning styles to which I had been exposed through previous study and work, such as the VAK (visual, auditory and kinaesthetic) model, the Myers-Briggs Type Indicator (MBTI) assessment, as well as other knowledge I has assimilated through discussions with friends and colleagues over the years in educational psychology and sensemaking, influenced the concept of learning by doing. 
After I had realised that reflective practice was a significant component of the research design, I began to actively explore tools for reflection, drawing from my past experiences as well as seeking out other tools to use. These tools included journal writing, unsent letters, interviews with self, filmmaking, posters, conversation, mind maps, games, automatic writing and presentations. I used any opportunity as a tool to help me understand my work more deeply and clearly. I developed some 3D models of the cultural heritage continuum model and uploaded them to my YouTube channel. I wanted to do more filmmaking, but it is a very time-consuming task. Other methods included removing myself from being in front of a computer and talking to a digital recorder, or drawing on $\mathrm{A} 3$ sheets of paper. The iterative nature of reflective practice had an influence on other quadrants, particularly the Theory/Scholarship quadrant where reflective practice as a method performed a major and central function in the research design as the pivotal method that supports the other methods chosen in the YouTube project.

\section{Spacetime and the Knot of Creation}

Practical implications of time are found in how long it will take to conduct the project (the duration), but also needs to take into consideration the placing of time (the time period) in which this research is conducted. The fixity of time and space, of timeliness and spheres of influence, tie in with sense-making of the symbols and language used by all the elements of research, including the subjects, the researcher, theory, and underlying principles. Fixity also refers to the purpose of the research project: the thesis. The threads that are drawn into the crafting of the thesis are bounded by the choices that are made by the researcher; however, the potential for generating more information and knowledge exists in how the research is used, and its transferability to new realms of time and space. Ultimately, this impacts on the transparency of the research and reveals the critical need for the researcher to explore and explain his/her impact on the research through the decisions made about its design. As time goes on, research reports are delivered, explored and tested by others in the discipline, perhaps discovered by others in other disciplines. Further into time, research might be re-discovered, or even lost and forgotten, but it is a timely presence of when the research actions occurred. Issues of space are bound by where a research phenomenon is contained, how it is accessed, and where in the system of understanding it can be placed. Issues of time, transparency, context and storage are referenced in the symbol located in the centre of the model - the Knot of Creation. 
Storage refers to how information and knowledge are used. The concept of storage links to how knowledge and information are both the fodder and product of activities. ${ }^{45}$ Knowledge is always in action but can be written down and codified, and then acts as information to inspire further knowledge activities. Recorded and codified information is therefore part of the continuous interaction between sense-making and knowledge generation. In the continuum, an information object has the potential to be captured, stored and managed in ways that can benefit individuals, groups, and communities, and push knowledge into plural spaces further out in space and time to become part of the collective memory and to inform other research practices and communities. ${ }^{46}$ The Knot of Creation is a visual representation of the recursive nature of knowledge and information. It is a Borromean knot of three entwined circles. A feature of the Borromean knot is that when one of the three circles is removed or cut, the relationship between them unravels. ${ }^{47}$

The three circles of the knot refer to the symbiotic and linked relationships between the dimensions of Create, Capture, and Organise. Each act of creation is also an act of capture and organisation - the activities of the continuum fused into a single moment of stored information and knowledge. These processes highlight and represent the multiple contexts and processes that help to place recorded information in spacetime and authenticity and reliability. In the case of research, the knot highlights the need to establish transparency and verifiability across all contexts - Create, Capture, and Organise. The knot can be used to design and test information systems, as well as research projects, but also be used as an instrument to examine historical recorded information contexts and processes. Pluralisation, omitted from the knot but vital to its purpose, refers to the potential impact and value of research and the storage (or not) of this research in shared collective memory. Therefore, the knot symbolises ever-evolving complexity, wherein the action

45 Brenda Dervin, "Sense-Making Theory and Practice: An Overview of User Interests in Knowledge Seeking and Use," Journal of Knowledge Management 2 no. 2 (1998): 36-46, doi:10.1108/13673279810249369.

46 Frank Upward, “The Records Continuum," in Archives : Recordkeeping in Society, ed. Sue McKemmish et al., vol. 24, Topics in Australian Library and Information Studies (Wagga Wagga, NSW: Centre for Information Studies, 2005), 197-222.

47 Jacques Lacan used the Borromean knot to explore and define the relationships between the symbolic, the real and the imaginary in psychoanalysis. Henri Cesbron Lavau, "Knot," ed. Alain de Mijolla, International Dictionary of Psychoanalysis, Gale Virtual Reference Library (Detroit, Michigan: Macmillan Reference USA, 2005), Gale Document Number: GALE|CX3435300773. 
of the three circles gives coherence and meaning to the knot itself. The multiple roles of the knot, as well as its metaphorical and literal presentation in the model, demonstrate the complexity of knowledge processes.

Understanding the goal of research and the role of the researcher through the four axes of the model helped me find a place for this new line of enquiry in academia, experience, scholarship, and praxis. The fit between methods and their paradigms needed to be positioned against how I collected, organised, and analysed data that took into account multiple points of view, potential outcomes and usefulness and how this would eventually be explained and reported. The outcomes of these reflections are the inclusion of domain expert case studies, used to support the Youser data and the analysis of the discourse about YouTube. Each set of analyses is a point of intersection between the different dimensions of cultural formation - Create, Capture, Organise, and Pluralise - with each performing multiple roles themselves.

\section{Conclusions and Possibilities}

The Research Design Model was developed in response to the complexities identified in undertaking research into social and technological complexity. The model has multiple informational heritages, but was heavily influenced from the outset by Giddens' double hermeneutic and information continuum theory and models. These two major influences highlight issues pertaining to individual and shared social realities and how experiences and interactions with tools under investigation might influence and impact on my experiences of conducting research and interacting with participants in interviews, as well as on YouTube itself. This impact helped me to identify how my experiences of the past and my world view influenced the way in which I approach the research and the role this plays in the progression of the research and its outcomes.

The research project described here, in which the model was conceptualised, was crafted around how to explore emergent activities in online spaces (such as social media) in relation to cultural heritage processes and formation. The topic, however, required a plan or design that would provide a framework within which to undertake the research. I needed to develop a way to explore complexity in social media spaces, while at the same time examine the role of continuum theory in the research. Issues with which I was grappling before the research started included how my interactions with the tools I was investigating would influence my experiences of conducting research; how my experiences of the past and my world view might influence how I 
approached the research; and how the research would develop as I learned more, and what that ultimately might mean for the research processes and outcomes.

In my attempts to parse these issues, the model was created reflexively as an exploration into the processes of research development, born of a need to understand what my role as researcher is to this (or to any) research project. Developing and using the model provided an opportunity to explore the multiple levels and interactions in my research. In applying the model in the context of a sole researcher, the model helped me to develop different ways of conceptualising the project, understanding the data and outcomes as well as the continuous interpretation of recorded information in the form of posters and papers. The role of the model in the YouTube case study was to help me make sense of my role as researcher and place myself into the history of knowledge and learning, as well as explore assumptions, tools and ways to communicate the research outcomes. There were some "failures" or unanticipated circumstances and situations in the project, but the model provided an opportunity to identify these issues, either during or after the project, and to turn them into opportunities for further research.

The development and use of the model played a vital role in the YouTube case study. It is an instrument for exploring potential via testing the relationships between experiential and theoretical contexts at any given point in the project. At the very beginning of my own research project I foresaw that a major advantage of the model as a tool that allows current and future stakeholders in a project to develop and reflect on the construction, purpose and outcome of a research project before it has even begun. Reflexivity is built into the model which means it can be used to help assess and success of construction, purpose and outcomes at different stages of a research project, and after it has concluded.

There is significant potential for using the model in collaborative and inclusive multi-team projects across disciplines and with partners in the community, industry and government. There is an urgent and growing need to understand and embrace the complex memory and archival needs of an expanding, technologically savvy and actively participative society. Recognition and inclusion of the multiple contexts or narratives that play out in online communities and spaces is essential to this mission. The model provides a way to design a research project by drawing on monuments of knowledge and learning from academia to identify potential research methods, methodologies, tools and community knowledge through an interwoven and non-linear development process. The model is intended to 
identify and address multiple points of view and seek to understand how they impact on the research, the researchers, the stakeholders or community, as well as practitioners within and across disciplines. It also provides a powerful analytical tool for exploring the assumptions and challenges of working with groups with varied and diverse ideas and practices, and for designing and implementing inclusive research.

\section{References}

Bastian, Jeannette Allis. "Reading Colonial Records Through an Archival Lens: The Provenance of Place, Space and Creation." Archival Science 6 (2006): 267-84. doi:10.1007/s10502-006-9019-1.

Burgess, Jean, and Joshua Green. YouTube: Online Video and Participatory Culture. Digital Media E Society. Cambridge, UK: Polity Press, 2009.

Carley, Kathleen, On the Persistence of Beliefs (Working Paper, Department of Social and Decision Sciences, Carnegie Mellon University, 1990) <http://alliance.casos.cs.cmu. edu/publications/papers/carley_1991_persistencebeliefs.PDF> [accessed 3 June 2014]

Dervin, Brenda. "Sense-Making Theory and Practice: An Overview of User Interests in Knowledge Seeking and Use.” Journal of Knowledge Management 2 no. 2 (1998): 36-46. doi:10.1108/13673279810249369.

Dredge, Stuart. "YouTube Aims to Tame the Trolls with Changes to Its Comments Section." The Guardian, November 7, 2013, sec. Technology. http://www. theguardian.com/technology/2013/nov/07/youtube-comments-trollsmoderation-google.

Elgesem, Dag. "What Is Special about the Ethical Issues in Online Research?" Ethics and Information Technology 4 no. 3 (2002): 195-203.

Gibbons, Leisa, “Testing the Continuum: User-Generated Cultural Heritage on YouTube." Archives and Manuscripts, 37 (2009), 90-113

Giddens, Anthony. The Constitution of Society: Outline of the Theory of Structuration. University of California Press, 1986.

Gilliland, Anne, and Sue McKemmish. "Building an Infrastructure for Archival Research." Archival Science 4 no. 3 (2004): 149-97. doi:10.1007/s10502-006-6742-6.

Hakim, Catherine. Research Design: Successful Designs for Social and Economic Research. 2nd ed. Social Research Today. London, UK: Routledge, 2000.

Jenkins, Henry, Ravi Purushotma, Katherine Clinton, Margaret Weigel, and Alice J Robinson. Confronting the Challenges of Participatory Culture: Media Education for the 21st Century. Chicago, IL: MacArthur Foundation, 2006.

Kaufer, David S., and Kathleen M. Carley, "Communication at a Distance." Hillsdale, NJ: Erlbaum, 11 (1993).

Ketelaar, Eric. "Archivalisation and Archiving." Archives and Manuscripts 27 (1999): 54-61.

Ketelaar, Eric. "Sharing: Collected Memories in Communities of Records." Archives and Manuscripts 33 (2005): 44-61.

Lange, Patricia. "Publicly Private and Privately Public: Social Networking on YouTube." Journal of Computer-Mediated Communication 13 no. 1 (2007): 361-80. doi:10.1111/ j.1083-6101.2007.00400.x.

Lavau, Henri Cesbron. “Knot.” Edited by Alain de Mijolla. International Dictionary of Psychoanalysis. Gale Virtual Reference Library. Detroit, Michigan: Macmillan Reference USA, 2005. Gale Document Number: GALE|CX3435300773. 
McKemmish, Sue, "Evidence of Me." Archives and Manuscripts, 45 (1996), 174-87. http://www.infotech.monash.edu.au/research/groups/rcrg/publications/ recordscontinuum-smckp1.html

McKemmish, Sue, Anne Gilliland-Swetland, and Eric Ketelaar. "Communities of Memory': Pluralising Archival Research and Education Agendas." Archives and Manuscripts 33 no. 1 (2005): 146.

McKemmish, Sue, Frada Burstein, Rosetta Manaszewicz, Julie Fisher, and Joanne Evans, "Inclusive Research Design: Unravelling the Double Hermeneutic Spiral." Information, Communication E' Society, 15 (2012), 1106-35 <http://dx.doi.org/10.10 80/1369118X.2012.707225>

McKemmish, Sue, and Anne Gilliland, "Archival and Recordkeeping Research: Past, Present and Future." In Research Methods: Information, Systems and Contexts, ed. by Kirsty Williamson and Graeme Johanson (Prahran, VIC: Tilde University Press, 2013), 79-112

Oliver, Gillian, "Investigating Information Culture: A Comparative Case Study Research Design and Methods." Archival Science, 4 (2004), 287-314 <http://dx.doi. org/10.1007/s10502-005-2596-6>

Onwuegbuzie, Anthony J., Rebecca M. Bustamante, and Judith A. Nelson, "Mixed Research as a Tool for Developing Quantitative Instruments.” Journal of Mixed Methods Research, 4 (2010), 56-78

Schauder, D., G. Johanson, and L. Stillman, "Sustaining and Transforming a Community Network: The Information Continuum Model and the Case of VICNET." The Journal of Community Informatics, 1 (2005) <http://ci-journal.net/index.php/ciej/ article/view/239> [accessed 25 July 2014]

Shah, C. "ContextMiner: Supporting the Mining of Contextual Information for Ephemeral Digital Video Preservation." International Journal of Digital Curation 4 no. 2 (2009): 171-83. doi:http://dx.doi.org/10.2218/ijdc.v4i2.106.

Strauss, Anselm L, and Juliet M Corbin. Basics of Qualitative Research: Techniques and Procedures for Developing Grounded Theory. 3rd ed. Thousand Oaks, California: Sage Publications, Inc., 2008.

Stillman, L. "Understandings of Technology in Community-Based Organisations: A Structurational Analysis" (Thesis PhD, Monash University, 2007)

Upward, Frank. "Structuring the Records Continuum (Part One): Postcustodial Principles and Properties." Archives and Manuscripts 24 no. 2 (1996). http:// infotech.monash.edu/research/groups/rcrg/publications/recordscontinuumfupp1.html.

Upward, Frank. "Structuring the Records Continuum (Part Two): Structuration Theory and Recordkeeping." Archives and Manuscripts 25 no. 1 (1997). http:// infotech.monash.edu/research/groups/rcrg/publications/recordscontinuumfupp2.html.

Upward, Frank. “The Records Continuum.” In Archives : Recordkeeping in Society, edited by Sue McKemmish, Michael Piggott, Barbara Reed, and Frank Upward, Topics in Australian Library and Information Studies, 24 (Wagga Wagga, NSW: Centre for Information Studies, 2005), 197-222.

Upward, Frank. "Continuum Mechanics and Memory Banks [Series of Parts]: Part 1: Multi-Polarity." Archives and Manuscripts, 33 (2005): 84-109

Upward, Frank, and Larry Stillman, "Community Informatics and the Information Processing Continuum", in 3rd Prato International Community Informatics Conference (CIRN 2006) (Monash University, 2006) <http://ccnr.infotech. monash.edu/conferences-workshops/prato2006papers.html> 
Upward, Frank, Sue McKemmish, and Barbara Reed. "Archivists and Changing Social and Information Spaces: A Continuum Approach to Recordkeeping and Archiving in Online Cultures.” Archivaria 72 (February 12, 2011). http://journals.sfu.ca/ archivar/index.php/archivaria/article/view/13364.

White, Kelvin L., and Anne J. Gilliland. "Promoting Reflexivity and Inclusivity in Archival Education, Research, and Practice.” The Library Quarterly 80 no. 3 (July 1, 2010): 231-48. doi:10.1086/652971.

Yin, Robert K. Case Study Research: Design and Methods. 3rd ed. Thousand Oaks, CA: Sage Publications, 2003. 


\title{
INVESTIGATING SOCIO-CULTURAL ASPECTS OF THE IMPLEMENTATION OF AN INTERNATIONAL ARCHIVAL DESCRIPTIVE STANDARD IN KOREA
}

\author{
Eunha (Anna) Youn
}

\begin{abstract}
This chapter presents and reflects upon a research project that used multiple research methods to describe the ways in which metadata schemas are closely related to the social values of an organisation and are understood as cultural outcomes of a given society. ${ }^{1}$ Based on the related assumption that information systems are social constructions and on ideas drawn from Actor Network Theory, it examined the adoption process of the international archival metadata standard, $\operatorname{ISAD}(G)$, in Korea and explored key factors that arose in adapting it to the Korean context. In particular it sought to produce some empirical data and provide a snapshot of specific Korean institutions regarding the ways in which archivists viewed and have approached integrating $\operatorname{ISAD}(G)$. The research involved analysing texts on Korean archival history, developing a metadata crosswalk, and undertaking ethnographic observation at two implementation sites. By using multiple research methods, it hoped to reveal the processes underlying which elements of the standard are adopted and which not, and how these are decided.
\end{abstract}

1 I am deeply grateful to Anne Gilliland for her careful readings and copyediting of drafts of this article. 


\section{Introduction}

Information studies, a field mainly concerned with the collection, organisation, retrieval and preservation of information, has for a long time been dominated by research which has emphasised "scientific" ways of arriving at an understanding of phenomena within the information world - pursuing efficiency, economic feasibility, and objectivity, especially in technological development, rather than focusing on the impact of personal or cultural perceptions. Research in archival studies has been similarly focused, attempting to gain quantitative and procedural data relating to archival use and practice. The field has paid little attention to discovering underlying meanings and cultural patterns or to understanding how technology and technologically-implemented standards impose social values on archival systems. For example, much research on national archival descriptive standards such as Encoded Archival Description (EAD), ${ }^{2}$ Rules for Archival Description (RAD) ${ }^{3}$ and the General International Standard Archival Description $(\operatorname{ISAD}(\mathrm{G}))^{4}$ has been conducted since the 1990s, but most studies have only focused on the technical dimension of the standards. ${ }^{5}$ The "social aspects" of implementations are simply treated as the context in which such standards are situated. This research picture may derive from inherent difficulties in examining the influence of social context over

2 http://www.loc.gov/ead/.

3 http://www.cdncouncilarchives.ca/archdesrules.html.

4 http://www.ica.org/10207/standards/isadg-general-international-standard-archivaldescription-second-edition.html.

5 Michael Cook, "The International Standard for the Description of Archives: A Progress Report," Information Development 8 no. 4 (January 1, 1992): 237-38; Paul Sillitoe, "ISAD $(\mathrm{G})$ - the Guiding Light? Issues in the Use of ISAD $(\mathrm{G})$ in the Preparation of a Repository Guide." In Scottish Universities Special Collections and Archives Group (SUSCAG) conference, Making The Most Of Automation: A Seminar On Users' Requirements From IT Applications In Archives And Special Collections, British Academy, 1996; Elizabeth Shepherd and Charlotte Smith, "The Application of ISAD $(G)$ to the Description of Archival Datasets," Journal of the Society of Archivists 21 (2000): 55-86; Elizabeth Shepherd and Rachael Pringle, "Mapping Descriptive Standards Across Domains: A Comparison of $\operatorname{ISAD}(\mathrm{G})$ and Spectrum," Journal of Society of Archivists 23 no.1 (2002): 17-34; Junte Zhang, "System Evaluation of Archival Description and Access," Ph.D. Dissertation, (Institute for Logic, Language and Computation, Universiteit van Amsterdam: Netherland), http://www.illc.uva.nl/Research/ Publications/Dissertations/DS-2011-04.text.pdf.

6 This concept was spotlighted by the U.S. National Science Foundation Workshop on the Social Aspects of Digital Libraries," held at the University of California Los Angeles in 1996. See Christine Borgman et al. Social Aspects of Digital Libraries Final Report to the National Science Foundation, November 1996, http://is.gseis.ucla.edu/ research/dig_libraries/UCLA_DL_Report.html. 
standards implementation and associated technological development or from a lack of appropriate research skills, but there is no doubt that few studies are interested in the interplay of archival practices between different cultures. Adopting Actor-Network Theory (ANT) as delineated by French sociologists Michael Callon and Brono Latour, who understand processes of technological innovation in terms of the relational ties within a network, the study discussed in this chapter attempted to provide a different kind of description of the roles of various cultural actors in technical innovation associated with standards implementation - examining how people modify their existing environment, add to it, and get rid of it. In other words, looking at how the actors attempt to foster localisation in various ways when a standardisation process imposes a global element on local practices.

The study described here attempted to understand the adoption process of the International Council on Archives (ICA)'s International Standard of Archival Description (General) (ISAD $(\mathrm{G})$ ) from both cultural and technical perspectives. It illustrates ways in which standards themselves are necessarily changed and partially re-appropriated within the social, cultural, and political contexts of the implementation setting, which itself is altered through the implementation. To address the issue thoroughly, the study used multiple research methods. Having multiple kinds of data that are derived from different research methods can be useful for providing insight into the connection between cultural phenomena and metadata systems and for showing a more holistic view of the standardisation process. This research used three different research methods: qualitative ethnography, analysis of texts regarding the history of Korean archival traditions, and a metadata crosswalk of the institutional systems. The first method was an ethnographic qualitative approach to establish the viability of the research's cross-cultural element through a close look at the relationship between practice and standards, and to examine the different cognitive dimensions of decisionmaking in two institutions. The research chose two archival sites in Korea. Both institutions are defined as research institutions supported by the government and were very receptive to any type of academic research examining their practices.

Since ethnography was used, it is important for me at this point to say something about my distinctive position as the person carrying out the research. I am a Korean who was born and grew up in South Korea. My formal education came from the Korean public education system and, therefore, shares the common social and cultural value system that Koreans are expected to have. I obtained my undergraduate and master's degrees in 
Korea, majoring in history. However, without knowledge of and experience in the Korean archival system, I moved to the U.S. to pursue first a master's and then a doctoral degree specialising in archival studies. Thus my professional orientation, skills and knowledge-base were developed in America. From this perspective, my position as a researcher was that of both an "insider" and an "outsider" in this particular setting.

Qualitative research is useful to uncover the social interaction of the cultural factors characteristic of the relevant phenomena and institutions, and makes it possible to position standardisation in an institutional context. In this study, it was essential that I capture various cultural patterns and understand subtle nuances in discussion and the meaning of people's behaviour in the course of the standardisation process. Ethnographic methods focus on generating detailed explanations and vivid descriptions of phenomena through the systematic collection of information about a particular person, social setting, event, or group through immersion, observation and interviews. It thus permits the researcher to effectively understand how the subject of study operates. In this case, I was interested in how the archivists in these two institutions understand $\operatorname{ISAD}(G)$, and their attitudes towards it. I was also interested in the social, cultural, and organisational factors that influenced the adoption of $\operatorname{ISAD}(\mathrm{G})$ in the two institutions studied. Researching these phenomena could not easily be reduced to numbers because the voices of people and their unique cultures are not clearly described in quantitative research. Qualitative research makes up for these weaknesses. The ethnographic method has the advantage of allowing the researcher simultaneously to experience the activities and observe multiple cultural phenomena across institutions. The study was largely explanatory, therefore, concentrating on social values and cultural influences embedded beneath the technical structure, which are far from known or understood. In addition to the qualitative methods, an analysis of Korean historical texts as well as metadata crosswalk analyses were used to obtain in-depth understanding about the processes used in each organisational setting and to overcome the weaknesses inherent in individual methods in addressing both technological and cultural perspectives.

In this chapter, I will first discuss the epistemological lineage of standardisation research as traced from both positivist and social constructivist perspectives. The next section will provide an overview of the research design, explaining how and why each method was chosen and combined with the other methods. The third section will show some of the findings from each method, and a discussion will follow in the final section. 


\section{The Theoretical Framing of Standardisation}

Traditionally, standardisation is believed to arise in response to the technical needs of society. In particular, in the late 20th century, when information technology moved quickly and official standards were created internationally, this approach received much attention from those in the fields which have a strong positivist tradition, such as economics, engineering, and political science. They understood standardisation as a process in which certain technical problems can be solved by devising an appropriate technical standard. In particular, Kling points out that information studies clung in the 1970s and 1980s to technological research that was mainly focused on technical aspects of computerised information systems and standards. ${ }^{7} \mathrm{~A}$ standard was considered necessary for facilitating the interaction between two systems in the exchange of information. It is assumed that there is a sender and a receiver of a message who each need to use the same set of communication standards. In this context, standards can be understood as rules that enable the sender and the receiver to interact. ${ }^{8}$ The development of a standard is explained in terms of the technical advantages and the economic benefits that the standard would produce. In short, standardisation is seen as an engineering problem. ${ }^{9}$

In this context, in defining the term "standard," many authors emphasise the importance of compatibility. Products are said to be compatible when their design is coordinated in some way, enabling them to work together. The compatibility, most of the time, is accompanied by an analysis of economic benefits. That is why mainstream economics literature approaches standardisation from a market viewpoint. Economic theory has studied why standards emerge, how technology is adopted, and how interest could be created. Farrell and Saloner compare standardisation using economic principles. ${ }^{10}$

7 Rob Kling, "Learning About Information Technologies and Social Change: The Contribution of Social Informatics," The Information Society 16 no. 3 (2000).

8 As Weitzel points out, "the term 'standard' refers to any technology or product incorporating technological specifications that provide for compatibility ...

Standardization is defined as the implementation of a standard or technology consistent with specifications in such a way as to provide for compatibility with a communication partner from the perspective of an individual technology user." Tim Weitzel, Economics of Standards in Information Networks (Frankfurt, Germany: Physica-Verlag, 2004), p.8.

9 Patrick Feng, "Studying Standardization: Review of the Literature." Proceedings of the Standardization and Innovation in Information Technology (SIIT2003) Conference, IEEE, 2003.

10 Joseph Farrell and Garth Saloner, "Standardization, Compatibility, and Innovation," The RAND Journal of Economics 16 no. 1 (1985); "Installed Base and Compatibility: 
They mainly focus on identifying the advantages and disadvantages of joining standards organisations and the mechanisms that can lead to excessive or insufficient standardisation through product design. ${ }^{11}$ According to Farrell and Saloner, technology gains an initial lead in the development of a standard and this triggers a process in which new members adopt the technology. The adoption of the technology in turn increases its attractiveness to third parties. This third party encourages new users to adopt the technology. This circular, yet linear model encourages different users to adopt the standard or sometimes to create a new one. ${ }^{12}$ Here, the basic economic assumption is that all people take equivalent steps to adopt standards and that the standard that is successful in the market place is better than any alternative standard.

However, a review of the literature indicates that economic arguments do not do sufficiently explain the standardisation process. Rather, social, political, cultural, and educational processes play important roles in the process of standardisation. Many scholars now pay attention to the importance of standardisation as a social process. What makes a good standard is no longer viewed as simply a matter of technical development because both the market and technological development are subject to the broad influence of socio-institutional forces. This idea is deeply related to the theory of social constructivism, whereby technology is seen as a social construction, and technological change is discussed with reference to social practices such as interpretation, negotiation and closure by the actors involved.

According to this view, technology is made up of different interpretations in terms of functional and social-cultural properties, rather than self-apparent and fixed properties. That is, facts about a technology are not objectively given by the technology itself, but are determined by how the technology is interpreted by relevant social groups. ${ }^{13}$ The positivistic view of standardisation mainly questions what the benefit of standardisation is and how it is created, but the socio-cultural view is more concerned with how

Innovation, Product Preannouncements, and Predation,” The American Economic Review 76 no. 5 (1986); "Coordination through Committees and Markets," The RAND Journal of Economics 19 no. 2 (1988); and "Converters, Compatibility, and the Control of Interfaces," The Journal of Industrial Economics 40 no. 1 (1992).

11 Carmen Matutesa and Pierre Regibeau, "A Selective Review of the Economics of Standardization Entry Deterrence, Technological Progress and International Competition," European Journal of Political Economy 12 (1996).

12 W. Brian Arthur, "Competing Technologies, Increasing Returns, and Lock-in by Historical Events," The Economic Journal 99 (1989).

13 Arthur, "Competing Technologies." 
standardisation occurs in society or within organisations as well as how the social elements are inter-related, and influence, construct, and are maintained in the process.

Rogers' diffusion of innovations theory, first published in the 1970s, ${ }^{14}$ provides a starting point in terms of addressing the socio-cultural dimensions of standardisation. The diffusion of innovations can be described as the spread of abstract ideas and concepts, technical information, standards and actual practices within a social system, where the spread denotes flow or movement from a source to an adopter, typically via communication and influence. An innovation is described as an idea that is perceived to be new to a particular person or group of people. Contemplating the standardisation of an information system inevitably involves innovation and diffusion since the system is always seen as new by local practice. Using this approach in an explanation of the successful adoption, or rejection of a standard is helpful to understand how the cultural actors (e.g., ideas, values, and attitudes) influenced the standard's adoption, and why some institutions accepted while others resisted the new standard. Rogers assumed that unlimited communication within a social system facilitates the diffusion of a technology. In contrast to other existing theories, he pointed out that the behaviour of the adopters is not necessarily rational but is more oriented to the cultural and social context surrounding the technology. ${ }^{15} \mathrm{He}$ focused on sociocultural aspects that are assumed to influence the diffusion of technology and from this we can understand his theory in terms of the diffusion of archival descriptive standardisation. The first step undertaken by the ICA Ad Hoc Commission in developing $\operatorname{ISAD}(\mathrm{G})$ was to conduct a comparative analysis of the three major Anglo-American data content standards, the U.S.'s Archives, Personal Papers and Manuscripts (APPM), Canada's Rules for Archival Description (RAD), and Great Britain's Manual of Archival Description (MAD). The standard was officially adopted by the International Council on Archives (ICA) in 1994 and was translated into five European

14 Everett M. Rogers, Diffusion of Innovations $5^{\text {th }}$ ed. (New York: The Free Press, 2003).

15 An alternative view of innovation is proposed in the actor network theory. Arthur Tatnall and Anthony Gilding, "Actor-Network Theory and Information Systems Research" (paper presented at the 10th Australasian Conference on Information Systems, Melbourne, Australia, 1999). The concept of translation that can be defined as: "... the means by which one entity gives a role to others," see Vladislav Fomin and Keil Thomas, "Standardization: Bridging the Gap between Economic and Social Theory," in Proceedings of the Twenty-first International Conference on Information Systems (Brisbane, Queensland, Australia: Association for Information Systems, 2000). 
languages and distributed worldwide. ${ }^{16}$ The emergence of $\operatorname{ISAD}(\mathrm{G})$ was an innovative event in archival practices and was diffused through the development of new compliant archival descriptive standards by nations in Asia, Europe, Africa, Australia, and America. A study on standardisation using $\operatorname{ISAD}(\mathrm{G})$, therefore, can potentially illustrate how technological innovations were diffused and localised in particular settings.

Actor-network theory (ANT) is also concerned with the processes by which scientific disputes become closed, ideas accepted, and tools and methods adopted - that is, with how decisions are made relating to knowledge development. ${ }^{17}$ Callon and Latour explain that the theory is concerned with studying the mechanics of power as this occurs through the construction and maintenance of networks. It is concerned with tracing the transformation of these heterogeneous networks that are made up of people, organisations, agents, machines and many other objects. ${ }^{18}$ The theory explores the ways

16 The commission identified 20 data elements that appeared in all three or for which there was substantial overlap and recognised this as the main framework of the $\operatorname{ISAD}(\mathrm{G})$. The traditional Canadian concept of Total Archives is codified in detail in the structure of the elements, the British idea of unit analysis is specified in the framework, and the U.S. tradition of highlighting bibliographic standards are the basic principles of $\operatorname{ISAD}(\mathrm{G})$. Also, according to the website of ICA/CDS, for 2000-2004, 11 of 13 full members came from Western Europe, the United States, and Australia. 7 of the 22 members were from non-Western countries, but 5 were only corresponding members. There were 22 committee members of ICA/CDS: 11 from Europe, 2 from the U.S., 2 from Australia, 3 from Latin America, 3 from Africa, and 1 from Asia. Therefore it could be speculated that the efforts to build a global standard underpinning diverse schemas for archival description were heavily influenced by Western practices and ideas about archival description. Consequently, it might be argued that $\operatorname{ISAD}(\mathrm{G})$, as a global standard for archival description, is less a global standard in terms of meaning that it takes into account all possible concepts of archival description from around the globe, but is rather a standard developed for the globe but based on the archival principles of certain Western archival traditions and even within that, on localised ideas of a few countries and institutions; Victoria Irons Walch for the Working Group on Standards for Archival Description with contributions by Marion Matters. Standards for Archival Description: A Handbook (Chicago: SAA, 1994); Wendy Duff and Kent Haworth, "The Reclamation of Archival Description: The Canadian Perspective," Archivaria 31 (1990-91): 26-35; Michael Cook, "Description Standards: The Struggle Towards the Light," Archivaria no. 34 (Summer 1992): 50-57; Shepherd and Pringle, "Mapping Descriptive Standards Across Domains"; Steven Hensen, "The First Shall Be First: APPM and Its Impact on American Archival Description," Archivaria 35 (1993): 64-70; See also, website of International Council on Archives Committee on Descriptive Standards, "History of ICA/CDS," http://www.icacds.org.uk/eng/history.htm.

17 Nancy Van House, "Actor-Network Theory, Knowledge Work, and Digital Libraries," (Berkeley, CA: the UC Committee on Research under their Research Bridging Grant Program, 1999-2001).

Tatnall and Gilding, "Actor-Network Theory and Information Systems Research." 
in which the networks of relations are inter-related, how they emerge, how they are constructed and maintained, how they compete with other networks, and how they survive over time. The important thing is the role of the actors, who have the power to make a change in the network by deciding to accept or reject new elements into the network. Latour understands that an innovation depends on the people (actors), who may react to it in different ways. People modify it, add to it, appropriate it and get rid of it. That is, when the standardisation process imposes the global element on local practice, the actors attempt to foster localisation in various ways. The actors are not passive objects that only accept. Rather, Latour argues, the actor only accepts the change if other actors can be persuaded to perform the appropriate actions for this to occur. In this case, the adoption of an innovation comes as a consequence of the actions of everyone in the chain of actors who has anything to do with it. ${ }^{19}$

Following Callon and Latour's argument, ${ }^{20}$ the study investigated how this can occur by comparing two case studies, Archives A and Archives B in Korea, in order to reveal how new standards feed off previous standards and cultural practices. Many decisions in the standardisation process are made based on multiple (possibly conflicting) values that are consciously and unconsciously at work in institutions. These decisions are associated with previously identified cultural elements, such as identity, roles, cultural norms, personal beliefs, education, values, and individual's spontaneous ways of thinking about the standard. Following actor-network theory, this research attempts to illustrate how the standards function through a distributed work of a multitude of heterogeneous actors and also how the standards themselves are necessarily changed and partially re-appropriated in the existing social, cultural, and political contexts such as a set of thoughts, behaviours, and tastes ${ }^{21}$. In contrast to other theories, by highlighting the role of technological actors in the innovation process, actor-network theory reveals how standards interplay with multiple heterogeneous local actors.

19 Tatnall and Gilding, "Actor-Network Theory and Information Systems Research."

20 Michel Callon, "Some Elements of a Sociology of Translation: Domestication of the Scallops and the Fishermen of St Brieuc Bay." First published in J. Law, Power, Action and Belief: A New Sociology of Knowledge? (London: Routledge, 1986), pp.196-223, https://bscw.uniwuppertal.de/pub/nj_bscw.cgi/d8022008/Callon_ SociologyTranslation.pdf; Bruno Latour, Science in Action: How to Follow Scientists and Engineers through Society (Cambridge, MA: Harvard University Press, 1987).

21 Stefan Timmermans and Marc Berg, "Standardization in Action: Achieving Local Universality through Medical Protocols," Social Studies of Science 27 no. 2 (1997). 


\section{Research Design}

\section{Research Sites}

The study examined two archives in Korea. Korea has maintained a welldeveloped central government for over thirteen hundred years, and the techniques, forms, rules, and regulations for managing records in old Korea became highly developed over the course of its history. In recent decades, however, it has adopted the ideas and practices developed in Western countries. For this reason, I judged Korea to be a significant jurisdiction for my research and accordingly chose two prominent institutions, referred to here as Archives A and Archives B, as case studies. Archives A is a research institution built in 1946 to promote Korean historical research by preserving historical resources, publishing academic literature, and supervising K-12 education. Its holdings include almost 153,701 volumes of rare books, 48,930 historical documents, and 24,043 audiovisual materials. These come in various formats - monographs, stone and metal inscriptions, calligraphic and painted records, periodicals, maps, photographs, music scores, slides, audio and video, and microfilm. Almost half of its staff members are professional historians, but without formal archival education. The institution rarely employs people with degrees in library or archival science. Only two staff members have completed university archival education, thereby being exposed to Western archival techniques. Archives A adopted ISAD $(G)$ in 2007 while building its Electronic Archives System (EAS), to modernise and streamline the institution's existing archival system.

Archives B was founded in December 2007. A government archives, it holds more than 8 million items, including 735,000 paper documents, 7,121,000 electronic documents, 775,000 audiovisual recordings, and 8,115 gifts. A total of $95 \%$ of the holdings are records created between 2003 and 2008 under the Public Records Management Act. The holdings are thus relatively homogeneous modern bureaucratic records, particularly compared to those in Archives A. Most of the staff members have completed archival and records management education programs in universities accredited by the government, and some have master's degrees in library science or history. Archives B adopted ISAD(G) in 2007 during a project to build an archival management system (AMS) for the long-term preservation of electronic and hardcopy government records. 
Each of these archives is unique and interesting while at the same time clearly distinct in terms of size, staff, history, organisational structure, and mission. During my data collection, it became clear that different ways of thinking were embedded in the systems of each institution's subdivisions. Each institutional setting, therefore, provided an opportunity to observe how the contextual factors of the systems were related to the adoption of $\operatorname{ISAD}(\mathrm{G})$. The case study method often aims to provide a broad social view and using multiple cases can reveal flaws in idiographic understanding drawn from single cases. According to Yin, multiple case studies are likely to be stronger than the classic single case study. ${ }^{22}$ By broadening the topic coverage, multiple case studies can show how changes occur in both different and similar ways. Thus, these two archives provide an opportunity to discover variations in the standardisation process and allow a comparison that should reveal the contextual factors that affect the process.

\section{Procedures}

I visited the sites twice each week for four months and observed six hours per day at each site. This time included attendance at seminars, conferences, and department meetings. After spending a significant number of hours in the institutions, in-depth interviews of key informants were conducted. ${ }^{23}$

1) Qualitative observation of research subjects: All formal field interviews and observations were recorded through audiotaping and note-taking. Key informants were identified from the organisational chart and job descriptions and selected based on their involvement in the standards implementation project and willingness to participate in the study. In order to become familiar with the environment of each setting, I spent significant time with the subjects and tried to attend meetings and events with them. I did not participate in the practices of the subjects but observed them from a distance. Neither institution allowed me to participate in actual work,

22 Stefan Timmermans and Marc Berg, "Standardization in Action."

23 For practical guidance in capturing the cultural implications hidden in the standard process, I used Martyn Hammersley and Paul Anthony Atkinson's Ethnography: Principles in Practice $3^{\text {rd }}$. ed. (London: Routledge, 2007, and Norman K. Denzin and Yvonna A. Lincoln's Handbook of Qualitative Research $4^{\text {th }}$ ed. (Thousand Oaks, CA: Sage, 2011) as frameworks for the interpretive research. Using Dorothy E. Smith's Institutional Ethnography: Sociology for People (Lanham, MD: Gender Lens Series, AltaMira Press, 1995) as the cognitive framework and Stephen Schensul and Jean Schensul's Essential Ethnographic Method: Observation, Interview, and Questionnaires (Lanham, MD: AltaMira Press, 1999). 
such as doing description, entering metadata into the system, or arranging records, thus observations of formal and informal conversations were carefully conducted and audiotaped. In Archives A, most observations were carried out in the staffroom of the department; this included conversations among staff, staff meetings, and personal education and tutoring time. In Archives B, the researcher spent most of the time observing activities in the main department office and also closely observing the work process, staff meetings and informal chatting. In addition to undertaking content analysis of relevant texts (described below) and interviews, the researcher made field notes describing relevant observations, such as unofficial department meetings, conferences, seminars for staff education, expert meetings, and staff presentations for public information at the sites.

2) Interviews: The interview protocol used open-ended questions, beginning with general questions about the respondents' personal background and experience at the institution. Twenty-three face-to-face, one-on-one interviews were conducted, each lasting between ninety minutes and two hours. Informal interviews were conducted as needed. In total, eight interviews were carried out in Archives A and fifteen in Archives B. Formal interviews were scheduled a month after the observation began. Interviews were important as methods of understanding the system, standards, and, especially, the workflows from the respondents' point of view. This gave more detailed information about how the respondents understand and use their metadata system in their everyday practice. Through the interviews, I not only acquired a narrative from each staff member but also obtained written materials such as presentation drafts, internal reports, and examples of each archives' metadata schema and system manuals.

3) Coding: Coding and memo writing in fieldnotes were the two techniques used in the analysis of the fieldwork data. I initially used coding to categorise and find patterns in the data. Line-to-line coding with different coloured tags was useful in comparing the data elements across institutions and discovering similarities and differences in the understanding and implementation of the standard. A qualitative data analysis software program was available but I chose not to use it in the study in order to ensure that the exact meanings from each element were captured. Sometimes qualitative data analysis software can miss subtle nuances in the words and behaviours of individuals being observed. 


\section{Analysis of Texts on Korean Historical and Archival Practices}

This method was used to understand system development within the broad context of Korean politics, society, and history. The assumption behind the use of the method was that archival traditions are a product of a complex mixture of history, politics, technology, and culture. These traditions are inevitably structured or limited by institutional development and are, therefore, relevant to the metadata system analysis as well as the cultural analysis of the institutions that I studied. The method was intended to identify the tradition of archival management in Korean history and to explore how the archives evolved and what archival traditions existed in Korea. To do this, the study examines the literature on Korean history and on Korean archival practices, since the history of archival management occurs at the nexus between the two. The following rationales and questions underlay the choice of method:

1. Understanding the archival institution's history in order to provide rich contextual information about the creation and operation of the two archives being studied. How have they been shaped or reshaped in modern Korean history?

2. Investigating the framework for archival practices before $\operatorname{ISAD}(G)$ was introduced to Korea. What were the local descriptive practices before the international standard was introduced?

3. Identifying the historical archival tradition in order to understand modern Korean archival management and the extent to which it may or may not have moved away from its traditional roots (e.g., examining prior practices such as the tradition of compilation, the origins of Sillok (實錄, annals), the roles and Sa-kwan (史官, a court diarist), and the early features of the archival tradition. ${ }^{24}$

24 The official annals of the old Korean dynasties, known as sillok, in Korean, were created under the archival records management system in Korea. The Annals of the Joseon Dynasty, the Joseon Wangjo Sillok (朝鮮王朝實錄), comprise 1,893 books and cover the entire history of the Joseon Dynasty, 472 years from 1392 to 1864 . Traditional Korean archival processes were oriented towards producing annals of the dynasty (Sillok). This process was carried out by court diarists or Sakwan (史官) whose works were to create court diaries, Sacho (史草) through collecting, appraising, organising, and compiling the original documents. This creation of official annals of the dynasty had a great impact on the entire archival process of record keeping in the Dynasty and was developed within the compilations tradition of historical writing. The compilation processes for 
Some of the study's research draws from as far back as the medieval Koryo Dynasty. What archival practices have historically been implemented in Korea? What characteristics and values are found in the history of Korean record keeping practice?

The purpose of this method was to describe the interplay between the historical development of descriptive archival practice and the role of the institutions in question since political, economic, and technological changes have led to reforms in records management and have affected the missions of these institutions. For example, Archives A originated in a research centre built by the Japanese colonial government to collect historical materials justifying their colonial rule. Archives B stemmed from the records management reforms of the Korean government in the 2000s. Thus, the two archival institutions were created under completely different social and political circumstances. These differences greatly influence the mission of the institutions, their holdings, organisational structure and culture, workflows, and, most importantly, their ways of describing archival materials.

To obtain historical information, the researcher collected publications from archival institutions such as Kyujang-gak (奎章閣), Jangseo-gak (藏 書閣), and the National Institutes of Korean History (國史編纂委員). All have functioned as archives or libraries, preserving archival records as well as publishing journals, newsletters, proceedings, and annals about their holdings. My analysis indicated, however, that while there is widespread agreement on the need to study the history of the Korean archival tradition, there has been little investigation into the historical documentary resources for this purpose. Much of my research analysed secondary history literature such as journal articles, dissertations, theses, and other academic publications, newsletters and magazines. It also drew upon interviews with historians as well as their books and newspaper and magazine articles on the history of archives. These resources are valuable in capturing the archival institution's history and often provided extensive bibliographic information such as footnotes useful for delving further into Korea's archival tradition.

publishing Sillok began with documentation of the king's business, and largely involved the process of records creation. The Sakwans' tasks included making authentic archival records through the process of careful documentation every day, and they were present at almost all of the official business conducted by the king in his court. To ensure thorough documentation, they recorded both his conversations with officials and his facial expressions. As a result, Sacho, official court documents written by Sakwan, include daily accounts of state affairs as well as diplomatic affairs, the economy, religion, meteorological phenomena, the arts, and daily life, among other things. See Eunha Youn, "Archival Traditions in Korean History: From Medieval Practice to the Contemporary Public Records Management Act," Archival Science 23 (2013): 23-44. 


\section{Crosswalk Analysis of the Metadata Schemas}

The historical analysis and ethnography together provided historical and cultural context for the institutions and suggested the social implications of their institutional context. The third method used in this study was the development of metadata crosswalks for analysing the two different implementations of $\operatorname{ISAD}(\mathrm{G})$. The crosswalk method was used for the following reasons:

1. To identify the characteristics of the systems: it provides an overview of the metadata schema, its elements and why they were chosen, and its technical development. For example, Archives A initially created 7 areas, 25 elements, and 123 sub-elements in its metadata schema and modified them later to accommodate the wide range of bibliographic data in its earlier system, while Archives $\mathrm{B}$ created 7 areas and 28 elements to attempt to maintain all the elements delineated in $\operatorname{ISAD}(\mathrm{G})$.

2. To explain in detail how $\operatorname{ISAD}(\mathrm{G})$ influenced the development of archival metadata in these Korean archives.

3. To identify the issues behind the design of the metadata schemas that were implemented: How or to what degree was the Korean item-level descriptive tradition reconciled with the collectivelevel description of $\operatorname{ISAD}(\mathrm{G})$ ? What kind of technical and legal issues arose during system design? Are the archives considering integrating their system with other information systems? Do they have any legal obligation to support citizens in retrieving information or records from their holdings?

Metadata crosswalks enable the use of elements defined in one metadata standard to interact with or be compared to other metadata standards. Devising crosswalks involves specifying a mapping of each element in the source metadata standard to an equivalent element in the target metadata standard. Translating one metadata element to another in a different standard allows different schemas to communicate with each other and for a user to search multiple databases with a single query. However, no particular crosswalk method has yet been widely adopted because metadata standards are often developed independently and locally and are specified differently using specialised terminology, methods, and processes. This study involved several crosswalk analyses between the EAS and $\operatorname{ISAD}(\mathrm{G})$, between the AMS and $\operatorname{ISAD}(G)$, and between the two institutions' legacy systems and $\operatorname{ISAD}(G)$. 
Each analysis included an overview of the system development, an actual mapping to $\operatorname{ISAD}(\mathrm{G})$ and conclusions regarding the key issues and characteristics of the systems. The crosswalk analyses comprised three phases: the first included the identification of the metadata schemas being used (e.g., edition, purpose, and author) at the general level; the numbers of elements, the unique identifier for each metadata elements (e.g., tag, label, identifier, and field name), and the required elements in deciding whether a metadata element is mandatory or optional and whether the element is repeatable or non-repeatable. The second stage examined the specific features of each identifier in its definition and actual use. The third stage mapped elements of the institutional metadata implementations to those of $\operatorname{ISAD}(\mathrm{G})$.

Stage 1: In May 2009, the first and second versions of the AMS and EAS metadata schemas were obtained directly from two research sites. ${ }^{25}$ The metadata elements comprising the selected metadata schemas were individually examined and compared.

Stage 2: After discovering the general features of the schemas, along with the analysis of the 26 elements and 6 areas of $\operatorname{ISAD}(\mathrm{G})$, the study examined the values of the individual elements of the EAS and AMS, such as free text, numeric range, date, and controlled vocabulary. Based on this analysis, the study explored how these elements are related to the organisational cultures, communication patterns and workflows of the parent archives and attempted to explain why standardisation is not a linear process.

Stage 3: A crosswalk analysis was performed by mapping the metadata elements from each institution's metadata standard to $\operatorname{ISAD}(\mathrm{G})$. In addition to creating a metadata crosswalk between the standards, the study also developed an overview of the development of the system within which each schema was implemented, and a content analysis of the prior systems.

25 The definitions of variables for the crosswalk are the following: 1 . ISAD $(G)$ : the General International Standard Archival Description (2nd edition) is a set of general rules that should be included in archival description as approved by the International Council of Archives (ICA/CIA) in 2000. It consists of 26 elements and 7 information areas that may be combined to constitute the description of archival materials. 2 . The Electronic Archival System of Archives A (EAS, 2nd edition) comprises a set of metadata elements that describe many of the archival resources in Archives A. Predicated on multi-level description, it includes elements describing archival resources, such as video, sound, image, documents, and microfilm. Metadata records based on EAS are intended to be used in promoting user accessibility and streamlining electronic systems to facilitate the business workflow of Archives A. 3. The Archival Management System of Archives B, (AMS) was developed to achieve the long-term preservation of archival materials, both in electronic and hardcopy, held by Archives B. To enhance accessibility and meet the legal requirements governing the institution, it provides 28 elements, 4 of which are defined as core elements for describing the records. 
Documentary sources associated with the system development were collected, such as system manuals, description guidelines, task manuals, and reports on project plans and designs. Both institutions published official guidelines describing system operation and a descriptive manual defining what values should be entered into the metadata system and how each metadata element could be used. The manuals were designed to produce a common understanding among all staff members regarding the usage of each element and, ultimately, to standardise it by restricting the values that could or should be entered.

\section{Findings}

Within the context of their particular historical and social circumstances, my study attempted to examine in detail how two different archival repositories in Korea, Archives A and Archives B, adopted ISAD(G). The analysis of Korean history literature revealed that in Korea, interest in archival description has been surprisingly low, particularly concerning its scope and methods. It also determined that the ideas of keeping the original documents that were generated directly out of bureaucratic activity, and providing public access to them were not part of traditional Korean archival practice. Rather, it was believed that important archival records should be compiled for future use and then replicated, with copies stored in multiple locations. This reflected Korea's history of frequently being invaded and how securing records and safely handing them down to subsequent generations was the most vital factor in archival management. The idea of using archival description to enhance public access was rarely viewed as necessary throughout the 20 th century. The introduction of $\operatorname{ISAD}(\mathrm{G})$ to archives brought an innovative change in attitudes to archival materials and triggered the systematic restructuring of the entire archival system and the archival description of both institutions.

The metadata crosswalk analysis found that the specific implementations of $\operatorname{ISAD}(\mathrm{G})$ in Archives A and Archives B were primarily determined by balancing different descriptive imperatives for collective and item-level description. For instance, Archives A included a total of 48 elements while $\operatorname{ISAD}(\mathrm{G})$ contains 26 elements. Archives A has defined in-house practice and identified new information units of specific relevance to them. As a result, most of the elements were derived from the previous systems. For example, 32 elements of the schema were identical with those of $\operatorname{ISAD}(G)$ in use and meaning and 4 elements roughly corresponded to $\operatorname{ISAD}(G)$ elements. 
12 new elements were added to cover classifications, title elements, and information sources that were mainly inherited from their legacy systems. Unlike Archives A, the basic principles of $\operatorname{ISAD}(\mathrm{G})$ were fully reflected in the system of Archives B. Archives B created 28 elements. All 7 areas and 26 elements of $\operatorname{ISAD}(\mathrm{G})$ were implemented in the Archives B schema and only 2 elements were added into the system. The absence of legacy systems and homogeneous collections of records in Archives B facilitated smooth adoption of the standard. These differences in standard adoption between the institutions show that direction of the standardisation is not a simple and linear one that progresses from the basic principles of archival description to system design. The study found that the direction of the standardisation is highly dependent on an institution's sociocultural values.

The ethnographic analysis identified that particular cultural norms and organisational values are often embedded in the process of adopting ISAD $(G)$. They can strongly influence the decisions, directions and methods associated with standards implementation because the standardisation process consists of a series of choices and a full range of procedural communication. For example, the communication of Archives B is more strategic and taskoriented. Their culture is more strategic in nature because their hierarchy exerts a strong influence, with the intention of tightly controlling the staff's behaviour and work performance. The organisational culture highlights prestige, control, authority, teamwork, personal achievement, tolerance, respect, commitment, politeness, and harmony; with each supporting the other (e.g., authority and commitment, and teamwork and harmony). They also use more formalised and codified communication in the form of letters, memos, reports, websites, and regular advertising to convey their agenda to the staff. This both minimises and negotiates internal conflict. Within this culture, the standardisation process and its coordination are seen as part of the larger process to regulate the work activities of the institution and its efforts to renovate their system so that results are aligned with organisational goals. Consequently, the adoption of the standard and the resultant organisational changes are coordinated and tightly controlled in a sophisticated manner by vertical communication.

On the other hand, informal social controls were more prominent at Archives A because the values of autonomy and independence are evident in the performance of all functions of the organisation at the site. This autonomy promotes considerable flexibility and creativity, which are essential for promoting work performance. Also, the culture requires very little in the way of interaction in order for staff to perform their tasks. The limited need 
for interaction normally brings about few opportunities for direct conflict; but, if any occur, due to the lack of the authority to negotiate the conflict and prior consensus-building, such upheavals as drastic system changes and modification of work processes are poorly accepted in the institution. Allocating time to understand the changes and internalise reform processes are key elements, therefore, in achieving successful adoption.

\section{Conclusions}

The study discussed in this chapter has highlighted the importance of socio-cultural studies in understanding not only the technical aspects of implementing a metadata schema standard within a system but also the social aspects of the adoption of that standard. The study used multiple methods to help the researcher better understand the different aspects of institutional environments. By combining technical and cultural approaches, it overcame the weakness of any single research method, particularly those of the kinds of quantitative methods often used in research relating to technological applications. The data collected in this way enabled me to gain a holistic view of the standards implementation process in both institutions that I examined. In both institutions attitudes toward $\operatorname{ISAD}(\mathrm{G})$ were highly positive, and the staff were ready to implement it within their systems. However, the adoption process and standardisation results clearly differ between both institutions. A crosswalk analysis of metadata schemas revealed how and why the systems were designed differently and what principles and technologies governed the systems. The qualitative ethnographic method revealed significant differences in organisational culture, communication patterns, and professional identity on the part of the staff, and these became clearly evident whenever the staff encountered changes during adoption of the standard or determined the direction of that standardisation.

While the methods used and data collected thereby demonstrated several means of understanding the characteristics of the standardisation process and how its implementation is influenced by culture, the research design (i.e., how the methods were implemented and the data triangulated) proved to have several limitations. First, the actual observation and fieldwork were conducted at the end of the adoption process and while it was relatively easy to observe the final project outcomes, the researcher did not get a chance to observe and capture the entire process of standardisation in detail. This resulted in an unfortunate inability to capture consistent data on the conversations among staff regarding the initial system design, and observation of 
the decision process at each stage. In both institutions, system development, as one of their largest projects, was expected to take more than three years, so ideally I would have needed to spend a long time in the field to capture all the necessary data and to observe all the dynamics. Instead, additional data such as in-depth interviews and the collection of internal reports and publications replaced actual anthropological observation. This illustrates how, in ethnographic research, it is significant when the researcher enters the research site, how long he or she stays in the field, and what he or she decides or is able to observe. Secondly, I paid more attention to processing archivists than to reference archivists or librarians. The reference perspective, particularly on the use of item-level description based on $\operatorname{ISAD}(\mathrm{G})$, was not well represented. Thirdly, in terms of metadata analysis, the crosswalk approach worked well, up to a point. There were problems with the subjectivity of the analysis because individual elements from the different metadata schemes were not perfectly equivalent, thus requiring me to exercise my own judgment about correspondences or lack thereof.

Nevertheless, although there were several limitations in applying each research method, as the above discussion indicates, the multiple methods approach had important advantages in its ability to uncover how all these cultural and technical aspects relate to each other and fit together in an implementation context. Even though such multifaceted research designs can be complicated to apply, they are undoubtedly able to reveal more of the complex nature of that context than a single-method study could.

\section{References}

Arthur, W. Brian. "Competing Technologies, Increasing Returns, and Lock-in by Historical Events," The Economic Journal 99 (1989).

Borgman, Christine, et al. Social Aspects of Digital Libraries Final Report to the National Science Foundation, November 1996, http://is.gseis.ucla.edu/research/dig_libraries/ UCLA_DL_Report.html

Cook, Michael. “Description Standards: The Struggle Towards the Light," Archivaria no. 34 (Summer 1992): 50-57.

Callon, Michel. "Some Elements of a Sociology of Translation: Domestication of the Scallops and the Fishermen of St Brieuc Bay." First published in J. Law, Power, Action and Belief: A New Sociology of Knowledge? (London: Routledge, 1986), pp.196-223, https://bscw.uniwuppertal.de/pub/nj_bscw.cgi/d8022008/Callon_ SociologyTranslation.pdf

Cook, Michael. "The International Standard for the Description of Archives: A Progress Report," Information Development 8 no. 4 (January 1, 1992): 237-38.

Denzin, Norman K. and Yvonna A. Lincoln's Handbook of Qualitative Research $4^{\text {th }} \mathrm{ed}$. (Thousand Oaks, CA: Sage, 2011). 
Duff, Wendy and Kent Haworth. "The Reclamation of Archival Description: The Canadian Perspective," Archivaria 31 (1990-91): 26-35.

Farrell, Joseph and Garth Saloner. "Coordination through Committees and Markets," The RAND Journal of Economics 19 no. 2 (1988).

Farrell, Joseph and Garth Saloner. "Installed Base and Compatibility: Innovation, Product Preannouncements, and Predation," The American Economic Review 76 no. 5 (1986).

Farrell, Joseph and Garth Saloner. "Standardization, Compatibility, and Innovation," The RAND Journal of Economics 16 no. 1 (1985).

Farrell, Joseph and Garth Saloner. "Converters, Compatibility, and the Control of Interfaces," The Journal of Industrial Economics 40 no. 1 (1992).

Feng, Patrick. "Studying Standardization: Review of the Literature." Proceedings of the Standardization and Innovation in Information Technology (SIIT2003) Conference, IEEE, 2003.

Fomin, Vladislav and Keil Thomas. "Standardization: Bridging the Gap between Economic and Social Theory," in Proceedings of the Twenty-first International Conference on Information Systems (Brisbane, Queensland, Australia: Association for Information Systems, 2000).

Hammersley, Martyn and Paul Anthony Atkinson. Ethnography: Principles in Practice $3^{\text {rd }}$. ed. (London: Routledge, 2007).

Hensen, Steven. "The First Shall Be First: APPM and Its Impact on American Archival Description," Archivaria 35 (1993): 64-70.

Kling, Rob. "Learning About Information Technologies and Social Change: The Contribution of Social Informatics," The Information Society 16 no. 3 (2000).

Latour, Bruno. Science in Action: How to Follow Scientists and Engineers through Society (Cambridge, MA: Harvard University Press, 1987).

Matutesa, Carmen and Pierre Regibeau. "A Selective Review of the Economics of Standardization Entry Deterrence, Technological Progress and International Competition," European Journal of Political Economy 12 (1996).

Rogers, Everett M. Diffusion of Innovations $5^{\text {th }}$ ed. (New York: The Free Press, 2003).

Schensul, Stephen and Jean Schensul. Essential Ethnographic Method: Observation, Interview, and Questionnaires (Lanham, MD: AltaMira Press, 1999).

Shepherd, Elizabeth and Rachael Pringle. "Mapping Descriptive Standards across Domains: A Comparison of ISAD $(G)$ and Spectrum," Journal of the Society of Archivists 23 no. 1 (2002): 17-34.

Shepherd, Elizabeth and Charlotte Smith. "The Application of $\operatorname{ISAD}(\mathrm{G})$ to the Description of Archival Datasets," Journal of the Society of Archivists 21 (2000): 55-86.

Sillitoe, Paul. "ISAD $(\mathrm{G})$ - the Guiding Light? Issues in the Use of ISAD $(\mathrm{G})$ in the Preparation of a Repository Guide." In Scottish Universities Special Collections and Archives Group (SUSCAG) conference, Making The Most Of Automation: A Seminar On Users' Requirements From IT Applications In Archives And Special Collections, British Academy, 1996.

Smith, Dorothy E. Institutional Ethnography: Sociology for People (Lanham, MD: Gender Lens Series, AltaMira Press, 1995).

Tatnall, Arthur and Anthony Gilding, "Actor-Network Theory and Information Systems Research" (paper presented at the 10th Australasian Conference on Information Systems, Melbourne, Australia, 1999).

Timmermans, Stefan and Marc Berg. "Standardization in Action: Achieving Local Universality through Medical Protocols,” Social Studies of Science 27 no. 2 (1997). 
Van House, Nancy. "Actor-Network Theory, Knowledge Work, and Digital Libraries" (Berkeley, CA: the UC Committee on Research under their Research Bridging Grant Program, 1999-2001).

Walch, Victoria Irons for the Working Group on Standards for Archival Description with contributions by Marion Matters. Standards for Archival Description: A Handbook (Chicago: SAA, 1994).

Weitzel, Tim. Economics of Standards in Information Networks (Frankfurt, Germany: Physica-Verlag, 2004).

Youn, Eunha. "Archival Traditions in Korean History: From Medieval Practice to the Contemporary Public Records Management Act," Archival Science 23 (2013): 23-44. 


\title{
BIBLIOMETRIC ANALYSIS AS A TOOL IN UNDERSTANDING THE DEVELOPMENT OF ARCHIVAL THOUGHT
}

\author{
Kimberly Anderson
}

\begin{abstract}
Bibliometric analysis, the systematic statistical analysis of publications, is a well-established method in information studies. It is usually employed to examine citation patterns and to identify significant publications. However, bibliometric analysis can also be employed to develop an understanding of social and geographical influences on the development of theory and thought. In a field like archival studies where mentoring and practica are a large part of learning, person-to-person relationships may be more significant than publication-to-publication relationships. This chapter describes a method in which bibliometric analysis is expanded to include the analysis of allusion, informal acknowledgement, interpersonal relationships, and shared geographical and institutional connections between authors and their influences. The author draws from her research on the social aspects of appraisal learning to demonstrate the strengths and limitations of the method and to provide a case study of this method in action.
\end{abstract}

\section{Introduction to Bibliometrics ${ }^{1}$}

Archival studies, like the broader information studies domain, has particular frameworks and objects of study, yet finds itself abutting many other fields. Archival studies scholars and practitioners know what the central concerns of

1 The author is grateful to the editors for their encouragement and wishes to thank Dietmar Wolfram for his guidance on relevant literature. 
the field are (e.g., archival evidence, recordkeeping practices, recordkeeping systems, the characteristics of records, the records-memory nexus, etc.) but the exact boundaries are disputed. As an academic field archival studies has been closely associated with the disciplines of history and law among others. Understanding where these dividing lines occur is challenging in part because the field is still finding its place in the broader academic landscape. In order to claim a place, fields must be able to delineate themselves from one another. These borders can be understood through examination of the shape of the field and its growth - where ideas come from, how they are normalised, what influences are at work, and so on.

Bibliometrics can provide a clearer understanding of the history of both the profession and the scholarly field when combined with other methods. Bibliometric analysis also reveals both the emergence and diffusion of new ideas. In cases where the assertions seem to be obvious bibliometric data can provide evidence in support of this "common sense." The ability to construct comparable data sets is one of the strengths of the method. In order to generate comparable data, practitioners of the bibliometric method must follow its basic rules. Provided the rules are consistent, these data sets can be stacked with one another over time to identify patterns that support assertions about the field. Bibliometric methods are not commonly used in archival studies, so the chapter explains the theoretical framing, basic concepts, rules, and applications of bibliometrics and its utility for the field.

\section{What is Bibliometrics?}

"Bibliometrics" refers to a body of mathematical and statistical methods that examine the structure of publications with largely consistent form, such as most scholarly communication. ${ }^{2}$ Bibliometrics is commonly used to understand the structural features of publications for application in information retrieval, assessment of scholarly impact, discovery of social networks, and the discovery of new subfields or scholarly insights. Jean Tague-Sutcliffe explains it as "the study of the quantitative aspects of the production, dissemination, and use of recorded information. It develops mathematical models and measures for these processes and then uses the models and measures for prediction and decision making." ${ }^{3}$ Typical structural aspects

2 Dorothy H. Hertzel, "Bibliometric Research: History," Encyclopedia of Library and Information Sciences, Taylor \& Francis, December 9, 2009. http://www.tandfonline.com/ doi/abs/10.1081/E-ELIS3-120009034.

3 Jean Tague-Sutcliffe, "An introduction to informetrics,." Information Processing \& Management 28 no. 1 (1992): 1. 
include the order of authors, outgoing citations (who and what the work being analysed cites), incoming citations (who and what cite the work being analysed), length, and type of publication. For academics, bibliometric analysis is frequently used by university administration to assess performance in research. Certain bibliometric calculations (discussed in "Bibliometric Laws, Algorithms, and Applications") are used to quantify the quality of research.

Bibliometrics belongs within the family of methods known as informetrics. Informetrics refers to the mathematical and statistical evaluation of patterns "that show up not only in publications, but also in many aspects of life, as long as the patterns deal with information." ${ }^{\prime \prime}$ The other common, but less broad, information "-metrics" are scientometrics and webometrics. Scientometrics is the study of the process of scientific knowledge production through application of quantitative methods with the goal of making scientific activity more efficient. ${ }^{5}$ This kind of study often employs bibliometric methods, but also incorporates other quantitative measures such as comparing funding amounts or incorporating demographics of scientists. ${ }^{6}$ Webometrics is a quantitative measure of aspects of the web such as web site structures, hyperlinks, search engine results, and words in web pages (web page content analysis). ${ }^{7}$ Webometrics is not limited to semantic analysis and can also include study of technology structures and page usage. ${ }^{8}$ It is essentially bibliometrics applied to the web instead of to traditional publications.

The classic example of bibliometrics is Don Swanson's work on Raynaud's Syndrome and fish oil. Using bibliometrics, Swanson found a previously undiscovered connection between the potential use of dietary fish oil and the treatment of Raynaud's Syndrome. ${ }^{9}$ Specifically, the literature on fish oil indicated that it could reduce blood viscosity and platelet aggregability, while the literature on Raynaud's syndrome discussed the disease's association with

4 Virgil Pasquale Diodato, Dictionary of Bibliometrics,. New York: Haworth Press, 1994, p. ix.

5 Yuri V. Granovsky, "Is It Possible to Measure Science? V. V. Nalimov's Research in Scientometrics," Scientometrics 52 no. 2 (2001): 134.

6 Diodato, Dictionary of Bibliometrics, p.x.

7 Michael Thelwall, Introduction to Webometrics: Quantitative Web Research for the Social Sciences, San Rafael, CA: Morgan \& Claypool Publishers, 2009, p. 1.

8 Radek Malinsky and Ivan Jelinek, "Improvements of Webometrics by Using Sentiment Analysis for Better Accessibility of the Web," In Current Trends in Web Engineering: 10th International Conference on Web Engineering ICWE 2010 Workshops, Vienna, Austria, July 2010, Revised Selected Papers, Florian Daniel, ed. Vienna: Springer, 2010, p.582.

9 Don R. Swanson, "Fish Oil, Raynaud's Syndrome, and Undiscovered Public Knowledge," Perspectives in Biology and Medicine 30 no. 1 (1986): 7-18. 
high blood viscosity and platelet aggregability. ${ }^{10}$ However, these literatures were disconnected and did not cite one another, and fish oil was not used in the treatment of Raynaud's. Using co-citation analysis, Swanson identified literature on Raynaud's Syndrome and a body of literature on fish oil. Co-citation analysis presumes that a relationship between two documents exists if they are both cited within a third document. ${ }^{11}$ Co-citation analysis in this case revealed a number of connections between literature on fish oil and Raynaud's Syndrome that led to Swanson hypothesising that fish oil might be used in the treatment of Reynaud's Syndrome. Swanson was careful to observe that this hypothesis was already implicit in the literature and he merely made it explicit. ${ }^{12}$ The amazing thing is that Swanson was not a physician, yet his analysis of the literature revealed a possible disease treatment. Swanson's method of using bibliometrics for literature-based discovery has subsequently been used in other studies. ${ }^{13}$

\section{History and Philosophies of Bibliometrics}

The origin of "bibliometrics" is ambiguous as it arises out of practices that were modern forerunners to bibliometric analysis as we know it today. The term itself has been credited both to Paul Otlet (1934) and, more commonly, to Alan Pritchard (1969). ${ }^{14}$ Pritchard offered the term as a replacement to "statistical bibliography," which he argued was ambiguous in its meaning. In his history of bibliometrics, Robert Broadus notes that counting and measuring publications is not a recent endeavour. Drawing on examples from the library of Alexandria, an 1837 comparison of the top libraries in the world, and an 1881 study of medical literature, Broadus argues that bibliometrics already had "a respectable history" by the time Pritchard was associated with the term. ${ }^{15}$

10 Swanson, “Fish Oil," p. 7-8.

11 Nicola De Bellis, Bibliometrics and Citation Analysis: From the Science Citation Index to Cybermetrics. Lanham, MD: Scarecrow Press, 2009, p. 158.

12 Swanson, "Fish Oil," 13.

13 See Kenneth A. Cory, "Discovering Hidden Analogies in an Online Humanities Database," Computers and the Humanities 31 no. 1 (1997): 1-12; Roy Davies, "The Creation of New Knowledge by Information Retrieval and Classification," Journal of Documentation 45 (December, 1989): 273-301; and Swanson's other study - Don R. Swanson, "Migraine and Magnesium: Eleven Neglected Connections," Perspectives in Biology and Medicine 31 (Summer, 1988): 526-557.

14 William W. Hood and Concepción S. Wilson, "The Literature of Bibliometrics, Scientometrics, and Informetrics," Scientometrics 52 no. 2 (2001): 292-293.

15 Robert N. Broadus, "Early Approaches to Bibliometrics." Journal of the American Society for Information Science 38 no. 2 (1987): 129. 
At its heart bibliometric analysis is an expression of a quantitative lens for viewing information and human interactions. It relies on reducing the complexity of human interaction to a mathematical (i.e., simple) model. De Ballis' textbook on bibliometrics explains:

Classic bibliometric distributions ... provide a general framework in which the discouraging individuality of documentation processes is reduced to manageable sets of mathematical functions useful to 1) replace inexact empirical formulations with exact mathematical concepts so as to enhance the mutual transparency and comparability of competing models (just a first humble step toward a yet-to-be-developed "grand bibliometric theory"); 2) specify the conditions of applicability of standard statistical tools to the analysis of specific datasets, thereby helping estimate random errors in the measurement of information flows; and 3) connect the mathematical structure of bibliometric processes with that of extra-bibliometric phenomena, such as the patterns emerging in the study of economically and biologically complex systems, so as to help clarify problems having common characteristics and promote the development of common methodologies for their resolution. ${ }^{16}$

Bibliometrics is a product of the knowledge society in which the rise of information dissemination and the production of scientific knowledge become important management concerns for nation-states. As a theoretical construct, bibliometrics frames the world of scholarly discourse in terms of quantitative characterisation, thus enabling nations to numerically measure their scientific output. Specifically, it sets forth probabilistically true ideas about the "express patterns, tendencies, and regularities that are said to be inherent in the phenomena." ${ }^{17}$ The most common criticism of bibliometrics is that quantification (as in citation analysis) does not equate to meaning. While it is the case that each citation is evidence of the act of citing, the quantity or dearth of citations to a particular scholar or that scholar's work may bear no correlation to a positive relationship between the citer and the cited. It is possible, therefore, to determine that something has been cited, but it is not always possible via bibliometrics to determine why the work was selected.

Bibliometric indicators are now one of the common measures of scholarly output. This reliance on the quantification of a scholar's work is not without criticism:

16 De Ballis, Bibliometrics, 76.

17 Alvin M. Schrader, “Teaching Bibliometrics,” Library Trends 30 no. 1 (1981): 151. 
Concerns surround how such interest from researchers, scientific policymakers, and businesses involved in commissioning research threatens the integrity of the field (Glänzel, 1996; Glänzel \& Schoepflin, 1994). Such concerns are as follows: the misuse of bibliometric research results; disregard for bibliometric consensus on standards; and the inaccurate dissemination of concepts, results, and methods outside of the bibliometric field. ${ }^{18}$

Paul Wouters, writing from a history of science and technology perspective, adds the argument that the act and meaning of citing itself has changed since the development of the Science Citation Index and it is now enmeshed in what he terms the "Citation Culture." ${ }^{19}$ Wouters' argument is not only that citing behaviour (the meaning behind a citation) is idiosyncratic to each citer and scholarly field, but also that citation analysis is not actually objective - the citation counts are not a product of the authors but of the indexers who created the index. ${ }^{20} \mathrm{~A}$ further complication is the presence of self-citations, which might artificially inflate the degree of influence properly attributed to an author or a work.

Citations are a social gesture - an intentional public connection between authors as demonstrated by the citing author. ${ }^{21}$ These connections can happen for many reasons. Some of these reasons do show the positive influence of another work: the author wants to demonstrate knowledge of the literature of the field and so cites a "classic" work; the author wants to give credit to another author for his/her idea or contribution to the field; an author self-cites because the author's own work is the most appropriate source. Sometimes something is cited for a negative reason, such as when the citing author is critical of the cited work. There are also ethically dubious reasons: selfciting to increase citations counts, or an author cites a work published in the same journal because the journal editor requires or encourages it to increase the journal's impact factor. There are many other reasons something might be cited, but as a rule a citation does not necessarily indicate the scholarly

18 Koen Jonkers and Gemma E. Derrick, "The Bibliometric Bandwagon:

Characteristics of Bibliometric Articles Outside the Field Literature," Journal of the American Society for Information Science and Technology 63 no. 4 (2012): 829-836. doi:10.1002/asi.22620.

19 Paul Wouters, “The Citation Culture," Ph.D. thesis, Universiteit van Amsterdam, 1999.

20 Wouters, "Citation Culture," 8.

21 Kimberly D. Anderson, "Appraisal Learning Networks: How University Archivists Learn to Appraise Through Social Interaction," Ph.D. dissertation, University of California, Los Angeles, 2011, p. 98. 
quality of the cited work. This is the primary reason why the application of bibliometrics (specifically, impact factor and citation analysis) has been controversial in measuring scholarly output.

Regardless of the meaning of the citation in regards to the quality of the cited work, the citation can be used to determine the "presence" of the work and/or author within the particular field or subfield. The citing author is always aware of the cited work even if that awareness is only superficial. If "awareness" is considered to be a kind of influence, then it is entirely possible to use citation analysis to measure the social presence or general influence an author or work has in a limited domain. For this heuristic, the kind of influence (good or bad) may be unimportant.

\section{Bibliometric Laws, Algorithms, and Applications}

There are two main kinds of bibliometrics: descriptive and evaluative. Descriptive bibliometrics looks at the social, geographic, and temporal features of publications. Evaluative bibliometrics looks at the productivity and quality, as measured by citations, of individual authors and publication venues, such as journals. Bibliometrics applied in this way is frequently used to evaluate scientific output. ${ }^{22}$ Research performance can be evaluated through conducting a citation count, a method called citation analysis, which is then used to formulate an individual author's scholarly productivity as well as each journal's impact factor.

\section{Impact Factor, Eigenfactor, and h-Index}

Eugene Garfield introduced the concept of an impact factor in 1955 as a result of his work indexing scientific literature using punch cards. ${ }^{23}$ Garfield and Irving H. Sher developed the Journal Impact Factor in the early 1960s while they were selecting journals for the Science Citation Index. They needed to include the most important journals, but didn't want to rely solely on citation or publication counts since those metrics would exclude small but significant journals. ${ }^{24}$ The Journal Impact Factor quantifies the significance of a particu-

22 Thed van Leeuwen, "Descriptive Versus Evaluative Bibliometrics," In Handbook of Quantitative Science and Technology Research, Henk F. Moed, Wolfgang Glänzel, and Ulrich Schmoch, eds., Springer Netherlands, 2005, p. 374.

23 Eugene Garfield, “The History and Meaning of the Journal Impact Factor," JAMA 295 no. 1 (January 4, 2006): 90-93.

24 Eugene Garfield, “Journal Impact Factor: A Brief Review,” Canadian Medical Association Journal 161 no. 8 (October 19, 1999): 979-980. 
lar journal based on the frequency of citations for an average article within the journal. ${ }^{25}$

The Eigenfactor ${ }^{T M}$ metric is based on network ranking schemes (eigenvector centrality methods) in which "journals are considered to be influential if they are cited often by other influential journals." ${ }^{26}$ It was developed by Carl Bergstrom, Jevin West, and Marc Wiseman as an alternative to the journal impact factor. ${ }^{27}$

The Hirsch Citation Index (b-index) is a number that represents the numerically highest combination of citations and publications in which the numbers are equal to each other. It serves as an index to measure a person's scientific output against other's output. Hirsch developed the $\mathrm{h}$-index as an alternative to other bibliometric indices that had methodological problems due to heterogeneous productivity, impact, and count of citations. ${ }^{28}$ It works as follows:

Person A (h-index of 1): One publication with zero citations, one publication with five citations, and one publication with one citation.

Person A cannot have an h-index of five because this person does not have five publications that were each cited five or more times.

Person B (h-index of 2): two publications with zero citations, one publication with five citations, and three publications with two citations.

Person B has an h-index of 2 because the largest combination in which the papers and citations to those papers are equal to each other is two.

\section{Power-Law Distributions in Bibliometrics}

Power-Laws are probability distributions in which one element has an exponential relationship to another element. So, if there is a change in the first

25 Richard Van Noorden, "Metrics: A Profusion of Measures." Nature 465 no. 7300 (2010): 864-866.

26 University of Washington, "Methods," eigenFACTOR.org, 2012. http://www. eigenfactor.org/methods.php.

27 Carl T. Bergstrom, Jevin D. West, and Marc A. Wiseman, "The Eigenfactor ${ }^{\mathrm{TM}}$ Metrics," The Journal of Neuroscience 28 no. 45 (November 5, 2008): 11433-11434.

28 Jorge E. Hirsch, "An Index to Quantify an Individual's Scientific Research Output," Proceedings of the National Academy of Sciences of the United States of America 102, no. 46 (November 15, 2005): 16569-16572. 
element, then the second element will change proportionally. When plotted normally, these relationships have a long or "heavy tail." When plotted on logarithmic scales, these relationships appear as a straight line. ${ }^{29}$ In bibliometrics the data elements are usually the following pairs:

- citations for journal : citations to all journals in data set

- citations to author : citations to all authors in data set

- citations to article : citations to all articles in data set

Three power-law distributions are regularly used in bibliometric analysis: Lotka's Law, Zipf's Law, and Bradford's Law.

\section{Lotka's Law ${ }^{30}$}

Lotka's law is a power law concerning authors. In brief, it is the idea that a small number of authors account for the majority of publications. ${ }^{31}$

Figure 27.1: Sample Lotka Distribution Based on Hypothetical Authors and Articles

\section{LOTKA DISTRIBUTION}

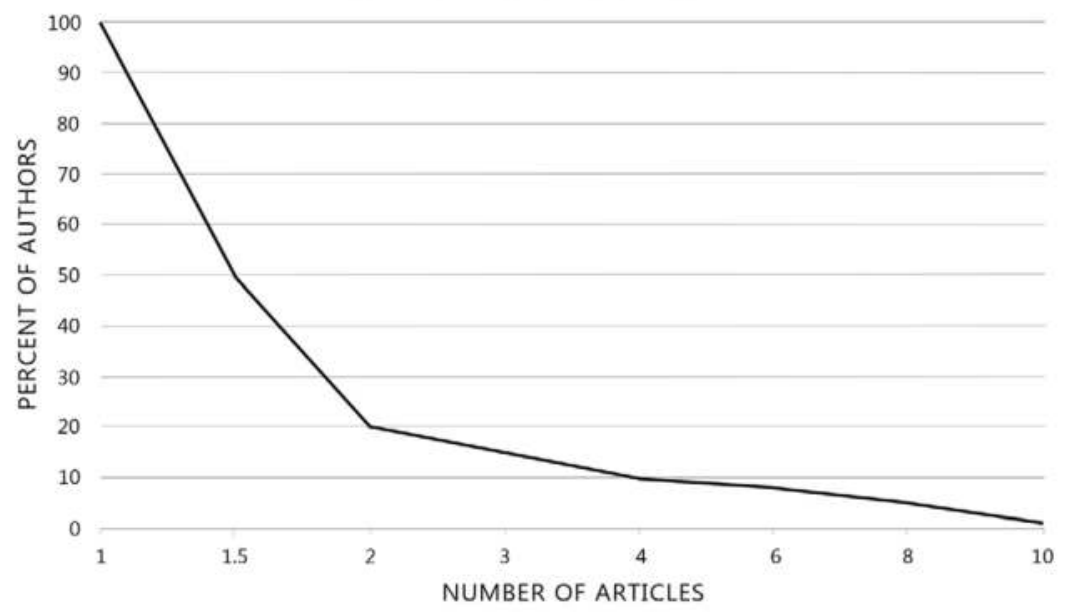

29 Mark A. J. Newman, "Power Laws, Pareto Distributions and Zipf's Law," Contemporary Physics 46 no. 5 (2005): 323-351.

30 The canonical text is Alfred J. Lotka, "The Frequency Distribution of Scientific Productivity," Journal of the Washington Academy of Sciences 16 no. 12 (1926): 317-324.

31 Christine L. Borgman and Jonathan Furner, "Scholarly Communication and Bibliometrics," Annual Review of Information Science and Technology 36 (2002): 3-72, p. 26. 


\section{Bradford's Law ${ }^{32}$}

Bradford's Law is a power law concerning journals within a subject domain. It posits that literature in a particular subject will scatter across journals using a distribution of $1: n: n^{2}$. A single journal is assigned to the top zone as being the journal with the most relevant literature to the subject. A second zone has much of the relevant literature, but the third zone consists of the bulk of the journals that have little relevant material. ${ }^{33}$

Figure 27.2: Bradford Distribution Showing the Three Journal Zones

\section{BRADFORD DISTRIBUTION}

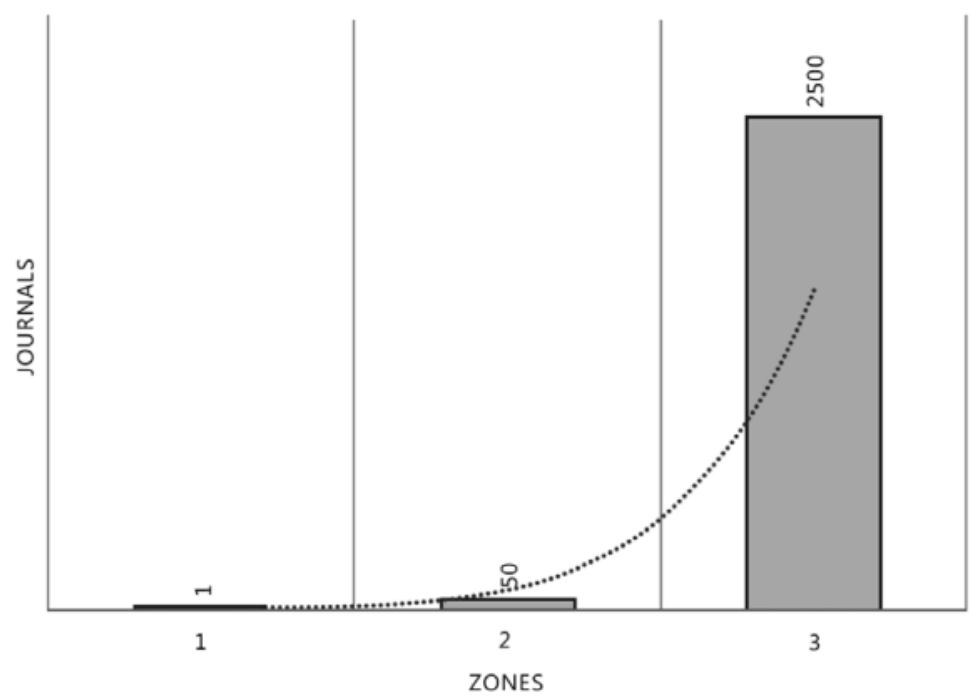

\section{Zipf's Law}

Zipf's law is a power law concerning words. In brief, it is the phenomenon that a few words appear very frequently while others occur rarely. ${ }^{34}$

32 The canonical text is Samuel C. Bradford, "Sources of Information on Specific Subjects," Engineering 137 no. 3550 (1934): 85-86.

33 Ye-Sho Chen and Ferdinand F. Leimkuhler, "A Relationship Between Lotka's Law, Bradford's Law, and Zipf's Law," Journal of the American Society for Information Science 37 no. 5 (September 1986): 307-314.

34 Paul E. Black, "Zipf's Law," Dictionary of Algorithms and Data Structures. U.S. National Institute of Standards and Technology, 2009. http://www.nist.gov/dads/HTML/ zipfslaw.html. 
Figure 27.3: Zipf Distribution for the Word Frequencies in this Chapter. Half of the Words in the Chapter are Plotted in the Long Tail to the Right of the Arrow.

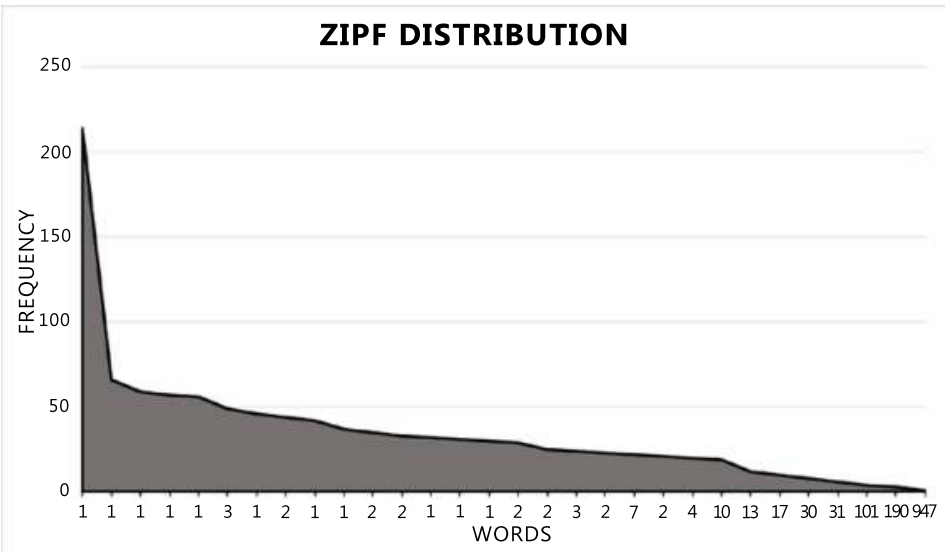

These are the most common metrics and phenomena associated with bibliometric analysis. Others include self-citing rate, immediacy index, percentage not cited, and citing half-life. ${ }^{35}$

\section{Sample Studies}

\section{Classic Citation Analysis: Excellence in Research for Australia (ERA)}

A sample evaluative bibliometric study that uses citation analysis is the Australian Research Council's "Excellence in Research for Australia" (ERA). ${ }^{36}$ This is a complex study with multiple components, one of which is employing citation analysis to identify significant journals. ${ }^{37}$ The first exercise in 2010 used bibliometrics to rank journals as to quality in four tiers, but abandoned the rankings for 2012. ERA 2012 used a comparison tool called the "journal indicator" instead. The journal indicator generates a table

35 Ming-Yueh Tsay, "An Overview of the Journal Impact Indicators," Journal of Educational Media E Library Sciences 49 no. 2 (Winter 2011): 210-214.

36 Australian Research Council, Excellence in Research for Australia (ERA). Commonwealth of Australia, June 12, 2012. http://www.arc.gov.au/era/default.htm.

37 Australian Research Council, ERA 2010 Citation Benchmark Methodology. Excellence in Research for Australia. Commonwealth of Australia, 2010. http://www.arc.gov.au/pdf/ era_2010_citation_benchmark_methods.pdf. 
unique to each unit of evaluation that shows 1) a descending list of journals (by number of articles) in which that unit has published, 2) the percentage of articles contributed to the journal by that unit, and 3) the percentage of total articles from the unit that were published in that journal. ${ }^{38}$ Although what the citations mean in terms of journal quality has changed between 2010 and 2012, the bibliometric methodology of citation analysis remained the same.

The Relative Citation Impact (RCI) was determined for each journal against both world and Australian benchmarks. The RCI is a calculation of the impact factor of a journal in relation to other journals in its field or subfield for a given time frame. So if the average number of citations per journal in a particular subfield for a specific year is 50 and a journal being analysed from that subfield in the same has 100 citations, its RCI would be 2. The RCI is a useful metric because it accounts for differing citation patterns between subfields and disciplines and is therefore an improvement on a straight count. The ERA project was developed to evaluate Australian research impact and quality, so this project measures RCI twice - a domestic $\mathrm{RCI}$ and a world RCI. The domestic RCI limits the pool of comparative journals to those from Australia while the world RCI includes journals from outside the country. This enables the ERA project to assess if a particular journal has a different impact globally than it does domestically.

Scopus was used to tabulate world citations and all citations, including selfcitations, were counted. The window for which citations were examined was 1 January 2003 to 1 March 2010 for ERA 2010. ${ }^{39}$ For ERA 2012 this window was 1 January 2005 to 31 December $2010 .{ }^{40}$ Discipline-specific benchmarks were created to accommodate the different citation and publication styles of each discipline. Journals were compared to the benchmarks created for their discipline rather than using a global benchmark. Multi-disciplinary journals were accommodated by tabulating the percentage of their output in each relative discipline. Journal articles submitted to the project by the authors' institutions could be assigned up to three discipline codes. Submitting institutions were asked to weight the assignments by percentage. So, an article submitted by institution A could be apportioned $40 \%$ to "Public Health and

38 Australian Research Council, ERA 2012 Refinements, Excellence in Research for Australia. Commonwealth of Australia, 7 February 2012. http://www.arc.gov.au/era/ era_2012/archive/faq.htm\#refinements.

39 Australian Research Council, ERA 2010 Citation Benchmark Methodology, 1.

40 Australian Research Council, ERA 2012 Evaluation Handbook, Excellence in Research for Australia. Commonwealth of Australia, 2012, p. 96. http://www.arc.gov.au/pdf/ era12/ERA_2012_Evaluation_Handbook_final_for_web_protected.pdf. 
Sciences" and 60\% to "Clinical Services." If the article had authors at another institution, the other institution could also submit the article with its own apportionment scheme. ${ }^{41}$ In addition, benchmarks were created for each year and each journal was compared to other journals within the year so as to avoid bias through the natural accumulation of citations over time.

The specific calculations used to assess each journal's ranking were as follows: ${ }^{42}$

\section{Citations per paper (CPP):}

$$
c p p=\frac{\text { sum of cites for all eligible papers }}{\text { total number of eligible papers }}
$$

Centile thresholds: a calculation of the raw citation counts required for each journal to be in the top $1,5,10,25$, and $50^{\text {th }}$ percentiles of the set.

\section{Relative Citation Impact (RCI) to be calculated for both Australia and the world:}

Part 1 - Calculate raw RCI for each article

$$
\begin{gathered}
\mathrm{RCI}(\text { world })=\frac{{\text { number of } \text { citations }_{\text {article } x}}_{\text {world } \mathrm{cpp}}}{\mathrm{RCI}(\text { Australia) }=} \\
\text { Aumber of } \text { citations }_{\text {article } x} \\
\text { Australia cpp }
\end{gathered}
$$

Part 2 - Apply apportionment for specific discipline area

Apportioned RCI(World)=RCI(World)*apportionment

Apportioned RCI(Australia)=RCI(Australia)*apportionment

Part 3 -Average the calculations in Part 2 to obtain average RCI

$$
\text { Average RCI }=\frac{\left(\frac{\text { number of } \text { citations }_{\text {article } x}}{c p p}\right) * \text { apportionment }}{\text { total apportioned count of articles }}
$$

41 Australian Research Council, ERA 2012 Evaluation Handbook. 6.

42 Australian Research Council, ERA 2012 Evaluation Handbook, 8. 
After calculating the average $\mathrm{RCI}$, the project then grouped articles into quality classes ranging from 0 impact to an $\mathrm{RCI}$ of over 8 .

This project has been successful in identifying the bibliometric features of Australian scholarship and journals. The Australian Research Council has used the results to assess research performance and has identified universities producing at or above the world standard. The 2012 National Summary indicates that there are "more researchers, more outputs, and more outputs per researcher" than in 2010 study. ${ }^{43}$ This, combined with an increase in "quality and diversity" is the foundation of Science and Research Minister Chris Evans' 2012 claim that the government's investment in research is working. ${ }^{44}$ However, not everyone agrees with Evans' claim. The National Tertiary Education Union, the national union for staff involved in college and university education, has created a website called "ERAWatch" that is focused on identifying how ERA impacts academic work. ${ }^{45}$ Among their concerns are that ERA's exclusion of "non-traditional outputs" and intense focus on journal articles will disadvantage particular disciplines and kinds of research, that it introduces or intensifies managerialist practices in regards to research performance," and uncertainty about how ERA will be leveraged in the academic workplace particularly in regards to evaluation and funding. ${ }^{46}$

From a purely bibliometric methods perspective, the ERA project is very important for archival studies scholarship as it is the only freely available source to identify impact for a comprehensive array of archival studies journals. The following table shows the array of journals specific to archival studies that are indexed in at least one of Scopus, Web of Science, or ERA. Journals were found by searching the title lists for "archiv"” and "record"." Journals that were not specific to archival studies were excluded.

43 Australian Research Council, 2012 Outcomes: National Overview, Excellence in Research for Australia. Commonwealth of Australia, 2012, p. 1. http://www.arc.gov. au/pdf/era12/NATIONAL.pdf.

44 Senator the Hon. Chris Evans, Minister for Tertiary Education, Skills, Science and Research, Record Investment Lifts University Research Rankings. Media Release, 6 December 2012. http://minister.innovation.gov.au/chrisevans/MediaReleases/ Pages/Recordinvestmentliftsuniversityresearchrankings.aspx.

45 National Tertiary Education Union, ERAWatch, 2013. http://www.erawatch.org.au/.

46 Jen Tsen Kwok, Impact of ERA Research Assessment on University Behaviour and Their Staff (South Melbourne, VIC: National Tertiary Education Union, 2013): 5, 18, 19. http://www.nteu.org.au/library/view/id/3800. 
Table 27.1: Archival journal indexing

\begin{tabular}{|c|c|c|c|}
\hline Journals & SCOPUS* & ERA 2012\# & Web of Science $\dagger$ \\
\hline $\begin{array}{l}\text { African Journal of Library Archives } \\
\text { and Information Science }\end{array}$ & 2008-ongoing & - & SSCI \\
\hline American Archivist & $\begin{array}{l}\text { 1971, 1974, 1991, } \\
\text { 1994, 1996, 2004, } \\
\text { 2007, } \\
\text { 2010-ongoing }\end{array}$ & $2005-2012$ & - \\
\hline Archival Informatics Newsletter & $1987-988$ & inactive & - \\
\hline Archival Issues & - & $2005-2012$ & - \\
\hline Archival Science & 2001-ongoing & $2005-2012$ & - \\
\hline Archivaria & $\begin{array}{l}\text { 1980, 1989-1990, } \\
1996-2009\end{array}$ & $2005-2012$ & - \\
\hline $\begin{array}{l}\text { Archives: Journal of the British } \\
\text { Records Association }\end{array}$ & - & $2005-2012$ & - \\
\hline Archives and Manuscripts & - & $2005-2012$ & - \\
\hline Archives and Museum Informatics & 1989-1995, 2001 & inactive & - \\
\hline Journal of Archival Organization & - & $2005-2012$ & - \\
\hline Journal of the Society of Archivists & $\begin{array}{l}\text { 1974, 1985, } \\
\text { 1996-ongoing }\end{array}$ & $2005-2012$ & AHCI \\
\hline Library and Archival Security & 2006-ongoing & $2005-2012$ & - \\
\hline $\begin{array}{l}\text { Prologue: Quarterly Journal of the } \\
\text { National Archives (USA) }\end{array}$ & $\begin{array}{l}\text { 1977, 1980, 1982, } \\
\text { 1987, 2000, } \\
\text { 2006-ongoing }\end{array}$ & $2005-2012$ & AHCI \\
\hline $\begin{array}{l}\text { Provenance: The journal of Public } \\
\text { Record Office Victoria }\end{array}$ & - & $2005-2012$ & - \\
\hline $\begin{array}{l}\text { Restaurator: International Journal } \\
\text { for the Preservation of Library and } \\
\text { Archival Material }\end{array}$ & 1988-ongoing & $2005-2012$ & SSCI \\
\hline $\begin{array}{l}\text { Watermark: Newsletter of Archivists } \\
\text { and Librarians in the History of the } \\
\text { Health Sciences }\end{array}$ & $\begin{array}{l}\text { 1908-1910, } \\
\text { 1982-1983, } \\
\text { 1995-1999, } \\
\text { 2004-ongoing }\end{array}$ & - & - \\
\hline
\end{tabular}

* Elsevier, Scopus Title List. September 2013. http://www.elsevier.com/online-tools/ scopus/content-overview.

\# Australian Research Council, ER A 2012 Journal List, 14 November 2012. http:// www.arc.gov.au/xls/era12/ERA2012JournalList.xlsx.

† Thomson-Reuters, "Master Journal List.” Web of Science, 13 April 2012. http:// science.thomsonreuters.com/cgi-bin/jrnlst. 


\section{Expanded Citation and Acknowledgement Analysis: Appraisal Learning Networks}

I conducted an analysis of appraisal literature published in The American Archivist and Archivaria between 1995 and 2009. ${ }^{47}$ The goal of this analysis was to expose formal information flows and relationships in appraisal thought and dissemination. Using simple counting as my main measure, I tracked the number of times a "social other" was gestured towards (e.g., cited, acknowledged, or thanked). ${ }^{48}$ The resultant numbers were used to assess, within a limited domain, the social presence of those receiving attribution.

My interest in bibliometrics as a method for archival studies research arose organically out of a pilot study I conducted in 2008 in which I interviewed archivists about their experiences learning how to conduct appraisal - the process of evaluating materials for their archival value. In this study, four of the eight participants volunteered that they relied on reading professional literature and used specific works to guide their practice. Furthermore, the participants also expressed personal admiration for some of the authors of these works. In many cases the authors were personally known to the interviewees as a result of their participation in professional activities and organisations. The archival world is relatively small, and the world of those who are professionally engaged is even smaller. Consequently, there is overlap and bridging between authors and readers that occurs outside of what is evident by analysing publications. In this setting, bibliometrics becomes just another way to study social interactions between a relatively small set of people.

Professions rely heavily on mentoring and apprenticeship models. Mentoring frequently occurs in even the smallest social units - those involving only two people. These dyads might include student and teacher, student and supervisor, employee and employee, employee and supervisor, and so on. In my bibliometric research I have seen all of these dyads in play via acknowledgements within the publications themselves. Bibliometric analysis provides a non-intrusive way to trace the significance of these types of relationships. Furthermore, no other records of such interpersonal connections may exist. We cannot assume, for example, that two people working together feel that they have helped or influenced each other. However, when a formal acknowledgment is written into a publication, we can take

47 Anderson, "Appraisal Learning Networks." I used a total of five methods for this research: bibliometrics, qualitative interviews, survey, social network analysis, and a modified form of participatory research.

"Social Other" is used to refer to other people, social groups, or their works. 
this as evidence that the awareness of the other person is significant enough to warrant formal recognition.

\section{Method}

One of the great advantages of using quantitative methods such as citation analysis and bibliometrics is that they yield data that are easily comparable to previous data sets. My research on appraisal learning through social interaction made use of a previous study conducted by Anne Gilliland-Swetland covering the years 1972 to 1994, thus enabling me to observe changes in citation behaviour over time. ${ }^{49}$ This type of longitudinal analysis is more difficult with qualitative and/or non-statistical studies where variables cannot be re-manipulated. These two studies have somewhat differing goals and so the methodology was changed slightly. Gilliland-Swetland's primary goal was to use citation analysis as a means to identify experts within a domain. Once experts were identified from the literature, she then used a snowball sample to identify additional experts (see more detailed discussion in Gilliland's chapter in this volume). ${ }^{50}$ Her 1995 work built on the method she developed for her previous 1992 study exploring the corpus of literature on archival automation and electronic records. ${ }^{51}$

While both studies address appraisal and engage with the issue of expertise, the focus of my study was influence rather than expertise - positing that a person can be influential without being expert. In my study I combined bibliometric analysis (citation analysis in addition to counting allusion, acknowledgment and explicit thanks) with feedback from "lay experts" people who appraise and select within university archives settings but who may or may not have published in this area. These experts commented on the bibliometric findings and indicated whether or not they had heard of the author or work and would recommend the author or work to a colleague. Their perception of significance and concomitant recommendations served as a measure of how well bibliometric analysis predicts influential people and texts in practice.

49 Anne Gilliland-Swetland, "Development of an Expert Assistant for Archival Appraisal of Electronic Communications: an Exploratory Study," Ph.D. dissertation, University of Michigan, 1995.

50 Gilliland-Swetland, Development of an Expert Assistant, 65.

51 Anne J. Gilliland-Swetland, "Archivy and the Computer: A Citation Analysis of North American Archival Periodical Literature,” Archival Issues 17 no. 2 (1992): 95-112. 


\section{Study Design}

Both studies used similar parameters. Gilliland-Swetland based her selection on Richard Cox's 1987 assessment of American archival literature and I based mine on the Gilliland-Swetland study. ${ }^{52}$ I began the project by attempting to analyse all of the kinds of journals included in the Gilliland-Swetland study, i.e., those published by major North American archival associations, and some that had begun publication right at the end of the time period she worked with. I started the study by examining four journals: SAA Nerwsletter, Archival Outlook, Archival Science, and Provenance. Out of the twenty-five issues I initially examined, fifteen qualifying articles were found which were later excluded from my final analysis when I abandoned the inclusion of these journals in the dataset (See Table 2).

Table 27.2: Publications analysed

\begin{tabular}{|l|l|}
\hline Gilliland-Swetland, 1972-1994* & Anderson, 1995-2009\# \\
\hline American Archivist & American Archivist \\
\hline Archival Issues & Archivaria \\
\hline Archivaria & \\
\hline Provenance & \\
\hline Public Historian & \\
\hline Monographs published in North America & \\
\hline
\end{tabular}

* Gilliland-Swetland, "Development of an Expert Assistant," 67.

\# Anderson, "Appraisal Learning Networks," 99.

After beginning the work, I found that this scope was too broad for my intent to use the bibliometric analysis as a single component of a multimethod study. In particular, I sought to get study participants' opinions on the bibliometric findings and so wanted to focus on material that was likely to be familiar to the participants. I selected The American Archivist and Archivaria as the two journals most likely to be read by North American practitioners since they are published by the two largest archival professional societies in the region: the Society of American Archivists and the Association of Canadian Archivists respectively. Consequently I imposed limitations on the materials analysed.

52 Richard J. Cox, "American Archival Literature: Expanding Horizons and Continuing Needs, 1901-1987." The American Archivist 50 no. 3 (1987): 306-323 as cited in Gilliland-Swetland, 1995, p. 67. 
Each study identified conditions that must be met before a work would be analysed for the study. These were largely kept the same to enable comparison between the two studies. My study had more inclusive parameters, however, because I also wanted to identify publications in which appraisal was a major component regardless of the focus of the work.

Table 27.3: Criteria for bibliometric analysis

\begin{tabular}{|c|c|c|}
\hline & Gilliland-Swetland's Criteria (1995)* & Anderson's Criteria (2011)\# \\
\hline Journals & $\begin{array}{l}\text { (apprais* OR select* OR } \\
\text { "documentation strategy" OR } \\
\text { "documentation plan*") must appear at } \\
\text { least three times in the abstract, title, } \\
\text { first paragraph or last paragraph }\end{array}$ & $\begin{array}{l}\text { (apprais* OR select* OR } \\
\text { "documentation strategy" OR } \\
\text { "documentation plan" OR } \\
\text { "collecting") must appear at } \\
\text { least two times in the abstract, } \\
\text { title, first paragraph or last } \\
\text { paragraph }\end{array}$ \\
\hline Monographs & $\begin{array}{l}\text { [ "apprais*” OR “sampl*” OR } \\
\text { ("select*" AND “archiv"”), } \\
\text { (("valuation" OR "assessment") AND } \\
\text { "archives") OR “documentation } \\
\text { strategy" OR "documentation plan*”] } \\
\text { appear in either the OCLC title } \\
\text { statement, the index terms assigned } \\
\text { by Library of Congress Subject } \\
\text { Headings (LCSH), or both }\end{array}$ & N/A \\
\hline
\end{tabular}

* Gilliland-Swetland, "Development of an Expert Assistant," 68.

\# Anderson, "Appraisal Learning Networks," 100. The study only required two appearances of these words, but the text incorrectly states that the threshold was three appearances.

\section{The Kinds of Counting}

Each article (including review articles) in The American Archivist and Archivaria between 1995 and 2009 was examined for the criteria I had established in advance (see Table 3). If the article qualified it was reviewed for the following:

1. Traditional citations

2. Non-traditional references to work

3. Acknowledgements

Traditional citations are formal attributions of credit to another work. A sub-category was created for each of the following kinds of citations: 
footnote/endnote, bibliography, and appearance in the works cited or references list. Non-traditional citations are those that involve allusion only, quotation only (usually in the form of epigrams), or an in-text mention without a formal citation. Allusions were primarily passing references to some canonical literature or the author's own previous work.

Acknowledgements were further broken down into: colleague/co-presenter, exemplum, granting agency, professor of citing author, recognition of work (assumed positive), recognition of work (negative), research subject/ interviewee, and direct thank you. These categories developed as I analysed the data. I began the study assuming that "acknowledgement" was a sufficient degree of granularity but rapidly found out that this was not the case. This study did not measure how heavily each source was used within one of the analysed articles, so only one traditional or non-traditional citation was counted for each unidirectional pairing. However, each different kind of role a person plays in relationship to a given work and its author was counted as an instance of that kind of acknowledgement. For example, Terry Cook received acknowledgement twice in Eric Ketelaar's "Archivistics Research Saving the Profession" - once as a general recognition of his work and once explicitly as a co-presenter or colleague. ${ }^{53}$

This kind of bibliometric analysis requires familiarity with the literature being indexed $-4.4 \%$ of the total social gestures would be missed if the researcher were unable to pick up on allusions within the field (see Table 4).

\section{Analysing the Data}

Once the data was gathered it was analysed using simple descriptive statistics with the goal of identifying the human and document features of appraisal scholarship. The study was managed through a Microsoft Access database. Tables were created for the article to be analysed, the works cited, authors, publishers, publications, institutions, citations, and acknowledgements. Working with a research assistant, ${ }^{54}$ we populated the database and conferred to clarify the study parameters as the data emerged. We had to set the following rules as our work progressed (listed below in the order in which we developed them):

53 Eric Ketelaar, "Archivistics Research Saving the Profession," The American Archivist 63 no. 2 (2000): 322-340.

54 Amy Croft. 
1. "Author" is used to refer to the person responsible for the work.

2. Ignore intermediary works. If a citation took the form of "AAAA as cited in BBBB [book chapter] in CCCCC [book]" or "AAAA as cited in $\mathrm{BBBB}$ [article/book]" only AAAA was tracked as that was the item of most interest to the citing author.

3. Analyse review articles, but not book reviews.

4. When citing conference presentations, treat the conference as the publication.

5. For single author texts, the citation is counted towards the book as a whole rather than an individual chapter.

6. Translators were not counted as authors.

7. Authorship of websites is attributed to either the organisation or project associated with the website.

8. Do not analyse meeting minutes.

9. For interviews, both interview and interviewee count as author.

10. The "thank you" acknowledgement is used for direct thank-yous, not just a recognition that was generally present in the text.

11. "Recognition of Work" is a non-specific mention of a person's contributions. The person is recognised as influential or significant, but this type of recognition does not necessarily imply endorsement.

We also discovered a flaw in our inclusion method. Sometimes the word stems (see Table 3) would appear only because the author was comparing his or her subject to, or differentiating it from, appraisal and selection. This was the case for Tyler Walters' 1995 work on archival preservation in which the word stems were observed three times - enough to qualify under both the Anderson and Gilliland-Swetland criteria. However, Walters mentions appraisal to distinguish it from his subject of preservation in the last paragraph of his essay: "Preservation is not an isolated function, like acquisition or reference, nor is it a skill with a restricted domain, like conducting appraisal or administering access. ${ }^{\text {55 }}$ Another anomaly was Patricia Galloway's 2006

55 Tyler O. Walters, "Thinking About Archival Preservation in the '90s and Beyond: Some Recent Publications and Their Implications for Archivists." The American Archivist 58 no. 4 (1995): 476-493. 
work on Dunbar Rowland. ${ }^{56}$ This article met the threshold via a sentence in the last paragraph: "Hence collecting proverbially dusty noncurrent records will always be easier than collecting records that are in demand." ${ }^{\prime 7}$ In this case, Galloway's discussion of Dunbar Rowland and his contributions to the State Archives of Mississippi is relevant to a study of archival appraisal literature. However, this one article cited Dunbar Rowland as an author 21 times, which dramatically skewed the results so that Rowland was tied with Luciana Duranti for fourth place in the list of most-cited authors in my study. ${ }^{58}$ The Walters example can be avoided in other studies in which the articles are analysed by hand if a subjective judgment of relevance is used by the researcher prior to inclusion in the data set. The Galloway example is trickier. However, in this particular case Rowland was only cited in Galloway's article so her article could be treated as an anomaly and not used in the data set.

\section{Findings}

There are two general kinds of findings from this study - those pertaining to works, and those pertaining to people. Findings about the works themselves arise solely from citation analysis. Findings about people arise from the combination of citation analysis and broader bibliometrics that also measured acknowledgements, allusion, etc. Findings were discovered by running queries through Access and then performing simple calculations on the resultant counts. I ended up analysing 76 articles, which produced 2703 citations to 2538 individual works. Each article cited between 0 and 130 works, with 36 citations as the average. ${ }^{59}$ In contrast, Gilliland-Swetland's study identified 93 works (articles from the 5 journals and applicable monographs) that each cited an average of 18 works. ${ }^{60}$ All but one (Lewison - "Archival Sampling" in Table 5) of the top articles identified in the Gilliland-Swetland study were still being cited in my study. Additionally, two new articles published since 1994 also received more than 10 citations. Also of significance

56 Patricia Galloway, "Archives, Power, and History: Dunbar Rowland and the Beginning of the State Archives of Mississippi (1902-1936)." The American Archivist 69 no. 1 (2006): 79-116.

57 Galloway, "Archives, Power, and History," 116.

58 Anderson, “Appraisal Learning Networks," 152.

59 Anderson, "Appraisal Learning Networks," 145. The original text contains a mathematical error.

60 Gilliland-Swetland, "Development of an Expert Assistant," 84. 
is that Gilliland-Swetland's study included three more journals than did my study, yet the most-cited articles are similar between the two studies. Six of the 15 articles receiving a total of 10 or more citations in sum between the two studies also met this threshold independently in both studies. These six articles have remained amongst the most important appraisal articles for the past 37 years (see Tables 4, 5, and 6).

Findings about people are enhanced by the inclusion of acknowledgement analysis. The most common kind of acknowledgment was the reference to a person's entire body of work. If this type of acknowledgement does not take the form of a formal citation, then the acknowledgment is missed in typical citation analysis. In my study, "recognition of work" acknowledgements accounted for 260 discrete connections, or $53.7 \%$ of the total acknowledgements and $12.2 \%$ of the total (3181) citations. In traditional citation analysis, informal recognition of work would not count as a citation, nor would any of the other forms of acknowledgement measured. Had I used traditional citation analysis alone I would have missed 484 acknowledgements and 106 non-traditional citations accounting for $16.1 \%$ of the total (3659) social gestures (see table 27.7)

Table 27.4. Comparison of publications receiving 10 or more citations in Gilliland-Swetland and Anderson studies

\begin{tabular}{|c|c|c|c|c|c|}
\hline $\begin{array}{l}\text { Total } \\
\text { Citations }\end{array}$ & $\begin{array}{l}\text { Change } \\
1995 \mathrm{v} \text {. } \\
2011\end{array}$ & Author & Title & $\begin{array}{l}\text { Gilliland- } \\
\text { Swetland } \\
1995\end{array}$ & $\begin{array}{l}\text { Anderson } \\
2011\end{array}$ \\
\hline 27 & $\uparrow$ & Jenkinson & $\begin{array}{l}\text { A Manual of Archives } \\
\text { Administration, 1922, 1937, } 1966 .\end{array}$ & 12 & 15 \\
\hline 27 & $\downarrow$ & Schellenberg & $\begin{array}{l}\text { Modern Archives: Principles } \\
\text { EO Techniques. Chicago, Ill.: } \\
\text { University of Chicago Press, } 1956 .\end{array}$ & 24 & 3 \\
\hline 25 & $\uparrow$ & Ham & $\begin{array}{l}\text { "The Archival Edge," The } \\
\text { American Archivist 38, no. } 1 \\
\text { (1975): 5-13. }\end{array}$ & 12 & 13 \\
\hline 24 & $\downarrow$ & Schellenberg & $\begin{array}{l}\text { The Appraisal of Modern Public } \\
\text { Records, Bulletins of the National } \\
\text { Archives (Washington: U.S. } \\
\text { Govt. Print. Off., 1956). }\end{array}$ & 14 & 10 \\
\hline $21+$ & $\uparrow$ & Cook & $\begin{array}{l}\text { "Mind Over Matter: Towards } \\
\text { a New Theory of Archival } \\
\text { Appraisal." In The Archival } \\
\text { Imagination: Essays in Honour } \\
\text { of Hugh A. Taylor, edited by } \\
\text { Barbara Craig, 38-70. Ottawa: } \\
\text { Association of Canadian } \\
\text { Archivists, } 1992 .\end{array}$ & $*$ & 21 \\
\hline
\end{tabular}




\begin{tabular}{|c|c|c|c|c|c|}
\hline $\begin{array}{l}\text { Total } \\
\text { Citations }\end{array}$ & $\begin{array}{l}\text { Change } \\
1995 \mathrm{v} \text {. } \\
2011\end{array}$ & Author & Title & $\begin{array}{l}\text { Gilliland- } \\
\text { Swetland } \\
1995\end{array}$ & $\begin{array}{l}\text { Anderson } \\
2011\end{array}$ \\
\hline $20+$ & $\uparrow$ & Samuels & $\begin{array}{l}\text { Varsity Letters: Documenting } \\
\text { Modern Colleges and Universities. } \\
\text { Lanham, MD: The Scarecrow } \\
\text { Press, Inc., } 1992 .\end{array}$ & $*$ & 20 \\
\hline $18+$ & $\uparrow$ & Booms & $\begin{array}{l}\text { "Society and the Formation of a } \\
\text { Documentary Heritage: Issues } \\
\text { in the Appraisal of Archival } \\
\text { Sources." Archivaria } 24 \text { (Summer } \\
\text { 1987): 69-107. }\end{array}$ & $*$ & 18 \\
\hline 16 & $\downarrow$ & Bauer & $\begin{array}{l}\text { "The Appraisal of Current and } \\
\text { Recent Records," The National } \\
\text { Archives Staff Information Circulars, } \\
13 \text { (June 1946): 1-25. }\end{array}$ & 12 & 4 \\
\hline 15 & $\downarrow$ & $\begin{array}{l}\text { Haas, } \\
\text { Samuels \& } \\
\text { Simmons }\end{array}$ & $\begin{array}{l}\text { Appraising the Records of Modern } \\
\text { Science and Technology: A Guide. } \\
\text { Cambridge, Mass.: Massachusetts } \\
\text { Institute of Technology, } 1985 .\end{array}$ & 10 & 5 \\
\hline 14 & $\downarrow$ & Rapport & $\begin{array}{l}\text { "No Grandfather Clause: } \\
\text { Reappraising Accessioned } \\
\text { Records," American Archivist 44, } \\
\text { no. } 2 \text { (1981): 143-150. }\end{array}$ & 11 & 3 \\
\hline 14 & $\uparrow$ & Samuels & $\begin{array}{l}\text { "Who Controls the Past," } \\
\text { American Arcbivist } 49 \text { (Spring } \\
\text { 1986): 109-124. }\end{array}$ & unranked & 14 \\
\hline 14 & $\downarrow$ & Steinwall & $\begin{array}{l}\text { "Appraisal and the FBI Files Case: } \\
\text { For Whom Do Archivists Retain } \\
\text { Records?" American Archivist 49, } \\
\text { no. } 1 \text { (Winter 1986): } 52-63 .^{\dagger}\end{array}$ & 12 & 2 \\
\hline 13 & $\downarrow$ & Lewison & $\begin{array}{l}\text { "Archival Sampling," American } \\
\text { Archivist } 20 \text { (Fall 1957): 291-312. }\end{array}$ & 13 & 0 \\
\hline 12 & NA & Duranti & $\begin{array}{l}\text { "The Concept of Appraisal and } \\
\text { Archival Theory," American } \\
\text { Archivist 57, no. } 2 \text { (Spring 1994): } \\
\text { 328-345. }\end{array}$ & - & 12 \\
\hline 11 & NA & Brown & $\begin{array}{l}\text { "Macro Appraisal Theory and } \\
\text { Context of the Public Records } \\
\text { Creator," Archivaria } 40 \text { (Fall 1995): } \\
\text { 121-170. }\end{array}$ & - & 11 \\
\hline
\end{tabular}

* Indicates a publication that was recommended by the experts interviewed in Gilliland-Swetland's study, but was not otherwise quantified.

$\dagger$ This is listed in Gilliland-Swetland as "Hammet, Hobson, and Hindus. 'FBI Case Files Appraisal,' American Archivist (1980).” 
Table 27.5. Top ranked publications in Gilliland-Swetland Study ${ }^{\star}$

\begin{tabular}{|l|l|l|l|}
\hline Rank & Works Cited & Author & Citations \\
\hline 1 & Modern Archives: Principals and Techniques (1956) & Schellenberg & 24 \\
\hline 2 & The Appraisal of Modern Public Records (1956) & Schellenberg & 14 \\
\hline 3 & Archives \& Manuscripts: Appraisal and Accessioning (1977) & Brichford & 13 \\
\hline 3 & Archival Sampling (1956) & Lewison & 13 \\
\hline 4 & Appraisal of Current and Recent Records (1946) & Bauer & 12 \\
\hline 4 & "The Archival Edge" (1975) & Ham & 12 \\
\hline 4 & $\begin{array}{l}\text { "Appraisal and the FBI Files Case: For Whom Do Archivists } \\
\text { Retain Records?" (1986) † }\end{array}$ & Steinwall & 12 \\
\hline 4 & Manual of Archives Administration (1922, 1937, 1966) & Jenkinson & 12 \\
\hline 5 & "No Grandfather Clause" (1982) & Rapport & 11 \\
\hline 6 & Appraising the Records of Modern Science and Technology (1985) & $\begin{array}{l}\text { Haas, Samuels, } \\
\text { Simmons }\end{array}$ & 10 \\
\hline
\end{tabular}

* Gilliland-Swetland, "Development of an Expert Assistant," 91.

† This is listed in Gilliland-Swetland as "Hammet, Hobson, and Hindus. 'FBI Case Files Appraisal,' American Archivist (1980).”

Table 27.6. Top Ranked Publications in Anderson Study

\begin{tabular}{|c|c|c|c|c|c|}
\hline \multicolumn{3}{|l|}{ Rank } & \multirow[t]{2}{*}{ Works Cited } & \multirow[t]{2}{*}{ Author } & \multirow{2}{*}{$\begin{array}{l}\text { Total } \\
\text { Citations }\end{array}$} \\
\hline Overall & $\begin{array}{l}\text { American } \\
\text { Archivist }\end{array}$ & Archivaria & & & \\
\hline 1 & 3 & 1 & $\begin{array}{l}\text { "Mind over Matter: Towards } \\
\text { a New Theory of Archival } \\
\text { Appraisal" (1992) }\end{array}$ & Cook & 21 \\
\hline 2 & 1 & 2 & $\begin{array}{l}\text { Varsity Letters: Documenting } \\
\text { Modern Colleges and Universities } \\
\text { (1992) }\end{array}$ & Samuels & 20 \\
\hline 3 & 6 & 1 & $\begin{array}{l}\text { "Society and the Formation of a } \\
\text { Documentary Heritage: Issues in } \\
\text { the Appraisal of Archival Sources" } \\
\text { (1987) }\end{array}$ & Booms & 18 \\
\hline 4 & 2 & 3 & $\begin{array}{l}\text { A Manual of Archive } \\
\text { Administration, 2nd ed. (1937) }\end{array}$ & Jenkinson & 15 \\
\hline 5 & 4 & 3 & "The Archival Edge" (1975) & Ham & 13 \\
\hline 5 & 4 & 3 & "Who Controls the Past?" (1986) & Samuels & 13 \\
\hline 6 & 3 & 5 & $\begin{array}{l}\text { "The Concept of Appraisal and } \\
\text { Archival Theory" (1994) }\end{array}$ & Duranti & 12 \\
\hline 7 & 0 & 2 & $\begin{array}{l}\text { "Macro Appraisal Theory and } \\
\text { Context of the Public Records } \\
\text { Creator" (1995) }\end{array}$ & Brown & 11 \\
\hline 7 & 5 & 4 & $\begin{array}{l}\text { Modern Archives: Principles and } \\
\text { Techniques (1956) }\end{array}$ & Schellenberg & 11 \\
\hline 8 & 5 & 5 & $\begin{array}{l}\text { The Appraisal of Modern Public } \\
\text { Records (1956) }\end{array}$ & Schellenberg & 10 \\
\hline
\end{tabular}


Table 27.7. Count of citation and acknowledgment types in Anderson study

\begin{tabular}{|c|c|c|c|}
\hline & Kind of Social Gesture & Count & Total (3659) \\
\hline \multirow{3}{*}{ 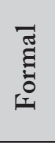 } & Bibliography & 16 & \\
\hline & Footnote/endnote & 2575 & 2591 \\
\hline & Works Cited/References & 0 & \\
\hline \multirow{3}{*}{ 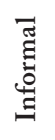 } & Allusion & 14 & \multirow{3}{*}{106} \\
\hline & In-text mention only & 65 & \\
\hline & Quotation only/epigram & 9 & \\
\hline \multirow{9}{*}{ 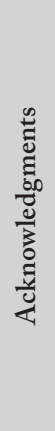 } & Colleague/Co-Presenter & 90 & \multirow{9}{*}{484} \\
\hline & Exemplum & 8 & \\
\hline & Granting Agency & 8 & \\
\hline & Mention of body of work (negative) & 2 & \\
\hline & Professor citing author & 6 & \\
\hline & Quotation & 6 & \\
\hline & Recognition of Work & 260 & \\
\hline & Research Subject/Interviewee & 8 & \\
\hline & Thank You & 96 & \\
\hline
\end{tabular}

Access to longitudinal data enabled me to observe shifts in influence over time. ${ }^{61}$ In my study the five ranks for most-cited individuals were (in order from most to least cited): Terry Cook, Richard Cox, David Bearman, Luciana Duranti, Dunbar Rowland, and W. Kaye Lamb. GillilandSwetland's top five ranks were held by (in order from most to least cited): Theodore Schellenberg, Helen Samuels, Maynard Brichford, Terry Cook, M. H. Fishbein, F. Gerald Ham, and David Bearman. David Bearman and Terry Cook both reached higher ranks in the 2011 study while Richard Cox went from rank 8 in 1995 to rank 2 in 2011. Although there also seems to be greater exchange and influence between Canada and the US in the later study, it is not possible to isolate "country" as a variable because GillilandSwetland's study involved more publications than Anderson's and did not differentiate between citations in American and Canadian journals (see tables 8 and 9). 
Table 27.8. Most-cited people in Gilliland-Swetland study

\begin{tabular}{|l|l|l|c|}
\hline Rank & Name (Last, First) & Country & Citations \\
\hline 1 & Schellenberg, T. & USA & 41 \\
\hline 2 & Samuels, H. & USA & 37 \\
\hline 3 & Brichford, M. & USA & 30 \\
\hline 4 & Cook, T. & CAN & 28 \\
\hline 4 & Fishbein, M. & USA & 28 \\
\hline 4 & Ham, F. G. & USA & 28 \\
\hline 5 & Bearman, D. & USA/CAN & 23 \\
\hline
\end{tabular}

Table 27.9. Most-cited people in Anderson study

\begin{tabular}{|l|l|l|c|c|c|}
\hline & & & \multicolumn{2}{|c|}{ Citations } & \\
\hline Rank & Name (Last, First) & Country & Amer. Arch. & Archivaria & TOTAL \\
\hline 1 & Cook, T. & CAN & 22 & 56 & 78 \\
\hline 2 & Cox, R. & USA & 34 & 19 & 53 \\
\hline 3 & Bearman, D. & USA/CAN & 23 & 18 & 41 \\
\hline 4 & Duranti, L. & CAN & 10 & 21 & 31 \\
\hline 5 & Eastwood, T. & CAN & 12 & 14 & 26 \\
\hline 5 & Samuels, H. & USA & 13 & 13 & 26 \\
\hline
\end{tabular}

The list of most acknowledged people is significantly different from the list of people most-cited. The top five ranks for most-acknowledged individuals were (in order from most to least acknowledged): Terry Cook, Helen Samuels, Theodore Schellenberg, Richard Cox, Luciana Duranti, Terry Eastwood, Verne Harris, Margaret Hedstrom, and Heather MacNeil. Only three names appear on both lists in my study (Cook, Cox, and Duranti). I think it is significant that all three people have been senior faculty members in North American graduate archival education programs, prolific scholars, and former practitioners - thus having several different areas of professional influence. However, the field is small and appraisal experts are an even smaller subset, so it may be that experts in a small sub-field are likely to overlap in their roles. Further analysis is needed to see if gender is a significant factor in the appearance of more women in the most acknowledged list than in the most cited list. 
Table 27.10. Most-acknowledged people in Anderson study

\begin{tabular}{|l|l|l|c|c|c|l|}
\hline & & & \multicolumn{3}{|c|}{ Acknowledgements } & \\
\hline Rank & Name (Last, First) & Country & Amer. Arch. & Archiv. & Total & $\%$ \\
\hline 1 & Cook, Terry & CAN & 8 & 20 & 28 & 5.8 \\
\hline 2 & Samuels, Helen & USA & 5 & 6 & 11 & 2.3 \\
\hline 3 & Schellenberg, Theodore & USA & 4 & 6 & 10 & 2.1 \\
\hline 4 & Cox, Richard J. & USA & 4 & 3 & 7 & 1.4 \\
\hline 5 & Duranti, Luciana & CAN & 2 & 4 & 6 & 1.2 \\
\hline 5 & Eastwood, Terry & CAN & 3 & 3 & 6 & 1.2 \\
\hline 5 & Harris, Verne & ZAF & 2 & 4 & 6 & 1.2 \\
\hline 5 & Hedstrom, Margaret & USA & 3 & 3 & 6 & 1.2 \\
\hline 5 & MacNeil, Heather & CAN & 1 & 5 & 6 & 1.2 \\
\hline
\end{tabular}

The major bibliometric analyses have been done with large sets of publications and authors (e.g., Swanson's Raynaud's and fish oil finding). Archival studies is too small a field for that type of analysis to be worthwhile. However, "altmetrics" (alternatives to biblio- and the other traditional metrics) such as acknowledgement analysis, when combined with qualitative social science and humanities methods, can produce a more fine-grained picture of the networks of scholarship and practice.

The final stage in my bibliometric research was seeking commentary on the most cited authors and publications from study participants who had already completed an interview and a survey with me. ${ }^{62} \mathrm{I}$ wanted to get a sense of whether influence in the world of publications would correspond to practitioners' experiences of influential people and publications. There was no direct correlation in that U.S. authors were more readily recognised by the participants and a majority of them stated that they would only recommend three of the works be read by others in the field..$^{63} \mathrm{I}$ made a, perhaps erroneous, assumption that information flows (and accordingly influence) happened across national borders within archival studies. My basis for this assumption is that Canadian and American practices do

62 Anderson, “Appraisal Learning Networks," 174-179.

63 These works were: Helen Willa Samuels, Varsity Letters: Documenting Modern Colleges and Universities. Lanham, MD: The Scarecrow Press, Inc., 1992; F. Gerald Ham, Selecting and Appraising Archives and Manuscripts, Archival Fundamentals Series. Chicago Ill.: Society of American Archivists, 1993; and Lewis Bellardo and the Society of American Archivists. A Glossary for Archivists, Manuscript Curators, and Records Managers Chicago: Society of American Archivists, 1992. 
influence each other in the literature. Therefore, I surmised, American practitioners would be aware of major people and works in Canada. Within my very small sample this was generally not the case. Surprisingly, the works that the practitioners would recommend to others were all nearly 20 years old at the time of my study. I believe these results may be due to the heterogeneity of the participating archivists' graduate and continuing education experiences. While too few archivists participated for these findings to have statistical merit, combining the bibliometric analysis with feedback from practicing archivists enabled some differences to emerge; namely, that there is possibly a formal influence network (publications and the people cited or acknowledged in them), and an informal influence network (people directly interacting in social groups).

\section{Conclusion}

My study benefitted greatly from comparison to Gilliland-Swetland's study. Although we had somewhat different goals, I was able to use her findings and methodology to inform my own thus creating an analysis that extends over 37 years. Bibliometric analysis relies heavily on comparable data sets something we do not have much of in archival studies scholarship, which often favours qualitative methods and surveys over other quantitative or mixed methods. The exposure of the resulting data in table form as well as detailed notes on formulas and constraints used in gathering and analysing the data enable this type of comparison. Bibliometrics is still a method that has been rarely used in archival studies but it can be very powerful if it is applied in full recognition of its limitations and the dangers of comparing apples and oranges. Bibliometrics can give us a sense of how a field is changing over decades. It can also support the identification of canonical pieces and of archival "elders" who have influenced generations of practitioners and scholars alike. While these aspects can be studied through humanistic and qualitative methodologies, the advantage that bibliometrics brings as a method is that it circumvents the constraints of working with people. Because it consists of textual analysis, it can be done at any time and to any degree of granularity without fatiguing or inconveniencing research participants. It is not bound by any particular social network so the researcher doesn't have to have an "in" to begin work. The data can be easily manipulated again in future studies or combined with other methods. It is also highly replicable and verifiable. 
Bibliometrics does have its limitations. As a quantitative method, bibliometrics has the disadvantages of all quantitative studies. In short - data is easily counted and analysed, but its meaning isn't clear. A traditional citation indicates nothing more than that the author is aware of the other work to some unspecified degree. The acknowledgements used in my study are a clearer indication of positive influence but even they are limited. Did the author thank Person A for various "political" reasons, but thanked Person B because of genuine appreciation for Person B's assistance? It is impossible to tell this through bibliometric analysis. However, the limitations of bibliometrics can be ameliorated through combining or triangulating the method with qualitative and/or humanistic textual studies. Meaning must be brought to bear on the bibliometric data through qualitative means. Pairings that would work especially well with bibliometrics are literature reviews (a qualitative assessment of a domain by an expert) and interviews with readers and/or authors (qualitative assessments of impact, intent, and the politics of citation).

Elsewhere in information studies, bibliometrics is used in close alliance with information retrieval. It can be used to test and design information retrieval systems as well as quantify scholarly output. As linked data usage and studies of digital archives become more prevalent within the field, bibliometrics and indeed webometrics may be similarly applied in archival studies. Webometrics may assist archives in gaining a clearer and unobtrusive picture of how forms of archival description (finding aids, catalogue entries, blogs, etc.) are actually used. Bibliometrics may assist in measuring the spread (i.e., the influence in a basic sense) of archival materials. Archivists currently rely on researchers or, in organisational archives, administrative users to provide archivists with feedback on how the materials have been used or repurposed. In the meantime, the method outlined here works well for understanding relationship structures and flows of influence amongst archivists and archival studies scholars. By including all the non-traditional citation counts and acknowledgements, it is possible to provide one measure of just how much of the field's intellectual work is shaped by individual relationships and human mediation rather than sole dependence on texts. 


\section{References}

Anderson, Kimberly D. “Appraisal Learning Networks: How University Archivists Learn to Appraise Through Social Interaction." Ph.D. dissertation, University of California, Los Angeles, 2011.

Australian Research Council. 2012 Outcomes: National Overview. Excellence in Research for Australia. Commonwealth of Australia, 2012. http://www.arc.gov.au/pdf/era12/ NATIONAL.pdf.

Australian Research Council. ERA 2010 Citation Benchmark Methodology. Excellence in Research for Australia. Commonwealth of Australia, 2010. http://www.arc.gov.au/ pdf/era_2010_citation_benchmark_methods.pdf.

Australian Research Council. ERA 2012 Evaluation Handbook. Excellence in Research for Australia. Commonwealth of Australia, 2012. http://www.arc.gov.au/pdf/era12/ ERA_2012_Evaluation_Handbook_final_for_web_protected.pdf.

Australian Research Council. ERA 2012 Refinements. Excellence in Research for Australia. Commonwealth of Australia, 7 February 2012. http://www.arc.gov.au/ era/era_2012/archive/faq.htm\#refinements.

Australian Research Council. ERA 2012 Journal List. 14 November 2012. http://www. arc.gov.au/xls/era12/ERA2012JournalList.xlsx.

Australian Research Council. Excellence in Research for Australia (ERA). Commonwealth of Australia, June 12, 2012. http://www.arc.gov.au/era/default.htm.

Bellardo, Lewis and the Society of American Archivists. A Glossary for Archivists, Manuscript Curators, and Records Managers. Chicago: Society of American Archivists, 1992.

Bergstrom, Carl T., Jevin D. West, and Marc A. Wiseman. "The Eigenfactor ${ }^{\mathrm{TM}}$ Metrics." The Journal of Neuroscience 28 no. 45 (November 5, 2008): 1143311434.

Black, Paul E. “Zipf's Law.” Dictionary of Algorithms and Data Structures. U.S. National Institute of Standards and Technology, 2009. http://www.nist.gov/dads/HTML/ zipfslaw.html.

Borgman, Christine L. and Jonathan Furner. "Scholarly Communication and Bibliometrics.” Annual Review of Information Science and Technology 36 (2002): 3-72.

Bradford, Samuel C. "Sources of Information on Specific Subjects," Engineering 137, no. 3550 (1934): 85-86.

Broadus, Robert N. "Early Approaches to Bibliometrics." Journal of the American Society for Information Science 38, no. 2 (1987): 127-129.

Chen, Ye-Sho and Ferdinand F. Leimkuhler. "A Relationship between Lotka's Law, Bradford's Law, and Zipf's Law." Journal of the American Society for Information Science 37 no. 5 (September 1986): 307-314.

Cory, Kenneth A. "Discovering Hidden Analogies in an Online Humanities Database." Computers and the Humanities 31 no. 1 (1997): 1-12.

Cox, Richard J. "American Archival Literature: Expanding Horizons and Continuing Needs, 1901-1987." American Archivist 50 no. 3 (1987): 306-323.

Davies, Roy. "The Creation of New Knowledge by Information Retrieval and Classification." Journal of Documentation 45 (December, 1989).

De Bellis, Nicola. Bibliometrics and Citation Analysis: From the Science Citation Index to Cybermetrics. Lanham, MD: Scarecrow Press, 2009.

Diodato, Virgil Pasquale. Dictionary of Bibliometrics. New York: Haworth Press, 1994.

Elsevier. Scopus Title List. September 2013. http://www.elsevier.com/online-tools/scopus/ content-overview. 
Evans, Senator the Hon. Chris, Minister for Tertiary Education, Skills, Science and Research. Record Investment Lifts University Research Rankings. Media Release, 6 December 2012. http://minister.innovation.gov.au/chrisevans/MediaReleases/ Pages/Recordinvestmentliftsuniversityresearchrankings.aspx.

Galloway, Patricia. "Archives, Power, and History: Dunbar Rowland and the Beginning of the State Archives of Mississippi (1902-1936).” The American Archivist 69 no. 1 (2006): 79-116.

Garfield, Eugene. “Journal Impact Factor: A Brief Review.” Canadian Medical Association Journal 161 no. 8 (October 19, 1999): 979-980.

Garfield, Eugene. “The History and Meaning of the Journal Impact Factor.” JAMA 295 no. 1 (January 4, 2006): 90-93.

Gilliland-Swetland, Anne J. "Archivy and the Computer: A Citation Analysis of North American Archival Periodical Literature.” Archival Issues 17 no. 2 (1992): 95-112.

Gilliland-Swetland, Anne. "Development of an Expert Assistant for Archival Appraisal of Electronic Communications: an Exploratory Study". Ph.D. dissertation, University of Michigan, 1995.

Granovsky, Yuri V. "Is It Possible to Measure Science? V. V. Nalimov's Research in Scientometrics." Scientometrics 52 no. 2 (2001): 127-150.

Ham, F. Gerald. Selecting and Appraising Archives and Manuscripts, Archival Fundamentals Series. Chicago IL: Society of American Archivists, 1993.

Hertzel, Dorothy H. "Bibliometric Research: History." Encyclopedia of Library and Information Sciences. Taylor \& Francis, December 9, 2009. http://www.tandfonline. com/doi/abs/10.1081/E-ELIS3-120009034.

Hirsch, Jorge E. "An Index to Quantify an Individual's Scientific Research Output." Proceedings of the National Academy of Sciences of the United States of America 102 no. 46 (November 15, 2005): 16569-16572.

Hood, William W. and Concepción S. Wilson. "The Literature of Bibliometrics, Scientometrics, and Informetrics." Scientometrics 52 no. 2 (2001): 291-314.

Jonkers, Koen and Gemma E. Derrick. "The Bibliometric Bandwagon: Characteristics of Bibliometric Articles outside the Field Literature." Journal of the American Society for Information Science and Technology 63 no. 4 (2012): 829-836. doi:10.1002/asi.22620

Ketelaar, Eric. "Archivistics Research Saving the Profession." The American Archivist 63 no. 2 (2000): 322-340.

Kwok, Jen Tsen. Impact of ERA Research Assessment on University Behaviour and Their Staff. South Melbourne, VIC: National Tertiary Education Union, 2013. http:// www.nteu.org.au/library/view/id/3800.

Lotka, Alfred J. "The Frequency Distribution of Scientific Productivity." Journal of the Washington Academy of Sciences 16 no. 12 (1926): 317-324.

Malinksy, Radek and Ivan Jelinek. "Improvements of Webometrics by Using Sentiment Analysis for Better Accessibility of the Web." In Current Trends in Web Engineering: 10th International Conference on Web Engineering ICWE 2010 Workshops, Vienna, Austria, July 2010, Revised Selected Papers. Florian Daniel, ed. Vienna: Springer, 2010.

National Tertiary Education Union. ERAWatch. 2013. http://www.erawatch.org.au/.

Newman, Mark A. J. "Power Laws, Pareto Distributions and Zipf's Law." Contemporary Physics 46 no. 5 (2005): 323-351.

Samuels, Helen Willa. Varsity Letters: Documenting Modern Colleges and Universities. Lanham, MD: The Scarecrow Press, Inc., 1992.

Schrader, Alvin M. "Teaching Bibliometrics.” Library Trends 30 no. 1 (1981): 151-172.

Swanson, Don R. "Fish Oil, Raynaud's Syndrome, and Undiscovered Public Knowledge." Perspectives in Biology and Medicine 30 no. 1 (1986): 7-18. 


\section{Chapter 27}

Swanson, Don R. "Migraine and Magnesium: Eleven Neglected Connections." Perspectives in Biology and Medicine 31 (Summer, 1988): 526-557.

Tague-Sutcliffe, Jean. "An introduction to Informetrics." Information Processing E Management 28 no. 1 (1992): 1-3.

Thelwall, Michael. Introduction to Webometrics: Quantitative Web Research for the Social Sciences. San Rafael, CA: Morgan \& Claypool Publishers, 2009.

Thomson-Reuters. “Master Journal List.”Web of Science. 13 April 2012. http://science. thomsonreuters.com/cgi-bin/jrnlst.

Tsay, Ming-Yueh. "An Overview of the Journal Impact Indicators." Journal of Educational Media E' Library Sciences 49 no. 2 (Winter 2011): 210-214.

University of Washington. "Methods." eigenFACTOR.org, 2012. http://www.eigenfactor. org/methods.php.

van Leeuwen, Thed. "Descriptive Versus Evaluative Bibliometrics." In Handbook of Quantitative Science and Technology Research. Henk F. Moed, Wolfgang Glänzel, and Ulrich Schmoch, eds. Springer Netherlands, 2005.

van Noorden, Richard. "Metrics: A Profusion of Measures.” Nature 465 no. 7300 (2010): 864-866.

Walters, Tyler O. "Thinking About Archival Preservation in the'90s and Beyond: Some Recent Publications and Their Implications for Archivists." The American Archivist 58 no. 4 (1995): 476-493.

Wouters, Paul. “The Citation Culture.” Ph.D. thesis, Universiteit van Amsterdam, 1999. 
Chapter 28

\title{
PUBLIC DIGITAL RECORDS MANAGEMENT IN THE UGANDAN PUBLIC SERVICE
}

\section{A Methodological Approach}

\author{
David Luyombya
}

Abstract: Governments worldwide are concerned about the proper management of digital records. Over the last three decades the Ugandan government has been implementing a wide range of reforms cutting across the entire public service with a focus on improving service delivery. ${ }^{1}$ Hence, the Ugandan public service (UPS) adopted information and communications technology (ICT) to revolutionise the way government operates and to enhance relationships between the government and citizens, between the government and the business community and between its departments. Developments in ICT have significantly changed the way information is collected, stored, processed, disseminated and used, thus making the acquisition and application of modern technology the basic determinants of modernisation and growth of the UPS. ${ }^{2}$ Through the utilisation of ICT, digital records have been generated; however, until this study there was a lack of detailed research addressing digital records management in Uganda and practical solutions to the problems of digital records management. This chapter presents the methodology adopted for investigating digital records management within the UPS. Existing digital records management systems and methods of managing digital records,

1 Government of Uganda, MoPS, Public Service Reform Programme Strategic Framework (2005/6-2009/10). Kampala: MoPS, 2005.

2 Venansius Baryamureeba, "ICT-enabled Services: A Critical Analysis of the Opportunities and Challenges in Uganda," International Journal of Computing and ICT Research 4 (2008): 215-235. 
ICT infrastructure and human resource capacity were studied, examined and evaluated for their suitability for digital records management. The study adopted a mixed-method research approach involving the use of quantitative and qualitative methods to assess the existing digital records management framework - how it functioned, interlinked or coordinated in managing digital records. The study population included a critical-case sampling of UPS institutions and the use of a multiple-case replication design to collect data from these cases. The technical distinctiveness of digital records management and its ability to yield many more variables necessitated the use of multiple sources of evidence, with data needing to converge in a triangulating fashion. The study used interpretative analysis, analysing quantitative data using SPSS and qualitative data by categorising data into themes. The study conducted document analysis by identifying trends, status and challenges in managing digital records management.

\section{Introduction}

This chapter reports on the research methodology employed in the study of public digital records management in the Ugandan public service (UPS). ${ }^{3}$ This was the first study to assess the extent to which a framework exists in Uganda for the creation and management of digital records and to determine whether digital records management strategies capable of capturing, maintaining and providing access to digital records over time are in place. This chapter describes the overall research design as well as the data collection methods and techniques used in the data analysis. It focuses on a description and justification of the methodological choice made in this study.

Uganda recognised information and communications technology (ICT) as a tool to improve the efficiency and effectiveness of government and its numerous ICT initiatives since the 1990s manifest this. ${ }^{4}$ This recognition led the Government of Uganda (GoU) to adopt an ICT framework in 2003 with the main goal of promoting the development and effective utilisation of ICT. ${ }^{5}$ The vision of the framework is a Uganda where the overall national growth and development is sustainably enhanced, promoted and accelerated by the application and use of ICT. This is an umbrella framework covering a wide range of issues, such as establishing conditions for the growth of

3 David Luyombya, Framework for Effective Public Digital Records Management in Uganda, $\mathrm{PhD}$ thesis, University College London, 2010.

4 David Wafula and Norman Clark, "Science and Governance of Modern Biotechnology in Sub-Saharan Africa - The Case for Uganda”, Journal of International Development (2005): 679-694.

5 Government of Uganda, MoWHC, National Information and Communication Technology Policy, Kampala: MoWHC, 2003. 
digital societies and economies, e-administration and ICT-based services. The purpose of the framework was to provide focused direction on information management, of which records management would be a part. Inevitably, the adoption of this framework was preparing Uganda for the introduction of e-governance. ${ }^{6}$ In pursuing the e-governance strategy, the government aimed at introducing new technologies to facilitate inter- and intra-agency communication and cooperation, and consequently to support widespread use of electronic services. ${ }^{7}$ The overall goal was to promote the development and effective utilisation of ICT such that a quantifiable impact is achieved throughout Uganda. ${ }^{8}$

The adoption of ICT in service delivery, which is in line with the New Public Management (NPM) strategy, has resulted in the creation and use of digital records in the UPS. The increased use of ICT to conduct business and transmit information contributes to the need to manage the resultant records. Without an appropriate supportive framework for adoption and absorption, it can be argued that the UPS will most likely lose significant public records during their capture, storage, retrieval and disposal, a situation which will undermine the country's governance process in the digital era. The above concerns led this study to investigate the readiness of the UPS to manage digital records to support governance.

\section{Background to the Study}

Uganda's economic history has gone through four distinct periods since independence from Britain in 1962. Between 1960 and 1970, real GDP grew at an average rate of $4.8 \%$ and GDP per capita grew at $3 \%$ per annum. ${ }^{9}$ From 1971, the situation changed drastically. The economy experienced the absence of sound macro-economic policies to address the strategic bottlenecks that had constrained Uganda's socio-economic development since independence, including inadequate infrastructure, lack of industrialisation,

6 Richard Heeks, “Information Systems and Developing Countries: Failure, Success and Local Improvisations", Information Technology for Development 7 no.1 (2001): 3-16.

7 Venansius Baryamureeba, "ICT as an Engine for Uganda's Economic Growth: The Role of and Opportunities for Makerere University," International Journal of Computing and ICT Research, 1 no.1 (2007): 1-48.

8 Baryamureeba,"'ICT as an Engine for Uganda's Economic Growth”.

9 Ritva Reinikka and Paul Collier, eds., Uganda's Recovery: The Role of Farms, Firms and Government, Washington, DC: The World Bank, 2001, p.26. 
and an underdeveloped services sector. ${ }^{10}$ The productive sectors were ignored in pursuit of informal trade, as most skilled personnel fled the country to escape the economic mismanagement and civil unrest, in which they were often caught as soft targets. ${ }^{11}$ Weak public sector management and administration was prevalent and standards generally collapsed in all sectors of the economy. Virtually no intellectual or physical control of records existed. ${ }^{12}$

For most of the 1970s and 1980s the country suffered severe macro-economic imbalances, including high rates of inflation and balance of payments deficits, because the growth of nominal aggregate demand consistently outstripped the growth of real supply in the economy. ${ }^{13}$ As a result, the application of ICT to increase administrative efficiency was ignored and the records management function represented a low priority in the UPS. ${ }^{14}$

By 1980, the need to rehabilitate the economy had become obvious. The government had come to realise that the public service (the implementing machinery of government) had become inefficient, ineffective and was generally not delivering. ${ }^{15}$ Structural reforms, including the Structural Adjustment Programmes (SAPs), the Economic Recovery Programme (ERP), and the Poverty Eradication Action Plan (PEAP) were introduced in 1981 to encourage economic growth in the UPS. ${ }^{16}$ The reforms were a deliberate intervention to ensure that the UPS had the required capacity to deliver public services efficiently and effectively. These have continued to date, with the main focus being on capacities and infrastructure to strengthen the success of the reform programme. The reforms have included activities that relate to helping in initiating, importing, modifying and diffusing new technologies and promoting impeccable levels of integrity, transparency and accountability; and the need for value for money in the

10 Government of Uganda, MoPS, Uganda Public Service Review and Reorganisation Commission (UPSRRC): Report of the Public Service Review and Reorganisation Commission, 1989/1990, Main Report, 1, Kampala: MoPS, 1990, p. 245.

11 E.A. Brett, "Adjustment Policy and Institutional Reform: Rebuilding Organisational Capacity in Uganda," In P. Langseth, J. Katorobo and E.B.J. Munene (eds.), Uganda: Landmarks in Rebuilding a Nation, 1997, pp.30-37.

12 J.R. Ikoja-Odongo, A Study of Information Needs and Uses of the Information Sector of Uganda, Ph.D. thesis, University of Zululand, 2002, p.75.

13 Brett, "Adjustment Policy."

14 Government of Uganda, MoPS, Public Service Reform.

15 Alcira Kreimer, Uganda Post-conflict Reconstruction: Country Case Study Series. Washington, DC: The World Bank, 2000, p.36.

16 United Kingdom, Department of International Development (DFID), Eastern Africa, Uganda Brief Review of UK Support to the Uganda Public Service Reform Programme 1990 to 2000. DFIDEA (U), March, 2001. 
use of public resources. ${ }^{17}$ The donor community, especially the World Bank (WB) and the International Monetary Fund (IMF), responded positively to the government's wish to reform the economy and to enhance corporate governance and accountability. ${ }^{18}$ The structural adjustment measures focused on aligning technologies to the business process as well as safeguarding information and information systems, and identified communication facilities as essential to improving service delivery. Through the use of ICT initiatives, accountability and transparency constituted the main and core objectives of the reform programme. ${ }^{19}$

After introducing a series of structural adjustment measures, the GoU formulated the first national development plan, Vision 2025, and launched it in February 1999 and the second Vision 2040 was launched in 2013. These two Visions provide an overview of long-term goals and aspirations to be achieved by 2025 with full operationalisation by 2040 . The visions recognise that ICT plays a vital role in the process of modernising a country. The focal elements of this aspiration include improving the country's competitiveness by technological innovation in the collection, storage, processing, transmission and presentation of information. This has resulted in growth in the level of business and government activity conducted by electronic systems. The latter element has led the UPS to focus on shifting from manual to digital methods of records creation and storage $\mathrm{e}^{20}$ and be able to store and access digital records in the future with as much ease as with paper records today.

The challenges for the UPS are, in particular, that:

- electronic systems change rapidly and become obsolete - this means that the digital records they generate are not stable and can become inaccessible over time through inaction;

- it is neither practical nor cost-effective to replicate digital records in paper form - this means that they need to be preserved, stored and managed in digital form;

- digital records can often be changed without detection thus compromising their integrity and evidentiary value, meaning that authentication measures are required; and

17 Kreimer, Uganda Post-conflict Reconstruction.

18 Arne Bigsten and Steve Kayizzi-Mugerw, Is Uganda an Emerging Economy? A Report for the OECD Project "Emerging Africa", Uppsala, Sweden: Nordic African Institute, 2001.

19 Government of Uganda, MoICT, National E-government Framerwork, 2009.

20 Government of Uganda, MoPS, Public Service Reform. 
- $\quad$ systems for managing digital records must be designed for long-term preservation, meaning that digital records must be appropriately stored and managed if long-term access is to be guaranteed.

Although the use of ICTs may enhance the UPS business processes, operations and service delivery, it is crucial to address issues pertaining to managing the resultant digital records adequately if the use of ICTs is to be effective. These challenges led this study to question whether Uganda's ICT management initiatives support digital records management and whether they reflect a well-conceived understanding of the importance of digital records. Although studies ${ }^{21}$ have been undertaken in other African countries such as Botswana, Namibia and South Africa, detailed research addressing digital records management in Uganda and seeking practical solutions to the problem of the management of digital records in the country has been lacking.

\section{New Public Management in Uganda}

New Public Management was introduced with the aims of revitalising the efficiency of public business processes and of enhancing cost-effectiveness in government. ${ }^{22}$ The introduction of NPM in Uganda can be traced to the recommendations of the 1990 Uganda Public Service Review and Reorganisation Commission (UPSRRC) report that concluded that the UPS had decayed. ${ }^{23}$ In response, Uganda adopted several programmes, including NPM, through which it hoped to use ICT to attain efficient service delivery. ${ }^{24}$ As in other countries worldwide, NPM in Uganda served as a catalyst for using ICT systems. ${ }^{25}$ It formed the basis for applying ICTs in the delivery of public services. ${ }^{26}$ Through NPM, the GoU applied ICTs

21 Segomotso Keakopa, The Management of Electronic Records in Botswana, Namibia and South Africa, PhD thesis, University of London, 2007.

22 E. Ciulla Kamarck, Government Innovation Around the World, Institute for Democratic Governance and Innovation, John F. Kennedy School of Government: Harvard University, 2004, p.2.

23 Government of Uganda, MoPS, 1990.

24 Government of Uganda, MoPS, A Handbook of Results Oriented Management: A Guide for Public Service Managers, Kampala: MoPS, 2002.

25 Peter Langseth, "Civil Service Reform in Uganda: Lessons Learned," Public Administration and Development 15 no.4 (1995): 365-390.

26 James Matovu, "Towards Management Information System in Public Administration in Uganda and South Africa," Proceedings of 4th Annual DLIS-LISA Conference, University of Zululand, 8 September 2003, pp.52-60. 
as a strategy for ensuring efficiency in the delivery of public services. ${ }^{27}$ Developments in ICTs gave rise to a new set of information systems, broadly referred to as management information systems (MIS). These formed the basis for improving the management of public information. The MIS focused on networking public workstations to provide an effective way of informationsharing. Through the management of information and control systems, the government aimed to enhance the use of available technologies. ${ }^{28}$ Although the NPM initiative was committed to better delivery of public services, accurate and complete records remained elusive.

\section{Goal and Objectives of the Study}

The goal of the study was to establish whether Uganda had a framework for digital records management that could provide strategies for the effective management of digital records. Its objectives were to:

1. examine the state of digital records management in the UPS;

2. reveal the factors preventing the effective management of digital records in the UPS; and

3. discover how efficiently digital records management could be established in the UPS in order to develop a framework for improvement.

The study was trying to find answers to the following three questions, which were further broken down into several sub-questions:

1. What is the state of the digital records created and held in ICT systems in the UPS?

1.1. How widespread is the use of ICT systems in the UPS?

1.2. What technology is used, and has the use of such technology allowed digital records to be created and managed in the UPS context?

1.3. Why is the state of digital records management important and significant for the UPS?

2. What are the factors affecting the management of digital records in the UPS?

27 Government of Uganda, MoPS, 2002.

28 United Kingdom, DFID, Eastern Africa. 
2.1. What is the impact of ICTs on records management processes?

2.2. What are the challenges affecting the management of digital records in the UPS

3. What measures for the capture, long-term safeguarding and accessibility of digital records exist in the UPS and what frameworks are needed for the future?

3.1. What measures already exist for digital records management in the UPS?

3.2. What are the digital records management best practices across the region?

3.3. What strategies and measures will help improve the management of digital records for the efficient delivery of public services in the UPS?

\section{Review of the Methods Used to Study Records Management in the UPS}

Case studies constituted the research design that underpinned this study. Case study approaches are well suited to information systems research where a phenomenon is supposed to be studied in real life and in its natural environment. ${ }^{29}$ As Ngulube ${ }^{30}$ and Yin $^{31}$ state, different data collection techniques are applied in research in order to capture the complexity and uniqueness of the settings of interest. Triangulating data yielded by multiple methods that corroborate one another or that address different aspects of a research question makes it possible to confirm apparent findings and to discern variances and complexities. ${ }^{32}$ What is significant in general in the use of qualitative methods is that it involved systematic and detailed study of individuals in natural settings, often using open-ended interviews in order to bring out interviewees'

29 Myers and Avison, 2002; Robert K.Yin, Case Study Research: Design and Methods, 4th ed. Thousand Oaks, CA: Sage, 2009, p.2.

30 Patrick Ngulube, "Mapping Mixed Methods Research in Library and Information Science Journals in Sub Saharan Africa, 2004-2008, The International Information E Library Review 42 (2010): 52-261.

31 Yin, Case Study Research, 46-49, 118-123.

32 R.S.O. Onyango, "Data Collection Instruments in Information Sciences," In L.O. Aina, Research Information Science: An African Perspective, Ibadan: Stiling-Horden Publishers, 2002, p.102. 
experiences and perspectives on specific situations, events or issues. ${ }^{33} \mathrm{I}$ asked questions, therefore, around what, when, where and why. Quantitative research, on the other hand, focused on aspects that related to answering questions such as 'How?' and 'How much?' as opposed to 'Why?'

The study adopted a mixed or multiple-methods approach and used qualitative and quantitative techniques to explore in depth the issues of digital records management activities in the UPS. I used both qualitative and quantitative methods in the collection and analysis of data to complement each other and to provide a single, well-integrated picture of the situation. Collecting diverse types of data using different methods provided this study with a broader understanding of digital records management. The rationale for using both methods was that neither a quantitative nor a qualitative method alone would have been sufficient to capture the trends and details of digital records management. When used in combination, quantitative and qualitative methods complemented each other and allowed for a more robust analysis of digital records management. ${ }^{34}$

By using quantitative methods, I was able to make precise measurements of some of the variables of digital records management. The techniques used consisted of counting the frequency of variables and then presenting these frequencies as summaries in tables and graphs. The aim was to analyse the digital records management technologies in the UPS and explore them by systematic measurement rather than to seek and interpret the meanings that people attached to their own actions. I also used quantitative methods to collect data in Uganda to establish the extent of utilisation of ICTs and how these had resulted in the creation, use and management of digital records. The quantitative focus was on aspects of digital records management that were quantifiable through measuring and assigning numbers to them.

Most quantitative methods, however, do not deal with a rich description of data, ${ }^{35}$ hence they could not provide explanations for and interpretations of the digital records management phenomena in this study. In order to interpret and describe how digital records were established and managed in the UPS, I applied qualitative methods. As a result, the qualitative methods

33 Joseph A. Maxwell, Qualitative Research Design: An Interactive Approach, 2nd ed. Thousand Oaks, CA: Sage, 2005.

34 M.B. Miles and A. M. Huberman, Qualitative Data Analysis: An Expanded Sourcebook, 2nd ed. Thousand Oaks, CA: Sage, 1994, p.5.

35 Nataliya V. Ivankova, John W. Creswell and Sheldon L. Stick, "Using Mixed-Methods Sequential Explanatory Design: From Theory to Practice," Field Methods 18 no.1 (2006): 3-20. 
were alternated with the quantitative methods in order to triangulate the data sources. This triangulation of data sources enabled the contrasting and validation of the views of the respondents that were collected from different sources and yielded a clear picture of how digital records were managed. The study sample included a number of different levels of managers in different organisational positions. These managers were interviewed in order to gather a variety of perspectives on the research problem.

The different levels of managers comprise the high-level administrators such as the head of agency, senior manager, middle manager, ICT manager and the records managers. In this study, 'head of agency' refers to the Permanent Secretary; 'senior manager' refers to a head of department, commissioner or director for ministries that have directorates; 'middle manager' refers to a principal officer/analyst; and 'ICT manager' means the information scientist/ information technologist or systems analyst and/or network administrator, system administrator and principal systems officer or information manager, depending on the structure of the ministry. In general, 'ICT manager' refers to the person in charge of ICT services and the 'records manager' is the person in charge of the records department.

In other words, I used qualitative methods to capture the existing digital records management framework, including how it functioned, interlinked or coordinated in managing digital records. This enabled me to make comparisons across data to generate all-embracing conclusions. The qualitative methods enabled me to have a comprehensive understanding of the management of digital records from the experiences of those respondents who create, use and manage them. Triangulation was, therefore, required to raise the study above personal bias that can stem from using single methodologies. Personal bias could also have resulted from lack of comprehensive knowledge about digital records management due to failure to use various approaches. Because of the technical distinctiveness of digital records management and its ability to yield many variables, an essential tactic was for me to use multiple converging sources of evidence. This was in line with the views of authors such as Guba, ${ }^{36}$ who have reported that the credibility of the data gathered in research is enhanced if it can be confirmed from several sources. Additionally, by triangulating data collection methods, I was able to provide both the macro- and micro-level perspectives on how digital records are established and managed.

36 Egon G.Guba, "Criteria for Establishing the Trustworthiness of Naturalistic

Enquiries," Educational Communication and Technology Journal, 29 no.2 (1981): 75-91. 


\section{Population of the Study}

The study looked at the existing digital records management systems and methods for managing digital records in the public agencies, the ICT infrastructure within them, as well as the human resource capacity with a view to evaluating and examining their suitability for the management of digital records in the UPS. I developed criteria for the examination and evaluation of the digital records management framework environment to establish the state of the existing digital records and the challenges faced. I included various levels of managers because the management of records requires a team of people working together and contributing their own unique knowledge and skills to the process. ${ }^{37}$

\section{The Multiple Case Study Research Design}

This study adopted a multiple-case study research design and the conclusions from one case were compared and contrasted with the results from the others. ${ }^{38}$ Critical-case sampling was used. These replication procedures were intended to lead me to the development of a rich theoretical framework. Eventually, multiple cases allowed for a single set of cross-case conclusions. The case study method provided a useful means to investigate and describe the establishment and management of digital records in their natural setting. This enabled the study to capture and understand the management of the records in the context within which they were established and used. In this regard, the case study research design provided real-life situations and enabled me to explore the lived experiences of respondents in relation to the management of digital records.

Specifically, the study sought to identify the current technologies, capacities and processes utilised to manage digital records in the UPS. As a result, it identified the strengths and shortcomings of the Ugandan approach to digital records management with a view to contributing towards building ideal capacities and processes for effective digital records management. While the focus of the study was the UPS, it sought additional data from the national archives services of the 13 member states ${ }^{39}$ of the East and Southern Africa

37 Philip C. Bantin, Understanding Data and Information Systems for Recordkeeping, London: Facet, 2008.

38 Yin, Case Study Methods.

39 ESARBICA member countries include Angola, Botswana, the Comoros, Kenya, Lesotho, Malawi, Mozambique, Namibia, South Africa, Swaziland, Seychelles, Tanzania, Zambia, Zanzibar and Zimbabwe that are neighbours to Uganda. 
Regional Branch of the International Council on Archives (ESARBICA). ESARBICA is one of the branches of the International Council on Archives (ICA), which is a network of institutions and professionals dedicated to the advancement of archives through international cooperation. The archival populations selected to be part of the study population were those in the ESARBICA countries that responded to the questionnaires. I chose the archival institutions on the basis of the need to learn from their varied experiences in managing records, generally, and digital records, in particular. The reason for this selection was that the national archival institutions within the ESARBICA countries are involved in debates seeking solutions to digital records management. ${ }^{40}$ Therefore, the data derived from these institutions enabled the study to meet its broad objective of understanding the requisite technologies, capacities and strategies for effective digital records management. The digital records management initiatives within this region provided lessons to Uganda to enhance its records management framework.

\section{Selection of Case Study Institutions}

According to Flyvebjerg, ${ }^{41}$ the choice of cases can be identified by looking at those that are either 'most likely' or 'least likely' to clearly confirm, refute or challenge the phenomena under study. I originally selected four case study agencies from the UPS to provide the greatest possible amount of information on their activities regarding the creation and management of digital records. The choice of the cases also sought to maximise opportunities to elicit data regarding the capacity of the UPS to meet the requirements for managing digital records. The agencies chosen were the Ministry of Information and National Guidance (whose mandate is to oversee media and information matters, especially government communications); the Ministry of Works, Housing and Communications (MoWHC) (which was overseeing the national ICT strategy before June 2006 when this role was passed on to the Ministry of Information and Communication Technology (MoICT)); the Ministry of Public Service (MoPS) (responsible for information management and public records); and the Ministry of Justice and Constitutional Affairs (MoJCA) (responsible for overseeing the national legislative framework).

40 International Records Management Trust (IRMT), Fostering Trust and Transparency in Governance: Investigating and Addressing the Requirements for Building Integrity in Public Sector Information Systems in the ICT Environment Final Report, London: IRMT, 2009.

41 Bent Flyvebjerg, "Five Misunderstandings about Case-Study Research," Qualitative Inquiry, 12 no.2, (2006): 219-245. 
The study focused on these agencies because, in one way or another, they contribute to information management of which digital records are a part, either in terms of policy design or by overseeing the management of records. For instance, the MoWHC is responsible for issuing ICT implementation guidelines, while the MoPS is expected to issue records and archives guidelines. Furthermore, these agencies were selected because the Uganda ICT Master Plan recognised the vital role that they play in information management. ${ }^{42}$ Additionally, the issue of practicality influenced me to include them in the sample - these cases presented a reasonable level of accessibility owing to the contacts I had in these agencies, thus making the request for information convenient and easy.

I also identified other agencies that would yield rich information and purposively selected them on an ongoing basis during data collection. The strategy I used to select these agencies was critical-case sampling, which permits logical generalisation and maximum application of information to other cases. ${ }^{43}$ In this study, the critical-case sampling strategy involved selecting agencies that had an ICT project or coordinated ICT-related projects.

For the purposes of gaining knowledge of the types of technology and their usage in records management, the study targeted four levels of managers in each of the UPS agencies selected. It focused on those departments responsible for implementing their respective institutional ICT strategies and action plans with a view to identifying their ICT capacities and strategies and how they managed the resulting digital records.

\section{Sampling and Sampling Strategies}

The case study agencies and respondents were selected using purposive sampling, which was used to detect cases within a wide range of situations. This provided the richness and the depth of the case description. Institutions were chosen because they had particular features or characteristics that enabled a detailed exploration of the digital records management phenomenon. As Marshall and Rossman noted, ${ }^{44}$ valuable information is gained from people selected on the basis of the positions they hold in

\footnotetext{
42 Government of Uganda, MoICT, Uganda ICT Master Plan and E-Government Feasibility Study. Uganda: USTDA, 2006.

43 M.Quinn Patton, Qualitative Research and Evaluation Methods, 3rd ed., Thousand Oaks, CA: Sage Publications, 2002, pp. 555-556.

44 C. Marshall and G.B. Rossman, Designing Qualitative Research, 3rd ed., Thousand Oaks, CA: Sage, 1999, p.113.
} 
the administrative realms of their institutions. I selected respondents who were knowledgeable about records management, ICT and e-governance and who could provide lived and professional experience. The respondents provided relevant data on the ways digital records were managed and the institutional capacities that were required for their effective management. The combined knowledge of these officers with respect to ICT and records management enabled the study to attain in-depth knowledge of the digital records management phenomenon.

Besides the purposive sampling method, this study also relied on the Records Management Capacity Assessment System (RMCAS). The RMCAS is an assessment tool developed between 2002 and 2004 by the International Records Management Trust (IRMT) to support governments in assessing the quality of their records systems, identifying weaknesses, and introducing appropriate solutions. ${ }^{45}$ It assesses records management systems based on the capacity levels of managers and establishes a link between the strengths and weaknesses in digital records management infrastructures and systems. It also highlights areas where capacity-building may be needed in relation to the management of the environment of policies, procedures, skills and resources. The greatest advantage of the RMCAS is its ability to assess the strengths and weaknesses of records management systems by grouping respondents according to their expertise and competencies. ${ }^{46}$ The RMCAS provided a means of evaluating whether the existing ICT infrastructure, legal and regulatory frameworks, resources and capacity were adequate to manage digital records created in the UPS. Thus, the adoption of the RMCAS framework enabled this study to explore the way records are managed in the public sector settings from the policy-makers down to the records users.

The decision to interview various levels of managers was in recognition of the fact that there are many actors whose cooperation is necessary when streamlining the capacities of public sector records management. This results from the fact that in an organisation, differences will exist in values, roles, perception and interests regarding digital records management depending on the employment position or level. By combining qualitative methods that support the understanding of individual cases in depth with quantitative approaches that make systematic comparisons and by complementing the

45 IRMT, Records Management Capacity Assessment System (RMCAS), 2005, http://www. nationalarchives.gov.uk/rmcas/.

46 Andrew Griffin, "Records Management Capacity Assessment System (RMCAS)," Archival Science 4 (1-2 (2004): 71-97. 
selection of cases with the choices arrived at by the RMCAS, this study sought to understand all the requirements that would contribute to the efficient management of digital records in Uganda.

\section{Sample Size}

The study adopted a non-statistical sample. It required that some subjects respond to the questionnaires while others were interviewed. The target population included ICT and records managers, senior and middle managers; and archivists from ESARBICA member states. I distributed 75 questionnaires to 23 fully-fledged ministries across the entire government of Uganda; these ministries formed the UPS, the focus of this study. 51 (68\%) of the questionnaires were returned. A total of 24 (32\%) questionnaires were not returned. I mailed 13 questionnaires to cover the ESARBICA member states and 6 (46\%) were returned.

The original plan was to select two respondents from each managerial level for each of the four ministries, which would lead to a minimum sample size of 32 interviews to be conducted in Uganda. However, in the field, the number of respondents changed to 40 . This resulted from identifying other information-rich cases. I held interviews with five chief archivists from the ESARBICA region, one lecturer from Moi University Kenya, two senior lecturers from the University of Botswana and one archives researcher from University of Zululand in South Africa.

\section{Data Collection Methods and Instruments}

In using this mixed-research strategy, I focused on both primary and secondary methods of gathering information and data. In the primary method, I engaged in the collection of data and interviewing key informants. Secondary methods involved me in reading both published and unpublished literature and official government documents. These methods are discussed below.

\section{Desk-based Research and Reading List Guide}

Desk-based research dealt with secondary data. In the process of gathering secondary data, I read peer-reviewed articles and literature deemed relevant to digital records management to build up validity and the theoretical context. Considering the digital records management phenomenon as being a fairly new concept, I took into account the importance of identifying the 
background information related to its development. Reviewing relevant literature, therefore, provided the link between the past and the present relating to ICT and digital records management development. The documents took various forms, including textbooks relevant to records and information management, monographs, scholarly journals, dissertations, GoU publications and policy reports, conference proceedings and public reports. I referred to scholarly journal literature because of its currency, while using public reports for their focus and topicality. A number of doctoral dissertations were also consulted.

The study made good use of the Internet to access current literature in full-text journals. It was possible to conduct a comprehensive review of the documents with the help of the reading list guide. I identified and read documents, both published and unpublished, and extracted relevant information indicating digital records management developments and relationships. Data from these sources helped me to identify gaps, trends and theory relevant to digital records management.

Through reviewing literature, the study analysed the capacities, strategies and frameworks required to manage digital records. Documentary research helped me to narrow and delineate the research problem and to develop a firm understanding of the subject under study. It also enabled me to supplement data obtained through interviewing and questionnaires, thus arriving at a profound understanding of the ways in which digital records are managed and the institutional frameworks currently in place for the purpose.

Relying on documents was a conscious decision but also posed a problem as documents did not provide a complete picture of events. Some issues, especially those related to policy and political commitment, were not detectable from an examination of official records. To compensate for such gaps in the records, the questionnaires provided an important sub-text to the documents.

\section{Field-based Research}

In the field, I basically applied two methods to conduct the study, as explained below.

\subsection{Self-administered Questionnaire}

I collected quantitative data through a self-administered questionnaire. The questionnaire was distributed in Uganda and to the ESARBICA states and targeted those departments that promoted the ideals and processes of digital 
records management. I chose to use this method because it allowed the collection of large amounts of data in a short period. ${ }^{47}$ The questionnaire was developed to enable the collection of standardised and general information about the types of ICTs used for the daily administration of public affairs and those used to manage records, among other things. It comprised both openand closed-ended questions to give respondents a chance to state their views. It also provided a systematic means of collecting data that assessed Uganda's and ESARBICA's capacities, strategies and institutional frameworks for digital records management. The completed questionnaires elicited a broad picture of the state of ICT in the UPS and indicated the technologies applied in the management of digital records from the perspective of the users and those who harness the technology.

I pre-tested the UPS questionnaire in one of the case-study institutions to eliminate vague or ambiguous questions and to ascertain its validity and reliability. The purpose of pre-testing the questionnaire was to determine whether or not the data it would produce would be sufficient for the study. The results of the pre-testing determined the level and depth of the revisions that needed to be made on the questionnaire.

The main problem of data collection by questionnaire was the complexity of obtaining responses. To maximise the response rate, I made follow-up telephone calls and physical visits to the respondents with a view to reducing the non-response rate. As a result of vigorous follow-ups, the questionnaire had a $68 \%$ response rate, which complemented the data collection process in the UPS. However, the response rate to the questionnaire sent to the ESARBICA countries was only $46 \%$.

The other problem with the questionnaire was the limited kind of information it provided. The information obtained from the questionnaire did not give a sufficiently holistic picture to enable full understanding of what was happening in the phenomenon of digital records management. I resolved this situation by carrying out in-depth interviews.

\subsection{In-depth Interviews}

I collected qualitative data through in-depth interviews with open-ended questions. Interviews in this study were composed of semi-structured questions, which allowed for precise and deep insight into the management of digital records. I piloted the interview schedule in two UPS agencies before undertaking the actual data collection. The pilot testing was done to

47 C.A. Moser and G. Kalton, Survey Methods in Social Investigation, Hampshire: Gower Publishing, 1979, p.41. 
address complexities and ensure the clarity of the questions posed. I evaluated the questions against the data collected to establish whether they generated the type of data that I needed. After piloting the study instrument, I revised the interview questions to remove elements found to be elements and improved the wording for clarity. The pilot study confirmed that the sampling methods functioned in the ways expected.

The interviews helped me to understand the lived experiences of the respondents. In-depth interviews allowed a new line of inquiry to uncover issues that did not appear to be exhaustively covered in the questionnaire. Through the interviews, the study was able to discover the respondents' views. It also enabled me to understand the pressing need for UPS agencies and organisations to be able to create, store and manage their digital records appropriately with the aim of ensuring that they are accessible in the future as part of a corporate and community memory.

I conducted interviews in the selected Ugandan agencies with a focus on one head of agency, two senior managers, two middle managers, one ICT officer, and two records officers. The reason for choosing at least two respondents for each level of management derived from Zaltman and Duncan's argument that in attempting to understand an organisation, a good rule of thumb would be to talk to at least two people at the same organisational level. ${ }^{48}$ Zaltman and Duncan further argued that gathering views from more than one person at each level in the organisation is helpful in understanding the phenomenon under study since different people at the same level might have different perceptions of the organisation. ${ }^{49} \mathrm{I}$ chose this approach for two reasons: first, I assumed that the interview subjects were knowledgeable about issues relating to the management of digital records; and second, the purpose of the interview was to elicit the analytical views and understanding of the interviewees.

Interviewing allowed for interaction between me and the interviewees to explore the meaning of questions posed and answers proffered and to resolve any ambiguities or misinterpretation. As a result, extended interview sessions also allowed comments on themes and discussions of emerging patterns that contributed to the enrichment of results.

Interviews were tape recorded after the respondents had given their consent. Slater suggests that when the respondents' words are recorded,

48 G. Zaltman and R. Duncan, Strategies for Planned Change, New York, NY: John Wiley and Sons, 1977, p.45.

49 Zaltman and Duncan, Strategies for Planned Change. 
they offer the opportunity to explore the experiences of the respondents. ${ }^{50}$ Recorded interviews allowed me to return to the gathered data in its original form as often as possible, which enabled me to understand more fully all the views raised. I also took notes during the interviews regarding the participants' non-verbal behaviour, which would not be captured through tape-recording.

The collection of data through interviews was concluded when theoretical saturation was reached. That point occurred when new interviews ceased to yield any new data on the themes and issues under discussion.

\section{Data Analysis and Presentation}

Interpretive analysis approaches were followed to present the research findings.

\section{Documentary Data Analysis}

I analysed documents for relevant information relating to digital records management. I determined preliminary units of data and consequently created broad categories of data from the reviewed documents. The documents related to NPM and its impact on public service reforms; how digital records are created, indexed, stored and retrieved; and the existing policy and standards related to the creation and management of digital records. I carried out a detailed analysis involving identifying trends, status and challenges to managing digital records. This formed the basis for understanding the existing framework for digital records management in the UPS. Data results were presented as descriptive statements.

\section{Quantitative Data Analysis}

Quantitative data analysis entailed categorising and summarising data in order to find answers to the research questions. With the help of a Quantitative Economics student, I analysed the quantitative data from both the questionnaires and interviews using the Statistical Package for Social Sciences (SPSS). The statistical package facilitated the breakdown of categories of data and helped to organise the data more quickly. The reason for this choice was that the results of the study establishing the patterns of ICT usage and its utilisation in the management of records could be shown in descriptive detail

50 M. Slater, 'Qualitative Research', in M. Salter, ed, Research Methods in Library and Information Studies (London: Library Association, 1990), pp.107-127. 
in tables, graphs and illustrations. With the results from the SPSS analysis it was possible, for example, to rank the Ugandan agencies according to what ICT technologies are available. The statistics revealed which institutions had a high level of capacity for digital records management and the potentially significant contrasts between them became apparent. Quantitative results were also presented with descriptive statements.

\section{Qualitative Data Analysis}

The qualitative data collected in the study was analysed through data reduction, editing and categorising into themes that were in line with the objectives of the study. According to Miles and Huberman, ${ }^{51}$ data reduction is "a form of analysis that sharpens, sorts, focuses, discards and reorganises data in such a way that 'final' conclusions can be drawn and verified." While I quantified the descriptive data using frequencies, the bulk of the analysis was interpretive to enable the discovery of concepts and relationships in the raw data. This provided me with insight into and an understanding of the framework for digital records management in the UPS.

I began the initial coding of each interview transcript with manual annotation of scripts during a process of close reading, line by line, to highlight each concept and label it. Lincoln and Guba ${ }^{52}$ argue that this process is necessary to confirm interpretations and coding decisions, including the development of categories. Subsequent iterations of reading and coding of each interview transcript in a constant comparison with previous interview transcripts and coding allowed the emergence of categories and themes. Data themes were developed according to the research questions and these allowed me to maintain an audit trail, tracking the development of analysis with annotations for major decisions. I applied themes of varying sizes, words and phrases connected to records management to identify relevant subject areas in the data.

Interview results were presented using relevant and substantive quotations. The 'voice' of various respondents was deliberately presented through the use of quotations clearly stating whose opinion was being represented. Reproducing the words of participants communicated their attitudes and depth of feeling while simultaneously advancing the argument being made. I did not,

51 Miles and Huberman, Qualitative Data Analysis.

52 Yvonna S. Lincoln and Egon G. Guba, Naturalistic Inquiry, Beverly Hills, CA: Sage, 1985. 
however, rely on interview material for factual information, but rather to help me understand the perspective of various respondents.

\section{Data Quality Control}

I piloted the interview guide and pre-tested the questionnaire in order to achieve content validation. I achieved content validity by ensuring that the questions were related to digital records management in a public service setting. Triangulation, a combination of multi-method and multi-data/ evidence sources, increased both the validity and reliability of evaluation of data. ${ }^{53}$ The multiple sources of evidence provided multiple measures of the phenomenon of digital records management in the UPS. Triangulation ensured that the case study on digital records management rendered participants' perspective accurately. By combining multi-methods, observers and data sources, I was able to overcome the intrinsic bias that comes from singlemethod, single-observer and single-theory studies. ${ }^{54}$ Different types of data were able to provide cross-data consistency checks.

In data analysis, the strategy of triangulation provided diverse ways of looking at the phenomenon of digital records management. It also added to credibility by strengthening my confidence in the conclusions that were drawn. Data analysis, including coding, tabulation and the use of descriptive statements, ascertained the quality of the findings.

\section{Major Findings of the Study}

This study established that, despite attempts to improve ICT capabilities and infrastructure in Uganda, no corresponding action plan existed for digital records management. A strategic plan for records management was also lacking and the creation and keeping of records relied on individual government officers. While the records manager maintained records, there was no formal policy stating what digital records are and why it is important to manage them. The head of the agency and senior managers disagreed on what the digital records management strategic plan or policy would entail. On the other hand, the ICT and middle managers expressed lack of understanding of what a digital records management policy is and how it could be implemented. Most levels of managers thought the presence of ICT was

53 Yin, Case Study Research, 46-49, 118-123; Patton, Qualitative Research and Evaluation Methods, 555-556.

54 N.K. Denzin and Y.S. Lincoln, eds., The Sage Handbook of Qualitative Research. 3rd ed. Thousand Oaks, CA: Sage, 2005, p.453. 
enough to create and manage digital records. However, the records managers were of the view that there was some level of electronic informationsharing and transfer between ministries but that digital records as such were not specifically addressed.

It was found that ICT was being integrated into the UPS business processes without the necessary framework preparations and required arrangements for digital records management. It was also found that ICT initiatives were not coordinated and that there was no plan regarding how digital records would be managed. The overall national ICT structure itself failed to address the requirement for digital records management even though authors such as Gilliland and McKemmish ${ }^{55}$ suggest that technology should proactively shape records management processes. Furthermore, substantial challenges existed regarding the management of the records that were created and held in digital systems. Specifically, there were reports of loss of data, poor accountability and failure to guarantee satisfactory access to required information, especially where digital systems were used to create and keep records. Even since this study was conducted in 2010, there have been no developments in terms of national records management policy.

\section{Conclusion}

By applying the above methodological approach, the study was able to reflect on and analyse an ongoing process within a specific context that had both immediate and future implications for professional record-keeping practices and strategic positioning. The great advantage of the mixed method was the flexibility to approach this study through diverse ways. Each method provided a unique path and language to evaluate the digital records management phenomenon in the UPS. Nevertheless, a mixedmethods approach presented its own challenges. One of the challenges was the development of diverging interpretations. However, the emergence of diverging interpretations was a critical learning experience in that it provided an opportunity for me as the researcher to witness the intricacy of the digital records management phenomenon under inquiry and to develop a richer description and explanation through crosschecking and reflection. It also revealed multiple layers of views about digital records management held by each level of management and this reflected each respondent's unique experience in the context of digital records management. The understanding

55 Anne Gilliland and Sue McKemmish, "Building an Infrastructure for Archival Research”, Archival Science, 4 nos. 3-4 (2004): 149-197. 
gained through the mixed-methods research approach enabled this study to propose an indicative framework for managing digital records in the UPS which could not have been so conclusively established using other methods.

\section{References}

Bantin, Philip C. Understanding Data and Information Systems for Recordkeeping. London: Facet, 2008.

Baryamureeba, Venansius. "ICT as an Engine for Uganda's Economic Growth: The Role of and Opportunities for Makerere University”. International Journal of Computing and ICT Research 1 no.1 (2007): 1-48.

Baryamureeba, Venansius. "ICT-enabled Services: A Critical Analysis of the Opportunities and Challenges in Uganda”. International Journal of Computing and ICT Research 4 (2008): 215-235.

Bigsten, Arne and Steve Kayizzi-Mugerwa. Is Uganda an Emerging Economy? A Report for the OECD "Project Emerging Africa". Uppsala, Sweden: Nordic African Institute, 2001.

Brett, E.A. "Adjustment Policy and Institutional Reform: Rebuilding Organisational Capacity in Uganda”. In Peter Langseth, J. Katorobo and E.B.J. Munene, eds. Uganda: Landmarks in Rebuilding a Nation, 1997, pp.30-37.

Flyvebjerg, Bent. "Five Misunderstandings about Case-Study Research". Qualitative Inquiry 12 no.2, (2006): 219-245.

Gilliland, Anne and Sue McKemmish. 2004. "Building an Infrastructure for Archival Research”, Archival Science 4 nos.3-4 (2004): 149-197.

Government of Uganda, MoICT. National E-government Framework, 2009.

Government of Uganda, MoFPED. Vision 2025: A Strategic Framework for National Development. Kampala: MoFPED, 1999.

Government of Uganda, MoICT. Uganda ICT Master Plan and E-Government Feasibility Study. Uganda: USTDA, 2006.

Government of Uganda, MoPS. A Handbook of Results Oriented Management: A Guide for Public Service Managers. Kampala: MoPS, 2002.

Government of Uganda, MoPS. Public Service Reform Programme Strategic Framework (2005/6-2009/10). Kampala: MoPS, 2005.

Government of Uganda, MoPS. Uganda Public Service Review and Reorganisation Commission (UPSRRC): Report of the Public Service Review and Reorganisation Commission, 1989/1990, Main Report, 1. Kampala: MoPS, 1990.

Government of Uganda, MoWHC.National Information and Communication Technology Policy. Kampala: MoWHC, 2003.

Griffin, Andrew. "Records Management Capacity Assessment System (RMCAS)”. Archival Science 4 (1-2 (2004): 71-97.

Guba, EgonG. "Criteria for Establishing the Trustworthiness of Naturalistic Enquiries". Educational Communication and Technology Journal 29 no.2 (1981): 75-91.

Heeks, Richard. "Information Systems and Developing Countries: Failure, Success and Local Improvisations”. Information Technology for Development 7 no.1 (2001): 3-16.

Ikoja-Odongo, J. R. A Study of Information Needs and Uses of the Information Sector of Uganda, $\mathrm{PhD}$ thesis, University of Zululand, 2002.

International Records Management Trust (IRMT), Fostering Trust and Transparency in Governance: Investigating and Addressing the Requirements for Building Integrity in Public Sector Information Systems in the ICT Environment Final Report. London: IRMT, 2009.

International Records Management Trust (IRMT), Records Management Capacity 
Assessment System (RMCAS), 2005, http://www.nationalarchives.gov.uk/rmcas/.

Ivankova, Nataliya V., John W. Creswell and Sheldon L. Stick. "Using Mixed-Methods Sequential Explanatory Design: From Theory to Practice”. Field Methods 18 no.1 (2006): 3-20.

Kamarck, E. Ciulla. Government Innovation Around the World. Institute for Democratic Governance and Innovation, John F. Kennedy School of Government: Harvard University, 2004.

Keakopa, Segomotso. The Management of Electronic Records in Botswana, Namibia and South Africa, $\mathrm{PhD}$ thesis, University of London, 2007.

Kreimer, Alcira. Uganda Post-Conflict Reconstruction: Country Case Study Series.

Washington, DC: The World Bank, 2000.

Langseth, Peter. "Civil Service Reform in Uganda: Lessons Learned”. Public Administration and Development 15 no.4 (1995): 365-390.

Lincoln, Yvonna S. and E. G. Guba, Naturalistic Inquiry. Beverly Hills, CA: Sage, 1985.

Luyombya, David. Framework for Effective Public Digital Records Management in Uganda. Ph.D. thesis, University College London, 2010.

Marshall, C. and G. B. Rossman, Designing Qualitative Research. 3rd ed. Thousand Oaks, CA: Sage, 1999.

Matovu, James. "Towards Management Information System in Public Administration in Uganda and South Africa". Proceedings of 4th Annual DLIS-LISA Conference, University of Zululand, 8 September, 2003, pp.52-60.

Maxwell, Joseph A. Qualitative Research Design: An Interactive Approach. 2nd ed. Thousand Oaks, CA: Sage, 2005.

Miles, M.B. and A. M. Huberman. Qualitative Data Analysis: An Expanded Sourcebook. 2nd ed. Thousand Oaks, CA: Sage, 1994.

Moser, A.A. and G. Kalton, Survey Methods in Social Investigation. Hampshire: Gower Publishing, 1979.

Neuman, L.W. Social Research Methods: Qualitative and Quantitative Approaches. Boston: Pearson Education, 2003.

Ngulube, Patrick. "Mapping Mixed Methods Research in Library and Information Science Journals in Sub Saharan Africa, 2004-2008. The International Information E Library Review 42 (2010): 52-261.

Onyango, R.S.O. “Data Collection Instruments in Information Sciences”. In L.O. Aina, Research Information Science: An African Perspective. Ibadan: Stiling-Horden Publishers, 2002.

Patton, M. Quinn, Qualitative Research and Evaluation Methods. 3rd ed. Thousand Oaks, CA: Sage Publications, 2002.

Reinikka, Ritva and Paul Collier (eds.). Uganda's Recovery: The Role of Farms, Firms and Government. Washington, DC: The World Bank, 2001.

Slater, M. "Qualitative Research", in M. Salter (ed.), Research Methods in Library and Information Studies. London: Library Association, 1990, pp.107-127.

United Kingdom, Department of International Development (DFID). Eastern Africa, Uganda, Brief Review of UK Support to the Uganda Public Service Reform Programme 1990 to 2000, DFIDEA (U), March, 2001.

Wafula, David and Norman Clark. "Science and Governance of Modern Biotechnology in Sub-Saharan Africa - The Case for Uganda", Journal of International Development (2005): 679-694.

Yin, Robert K. Case Study Research: Design and Methods. 4th ed. Thousand Oaks, CA: Sage, 2009.

Zaltman, G. and R. Duncan. Strategies for Planned Change. New York, NY: John Wiley and Sons, 1977. 
Chapter 29

\title{
DOCUMENTING COMMUNITIES OF PRACTICE
}

Making the Case for Archival Ethnography

\author{
Karen F. Gracy
}

(C) Springer 2006

This chapter was originally published under the same title in Archival Science 2004, 4 (3-4): 335-365. The digital rights to the chapter are owned or controlled by Springer, and unfortunately it was not possible to include this chapter in the digital edition of Research in the Archival Multiverse.

The chapter can be obtained through Springer at http://link.springer.com/article/10.1007/s10502-005-2599-3 or through purchasing a print copy of Research in the Archival Multiverse. 



\title{
ABORIGINAL COMMUNITY ARCHIVES
}

\section{A Case Study in Ethical Community Research}

\author{
Kirsten Thorpe
}

\begin{abstract}
Aboriginal individuals and communities are heavily documented in archival collections throughout Australia. In New South Wales (NSW) significant material has been collected on Aboriginal communities that document personal, family and community histories. Many Aboriginal people are accessing these collections and returning copies to their communities so that they can be used as a local resource. In addition to this, there is strong community interest in establishing Aboriginal community archives. As a case study in ethical community research, this chapter will discuss the research approach, design and methods that were employed to explore the archival needs of the Aboriginal community in Singleton, NSW. The chapter will discuss the rationale for designing community-based research that is respectful of the Aboriginal community. In the case study, an ethical community research approach was vital to the full engagement and participation of the community in identifying issues and aspirations relating to the establishment of an Aboriginal community archive, and exercising control over the management of their cultural heritage. In exploring this approach the chapter will provide insight into the methods and techniques used, and their effectiveness in conducting partnership research. In addition, it will discuss the importance of self-reflection and immersive research methods as essential components of conducting meaningful and outcomebased community research.
\end{abstract}




\section{Introduction}

Aboriginal individuals and communities are heavily documented in archival collections throughout Australia. In New South Wales (NSW) significant material has been collected on Aboriginal communities that document personal, family and community histories. This material is predominantly created by non-Aboriginal people about Aboriginal people. There is a strong interest being expressed by various Aboriginal communities in NSW for community archives to be established. ${ }^{1}$ They are looking both to create and manage their own materials and to obtain copies of material held in other archives that relate to their communities. ${ }^{2}$ Opportunities exist for the Australian archival community to work with Aboriginal communities to create an archival system that is respectful of Aboriginal community protocols and needs, but that also uses archival knowledge, experience, theory and practice. Indeed, over the past 20 years, the Australian archival profession and archival institutions have made significant progress in increasing access to collections and participating in discussions about issues and the records they hold that are of importance to Aboriginal people. This level of commitment was in the most part a response to government enquiries that identified the importance of records for Aboriginal people for recovering and maintaining identity, particularly in cases where community members had been affected by past government policies. As the number of Aboriginal people who engage with archival institutions and the profession increases, however, so does the demand for Aboriginal participation in decision-making

1 Jennifer Ingall, "Moree's Indigenous History Collection to be Part of a New Cultural Exhibition Centre," Australian National Broadcasting Service (ABC) New England Northwest, September 2, 2010, http://www.abc.net.au/local/ stories/2010/09/02/3000606.htm; Australian Museum and Wonnarua People, "Wonnarua People Visit the Collection," Australian Museum (2009), http:// australianmuseum.net.au/movie/Wonnarua-Visit; Larissa Beherendt, "An Aboriginal Keeping Place (Proposal)," http://www.thekeepingplace.org/proposal.html; Australian Institute of Aboriginal and Torres Strait Islander Studies (AIATSIS), Proceedings of the Symposium on Information Technologies and Indigenous Communities, Canberra July 13-15, 2010, http://www.aiatsis.gov.au/research/symposia/Digi10.html; Museums and Galleries NSW, Keeping Places and Beyond: Building cultural futures in NSW http:// mgnsw.org.au/media/uploads/files/keeping_places_and_beyondnew2.pdf.

2 Terri Janke, Our Culture, Our Future: Report on Australian Indigenous Cultural and Intellectual Property. Michael Frankel, Solicitors, 1998; Jane Anderson, "Access and Control of Indigenous Knowledge in Libraries and Archives: Ownership and Future Use." Proceedings from Correcting Course: Rebalancing Copyright for Libraries in the National and International Arena, Columbia University, 2005; Alana Garwood-Houng, "Protocols: Meeting the Challenges of Indigenous Information Needs," Australian Academic \& Research Libraries 36 no.2 (2005): 143-151. 
and input into the creation of archival systems and processes that support Aboriginal community and individual needs.

In 2010, I undertook a research project titled Creating an Aboriginal Community Archive in New South Wales as part of a Masters Honours degree. This project explored how ideas can be exchanged between archival professionals and an Aboriginal community in relation to the management of community records. In particular, it sought to explore the needs of Aboriginal people and communities in NSW regarding the creation of an Aboriginal community archive, and to address the question: How can the archival profession and Aboriginal communities work together to create sustainable community archival programs based on Aboriginal protocols?

When designing the research project, I spent considerable time reflecting on my own professional experiences working with Aboriginal and Torres Strait Islander people and archives. I felt passionately that the research approach, design and results should be of benefit to the community within which I undertook the research. In this chapter, I will discuss this project as a case study in community research approaches and methods, with particular emphasis on the ethical considerations of conducting partnership research with Aboriginal communities. I will review the community-centred research design and outline the case study, data collection and analysis methods that were used to examine the archival needs of the Aboriginal community in Singleton, including the literary warrant analysis and literature review that I employed. Finally I will discuss the ongoing process of using self-reflection to question and refine my approaches to the research.

I will use a number of terms in this chapter that have broad and sometimes contested meanings in the archival, and broader, information professions. ${ }^{3}$ These include: "community archive" and "living archive", and other terms specific to the management of Aboriginal archival materials: "digital repatriation" and "Aboriginal protocols". While my focus is not to analyse and discuss the debates around these broad conceptual frameworks, I will briefly introduce the use of terms as they relate to the research undertaken in this chapter.

3 Anne Gilliland and Andrew Flinn, "The Wonderful and Frightening World of Community Archives: What Are We Really Talking About?" Keynote address, Nexus, Confluence, and Difference: Community Archives meets Community Informatics: Prato CIRN Conference Oct 28-30 2013, Larry Stillman, Amalia Sabiescu, Nemanja Memarovic, eds, Centre for Community Networking Research, Centre for Social Informatics, Monash University. ISBN 978-0-9874652-1-4, http://www.ccnr.infotech.monash.edu.au/assets/ docs/prato2013_papers/gilliland_flinn_keynote.pdf. 
The term "community archive" is used in the context of an "Aboriginal community archive" and refers to records that are considered to be significant to a local Aboriginal community group. These materials encompass multiple forms and types of records and relate broadly to the local community: the land, its people, language, knowledge and histories. These records might be sourced from multiple places nationally and internationally besides Aboriginal keeping places, including museums, archives, libraries or other private repositories, and local historical societies, and could include business records in community organisations, or records created specifically to be a part of the community archive. ${ }^{4}$ While the physical materials might reside in any of these locations, duplicate copies might be made, digitally or otherwise, and "returned" to the community archive, thus drawing together and reconnecting the fragments of Aboriginal history and knowledge to support local access and community identity. The records might be owned by the Aboriginal community, or by government or other copyright holders. Since an underlying principle of the Aboriginal community archive is the notion of broad communal ownership, and community responsibilities for the management of Aboriginal knowledge over time, this could also mean that such a community archive would need to be governed progressively by key community representatives (such as recognised community elders) over time.

The term "living archive" refers to an Aboriginal community archive containing both tangible and intangible records. The living Aboriginal archive holds records that may be transmitted orally by members of the community or passed on through art, dance or storytelling - that is, they are not captured in particular physical or digital form but are transmitted through interaction and connections between people. ${ }^{5}$ In addition, the living archive is considered to be not only a place for storing or gathering materials, but also a place where information can be contested. ${ }^{6}$ Multiple sources of records can be gathered, analysed, debated and new layers of information captured on their context. This process, which will be further explored in this chapter, is of particular importance in cases where Aboriginal people were subjects of records and where information might have been recorded incorrectly or from

4 Andrew Flinn, "Community Histories, Community Archives: Some Opportunities and Challenges," Journal of the Society of Archivists 28 no.2 (2007): 151-176.

5 Sue McKemmish, Anne Gilliland-Swetland, and Eric Ketelaar, "'Communities of Memory': Pluralising Archival Research and Education Agendas," Archives and Manuscripts 33 no.1 (2005): 146.

6 Eric Ketelaar, "A Living Archive, Shared by Communities of Records", Jeannette A. Bastian and Ben Alexander, eds. Community Archives: The Shaping of Memory, London: Facet Publishing, 2009. 
different perspectives. In this context, the living archive provides a space in which voice and representation can be given to the experiences of Aboriginal people.

The term "digital repatriation" refers to the copying and digital return of records to the communities which created them or to which they relate and is a key element in the return of knowledge to a community archive. ${ }^{7}$ It supports the notion that an Aboriginal community archive could comprise digital copies as well as virtual links to collections. Such digitised records or digital objects could be returned from multiple places, including archives, libraries, museums or other private repositories.

The term "Aboriginal protocols" refers to specific protocols that have been developed to guide the Australian information professions around engagement between libraries, archives and information services with Aboriginal people, collections and services. These protocols have been developed out of recognition of the history of Aboriginal dispossession and discrimination in Australian society since the onset of colonisation in 1788. The principle underlying the development of Aboriginal protocols is to develop a set of ethical guidelines and principles that respect Aboriginal cultural beliefs and practices. The development of protocols specific to the archival profession will be explored more in this chapter.

\section{Placing Myself Within the Research}

As is customary in Aboriginal communities, I begin by introducing myself. As an archivist and member of the NSW Aboriginal community, it is important that I position myself within this exploration of ethical community research since my experiences and self-reflections provide the foundations for the approaches and methods used to investigate my research question.

I have been deeply immersed in working with Aboriginal communities and archives in NSW for the past decade. In 1999, I received an Aboriginal Cadetship to train as an archivist within the State Records Office NSW (the State archives). The Cadetship was an outcome of the Aboriginal Cadetship Project - Aboriginal and Torres Strait Islander Recruitment and Career Development Strategy that was based on an agreement between the federal Department of Employment, Education, Training and Youth Affairs and the Australian Library and Information Service (ALIA). ALIA worked

7 Kimberly Christen, “Opening Archives: Respectful Repatriation," American Archivist 74 no.1 (2011): 185-210. 
with the Australian Society of Archivists (ASA) in applying the strategy in the archives sector. In my role as the Archivist - Aboriginal Liaison, I assisted Aboriginal people in gaining access to the NSW State Records Office and negotiating appropriate access to records relating to them, their families and communities. This role required me to navigate and balance community needs and protocols with the requirements of the NSW State Records Act of $1998 .{ }^{8}$

The Aboriginal people who were documented in the records were entering the reading room requesting access to their files. The records were, in many cases, closed to public access due to the sensitive and personal nature of the information contained within them, and clients had to seek permission to access them via the NSW Department of Aboriginal Affairs (DAA). Access directions were set by the Department of Aboriginal Affairs under Part 6 of the State Records Act. ${ }^{9}$ When I began my cadetship, clients would need to contact the then Department of Aboriginal Affairs (now the Office of Aboriginal Affairs) and complete an "Application for Personal Family History Research" and bring a letter of permission to view original files held at the archives. The letter was required for access to all closed materials, including material that might relate to the client individually as the subject of the file. In cases where the files related to a family member or descendant, the client would need to obtain permission from the subject of the file (that is, the person to whom the file related) in order to view the file. In cases where the subject was deceased, the application needed to be supported by the eldest living relative, or next of kin. This added levels of bureaucracy and form-filling in order to access information about clients' own families that often resulted in frustration and distrust of the archives on the part of the Aboriginal clients making the requests. They felt that the archival processes were mirroring the treatment that they, or their family, had endured whilst under the control of the Aborigines Welfare and Protection Boards.

The NSW Aborigines Protection and Welfare Boards, which operated in NSW from 1883 to 1969, had a major impact on the lives and aspirations of Aboriginal people and communities. The Board's duties were far reaching and included: the ability for the Minister to distribute monies voted by Parliament for the relief of Aboriginal people; the role of distributing blankets, clothing and relief to Aboriginal people; provision for the custody,

8 New South Wales. State Records Act 1998, Act No. 17, http://www.austlii.edu.au/au/ legis/nsw/consol_act/sra1998156/.

9 New South Wales. State Records Act 1998. 
maintenance and education of the children of Aboriginal people; the management and regulation of reserves; and the general supervision of care over all matters affecting the interests and welfare of Aboriginal people with the intention of: "protecting them against injustice, imposition and fraud". ${ }^{10}$ The Aborigines Protection and Welfare Boards defined Aboriginal people according to their "percentage" of "Aboriginal blood", applying the derogatory categories of "Full-blood", "Half-Caste" and "Octoroon" as determinants of the future of specific Aboriginal people who came under the Board's control. These categories were forced on Aboriginal people; it was not, and is not, the way that the community defines themselves. A significant group of people affected by the policies were those children removed by the Board (the Stolen Generations) and their families to be apprenticed or brought up in government institutions, or to be fostered, adopted or assimilated into the mainstream community:

What happened to us as Aboriginal people was unprecedented. Our identity as Aboriginal people, our culture, our land, our mothers, families and communities, were forcibly and often brutally removed from us as little children. We were systematically punished for being ourselves, until we learned to act like non-Aboriginal people. Often the process of removal was designed to prevent us from ever finding our way home and also to prevent our families from being able to find us. Then, as a nation we went on to pretend that this never happened, right up until last year, when Prime Minister Rudd made the Apology to the Stolen Generations. The truth about what happened to us can no longer be denied. ${ }^{11}$

Clients accessing the State archives do so for a variety of reasons, including family history, native title research, reconnecting with families and communities after forced removal by government, rejuvenation of languages and culture, and, investigation and research for supporting claims, such as claims submitted to the NSW Aboriginal Trust Fund Repayment Scheme (ATFRS) which began in 2003 to repay monies held in trust by government through the Aborigines Protection Board that functioned between 1883 and 1969.

10 State Records NSW, "Board for the Protection of Aborigines," http://investigator. records.nsw.gov.au/Entity.aspx?Path=\%5CAgency\%5C559

11 Lorraine Peeters, "The Marumali Program: An Aboriginal Model of Healing," Working Together (2010): 285, http://www.wimmerapcp.org.au/wp-gidbox/uploads/2014/04/ working_together_full_book.pdf\#page=319 
Many of the challenges with which I met while working as the Archivist - Aboriginal Liaison required broader considerations and solutions that could not be provided by the traditional methods and approaches that were promoted in Australian archival theory and practice. For example, these tensions arose in relation to the kinds of reference services that were offered and the amount of support required when assisting clients who were viewing their own, or family members' files. Other factors, such as the description of files and use of derogatory language in finding aids also brought challenges for access. If the services offered by the State archives were to be meaningful to Aboriginal people who were documented in the records, then a new service design was needed. New procedures, practices and protocols were implemented to address the unique nature and sensitivity of the information collected and people's access needs. For example, State Records NSW implemented Protocols for staff working with Indigenous people to guide staff with culturally appropriate services for the Aboriginal community of NSW. ${ }^{12}$ During this time, State Records NSW under the operation of the ATFRS, also completed an in-depth indexing project to provide more accurate access to the collection, which avoided release of sensitive third party information, and a major project to digitise the records to enable a mediated system of access through Aboriginal Affairs NSW.

A large part of my job was to warn and then debrief clients about the nature of the records. I would explain that the records were sensitive, and that the language that was used was often discriminatory and offensive in today's context. I would explain to clients that the records were poorly kept and that some had been destroyed and thus had not survived to be kept in the State archives. I recall situations where clients were deeply afraid of accessing their records since the Board had threatened them that deeply personal information had been recorded about their personal lives, only to find out that no such information was captured. In other cases, clients visiting the archives were shocked to find that no records existed that documented themselves or their families' contact with the Board. Others were offended and traumatised by the level of surveillance of their personal affairs that was documented in the files. The emotions were real - fear, anger, sadness, grief, distress and frustration. Sometimes the response to the records was total dismay that the Government recorded absolute untruths - that the archival records contained information that they knew to be wrong. Some extreme

12 State Records NSW, "Protocols for Staff Working with Indigenous People," 2008, http://www.records.nsw.gov.au/state-archives/resources-for/indigenous-people/files/ Protocols\%20for\%20Staff\%20Working\%20with\%20Indigenous\%20People.pdf/view. 
example of this included incorrect facts recorded relating to deaths of family members, including parents of children who had been removed from their families. And the frustration of no records existing often affected clients as heavily as did the records of surveillance and control. How could my mother have been removed from her family and community, and work all those years under the eyes of the Protection Board, and no records exist? Situations like this occurred frequently where a person was named only in an index, and no other information was ever found.

As an archivist I was not trained in how to be responsive to this. My skills in dealing with these situations came from my own personal background, as an Aboriginal person and member of the NSW Aboriginal community. My skills also came from my training in Aboriginal studies at the Wollotuka Institute at the University of Newcastle, and my subsequent undergraduate study in social science and sociology. My responsiveness drew on deep understandings of respect and obligation instilled in me from my teachers in the Aboriginal community and from my own personal experience of archives. My Mother's Grandmother, Violet May Newlin, appears on a list created by the Aborigines Protection Board for monies held in Trust with a figure of $£ 10 / 9 / 1$ still owed to her $1938 .{ }^{13}$ From the Board's Ledgers we know that these were Child Endowment funds. We also know that the policy at the time was that the Board managed the funds if they decided that an Aboriginal mother was unfit to manage their own monies. No other information exists in the archives as to why Violet's money was held by the Board. There is no perspective from Violet in the records. Her children are not named. There is no conversation with Violet about her needs, her ability to manage these funds, or how the money could have helped her in times of need. This information exists in our family's oral records but there is no link from these records to the records held in the State archives. If these kinds of absences exist in the wider collection of records, they are not only 'imperfect' evidence $^{14}$; they are also an incomplete record of the history of Aboriginal people in NSW. They allow the perspectives of public servants, administrators and other officials to be the only voices that are heard, whilst Aboriginal perspectives are absent and missing from the official records. These absences

13 State Records NSW, NRS 1063, "Special Bundles. Report and Recommendations of the Public Service Board on Aborigines Protection, 1940” [6/4501.1] Appendix C: Aborigines Protection Board, Trust Accounts - Endowee's Balances as at 31st July 1938.

14 David Roberts, "Imperfect Evidence”, Vital Signs Magazine 9, September 2006, http:// www.records.nsw.gov.au/documents/vital-signs/issue-09/Vital\%20Signs\%20Issue\%20 9\%20-\%20Directors\%20Letter.pdf 
not only affect Aboriginal people in terms of connecting them with their history and developing understandings of the past, they also support a distorted picture of our national history.

Clients visited the State archives and left with copies of material they sought, if it existed. Where these materials subsequently went and how Aboriginal people responded to the information they contained are important areas that up until recently have not been thoroughly researched by the archival profession. Much of the literature produced by archival institutions and professionals tends to focus on service models designed by archives for Aboriginal people such as indexes, finding aids and outreach, and not on community and individual use of the records after accessing the archive. In my Masters research I aimed to examine the potential for creating an Aboriginal community archive in NSW by and for Aboriginal people.

\section{Setting the Scene - Literary Warrant Analysis, Literature Review and Self-Reflection}

\section{Literary Warrant Analysis}

Literary warrants are found in authoritative sources that constitute mandates for action in organisations, professions and communities. Such authoritative sources include lore, laws, protocols and conventions, professional best practice, domain experts, traditional knowledge holders, and the scholarly literature. ${ }^{15} \mathrm{I}$ employed literary warrant analysis in my research project to identify the key drivers for action in relation to Aboriginal protocols and Aboriginal community archives. According to McKemmish:

... the literary warrant for professional practice is made up of authoritative sources, which are recognised and valued by practitioners. Such authoritative sources are found in the law, codes of ethics, standards, the professionally and scholarly literature, and literary texts. Analysis of literary warrant for professional practice establishes the "mandates" for best practice, and identifies its conceptual and theoretical frames of reference. ${ }^{16}$

15 Livia Iacovino, "Multi-method Interdisciplinary Research in Archival Science: The Case of Recordkeeping, Ethics and Law," Archival Science 4 nos.3-4 (2004): 273.

16 Sue McKemmish, "Placing Records Continuum Theory and Practice," Archival Science 1 no.4 (2001): 334. 
In relation to its application in archival research, literary warrant analysis involves:

... identification of social mandates for personal recordkeeping through analysis of social texts, and creative and reflective writings. Analysis of literary warrant to establish recordkeeping requirements. Analysis of standards, statements of best practice, and research reports to identify recordkeeping metadata requirements. ${ }^{17}$

A more detailed discussion of literary warrant analysis can be found in the chapter by Duff and Cumming in this volume. I believed that literary warrant analysis would be important in identifying the "authoritative sources" of the intersection between Indigenous peoples and archives, even though there was not an existing precedent for such an application.

The literary warrant analysis in my research explored mandates and frameworks for developing archival services for Aboriginal people and communities. Sources of literary warrant include international and local conventions and protocols, such as the 2007 United Nations' Declaration on the Rights of Indigenous Peoples, which provides a roadmap for archival institutions to embed in their practice the inherent rights of Indigenous peoples including self-determination, and the right to maintain distinct cultural identities, ${ }^{18}$ and Indigenous protocols for libraries and archives. An example of the latter are the Aboriginal and Torres Strait Islander Library and Information and Resource Network (ATSILIRN) published Protocols designed to:

... guide libraries, archives and information services in appropriate ways to interact with Aboriginal and Torres Strait Islander people in the communities which the organisations serve, and to handle materials with Aboriginal and Torres Strait Islander content. ${ }^{19}$

17 Gilliland and McKemmish, "Building an Infrastructure for Archival Research," 180.

18 Monash University Trust and Technology Project, "Position Statement on Indigenous Human Rights and the Archives," 2009, http://www.infotech.monash.edu.au/ research/centres/cosi/projects/trust/deliverables/human-rights.html; United Nations General Assembly, United Nations Declaration on the Rights of Indigenous Peoples. Washington, D.C.: United Nations, 2007, http://www.un.org/esa/socdev/unpfii/ documents/DRIPS_en.pdf.

19 Aboriginal and Torres Strait Islander Library and Information Resource Network (ATSILIRN), ATSILIRN Protocols, 2005. http://www1.aiatsis.gov.au/atsilirn/ protocols.atsilirn.asn.au/index0c51.html?option=com_f. 
Of particular relevance to this research are the Protocols for Native American Archival Materials, ${ }^{20}$ which demonstrate the international relevance of the recognition of Indigenous rights in archives and the desire of Indigenous peoples to work in partnership with archival institutions in managing their cultural heritage material. Such protocols are also critical in the areas of archival research and education. Other sources of literary warrant analysed in my research include the research literature reporting on the outcomes of related Australian research projects, for example the Statement of Principles relating to Archives and Indigenous knowledge and the Position Statement: Human Rights, Indigenous Communities in Australia and the Archives that were developed as part of the Trust and Technology Project. ${ }^{21}$

\section{Literature Review}

The literature review I undertook reported on complementary research into Indigenous archives, discussing the Trust and Technology Project relating to Koorie people and records in Victoria, ${ }^{22}$ Faulkhead's Narratives of Koorie Victoria, and Nakata and colleagues' work on digital repatriation of Indigenous knowledge from libraries and archives to communities. ${ }^{23}$ These projects provide strong foundations for action in relation to recognising the need for communities to have engagement with material that relates to them, and for archival institutions to create systems for the capture of additional context and narratives relating to the records. These would include

20 First Archivist Circle, Protocols for Native American Archival Materials, 2007, http:// www2.nau.edu/libnap-p/protocols.html; Jennifer R O'Neal, “'The Right to Know': Decolonizing Native American Archives," Journal of Western Archives 6 no.1 (2015): 2; Allison Boucher Krebs, "Native America's Twenty-first-century Right to Know," Archival Science 12 no.2 (2012): 173-190; Jennifer R O’Neal, “Respect, Recognition, and Reciprocity: The Protocols for Native American Archival Materials," in Identity Palimpsests: Archiving Ethnicity in the US and Canada, Dominique Daniel and Amalia Levi, eds. (Chicago, IL: Society of American Archivists, 2014), pp.125-142.

21 Sue McKemmish, Shannon Faulkhead, Livia Iacovino and Kirsten Thorpe, "Australian Indigenous Knowledge and the Archives: Embracing Multiple Ways of Knowing and Keeping," Archives \& Manuscripts 38 no.1 (2010): 27-50; Sue McKemmish, Shannon Faulkhead, and Lynette Russell, "Distrust in the Archive: Reconciling Records," Archival Science 11 nos.3-4 (2011): 211-239.

22 Monash University Trust and Technology Project. Final Report, 2009. http://www. infotech.monash.edu.au/research/centres/cosi/projects/trust/final-report/

23 Martin Nakata, Vicky Nakata, Gabrielle Gardiner, Jill McKeough, Alex Byrne and Jason Gibson, "Indigenous Digital Collections: An Early Look at the Organisation and Culture Interface," Australian Academic \& Research Libraries 39 no.4 (2008): 223-236. 
the vital right of reply, a right that could assist in healing and archival reconciliation. ${ }^{24}$ The literature review indicated that if these measures are to be achieved there needs to be a shift in traditional archival practice and theory so that they incorporate Indigenous perspectives and ways of viewing the archive.

As well as placing the research in the context of related research, the literature review explored theoretical frameworks and concepts relevant to interpreting the case study findings and to addressing the research question. It drew together data about current knowledge of the research topic. ${ }^{25}$ It also contextualised the case study by exploring the historical situation under which material in archives was collected and the power relationships that are reflected in that material, while providing current context through the responses of the archival and allied professions to issues associated with managing sensitive records relating to Aboriginal and Torres Strait Islander communities held in their institutions. As well as contextualising the archival environment, the review also revealed Indigenous responses to archives and archival science that had led to the development of protocols and various initiatives of engagement with Indigenous communities. The literature review also explored the political nature of records held in archives relating to Aboriginal people, the records of surveillance, the documentary bias of the records, and the lack of Indigenous perspectives or narratives surrounding the history and experiences of Aboriginal people. It included a discussion on emerging postcolonial and postmodern literature that decodes the creation of records ${ }^{26}$ and calls for a major reassessment of traditional archival practice to allow multiple perspectives to be recorded alongside the records and more holistic engagement between communities and archival subjects.

In particular, reviewing this literature highlighted the need for Aboriginal communities to be equal partners in research - the aim of my research. The literature review drew on materials that discussed the role that archives can play in relation to healing and reconciliation. The term "archival reconciliation" refers to the potential for the archival community — comprising both archival professionals and archival institutions - to use archives as tools for

24 Sue McKemmish, "Evidence of Me ... in a Digital World" in I, Digital, Christopher A. Lee, ed. Chicago, IL: Society of American Archivists, 2011, p.1.

25 Lawrence A. Machi and Brenda T. McEvoy, The Literature Review: Six Steps to Success, Thousand Oaks, CA: Sage, 2009, p.156

26 Michael Piggott, "Archives and Memory," in Archives: Recordkeeping in Society. Wagga Wagga, New South Wales: Charles Stuart University, 2005, p.285. 
redress and reconciliation. It acknowledges the dual role of records as instruments of oppression and surveillance, and also as sources of evidence for past injustices that can assist with redress. ${ }^{27}$ It also pointed to the potential for the records continuum model to assist in the creation of Aboriginal community archives, and for emerging archival theory such as the concept of the archival multiverse ${ }^{28}$ to provide essential frameworks to articulate archival programs based on local community needs and protocols. This formed a critical underpinning for my reflections on future possibilities.

\section{Self-reflection}

Self-reflection was an essential part of my research project. I am one of only a small number of Aboriginal people who have been employed in archives in Australia, and who have undertaken courses recognised by the Australian Society of Archivists (ASA) as a professional qualification. I have always felt a strong obligation to respond to the concerns expressed by members of the many Aboriginal communities with whom I have worked about issues they face in accessing records. I am privileged to have had the opportunity to work with these Elders and community members and to discuss with them archival issues and their aspirations about archives. I have also seen a lot of frustration, pain and trauma experienced by members of the Aboriginal community who have not had positive experiences in connecting with archives. I feel that these experiences, and my ability to reflect on them, add depth and meaning to my research. Research is never perspective, opinion or culture lens-neutral. Reflexivity allows for the: "continual monitoring of, and reflection on, the research process." ${ }^{29} \mathrm{I}$ found the practice of self-reflection to be an important and valued method within this research project. Suggesting that reflexivity "adds validity and rigour in research by providing information about the contexts in which data are located,"30 Etherington observes that:

... during the postmodern era, we have been encouraged to view all that has gone before as important 'stories' that were constructions of their time. All of those stories have served a purpose and are part of where

27 McKemmish et al., "Distrust in the Archive."

28 Sue McKemmish, "Evidence of Me...in a Digital World," 11; Archival Education Research Institute (AERI), Pluralizing the Archival Curriculum Group (PACG), "Educating for the Archival Multiverse," American Archivist 73 (2011): 69-101.

29 Sapsford and Jupp, Data Collection and Analysis, 89.

30 Etherington, Becoming a Reflexive Researcher, 284. 
we are today ... As well as gathering local stories, narrative research encourages the inclusion of the researcher's story, thus making transparent the values and beliefs that are held, which almost certainly influence the research process and its outcomes. ${ }^{31}$

Tuhiwai Smith wrote of the need for insider self-reflexivity when working with communities, noting that, "The critical issue for insider research is the constant need for reflexivity."32 Self-reflexivity as part of a research methodology also produces some of the more interesting results, as shown by Faulkhead et al. when working with Aboriginal communities in Victoria. ${ }^{33}$ This self-reflexivity has been applied throughout this research, from critiquing relevant archival literature through to my interactions with the community in the case study.

Relationships are an important factor when working with Aboriginal people and communities, and in the building of relationships people tend to exchange information on family connections and backgrounds. This also argues for self-reflection. Faulkhead notes that it is important when working with Aboriginal communities to be able to step back and reflect on the conversations. ${ }^{34}$ You have to take the pain, the politics and the passion along with the research while keeping focus on the research questions in hand. I found this to be an important aspect of self-reflection with this research. More broadly, self-reflection has allowed me to think deeply about the gaps that exist for Aboriginal communities in relation to community archives. The literary warrant analysis and literature review, the case study in Singleton and my experiences working as an Aboriginal archivist together provide a strong foundation for reflection on the archival profession's responses to Indigenous people and archives.

31 Kim Etherington, Becoming a Reflexive Researcher-Using Our Selves in Research. Jessica Kingsley Publishers, 2004, p.27.

32 Linda Tuhiwai Smith, Decolonizing Methodologies: Research and Indigenous Peoples, Zed Books, 1999, p.137.

33 Shannon Faulkhead, Lynette Russell, Diane Singh, and Sue McKemmish, "Is Community Research Possible within the Western Academic Tradition?” in Researching with Communities: Grounded Perspectives on Engaging Communities in Research, Andy Williamson and Ruth DeSouza, eds., Auckland: Muddycreekpress, 2007, p.4.

34 Faulkhead et al., "Is Community Research Possible," 60 and 64; Shannon Faulkhead, "Narratives of Koorie Victoria." Ph.D. dissertation, Centre for Australian Indigenous Studies, Faculty of Arts, Monash University, 2008, p.204. 


\section{The Research Project: Research Design and Methods}

The primary data for my research project came from a case study undertaken in partnership with the Ungooroo Corporation in Singleton in the Hunter Valley region of NSW, and reflection on my own experiences as an Aboriginal archivist as outlined above. The Aboriginal community in Singleton was selected because of my personal and professional links to the community, and my longstanding relationship with its members. My research also drew extensively on secondary sources through a literature review, and literary warrant analysis. The data from these various sources was brought together to explore:

- Community needs in creating a community archive

- Community protocols in relation to the current management of community records/knowledge

- Differences between actual community needs and the perceived needs constructed within traditional archival practice

- Emerging archival theory and practice in the formation of holistic archives that are inclusive of multiple perspectives and local community needs.

The research aimed to provide new insights into the importance of establishing meaningful exchanges of ideas between the archival profession and Indigenous communities so that Aboriginal community protocols can inform archival practice and create new ways of keeping community records. The research project was designed within a framework of ethical community research that respects and responds to the need to return research material to the community for reciprocal benefit, and specifically employing partnership research with the aim of creating outcomes specific to the partner community. As partnership research it was also significant in the development of a community archive in Singleton, in assisting other Aboriginal communities in creating their own community archives relevant to their needs and protocols, and in informing professional discourse about issues and challenges in this research area with the aim of bringing about change. The case study also produced a new literary warrant for the archival profession by identifying a mandate for action in relation to the creation of Aboriginal community archives. Finally, it proposed recommendations for future research in relation to Indigenous people and archives. 


\section{Ethical Considerations for Partnership Research in Indigenous Communities}

In the publication, We Don't Like Research: But in Koori Hands it Could Make a Difference, Salina Bernard from Victorian Aboriginal Community Controlled Health Organisation Inc. notes, perhaps stating the obvious, that:

I found that Aboriginal Community controlled research is the most beneficial research that can be done because it has the involvement, commitment and the participation of the local people which can open up the doors for the research. ${ }^{35}$

Another way of achieving community participation is through partnership research where both the researcher and community representatives work together to achieve shared outcomes. This is the approach taken with the Singleton community, an approach that also respects and takes guidance from the Australian Institute of Aboriginal and Torres Strait Islander Studies (AIATSIS) Guidelines for Ethical Research. The guidelines state that:

It is essential that Indigenous peoples be participants in any research project that concerns them, sharing an understanding of the aims and methods of the research, and sharing the results of this work ... The principles of the Institute's Guidelines for Ethical Research in Indigenous Studies are founded on respect for Indigenous peoples' inherent right to self-determination, and to control and maintain their culture and heritage. ${ }^{36}$

Research output is more meaningful because it is based on community involvement, commitment and participation. It involves "reciprocal responsibilities" where the community and academic both benefit from the sharing of knowledge and research collaboration, ${ }^{37}$ and where Indigenous perspectives

35 Victorian Health Koori Health Research and Community Development Unit, "We Don't Like Research - But in Koori Hands It Could Make a Difference." Melbourne: VicHealth Koori Health Research and Community Development Unit \& University of Melbourne (2000): 1-32.

36 Australian Institute of Aboriginal and Torres Strait Islander Studies (AIATSIS), “Guidelines for Ethical Research in Indigenous Studies,” 2011, http://www.aiatsis.gov. au/research/docs/ethics.pdf.

37 Michael Christie, "Yolngu Studies: A Case Study of Aboriginal Community Engagement," Gateways: International Journal of Community Research and Engagement 1 (2008): 31-47. 
permeate the research methods. ${ }^{38}$ Reciprocal responsibilities or reciprocity within research are outlined by the National Health and Medical Research Council Australia in the following way:

In the research context, reciprocity implies inclusion and means recognising partners' contributions, and ensuring that research outcomes include equitable benefits of value to Aboriginal and Torres Strait Islander communities or individuals.

... Reciprocity requires the researcher to demonstrate a return (or benefit) to the community that is valued by the community and which contributes to cohesion and survival. ${ }^{39}$

There is a close linkage between the research process and the potential for this information to be used by the community for community development and social change. ${ }^{40}$ The research design used in my research project recognised this link, whilst taking on the challenge posed by McKemmish et al. to archival researchers: “... experiment with inclusive and innovative research designs that respect, empower and facilitate the full and equal participation of the communities engaged in the research." ${ }^{.11}$ Ask First: A Guide to Respecting Indigenous Heritage Places and Values stresses the importance of Aboriginal participation in discussions about Aboriginal land management issues. In the guide, Charley notes that:

By using this guide and working together, we can ensure that the rights and interests of Indigenous people in maintaining their heritage is accepted and respected. I have no doubt that in doing so, all Australians will benefit. ${ }^{42}$

38 Lester-Irabinna Rigney, "Internationalization of an Indigenous Anticolonial Cultural Critique of Research Methodologies: A Guide to Indigenist Research Methodology and its Principles", Wicazo Sa Review 14 no.2 (1999): 109-121.

39 National Health and Medical Research Council (Australia), Values and Ethics: Guidelines for Ethical Conduct in Aboriginal and Torres Strait Islander Health Research, The Council, 2003.

40 John Henry, Terry Dunbar, Allan Arnott, Margaret Scrimgeour, Sally Matthews, Lorna Murakami-Gold and Allison Chamberlain, "Indigenous Research Reform Agenda”, Rethinking Research Methodologies. Casuarina, NT: Cooperative Research Centre for Aboriginal Health, 2002.

41 Sue McKemmish, Anne Gilliland-Swetland and Eric Ketelaar, "Communities of Memory," 146.

42 Australian Heritage Commission, "Ask First: A Guide to Respecting Indigenous Heritage Places and Values," Canberra: Australian Heritage Commission, 2002. 
My rationale for using a community-based partnership research approach follows this guidance. Working with an Aboriginal community with all rights and interests respected makes it possible to achieve the aim of creating research that benefits the partner community, the researcher, and the broader Australian community. As an Aboriginal researcher within the field of archival science, I also believe that the more researchers and communities that engage in partnerships, the greater the likelihood of meeting McKemmish et al.'s challenge.

\section{Community-centred Research Design}

I employed a research methodology that seeks to be respectful to both the Aboriginal community and to the academic traditions of archival science. A community-centred research design set the landscape for creating an approach that balanced the needs of both community and academia and guided the way in which I conducted the research with the community, for example, in making sure that community aspirations were a core part of the questions being researched. Similarly, the research methods used, i.e., the techniques and tools for data collection and analysis, were also designed to be respectful of the community and encourage safe and open discussions. My research design responded to the concerns raised by Aboriginal communities about ethically based research being undertaken with communities. My aim was to undertake research in partnership with a community for mutual benefit rather than conducting research about a community. The methodology was guided by two main principles. The first principle related to designing research in which Aboriginal people actively participate and which results in valuable insight into community perceptions on archives. Secondly, the research aimed to be transformative, to provide new perspectives and insights into archival literature that can be utilised to create change for Aboriginal communities and archives.

In community-centred research, the research outcomes are designed to assist the community in meaningful ways. This could be to lobby the local council, write funding applications, or draw together material significant to the community - as my research did by providing a basis for developing a community archive. As Faulkhead notes:

The premise or intention of community research is to do no harm, physical or emotional, to the community being researched, and to 
produce outcomes that benefit the community. It is a research type that is respectful of the community and the knowledge they have. ${ }^{43}$

The methods of data collection and analysis employed included a case study approach, literary warrant review, literature review and self-reflection.

In accordance with the conditions they placed on agreeing to be part of the research, the results of the research were returned to the Aboriginal community in Singleton. This approach is an important aspect of any community research. Gilliland and McKemmish reinforce the importance of community involvement in the research process, and the need to negotiate ownership of the data created:

Working with communities, particularly vulnerable communities, brings into play a range of ethical considerations including what constitutes ethical research behaviour in terms of the culture and values of the community involved, and issues relating to the ownership of data gathered during the research, access and intellectual property rights, and the appropriation of traditional knowledge through unethical research practice. ${ }^{44}$

I have maintained contact with the Ungooroo Corporation through continued discussions about archives. The draft results from the case study were returned to Ungooroo for feedback, additions, changes and validation of what was reported. Sapsford and Jupp identify this method of "respondent validation" as an important part of the data analysis phase of research that ensures correct interpretation of the data collected. ${ }^{45}$ The final research outcomes were returned to the community on completion of the research and they will be further developed to assist the community in creating an Aboriginal community archive. Discussions on how this will be achieved continue with the Singleton Aboriginal community. The community will also be able to use the research outcomes to support future applications and research in developing their community archive. This approach was in line with my approved University ethics application for research with the community.

43 Faulkhead, "Narratives of Koorie Victoria."

44 Anne Gilliland and Sue McKemmish, "Building an Infrastructure for Archival Research," Archival Science 4 nos.3-4 (2004): 149-197.

45 Roger Sapsford and Victor Jupp, eds, Data Collection and Analysis, Thousand Oaks, CA, Sage, 2006, p.91. 


\section{The Case Study - The Aboriginal Community in Singleton}

Case study methods assist in broadening understandings of "real life" situations and in investigating research questions in relation to social phenomena while retaining the holistic and meaningful characteristics of the inquiry. ${ }^{46}$ An in-depth case study can assist in providing valuable insights into relationships and processes and can: “... unravel the complexities of a given situation. ${ }^{37}$ Case study research can also examine an individual community, institution or process. ${ }^{48}$ This research worked with a specific institution within a local Aboriginal community, the Singleton Aboriginal community. Community members were asked, through focus group and other discussions, about their perceptions of archives and their archival needs. Focus groups can draw together members of the community to discuss their collective opinion on a topic, ${ }^{49}$ and provide an opportunity for open-ended questions to be asked by both researcher and community members. Community needs and attitudes can thereby by identified..$^{50}$ This line of enquiry allows for new insights to be generated in relation to the research question. ${ }^{51}$

According to the Wonnarua dreamtime, the Hunter Valley was created by the great spirit Baiame (Byamee). Before Baiame there was nothing, everything was sleeping. Baiame awoke and created everything, the mountains, plains, rivers and every living thing. ${ }^{52}$ The Wonnarua are the traditional owners of the Singleton area in the Hunter Valley. Wonnarua descendant and author, James Wilson-Miller, has written an important history of the area, Koori: A Will to Win (1985). Miller outlines the history of his family, and the strength and resilience to maintain the culture of the wider Aboriginal community of Singleton. Many other Aboriginal people, who are not traditional owners, also call Singleton home. While there are a number of Aboriginal community organisations in Singleton, the main Corporations are Ungooroo and Wonnarua Nation. The Ungooroo Corporation began to

46 Robert K. Yin, ed, Case Study Research: Design and Methods, Vol. 5. Thousand Oaks, CA: Sage, 2003,p.2.

47 Martyn Denscombe, The Good Research Guide for Small-scale Social Research Projects, Maidenhead, Berks.: Open University Press, 2007, p.37.

48 Gilliland and McKemmish, "Building an Infrastructure for Archival Research," 183.

49 Denscombe, The Good Research Guide, 179.

50 Gilliland and McKemmish, “Building an Infrastructure for Archival Research,” 186.

51 Yin, Case Study Research, 2; 90.

52 Wonnarua Nation Aboriginal Corporation, Wonnarua Nation website, http://www. wonnarua.org.au/index.html. 
develop programs in 1994 that benefit the local Aboriginal community. ${ }^{53}$ The Wonnarua Nation Corporation was formed in 1999 to represent the traditional landowners of the area. ${ }^{54}$ The Ungooroo Corporation and Wonnarua Nation Corporation are both actively engaged in discussions about the management of local cultural heritage. The Ungooroo Corporation has a strong focus on developing art programs within the community and has the core value and philosophy of, "commitment to, and respect for, our culture and heritage (past, present and future)." 55 The Wonnarua Nation Corporation is actively involved in working with the Australian Museum for the return of the Morrison Collection of local artefacts. ${ }^{56}$

The demographics of Singleton include a population of approximately 22,000 people. The Aboriginal population of the town is approximately $2.2 \%$ of this population, which is slightly higher than the NSW average of 1.9\%. ${ }^{57}$ Mining and defence are two major employment sectors in Singleton. The town maintains it historical roots in farming and it is in close proximity to the wine industry in the Hunter Valley.

\section{Data Collection}

Community consultation identified the Ungooroo Corporation as the most appropriate organisation to represent the needs of the Singleton Aboriginal community regarding archival issues. Determining who can speak for and represent the interests of a community can be a challenging task. It is important to take the time to speak to a number of community organisations and representatives to ascertain who has the authority to speak on behalf of community interests. Input should be sought from a number of key community representatives and organisations about the best fit for the research. Through the Ungooroo Corporation's network of members, Aboriginal community representatives who had used archival services and/or Aboriginal knowledge were identified as well as Aboriginal Elders who were holders/custodians of Aboriginal knowledge. I visited the Ungooroo Corporation prior to organising the focus group to introduce myself and to present and discuss

53 Ungooroo Aboriginal Corporation, Ungooroo website, http://www.ungooroo.com.au/ Files/aboutus.html.

54 Wonnarua Nation website.

55 Ungooroo website.

56 Australian Museum and Wonnarua People, "Wonnarua People Visit the Collection," Wonnarua.

57 Singleton Tourism, "The Joe Governor Story, Brochure on the History of Joe Governor," http://121.50.208.46/singleton/Joe\%20Governor.pdf. 
my proposed research with appropriate staff. This meeting was important in building a relationship with Ungooroo. It also helped in finding out the Corporation's research-related needs whilst identifying my own.

In line with my approved ethics application for research, I consulted with the Ungooroo Corporation as to the best way to approach and invite community members to attend the focus group. I provided copies of my Explanatory Sheet for Focus Group Participants and an information sheet on Discussion topics for Focus Group to Ungooroo and stamped envelopes for circulation to the community prior to the day (See Appendix). The Ungooroo Corporation then circulated a flyer to advertise the event to community members.

The focus group was held on Thursday 3 June 2010 at Ungooroo in a comfortable setting over morning tea, and included staff from Ungooroo and members of the local Aboriginal community. There were some visitors at the beginning of the session who wanted to attend, but unfortunately they could not stay for the focus group discussion. This illustrated the need for further engagement to be undertaken with the community in the future through individual interviews since unfortunately additional interviews were outside the scope of the research at the time. To begin the meeting I gave some background information about who I am, and what I hoped to achieve. The broad questions were outlined on the information sheet, and I asked for any questions, concerns or ideas about the planned method and/ or the research itself. I explained that I would be taking notes from the discussions and would use these as a means of data gathering to identify the group's broad perceptions of archives. Consent forms were provided to the group, and contents explained, before they were signed by participants (see Appendix One: Explanatory Sheet and Discussion Topics for further details).

The conversations were initiated by the questions in the Discussion Topics for Focus Group (Appendix One), and grew into a discussion about the desire of the community to create a community archive. The nature of the conversations drifted off topic from time to time to discuss other aspects relating to the community. As is often the case, discussions about archives led to questions about collections and where information is held, where to access family history information, and how to go about it. The discussions that were specific to individuals, or were outside the scope of this research were not included in the notes or the data for the research project. Throughout the focus group I took the time to reiterate the opinions and sentiments that I was hearing to ensure that I had captured them correctly. This also allowed the focus group to reinforce where there was consensus on the identified themes. As 
part of the ethics process, participants were given the option to be identified or de-identified in the research. This option was included to ensure that participants could provide input anonymously if they wished, or alternatively, could be acknowledged for their input if they so desired. Negotiating these ethical concerns built a more trusted environment for conversation. All the group discussions belong to the entire group. The only participants who have been individually identified are community Elder Irene (Rene) Molineaux and community member Jenny Campbell. I conducted a follow-up interview with Rene because of her passion for researching family history.

\section{Data Analysis}

Aboriginal participation in the data gathering and analysis processes, and in the presentation of the findings in a way that was meaningful to the community were integral to the research. In analysing the data collected from the focus group, I was able to draw out and identify themes around community needs and perceptions of the archives, utilising diagrams to report and communicate the areas identified in the focus group (see Figure 30.1). This technique has benefits as it visually represents the views of the focus group, and provides a framework for further discussions to take place to expand these themes and discuss protocols surrounding each of the areas. It also has the potential benefit for the community of being a mechanism for promoting a community archive, e.g., in the form of a poster, or on their web site. The diagrams are also helpful in illustrating the holistic and connected nature of the perceptions of the community archive. These diagrams could also be shared with other Aboriginal communities to assess the relevance to their community perceptions and needs.

Data collected from the focus group revealed the political nature of the research question, and the ever-present discussions about past histories of dispossession and removal of Aboriginal children from their families. It also identified a desire from the community to access records and then bring copies of records held in archival institutions back into community settings for discussion and debate. In addition, it identified the need for relationships to be built between archival institutions and community archives to deepen the context and understanding of existing records. 
Figure 30.1: Aboriginal Community Archive Model

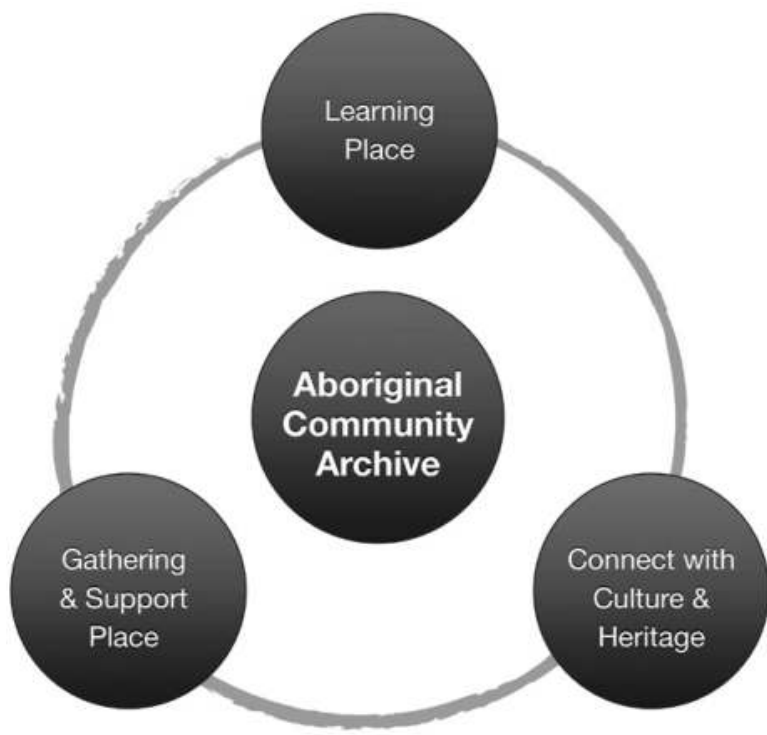

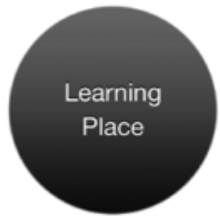

$\longrightarrow$ Teaching culture \& history

$\longrightarrow$ Educational tourism

$\longrightarrow$ Identity, Language revival

$\longrightarrow$ Community authority

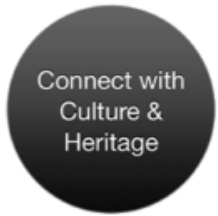

$\longrightarrow$ Gathering, managing \& preserving history

$\longrightarrow$ Repatriation of cultural material

$\longrightarrow$ Community perspectives of history

$\longrightarrow$ Acknowledging places
Gathering

\& Support

Place

-

Exhibitions

Connect families Link Up services

$\longrightarrow$ Private space

$\longrightarrow$ Events 
Figure 30.2: Archival Institutions Working in Partnership with Aboriginal Community Archives

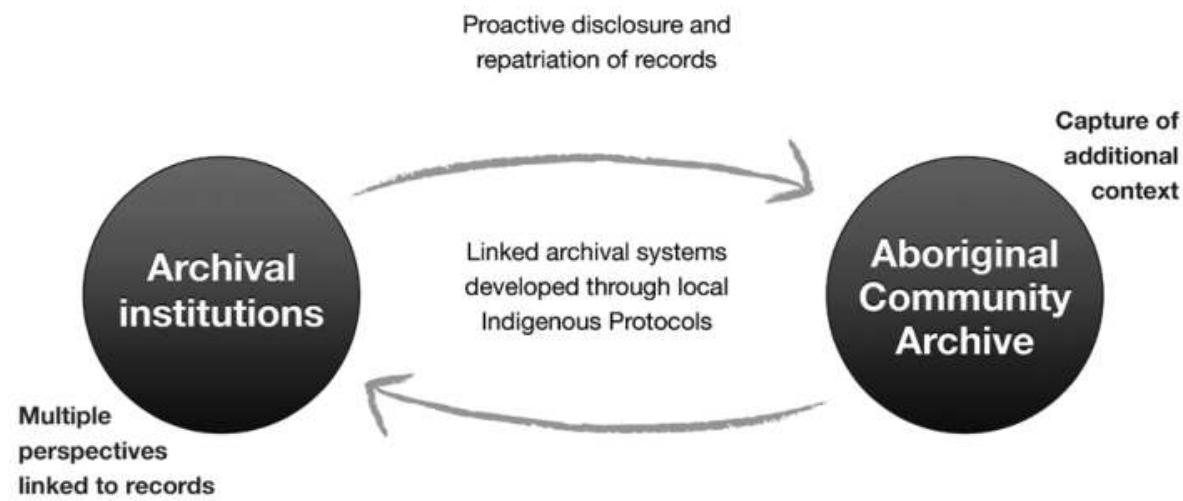

Additional context integrated into archival institution

The data collected relating to Figure 30.2 identified a number of key themes. These themes included: the need for archival systems to be developed to capture responses to records so that multiple perspectives can be linked back to records held in archival institutions; the need for archival institutions to develop relationships and mechanisms to be able to respond to community needs and aspirations for managing existing archival collections; and finally the need for Aboriginal community archives to develop appropriate governance structures to manage and oversee community archives so that rich partnerships are built with archival institutions.

\section{Evaluation of Research Methods}

How effective was my approach to ethical community research, research design and methods in addressing my research question? Undertaking a case study within a community-centred participatory approach was an excellent way of gaining a rich understanding of the importance of records for the Aboriginal people in Singleton, their expressed desires to have material relating to their cultural heritage and history available for ongoing engagement within their own community, and the role they envisaged for a community archive.

The literary warrant analysis provided mandates for such action, particularly the UN Declaration on the Rights of Indigenous Peoples; the ASTILIRN 
Protocols, the Protocols for Native American Archival Materials, and the Trust and Technology Project's Statement of Principles and Position Statement on Human Rights, Indigenous Communities and the Archives emanating from the Trust and Technology Project. ${ }^{58}$ It should no longer be the case that Aboriginal people locate records "by chance." Communities should be informed of resources that relate to them. If reciprocal relationships are established and maintained with Aboriginal communities, an Aboriginal community archive could update and reinvigorate records held in archives and the voices and narratives of Aboriginal people could be returned to the institutional archives and new layers of meaning recorded alongside official government records.

The literature review highlighted the importance of shared spaces being created, based on mutual respect and understanding, between the archival profession and Aboriginal communities. In particular, it underscored how the archival profession and Aboriginal communities can work together to find archival solutions that better meet community needs and respect community protocols. It was also important to look at the relevant literature as a source of data that supported the case study in my research project. My review included archival research; related literature that provides contextual background to the importance of archives for Aboriginal people; literature about the type of Aboriginal archival services currently provided by archival institutions, and the relationships between the archival profession and Aboriginal communities; and the broader archival and Indigenous studies literature, including writings that form part of the postmodern and postcolonial discourse.

Self-reflection has allowed me to think deeply about the gaps that exist for Aboriginal communities in relation to their relationship with archival institutions and the dearth of community archives. This was essential for understanding the archive as a political tool, and records as instruments of control and oppression of the Aboriginal community through the operation of the Aborigines Protection and Welfare Boards. Self-reflection pointed to the need to build strong and ongoing relationships between archival institutions and Aboriginal communities that would assist archives in proactively disclosing information to the so-called subjects of records.

The focus group technique proved to be very effective for collecting and analysing data relating to the part of my research question that focused on

58 McKemmish et al., "Australian Indigenous Knowledge and the Archives;" McKemmish, Faulkhead, and Russell, "Distrust in the Archive." 
Aboriginal community needs and protocols for archives, especially when combined with graphic representations and communication of the outcomes.

An example of the use of diagrams to represent and communicate community perceptions and needs was provided in Figure 1, which presents an Aboriginal community archive model. A further example was provided in Figure 2, which represents the types of relationships that need to be formed with archival institutions for the community to provide feedback and guidance and advice in relation to local protocols. This representation is informed broadly by the case study, the literary warrant analysis and literature review, and self-reflection on my own deep immersion in working with Aboriginal communities and archives in NSW over the past decade.

The literary warrant analysis and literature review, the case study in Singleton and my experiences working as an Aboriginal archivist provided a strong foundation for reflecting on the archival profession's responses to Aboriginal people and archives. Three main themes were identified through this research in terms of the nature of the proposed Aboriginal community archive. It should be:

- A learning place,

- A gathering and support place, and

- A place to connect with culture and heritage.

\section{Conclusion}

I believe that the establishment of Aboriginal community archives could provide a place for sustained and transformed engagement between those formerly regarded as the subject of the record and the record itself, and in so doing could provide Aboriginal people with a suite of rights in records beyond access rights. The history of Aboriginal people in NSW uncovers many issues relating to how records were created, and how they are accessed, kept and used in contemporary contexts. Building Aboriginal community archives provides many opportunities, including a strengthening of relationships between the archival profession and Aboriginal communities, to share the diverse stories of the history of the Australian nation.

I would argue that most exchanges between archival institutions and communities occur within traditional archival frameworks that give priority to accessing material rather than to building ongoing engagement between Aboriginal communities and the archives. Aboriginal responses to the archive and archival science encourage a rethinking of protocols around 
access and use of records. Although the idea of refiguring the archive presents challenges for traditional theory and practice, it also provides enormous potential for the acknowledgement of Indigenous human rights and records, and the provision of a place and space of healing for Aboriginal and Torres Strait Islander communities.

Following an ethical, community-centred approach, integrating an appropriate set of methods and techniques in my research design, and engaging in deep, reflective research practice, I hope to have made a contribution to new and emerging discourses that might frame opportunities for Aboriginal and Torres Strait Islander communities to work together with the archival profession to create sustainable community archival programs based on Aboriginal protocol and local needs.

\section{Acknowledgements}

I would like to acknowledge and pay my respects to Elders past and present from Sydney to Singleton and Port Stephens - and to all of the wonderful communities that I have visited since working as an Archivist in NSW. I pay my respects also to the people who are documented in the State Archives of New South Wales. I thank the Ungooroo Corporation for their time and support in hosting the focus group in Singleton and for being a part of the discussions about community archives. Thank you to Rene Molineaux, Denise Hedges, Jenny Campbell and Taasha Layer for making me feel welcome at Ungooroo. I hope that we can continue working on a project together for the establishment of a community archive in Singleton.

Since writing this thesis in 2010, the Singleton community lost an important Elder in the community with the passing of Rene Molineaux. Her obituary stated that, "Aunty Rene Molineaux will be remembered as a great advocate for Aboriginal people and a passionate educator". ${ }^{59}$ Rene was incredibly supportive to me - from the time I was in secondary education through to undertaking this Master's research. I will be forever grateful for her time, her knowledge and spirit in educating me about Aboriginal perspectives and issues.

59 Sam Rigney, “OBITUARY: A Fighter for Education of Her People,” 2011, http://www. theherald.com.au/story/440592/obituary-a-fighter-for-education-of-her-people/. 


\section{Appendix: Explanatory Sheet and Discussion Topics}

\section{Creating an Aboriginal Community Archive in New South Wales}

\section{Explanatory Sheet for Focus Group Participants}

Dear potential participants,

My name is Kirsten Thorpe and I am undertaking my Masters of Information Management and Systems (MIMS) (Professional) through the Faculty of Information Technology. My supervisors are Professor Sue McKemmish, Faculty of Information Technology (FIT) and Shannon Faulkhead, Centre for Australian Indigenous Studies (CAIS), Monash University.

My research question is:

How can the archival profession assist Aboriginal communities to create sustainable community archival programs based on Aboriginal protocol?

Aboriginal individuals and communities are heavily documented in archival collections throughout Australia. In New South Wales (NSW) significant material has been collected on Aboriginal communities that document both personal, family and community histories. This material is predominately created by non-Aboriginal people about Aboriginal people.

Over the past 20 years the Australian archival profession, and recordholding agencies represented in the profession, have made significant progress in increasing access to collections and participating in discussions about Aboriginal issues with archival institutions and the records they hold. As the number of Aboriginal people who engage with archival institutions and the profession increases so does the need for Aboriginal participation in decision making and input into the creation of archival systems and processes that support Aboriginal community needs.

The research, Creating an Aboriginal community archive in New South Wales will be a case study of how ideas can be exchanged between the archival profession and an Aboriginal community in relation to the management of community records. 
I am currently organising focus groups for this research with Aboriginal community representatives who have used archival services and/or Indigenous knowledge, and Aboriginal Elders who are holders/custodians of Aboriginal knowledge.

You have been approached as a potential focus group participant. The focus group will be around your experiences of being someone who may or may not have any knowledge of conventional archival services but has Aboriginal knowledge. If you agree to take part in this focus group, there will be time available prior to the focus group to outline the processes and to answer any questions that you may have.

\section{Focus Group provisions}

Participation in the focus group and the project is voluntary. The participant can withdraw at any stage prior to the completion of the thesis.

Confidentiality - it is preferred that the participants be listed in the thesis, however if confidentiality is requested it will be respected in accordance with the consent from.

Participants will receive a copy of the agreed transcript or notes from the focus group.

The focus group discussion will only be audio recorded if agreed to by all participants.

The researcher's copy of the audio recording, and transcript, or research notes will be accessed and used in accordance with the participant's consent form.

At the completion of the research, the researcher's copy of the audio recording and transcript will be stored securely at Monash University for a period of five years at which time they will be either destroyed, or placed within the Koorie Heritage Trust Inc. Collection if agreed to by all participants.

The participant may receive a copy of the audio recording if they wish and provided other members of the focus group consent to members of the group receiving copies.

Any access, other than the researchers, of the researcher's copy of the audio recordings and transcripts will only be given with permission of the participant. 


\section{Creating an Aboriginal Community Archive in New South Wales}

\section{Discussion Topics for Focus Group}

\section{Community Needs}

How might an archive assist the local community? What would be the perceived benefits?

How does the community see an archive working? Who would store information in the archive?

Who would manage the information?

Does an archive already exist in the community and how is that managed? Who would be the key stakeholders of the local Aboriginal community archive? Who would be the main users of the local Aboriginal community archive?

What benefits could an archive bring to the community?

What history of the area may have an impact on the management of the archive?

\section{Community Protocols}

What structures would be put in place to ensure that the archive would be managed in a way that is based on community protocols?

What protocols should be considered in relation to the management of the archive?

What protocols should be considered in relation to access to material held in the collection?

Would some of the material in the collection be considered sensitive? And if so, how would this be managed in terms of Aboriginal protocol? 


\section{References}

Aboriginal and Torres Strait Islander Library and Information Resource Network (ATSILIRN). ATSILIRN Protocols, 2005, http://www1.aiatsis.gov.au/atsilirn/ protocols.atsilirn.asn.au/index0c51.html?option=com_f.

Anderson, Jane. "Access and Control of Indigenous Knowledge in Libraries and Archives: Ownership and Future Use," in Proceedings from Correcting Course: Rebalancing Copyright for Libraries in the National and International Arena, Columbia University, 2005.

Archival Education Research Institute (AERI), Pluralizing the Archival Curriculum Group (PACG). "Educating for the Archival Multiverse." American Archivist 73 (2011): 69-101.

Australian Heritage Commission. Ask First: A Guide to Respecting Indigenous Heritage Places and Values. Canberra: Australian Heritage Commission, 2002.

Australian Institute of Aboriginal and Torres Strait Islander Studies (AIATSIS). Guidelines for Ethical Research in Indigenous Studies, 2011, http://www.aiatsis.gov.au/research/ docs/ethics.pdf.

Australian Institute of Aboriginal and Torres Strait Islander Studies (AIATSIS). Proceedings of the Symposium on Information Technologies and Indigenous Communities, Canberra July 13 - 15, 2010, http://www.aiatsis.gov.au/research/ symposia/Digi10.html.

Australian Museum and Wonnarua People. "Wonnarua People Visit the Collection." Australian Museum (2009), http://australianmuseum.net.au/movie/Wonnarua-Visit.

Beherendt, Larissa. An Aboriginal Keeping Place (Proposal), http://www.thekeepingplace. org/proposal.html.

Christen, Kimberly. “Opening Archives: Respectful Repatriation.” American Arcbivist 74 no.1 (2011): 185-210.

Christie, Michael. "Yolngu Studies: A Case Study of Aboriginal Community Engagement." Gateways: International Journal of Community Research and Engagement 1 (2008): 31-47.

Denscombe, Martyn. The Good Research Guide for Small-scale Social Research Projects, Maidenhead, Berks.: Open University Press, 2007.

Etherington, Kim. Becoming a Reflexive Researcher - Using Our Selves in Research. Jessica Kingsley Publishers, 2004.

Faulkhead, Shannon. "Narratives of Koorie Victoria." Ph.D. dissertation, Centre for Australian Indigenous Studies, Faculty of Arts, Monash University, 2008.

Faulkhead, Shannon, Lynette Russell, Diane Singh, and Sue McKemmish. "Is Community Research Possible within the Western Academic Tradition?” Williamson, Andy and Ruth DeSouza, eds. Researching with Communities: Grounded Perspectives on Engaging Communities in Research. Auckland: Muddycreekpress, 2007.

First Archivist Circle. Protocols for Native American Archival Materials, 2007, http://www2. nau.edu/libnap-p/protocols.html.

Flinn, Andrew. "Community Histories, Community Archives: Some Opportunities and Challenges." Journal of the Society of Archivists 28 no.2 (2007): 151-176.

Garwood-Houng, Alana. "Protocols: Meeting the Challenges of Indigenous Information Needs." Australian Academic \& Research Libraries 36 no. 2 (2005): 143-151.

Gilliland, Anne J. and Andrew Flinn. "The Wonderful and Frightening World of Community Archives: What Are We Really Talking About?” Keynote address, Nexus, Confluence, and Difference: Community Archives meets Community Informatics: Prato CIRN Conference Oct 28-30 2013, Larry Stillman, Amalia Sabiescu, Nemanja 
Memarovic, eds, Centre for Community Networking Research, Centre for Social Informatics, Monash University. ISBN 978-0-9874652-1-4, http://www.ccnr. infotech.monash.edu.au/assets/docs/prato2013_papers/gilliland_flinn_keynote.pdf. Gilliland, Anne, and Sue McKemmish. "Building an Infrastructure for Archival Research." Archival Science 4 nos.3-4 (2004): 149-197.

Henry, John, Terry Dunbar, Allan Arnott, Margaret Scrimgeour, Sally Matthews, Lorna Murakami-Gold, and Allison Chamberlain. "Indigenous Research Reform Agenda." Rethinking Research Methodologies. Casuarina, NT: Cooperative Research Centre for Aboriginal Health, 2002.

Iacovino, Livia. "Multi-method Interdisciplinary Research in Archival Science: The Case of Recordkeeping, Ethics and Law.” Archival Science 4 nos.3-4 (2004): 267-286.

Ingall, Jennifer. "Moree's Indigenous History Collection to be Part of a New Cultural Exhibition Centre," Australian National Broadcasting Service (ABC) New England Northwest, September 2, 2010, http://www.abc.net.au/local/ stories/2010/09/02/3000606.htm.

Janke, Terri. Our Culture, Our Future: Report on Australian Indigenous Cultural and Intellectual Property. Michael Frankel, Solicitors, 1998.

Ketelaar, Eric. "A Living Archive, Shared by Communities of Records", in Jeannette A. Bastian and Ben Alexander, eds. Community Archives. Facet Publishing, 2009.

Krebs, Allison Boucher. “Native America's Twenty-first-century Right to Know." Archival Science 12 no.2 (2012): 173-190.

Machi, Lawrence A., and Brenda T. McEvoy. The Literature Review: Six Steps to Success. Thousand Oaks, CA: Sage, 2009.

McKemmish, Sue. "Evidence of Me ... in a Digital World," in Lee, Christopher A. ed. I, Digital. Chicago, IL: Society of American Archivists, 2011.

McKemmish, Sue. "Placing Records Continuum Theory and Practice." Archival Science 1 no.4 (2001): 333-359.

McKemmish, Sue, Shannon Faulkhead, Livia Iacovino and Kirsten Thorpe. "Australian Indigenous Knowledge and the Archives: Embracing Multiple Ways of Knowing and Keeping." Archives E̋ Manuscripts 38 no.1 (2010): 27-50.

McKemmish, Sue, Shannon Faulkhead, and Lynette Russell. "Distrust in the Archive: Reconciling Records.” Archival Science 11 no.3-4 (2011): 211-239.

McKemmish, Sue, Anne Gilliland-Swetland and Eric Ketelaar. "Communities of Memory': Pluralising Archival Research and Education Agendas." Archives and Manuscripts 33 no.1 (2005): 146-175.

Monash University Trust and Technology Project. Final Report, 2009, http://www. infotech.monash.edu.au/research/centres/cosi/projects/trust/final-report/

Monash University Trust and Technology Project. "Position Statement on Indigenous Human Rights and the Archives," 2009, http://www.infotech.monash.edu.au/ research/centres/cosi/projects/trust/deliverables/human-rights.html.

Museums and Galleries NSW, Keeping Places and Beyond: Building Cultural Futures in NSW, http://mgnsw.org.au/media/uploads/files/keeping_places_and_beyondnew2.pdf.

Nakata, Martin, Vicky Nakata, Gabrielle Gardiner, Jill McKeough, Alex Byrne and Jason Gibson. "Indigenous Digital Collections: An Early Look at the Organisation and Culture Interface.” Australian Academic \& Research Libraries 39 no.4 (2008): 223-236.

National Health and Medical Research Council (Australia). Values and Ethics: Guidelines for Ethical Conduct in Aboriginal and Torres Strait Islander Health Research. The Council, 2003.

New South Wales. State Records Act 1998, Act No. 17. http://www.austlii.edu.au/au/legis/ nsw/consol_act/sra1998156/. 
O’Neal, Jennifer R. "Respect, Recognition, and Reciprocity: The Protocols for Native American Archival Materials," in Identity Palimpsests: Archiving Ethnicity in the US and Canada, Dominique Daniel and Amalia Levi, eds. (Chicago: Society of American Archivists, 2014), pp.125-142.

O’Neal, Jennifer R. “'The Right to Know': Decolonizing Native American Archives." Journal of Western Archives 6 no. 1 (2015): 2.

Peeters, Lorraine. "The Marumali Program: An Aboriginal Model of Healing." Working Together (2010): 285, http://www.wimmerapcp.org.au/wp-gidbox/uploads/2014/04/ working_together_full_book.pdf\#page=319.

Piggott, Michael. "Archives and Memory," in Archives: Recordkeeping in Society (Wagga Wagga, New South Wales: Charles Stuart University, 2005), pp. 299-328.

Rigney, Lester-Irabinna. "Internationalization of an Indigenous Anticolonial Cultural Critique of Research Methodologies: A Guide to Indigenist Research Methodology and its Principles." Wicazo Sa Review 14 no.2 (1999): 109-121.

Rigney, Sam. “OBITUARY: A Fighter for Education of Her People,” 2011, http://www. theherald.com.au/story/440592/obituary-a-fighter-for-education-of-her-people/.

Roberts, David. “Imperfect Evidence”, Vital Signs Magazine 9, September 2006, http:// www.records.nsw.gov.au/documents/vital-signs/issue-09/Vital\%20Signs\%20Issue\%20 9\%20-\%20Directors\%20Letter.pdf.

Sapsford, Roger, and Victor Jupp, eds. Data Collection and Analysis. Thousand Oaks, CA, Sage, 2006.

Singleton Tourism. "The Joe Governor Story, Brochure on the History of Joe Governor," http://121.50.208.46/singleton/Joe\%20Governor.pdf

Smith, Linda Tuhiwai. Decolonizing Methodologies: Research and Indigenous Peoples. Zed Books, 1999.

State Records NSW. State Records Website, "Board for the Protection of Aborigines", http://investigator.records.nsw.gov.au/Entity.aspx?Path=\%5CAgency\%5C559.

State Records NSW. "Protocols for Staff Working with Indigenous People," 2008, http:// www.records.nsw.gov.au/state-archives/resources-for/indigenous-people/files/ Protocols\%20for\%20Staff\%20Working\%20with\%20Indigenous\%20People.pdf/view

State Records NSW, NRS 1063. "Special Bundles. Report and Recommendations of the Public Service Board on Aborigines Protection, 1940.” [6/4501.1] Appendix C: Aborigines Protection Board, Trust Accounts - Endowee's Balances as at 31st July 1938.

Ungooroo Aboriginal Corporation. Ungooroo website. http://www.ungooroo.com.au/ Files/aboutus.html.

United Nations General Assembly. United Nations Declaration on the Rights of Indigenous Peoples. (Washington, D.C.: United Nations, 2007), http://www.un.org/esa/socdev/ unpfii/documents/DRIPS_en.pdf.

Victorian Health Koori Health Research and Community Development Unit. "We Don't Like Research - But in Koori Hands It Could Make a Difference." Melbourne: VicHealth Koori Health Research and Community Development Unit E University of Melbourne (2000): 1-32.

Wonnarua Nation Aboriginal Corporation. Wonnarua Nation website, http://www. wonnarua.org.au/index.html.

Yin, Robert K., ed. Case Study Research: Design and Methods. Vol. 5. Thousand Oaks, CA: Sage, 2003. 


\title{
Chapter 31
}

\section{EXAMINATIONS OF INJUSTICE}

\section{Methods for Studying Archives in a Human Rights Context}

\author{
Lorraine Dong, Joel A. Blanco-Rivera, Michelle Caswell and \\ Joanna Steele
}

\begin{abstract}
The authors of this chapter are archival researchers who strive to integrate a philosophy of activism and social justice into their research. The social justice approach to archives involves the goals of resource equity, frameworks for understanding oppression, and means for individuals and communities to present their own viewpoints and representations. Portions of the authors' research serve as illustrative case studies of various qualitative methods that can be utilised to examine the role of archives in human rights and social justice activism and research. The case studies, while a reflection of a diversity of research interests, are connected by a concern for conducting research rooted in human rights activism. The authors' research questions and methods aim to identify and acknowledge issues of power and inequity in archival contexts, as well as to encourage the proactive building of archives and archival communities. Each researcher uses triangulation to engage with the complex networks of stakeholders, documents and actions involved in the processes of truth-telling and healing. The qualitative methods discussed include discourse and textual analyses, semi-structured interviews, and participant observation. Such methods are representative of the types of approaches that can be used to situate records, institutions and people in relation to one another and within the human rights context.
\end{abstract}




\section{Introduction}

The social justice approach to archives involves striving for resource equity, frameworks for understanding oppression, and means for individuals and communities to present their own viewpoints and representations. ${ }^{1}$ Social justice can be furthered through archival principles and institutional missions, the records themselves and the activism of archival practitioners, educators, and researchers. Archival educators have begun to address how to implement a social justice framework in the classroom and activism in the field. ${ }^{2}$ Similarly, archival researchers are seeking how to integrate a philosophy of activism in their work. The case studies in this chapter reflect a diversity of geographical and temporal interests, but share a concern for conducting research rooted in human rights activism. The authors' research questions and methods aim to identify and acknowledge issues of power and inequity in archival contexts. Such research resides within a current postmodernist trend in archival studies that acknowledges the power of archives and the archivist and encourages the proactive building of community archives and archival communities. ${ }^{3}$

In his 2005 presidential address at the Society of American Archivists annual meeting, Randall Jimerson underscored that archives are not neutral institutions. Arguing that the record-making process is steeped in economic and social power dynamics, Jimerson points to archival activities as being subject to the same socioeconomic forces. ${ }^{4}$ Unburdened by the untenable

1 Anthony W. Dunbar, "Introducing Critical Race Theory to Archival Discourse: Getting the Conversation Started," Archival Science 6 (2006): 109-129.

2 See Kelvin White and Anne Gilliland, "Promoting Reflexivity and Inclusivity in Archival Education, Research and Practice,” Library Quarterly 80 no. 3 (2010): 231-248; Anne Gilliland, "Neutrality, Social Justice, and the Obligations of Archival Education and Educators in the Twenty-First Century," Archival Science 11 (2011): 193-209; Caswell et al., "Implementing a Social Justice Framework in an Introduction to Archives Course: Lessons from Both Sides of the Classroom," InterActions 8 no. 2 (2012), http://www.escholarship.org/uc/item/2jx083hr.

3 See Frank Upward, "Modeling the Continuum as Paradigm Shift in Recordkeeping and Archiving Processes, and Beyond - a Personal Reflection," Records Management Journal 10 no. 3 (2000): 115-139; Terry Cook, "Archival Science and Postmodernism: New Formulations for Old Concepts," Archival Science 1 (2001): 3-24; Sue McKemmish, "Placing Records Continuum Theory and Practice," Archival Science 1 no. 4 (2002): 333 359; Isto Huvila, "Participatory Archive: Toward Decentralised Curation, Radical User Orientation and Broader Contextualisation of Records Management," Archival Science 8 no.1 (2008): 15-36; Katie Shilton and Ramesh Srinivasan, "Participatory Appraisal and Arrangement for Multicultural Archival Collections," Archivaria 63 (2008): 87-101.

4 Randall C. Jimerson, "Embracing the Power of Archives," Presidential address to the Society of American Archivists, 2005, http://www.archivists.org/governance/ presidential/jimerson.asp\#_ftn42. 
notion of remaining passive and objective caretakers of records, archivists can choose to work within a social justice framework. ${ }^{5}$ Inspired by a speech by historian Howard Zinn in 1970, archivist Gerald Ham called for the "active archivist" who would redress imbalances in the archival record by taking a proactive stance in documenting society. ${ }^{6}$ Archivists such as Verne Harris, Terry Cook and many others have exhorted the field to "embrace the role of memory activist."7 Jimerson urges archivists to think reflexively on their roles as selectors and interpreters of records, and, consequentially, as gatekeepers of what becomes part of our cultural record. Archivists can give voices to those who have been silenced or ignored by dominant groups, and assist in holding leaders and institutions accountable for their actions. By protecting and making documents available, "[a]rchives document and protect the rights of citizens."

Archivists who specialise in human rights work require specific skills and knowledge that will facilitate the social justice mission. The responsibilities of human rights archivists include, but are not limited to, the ability to "ensure historical accountability, retain memory of the victims and survivors, support prosecution, document the extremes of repression, and chronicle the individual's power against the state." Since human rights archivists focus on functioning as facilitators for justice, an understanding of legal recordkeeping and how to maintain the evidential value of records (for burden of proof purposes) is critical. ${ }^{10}$

5 Archivists continue to discuss what is meant by "social justice" within the field. See Wendy M. Duff, Andrew Flinn, Karen Emily Suurtamm, and David A. Wallace, "Social Justice Impact of Archives: A Preliminary Investigation," Archival Science 13 no. 4 (2013): 317-348, which elaborates on "the multiple relationships between archives and social justice" and includes a section on an archival social justice framework.

$6 \quad$ F. Gerald Ham, "The Archival Edge," The American Archivist 38 no. 1 (1975): 6. Cf., Howard Zinn, "Secrecy, Archives and the Public Interest," in The Zinn Reader: Writings on Disobedience and Democracy (New York: Seven Stories, 1997), pp.516-528.

7 Verne Harris, "Ethics and the Archive: 'An Incessant Movement of Recontextualisation,'” in Controlling the Past: Documenting Society and Institutions, Terry Cook, ed. Chicago, IL: Society of American Archivists, 2011, p.346. Cf., Randall Jimerson, Archives Power: Memory, Accountability, and Social Justice, Chicago, IL: Society of American Archivists, 2009; Terry Cook, "What is Past is Prologue: A History of Archival Ideas Since 1898, and the Future Paradigm Shift," Archivaria 43 (Spring 1997): 17-63.

8 Jimerson, "Embracing the Power of Archives."

9 Tom Adami, "Future Perfect? Peacekeeping, Peacebuilding and Archives - The United Nations in Sudan," Journal of the Society of Archivists 30 no. 1 (2009): 20.

10 Tom Adami and Martha Hunt, "Genocidal Archives: The African Context - Genocide in Rwanda," Journal of the Society of Archivists 26 no. 1 (2005): 106. 
All archives are essential for the development of institutional infrastructures since they serve as keepers of the past, and the records they contain have the potential to be used by new communities of users for reconciliatory or cathartic purposes. Helen Forde notes that:

The essential role of the archivist in the prevention of human rights abuse is being recognised increasingly, whether the issue is the aftermath of mass genocide or the injustices suffered by individuals let down by the society in which they live. ${ }^{11}$

Archival activism for social justice is not without ongoing practical and ethical questions, however.

Discussions regarding the extent of archivists' responsibilities for advancing social justice, especially given the tradition of professional neutrality for archivists, have implications for the pedagogical approaches used in archival studies programs. Archival academic Anne Gilliland suggests that in order for graduate students to become activist archivists who mindfully strive for social justice in their scholarly and professional work, there are several skills and characteristics they must develop. ${ }^{12}$ In particular, they must demonstrate self-reflexivity, sensitivity to multiple perspectives, and critical inquiry. They must also have proficiency in their understanding and application of appropriate methodologies and methods to their research; with these skills, they will bring rigour, accountability and an evidence-based approach to their work.

In this chapter, the authors present portions of their own research as illustrative case studies that introduce several different qualitative methods that can be used to examine the role of archives in human rights and social justice activism and research. Joel Blanco-Rivera discusses ongoing research regarding transitional justice in Latin America and the growing recognition of the importance of archives within this area of study. Case study methodology and a triangulation of methods (here, qualitative analysis of textual documents and semi-structured interviews) are highlighted as a means to assess the impact of archives on cases of human rights abuses. Michelle Caswell approaches Khmer Rouge records in Cambodia through a social life of records methodology and a variety of qualitative methods that include participant observation, semi-structured interviews and visual analysis. Her

11 Helen Forde, “'We Must Remember Our Past so that We Do Not Repeat It', Journal of the Society of Archivists 25 no. 2 (2004): 122.

12 Gilliland, "Neutrality, Social Justice, and the Obligations," 200. 
research articulates how the archivist-researcher can enable records to "perform" human rights. Lorrie Dong discusses the discipline of historiography and specifically the social actors approach to critical discourse analysis that was applied as part of a suite of methods to identify the participatory role of records in hegemonic institutional practices. She uses discourse analysis to demonstrate how categories and labels found in old records from a mental hospital are social constructions that had an impact upon patients in the past and continue to be a part of the human rights dialogue today. Finally, Joanna Steele presents her research on how human rights documentation needs are changing archival practices. Using a postcustodial lens, she employs observation, interviews and discourse analysis to study human rights archives and their evolving practices. Figure 31.1 groups the authors' qualitative methods, and provides a brief description of each as applied in these particular studies, including the potential challenges when applying these methods in a human rights context.

Figure 31.1. Qualitative Methods Employed by Authors

\begin{tabular}{|l|l|l|}
\hline Method group & Description & Limitations \\
\hline $\begin{array}{l}\text { Record analysis (textual, } \\
\text { visual, critical discourse) }\end{array}$ & $\begin{array}{l}\text { The in-depth study of the } \\
\text { texts, forms, structures, } \\
\text { and purposes of records } \\
\text { in order to discern their } \\
\text { themes, discursive practices, } \\
\text { instrumentality, performative } \\
\text { role, the import of their } \\
\text { silences, and their impact. }\end{array}$ & $\begin{array}{l}\text { Privacy restrictions; ethical } \\
\text { considerations of opening } \\
\text { sensitive records; potential } \\
\text { language barriers; institutional } \\
\text { perspective of records }\end{array}$ \\
\hline Observation (participant) & $\begin{array}{l}\text { A type of field research to } \\
\text { examine directly human } \\
\text { interactions with records }\end{array}$ & $\begin{array}{l}\text { Researcher needs to be reflexive } \\
\text { of her presence; access may be } \\
\text { limited/denied due to researcher's } \\
\text { status in studied community }\end{array}$ \\
\hline $\begin{array}{l}\text { Interviews } \\
\text { (semi-structured) }\end{array}$ & $\begin{array}{l}\text { Conversations in which } \\
\text { the researcher seeks out } \\
\text { interviewees' viewpoints } \\
\text { regarding certain topics }\end{array}$ & $\begin{array}{l}\text { Interviewee's responses might } \\
\text { be affected by his relationship } \\
\text { with the interviewer; interviewee } \\
\text { anonymity may be required/ } \\
\text { requested; ethical implications } \\
\text { of maintaining informed consent } \\
\text { throughout research process }\end{array}$ \\
\hline
\end{tabular}

To elucidate the relationships between social justice and archives, the authors all found it necessary to analyse the records that are central to the human rights work being done. However, the records, which have organisational origins (e.g., governmental, legal, institutional) cannot tell the whole story, so the four contributors to this chapter turned to other methods as well. Besides records analysis, they conducted interviews and/or observations 
in order to collect and analyse other perspectives from individuals actively interacting with the archival collections. When archival researchers employ a variety of methods, they are able to better address the past, present, and future work of records in social justice contexts.

\title{
Studying the Roles of Archives in Transitional Justice
}

\author{
Joel A. Blanco-Rivera
}

Since the early 1980s, the study of transitional justice has emerged as a multidisciplinary field of scholarship that addresses important questions about societies' transitions from authoritarian regimes and other violent conflicts. ${ }^{13}$ Political scientists such as Guillermo O'Donnell, Philippe C. Schmitter, and Samuel P. Huntington have discussed how these transitions are characterised by negotiations and politics. ${ }^{14}$ Scholars in legal studies have discussed whether retributive justice is feasible during transitions, ${ }^{15}$ while others have analysed the implementation of truth commissions. ${ }^{16}$ Additionally, the field has addressed the topic of memory and trauma. ${ }^{17}$

13 A very good definition of the term transitional justice is provided by the Encyclopedia of Genocide and Crimes Against Humanity, Detroit, MI: Macmillan Reference, 2004, p. 1045 , which defines the term as "a field of study and inquiry focused on how societies address legacies of past human rights abuses, mass atrocity, or other forms of severe social trauma, including genocide or civil war, in order to build a more democratic, just, or peaceful future." For a historical background of the field of study see Paige Arthur, "How "Transitions" Reshaped Human Rights: A Conceptual History of Transitional Justice," Human Rights Quarterly 31 (2009): 321-367. Also see Jon Elster, Closing the Books: Transitional Justice in Historical Perspective, Cambridge: Cambridge University Press, 2004.

14 See Guillermo O'Donnell and Philippe C. Schmitter, Transitions from Authoritarian Rule: Tentative Conclusions about Uncertain Democracies, Baltimore, MD: John Hopkins University Press, 1986 and Samuel P. Huntington, The Third Wave: Democratization in the Late Twentieth Century, Norman, OK: University Oklahoma Press, 1991.

15 See Ruti G. Teitel, Transitional Justice, Oxford: Oxford University Press, 2000; José Zalaquett, "Balancing Ethical Imperatives and Political Constraints: The Dilemma of New Democracies Confronting Past Human Rights Violations," Hastings Law Journal 43 (August 1992); Juan Méndez, Accountability for Past Abuses, Working Paper \#233, Notre Dame, IN: The Helen Kellogg Institute for International Studies, 1996.

16 See Priscilla B. Hayner, Unspeakable Truths: Transitional Justice and the Challenge of Truth Commissions, 2nd ed., New York: Routledge, 2011; Mark Freeman, Truth Commissions and Procedural Fairness, Cambridge: Cambridge University Press, 2006.

17 This literature includes: Jeffrey K. Olick, The Politics of Regret: On Collective Memory and Historical Responsibility, New York: Routledge, 2007; Elizabeth Jelin, State Repression and the Labors of Memory, Minneapolis, MN: University of Minnesota Press, 2003; Paul Williams, Memorial Museums: The Global Rush to Commemorate Atrocities, Oxford: Berg, 2007. 
While the scholarship of transitional justice has grown significantly, it was not until the late 2000s that academics began to address more forcefully questions about research methods. Most of the literature, particularly early scholarship, focused on anecdotal evidence. Nevertheless, more recent works have applied specific research methods. Recognising the need to study the impact of transitional justice and the importance of addressing the questions about the different outcomes of the implementation of mechanisms, Assessing the Impact of Transitional Justice: Challenges for Empirical Research ${ }^{18}$ presents essays from fourteen scholars who provide their analysis of the field. Methods discussed in the book include oral history, ${ }^{19}$ analysis of confessions from perpetrators of human rights abuses, ${ }^{20}$ and comparative qualitative research. ${ }^{21}$ In one of the essays, Neil Kritz discusses how empirical research contributes to the understanding of policy making in countries under transition. Furthermore, he highlights topics from the field of transitional justice in need of additional research, one of which is "public access to security files." ${ }^{22}$ Because of the important role of records in the implementation of transitional justice mechanisms, the contribution of archivists in these conversations is fundamental.

Research topics that explore the social and political implications of records and recordkeeping have been part of the diverse number of topics studied by archivists since the mid-1990s. ${ }^{23}$ Case study methodology has been widely applied to the field of archival studies as one of the best approaches to study records and recordkeeping. Archival literature about accountability also builds mainly from case studies. A good example is the collection of essays,

18 Hugo Van Der Merwe, Victoria Baxter and Audrey R. Chapman, eds. Assessing the Impact of Transitional Justice: Challenges for Empirical Research, Washington, DC: United States Institute of Peace, 2008.

19 Matilde González, "Local Histories: A Methodology for Understanding Community Perspectives on Transitional Justice," in Van Der Merwe et al., Assessing the Impact of Transitional Justice: $295-311$.

20 Leigh A. Payne, "Confessional Performances: A Methodological Approach to Studying Perpetrators' Testimonies," in Van Der Merwe et al., Assessing the Impact of Transitional Justice: 227-247. Payne also wrote a book on this topic. See Leigh A. Payne, Unsettling Accounts: Neither Truth nor Reconciliation in Confessions of State Violence, Durham, NC: Duke University Press, 2008.

21 Victor Espinoza Cuevas and María Luisa Ortiz Rojas, "Practical Considerations in Comparative Research: Approaching Problems from the Bottom and from Within," in Van Der Merwe et al., Assessing the Impact of Transitional Justice, 313-324.

22 Neil Kritz, "Policy Implications of Empirical Research on Transitional Justice," in Van Der Merwe et al., Assessing the Impact of Transitional Justice: 19.

23 Anne Gilliland and Sue McKemmish, "Building an Infrastructure for Archival Research,” Arcbival Science 4 (2004): 152. 
Archives and the Public Good, in which Richard Cox and David A. Wallace recognise the importance of closer study of particular cases: "Accountability as an idea suggesting the importance of records, needs to be explored in greater detail so that all its ramifications are understood." ${ }^{24}$ Similarly, Wallace underscores the need for close study of both context and content of records to better assess the relations between archives and justice:

... I believe that the connection between archives and justice depends, in part, upon a sharp examination of what types of archives survive, which do not, and why this is the case. It also depends in part on sharp examinations of 'document viewing': Who gets to create, edit, use, withhold, and destroy? And to what effect? ${ }^{25}$

Archivists can also use this approach to assess how the historical record can open the doors to mechanisms of accountability for human rights violations, regardless of whether the mechanism be criminal prosecution, truth commission or the work of human rights organisations and social movements.

Within the case study methodology, what specific methods can be implemented in this context? One strategy can be triangulation between qualitative analysis of textual documents and semi-structured interviews. Although the former method has some similarities to content analysis, particularly regarding categorisation, qualitative analysis of content goes beyond quantitatively extracting particular themes or words. Yan Zhang and Barbara Wildemuth explain that this qualitative approach "pays attention to unique themes that illustrate the range of the meanings of the phenomenon."26 Using this approach, the following general questions can be explored: what do these documents tell us about the role of archives in investigations of past human rights abuses? What impact did records have on a particular case?

I applied methods of qualitative analysis of textual documents and semistructured interviews for my dissertation research on the study of the work of the National Security Archive (NSA) in transitional justice mechanisms

24 Richard J. Cox and David A. Wallace, eds., Archives and the Public Good: Accountability and Records in Modern Society, Westport, CT: Quorum Books, 2002, p.3.

25 Wallace, "Historical \& Contemporary Justice and the Role of Archivists," in Arkiv, Demokrati Og Rettferd [Archives, Justice, Democracy](Oslo, Norway: ABM-Utvikling, 2006), 14-27.

26 Yan Zhang and Barbara M. Wildemuth, "Qualitative Analysis of Content," in Barbara M. Wildemuth, ed. Applications of Social Research Methods to Questions in Information and Library Science, Westport, CT: Libraries Unlimited, 2009, p.309. 
in Latin America. ${ }^{27}$ The NSA, a non-governmental research institute based in Washington D.C., has been actively involved with the ongoing struggle over accountability for past human rights abuses in the region. The NSA has collaborated with most of the truth commissions in Latin America, providing declassified U.S. government records and advocating for the declassification of secret records in the archives of U.S. government agencies. The NSA has also assisted in the recovery, organisation and use of discovered archives such as the Archives of the Guatemalan National Police. Declassified records housed at the NSA have been presented in multiple human rights trials, and members of the organisation have also testified as expert witnesses in a number of these trials. ${ }^{28}$

Most of the research focused on studying legal cases in El Salvador, Peru, Uruguay, Argentina and Guatemala. These cases were identified through the National Security Archive's Electronic Briefing Books and blog, and my own research. For each case, I studied the court decisions and performed additional research with the study of news, reports and secondary sources. The semi-structured interviews served to complement the findings from the qualitative analysis of the documents. The interviews included members of the National Security Archive who were directly involved with the cases I studied, particularly the cases in Guatemala. I also interviewed the director of the Historical Archives of the Guatemalan National Police and one lawyer who participated in one legal case in Guatemala. I identified the individuals through the reading of publications, news reports and resources from the NSA's website.

During my close reading of the documents I identified relevant themes by employing the following questions:

1. What does the document say about the National Security Archive?

2. What impact do the records provided by the National Security Archive have on mechanisms of accountability for past human rights violations in Latin America?

This approach was particularly useful for the analysis of final court decisions. For each court decision, I identified any reference to the National

27 Joel A. Blanco-Rivera, "Archives as Agents of Accountability and Justice: An Examination of the National Security Archive in the Context of Transitional Justice in Latin America," Ph.D. dissertation, University of Pittsburgh, 2012.

28 See for example Kate Doyle, "Un Testimonio Inédito de Kate Doyle, del National Security Archive. ¿Por qué Testifique Contra Dos Dictadores: Fujimori y Ríos Montt?” Emequis (September 28, 2009): 19-26. 
Security Archive and/or to records provided by the organisation. I also searched and retrieved news about the legal case and identified those who mentioned the Archives participation. This approach, along with the information obtained from publications, web postings, additional reports, and semi-structured interviews, provided an important context within which to answer the main research question.

Through the application of these methods, I was able to identify three main themes related to the roles of archives and records in the implementation of transitional justice mechanisms. First, that one of the archivist's main contributions to these mechanisms is the understanding of provenance, custody, and record-keeping. The main contribution of the records presented in the legal cases was that they proved that human rights violations were part of systematic and well-planned counterinsurgency strategies implemented by the State. In other words, they provided a context to specific human rights abuses. Second, one of the most powerful elements found through this research was how records from different countries were used in human rights investigations to establish strong documentary evidence. In Guatemala, declassified U.S. government records and documents from the Archives of the Guatemalan National Police were critical to proving the authenticity of the Diario Militar, a Guatemalan military logbook made public by the NSA in 1999 and exposing details about the fate of 183 victims. ${ }^{29}$ Third, this research underscored that access to government information is a fundamental human right, and that the State has a responsibility to open its archives and confront the past.

In their article "Social Justice Impact of Archives: A Preliminary Investigation," ${ }^{30}$ Wendy Duff, Andrew Flinn, Karen Suurtamm and David A. Wallace present the first step toward a more concrete conceptualisation of the relationship between social justice and archives. The authors explain that the impacts of social justice are "complex and multidimensional." They highlight the complexities of understanding the impacts of archival approaches to social justice, since these impacts are both tangible and intangible. However, this does not preclude researchers from identifying the impacts of archives in social justice issues. The same applies to the study of the relationships between archives and transitional justice. The three main

29 For more information on the Diario Militar case before the Inter-American Commission on Human Rights and the Inter-American Court of Human Rights see Blanco-Rivera, "Archives as Agents of Accountability and Justice," 176-186.

30 Duff et al., "Social Justice Impact of Archives".

31 Duff et al., "Social Justice Impact of Archives,” 319. 
themes explained above demonstrate that archives play an important role in transitional justice mechanism. The impacts include specific results such as the use of records as evidence to successfully prosecute perpetrators of human rights violations. Intangible impacts can be found in personal stories of people whose loved ones were victims of repression and who have been able to find information about their relatives in the archives. ${ }^{32}$

The growth of human rights trials in Latin America since the late 1990s provides very valuable datasets that can be used to address the above questions. An analysis of legal complaints, trial transcripts, and court decisions provides archival researchers with an opportunity to identify and understand how archival ideas of context, provenance, and custody might have an impact upon the legal process. ${ }^{33}$ Moreover, the study of how these documents became accessible in the first place allows researchers to assess the ways in which social and political factors affect access to archives and efforts toward the right to know.

\section{Following the Thing: The Social Life of Records Approach for the Study of Archives and Human Rights}

\section{Michelle Caswell}

Using photographic records created by the Khmer Rouge in Cambodia as a case study, this discussion outlines how a social life of objects approach can be deployed for research at the nexus of archival studies and human rights. ${ }^{34}$ By coupling ethnographic methods from the social sciences with analytical methods from the humanities, researchers can trace the social life of records,

32 See for example, Carlos Osorio, "Children of the Disappeared: "The Documents Enable Us to Touch Them...", Unredacted: The National Security Archive, Unedited and Uncensored (blog), December 17, 2009, http://nsarchive.wordpress.com/2009/12/17/thedocuments-enable-us-to-touch-them $\% \mathrm{E} 2 \% 80 \% \mathrm{~A} 6 /$.

33 For example, see the arrest warrants issued on March 30, 2001 by Spanish Judge Eloy Velasco Nuñez against the intellectual authors of the assassinations of six Jesuit priests, a housekeeper and her daughter in El Salvador on November 16, 1989. In this case declassified U.S. government records provided important evidence about the planning of the murders. http://www.cja.org/downloads/ JesuitsArrestWarrants.pdf.

34 For a more detailed analysis, see the subsequently published monograph based on this research. Michelle Caswell, Archiving the Unspeakable, Madison, WI: University of Wisconsin Press, 2014. 
producing scholarship that not only furthers the field of archival studies, but also constitutes a form of human rights activism.

Arising first out of anthropology, but taken up by visual studies, science and technology studies, and cultural studies, the social life of objects approach traces the trajectory of items or images as they exchange hands, take on different meanings, and are assigned different values depending on context. Arjun Appadurai contends that, "commodities, like persons, have social lives," as well as a "cultural biography," "a career," "a life history" and a "trajectory." ${ }^{35} \mathrm{He}$ shifts the methodological attention away from people and towards the objects with which they interact. He encourages scholars to "follow the thing," revealing both a theoretical framework (that objects are imbued with certain power) and a methodological framework (that we can ask certain types of questions to uncover that power). Scholars in visual culture have since refined Appadurai's approach, turning attention to visual "things" in particular. Building on a career exploring the power of iconography, W.J.T. Mitchell invites us to think as if photographs had desires: "What do pictures want?" he asks. ${ }^{36}$ Delineating Mitchell's methodology, Gillian Rose asks, "What happens if ... we start to think of visual materials less as texts to be decoded for their meaning, and more as objects with which things are done?" ${ }^{37}$ Rose outlines three key elements of this approach: materiality, or how images "look and feel" within particular places and times; performativity, or how images perform social functions as they are viewed by people in various contexts, and mobility, or how images are re-contextualised and reimagined as they travel. ${ }^{38}$ In this light, photographs may be attributed with instrumentality in the sense that they serve as instruments through which social interaction occurs.

Although not explicitly identified as such, this social life of objects approach has also filtered down to both information studies through the work of John Seely Brown and Paul Duguid ${ }^{39}$ and to archival studies, most notably in the Australian records continuum model and Eric Ketelaar's work

35 Arjun Appadurai, ed. The Social Life of Things: Commodities in Cultural Perspective, Cambridge: Cambridge University Press, 1986.

36 W.J.T. Mitchell, What Do Pictures Want?: The Lives and Loves of Images, Chicago, IL: University of Chicago Press, 2005.

37 Gillian Rose, Visual Methodologies: An Introduction to the Interpretation of Visual Materials, London: Sage, 2007, p.217.

38 Rose, Visual Methodologies, 220. In its insistence on the role of nonhuman actors, this approach resembles Actor Network Theory (ANT) as articulated by Bruno Latour.

39 John Seely Brown and Paul Duguid, “The Social Life of Documents," First Monday 1:1 (1996), http://firstmonday.org/htbin/cgiwrap/bin/ojs/index.php/fm/article/view/466/387. 
on the activation of records. First proposed by Frank Upward and further articulated by Sue McKemmish and others, the continuum model is characterised by the transformative nature of records and recordkeeping within multiple and interacting dimensions such that, "in continuum terms, while a record's content and structure can be seen as fixed, in terms of its contextualization, a record is 'always in a process of becoming."'40 In this view, archives are not stable entities to be tapped for facts, but rather, are key players in a constantly shifting process of re-contextualisation. Adding to this view (though not a continuum theorist per se), Ketelaar sees records as dynamic objects shifting with each new use, influencing human behaviour and culture, and being transformed along the way. ${ }^{41}$

In employing the social life of objects framework in work on archives and human rights, researchers posit that records documenting human rights abuse are objects with a social life whose meaning and uses evolve and are transformed as they migrate through formats, space, and time. For example, my research followed several activations of a collection of photographic mug shots of political prisoners taken by the Khmer Rouge regime in Cambodia. ${ }^{42}$ I showed how these mug shots are used to construct meaning by/for particular groups of people (the international community, survivors, the families of victims) at particular times (the tribunal, the retrospective shaping of collective memory) in particular realms (political, cultural, and religious). By using a variety of methods (including ethnographic observation, semi-structured interviews, and visual and textual analysis) to trace the social life of these records documenting human rights abuse, we see how, in the vein of Ketelaar, these activations influence all future activations, such that our future readings of the records are bound to their current and past uses.

In using the social life of records methodological framework, no single method is adequate to fully capture the social life of the record as it moves through time and space; rather, the research methods are dictated by the

40 Sue McKemmish, "Placing Records Continuum Theory and Practice," Archival Science 1 (2002): 335 .

41 Despite sharing the word "life," the social life of objects approach, when placed within the framework of existing archival theory, is not necessarily bound to the records life cycle model, but can be used to describe records within the records continuum as well.

42 The term "mug shot" describes a genre of standardised police photography that depicts the faces of arrested individuals. The genre was standardised by Alphonse Bertillon, a French police clerk, in 1888 and quickly spread throughout the world. For more a history of the genre and the term, see: Michelle Caswell, Archiving the Unspeakable, Madison: University of Wisconsin Press, 2014. 
format, media, use, and flow of the records. Given the use of methods that draw from both the social sciences and the humanities, this approach calls for flexibility and a wide breadth of methodological training on the part of the researcher. While developing the ability to switch seamlessly between social science and humanities methods might be burdensome, that ability also allows for creativity in response to the specificities of each case.

In this particular case, I used participant observation, semi-structured interviews, and visual and textual analyses. First, I employed participant observation by directly observing and participating in archival interventions with the mug shots at the Documentation Center of Cambodia (DC-Cam) and the Tuol Sleng Genocide Museum in Phnom Penh. In this capacity, I gained firsthand knowledge of how archivists and museum staff interact with the mug shots through arrangement, description, digitisation, display, publication and reference. This includes tracing the ways in which DC-Cam staff distribute copies of the mug shots through publications such as monthly newsletters and monographs, how such photographs are contextualised in publications, museum displays and in digital databases, and how staff respond to reference requests from survivors of the regime inquiring about the fate of family members.

Next, I conducted semi-structured interviews with a dozen participants in the creation of DC-Cam. While I prepared by composing a series of questions, I also tailored questions to my subjects and had the flexibility to ask follow-up questions. These interviews allowed me to get a sense of how archivists, activists, and academics interacted with the records and their importance for scholarship, legal adjudication, and activism. A significant language barrier, however, prevented me from interviewing many Cambodians and was a limitation posed by this method.

Building on my training in the humanities, I engaged in both visual and textual analysis. I analysed the Tuol Sleng mug shots themselves, and more recent photographs that have incorporated the mug shots into images that depict survivors and victims' family members witnessing the mug shots. This analysis allowed me to examine the visual impact of the records, as well as to view them as historiographical evidence. I also conducted textual analysis of court transcripts, survivor autobiographies, and DC-Cam newsletter stories that explicitly describe interaction with the mug shots. This method limited me to stories that are already written down and translated into English, but it also uncovered reoccurring themes related to the ways in which survivors and victims' family members respond to records. Using a plurality of methods in combination can help overcome weakness in any 
single method, allowing a more complex picture of the social life of the record to emerge.

The iconic mug shot of Tuol Sleng victim Chan Kim Srun holding her infant son serves as a key example illustrating the ways in which these methods can be triangulated to offer new insight into the social life of records. Through visual discourse analysis, I was able to uncover the ways in which the Khmer Rouge used the bureaucratic creation of photographs such as this one to transform those arrested into enemies of the state who could be eliminated. Through direct observation, I noted how archivists at DC-Cam reprinted this photograph in multiple contexts and how visitors to the Tuol Sleng Genocide Museum responded to it. Through semistructured interviews with archivists and historians, I was able to uncover the story of who Chan Kim Srun was, why she was arrested and when she died, as well as how her adult daughter only learned of her demise at Tuol Sleng recently through the reprinting of the mug shot in a DC-Cam publication. I was then able to employ a second layer of visual discourse analysis to a photograph subsequently taken by DC-Cam staff of Chan Kim Srun's granddaughter looking at the original mugshot to uncover the ways in which such records are repurposed to "perform" human rights in contemporary contexts.

Some anthropologists have recently used the term the "the performance of human rights" to refer to ways in which survivors and victims' family members publicly engage in political action in order to prevent future abuse. ${ }^{43}$ Through this performative lens, the Tuol Sleng mug shots become active agents in the performance of human rights in their reuse in photographs of survivors and victims' families looking at them. The mug shots are being incorporated into new records that document the act of witnessing. Drawing on the work of Andrea Noble and Susan Slyomovics, this framework examines how the creation of new records of witnessing constitutes a performative deployment of the archives for political engagement. ${ }^{44}$ The staging of the photograph documenting the witnessing of the record is an act of political activism. Through their strategic deployment, the photographs become active agents in the human rights struggle in that they perform the act of

43 Susan Slyomovics, The Performance of Human Rights in Morocco, Philadelphia: University of Pennsylvania Press, 2005.

44 Andrea Noble, "Traveling Theories of Family Photography and the Material Culture of Human Rights in Latin America," Journal of Romance Studies 8 no.1 (2008): 44. Noble is primarily concerned with family portraits rather than mug shots, but the similarities are evident. 
bearing witness. ${ }^{45}$ Finally, by drawing attention to the deployment of records for the performance of human rights, this research also constitutes a type of political activism. The researcher does not claim to be a neutral observer of the record as it is acted upon by others, but an active participant in the social life of the record, contributing to its significance - and ultimately its agency — in the struggle for human rights.

\title{
Applying Critical Discourse Analysis toward Institutional Historiography
}

\author{
Lorrie Dong
}

This section examines historiography and the social actors approach to critical discourse analysis (CDA) as a discipline and method, respectively, for studying the historical development of institutions within a human rights context. Historiography, which is broadly defined as the study of how the historical texts of a particular topic or discipline were written, is useful for examining changes in social norms and institutional practices over time. According to Ernst Breisach, the postmodern turn in historiography emphasises that, "historians [must] become archaeologists of linguistic structures of power and oppression encrusted in writings and institutions." ${ }^{36}$ Postmodern historiography posits that the factual reconstruction of history through records is impossible; therefore, readers should read texts only as truth claims. ${ }^{47}$ In practice, a historiographer contextualises and interprets institutional records within historical frameworks, and looks for the embedded social, economic, or intellectual influences on the text. As a result, historical documents that were not created expressly

45 Here, I am indebted to Frances Guerin and Roger Hallas's assertion that Holocaust testimony reveals the "ultimate agency of the image in the performative act of bearing witness to historical trauma." Frances Guerin and Roger Hallas, "Introduction," in The Image and the Witness: Trauma, Memory, and Visual Culture, New York: Wallflower Press, 2007, p.11.

46 Ernst Breisach, Historiography: Ancient, Medieval, \& Modern, Chicago: University of Chicago Press, 2007, p.423.

47 For an examination on the "linguistic turn" in historical studies, see Elizabeth A. Clark, History, Theory, Text: Historians and the Linguistic Turn, Cambridge: Harvard University Press, 2004. The influence of de Saussure on how literary theorists, historians, anthropologists, and sociologists treat the relationship between what is presented (the "text") and "reality" cannot be overstated. 
to be historical accounts can serve as "the evidence of witnesses in spite of themselves." ${ }^{48}$

Critical discourse analysis (CDA), which is both a method and an overarching paradigm, assumes that discursive practices are both socially conditioned and socially constitutive. ${ }^{49}$ The social actors approach is one method of analysis used in CDA that is particularly well-suited for examining nonhuman actants, such as records, that are involved in discursive practices. Institutional texts, which are often records of everyday routines, can individually and especially collectively convey the cultural life of a particular community, albeit from the perspective of the dominant group. The writing of the records, and other daily practices, naturalise and reify the control of governing bodies such as professional societies or administrations. Conducting CDA of institutional texts requires looking at both how authorities can exhibit and legitimise their dominance through language, and the social implications of it. ${ }^{50}$ I use examples of the application of CDA to state mental hospital records to demonstrate how this research method, and historiography in general, are valuable tools within a more comprehensive battery of epistemological methods for archival scholars to unearth entrenched inequities in power.

In order to better understand the contributing role of records and recordkeeping practices to historical social marginalisation and hegemonic patterns, my research involved a longitudinal analysis of the text and materiality of documents from a state mental hospital in the American South. The institution, which was created at the end of the Civil War, is notable for being a hospital that exclusively served African American; it was not until after the Civil Rights Act of 1964 that the hospital was opened to all state residents regardless of ethnicity or race. Since the mid- $20^{\text {th }}$ century, mental hospitals have been a focus of study for sociologists to examine issues of agency, power, and knowledge. Erving Goffman called the mental hospital a "total institution" because it is at once a physical place and a set of ideals that classifies and removes a subset of people from the rest of society. ${ }^{51}$ Inhabitants of total

48 Marc Bloch, The Historian's Craft, New York: Alfred A. Knopf, 1962, p.61.

49 Norman Fairclough and Ruth Wodak, "Critical Discourse Analysis," in Discourse as Social Interaction, Teun Van Dijk, ed. Thousand Oaks, CA: Sage, 1997, p.258.

50 Teun Van Dijk, "Principles of Critical Discourse Analysis," in Discourse Theory and Practice, Margaret Wetherell, Stephanie Taylor, and Simeon J. Yates, eds. Los Angeles, CA: Sage, 2001, p.303.

51 Erving Goffman, Asylums: Essays on the Social Situations of Mental Patients and Other Inmates. New York: Anchor, 1961. Peter Sedgwick argues that Goffman's idea of a "final and ultimate paradigms of human existence," e.g., the "total institution," gave the public a dangerous sense of resignation about mental health care. He criticises anti-psychiatry 
institutions are required to adhere to a regimented lifestyle, often marked by a lack of privacy or possessions. Michel Foucault similarly viewed institutions of confinement as tools of segregation and alienation. ${ }^{52} \mathrm{He}$ argued that hospitals in particular were isolated and "marked" communities. ${ }^{53}$

I used the social actors approach to CDA to examine a variety of records types, but here I focus on patient admission registers and, in particular, the categories of recorded information therein. Geoffrey Bowker and Susan Leigh Star argue that "one can read a surprising amount of social, political, and philosophical context from a set of categories - and that in many cases the classification system in practice is all that we have to go on." ${ }^{54}$ Language employed in records by specialists, such as psychiatric staff members, can act as "symbolic violence" that perpetuates cultural hegemonies and affects how controlled populations and individuals are treated..$^{55}$

The social actors approach calls for the identification of seven "core elements" in texts, although all elements are not always available in a given text: actions, performance modes, actors, actors' presentation styles, times of the constituted social practices, social spaces, and resources required by the actors. ${ }^{56}$ Using the CDA elements as a starting point to analyse the language, materiality, and organisation of institutional records, researchers can tease out emerging discourses of authorisation and legitimisation of hierarchies. As an example, I present an entry from an early patient admission register (1866-1887) (Figure 31.2).

The hospital administration ostensibly decided on the categories seen in the register to keep track of incoming patients and their ongoing mental and physical statuses in a systematic manner. The volume was printed for the hospital by a local bindery. The following table is a summary of my findings when I used the core elements framework to examine the hospital register's categories and how they were utilised over two decades (Table 31.1).

sociologists for perpetuating the notion that mental illness is not a "real" illness but entirely socially constructed, and correctly predicted social conservatives would employ anti-psychiatry theories to justify cutting mental health services, as they were in the UK and the US in the 1980s. Peter Sedgwick, Psycho Politics: Laing, Foucault, Goffman, Szasz, and the Future of Mass Psychiatry, New York: Harper \& Row, 1982.

52 Michel Foucault, History of Madness, London: Routledge, 2006, p.79.

53 Michel Foucault, Discipline and Punish, New York: Vintage Books, 1995, p.198.

54 Geoffrey Bowker and Susan Leigh Star, Sorting Things Out: Classification and Its Consequences. Cambridge, MA: MIT Press, 1999, p.55.

55 Pierre Bourdieu, Language and Symbolic Power, Cambridge, MA: Harvard University Press, 1991, p.23.

56 Ruther Wodak and Michael Meyer, Methods of Critical Discourse Analysis, Thousand Oaks, CA: Sage, 2009, p.30. 
Figure 31.2: Patient Entry from 1882 In-patient Admission Register.

Image and digital redactions by Lorrie Dong.

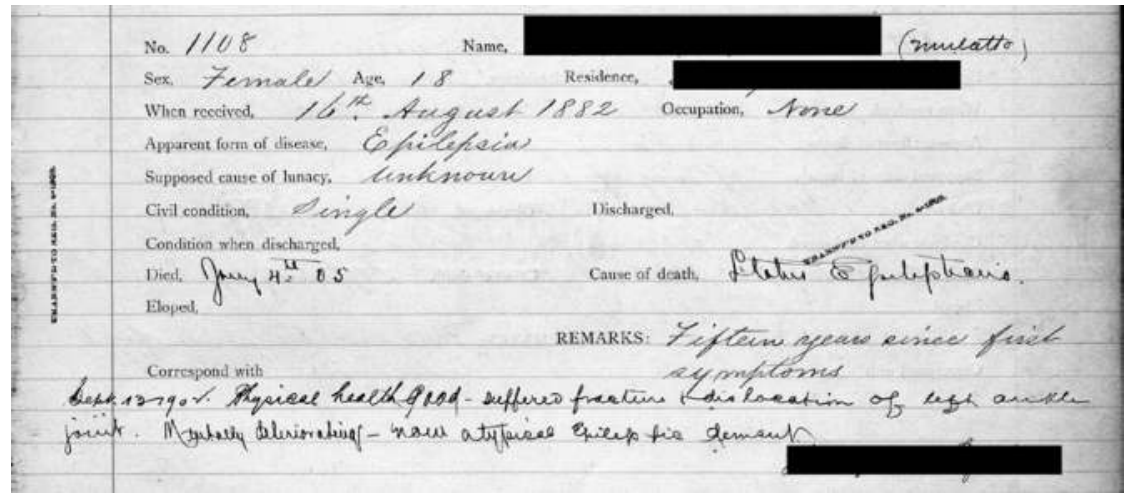

In the above example, I discuss information recorded in the early register. Additionally, archival researchers can look for content that intentionally has been excluded or excised from individual records and aggregations of records. Jacques Derrida implies that archives researchers can come to understand systems of institutional thought by looking at what content has been purposely excluded from material records because institutional creators considered it trivial information. ${ }^{57}$ Similarly, Michel-Rolph Trouillot posits that there are four junctures for potential silences in the production of history: the moment of source-making, archival creation, retrieval for the construction of narratives, and history-making retrospection..$^{58}$ Of particular interest for this section are the first two possible moments of silence - the creation of records and the keeping of them. Ann Laura Stoler notes that, "Information out of place underscores what categories matter, which ones become common sense and then fall out of favor. Not least, they provide road maps to anxieties that evade more articulate form. ${ }^{\prime 59}$ For example, after the site of my research was desegregated in the mid-1960s, the registers ceased to have a printed category dedicated to describing a patient's race. However, in 1969, a register (1967-1969) has for a brief time an unlabelled column at the margins of each page that has been reserved to note whether the individual is "W" for "White" or "N" for "Negro" (Figure 31.3).

57 Jacques Derrida, La Carte Postale: de Socrate à Freud et Audela, Paris: Flammarion, 1980.

58 Michel-Rolph Trouillot, Silencing the Past: Power and the Production of History, Boston: Beacon Press, 1995.

59 Ann Laura Stoler, "Colonial Archives and the Arts of Governance: On the Content in the Form," in Archives, Documentation, and Institutions of Social Memory: Essays from the Sawyer Seminar, Francis X. Blouin, Jr., ed. Ann Arbor: University of Michigan Press, p.275. 


\title{
Table 31.1. Findings based on the application of the CDA core elements framework to hospital register categories.
}

\author{
Element \\ Synopsis \\ Actions \\ The purpose of the categories is to classify incoming patients for \\ administrative accountability to the state. Unlike later registers, this register \\ is the only formal documentation of patients throughout their entire \\ institutionalisation; therefore, it may also have been used for patient care \\ purposes.
}

Modes

The provided space for each patient entry is fifteen lines, although some entries have extensive patient updates written tightly in the margins or in the correspondence section. The staff increasingly repurposed the latter to record hereditary predispositions to mental illness and the frequency and length of "attacks" (or "symptoms," as they are called later), thus suggesting a growing medical interest in studying familial and individual patterns of mental disease.

Actors Besides the doctors and staff members who wrote in the registers and the state government that required the records, there are the implicated or silent actors, the patients, whose bodies and minds were examined and recorded. Expanding upon the element, I also consider the actants that create and reproduce social structures - in this case, the register itself. The register assisted the staff in determining where to place patients and how to care for them. Keeping in mind that patients often were institutionalised for their entire lives, some individuals fell under the care of multiple staff members over many decades, with the registers being the only source of written information about them.

Styles The diction of the category titles indicates the inchoate state of American psychiatry at the time of the register's creation. Disease diagnoses and etiology were uncertain, as seen in categories such as "Apparent form of disease" and "Supposed cause of lunacy" (emphasis mine). Toward the end of the $19^{\text {th }}$ century, more technical and detailed medical terminology appears under these categories, which coincides with psychiatry's growth as a discipline.

Times Once the hospital determined its register categories, this register - by simply being a part of the facility's everyday practices - helped perpetuate the use of the same recordkeeping system for 21 years, even when the categories became insufficient for the hospital's medical and administrative needs. Later registers speak to the changes in institutional psychiatry, and include categories for legal statuses, race, syphilis testing, and addictions, among others.

Spaces Based on the purpose of the register and the critical information it held, the volume most likely had a dedicated space within the hospital. From the text alone, it is impossible to determine if recordkeeping in the register took place near patient intake or at a separate location. By the mid- $20^{\text {th }}$ century, the hospital had a separate building for medical record management and storage.

Resources Again, the materiality of the actant comes into play here. The register is a single bound volume, limiting the space for the hospital personnel to write information about the patients and perpetuating the use of certain categories over an extended period of time. 
Figure 31.3: Excerpt from 1969 Register. Photo by Lorrie Dong.

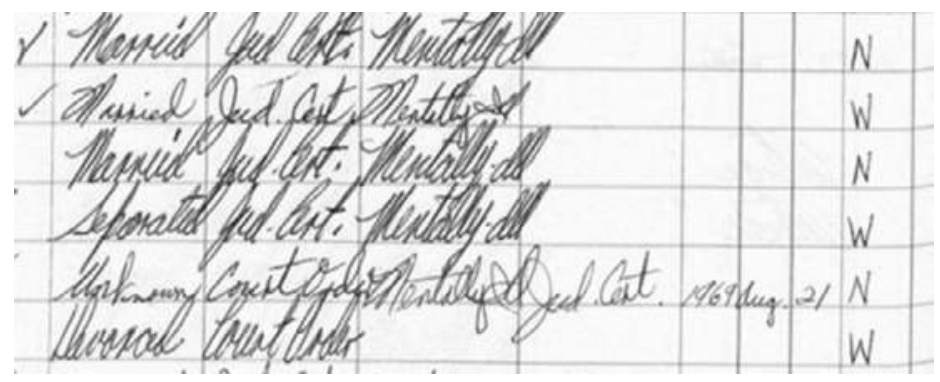

Race, while no longer a factor for admission to the hospital, still mattered to the administration. Many questions remain regarding why the race category persisted in a less formalised form. Based on the source material alone, it is unclear who authorised the creation of the unnamed category, when the race notations were added, and the administrative and/or broader social reasons for adding racial information.

The unanswered questions about recordkeeping intent point to the primary limitation of conducting a historiography of institutional records the narrow content of the source material. If such texts are the sole source of data, researchers focusing on human rights issues are restricted to institutional viewpoints. In order to ameliorate this limitation, the researcher can look for silences in the institutional records. Prior archival research to "explore silences in historical accounts" ranges from the Tuskegee syphilis experiments to apartheid. ${ }^{60}$ With the Nelson Mandela prison records, Harris notes the gaps in the collection and the uncertainty of whether or not those files were intentionally lost in the regime change. ${ }^{61}$ For institutional creators, the missing information could be considered either trivial or dangerous. What institutions might justify as unimportant might at the same time point to more expansive questions of legal and ethical accountability. In her study of eugenics in the U.S., Rebecca Kluchin notes the lack of evidence in medical records regarding the circumstances surrounding sterilisation operations. She examined legal sources (e.g., affidavits, trial transcripts) of sterilised women to gain a better understanding of the

60 Ronald Doel and Thomas Söderqvist, The Historiography of Contemporary Science, Technology, and Medicine, New York: Routledge, 2006, p.3.

61 Verne Harris, "Ethics and the Archive". 
lack of informed consent in these cases. ${ }^{62}$ When possible, additional data collection methods such as interviews and observation focusing on the implicated voices can provide further perspectives on institutional power.

While it is insufficient to examine only institutional records in order to understand social, economic, and political hegemony from multiple perspectives, it is a starting point for researchers to grasp the tacit pervasiveness of marginalising practices. Reading mental hospital records as historical narratives can provide insight into institutional perspectives on race, mental illness, and other categorisations. My longitudinal critical discourse analysis of the patient admission registers, a small portion of which I presented here, allowed me to connect the daily practice of classifying individuals at one location with larger political structures and actors. The registers were primarily for governmental accountability and a factor in ensuring continued financial support from the state. Furthermore, by looking at the changing modes and styles of the register's text, I could trace the parallel development of psychiatry as a burgeoning medical discipline and the increasing specificity of diagnoses, etiologies, and treatments. The records themselves helped the institution hold control over patients' bodies and minds. Now, researchers have the opportunity to utilise these and other historical institutional records in order to begin addressing the power inequities that are a part of the records' provenance.

\title{
Human Rights Archives: Pushing the Postcustodial Boundaries
}

\author{
Joanna Steele
}

Human rights archives have received a fair amount of coverage from archival scholars who are predominantly interested in internationally known cases of conflict, atrocity, and judicial redress. The archival literature addresses concerns in the context of various national efforts to come to terms with the past, including truth commissions and international tribunals. ${ }^{63}$ Less

62 Rebecca M. Kluchin. "Locating the Voices of the Sterilized," The Public Historian 29 no. 3 (2007): 131-144.

63 See Tom Adami, “'Who Will be Left to Tell the Tale?' Recordkeeping and International Criminal Jurisprudence," Archival Science 7 (2007): 213-221; Tom Adami and Martha Hunt, "Genocidal Archives: The African Context - Genocide in Rwanda," Journal of the Society of Archivists 26 no. 1 (2005): 105-121; Verne 
has been written, however, about archiving human rights as a social movement, which would encompass "the (often digital) traces of these campaigns and networks, as well as other non-elite, non-institutional communities and groups." ${ }^{64}$ I set out to investigate the emergence of archival initiatives by university libraries to collect human rights documentation in order to understand how such archives were situating themselves in the complex landscape of human rights. This section will examine human rights archives as subject through a qualitative research approach that paired a single case study with a broader analysis of the collection policies of several such archives.

\section{A Postcustodial Framework}

This study has its roots in postcustodialism, a term that refers to a conceptual shift in archival thinking spurred by the information revolution of the end of the $20^{\text {th }}$ century. Gerald Ham coined this new period in archival history - characterised by vast quantities of data and the proliferation and decentralisation of collections - the "post-custodial era." ${ }^{\text {5 }}$ Terry Cook cited postcustodialism as a paradigm shift, signalling movement away from a focus on archival records themselves to an analysis of the social contexts in which records are created. He also used the term to recognise archivists as "active shapers of the archival heritage," as opposed to "passive keepers [or custodians] of an entire documentary residue left by creators." ${ }^{" 6}$ Postcustodialism can also be understood as part of an evolving archival framework around custody. Jeannette Bastian traces how this framework has "evolved in

Harris, Archives and Justice: A South African Perspective, Chicago, IL: Society of American Archivists, 2007; Verne Harris, "Review of Final Acts: A Guide to Preserving the Records of Truth Commissions by Trudy Huskamp Peterson," Archives and Manuscripts 33 no. 2 (2005): 181-185; Eric Ketelaar, "Archives as Spaces of Memory," Journal of the Society of Archivists 29 no. 1 (2008): 9-27; Trudy Huskamp Peterson, Final Acts: A Guide to Preserving the Records of Truth Commissions, Baltimore: The Johns Hopkins University Press, 2005; Trudy Huskamp Peterson, Temporary Courts, Permanent Records, Woodrow Wilson Center for Scholars, 2008, http://www.wilsoncenter.org/sites/default/files/TCPR_Peterson_HAPPOP02. pdf.; Antonio Gonzalez Quintana, Archival Policies in the Defense of Human Rights, Paris: International Council on Archives, 2009.

64 Andrew Flinn, "Other Ways of Thinking, Other Ways of Being. Documenting the Margins and the Transitory: What to Preserve, How to Collect," in What are Archives? Cultural and Theoretical Perspectives: A Reader, Louise Craven, ed., Burlington, VT: Ashgate, 2008, 110.

65 F. Gerald Ham, "Archival Strategies for the Post-Custodial Era," The American Archivist 44 no. 3 (1981): 207.

66 Terry Cook, "What is Past is Prologue," 46. 
response to societal needs" ${ }^{37}$ from the 20th into the 21st century. Arising out of a debate in the 1990s over the distributed management of electronic records, postcustodialism has evolved into an archival approach that values access over possession and whose influence pervades much of the current literature on independent or community archives. ${ }^{68}$ By applying a postcustodial framework to human rights archives, I was able to identify how archival practice is being challenged by changing societal needs to document the subject of human rights.

\section{The Human Rights Documentation Initiative}

I began my research with a case study of the Human Rights Documentation Initiative (HRDI) at the University of Texas (UT) at Austin because of its explicit mission to document human rights by building digital archival partnerships with grassroots human rights organisations using a postcustodial archives model. Founded in 2008, the HRDI's collecting focus is on the fragile, vulnerable digital audio visual material and ephemeral electronic web content created and captured by human rights organisations. The case of the HRDI, bringing together a human rights collecting scope within a postcustodial framework, presented a unique research opportunity to explore the fit of such a model within a human rights context.

To gain a broad perspective in tracing the motivations behind the establishment of this human rights archives, I conducted interviews with multiple stakeholders, including the head of UT libraries, archivists, an HRDI partner, a systems analyst, UT faculty, and graduate interns. I organised my interview questions under the following themes to operationalise the notion of "position" in the human rights landscape: mission, defining or parsing "human rights," alignment with human rights discourse, alignment with communities, advocacy, and role(s) of archival staff. Over a two-week period, I observed the daily operations of the HRDI, attended staff meetings, and shadowed the human rights archivist. To compare the information I was gathering through interviews and observation to official policies, I also examined documents and web content, including grant proposals and reports, press releases and library newsletters, the HRDI blog, meeting minutes, metadata guidelines, and the HRDI thesaurus. I used discourse

67 Jeannette A. Bastian, “Taking Custody, Giving Access: A Postcustodial Role for a New Century," Archivaria 53 (2004): 80-81.

68 Terry Cook, "What is Past is Prologue: A History of Archival Ideas Since 1898, and the Future Paradigm Shift," Archivaria 43 (1997): 17-63. 
analysis ${ }^{69}$ to understand the context-specific discourse captured in the interviews and observation, as well as how the global language of human rights is taken up in the archives, as expressed in the HRDI's public statements and web presence.

The interdisciplinary field of human rights has its own distinct, yet complex and contested, discourse, where language is used to carve out one's position within the landscape. Word choice, frames, and verbal claims hold tacit meanings that can be deconstructed through a cyclical process of coding and analytic memoing. I first coded the interviews according to the themes mentioned above. Then in an iterative process, I systematically searched the interview transcripts to locate terms or phrases used by interviewees that reflected those themes. When necessary, I recalibrated my codes to include the particular language used by interviewees, for example "post-custodial model," "partnership," and "capacity building." I then compared the alignment of these thematic codes with the larger discourses in human rights scholarship, gleaned from a previous literature review, to determine the extent of overlap or gaps. The range of sources I consulted, from informal interactions with staff to formal documentation, were critical in gaining a holistic understanding of the HRDI, as it is situated in the larger university and in the broader realm of human rights documentation.

Shadowing the archivist revealed the daily operations of the HRDI and the interwoven functions of the different stakeholders with respect to the archives. Combining these observations with interviews provided me with the option to ask follow-up questions about how the HRDI staff dealt with unforeseen challenges, from technical operations in Kigali, Rwanda, to decisions about what material should be made available online and the look and feel of those collections on the web. By digging into such practicalities, I was able to locate the negotiations that exemplify a post-custodial relationship between a partner organisation and an archives.

\section{Analysis of Archival Collection Policies}

In the second phase of research, I analysed the collecting policies of four human rights archives, including the HRDI, to better understand their appraisal criteria and to gauge archival administrators' engagement with the complexities of human rights discourse. Archival collecting policies are a public statement reflecting an institution's mission and goals, but they can

69 Mats Alvesson and Dan Karreman, "Varieties of Discourse: On the Study of

Organizations through Discourse Analysis," Human Relations 53 (2000): 1129-1135. 
also be used by archivists as a tool to navigate the subject being documented. I chose collecting policies as my unit of analysis because they embody archivists' intellectual attempts to represent the concept of human rights. Speaking to what he sees as a gap between appraisal theory and application, Timothy Ericson states that the collecting policy is the space where archivists should overcome generality to define, specify, and delineate in meaningful terms the phenomena that they are hoping to document. This work of defining is "a painstaking, slow and difficult process ... still, it must be done." ${ }^{70}$ Ericson takes us back to Gerald Ham's simple precept: "[C]onceptualization must precede collection." ${ }^{\prime 1}$

Collecting policies as interface present an opportunity for archivists to "confront the interpretive nature of their work and exploit opportunities to place themselves visibly in the interfaces they construct." ${ }^{\prime 2}$ They help make the appraisal process visible - "they transform appraisal decisions from value judgments to policy choices." 73 Thus I analysed not only the archives' formal statements but also the interfaces through which they present their collections online by comparing the archives' articulations of mission, collecting scope, geography, users, collecting focus, selection criteria, formats, definitions of human rights, ethics, and treatment of human rights debates. Having broken down the policies into these categories, ${ }^{74} \mathrm{I}$ was able to identify policy choices that intersected with themes from my review of archival scholarship on human rights, specifically how archivists assess potential costs of archival work and how archives share control and authority.

Qualitative research employing interviews, observation, and discourse analysis is subject to limitations stemming from a reliance on the researcher as the main instrument of data collection and analysis. I was particularly aware of personal subjectivities arising from knowing one of my research subjects,

70 Timothy Ericson, "At the 'Rim of Creative Dissatisfaction': Archivists and Acquisition Development," Archivaria 33 (Winter 1991-92): 73.

71 Ericson quotes F. Gerald Ham, “The Archival Edge,” 12.

72 Margaret Hedstrom, "Archives, Memory, and Interfaces with the Past," Archival Science 2 (2002): 21.

73 Jennifer A. Marshall, "Toward Common Content: An Analysis of Online College and University Collecting Policies," American Archivist 65 (2002): 232.

74 Categories, in part, were drawn from Bruce Montgomery's account of the development of an international human rights collecting policy by the Archives, University of Colorado at Boulder Libraries, in "Archiving Human Rights: A Paradigm for Collection Development," The Journal of Academic Librarianship 22 no. 1 (1996): 87-96. 
through whom I was able to gain entrée into the HRDI community. I made efforts to be transparent about this connection and, more importantly, to involve my research informants in reflective thinking in order not to make the analysis solely about archivists and their practices but rather to grapple together over the issues. I balanced a case study with analysis of the collecting policies of multiple archives to capture both the depth and breadth of approaches to human rights archiving. The main limitation of this study was my inability to get access to more partner organisations, whose members were primarily located outside the U.S. These contacts would have provided a fuller picture of the motivations, apprehensions, and ongoing negotiations of the archives-NGO partnerships. Additionally, gathering information on subjects that were new and evolving made it difficult to ensure access to the latest information. Some of the human rights archives whose collection policies I analysed have since gone through transitions and/or further developed their collections.

Ultimately, I discovered the potential of human rights archives to illuminate how postcustodialism is evolving to meet today's societal needs through a model of equitable partnerships between archival institutions and human rights NGOs. This finding clarifies how postcustodialism has come to represent not only more inclusive notions of access and appraisal ${ }^{75}$ but also a model of archiving where neither ownership of the records, nor physical possession, nor intellectual control is wholly transferred to the archives. This research particularly identifies an archival model based on relationships of mutual trust and capacity building, the reorientation of records ownership made possible by digital technologies, and the opening up of authorship to record subjects and/or record creators through the sharing of expertise, whether it be content, context, technical, preservation, or access. These dynamic partnerships can foster a sense of community ownership of records and lessen the distance between the record and its originating context. Both of these aspects are crucial to the interpretation of human rights records in a way that is respectful of the sensitivities and historicity of trauma. Human rights collections have proven to be a fruitful space in which to test the boundaries of the postcustodial shift in archives.

75 These trends are also mentioned by Ciaran Trace in "On or Off the Record? Notions of Value in the Archive," in Currents of Archival Thinking, Terry Eastwood and Heather MacNeil, eds. (Santa Barbara, CA: Libraries Unlimited, 2010), 47-68. 


\section{Conclusion}

The case studies presented in this chapter remind us of the potential power of records to effect change that was unintended by their creators. Records that once served as tools of control are now being used to facilitate social justice efforts. Recordkeeping bodies are being held accountable by means of their own records, which are acting in a number of capacities ranging from legal evidence for transitional justice to components of emergent narratives by formerly silenced communities.

Archivists and archives are critical to the social justice process, and in turn the social justice ethos is changing the archival mission. In addition to the professional archivists who have the ability to change how and why records are preserved and made accessible for human rights purposes, archival researchers are responsible for examining and illuminating the relationships between archives and human rights goals as well as the interaction effects between archives and human rights. Human rights archival researchers bring to the forefront the power and responsibility of archives to help to achieve social justice. By contributing to an increasingly robust corpus of literature on the study of documents and social justice, these researchers are at once advocates for archives as social institutions and activists who draw attention to recorded injustices. In order to be effective voices in the human rights dialogue within archival studies and beyond, researchers must design their studies in a manner that allows them to explore in-depth historical contexts, multiple perspectives, and the evolving relationships between records and people. As a result, the methods tend to be cumulative and qualitative, and to encourage iterative and reflective work.

A diverse array of methods is necessary to trace the complex networks of stakeholders, documents, and actions involved in the processes of truth-telling and healing. The four illustrative case studies presented in this chapter each use variations of triangulation to engage with a corpus of perspectives that are as diverse and comprehensive as possible. The qualitative application of methods discussed here - discourse and textual analyses, semi-structured interviews, and participant observation - are representative of the types of approaches that can be used to situate records, institutions and people in relation to one another and within the human rights context. As indicated by all four authors, any single method would have been inadequate to address fully their research goals. Textual analysis, for example, may only offer a particular perspective, and often needs to be used in conjunction with other methods such as a broader material systems approach, observations, interviews or 
oral narratives. By utilising a combination of methods, each author has been able to address the roles of records, their custodians, and their users in the ongoing social justice dialogue. Blanco determined how archives and records are integral to carrying out transitional justice in Latin America by analysing the popular, legal, and oral narratives that documented the situations. Through record analysis and her interactions with people, Caswell investigated the active engagement of both records and archivist-researchers in human rights work. While critical discourse analysis helped Dong construct how institutional records played a part in hegemonic control in the past, it was her interviews with current hospital record stakeholders that pointed her toward the potential continued use of records as archival objects to help against injustices. Steele's multiple methods approach allowed her to analyse a wide variety of viewpoints to come to the conclusion that a postcustodial model for human rights archives provides the necessary flexibility to accommodate the diverse and complex needs of human rights actors from multiple organisations.

Finally, self-reflexivity, whether fostered through autoethnography, memo writing, journaling, or another method, is a key component to any activist research design. By reflecting on their roles as subject-participants within the research process, researchers are better able to acknowledge their own limitations as observers and to accept multiple, and possibly conflicting, viewpoints and narratives. The public recognition of multiple perspectives is a means for archival researchers to further social justice, and is imperative for a pluralistic society.

\section{References}

Adami, Tom. "Future Perfect? Peacekeeping, Peacebuilding and Archives - The United Nations in Sudan," Journal of the Society of Archivists 30 no. 1 (2009): 3-26.

Adami, Tom. "Who Will be Left to Tell the Tale?' Recordkeeping and International Criminal Jurisprudence," Archival Science 7 (2007): 213-221

Adami, Tom and Martha Hunt. "Genocidal Archives: The African Context - Genocide in Rwanda," Journal of the Society of Archivists 26 no. 1 (2005): 105-121.

Alvesson, Mats and Dan Karreman. "Varieties of Discourse: On the Study of

Organizations through Discourse Analysis," Human Relations 53 (2000):

1129-1135.

Appadurai, Arjun, ed. The Social Life of Things: Commodities in Cultural Perspective.

Cambridge: Cambridge University Press, 1986.

Arthur, Paige. "How “Transitions” Reshaped Human Rights: A Conceptual History of

Transitional Justice," Human Rights Quarterly 31 (2009): 321-367.

Bastian, Jeannette A. "Taking Custody, Giving Access: A Postcustodial Role for a New

Century," Archivaria 53 (2004): 76-93. 
Blanco-Rivera, Joel A. "Archives as Agents of Accountability and Justice: An

Examination of the National Security Archive in the Context of Transitional Justice in Latin America." Ph.D. dissertation, University of Pittsburgh, 2012.

Bloch, Marc. The Historian's Craft. New York: Alfred A. Knopf, 1962.

Bourdieu, Pierre. Language and Symbolic Power. Cambridge: Harvard University Press, 1991.

Bowker, Geoffrey and Susan Leigh Star. Sorting Things Out: Classification and Its Consequences. Cambridge: MIT Press, 1999.

Breisach, Ernst. Historiography: Ancient, Medieval, \& Modern. Chicago: University of Chicago Press, 2007.

Caswell, Michelle. Archiving the Unspeakable. Madison, WI: University of Wisconsin Press, 2014.

Caswell, Michelle, Giso Broman, Jennifer Kirmer, Laura Martin and Nathan Sowry. "Implementing a Social Justice Framework in an Introduction to Archives Course: Lessons from Both Sides of the Classroom." InterActions 8 no. 2 (2012). http:// www.escholarship.org/uc/item/2jx083hr.

Clark, Elizabeth A. History, Theory, Text: Historians and the Linguistic Turn. Cambridge: Harvard University Press, 2004.

Cook, Terry. "Archival Science and Postmodernism: New Formulations for Old Concepts." Archival Science 1 no. 1 (2001): 3-24.

Cook, Terry. "What is Past is Prologue: A History of Archival Ideas Since 1898, and the Future Paradigm Shift," Archivaria 43 (Spring 1997): 17-63.

Cox, Richard J. and David A. Wallace, eds. Archives and the Public Good: Accountability and Records in Modern Society. Westport, CT: Quorum Books, 2002.

Cuevas, Victor Espinoza and María Luisa Ortiz Rojas. "Practical Considerations in Comparative Research: Approaching Problems from the Bottom and from Within," in Hugo Van Der Merwe, Victoria Baxter and Audrey R. Chapman, eds. Assessing the Impact of Transitional Justice: Challenges for Empirical Research (Washington, DC: United States Institute of Peace, 2008), 313-324.

Derrida, Jacques. La Carte Postale: de Socrate à Freud et Audela. Paris: Flammarion, 1980.

Doel, Ronald and Thomas Söderqvist. The Historiography of Contemporary Science, Technology, and Medicine. New York: Routledge, 2006.

Doyle, Kate. “Un Testimonio Inédito de Kate Doyle, del National Security Archive. ¿Por qué Testifique Contra Dos Dictadores: Fujimori y Ríos Montt?” Emequis (September 28, 2009): 19-26.

Duff, Wendy M., Andrew Flinn, Karen Emily Suurtamm, and David A. Wallace. "Social Justice Impact of Archives: A Preliminary Investigation." Archival Science 13 no. 4 (2013): 317-348. DOI: 10.1007/s10502-012-9198-x.

Dunbar, Anthony W. "Introducing Critical Race Theory to Archival Discourse: Getting the Conversation Started." Archival Science 6 (2006): 109-129.

Elster, Jon. Closing the Books: Transitional Justice in Historical Perspective. Cambridge: Cambridge University Press, 2004.

Encyclopedia of Genocide and Crimes Against Humanity. Detroit, MI: Macmillan Reference, 2004.

Ericson, Timothy. "At the 'Rim of Creative Dissatisfaction': Archivists and Acquisition Development," Archivaria 33 (Winter 1991-92): 66-77.

Fairclough, Norman and Ruth Wodak. "Critical Discourse Analysis," in Discourse as Social Interaction, Teun Van Dijk, ed. Thousand Oaks, CA: Sage, 1997.

Flinn, Andrew. "Other Ways of Thinking, Other Ways of Being. Documenting the Margins and the Transitory: What to Preserve, How to Collect," in What are 


\section{Chapter 3 I}

Archives? Cultural and Theoretical Perspectives: A Reader, Louise Craven, ed.

(Burlington, VT: Ashgate, 2008), 109-128.

Foucault, Michel. Discipline and Punish. New York: Vintage Books, 1995.

Foucault, Michel. History of Madness. London: Routledge, 2006.

Forde, Helen. "We Must Remember Our Past so that We Do Not Repeat It', Journal of the Society of Archivists 25 no. 2 (2004): 117-122.

Freeman, Mark. Truth Commissions and Procedural Fairness. Cambridge: Cambridge University Press, 2006.

Gilliland, Anne. "Neutrality, Social Justice, and the Obligations of Archival Education and Educators in the Twenty-First Century." Archival Science 11 (2011): 193-209.

Gilliland, Anne and Sue McKemmish. "Building an Infrastructure for Archival Research,” Archival Science 4 (2004): 149-199.

Goffman, Erving. Asylums: Essays on the Social Situations of Mental Patients and Other Inmates. New York: Anchor, 1961.

González, Matilde. "Local Histories: A Methodology for Understanding Community Perspectives on Transitional Justice," in Hugo Van Der Merwe, Victoria Baxter, and Audrey R. Chapman, eds., Assessing the Impact of Transitional Justice: Challenges for Empirical Research (Washington, D.C.: United States Institute of Peace Process, 2008), 295-311.

Guerin, Frances and Roger Hallas. "Introduction," in The Image and the Witness: Trauma, Memory, and Visual Culture. New York: Wallflower Press, 2007.

Ham, F. Gerald. "The Archival Edge," The American Archivist 38 no. 1 (1975): 5-13.

Ham, F. Gerald. "Archival Strategies for the Post-Custodial Era," The American Archivist 44 no. 3 (1981): 207-216.

Harris, Verne. "Ethics and the Archive: 'An Incessant Movement of Recontextualisation,"' in Controlling the Past: Documenting Society and Institutions, Terry Cook, ed. Chicago, IL: Society of American Archivists, 2011, 357-360.

Hayner, Priscilla B. Unspeakable Trutbs: Transitional Justice and the Challenge of Truth Commissions, 2nd ed. New York: Routledge, 2011.

Hedstrom, Margaret. "Archives, Memory, and Interfaces with the Past," Archival Science 2 no. 1-2 (2002): 21-43.

Huntington, Samuel P. The Third Wave: Democratization in the Late Twentieth Century. Norman: University Oklahoma Press, 1991.

Huvila, Isto. "Participatory Archive: Toward Decentralised Curation, Radical User Orientation and Broader Contextualisation of Records Management. Archival Science 8 no. 1 (2008): 15-36.

Jelin, Elizabeth. State Repression and the Labors of Memory. Minneapolis, MN: University of Minnesota Press, 2003.

Jimerson, Randall C. Archives Power: Memory, Accountability, and Social Justice. Chicago, IL: Society of American Archivists, 2009.

Jimerson, Randall C. "Embracing the Power of Archives." Presidential address to The Society of American Archivists, 2005, http://www.archivists.org/governance/ presidential/jimerson.asp\#_ftn42.

Ketelaar, Eric. “Archives as Spaces of Memory," Journal of the Society of Archivists 29 no. 1 (2008): 9-27.

Kluchin, Rebecca M. "Locating the Voices of the Sterilized." The Public Historian 29 no. 3 (2007): 131-144.

Kritz, Neil. "Policy Implications of Empirical Research on Transitional Justice," in Hugo Van Der Merwe, Hugo, Victoria Baxter and Audrey R. Chapman, eds. Assessing the Impact of Transitional Justice: Challenges for Empirical Research. Washington, DC: United States Institute of Peace, 2008. 
Marshall, Jennifer A. "Toward Common Content: An Analysis of Online College and University Collecting Policies," The American Archivist 65 no. 2 (2002): 231-256.

McKemmish, Sue. "Placing Records Continuum Theory and Practice." Archival Science 1 no. 4 (2002): 333-359.

Méndez, Juan. Accountability for Past Abuses, Working Paper \#233. Notre Dame, IN: The Helen Kellogg Institute for International Studies, 1996.

Mitchell, W.J.T. What Do Pictures Want?: The Lives and Loves of Images. Chicago, IL: University of Chicago Press, 2005.

Montgomery, Bruce. "Archiving Human Rights: A Paradigm for Collection Development," The Journal of Academic Librarianship 22 no. 1 (1996): 87-96.

Noble, Andrea. "Traveling Theories of Family Photography and the Material Culture of Human Rights in Latin America," Journal of Romance Studies 8 no.1 (2008).

O'Donnell, Guillermo and Philippe C. Schmitter. Transitions from Authoritarian Rule: Tentative Conclusions about Uncertain Democracies. Baltimore, MD: John Hopkins University Press, 1986.

Olick, Jeffrey K. The Politics of Regret: On Collective Memory and Historical Responsibility. New York: Routledge, 2007.

Payne, Leigh A. "Confessional Performances: A Methodological Approach to Studying Perpetrators'Testimonies," in Hugo Van Der Merwe, Hugo, Victoria Baxter and Audrey R. Chapman, eds. Assessing the Impact of Transitional Justice: Challenges for Empirical Research (Washington, DC: United States Institute of Peace, 2008), 227-247.

Payne, Leigh A. Unsettling Accounts: Neither Truth nor Reconciliation in Confessions of State Violence. Durham, NC: Duke University Press, 2008.

Peterson, Trudy Huskamp. Final Acts: A Guide to Preserving the Records of Truth Commissions. Baltimore, MD: The Johns Hopkins University Press, 2005.

Peterson, Trudy Huskamp. Temporary Courts, Permanent Records. Woodrow Wilson Center for Scholars, 2008, http://www.wilsoncenter.org/sites/default/files/TCPR_Peterson_ HAPPOP02.pdf.

Quintana, Antonio Gonzalez. Archival Policies in the Defense of Human Rights. Paris: International Council on Archives, 2009.

Rose, Gillian. Visual Methodologies: An Introduction to the Interpretation of Visual Materials. London: Sage, 2007.

Sedgwick, Peter. Psycho Politics: Laing, Foucault, Goffman, Szasz, and the Future of Mass Psychiatry. New York: Harper \& Row, 1982.

Shilton, Katie and Ramesh Srinivasan. "Participatory Appraisal and Arrangement for Multicultural Archival Collections.” Archivaria 63 (2008): 87-101.

Slyomovics, Susan. The Performance of Human Rights in Morocco. Philadelphia: University of Pennsylvania Press, 2005.

Stoler, Ann Laura. "Colonial Archives and the Arts of Governance: On the Content in the Form," in Archives, Documentation, and Institutions of Social Memory: Essays from the Sawyer Seminar, Francis Blouin, ed. Ann Arbor: University of Michigan Press.

Teitel, Ruti G. Transitional Justice. Oxford: Oxford University Press, 2000.

Trace, Ciaran. "On or Off the Record? Notions of Value in the Archive," in Currents of Archival Thinking, Terry Eastwood and Heather MacNeil, eds. (Santa Barbara, CA: Libraries Unlimited, 2010), 47-68.

Upward, Frank. "Modeling the Continuum as Paradigm Shift in Recordkeeping and Archiving Processes, and Beyond - a Personal Reflection." Records Management Journal 10 no. 3 (2000): 115-139.

Trouillot, Michel-Rolph. Silencing the Past: Power and the Production of History. Boston: Beacon Press, 1995. 


\section{Chapter 3 I}

Van Der Merwe, Hugo, Victoria Baxter and Audrey R. Chapman, eds. Assessing the Impact of Transitional Justice: Challenges for Empirical Research. Washington, DC: United States Institute of Peace, 2008.

Van Dijk, Teun. "Principles of Critical Discourse Analysis," in Discourse Theory and Practice, eds. Margaret Wetherell, Stephanie Taylor, and Simeon J. Yates. Los Angeles: Sage, 2001.

Wallace, David. "Historical and Contemporary Justice and the Role of Archivists," in Arkiv, Demokrati Og Rettferd [Archives, Justice, Democracy] (Oslo, Norway: ABMUtvikling, 2006), 14-27.

White, Kelvin and Anne Gilliland. "Promoting Reflexivity and Inclusivity in Archival Education, Research and Practice.” Library Quarterly 80 no. 3 (2010): 231-248.

Williams, Paul. Memorial Museums: The Global Rush to Commemorate Atrocities. Oxford: Berg, 2007.

Wodak, Ruther and Michael Meyer. Methods of Critical Discourse Analysis. Thousand Oaks, CA: Sage, 2009.

Zalaquett, José. "Balancing Ethical Imperatives and Political Constraints: The Dilemma of New Democracies Confronting Past Human Rights Violations," Hastings Law Journal 43 (August 1992).

Zhang, Yan and Barbara M. Wildemuth. "Qualitative Analysis of Content," in Barbara M. Wildemuth, ed. Applications of Social Research Methods to Questions in Information and Library Science. Westport, CT: Libraries Unlimited, 2009.

Zinn, Howard. "Secrecy, Archives and the Public Interest," in The Zinn Reader: Writings on Disobedience and Democracy. New York: Seven Stories, 1997. 
Chapter 32

\title{
EXAMINING ACTIVISM IN \\ PRACTICE
}

\section{Research Design for an Exploratory Study of the Practical Applicability of Archival Activism}

\author{
Joy R. Novak
}

\begin{abstract}
While recent scholarship has defined activism in the context of the archival profession, there has been little empirical examination of the application of such activism in archival practice. This paper details and reflects upon the effectiveness of the design of a qualitative study examining archival activism in terms of the extent to which it has been accepted by professional archivists. The study employed in-depth interviews and focus groups with practising archivists. Participants were asked to draw upon their own professional experience to evaluate the applicability of key concepts associated with archival activism that had been derived from current discourse.
\end{abstract}

\section{Introduction}

The social role of archives ${ }^{1}$ has increasingly been examined in the archival literature, ${ }^{2}$ including the implied responsibility of archival practice to address political and social inequalities. Understanding the significance of the role played by archives in the establishment of social power structures and community development has been the primary justification given for reflections on archival activism (i.e., proactive archival practice that promotes diversity,

1 i.e., the functions archival records and/or institutions serve individuals, communities and society at large such as being sites of cultural memory.

2 i.e., published scholarship about archives and/or archival records regardless of discipline. 
transparency, accountability and social justice). Yet despite this discourse on archives and activism, ${ }^{3}$ few empirical studies have attempted to measure the extent to which practising archivists have accepted this social role or incorporated such activism into practice. Scholarship that has explored the practical application of activism in archives has predominately focused on case studies or on proposing methods rather than on measuring trends in practice across the field. ${ }^{4}$ Empirical study is necessary to evaluate the acceptance of the concept among those practising in the field.

This chapter details and reflects upon the design of a qualitative study that examined practising archivists' perspectives on constituent concepts of archival activism that were derived from current archival scholarly and professional literature. Because there has been little examination of the practical application of activism, the study not only attempted to examine archival activism empirically but also to provide a methodological model that might be used in future research on the subject. This chapter, therefore, addresses the development of the research design, framework, and interview instrument that I used. In addition, it evaluates the research design by considering the completed study findings in order to examine the extent to which the

3 Terry Cook and Joan M. Schwartz, "Archives, Records, and Power: From (Postmodern) Theory to (Archival) Performance." Archival Science 2 (2002): 171-185; Verne Harris, Archives and Justice: A South African Perspective (Chicago, IL: Society of American Archivists, 2007); Randall C. Jimerson, Archives Power: Memory, Accountability, and Social Justice (Chicago, IL: Society of American Archivists, 2009). Scholarship addressing the social and cultural significance of archives includes Francis X. Blouin, Jr., "Archivists, Mediation, and the Constructs of Social Memory," Archival Issues 24 (1999): 101-112; Mike Featherstone, “Archiving Cultures," Journal of Sociology (January/March 2000): 161-184; Kenneth E. Foote, "To Remember and Forget: Archives, Memory, and Culture," American Archivist 53 (Summer 1990): 378-92; Berndt Fredriksson, "The Changing Role of Archivists in the Contemporary Society," Comma: International Journal of Archives 1 no.2 (2002): 37-43; Mark Greene, "The Messy Business of Remembering: History, Memory, and Archives," Archival Issues 28 no. 2 (2003-2004): 95-104; David Lowenthal, "Archives, Heritage, and History," In Archives, Documentation and Institutions of Social Memory, William G. Rosenberg and Francis X. Blouin, Jr., eds. (Ann Arbor, MI: University of Michigan Press, 2006), pp. 43-45; Archives and the Public Good: Accountability and Records in Modern Society Richard J. Cox and David A. Wallace, eds. Westport, CT: Quorum Books, 2002.

4 Examples of case studies or proposed methodologies integrating concepts of archival activism include: Wendy M. Duff and Verne Harris, "Stories and Names: Archival Description as Narrating Records and Constructing Meanings," Archival Science 2 (2002): 263-285; Max J. Evans, "Archives of the People, by the People, for the People," American Archivist 70 (Fall/Winter 2007): 387-400; Ghetu Magia Krause and Elizabeth Yakel, "Interaction in Virtual Archives: The Polar Bear Expedition Digital Collections Next Generation Finding Aid," American Archivist 70 (Spring/Summer 2007): 282-314; Michelle Light and Tom Hyry, "Colophons and Annotations: New Directions for the Finding Aid," American Archivist 65 (Fall/Winter 2002): 216-230. 
methods used were effective in generating data to answer the research questions that guided the study.

\section{Research Design}

The principle aims of the study were to evaluate the extent to which the notion of archival activism was accepted among practising archivists and the applicability of such activism in practice. Its primary research questions were:

- To what extent, if at all, do practising archivists believe that archival activism, as defined by current scholarship, is appropriate and/or applicable to archival practice? If considered appropriate, to what extent, if at all, is it feasible or realistic to incorporate archival activism into practice?

- Which, if any, core concepts of activism are most accepted among practising archivists?

- To what extent, if at all, do practising archivists believe they have agency to effect social change through their practice?

- What variables, if any, contribute to an acceptance of activism among archivists?

The challenge posed by the notion of archival activism to archival traditions makes research design on the topic uniquely complex. Archival activism assumes that archivists in their practice actively engage with records and the public to promote social justice, which seemingly contradicts the traditional understanding of archivists as objective custodians of records. ${ }^{5}$ Passivity in archival practice has been further encouraged by the influence of positivism on the field of modern history, which promoted "scientific"

5 S. Muller, J.A. Feith and R. Fruin, Manual for the Arrangement and Description of Archives, New York: H.W. Wilson Company, 1968; Hilary Jenkinson, A Manual of Archive Administration: Including the Problems of War Archives and Archive Making, London: Clarendon Press, 1922; Theodore Schellenberg, The Management of Archives, Columbia University Press, 1965. For a discussion on the significance of these works, see Michael Duchein, "Theoretical Principles and Practical Problems of Respect des Fonds in Archival Science," Archivaria 15 (1983): 64-82; Anne Gilliland-Swetland, Enduring Paradigm, New Opportunities: The Value of the Archival Perspective in the Digital Environment. (Washington, D.C.: Council on Library and Information Resources, 2000); Anne J. Gilliland and Kelvin White, "Perpetuating and Extending the Archival Paradigm: The Historical and Contemporary Role of Professional Education and Pedagogy," InterActions: UCLA Journal of Education and Information Studies 5 iss. 1 (2009): 1-23. 
historical methods that placed increased emphasis on archival research and analysis. Understanding historical research and archival practice as a science seeks to remove human subjectivity from how archival records, considered to be indisputable evidence, are managed and interpreted. Working within this positivist framework, intervention by the archivist is constrained only to what is essential in order to ensure that this evidence remains intact and unaltered. ${ }^{6}$ Taking this stance to its logical conclusion, archivists would play no active role in record creation, management or dissemination and have little agency or indeed imperative to have other kinds of social impact through their practice.

Scholars from multiple disciplines, including scholars in archival science as well as historians and cultural theorists, have challenged this positivist understanding of archives, exploring the political and cultural functions of archives in society. Conceptual critiques argue that archivists and archival practice can never be neutral since archival work involves active engagement with the record. Largely drawing from postmodern theory, they contest the positivist view of "records as truth." They also argue that records are not static, but are constantly evolving and continually interpreted with each use. Archivists in this view play an active role in shaping and interpreting these evolving records and every decision they make could be considered to be a subjective intervention that ultimately influences the record. ${ }^{7}$ Disciplinary scholarship critical of archival traditions has also examined the social impact of the traditionalist "value neutral" stance of the archivist, primarily arguing that such an approach to practice demonstrates in various ways an imbalance of social power. Post-colonial scholars in particular have explored the ways in which archives, and perhaps more importantly, the archiving process, have been used to inscribe power, not only by archiving the records of those in power, but also by implementing practices that privilege those in power. ${ }^{8}$ Some scholars have addressed the topic of archives and activism

6 Anne J. Gilliland and Kelvin White. "Perpetuating and Extending the Archival Paradigm". For a discussion of the influence of academic historians on the archival field, see also Patrick M. Quinn, "Archivists and Historians: The Times They Are A-Changin', Midwestern Archivist 2 no. 2 (1977): 5-13.

7 Harris, Archives and Justice; Schwartz and Cook, "Archives, Records and Power"; Terry Cook, "Fashionable Nonsense or Professional Rebirth: Postermodernism and the Practice of Archives," Archivaria 51 (Spring 2001): 126-151; Tom Nesmith, "Seeing Archives: Postmodernism and the Changing Intellectual Place of Archives," American Archivist 65 (Spring/Summer 2002): 24-41.

8 Jeannette Allis Bastian, "Whispers in the Archives: Finding the Voices of the Colonized in the Records of the Colonizer," In Political Pressure and the Archival Record, 
more directly, exploring the ways in which proactive archival practice can promote diversity, transparency, accountability and social justice. ${ }^{9}$

The study employed a qualitative, person-centred approach (examining the topic - the applicability of archival activism - by exploring individual participants' personal perceptions of the archival activism) in order to encourage participants to draw upon their professional experience in evaluating concepts central to archival activism. The use of this person-centred framework was largely influenced by my own understanding of archival activism since my attitude towards the concepts is strongly related to my personal and professional background. Given my own experience, I had believed that participants would relate their personal or professional backgrounds to the concepts during their discussion, regardless of their attitude towards archival activism. ${ }^{10}$ This was ultimately reflective of my assumptions of the significance of personal experience on practice.

Regarding the research design, I first derived a framework of core concepts central to archival activism from scholarship addressing the social role of archives and/or archivists to be used in the interview instrument. I then used this instrument in both individual and group (focus groups and video conferences) interviews to examine the above research questions comprehensively. The interviews in this study were guided or semi-structured, which allowed flexibility to elicit more in-depth user response while still giving guidance to address key issues to compare the data between participants. Because of such advantages, interviews are often used for exploratory and

Margaret Procter, Michael Cook and Caroline Williams, eds. (Chicago: Society of American Archivist, 2005), pp. 25-43; Ann Laura Stoler, "Colonial Archives and the Arts of Governance," Archival Science 2 (2002): 87-109.

9 See, for example, Duff and Harris, "Stories and Names"; Jimerson, Archives Power; Randall C. Jimerson, "Archives for All: Professional Responsibility and Social Justice," American Archivist 70 (Fall/Winter 2007): 252-281, and "Embracing the Power of Archives," American Archivist 69 (Spring/Summer 2006): 19-32.

10 My initial interest in becoming an archivist grew out of my undergraduate work as a history major, which introduced me to the special collections library at my university. As I began working in archival collections, I became increasingly aware of the social power of archival work in terms of the extent to which archivists determined which documents are preserved and made available to researchers. For both my bachelors and masters degrees, my primary research focus was gender history. This research, together with my growing involvement with LGBTQ archives and politics, helped me to develop an understanding of archivists' potential agency as activists who could preserve and bring visibility to histories or communities traditionally excluded from archives. For more than eight years, I was the collections manager for a special collection with a social justice mission. I entered the doctoral programme at UCLA to research further the role of archives with regard to traditionally marginalised communities, primarily focusing on LGBTQ archives. 
inductive studies that focus on understanding how people view and interpret their world. ${ }^{11}$ For example, in their 2007 study, Yakel and Torres examined genealogists' relationship with the records they use in their family history research. Yakel and Torres used in-depth interviews with genealogists to explore not only their information seeking behaviour and management but also their perceptions and understanding of their work. ${ }^{12}$

Although the same set of questions was used for both interview formats, group interviews were used in addition to the individual interviews in the hope that group dynamics would bring multiple perspectives to the topic and encourage further comments and examples between peers. Manuel and Beck suggest that focus groups are particularly popular and effective for library science since the groups focus on perceptions and allow immediate feedback on an issue..$^{13}$ In their 2008 study, Duff et al. used semi-structured scripts with five focus groups designed to examine the viewpoints and values of archivists regarding their perceptions of user studies. They offer an in-depth review of the effectiveness of focus groups for their research, suggesting that they offered a way to explore archivists' feelings more fully and encourage diverse and substantial discussion between participants. ${ }^{14}$

\section{Defining Activism: Building a Conceptual Framework}

Instead of using a single, all-encompassing definition, I drew core concepts of archival activism from current archival scholarship. Employing a conceptual framework (in which both the study instrument and data analysis were organised around key themes of archival activism) was appropriate for

11 Susan E. Beck and Kate Manuel, Practical Research Methods for Librarians and Information Professional,. New York, NY: Neal-Schuman Publishers, Inc., 2008.

12 Elizabeth Yakel and Deborah A. Torres. "Genealogists as a 'Community of Records," American Archivist, (Spring/Summer 2007): 93-113. Other examples of archival research employing interview methods include: Wendy M. Duff and Catherine A. Johnson. "Where is the List with All the Names? Information-Seeking Behavior of Genealogists," American Archivist (Spring/Summer 2003): 79-95; Barbara C. Orbach, "The View From the Researcher's Desk: Historians' Perceptions of Research and Repositories," American Archivist (Winter 1991): 28-43; Elizabeth Yakel and Deborah A. Torres. "AI: Archival Intelligence and User Expertise," American Archivist (Spring/ Summer 2003): 51-78.

13 Beck and Manuel, Practical Research Methods for Librarians and Information Professionals.

14 Wendy M Duff, Jean Dryden, Carrie Limkilde, Joan Cherry, and Ellie Bogomazova, "Archivists' Views of User-Based Evaluation: Benefits, Barriers, and Requirements," American Archivist (Spring/Summer 2008): 144-166. 
such a qualitative study because it allowed a more comprehensive and richer examination of activism. Concepts could thus be identified within the data in varying degrees and combinations. For example, it was flexible enough to allow participants to be able to identify strongly with some concepts and less so with others rather than only being able to signify their agreement or disagreement with a more singular definition. Broadening the definition of activism, therefore, conceptually allowed for a more thorough and detailed analysis of the multifaceted ways in which archival activism has been expressed among practising archivists.

To identify the concepts, I explored a range of scholarly and professional literature, both from within and from outside Information Studies (including Archival Science). This examination was not limited to works focusing directly on archival activism or archives and social justice. It also included works exploring the social and cultural significance of archives in society since such discussions ultimately imply a responsibility on the part of archivists to fulfil these social roles. The distinct common themes that emerged from this literature analysis became the core concepts of archival activism for this study.

While including a range of diverse literature in the analysis, I drew heavily on the work of archival scholar Randall Jimerson when developing the core concepts. Not only did Jimerson focus on the intersection of archives and social justice in his 2009 work, Archives Power: Memory, Accountability, and Social Justice, he also discussed the social power of archives and archivists in his 2005 Society of American Archivists (SAA) Presidential Address as well as a 2006 SAA conference. ${ }^{15}$ Both of these presentations addressing the social responsibilities of archivists can be read as calls for practising archivists to use their practice to promote social justice. Jimerson identifies four key ways in which archives can be used to protect the public interest:

- by holding political and social leaders accountable for their actions,

- by resisting political pressure in order to support open government,

- by redressing social injustices, and

- by documenting underrepresented social groups and fostering ethnic and community identities. ${ }^{16}$

As a result of this approach, accountability was defined in my study as maintaining, preserving and making accessible records that document

15 Jimerson, Archives Power; Jimerson, “Archives for All”; Jimerson, "Embracing the Power of Archives."

16 Jimerson, “Archives for All," 256. 
criminal, unethical or other actions to hold governments, political or cultural leaders, or other institutions or people in power accountable. Although Jimerson considered redressing social injustice to be a separate issue, for the purposes of this study, this concept was merged with accountability because archivists' ability to redress social injustice primarily involves maintaining and making accessible records documenting such abuses in order to enforce accountability and thereby enable compensation. ${ }^{17}$ Diversity/inclusivity is conceived of as the aim to actively document communities or cultures that have traditionally been excluded from the historical narrative. ${ }^{18}$ Open government refers to supporting transparency of government action by ensuring access to government records. ${ }^{19}$ In addition to these three concepts, an essential concept that was identified was social power. Central to any definition of archival activism is an awareness of the social power of archives and an

17 See also Greg Bradsher, "Turning History into Justice: The National Archives and Records Administration and Holocaust-Era Assets, 1996-2001," In Arcbives and the Public Good: Accountability and Records in Modern Society, Richard J. Cox and David A. Wallace, eds. (Westport, CT: Quorum Books, 2002), pp. 177-203; Richard J. Cox and David A. Wallace, "Introduction," In Archives and the Public Good: Accountability and Records in Modern Society; John M. Dirks, "Accountability, History and Archives: Conflicting Priorities or Synthesized Strands?” Archivaria 57 (Spring 2004): 29-45; Verne Harris, "The Archival Sliver: Power, Memory, and Archives in South Africa," Archival Science 2 (2002): 63-86; Chris Hurley, "Recordkeeping and Accountability," in Archives: Recordkeeping in Society, edited by Sue McKemmish, Michael Piggott, Barbara Reed and Frank Upward, Wagga Wagga, New South Wales: Charles Stuart University, 2005; Jennifer Osorio, "Proof of a Life Lived: The Plight of the Braceros and What it Says About How We Treat Records," Archival Issues 292 (2005): 97-104; Ian E. Wilson, “Peace, Order and Good Government': Archives in Society," Archival Science 11 (2011): 235-244.

18 Phillip Alexander and Helen W. Samuels, "The Roots of 128: A Hypothetical Documentation Strategy," American Archivist 50 (Fall 1987): 518-31; Hans Booms, "Uberlieferungsbildung: Keeping Archives as a Social and Political Activity," Archivaria 33 (Winter 1991-1992): 25-33; Terry Cook, "Documentation Strategy," Archivaria 34 (Summer 1992): 181-191; Richard J. Cox, "The Documentation Strategy and Archival Appraisal Principles: A Different Perspective,” Archivaria 38 (Fall 1994): 11-36; Timothy Ericson, "To Approximate June Pasture: The Documentation Strategy in the Real World," Archival Issues 22 no. 1 (1997): 5-20; Flinn, "An Attack on Professionalism and Scholarship?”; Jeannette A. Bastian, "Play Mas': Carnival in the Archives and the Archives in Carnival: Records and Community Identity in the US Virgin Islands," Archival Science 9 (2009): 113-125; Alana Kumbier, "Ephemeral Material: Developing a Critical Archival Practice," PhD, diss., The Ohio State University, 2009; Elizabeth W. Adkins, "Our Journey Toward Diversity - and a Call to (More) Action," American Archivist 71 (Spring/Summer 2008): 21-49; Helen W Samuels, "Who Controls the Past," American Archivist 49 (1986): 109-124.

19 Harris, Archives and Justice; Loris Williams, Kristen Thorpe, and Andrew Wilson, "Identity and Access to Government Records: Empowering the Community," Archives and Manuscripts 34 no. 1 (May 2006): 8-30. 
understanding that archivists have agency (i.e., the capacity to exert action) in their practice to affect society at large..$^{20}$ The other key concepts of activism gleaned from current scholarship were neutrality/archival transparency and community engagement. Archival transparency involves archivists disclosing details about their personal background as well as the extent and nature of their intervention with the records in order to provide users with the context necessary to understand how they may have shaped the record. This concept particularly challenges the traditional understanding of archival neutrality (i.e., attempting to remove personal perspectives or beliefs from decisions made by archivists in archival practice) as it requires an acceptance that individual perspectives can have an impact upon the record and how it is understood, managed and made available. ${ }^{21}$ Community engagement refers to archival projects that encourage community participation in the archival process, particularly in the area of description. ${ }^{22}$

\section{Pilot Study and Instrument Development}

Because of the potential for resistance to the concept of activism on the part of interviewees, the framework used to construct the interview instrument outlined above was essential. Moreover, I wished to avoid any unintended

20 Jimerson, Archives and Power, XVII; Gilliland, "Pluralizing Archival Education"; Cook and Schwarz, "Archives, Records, and Power"; Hans Booms, "Überlieferungsbildung." See also Hans Booms, "Society and the Formation of a Documentary Heritage: Issues in the Appraisal of Archival Sources," Archivaria 24 (Summer 1987): 69-107; Philip P. Mason, "Archives in the Seventies: Promises and Fulfillment," American Archivist 44 no. 3 (Summer 1981): 225-241; F. Gerald Ham, "The Archival Edge," American Archivist, (January 1975): 5-13; F. Gerald Ham, "Archival Strategies for the Post-Custodial Era," American Archivist 44 (Summer 1981): 207-216; Trudy Huskamp Peterson, "The National Archives and the Archival Theorists Revisited, 1954-1984," American Archivist 49 no. 2 (Spring 1986): 125-133.

21 Flinn, "An Attack on Professionalism and Scholarship"; Harris, Archives and Justice; Gilliland "Proposition, Pluralizing Archival Education: A Non-Zero-Sum"; Anne Gilliland, "Neutrality, Social Justice and the Obligations of Archival Education in the Twenty-first Century," Archival Science (2011): 193-209.

22 Michelle Light and Tom Hyry, "Colophons and Annotations: New Directions for the Finding Aid,” American Archivist 65 (Fall/Winter 2002): 216-230; Wendy M Duff and Verne Harris, "Stories and Names: Archival Description as Narrating Records and Constructing Meanings," Archival Science 2 (2002): 263-285; Flinn, "An Attack on Professionalism and Scholarship?”; Ghetu Magia Krause and Elizabeth Yakel, "Interaction in Virtual Archives: The Polar Bear Expedition Digital Collections Next Generation Finding Aid"; Prescott, "The Textuality of the Archive”; Evans, "Archives of the People, by the People, for the People."Mick Gooda, "The Practical Power of Human Rights: How International Human Rights Standards Can Inform Archival and Record Keeping Practices," Archival Science (December 2011): 1-21. 
influence over the participants' reactions to the study and consequently, their responses as a result of my choice of wording of, or directions taken by the interview questions. A pilot study consisting of a few individual interviews was conducted, therefore, with the primary aim of refining the interview instrument. Because the goal of the pilot study was to test the usability of the questions, interviews were followed by debriefings of interviewees.

The initial approach to the study avoided any terms related to activism or social justice as these might immediately be rejected because of their potential challenge to more traditional archival thinking. Yet it remained a possibility that participants might oppose the idea of 'activism' while still demonstrating an awareness of their social role and an acceptance of the concepts of archival activism as defined in the archival literature. The questions for the pilot study, therefore, encouraged archivists to reflect on the role of their practice in society without using terminology connoting activism (such as social power). Additional questions asked about the motivations behind decisions that archivists make in everyday practice (See Appendix 1).

The aim of the approach employed in the original interview instrument was to encourage responses that demonstrated participants' understanding of the social role of their work as archivists as well as to elicit their personal motivations behind their practice. Such data could be used to evaluate the extent to which participants' understanding of their work and their professional motivations reflected concepts of archival activism. However, this approach proved problematic as participants were not asked about archival activism directly and, consequently, their responses were not necessarily indicative of their perceptions of the topic. This became apparent in postinterview debriefing. For example, one participant articulated an acceptance of many of the concepts of activism in the debriefing, including efforts to actively pursue acquisitions related to communities traditionally excluded from archives. When asked why this was not expressed during the interview, the participant said that she did not think this area of her work was relevant to any of the questions asked. While the debriefings demonstrated that the indirectness of the questions could result in participants inadvertently failing to demonstrate an acceptance of activism, it was also possible that participants might purposefully avoid vocalising support of activism as such ideas might be deemed inappropriate by some within the field. As this approach did not directly address the topic of archival activism, it would be difficult to determine the extent to which the participants' responses fully reflected their perspectives on the topic of archival activism. Furthermore, this approach would have required significant interpretation of the participants' responses, 
which might not have always resulted in an accurate assessment of their perspectives.

The finalised interview instrument ultimately took a much more direct approach to addressing the topic of archival activism by providing participants with summaries and quotations from the scholarship on archival activism and asking them to evaluate the literature based on their professional experience (Appendix II). The summaries and quotations were used in an effort to provide fuller context and avoid any immediate responses that participants might have to specific terms that they regarded as "loaded." Before focusing on the scholarship on activism, the participants were given a copy of the Society of American Archivists' Code of Ethics to review (the version that was current at the time) and were then asked about the extent to which they felt the Code was significant and applicable to daily practice. The purpose of examining the code of ethics was to evaluate the extent to which they perceived currently accepted professional ethics as applicable to everyday practice. The discussion then focused on activism, beginning with the broader idea of social power and consciousness before moving on to the other 5 core concepts. For each, the summaries or quotations were read and the participants were asked if they agreed or disagreed with the concepts or statements; if they thought they were appropriate for the profession; and the extent to which they were feasible or realistic to integrate into practice. This interview approach helped to ensure that the topic of archival activism was discussed directly and that the participants were given the opportunity to reflect on each key concept.

The interview also included two introductory professional and educational questions used to examine the participants' motivations for entering the field as well as the extent to which they felt their educational background influenced their daily practice. In her examination of archival activism, Anne Gilliland claims that archivists' personal histories are often directly linked to their acceptance of activism, in part because archives' role in promoting social justice can be a motivation for entering the field. Gilliland further suggests there has been a lack of research on the relationship between archivists' personal histories and their practice, demonstrating a need for such a biographical framework in research. ${ }^{23}$ While a Masters of Library and Information Science or equivalent information profession-based degree has become widely accepted as an education standard for the American archival

23 Anne J. Gilliland, "Neutrality, Social Justice and the Obligations of Archival

Educators and Education in the Twenty-first Century," Archival Science 11 nos. 3-4

(2011): 193-209. 
profession, archivists continue to enter the field from different backgrounds and, consequently, archivists' familiarity with existing archival scholarship may vary significantly. Since resistance to archival activism might stem from the challenge that the concept poses to archival traditions, examining participants' archival education and relationship to archival scholarship was an important variable to isolate and examine.

Retrospectively, the significance of negotiating the extent to which activism would be discussed directly in the interview instrument was likely influenced by my own professional, academic and personal background. Even though I did not personally find the concepts threatening, I knew through my experience in the field that many archivists did adhere very closely to tradition and would find these concepts challenging. This experience was the primary reason for the initial attempt to strip the instrument of any direct discussion of archival activism. I believe that one of the advantages of the direct focus on the literature in the final interview instrument was that it shielded my own position on activism from participants. Within the framework of the study, participants were specifically asked to evaluate the concepts of archival activism drawn from current scholarship, as opposed to evaluating definitions or models I personally wrote or developed. This approach gave participants less access to my own position on archival activism, which helped to limit the extent to which participants' perception of my position might influence their responses. For example, if a participant thought I was a strong advocate of archival activism, he/she might respond with more support of archival activism than he/she actually felt because he/ she thought it was the response I wanted to hear. For this same reason, I did not disclose that I was a practising archivist to the study participants despite my personal belief in transparency. While my professional experience might have made participants more comfortable, I felt that any potential benefit would be counterbalanced or negated if they asked where I worked and realised that my own repository has a specific focus on social justice.

\section{Identifying the Participant Pool}

Since the study focused on activism in practice, and in accordance with the types of settings in which American archivists typically work, the subject pool was restricted to archivists who were managing and maintaining archival repositories or special collections. It did not include archival educators or scholars who were not currently practitioners. Practising archivists were defined as any professionals working with primary documents, including 
both traditional institutional records as well as personal papers such as correspondence, scrapbooks, and ephemera. ${ }^{24}$ Similarly, repositories were broadly defined to include, but not be limited to, local, state or federal archives; university archives; community-based archives and historical societies; and special collections and manuscript libraries.

The study subjects were archivists practising in the Midwest region of the United States, as defined by the states represented by the Midwestern Archives Conference (MAC), one of two major regional archives associations in the United States. The region was chosen because it includes rural and urban communities with diverse types of institutions with archives or historical collections including major research universities, local historical societies, state and local government offices, and religious bodies. It is diverse politically, representing both politically conservative and liberal states and regions. While the American east and west coasts are often perceived as being more liberal or progressive, the Midwest is commonly known as the 'Heartland' or 'Middle America,' implying an inherent belief that the region is more representative of the country's perspective. While this depiction may be a stereotype, the decision to focus on the Midwest was made to avoid the potential critique of regional bias. Further studies focusing on other regions might be able to determine if such a bias does exist.

\section{Sampling and Recruitment}

Random sampling for participant recruitment was generated from a master list of repositories located in the 13 states represented in MAC and gathered from three directories: The Directory of Repositories of Primary Sources, which includes a list of over 5000 websites for archival and manuscript repositories; the membership directory for the SAA; and the membership directory for MAC. The SAA and MAC membership directories were only used to identify additional repositories in the region. No individual membership information was collected. The Repository of Primary Sources directory includes corporate, regional, public and private collections, and there are 642 repositories in the states in the MAC region listed in the directory. ${ }^{25} \mathrm{~A}$ search by

24 Professional titles were not considered when determining eligibility as professional responsibilities are not consistently tied to specific titles. See Caroline Williams, "Personal Papers: Perceptions and Practice" in What are Archives? A Cultural and Theoretical Perspectives: A Reader, Louise Craven, ed. (Hampshire, England: Ashgate Publishing Limited, 2008), pp. 7-30.

http://www.uiweb.uidaho.edu/special-collections/Other.Repositories.html. 
state was used to locate any repositories represented in the SAA or MAC directories that were not included on the Directory of Repositories of Primary Sources. The repositories names were all entered into an Excel spreadsheet and sorted using the randomisation feature to generate a random sample.

For individual interview and videoconference recruitment, a participation request was sent to the general contact address of any repository that had been randomly selected from the master inventory. This initial contact offered a brief description of the project as well as the qualifications for participation. For the focus groups conducted at the MAC and SAA conferences, participants were recruited by posting an inquiry to the MAC, SAA and the SAA roundtable email listservs. ${ }^{26}$ In addition, focus group recruitment emails were sent to the general contact address for all the repositories in the city hosting the conference. For the local focus groups, recruitment emails were sent to all the repositories in the selected cities from the master list of the repositories. Once the repository had been contacted for a local focus group, it was removed from the master inventory as a potential contact for individual interviews or videoconference recruitment.

To encourage participation, both individual and group interview participants were entered into a raffle for a $\$ 50$ gift certificate for participation.

\section{Individual Interviews}

Individual interviews were conducted by telephone as it would not require travelling on the part of either interviewees or the researcher but would still support a broader base of participation. The interviews were audio-recorded and were approximately 60 minutes long. Prior to the interview, participants were sent an email with an informed consent form and a link to the demographic survey. The first question on the survey consisted of a yes-no checkbox asking if the participant had read and fully understood the consent form. The survey contained personal and professional demographics questions including gender identification, ethnicity, age, degrees earned (including year), and undergraduate and graduate fields of studies. The demographic questions were only used as variables in analysing the data of the study and did not affect recruitment or participation in any way.

26 The listservs to which recruitment emails were sent include the Public Library Archives/Special Collections Roundtable; Issues \& Advocacy Roundtable; Human Rights Archives Roundtable; Reference, Access and Outreach Roundtable; Lone Arrangers Roundtable; Archives Management Roundtable; and the Labor Archives Roundtable. In addition, a MAC conference organiser forwarded a recruitment email to the entire MAC mailing list. 


\section{Group Interviews}

Group interviews were also audio-recorded. Participants were sent the informed consent and a link to the demographic survey in an email prior to the group interview. Due to time considerations, participants were asked to participate in a brief telephone interview prior to the group session in order to address the two professional and educational background questions, as these would take up a significant amount of the group time while not encouraging significant group discussion. Asking these questions individually in a preliminary phone interview, usually lasting no longer than 10 minutes, made the most productive use of the group discussion time. This proved to be effective and helped to keep the group session at approximately 90 minutes.

Because of the large size of the region, focus groups were organised around the Midwestern Archives Conference (MAC) Annual Meeting and the SAA Annual Conferences. These events bring potential participants to a centralised location, allowing for more regional diversity within groups. Since limiting focus groups to conference participants had the potential to bias results as participants might be more aware of trends in the field due to such recent conference attendance, several local focus groups in cities throughout the region had also been planned. Cities were originally selected based on diversity in size, demographics and the number of repositories in the region.

Because of the logistical difficulty of organising focus group sessions, the design of the study was subsequently modified to include videoconferences in an attempt to conduct more group interview sessions without requiring participants to meet at a central location. Videoconference participation included taking the online survey and participating in the preliminary telephone interview.

\section{Modifications to Recruitment and Research Methods}

The logistical difficulties of organising the focus groups necessitated modification to recruitment and research methods. Organising the local focus groups proved especially difficult as the participant pool was limited by the number of repositories in the region. Attempts were made to organise focus groups in three cities: Springfield (IL), Milwaukee and Indianapolis. However, no more than one person expressed interest in participating in any single city, so the local focus groups were not conducted. This ultimately 
resulted in both a modification to recruitment and to the group interview methods. With regard to recruitment, the participants who did respond with interest in the local focus groups were asked if they were interested in participating in the individual phone interview as an alternative. The methods of the study were further modified to use videoconference as the format for conducting group interviews instead of focus groups because recruitment would not then be limited by location. Videoconference recruitment followed the same sampling methods as the individual interview recruitment.

\section{Evaluation of Research Design}

\section{i. Measuring the Acceptance of Archival Activism}

The primary aim of the study was to measure the extent of acceptance of archival activism among practising archivists, which included identifying the concepts most accepted as well as variables that correlate to archivists' acceptance of the notion. The research design was successful in exploring archivists' perceptions of archival activism and many themes emerged from this data that suggested conclusions related to practising archivists' support of archival activism. However, while the interview instrument approached the concepts directly by using quotes and summaries from the scholarship, the participants' full responses often demonstrated that they understood the concepts differently from how they were defined in published scholarship. This range in interpretations of the concepts ultimately made it difficult to measure individual participant's acceptance of the concepts consistently and to compare acceptance between participants. Voiced agreement was not necessarily indicative of support if the full response from an individual demonstrated an understanding that diverged from the scholarship. Furthermore, comparison of participants' acceptance was also more complicated and potentially problematic because participants did not necessarily expand on their answers to the same extent. It was possible, therefore, that some participants who had contradictory understandings of the concepts simply did not indicate this in their responses.

Overall, the interview instrument was much more effective in identifying themes that indicated rejection or acceptance of archival activism among participants. The findings were used to create an acceptance key to identify rejection or support of the participants' acceptance of the concepts of activism which facilitated analysis and evaluation of the data. 


\section{ii. Summary of Findings}

While the findings of the study are not generalisable to the wider community of practising archivists because of the limited number of participants, a comparison of the acceptance of the different concepts facilitates analysis of the concepts themselves as well as of the interview instrument, which may be significant for future research. Although participants voiced overwhelming support for concepts such as diversity/inclusivity, community engagement, accountability and open government, their responses demonstrated that many did not strongly support individual concepts as they were defined in the archival literature. Overall, no concept as thus defined was strongly accepted by the majority of participants, with most being moderately accepted.

The concept most accepted was diversity/inclusivity, which not only had the most participants indicating high acceptance (12) but also had only 1 participant indicating no acceptance. An indication of high acceptance voiced support of active collecting, which would include both pursuing the acquisition of diverse collections as well as assisting communities in their own documentation to increase diversity/inclusivity in the historical record. While many participants indicated that budget, space or staff limitations restricted the extent to which they could actively collect diverse materials for their collections, many also indicated their support for projects which would assist communities in documenting themselves. The appropriateness of this work was addressed directly in a follow-up question that asked participants to focus on a specific effort relating to diversifying collections. This strategy helped to differentiate between different levels of acceptance, and highlights the need for even more experientially-based or critical incident-type questions in future interview instruments.

Participants were most divided over the concept of social power. 10 participants demonstrated high acceptance of the concept, while 8 rejected it, some very clearly so. Almost all of the participants who did not support the concept recognised the potential for archivists to wield social power but believed that they should not exercise that power (primarily out of concern that it would interfere with archivists' neutrality). Similarly, participants were very divided on the concept of neutrality/archival transparency, with 11 indicating high, 7 moderate, and 6 no acceptance. As with the concept of social power, those rejecting the concept of archival transparency did so with the belief that it too might result in archivists shaping or exerting undue influence over the records and how they might be interpreted. 
Participants voiced almost unanimous, and often very strong, support of the concepts of community engagement, accountability and open government; however, only a few participants indicated high acceptance for each concept as defined by the scholarship. With the concept of community engagement, participants generally did not indicate clear rejection but demonstrated an understanding of the concept that was not as theoretical, proactive or engaged with the community as how it was being discussed in the archival literature. In the case of accountability and open government their support was limited by the extent to which they felt archivists had agency in these areas. Like social power and neutrality/archival transparency, accountability also had a clear indicator of rejection (i.e., the belief that archivists should not interpret or provide any value judgment on the records) and a similarly high number of participants indicating no acceptance (6). Ultimately, these findings demonstrate the extent to which the participants' understanding of the concepts differed from the published scholarship on archival activism.

\section{iii. Advantages of Methods}

The design of the study was successful in generating significant data on participating practising archivists' perspectives on the concepts of archival activism as drawn from current published scholarship. The interviews, both individual and group, also demonstrated ways in which practising archivists see the concepts currently being incorporated into practice. In some cases, such examples have not been discussed in current scholarship, perhaps because it has primarily been generated by academics, not practising archivists. In addition, the format of the interviews gave the participants the opportunity to express the extent to which they believe such ideas are practical and realistic in terms of everyday practice, which is also an area where their perception as practising archivists and those of academics can differ significantly.

Regarding the advantages of interview formats, while individual interviews could generate significant data, group interviews had the potential to generate more detailed, richer data as they not only recorded the primary responses but also the other participants' feedback, comments and shared experiences. However, the group format could also be problematic as individual participants' responses could influence overall results to some degree. For the purposes of an exploratory study, the benefits of the data produced 
from the more in-depth discussions outweighed the few instances of potentially inflated agreement.

\section{iv. Limitations and Challenges of the Research Design}

The most significant limitation to the study was the use of small-scale research methods. Since the study explored the participants' perceptions of archival activism, it was more important to have richer data from a smaller number of participants than less in-depth data from a larger group of participants. However the small scale of the project limited the extent to which the findings could be generalised to a larger population of practising archivists.

The primary challenge of the research design involved participant recruitment, specifically for the group interviews. While the response rate for individual interviews had been low, ${ }^{27}$ more recruitment emails could be sent out to secure individual interview participants. However, the focus group protocol limited recruitment to the repositories within the selected cities and also required participants' ability to meet at a centralised location at a specific time. ${ }^{28}$ Overall, the low recruitment response rate is possibly indicative of both low practitioner interest in participating in a study of archival activism as well as limited availability for participation. It is difficult to determine whether the recruitment would have been more successful had the recruitment documents not mentioned activism. Three initial respondents said they did not consider themselves 'activists' or did not think their work 'reflected archival activism.' Despite reassurance that this was not a requirement of participation, these individuals did not wish to participate. It is impossible to know how many other recipients of the emails might have not responded because they thought it was necessary to identify as an activist to participate. However, without a further study targeted on recruitment, it would be impossible to determine if such response was due to the topic of the study.

27 Of the repositories contacted, individuals from only $32.5 \%$ responded and only $16 \%$ participated.

28 Only $13.5 \%$ responded and only $4 \%$ participated. 


\section{Conclusions}

\section{i. Framework}

While the research design remained person-centred, the participants' responses were overall less personally reflective than I had anticipated. Instead of approaching the concepts by discussing their professional and personal backgrounds, most participants instead focused on the practical or logistical aspects. A small minority of participants did relate some of their responses to their personal experiences; however, participants' perspectives on archival activism appeared less influenced by personal and professional history than I expected. This unexpected trend ultimately made the findings seem less "person-centred" than I had envisaged when designing the study. Retrospectively, I think this may be attributed to the interview instrument design, specifically in the clear separation of biographical and educational questions from concept questions. While I intended to use these introductory questions to both identify variables and frame the rest of the discussion, this approach may have encouraged some participants to contain their personal reflections more to the 'biographical' questions. However, I do not believe any shortcomings in the person-centred framework negatively impacted the goals of the study as participants still offered significant reflections on their perceptions on the concepts of archival activism, even if most were more practical than personal.

\section{ii. Future Research}

The results of this study demonstrate several areas for further examination. First, there is a need to conduct a study specifically on recruitment methods for studies related to "controversial" topics such as archival activism. Considering the recruitment challenges, a study focused specifically on recruitment methods which compared response rates between recruitment documents using the word "activism" and some kind of alternative phrasing would have been valuable in refining a more effective recruitment tool. Future larger scale studies on archival activism would require more participants, thus increasing the need for as high a participant response rate as possible.

Another potential study would specifically focus on self-identified 'activists' to examine the extent to which those who did feel dedicated to these issues believed they had agency and that the identified concepts were feasible. 
Such a study would help minimise the unknown factor of participants' potential complacency in evaluating the feasibility of archival activism, since participants with less acceptance of archival activism might be more prone to consider some concepts unfeasible if they are less motivated to commit the necessary time or resources. Also, because of the lack of racial diversity among participants, future studies might also benefit from targeted recruitment of participants of colour by reaching out directly to the Archivists of Color Roundtable for assistance. As the current study was regional, there is also need to expand the study geographically to determine if the results were influenced by the region that was chosen. However, in addition to conducting a national study, a comparative international study in countries with different archival traditions or recordkeeping histories would also be informative. Examples might include countries whose archival professions are not as closely tied with the history profession or countries previously under government regimes that tightly controlled how records and archives are managed. Such a comparative study would indicate the extent to which perceptions are shaped by archival professional traditions as well as by national political histories.

As the interview instrument was based around concepts derived from published scholarship, often using quotes from the literature on archival activism in an attempt to examine the concepts directly, this framework might have seemed to isolate the concepts from practice, potentially influencing participants' perceptions of the concepts. Some participants may have responded in part to the academic language of the instrument in addition to the concepts themselves. Future studies might benefit from using frameworks that situate the concepts within practical scenarios for participants to evaluate. For example, the participants could be given an example of a practice that would be considered to be archival activism, as defined by the scholarship, and asked to what extent they think it is appropriate and why. However, the acceptance key developed from the findings of this initial exploratory study of the concepts will enable more directed and efficient interview instruments for future studies using such alternative frameworks.

\section{iii. Core Values of Archivists}

In 2011, the Society of American Archivists approved a statement on the Core Values of Archivists to accompany the Code of Ethics. The Core Values focus on the following aspects of practice: Access and Use, Accountability, Advocacy, Diversity, History and Memory, Preservation, Professionalism, 
Responsible Custody, Selection, Service, and Social Responsibility. The purpose of this statement is "to remind archivists why they engage in their professional responsibilities and to inform others of the basis for archivists' contributions to society" and "to provide guidance by identifying the core values that guide archivists in making such decisions and choices." While this current study did not aim to examine these core values specifically, many of the concepts of archival activism that were explored in the study discussed here did address values such as accountability, diversity, service and social responsibility. Because of this overlap, the findings of this study suggest how and the extent to which these Core Values might be perceived and accepted in certain setting. Potentially, the research design and the acceptance key developed through this study could be used to design a study specifically to evaluate the reception of these Core Values among practising archivists throughout the field.

\section{iv. Significance of Study}

The study outcomes contribute to the scholarship on archival activism in several ways. Perhaps the most significant contribution is the development of the acceptance key that can be used in future studies to identify acceptance or rejection of key concepts of archival activism. Having this tool to analyse data on archival activism allows for employing a wider range of research methods in future studies. While the questions in this study were openended and allowed participants to expand on their responses, the interview instrument used a set script to insure that participants answered the same questions to facilitate data analysis. With an existing tool to measure the data, future studies' can have more flexibility in the instrument, allowing for more participatory methods in which the participants have a larger role in the direction of the study. For example, participants could be given scenarios that had social justice implications and asked what they would do in that situation, allowing them to express their approach to practice in their own words. The acceptance key produced by this completed study can be used to both develop such a study instrument and evaluate the collected data. Overall, the outcomes of this study can be used to further rigorous research on archival activism, including evaluating the impact of advocacy on such activism and supporting paradigm shifts in archival practice that promote social justice. 


\section{Appendix One - Pilot Study Interview Questions}

1. Why did you become an archivist?

2. What do you think is the primary role of Archives in Society? For individual and communities

3. What do you think is the primary role of an archivist? What is the most significant functions the profession serves society? Has your understanding of the field changed throughout the course of your career?

4. What aspect of archival work do you think is the most important and why?

5. What do you feel is your biggest motivation in your work? What factors motivates your work and decisions in the field?

6. To what extent do you think that your archival education shapes your work?

7. To what extent do you think your own perspective or background has influenced your practice?

8. How do you decide what collections are prioritised for collecting and processing? Do you have an example of a collection that you thought was really important to collect or prioritise for processing?

9. Can you describe a project that you've worked on as an archivist that you've felt was especially significant for you personally?

\section{Appendix Two - Final Interview Schedule}

BACKGROUND QUESTIONS: Before we talk about the literature on archival activism, I would like to start with a brief discussion on your background and your experience in the field:

1. Why did you become an archivist?

2. How has your archival education or training influenced your archival practice? What concepts or topics addressed in your education have you found to be the most important in your everyday practice?

CODE OF ETHICS: I'd like to briefly discuss the current SAA 'Code of Ethics," Here is a copy of the existing SAA code of ethics for you to look over. 
3. To what extent do you think the ethics discussed here apply to everyday practice? Are there any points that are particularly important to your practice? Are there any points that you think are not applicable or inappropriate to your practice?

THEMES OF ARCHIVAL ACTIVISM: Let's move on to the discussion of the literature on archival activism, starting with some larger concepts before moving onto more specific elements.

SOCIAL POWER/SOCIAL CONCIOUSNESS: The discussions of archival activism argue that archivists wield a significant amount of social power in their work with records: appraising, collecting, preserving, describing and making them accessible. For example, archival scholars Terry Cook and Joan Schwartz claim archivists have the "power to make records of certain events and ideas and not others, power to name, label, and order records to meet business, government, or personal needs, power to preserve the record, power to mediate the record, power over access, and power over individual rights and freedoms, over collective memory and national identity."

4. To what extent do you believe archivists can or do exert such social power through archival practice? Do you think this idea of the social power of archivists applies to your own work?

A key argument for archival activism is that archival practice has traditionally created an imbalance of such social power. The most recent draft of the "Values Statement" prepared in 2010 by the Task Force on Developing a Statement of Core Values for Archivists, specifically points out this imbalance: "Since ancient times, archives have afforded a fundamental power to those who control them."

5. Do you agree that traditional archival practice has created an imbalance of social power? Why or why not? To what extent do you believe archivists have agency in supporting or even exercising social power?

NEUTRALITY: Another issue central to the discussion of archival activism is the professional role of neutrality in archival practice, which can be summarised by Mark Greene in his the 2008 SAA Presidential address. Greene claimed "Our values include a recognition, acceptance, and deliberate application of our own agency in the work we do with records and users. This simply means that we are not neutral or objective protectors and transmitters of primary sources, but shapers and interpreters of the sources as well." 
Do you agree with this assessment of archival neutrality? Why or why not? What do you think is the role of neutrality in archival practice? How do you deal with your personal perspectives in your practice?

6. The discussion of activism has suggested the need for archivists to be transparent about their perspectives and the influence they might have on shaping the record. One option proposed was to include a biographical note about the archivists in the finding aid. Do you think that this would be appropriate? Do you see any benefit of doing this? Limitations?

DIVERSITY/INCLUSIVITY: The discussion of archival activism identifies ways in which archivists can be more proactive in practice. One way is to actively give voice to largely marginalised or undocumented communities. This focus on diversity and inclusivity was included in the recent 2010 draft of the "Values Statement", which includes diversity as a core value. It states that "Archivists embrace the importance of deliberately acting to identify (even create) materials documenting those whose voices have been overlooked or marginalized."

7. Do you agree that this should be a priority for archivists? Why or why not? To what extent do you think it is appropriate or feasible for archivists to actively seek out collections or assist in the creation of documentation of under-represented communities?

One example might be: Assisting communities in documenting their own culture. Do you think this is an appropriate role? Realistic?

COMMUNITY ENGAGEMENT: Along with the emphasis on more diverse representation in the archival record, the discussion of archival activism has also addressed the significance of incorporating insider voices into description. This can include participatory archival projects, which would encourage users with insider knowledge of the subject/community/collection to contribute to the description.

8. Do you agree that this should be a priority? Why or why not? Do you think that this type of project would be beneficial to archival work? Do you think it is realistic? Have you had experience doing this?

ACCOUNTABILITY AND OPEN GOVERNMENT: Another issue central to archival activism is the significance of archivists in holding 
governments, political or cultural leaders, or other institutions or people in power accountable for their actions. This includes maintaining, preserving and making accessible records that document criminal, unethical or other un-just actions.

9. Do you agree that this is the responsibility of archivists? To what extent, if any, do you feel archivists should consider accountability when making professional decisions?

Along with accountability, most scholarship on archival activism also promotes open government, suggesting archivists should support transparency of government action by ensuring access to government records.

10. To what extent, if any, do you agree that supporting open government should be a responsibility or priority of archivists?

CONCLUDING QUESTIONS: Overall, do you think that the ideas and aspirations addressed in the discussion of archival activism are appropriate for everyday practice? Why or why not?

Are there certain areas of practice for which activism might be more appropriate? Where it might be less appropriate?

To what extent do you think archivists have the capacity or agency to use their practice as a form of activism?

Thank you for agreeing to participate in this research. If you have any questions, please feel free to contact me at any time. I also encourage you to contact me if you think of anything you may like to add that we did not address in our discussion today.

\section{References}

Adkins, Elizabeth W. “Our Journey Toward Diversity - and a Call to (More) Action.” American Archivist 71 (Spring/Summer 2008): 21-49.

Alexander, Phillip, and Helen W. Samuels. "The Roots of 128: A Hypothetical Documentation Strategy." American Archivist 50 (Fall 1987): 518-31.

Bastian, Jeannette A. "Play Mas': Carnival in the Archives and the Archives in Carnival: Records and Community Identity in the US Virgin Islands." Archival Science 9 (2009): 113-125.

Bastian, Jeannette A. "Whispers in the Archives: Finding the Voices of the Colonized in the Records of the Colonizer." In Political Pressure and the Archival Record, edited by Margaret Procter, Michael Cook and Caroline Williams (Chicago: Society of American Archivist, 2005), 25-43.

Beck, Susan E. and Kate Manuel, Practical Research Methods for Librarians and Information Professionals. New York, NY: Neal-Schuman Publishers, Inc., 2008. 
Blouin, Francis X. Jr. "Archivists, Mediation, and the Constructs of Social Memory." Archival Issues 24 (1999): 101-112.

Boles, Frank. “Just a Bunch of Bigots': A Case Study in the Acquisition of Controversial Material." Archival Issues 19 (1994): 53-65.

Booms, Hans. "Society and the Formation of a Documentary Heritage: Issues in the Appraisal of Archival Sources.” Archivaria 24 (Summer 1987): 69-107.

Booms, Hans. "Uberlieferungsbildung: Keeping Archives as a Social and Political Activity." Archivaria 33 (Winter 1991-1992): 25-33.

Bradsher, Greg. "Turning History into Justice: The National Archives and Records Administration and Holocaust-Era Assets, 1996-2001." In Archives and the Public Good: Accountability and Records in Modern Society, edited by Richard J. Cox and David A. Wallace (Westport, CT: Quorum Books, 2002), 177-203.

Cook, Terry. "Documentation Strategy." Archivaria 34 (Summer 1992): 181-191.

Cook, Terry. "Fashionable Nonsense or Professional Rebirth: Postermodernism and the Practice of Archives." Archivaria 51 (Spring 2001): 126-151.

Cook, Terry. "Remembering the Future: Appraisal of Records and the Role of Archives in Constructing Social Memory." In Archives, Documentation and Institutions of Social Memory: Essays from the Sawyer Seminar (Ann Arbor, MI: University of Michigan Press, 2007), 169-181.

Cook, Terry. "What is Past is Prologue: A History of Archival Ideas Since 1989, and the Future Paradigm Shift.” Archivaria 43 (Spring 1997): 17-63.

Cook, Terry and Joan M. Schwartz, "Archives, Records, and Power: From (Postmodern) Theory to (Archival) Performance." Archival Science 2 (2002): 171-185.

Cox, Richard J. "The Archivist and Collecting: A Review Essay." American Archivist 59 (Fall 1996): 496-512.

Cox, Richard J. “The Documentation Strategy and Archival Appraisal Principles: A Different Perspective.” Archivaria 38 (Fall 1994): 11-36.

Cox, Richard J., and David A. Wallace. "Introduction." In Archives and the Public Good: Accountability and Records in Modern Society, edited by Richard J. Cox and David A. Wallace. Westport, CT: Quorum Books, 2002.

Craven, Louise. "From the Archivist's Cardigan to the Very Dead Sheep: What are Archives? What are Archivists? What do They Do?" In What are Archives?: Cultural and Theoretical Perspectives: A Reader, edited by Louise Craven (Hampshire, England: Ashgate Publishing Limited, 2008), 7-30.

Cvetkovich, Ann. An Archive of Feelings: Trauma, Sexuality, and Lesbian Public Cultures. Durham, NC: Duke University Press, 2003.

Dirks, John M. "Accountability, History and Archives: Conflicting Priorities or Synthesized Strands?” Archivaria 57 (Spring 2004): 29-45.

Duchein, Michael. "Theoretical Principles and Practical Problems of Respect des Fonds in Archival Science," Archivaria 15 (1983): 64-82.

Duff, Wendy M., Jean Dryden, Carrie Limkilde, Joan Cherry, and Ellie Bogomazova. "Archivists' Views of User-Based Evaluation: Benefits, Barriers, and Requirements." American Archivist 71(Spring/Summer 2008): 144-166.

Duff, Wendy M. and Verne Harris. "Stories and Names: Archival Description as Narrating Records and Constructing Meanings.” Archival Science 2 (2002): 263-285.

Duff, Wendy M. and Catherine A. Johnson. "Where is the List with All the Names? Information-Seeking Behavior of Genealogists.” American Archivist 66 (Spring/ Summer 2003): 79-95.

Duranti, Luciana. “The Concept of Appraisal and Archival Theory." American Archivist 57 (Spring 1994): 328-344. 
Eastwood, Terry. "Reflections on the Goal of Archival Appraisal in Democratic Societies." Archivaria 54 (Fall 2002): 59-71.

Ericson, Timothy. "To Approximate June Pasture: The Documentation Strategy in the Real World.” Archival Issues 22 no. 1 (1997): 5-20.

Evans, Max J. "Archives of the People, by the People, for the People." American Archivist 70 (Fall/Winter 2007): 387-400.

Fear, Kathleen and Conway, Paul, "Valuing the American Archivist: An Interpretation of SAA's First Readership Survey.” American Archivist (Fall/Winter 2011): 685-701.

Featherstone, Mike. "Archiving Cultures.” Journal of Sociology (January/March 2000): 161-184.

Flinn, Andrew. "'An Attack on Professionalism and Scholarship'?: Democratising Archives and the Production of Knowledge." Ariadne 62 (January 2010).

Foote, Kenneth E. "To Remember and Forget: Archives, Memory and Culture." American Archivist 53 (Summer 1990): 378-92.

Fredriksson, Berndt. "The Changing Role of Archivists in the Contemporary Society." Comma: International Journal of Archives 1 no.2 (2002): 37-43.

Gilliland, Anne J. "Neutrality, Social Justice and the Obligations of Archival Education in the Twenty-first Century." Archival Science (2011): 193-209.

Gilliland, Anne J. "Pluralizing Archival Education: A Non-Zero-Sum Proposition," Chapter 10 in Through the Archival Looking Glass: A Reader on Diversity and Inclusion, Mary Caldera and Kathy Neal, eds. (Chicago, IL: Society of American Archivists, 2014), 231-268.

Gilliland, Anne J., and Kelvin White. "Perpetuating and Extending the Archival Paradigm: The Historical and Contemporary Role of Professional Education and Pedagogy." InterActions: UCLA Journal of Education and Information Studies 5 iss.1 (2009): 1-23.

Gilliland-Swetland, Anne. Enduring Paradigm, New Opportunities: The Value of the Archival Perspective in the Digital Environment. Washington, D.C.: Council on Library and Information Resources, 2000.

Gilliland-Swetland, Luke J. “The Provenance of a Profession: The Permanence of the Public Archives and Historical Manuscripts Traditions in American Archival History." American Archivist 54 (Spring 1991): 160-175.

Gooda, Mick. "The Practical Power of Human Rights: How International Human Rights Standards Can Inform Archival and Record Keeping Practices." Archival Science (December 2011): 1-21.

Gracy, Karen F. "Documenting Communities of Practice: Making the Case for Archival Ethnography.” Archival Science 68 no. 2 (2004): 335-365.

Greene, Mark. "The Messy Business of Remembering: History, Memory, and Archives." Archival Issues 28 no. 2 (2003-2004): 95-104.

Greene, Mark, and Dennis Meissner. "More Product, Less Process: Revamping Traditional Processing." American Archivist 68 no. 2 (Fall/Winter 2005): 208-263.

Hackman, Larry J., and Joan Warnow-Blewett. "The Documentation Strategy Process: A Model and A Case Study." American Archivist Winter 1987: 12-47.

Ham, F. Gerald. "Archival Strategies for the Post-Custodial Era." American Archivist 44 (Summer 1981): 207-216.

Ham, F. Gerald. “The Archival Edge.” American Archivist (January 1975): 5-13.

Harris, Verne. Archives and Justice: A South African Perspective. Chicago, IL: Society of American Archivists, 2007.

Harris, Verne. "The Archival Sliver: Power, Memory, and Archives in South Africa." Archival Science 2 (2002): 63-86. 
Hill, Amanda. "Serving the Invisible Researcher: Meeting the Needs of Online Users." Journal of the Society of Archivists 25 no.1 (2004): 139-148.

Hurley, Chris. "Recordkeeping and Accountability." In Archives: Recordkeeping in Society, edited by Sue McKemmish, Michael Piggott, Barbara Reed and Frank Upward. Wagga Wagga, New South Wales: Charles Stuart University, 2005.

Jenkinson, Hilary. A Manual of Archive Administration: Including the Problems of War Archives and Archive Making. London: Clarendon Press, 1922.

Jenkinson, Hilary. "Reflections of an Archivist." In A Modern Archives Reader: Basic Readings on Archival Theory and Practice, Maygene F. Daniels and Timothy Walch, eds. (Washington D.C.: National Archives and Records Administration, 1984, reprinted in 2004), 20.

Jimerson, Randall C. "American Archivists and the Search for Professional Identity." In Randall C. Jimerson ed. American Archival Studies: Readings in Theory and Practice (Chicago, IL: Society of American Archivists, 2000), 1-17.

Jimerson, Randall C. "Archives for All: Professional Responsibility and Social Justice." American Archivist 70 (Fall/Winter 2007): 252-281.

Jimerson, Randall C. "Embracing the Power of Archives." American Archivist 69 (Spring/ Summer 2006): 19-32.

Jimerson, Randall C. Archives Power: Memory, Accountability, and Social Justice. Chicago, IL: Society of American Archivists, 2009.

Johnson, Elizabeth Snowden. "Our Archives, Our Selves: Documentation Strategy and the Re-Appraisal of Professional Identity." American Archivist 71 (Spring/Summer 2008): 190-202.

Kaplan, Elisabeth. "We Are What We Collect, We Collect What We Are: Archives and the Construction of Identity." American Archivist 63 (Spring/Summer 2000): 126-151.

Kaplan, Elisabeth. "Many Paths to Partial Truths: Archives, Anthropology, and the Power of Representation." Archival Science 2 (2002): 209-220.

Ketelaar, Eric. "Archival Temples, Archival Prisons: Modes of Power and Protection." Archival Science 2 (2002): 221-238.

Krause, Ghetu Magia, and Elizabeth Yakel. "Interaction in Virtual Archives: The Polar Bear Expedition Digital Collections Next Generation Finding Aid." American Archivist 70 (Spring/Summer 2007): 282-314.

Kumbier, Alana. "Ephemeral Material: Developing a Critical Archival Practice." PhD, diss., The Ohio State University, 2009.

Light, Michelle, and Tom Hyry. "Colophons and Annotations: New Directions for the Finding Aid." American Archivist 65 (Fall/Winter 2002): 216-230.

Lowenthal, David. "Archives, Heritage, and History." In Archives, Documentation and Institutions of Social Memory, William G. Rosenberg and Francis X. Blouin, Jr., eds. (Ann Arbor, MI: University of Michigan Press, 2006), 43-45

MacNeil, Heather. "Trusting Records in a Postmodern World." Archivaria 51 (Spring 2001): 36-47.

Mason, Jennifer. "Qualitative Interviewing: Asking, listening and interpreting." In Qualitative Research in Action, Tim May, ed. (Thousand Oaks, California: Sage Publications, 2002), 225-241.

Mason, Philip P. "Archives in the Seventies: Promises and Fulfillment." The American Archivist 44 no. 3 (Summer 1981): 199-206.

Millar, Laura. "An Obligation of Trust: Speculations of Accountability and Description." American Archivist 69 (Spring/Summer 2006): 60-78.

Muller, S., Feith, J.A., and Fruin, R. Manual for the Arrangement and Description of Archives. New York: H.W. Wilson Company, 1968. 
Nesmith, Tom. "Reopening Archives: Bringing New Contextualities into Archival Theory and Practice." Archivaria 60 (Fall 2005): 259-274.

Nesmith, Tom. "Seeing Archives: Postermodernism and the Changing Intellectual Place of Archives.” American Archivist 65 (Spring/Summer 2002): 24-41.

Orbach, Barbara C. “The View From the Researcher's Desk: Historians' Perceptions of Research and Repositories." American Archivist 54 (Winter 1991): 28-43.

Osorio, Jennifer. "Proof of a Life Lived: The Plight of the Braceros and What it Says About How We Treat Records," Archival Issues 29 no.2 (2005): 97-104.

O'Toole, James. "Archives and Historical Accountability: Toward a Moral Theology of Archives." Archivaria 58 (Fall 2004): 4-19.

Peterson, Trudy Huskamp. "The National Archives and the Archival Theorists Revisited, 1954-1984.” American Archivist 49 no. 2 (Spring 1986): 125-133.

Pylypchuk, JoAnne. "A Documentary Approach to Aboriginal Archives." Archivaria 33 (Winter 1991-1992): 117-124.

Quinn, Patrick M. "Archivists and Historians: The Times They Are A-Changin'." Midwestern Archivist 2 no. 2 (1977): 5-13.

Samuels, Helen W. "Who Controls the Past." American Archivist 49 (1986): 109-124.

Schellenberg, Theodore. The Management of Archives. New York: Columbia University Press, 1965.

Stoler, Ann Laura. "Colonial Archives and the Arts of Governance." Archival Science 2 (2002): 87-109.

Tough, Alistair G. "The Post-custodial/Pro-custodial Argument from a Records Management Perspective." Journal of the Society of Archivists 25 no.1 (2004): 19-26.

Trace, Ciaran B. "What is Recorded is Never Simply 'What Happened': Record Keeping in Modern Organizational Culture.” Archival Science 2 (2002): 137-159.

Wallace, David A. "Historical and Contemporary Justice and the Role of Archivists." Arkiv, Demokrati og Rettferd. 2005.

Williams, Caroline. "Personal Papers: Perceptions and Practice." In What are Archives? A Cultural and Theoretical Perspectives: A Reader, Louise Craven, ed.. Hampshire, England: Ashgate Publishing Limited, 2008.

Williams, Loris, Kristen Thorpe, and Andrew Wilson. "Identity and Access to Government Records: Empowering the Community." Archives and Manuscripts 34 no. 1 (May 2006): 8-30.

Wilson, Ian E. "Peace, Order and Good Government: Archives in Society." Archival Science 11 (2011): 235-244.

Yakel, Elizabeth. "Archival Representation." Archival Science 3 (2003): 1-25.

Yakel, Elizabeth and Deborah A. Torres. "AI: Archival Intelligence and User Expertise." American Archivist 66 (Spring/Summer 2003): 51-78.

Yakel, Elizabeth and Deborah A. Torres. "Genealogists as a 'Community of Records'." American Archivist 70 (Spring/Summer 2007): 93-113.

Zinn, Howard. "Secrecy, Archives, and the Public Interest." Midwestern Archivist 2, no. 2 (1977): 14-26. 
Chapter 33

\title{
USING GROUNDED THEORY TO UNDERSTAND THE ARCHIVAL NEEDS OF GEOLOGISTS
}

\author{
Sarah Ramdeen and Alex H. Poole
}

\begin{abstract}
Unpacking "small science," this chapter focuses on the recordkeeping practices of geologists as embodied by their field notebooks. Used to record observations and to document data collection, field notebooks represent "dark data" secreted in the "long tail" of science. This example addresses geologists' use and reuse of field notebooks, their provisions for long-term preservation (if any), and their understanding of archival and archiving principles and practices. The chapter begins by reviewing relevant literature on dark data and its reuse, on geologists, and on archiving. Second, it lays out the basic framework of Grounded Theory and suggests that the constructivist variant of Grounded Theory advocated by Kathy Charmaz is most appropriate for this research question. Third, it weaves together the theoretical insights of Grounded Theory with a specific delimited case example centred on geologists and archiving practices. It then describes the various stages of Grounded Theory and the ways in which these iterative and symbiotic stages relate to our work with geologists and their field notebooks. It presents preliminary results from that work and links those results to archival concepts. Finally, it engages the broader challenge of reflective and interpretive theory development and underscores the potential of constructivist Grounded Theory to facilitate new communication - and collaboration - between archivists and practitioners of "small science."
\end{abstract}

In the end, inquiry takes us outward yet reflecting about it draws us inward. ${ }^{1}$

1 Kathy Charmaz, Constructing Grounded Theory: A Practical Guide Through Qualitative Analysis, London: Sage Publications, 2006, p.149. 


\section{Introduction and Context}

In their daily lives, citizens and scholars alike may underestimate the importance and impact of geological data. But as the United States National Resource Council concludes, geological materials can be used and reused "to address issues that are important to all." They lend themselves to hazard assessment, to basic and applied scientific research, to discovery, assessment, and enhanced use of national resources, and to education and public awareness. The reuse of geological data can prove pivotal, for instance by aiding emergency responses to natural disasters, by helping scientists and policymakers to re-evaluate risk potential following earthquakes, and by supporting researchers to grapple with climate change. On a day-to-day level, however, scientists frequently reuse geological materials to verify the locations of ambiguous data points mentioned in professional publications. These stakeholders verify such data point locations by revisiting raw data - data frequently captured and preserved only in the field notebooks of academic or professional geologists.

For the subdomains focused on in this research, fieldwork often involves hiking to a remote location with tools such as a Brunton (a compass-like device), a rock hammer, a hand lens, a vial of acid and a field notebook (also called field notes). Perhaps the most important of these materials, the field notebook is used to record scientific observations and to document the process of data collection. These notebooks often constitute the only record of a geologist's experiences in the field until these data are distilled into a published report. Field notebooks include notes about locations visited, lithography observed, and station identification for samples collected (rocks, fossils, etc.). They may also include sketches and other information about the events of the trip; in this way, a field notebook may resemble a diary. Field notebooks constitute a pivotal part of the work of those scientists who have been trained similarly and who conduct research in one to two-person teams. These scientists represent the backbone of so-called small science. ${ }^{3}$ Small science is

2 National Research Council, Geoscience Data and Collections - National Resources in Peril, Washington, D.C., National Academies Press, 2002, p.16.

3 As Heidorn notes apropos of small science, "The majority of the work done by scientists is conducted in relatively small projects with one lead researcher with part-time commitment to the project and perhaps two or three graduate students or part-time staff scientists." Bryan Heidorn, "Shedding Light on the Dark Data in the Long Tail of Science," Library Trends 57 no. 2 (2008): 280. In small science, data sharing is akin to a "cottage industry" predicated upon personal communication and professional relationships. See Melissa H. Cragin, Carole L. Palmer, Jacob R. Carlson and Michael Witt, "Data Sharing, Small Science and Institutional Repositories," Transactions of 
"hypothesis-driven research led by a single investigator, in which progress and reward are contingent on generating and analyzing one's own data." But this small science data too frequently is overlooked or lost: it becomes "dark."

This chapter describes the process of conducting a research project using Grounded Theory to study archiving practices of small science. It focuses on geologists who are actively conducting research and who use field notebooks in their everyday work for a multiplicity of purposes ranging from basic reference to historical research, and mapping projects to verifying secondary and derived data against the source. These users may need data hidden away in field notebooks to develop new research questions, to embark upon new research projects, and to verify previous findings. Thus we ask: what are the archiving practices of geologists?

To address this research question, we examine geologists' conceptions of "archives" and "archiving" as well as their level of awareness of the possible (reuses) of archival materials such as field notebooks. If geologists are aware of these archived notebooks, do they reuse them and if so, how? We also analyse the searching processes of those geologists who do reuse field notebooks and discuss how best to foster such reuse. Finally, we solicit geologists' opinions on the long-term preservation of their artefacts and on the perceived roles and responsibilities played by archives and archivists. We seek not only to flesh out the processes followed by geologists as they search for and retrieve field notebooks qua archival materials, but also to develop theory on their information needs and skills.

\section{Literature Review}

A complex field of study, geology encompasses subdomains such as sedimentary geology, structural geology and palaeontology. Each of these subdomains maintains its own standards for how data is collected, processed and

the Royal Society A 368 no. 1926 (September 13, 2010): 4023-4038. By contrast, larger science endeavours often have the resources (funding and staff time) to care for these materials. See M. A. Parsons and P.A. Fox, "Is Data Publication the Right Metaphor?" Data Science Journal 12 no. 0 (2013). Along these lines, state geological surveys often contain collections built on the donations of physical data from individual scientists, which "each has their own method of documentation, data collection, and curation." See Sarah Ramdeen, "Preservation Challenges for Geological Data at State Geological Surveys," manuscript submitted for publication.

4 Cragin et al., "Data Sharing, Small Science and Institutional Repositories," 4024.

5 "Dark data may be positive or negative research findings or from either 'large' or 'small" science," Heidorn, “Shedding Light," 281. 
transformed into knowledge. But all three of these subdomains commit to fieldwork and thus to the use of field notebooks. Undergraduate and graduate education in geology - as well as geologists' subsequent socialisation into a relevant community of practice - teaches aspiring geologists in these subdomains to make and to keep detailed field notes as a crucial part of their professional development. The US Geological Survey (USGS)'s Geologic Materials Repository Working Group (GMRWG) and the National Research Council (NRC) of the National Academies both classify field notebooks under the umbrella of scientific data. ${ }^{6}$ The GMRWG characterises such data as "ancillary geologic records" that prove indispensable for documenting sample collections and research projects. ${ }^{7}$ Field notebook data often remains implicit knowledge not associated with a digital environment (e.g., electronic data sources or software or analysis tools), which militates against automated capture. ${ }^{8}$ Although digital capture offers promise, thus far such tools have failed to interoperate in organised data collection. ${ }^{9}$

Archivists should be interested in geological data as they may be able to provide much needed expertise. Data engenders new research but can be used to conduct internal and external reliability and validity checks. ${ }^{10}$ But geological data has long been at risk of loss. As Jillian Wallis et al., Catherine Marshall, and Kalpana Shankar note, archivists usually receive

6 U.S. Geological Survey. The USGS Geologic Collections Management System (GCMS): A Master Catalog and Collections Management Plan for USGS Geologic Samples and Sample Collections, by the Geologic Materials Repository Working Group, preprint, submitted January $25^{\text {th }}, 2013$.

7 Geologic Materials Repository Working Group, The USGS Geologic Collections Management System, 14.

8 T.V. Loudon and J.L. Laxton, "Steps Toward Grid-based Geological Survey: Suggestions for a Systems Framework of Models, Ontologies, and Workflows," Geosphere 3 no. 5 (2007): 319-336.

9 D. Vieira, M. Mookerjee and M. Swathi, "Incorporating Geoscience, Field Data Collection Workflows into Software Developed for Mobile Devices," poster presented at the American Geophysical Union Fall Meeting, San Francisco, CA, December, 2014. A recent National Science Foundation-funded field trip brought together geologists from the subdomains of structural geology, palaeontology, and sedimentology with information scientists, computer scientists, and other social scientists. Geologists tested various field notebook applications (on tablets, rugged laptops, and smartphones). They had many comments about the disruption of scientific workflows during data collection as well as a lack of semantic understanding related to differences among subdomains.

10 M. F. Perutz, Is Science Necessary? Essays on Science and Scientists, New York: E.P. Dutton: 1989. For a comprehensive review of science data, sharing, and reuse (and the relevance of archival principles to these issues), see Alex H. Poole, "How Has Your Science Data Grown? Digital Curation and the Human Factor: a Critical Literature Review," Archival Science (2014): 1-39. 
scientists' data only after their findings have been published or after the researcher retires. ${ }^{11}$ As a result, important information regarding the context, origins, and provenance of scientists' records - information imperative for future reuse - may be lost. Geological data is effectively incomplete without accompanying documentation, i.e., "a complete set of notes characterizing the site from which it was taken and the conditions under which it was collected." ${ }^{2}$ Moreover, publications represent only "a distillation and stylized version of the processes by which the primary documents are created"; they are not the archival record per se. ${ }^{13}$ Publication occurs semi-regularly and thus published documents cannot depict the ongoing practices implicated in the scientific endeavour. Finally, these documents represent science in its most idealised, linear form. Many artefacts such as field notebooks remain understudied.

Personal scientific records exemplify what Bryan Heidorn calls "dark data" that remain sequestered in the "long tail" of science. ${ }^{14}$ Shankar identifies a paradox: "these records which are of grave import to the research enterprise become 'nothing': They are created day after day, 'written up' in conference papers and posters, and ultimately shelved in countless laboratories, never to be consulted again." ${ }^{15}$ Dark data, as Heidorn contends, accumulates because "no one is paying attention." 16 The work of scholars

11 Jillian Wallis, Christine Borgman, Matthew Mayernik and Alberto Pepe, "Moving Archival Practices Upstream: An Exploration of the Life Cycle of Ecological Sensing Data in Collaborative Field Research," International Journal of Digital Curation 1 no. 3 (2008): 114-126; Cathy C. Marshall, "From Writing and Analysis to the Repository: Taking the Scholars' Perspective on Scholarly Archiving," in Proceedings of JCDL '08, June 16-20, 2008, Pittsburgh, PA, 2008, pp. 251-260; Kalpana Shankar, "Order from Chaos: The Poetics and Pragmatics of Scientific Recordkeeping," Journal of the American Society for Information Science and Technology 58 no. 10 (2007): 1457-1466.

12 W.L. Lee, B.M. Bell, B.M. and J.F. Sutton, eds., Guidelines for Acquisition and Management of Biological Specimens, Lawrence, KS: Association System of Collections, 1982, p.15. Historically the knowledge captured by geologists in their notebooks has been seen as a value-added tool for better using collections. For example, William 'Strata' Smith donated his palaeontological collection to the British Museum in 1817. The museum later hired Smith to organise the collection for public access and use as it did not have the same value without his input. This was seen as a key contribution to scientific knowledge. See "Letter to the Editor," Philosophical Magazine 50 (1817), 269-74. While museums and other scientific organisations would become homes for scientific collections during the nineteenth and twentieth centuries, of course, the process for gathering data still resides with individual scientists (and their notebooks).

13 Shankar, "Order from Chaos," 1458.

14 Heidorn, "Shedding Light," 280-299.

15 Shankar, "Order from Chaos," 1459.

16 Heidorn, "Shedding Light," 290. 
such as Wallis and her colleagues, Marshall, Shankar, and Heidorn suggests an urgent need for scholars to address the "upstream" practices of natural scientists, especially this abundant dark data. The long tail of science serves as a "breeding ground" for new and innovative science, despite being often "less well planned, more poorly curated and less visible to other scientists." 17 Such information tends to be underutilised at best and lost at worst.

Researchers in archives have not yet tackled geologists' recordkeeping practices, much less those that occur upstream. In the broader field of Library and Information Science, Julie Bichteler and Dederick Ward, Bichteler, and Lura Joseph among others, have addressed various aspects of geologists' information-seeking practices. ${ }^{18}$ Similarly, with the notable exceptions of Jenny Bunn (see her chapter in this volume), Karen Gracy and Paul Conway, few archives scholars have employed Grounded Theory as an analytical tool. ${ }^{19}$ Still, Anne Gilliland and Sue McKemmish make a persuasive case for adding Grounded Theory to archival scholars' toolkits. ${ }^{20}$ To this point, then, the geological and archival literatures remain siloed but they might profitably be brought together under the auspices of constructivist Grounded Theory, specifically to examine the archiving practices of geologists regarding field notebooks.

\section{Grounded Theory}

Developed by sociologists Barney Glaser and Anselm Strauss in the 1960s and codified in The Discovery of Grounded Theory (1967), Grounded Theory is a systematic and purposeful research method used both to gather data from the field and to develop theories directly from them. ${ }^{21}$ Based on their experiences with terminal hospital patients, Glaser and Strauss promulgated

17 Heidorn, "Shedding Light," 282-283.

18 Julie Bichteler, "Geologists and Gray Literature," Science E̋ Technology Libraries 11 no. 3 (1991): 39-50; Julie Bichteler and D. Ward, "Information-Seeking Behavior of Geoscientists," Special Libraries 80 no. 3 (1989): 169-78; L.E. Joseph, "InformationSeeking and Communication Behavior of Petroleum Geologists," Science Eऽ Technology Libraries 21 no. 3 (2001): 47-62.

19 Karen F. Gracy, "Documenting Communities of Practice: Making the Case for Archival Ethnography," Archival Science 4 no. 3-4 (2004): 335-365; Paul Conway, "Modes of Seeing: Digitized Photographic Archives and the Experienced User," American Archivist 73 no. 2 (2010): 425-462.

20 Anne J. Gilliland and Sue McKemmish, "Building an Infrastructure for Archival Research," Archival Science 4 no. 3 (2004): 149-197.

21 B.G. Glaser and A.L. Strauss, The Discovery of Grounded Theory: Strategies for Qualitative Research, Chicago: Aldine Publishing Company, 1967. 
Grounded Theory as a response to what they perceived to be the positivist hegemony undergirding the social sciences in the mid-twentieth century. Positivism enshrined objectivity, neutrality, replicability and falsification as primary concerns of empirical research. Glaser and Strauss both challenged the prevailing methodological consensus among scientists and delineated strategies for engaging in qualitative research. Their work combated longstanding arguments that qualitative methods remained impressionistic; that qualitative efforts represented mere preludes to quantitative strategies; that qualitative methods could not generate theory; that the stages of data collection and data analysis remained discrete; and that the division between theory and research needed to be upheld. ${ }^{22}$

Over the course of more than four decades, Grounded Theory has become a contested methodology (see Jenny Bunn's chapter for more discussion on this point). ${ }^{23}$ Researchers continue to differ over the proper execution of Grounded Theory methods as well as what constitutes "finished" Grounded Theory. J.W. Creswell insists that conclusions reached through Grounded Theory remain only "suggestive, incomplete, and inconclusive." ${ }^{24}$ Despite their original collaboration, Glaser and Strauss ultimately differed over the conduct and significance of Grounded Theory. For example, Glaser added a distinction between substantive codes and theoretical codes as well as a distinction between open and selective coding. Conversely, by 1990 Strauss and Juliet Corbin had developed a purportedly "systematic" Grounded Theory that prescribed that the coding process centre on axial coding (a position they subsequently dropped); finally, they created a "conditional matrix" to analyse conditions and consequences ranging from the immediate situational to the international..$^{25}$

Students of Glaser or Strauss or both such as Kathy Charmaz (constructivist) and Adele Clarke (postmodernist) spearheaded a new generation of Grounded Theorists. Charmaz asserts that neither data nor theories may be discovered; rather, "We construct our grounded theories through our past

22 Charmaz, Constructing Grounded Theory (2006 edition), 4-6.

23 J.A. Wasserman, J.M. Clair and K.L. Wilson, "Problematic of Grounded Theory: Innovations for Developing an Increasingly Rigorous Qualitative Method," in W.P. Vogt, ed., Data Collection: Data Collection in Survey and Interview Research, Los Angeles: Sage Publishing, 2010, p.403.

24 J.W. Creswell, "Five Qualitative Approaches to Inquiry," in Qualitative Inquiry and Research Design: Choosing Among Five Traditions, J.W. Cresswell, ed., Thousand Oaks, CA: Sage, 2007, p.66.

25 Ian Dey, Grounding Grounded Theory: Guidelines for Qualitative Inquiry, San Diego: Academic Press, 1999, pp.9-11. 
and present involvements and interactions with people, perspectives, and research practices." ${ }^{26}$ Meanwhile, Adele Clarke proposes "to supplement basic grounded theory with a situation-centred approach that in addition to studying action also explicitly includes the analysis of the full situation, including discourses - narrative, visual, and historical."27 Each of these scholars has pushed Grounded Theory in new and productive ways.

Constructivist Grounded Theory "aspires to understand, and eventually generate theory from, participants' perspectives and interpretations, from how they 'construct' their worlds." ${ }^{28}$ It guides researchers in their direct data collection, their management of data analysis, and their development of a theoretical framework that explains the process being studied. ${ }^{29}$ Hence it was an ideal approach for this study.

The conduct of Grounded Theory research involves iteratively collecting, coding, and analysing data and marshalling the information gathered to determine what data to collect next and where best to find it in order to develop a theory as it is emerging from the data. ${ }^{30}$ Potential data sources include documents, surveys, observations, and interviews. These data, "detailed, focused, and full," may push the researcher to reconsider her data collection methodology or to consider themes emerging from existing data or both. ${ }^{31}$ Grounded Theory promotes both flexibility and focus through "thick" description. ${ }^{32}$ To place gathered data in context, a researcher should start with concepts that promote sensemaking in the context of the study. She should conclude inductively by making theorised connections between what she sees in the data and the larger world she seeks to describe. ${ }^{33}$

Though this study's use of Grounded Theory is rooted in the work of Glaser and Strauss, it relies primarily upon the framework of Grounded Theory as propagated by Kathy Charmaz. Charmaz advocates a Grounded Theory perspective that focuses on individuals over methods and that

26 Charmaz, Constructing Grounded Theory (2006 edition), 10.

27 Adele Clarke, Situational Analysis: Grounded Theory after the Postmodern Turn, Thousand Oaks: Sage, 2005, xxxii.

28 Helen Simons, Case Study Research in Practice, Los Angeles: Sage Publications, 2009, p.124.

29 Kathy Charmaz, "Qualitative Interviewing and Grounded Theory Analysis," in J.F. Gubrium and J.A. Holstein, eds. The Handbook of Interview Research: Context and Method, Thousand Oaks: Sage Publications, 2002, p.675.

30 Glaser and Strauss, The Discovery of Grounded Theory, 45.

31 Charmaz, Constructing Grounded Theory (2006 edition), 14.

32 Clifford Geertz, The Interpretation of Cultures. New York: Basic Books, 1973.

33 Charmaz, Constructing Grounded Theory (2006 edition), 133. 
respects diverse local worlds and multiple realities. Finally, Charmaz's constructivist Grounded Theory embraces a strategy of active coding that reveals the researcher's agency in bringing queries to the data, in making decisions about categories, and in accepting and reflecting upon the personal values and experiences she brings to her analysis. ${ }^{34}$

\section{The Praxis of Grounded Theory}

Proponents of grounded theory "share a conviction with many other qualitative researchers that the usual canons of 'good science' ... require redefinition in order to fit the realities of qualitative research and the complexities of social phenomena." ${ }^{35}$ Our study on field notebooks is used to exemplify the process of conducting Grounded Theory research. In this case, one of the authors, Ramdeen, worked as a professional geologist before entering the field of Library and Information Science. As such, she brought to this study her own broad knowledge of the field of geology and her particular situated experience with both creating and using field notes. More specifically, this study was inspired by a conversation. Knowing of Ramdeen's work as a librarian and archivist, an academic geologist contacted her to ask whether she knew of an appropriate place to deposit field notebooks for long-term preservation upon a faculty member's retirement. This geologist thought that field notebooks are quite valuable and typically searched for them via personal networking. In other words, he asked researchers in his domain directly to share their materials. But he expressed some confusion: where did such materials belong other than in a scholar's personal collection?

Despite Ramdeen's own intellectual and professional background, however, she had not considered the issue; in fact, she had assumed that such notebooks belonged in the archives or libraries in the faculty member's home institution. Thus she felt unsure how best to advise him. Subsequently reflecting upon this conversation, we wondered whether any scholars had addressed the problem as specified above not from the point of view of the home organisation, but rather from the perspective of the user-geologist. Indeed, the conversation engendered two important if overlooked questions that we returned to time and again. First, in what types of venues, archival

34 Creswell, "Five Qualitative Approaches to Inquiry," 65-66.

35 Corbin, Juliet, and Anselm Strauss, "Grounded Theory Research: Procedures, Canons, and Evaluative Criteria," Qualitative Sociology (1990): 4. 
or otherwise, should geologists' field notebooks be archived? Second, what strategies do geologists employ to search for these items?

After discerning a general subject or problem, the researcher next ensures that Grounded Theory is the appropriate method for her purposes. Given the lack of scholarly work done on geologists' archiving practices, Grounded Theory - specifically constructivist Grounded Theory - seemed ideal. This decision had important ramifications; as Kathy Charmaz notes, "How you collect data affects which phenomena you will see, how, where, and when you will view them, and what sense you will make of them." ${ }^{36}$ Following the guidelines of Grounded Theory, we avoided theoretical preconceptions and being heavily influenced by the relevant literature at the outset, thus ensuring we would not subsequently force our data to "fit." 37

Having determined a general subject or problem and having verified the appropriateness of employing Grounded Theory, the researcher transitions into sampling. Sample selection relies upon the type of study conducted, the research questions asked, and the type of evidence needed by the investigator. ${ }^{38}$ "All sampling," Lincoln and Guba argue, "is done with some purpose in mind." 39 Theoretical sampling is "the process of data collection whereby the researcher simultaneously collects, codes, and analyzes the data in order to decide what data to collect next." ${ }^{\text {"0 }}$ Sampling continues until the researcher has reached theoretical saturation, the point at which similarities are registered and new data does not compel the development of new categories. ${ }^{41}$

We began our sampling by contacting the researcher who first broached the subject with Ramdeen. He elaborated upon his process of searching for and using field notebooks. Better still, he referred us to other geologists who he thought would provide insight, thereby jumpstarting our sampling process. For instance, his referral led us to an interview with a geologist stationed with the state government who had recently developed a process to capture field notes digitally.

36 Charmaz, Constructing Grounded Theory (2006 edition), 15.

37 As Bernard and Ryan note, however, "purely" inductive studies are impossible. See H. Russell Bernard and Gery W. Ryan, Analyzing Qualitative Research, Thousand Oaks: Sage, 2010.

38 Alison Pickard, Research Methods in Information, Chicago: Neal-Shuman, 2013.

39 Yvonna S. Lincoln and Egon Guba, Naturalistic Inquiry, Newbury Park, CA: 1985, p.199.

40 I.T. Coyne, "Sampling in Qualitative Research: Purposeful and Theoretical Sampling; Merging or Clear Boundaries?” Journal of Advanced Nursing 26 no. 3 (1997): 625.

41 Glaser and Strauss, The Discovery of Grounded Theory, 61. 
Initial sampling persisted by way of personal contacts, snowball recruitment, and networking and informal conversations with professionals at the Geological Society of America's annual meeting. One concern persisted as we proceeded with our sampling. First, we considered how to circumscribe our sample population - or if we should do so at all. On the one hand, a researcher hopes data will prove flexible and help to answer her research question(s). On the other, describing the studied group may be hindered if the population is too dynamic. Geology as a discipline comprises not only many sub-disciplines, but also is only one part of the larger field of Earth Sciences. While originally planning on targeting sedimentary and structural geologists, our initial sample included two palaeontologists. We also spoke to scholars outside of academia, namely geologists working in state or federal government or in the private sector, each of whom brought a different perspective on the process. Every one of these geologists used field notebooks. Validity was further supported through triangulation, which can "potentially generate ... 'holistic work' or 'thick description." ${ }^{\text {'42 }}$ We felt that we had achieved saturation in our data collection.

In Grounded Theory, sampling exists in a reciprocal relationship with data collection. A researcher should ferret out diverse data sources, begin collection with unstructured methods, and refine her methods of observation as her theory emerges. ${ }^{43}$ She is "an active sense maker" who seeks to discern truth but who also engages in self-reflexivity. ${ }^{44}$ Finally, she should evaluate her data for its depth and its scope as well as for its reflection of events.

Dynamic and iterative, qualitative interviewing depends upon understanding experiences and upon reconstructing events. ${ }^{45}$ It necessitates "more intense listening than normal conversations, a respect for and curiosity about what people say, a willingness to acknowledge what is not understood, and the ability to ask about what is not yet known." ${ }^{\text {"6 }}$ Interviews allow an investigator to integrate multiple perspectives, to discern processes, and to

42 Todd Jick, "Mixing Qualitative and Quantitative Methods: Triangulation in Action," Administrative Science Quarterly (1979): 609.

43 Dey, Grounding Grounded Theory, 6.

44 J.M. Johnson, "In-Depth Interviewing," printed in J.F. Gubriuym and J.A. Holstein (eds.), Handbook of Interview Research, Thousand Oaks, CA: Sage, 2002, p.105.

45 Herbert J. Rubin and Irene S. Rubin, Qualitative Interviewing: The Art of Hearing Data, Thousand Oaks: Sage, 2005.

46 Rubin and Rubin, Qualitative Interviewing: The Art of Hearing Data, 14. 
develop holistic descriptions. ${ }^{47}$ Constructivist grounded theorists "attend to the situation and construction of the interview, the construction of the research participant's story and silences, and the interviewer-participant relationship as well as the explicit content of the interview." ${ }^{38}$ The questions the researcher poses may evolve. ${ }^{49}$ Such interviews are relatively lengthy; take place one-on-one and face-to-face; allow a greater expression of the interviewer's self; and demonstrate the participants' personal commitment. ${ }^{50}$ Additionally, observations allow a researcher to accrue information about activities that participants may find difficult to describe. Body language and gestures provide rich data.

Semi-structured interviews "involve less rigidity and more leeway than structured interviews but are more organized and systematic than unstructured interviews in developing the conversation. ${ }^{51}$ Our semi-structured interviewing strategy took root in a guiding set of broad questions that were refined iteratively. We used an interview guide to ensure questions and concepts were clear. In her previous experience, Ramdeen found that the use of archival and LIS terminology often led to confusion with scientists. We posed these broad questions to the first set of geologists and subsequently determined areas either important to add because interviewees highlighted their importance or because they stood out when we reviewed our notes. Serendipity also played an important role. After one interview, for example, we realised that we had not asked a single one of our questions. Rather, the interviewee had addressed these questions (and a good deal more) as part of the flow of conversation.

Many of the participants consented to secondary interviews, which allowed for follow-up, clarification, and verification of statements. In one particularly striking - and serendipitous - case, the interviewee requested that one of our researchers meet with him in his office where he showed her documents and described geological field techniques not amenable to articulation over the phone. Furthermore, we followed the prescriptions on interaction suggested by Charmaz in order to build trust.

47 Robert Weiss, Learning from Strangers: The Art and Method of Qualitative Interviewing, New York: Free Press, 1994.

48 Kathy Charmaz, Constructing Grounded Theory, Thousand Oaks: Sage, 2014, p. 291.

49 J.M. Corbin and A.L. Strauss, Basics of Qualitative Research: Techniques and Procedures for Developing Grounded Theory, Los Angeles: Sage, 2008, p.27.

50 Johnson, “In-Depth Interviewing," 103-119.

51 Lili Luo and Barbara Wildemuth, "Semistructured Interviews," in Barbara Wildemuth, ed., Applications of Social Research Methods to Questions in Information and Library Science, Westport: Libraries Unlimited, 2009, p.233. 
On the other hand, we gathered data outside of our interviews, namely documentation. Documents may be a useful data source. ${ }^{52}$ They "not only serve as records, but also explore, explain, justify, and/or foretell actions." ${ }^{53}$ For example, one participant mailed us colour scans of a field notebook he had recently used in a project. Reviewing the documents, one of our researchers recalled discussing the study with a faculty member not affiliated with the study. The professor had asked about the archival process for field notebooks: specifically, what preservation strategies might be needed, especially related to potential digitisation of these artefacts. At the time, Poole thought these questions likely fell outside the scope of the project. Seeing the scanned selections from the notebooks, though, he reconsidered ultimately and he addressed this connection in his memo writing.

The iterative nature of the process became evident. For example, we initially requested that participants speak about their own experiences in creating and maintaining field notebooks. Unfortunately, this topic yielded information that fell outside the scope of our research question. We retrenched, therefore, instead querying researchers about their broad uses of field notebooks. Grounded Theory encourages this sort of interrogative evolution.

\section{Exemplary Memo Excerpt 1}

I wish I had asked the subject to clarify what he considered to be included in the greater concept of a field notebook - one thing that came up as a separate but associated artefact was a printed map that accompanied the notebook when in the field. This has details and notes written on it at stations and locations beyond those included in the notebook. But is it usually stored separately? Should it be considered a different document?

52 Ian Hodder observes: "the written text is an artifact, capable of transmission, manipulation, and alteration, of being used and discarded, reused and recycled - 'doing' different things contextually through time." See Ian Hodder, "The Interpretation of Documents and Material Culture," printed in Norman K. Denzin and Yvonna S. Lincoln, eds., Handbook of Qualitative Research (Thousand Oaks: Sage, 2000), 704. Charmaz goes further: "A study of what a document does can include the following: 1) what its originators intended to accomplish;2) the process of producing the document; 3) what and whom the document affects; 4) how various audiences interpret it; 5) how, when, and to what extent these audiences use the document." See Charmaz, Constructing Grounded Theory (2014 edition), 46. Documents "not only serve as records, but also explore, explain, justify, and/or foretell actions." Charmaz, Constructing Grounded Theory (2014 edition), 46.

Charmaz, Constructing Grounded Theory (2014 edition), 46. 
Proceeding in tandem with sampling and data collection, memo writing constitutes a key part of Grounded Theory work. It frees the researcher "to theoretically develop ideas (codes), with complete freedom into a memo fund, that is highly sortable." ${ }^{54}$ Whether paragraphs or pages, memos constitute the foundation of the emerging theory; they contain the "logic of analysis" in the categories, properties, and exploration of their interrelationships. ${ }^{55} \mathrm{Memos}$ allow the researcher to "define the properties of each category; specify conditions under which each category develops, is maintained, and changes; and note the consequences of each category and its relationship with other categories. ${ }^{26}$ They proved invaluable throughout our work; memos also funnel into the process of concept development, a process addressed below.

During the data collection process, a researcher continually and iteratively writes memos. These in turn influence the sampling process. Before conducting our interviews, in fact, we had already captured data through memo writing. In particular, Ramdeen reflected on the conversation that had first piqued our interest in the topic of archiving geological artefacts such as field notebooks. Further, she ruminated over her own (rather limited) knowledge of field notebooks. These reflections influenced our decisions both in the sampling process and in question development. Complementing this preinterview memoing, we wrote memos both on our formal interviews and on our casual conversations. Through these outlets we tested ideas and suggested holistic connections.

\section{Exemplary Memo Excerpt 2}

Our third participant emphasized the importance of historical field notebooks (notebooks from the late 1800s and early 1900s) as part of his search process. What connections can I make between his search strategy and the search processes outlined by my other geologists? Are other geologists aware of this possibility for use or reuse? Do they avoid archives because they believe archives would not have field notebooks or do geologists simply not value older notebooks?

54 B.G. Glaser, Theoretical Sensitivity: Advances in the Methodology of Grounded Theory, Mill Valley: The Sociology Press, 1978, p.83.

55 G.G. Darkenwald, "Field Research and Grounded Theory," printed in H.B. Long, R. Hiemstra, et al. eds., Changing Approaches to Studying Adult Education, San Francisco: Jossey-Bass Publishers, 1980, p.74.

56 Charmaz, "Qualitative Interviewing," 687. 
Finally, we maintained notes during the interview process and subsequently used them in our reflective memo writing. During and after each conversation as well as during the transcription process we wrote memos encapsulating our thoughts and reactions. We transcribed the interviews; transcription funnelled into coding as we processed the individual interviews and determined next steps for sampling.

Perhaps the most important stage of Grounded Theory, coding, involves "naming segments of data with a label that simultaneously categorizes, summarizes, and accounts for each piece of data." ${ }^{57}$ An emergent process, coding links data collection and emergent theory ${ }^{58}$ Codes emerge from the language, meanings, and perspectives of the researcher and her participants; as a result, it facilitates examination of hidden assumptions in the language of the researcher or her participants or both. Overall, coding involves Glaser and Strauss's notion of "constant comparison," an ongoing process of juxtaposing new and old data and reassessing codes and focused concepts derived from those codes. ${ }^{59}$ As Charmaz concludes, "coding and recoding not only leads you in new directions but also directly to theoretical sampling of your new categories as well." ${ }^{\prime 0}$

Various Grounded Theory researchers have adduced four classes of coding: initial, focused, axial, ${ }^{61}$ and theoretical. During initial coding, the researcher scrutinises fragments of data, e.g., words, lines, or segments, through a close reading of the data while remaining open to all possible theoretical avenues. This process helps the researcher to discern early on any gaps in the data. Initial coding should be done with speed and spontaneity in order to keep a fresh perspective: some codes will fit while others will need refinement. ${ }^{62}$

Some researchers favour coding programs such as NVivo or ATLAS.ti, yet coding by hand using Excel better suited this research team. While such automated tools offer advanced options for coding process, they can be difficult to manage for the uninitiated research team. Software programs engender concerns over hamstringing the analysis and settling for superficiality as

57 Charmaz, Constructing Grounded Theory (2006 edition), 43.

58 Charmaz, Constructing Grounded Theory (2006 edition), 46.

59 Glaser and Strauss, The Discovery of Grounded Theory, 106.

60 Charmaz, Constructing Grounded Theory (2006 edition), 70.

61 In axial coding, the researcher pinpoints one open coding category on which to focus (the "core") and then returns to data to construct categories that pivot around this "core." We found this approach unduly constricting and chose not to engage in it. 
well as shoehorning qualitative analysis into a single method. More importantly, hand coding allowed us more flexibility in how we sorted and reorganised the codes as we moved into the conceptualising and theorising phases.

During initial coding we reviewed the data line-by-line, identifying the process or topic represented in the data. We avoided undue specificity during this process as these codes represented ideas that would lead to categories later during focused coding. During the initial coding, perhaps reflecting our lack of experience with Grounded Theory, one of our researchers found herself giving a line of text more than one code. All the same, we endeavoured to capture our impressions of the data and their implications without overanalysing our thoughts. In the initial process, we reused codes occasionally, but overall we abjured preconceived standards for coding the text. Coding terms included "drawings and text," "interpretations of what seeing," "problems with personal shorthand," "not taught how to search," "spatial searching," "older generation more knowledgeable of searching," and "weighing the value against crunch time." In developing these codes we followed Charmaz's exhortation to "Pursue telling terms."

In the most specific variety of initial coding, line-by-line, the researcher simply names each line. The researcher breaks data into its components, pinpoints the actions upon which they rest, looks for tacit assumptions, unpacks implicit actions and meanings, compares data, discerns any gaps in the data, and ponders the larger significance of points. ${ }^{64}$ Through lineby-line coding, the researcher teases out implicit concerns and also focuses on the nuances of language. Line-by-line coding helps her to refocus her subsequent interviews.

We next leveraged initial codes to help identify gaps in the data we had collected. We also elicited categories that we hoped to explore in future sampling. For instance, one interviewee mentioned the Field Records Collection of the United States Geological Survey (USGS) (http://www.cr.usgs.gov/ fieldrecords/). The USGS system provides users with the ability to search by keyword(s), but its finding aids fail to offer basic functionalities such as searching by geographical region or by geological terms. Struck by this interviewee's knowledge of and engagement with an archival affordance as fundamental as a finding aid-though he did not know exactly what a "finding aid" was - we wondered how many geologists (and others outside

63 Charmaz, Constructing Grounded Theory (2006 edition), 57.

64 Charmaz, Constructing Grounded Theory (2006 edition), 50-53. 
of archives) might know about finding aids. ${ }^{65}$ Even in the case above, the interviewee requested information on finding aids in order to create one for himself, not because he thought external archives or archivists might be helpful allies, a most useful as well as serendipitous piece of information for us as researchers. Similarly, in the case of those geologists who were aware of the existence of finding aids, we wondered how useful they had found them to be. Thus this initial coding work helped us to develop a category and subsequently to refine it over the course of our other interviews.

More directed, selective, and conceptual than initial coding, focused coding represents a move toward synthesis. Focused coding permits the researcher to "sift" through her most frequently emerging initial codes and to revisit their effectiveness. In other words, comparing data with data provides the analytical foundation for focused codes; the researcher then compares her data to her emerging codes and refines them further. Finally, theoretical coding follows focused coding. It allows the researcher to clarify possible relationships among categories developed in focused coding: theoretical codes are integrative and provide form to focused codes. Theoretical codes add precision, clarity and cogency.

Following Charmaz, after the initial coding of each interview we created a secondary worksheet in which we first sorted the codes and then developed theoretical codes. We began to identify relationships suggested by the codes. These relationships included groupings such as "examples of use of field notebooks," "expressions of value," "search process," and "barriers." For each of these theoretical codes we created descriptions. For instance, we described "barriers" as "Examples or comments about the barriers to access or other problems encounter while using, searching or managing field notebooks includes duplicates from searching. Not all barriers are related to searching, some are about management/preservation."

During the coding process, we continually wrote memos. Three types of memos are useful: code memos, theoretical memos, and operational or procedural memos. Code memos describe and detail codes and their underpinning assumptions. Theoretical memos suggest ideas about codes and their (inter) relationships. Operational or procedural memos deal with the methodological aspect of the project and thus with the entire procedure of the study.

65 The Society of American Archivists Glossary of Archival and Records Terminology defines a finding aid as: "n. - 1. A tool that facilitates discovery of information within a collection of records. - 2. A description of records that gives the repository physical and intellectual control over the materials and that assists users to gain access to and understand the materials." See http://www2.archivists.org/glossary/terms/f/finding-aid. 
First, we created memos to reflect on our initial codes, pausing intermittently to reflect on what the data represented. We used theoretical memos to describe relationships: we thought about why certain codes should be grouped together and what those groupings represented. We also wrote memos about codes that did not seem to "fit" neatly into a single category (for example, "reaction to error processing") or codes that seemed effective outliers.

Procedural memos lent themselves to capturing the details of our research process. We periodically revisited these memos to refresh our memory and to reflect on the steps we had taken (and their sequence). This saved time and effort, which proved particularly useful as our codebook evolved into a far larger document over the course of our analysis.

Despite a researcher's conscientiousness, problems may crop up. A researcher might code at an overly general level, neglect to identify actions and processes in favour of topics, overlook the specific ways persons construct their actions and processes, foreground personal or disciplinary instead of participants' concerns, code out of context, or use codes to summarise rather than to analyse ${ }^{66}$ As a self-checking mechanism, the researcher should consider four questions: How does her coding reflect the incident or described experience? Do her analytic constructions begin at this point? Has she forged clear connections between data and codes? Has she refrained from translating language into academese ${ }^{67}$

Varying in their degree of abstraction, categories and properties emerge from the data during coding. They emerge initially at a low level of abstraction; over the course of the process, however, they become increasingly abstract and allow integrating concepts to emerge. As Charmaz notes, "the properties of the category remain implicit until theoretical sampling and interpretive rendering make them explicit." ${ }^{\prime 8}$ In Grounded Theory, categories channel into the development of concepts and concepts in turn channel into theory development. "We choose to raise certain categories to concepts because of their theoretical reach, incisiveness, generic power, and relation to other categories. ${ }^{19}$ Elevating categories to concepts involves further analytic refinement and delineating their relationships to other concepts.

66 Charmaz, Constructing Grounded Theory (2006 edition), 68-69.

67 Charmaz, Constructing Grounded Theory (2006 edition), 68-69.

68 Charmaz, Constructing Grounded Theory (2006 edition), 68-69.

69 Charmaz, Constructing Grounded Theory (2006 edition), 139. 
Concepts are the base of theories but they must be connected and supported by the memos and by other collected data. ${ }^{70}$ Concepts generated for theory development should be of two types: analytic ("sufficiently generalized to designate characteristics of concrete entities, not the entries themselves") and sensitising ("yield[ing] a 'meaningful' picture, abetted by apt illustrations that enable one to grasp the reference in terms of one's own experiences"). ${ }^{71}$ For the constructivist grounded theorist, these concepts should represent the data as opposed to "discovering order within the data." 72 These concepts, underpinned by the accumulation of interrelated categories and properties, ultimately funnel into hypothesis development. In the end, a theoretical framework will emerge. ${ }^{73}$ In the development of theory, moreover, concepts should apply to all cases in a generalised way; they should characterise the data gathered and not be examples of the data themselves. ${ }^{74}$ But this development must be balanced with theoretical saturation. In other words, categories must be saturated (i.e., further sampling yields no additional data) before theory development is broached. Corbin and Strauss advocate selecting a "core category" that represents the main theme of the research. This core category should be abstract, indicated by all or most of the cases, not forced; it also should expand as relationships to other categories are examined..$^{75}$ For example, we found the concept of barriers - barriers that hinder the discovery of field notebooks by potential users - to be sufficiently broad to capture the statements made by participants as they recounted their search experiences. This potential core concept relates to numerous other developing categories, including geologists' search skills, the archival processes necessary to manage these materials, and geologists' opinions on the ways in which these materials are or should be managed.

In developing our study, we confronted challenges and even limitations. In our initial coding work, for example, we were concerned about both being too general and overlooking a major category or conversely, being too specific and premising undue granularity in our coding. We also worried that Ramdeen's knowledge of geology and mapping processes influenced our processing of comments. Third, we tried diligently to eschew any pre-existing

70 Corbin and Strauss, Basics of Qualitative Research, 103.

71 Glaser and Strauss, The Discovery of Grounded Theory, 38.

72 Charmaz, Constructing Grounded Theory (2006 edition), 139-140.

73 Glaser and Strauss, The Discovery of Grounded Theory, 40.

74 Corbin and Strauss, Basics of Qualitative Research, 103; Glaser and Strauss, The Discovery of Grounded Theory, 38.

75 Corbin and Strauss, Basics of Qualitative Research, 104-105. 
categories for imposing order on the data; rather, we developed our ideas from the data. Finally, we worried about achieving a sufficiently abstract level of conceptualisation when working at theory formation. ${ }^{76}$

Concluding a study predicated upon Grounded Theory is challenging. But certain heuristics may be invoked. For example, a researcher may conclude data collection when those data fail to produce further conceptual variation (theoretical saturation), when she pinpoints a core category or particular narrative for study, when she completes integrating analysis into her narrative, when she finishes using memos and coded data to amplify or to modify her resulting analysis, or when she believes a satisfactory theory has emerged. ${ }^{77}$

Perhaps inevitably, we struggled to learn how and when to stop coding data. The open-endedness of Grounded Theory can prove vexing: how much time and resources must (or indeed, can) be invested to ensure a "finished" Grounded Theory? As we passed through the initial sampling and data collection, for instance, several questions remained. During the coding process some text received the code "older generation more knowledgeable of searching." Thus we wondered whether to include the age range or years of academic or professional experience of our participants. Indeed, the sample set did not include geologists nearing retirement or those who had recently retired. A consideration of generational change might usefully capture geologists' opinions on the digital search process and even on the digitisation of field notebooks.

\section{Exemplary Memo Excerpt 3}

[Digitisation] is still an area of the study that has not been fully explored. It was a question raised by participants and they think it's important. Should we ask about the search process before databases? Might older participants have had experience with archives or archiving? Where did these geologists deposit their field notebooks when they retired or where do they plan to (if they do in fact plan)? If they intend to retain them, what is their long-term plan (if any)?

76 Glaser, Theoretical Sensitivity, 143.

77 Dey, Grounding Grounded Theory, 9. 


\section{Selected Findings: Developing Theory and Telling Stories with Data}

The archive's relationship to field notebooks coalesced as a major concept. Geologists use field notebooks for diverse reasons. Field notebooks and their contents constitute historical documents as well as data. They may represent the only written record that "transforms" a rock into scientific data: without location information or metadata, after all, rocks are just rocks.

Some scientific data invariably is 1 lost. $^{78}$ Some of our participants alluded to such losses, citing failed searches or barriers to searching or access. On the other hand, we discerned a number of examples of discovery and use of scientific datasets that demonstrates their value. These findings buttress the need for additional curation. Several participants identified curation needs or overarching policies that would improve their ability to retrieve these items, for instance employing new technologies or setting priorities for archiving dark datasets.

But in addressing existing or potential archiving practices, our participants never reached consensus. Many geologists differ over optimal disciplinary communication practices. Participants showed considerable uncertainty over a range of basic archiving issues: whether field notebooks constituted archival documents, whether these artefacts belonged in archives, and if they do, where and when they should be deposited.

Instead of relying upon archivists and archives, nearly all of our participants depended upon personal networks in every stage of their research processes. These personal networks prevailed instead of formalised search processes - perhaps not surprising given the socialisation process that transpires among these scientists during their education and early career training.

Even when the researcher lacked a direct personal connection, her tacit knowledge of the field was crucial in locating field notebooks. Still, personal networks confront limits as well. One participant, for example, spoke about his experience with a scholar visiting from another region. This scholar unsurprisingly lacked the same knowledge of local geologists and their practices as our interviewee and thus struggled to find relevant materials.

78 Heidorn, "Shedding Light on the Dark Data," 280-299; Shankar, “Order from Chaos," 1457-1466. 


\section{Exemplary Participant Quotation 1}

"For what we are doing, typically our field areas are based on geographical location. Whether it is a county, you know you're working on a geological map of a certain county ... [I]t is always latitude-longitude bounded."

Notwithstanding their heavy reliance upon personal networks, geologists expressed numerous opinions on the affordances they wanted in a standard search interface. But no such search mechanisms whether digital or analogue seem currently to exist. The lack of such affordances stems from two as yet unsolved problems. First, most organisations have not set priorities - much less codified those priorities into policies - that address the massive investment of resources necessary to give these scientists the formal searchable system they desire. Second, scientists rarely have the archival skills necessary to deal with their field notebooks, even if they know precisely what they want to do with them. Finally, current finding aids are generally ill-suited for scientific research. Instead they foreground historical information and are based on the name(s) of the scientist(s) who wrote them. This is less than ideal for locating scientific collections. Participants wanted to search by geographic location(s) and by geological term(s). The familiar question re-emerges: what roles can archivists play to ameliorate this situation?

\section{Exemplary Participant Quotation 2}

[In reference to temporary identification numbers assigned to samples gather in the field.] "Those numbers, field numbers ... some people around the country had used those and I learned [in the] last couple years that they have actually published on those, on the basis of field numbers instead of requesting that we did give it a catalogue number. So that cost quite a bit of a furore realizing that they were as irresponsible as that, so we've got to coordinate with what has been published ... [as] they used a different numbering system. That will be a special project that will take quite some time but again, we couldn't do that without X's field notebooks."

Our participants evinced mixed feelings about archives and archivists. One eschewed the notion of storing field notebooks in an archive, instead insisting that field notebooks should not be separated from their datasets (i.e., the rock and fossil collections they describe). After all, these notebooks contain 
raw data and valuable metadata pertaining to the physical samples. But the narrative and historical value of these works militates against such a strategy. Indeed, field notebooks created by geologists in the early United States possess historical information valuable far beyond the domain of geology. Should such field notebooks be deposited in an archive in order to facilitate scholarly discovery by both historians and scientists? In a digital age, it needs not be an either/or situation; various stakeholders need to communicate about this issue.

\section{Exemplary Participant Quotation 3}

"I just opened the drawer and looked down and thought my god, there're the notes of $[X]$ and $[Y]$ and $[Z]$, these were things from the 1800 s and early 1900s that nobody knew they were there. He had inherited them from other people and obviously someone known they were there originally ... They badn't been seen in over a generation for sure."

On the other hand, one participant spoke of a set of field notebooks deposited in an archive that was "lost" for generations. In fact, the notebooks disappeared only because nobody thought to look for them (or perhaps knew how); they were "rediscovered" by happenstance. Also complicating efforts to track notebooks, although they continue with the same specialty geologists often work for more than one organisation during their careers. Thus tracking artefacts such as field notebooks becomes even more daunting.

\section{Exemplary Participant Quotation 4}

The notebooks are in"different places - some of them in the Smithsonian, some of it here, some of it's in his university town. Some of its with family, so there's another problem for grasping at historical sense of where's the body of work is, it's all over the place... [E]very now and then I get a letter from $[X]$ - eureka we made another discovery and now I know what he did from 1922 to 1928 or something like that, missing periods of time gets flled in with little discover moments."

By dint of their education and socialisation into given communities of practice, geologists think in a certain way about their artefacts and how to 
preserve them. ${ }^{79}$ Archivists undergo a rather different process of education and socialisation and thus think in their own way about artefacts and how best to archive them. These patterns of thought may not - are perhaps likely not - to match. A starting point for productive dialogue presents itself: how can archivists think like geologists and create tools (particularly finding aids) that mimic the processes by which geologists search for and use a specific form of information, the field notebook. Overall, geologists talk in very similar ways if not always in the same actual language about their needs in the archiving process. Even so, they have been unable to bridge the fields of geology and archives to realise their commonalities - as researchers, propitiously, we did so.

These areas represent only the beginning of the rich rewards Grounded Theory confers on the researcher. More areas surely remain yet to be explored; we anticipate bolstering our findings as we iteratively employ the method to talk to more geologists and to integrate new information to our extant observations. Similarly, we shall continue to demonstrate to the geological community the efficacy of Grounded Theory and perhaps more importantly, the potential for partnerships between the geological and archival communities.

We continue to engage in coding data, but in line with Charmaz's approach to constructivist Grounded Theory, we have also made the transition into theory development. We began with the concepts developed in the theoretical coding process and through writing, discussing, and reviewing the data have moved to explore where the data, the codes, and our reflections will direct us. Our efforts coalesced into a document in which we examined relationships emerging among these concepts and wrote in free form what these relationships suggest on a higher level. We sought to explain what we were seeing and at the same time we deliberated over potential underlying causes. Some ideas may push us to collect additional data to address questions raised or refined by the processes we analysed. Thus we may revisit early participants or engage new ones. For example, we might need to probe related domains beyond those already explored (palaeontologists and geologists conducting mapping research): how do

79 On communities of practice, see Etienne Wenger, "Communities of Practice:

The Key to Knowledge Strategy,” printed in Eric L. Lesser, Michael A. Fontaine, and Jason A. Slusher (eds.), Knowledge and Communities (Boston: ButterworthHeinemann, 2000), 3-20, and E. Davenport and Hall, H., "Organizational Knowledge and Communities of Practice," Annual Review of Information Science and Technology 36 (2002): 171-227. 
their archiving needs differ from those of scientists in cognate domains? Hence our Grounded Theory process remains, as it should, open-ended and embracing diversity and multiplicity.

But to meet the challenges underscored in this study, geologists and archivists must learn more about each other and then collaborate to improve the accessibility of field notebooks, namely by exploring both informal and formal ways that geologists search for information. Archival scholars may be well-suited for the task, having accrued nearly three decades of literature on archival users and use. Moreover, these scholars and practitioners must educate geologists on basic archival principles endemic to the prosaic needs of academic and professional geologists. Archival education might be incorporated as a module into beginning geological curricula such as courses on research methods. This information might be leveraged to create teaching tools; traditionally, archival principles and practices are not taught to geologists (and vice versa). Thus collaborative learning and collaborative creation of finding aids may be the best strategy. For their part, archivists must focus not only on artefacts birthed by digital technologies (the future may see born-digital field notebooks, for instance), but also on so-called "traditional" materials such as handwritten field notebooks. These notebooks contain evidence of considerable evidential and information value.

\title{
Exemplary Participant Quotation 5
}

\begin{abstract}
"I would keep any of the geo, any of the palaeontology notebooks I came upon because I don't want to put myself in a judgmental position ... that is a lot of responsibility to say this is not good enough or not enough data... It's just too much responsibility."
\end{abstract}

In general archival scholars and geologists must focus on moving archival processes upstream. First, they should explain to geologists the vital importance of appraisal and of establishing and retaining intellectual and physical control of geological materials such as field notebooks. Similarly, geologists must learn about standard practices of arrangement and description, access and availability. Third, archivists must imbue geologists as both creators and users of archives with an appreciation of the role of original order, respect des fonds, and provenance and in turn, how these principles ensure the trustworthiness and authenticity of a notebook's content. Fourth, working together archivists and geologists must determine that enough context information is available to all users and re-users. Finally, archivists must 
engage in more concentrated outreach toward the geological community. Archivists and geologists must sell the importance of their respective professional work to each other. Only with a cross-pollination of archival and geological knowledge can geologists and historians optimise the use and reuse of such valuable evidential artefacts as field notebooks. ${ }^{80}$

\section{Reflective Theory Development}

Theories "are essentially conceptual systems designed to be useful in identifying, organizing, and ... explaining or predicting some defined portion of the experienced world." ${ }^{11}$ Positivist theory "seeks causes, favors deterministic explanations, and emphasizes generality and universality." 82 Positivism's objectivism minimises the importance of the social context that envelops the data, the researcher's influence, and the quotidian interactions between the researcher and her participant(s). The researcher "becomes more of a conduit for the research process rather than a creator of it." ${ }^{\text {83 }}$

On the other hand, interpretive theory presupposes "emergent, multiple realities; indeterminacy; facts and values as inextricably linked; truth as provisional; and social life as processual." ${ }^{24}$ Its goals include conceptualising the given phenomenon, propagating theoretical claims vis-à-vis scope, depth, power, and relevance, recognising one's subjectivity, and propagating an interpretation. Charmaz argues that interpretive theorising allows a researcher to transcend the individual and micro levels. ${ }^{85}$

80 Within scientific institutions, there is often a lack of specialists trained in managing information: "Calling your spare researcher or technician a 'curator' doesn't confer the ability to curate." See M.S. Taylor, "What Will Happen to the Universities' Geological Collections in the Post-Oxburgh World?” Geology Today 4 no. 4 (1988): 119. In "Current Trends and Future Directions in Data Curation Research and Education" Journal of Web Librarianship 6 no. 4 (2012), 305-320, N. Weber, C.L. Palmer, and T.C. Chao call for specialised training to enable data management in a scientific organisation. A widely-observed need exists for individuals that can straddle scientific and technological domains. These tasks need to be built into the workflow and organisational structure. This role might be filled by archival professionals.

81 J. Jaccard and J. Jacoby, "The Nature of Understanding," in Theory Construction and Model-Building Skills: A Practical Guide for Social Scientists, New York: Guilford Press, 2010, 15.

82 Charmaz, Constructing Grounded Theory (2006 edition), 125-126.

83 Charmaz, Constructing Grounded Theory (2006 edition), 132.

84 Charmaz, Constructing Grounded Theory (2006 edition), 126-127.

85 Charmaz, Constructing Grounded Theory (2006 edition), 128. 
A social constructivist perspective flows naturally from interpretive theory. It pivots around a reflexive approach toward both processes and products. Constructivism maintains that reality - and thus meaning - is a construction of the human mind rooted in a particular time and place: "reality" changes along with its social context. ${ }^{86}$ Constructivist Grounded Theory "places priority on the phenomena of study and sees both data and analysis as created from shared experiences and relationships with participants and other sources of data. But even constructivist Grounded Theory is no panacea in qualitative research: in the end, "Theory generation continues to be the unfilled promise and potential of grounded theory." ${ }^{87}$

Nonetheless, Grounded Theory offers no mean return on investment. The lengthy process and the iterative interrogation of the data imbues the researcher with more confidence in her results, especially when she juxtaposes Grounded Theory with less rigorous methods such as conducting only a survey or only a series of interviews. Grounded Theory can bolster a study's credibility, resonance, originality, and usefulness. But Grounded Theory demands many resources; to say the least, it requires a researcher to balance planning and creative work.

The difficulty of maintaining such a balance perhaps culminates even before developing theory. Bluntly put, the coding process is the most difficult part of Grounded Theory. The researcher immerses herself in the data with but the vaguest of notions of what might come of her work. Ideally, the iterative process of Grounded Theory work helps the researcher to find common threads in her interviews and observations. But as Charmaz reminds us, "The more unproblematic - that is, routine, familiar, and ordinary - observed events seem to you, the more problematic creating an original conceptual analysis of them will be. ${ }^{18}$ Geologists and archivists both guard against insignificant results, whether contradictory or confirmatory. However, confusion and indeed failure may be as instructive as significant confirmation, especially in mid-range theory-building. As such, Grounded Theory represents an analytical tool not to be neglected. In this case, its use serves geologists and archivists both.

86 Jaccard and Jacoby, "The Nature of Understanding," 7.

87 Charmaz, Constructing Grounded Theory, (2006 edition), 135.

88 Charmaz, Charmaz, Constructing Grounded Theory, 53. 


\section{References}

Bernard, H. Russell and Gery W. Ryan. Analyzing Qualitative Research. Thousand Oaks: Sage, 2010.

Bichteler, Julie. "Geologists and Gray Literature." Science E Technology Libraries 11 no. 3 (1991): 39-50.

Bichteler, J., and D. Ward. "Information-Seeking Behavior of Geoscientists." Special Libraries 80 no. 3 (1989): 169-78.

Charmaz, Kathy. "Qualitative Interviewing and Grounded Theory Analysis." In The Handbook of Interview Research: Context and Method edited by J.F. Gubrium and J.A. Holstein, 675-694. Thousand Oaks: Sage Publications, 2002.

Charmaz, Kathy. Constructing Grounded Theory: A Practical Guide Through Qualitative Analysis. London: Sage Publications, 2006.

Clarke, Adele. Situational Analysis: Grounded Theory after the Postmodern Turn. Thousand Oaks: Sage, 2005.

Conway, Paul. "Modes of Seeing: Digitized Photographic Archives and the Experienced User." American Archivist 73 no. 2 (2010): 425-462.

Corbin, J.M., and A.L. Strauss. Basics of Qualitative Research: Techniques and Procedures for Developing Grounded Theory. Los Angeles: SAGE Publications, 2008.

Coyne, I.T. "Sampling in Qualitative Research; Purposeful and Theoretical Sampling; Merging or Clear Boundaries?” Journal of Advanced Nursing 26 no. 3 (1997): 623-630.

Cragin, Melissa H., Carole L., Palmer, Jacob R. Carlson and Michael Witt. "Data Sharing, Small Science and Institutional Repositories."Transactions of the Royal Society A 368 no. 1926 (September 13, 2010): 4023-4038.

Creswell, J.W. "Five Qualitative Approaches to Inquiry." In Qualitative Inquiry and Research Design: Choosing Among Five Traditions, edited by J.W. Cresswell (Thousand Oaks, CA: Sage, 2007), 53-84.

Darkenwald, G.G. "Field Research and Grounded Theory." In Changing Approaches to Studying Adult Education, H.B. Long, R. Hiemstra \& Associates, eds. (San Francisco: Jossey-Bass Publishers, 1980), 63-77.

Davenport, E., and H. Hall. "Organizational Knowledge and Communities of Practice," Annual Review of Information Science and Technology 36 (2002): 171-227.

Dey, Ian. Grounding Grounded Theory: Guidelines for Qualitative Inquiry. San Diego: Academic Press, 1999.

Geertz, Clifford. The Interpretation of Cultures. New York: Basic Books, 1973.

Gilliland, Anne and Sue McKemmish. "Building an Infrastructure for Archival Research." Archival Science 4 no. 3 (2004): 149-197.

Glaser, B.G. Theoretical Sensitivity: Advances in the Methodology of Grounded Theory. Mill Valley: The Sociology Press, 1978.

Glaser, B.G., and A.L. Strauss. The Discovery of Grounded Theory: Strategies for Qualitative Research. Chicago: Aldine Publishing Company, 1967.

Gracy, Karen F. "Documenting Communities of Practice: Making the Case for Archival Ethnography.” Archival Science 4 no. 3-4 (2004): 335-365.

Heidorn, P. Bryan. "Shedding Light on the Dark Data in the Long Tail of Science." Library Trends 57 no. 2 (2008): 280-299.

Hodder, Ian. "The Interpretation of Documents and Material Culture," in Norman K. Denzin and Yvonna S. Lincoln, eds. Handbook of Qualitative Research (Thousand Oaks: Sage, 2000), 703-715.

Jaccard, James, and Jacob Jacoby. Theory Construction and Model-Building Skills: A Practical Guide for Social Scientists. New York: Guilford Press, 2010. 
Jick, Todd. "Mixing Qualitative and Quantitative Methods: Triangulation in Action," Administrative Science Quarterly (1979): 602-611.

Johnson, J.M. “In-Depth Interviewing." In Handbook of Interview Research: Context $\mathcal{E}^{\circ}$ Method, J.F. Gubrium and J.A. Holstein, eds. (Thousand Oaks, CA: Sage, 2002), 103-119.

Johnson, J.M. "In-Depth Interviewing." in Handbook of Interview Research, J.F. Gubriuym and J.A. Holstein, eds. (Thousand Oaks: Sage, 2002), 103-119.

Joseph, L. E. "Information-Seeking and Communication Behavior of Petroleum Geologists." Science E Technology Libraries 21 no. 3 (2001): 47-62.

Lee, W.L., Bell, B.M., and Sutton, J.F., eds. Guidelines for Acquisition and Management of Biological Specimens. Lawrence, KS: Association System of Collections, 1982.

Lincoln, Yvonna S., and Guba, Egon, Naturalistic Inquiry. Newbury Park, CA: 1985.

Lofland, J., D. Snow, L. Anderson, and L.H. Lofland. Analyzing Social Settings: A Guide to Qualitative Observation and Analysis. Belmont: Wadsworth/Thomson Learning, 2006.

Loudon, T.V., and J.L. Laxton. "Steps Toward Grid-based Geological Survey: Suggestions for a Systems Framework of Models, Ontologies, and Workflows," Geosphere 3 no. 5 (2007): 319-336.

Luo, Lili, and Barbara Wildemuth. "Semistructured Interviews," in Barbara Wildemuth, ed., Applications of Social Research Methods to Questions in Information and Library Science (Westport: Libraries Unlimited, 2009), 232-241.

Marshall, Cathy C. "From Writing and Analysis to the Repository: Taking the Scholars' Perspective on Scholarly Archiving." In Proceedings of JCDL '08, June 16-20, 2008, Pittsburgh, PA, 2008, pp. 251-260.

National Research Council. Geoscience Data and Collections - National Resources in Peril. Washington, D.C., National Academies Press, 2002.

Parsons, M.A., and Fox, P.A. "Is Data Publication the Right Metaphor?" Data Science Journal 12 , no. 0 (2013).

Perutz, M.F. Is Science Necessary? Essays on Science and Scientists. New York: E.P. Dutton: 1989.

Pickard, Alison. Research Methods in Information. Chicago: Neal-Shuman, 2013.

Poole, Alex H. "How Has Your Science Data Grown? Digital Curation and the Human Factor: a Critical Literature Review," Archival Science (2014): 1-39.

Ramdeen, S. "Preservation Challenges for Geological Data at State Geological Surveys." GeoResJ 6 (2015), in press.

Rubin, Herbert J., and Irene S. Rubin. Qualitative Interviewing: The Art of Hearing Data. Thousand Oaks: Sage, 2005.

Shankar, Kalpana. "Order from Chaos: The Poetics and Pragmatics of Scientific Recordkeeping." Journal of the American Society for Information Science and Technology 58 no.10 (2007): $1457-1466$.

Simons, Helen. Case Study Research in Practice. Los Angeles: Sage Publications, 2009.

Taylor, M.S. "What Will Happen to the Universities' Geological Collections in the PostOxburgh World?” Geology Today 4 no. 4 (1988): 119-120.

U.S. Geological Survey. The USGS Geologic Collections Management System (GCMS): A Master Catalog and Collections Management Plan for USGS Geologic Samples and Sample Collections. By the Geologic Materials Repository Working Group. Preprint, submitted January $25^{\text {th }}, 2013$.

Vieira, D., M. Mookerjee and M. Swathi, "Incorporating Geoscience, Field Data Collection Workflows into Software Developed for Mobile Devices," Poster presented at the American Geophysical Union Fall Meeting, San Francisco, CA, December, 2014. 


\section{Chapter 33}

Wallis, Jillian, Christine Borgman, Matthew Mayernik, Matthew and Alberto Pepe. "Moving Archival Practices Upstream: An Exploration of the Life Cycle of Ecological Sensing Data in Collaborative Field Research." International Journal of Digital Curation 1 no.3 (2008): 114-126.

Wasserman, J.A., J.M. Clair, and K.L. Wilson. "Problematic of Grounded Theory: Innovations for Developing an Increasingly Rigorous Qualitative Method." In Data Collection: Data Collection in Survey and Interview Research, W.P. Vogt, ed., vol. II (Los Angeles: Sage Publishing, 2010), 401-427.

Weber, N., C.L. Palmer and T.C. Chao. "Current Trends and Future Directions in Data Curation Research and Education," Journal of Web Librarianship 6 no. 4 (2012): 305-320.

Weiss, Robert. Learning from Strangers: The Art and Method of Qualitative Interviewing. New York: Free Press, 1994.

Wenger, Etienne. “Communities of Practice: The Key to Knowledge Strategy," in Eric L. Lesser, Michael A. Fontaine and Jason A. Slusher, eds. Knowledge and Communities (Boston: Butterworth-Heinemann, 2000), 3-20. 
Chapter 34

\title{
APPLYING Q METHOD TO ARCHIVAL RESEARCH
}

\section{"A Quantitative Approach to Qualitative Study"}

\author{
Amber L. Cushing
}

\begin{abstract}
Developed in 1935, Q method has been described as a method used to measure individuals' points of view and/or opinions on a specific topic or issue at hand. While $\mathrm{Q}$ method uses quantitative statistical analysis, it is often used to address qualitative research questions, due to its ability to explore opinion and intent, while providing the participants' personal frames of reference during the study. Q method involves the development of a corpus of statements, participants' rankings of the statements, and statistical analysis. In this chapter, I detail the history, epistemology, and mechanics of $\mathrm{Q}$ method, while explaining my use of $\mathrm{Q}$ method in an archival research study, and my reflections on the experience. While somewhat obscure, Q method has potential for use in archival studies research, especially for collecting data about user preferences related to archival practice.
\end{abstract}

\section{Introduction}

"This is not going to be a statistical article," was how William Stephenson began a 1935 article in which he first introduced Q method. ${ }^{1}$ It is a fitting description of how to begin an introduction of $\mathrm{Q}$ method to archival studies. While not considered a qualitative technique, $\mathrm{Q}$ method is used

1 William Stephenson, “Correlating People Instead of Tests," Character and Personality 4 (1953): 17-24. 
to measure human subjectivity, ${ }^{2}$ or individuals' points of view on a specific topic, which are typically captured using qualitative techniques. With the goal of exploring individuals' opinions about how they select personal digital items for preservation, I used $\mathrm{Q}$ method as a complement to participant interviews. While the concepts of archival selection and archival appraisal ${ }^{3}$ are frequently explored and discussed in archival studies, ${ }^{4} \mathrm{Q}$ method has not previously been applied to this end, but has great potential applicability. This chapter introduces the method to archival studies. It also discusses why I chose $\mathrm{Q}$ method, how I implemented it, and my reflections on how my $\mathrm{Q}$ method study affected the future direction of my research on preservation and personal recordkeeping.

\section{Archival Studies and Information Studies Research}

My goal for this research project arose out of a desire to understand how individuals select which of their digital items, to preserve and maintain. In work discussing personal digital archiving, several studies have found that one of the largest obstacles in the practice of personal digital archiving was an individual's ability to determine whether his or her digital item had enough value to maintain over time, and that individuals did not like to engage in decisions to maintain material because they found it cognitively difficult. ${ }^{5}$

2 Stephenson's use of subjectivity refers to an "operant." This has roots in behaviourism, in which an operant is a naturally occurring behaviour without external cause. The operant is defined by its relationship with and impact on a specific environment. Thus, subjectivity refers to an unprompted behaviour that occurs in and due to a specific environment. Simon Watts, "Subjectivity as Operant: a Conceptual Exploration and Discussion," Operant Subjectivity: the International Journal of $Q$ Methodology, 35 (2011): 38-39.

3 "The process of identifying materials offered to an archives that have sufficient value to be accessioned," Glossary of Archival and Records Terminology, s.v. "appraisal," by Richard Pearce Moses, http://www2.archivists.org/glossary/terms/a/appraisal.

4 "The body of knowledge that supports the practice of appraising, acquiring, authentication, preserving, and providing access to recorded materials; archival science," Glossary of Archival and Records Terminology, s.v. "archival studies," by Richard Pearce Moses, http://www2.archivists.org/glossary/terms/archival-studies.

5 Catherine C. Marshall, Sara Bly, and Francoise Brun-Cottan, "The Long Term Fate of Our Personal Digital Belongings" (Paper presented at the Archiving 2006, Ottawa, Canada: Society for Imaging Science and Technology, 2006); Catherine C. Marshall, "How People Manage Information over a Lifetime," in Personal Information Management ed. William Jones and Jamie Teevan (Seattle, WA: University of Washington Press, 2007), 57-75; Catherine C. Marshall, "Rethinking Personal Digital Archiving Part I: Four Challenges from the Field," D-lib Magazine 14 (2008): http://www.dlib.org/dlib/march08/marshall/03marshall-pt1.html; Catherine C. 
Archival appraisal had yet to be addressed at the individual level, from the perspective of the creator, and my research was an attempt to address that obstacle. I speculated that if archivists could better understand how individuals determined value in their personal digital items, then they could use this knowledge in their work with donors of digital material. Archival scholars had already suggested that archivists should work with creators of digital content early in the pre-custodial phase so that the digital items would not become obsolete before they were donated to an institutional archive. ${ }^{6}$ The ability to understand individual concepts of value might also allow archivists to develop tools that would streamline the "cognitively difficult" decisions. I imagined my research contributing to something akin to a personality quiz that one might see in a magazine: take a short quiz to determine your main values (from a set of common values) that you perceive your digital items to hold, determine which items hold these values.

I hypothesised that there were likely several common values that might emerge and that individuals would usually fall into one of a few categories associated with these values. I set out to find a method that would allow me to understand individuals' values and summarise them, so I could determine the characteristics of this group of common values. Again, I envisioned this as a Myers-Briggs type indicator assessment. ${ }^{7}$ Are you an INFJ? An ESTP? An ISTP? And so forth. I just needed to discover the method that would allow me to do this. However, there was a challenge: I did not want to force participants into an artificial personal archiving environment. After all, I was most interested in how individuals' perceived value, not necessarily in how they archived and preserved personal material, because the act of archiving caused the cognitive difficulty reported by Marshall. ${ }^{8}$ While previous research, mostly conducted in the field of

Marshall, "Rethinking Personal Digital Archiving Part II: Implications for Services, Applications, and Institutions," D-lib Magazine 14 (2008): http://www.dlib.org/dlib/ march08/marshall/03marshall-pt2.html.

6 Adrian Cunningham, "The Archival Management of Personal Records in Electronic Form: Some Suggestions," Archives and Manuscripts 22 no. 1 (1994): 94-105; Susan Thomas, "PARADIGM: A Practical Approach to the Preservation of Personal Digital Archives" (Manchester, UK: Bodleian Library, 2007); Jeremy Leighton John, Ian Rowland, Peter Williams, and Katrina Dean, "Digital lives: Personal Digital Archives for the 21st Century: An Initial Synthesis” (London, UK: British Library, 2010).

7 The Myers-Briggs Type Indicator (MBTI) assessment is a popular psychometric questionnaire used to measure personality traits.

8 Marshall, "Rethinking Personal Digital Archiving Part I: Four Challenges from the Field,” Marshall, "Rethinking Personal Digital Archiving Part II: Implications for Services, Applications, and Institutions." 
human computer interaction, had explored an individual's relationship to a computer or a physical device, digital items were different because they are not tangible. ${ }^{9}$ I wanted to situate my study in individuals' cognitive environments, before the act of archiving and preservation began. Interviews (as a method) were a good place to start, but the method did not allow for the standardisation I sought in order to characterise the common value sets.

My research agenda had led me to reading about the concept of selfextension to possessions. A concept promoted by scholar Russell Belk in the field of consumer behaviour, self-extension to possessions assumes that individuals understand that their possessions can contribute to their identity, and are therefore imbued with value and meaning. ${ }^{10}$ The value of possessions was important in consumer behaviour (a branch of marketing) because it helped researchers understand what people valued and why, which in turn helped companies promote products based on that value. For example, if an individual values a car because it makes him or her feel strong, then a car commercial could be developed that promoted the car as a symbol of strength, which could sell more cars. In addition, after reading the work of Lita Furby on the definition of possessions, I understood that if an object was considered to be a possession, then it was valued by an individual and had distinctive characteristics compared to other objects, mainly by the act of claiming the object. ${ }^{11}$ In other words, the label of "possession" makes the object more valuable than just recognising it as an object.

At that point, I had yet to encounter the concept of self-extension being applied to digital possessions. This concept of "digital possessions" sparked research questions that became part of my research agenda: what characterises digital items that have more value than others? Do Furby's findings extend to the digital environment? What characterises digital possessions?

9 Sherry Turkle, The Second Self: Computers and the Human Spirit (Cambridge, MA: MIT Press, 2005).

10 Russell Belk, "Possessions and the Extended Self," Journal of Consumer Research 15 (1988): 139-168, Eugene Sivadas and Karen Machleit, "A Scale to Determine the Extent of Object Incorporation in the Extended Self," paper presented at the Annual Meeting of the American Marketing Association's Winter Educator's Conference, St. Petersberg, FL, 1994.

11 Lita Furby, "Possession in Humans: an Exploratory Study of its Meaning and Motivation," Social Behavior and Personality 6 no.1 (1978): 49-65. 


\section{History}

William Stephenson, the founder of Q method, began his academic study as a student of Charles Spearman, who is most known for his work in methods using factor analysis. ${ }^{12}$ Stephenson was frustrated with Spearman's R factor analysis for several reasons. Researchers would typically use $\mathrm{R}$ to learn how variables differed, with the goal of reducing data. ${ }^{13}$ Using $\mathrm{R}$ factor analysis, a researcher could move from a set of correlated variables to a smaller, more efficient set of uncorrelated variables. However, a researcher could only isolate one variable at a time, which required the use of large populations of participants. Stephenson argued that this was inefficient because it slowed the pace of research and in running experiments with large populations, the process becomes routine and the research is less able to recognise the intricacies that characterise human behaviour..$^{14}$ Further, due to the large population required, individuals were typically given written tests, outside of the lab, where the researcher was unable to control the environment completely.

Stephenson proposed a new method that measured how individuals (versus factors) were alike instead of different, required fewer participants, measured multiple variables simultaneously, and allowed the researcher to conduct the experiment in the lab, where the environment could be controlled..$^{15}$ In addition, Stephenson wanted to remain close to his participants, as he believed this provided a better opportunity for learning more about their viewpoints on the issues at hand. Stephenson also desired a method that he could use to measure opinion, without removing the subject's frame of reference from inquiry, ${ }^{16}$ for example, by asking participants their opinions about a hypothetical situation versus asking them about their opinions about a situation specifically relevant to their own experiences.

What Stephenson referred to in 1935 as "the new factor technique" eventually came to be known as $\mathrm{Q}$ method. With $\mathrm{Q}$ method, subjects are correlated

12 "A technique used to identity factors that statistically explain variation and covariation among measures," Samuel B. Green and Neil J. Salkind, Using SPSS for Windows and Macintosh: Analyzing and Understanding Data, $5^{\text {th }}$ ed. (Upper Saddle River, NJ: Pearson Education Inc., 2008), 313.

13 Steven R. Brown, "The History and Principles of QMethodology in Psychology and the Social Sciences," unpublished manuscript, http://facstaff.uww.edu/cottlec/ QArchive/Bps.htm.

14 Stephenson, "Correlating People Instead of Tests."

15 Stephenson, "Correlating People Instead of Tests."

16 Paul Robbins and Rob Krueger, "Beyond Boas? The Promise and Limits of QMethod in Human Geography,” Professional Geographer 52 no.4 (2000): 636-648. 
to allow for the emergence of common opinions, points of view preferences, or "tastes." ${ }^{17}$ While Q method gained in popularity after Stephenson's initial writings on the method in the 1930s, fewer researchers used Q method when computers gained in popularity as a statistical tool, beginning in the 1970s. ${ }^{18}$ Even today, few commercial statistical software packages allow for data collection or analysis using $\mathrm{Q}$ method or $\mathrm{Q}$ factor analysis. Without the use of computers, one needs a large table designed with distribution boxes upon which subjects can sort cards and complete the statistical analysis "by hand," which can be time consuming. Barriers of space, time, and the ability of subjects to travel to a designated space may hinder a project. Many Q methodologists consider one statistical program, PQMethod, to be an excellent analytical tool, in large part due to the fact that it was created by a practicing Q methodologist. However, PQMethod was written in Fortran, a computer code developed in 1956, and it can only be accessed with modern computer operating systems by using a program emulator. These two technological characteristics of conducting a $\mathrm{Q}$ method study have been linked to the method falling out of favour for many researchers with the rise of the personal computer. ${ }^{19}$

\section{Epistemological Lineage}

Epistemologically, "Q methodology ruptures the boundaries between the scientific and interpretive frameworks." ${ }^{20} \mathrm{Q}$ method's statistical analysis has roots in logical positivism, which posits basing all knowledge on relevant experiences. The building of the concourse, or the range of items that are sorted, is rooted in interpretivism, ${ }^{21}$ which focuses on the role of the actor's beliefs in understanding a situation or event. As a result, Q method is often referred to as a quantitative method (due to the factor analysis) used to explore qualitative issues (human subjectivity). Concourse theory

17 Fred N. Kerlinger, Foundations of Behavioral Research (New York: Holt, Rinehart, and Winston), 517.

18 Steven R. Brown, “A Primer on QMethodology," Operant Subjectivity: the International Journal of Q Methodology 16(1993): 91-138.

19 Brown, "A Primer on QMethodology."

20 Irvin Goldman, "QMethodology as Process and Context in Interpretivism, Communication and Psychoanalytic Psychotherapy Research," The Psychological Record 49 (1999): 594.

21 Jonathan Grix, "Introducing 'Hard' Interpretivism and Q Methodology: Notes From a Project on County Sport Partnerships and Governance," Leisure Studies 29 issue 4 (2010): 459. 
refers to the subjective ideas and/or thoughts of individuals from which the researcher builds the $\mathrm{Q}$ sample, which is sorted by subjects in a $\mathrm{Q}$ study. For example, if one wanted to explore paint colour preferences (as Stephenson discussed in his 1935 article), the concourse would be all paint samples in existence and the Q sample would be a narrowed version of that, or of the paint samples that Stephenson chose to have individuals sort. While a concourse is built empirically, it is influenced by cultural norms and values. $Q$ method uses the scientific method to garner results but it is ultimately an exploration of subjectivity, through qualitative interviewing. ${ }^{22}$

According to Stephenson, Q method incorporates the philosophical ideas of Kaufmann, Schlick and Kantor, ${ }^{23}$ all of whom were philosophers of science associated with logical positivism (also known as logical empiricism). All emphasised the importance of observable data and the use of standard language in scientific work coupled with mathematical constructs. Consequently Stephenson emphasised the importance of the scientific method in Q method: "facts are gathered and a theory induced to explain them." ${ }^{24}$ However, Stephenson also stated that $\mathrm{Q}$ method emphasises an individual's self-concepts about a certain issue and that the individual's selfconceptions "are facts to be discovered about him." 25 Thus, through an individual's viewpoints and opinions, the researcher can learn which facts to explore next about the individual or set of individuals.

\section{My Introduction to Q Method}

I was first introduced to a $\mathrm{Q}$ sort (labelled as such) when reading a study conducted by psychologist Ernst Prelinger. ${ }^{26}$ In this study, Prelinger conducted a basic Q sort (without Q factor analysis) with individuals, in order to explore aspects of self that extended beyond the physical body. He developed the sorting sample by asking 100 college students to make a list of terms that represent ideas or concepts that they believed to be "a part of their own selves." From these lists, Prelinger developed a list of 160 items that he put on cards. He then

22 Goldman, "QMethodology as Process and Context in Interpretivism, Communication and Psychoanalytic Psychotherapy Research."

23 William Stephenson, The Study of Behavior; Q-technique and its Methodology (Chicago, Il: University of Chicago Press, 1953).

24 Stephenson, The Study of Behavior, 341.

25 Stephenson, The Study of Behavior, 342.

26 Ernst Prelinger, "Extension and Structure of the Self," Journal of Psychology 47 (1959): 13-23. 
divided the cards into eight categories: psychological or intra-organismic processes (e.g., the concept of consciousness), body parts, objects within the close physical environment, objects from the distant physical environment, personal identifying characteristics and attributes, possessions and productions, other people, and abstract ideas. ${ }^{27} \mathrm{He}$ then used a convenience sample of 60 soldiers, who sorted the items into piles labelled "part of my Self" and "not part of my Self." ${ }^{\prime 2}$ The card sorting activity was then repeated, and participants were asked to sort the two piles into two more piles based upon whether they felt "sure" or "not so sure" about the cards in the "part of Self" and "not part of Self" piles. The piles were then labelled with a score of $0-3$ with cards that scored a 0 or 1 (cards that were not part of my self) and cards scoring a 2 or 3 (cards that were considered to be part of my self).

According to Prelinger, the fact that the participants were able to sort the cards into piles representing themselves and not representing themselves implied that subjects perceived a boundary between self and not self..$^{29} \mathrm{Next}$, Prelinger tested the significance of the variance between the means of the eight item categories by comparing the scores given to each card with the total scores within the item groups. The results were significant, and demonstrated a rank order among item groups. Body parts were the item most considered to be part of the self, followed by psychological and intra-organismic processes, personal identifying characteristics, and possessions and productions. ${ }^{30}$ The scores for these items were above 1.5 , which Prelinger considered the border between self and not self (the items were given scores of 1-3). Items falling below the 1.5 mark included abstract ideas, other people, objects in the close physical environment, and objects in the distant physical environment. Note that Prelinger did not conduct a true $\mathrm{Q}$ method study using a legitimate distribution, $\mathrm{Q}$ factor analysis, or factor interpretation. ${ }^{31}$ However, I was intrigued by the use of the $\mathrm{Q}$ sort to understand individuals' opinions and attempted to incorporate the method into the design of my study.

I was also influenced by the work of Kleine (nee Schultz), which I discovered when searching for dissertations listing $\mathrm{Q}$ method as a keyword in the Proquest Dissertation and Theses Database. ${ }^{32}$ Kleine was a consumer behav-

27 Prelinger, "Extension and Structure of the Self," 14.

28 Prelinger, "Extension and Structure of the Self," 15.

29 Prelinger, "Extension and Structure of the Self".

30 Prelinger, "Extension and Structure of the Self".

31 Prelinger, "Extension and Structure of the Self".

32 Susan Schultz, "An Empirical Investigation of Person-Material Possession Attachment.” PhD. Diss., University of Cincinnati, 1989. 
iourist who conducted research similar to Belk's work on self-extension to possessions. ${ }^{33}$ In her dissertation, Kleine aimed to explore individual attachment to material goods. She utilised $\mathrm{Q}$ method to determine whether strong attachments reflected specific self-developmental processes compared with weak attachments, which would not reflect these specific developmental processes. ${ }^{34}$ Kleine is a well-known expert in the study of possession attachment in consumer behaviour. Her concept of possession attachment is very similar to that of self-extension to possessions. ${ }^{35} \mathrm{I}$ contacted her and she ultimately became a member of my dissertation committee and helped to guide the development of this study on the characteristics of self-extension to digital possessions, using $\mathrm{Q}$ method. I used $\mathrm{Q}$ method in combination with interviews to answer several research questions. This chapter focuses on a single research question and details how I used Q method to answer the following: what characterises self-extension in digital environments?

\section{Study Design}

\section{Step 1. Develop a Q Sample from a Concourse}

The first step in Q method is to develop a Q sample. The Q sample is a collection of items drawn from a concourse. According to Brown, the concourse has been defined as "the flow of communicability surrounding any topic." 36 The Q researcher scales down the concourse to develop a Q sample: a representative sample of statements about an issue. Most often, a researcher develops a concourse by conducting interviews with individuals. If the concourse consists of statements, those statements are usually direct quotes from these interviews. In addition, researchers could also create a concourse by asking individuals to make a list of words or, a concourse could be developed

33 Belk, "Possessions and the Extended Self."

34 Schultz, "An Empirical Investigation of Person-Material Possession Attachment."

35 Attachment to possessions includes self-extension to possessions as well as other characteristics and can thus be conceived of as an "extreme" form of self-extension. Possession attachment has been defined as "a multi-faceted property of the relationship between a specific individual or group of individuals and a specific, material object that an individual has psychologically appropriated, decommodified, and singularized through a person-object interaction," Susan Kleine and Stacey Baker, "An Integrative Review of Material Possession Attachment," Academy of Marketing Science Review 1 (2004).

Brown, "A Primer of QMethodology." 
from newspaper commentaries, televisions interviews, or essays. ${ }^{37}$ The $\mathrm{Q}$ sample usually includes frequently occurring statements.

For my study, I developed a Q sample from data developed from 23 interviews, using a hierarchy. During the interviews, I asked participants to describe the digital items they considered to be their possessions and why they considered the digital items to be possessions. I asked participants to define the term "digital possession," and describe the characteristics of digital possessions. As a prompt to explore self-extension to digital possessions, I also asked if the digital possessions represented something, and if so to describe what. The questions were developed based on the questions that Furby used in her study exploring the definitions of physical possessions. ${ }^{38}$ After conducting and analysing the interviews, I was able to develop a hierarchy to organise my $\mathrm{Q}$ sample:

Figure 34.1. Q Sample Hierarchy, Interpretation of Statements

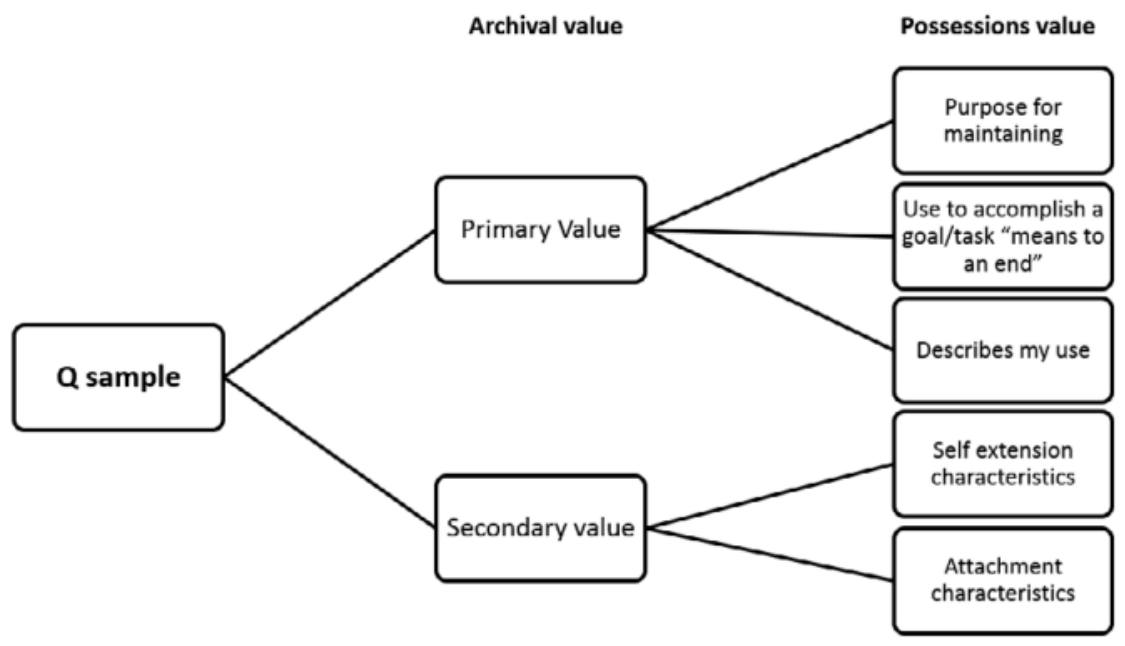

When participants described digital possessions and representations and/or relationships with digital possessions, their statements were often associated with value and aligned with categories connected with archival studies. I was able to categorise the statements using the concepts of secondary and primary value that are associated with archival appraisal. ${ }^{39}$ Primary

37 Brown, "A Primer of QMethodology."

38 Furby, "Possession in Humans: an Exploratory Study of its Meaning and Motivation."

39 "The value of records derived from the original use that caused them to be created," Glossary of Archival and Records Terminology, s.v. "primary value," by Richard Pearce 
value, or value that is derived from the original use of the item, was further refined by a few common characteristics, all of which were found in Furby's study that identified the characteristics of possessions. Secondary value, or value that is based on a purpose other than that for which the item was originally created was refined as a characteristic already associated with selfextension to possessions or which attachment to possessions. From this data and using the hierarchy, I developed 60 statements that interview participants used to characterise digital possessions. Kerlinger, as well as McKeown and Thomas suggest that the minimum number of items (cards to sort) in a $\mathrm{Q}$ sample to be 60 , so I used this standard. ${ }^{40}$ Most of these statements used natural language derived from participant quotes.

\section{Step 2. Develop Conditions of Instruction and Q Sort Conditions}

As mentioned above, I was using $\mathrm{Q}$ method to explore the research question what characterises self-extension in digital environments? In order to respond to that question, I developed two sets of directions, known in Q method as "conditions of instruction" to supply to participants as they ranked the statements about digital possessions:

1. Reflect on your digital possession that most reinforces your identity to you. Sort the cards according to "least representative of my view" to "most representative of my view."

2. Reflect on the digital possession that most displays your identity to others and sort the cards according to "least representative of my view" to "most representative of my view."

In accordance with $\mathrm{Q}$ method, I provided each of the 48 participants (a new sample that did not overlap with interview participation) with conditions of instruction so that each participant knew how to rank the Q sample. Participants ranked the $\mathrm{Q}$ sample twice, using the first and then the second set of directions.

Moses accessed August 6, 2013, http://www2.archivists.org/glossary/terms/a/archivalstudies. "The usefulness or significance of records based on purposes other than that for which they were originally created," Glossary of Archival and Records Terminology, s.v. “secondary value," by Richard Pearce Moses accessed August 6, 2013, http://www2. archivists.org/glossary/terms/a/archival-studies. Furby, "Possession in Humans: an Exploratory Study of its Meaning and Motivation."

40 Kerlinger, Foundations of Behavioral Research, Bruce McKeown and Dan Thomas, $Q$ Methodology (Newbury Park, CA: Sage, 1988). 
In addition to developing the directions for sorting, the Q researcher must also determine the $\mathrm{Q}$ sort conditions. In $\mathrm{Q}$ method, participants rank the $\mathrm{Q}$ sample along a predetermined distribution; the quasi-normal distribution being the most commonly used in the method (and the distribution I used in my study). The quasi-normal distribution is the shape of the diagram upon which the participant sorts the statement cards. The "V" shape made by the boxes in the columns, visible in Figure 34.2, are referred to in Q method as "quasi-normal" because previous studies have found that most sorting tasks tend to move toward resembling this shape. ${ }^{41}$ This is because participants tend to feel strongly about a few statements, while most statements fall somewhere in the middle.

\section{Figure 34.2. Sorting along the Quasi-Normal Distribution}

This figure displays a screenshot of sorting statements using the software program Flash Q, which was specifically developed for Q method.

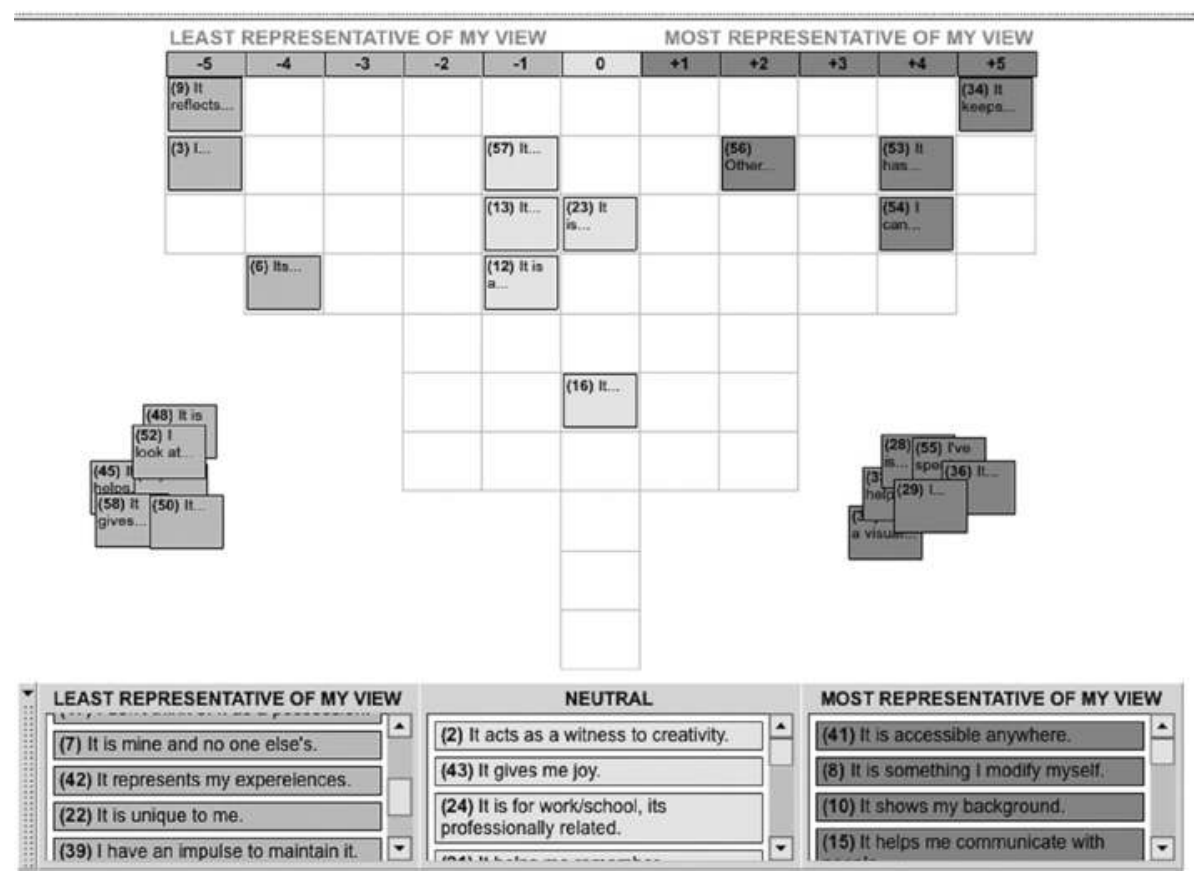

\section{Step 3. Data Collection}

Forty-eight participants sorted the 60-item Q sample using Flash Q (see Figure 34.2). The sample age of populations was developed using Levinson's

41 Brown, "A Primer of QMethodology." 
theory of cross-era transitions. According to Levinson, the life cycle is made up of eras and cross-era transitional periods lasting about five years and including a two-year range-above and below the average of five years. ${ }^{42}$ Prior research has shown that individuals tend to assess their personal archives during life transitions, such as a move or retirement, which are likely to occur during these ages, especially for young adult transition and late adult transition. ${ }^{43}$ Therefore, quota sampling was used to create 6 sample groups. The samples were stratified according to sex and age (see Table 34.1). Each participant was then provided with the study's conditions of instruction.

Table 34.1. Study Sample Groups

\begin{tabular}{|c|c|c|}
\hline Group & Age & Size \\
\hline \multicolumn{3}{|c|}{ Part 1: Interviews } \\
\hline A & & 8 \\
\hline B & & 7 \\
\hline C & & 8 \\
\hline \multicolumn{3}{|c|}{ Part 2: Qmethod } \\
\hline D & $18-24$ & 16 \\
\hline E & $38-47$ & 16 \\
\hline F & $58-67$ & 16 \\
\hline
\end{tabular}

Once revealing their digital possession, the participant began using Flash Q to sort the 60-item Q sample statement along the quasi-normal distribution. I observed the sorting, took notes on any utterances and answered any clarifying questions that participants might have had. The use of Flash Q allowed me flexibility in working with participants, as opposed to a traditional paper sort, that required a large table. I was able to use any device and meet participants anywhere there was wireless access, as I ran Flash $Q$ from the servers of my institution. This was more convenient for participants because I could meet them at a public location of their choosing.

42 Daniel J. Levinson, "A Theory of Life Structure Development in Adulthood," in Higher Stages of Human Development, ed. Charles N. Alexander and Ellen J. Langer (New York, NY: Oxford University Press, 1990).

43 Steve Whittaker and Julie Hirschberg. "The Character, Value and Management of Personal Paper Archives," paper presented at the SIGCHI Conference on Human Factors in Computing Systems, Seattle, WA, 2005. 
Most participants adapted quickly to the sorting exercise, with a few uttering that they wished they could just sort into two piles, instead of structuring the statements according to a -5 to +5 distribution. I believe that this is where $\mathrm{Q}$ method was particularly useful: participants were challenged to spend more time thinking about their opinions regarding the statements, rather than just ranking them into a positive and negative (2 pile) sort. I was able to obtain data about opinions and viewpoints that might not be as easily discernible in an interview.

Most other utterances were not useful in my final data analysis, except for one made by a male participant in the "late adult" age group. He remarked that he had spent less of his lifetime with digital possessions than he had spent with physical possessions, a factor to which he attributed to his negative rankings for many of the statements. Many of the statements to which he referred were linked to self-extension. While $\mathrm{Q}$ methodologists have mixed feelings about whether or not it is necessary to observe participant sorting, I believe it was useful because I was able to gain this comment from the participant.

\section{Step 4. Q Factor Analysis}

The data were analysed using the computer program PQ Method. PQ Method provided factor loadings for each item in the $\mathrm{Q}$ sample. While Flash Q is a data collection computer program, PQMethod is a statistical analysis computer program. Like any statistical software, these software programs made my data collection and data analysis more efficient, allowing me to arrive at results (the factor loadings) sooner.

Factor loadings are clusters of individuals who share similar opinions about the sorting tasks. Brown explained that factors could be conceived of as "qualitative categories of thought" because they represent a way of thinking about the issue at hand that all individuals who load on the Factors have in common. ${ }^{44}$ For example, in sorting task 1 , in which participants were asked to rank the statement cards according to the digital possession that most reflected their identity back to them, all the participants who similarly ranked the statement cards loaded onto the same factor. Thus, a factor represents a cluster of participants who share the same opinion about the characteristics of their chosen digital possession that most reflects their identity back to them. These participants shared the same opinion about

44 Brown, "A Primer on QMethodology." 
the characteristics of their digital possession even though they each chose different digital possessions that were relevant to them. For example, factor one could represent five participants who believe that the digital possession that most reflects their identity back to them "represents their personality" (one of the statement cards). However, one participant on the factor could have chosen a specific digital photo and another participant could have chosen a digital music collection. What the participants on this factor have in common is how they conceive of their own digital possessions that reflect their identity back to them, which is that the digital possession reflects their identity back to them by "reflect[ing] their personality."

In order to understand $\mathrm{Q}$ factors, I used a point of reference: the icebreaker game called "Four Corners," that I often played as a child in school. In this icebreaker, each corner is designated with a topic. Participants are instructed to go to the topic corner of the room with which they feel they best align. For example, if the question posed to party goers was "what is your favourite season?” corners would be designated Spring, Summer, Winter and Fall. Participants are supposed to go to the corner of the season they like best and talk about why they like that season with others who chose the same corner. I visualised my factors as corners and my study participants moving to the corners in which they most believed/agreed.

Defining sorts for each factor (cluster of individuals who similarly ranked the statement cards for each sorting task) was based on Schlinger's formula to determine significant Factor loading: $3^{*} 1 /{ }$. ${ }^{45}$ A student of Q Method inventor William Stephenson, Schlinger stated that, "there is not a simple, agreed-upon formula for determining the standard error of a factor loading. Most researchers use some rough rule-of-thumb to establish a criterion of significance. A guide this researcher has found to be both expedient and workable is ... $3^{*} 1 / \sqrt{ }$ n." ${ }^{\prime 46}$ As students who worked with Stephenson tend to be regarded as the best resources for the intricacies of $\mathrm{Q}$ method, Dr. Kleine suggested I use this formula. Only factors (clusters of individuals who similarly ranked the statement cards for each sorting task) on which more than two participants loaded were considered representative of a defined opinion in each sorting task. ${ }^{47}$

45 Mary Jane Schlinger, “Cues on QTechnique," Journal of Advertising Research 9 no.3 (1969): 53-60.

46 Schlinger, "Cues on QTechnique," 56.

47 Steven Brown, Political Subjectivity: Applications of QMethodology in Political Science (New Haven, CT: Yale University Press, 1980). 


\section{Step 5. Interpretation of Factors}

A five-factor solution for sorting task one and a three factor solution for sorting task two (Reflect on the digital possession that most displays your identity to others ... ). PQ method then provides the highest and lowest ranked statements for each factor. These are the distinguishing statements for the factor because they received the highest average rank from the participants who were defined by the factor. Upon exploration of the statements, the researcher can understand what most defines the factor, or opinion about the sorting task. For example: Table 34.2 displays the distinguishing statements for factor 1 , sorting task 2 . In exploring the statements, I would compare the statement with the Q sample hierarchy (Figure 34.1) to see where the statement was listed, and ask myself the following questions: was it associated with a primary or secondary value? Was it associated with self-extension or attachment? What do the top statements have in common? How are they different? How do the rankings compare to the rankings on other factors? Are they complete opposites?

Table 34.2. Distinguishing Statements for Factor 1, Sort Task 2 (and Their Loadings on Other Factors)

\begin{tabular}{|c|c|c|c|c|}
\hline \multirow[t]{3}{*}{ \# } & \multirow[t]{3}{*}{ Statements } & \multicolumn{3}{|c|}{ Factors } \\
\hline & & 1 & 2 & 3 \\
\hline & & Rank & Rank & Rank \\
\hline 17 & It helps me achieve my goals. & 5 & 0 & -2 \\
\hline 37 & It allows me to reflect on things. & 5 & 1 & -2 \\
\hline 4 & It helps me forget. & 5 & 0 & 3 \\
\hline 20 & It represents change in me. & 4 & -3 & 0 \\
\hline 38 & It's useful as a record. & 4 & -2 & 2 \\
\hline 59 & I wouldn't feel right deleting it. & 4 & 1 & 0 \\
\hline 52 & I look at it/open it frequently. & 3 & 0 & -1 \\
\hline 27 & It represents a side of me. & 3 & -2 & -5 \\
\hline 7 & It is mine and no one else's. & 3 & 0 & -4 \\
\hline 24 & It's for work/school, it's professionally related. & -3 & 3 & 3 \\
\hline 26 & It's nostalgia. & -3 & -5 & 5 \\
\hline 18 & It represents a history/chronology. & -4 & 0 & -1 \\
\hline 55 & I've spent a lot of time with it. & -4 & 1 & 0 \\
\hline 33 & It helps me function. & -4 & 2 & 0 \\
\hline 2 & It acts as a witness to creativity. & -5 & 5 & 5 \\
\hline 51 & It's easy to use. & -5 & 5 & -5 \\
\hline
\end{tabular}


I then summarised each factor, using the statements and their associations as my guide. Summarisation can also help the researcher choose a name to describe the factor. I named factor one, sort task two (Table 34.2) "accomplishment aid" because the statements suggest that the participants on this factor share the viewpoint that a possession can act as an aid to accomplishing a task or goal, which happens to correlate with one of Furby's definitions of physical possessions. ${ }^{48}$

After exploring and summarising each factor, I created a table so that the factor interpretations could be visually compared. While this is not necessary to complete a $\mathrm{Q}$ method study, I found it to be a useful way to describe my results (see Table 34.3).

Table 34.3. Summary of All Factors, Sorting Task 2

\begin{tabular}{|c|c|c|c|c|}
\hline \multicolumn{2}{|c|}{ Factor } & Statement summary & Possessions level & Archival level \\
\hline \multirow[t]{6}{*}{1} & \multirow[t]{3}{*}{+} & "Means to an end" & Use to accomplish a goal/task & Primary \\
\hline & & Represents me & Self-extension & Secondary \\
\hline & & Description & Description and use & Primary \\
\hline & \multirow[t]{3}{*}{-} & Description & Description and use & Primary \\
\hline & & "Means to an end" & Use to accomplish a goal/task & Primary \\
\hline & & Attachment & Attachment & Secondary \\
\hline \multirow[t]{8}{*}{2} & \multirow[t]{4}{*}{+} & Represents identity & Self-extension & Secondary \\
\hline & & Description & Description and use & Primary \\
\hline & & Meaning & Self-extension & Secondary \\
\hline & & Use & Description and use & Primary \\
\hline & \multirow[t]{4}{*}{-} & Emotional relationship & Attachment & Secondary \\
\hline & & Evidence & Self-extension & Secondary \\
\hline & & Description & Description and use & Primary \\
\hline & & Purpose for maintaining & Purpose for maintaining & Primary \\
\hline \multirow[t]{4}{*}{3} & \multirow[t]{4}{*}{+} & Personal history & Attachment & Secondary \\
\hline & & Shows identity & Self-extension & Secondary \\
\hline & & "Means to an end" & Use to accomplish a goal/task & Primary \\
\hline & & Purpose for maintaining & Purpose for maintaining & Primary \\
\hline
\end{tabular}

48 Schlinger, “Cues on QTechnique," 56. 


\section{Reflection}

\section{Study Design and Results}

One of the most useful things I did when preparing for the study detailed above was to complete a small pilot study. In the pilot study, I directed participants to sort digital items, rather than statements about digital items. While I was successfully able to produce salient results from the pilot study, problems in the study design became evident. During the sort, participants expressed that they did not have many of the 60 items in the Q sort, especially the participants who were non-adopters of social media. As a result, some of the $\mathrm{Q}$ sort items were placed on the distribution with little care, due to the lack of opinion on these items. It was difficult to design a $\mathrm{Q}$ sample of items that would apply to every subject. In addition, some of the data were difficult to interpret because they were not mutually exclusive, for example, digital photos appeared in more than one Q sample item. Finally, I noticed participants began to grow fatigued after conducting 4 sorts.

Moving forward, I decided to redesign the study to account for these issues. Instead of directing participants to sort actual possessions, I designed the interviews to gather information about participants' understandings of digital possessions, including their definition of the term and personal meaning of their digital possessions. I redesigned the $\mathrm{Q}$ sample as a hybrid design of participants' statements about the characteristics and perceived value of digital possessions and interpretations of participants' characteristics and perceived value of digital possessions from previously published literature. I also redesigned the conditions of instruction to instruct subjects to reflect on one of their digital possessions in order to complete the sort. This addressed the problem of participants sorting possessions they might not have, as they decided which of their own digital possessions to use as a marker as they completed the sorts. I also designed the conditions to address digital possessions that "most reinforce your identity to you," and "display your identity to others."

The pilot study was also useful in helping me to practice good Q methodological procedure. I conducted the pilot study using the paper cards/large table method and learned that this made it difficult to be flexible enough to meet participants' needs (i.e., meeting somewhere other than a designated room with a large table). This led me to the sort program Flash $\mathrm{Q}$, which made data collection more efficient. 
Upon reflection, I am satisfied with my final study and believe that the method allowed me to obtain useful data that I might not have been able to obtain using another method. I also believe my $\mathrm{Q}$ method study and qualitative interviews complemented each other because they allowed me to explore the research questions from different angles, and compare the data, which I believe led to more fruitful results. I learned that a major downside to using Q method is its obscurity. It can be difficult to explain a new method to colleagues at conferences and in article drafts. I often found that other scholars would get caught up in trying to understand the method, or thought that they understood the method, when they actually did not completely comprehend it, which overshadowed my findings. Although this created several hurdles to explaining my research, I do believe that I was able to learn from my repeated need to explain $\mathrm{Q}$ method, which allowed me to eventually explain it more succinctly.

Going forward, I would suggest that anyone who wants to conduct a study using $\mathrm{Q}$ method seek out a qualified Q method researcher for a mentor/ guide. I was extremely fortunate to have worked with such a researcher. She was gracious enough to offer me pointers on how to explain the method and my findings, as well support my choices for rotation and Schlinger's significance factor. ${ }^{49}$ In addition, those attempting to implement $\mathrm{Q}$ method for the first time may wish to sign up for the QMethod listserv (Q-METHOD@ LISTSERV.KENT.EDU). ${ }^{50} \mathrm{Q}$ Method researchers on the list are generally very helpful in their suggestions and welcoming to new researchers.

\section{Criticisms of Q Method}

Criticism of Q method began shortly after Stephenson first introduced it to the scientific community in 1935, due to Stephenson's initial description of $\mathrm{Q}$ factor analysis as an inversion of $\mathrm{R}$ factor analysis. ${ }^{51}$ This description was not completely correct because $\mathrm{Q}$ is not actually an inversion of $\mathrm{R}$. In an $\mathrm{R}$ correlation, the columns of the data matrix represent different units of measurement. For example, column A could represent height and column $\mathrm{B}$ could represent the score from an intelligence test. In a $\mathrm{Q}$ data matrix, the columns represent a common unit of measurement: how important the issue at hand is to the participant. For example, in a $\mathrm{Q}$ matrix, column $\mathrm{A}$

49 Schlinger, "Cues on QTechnique."

50 One can join the listserv at the QMethodology Network website: http://www.lsoft. com/SCRIPTS/WL.EXE?SL1=Q-METHOD\&H=LISTSERV.KENT.EDU

51 McKeon and Thomas, Q Methodology. 
could represent opinions associated with dogs and column B could represent opinions associated with cats. Both columns represent the individual's opinions about an issue: pets. Once researchers stopped describing $\mathrm{Q}$ factor analysis as a simple inversion of $\mathrm{R}$ factor analysis, much of the outright criticism of $\mathrm{Q}$ method ceased, yet comparisons between the two methods are still made. ${ }^{52}$ Lasting criticism of $\mathrm{Q}$ method is identified in the specific steps taken when conducting the method, highlighted below.

In $\mathrm{R}$ factor analysis, the researcher builds meaning into each question or scale in an instrument. Therefore, one can criticise that "you can only ever get back what you put in"; that is to say, results will only reflect the meaning the researcher imbues in the instrument. ${ }^{53}$ However, if a proper Q sample is carefully created to include representations of all know segments involved within a known issue, the researcher should be able to address this concern. The researcher can configure the Q sample items in any manner he sees fit, allowing for a multitude of arrangements. This is therefore less restrictive than are questions in an $\mathrm{R}$ factor analysis. ${ }^{54}$

The forced distribution has faced criticism related to the requirement that participants align the $\mathrm{Q}$ sample cards using a certain design. According to Brown, research has found that individuals tend to naturally establish a midpoint consistent with a normal curve (many items near the midpoint, fewer items the further one extends from the midpoint). ${ }^{55}$ Cottle and McKeown (1980) found that the shape of the distribution does not affect the factors that will emerge from Q factor analysis. ${ }^{56}$

Using theoretical rotation, the original factor loadings matrix can be rotated in order to "make sharper distinctions in the meaning of the factors. ${ }^{" 57}$ When factors appeared with medium loadings, a rotation of the factors could allow for more high and low loading on factors, thus allowing for a more simple interpretation. ${ }^{58}$ While some criticise that rotation allows the researcher to "force" the data to conform to a predetermined

52 McKeon and Thomas, Q Methodology.

53 Amy Hogan, "Users' Metaphoric Interaction with the Internet," Ph.D. dissertation, University of Bath, 2008, 290.

54 Hogan, "Users' Metaphoric Interaction with the Internet."

55 Brown, "A Primer on QMethodology."

56 Charles Cottle and Bruce McKeown, "The Forced-Free Distinction in QTechnique: A Note on Unused Categories in the Q Sort Continuum," Operant Subjectivity 3 no. 2 (1980): 58-63.

57 Sam K. Kachigan, Multivariate Statistical Analysis (New York, NY: Radius Press, 1991), 248.

58 Kachigan, Multivariate Statistical Analysis. 
notion, rotating the matrix in order to force the data is not a proper use of the technique..$^{59} \mathrm{PQMethod} \mathrm{automatically} \mathrm{uses} \mathrm{a} \mathrm{varimax} \mathrm{rotation} \mathrm{to} \mathrm{better}$ focus participants on factors. Varimax is the name of the specific formula used to rotate the data, it is a popular choice among statisticians due to its ability to maximise factor loadings. This is the standard used, except in cases where the researcher would like to explore a hunch. For example, if a researcher would like to highlight the strength between two participants, PQMethod will allow the researcher to input specific rotation directions to focus the two participants, but again this should only be used to explore specific issues. Theoretical rotation simply changes the vantage point from which the data is viewed. Visually, one could imagine the factors laid out on a cross plot graph and rotated in order to shift the frame of reference from which the data is viewed. ${ }^{60}$

Robbins and Krueger assert that $\mathrm{Q}$ method is problematic because the researcher can never fully remove her bias when interpreting the data. ${ }^{61}$ Hogan argues that researchers are able to interpret the factors derived from Q factor analysis without a priori definitions, allowing for removal of the bias that plagues the interpretation of $\mathrm{R}$ factor analysis. ${ }^{62} \mathrm{With}$ an open mind, an interpretation of factors produced from $\mathrm{Q}$ method allows the researcher to discern the association between ideas of individuals in new ways. ${ }^{63}$ Watts and Stenner stated that $Q$ method "makes no claim to have identified viewpoints that are consistent within individuals across time." ${ }^{64}$ As viewpoints can change over time, a $\mathrm{Q}$ method study may not be replicable. This criticism acts as an example of how $\mathrm{Q}$ method has been referred to as "a quantitative approach to qualitative study": When evaluating Q method, the goal is not only validity and reliability as with quantitative study, but also credibility and dependability, a criterion for qualitative study. ${ }^{65}$

59 Hogan, "Users' Metaphoric Interaction with the Internet."

60 McKeown and Thomas, Q Methodology.

61 Robbins and Krueger, "Beyond Boas? The Promise and Limits of Q Method in Human Geography."

62 Hogan, "Users' Metaphoric Interaction with the Internet."

63 Robbins and Krueger, "Beyond Boas? The Promise and Limits of QMethod in Human Geography."

64 Simon Watts and Paul Stenner, "Doing QMethodology: Theory, Method and Interpretation," Qualitative Research in Psychology 2 (2005): 67-91.

65 Juliet Corbin and Anselm Strauss, Basics of Qualitative Research (Los Angeles, CA:

Sage, 2008), 307-9. 


\section{On the Use of Q Method in Archival Studies}

As demonstrated by the study above, $\mathrm{Q}$ method can be used to explore any aspect of opinion and viewpoints on issues. While much of the previous archival research that involves human subjects has utilised purely qualitative methods, Q method is adaptable to answer research questions posed in archival studies quantitatively. An understanding of similar opinions and viewpoints can lead to further research on intent, such as information seeking behaviour, and user studies in archives. For example, a research question that starts: "how do individuals understand ..." could potentially be addressed using Q method. Q method could be of use in archival user studies, understanding how archivists work or even exploring job satisfaction. All these research topics rely on individual understanding and opinions, and are thus suited to $\mathrm{Q}$ method. Exploratory research in LIS with human participants can also be complemented by the use of Q method - before behaviour patterns are established, it can be useful to explore thoughts and opinions that might influence behaviour.

In the study I detailed in this chapter, I describe how I used Q method to explore individuals' opinions about self-extension in the digital environment. This has implications for archival studies because understanding how individuals relate to their digital possessions can be utilised to explore the preservation of personal digital material. Cunningham has written about the importance of the opinion of future donors to archival collections, which was briefly explored in the PARADIGM project. ${ }^{66}$ As this research is exploratory, $\mathrm{Q}$ method is a useful tool to investigate the research topic. As exploratory research moves the field forward in different directions, $\mathrm{Q}$ method should be considered among the many methodological tools available to the archival studies researcher. Q method may have specific potential for use in appraisal research. If archival appraisal can be understood as a subjective process, then $\mathrm{Q}$ method, which measures subjectivity, could be used to explore subtle differences in opinion about understandings of professional archival appraisal methods.

66 Cunningham, "The Archival Management of Personal Records in Electronic Form: Some Suggestions"; Thomas, "PARADIGM: A Practical Approach to the Preservation of Personal Digital Archives." 


\section{Conclusion}

Using Q method, I had the opportunity to learn a lot about a method I had yet to see used in archival studies, and that I could introduce to archival researchers. This process taught me that to use this method, I needed to become somewhat of an expert in order to answer the questions and challenges that come with the introduction of a new method to a field of study. Before my endeavour with $\mathrm{Q}$ method, I viewed methods as a tool I could use to get what I wanted (findings). And typically, methodology was not the area of research in which I liked to dwell, as I viewed the methods process of research as the metaphorical small town one drives through to get to their true destination. My need to become an expert in $\mathrm{Q}$ method caused me to slow down the research process and really understand the method, its abilities, and the pros/cons of utilising the method, and the evolution of the method over time. Learning the details of the evolution and history of the method allowed me to understand why the method existed in its current state, which better helped me explain why it was the best method to use to answer my research question.

Whether the reader chooses to use $\mathrm{Q}$ method or not, I suggest that engaging with a method enough to become well-versed is important when undergoing a significant study. This process can teach the researcher to better understand her research, and research in general.

\section{References}

Belk, Russell W. "Possessions and the Extended Self." Journal of Consumer Research 15

(1988): 139-168.

Belk, Russell W. and Mark Austin. "Organ Donation Willingness as a Function of

Extended Self and Materialism." In Advances in Health Care Research, edited by M.

Venkatesan and Wade Lancaster (Toldeo, OH: Association for Health Care, 19860, 84-88.

Block, Jack. "A Comparison of the Forced and Unforced Q-sorting Procedures."

Educational and Psychological Measurement 16 (1956): 481-493.

Brown, Stephen R. “A Primer on QMethodology.” Operant Subjectivity 16 (1993): 91-138.

Brown, Stephen R. "The History and Principles of QMethodology in Psychology And the

Social Sciences” (unpublished manuscript, 1997), posted at http://facstaff.uww.edu/ cottlec/QArchive/Bps.htm.

Cottle, Charles E., and Bruce McKeown. "The Forced-free Distinction in QTechnique: A

Note on Unused Categories in the Q Sort Continuum." Operant Subjectivity 3 no. 2 (1980): 58-63.

Cunningham, Adrian. “The Archival Management of Personal Records in Electronic Form: Some Suggestions.” Archives and Manuscripts 22 no. 1 (1994): 94-105.

Furby, Lita. "Possession in Humans: An Exploratory Study of Its Meaning and 
Motivation." Social Behavior and Personality 6 no. 1 (1978): 49-65.

Goldman, Irvin. "QMethodology as Process and Context in Interpretivism, Communication and Psychoanalytic Psychotherapy Research.” The Psychological Record 49 (1999): 589-604.

Grix, Jonathan. "QMethodology: Notes from a Project on County Sport Partnerships and Governance.” Leisure Studies 29 no. 4 (2010): 457-467.

Hogan, Amy Louise. "Users' Metaphoric Interaction with the Internet." University of Bath, 2008.

Kachigan, Sam Kash. Multivariate Statistical Analysis. Second edition. New York, NY: Radius Press, 1991.

Kerlinger, Fred N. Foundations of Behavioral Research. 3rd ed. Fort Worth, Texas: Holt, Rinehart and Winston, Inc., 1986.

Kleine, Susan and Stacey Baker, "An Integrative Review of Material Possession Attachment." Academy of Marketing Science Review 1(2004) accessed 6 August 2013 at http://www.amsreview.org/articles/kleine01-2004.pdf

Kleine, Susan Schultz, Robert Kleine, and Chris T. Allen. "How Is a Possession 'Me' or 'Not Me'? Characterizing Types and an Antecedent of Material Possession Attachment." Journal of Consumer Research 22 (December 1995): 327-343.

Marshall, Catherine C. "Rethinking Personal Digital Archiving Part I: Four Challenges from the Field." D-Lib Magazine, 2008. http://www.dlib.org/dlib/ march08/marshall/03marshall-pt1.html

Marshall, Catherine C. "Rethinking Personal Digital Archiving Part II: Implications for Services, Applications and Institutions.” D-Lib Magazine, 2008. http://www. dlib.org/dlib/march08/marshall/03marshall-pt2.html

Marshall, Catherine C. "How People Manage Information over a Lifetime.” In Personal Information Management, 57-75. Seattle, Washington: University of Washington Press, 2007.

Marshall, Catherine C., S. Bly, and F. Brun-Cottan. "The Long Term Fate of Our Digital Belongings: Toward a Service Model for Personal Archives.” In Proceedings of Archiving 2006 (Ottawa, Canada: Society for Imaging Science and Technology, 2006), 25-30.

McKeown, Bruce, and Dan Thomas. Q Methodology. Quantitative Applications in the Social Sciences 66. Newbury Park: Sage, 1988.

Morgan, Amy. "The Evolving Self in Consumer Behavior: Exploring Possible Selves." Advances in Consumer Research 20 (1993): 429-432.

Patrick, Vanessa M., Deborah J. MacInnis, and Valerie S. Folkes. "Approaching What We Hope for and Avoiding What We Fear: The Role of Possible Selves in Consumer Behavior." Advances in Consumer Research 29 (2002): 270-276.

Prelinger, Ernst. "Extension and Structure of the Self." Journal of Psychology 47 (1959): 13-23.

Robbins, Paul, and Rob Krueger. "Beyond Boas? The Promise and Limits of QMethod in Human Geography.” Professional Geographer 52 no. 4 (2000): 636-648.

Schultz, Susan E. "An Empirical Investigation of Person-material Possession Attachment." University of Cincinnati, 1989.

Sivadas, Eugene, and Karen Machleit. "A Scale to Determine the Extent of Object Incorporation in the Extended Self." In Marketing Theory and Applications, 5 (St. Petersburg, FL: American Marketing Association, 1994), 143-149.

Sivadas, Eugene, and Ravi Venkatesh. "An Examination of Individual and Objectspecific Influences on the Extended Self and Its Relation to Attachment and Satisfaction." Advances in Consumer Research 22 (1995): 406-412. 
Stephenson Research Center, and R. Logan. Workshop of Q Methodology by Steven Brown, Chapters 1-8. Videorecording, 1996. http://www.qmethod.org/howto.

Stephenson, William. "Correlating Persons Instead of Tests." Character and Personality 4 (1935): 17-24.

Stephenson, William. The Study of Behavior; Q-technique and Its Methodology. Chicago: University of Chicago Press, 1953.

Thomas, Susan. PARADIGM: A Practical Approach to the Preservation of Personal Digital Archives. Manchester, UK: Bodleian Library, 2007.

Watts, Simon, and Paul Stenner. "Doing QMethodology: Theory, Method and Interpretation." Qualitative Research in Psychology 2 (2005): 67-91. doi:10.1191/1478088705qp022oa. 


\section{Part V}

\section{Biographical Notes on Contributors}

Amelia Acker is an assistant professor at the University of Texas at Austin in the School of Information. She holds a Ph.D. in Information Studies and an MLIS with a specialisation in archival studies from the University of California, Los Angeles. Dr. Acker's research interests include information infrastructure studies, archival science, and data studies, specifically the material production and transmission of information objects in wireless communication systems over time. Before joining the University of Texas, Dr. Acker served as the lead faculty of the Archives and Information Science specialisation at the University of Pittsburgh.

Kimberly Anderson is digital initiatives archivist at Iowa State University. Prior to returning to practice, she was an assistant professor and director of the Archival Studies Program in the University of Wisconsin, Milwaukee's School of Information Studies. She earned her doctorate in Information Studies and her MLIS with a specialisation in archival studies at the University of California, Los Angeles. Her research interests are influenced by her practice - previous and current - in university archives, special collections, police records and law libraries. She studies intersections between archival appraisal, the sociocultural aspects of recordkeeping, and archival education and learning. 
Anjali Arondekar is an associate professor of Feminist Studies at the University of California, Santa Cruz. Her research engages the poetics and politics of sexuality, colonialism and historiography. She is the author of For the Record: On Sexuality and the Colonial Archive in India (Duke University Press, 2009), winner of the Alan Bray Memorial Book Award for best book in lesbian, gay, or queer studies in literature and cultural studies, Modern Language Association, 2010. Her second book-project, Abundance: On Sexuality and Historiography (forthcoming from Duke University Press), grows out of her interest in the figurations of sexuality, ethics and collectivity in colonial British and Portuguese India.

Jeannette Allis Bastian is a professor at the Graduate School of Library and Information Science, Simmons College, Boston, Massachusetts where she directs their archives education programme. She was the Territorial Librarian and Archivist of the United States Virgin Islands from 1987 to 1998 and received her Ph.D. from the University of Pittsburgh in 1999. Her research interests and writings are in the areas of post-colonialism, collective memory and archival education. Her publications include West Indian Literature: An Index to Criticism, 1930-1975 (J. Allis, 1981), Owning Memory, How a Caribbean Community Lost Its Archives and Found Its History (2003), Archival Internships: A Guide for Faculty, Supervisors, and Students (2008 with Donna Webber), and an edited volume with Ben Alexander, Community Archives, The Shaping of Memory (2009).

Joel A. Blanco-Rivera is an assistant professor at the University of Puerto Rico Graduate School of Information Sciences and Technologies, where he teaches courses on archives management and digital libraries. His research interests are archives and transitional justice in Latin America, freedom of information, and social memory.

JENNy Bunn is a lecturer on the archives and records management programme at University College London, where she has recently completed a $\mathrm{PhD}$ in Archival Studies. Prior to embarking on her $\mathrm{PhD}$ she worked extensively in a number of archival institutions including The National Archives, the Victoria and Albert (V\&A) Museum and The Royal Bank of Scotland Group Archives. 
Michelle Caswell, PhD, is an assistant professor in the department of information studies at the University of California, Los Angeles. She is the author of the book Archiving the Unspeakable: Silence, Memory, and the Photographic Record in Cambodia (University of Wisconsin Press, 2014) and the guest editor of a special double issue of Archival Science on archives and human rights. She is also the co-founder and a board member of the South Asian American Digital Archive (http://www.saada.org).

Kate Cumming works in the Government Recordkeeping programme of the State Records Authority of New South Wales where her main areas of responsibility concern digital recordkeeping. She holds a $\mathrm{PhD}$ from Monash University. Her dissertation examined the roles and purposes of recordkeeping metadata. She has a long-standing involvement with the Standards Australia Records Management Committee and has been on the editorial board of the Records Management Journal since 2004.

Amber L. Cushing is a lecturer at the School of Information and Communication Studies at University College Dublin, where she also serves as Director of the PhD programme and Director of the Msc in Digital Curation programme. Her research interests explore the intersection of personal information management, digital curation and cultural heritage. Cushing holds a PhD in Information Science from the University of North Carolina at Chapel Hill, an MS in Library and Information Science from Simmons Graduate School of Library and Information Science with a concentration in Archives Management, and a BA in History from Mount Holyoke College.

Lorraine Dong earned her Ph.D. from the University of Texas at Austin's School of Information. She is currently a post-doctoral scholar for a Mellonfunded project to develop a digital archives model and a corresponding set of privacy and access recommendations for historical mental institution records. Lorraine was a recipient of the Institute of Museum \& Library Services Doctoral Preservation Fellowship, 2008-2012; the William Powers, Jr. Graduate Fellowship, 2012-2013; and the Beta Phi Mu Eugene Garfield Doctoral Dissertation Fellowship, 2014-2015. Her research interests include the social ecologies of health records, community archives-creation, and international preservation collaborations. 
Wendy M. Duff is a professor at the University of Toronto School of Information. She obtained her Ph.D. (1996) from the University of Pittsburgh where she also served as the project coordinator for the Pittsburgh Project. Her dissertation "The Influence of Literary Warrant on the Acceptance and Credibility of the Functional Requirements for Recordkeeping" was the first investigation of power of warrant in the archival literature. She is currently the Director of the Digital Curation Institute, and teaches archives and records management with a focus on access to archival materials. She is a founding member of AX-SNet, an evolving international team of researchers interested in facilitating access to primary materials. Her current research focuses on archival users, access to archival material, digital curation, and the convergence of libraries, archives and museum.

Anthony W. Dunbar (Tony) is a diversity expert and consultant, critical theorist, interdisciplinary social scientist, activist/community organiser, educator, and media production enthusiast. His education includes a Ph.D. in Information Studies and a Master's in Library and Information Science; and Master Degrees in Education and Communication and Training. He has also been engaged in multiple community based activities such as volunteer coordinator for the Pan-African Film \& Arts Festival, mentorship coordinator for the Los Angeles Urban League, volunteer work in Los Angeles public libraries, civil rights projects in Chicago, as well as supporting various campus student groups as those groups address issues that affect students of colour.

Luciana Duranti is chair and professor of Archival Studies at the University of British Columbia (UBC). She is Director of the UBC Centre for the International Study of Contemporary Records and Archives (CISCRA) and of several research projects about the authenticity and preservation of digital records, including four phases of InterPARES (International research on Permanent Authentic Records in Electronic Systems), Digital Records Forensics, and Records in the Cloud. She is also co-Director of the research project on the Law of Evidence in the Digital Environment.

Joanne Evans is an ARC Future Fellow at the Centre for Organisational and Social Informatics, Faculty of Information Technology, Monash University. She has many years of experience in archival systems development, 
and the technologies she has been involved in designing and developing have been deployed in a number of research projects as well as in small archives settings. She undertook her doctoral research at Monash University from 2003-2007 as part of the ARC Linkage-funded Clever Recordkeeping Metadata Project, and received a Vice Chancellor's Commendation for Doctoral Thesis Excellence in 2008. Her research interests lie in the multifarious roles metadata plays in creating, managing, and sustaining information infrastructure and systems, and in her 2015-2018 Future Fellowship research program is exploring the development of a participatory archival design methodology.

Shannon Faulkhead is a Koorie woman from Victoria Australia, and her Ph.D. thesis from Monash University was titled "Narratives of Koorie Victoria". Shannon's research concentrates on the location of Koorie peoples and their knowledge within the broader Australian society and its collective knowledge as reflected through narratives and records. While Shannon's research is multi-disciplinary in nature, to date it has centred on community and archival collections of records, and has been situated within the disciplines of Indigenous studies and archival science. Shannon's appointment as the Finkel Fellow, attached to the Monash Country Lines Archive, allows for greater exploration and development in the area of Indigenous ways of archiving.

Jonathan Furner is chair and professor of Information Studies at the University of California, Los Angeles (UCLA), and a faculty affiliate of UCLA's Center for Digital Humanities. He studies the history and philosophy of cultural stewardship, and teaches classes on the representation and organisation of archival records, library materials, and museum objects. $\mathrm{He}$ has published over fifty papers on these and related topics, frequently using conceptual analysis to evaluate the theoretical frameworks, data models, and metadata standards on which information access systems rely.

Leisa Gibbons is an assistant professor in cultural heritage informatics at Kent State University. Leisa's research specialisations include theoretical explorations of continuum informatics, the records continuum model, archival methodologies and research design, and use of social media and emergent technologies for memory-making. Leisa's $\mathrm{Ph} . \mathrm{D}$. was completed 
at Monash University and examined how continuum models can be used to design research, and in doing so explored YouTube from a continuum perspective as an emergent archival space. Leisa has had extensive experience as a recordkeeping and archives consultant working with community organisations in the not-for-profit sector in Australia.

Anne J. Gilliland is professor and director of the Archival Studies specialisation in the Department of Information Studies, as well as director of the Center for Information as Evidence, Graduate School of Education \& Information Studies at the University of California Los Angeles (UCLA). She is a faculty affiliate of UCLA's Center for Digital Humanities. She is also the director of the Archival Education and Research Initiative (AERI), a consortium dedicated to the advancement of education and research in archival studies, broadly defined, and the development of a global scholarly community in archival studies. Her recent work has been addressing recordkeeping and archival systems and practices in support of human rights and daily life in post-conflict settings; the role of community memory in promoting reconciliation in the wake of ethnic conflict; bureaucratic violence and the politics of metadata; digital recordkeeping and archival informatics; and research methods and design in archival studies. She is a fellow of the Society of American Archivists.

Karen F. Gracy is an associate professor in Library and Information Science at Kent State University. She possesses an MLIS and Ph.D. in Library and Information Science from the University of California, Los Angeles and an M.A. in critical studies of Film and Television from UCLA. Recent publications have appeared in JASIST, Archival Science, American Archivist, Journal of Library Metadata, and Information and Culture. Dr. Gracy's scholarly interests are found within the domain of cultural heritage stewardship, which encompasses a broad range of activities such as preservation and conservation processes and practices, digital curation activities that consider the roles of heritage professionals and users in the lifecycle of objects and records, as well as knowledge representation activities such as definitions of knowledge domains, development of standards for description, and application of new technologies to improve access to cultural heritage objects. She teaches in the areas of preservation and archiving, with a focus on moving image archives and digital preservation issues. 
Randolph C. Head is professor of History at the University of California, Riverside, where he specialises in the history of early modern Europe and its institutional cultures. He is the author of several books on the history of Switzerland and Swiss political culture, and recently edited a collection of articles about European archives, "Archival Knowledge Cultures in Europe, 1400-1900", which appeared as a special issue of the journal Archival Science. Professor Head is currently writing a comparative study of early modern archives in Europe, which he hopes to follow with a collaborative comparative study of pre-modern archiving practices around the world.

Perka Hentronen is Adjunct Professor in the School of Information Sciences in the University of Tampere (Finland) and has a Ph.D. in information studies. His special field is electronic records management. Henttonen has published research about requirements for electronic records management systems, metadata, knowledge organisation, and Finnish archival history. Before his university career, Henttonen worked in the National Archives and Military Archives of Finland. Besides scholarly publications, he regularly writes for professional journals and is a frequent speaker at national and international conferences.

Hans Hofman has recently retired as senior advisor on digital longevity at the Nationaal Archief of the Netherlands. He has been involved in several programmes in the area of e-government with respect to recordkeeping, metadata, digital preservation and open standards. At the International level he represents since 2000 the Netherlands (and the National Archives) in ISO TC46/SC11 on Records and Archives Management, in which committee he is chairing the Working Group on Records Metadata as well as the Working Group on risk management related to records systems. He acts also as the liaison between ISO and the International Council on Archives (ICA), he is a member of the Programme Committee of the ICA and he is a member of the review group for MoReq2010.

Eric Ketelaar is Professor Emeritus at the University of Amsterdam. From 1997 to 2009 he was Professor of Archivistics in the Department of Media Studies of the University of Amsterdam. As an honorary fellow of his former department he continues his research, which is concerned mainly with the social and cultural contexts of records creation and use. Eric Ketelaar 
was General State Archivist (National Archivist) of The Netherlands from 1989-1997 and held the archivistics chair in the Department of History, University of Leiden, 1992-2002. He was visiting professor at the University of Michigan (Ann Arbor), Gakushuin University (Tokyo), the University of Toronto and Monash University (Melbourne), where he continues to be involved as a Senior Research Fellow in Monash's Centre for Organisational and Social Informatics. He was one of the three founding editors-in-chief of Archival Science.

Andrew J LaU is the Program Director for Instructional Content Development at UCLA Extension and lecturer at the University of Maryland College of Information Studies. He received his Ph.D. in Information Studies and his Master's degree in Library and Information Science with a specialisation in archival studies from the University of California, Los Angeles. Dr. Lau's research interests include documentation practices in contemporary art, community archives, and postcolonial archives and recordkeeping. In addition, his interests include research and practice in the areas of information technology, instructional design, and online pedagogies in continuing and professional educational settings.

Jamie A. Lee, Assistant Professor of Digital Culture, Information and Society in the School of Information at the University of Arizona, attends to storytelling, archives, multimodal media-making contexts, bodies, and onging analyses of the ways humans know and move in the world. Her work is intricately woven through the intersections of archival studies, media studies, digital and visual culture, information, society, and the body. Studying her hands-on archival work through archival theory and practice that emerges from community contexts, she engages theories of affect and embodiment, archival and queer theories, somatechnics, and decolonising methodologies. Lee has developed a Queer/ed Archival Methodology, Q/M, for archivists to utilise in new and existing archival productions to offer a way of thinking critically about archival practices and productions as radically open, productive, and generative spaces that many multiply-situated bodies might fit into, even complicatedly. Since 1991, Lee has worked in film/video/TV and has owned and operated a multimedia production firm. As an award-winning social justice filmmaker, Lee's work has screened on PBS, Free Speech TV, and at film festivals and conferences throughout North America and Europe. She presented at the 2008 Women's World Congress in Madrid, Spain and was the 
keynote speaker at the 2009 New Directions in Critical Theory Conference. She is the recipient of the 2009 Arizona Commission on the Arts Artist Project Grant and a 2010 Arizona Governors Arts Award nominee for her work connecting her artform to community activism.

Zhiying Lian is a professor in the School of Library, Information and Archival Studies at Shanghai University of China. She earned her Ph.D. in Archival Science from Renmin University of China. From August 2012 to August 2013, she was a visiting scholar at the University of California, Los Angeles. Her research interests focus on social media and archives services, the organisational culture of archives, right of access to government electronic records and community archives.

David Luyombya holds a Ph.D. in archives studies from University College London (2010). He is Chair of the Department of Records and Archives Management at the East African School of Library and Information Science, College of Computing and Information Science, Makerere University, Uganda. His thesis "Framework for Effective Public Digital Records Management in Uganda" was the first archival graduate study in Uganda. He is also the chair of the Standards Committee of the Uganda Records and Archivists Association. He served as National Coordinator for the IDRCfunded Aligning Records Management with ICT, e-Government and Freedom of Information in East Africa research project (2010). He has also established records management systems for a number of institutions in Uganda including Bank of Uganda, Shell Uganda Limited, DFCU Bank and the Uganda Population Secretariat. His works appear in Comma, ESARBICA Journal, Records Management Journal and Information Development Journal.

Heather MacNeil is a professor in the Faculty of Information at the University of Toronto where she teaches courses on archival concepts and issues, the arrangement and description of archival documents, and the trustworthiness of records from different disciplinary perspectives. Her research and publications focus on the historical and theoretical foundations of the archival discipline, the links between the archival discipline and other humanities disciplines concerned with the representation and preservation of texts and documents, and the impact of changing social cultures 
and technological environments on our understanding of authenticity and trust. She is a fellow of the Association of Canadian Archivists.

Sue McKemmish is the Chair of Archival Systems and Director of the Centre for Organisational and Social Informatics at Monash University in Australia. Her research relates to the records continuum, the use of metadata in recordkeeping and archival systems, social justice and human rights issues as they relate to archives and records, community archives, Australian Indigenous archives, and the development of more inclusive archival educational programmes to meet the needs of diverse communities. She is a Laureate of the Australian Society of Archivists.

Giovanni Michetti is an assistant professor of Archival Studies at Sapienza University of Rome. His research interests include records management, archival description and digital preservation. He has been involved in national and international initiatives on digital preservation (including ERPANET, CASPAR and InterPARES). He works actively in the standardisation area: as the Chair of the committee "Archives and Records Management" of the Italian Standards Organisation (UNI) and the Italian representative in ISO TC46/SC11, he has contributed to the development of both national and international standards in the field.

Joy R. Novak earned her M.A. in Public History at the University of California, Riverside and Ph.D. in Information Studies at the University of California Los Angeles, concentrating her research on archival activism. Throughout her career, she has worked in a variety of archival settings including a state historical society, special collections, public libraries and community-based archives. From 2007-2015, she was the Collections Manager for the Center for the Study of Political Graphics in Culver City, California. She is currently the Head of Collection Management and Outreach for Special Collections at Washington University in St. Louis.

Alex H. Poole is Assistant Professor at Drexel University's College of Computing and Informatics. He received his $\mathrm{PhD}$ from the University of North Carolina at Chapel Hill. Poole's research interests centre on archives and records management, digital curation, digital humanities, pedagogy, and 
diversity and inclusivity. His work has been published in Digital Humanities Quarterly, The American Archivist, and Archival Science and is forthcoming in The Journal of Documentation and in The Journal of the Association for Information Science and Technology. He received the Theodore Calvin Pease Award from the Society of American Archivists for his article "The Strange Career of Jim Crow Archives: Race, Space, and History in the Mid-Twentieth-Century American South." Poole earned a BA from Williams College (Highest Honours, History), an MA from Brown University (History), and an MLS (Beta Phi Mu) from the University of North Carolina at Chapel Hill.

Sarah Ramdeen is a doctoral candidate at the School of Information and Library Science at the University of North Carolina in Chapel Hill. Her research interests include information seeking behaviour of geologists when they are searching for physical sample materials, and the stewardship of geological data. Sarah has a B.S. in Geology and a M.S. in Library and Information Studies, both from Florida State University. She was the Project Manager for the ILMS funded ELIME-21 programme and a Research Data Alliance/US Fellow. She is currently a research assistant on a UNC climate study on increasing the representation and advancement of women in academic science and engineering careers.

Joanna STEele has an MLIS from the University of California, Los Angeles, and an MA in Information from the University of Michigan, Ann Arbor. She was a recipient of the IMLS-sponsored doctoral fellowship "Building the Future of Archival Education and Research." Her research examined how non-governmental organisations use satellite technologies to monitor human rights violations.

Anneli Sundevist, B.A., M.A., Ph.D. is associate professor in archivistics at Oslo and Akershus University College of Applied Sciences. Her teaching areas include archival theory, archival description, information access, and theory and methodology. Her research interests are information seeking, use and access, information representation and material aspects of records. At present she is working with a regional research project about digital archiving in private organisations, and she is a member of the InterPARES Trust-team. She has previously worked as a professional archivist. She is a member of the editorial board of the Swedish scholarly journal Arkiv, Sambälle E Forskning 
and of the committee of Näringslivsarkiv i Norrland (Business Archives Norrland).

Kirsten Thorpe is a descendant of the Worimi people of Port Stephens, New South Wales (NSW), Australia. Kirsten holds a Masters of Information Management and Systems (Professional) from Monash University, which included a Minor Thesis titled "Creating an Aboriginal Community Archive in New South Wales". Kirsten's professional and research interests relate to the return of archival sources of material to Aboriginal and Torres Strait Islander communities. She is also interested in the opportunities that the digital domain presents for Aboriginal and Torres Strait Islander communities to be actively involved in managing their cultural heritage resources. Kirsten is the Manager, Indigenous Services at the State Library of New South Wales, Australia. Kirsten is the Manager, Indigenous Services, Division of the Mitchell Librarian and Education \& Scholarship, State Library of New South Wales.

Frank Upward is a former senior lecturer at Monash University where he delivered courses relating to archival management, document and records management, information management and information systems. He is currently a principal researcher at their Centre for Social and Organisational Informatics and co-director of the Recordkeeping Institute.

Kelvin White is an associate professor in the School of Library and Information Studies and African and African American Studies as well as Associate Dean of Faculty and Community for the College of Arts and Sciences at the University of Oklahoma (Norman). Using social justice as a framework, his work examines the interconnections between the social, cultural, and historical contexts in which archival and recordkeeping activities exist and the implications they have for marginalised or underrepresented communities.

Eunha (Anna) Youn is an assistant professor at Chonbuk National University in Korea. Her research focuses on culture, society and archival technology, and especially in the effects of cultural elements on the archival standards. She is currently interested in the role of archives in the community building movement of the city of Seoul and also researches the implications of FOIA on public records management. 


\section{Research in the Archival Multiverse}

\section{Edited by Anne J Gilliland, Sue McKemmish and Andrew J Lau}

Within the past 15 years, the field of archival studies around the world has experienced unprecedented growth, both within the academy and the profession, and archival studies graduate education programs today have among the highest enrolments in any information field. During the same period, there has also been unparalleled expansion and innovation in the diversity of methods and theories being applied in archival scholarship. Global in scope, Research in the Archival Multiverse compiles critical and reflective essays across a wide range of emerging research areas and interests in archival studies with the aim of providing current and future archival academics with a text addressing possible methods and theoretical frameworks that have been and might be used in archival scholarship. More than a collation of research methods for handy reference, this volume advocates for reflexive research practice as a means by which to lay bare the fuzziness and messiness of research. Whereas research in the form of published research papers and juried conference presentations provides a view of the study framed in terms of research questions and findings, reflexive research practice reveals the context of the study and chains of situations, choices, and decisions that influence the trajectories of the studies themselves. Such elucidations from the position of the researcher are instructive for others, who may be inspired to apply or adapt the method for their own research.

Anne J Gilliland is Professor and Director, Archival Studies specialisation, Department of Information Studies, and Director, Center for Information as Evidence, Graduate School of Education \& Information Studies, University of California Los Angeles (UCLA).

Sue McKemmish is Chair of Archival Systems and Director, Centre for Organisational and Social Informatics, Monash University.

Andrew J Lau is Program Director for Instructional Content Development, UCLA Extension, and Lecturer, the University of Maryland College of Information Studies.

MONASH University Publishing
ISBN: 978-1-876924-67-6 (pb)

ISBN: 978-1-876924-70-6 (PDF)

ISBN: 978-1-925377-68-2 (ePub)

ISBN: 978-1-925377-69-9 (Knowledge Unlatched open access PDF) 